Prepared in cooperation with the city of Santa Barbara

\title{
Santa Barbara and Foothill Groundwater Basins Geohydrology and Optimal Water Resources Management- Developed using Density Dependent Solute Transport and Optimization Models
}
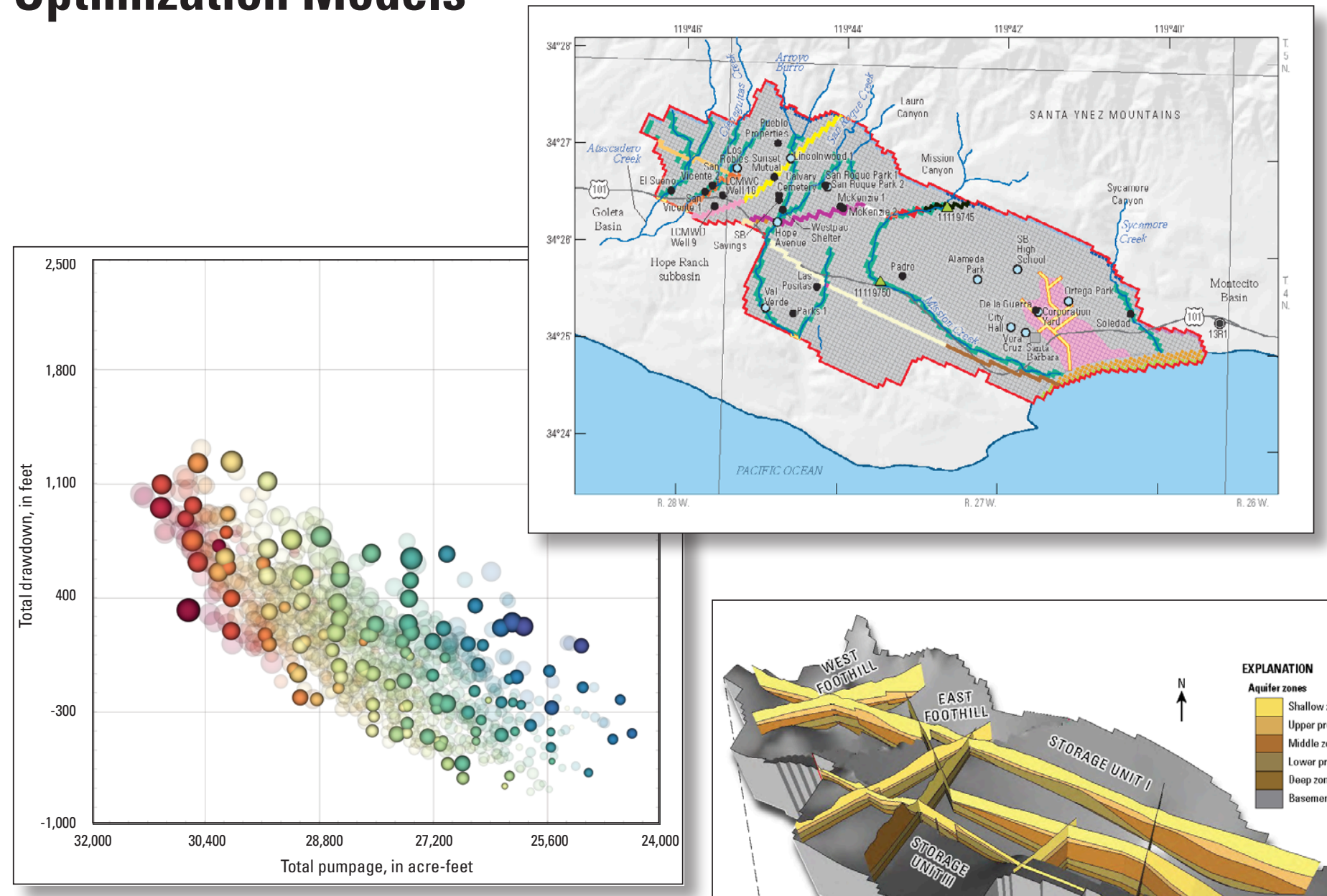

Scientific Investigation Report 2018-5059

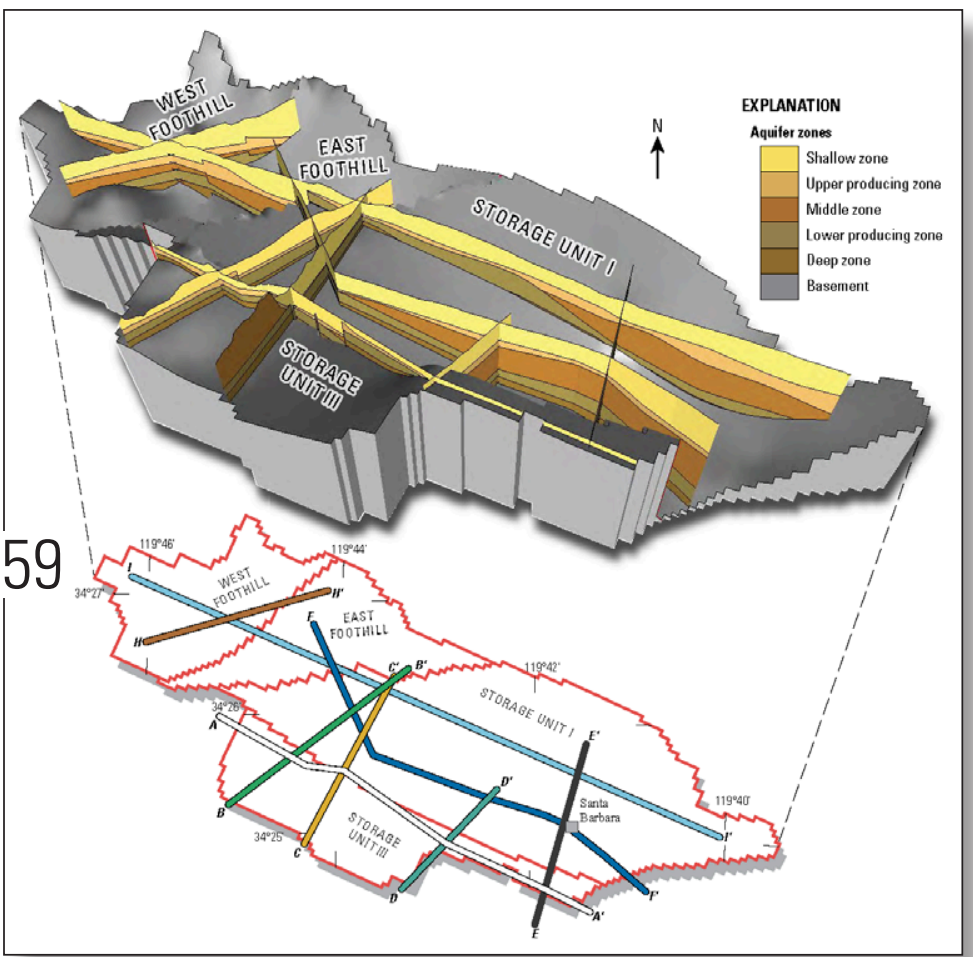



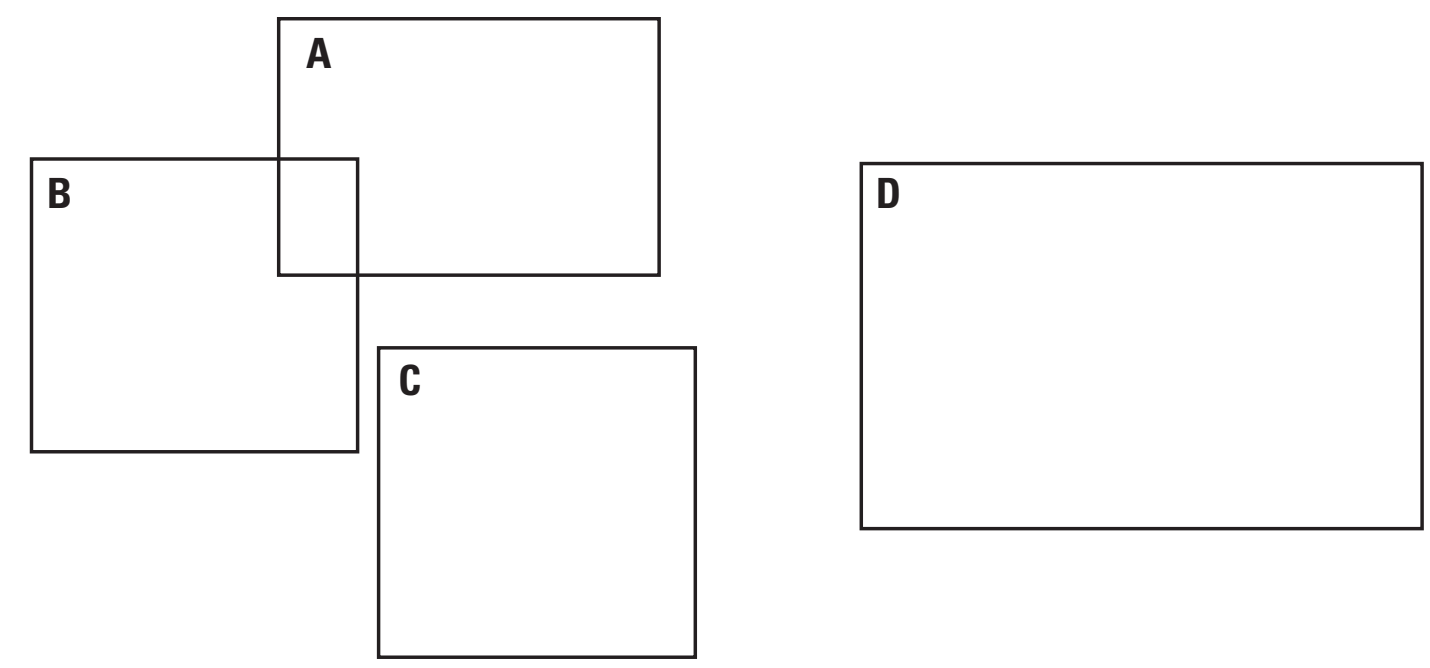

\section{Cover photographs:}

Front cover: $A$, Distribution of flow barriers, general-head boundaries, drains, small-catchment recharge, and creek recharge in the Santa Barbara flow and transport model, Santa Barbara, California.

$B$, Santa Barbara multi-object management model, Santa Barbara, California.

$C$, Hydrogeologic framework model results by fence diagram, Santa Barbara and Foothill groundwater basins, Santa Barbara, California: $A$, geometric framework model showing subsurface extent of the five aquifer zones, and $B$, texture model showing the distribution of coarse- and fine-grained material in each aquifer zone, with percentcoarse intervals for drillers' well logs and geophysical e-logs (locations of all logs are also shown on the inset diagram). Fence diagrams shown with five times vertical exaggeration.

Back cover: D, Photograph showing mission reflection in pond (Photograph taken by Claudia C. Faunt, U.S. Geological Survey). 


\section{Santa Barbara and Foothill Groundwater Basins Geohydrology and Optimal Water Resources Management-Developed using Density Dependent Solute Transport and Optimization Models}

Edited by Tracy Nishikawa

Chapter A

Introduction and Overview of Geology and Hydrogeology

By Scott R. Paulinski and Tracy Nishikawa

Chapter B

Overview of Hydrogeologic Framework Model

By Geoffrey Cromwell and Scott E. Boyce

Chapter C

Numerical Model of Groundwater Flow and Solute Transport

By Scott R. Paulinski

Chapter D

Multi-Objective Simulation-0ptimization Model

By Zachary P. Stanko and Tracy Nishikawa

Prepared in cooperation with the city of Santa Barbara

Scientific Investigations Report 2018-5059 


\section{U.S. Department of the Interior RYAN K. ZINKE, Secretary}

\section{U.S. Geological Survey James F. Reilly II, Director}

\section{U.S. Geological Survey, Reston, Virginia: 2018}

For more information on the USGS - the Federal source for science about the Earth, its natural and living resources, natural hazards, and the environment-visit https://www.usgs.gov or call 1-888-ASK-USGS.

For an overview of USGS information products, including maps, imagery, and publications, visit https://store.usgs.gov.

Any use of trade, firm, or product names is for descriptive purposes only and does not imply endorsement by the U.S. Government.

Although this information product, for the most part, is in the public domain, it also may contain copyrighted materials as noted in the text. Permission to reproduce copyrighted items must be secured from the copyright owner.

Suggested citation:

Nishikawa, T., ed., 2018, Santa Barbara and Foothill groundwater basins Geohydrology and optimal water resources management-Developed using density dependent solute transport and optimization models, U.S. Geological Survey, Scientific Investigations Report 2018-5059, 4 chap. (A-D), variously paged, https://doi.org/10.3133/sir20185059. 


\section{Contents}

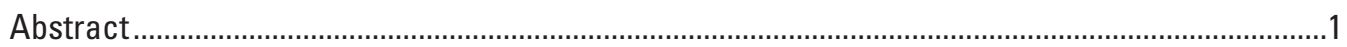

Chapter A: Introduction and Overview of Geology and Hydrogeology ..........................................

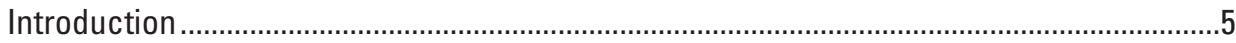

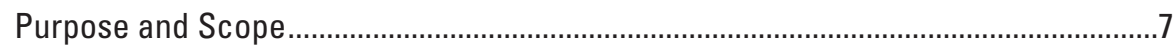

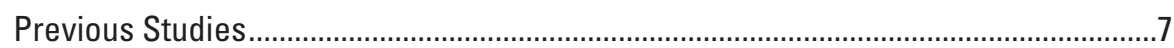

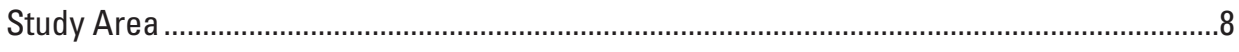

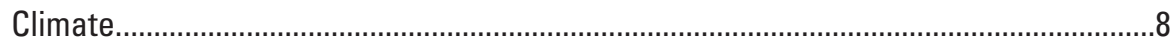

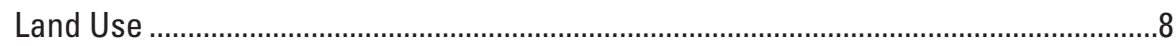

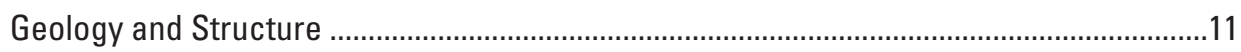

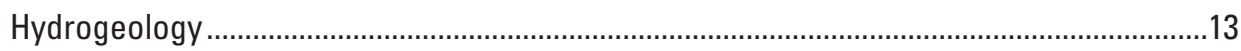

Description of the Aquifer System .............................................................................13

Faults and Groundwater Flow ............................................................................13

Hydrogeologic Zones...................................................................................... 13

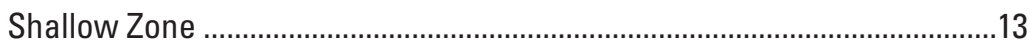

Upper Producing Zone ............................................................................13

Middle Zone ..........................................................................................13

Lower Producing Zone ..........................................................................19

Deep Zone ............................................................................................19

Pre-Development Recharge and Discharge............................................................19

Post-Development Effects on Groundwater System..................................................20

Pre-Development Groundwater Movement .................................................................20

Post-Development Groundwater Levels and Movement .............................................23

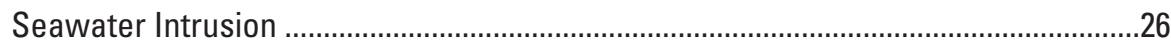

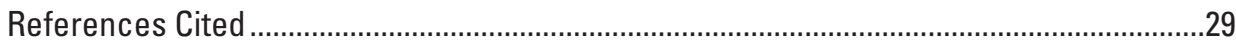

Chapter B: Overview of Hydrogeologic Framework Model ..........................................................

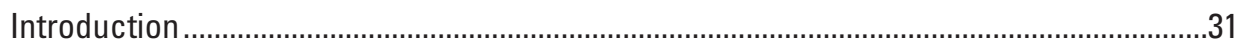

Hydrogeology and Structure of the Santa Barbara Study Area.......................................31

Construction of the Hydrogeologic Framework Model.....................................................34

Geometric Framework ..........................................................................................

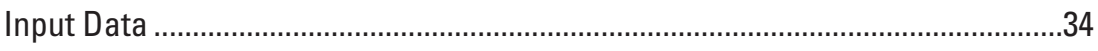

Structures and Aquifer Zones .........................................................................

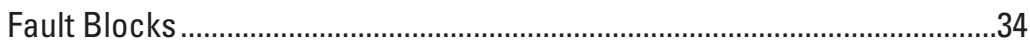

Aquifer-Zone and Bedrock-Horizon Modeling ............................................36

Geometric Framework Model Description............................................................36

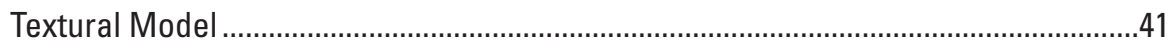

Textural Classification of Borehole Data.............................................................42

Textural Property Modeling ...................................................................................4

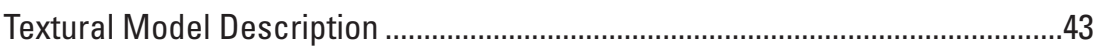

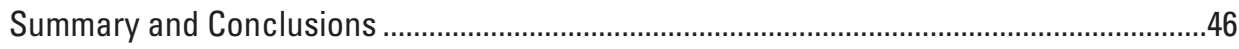

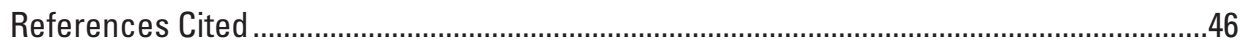

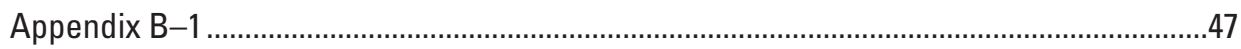




\section{Contents-Continued}

Chapter C: Numerical Model of Groundwater Flow and Solute Transport ....................................119

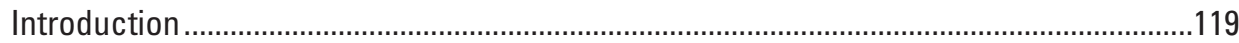

Groundwater Model Development..................................................................................119

Spatial and Temporal Discretization ........................................................................119

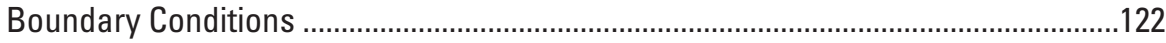

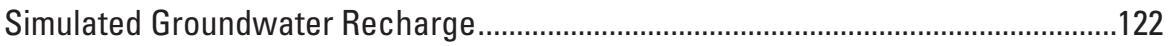

Simulated Groundwater Discharge ........................................................................124

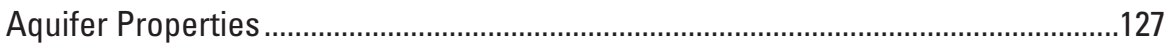

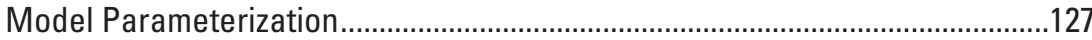

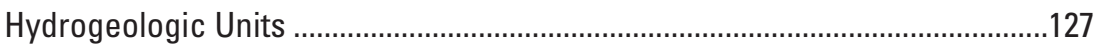

Hydraulic and Transport Properties ................................................................131

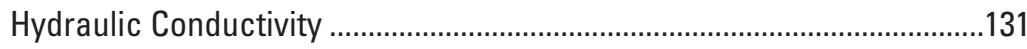

Conductance Properties ..........................................................................132

Creek Properties.................................................................................132

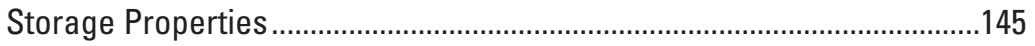

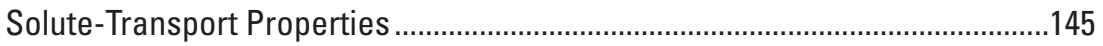

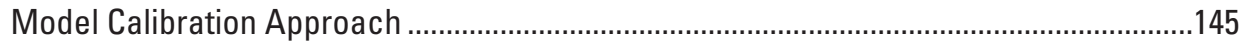

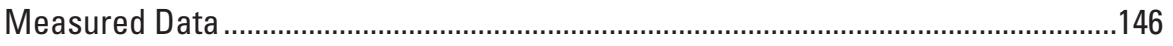

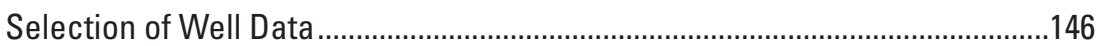

Water-Level Data............................................................................................146

Chloride-Concentration Data ...................................................................146

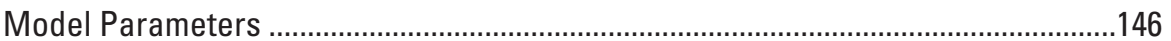

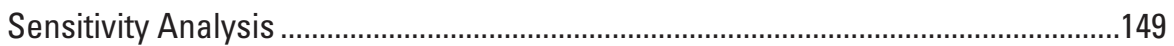

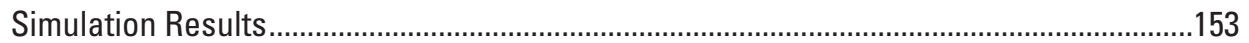

Groundwater Budget .....................................................................................153

Simulated Groundwater Flow and Movement..........................................................161

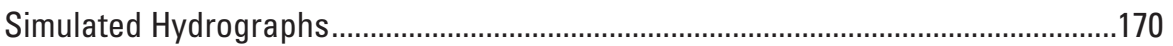

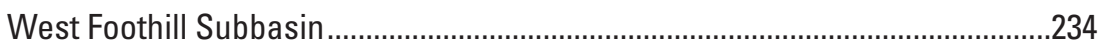

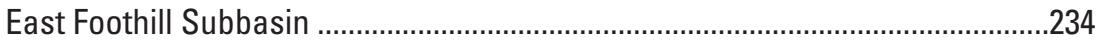

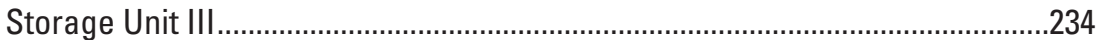

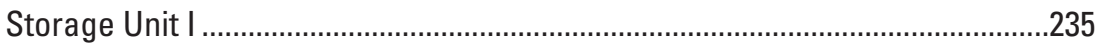

Water-Level Data Fit .........................................................................................236

Simulated Chloride Concentrations and Model Data Fit ..............................................237

Model Limitations and Appropriate Use......................................................................246

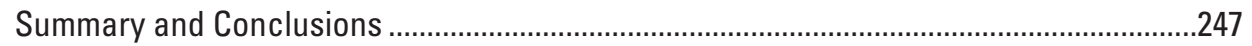

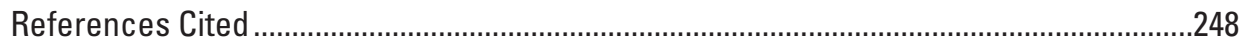

Chapter D: Multi-Objective Simulation-Optimization Model ........................................................251

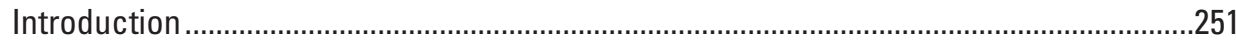

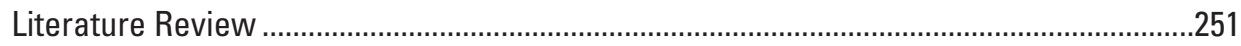

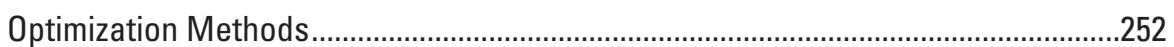

Groundwater-Management Models ....................................................................253

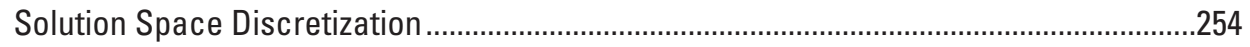




\section{Contents-Continued}

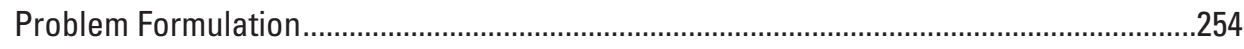

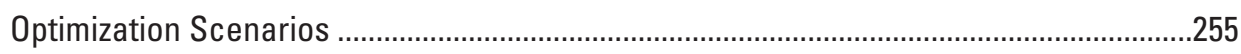

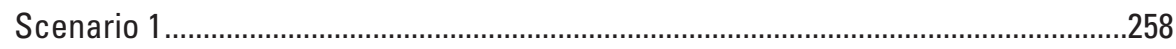

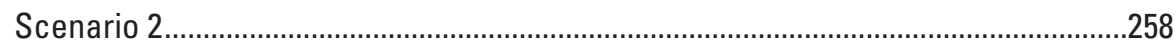

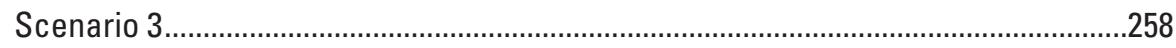

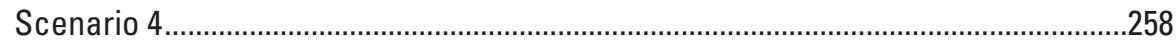

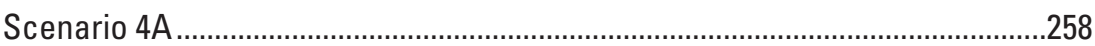

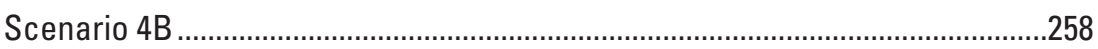

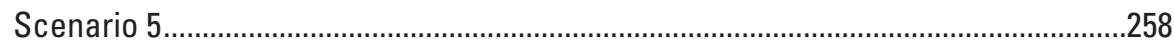

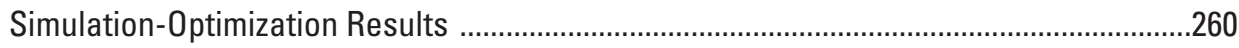

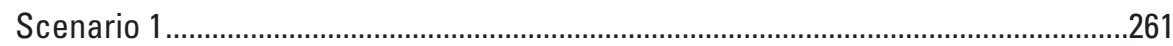

Scenario 2 271

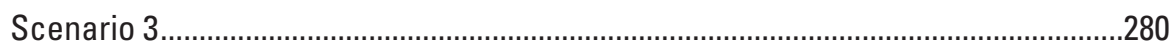

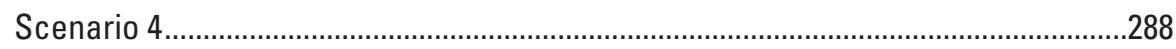

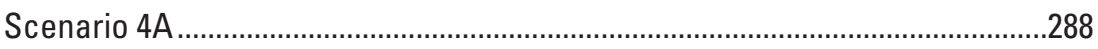

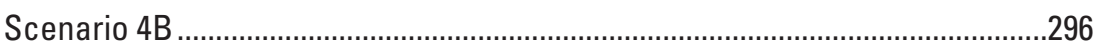

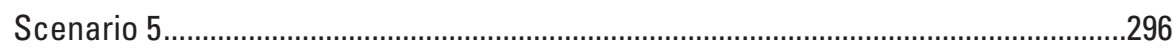

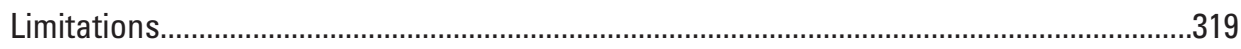

Summary and Conclusions .............................................................................................

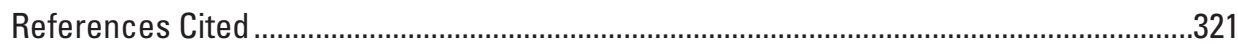

Appendix D-1: Schedule 1D Pumpage, by Well, Santa Barbara Multi-Objective

Management Model, Santa Barbara, California ..............................................................323

Appendix D-2: Schedule 2D Pumpage, by Well, Santa Barbara Multi-Objective

Management Model, Santa Barbara, California .................................................................335

Appendix D-3: Scenario 3 Pumpage, by Well, Santa Barbara Multi-Objective

Management Model, Santa Barbara, California ..................................................................34

Appendix D-4: Scenario 4B Decision Rules, Santa Barbara Multi-Objective Management Model, Santa Barbara, California ...............................................................360

\section{Figures}

\section{Chapter A}

1. Map showing the location and general features of the Santa Barbara and Foothill groundwater basins, Santa Barbara, California .................................................

2. Graphs showing precipitation measured at the Santa Barbara County building (1899-2013), by water year, Santa Barbara, California ........................................................

3. Map showing the distribution of land-use patterns, North American Land Cover Dataset 1992, Santa Barbara, California ......................................................................10

4. Map showing surface geology and faults of the Santa Barbara area, California ...........12

5. Map showing the Santa Barbara and Foothill groundwater subbasins, creeks, faults, production wells, and drains, Santa Barbara, California ......................................14

6. Maps showing the lateral extent of the upper, middle, and lower producing zones in the Santa Barbara and Foothill groundwater basins, Santa Barbara, California........15 


\section{Figures-Continued}

7. Cross section through Storage Unit I from the northwest to the southeast coast, showing resistivity logs for selected wells and aquifer-zone horizons defined by the hydrogeologic framework model, Santa Barbara groundwater basin, Santa Barbara, California.....

8. Graph showing estimated and reported total annual pumpage in the Santa Barbara and Foothill groundwater basins, water years 1929-2013, Santa Barbara, California...

9. Map showing parcels with septic tanks, Santa Barbara, California

10. Maps showing groundwater-level contours of the lower producing zone in the Santa Barbara and Foothill groundwater basins, Santa Barbara, California

11. Graph showing water-level elevations from selected wells in Storage Unit I, Storage Unit III, and Foothill groundwater basin, Santa Barbara, California

12. Map showing locations of coastal monitoring wells with associated peak, measured chloride concentrations, Santa Barbara groundwater basin, Santa Barbara, California

13. Graphs showing time-varying chloride concentrations from coastal wells, Santa Barbara groundwater basin, Santa Barbara, California.

\section{Chapter B}

1. Geologic map showing the hydrogeologic framework model domain, subbasins and storage units, and major faults, Santa Barbara and Foothill groundwater basins, Santa Barbara, California

2. Map showing aquifer-zone groupings organized by mapped geologic units, Santa Barbara and Foothill groundwater basins, California; cross-section lines show locations of previously published cross sections used in the hydrogeologic framework model

3. Map showing aquifer-zone groupings with drillers' well logs and geophysical e-logs used in the hydrogeologic framework model for Storage Unit I, Storage Unit III, and the Foothill subbasins, Santa Barbara and Foothill groundwater basins, California

4. Maps showing interpolated elevations of hydrogeologic framework model horizons with subsurface data points, Santa Barbara and Foothill groundwater basins, Santa Barbara, California

5. Images showing hydrogeologic framework model results by fence diagram, Santa Barbara and Foothill groundwater basins, Santa Barbara, California

6. Example of logs from one borehole showing the textural variation and interpretation of borehole sediments with depth.

7. Diagrams showing a cross section through the hydrogeologic framework model and geophysical resistivity e-logs shown for select boreholes and approximate extent of the middle producing zone, Santa Barbara and Foothill groundwater basins, Santa Barbara, California

\section{Chapter C}

1. Map showing distribution of flow barriers, general-head boundaries, drains, small-catchment recharge, and creek recharge in the Santa Barbara flow and transport model, Santa Barbara, California

2. Map showing the rates of small-catchment recharge for the Santa Barbara flow and transport model, Santa Barbara, California.

3. Maps showing areas of potential groundwater recharge for the Santa Barbara flow and transport model, Santa Barbara, California. 


\section{Figures-Continued}

4. Map showing the five regions used in the Santa Barbara flow and transport model, Santa Barbara, California.

5. Map showing the 14 subregions used in the Santa Barbara flow and transport model, Santa Barbara, California.

6. Map showing the two areas used in the Santa Barbara flow and transport model, Santa Barbara, California. 130

7. Diagram showing hydrogeologic units defined for the Santa Barbara flow and transport model, Santa Barbara, California

8. Map showing calibrated horizontal-flow-barrier hydraulic-characteristic values (per day), Santa Barbara flow and transport model, Santa Barbara, California.

9. Map showing monitoring wells with available water-level data and Mission Creek segments where infiltration rates were estimated by McFadden and others (1987), Santa Barbara and Foothill groundwater basins, Santa Barbara, California

10. Map showing monitoring wells with chloride concentration data near the coast of the city of Santa Barbara, California

11. Graphs showing composite scaled sensitivity values for the Santa Barbara flow and transport model, Santa Barbara, California

12. Graph showing normalized composite scaled sensitivity values, grouped by parameter types for all simulated values, hydraulic head, drawdown, chloride concentration, and change in chloride concentration, Santa Barbara flow and transport model, Santa Barbara, California

13. Histograms showing the distribution of hydraulic conductivity values used in the Santa Barbara flow and transport model, Santa Barbara, California

14. Graphs showing simulated annual hydrologic budget for two simulation periods, Santa Barbara flow and transport model, Santa Barbara, California.

15. Graph showing simulated cumulative change in groundwater storage, 1929-2013, Santa Barbara flow and transport model, Santa Barbara, California...

16. Maps showing contours of simulated initial-condition (average 1929) hydraulic head for producing zones of the Santa Barbara groundwater basin, California

17. Maps showing contours of simulated July 1990 hydraulic head for the producing zones of the Santa Barbara groundwater basin, California.

18. Maps showing contours of simulated drawdown between average 1929 and July 1990 hydraulic heads for the producing zones of the Santa Barbara groundwater basin, California

19. Graphs showing simulated hydraulic heads and measured water levels for selected wells in the Santa Barbara groundwater basin, California, by subbasin .......171

20. Graph showing simulated hydraulic heads compared with measured water levels by subbasin and well type for the Santa Barbara flow and transport model, Santa Barbara, California

21. Maps showing simulated chloride concentration contours for different years and producing zones for the Santa Barbara groundwater basin, California.

22. Graphs showing simulated and measured chloride breakthrough curves for selected wells in Storage Unit I, Santa Barbara groundwater basin, California...

23. Graph showing simulated chloride concentrations compared with measured chloride concentrations for selected monitoring wells in Storage Unit I, Santa Barbara groundwater basin, California . 


\section{Figures-Continued}

\section{Chapter D}

1. Graph showing the concepts of Pareto-optimality are shown with two generic objectives, which are assumed to be minimization; no single point is optimal because a decrease in one objective causes an increase in the other, so any point on the curve is considered Pareto-optimal (Kollat and Reed, 2006).

2. Map showing the locations of selected production wells, monitoring wells, and chloride monitoring wells, Santa Barbara and Foothill groundwater basins, Santa Barbara, California

3. Hydrograph showing measured water levels for wells 4N/27W-15K1, 4N/27W-16R1, 4N/27W-22G4, and 4N/27W-23E5 in Storage Unit I, Santa Barbara groundwater basin, Santa Barbara, California .....

4. Graph showing selected Pareto-optimal solutions for scenario 1, Santa Barbara multi-objective management model, Santa Barbara, California.

5. Graph showing scaled objective scores for schedules $1_{A}$ through $1_{E^{\prime}}$ Santa Barbara multi-objective management model, Santa Barbara, California

6. Graphs showing quarterly pumpage for Storage Unit I and Foothill groundwater basin, Santa Barbara multi-objective management model, Santa Barbara, California

7. Maps showing contours of simulated hydraulic head after 10 years for schedule $1_{D}$, Santa Barbara multi-objective management model, Santa Barbara, California

8. Cross section and maps showing simulated year-10 chloride concentration distribution for Storage Unit I, schedule $1_{D^{\prime}}$ Santa Barbara multi-objective management model, Santa Barbara, California

9. Graph showing simulated chloride breakthrough curves for selected monitoring wells for schedule $1_{D}$, Santa Barbara multi-objective management model, Santa Barbara, California

10. Graph showing selected Pareto-optimal solutions for scenario 2, Santa Barbara multi-objective management model, Santa Barbara, California.

11. Graph showing scaled objective scores for schedules $2_{\mathrm{A}^{\prime}} 2_{\mathrm{B}^{\prime}}, 2_{\mathrm{Ct}^{\prime}} 2_{\mathrm{Cm}^{\prime}}, 2_{\mathrm{D}^{\prime}}$ and $2_{\mathrm{E}^{\prime}}$ Santa Barbara multi-objective management model, Santa Barbara, California

12. Graphs showing scenario 2 quarterly pumpage for Storage Unit I and Foothill groundwater basin, Santa Barbara multi-objective management model, Santa Barbara, California

13. Maps showing contours of simulated hydraulic head after 10 years for scenario 2, schedule $2_{D}$, Santa Barbara multi-objective management model, Santa Barbara, California

14. Cross section and maps showing simulated year-10 Storage Unit I chloride concentration distribution for schedule 2 , Santa Barbara multi-objective management model, Santa Barbara, California

15. Graph showing simulated chloride breakthrough curves for selected monitoring wells for schedule ${ }_{\mathrm{D}}$, Santa Barbara multi-objective management model, Santa Barbara, California

16. Graphs showing simulated drawdowns with respect to 1998 water levels for selected wells and total pumpage equaling 484 acre-feet per year, scenario 3 , Santa Barbara multi-objective management model, Santa Barbara, California 


\section{Figures-Continued}

17. Maps showing cContours of simulated year-10 drawdowns with respect to 1998 water levels, assuming typical conditions, scenario 3, Santa Barbara multi-objective management model, Santa Barbara, California.

18. Maps showing contours, assuming a typical climatic condition, scenario 3 , Santa Barbara multi-objective management model, Santa Barbara, California

19. Maps showing contours of simulated year- 10 drawdown with respect to 1998 water levels, assuming dry climatic condition, scenario 3, Santa Barbara multi-objective management model, Santa Barbara, California

20. Maps showing contours; assuming a dry climatic condition, scenario 3, Santa Barbara multi-objective management model, Santa Barbara, California, of simulated year-10 chloride concentrations.

21. Graphs showing subsets of Pareto-optimal solutions, Santa Barbara multi-objective management model, Santa Barbara, California

22. Graphs showing example decision-rule curves for wells 4N/27W-23E3 and $4 \mathrm{~N} / 27 \mathrm{~W}-23 \mathrm{E} 5$ used to assess total pumpage from Storage Unit I on the basis of observed chloride concentrations in selected monitoring wells, Santa Barbara multi-objective management model, Santa Barbara, California...

23. Graphs showing the example decision-rule curves for the Los Robles well, Santa Barbara multi-objective management model, Santa Barbara, California

24. Graphs showing selected Pareto-optimal solutions relative to seawater intrusion, maximum drawdown, total pumpage, and total drawdown, Santa Barbara multiobjective management model, Santa Barbara, California.

25. Maps showing contours of simulated hydraulic heads for year two, Santa Barbara multi-objective management model, Santa Barbara, California, for pumping schedule $5 A_{D}$

26. Maps showing contours of simulated hydraulic heads for year two, Santa Barbara multi-objective management model, Santa Barbara, California, for pumping schedule $5 B_{D}$

27. Maps showing contours of simulated hydraulic heads for year two, Santa Barbara multi-objective management model, Santa Barbara, California, for pumping schedule $5 C_{D}$

28. Maps showing contours of simulated chloride concentrations for year two, Santa Barbara multi-objective management model, Santa Barbara, California

29. Graphs showing pumping schedules $A$ and $B$ (maximum pumpage and minimum seawater intrusion, respectively), Storage Unit I and Foothill groundwater basin, Santa Barbara multi-objective management model, Santa Barbara, California

30. Graphs showing scaled objective scores by scenario 5 pumping schedule used in the Santa Barbara multi-objective management model, Santa Barbara, California .....

31. Graphs showing breakthrough curves of simulated chloride concentrations for selected monitoring wells, Santa Barbara multi-objective management model, Santa Barbara, California....

D1-1. Graph showing optimal quarterly pumpage for the Alameda Park well, schedule $1_{D^{\prime}}$ Santa Barbara, California.

D1-2. Graph showing optimal quarterly pumpage for the City Hall well, schedule $1_{D^{\prime}}$ Santa Barbara, California... 


\section{Figures-Continued}

D1-3. Graph showing optimal quarterly pumpage for the Corporation Yard well, schedule $1_{D}$, Santa Barbara, California....

D1-4. Graph showing optimal quarterly pumpage for the Ortega Park well, schedule $1_{D^{\prime}}$ Santa Barbara, California

D1-5. Graph showing optimal quarterly pumpage for the Santa Barbara High School well, schedule $1_{D^{\prime}}$ Santa Barbara, California

D1-6. Graph showing optimal quarterly pumpage for the Vera Cruz well, schedule $1_{D^{\prime}}$ Santa Barbara, California

D1-7. Graph showing optimal quarterly pumpage for the Hope Avenue well, schedule $1_{D}$, Santa Barbara, California.

D1-8. Graph showing optimal quarterly pumpage for the Lincolnwood 1 well, schedule $1_{D^{\prime}}$ Santa Barbara, California.

D1-9. Graph showing optimal quarterly pumpage for the Los Robles well, schedule $1_{D^{\prime}}$ Santa Barbara, California

D1-10. Graph showing optimal quarterly pumpage for the San Roque Park 2 well, schedule $1_{D}$, Santa Barbara, California.....

D1-11. Graph showing optimal quarterly pumpage for the Val Verde well, schedule $1_{D^{\prime}}$ Santa Barbara, California.

D2-1. Graph showing optimal quarterly pumpage for the Alameda Park well, schedule 2, Santa Barbara, California.

D2-2. Graph showing optimal quarterly pumpage for the City Hall well, schedule $2_{D^{\prime}}$ Santa Barbara, California

D2-3. Graph showing optimal quarterly pumpage for the Corporation Yard well, schedule $2{ }_{D}$, Santa Barbara, California.

D2-4. Graph showing optimal quarterly pumpage for the Ortega Park well, schedule ${ }_{D^{\prime}}$ Santa Barbara, California

D2-5. Graph showing optimal quarterly pumpage for the Santa Barbara High School well, schedule 2, Santa Barbara, California

D2-6. Graph showing optimal quarterly pumpage for the Vera Cruz well, schedule $2_{D^{\prime}}$ Santa Barbara, California

D2-7. Graph showing optimal quarterly pumpage for the Hope Avenue well, schedule ${ }_{\mathrm{D}}$, Santa Barbara, California.

D2-8. Graph showing optimal quarterly pumpage for the Lincolnwood 1 well, schedule ${ }_{D^{\prime}}$ Santa Barbara, California.

D2-9. Graph showing optimal quarterly pumpage for the Los Robles well, schedule ${ }_{D^{\prime}}$ Santa Barbara, California.

D2-10. Graph showing optimal quarterly pumpage for the San Roque Park 2 well, schedule ${ }_{0}$, Santa Barbara, California.

D2-11. Graph showing optimal quarterly pumpage for the Val Verde well, schedule ${ }_{\mathrm{D}}$, Santa Barbara, California..

D3-1. Graph showing quarterly pumpage for the Alameda Park well, scenario 3, typical conditions, Santa Barbara, California

D3-2. Graph showing quarterly pumpage for the City Hall well, scenario 3, typical conditions, Santa Barbara, California

D3-3. Graph showing quarterly pumpage for the Corporation Yard well, scenario 3, typical conditions, Santa Barbara, California 


\section{Figures-Continued}

D3-4. Graph showing quarterly pumpage for the Ortega Park well, scenario 3, typical conditions, Santa Barbara, California

D3-5. Graph showing quarterly pumpage for the Santa Barbara High School well, scenario 3, typical conditions, Santa Barbara, California

D3-6. Graph showing quarterly pumpage for the Vera Cruz well, scenario 3, typical conditions, Santa Barbara, California

D3-7. Graph showing quarterly pumpage for the Alameda Park well, scenario 3, drought conditions, Santa Barbara, California

D3-8. Graph showing quarterly pumpage for the City Hall well, scenario 3, drought conditions, Santa Barbara, California

D3-9. Graph showing quarterly pumpage for the Corporation Yard well, scenario 3, drought conditions, Santa Barbara, California

D3-10. Graph showing quarterly pumpage for the Ortega Park well, scenario 3, drought conditions, Santa Barbara, California

D3-11. Graph showing quarterly pumpage for the Santa Barbara High School well, scenario 3, drought conditions, Santa Barbara, California

D3-12. Graph showing quarterly pumpage for the Vera Cruz well, scenario 3, drought conditions, Santa Barbara, California...

D4-1. Graphs showing decision rules for total Foothill groundwater basin pumpage as a function of maximum drawdown at the city of Santa Barbara production wells assuming scenario 1 (typical) precipitation, Santa Barbara groundwater basin, Santa Barbara, California....

D4-2. Graphs showing decision rules for total Storage Unit I pumpage as a function of maximum drawdown at the city of Santa Barbara production wells assuming scenario 1 (typical) precipitation, Santa Barbara groundwater basin, Santa Barbara, California

D4-3. Graphs showing decision rules for total Foothill groundwater basin pumpage as a function of maximum drawdown at the city of Santa Barbara production wells assuming scenario 2 (dry) precipitation, Santa Barbara groundwater basin, Santa Barbara, California

D4-4. Graphs showing decision rules for total Storage Unit I pumpage as a function of maximum drawdown at the city of Santa Barbara production wells assuming scenario 2 (dry) precipitation, Santa Barbara groundwater basin, Santa Barbara, California

\section{Tables}

\section{Chapter A}

1. Velocity-profile testing results for the Corporation Yard well, Santa Barbara, California

2. City of Santa Barbara production wells and state well identifiers, Santa Barbara, California

\section{Chapter B}

1. Aquifer-zone groupings listed by mapped geologic unit and age, Santa Barbara and Foothill groundwater basins, California

2. Summary statistics of borehole depths by subbasin, Santa Barbara and Foothill groundwater basins, Santa Barbara, California. 


\section{Tables-Continued}

3. Unique lithology descriptions and modifiers with associated binary coarse or fine classification as identified in drillers' lithology logs, Santa Barbara and Foothill groundwater basins, Santa Barbara, California

B1-1. Calculated percent-coarse intervals for all drillers' logs and e-logs used to define textural properties of the hydrogeologic framework model.

\section{Chapter C}

1. SEAWAT Version 4 packages and processes used with the Santa Barbara flow and transport model, Santa Barbara, California

2. Coordinates of the Santa Barbara Flow and Transport Model grid, Santa Barbara, California

3. Calibrated groundwater flow and solute transport model parameter values, Santa Barbara flow and transport model, Santa Barbara, California.

4A. Simulated cumulative groundwater budget, 1929-71, Santa Barbara flow and transport model, Santa Barbara, California

4B. Simulated cumulative groundwater budget, 1972-2013, Santa Barbara flow and transport model, Santa Barbara, California

4C. Simulated water budget, July 1990, Santa Barbara flow and transport model, Santa Barbara, California

\section{Chapter D}

1. Maximum pumping capacities for city of Santa Barbara production wells, Santa Barbara, California

2. A summary of the optimization scenarios, Santa Barbara multi-objective management model, Santa Barbara, California

3. Values for the four objectives for selected Pareto-optimal pumping schedules, scenario 1, Santa Barbara multi-objective management model, Santa Barbara, California

4. Values for the four objectives for selected Pareto-optimal pumping schedules, scenario 2, Santa Barbara multi-objective management model, Santa Barbara, California

5. Objective values for scenario 5A, Santa Barbara multi-objective management model, Santa Barbara, California

6. Objective values for scenario $5 B$, Santa Barbara multi-objective management model, Santa Barbara, California

7. Objective values for scenario $5 \mathrm{C}$, Santa Barbara multi-objective management model, Santa Barbara, California 


\section{Conversion Factors}

U.S. customary units to International System of Units

\begin{tabular}{|c|c|c|}
\hline Multiply & By & To obtain \\
\hline \multicolumn{3}{|c|}{ Length } \\
\hline inch (in.) & 25.4 & millimeter $(\mathrm{mm})$ \\
\hline inch (in.) & 0.0254 & meter $(\mathrm{m})$ \\
\hline foot $(\mathrm{ft})$ & 0.3048 & meter $(\mathrm{m})$ \\
\hline mile (mi) & 1.609 & kilometer $(\mathrm{km})$ \\
\hline \multicolumn{3}{|c|}{ Area } \\
\hline acre & 4,047 & $\operatorname{meter}^{2}\left(\mathrm{~m}^{2}\right)$ \\
\hline square mile $\left(\mathrm{mi}^{2}\right)$ & 2.590 & square kilometer $\left(\mathrm{km}^{2}\right)$ \\
\hline \multicolumn{3}{|c|}{ Volume } \\
\hline acre-foot (acre-ft) & 1,233 & cubic meter $\left(\mathrm{m}^{3}\right)$ \\
\hline gallon per day (gal/d) & 3.78541 & liter per day $(\mathrm{L} / \mathrm{d})$ \\
\hline \multicolumn{3}{|c|}{ Flow rate } \\
\hline acre-foot per day (acre-ft/d) & 0.01427 & cubic meter per second $\left(\mathrm{m}^{3} / \mathrm{s}\right)$ \\
\hline acre-foot per year (acre-ft/yr) & 1,233 & cubic meter per year $\left(\mathrm{m}^{3} / \mathrm{yr}\right)$ \\
\hline foot per day (ft/d) & 0.3048 & meter per day $(\mathrm{m} / \mathrm{d})$ \\
\hline foot per year (ft/yr) & 0.3048 & meter per year $(\mathrm{m} / \mathrm{yr})$ \\
\hline gallon per day (gal/d) & 0.003785 & cubic meter per day $\left(\mathrm{m}^{3} / \mathrm{d}\right)$ \\
\hline
\end{tabular}

Temperature in degrees Celsius $\left({ }^{\circ} \mathrm{C}\right)$ may be converted to degrees Fahrenheit $\left({ }^{\circ} \mathrm{F}\right)$ as follows:

$$
{ }^{\circ} \mathrm{F}=\left(1.8 \times{ }^{\circ} \mathrm{C}\right)+32 .
$$

Temperature in degrees Fahrenheit $\left({ }^{\circ} \mathrm{F}\right)$ may be converted to degrees Celsius $\left({ }^{\circ} \mathrm{C}\right)$ as follows:

$$
{ }^{\circ} \mathrm{C}=\left({ }^{\circ} \mathrm{F}-32\right) / 1.8 \text {. }
$$

\section{Datum}

Vertical coordinate information is referenced to the North American Vertical Datum of 1988 (NAVD 88).

Horizontal coordinate information is referenced to the North American Datum of 1983 (NAD 83).

Elevation, as used in this report, refers to distance above the vertical datum. 


\section{Supplemental Information}

Concentrations of chemical constituents in water are given in either milligrams per liter (mg/L) or micrograms per liter $(\mu \mathrm{g} / \mathrm{L})$.

\section{Abbreviations}

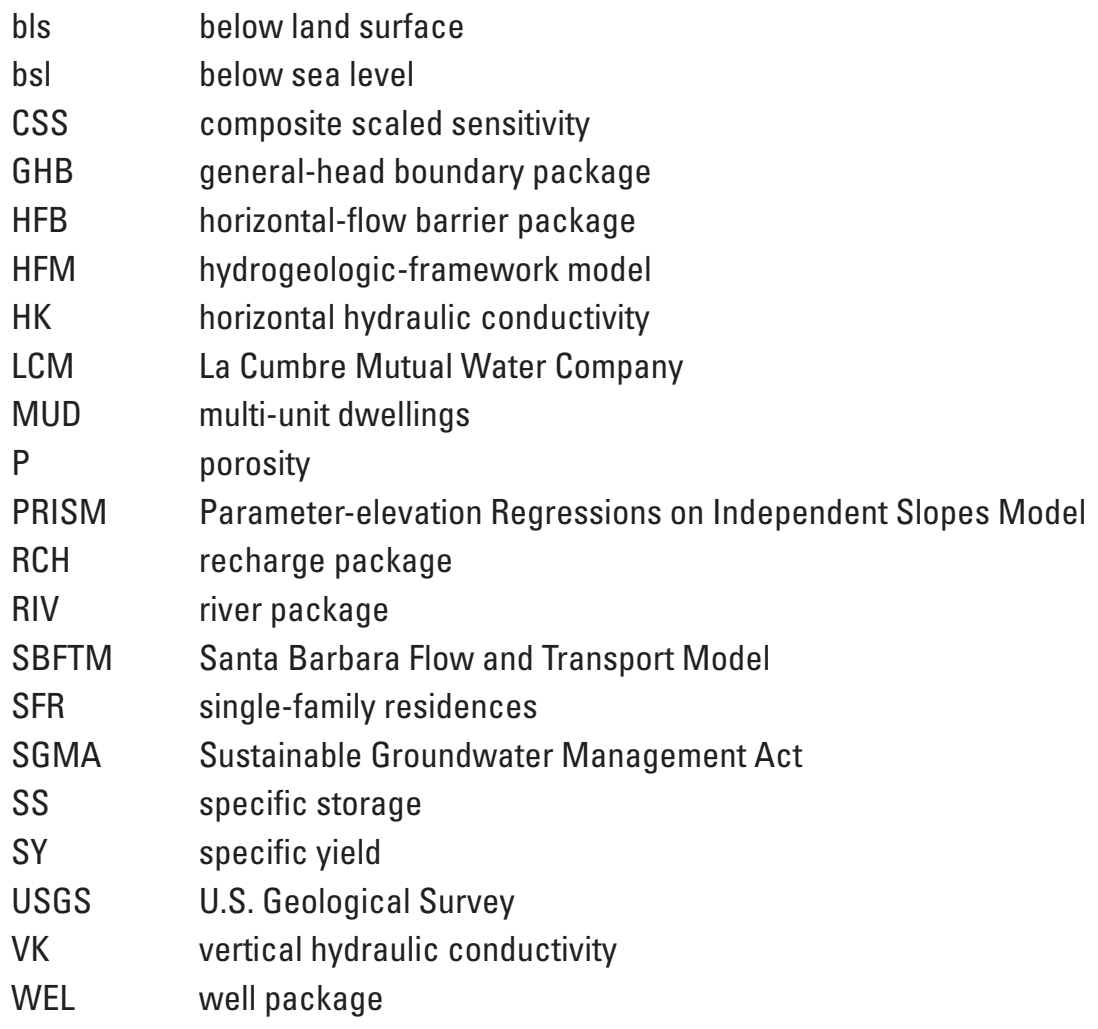




\section{Santa Barbara and Foothill Groundwater Basins Geohydrology and Optimal Water Resources Management-Developed using Density Dependent Solute Transport and Optimization Models}

\section{Abstract}

Groundwater has been a part of the city of Santa Barbara's water-supply portfolio since the 1800s; however, since the 1960s, the majority of the city's water has come from local surface water, and the remainder has come from groundwater, State Water Project, recycled water, increased water conservation, and as needed, seawater desalination. Although groundwater from the Santa Barbara and Foothill groundwater basins only accounts for a small percentage of the long-term supply, it is an important source of supplemental water during times of surface-water shortages. During the late 1980s and early 1990s, production wells extracted additional groundwater to compensate for drought related waterdelivery shortfalls from other sources; in response, water levels declined substantially in the Santa Barbara and Foothill groundwater basins (below sea level in the Santa Barbara groundwater basin).

In coastal basins that have groundwater extraction near shore, seawater intrusion is often a problem. Seawater intrusion in the Santa Barbara groundwater basin is thought to be more limited than in other coastal basins because of an offshore fault that acts as a partial barrier to groundwater flow. During the late 1980s and early 1990s, seawater intrusion was observed in the Santa Barbara groundwater basin, as indicated by increased chloride concentrations at several monitoring wells that ranged from $200 \mathrm{ft}$ to $1,300 \mathrm{ft}$ from the ocean and as close as 2,900 $\mathrm{ft}$ to the nearest pumping well. This demonstrated that seawater can intrude into the Santa Barbara groundwater basin when groundwater levels fall below sea level near the coast.

The city of Santa Barbara is interested in developing a better understanding of the sustainability of its groundwater supplies. In 2014, California adopted historic legislation to manage its groundwater: the Sustainable Groundwater Management Act (SGMA). The SGMA requires the development and implementation of "Groundwater Sustainability Plans" in 127 priority groundwater basins; although Santa Barbara was not a designated priority basin, the city is taking steps to achieve sustainability. Sustainability was defined in the SGMA in terms of avoiding undesirable results: significant and unreasonable groundwater-level declines, reduction in groundwater storage, seawater intrusion, water-quality degradation, land subsidence, and surface-water depletion.
In this project, a cooperative study between the U.S. Geological Survey (USGS) and the city of Santa Barbara, sustainable yield is defined as the volume of groundwater that can be pumped from storage without causing waterlevel drawdowns and the associated increases in seawater intrusion (as indicated by increases in measured chloride concentrations) at selected wells. In order to estimate the sustainability of Santa Barbara's groundwater basins, a three-dimensional density-dependent groundwater-flow and solute-transport model (the Santa Barbara Flow and Transport Model, or SBFTM) was developed on the basis of an existing groundwater-flow model. To simulate seawater intrusion to the Santa Barbara Basin under various management strategies, the SBFTM uses the USGS code SEAWAT to simulate salinity transport and variable-density flow. The completed SBFTM was coupled with a management optimization tool, in this case a multi-objective evolutionary algorithm, to determine optimal pumping strategies that maximize the sustainable yield and at the same time satisfy user-defined drawdown and chlorideconcentration constraints.

As part of this study, a three-dimensional hydrogeologic framework model was developed to quantify the extent and hydrogeologic characteristics of the Santa Barbara and Foothill groundwater basins and to help define the discretization and hydraulic properties used in the SBFTM. The development of the hydrogeologic framework model required the collection and reconciliation of geologic and geophysical data from existing maps, reports, and databases, along with geologic and hydrologic data from recently drilled wells. These data were integrated into a three-dimensional hydrogeologic framework model that defines the stratigraphy and geometry of the aquifer zones and the major geologic structures in the basin. The hydrogeologic framework model also quantifies the variation in sediment grain size within each aquifer zone as the percentage of coarse-grained sediment. Previous studies indicated that there are two principal waterproducing zones in the Santa Barbara groundwater basin, the upper and lower producing zones; an additional thin, productive zone was identified as part of this study. This "middle producing zone" is not as areally extensive as the upper and lower producing zones and only exists in the coastal part of Storage Unit I. These producing zones are bounded at depth by less productive shallow, middle, and deep zones. 
Two versions of the SBFTM were constructed: an initial-condition model and a modern transient model. The initial-condition model is a long-term transient model that simulates flow and solute-transport conditions during a period with limited anthropogenic influences preceeding the modern transient model. The simulation-transient model simulates flow and transport conditions from 1929 through 2013; however, because of data availability, the focus of the model calibration was 1972-2013. The SBFTM was calibrated to measured groundwater levels and drawdown, as well as measured chloride concentrations and change in concentrations, using a combination of automated and trialand-error parameter-estimation techniques.

A sensitivity analysis indicated that, in general, the SBFTM was most sensitive to recharge- and pumpingdistribution parameters, specifically those controlling the amount of small-catchment recharge and the distribution of water extraction by hydrogeologic layer for production wells. The model was also sensitive to parameters controlling stream-recharge rates, horizontal and vertical hydraulic conductivity, and porosity.

From 1929 to 1971 , most of the water entering the area represented by the SBFTM was from creek and smallcatchment recharge, and the majority of water leaving the SBFTM area was from pumping, discharge to creeks, and drains. In addition, about 37 percent of the total pumpage came from a net reduction in groundwater storage. From 1972 to 2013, the amount of water entering and leaving the SBFTM was fairly similar as that from 1929 to 1971 , except the reduction in pumpage added about 17,000 acre- $\mathrm{ft}$ of water to storage. During this later period, there were also times of storage loss. For example, during July 1990, a month when approximately 705 acre-ft of groundwater was pumped in the study area, the pumpage was much greater than all sources of recharge combined, and about 382 acre-ft of water was removed from groundwater storage.

Simulated hydraulic heads replicated the observed data to an acceptable matching of the measured water-level, flow direction, and vertical gradients. Simulated hydrographs for selected wells were in good agreement with the measured data, with an average residual of $-2.7 \mathrm{ft}$ and a standard deviation of $14.5 \mathrm{ft}$, indicating that the simulated heads, on average, underestimated the observed water levels. An examination of the model fit indicated that most of the discrepancies were lower simulated heads at wells proximal to production well sites.

The simulated chloride concentrations reasonably matched the rising limbs of the measured breakthrough curves in terms of timing and magnitude; however, the simulation overestimated the chloride concentrations on the falling limbs. The overestimation of low chloride concentrations was attributed to the model overestimating the advance of the chloride front during periods of heavy pumping and underestimating the retreat of the chloride front during periods of low pumping. These simulation errors would result in a conservative response by local water managers to seawater intrusion.

The SBFTM was used to develop a collection of predictive simulations optimized to produce pumping schedules that maximize yield, subject to a set of constraints and competing objectives. The simulations were grouped as scenarios that differed in their time horizon, initial conditions for groundwater levels and chloride concentrations, as well as precipitation, which was incorporated into the model through simulated recharge. Overall, five scenarios were developed in a multi-objective framework to obtain optimal pumping rates for all of the wells managed by the city, while minimizing excessive drawdown and seawater intrusion.

For the current study, complexities in the simulation model and the optimization formulation required additional considerations. Incorporating the solute-transport equations to simulate chloride transport added a highly nonlinear process that is solved iteratively in each time step of the groundwater-flow model. These nonlinearities, coupled with the highly refined grid in the current model, creates challenges for many traditional optimization methods. Therefore, an optimization method was needed that could address nonlinear relationships as well as a very large problem size. Lastly, the optimization problem was reformulated to include multiple objectives without requiring convergence to a single solution. This approach, guided by the city's objectives, allowed the maximum extraction of information from the complex simulation.

Borg, a multi-objective evolutionary algorithm, was chosen as the optimization algorithm for this study for several reasons: (1) it is very computationally efficient; (2) it can run in parallel; (3) it requires little user input; and (4) it can solve for multiple competing objectives. The first three points allow the algorithm to proceed toward the optimal solutions at the fastest possible rate. The fourth point is advantageous for large, complex optimization problems because it is difficult to formulate the optimization problem in a way that produces only one optimal solution.

The problem formulation consisted of four competing objectives and a constraint set in accordance with the main concerns of the city. The objectives were maximizing total pumpage, minimizing seawater intrusion, minimizing total drawdown in production wells, and minimizing the maximum drawdown. The constraints were pump capacity, meeting drinking-water standards for chloride, maintaining a specified minimum flowrate to a groundwater treatment plant, and maintaining minimum water levels in pumping wells. The decision variables either were quarterly pumpage by well or total pumpage by basin. 
Five optimization scenarios were developed that allow the decision makers to evaluate a range of optimal solutions for a variety of water levels and chloride concentrations as well as potential future climatic conditions. Three scenarios (1, 2 , and 5) were multi-objective optimization formulations that allowed for variations in management preferences and climatic conditions. The other two scenarios ( 3 and 4 ) were designed to examine the optimization results to answer specific questions. Scenario 1 described the best-case sustainable yield assuming a "full" basin (that is, high initial water levels) and typical climate conditions for 10 years. Scenario 2 also started with a "full" basin; however, this was followed by a 10-year drought. Scenario 3 determined if an "empty" basin (that is, low initial water levels) would recover to full conditions (1998 conditions) given climate assumptions and optimal pumping schedules from scenarios 1 and 2. Scenario 4 was designed to produce decision rules that can be used by water managers to help choose an optimal pumping schedule based on measured water-level or chloride data. Scenario 5 identified future pumping schedules based on short-term climate variations during a 2-year management horizon.

The results from scenarios 1 and 2 described the differences in maximum pumpage in the basin under typical and dry long-term climate projections, respectively. The scenario 1 results indicated the maximum 10-year pumpage of the basin was about 31,300 acre-ft under typical conditions and controlling simulated seawater intrusion and drawdowns. For scenario 2, less recharge over the 10-year dry climate produced a maximum pumpage estimate of 30,000 acre- $\mathrm{ft}$ to control seawater intrusion and drawdowns. The larger pumpage for scenario 1 resulted in more seawater intrusion, but less total drawdown, compared to that of scenario 2 .
Results for scenarios 3 and 4 showed the basin's response to management actions combined with climate projections. Both scenarios used the optimal pumping schedules and the 10-year climates from scenarios 1 and 2 . The scenario 3 results showed that under minimal pumping, the basin did not fully recover to 1998 water levels within 10 years under either climate scenario. The relatively larger recharge from the typical climate resulted in less drawdown at coastal monitoring wells after the 10 -year recovery period than that from the dry climate. The location of the seawater intrusion front was not appreciably different between the scenarios, however. Scenario 4 used the optimal results from scenarios 1 and 2 to produce decision-rule curves that illustrated the pumpage for each basin, given measured levels of chloride concentration or drawdown. This allowed the use of additional measurements at monitoring wells to assess future management decisions on the basis of the sensitivity of observations of drawdown and seawater intrusion to various pumping rates.

Scenario 5 allowed managers to investigate the effects of short-term climate variations on optimal pumping schedules. Three specific 2-year simulations were optimized: typical-todry (scenario 5A), dry-to-typical (scenario 5B), and dry-to-dry (scenario $5 \mathrm{C}$ ). The most noteable result from scenario 5 was the overall reduction in optimal pumpage for most schedules in scenario $5 \mathrm{C}$, when the climate is simulated as dry-to-dry. There are also many optimal pumping schedules that produced an overall increase in waterlevels over the two-year simulation period, regardless of climatic condition. Similar to scenario 2, the scenario $5 \mathrm{C}$ results represents conservative yield estimates under a minimal-precipitation climatic condition. 
4 Santa Barbara and Foothill Groundwater Basins Geohydrology and Optimal Water Resources Management 


\title{
Chapter A: Introduction and Overview of Geology and Hydrogeology
}

\author{
By Scott R. Paulinski and Tracy Nishikawa
}

\section{Introduction}

Groundwater has been a part of the city of Santa Barbara's water supply since the 1800 s, when it was the city's primary source of water. Surface water became the primary water source after the early 1910s. In 1991, the city completed construction of wastewater-treatment facilities that supply recycled water for irrigation purposes. The recycled-water project has the capacity to treat and deliver up to 1,400 acrefeet per year (acre-ft/yr) of recycled water. The city also completed construction of a desalination plant in 1992 (City of Santa Barbara, 2015), which was in long-term standby mode until the recent drought. The city reactivated the desalination plant and it became operational in spring 2017. In July 1997, the city gained access to California State Water Project water and is entitled to 3,300 acre-ft/yr, subject to availability (City of Santa Barbara, 2015). In a typical non-drought year, about 82 percent of the city's water supply comes from surface water; the remainder comes from groundwater, the State Water Project, and recycled water (City of Santa Barbara, 2015).

Despite the increased use of surface water, pumping in the Santa Barbara groundwater basin increased from less than $1,000 \mathrm{acre}-\mathrm{ft} / \mathrm{yr}$ in the late $1950 \mathrm{~s}$ to about 3,000 acre-ft/yr in the 1960s. Although groundwater only accounts for a small percentage of the long-term demand, it is an important source of supplemental water during water shortages. For example, groundwater was an important addition to the water supply during the severe drought in the late 1980s to early 1990s (Freckleton and others, 1998). Production wells extracted more groundwater to compensate for water-delivery shortfalls from other sources, and water levels declined noticeably in the Santa Barbara and Foothill groundwater basins (fig. 1).

In coastal basins with groundwater extraction near shore, seawater can intrude into freshwater resources. Many of the city of Santa Barbara's production wells are in the Santa Barbara groundwater basin between 3,500 and 5,000 feet (ft) from the ocean; these wells can be susceptible to seawater intrusion during times of intensive pumping, causing below-sea-level groundwater levels. Seawater intrusion into the Santa Barbara groundwater basin is thought to be limited by the presence of an assumed offshore fault, which acts as a partial barrier to groundwater flow. During the 1980s and early 1990 s, however, seawater intrusion was observed at several monitoring wells in the Santa Barbara groundwater basin between about 200 and 1,300 ft from the ocean and as close as 2,900 $\mathrm{ft}$ to the nearest pumping well (Martin, 1984). These observations demonstrated that seawater can intrude into the basin when water levels fall below sea level at the coastal boundary of the basin.

The city of Santa Barbara is interested in developing a better understanding of the sustainability of its groundwater supplies. In 2014, California adopted historic legislation to manage its groundwater: the Sustainable Groundwater Management Act (SGMA). The SGMA requires the development and implementation of "Groundwater Sustainability Plans" in 127 priority groundwater basins; although Santa Barbara was not a priority basin as of 2017, the city is taking steps to achieve sustainability. Sustainability is defined in the SGMA in terms of avoiding undesirable results: large and unreasonable groundwater-level declines, reduction in groundwater storage, seawater intrusion, water-quality degradation, land subsidence, and surface-water depletion (U.S. Geological Survey, 2018).

In order to estimate the sustainability of the Santa Barbara and Foothill groundwater basins, a three-dimensional, density-dependent solute-transport model was developed by the U.S. Geological Survey (USGS) in cooperation with city of Santa Barbara. For this work, sustainable yield is defined, with respect to seawater intrusion, as the volume of groundwater that can be pumped from storage without causing water-level drawdowns and associated increases in seawater intrusion (as indicated by increases in measured chloride concentrations) at selected wells. The groundwater-flow model developed by Freckleton and others (1998) provided the basis for solute-transport modeling. 


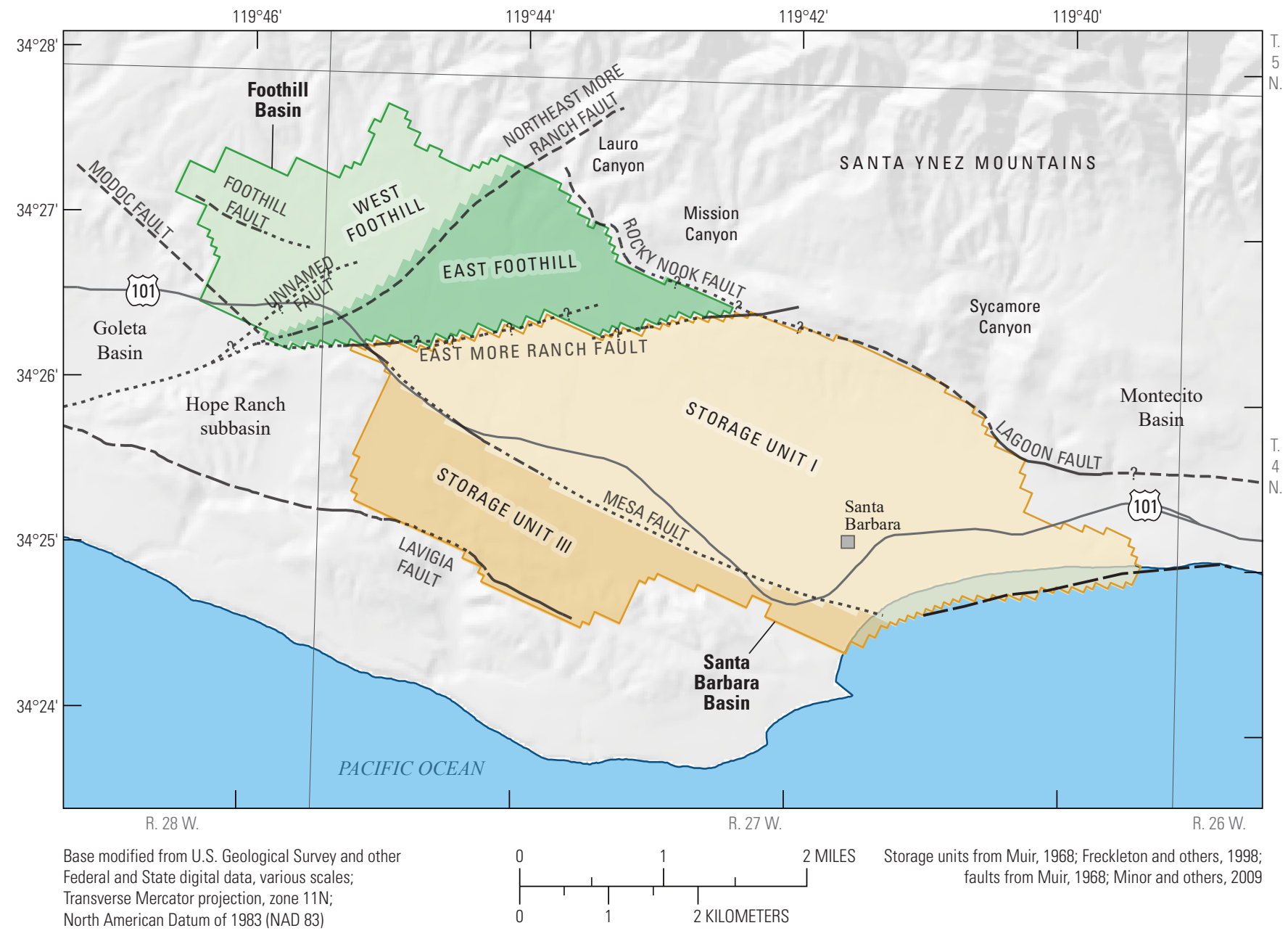

EXPLANATION

\begin{tabular}{|c|c|}
\hline \multicolumn{2}{|c|}{ Subbasins } \\
\hline \multicolumn{2}{|c|}{ West Foothill } \\
\hline & East Foothill \\
\hline & Storage Unit I \\
\hline & Storage Unit III \\
\hline & Foothill groundwater basin \\
\hline & Santa Barbara groundwater basin \\
\hline & Highway \\
\hline & City \\
\hline
\end{tabular}

Faults

- - - - Existence certain, location inferred

- -?- - Existence questionable, location inferred

........ Existence certain, location concealed

...?.... Existence questionable, location concealed

Existence certain, location accurate

- Existence certain, location approximate

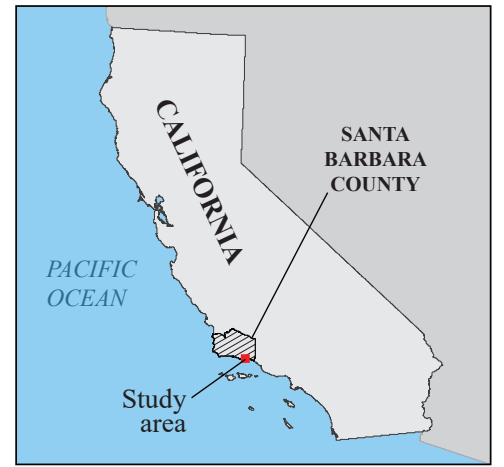

Figure 1. Location and general features of the Santa Barbara and Foothill groundwater basins, Santa Barbara, California. 


\section{Purpose and Scope}

This report describes the solute-transport model that was used in conjunction with a management optimization model, in this case a multi-objective evolutionary algorithm, to determine the optimal pumping strategies that maximize the sustainable yield yet satisfy user-defined drawdown and chloride-concentration constraints. In addition to developing the solute-transport and optimization models, new hydrogeologic framework and textural models of the Santa Barbara and Foothill groundwater basins were developed. These models were used in the construction of the solutetransport model.

The study is summarized in this four-chapter report describing the hydrogeology and the hydrogeologic framework, groundwater-flow, and solute-transport models of the Santa Barbara and Foothill groundwater basins. This chapter (chapter A) introduces the study, presenting a description of the study area, an overview of previous work, and an overview of the geology and hydrogeology of the study area. Chapter B presents a hydrogeologic framework model of the study area. This hydrogeologic framework model includes a three-dimensional interpretation of the geologic features associated with producing zones that supply most of the city of Santa Barbara's groundwater. The textural component of the hydrogeologic framework model provides a three-dimensional gradational distribution of the coarse and fine sediments in the Santa Barbara and Foothill groundwater basins. Chapter C presents the numerical groundwater-flow and solute-transport model of the Santa Barbara and Foothill groundwater basins (Santa Barbara Flow and Transport Model or SBFTM), which simulates groundwater flow and transport of chloride in the study area from 1929 through 2013. Chapter D presents a simulation-optimization model of the study area that enables use of the solute-transport model within a management optimization approach for water-management decision support.

\section{Previous Studies}

The Santa Barbara groundwater basin has been the subject of a number of USGS studies in the last 50 years. As groundwater levels declined throughout the 1960s, a study by the USGS, in cooperation with the Santa Barbara County Water Agency, developed the information needed to ensure sufficient water for the future needs of the county. A study of the geology, hydrogeology, water quality, and inflow/ outflow of the Santa Barbara and Montecito area (Muir, 1968) concluded that groundwater pumping had removed 6,000 acre-feet (acre-ft) of water from groundwater storage in the Santa Barbara area between 1959 and 1964. The study also concluded that seawater intrusion during this time was limited to near-shore shallow alluvial deposits and that there was no direct connection between the deeper water-bearing zones and the ocean. This lack of connection was attributed to an offshore fault.

In 1977, the USGS, in cooperation with the city of Santa Barbara, began to develop and implement a three-phase groundwater-monitoring program focused on "Storage Unit I" of the Santa Barbara groundwater basin (fig. 1 in Hutchinson, 1979). The monitoring program's main focus was to study the effects of pumping in Storage Unit I on groundwater levels and seawater intrusion. During phase 1 of the study, coastal monitoring wells were constructed and sampled to detect sea intrusion into the freshwater aquifer. The study concluded that observed elevated levels of chloride in coastal monitoring wells could either be due to connate water or to underflow across the offshore fault (Hutchinson, 1979). Phase 2 of the groundwater monitoring program focused on characterizing the geohydrology of the Santa Barbara groundwater basin (Martin, 1984). The number of monitoring wells in the Santa Barbara groundwater basin was increased from 17 to 30 , and data from the monitoring wells were used to assess the effects of pumping on water levels and water quality. The study concluded that several methods, including decreased pumpage, artificial recharge, and relocation of the city well field, could be used to displace intruded ocean water in the basin and force it seaward (Martin, 1984). A three-dimensional groundwaterflow model for Storage Unit I was developed for phase 3 of the study (Martin and Berenbrock, 1986). Results from the groundwater-flow model indicated that increased municipal pumpage resulted in increased inflow across the offshore fault at a rate of about 580 acre-ft/yr.

The USGS, in cooperation with the city of Santa Barbara, extended the three-phase groundwater study to include the Foothill groundwater basin, where additional groundwater monitoring wells were installed, a geohydrologic assessment was performed, and a three-dimensional, finite-difference, two-layer model was developed to confirm estimates of basin recharge and natural discharge and to evaluate water-level response to groundwater pumping (Freckleton, 1989).

In July and September of 1987, streamflow gains and losses were measured along Mission Creek (McFadden and others, 1991). As part of the study, water was released from a reservoir to Mission Creek; streamflow was measured at 10 sites downstream; and seepage was calculated along 10 segments of Mission Creek. The rate of streamflow gain or loss between stations varied greatly. Results along some segments were substantially different from a 1979 study by the USGS, when the water table was lower (Martin, 1984). 
In the 1990s, the USGS, in cooperation with the city of Santa Barbara, developed an area-wide groundwaterflow model of the Santa Barbara and Foothill groundwater basins, including the previously unmodeled Storage Unit III (Freckleton and others, 1998). During the simulation period, from 1978 to 1992, the model simulated water moving landward, across the offshore fault, to the Santa Barbara groundwater basin. Based on this groundwater-flow model, a two-dimensional simulation-optimization density-dependent groundwater-flow and transport model was developed to aid in making management decisions regarding the city of Santa Barbara's water resources (Nishikawa and Martin, 1998). Selected optimal pumping strategies were simulated to determine the effect on seawater intrusion during a 5-year management period.

\section{Study Area}

The study area is situated on a south-facing section of coastline in southeastern Santa Barbara County, in southern California about 85 miles (mi) west-northwest of Los Angeles. The study area includes the Santa Barbara and Foothill groundwater basins on the Santa Barbara coastal plain, a narrow strip of land about 2 mi wide between the Santa Ynez Mountains to the north and the Pacific Ocean to the south (fig. 1). The coastal plain extends beyond the study area to the Goleta groundwater basin to the west and the Montecito groundwater basin to the east.

The study area includes much of the city of Santa Barbara, which had an estimated population of 91,842 in 2015 (United States Census Bureau, 2017). The city of Santa Barbara covers approximately 19 square miles and has a population density of approximately 4,800 people per square mile.

\section{Climate}

The study area has a Mediterranean-like climate, with warm summers and mild winters. Average temperatures range from highs around 65 degrees Fahrenheit $\left({ }^{\circ} \mathrm{F}\right)$ and lows around $45^{\circ} \mathrm{F}$ in the winter to highs around $75^{\circ} \mathrm{F}$ and lows around $60^{\circ} \mathrm{F}$ in the summer (National Weather Service, 2016). The study area has distinct wet and dry seasons; between 1972 and 2013, about 83 percent of the rain fell between November and May. The average annual rainfall from 1899 to 2013 measured at a rain gage at the Santa Barbara County building in downtown Santa Barbara was 18.55 inches (in.; County of Santa Barbara, 2014). The least and most recorded precipitation in the city of Santa Barbara fell in water year 2007 (6.41 in.) and water year 1998 (46.97 in.), respectively (County of Santa Barbara, 2014; fig. 2A). From 1900 to 2013, the study area has experienced several sequences of wet and dry periods, as indicated by a cumulative-departure from the mean curve (fig. 2B).

\section{Land Use}

Land use during the study period (1929-2013) was relatively stable, and it was assumed that the 1992 land-use map was representative of the land-use conditions during the study period. In 1992, 70 percent of the land use was residential and commercial-industrial; 21 percent was shrubforest or grassland; 5 percent was planted or cultivated land or planted grasses; 2 percent was barren; and less than 1 percent was wetlands (fig. 3). Commercial-industrial land use was most prevalent in downtown Santa Barbara near the coast. Small parks and golf courses were scattered throughout the study area. Forests, shrubs, and grasslands characterized the parks, and planted grasses were used in the golf courses. Small ranches in the foothills of the northern region of the western study area accounted for much of the planted and cultivated land use. Wetlands were confined to areas along the coast in the eastern-most area of the study area. 

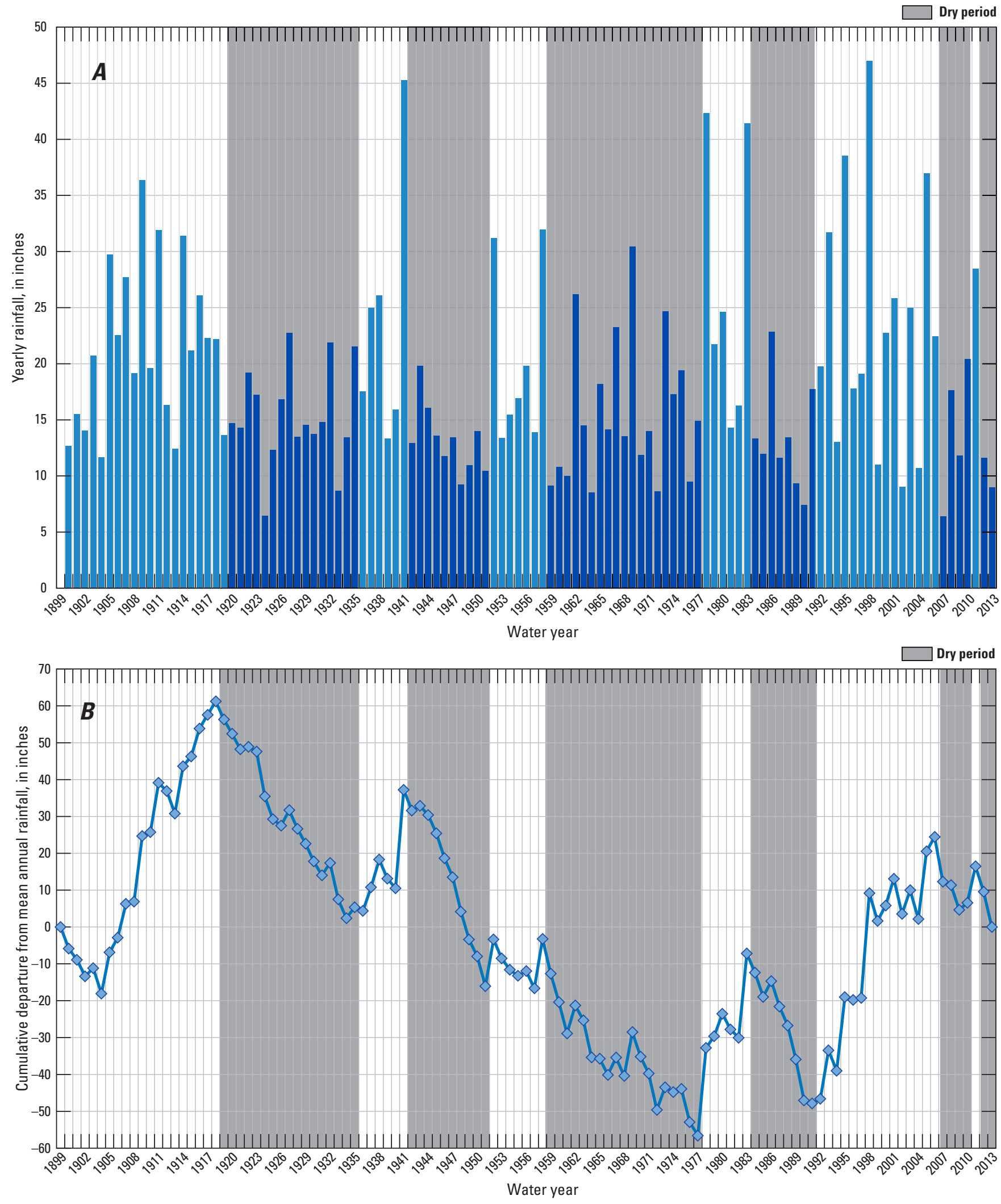

Figure 2. Precipitation measured at the Santa Barbara County building (1899-2013), by water year, Santa Barbara, California: $A$, total annual precipitation, and $B$, cumulative departure from mean. 

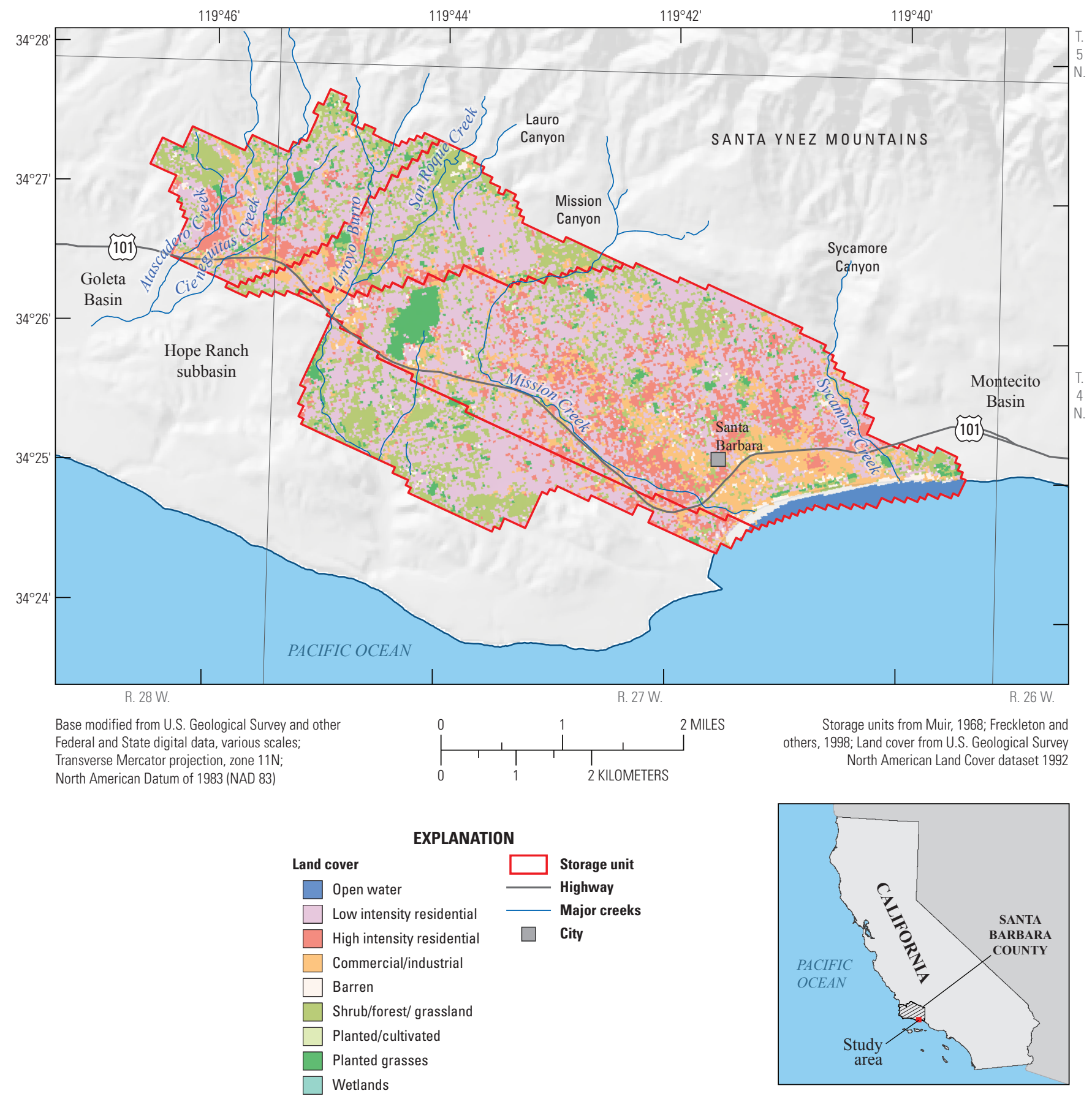

Figure 3. Distribution of land-use patterns, North American Land Cover Dataset 1992, Santa Barbara, California. 


\section{Geology and Structure}

Previous studies have described the geology and structure of the Santa Barbara groundwater basin in great detail (summarized by Minor and others, 2009). A general overview of the geology is presented in this report; the reader is referred to Minor and others (2009) for additional information.

The Santa Barbara groundwater basin is part of the coastal plain and is in the western Transverse Ranges physiographic province (Minor and others, 2009). The western Transverse Ranges consist mainly of variably deformed marine and nonmarine sedimentary rocks and deposits that range in age from Jurassic to the present. The coastal plain includes several mesas and hills that represent potentially active structures of the Santa Barbara fold and fault belt that transects the coastal plain. The study area is dominated by oblique-slip reverse and thrust faulting. Bounding the study area are the Rocky Nook and Lagoon faults to the north and the Lavigia and Modoc faults to the south (fig. 4). An unnamed fault, about $0.25 \mathrm{mi}$ offshore extending from the Mesa fault eastward, truncates deeper deposits so they lie against consolidated deposits on the seaward side of the fault. Outcrops of sedimentary rock can be observed in the local
Santa Ynez Mountains where oblique-slip reverse and thrust faulting has uplifted and overturned many of the formations (Minor and others, 2009).

The Santa Barbara groundwater basin consists of tertiary sedimentary rock overlain by unconsolidated to partially consolidated deposits. The sedimentary rock, mostly marine in origin, consists primarily of shale, mudstone, sandstone, and conglomerate.

Overlying the consolidated units are marine and nonmarine unconsolidated and partially consolidated deposits of the Santa Barbara Formation of late Pliocene and Pleistocene age and alluvium of Holocene age. A medium- to coarsegrained sand with fine gravel near the base of the Santa Barbara Formation extends inland several miles from the coast. Most of the surficial deposits in the study area are either Pleistocene moderately consolidated poorly sorted sands, gravels, and conglomerates deposited as alluvial fans from the Santa Ynez Mountains or Holocene and upper Pleistocene poorly consolidated silts, sands, and gravels deposited from modern drainages, alluvial fans, and floodplains (Minor and others, 2009). The study area also contains small areas of Holocene estuarine deposits of organically rich clay, silt, and sand along low-lying coastal areas (fig. 4). 


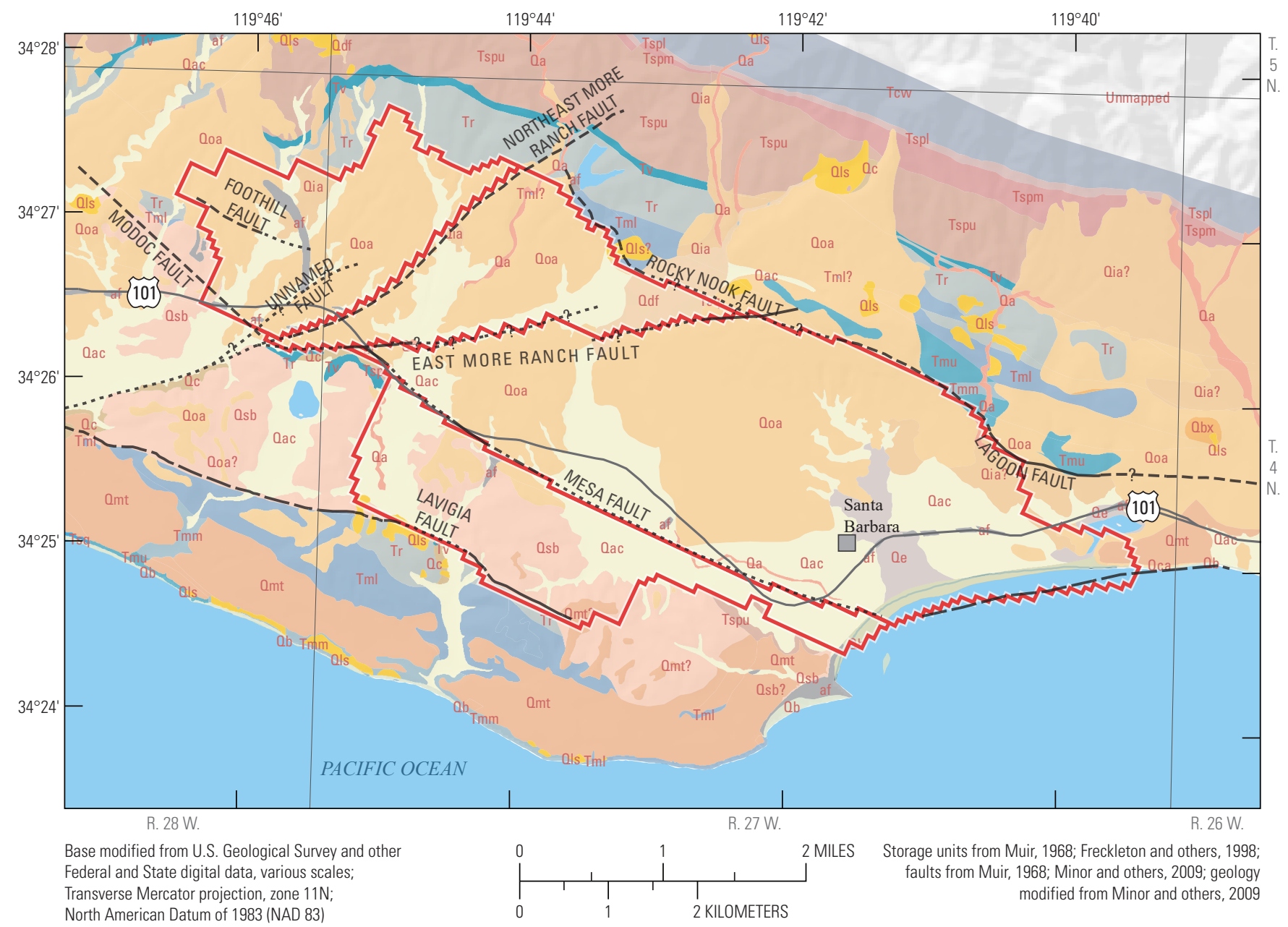

EXPLANATION

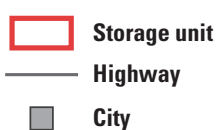

City

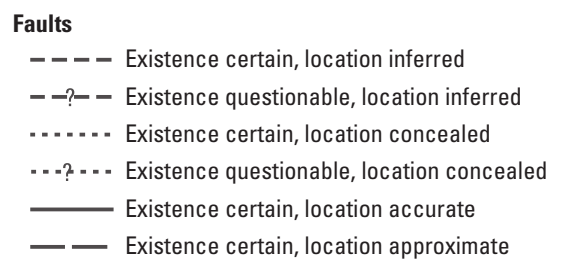

\author{
Geology \\ af - Artificial fill (Holocene) \\ Qa - Active channel alluvium (Holocene) \\ Qb - Beach deposits (Holocene) \\ Qe - Estuarine deposits (Holocene) \\ Qdf - Debris flow deposits (Holocene and upper Pleistocene?) \\ Oac - Alluvium and colluvium (Holocene and upper Pleistocene) \\ Qc - Colluvium (Holocene and upper Pleistocene) \\ Qls - Landslide deposits (Holocene and Pleistocene) \\ Qia - Intermediate alluvial deposits (upper Pleistocene) \\ Omt - Marine-terrace deposits (upper Pleistocene) \\ Qoa - Older alluvial deposits (upper and middle Pleistocene) \\ 0ca - Casitas Formation (upper and middle Pleistocene) \\ Qbx - Shale-clast sedimentary breccia (middle Pleistocene)
}

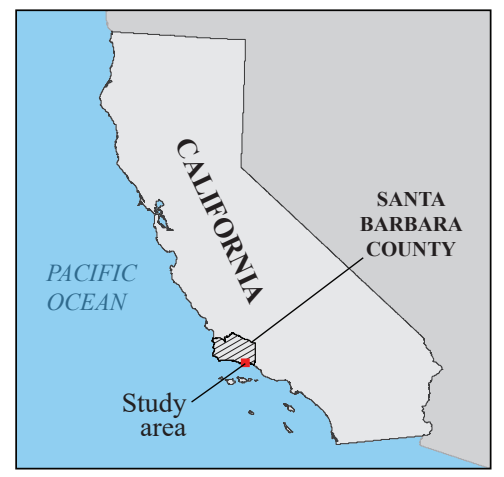

\begin{tabular}{l}
\hline Osb - Santa Barbara Formation (middle and lower Pleistocene) \\
\hline \hline Tsq - Sisquoc Formation (lower Pliocene and upper Miocene) \\
\hline \hline Tmu - Monterey Formation, upper siliceous unit (upper Miocene) \\
Tmm - Monterey Formation, middle shale unit (upper and middle Miocene) \\
\hline \hline Tml - Monterey Formation, lower calcareous unit (middle and lower Miocene) \\
\hline \hline Tr - Rincon Shale (lower Miocene) \\
Tv - Vaqueros Formation (upper Oligocene) \\
\hline \hline Tspu - Sespe Formation, upper sandstone and mudstone unit (upper Oligocene) \\
\hline \hline Tspm - Sespe Formation, middle conglomerate and sandstone unit (Oligocene) \\
\hline \hline Tspl - Sespe Formation, lower conglomerate and sandstone unit (lower Oligocene? and upper Eocene) \\
\hline
\end{tabular}

Figure 4. Surface geology and faults of the Santa Barbara area, California. 


\section{Hydrogeology}

The hydrogeology of the Santa Barbara and Foothill groundwater basins was defined by summarizing previously published research (for example, Muir, 1968; Martin and Berenbrock, 1986; Freckleton and others, 1998). In addition, a new hydrogeologic zone is described.

\section{Description of the Aquifer System}

There are two main groundwater basins in the study area that are separated by faults: the Santa Barbara groundwater basin and the Foothill groundwater basin (fig. 5). The Santa Barbara groundwater basin contains two subbasins, "Storage Unit I" and "Storage Unit III" (Muir, 1968). The Foothill basin is divided by a fault into two subbasins; for the purposes of this report, these subbasins are named the East and West Foothill subbasins (fig. 5). Storage Unit I underlies the main part of the city of Santa Barbara and is the main focus of this report; less emphasis is placed on Storage Unit III and the two Foothill subbasins. Storage Unit I and the Foothill subbasins are the main sources of potable groundwater for the city of Santa Barbara.

\section{Faults and Groundwater Flow}

In the study area, many of the faults separate the subbasins and act as partial barriers to groundwater flow (Freckleton and others, 1998). The Rocky Nook and Lagoon faults to the north and the Lavigia and Modoc faults to the south (fig. 4) are assumed to restrict groundwater flow into the basin and, therefore, define parts of the basin boundary. The East More Ranch fault acts as a groundwater barrier separating the Foothill groundwater basin from the Santa Barbara groundwater basin. The Mesa fault divides Storage Unit I from Storage Unit III in the Santa Barbara groundwater basin. Additionally, the Northeast More Ranch fault divides the Foothill groundwater basin into two subbasins, East Foothill and West Foothill (Minor and others, 2009, fig. 4). The Foothill fault and an unnamed fault could act as groundwater barriers in the West Foothill subbasin. The unnamed fault about $0.25 \mathrm{mi}$ offshore that extends from the Mesa fault eastward is thought to impede groundwater flow (Freckleton and others, 1998).

\section{Hydrogeologic Zones}

The Santa Barbara and Foothill groundwater basins consist primarily of unconsolidated silts, sands, and gravels deposited from drainages, alluvial fans, and floodplains. Based on electric and geologic logs, Martin and Berenbrock
(1986) divided the unconsolidated deposits into five zones representing the hydraulic characteristics of the layers: the shallow, upper producing, middle, lower producing, and deep zones. Based on additional analyses, the middle zone contains a thin, not very extensive, but productive zone, referred to as the "middle producing zone." The three main productive zones in the basins and their extents are shown on figure 6. A description of each of the zones and how the middle producing zone was defined is given below. Chapter B describes a hydrogeologic framework model defining the extent and characteristics of all the zones.

\section{Shallow Zone}

The shallow zone includes alluvium from land surface to the top of the upper producing zone. In general, it is composed of fine-grained deposits that confine or partly confine the upper producing zone.

\section{Upper Producing Zone}

The upper producing zone, one of the main water-bearing units in the study area, consists predominately of medium- to coarse-grained sand and fine gravel. It averages about $50 \mathrm{ft}$ thick (fig. 7). The upper producing zone extends through much of the Santa Barbara and Foothill groundwater basins and pinches out to the north near the Santa Ynez foothills in Storage Unit I and East Foothill subbasin and to the east of Las Positas Creek in Storage Unit III (fig. 6A).

\section{Middle Zone}

As defined by Martin and Berenbrock (1986), the middle zone consists of fine-grained deposits interspersed with sporadic coarse-grained water-bearing deposits, which confine or partially confine the lower producing zone. The middle zone separates the upper and lower producing zones in most of the study area. The middle zone pinches out in the northwestern part of Storage Unit I, where the upper and lower producing zones are in contact with each other. A downholevelocity survey of the Corporation Yard well (4N/27W-15Q10, fig. 5) and resistivity logs from several wells perforated in the middle zone indicated a thin, not very extensive, but productive zone of coarse-grained deposits at a depth of about 360-380 ft below NAVD 88 near the coast (Pueblo Water Resources, written commun., 2013). The results from the velocity survey indicated that 17 percent of total production from the Corporation Yard well was extracted from 410 to $430 \mathrm{ft}$ bls, 21 percent was from the upper producing zone, and 36 percent was from the lower producing zone (table 1); the balance of production was extracted from other formations. The middle producing zone was not identified in previous studies, but was identified in this study. 


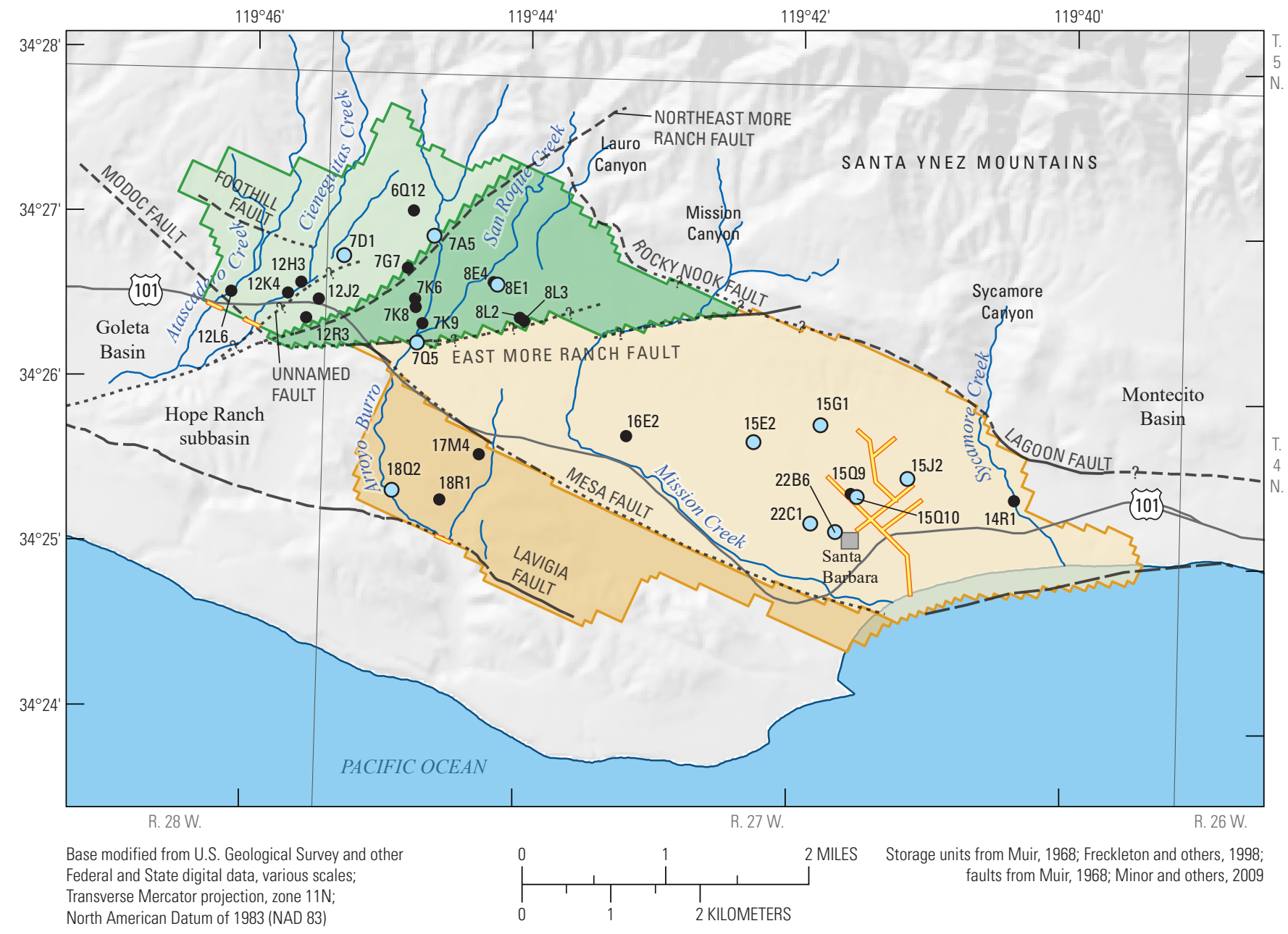

\section{EXPLANATION}
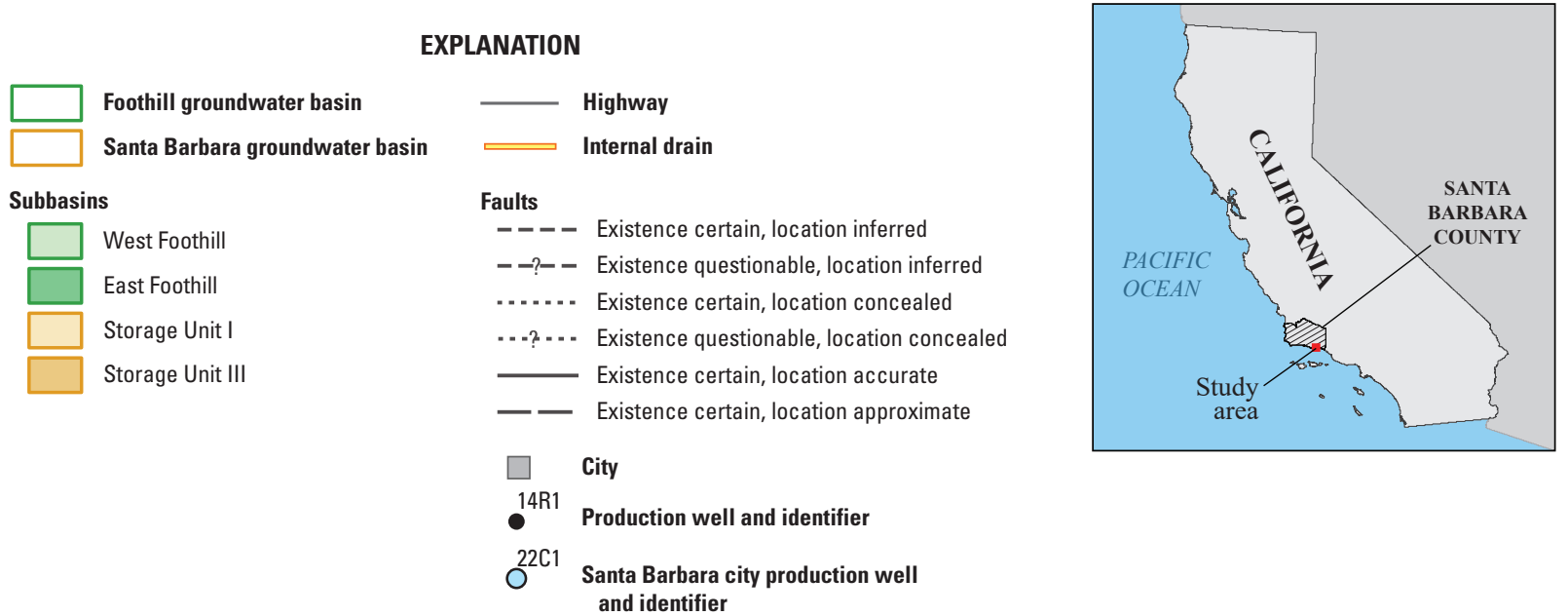

Figure 5. Santa Barbara and Foothill groundwater subbasins, creeks, faults, production wells, and drains, Santa Barbara, California. 

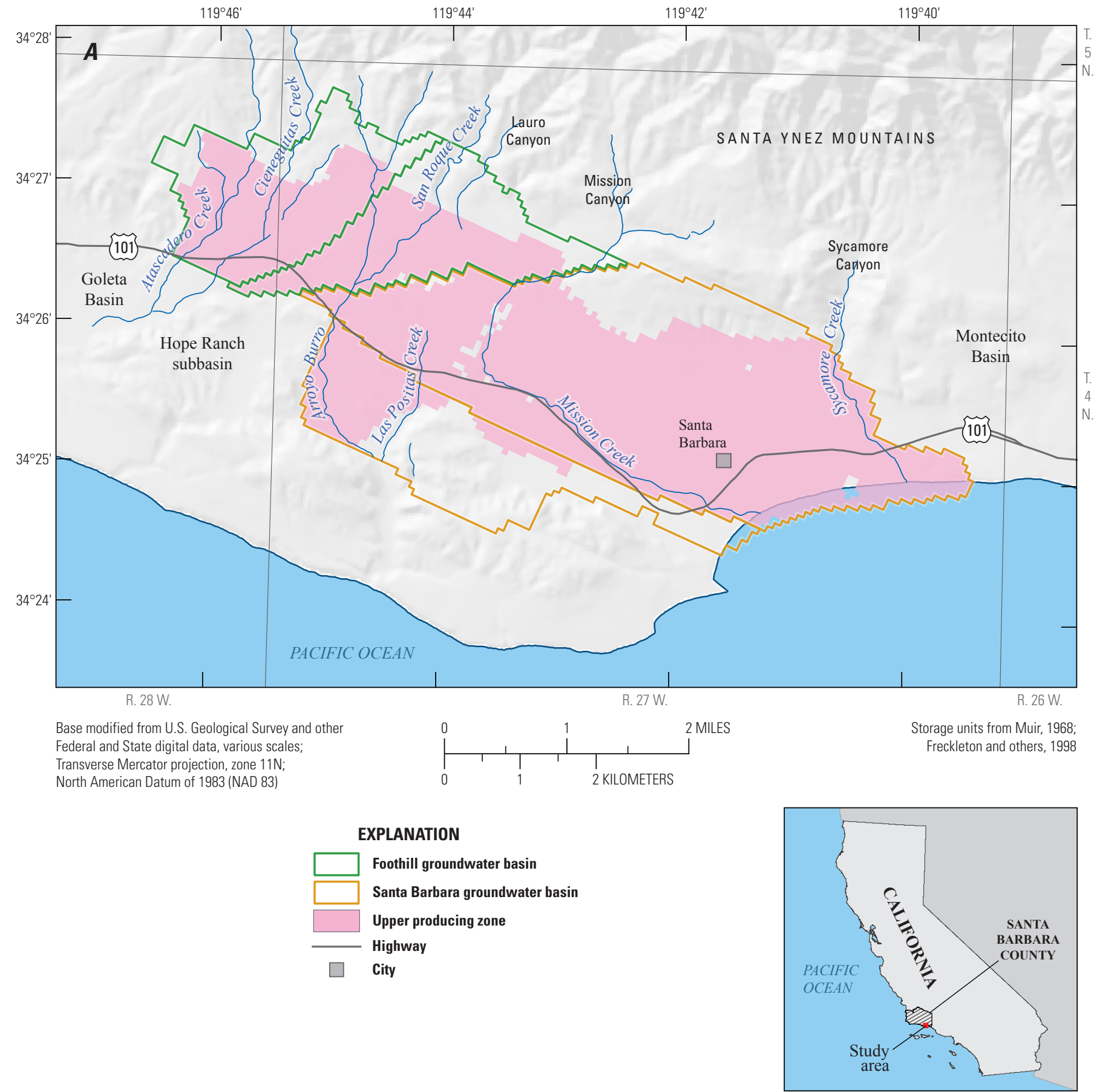

Figure 6. Lateral extent of the upper, middle, and lower producing zones in the Santa Barbara and Foothill groundwater basins, Santa Barbara, California: $A$, upper producing zone, $B$, middle producing zone, and $C$, lower producing zone. 


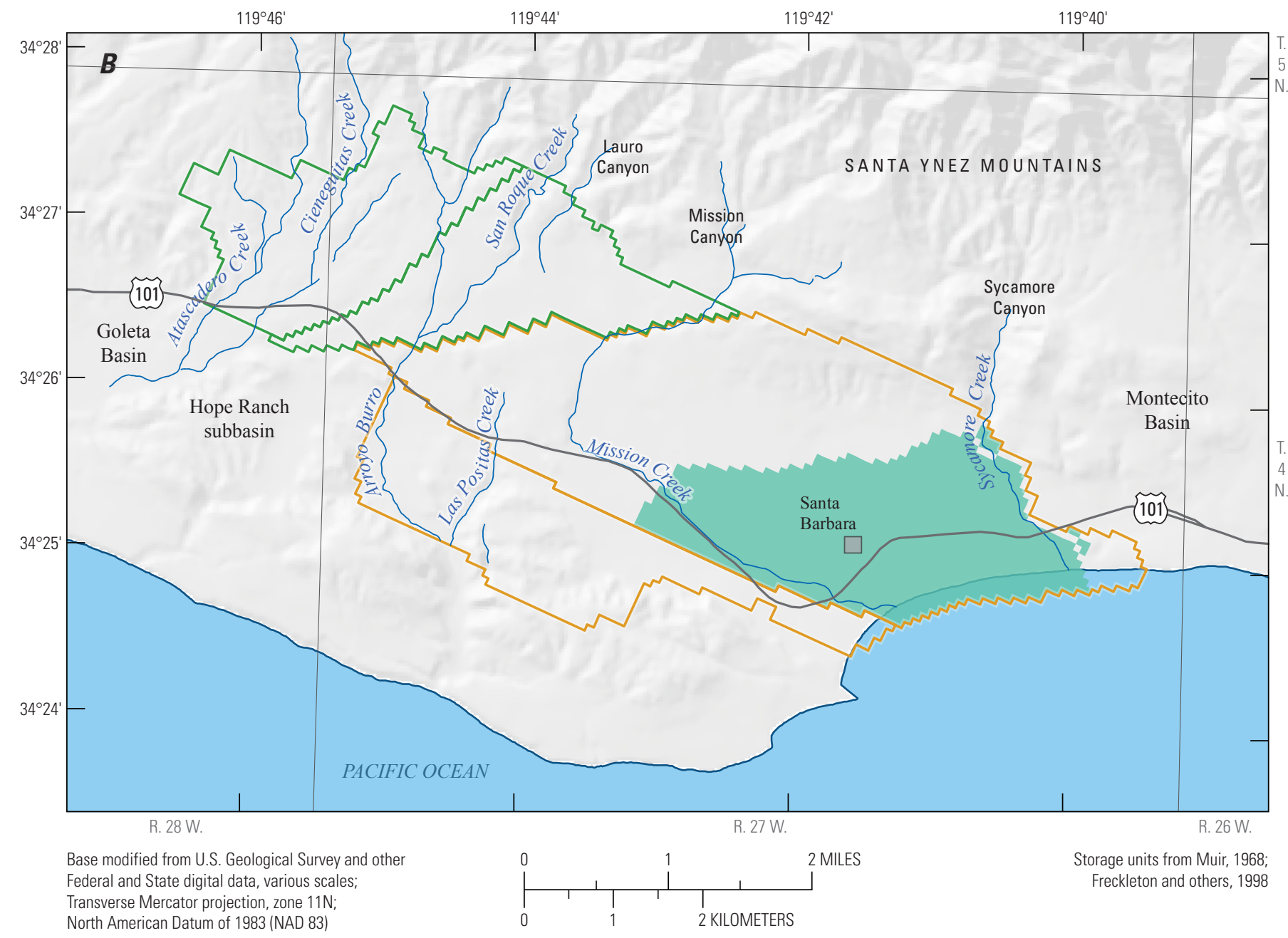

(1)

\section{EXPLANATION}

Foothill groundwater basin

Santa Barbara groundwater basin

Middle producing zone

Highway

City

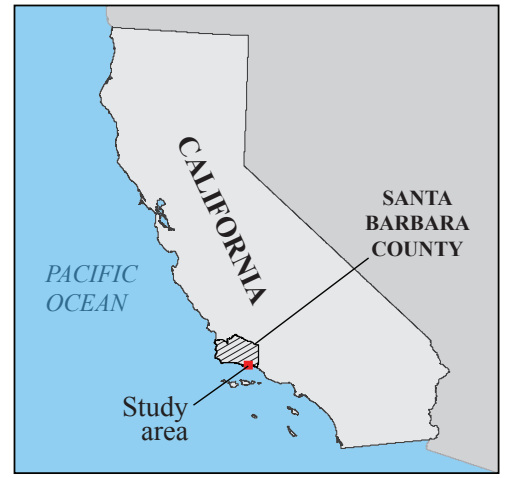

Figure 6. - Continued 


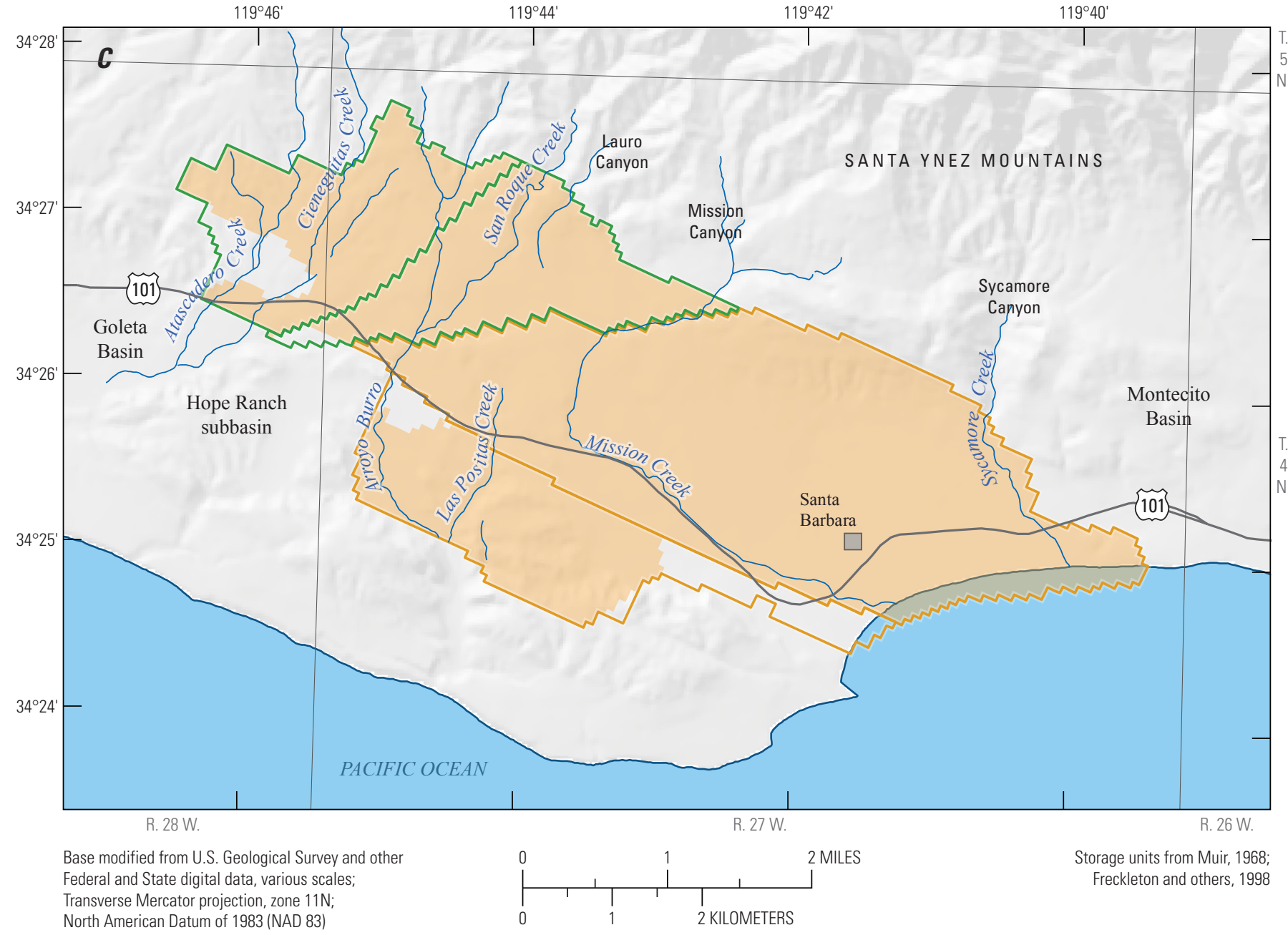

\section{EXPLANATION}

Foothill groundwater basin

Santa Barbara groundwater basin

Lower producing zone

Highway

City

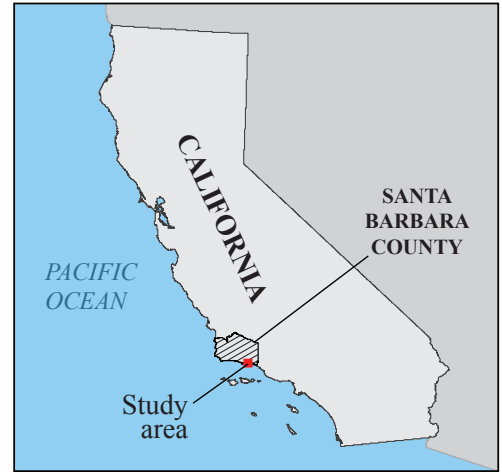

Figure 6. - Continued 

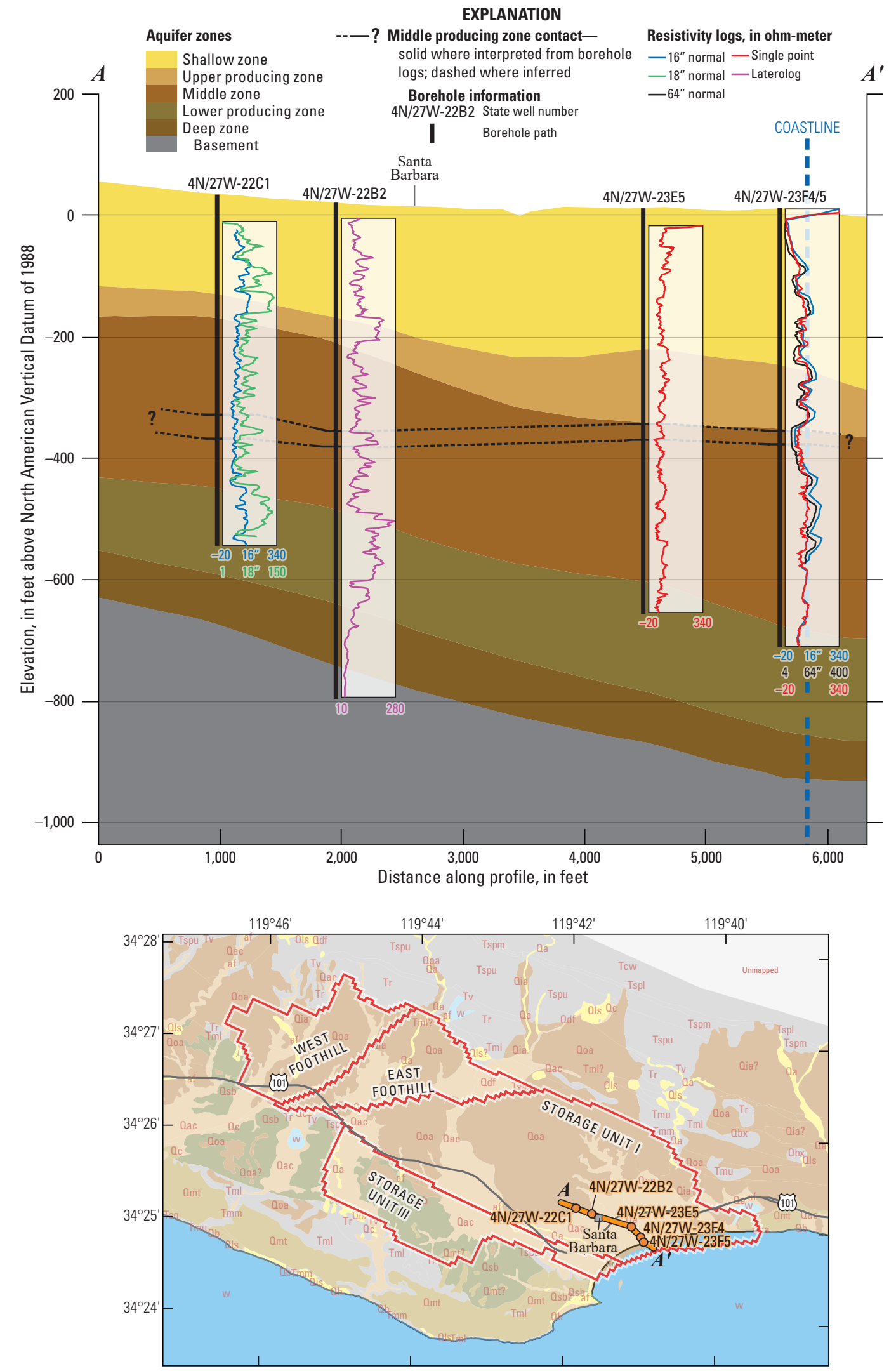

Figure 7. Storage Unit I from the northwest to the southeast coast (from left to right), showing resistivity logs for selected wells and aquifer-zone horizons defined by the hydrogeologic framework model (see chapter B), Santa Barbara groundwater basin, Santa Barbara, California. 
Table 1. Velocity-profile testing results for the Corporation Yard well, Santa Barbara, California.

[Pueblo Water Resources, written commun., 2013, table 3. Abbreviations: bls, below land surface; ft, feet; gpm, gallons per minute; - , not applicable]

\begin{tabular}{|c|c|c|c|c|c|c|}
\hline \multirow[b]{2}{*}{$\begin{array}{l}\text { Screen } \\
\text { intervals } \\
\text { (ft bls) }\end{array}$} & \multirow[b]{2}{*}{$\begin{array}{l}\text { Thickness } \\
\text { (ft) }\end{array}$} & \multicolumn{5}{|c|}{ Percentage of total production } \\
\hline & & $\begin{array}{c}\text { Step } 1 \\
411 \\
\text { gpm }\end{array}$ & $\begin{array}{c}\text { Step } 2 \\
602 \\
\text { gpm }\end{array}$ & $\begin{array}{c}\text { Step } 3 \\
817 \\
\text { gpm }\end{array}$ & $\begin{array}{l}\text { Step } 4 \\
1,006 \\
\text { gpm }\end{array}$ & Averages \\
\hline \multicolumn{7}{|c|}{ Upper producing aquifer zone } \\
\hline $190-205$ & 15 & 3 & 9 & 2 & 12 & 6 \\
\hline $225-270$ & 45 & 15 & 14 & 15 & 14 & 14 \\
\hline Subtotal & 60 & 17 & 23 & 16 & 25 & 21 \\
\hline \multicolumn{7}{|c|}{ Middle aquifer zone } \\
\hline $350-395$ & 45 & 10 & 9 & 20 & 7 & 12 \\
\hline $410-430$ & 20 & 16 & 16 & 18 & 17 & 17 \\
\hline $450-460$ & 10 & 3 & 6 & 5 & 6 & 5 \\
\hline $490-510$ & 20 & 14 & 11 & 4 & 13 & 10 \\
\hline Subtotal & 95 & 42 & 42 & 47 & 44 & 44 \\
\hline \multicolumn{7}{|c|}{ Lower producing aquifer zone } \\
\hline $580-605$ & 25 & 15 & 14 & 15 & 16 & 15 \\
\hline $615-655$ & 40 & 26 & 21 & 22 & 15 & 21 \\
\hline Subtotal & 65 & 41 & 35 & 36 & 31 & 36 \\
\hline Total & 220 & 100 & 100 & 100 & 100 & - \\
\hline
\end{tabular}

Resistivity logs for wells 4N/27W-22C1, -22B2, -22A2, and 4N/27W-23E5 display high resistivity readings in the middle zone at the interval around 360 to $380 \mathrm{ft}$ below NAVD 88 (fig. 7), indicating coarser sediments in this interval. Near the coastline, however, wells $4 \mathrm{~N} / 27 \mathrm{~W}-23 \mathrm{~F} 4$ and 4N/27W-23F5 showed low-resistivity readings over the same interval (fig. 7), which could be due to increased salinity or the presence of fine-grained materials. Groundwater samples collected from these wells have higher salinity levels at this interval, indicating that the resistivity drop is likely due to higher salinity levels and not fine-grained material. The higher salinity levels are confined to this narrow interval of coarsegrained materials because there are finer grained materials above and below this interval.

The coarse-grained deposits in the middle zone of Storage Unit I around 360-380 ft below NAVD 88 are defined as the middle producing zone. This analysis is based on the Corporation Yard well velocity survey, resistivity logs, and examination of drillers' logs throughout Storage Unit I, as described in chapter $\mathrm{B}$. The middle producing zone extends from the coastline in Storage Unit I to about 1-2 mi inland (fig. 6B), where it pinches out. It appears to terminate at the offshore fault to the southeast and at the Mesa fault to the southwest.

\section{Lower Producing Zone}

The lower producing zone, the third main water-bearing unit, consists of medium- to coarse-grained sand with fine gravel. The lower producing zone extends through much of the Santa Barbara and Foothill groundwater basins and thins or pinches out near the Santa Ynez foothills to the north and pinches out in southeastern Storage Unit III (fig. 6C). The lower producing zone thickens to about $200 \mathrm{ft}$ from north to south in Storage Unit I (fig. 7).

\section{Deep Zone}

In most of the study area, there is a deep zone of finegrained deposits of variable thickness below the lower producing zone (fig. 7). In general, these deposits have relatively low hydraulic conductivity, are reported to have poor water quality (Martin and Berenbrock, 1986), and are assumed to not be an important source of water.

\section{Pre-Development Recharge and Discharge}

The main components of pre-development groundwater recharge and discharge for the Santa Barbara and Foothill groundwater basins are stream (creek) leakage, smallcatchment recharge, areal recharge, underflow, and evapotranspiration. Ephemeral creeks originating from the Santa Ynez Mountains pass through the study area and empty into the Pacific Ocean. Creeks in the study area include Mission, Sycamore, Arroyo Burro, San Roque, Cieneguitas, and Atascadero Creeks (fig. 3). Depending on location, creeks can be either a source of groundwater recharge or mechanism of discharge. Martin (1984) identified segments of groundwater recharge and discharge along Mission Creek and estimated net recharge from Mission Creek to be $376 \mathrm{acre}-\mathrm{ft} / \mathrm{yr}$ on the basis of seepage-loss measurements. Freckleton and others (1998) estimated the long-term, mean groundwater recharge from creeks was about 925 acre-ft/yr, and the steady-state groundwater discharge to creeks was as high as 1,700 acre-ft/yr, yielding a net loss of groundwater to creeks of about 775 acre-ft/yr. Note that Freckleton and others (1998) used MODFLOW's Drain Package (Harbaugh and others, 2000) to simulate the groundwater discharge to creeks and the drain system beneath downtown Santa Barbara, but did not differentiate between these losses; therefore, the groundwater discharge to creeks was probably less than 1,700 acre-ft/yr.

For this study, small-catchment recharge is defined as runoff and underflow from the Santa Ynez Mountains and foothills. Muir (1968) estimated an average of approximately $300 \mathrm{acre}-\mathrm{ft} / \mathrm{yr}$ of recharge to the Santa Barbara groundwater basin as underflow from the consolidated rocks that form the majority of the boundary between the Santa Barbara groundwater basin and the Santa Ynez foothills. 
Areal recharge is the amount of direct infiltration from precipitation in the study area. As stated earlier, the average annual precipitation for the study area is 18.55 in. Not all precipitation that falls in the study area recharges the groundwater basins. A large percentage of precipitation runs off directly to the ocean, predominantly in the urban areas of the city, where much of the area is paved, and water runs through the city's drain system to the ocean. Some of the precipitation that infiltrates the vadose zone is evaporated or is transpired by plants before reaching the groundwater system. Freckleton and others (1998) simulated the total steadystate areal recharge to be about 1,100 acre-ft/yr for the Santa Barbara and Foothill groundwater basins.

Groundwater can leave the basins laterally as underflow across the offshore fault from the Santa Barbara groundwater basin, at Arroyo Burro Creek across the Lavigia fault in Storage Unit III, at Atascadero and Cieneguitas Creeks across the Modoc fault from the Foothill to the Goleta groundwater basin, or from Storage Unit I to the Montecito groundwater basin (fig. 5). Freckleton and others (1998) simulated predevelopment discharge across the offshore fault to be about 290 acre-ft/yr and discharge across the Lavigia fault to be about 24 acre-ft/yr. Freckleton and others (1998) did not simulate the flux across the Modoc fault and treated the boundary between Storage Unit I and Montecito groundwater basin as a no-flow boundary; however, no known geologic feature prevents flow between the two basins.

An area of the city of Santa Barbara was originally part of the Santa Barbara Estero, a marshy lagoon (Martin and Berenbrock, 1986). Because artesian groundwater conditions existed before development in much of this part of the basin, groundwater most likely exited the Santa Barbara groundwater basin through evapotranspiration in the Santa Barbara Estero. Martin and Berenbrock (1986) estimated the steady-state groundwater loss to the Estero was about 400 acre-ft/yr.

\section{Post-Development Effects on Groundwater System}

Development in the study area has had several effects on groundwater recharge, flow, and discharge. The major effect has been from groundwater pumping; however, development has had other effects as well. Limited groundwater pumpage data were available prior to 1947; records are more complete from 1947 to present. The available data show that groundwater pumpage has varied greatly over the past 85 years (fig. 8). From water year 1949 through water year 1991, there was substantial groundwater pumping in the study area, with pumpage averaging over 1,700 acre-ft/yr for the Santa Barbara and Foothill groundwater basins. For comparison, Freckleton and others (1998) reported that the total simulated outflow from the basins under pre-development conditions was about 2,030 acre-ft/yr. Since water year 1992, groundwater pumping in Storage Unit I has been reduced, with pumpage averaging less than $200 \mathrm{acre}-\mathrm{ft} / \mathrm{yr}$. There were three main periods of heavier pumping in the Foothill groundwater basin, from water years 1964 to 1970, 1985 to 1991, and 2008 to 2013 (fig. 8), with pumpage averaging 831, 1,005, and 869 acre-ft/yr, respectively. During dry years, groundwater pumping accounts for a substantial part of the discharge in Santa Barbara and Foothill groundwater basins.

Septic tanks are used for wastewater treatment in the parcels shown in figure 9. These data were provided by the Santa Barbara County Clerk-Recorder-Assessor's Mapping Division. There were 195 single-family residences (SFR), 16 multi-unit dwellings (MUD) with 2-4 units, one rest home, one restaurant, and one retail store with septic systems. Eckenfelder (1980, in table 4.5) reported an average septiceffluent flow of 90 gallons per day (gal/d) for each person in "better subdivisions." This average yields a total septic flowrate of about $65 \mathrm{acre}-\mathrm{ft} / \mathrm{yr}$, if one assumes 2.5 residents for each SFR (U.S. Census Bureau, 2017) and 10 people for each MUD, rest home, restaurant, and store (assuming 4 units in each MUD and 2.5 people in each unit).

The land use in the study area is predominantly residential and commercial (fig. 3). Below an elevation of $100 \mathrm{ft}$, storm sewers, city streets, and buildings prevent much infiltration of rain (Muir, 1968). In addition, the concrete lining of the lower section of Mission Creek has reduced groundwater interaction with the creek. Likewise, a network of drains was installed near land surface to dewater the Santa Barbara Estero area prior to development (fig. 5). This network continues to drain runoff and groundwater to the ocean during storm events.

\section{Pre-Development Groundwater Movement}

Martin and Berenbrock (1986) reported that under unpumped conditions (presumably similar to pre-development conditions) groundwater movement in Storage Unit I was generally from the northwest and north toward the Pacific Ocean. Martin and Berenbrock (1986, fig. 3) also indicated that pre-development groundwater movement in the Foothill groundwater basin was to the west-southwest across the Modoc fault. Pre-development groundwater movement in Storage Unit III was easterly toward the Pacific Ocean (Martin and Berenbrock, 1986, fig. 3). 


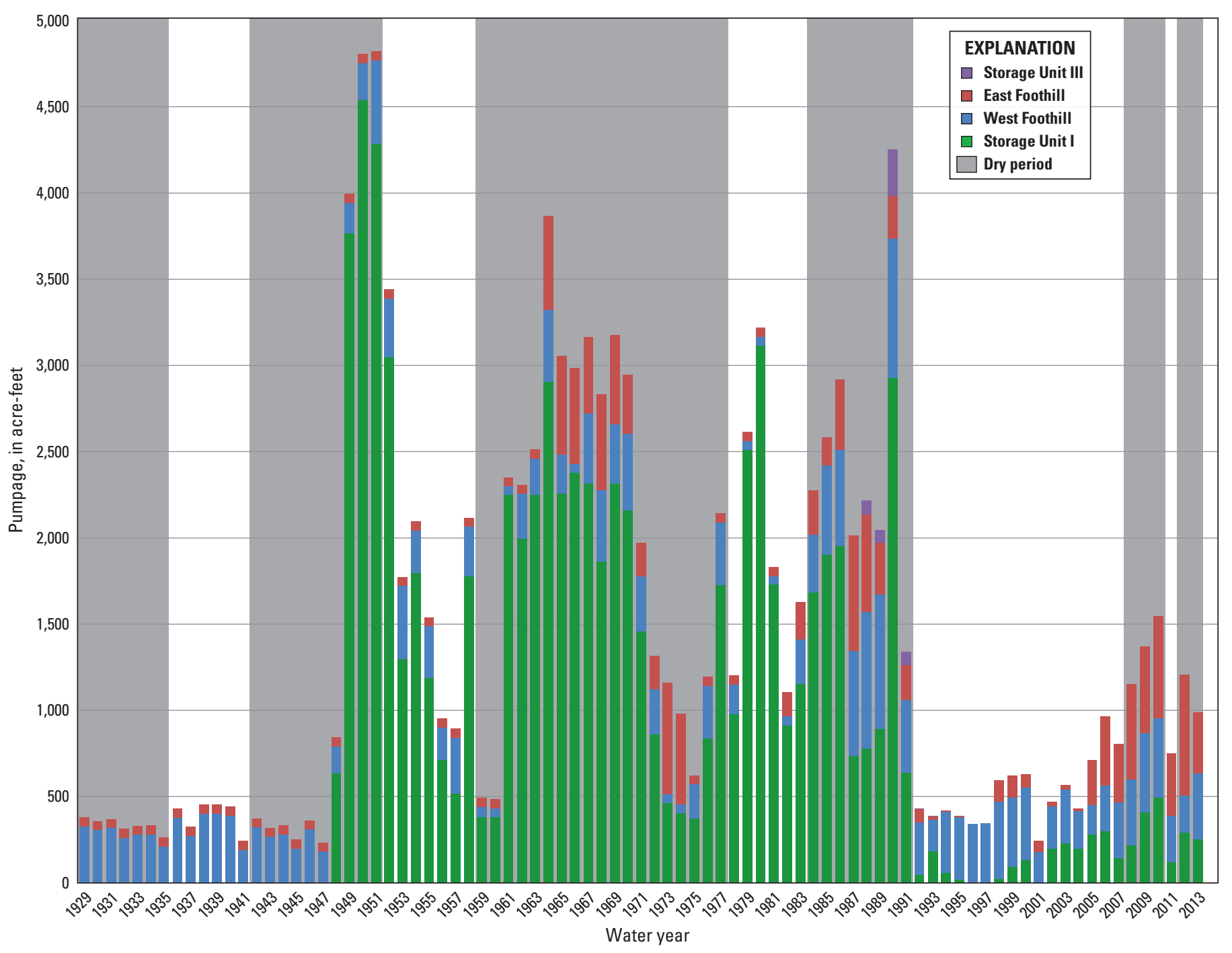

Figure 8. Estimated and reported total annual pumpage in the Santa Barbara and Foothill groundwater basins, water years 1929-2013, Santa Barbara, California. 

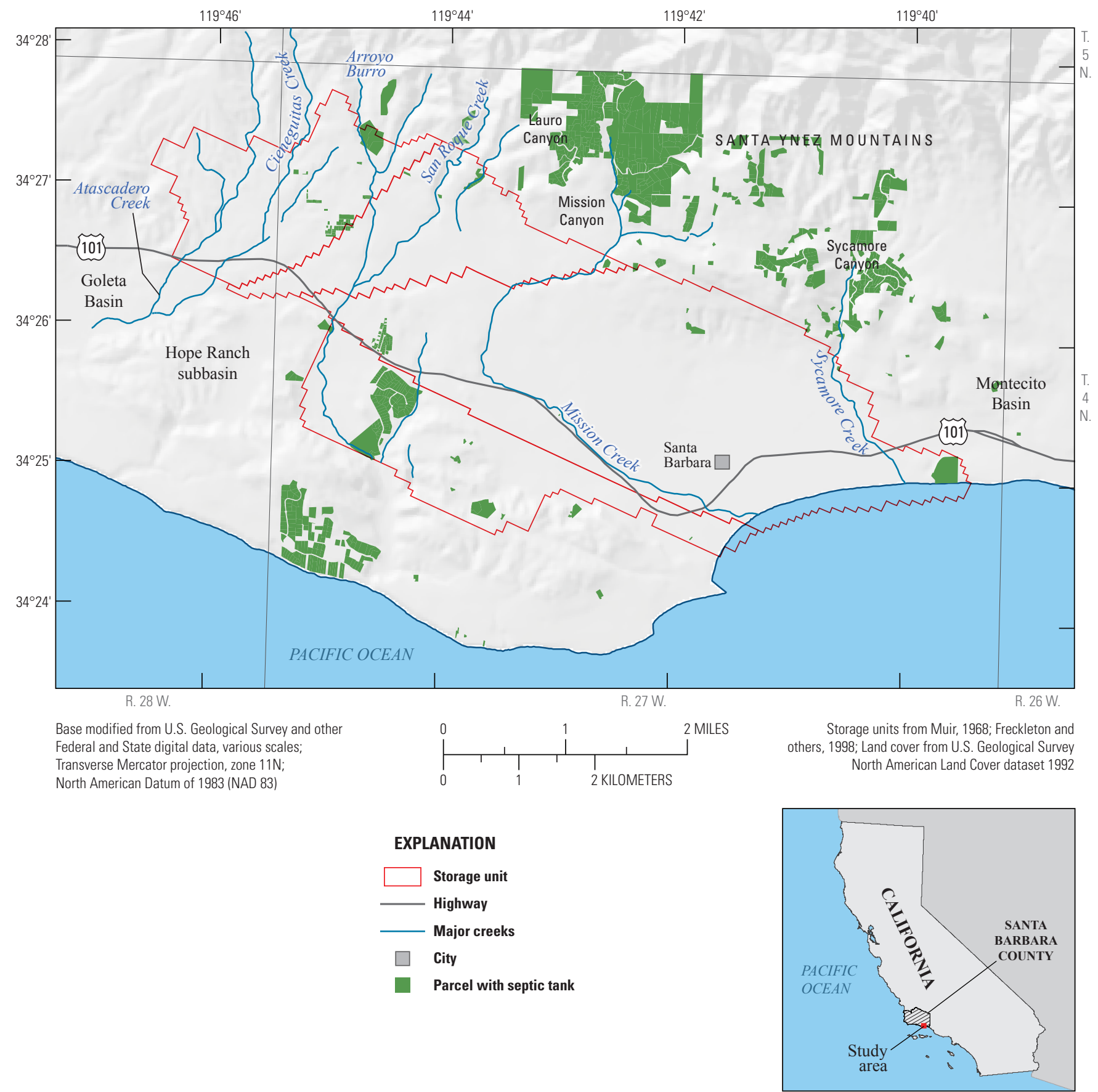

Figure 9. Parcels with septic tanks, Santa Barbara, California (parcel data from Santa Barbara County, Mapping Division, 2011). 


\section{Post-Development Groundwater Levels and Movement}

The groundwater-level contours of the lower producing zone, based on measured water levels from the summer of 1990 , the end of an extended period of high pumping, and from the spring of 1997, after several years of low pumping, are shown in figures $10 A$ and $B$, respectively. Water-level data from the lower producing zone were used because of limited data availability for the upper and middle producing zones; also, most groundwater was pumped from the lower producing zone.

The summer of 1990 was the end of a prolonged period of increased pumpage between 1985 and 1990 (fig. 8), when total annual pumping in the study area varied from 2,000 to 4,300 acre-ft/yr. Pumping in the Foothill groundwater basin resulted in water levels as low as about $70 \mathrm{ft}$ above NAVD 88 (fig. 10A). Flow directions in Foothill groundwater basin were generally from northeast to southwest, except near production wells, where flow directions were radially inward toward the production well. Pumping in Storage Unit I resulted in water levels as low as $90 \mathrm{ft}$ below NAVD 88 in the vicinity of production wells and in a large area of below sea level water levels in the southeastern part of Storage Unit I (fig. 10A). Flow in Storage Unit I was generally from northwest to southeast, except in the southeastern part, where flow directions were radially inward toward the production wells. The radial flow extended through much of the southeastern region of Storage Unit I between the Lagoon and Mesa faults.

In the spring of 1997, the flow direction in Foothill groundwater basin was from northeast to southwest; the highest heads were near the intersection of Mission Creek and Rocky Nook fault and were at least $170 \mathrm{ft}$ above NAVD 88 and could be much higher, and lowest heads were less than $100 \mathrm{ft}$ above NAVD 88 and were near the border of Foothill and Goleta groundwater basins (fig. 10B). Flow in Storage Unit I and Storage Unit III was from northwest to southeast; the highest heads were near the intersection of the East More Ranch and Mesa faults, and the lowest heads were near the coast.

Water levels in the Foothill groundwater basin decreased during the late 1980s through the early 1990s, which was followed by a recovery period with increasing water levels from the mid-1990s through 2004, and then by another period of decreasing water levels from 2005 through 2014. Monitoring well 4N/27W-08M6 (located adjacent to well 8M5 in fig. 10) is perforated in the upper producing zone and is in the middle of the East Foothill subbasin about $0.25 \mathrm{mi}$ southwest of the San Roque Park production wells (8E4; table 2) and about $0.4 \mathrm{mi}$ west of the McKenzie production wells (8L2, 8L3; fig. 5). The hydrograph for this well (fig. 11) shows drawdown from the late 1980s through the early 1990s during a period of high pumping from the McKenzie \#2 (8L3) well. Water levels dropped below $130 \mathrm{ft}$ during this period. As pumping decreased in this well during the mid-1990s, water levels in 4N/27W-08M6 recovered through 2004, when they peaked close to $180 \mathrm{ft}$. Beginning in 2005, the San Roque Park \#2 (8E4) well started pumping, leading to another period of drawdown through 2015, when water levels dropped below $120 \mathrm{ft}$.

Monitoring well 4N/28W-12H04 is perforated in the lower producing zone and is in the West Foothill subbasin about 0.2 mi north of La Cumbre Mutual Water Company Well \#16 production well (12J2) and about $0.25 \mathrm{mi}$ southwest of the Los Robles production well (7D1). Groundwater levels from this well indicated a similar drawdown and recovery pattern as monitoring well 4N/27W-08M6 (fig. 11).

Water levels in Storage Unit I fluctuated during much of the 1980s, such that water levels declined substantially between the late 1980s and early 1990s in response to groundwater pumping. Water levels started increasing in 1991 and continued to recover through 1999, when they leveled off and then slowly declined through 2014 in the lower part of the basin, compared to a more rapid decline in the upper part of the basin. Well $4 \mathrm{~N} / 27 \mathrm{~W}-16 \mathrm{C} 1$ is perforated in the lower producing zone and is representative of the inland part of Storage Unit I, upgradient of most of the production wells in Storage Unit I. Water levels in 4N/27W-16C1 fluctuated with pumping between 90 and $105 \mathrm{ft}$ from 1982 to 1988 (fig. 11). Water levels dropped abruptly in 1989 and were at $70 \mathrm{ft}$ by late 1990. Water levels recovered during the 1990s, peaking at about $115 \mathrm{ft}$ in 1999. From 1999 through 2015, water levels declined, and the annual fluctuations in water levels increased.

Well $4 \mathrm{~N} / 27 \mathrm{~W}-22 \mathrm{~A} 4$ is perforated in the lower producing zone between the main production wells in Storage Unit I and the coast. It is representative of the coastal part of Storage Unit I. In general, the water levels for this well were much lower than those measured in the inland part of Storage Unit I, Foothill groundwater basin, and Storage Unit III (fig. 11). During the late 1980s through 1990, water levels fluctuated greatly, varying from $20 \mathrm{ft}$ in 1989 to $-80 \mathrm{ft}$ in 1990 (fig. 11). Water levels recovered starting in 1990 , peaking at $52 \mathrm{ft}$ in 1996. From 1999 through 2009, water levels generally trended downward to about $25 \mathrm{ft}$ by 2014 . Water levels decreased to about $-60 \mathrm{ft}$ by late 2016 The below-sea-level water levels in the late 1980s to early 1990s, and again in the mid-2010s, indicate that seawater could have intruded the aquifer during these periods.

Water levels fluctuated more in the inland part of Storage Unit III than in the areas closer to the coast. Water levels at well 4N/27W-18Q4 in the inland part of Storage Unit III, near the Val Verde production well (18Q2), responded to nearby pumping, varying from a low of about $85 \mathrm{ft}$ in 1991 to a high of $102 \mathrm{ft}$ in 2005 (fig. 11). Water levels at well 4N/27W-21F1, downgradient from well 18Q4, were fairly steady between the early 1990s and 2010s, but water levels started to decline after 2010 (fig. 11). 


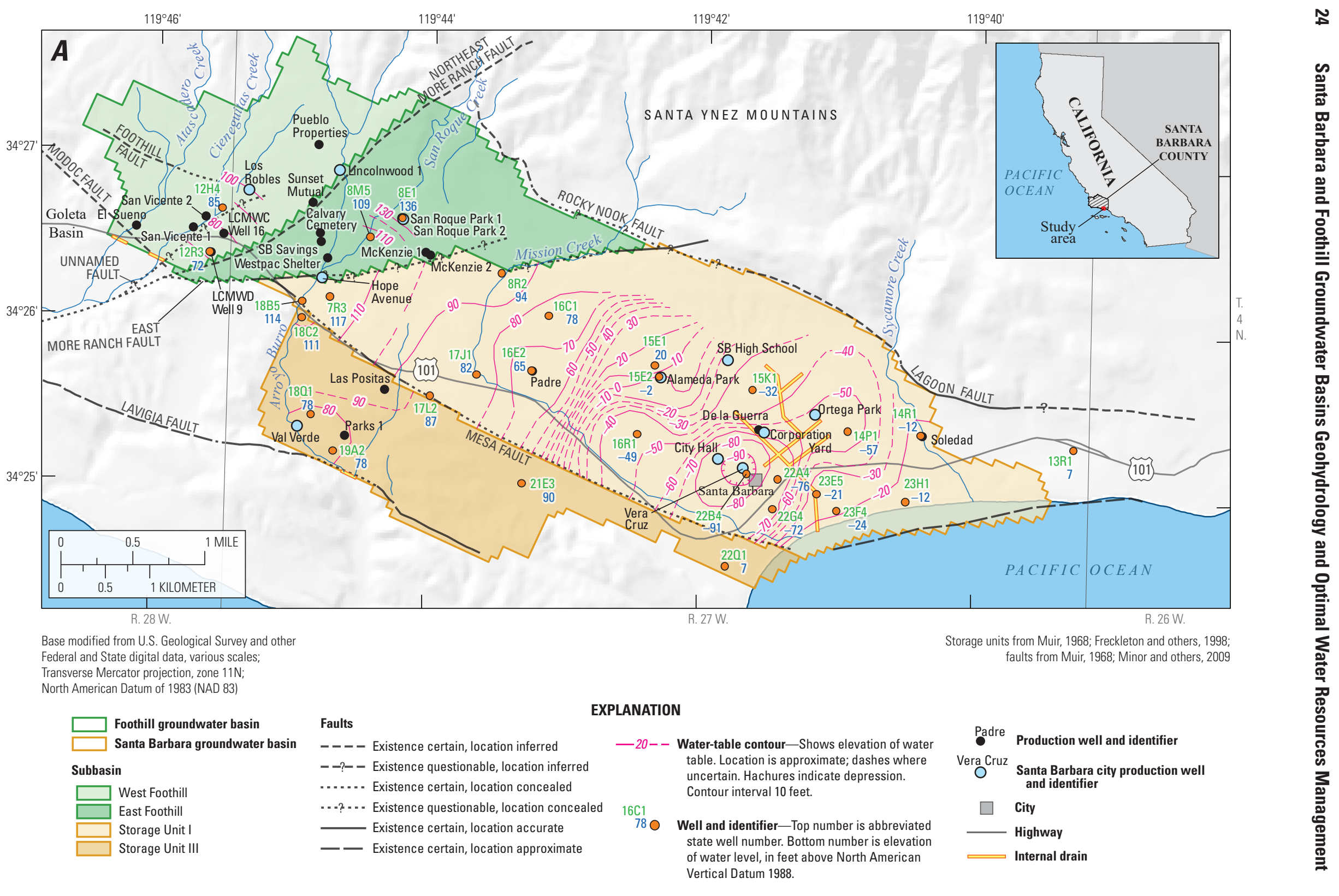

Figure 10. Groundwater-level contours of the lower producing zone in the Santa Barbara and Foothill groundwater basins, Santa Barbara, California: $A$, summer 1990, and $B$, spring 1997. 


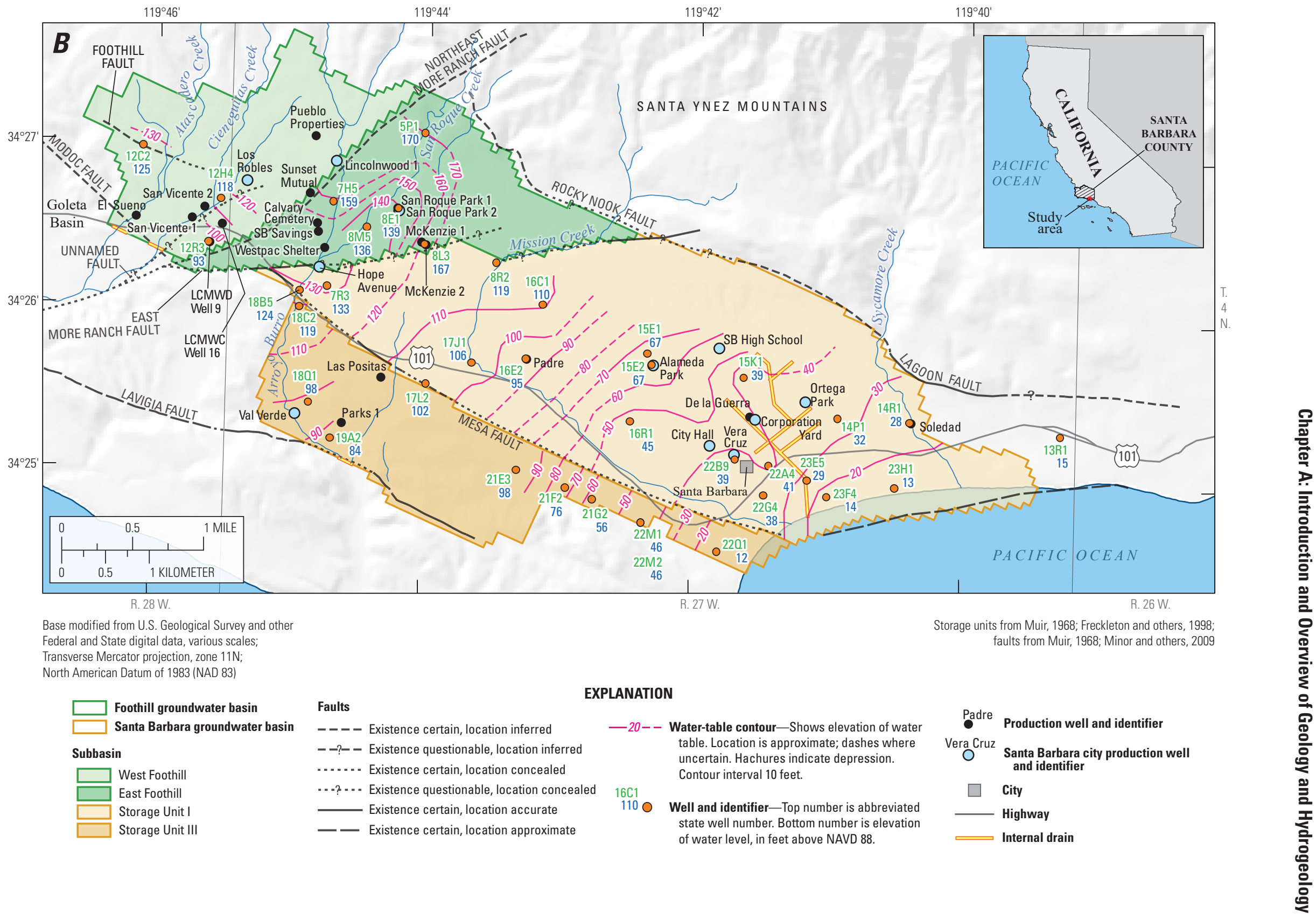

Figure 10. - Continued 
Table 2. City of Santa Barbara production wells and state well identifiers, Santa Barbara, California.

\begin{tabular}{lc}
\hline \multicolumn{1}{c}{ Common name } & State well number \\
\hline Alameda Park & 4N/27W-15E2 \\
City Hall & $4 \mathrm{~N} / 27 \mathrm{~W}-22 \mathrm{C} 1$ \\
Corporation Yard & $4 \mathrm{~N} / 27 \mathrm{~W}-15 \mathrm{Q} 10$ \\
Hope Avenue & $4 \mathrm{~N} / 27 \mathrm{~W}-07 \mathrm{Q} 5$ \\
Lincolnwood 1 & $4 \mathrm{~N} / 27 \mathrm{~W}-07 \mathrm{~A} 5$ \\
Los Robles & $4 \mathrm{~N} / 27 \mathrm{~W}-07 \mathrm{D} 1$ \\
Ortega Park & $4 \mathrm{~N} / 27 \mathrm{~W}-15 \mathrm{~J} 2$ \\
San Roque Park (2) & $4 \mathrm{~N} / 27 \mathrm{~W}-08 \mathrm{E} 4$ \\
Santa Barbara High School & $4 \mathrm{~N} / 27 \mathrm{~W}-15 \mathrm{G} 1$ \\
Val Verde & $4 \mathrm{~N} / 27 \mathrm{~W}-18 \mathrm{Q} 2$ \\
Vera Cruz & $4 \mathrm{~N} / 27 \mathrm{~W}-22 \mathrm{~B} 6$ \\
\hline
\end{tabular}

\section{Seawater Intrusion}

Because of the Santa Barbara groundwater basin's proximity to the Pacific Ocean and historical pumpage, seawater intrusion has been an important water-quality issue. Fortunately, the risk of seawater intrusion is mitigated by the (previously noted) unnamed offshore fault that truncates the water-bearing deposits of Storage Unit I, which are adjacent to consolidated rocks on the seaward side of the fault. The offshore fault acts as a partial barrier to groundwater movement; however, saltwater at depth in Storage Unit I indicates that saline groundwater can cross the offshore fault (Martin and Berenbrock, 1986).

Several of the city of Santa Barbara's production wells are within a mile of the ocean, including Vera Cruz (22B6), City Hall (22C1), Corporation Yard (15Q10), and Ortega Park (15J2) wells. Between 1985 and 1991, increased pumping rates in these wells caused notable decreases in water levels in the lower producing zone, with heads as low as $90 \mathrm{ft}$ bsl in the vicinity of these wells and more than $10 \mathrm{ft}$ bsl at the coast (fig. 10A). Relatively high salinity levels were observed in wells perforated in the middle and lower producing zones along the coast, including wells $4 \mathrm{~N} / 27 \mathrm{~W}-23 \mathrm{~F} 8,4 \mathrm{~N} / 27 \mathrm{~W}-$ 23F4, 4N/27W-23E5, and 4N/27W-23E1 (figs. 12, 13). Higher salinity levels were not observed at monitoring wells 4N/27W-22G3, 4N/27W-22G4, 4N/27W-22A4 (fig. 13),

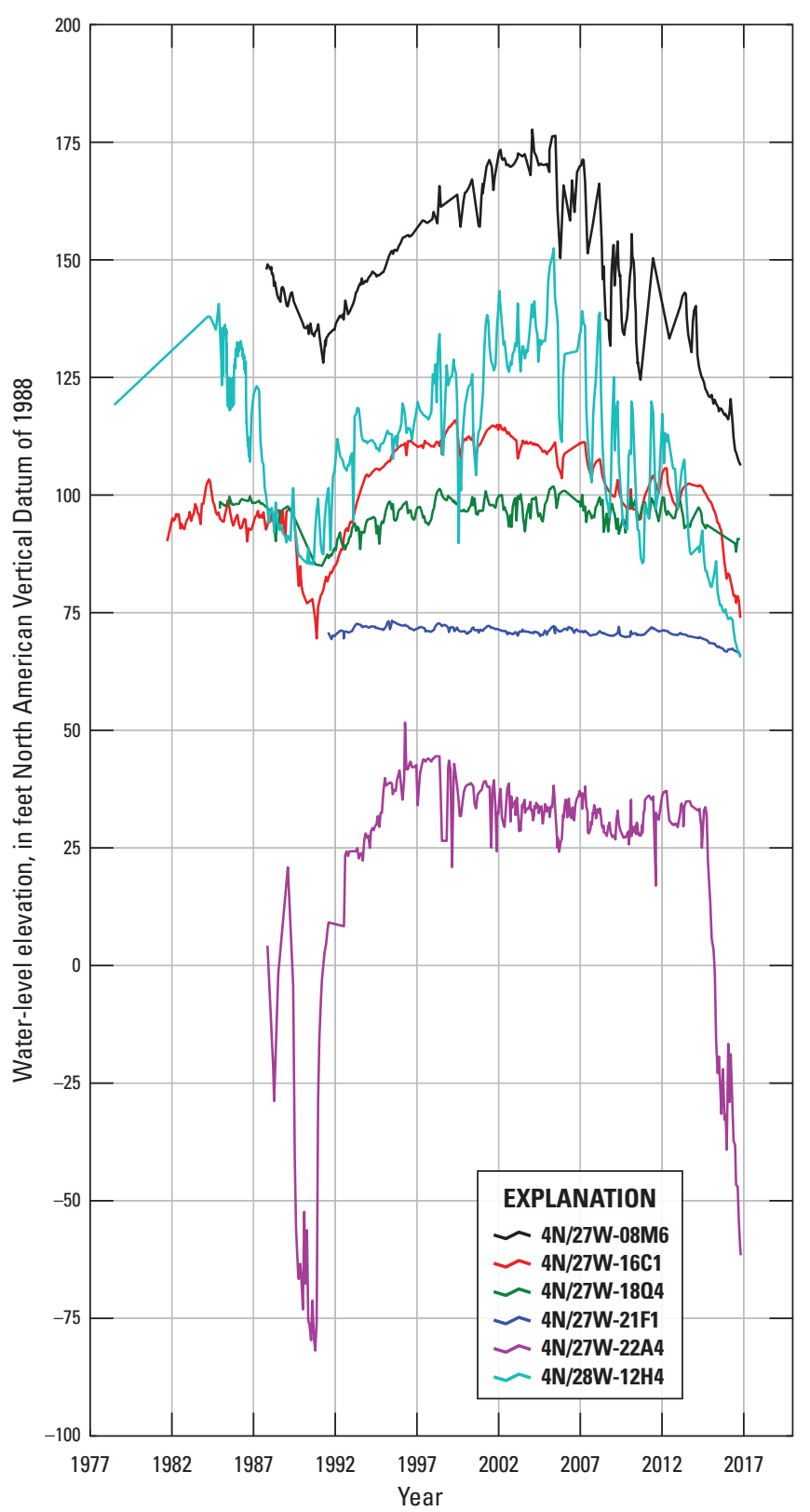

Figure 11. Water-level elevations from selected wells in Storage Unit I, Storage Unit III, and Foothill groundwater basin, Santa Barbara, California.

which are perforated in the middle and lower producing zone and are about $0.5 \mathrm{mi}$ from the coast and $0.25 \mathrm{mi}$ from the production wells (figs. 10B, 11). Although elevated chloride concentrations up to 1,600 milligrams per liter $(\mathrm{mg} / \mathrm{L})$ were recorded in some of the deeper wells (for example, well 4N/27W-22B8), the elevated chloride concentrations were most likely not from seawater intrusion. The presence of barium and boron in concentrations much greater than those in ocean water indicates that seawater intrusion was not the source of the elevated chloride concentrations in these deeper wells (Martin, 1984). 


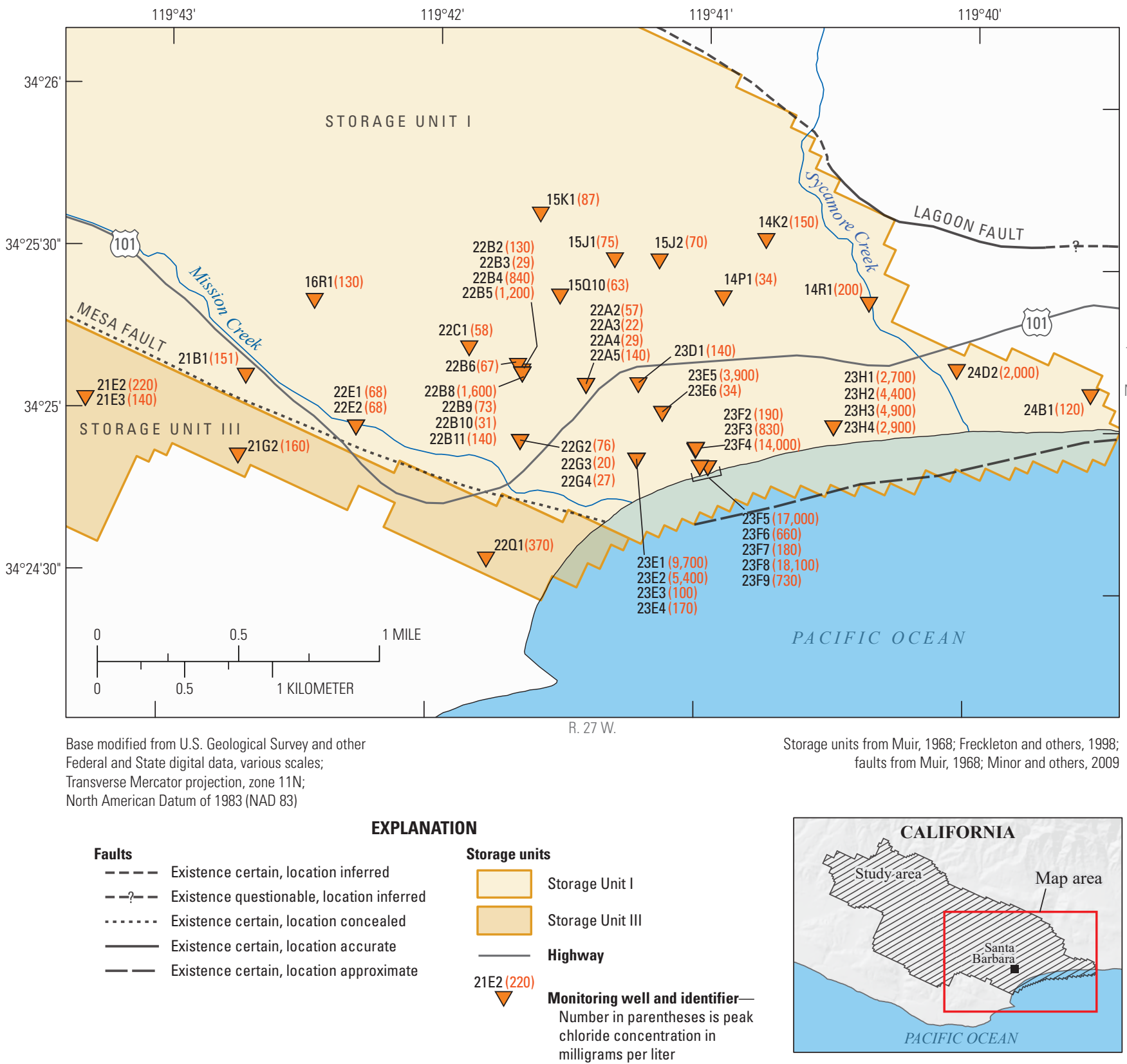

Figure 12. Locations of coastal monitoring wells with associated peak, measured chloride concentrations, Santa Barbara groundwater basin, Santa Barbara, California. 

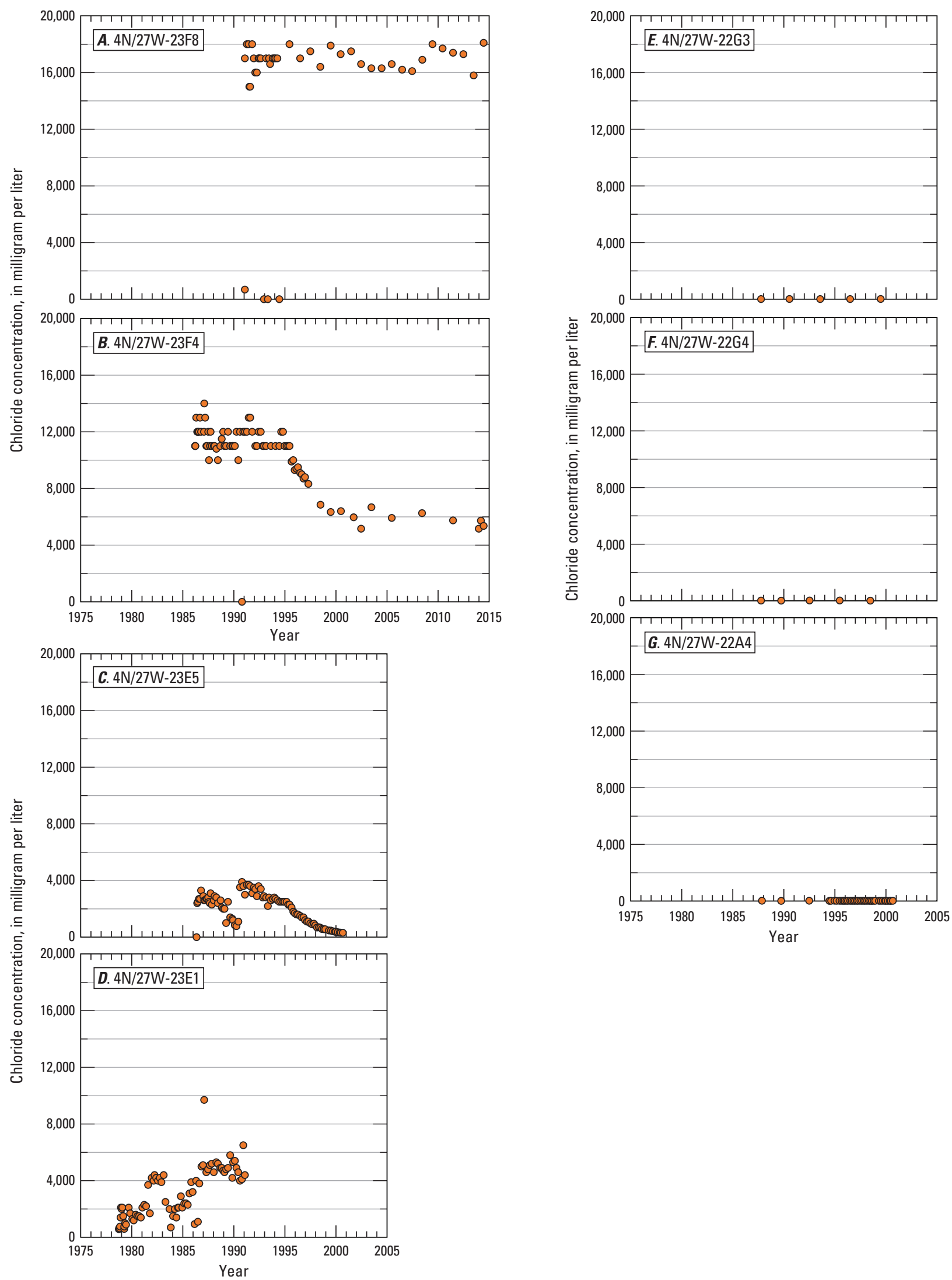

Figure 13. Time-varying chloride concentrations from coastal wells, Santa Barbara groundwater basin, Santa Barbara, California: $A, 4 \mathrm{~N} / 27 \mathrm{~W}-23 \mathrm{~F} 8 ; \quad B, 4 \mathrm{~N} / 27 \mathrm{~W}-23 \mathrm{~F} 4 ; C_{1}$ 4N/27W-23E5; $D, 4 \mathrm{~N} / 27 \mathrm{~W}-23 \mathrm{E} 1 ; E_{,}$NN/27W-22G3; $F, 4 \mathrm{~N} / 27 \mathrm{~W}-22 \mathrm{G} 4 ;$ and G, 4N/27W-22A4. 


\section{References Cited}

City of Santa Barbara, 2015, Water sources: Santa Barbara, Calif., Public Works Department, accessed April 2015 at http://www.santabarbaraca.gov/gov/depts/pw/resources/ system/sources/default.asp.

County of Santa Barbara, 2014, Rainfall-daily XLS data: Santa Barbara, Calif., Public Works, Water Resources Division, accessed November 2014 at http://www.countyofsb.org/pwd/pwwater.aspx?id=30042.

Eckenfelder, W.W., Jr., 1980, Principles of water quality management: Boston, CBI Publishing Company, Inc., $717 \mathrm{p}$.

Freckleton, J.R., 1989, Geohydrology of the Foothill groundwater basin near Santa Barbara, California: U.S. Geological Survey Water-Resources Investigations Report 89-4017, 46 p., https://pubs.er.usgs.gov/publication/wri894017.

Freckleton, J.R., Martin, P., and Nishikawa, T., 1998, Geohydrology of Storage Unit III and a combined flow model of the Santa Barbara and Foothill ground-water basins, Santa Barbara County, California: U.S. Geological Survey Water-Resources Investigations Report 97-4121, 80 p., http://pubs.er.usgs.gov/publication/wri974121.

Harbaugh, A.W., Banta, E.R., Hill, M.C., and McDonald, M.G., 2000, MODFLOW-2000, the U.S. Geological Survey modular ground-water modelUser guide to modularization concepts and the groundwater flow process: U.S. Geological Survey Open-File Report 2000-92, 121 p., https://pubs.er.usgs.gov/publication/ofr200092.

Hutchinson, C.B., 1979, Ground-water monitoring at Santa Barbara, California-Phase 1, Coastal monitor-well installation and initial measurements: U.S. Geological Survey Open-File Report 79-923, 36 p., https://pubs.er.usgs.gov/publication/ofr79923.

Martin, P., 1984, Ground-water monitoring at Santa Barbara, California; Phase 2-Effects of pumping on water levels and on water quality in the Santa Barbara ground-water basin: U.S. Geological Survey Water Supply Paper 2197, 31 p., https://pubs.er.usgs.gov/publication/wsp2197.
Martin, P., and Berenbrock, C., 1986, Ground-water monitoring at Santa Barbara, California; Phase 3Development of a three-dimensional digital ground-water flow model for Storage Unit I of the Santa Barbara groundwater basin: U.S. Geological Survey Water-Resources Investigations Report 86-4103, 58 p., https://pubs.er.usgs.gov/publication/wri864103.

McFadden, M.C., Polinoski, K.G., and Martin, P.M., 1991, Measurement of streamflow gains and losses on Mission Creek at Santa Barbara, California; July and September 1987: U.S. Geological Survey Water-Resources Investigations Report 91-4002, 15 p., https://pubs.er.usgs.gov/publication/wri914002.

Minor, S.A., Kellogg, K.S., Stanley, R.G., Gurrola, L.D., Keller, E.A., and Brandt, T.R., 2009, Geologic map of the Santa Barbara coastal plain area, Santa Barbara County, California: U.S. Geological Survey Scientific Investigations Map 3001, scale 1:25,000, 1 sheet, pamphlet, 38 p., https://pubs.er.usgs.gov/publication/sim3001.

Muir, K.S., 1968, Ground-water reconnaissance of the Santa Barbara-Montecito area, Santa Barbara County, California: U.S. Geological Survey Water Supply Paper 1859-A, 28 p., https://pubs.er.usgs.gov/publication/wsp1859A.

National Weather Service, 2016, Santa Barbara, California (047902) period of record monthly climate summary [1893-2016]: Western Regional Climate Center, accessed November 14, 2016, at http://www.wrcc.dri.edu/cgi-bin/cliMAIN.pl?ca7902.

Nishikawa, T., and Martin, P., 1998, Postaudit of optimal conjunctive use policies, in Loucks, E.D., ed., Water Resources in the Urban Environment, Chicago, June 7-10, 1998, Proceedings: Reston, Va., American Society of Civil Engineers, p. 591-596.

U.S. Census Bureau, 2017, Santa Barbara (city), California, QuickFacts: U.S. Census Bureau, accessed May 2017 at https://www.census.gov/quickfacts/table/ PST045215/0669070.

U.S. Geological Survey, 2018, Sustainable groundwater management: U.S. Geological Survey, accessed March 2018 at https://ca.water.usgs.gov/sustainable-groundwatermanagement/. 



\title{
Chapter B: Overview of Hydrogeologic Framework Model
}

\author{
By Geoffrey Cromwell and Scott E. Boyce
}

\section{Introduction}

This chapter describes the compilation of measured data and the construction of a hydrogeologic-framework model (HFM) of the Santa Barbara and Foothill groundwater basins. The development of the HFM required the compilation and reconciliation of geologic and geophysical data from existing maps, reports, and databases, which were then integrated along with data from recently drilled wells. The goal in developing an HFM is to define the three-dimensional (3D) geometry of the major geologic structures and stratigraphy. This HFM also expresses the variation of properties in each stratigraphic unit in the form of the percentage of coarse-grained deposits (texture). The HFM was developed using EarthVision, a threedimensional geologic-modeling software package (Dynamic Graphics, Inc., 2015).

\section{Hydrogeology and Structure of the Santa Barbara Study Area}

The HFM domain is composed of two separate groundwater basins, Santa Barbara (Hutchinson, 1979) and Foothill (Freckleton, 1989) groundwater basins, which are separated by the East More Ranch fault (fig. 1). Each of the groundwater basins is further subdivided into what can be called subbasins or storage units. The Santa Barbara groundwater basin is subdivided into Storage Unit I and Storage Unit III by the Mesa fault (Muir, 1968), and the Foothill groundwater basin is subdivided into the East and West Foothill subbasins by the Northeast More Ranch fault (see chapter A).

As described in chapter A, the two groundwater basins are composed of stratified unconsolidated deposits. These deposits have been classified in five aquifer zones that represent the overall hydraulic characteristics and depositional history of the unconsolidated material (see fig. 2; table 1). The five aquifer zones are the shallow zone, upper producing zone, middle zone, lower producing zone, and deep zone. Consolidated sedimentary rocks of Tertiary age are beneath the deep zone and also compose parts of the surrounding hills (Martin and Berenbrock, 1986). These consolidated rocks, predominantly marine in origin, are relatively impermeable, except for some sandstone layers and in fractured areas. Because of their low permeability, these consolidated units are considered the basement, or bedrock, of the HFM and form the lower boundary of the groundwater basins. 


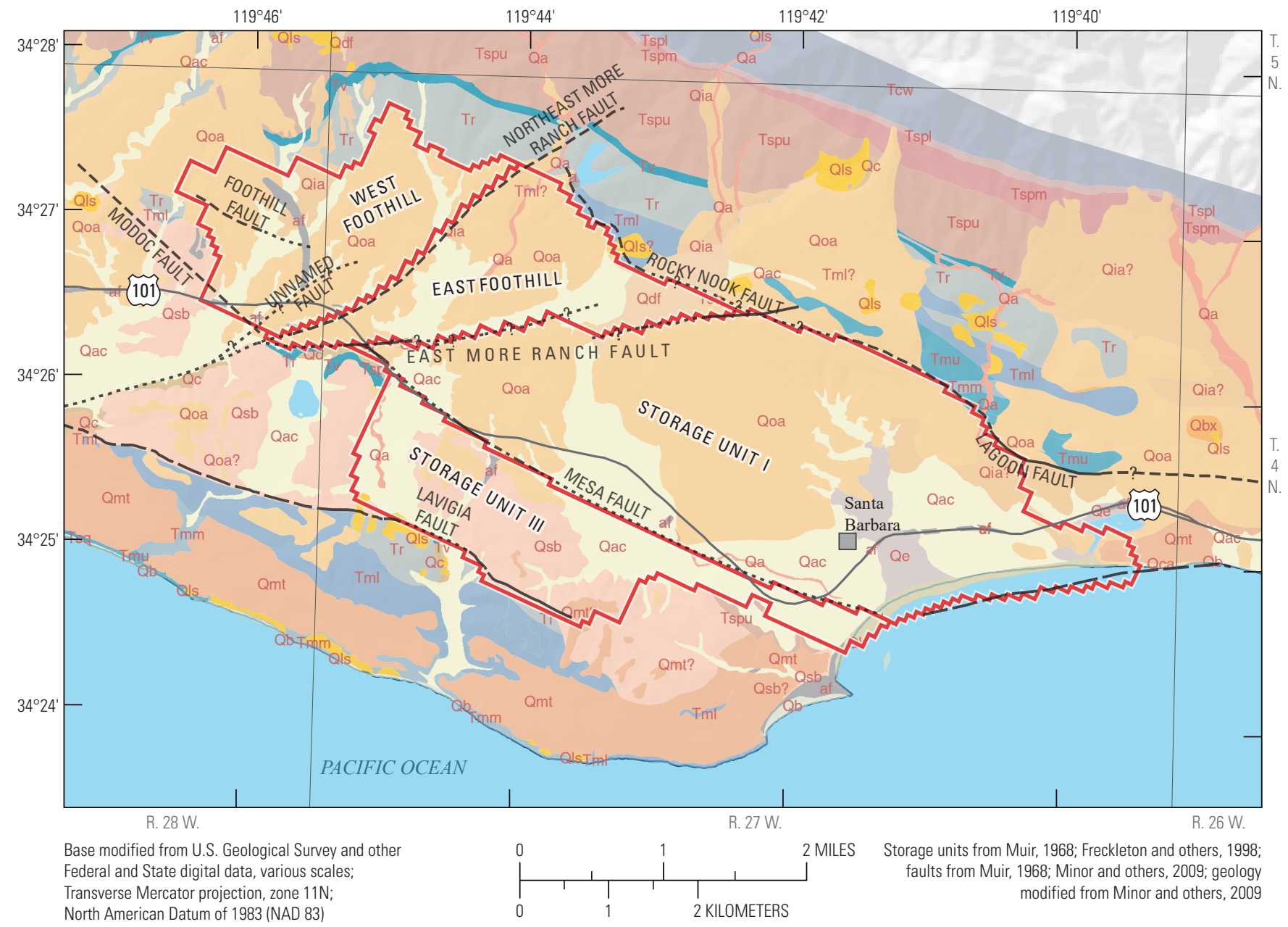

North American Datum of 1983 (NAD 83)

\section{EXPLANATION}

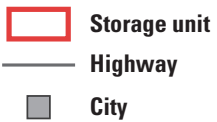

City

\section{Faults}

- - - Existence certain, location inferred

- -? - Existence questionable, location inferred

........ Existence certain, location concealed

...? -.. Existence questionable, location concealed

- Existence certain, location accurate

_ - Existence certain, location approximate

\footnotetext{
Geology

af - Artificial fill (Holocene)

Oa - Active channel alluvium (Holocene)

Qb - Beach deposits (Holocene)

Qe - Estuarine deposits (Holocene)

Qdf - Debris flow deposits (Holocene and upper Pleistocene?)

Qac - Alluvium and colluvium (Holocene and upper Pleistocene)

Qc - Colluvium (Holocene and upper Pleistocene)

Ols - Landslide deposits (Holocene and Pleistocene)

Qia - Intermediate alluvial deposits (upper Pleistocene)

Omt - Marine-terrace deposits (upper Pleistocene)

Qoa - Older alluvial deposits (upper and middle Pleistocene)

0ca - Casitas Formation (upper and middle Pleistocene)

Qbx - Shale-clast sedimentary breccia (middle Pleistocene)
}

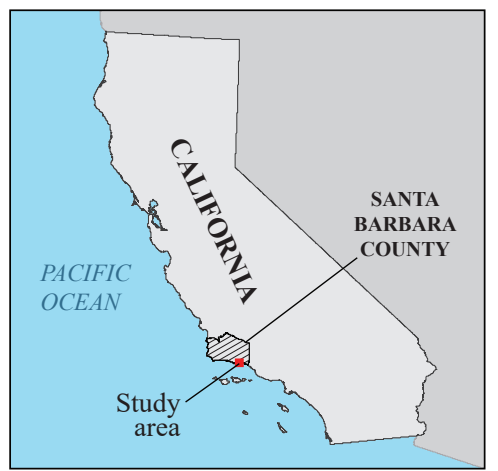

Qsb - Santa Barbara Formation (middle and lower Pleistocene)

Tsq - Sisquoc Formation (lower Pliocene and upper Miocene)

Tmu - Monterey Formation, upper siliceous unit (upper Miocene)

Tmm - Monterey Formation, middle shale unit (upper and middle Miocene)

Tml - Monterey Formation, lower calcareous unit (middle and lower Miocene)

$\mathrm{Tr}$ - Rincon Shale (lower Miocene)

Tv - Vaqueros Formation (upper Oligocene)

Tspu - Sespe Formation, upper sandstone and mudstone unit (upper Oligocene)

Tspm - Sespe Formation, middle conglomerate and sandstone unit (Oligocene)

Tspl - Sespe Formation, lower conglomerate and sandstone unit (lower Oligocene? and upper Eocene)

Tcw - Coldwater Sandstone (upper? and middle Eocene)

Figure 1. Hydrogeologic framework model domain, subbasins and storage units, and major faults, Santa Barbara and Foothill groundwater basins, Santa Barbara, California. 


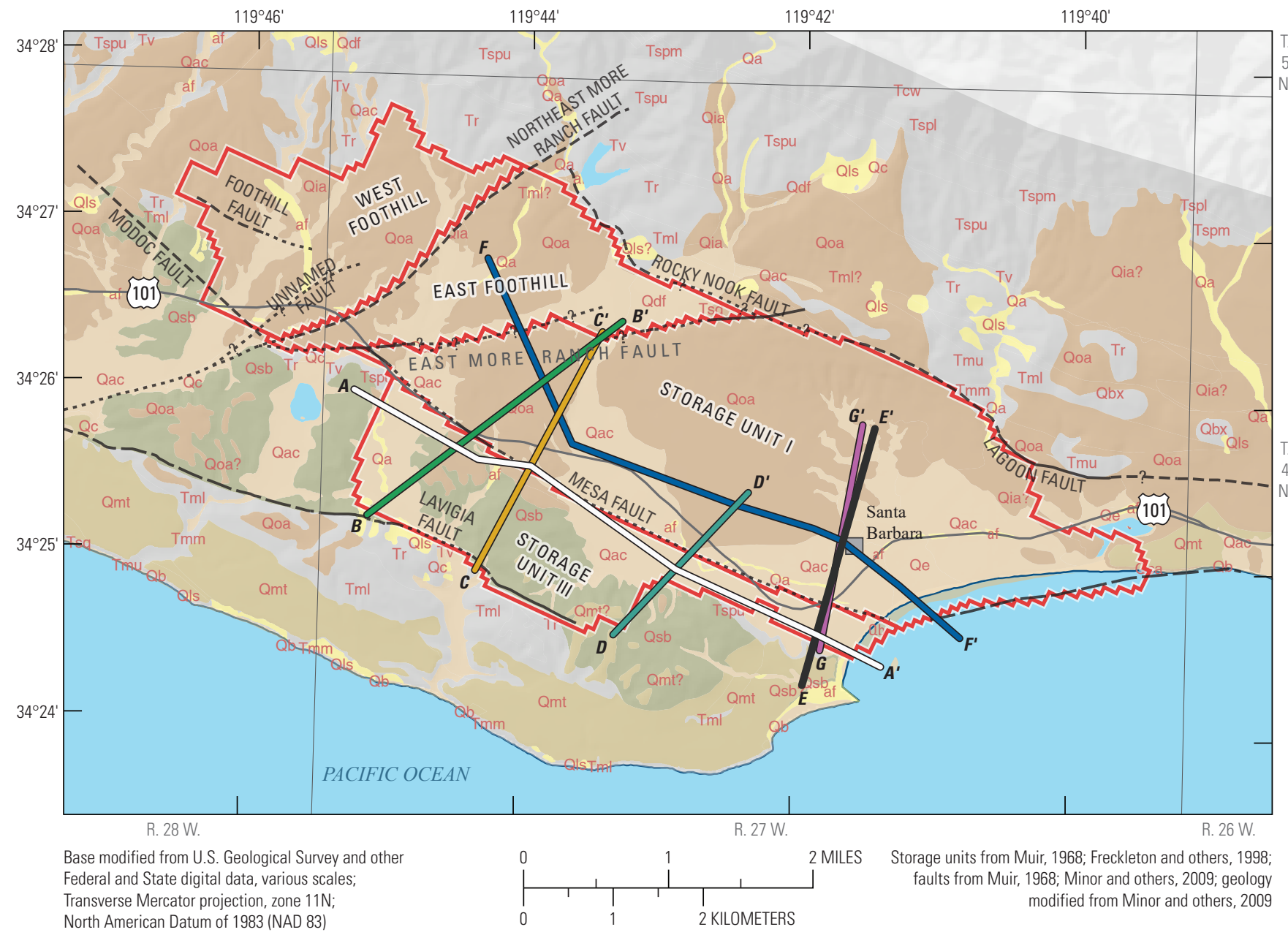

\section{EXPLANATION}

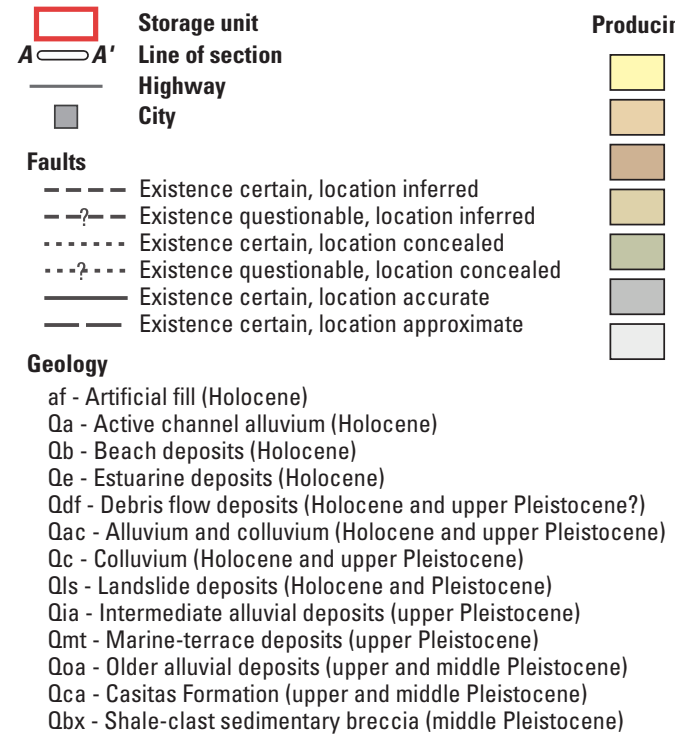

Producing zones

Shallow zone

Shallow and upper producing zones

Shallow, upper, middle, and lower producing zones

Middle and lower producing zones

Middle, lower, and deep producing zones

Bedrock

Unmapped

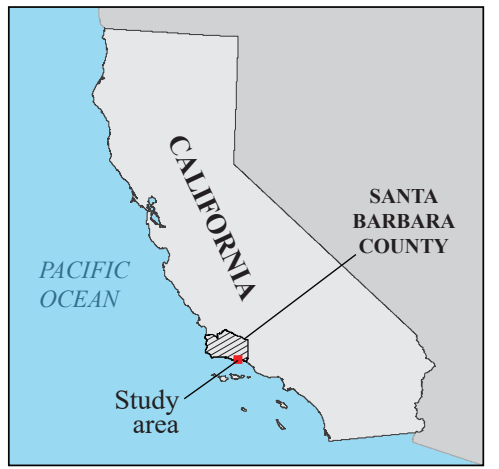

Qsb - Santa Barbara Formation (middle and lower Pleistocene)

Tsq - Sisquoc Formation (lower Pliocene and upper Miocene)

Tmu - Monterey Formation, upper siliceous unit (upper Miocene)

Tmm - Monterey Formation, middle shale unit (upper and middle Miocene)

Tml - Monterey Formation, lower calcareous unit (middle and lower Miocene)

Tr - Rincon Shale (lower Miocene)

Tv - Vaqueros Formation (upper Oligocene)

Tspu - Sespe Formation, upper sandstone and mudstone unit (upper Oligocene)

Tspm - Sespe Formation, middle conglomerate and sandstone unit (Oligocene)

Tspl - Sespe Formation, lower conglomerate and sandstone unit (lower Oligocene? and upper Eocene)

Tcw - Coldwater Sandstone (upper? and middle Eocene)

Figure 2. Aquifer-zone groupings organized by mapped geologic units, Santa Barbara and Foothill groundwater basins, California; cross-section lines show locations of previously published cross sections (Freckleton and others, 1998; Martin and Berenbrock, 1986) used in the hydrogeologic framework model. 
Table 1. Aquifer-zone groupings listed by mapped geologic unit and age, Santa Barbara and Foothill groundwater basins, California.

$[-$, not applicable $]$

\begin{tabular}{|c|c|c|}
\hline Aquifer zone & Geologic unit & Age \\
\hline Shallow & af, Qa, Qls, Qls? & Holocene \\
\hline Shallow and upper & $\begin{array}{l}\text { Qac, Qb, Qc, Qdf, } \\
\text { Qe }\end{array}$ & $\begin{array}{l}\text { Holocene and upper } \\
\text { Pleistocene }\end{array}$ \\
\hline $\begin{array}{l}\text { Shallow, upper, } \\
\text { middle, and lower }\end{array}$ & Qca, Qia, Qia?, Qoa & $\begin{array}{l}\text { Upper and middle } \\
\text { Pleistocene }\end{array}$ \\
\hline Middle and lower & Qmt, Qmt? & Upper Pleistocene \\
\hline $\begin{array}{l}\text { Middle, lower, and } \\
\text { deep }\end{array}$ & Qsb & $\begin{array}{l}\text { Middle and lower } \\
\text { Pleistocene }\end{array}$ \\
\hline Bedrock & $\begin{array}{l}\text { Tsq, Tml, Tmm, } \\
\text { Tmu, Tr, Tv, Tspu, } \\
\text { Tspm, Tspl, Tcw }\end{array}$ & $\begin{array}{l}\text { Miocene, Oligocene, } \\
\text { and Eocene }\end{array}$ \\
\hline
\end{tabular}

\section{Construction of the Hydrogeologic Framework Model}

The HFM has two main parts: (1) a geometric framework model showing the stratigraphic relationships of the aquifer zones; (2) a textural model detailing physical properties of the aquifer zones within the geometric framework.

\section{Geometric Framework}

The geometric framework model quantifies the surficial and subsurface extent of the four subbasins or storage units as defined by major faults, the distribution of consolidated rocks, and the extent and thickness of the five aquifer zones. Subbasin geometry was constructed by compiling and digitizing existing geologic maps, cross sections, textural descriptions from drillers' lithology logs, and geophysical logs. These data sets were interpolated to define the extent and geometry of the subbasins and the thickness of the aquifer zones within them.

\section{Input Data}

Previous studies have presented geologic maps and cross sections of the Santa Barbara region. The geologic map used for this study is of the Santa Barbara coastal plain area by Minor and others (2009; fig. 1). This map served as a reference to the location and dip of the major faults and to the surficial extent of the consolidated, Tertiary sedimentary rocks that define much of the HFM boundary. The subsurface extent of the aquifer zones was derived from previously published geologic cross sections and drillers' lithology logs and from geophysical electricity resistivity logs (e-logs) compiled as part of this study. Two sets of previously published geologic cross sections were digitized from Freckleton and others (1998; fig. 2, sections A-A' through E-E') and Martin and Berenbrock (1986; fig. 2, sections F-F' and G-G'). For a more detailed description of these sections, see Freckleton and others (1998) and Martin and Berenbrock (1986).

Drillers' lithology logs and e-logs were compiled from the California Department of Water Resources. Drillers' logs from a total of 159 boreholes in the study area (fig. 3) were obtained and entered in a new digital database containing well-construction information and subsurface-lithology characteristics (following the database design of Burow and others, 2004). Of those 159 boreholes, 40 had geophysical e-logs included with the drillers' lithology log. The deepest boreholes in Storage Unit I, East Foothill, Storage Unit III, and West Foothill had depths of 840, 660, 340, and 490 feet (ft), respectively (table 2 ).

\section{Structures and Aquifer Zones}

The HFM area was formed from a set of independent blocks defined by faults and, therefore, fault blocks that represent geologic materials. In this case, the geologic material consists of Tertiary consolidated rocks that form the bedrock and unconsolidated sedimentary deposits that are divided into five aquifer zones and are stacked chronologically to fill the volume of the HFM.

\section{Fault Blocks}

Each of the four subbasins are delineated by faults, some of which are estimated to have substantial vertical offset (Martin and Berenbrock, 1986; Freckleton and others, 1998). To preserve the measured offset across these faults in the HFM, each subbasin was defined as an independent fault block. Using EarthVision software, the elevation of the top of bedrock and each aquifer zone in a particular fault block were calculated independently and then combined to form a single model. This method prevents any aquifer-zone horizon data from one subbasin affecting the horizon data in another subbasin and, thus, preserves the expected geologic offsets between subbasins. 


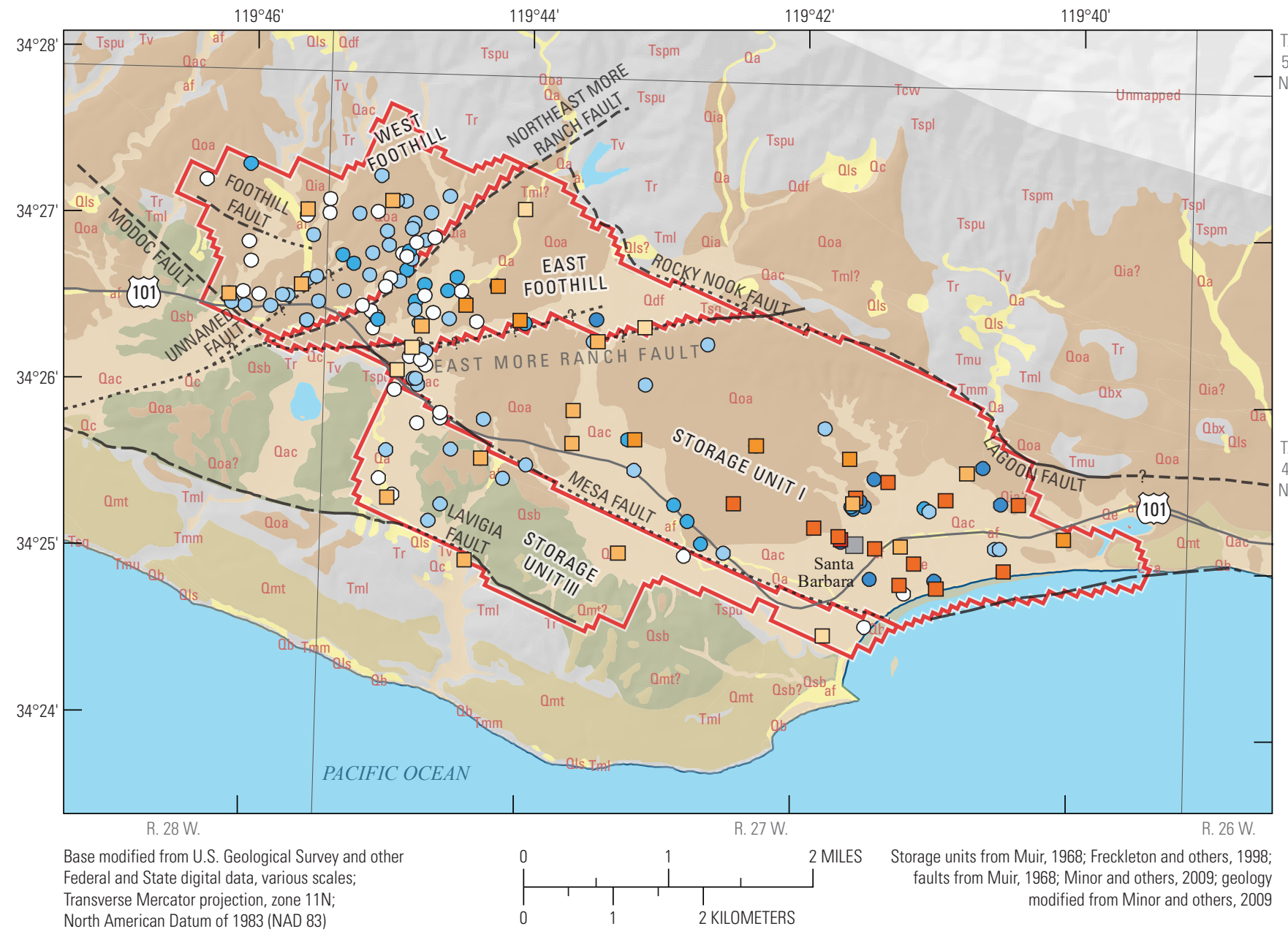

EXPLANATION

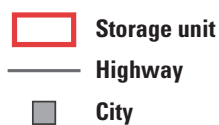

Faults

- - - - Existence certain, location inferred

- -? - Existence questionable, location inferred

........ Existence certain, location concealed

...? -... Existence questionable, location concealed

Existence certain, location accurate

—— Existence certain, location approximate

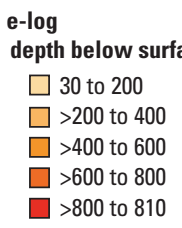

Producing zones

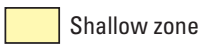

Shallow and upper producing zones

Shallow, upper, middle, and lower producing zones

Middle and lower producing zones

Middle, lower, and deep producing zones

Bedrock

Unmapped

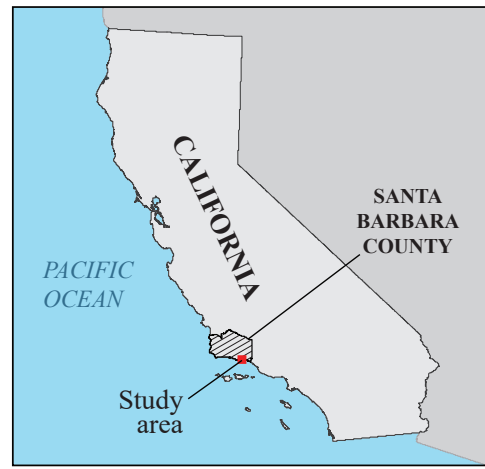

\section{Drillers' log}

depth below surface, in feet

00 to 200

$0>200$ to 400

$>>400$ to 600

$>>600$ to 800

$>>800$ to 840

Figure 3. Aquifer-zone groupings with drillers' well logs and geophysical e-logs used in the hydrogeologic framework model for Storage Unit I, Storage Unit III, and the Foothill subbasins, Santa Barbara and Foothill groundwater basins, California. 
Table 2. Summary statistics of borehole depths by subbasin, Santa Barbara and Foothill groundwater basins, Santa Barbara, California.

\begin{tabular}{lcccccc}
\hline \multirow{2}{*}{$\begin{array}{c}\text { Groundwater } \\
\text { subbasin }\end{array}$} & \multicolumn{5}{c}{ Depth statistic property, in feet } & \multirow{2}{*}{ Count } \\
\cline { 2 - 5 } & Minimum & Maximum & Median & Mean & Standard deviation & \\
\hline Storage Unit I & 5 & 840 & 420 & 452.9 & 213.7 & 60 \\
East Foothill & 90 & 660 & 280 & 323.8 & 182.7 & 29 \\
Storage Unit III & 5 & 340 & 210 & 188.6 & 93.12 & 18 \\
West Foothill & 90 & 490 & 245 & 254.4 & 99.0 & 52 \\
\hline
\end{tabular}

\section{Aquifer-Zone and Bedrock-Horizon Modeling}

The horizons for the five aquifer zones and the bedrock unit were constructed by interpolating and extrapolating a two-dimensional surface in EarthVision from subsurface data points. EarthVision uses a biharmonic cubic-spline algorithm that uses a minimum-tension (minimum curvature) gridding technique (Dynamic Graphics, Inc., 2015). Grid spacing for bedrock and the aquifer zones is about $328 \mathrm{ft}$ (100 meters) in the X and Y horizontal directions. Artificial control points were added in areas with limited subsurface data (that is, from drillers' logs or geologic cross sections) to improve constraint of horizon interpolations. When input data are sparse, the EarthVision gridding algorithm may extrapolate unreasonably high or low values, and in areas where data show abrupt changes in elevations, the horizon grid interpolation could be unrealistic; the addition of artificial control points helps minimize these effects. Control points also are used in the HFM to force horizon grids to pinch out where a unit is no longer present at depth. EarthVision stacks the aquifer-zone horizon grids in stratigraphic order on top of the bedrock horizon. In this manner, the total thickness of each zone is defined as the difference between the zone horizon and the upper surface of the zone below. Aquifer-zone and bedrock horizons and subsurface data used for horizon-grid interpolation are shown in figure 4.

Recall, there are five aquifer zones: the shallow zone, upper producing zone, middle zone, lower producing zone, and deep zone (see table 1 for a list of the geologic units in each zone). The shallow zone is composed of younger and older alluvium; the upper producing zone is only older alluvium. The middle, lower producing, and deep zones are composed of the Santa Barbara Formation and some older alluvial units. For a detailed discussion about the geology of the five zones, see Martin and Berenbrock (1986) and Freckleton and others (1998). As described in chapter A, near the ocean boundary, there is a small section of relatively high permeability rocks in the middle zone, referred to as the middle producing zone. Although a distinct hydrologic zone, the middle producing zone was not modeled independently, but was included in the HFM as part of the middle zone.

The five aquifer zones were identified by analyzing geophysical e-logs, surficial geologic maps (fig. 1), and published geologic cross sections. The upper contact of each aquifer zone was identified and digitized from geologic cross sections published by Martin and Berenbock (1986) and Freckleton and others (1998). Similarly, geophysical e-logs were digitized and examined, and the upper contact of each zone was identified. The geologic map was used to identify where the aquifer zones outcrop at land surface. The interpolated aquifer-zone horizons and subsurface data points are shown in figure $4 A-E$.

In this study, bedrock is defined as the relatively lesspermeable consolidated Tertiary rocks of Miocene age and older. In general, the upper contact of the bedrock unit was identified by the presence of shale (as identified in previous reports or interpreted in drillers' lithology logs and geophysical e-logs) or fine-grained lithologic layers of substantial thickness. Surficial and subsurface bedrock elevations were derived from drillers' lithology logs, geophysical e-logs, surficial geologic maps (fig. 1), and geologic cross sections from previous reports (Martin and Berenbock, 1986; Freckleton and others, 1998). Where bedrock outcrops at land surface, the total aquifer thickness was assumed to be zero. The interpolated bedrock horizon and subsurface data points are shown in figure $4 F$.

\section{Geometric Framework Model Description}

A fence diagram of the geometric framework model is shown in figure $5 \mathrm{~A}$. The cross sections in the fence diagram are the same as those shown in figure 2 (with the exception of G-G'), and with the addition of one cross section (H-H') cutting southwest-northeast through the Foothill groundwater basin and a second cross section (I-I') running northwestsoutheast through the entire study area.

Total aquifer thickness, as defined by land surface and top of the bedrock unit, varies considerably across the study area. The thickest part of the aquifer is at the coast, in the southeastern-most part of Storage Unit I, where the total thickness of unconsolidated sediment is about $1,100 \mathrm{ft}$. Aquifer thickness gradually decreases from southeast to northwest in Storage Unit I to a minimum of about $200 \mathrm{ft}$ along the boundary between the Storage Unit I and the Foothill groundwater basin. In Storage Unit III, the aquifer ranges in thickness from about 200 to $450 \mathrm{ft}$, except at the southeastern end, where the bedrock unit rises substantially, and only a thin veneer of shallow-zone sediments is present. Total aquifer thickness is relatively constant in the West Foothill and East Foothill subbasins, at about $330 \mathrm{ft}$ and $650 \mathrm{ft}$, respectively. In the northern section of both subbasins, bedrock rises rapidly in elevation toward land surface, and the thickness of unconsolidated sediments decreases in similar fashion. 

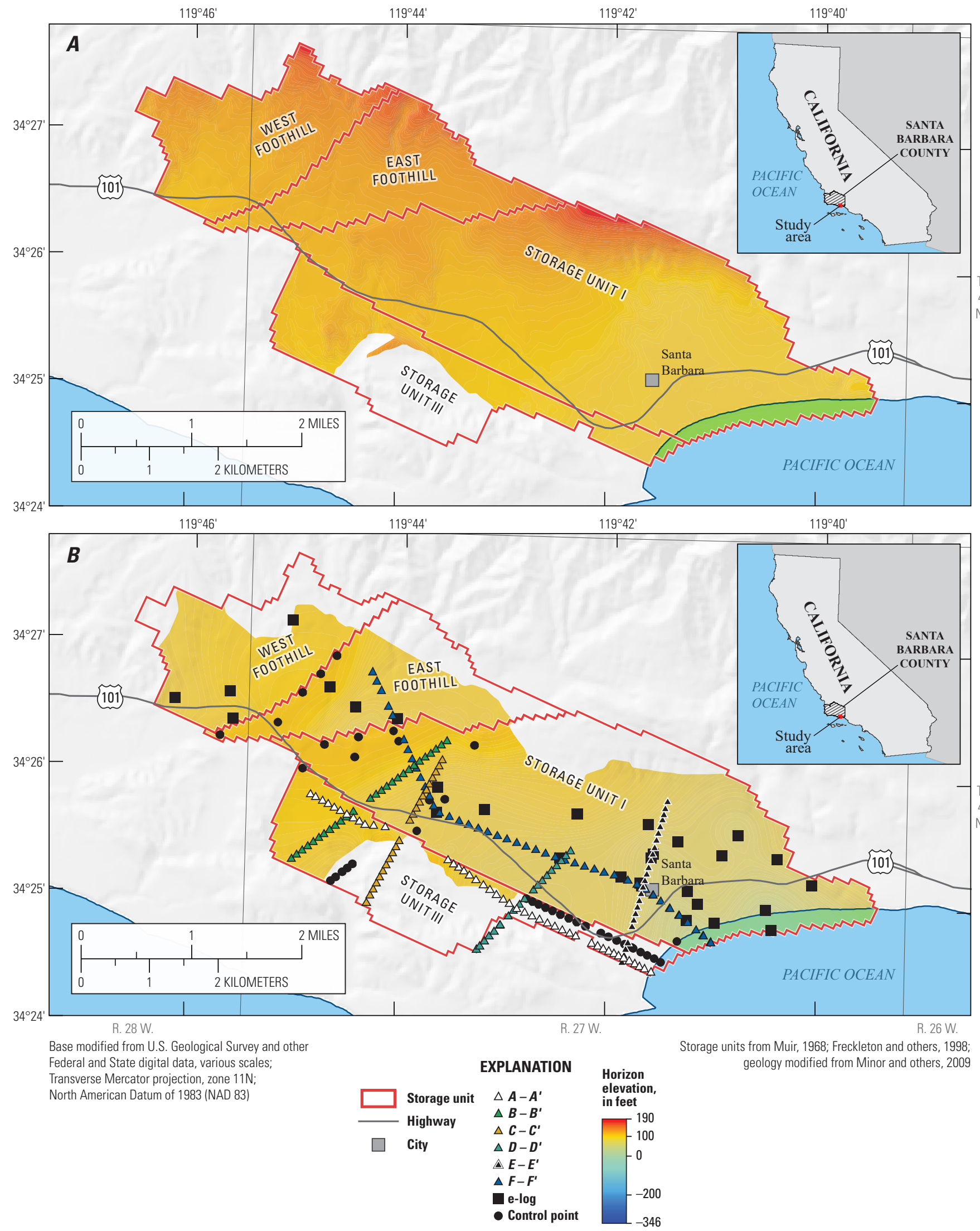

Figure 4. Interpolated elevations of hydrogeologic framework model horizons with subsurface data points, Santa Barbara and Foothill groundwater basins, Santa Barbara, California: $A$, shallow zone; $B$, upper producing zone; $C$, middle zone; $D$, lower producing zone; $E$, deep zone; and $F$, bedrock. Horizon elevation is feet above NAVD 88. 

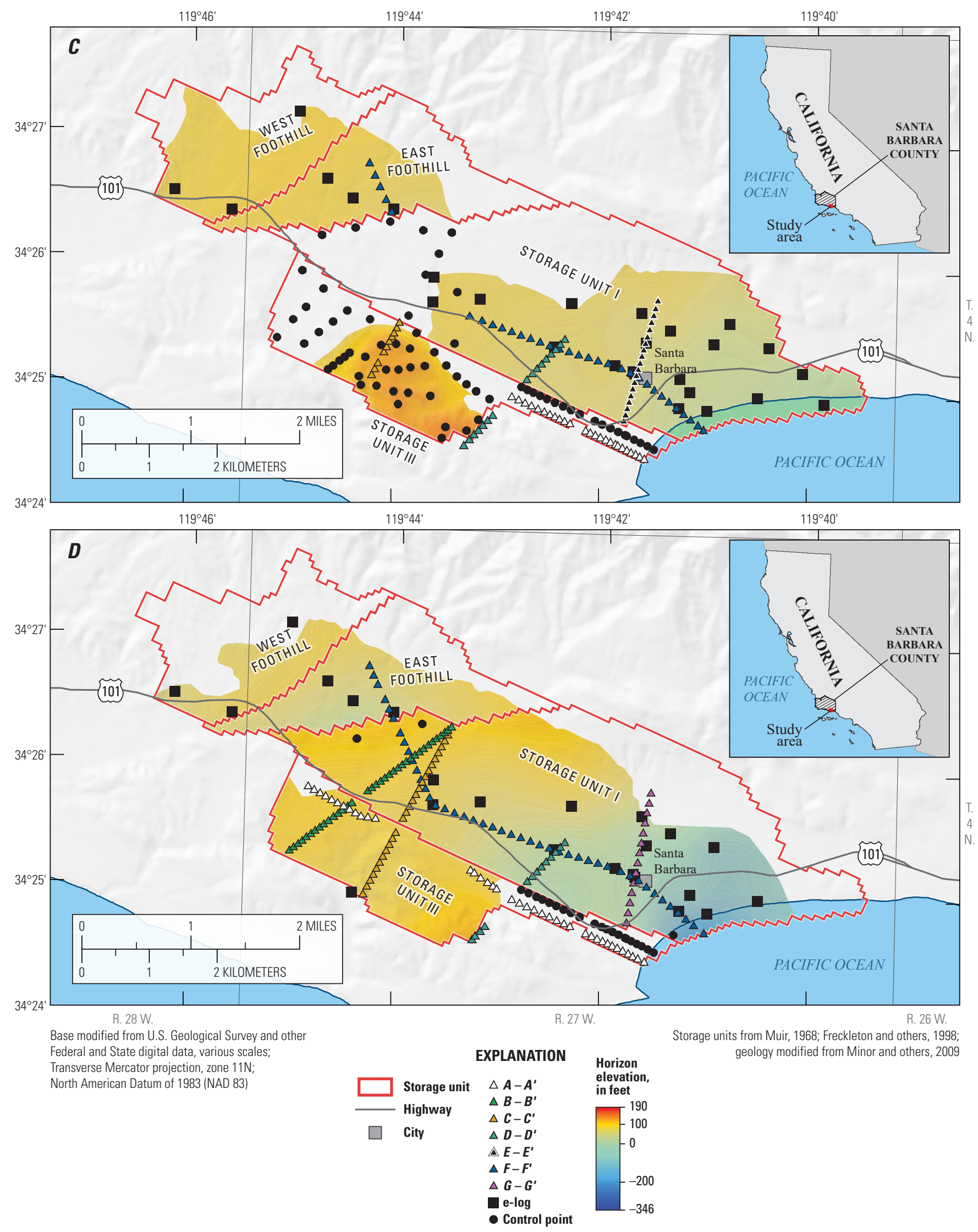

Figure 4. - Continued 

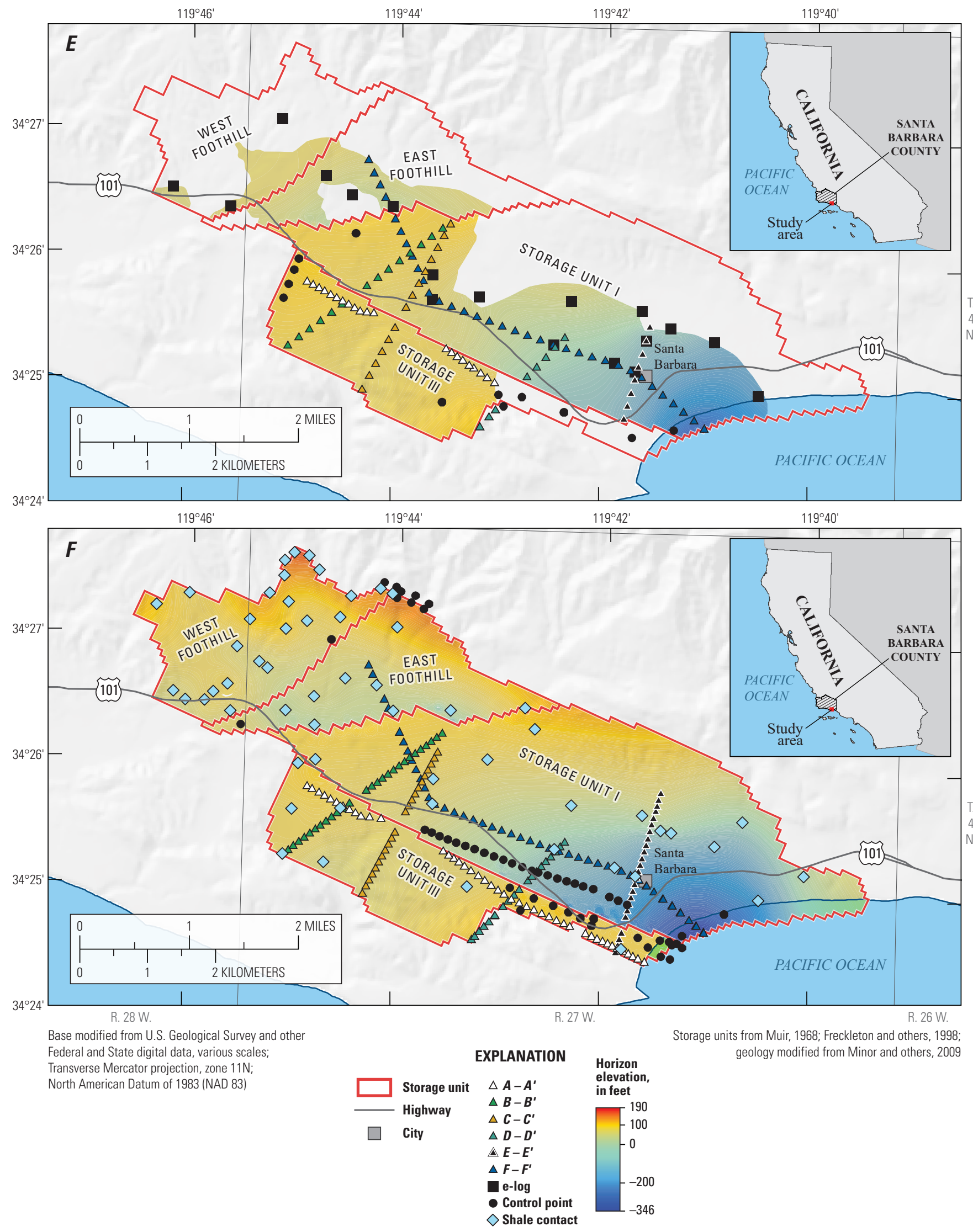

Figure 4. - Continued 


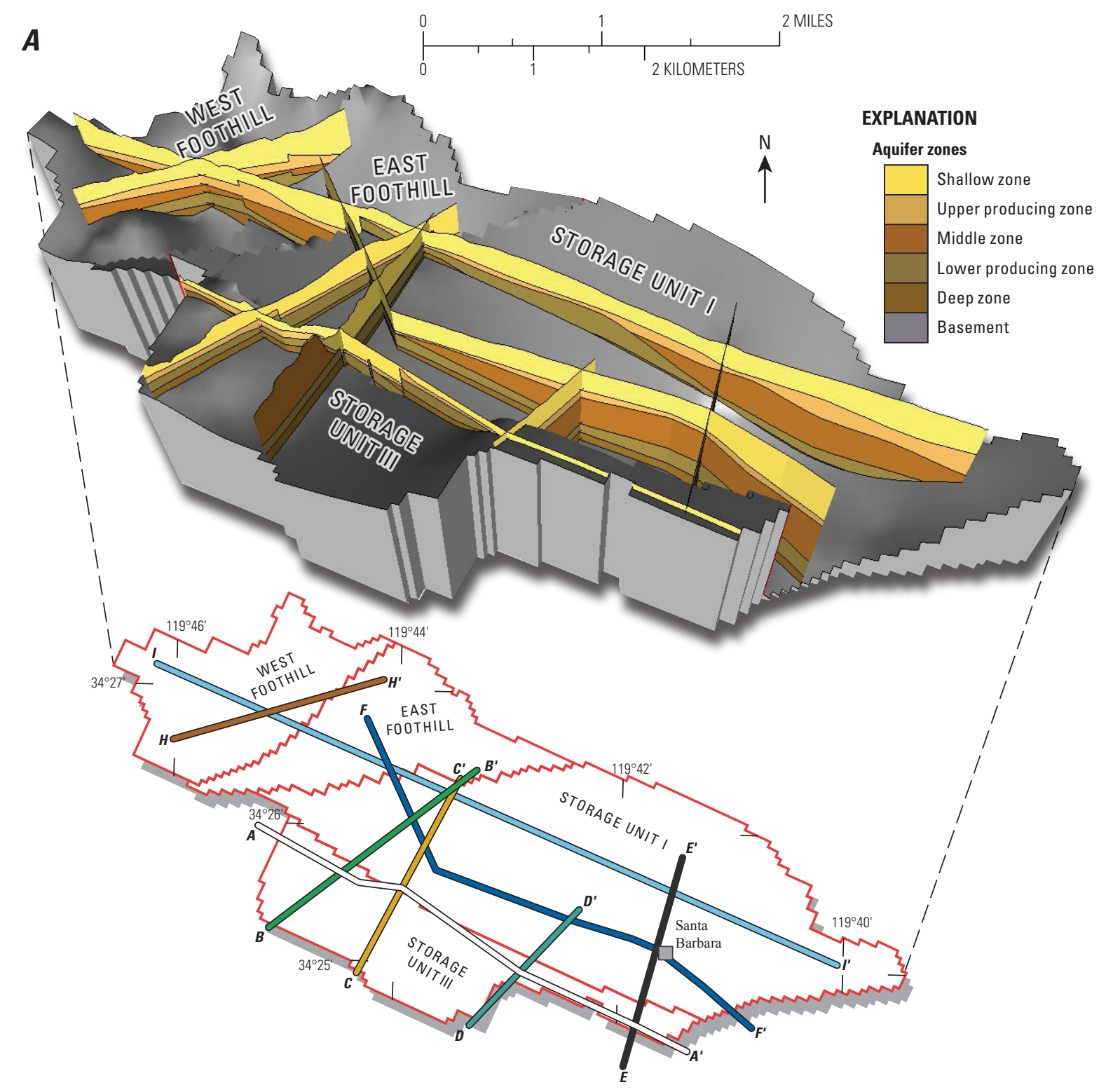

Figure 5. Hydrogeologic framework model results by fence diagram, Santa Barbara and Foothill groundwater basins, Santa Barbara, California: $A$, geometric framework model showing subsurface extent of the five aquifer zones, and $B$, texture model showing the distribution of coarse- and fine-grained material in each aquifer zone, with percent-coarse intervals for drillers' well logs and geophysical e-logs (locations of all logs are also shown on the inset diagram). Fence diagrams shown with five times vertical exaggeration.

In general, all five aquifer zones are present across the study area, with the exception of (1) the middle zone in Storage Units I and III and (2) the upper producing zone and shallow zone in the southeastern section of Storage Unit III. In Storage Unit I, the middle zone pinches out approximately
2-3 miles from the coast, and is absent further inland and in the northwestern part of Storage Unit III. In the southeastern part of Storage Unit III, the Santa Barbara Formation outcrops at land surface; therefore, the younger upper producing zone and shallow zone are absent. 


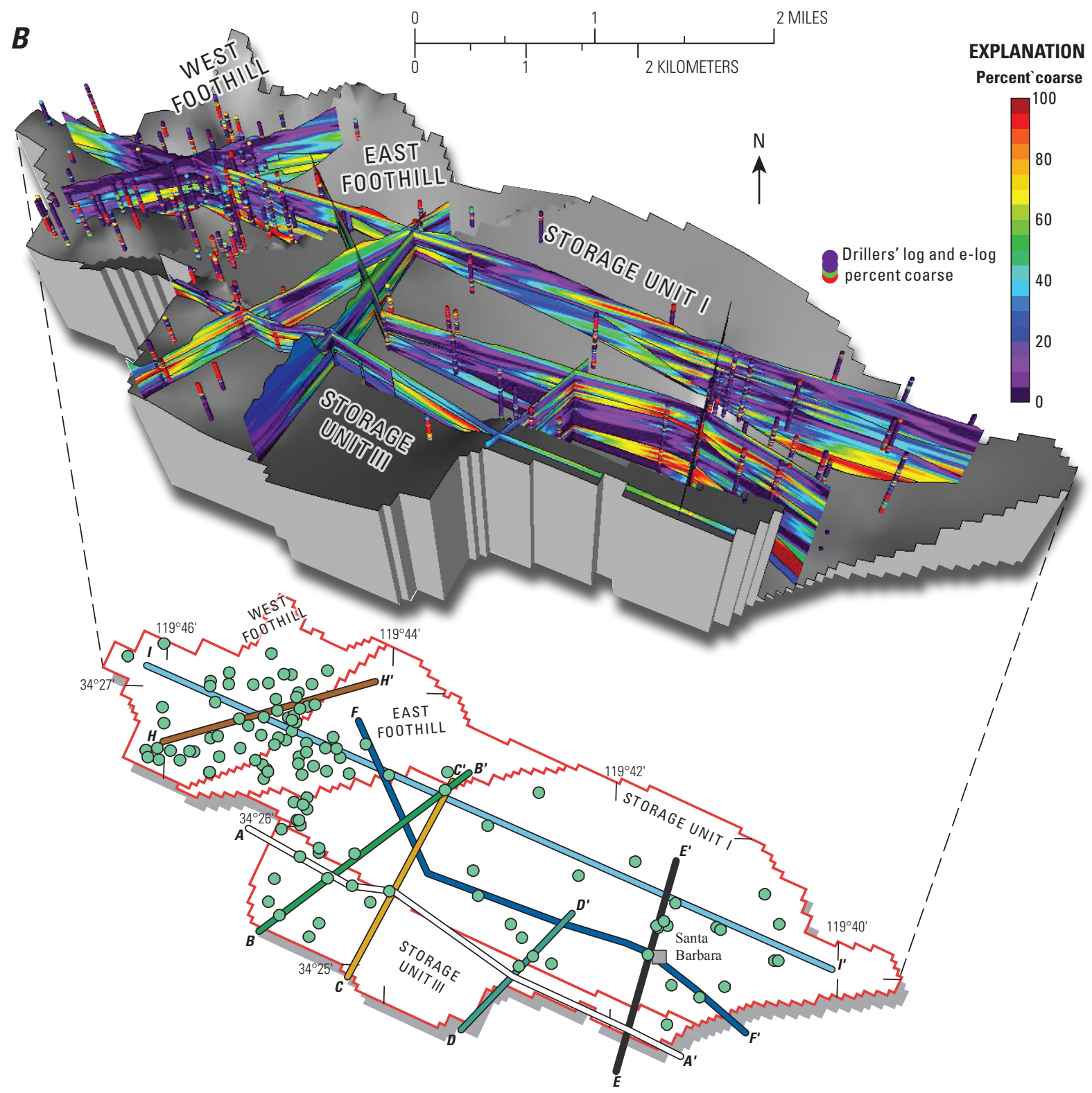

Figure 5. - Continued

\section{Textural Model}

The textural model quantifies the lateral and vertical distribution of aquifer materials that affect the rate, and possibly direction, of groundwater flow through the aquifer system. Variability in sediment texture (lithology type and grain size), as identified in drillers' lithology logs and geophysical e-logs, is ultimately a function of the depositional environment and geologic history of the area. An analysis of the spatial variability of lithology and grain size was done for the unconsolidated deposits that compose the five aquifer zones. The interpreted distribution of these textural properties can be used to define the distribution of hydrologic characteristics (such as horizontal and vertical hydraulic conductivity and groundwater storage), which can be used in a numerical groundwater-flow and solute-transport model. 


\section{Textural Classification of Borehole Data}

In order to describe the spatial variability in textural properties, the drillers' lithologic descriptions and geophysical e-logs were evaluated and classified by texture. Texture is defined as the percentage of coarse-grained sediment present in a specified subsurface-depth interval (Hanson and others, 1990; Laudon and Belitz, 1991; Faunt and others, 2009). Drillers' log depth-interval entries were categorized into 15 main lithology types and assigned one of 8 lithology modifiers (if applicable), resulting in a total of 62 unique lithology classes (table 3). Each of these unique lithology classes was further codified as either "coarse" or "fine" (representing sediment grain size) on the basis of the main lithology type and modifier. In this study, coarse-grained sediment was defined as that consisting principally of sand, gravel, boulders, or cobbles. Fine-grained sediment was defined as that consisting principally of clay, loam, mud, silt, or conglomerate. If either the lithology or its modifier was considered fine-grained, then the overall composition was considered to be fine-grained (for example, silty-sand and sandy-silt were considered fine-grained; table 3).

Geophysical e-logs measure the resistivity of rock and sediment adjacent to a borehole. Typically, greater resistance represents more permeable materials, such as sand; lower resistance indicates clays. However, if seawater is present or the sediments have a high salt concentration the resistance is substantially less, irrespective of the lithology. Combining resistivity values from e-log measurements with descriptions from drillers' logs often provides a more detailed description of borehole lithology. Using the associated drillers' log as a guide, a vertical line was subjectively fitted with the resistivity to the e-log, such that it delineated coarse- (right of the line) and fine- (left of the line) grained materials by depth. An example of this process is shown in figure 6, where a red line is fit across the black resistivity curve until the coarse (shaded) and fine (white) depth intervals generally correspond to the observations in the associated drillers' log.

Table 3. Unique lithology descriptions and modifiers with associated binary coarse or fine classification as identified in drillers' lithology logs, Santa Barbara and Foothill groundwater basins, Santa Barbara, California.

[Descriptions with no associated modifier are indicated by dashes (-) in the appropriate modifier cell.]

\begin{tabular}{|c|c|c|c|c|c|}
\hline Modifier & Lithology & Texture & Modifier & Lithology & Texture \\
\hline- & Boulders & Coarse & Clayey & Sand & Fine \\
\hline Clayey & Boulders & Fine & Cobbley & Sand & Coarse \\
\hline Sandy & Boulders & Coarse & Gravelly & Sand & Coarse \\
\hline Silty & Boulders & Fine & Muddy & Sand & Fine \\
\hline- & Clay & Fine & Pebbley & Sand & Coarse \\
\hline Cobbley & Clay & Fine & Silty & Sand & Fine \\
\hline Gravelly & Clay & Fine & Sticky & Sand & Fine \\
\hline Pebbley & Clay & Fine & - & Sandstone & Coarse \\
\hline Sandy & Clay & Fine & Clayey & Sandstone & Fine \\
\hline Silty & Clay & Fine & Cobbley & Sandstone & Coarse \\
\hline Sticky & Clay & Fine & Gravelly & Sandstone & Coarse \\
\hline- & Cobbles & Coarse & Pebbley & Sandstone & Coarse \\
\hline Clayey & Cobbles & Fine & Sandy & Sandstone & Coarse \\
\hline Gravelly & Cobbles & Coarse & - & Shale & Fine \\
\hline Sandy & Cobbles & Coarse & Clayey & Shale & Fine \\
\hline - & Conglomerate & Fine & Cobbley & Shale & Fine \\
\hline - & Gravel & Coarse & Gravelly & Shale & Fine \\
\hline Clayey & Gravel & Fine & Sandy & Shale & Fine \\
\hline Cobbley & Gravel & Coarse & Silty & Shale & Fine \\
\hline Pebbley & Gravel & Coarse & - & Silt & Fine \\
\hline Sandy & Gravel & Coarse & Clayey & Silt & Fine \\
\hline Silty & Gravel & Fine & Gravelly & Silt & Fine \\
\hline - & Hard pan & Fine & Sandy & Silt & Fine \\
\hline Sandy & Hard pan & Fine & Sticky & Silt & Fine \\
\hline Sandy & Loam & Fine & - & Siltstone & Fine \\
\hline- & Mud & Fine & Clayey & Siltstone & Fine \\
\hline Sandy & Mud & Fine & Sandy & Siltstone & Fine \\
\hline Silty & Mud & Fine & - & Top soil & Coarse \\
\hline Sticky & Mud & Fine & Clayey & Top soil & Fine \\
\hline - & Rock & Fine & Cobbley & Top soil & Coarse \\
\hline- & Sand & Coarse & Sandy & Top soil & Coarse \\
\hline
\end{tabular}


$\boldsymbol{A}$

WELL LOG

\begin{tabular}{|c|c|c|}
\hline \multicolumn{2}{|c|}{ Total depth: 630 feet } & \multirow{2}{*}{$\begin{array}{l}\text { Depth of completed well: } 630 \text { fee } \\
\text { Fine sand }\end{array}$} \\
\hline 0 & -50 & \\
\hline 50 & -140 & Clay \\
\hline 140 & -148 & Sand \\
\hline 148 & -164 & Swelling clay \\
\hline 164 & -166 & Gravel \\
\hline 166 & -214 & Fine sand \\
\hline 214 & -244 & Swelling clay \\
\hline 244 & -248 & Rock \\
\hline 248 & -294 & Swelling clay \\
\hline 294 & -316 & Fine sand \\
\hline 316 & -366 & Swelling clay \\
\hline 366 & -372 & Brown rock \\
\hline 372 & -406 & Swelling clay \\
\hline 406 & -408 & Rock \\
\hline 408 & -430 & Clay \\
\hline 430 & -440 & Gravel (waterless) \\
\hline 440 & -510 & Clay \\
\hline 510 & -518 & Large gravel \\
\hline 518 & -532 & Clay \\
\hline 532 & -614 & Gravel (rock) \\
\hline 614 & -625 & Clay \\
\hline 625 & -630 & Gravel \\
\hline
\end{tabular}

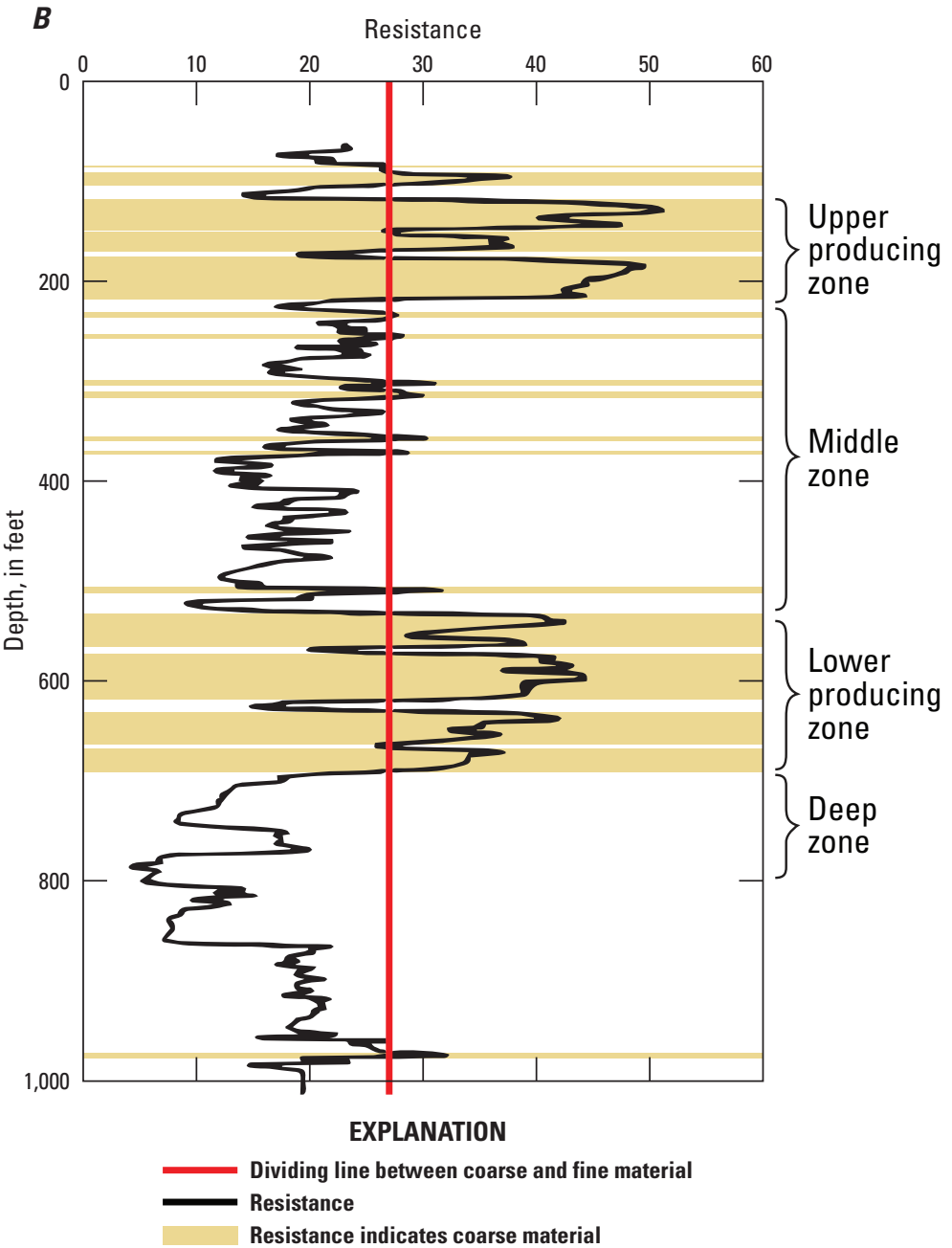

Figure 6. Example of logs from one borehole showing the textural variation and interpretation of borehole sediments with depth: $A$, drillers' lithology log, and $B$, geophysical resistivity e-log.

The percentage of coarse-grained deposits was calculated in 10-ft intervals both for the drillers' lithology logs and for geophysical e-logs. When the drillers' logs and geophysical e-logs were both available for a given borehole, the percentcoarse calculation from the e-log was preferentially used. The percent-coarse distribution for each borehole is shown in figure $5 B$ (appendix B-1).

\section{Textural Property Modeling}

The three-dimensional distribution of aquifer properties was interpolated from the percent-coarse values for each borehole using the EarthVision software. As stated earlier, the property gridding module in EarthVision uses a biharmonic cubic-spline algorithm. This algorithm is an iterative process that adjusts tension surfaces so that they honor the input data with minimum curvature. The percent-coarse distribution was modeled for each aquifer zone as quantified in the geometric framework model: percent-coarse data from each borehole were partitioned according to the zone they were in, allowing the gridding software to interpolate only the data from within a specific zone. In addition, the faults separating the subbasins act as barriers to interpolation within zones, maintaining fault offsets and modeling textural discontinuities.

\section{Textural Model Description}

A fence diagram showing the three-dimensional distribution of aquifer texture is shown in figure $5 B$. The cross-section profile locations, orientation, and vertical exaggeration (5 times) are the same as in figure $5 \mathrm{~A}$. The spatial pattern of the percentage of coarse-grained texture is heterogeneous in each aquifer zone, although in general, the textures in the upper and lower producing zones yielded higher percent-coarse values than the shallow, middle, and deep zones. Regions with a predominance of very coarsegrained material (greater than 90-percent coarse) are locally important, as indicated by concentrations of high percent coarse for boreholes in the Storage Unit I, Storage Unit III, and East Foothill subbasins. Overall, however, there appeared to be an intermediate predominance of coarse-grained material (30-70-percent coarse) throughout the study area. 


\section{Santa Barbara and Foothill Groundwater Basins Geohydrology and Optimal Water Resources Management}

Figure 7 shows the relationship between the geometric framework (fig. $7 A$ ) and textural property (fig. $7 B$ ) model outputs along a single cross section that cuts through Storage Unit I. Select geophysical resistivity e-logs used to quantify the aquifer-zone horizons and percent-coarse textural values are displayed. These e-logs show the textural variation of each aquifer zone by depth and how the aquifer-zone horizon elevations change along the cross section. The upper and lower producing zones were defined by distinct, highamplitude increases in the resistivity curves, indicating more permeable, very coarse-grained sedimentary material. This observation is shown in the textural model by a concentration of warm colors in the upper and lower producing zones. Conversely, the shallow, middle, and deep zones were characterized by smaller, low-amplitude shifts in resistivity, indicating less-permeable sediments with a lower percentage of coarse-grained material. These aquifer zones are indicated by cooler colors in figure $7 B$.
The middle producing zone is a thin, areally limited layer of coarse-grained deposits at a depth of about $360-380 \mathrm{ft}$ below NAVD 88 and extending 1-2 miles inland from the coast. Because of its limited areal extent, the middle producing zone was not modeled as an independent layer in the geometric framework model, but is shown in the cross section in figure 7. High-resistivity e-log readings in the middle zone indicate lenses of coarse-grained material, which were identified as the middle producing zone. The exception to this observation was from well 4N/27W-23F05, where a low-resistivity reading was interpreted to be seawater intrusion along the coarse-grained middle producing zone. 

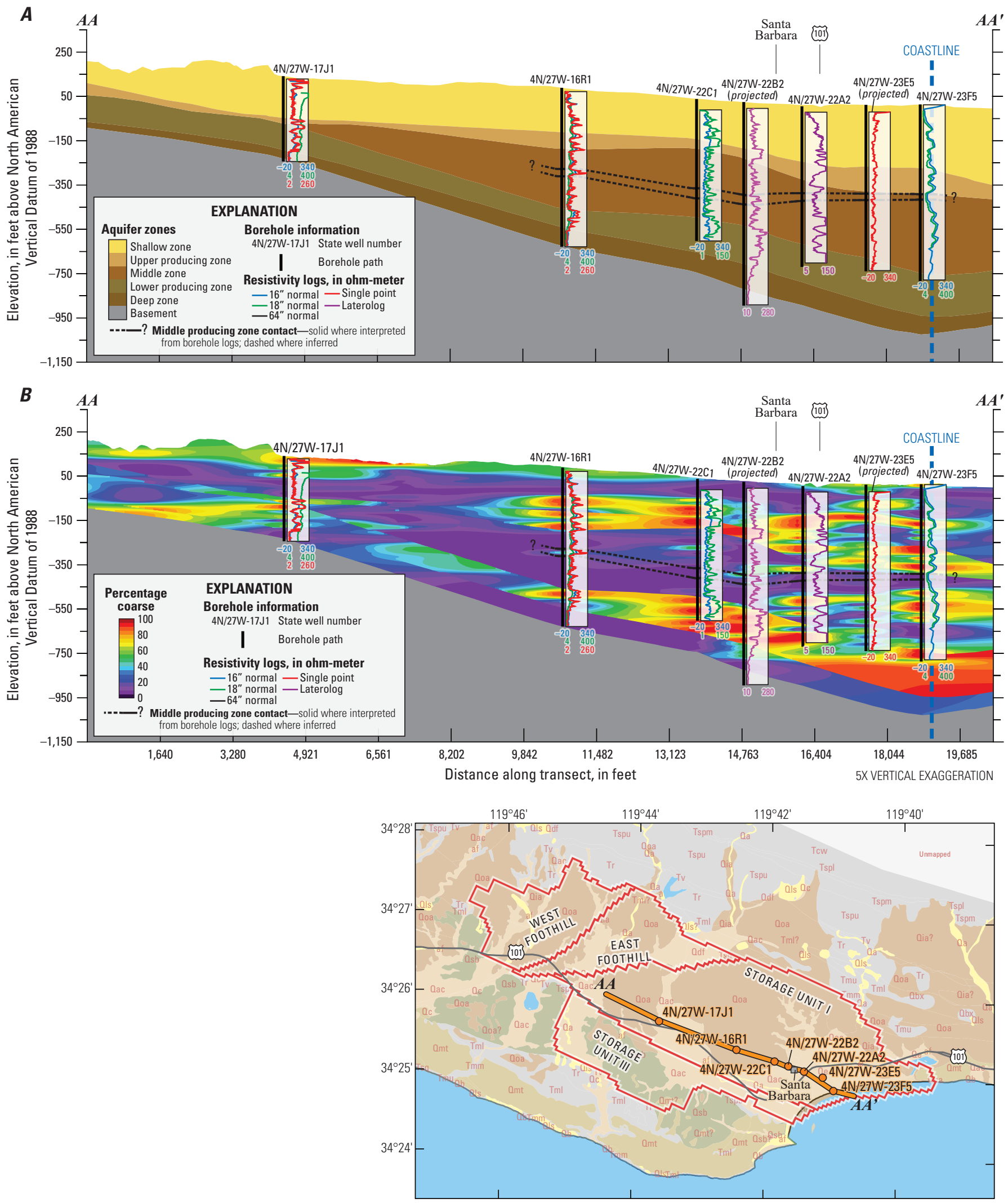

Figure 7. Cross section through the hydrogeologic framework model and geophysical resistivity e-logs shown for select boreholes and approximate extent of the middle producing zone, Santa Barbara and Foothill groundwater basins, Santa Barbara, California: $A$, aquiferzone horizons in the geometric framework model, and $B$, percent-coarse distributions in each aquifer zone in the textural model. 


\section{Summary and Conclusions}

This chapter presents an integrated three-dimensional hydrogeologic framework model (HFM) of the Santa Barbara and Foothill groundwater basins. The HFM has two parts: (1) a geometric framework model that quantifies the subsurface extent of the five aquifer zones in the study area with respect to major geologic structures and stratigraphy and (2) a texture model that characterizes the distribution of sedimentary lithology type and grain size in each aquifer zone. The geometric framework model incorporates previously published geologic maps, interpretive geologic cross sections, drillers' lithology descriptions, and geophysical resistivity e-log information in a three-dimensional digital representation of aquifer-zone horizons and major geologic structures. The texture model uses interpreted lithologic information from drillers' lithology logs and e-logs to interpolate the distribution of coarse- and fine-grained material throughout the aquifer zones defined in the geometric framework model.

The aquifer system in the Santa Barbara and Foothill groundwater basins was divided into five stratigraphic aquifer zones made up of unconsolidated sedimentary deposits of Pleistocene age and younger. The five aquifer zones are, from youngest to oldest, the shallow zone, upper producing zone, middle zone, lower producing zone, and deep zone. With few exceptions, the five aquifer zones are present throughout the study area, but vary in thickness between and within subbasins. The two main producing aquifer layers, the upper and lower producing zones, contain considerably more coarse-grained material than the other three zones. Thin lenses of coarse sediments are locally present in the shallow, middle, and deep zones and could be significant in terms of groundwater extraction, such as the middle producing zone in the middle zone.

\section{References Cited}

Burow, K.R., Shelton, J.L., Hevesi, J.A., and Weissmann, G.S., 2004, Hydrogeologic characterization of the Modesto area, San Joaquin Valley, California: U.S. Geological Survey Scientific Investigations Report 2004-5232, 54 p., http://pubs.usgs.gov/sir/2004/5232/.

Dynamic Graphics, Inc., 2015, EarthVision, accessed November 24, 2015, at http://www.dgi.com/earthvision/evmain.html.
Faunt, C.C., Belitz, Kenneth, and Hanson, R.T., 2009, Development of a three-dimensional model of sedimentary texture in valley-fill deposits of Central Valley, CA, USA: Hydrogeology Journal, v. 18, p. 625-649, https://doi.org/0.1007/s10040-009-0539-7.

Freckleton, J.R., 1989, Geohydrology of the Foothill groundwater basin near Santa Barbara, California: U.S. Geological Survey Water-Resources Investigations Report 89-4017, 46 p., https://pubs.er.usgs.gov/publication/wri894017.

Freckleton, J.R., Martin, P., Nishikawa, T., 1998, Geohydrology of Storage Unit III and a combined flow model of the Santa Barbara and foothill ground-water basins, Santa Barbara County, California: U.S. Geological Survey Water-Resources Investigations Report 97-4121, 85 p., http://pubs.er.usgs.gov/publication/wri974121.

Hanson, R.T., Anderson, S.R., and Pool, D.R., 1990, Simulation of ground-water flow and potential land subsidence, Avra Valley, Arizona: U.S. Geological Survey Water-Resources Investigations Report 90-4178, 41 p., http://pubs.er.usgs.gov/usgspubs/wri/wri904178.

Hutchinson, C.B., 1979, Ground-water monitoring at Santa Barbara, California-Phase 1, Coastal monitor-well installation and initial measurements: U.S. Geological Survey Open-File Report 79-923, 24 p., https://pubs.er.usgs.gov/publication/ofr79923.

Laudon, J., and Belitz, K., 1991, Texture and depositional history of late Pleistocene-Holocene alluvium in the central part of the western San Joaquin Valley, California: Environmental and Engineering Geoscience, v. 28, no. 1, p. 73-88, https://doi.org/10.2113/ggeosci.xxviii.1.73.

Martin, P., and Berenbrock, C., 1986, Ground-water monitoring at Santa Barbara, California; Phase 3Development of a three-dimensional digital ground-water flow model for Storage Unit I of the Santa Barbara groundwater basin: U.S. Geological Survey Water-Resources Investigations Report 86-4103, 58 p., https://pubs.er.usgs.gov/publication/wri864103.

Minor, S.A., Kellogg, K.S., Stanley, R.G., Gurrola, L.D., Keller, E.A., and Brandt, T.R., 2009, Geologic map of the Santa Barbara coastal plain area, Santa Barbara County, California: U.S. Geological Survey Scientific Investigations Map 3001, scale 1:25,000, 1 sheet, pamphlet, 38 p., https://pubs.er.usgs.gov/publication/sim3001.

Muir, K.S., 1968, Ground-water reconnaissance of the Santa Barbara-Montecito area, Santa Barbara County, California: Geological Survey Water-Supply Paper 1859-A, p. A1A28, https://pubs.er.usgs.gov/publication/wsp1859A. 


\section{Appendix B-1}

Table B1-1. Calculated percent-coarse intervals for all drillers' logs and e-logs used to define textural properties of the hydrogeologic framework model.

[Percent-coarse values are calculated for 10-foot intervals for the entire depth of each borehole. State well number is the state of California well identifier; interval midpoint depth is the midpoint of each 10-foot interval, in feet from top of borehole; PC is the percent-coarse value for each interval; log type defines whether the borehole is a drillers' lithology log (D) or a geophysical e-log (E).]

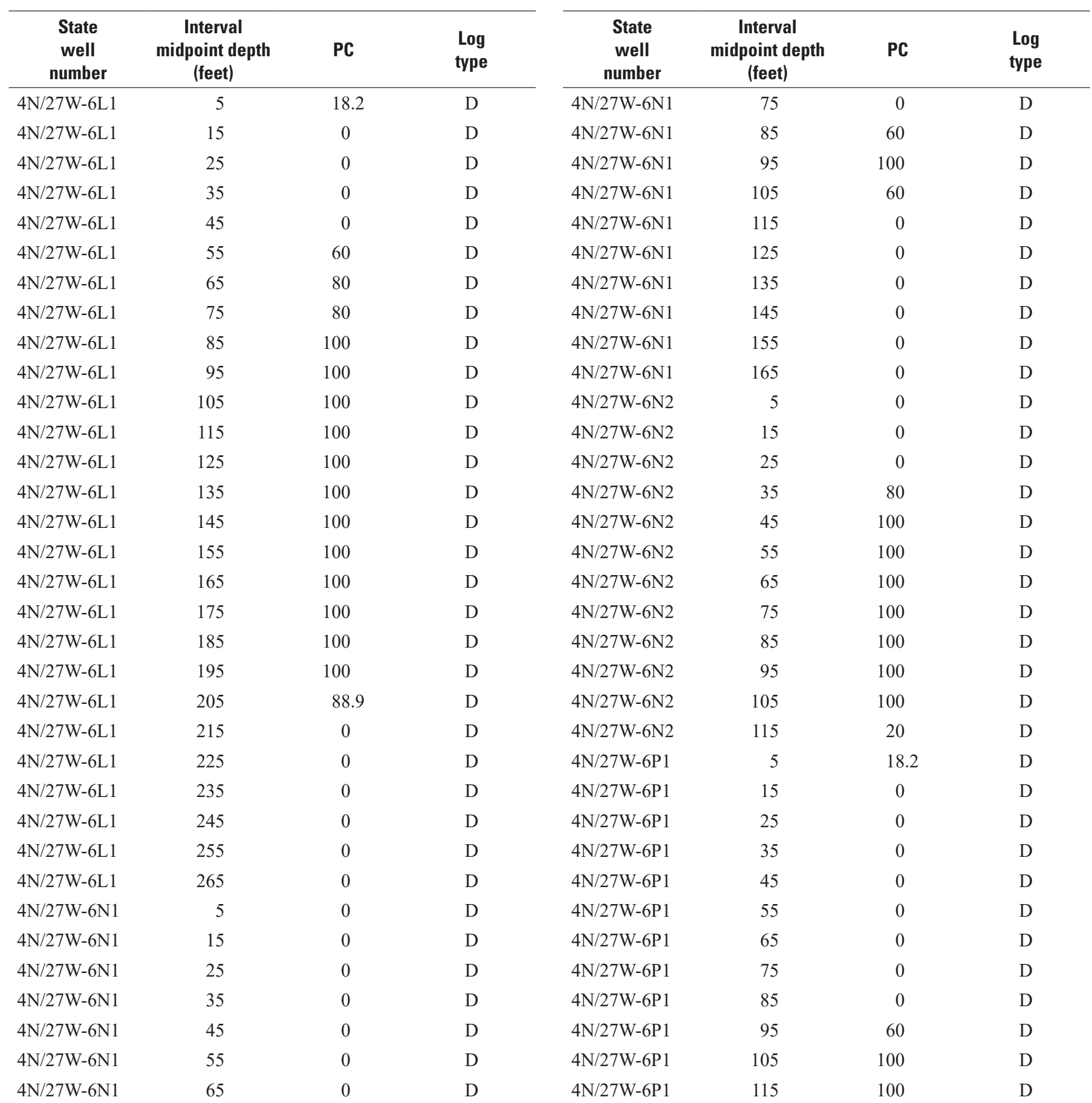


Table B1-1. Calculated percent-coarse intervals for all drillers' logs and e-logs used to define textural properties of the hydrogeologic framework model.-Continued

[Percent-coarse values are calculated for 10-foot intervals for the entire depth of each borehole. State well number is the state of California well identifier; interval midpoint depth is the midpoint of each 10-foot interval, in feet from top of borehole; PC is the percent-coarse value for each interval; log type defines whether the borehole is a drillers' lithology log (D) or a geophysical e-log (E).]

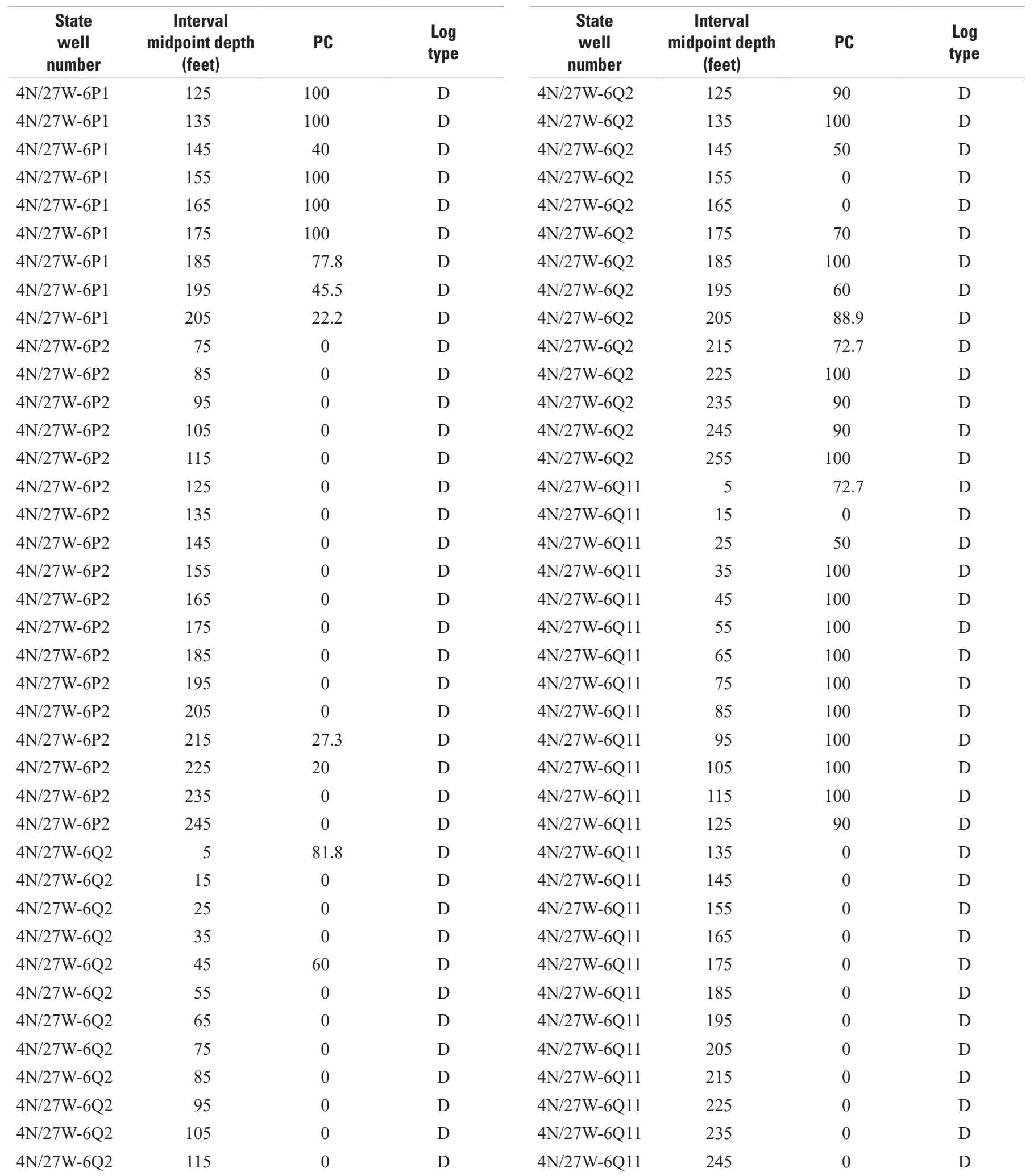


Table B1-1. Calculated percent-coarse intervals for all drillers' logs and e-logs used to define textural properties of the hydrogeologic framework model.-Continued

[Percent-coarse values are calculated for 10-foot intervals for the entire depth of each borehole. State well number is the state of California well identifier; interval midpoint depth is the midpoint of each 10-foot interval, in feet from top of borehole; PC is the percent-coarse value for each interval; log type defines whether the borehole is a drillers' lithology $\log (\mathrm{D})$ or a geophysical e-log (E).]

\begin{tabular}{|c|c|c|c|c|c|c|c|}
\hline $\begin{array}{c}\text { State } \\
\text { well } \\
\text { number }\end{array}$ & $\begin{array}{c}\text { Interval } \\
\text { midpoint depth } \\
\text { (feet) }\end{array}$ & PC & $\begin{array}{l}\text { Log } \\
\text { type }\end{array}$ & $\begin{array}{c}\text { State } \\
\text { well } \\
\text { number }\end{array}$ & $\begin{array}{c}\text { Interval } \\
\text { midpoint depth } \\
\text { (feet) }\end{array}$ & PC & $\begin{array}{l}\text { Log } \\
\text { type }\end{array}$ \\
\hline 4N/27W-6Q11 & 255 & 0 & $\mathrm{D}$ & $4 \mathrm{~N} / 27 \mathrm{~W}-6 \mathrm{R} 2$ & 245 & 0 & $\mathrm{D}$ \\
\hline 4N/27W-6Q11 & 265 & 0 & $\mathrm{D}$ & $4 \mathrm{~N} / 27 \mathrm{~W}-6 \mathrm{R} 2$ & 255 & 0 & $\mathrm{D}$ \\
\hline 4N/27W-6Q11 & 275 & 0 & $\mathrm{D}$ & $4 \mathrm{~N} / 27 \mathrm{~W}-6 \mathrm{R} 2$ & 265 & 60 & $\mathrm{D}$ \\
\hline 4N/27W-6Q11 & 285 & 0 & $\mathrm{D}$ & $4 \mathrm{~N} / 27 \mathrm{~W}-6 \mathrm{R} 2$ & 275 & 100 & $\mathrm{D}$ \\
\hline 4N/27W-6Q11 & 305 & 0 & $\mathrm{D}$ & $4 \mathrm{~N} / 27 \mathrm{~W}-6 \mathrm{R} 2$ & 295 & 100 & $\mathrm{D}$ \\
\hline 4N/27W-6Q11 & 315 & 90 & $\mathrm{D}$ & $4 \mathrm{~N} / 27 \mathrm{~W}-6 \mathrm{R} 2$ & 305 & 20 & $\mathrm{D}$ \\
\hline 4N/27W-6Q11 & 325 & 100 & $\mathrm{D}$ & $4 \mathrm{~N} / 27 \mathrm{~W}-6 \mathrm{R} 3$ & 5 & 0 & $\mathrm{D}$ \\
\hline 4N/27W-6Q11 & 335 & 100 & $\mathrm{D}$ & $4 \mathrm{~N} / 27 \mathrm{~W}-6 \mathrm{R} 3$ & 15 & 0 & $\mathrm{D}$ \\
\hline 4N/27W-6Q11 & 375 & 100 & $\mathrm{D}$ & 4N/27W-6R3 & 55 & 0 & $\mathrm{D}$ \\
\hline 4N/27W-6Q11 & 385 & 100 & $\mathrm{D}$ & $4 \mathrm{~N} / 27 \mathrm{~W}-6 \mathrm{R} 3$ & 65 & 0 & $\mathrm{D}$ \\
\hline 4N/27W-6Q11 & 395 & 90 & $\mathrm{D}$ & $4 \mathrm{~N} / 27 \mathrm{~W}-6 \mathrm{R} 3$ & 75 & 0 & $\mathrm{D}$ \\
\hline 4N/27W-6R2 & 5 & 54.5 & $\mathrm{D}$ & $4 \mathrm{~N} / 27 \mathrm{~W}-6 \mathrm{R} 3$ & 85 & 0 & $\mathrm{D}$ \\
\hline $4 \mathrm{~N} / 27 \mathrm{~W}-6 \mathrm{R} 2$ & 15 & 0 & $\mathrm{D}$ & $4 \mathrm{~N} / 27 \mathrm{~W}-6 \mathrm{R} 3$ & 95 & 0 & $\mathrm{D}$ \\
\hline $4 \mathrm{~N} / 27 \mathrm{~W}-6 \mathrm{R} 2$ & 25 & 0 & $\mathrm{D}$ & $4 \mathrm{~N} / 27 \mathrm{~W}-6 \mathrm{R} 3$ & 105 & 0 & $\mathrm{D}$ \\
\hline 4N/27W-6R2 & 35 & 0 & $\mathrm{D}$ & $4 \mathrm{~N} / 27 \mathrm{~W}-6 \mathrm{R} 3$ & 115 & 80 & $\mathrm{D}$ \\
\hline $4 \mathrm{~N} / 27 \mathrm{~W}-6 \mathrm{R} 2$ & 45 & 0 & $\mathrm{D}$ & $4 \mathrm{~N} / 27 \mathrm{~W}-6 \mathrm{R} 3$ & 125 & 100 & $\mathrm{D}$ \\
\hline 4N/27W-6R2 & 55 & 0 & $\mathrm{D}$ & $4 \mathrm{~N} / 27 \mathrm{~W}-6 \mathrm{R} 3$ & 135 & 80 & $\mathrm{D}$ \\
\hline $4 \mathrm{~N} / 27 \mathrm{~W}-6 \mathrm{R} 2$ & 125 & 0 & $\mathrm{D}$ & $4 \mathrm{~N} / 27 \mathrm{~W}-6 \mathrm{R} 3$ & 205 & 100 & $\mathrm{D}$ \\
\hline 4N/27W-6R2 & 135 & 70 & $\mathrm{D}$ & $4 \mathrm{~N} / 27 \mathrm{~W}-6 \mathrm{R} 3$ & 215 & 63.6 & $\mathrm{D}$ \\
\hline $4 \mathrm{~N} / 27 \mathrm{~W}-6 \mathrm{R} 2$ & 145 & 30 & $\mathrm{D}$ & $4 \mathrm{~N} / 27 \mathrm{~W}-6 \mathrm{R} 3$ & 225 & 90 & $\mathrm{D}$ \\
\hline $4 \mathrm{~N} / 27 \mathrm{~W}-6 \mathrm{R} 2$ & 155 & 0 & $\mathrm{D}$ & $4 \mathrm{~N} / 27 \mathrm{~W}-6 \mathrm{R} 3$ & 235 & 90 & $\mathrm{D}$ \\
\hline $4 \mathrm{~N} / 27 \mathrm{~W}-6 \mathrm{R} 2$ & 165 & 0 & $\mathrm{D}$ & $4 \mathrm{~N} / 27 \mathrm{~W}-6 \mathrm{R} 3$ & 245 & 100 & $\mathrm{D}$ \\
\hline $4 \mathrm{~N} / 27 \mathrm{~W}-6 \mathrm{R} 2$ & 175 & 0 & $\mathrm{D}$ & $4 \mathrm{~N} / 27 \mathrm{~W}-6 \mathrm{R} 3$ & 255 & 100 & $\mathrm{D}$ \\
\hline $4 \mathrm{~N} / 27 \mathrm{~W}-6 \mathrm{R} 2$ & 185 & 40 & $\mathrm{D}$ & $4 \mathrm{~N} / 27 \mathrm{~W}-6 \mathrm{R} 3$ & 265 & 100 & $\mathrm{D}$ \\
\hline $4 \mathrm{~N} / 27 \mathrm{~W}-6 \mathrm{R} 2$ & 195 & 0 & $\mathrm{D}$ & $4 \mathrm{~N} / 27 \mathrm{~W}-7 \mathrm{~A} 5$ & 155 & 0 & $\mathrm{D}$ \\
\hline $4 \mathrm{~N} / 27 \mathrm{~W}-6 \mathrm{R} 2$ & 205 & 88.9 & $\mathrm{D}$ & $4 \mathrm{~N} / 27 \mathrm{~W}-7 \mathrm{~A} 5$ & 165 & 0 & $\mathrm{D}$ \\
\hline 4N/27W-6R2 & 215 & 100 & $\mathrm{D}$ & 4N/27W-7A5 & 175 & 10 & $\mathrm{D}$ \\
\hline 4N/27W-6R2 & 225 & 60 & $\mathrm{D}$ & $4 \mathrm{~N} / 27 \mathrm{~W}-7 \mathrm{~A} 5$ & 185 & 44.4 & $\mathrm{D}$ \\
\hline $4 \mathrm{~N} / 27 \mathrm{~W}-6 \mathrm{R} 2$ & 235 & 0 & $\mathrm{D}$ & $4 \mathrm{~N} / 27 \mathrm{~W}-7 \mathrm{~A} 5$ & 195 & 0 & $\mathrm{D}$ \\
\hline
\end{tabular}


Table B1-1. Calculated percent-coarse intervals for all drillers' logs and e-logs used to define textural properties of the hydrogeologic framework model.-Continued

[Percent-coarse values are calculated for 10-foot intervals for the entire depth of each borehole. State well number is the state of California well identifier; interval midpoint depth is the midpoint of each 10-foot interval, in feet from top of borehole; PC is the percent-coarse value for each interval; log type defines whether the borehole is a drillers' lithology log (D) or a geophysical e-log (E).]

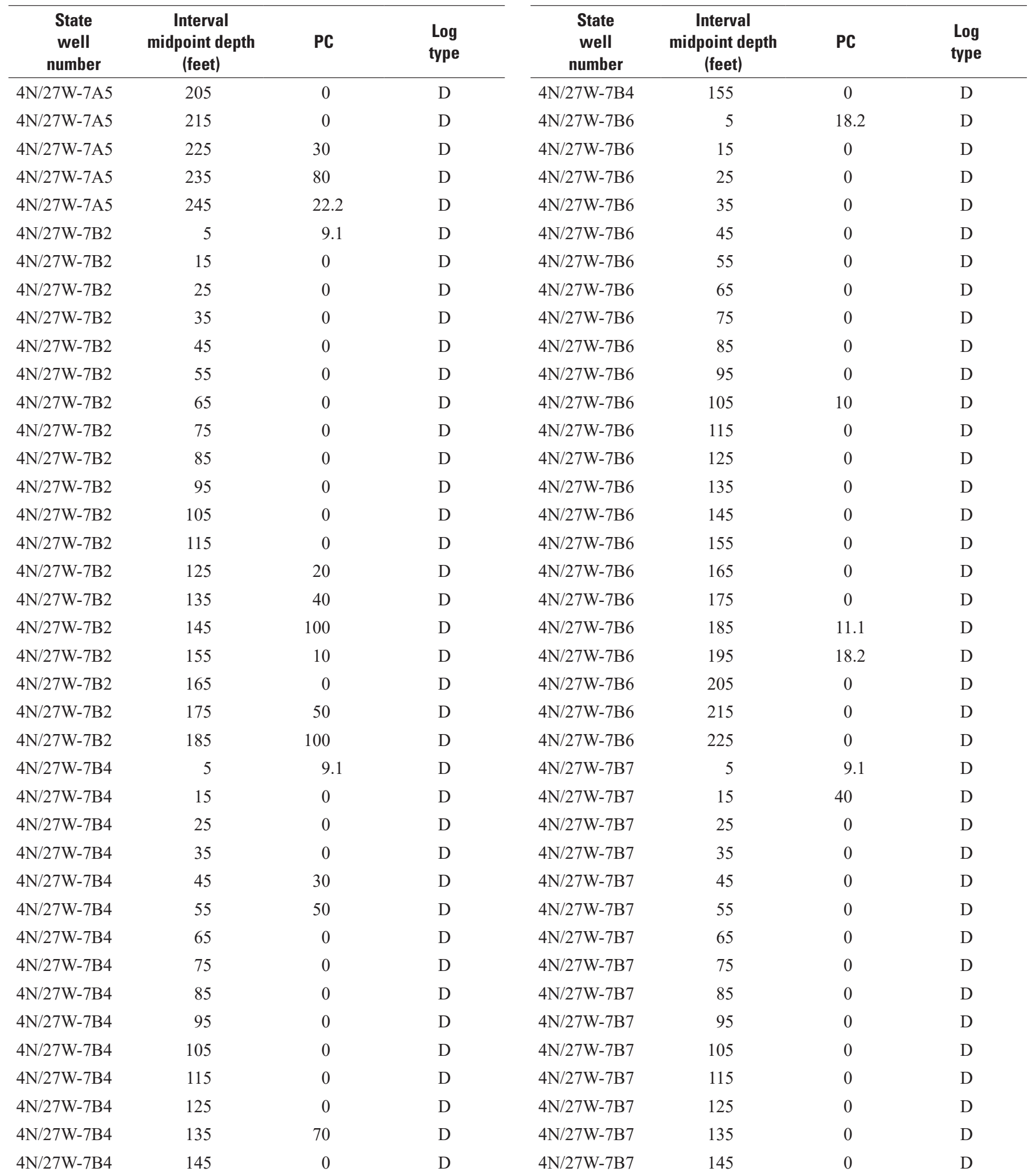


Table B1-1. Calculated percent-coarse intervals for all drillers' logs and e-logs used to define textural properties of the hydrogeologic framework model.-Continued

[Percent-coarse values are calculated for 10-foot intervals for the entire depth of each borehole. State well number is the state of California well identifier; interval midpoint depth is the midpoint of each 10-foot interval, in feet from top of borehole; PC is the percent-coarse value for each interval; log type defines whether the borehole is a drillers' lithology $\log (\mathrm{D})$ or a geophysical e-log (E).]

\begin{tabular}{|c|c|c|c|c|c|c|c|}
\hline $\begin{array}{c}\text { State } \\
\text { well } \\
\text { number }\end{array}$ & $\begin{array}{c}\text { Interval } \\
\text { midpoint depth } \\
\text { (feet) }\end{array}$ & PC & $\begin{array}{l}\text { Log } \\
\text { type }\end{array}$ & $\begin{array}{c}\text { State } \\
\text { well } \\
\text { number }\end{array}$ & $\begin{array}{c}\text { Interval } \\
\text { midpoint depth } \\
\text { (feet) }\end{array}$ & PC & $\begin{array}{l}\text { Log } \\
\text { type }\end{array}$ \\
\hline $4 \mathrm{~N} / 27 \mathrm{~W}-7 \mathrm{~B} 7$ & 155 & 0 & $\mathrm{D}$ & $4 \mathrm{~N} / 27 \mathrm{~W}-7 \mathrm{~B} 10$ & 295 & 81.8 & $\mathrm{D}$ \\
\hline 4N/27W-7B7 & 165 & 0 & $\mathrm{D}$ & 4N/27W-7B11 & 5 & 100 & $\mathrm{D}$ \\
\hline 4N/27W-7B7 & 175 & 0 & $\mathrm{D}$ & 4N/27W-7B11 & 15 & 100 & $\mathrm{D}$ \\
\hline 4N/27W-7B7 & 185 & 0 & $\mathrm{D}$ & $4 \mathrm{~N} / 27 \mathrm{~W}-7 \mathrm{~B} 11$ & 25 & 100 & $\mathrm{D}$ \\
\hline 4N/27W-7B7 & 205 & 0 & $\mathrm{D}$ & 4N/27W-7B11 & 45 & 100 & $\mathrm{D}$ \\
\hline 4N/27W-7B7 & 215 & 0 & $\mathrm{D}$ & 4N/27W-7B11 & 55 & 90 & $\mathrm{D}$ \\
\hline 4N/27W-7B7 & 225 & 40 & $\mathrm{D}$ & 4N/27W-7B11 & 65 & 0 & $\mathrm{D}$ \\
\hline 4N/27W-7B7 & 235 & 40 & $\mathrm{D}$ & $4 \mathrm{~N} / 27 \mathrm{~W}-7 \mathrm{~B} 11$ & 75 & 0 & $\mathrm{D}$ \\
\hline 4N/27W-7B10 & 25 & 0 & $\mathrm{D}$ & 4N/27W-7B11 & 115 & 0 & $\mathrm{D}$ \\
\hline $4 \mathrm{~N} / 27 \mathrm{~W}-7 \mathrm{~B} 10$ & 35 & 0 & $\mathrm{D}$ & 4N/27W-7B11 & 125 & 0 & $\mathrm{D}$ \\
\hline $4 \mathrm{~N} / 27 \mathrm{~W}-7 \mathrm{~B} 10$ & 45 & 0 & $\mathrm{D}$ & 4N/27W-7B11 & 135 & 0 & $\mathrm{D}$ \\
\hline 4N/27W-7B10 & 55 & 0 & $\mathrm{D}$ & $4 \mathrm{~N} / 27 \mathrm{~W}-7 \mathrm{~B} 11$ & 145 & 0 & $\mathrm{D}$ \\
\hline $4 \mathrm{~N} / 27 \mathrm{~W}-7 \mathrm{~B} 10$ & 65 & 0 & $\mathrm{D}$ & 4N/27W-7B11 & 155 & 0 & $\mathrm{D}$ \\
\hline $4 \mathrm{~N} / 27 \mathrm{~W}-7 \mathrm{~B} 10$ & 75 & 0 & $\mathrm{D}$ & 4N/27W-7B11 & 165 & 0 & $\mathrm{D}$ \\
\hline $4 \mathrm{~N} / 27 \mathrm{~W}-7 \mathrm{~B} 10$ & 85 & 0 & $\mathrm{D}$ & 4N/27W-7B11 & 175 & 0 & $\mathrm{D}$ \\
\hline $4 \mathrm{~N} / 27 \mathrm{~W}-7 \mathrm{~B} 10$ & 95 & 0 & $\mathrm{D}$ & 4N/27W-7B11 & 185 & 0 & $\mathrm{D}$ \\
\hline 4N/27W-7B10 & 105 & 0 & $\mathrm{D}$ & $4 \mathrm{~N} / 27 \mathrm{~W}-7 \mathrm{~B} 11$ & 195 & 0 & $\mathrm{D}$ \\
\hline $4 \mathrm{~N} / 27 \mathrm{~W}-7 \mathrm{~B} 10$ & 175 & 0 & $\mathrm{D}$ & $4 \mathrm{~N} / 27 \mathrm{~W}-7 \mathrm{~B} 11$ & 265 & 0 & $\mathrm{D}$ \\
\hline $4 \mathrm{~N} / 27 \mathrm{~W}-7 \mathrm{~B} 10$ & 185 & 0 & $\mathrm{D}$ & $4 \mathrm{~N} / 27 \mathrm{~W}-7 \mathrm{~B} 11$ & 275 & 0 & $\mathrm{D}$ \\
\hline $4 \mathrm{~N} / 27 \mathrm{~W}-7 \mathrm{~B} 10$ & 195 & 0 & $\mathrm{D}$ & 4N/27W-7B11 & 285 & 0 & $\mathrm{D}$ \\
\hline $4 \mathrm{~N} / 27 \mathrm{~W}-7 \mathrm{~B} 10$ & 205 & 0 & $\mathrm{D}$ & 4N/27W-7B11 & 295 & 0 & $\mathrm{D}$ \\
\hline $4 \mathrm{~N} / 27 \mathrm{~W}-7 \mathrm{~B} 10$ & 215 & 0 & $\mathrm{D}$ & 4N/27W-7B11 & 305 & 0 & $\mathrm{D}$ \\
\hline $4 \mathrm{~N} / 27 \mathrm{~W}-7 \mathrm{~B} 10$ & 225 & 0 & $\mathrm{D}$ & $4 \mathrm{~N} / 27 \mathrm{~W}-7 \mathrm{~B} 11$ & 315 & 0 & $\mathrm{D}$ \\
\hline $4 \mathrm{~N} / 27 \mathrm{~W}-7 \mathrm{~B} 10$ & 235 & 0 & $\mathrm{D}$ & $4 \mathrm{~N} / 27 \mathrm{~W}-7 \mathrm{~B} 11$ & 325 & 0 & $\mathrm{D}$ \\
\hline $4 \mathrm{~N} / 27 \mathrm{~W}-7 \mathrm{~B} 10$ & 245 & 0 & $\mathrm{D}$ & $4 \mathrm{~N} / 27 \mathrm{~W}-7 \mathrm{~B} 11$ & 335 & 0 & $\mathrm{D}$ \\
\hline $4 \mathrm{~N} / 27 \mathrm{~W}-7 \mathrm{~B} 10$ & 255 & 0 & $\mathrm{D}$ & 4N/27W-7B11 & 345 & 0 & $\mathrm{D}$ \\
\hline $4 \mathrm{~N} / 27 \mathrm{~W}-7 \mathrm{~B} 10$ & 265 & 0 & $\mathrm{D}$ & 4N/27W-7B11 & 355 & 0 & $\mathrm{D}$ \\
\hline 4N/27W-7B10 & 275 & 60 & $\mathrm{D}$ & $4 \mathrm{~N} / 27 \mathrm{~W}-7 \mathrm{~B} 11$ & 365 & 0 & $\mathrm{D}$ \\
\hline $4 \mathrm{~N} / 27 \mathrm{~W}-7 \mathrm{~B} 10$ & 285 & 20 & $\mathrm{D}$ & $4 \mathrm{~N} / 27 \mathrm{~W}-7 \mathrm{~B} 11$ & 375 & 0 & $\mathrm{D}$ \\
\hline
\end{tabular}


Table B1-1. Calculated percent-coarse intervals for all drillers' logs and e-logs used to define textural properties of the hydrogeologic framework model.-Continued

[Percent-coarse values are calculated for 10-foot intervals for the entire depth of each borehole. State well number is the state of California well identifier; interval midpoint depth is the midpoint of each 10-foot interval, in feet from top of borehole; PC is the percent-coarse value for each interval; log type defines whether the borehole is a drillers' lithology $\log (\mathrm{D})$ or a geophysical e-log (E).]

\begin{tabular}{|c|c|c|c|c|c|c|c|}
\hline $\begin{array}{l}\text { State } \\
\text { well } \\
\text { number }\end{array}$ & $\begin{array}{c}\text { Interval } \\
\text { midpoint depth } \\
\text { (feet) }\end{array}$ & PC & $\begin{array}{l}\text { Log } \\
\text { type }\end{array}$ & $\begin{array}{l}\text { State } \\
\text { well } \\
\text { number }\end{array}$ & $\begin{array}{c}\text { Interval } \\
\text { midpoint depth } \\
\text { (feet) }\end{array}$ & PC & $\begin{array}{l}\text { Log } \\
\text { type }\end{array}$ \\
\hline 4N/27W-7B11 & 385 & 0 & $\mathrm{D}$ & $4 \mathrm{~N} / 27 \mathrm{~W}-7 \mathrm{C} 6$ & 55 & 0 & $\mathrm{D}$ \\
\hline 4N/27W-7B11 & 395 & 0 & D & 4N/27W-7C6 & 65 & 0 & $\mathrm{D}$ \\
\hline 4N/27W-7B11 & 405 & 0 & D & $4 \mathrm{~N} / 27 \mathrm{~W}-7 \mathrm{C} 6$ & 75 & 0 & D \\
\hline 4N/27W-7B11 & 415 & 0 & D & $4 \mathrm{~N} / 27 \mathrm{~W}-7 \mathrm{C} 6$ & 85 & 0 & $\mathrm{D}$ \\
\hline 4N/27W-7B11 & 425 & 0 & D & $4 \mathrm{~N} / 27 \mathrm{~W}-7 \mathrm{C} 6$ & 95 & 0 & $\mathrm{D}$ \\
\hline 4N/27W-7B11 & 435 & 0 & D & $4 \mathrm{~N} / 27 \mathrm{~W}-7 \mathrm{C} 6$ & 105 & 0 & $\mathrm{D}$ \\
\hline 4N/27W-7B11 & 445 & 0 & D & $4 \mathrm{~N} / 27 \mathrm{~W}-7 \mathrm{C} 6$ & 115 & 0 & $\mathrm{D}$ \\
\hline 4N/27W-7B11 & 455 & 0 & D & 4N/27W-7C6 & 125 & 0 & $\mathrm{D}$ \\
\hline 4N/27W-7B11 & 465 & 0 & D & 4N/27W-7C6 & 135 & 0 & $\mathrm{D}$ \\
\hline 4N/27W-7B11 & 475 & 0 & D & $4 \mathrm{~N} / 27 \mathrm{~W}-7 \mathrm{C} 6$ & 145 & 0 & $\mathrm{D}$ \\
\hline 4N/27W-7B11 & 485 & 0 & D & $4 \mathrm{~N} / 27 \mathrm{~W}-7 \mathrm{C} 6$ & 155 & 0 & $\mathrm{D}$ \\
\hline 4N/27W-7C3 & 5 & 100 & D & $4 \mathrm{~N} / 27 \mathrm{~W}-7 \mathrm{C} 6$ & 165 & 0 & $\mathrm{D}$ \\
\hline $4 \mathrm{~N} / 27 \mathrm{~W}-7 \mathrm{C} 3$ & 15 & 30 & D & $4 \mathrm{~N} / 27 \mathrm{~W}-7 \mathrm{C} 6$ & 175 & 0 & $\mathrm{D}$ \\
\hline $4 \mathrm{~N} / 27 \mathrm{~W}-7 \mathrm{C} 3$ & 25 & 0 & D & $4 \mathrm{~N} / 27 \mathrm{~W}-7 \mathrm{C} 6$ & 185 & 0 & $\mathrm{D}$ \\
\hline $4 \mathrm{~N} / 27 \mathrm{~W}-7 \mathrm{C} 3$ & 35 & 0 & D & 4N/27W-7C6 & 195 & 0 & $\mathrm{D}$ \\
\hline $4 \mathrm{~N} / 27 \mathrm{~W}-7 \mathrm{C} 3$ & 45 & 0 & D & $4 \mathrm{~N} / 27 \mathrm{~W}-7 \mathrm{C} 6$ & 205 & 0 & $\mathrm{D}$ \\
\hline $4 \mathrm{~N} / 27 \mathrm{~W}-7 \mathrm{C} 3$ & 55 & 0 & D & $4 \mathrm{~N} / 27 \mathrm{~W}-7 \mathrm{C} 6$ & 215 & 0 & $\mathrm{D}$ \\
\hline $4 \mathrm{~N} / 27 \mathrm{~W}-7 \mathrm{C} 3$ & 65 & 0 & D & 4N/27W-7C6 & 225 & 0 & $\mathrm{D}$ \\
\hline $4 \mathrm{~N} / 27 \mathrm{~W}-7 \mathrm{C} 3$ & 75 & 0 & D & $4 \mathrm{~N} / 27 \mathrm{~W}-7 \mathrm{C} 6$ & 235 & 0 & $\mathrm{D}$ \\
\hline $4 \mathrm{~N} / 27 \mathrm{~W}-7 \mathrm{C} 3$ & 85 & 0 & D & $4 \mathrm{~N} / 27 \mathrm{~W}-7 \mathrm{C} 6$ & 245 & 66.7 & $\mathrm{D}$ \\
\hline $4 \mathrm{~N} / 27 \mathrm{~W}-7 \mathrm{C} 3$ & 95 & 0 & D & 4N/27W-7D1 & 5 & 0 & $\mathrm{D}$ \\
\hline $4 \mathrm{~N} / 27 \mathrm{~W}-7 \mathrm{C} 3$ & 105 & 0 & D & 4N/27W-7D1 & 15 & 0 & $\mathrm{D}$ \\
\hline $4 \mathrm{~N} / 27 \mathrm{~W}-7 \mathrm{C} 3$ & 115 & 0 & D & $4 \mathrm{~N} / 27 \mathrm{~W}-7 \mathrm{D} 1$ & 25 & 0 & $\mathrm{D}$ \\
\hline $4 \mathrm{~N} / 27 \mathrm{~W}-7 \mathrm{C} 3$ & 125 & 0 & D & 4N/27W-7D1 & 35 & 0 & $\mathrm{D}$ \\
\hline $4 \mathrm{~N} / 27 \mathrm{~W}-7 \mathrm{C} 3$ & 135 & 0 & D & 4N/27W-7D1 & 45 & 0 & $\mathrm{D}$ \\
\hline $4 \mathrm{~N} / 27 \mathrm{~W}-7 \mathrm{C} 3$ & 145 & 30 & D & 4N/27W-7D1 & 55 & 0 & $\mathrm{D}$ \\
\hline $4 \mathrm{~N} / 27 \mathrm{~W}-7 \mathrm{C} 3$ & 155 & 100 & D & $4 \mathrm{~N} / 27 \mathrm{~W}-7 \mathrm{D} 1$ & 65 & 0 & $\mathrm{D}$ \\
\hline $4 \mathrm{~N} / 27 \mathrm{~W}-7 \mathrm{C} 3$ & 165 & 100 & D & 4N/27W-7D1 & 75 & 0 & $\mathrm{D}$ \\
\hline $4 \mathrm{~N} / 27 \mathrm{~W}-7 \mathrm{C} 3$ & 175 & 100 & D & 4N/27W-7D1 & 85 & 0 & $\mathrm{D}$ \\
\hline $4 \mathrm{~N} / 27 \mathrm{~W}-7 \mathrm{C} 3$ & 185 & 100 & D & 4N/27W-7D1 & 95 & 0 & $\mathrm{D}$ \\
\hline $4 \mathrm{~N} / 27 \mathrm{~W}-7 \mathrm{C} 3$ & 195 & 36.4 & D & 4N/27W-7D1 & 105 & 0 & $\mathrm{D}$ \\
\hline $4 \mathrm{~N} / 27 \mathrm{~W}-7 \mathrm{C} 3$ & 205 & 0 & D & 4N/27W-7D1 & 115 & 0 & $\mathrm{D}$ \\
\hline $4 \mathrm{~N} / 27 \mathrm{~W}-7 \mathrm{C} 3$ & 215 & 27.3 & D & 4N/27W-7D1 & 125 & 0 & $\mathrm{D}$ \\
\hline $4 \mathrm{~N} / 27 \mathrm{~W}-7 \mathrm{C} 3$ & 225 & 100 & D & 4N/27W-7D1 & 135 & 0 & D \\
\hline $4 \mathrm{~N} / 27 \mathrm{~W}-7 \mathrm{C} 6$ & 5 & 18.2 & D & 4N/27W-7D1 & 145 & 0 & $\mathrm{D}$ \\
\hline $4 \mathrm{~N} / 27 \mathrm{~W}-7 \mathrm{C} 6$ & 15 & 0 & D & 4N/27W-7D1 & 155 & 0 & $\mathrm{D}$ \\
\hline $4 \mathrm{~N} / 27 \mathrm{~W}-7 \mathrm{C} 6$ & 25 & 0 & D & 4N/27W-7D1 & 165 & 0 & $\mathrm{D}$ \\
\hline $4 \mathrm{~N} / 27 \mathrm{~W}-7 \mathrm{C} 6$ & 35 & 0 & D & 4N/27W-7D1 & 175 & 0 & $\mathrm{D}$ \\
\hline 4N/27W-7C6 & 45 & 0 & D & $4 \mathrm{~N} / 27 \mathrm{~W}-7 \mathrm{D} 1$ & 185 & 0 & $\mathrm{D}$ \\
\hline
\end{tabular}


Table B1-1. Calculated percent-coarse intervals for all drillers' logs and e-logs used to define textural properties of the hydrogeologic framework model.-Continued

[Percent-coarse values are calculated for 10-foot intervals for the entire depth of each borehole. State well number is the state of California well identifier; interval midpoint depth is the midpoint of each 10-foot interval, in feet from top of borehole; PC is the percent-coarse value for each interval; log type defines whether the borehole is a drillers' lithology $\log (\mathrm{D})$ or a geophysical e-log (E).]

\begin{tabular}{|c|c|c|c|c|c|c|c|}
\hline $\begin{array}{c}\text { State } \\
\text { well } \\
\text { number }\end{array}$ & $\begin{array}{c}\text { Interval } \\
\text { midpoint depth } \\
\text { (feet) }\end{array}$ & PC & $\begin{array}{l}\text { Log } \\
\text { type }\end{array}$ & $\begin{array}{c}\text { State } \\
\text { well } \\
\text { number }\end{array}$ & $\begin{array}{c}\text { Interval } \\
\text { midpoint depth } \\
\text { (feet) }\end{array}$ & PC & $\begin{array}{l}\text { Log } \\
\text { type }\end{array}$ \\
\hline 4N/27W-7D1 & 195 & 0 & $\mathrm{D}$ & 4N/27W-7F5 & 105 & 0 & $\mathrm{D}$ \\
\hline 4N/27W-7D1 & 205 & 0 & $\mathrm{D}$ & $4 \mathrm{~N} / 27 \mathrm{~W}-7 \mathrm{~F} 5$ & 115 & 0 & $\mathrm{D}$ \\
\hline 4N/27W-7D1 & 215 & 100 & $\mathrm{D}$ & $4 \mathrm{~N} / 27 \mathrm{~W}-7 \mathrm{~F} 5$ & 125 & 0 & $\mathrm{D}$ \\
\hline 4N/27W-7D1 & 225 & 100 & $\mathrm{D}$ & $4 \mathrm{~N} / 27 \mathrm{~W}-7 \mathrm{~F} 5$ & 135 & 0 & $\mathrm{D}$ \\
\hline $4 \mathrm{~N} / 27 \mathrm{~W}-7 \mathrm{D} 1$ & 245 & 100 & $\mathrm{D}$ & $4 \mathrm{~N} / 27 \mathrm{~W}-7 \mathrm{~F} 5$ & 155 & 40 & $\mathrm{D}$ \\
\hline 4N/27W-7D1 & 255 & 90 & $\mathrm{D}$ & $4 \mathrm{~N} / 27 \mathrm{~W}-7 \mathrm{~F} 10$ & 5 & 36.4 & $\mathrm{D}$ \\
\hline 4N/27W-7D1 & 265 & 0 & $\mathrm{D}$ & $4 \mathrm{~N} / 27 \mathrm{~W}-7 \mathrm{~F} 10$ & 15 & 0 & $\mathrm{D}$ \\
\hline 4N/27W-7D1 & 275 & 100 & $\mathrm{D}$ & $4 \mathrm{~N} / 27 \mathrm{~W}-7 \mathrm{~F} 10$ & 25 & 0 & $\mathrm{D}$ \\
\hline 4N/27W-7D1 & 315 & 0 & $\mathrm{D}$ & 4N/27W-7F10 & 65 & 0 & $\mathrm{D}$ \\
\hline 4N/27W-7D1 & 325 & 0 & $\mathrm{D}$ & $4 \mathrm{~N} / 27 \mathrm{~W}-7 \mathrm{~F} 10$ & 75 & 0 & $\mathrm{D}$ \\
\hline 4N/27W-7D1 & 335 & 0 & $\mathrm{D}$ & $4 \mathrm{~N} / 27 \mathrm{~W}-7 \mathrm{~F} 10$ & 85 & 0 & $\mathrm{D}$ \\
\hline 4N/27W-7D1 & 345 & 0 & $\mathrm{D}$ & $4 \mathrm{~N} / 27 \mathrm{~W}-7 \mathrm{~F} 10$ & 95 & 0 & $\mathrm{D}$ \\
\hline $4 \mathrm{~N} / 27 \mathrm{~W}-7 \mathrm{D} 1$ & 355 & 0 & $\mathrm{D}$ & $4 \mathrm{~N} / 27 \mathrm{~W}-7 \mathrm{~F} 10$ & 105 & 60 & $\mathrm{D}$ \\
\hline $4 \mathrm{~N} / 27 \mathrm{~W}-7 \mathrm{D} 1$ & 365 & 0 & $\mathrm{D}$ & $4 \mathrm{~N} / 27 \mathrm{~W}-7 \mathrm{~F} 10$ & 115 & 50 & $\mathrm{D}$ \\
\hline 4N/27W-7D1 & 375 & 0 & $\mathrm{D}$ & $4 \mathrm{~N} / 27 \mathrm{~W}-7 \mathrm{~F} 10$ & 125 & 0 & $\mathrm{D}$ \\
\hline $4 \mathrm{~N} / 27 \mathrm{~W}-7 \mathrm{D} 1$ & 385 & 0 & $\mathrm{D}$ & $4 \mathrm{~N} / 27 \mathrm{~W}-7 \mathrm{~F} 10$ & 135 & 0 & $\mathrm{D}$ \\
\hline 4N/27W-7D1 & 395 & 0 & $\mathrm{D}$ & $4 \mathrm{~N} / 27 \mathrm{~W}-7 \mathrm{~F} 10$ & 145 & 0 & $\mathrm{D}$ \\
\hline 4N/27W-7D1 & 465 & 100 & $\mathrm{D}$ & $4 \mathrm{~N} / 27 \mathrm{~W}-7 \mathrm{~F} 10$ & 215 & 0 & $\mathrm{D}$ \\
\hline 4N/27W-7D1 & 475 & 90 & $\mathrm{D}$ & $4 \mathrm{~N} / 27 \mathrm{~W}-7 \mathrm{~F} 10$ & 225 & 0 & $\mathrm{D}$ \\
\hline $4 \mathrm{~N} / 27 \mathrm{~W}-7 \mathrm{~F} 5$ & 5 & 0 & $\mathrm{D}$ & $4 \mathrm{~N} / 27 \mathrm{~W}-7 \mathrm{~F} 10$ & 235 & 20 & $\mathrm{D}$ \\
\hline $4 \mathrm{~N} / 27 \mathrm{~W}-7 \mathrm{~F} 5$ & 15 & 20 & $\mathrm{D}$ & $4 \mathrm{~N} / 27 \mathrm{~W}-7 \mathrm{~F} 10$ & 245 & 0 & $\mathrm{D}$ \\
\hline $4 \mathrm{~N} / 27 \mathrm{~W}-7 \mathrm{~F} 5$ & 25 & 0 & $\mathrm{D}$ & $4 \mathrm{~N} / 27 \mathrm{~W}-7 \mathrm{~F} 10$ & 255 & 0 & $\mathrm{D}$ \\
\hline 4N/27W-7F5 & 35 & 0 & $\mathrm{D}$ & $4 \mathrm{~N} / 27 \mathrm{~W}-7 \mathrm{~F} 10$ & 265 & 0 & $\mathrm{D}$ \\
\hline 4N/27W-7F5 & 45 & 0 & $\mathrm{D}$ & $4 \mathrm{~N} / 27 \mathrm{~W}-7 \mathrm{~F} 10$ & 275 & 0 & $\mathrm{D}$ \\
\hline $4 \mathrm{~N} / 27 \mathrm{~W}-7 \mathrm{~F} 5$ & 55 & 30 & $\mathrm{D}$ & $4 \mathrm{~N} / 27 \mathrm{~W}-7 \mathrm{~F} 10$ & 285 & 0 & $\mathrm{D}$ \\
\hline $4 \mathrm{~N} / 27 \mathrm{~W}-7 \mathrm{~F} 5$ & 65 & 20 & $\mathrm{D}$ & $4 \mathrm{~N} / 27 \mathrm{~W}-7 \mathrm{~F} 10$ & 295 & 0 & $\mathrm{D}$ \\
\hline $4 \mathrm{~N} / 27 \mathrm{~W}-7 \mathrm{~F} 5$ & 75 & 0 & $\mathrm{D}$ & 4N/27W-7F11 & 5 & 9.1 & $\mathrm{D}$ \\
\hline 4N/27W-7F5 & 85 & 60 & $\mathrm{D}$ & 4N/27W-7F11 & 15 & 0 & $\mathrm{D}$ \\
\hline 4N/27W-7F5 & 95 & 10 & $\mathrm{D}$ & $4 \mathrm{~N} / 27 \mathrm{~W}-7 \mathrm{~F} 11$ & 25 & 0 & $\mathrm{D}$ \\
\hline
\end{tabular}


Table B1-1. Calculated percent-coarse intervals for all drillers' logs and e-logs used to define textural properties of the hydrogeologic framework model.-Continued

[Percent-coarse values are calculated for 10-foot intervals for the entire depth of each borehole. State well number is the state of California well identifier; interval midpoint depth is the midpoint of each 10-foot interval, in feet from top of borehole; PC is the percent-coarse value for each interval; log type defines whether the borehole is a drillers' lithology $\log (\mathrm{D})$ or a geophysical e-log (E).]

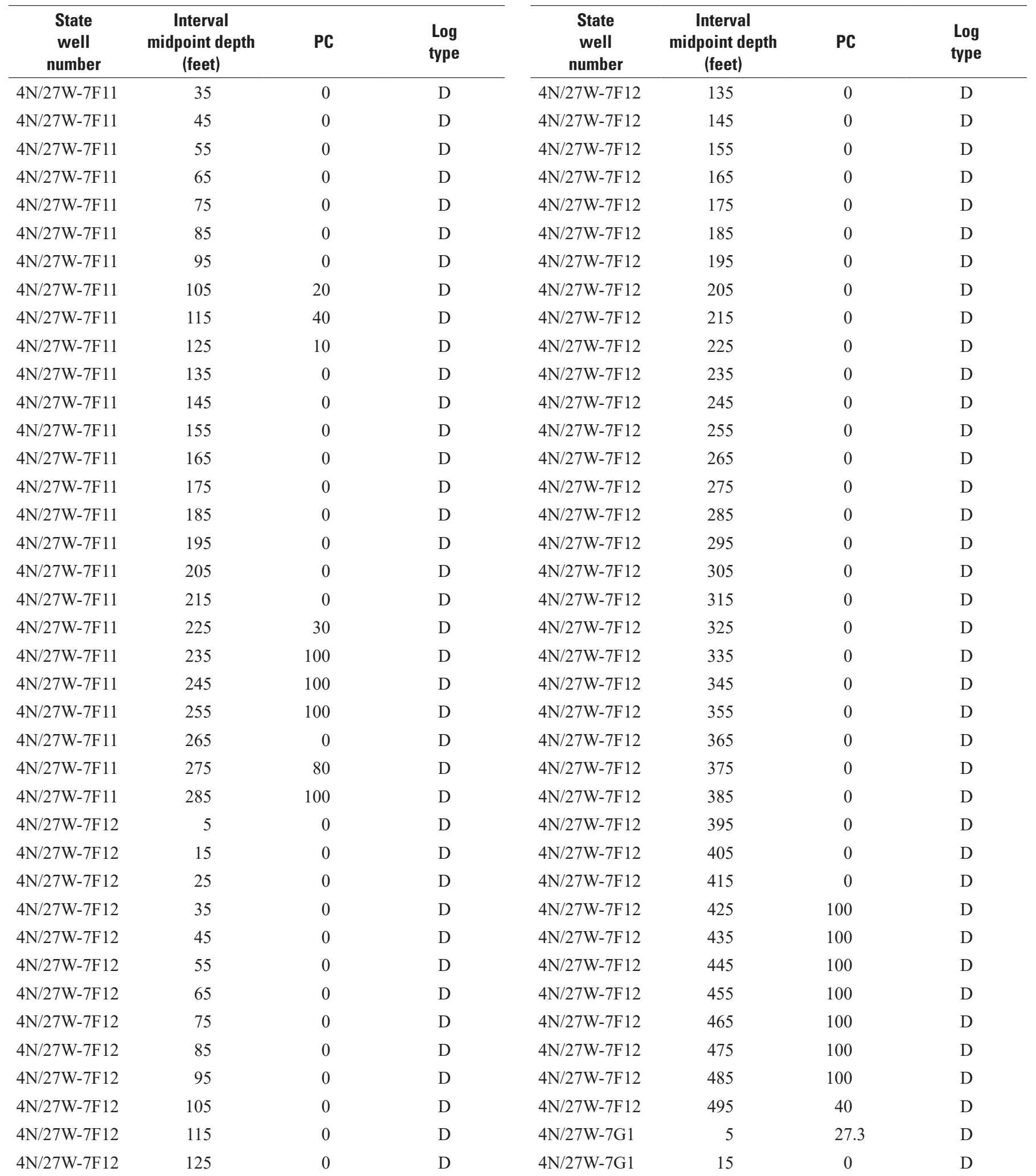


Table B1-1. Calculated percent-coarse intervals for all drillers' logs and e-logs used to define textural properties of the hydrogeologic framework model.-Continued

[Percent-coarse values are calculated for 10-foot intervals for the entire depth of each borehole. State well number is the state of California well identifier; interval midpoint depth is the midpoint of each 10-foot interval, in feet from top of borehole; PC is the percent-coarse value for each interval; log type defines whether the borehole is a drillers' lithology $\log (\mathrm{D})$ or a geophysical e-log (E).]

\begin{tabular}{|c|c|c|c|c|c|c|c|}
\hline $\begin{array}{c}\text { State } \\
\text { well } \\
\text { number }\end{array}$ & $\begin{array}{c}\text { Interval } \\
\text { midpoint depth } \\
\text { (feet) }\end{array}$ & PC & $\begin{array}{l}\text { Log } \\
\text { type }\end{array}$ & $\begin{array}{c}\text { State } \\
\text { well } \\
\text { number }\end{array}$ & $\begin{array}{c}\text { Interval } \\
\text { midpoint depth } \\
\text { (feet) }\end{array}$ & PC & $\begin{array}{l}\text { Log } \\
\text { type }\end{array}$ \\
\hline $4 \mathrm{~N} / 27 \mathrm{~W}-7 \mathrm{G} 1$ & 25 & 0 & $\mathrm{D}$ & $4 \mathrm{~N} / 27 \mathrm{~W}-7 \mathrm{G} 3$ & 65 & 0 & $\mathrm{D}$ \\
\hline 4N/27W-7G1 & 35 & 0 & $\mathrm{D}$ & 4N/27W-7G3 & 75 & 0 & $\mathrm{D}$ \\
\hline $4 \mathrm{~N} / 27 \mathrm{~W}-7 \mathrm{G} 1$ & 45 & 0 & $\mathrm{D}$ & $4 \mathrm{~N} / 27 \mathrm{~W}-7 \mathrm{G} 3$ & 85 & 0 & $\mathrm{D}$ \\
\hline 4N/27W-7G1 & 55 & 0 & $\mathrm{D}$ & 4N/27W-7G3 & 95 & 0 & $\mathrm{D}$ \\
\hline $4 \mathrm{~N} / 27 \mathrm{~W}-7 \mathrm{G} 1$ & 75 & 0 & $\mathrm{D}$ & $4 \mathrm{~N} / 27 \mathrm{~W}-7 \mathrm{G} 3$ & 115 & 0 & $\mathrm{D}$ \\
\hline 4N/27W-7G1 & 85 & 0 & $\mathrm{D}$ & $4 \mathrm{~N} / 27 \mathrm{~W}-7 \mathrm{G} 3$ & 125 & 30 & $\mathrm{D}$ \\
\hline $4 \mathrm{~N} / 27 \mathrm{~W}-7 \mathrm{G} 1$ & 95 & 20 & $\mathrm{D}$ & $4 \mathrm{~N} / 27 \mathrm{~W}-7 \mathrm{G} 3$ & 135 & 40 & $\mathrm{D}$ \\
\hline 4N/27W-7G1 & 105 & 0 & $\mathrm{D}$ & $4 \mathrm{~N} / 27 \mathrm{~W}-7 \mathrm{G} 3$ & 145 & 0 & $\mathrm{D}$ \\
\hline 4N/27W-7G1 & 145 & 60 & $\mathrm{D}$ & $4 \mathrm{~N} / 27 \mathrm{~W}-7 \mathrm{G} 3$ & 185 & 20 & $\mathrm{D}$ \\
\hline 4N/27W-7G1 & 155 & 100 & $\mathrm{D}$ & $4 \mathrm{~N} / 27 \mathrm{~W}-7 \mathrm{G} 3$ & 195 & 0 & $\mathrm{D}$ \\
\hline $4 \mathrm{~N} / 27 \mathrm{~W}-7 \mathrm{G} 1$ & 165 & 50 & $\mathrm{D}$ & $4 \mathrm{~N} / 27 \mathrm{~W}-7 \mathrm{G} 3$ & 205 & 0 & $\mathrm{D}$ \\
\hline 4N/27W-7G1 & 175 & 60 & $\mathrm{D}$ & $4 \mathrm{~N} / 27 \mathrm{~W}-7 \mathrm{G} 3$ & 215 & 0 & $\mathrm{D}$ \\
\hline 4N/27W-7G1 & 185 & 100 & $\mathrm{D}$ & 4N/27W-7G3 & 225 & 0 & $\mathrm{D}$ \\
\hline $4 \mathrm{~N} / 27 \mathrm{~W}-7 \mathrm{G} 1$ & 195 & 0 & $\mathrm{D}$ & $4 \mathrm{~N} / 27 \mathrm{~W}-7 \mathrm{G} 3$ & 235 & 0 & $\mathrm{D}$ \\
\hline 4N/27W-7G2 & 5 & 81.8 & $\mathrm{D}$ & $4 \mathrm{~N} / 27 \mathrm{~W}-7 \mathrm{G} 3$ & 245 & 0 & $\mathrm{D}$ \\
\hline $4 \mathrm{~N} / 27 \mathrm{~W}-7 \mathrm{G} 2$ & 15 & 0 & $\mathrm{D}$ & $4 \mathrm{~N} / 27 \mathrm{~W}-7 \mathrm{G} 3$ & 255 & 0 & $\mathrm{D}$ \\
\hline 4N/27W-7G2 & 25 & 0 & $\mathrm{D}$ & $4 \mathrm{~N} / 27 \mathrm{~W}-7 \mathrm{G} 3$ & 265 & 40 & $\mathrm{D}$ \\
\hline $4 \mathrm{~N} / 27 \mathrm{~W}-7 \mathrm{G} 2$ & 95 & 0 & $\mathrm{D}$ & $4 \mathrm{~N} / 27 \mathrm{~W}-7 \mathrm{G} 3$ & 335 & 30 & $\mathrm{D}$ \\
\hline $4 \mathrm{~N} / 27 \mathrm{~W}-7 \mathrm{G} 2$ & 105 & 0 & $\mathrm{D}$ & $4 \mathrm{~N} / 27 \mathrm{~W}-7 \mathrm{G} 3$ & 345 & 30 & $\mathrm{D}$ \\
\hline $4 \mathrm{~N} / 27 \mathrm{~W}-7 \mathrm{G} 2$ & 115 & 0 & $\mathrm{D}$ & $4 \mathrm{~N} / 27 \mathrm{~W}-7 \mathrm{G} 3$ & 355 & 55.6 & $\mathrm{D}$ \\
\hline $4 \mathrm{~N} / 27 \mathrm{~W}-7 \mathrm{G} 2$ & 125 & 0 & $\mathrm{D}$ & $4 \mathrm{~N} / 27 \mathrm{~W}-7 \mathrm{G} 6$ & 5 & 0 & $\mathrm{D}$ \\
\hline $4 \mathrm{~N} / 27 \mathrm{~W}-7 \mathrm{G} 2$ & 135 & 0 & $\mathrm{D}$ & 4N/27W-7G6 & 15 & 0 & $\mathrm{D}$ \\
\hline $4 \mathrm{~N} / 27 \mathrm{~W}-7 \mathrm{G} 2$ & 145 & 30 & $\mathrm{D}$ & 4N/27W-7G6 & 25 & 0 & $\mathrm{D}$ \\
\hline $4 \mathrm{~N} / 27 \mathrm{~W}-7 \mathrm{G} 3$ & 5 & 0 & $\mathrm{D}$ & 4N/27W-7G6 & 35 & 0 & $\mathrm{D}$ \\
\hline $4 \mathrm{~N} / 27 \mathrm{~W}-7 \mathrm{G} 3$ & 15 & 0 & $\mathrm{D}$ & 4N/27W-7G6 & 45 & 0 & $\mathrm{D}$ \\
\hline $4 \mathrm{~N} / 27 \mathrm{~W}-7 \mathrm{G} 3$ & 25 & 0 & $\mathrm{D}$ & $4 \mathrm{~N} / 27 \mathrm{~W}-7 \mathrm{G} 6$ & 55 & 0 & $\mathrm{D}$ \\
\hline $4 \mathrm{~N} / 27 \mathrm{~W}-7 \mathrm{G} 3$ & 35 & 0 & $\mathrm{D}$ & 4N/27W-7G6 & 65 & 0 & $\mathrm{D}$ \\
\hline 4N/27W-7G3 & 45 & 0 & $\mathrm{D}$ & 4N/27W-7G6 & 75 & 0 & $\mathrm{D}$ \\
\hline $4 \mathrm{~N} / 27 \mathrm{~W}-7 \mathrm{G} 3$ & 55 & 0 & $\mathrm{D}$ & 4N/27W-7G6 & 85 & 0 & $\mathrm{D}$ \\
\hline
\end{tabular}


Table B1-1. Calculated percent-coarse intervals for all drillers' logs and e-logs used to define textural properties of the hydrogeologic framework model.-Continued

[Percent-coarse values are calculated for 10-foot intervals for the entire depth of each borehole. State well number is the state of California well identifier; interval midpoint depth is the midpoint of each 10-foot interval, in feet from top of borehole; PC is the percent-coarse value for each interval; log type defines whether the borehole is a drillers' lithology log (D) or a geophysical e-log (E).]

\begin{tabular}{|c|c|c|c|c|c|c|c|}
\hline $\begin{array}{c}\text { State } \\
\text { well } \\
\text { number }\end{array}$ & $\begin{array}{c}\text { Interval } \\
\text { midpoint depth } \\
\text { (feet) }\end{array}$ & PC & $\begin{array}{l}\text { Log } \\
\text { type }\end{array}$ & $\begin{array}{c}\text { State } \\
\text { well } \\
\text { number }\end{array}$ & $\begin{array}{c}\text { Interval } \\
\text { midpoint depth } \\
\text { (feet) }\end{array}$ & PC & $\begin{array}{l}\text { Log } \\
\text { type }\end{array}$ \\
\hline 4N/27W-7G6 & 95 & 0 & $\mathrm{D}$ & 4N/27W-7G7 & 235 & 100 & $\mathrm{D}$ \\
\hline 4N/27W-7G6 & 105 & 0 & $\mathrm{D}$ & 4N/27W-7G7 & 245 & 100 & $\mathrm{D}$ \\
\hline 4N/27W-7G6 & 115 & 0 & $\mathrm{D}$ & 4N/27W-7G7 & 255 & 100 & $\mathrm{D}$ \\
\hline $4 \mathrm{~N} / 27 \mathrm{~W}-7 \mathrm{G} 6$ & 125 & 0 & $\mathrm{D}$ & $4 \mathrm{~N} / 27 \mathrm{~W}-7 \mathrm{G} 7$ & 265 & 40 & $\mathrm{D}$ \\
\hline 4N/27W-7G6 & 145 & 0 & $\mathrm{D}$ & 4N/27W-7G7 & 285 & 100 & $\mathrm{D}$ \\
\hline 4N/27W-7G6 & 155 & 0 & $\mathrm{D}$ & $4 \mathrm{~N} / 27 \mathrm{~W}-7 \mathrm{G} 7$ & 295 & 100 & $\mathrm{D}$ \\
\hline 4N/27W-7G6 & 165 & 0 & $\mathrm{D}$ & 4N/27W-7G7 & 305 & 90 & $\mathrm{D}$ \\
\hline 4N/27W-7G6 & 175 & 0 & $\mathrm{D}$ & 4N/27W-7G7 & 315 & 0 & $\mathrm{D}$ \\
\hline 4N/27W-7G6 & 215 & 0 & $\mathrm{D}$ & 4N/27W-7G7 & 355 & 0 & $\mathrm{D}$ \\
\hline 4N/27W-7G6 & 225 & 0 & $\mathrm{D}$ & 4N/27W-7G7 & 365 & 0 & $\mathrm{D}$ \\
\hline 4N/27W-7G6 & 235 & 0 & $\mathrm{D}$ & $4 \mathrm{~N} / 27 \mathrm{~W}-7 \mathrm{G} 7$ & 375 & 100 & $\mathrm{D}$ \\
\hline 4N/27W-7G6 & 245 & 44.4 & $\mathrm{D}$ & $4 \mathrm{~N} / 27 \mathrm{~W}-7 \mathrm{G} 7$ & 385 & 100 & $\mathrm{D}$ \\
\hline $4 \mathrm{~N} / 27 \mathrm{~W}-7 \mathrm{G} 7$ & 5 & 100 & $\mathrm{D}$ & $4 \mathrm{~N} / 27 \mathrm{~W}-7 \mathrm{G} 7$ & 395 & 100 & $\mathrm{D}$ \\
\hline $4 \mathrm{~N} / 27 \mathrm{~W}-7 \mathrm{G} 7$ & 15 & 100 & $\mathrm{D}$ & $4 \mathrm{~N} / 27 \mathrm{~W}-7 \mathrm{G} 7$ & 405 & 100 & $\mathrm{D}$ \\
\hline $4 \mathrm{~N} / 27 \mathrm{~W}-7 \mathrm{G} 7$ & 25 & 100 & $\mathrm{D}$ & $4 \mathrm{~N} / 27 \mathrm{~W}-7 \mathrm{G} 7$ & 415 & 100 & $\mathrm{D}$ \\
\hline 4N/27W-7G7 & 35 & 70 & $\mathrm{D}$ & 4N/27W-7G7 & 425 & 100 & $\mathrm{D}$ \\
\hline $4 \mathrm{~N} / 27 \mathrm{~W}-7 \mathrm{G} 7$ & 45 & 10 & $\mathrm{D}$ & 4N/27W-7G7 & 435 & 100 & $\mathrm{D}$ \\
\hline $4 \mathrm{~N} / 27 \mathrm{~W}-7 \mathrm{G} 7$ & 115 & 100 & $\mathrm{D}$ & $4 \mathrm{~N} / 27 \mathrm{~W}-7 \mathrm{H} 3$ & 65 & 20 & $\mathrm{D}$ \\
\hline $4 \mathrm{~N} / 27 \mathrm{~W}-7 \mathrm{G} 7$ & 125 & 100 & $\mathrm{D}$ & $4 \mathrm{~N} / 27 \mathrm{~W}-7 \mathrm{H} 3$ & 75 & 0 & $\mathrm{D}$ \\
\hline 4N/27W-7G7 & 135 & 50 & $\mathrm{D}$ & 4N/27W-7H3 & 85 & 0 & $\mathrm{D}$ \\
\hline $4 \mathrm{~N} / 27 \mathrm{~W}-7 \mathrm{G} 7$ & 145 & 0 & $\mathrm{D}$ & $4 \mathrm{~N} / 27 \mathrm{~W}-7 \mathrm{H} 3$ & 95 & 0 & $\mathrm{D}$ \\
\hline $4 \mathrm{~N} / 27 \mathrm{~W}-7 \mathrm{G} 7$ & 155 & 0 & $\mathrm{D}$ & $4 \mathrm{~N} / 27 \mathrm{~W}-7 \mathrm{H} 3$ & 105 & 0 & $\mathrm{D}$ \\
\hline $4 \mathrm{~N} / 27 \mathrm{~W}-7 \mathrm{G} 7$ & 165 & 30 & $\mathrm{D}$ & $4 \mathrm{~N} / 27 \mathrm{~W}-7 \mathrm{H} 3$ & 115 & 0 & $\mathrm{D}$ \\
\hline $4 \mathrm{~N} / 27 \mathrm{~W}-7 \mathrm{G} 7$ & 175 & 100 & $\mathrm{D}$ & $4 \mathrm{~N} / 27 \mathrm{~W}-7 \mathrm{H} 3$ & 125 & 0 & $\mathrm{D}$ \\
\hline $4 \mathrm{~N} / 27 \mathrm{~W}-7 \mathrm{G} 7$ & 185 & 100 & $\mathrm{D}$ & $4 \mathrm{~N} / 27 \mathrm{~W}-7 \mathrm{H} 3$ & 135 & 0 & $\mathrm{D}$ \\
\hline $4 \mathrm{~N} / 27 \mathrm{~W}-7 \mathrm{G} 7$ & 195 & 100 & $\mathrm{D}$ & $4 \mathrm{~N} / 27 \mathrm{~W}-7 \mathrm{H} 3$ & 145 & 0 & $\mathrm{D}$ \\
\hline 4N/27W-7G7 & 205 & 100 & $\mathrm{D}$ & $4 \mathrm{~N} / 27 \mathrm{~W}-7 \mathrm{H} 3$ & 155 & 0 & $\mathrm{D}$ \\
\hline $4 \mathrm{~N} / 27 \mathrm{~W}-7 \mathrm{G} 7$ & 215 & 100 & $\mathrm{D}$ & $4 \mathrm{~N} / 27 \mathrm{~W}-7 \mathrm{H} 3$ & 165 & 0 & $\mathrm{D}$ \\
\hline $4 \mathrm{~N} / 27 \mathrm{~W}-7 \mathrm{G} 7$ & 225 & 100 & $\mathrm{D}$ & $4 \mathrm{~N} / 27 \mathrm{~W}-7 \mathrm{H} 3$ & 175 & 0 & $\mathrm{D}$ \\
\hline
\end{tabular}


Table B1-1. Calculated percent-coarse intervals for all drillers' logs and e-logs used to define textural properties of the hydrogeologic framework model.-Continued

[Percent-coarse values are calculated for 10-foot intervals for the entire depth of each borehole. State well number is the state of California well identifier; interval midpoint depth is the midpoint of each 10-foot interval, in feet from top of borehole; PC is the percent-coarse value for each interval; log type defines whether the borehole is a drillers' lithology $\log (\mathrm{D})$ or a geophysical e-log (E).]

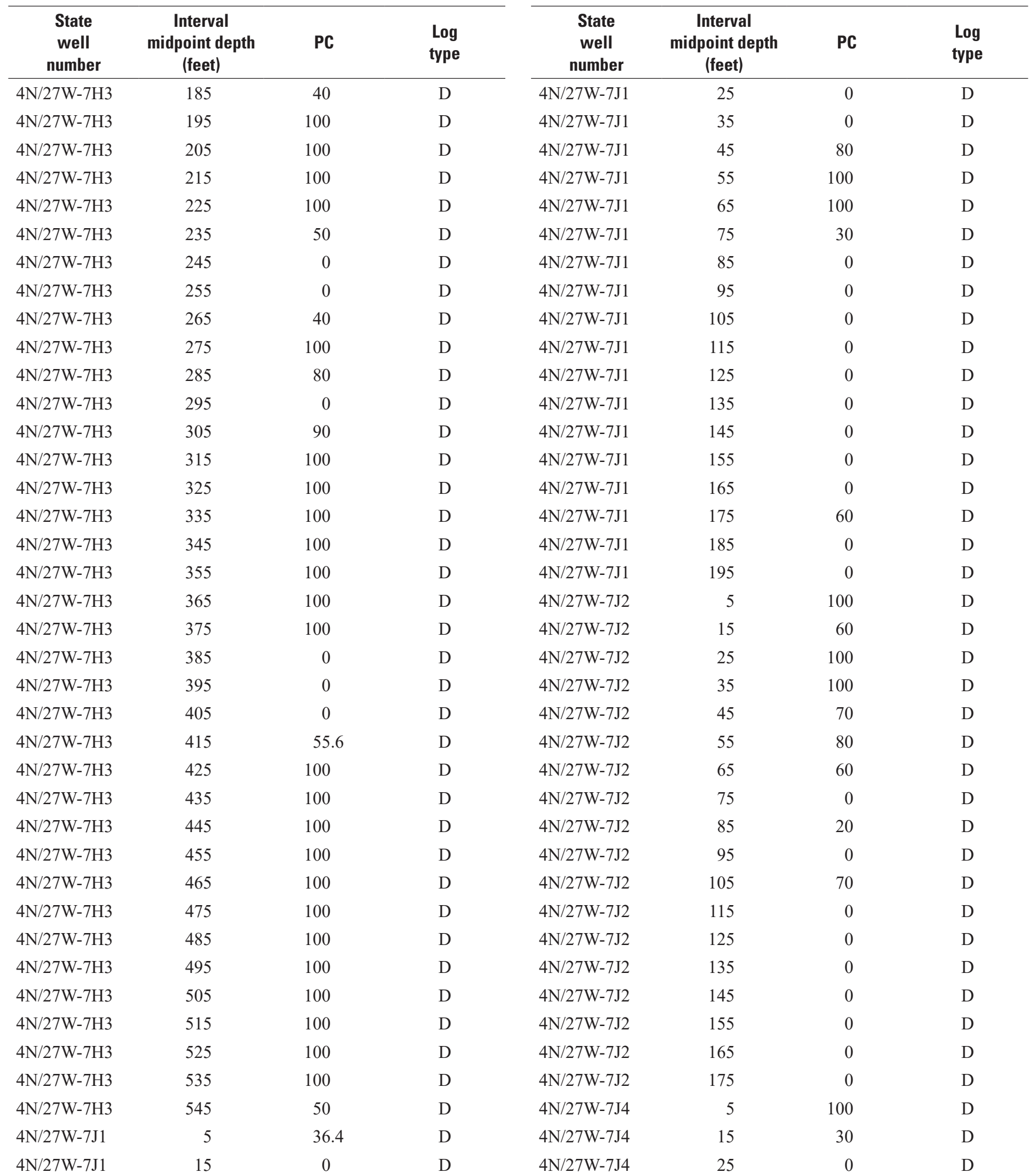


Table B1-1. Calculated percent-coarse intervals for all drillers' logs and e-logs used to define textural properties of the hydrogeologic framework model.-Continued

[Percent-coarse values are calculated for 10-foot intervals for the entire depth of each borehole. State well number is the state of California well identifier; interval midpoint depth is the midpoint of each 10-foot interval, in feet from top of borehole; PC is the percent-coarse value for each interval; log type defines whether the borehole is a drillers' lithology log (D) or a geophysical e-log (E).]

\begin{tabular}{|c|c|c|c|c|c|c|c|}
\hline $\begin{array}{c}\text { State } \\
\text { well } \\
\text { number }\end{array}$ & $\begin{array}{c}\text { Interval } \\
\text { midpoint depth } \\
\text { (feet) }\end{array}$ & PC & $\begin{array}{l}\text { Log } \\
\text { type }\end{array}$ & $\begin{array}{c}\text { State } \\
\text { well } \\
\text { number }\end{array}$ & $\begin{array}{c}\text { Interval } \\
\text { midpoint depth } \\
\text { (feet) }\end{array}$ & PC & $\begin{array}{l}\text { Log } \\
\text { type }\end{array}$ \\
\hline $4 \mathrm{~N} / 27 \mathrm{~W}-7 \mathrm{~J} 4$ & 35 & 80 & $\mathrm{D}$ & 4N/27W-7J5 & 5 & 18.2 & $\mathrm{D}$ \\
\hline $4 \mathrm{~N} / 27 \mathrm{~W}-7 \mathrm{~J} 4$ & 45 & 50 & $\mathrm{D}$ & 4N/27W-7J5 & 15 & 0 & $\mathrm{D}$ \\
\hline $4 \mathrm{~N} / 27 \mathrm{~W}-7 \mathrm{~J} 4$ & 55 & 0 & $\mathrm{D}$ & $4 \mathrm{~N} / 27 \mathrm{~W}-7 \mathrm{~J} 5$ & 25 & 0 & $\mathrm{D}$ \\
\hline $4 \mathrm{~N} / 27 \mathrm{~W}-7 \mathrm{~J} 4$ & 65 & 0 & $\mathrm{D}$ & 4N/27W-7J5 & 35 & 0 & $\mathrm{D}$ \\
\hline $4 \mathrm{~N} / 27 \mathrm{~W}-7 \mathrm{~J} 4$ & 85 & 0 & $\mathrm{D}$ & 4N/27W-7J5 & 55 & 0 & $\mathrm{D}$ \\
\hline 4N/27W-7J4 & 95 & 0 & $\mathrm{D}$ & 4N/27W-7J5 & 65 & 0 & $\mathrm{D}$ \\
\hline $4 \mathrm{~N} / 27 \mathrm{~W}-7 \mathrm{~J} 4$ & 105 & 0 & $\mathrm{D}$ & 4N/27W-7J5 & 75 & 0 & $\mathrm{D}$ \\
\hline $4 \mathrm{~N} / 27 \mathrm{~W}-7 \mathrm{~J} 4$ & 115 & 40 & $\mathrm{D}$ & $4 \mathrm{~N} / 27 \mathrm{~W}-7 \mathrm{~J} 5$ & 85 & 0 & $\mathrm{D}$ \\
\hline $4 \mathrm{~N} / 27 \mathrm{~W}-7 \mathrm{~J} 4$ & 155 & 60 & $\mathrm{D}$ & 4N/27W-7J5 & 125 & 0 & $\mathrm{D}$ \\
\hline $4 \mathrm{~N} / 27 \mathrm{~W}-7 \mathrm{~J} 4$ & 165 & 0 & $\mathrm{D}$ & $4 \mathrm{~N} / 27 \mathrm{~W}-7 \mathrm{~J} 5$ & 135 & 10 & $\mathrm{D}$ \\
\hline $4 \mathrm{~N} / 27 \mathrm{~W}-7 \mathrm{~J} 4$ & 175 & 90 & $\mathrm{D}$ & $4 \mathrm{~N} / 27 \mathrm{~W}-7 \mathrm{~J} 5$ & 145 & 40 & $\mathrm{D}$ \\
\hline $4 \mathrm{~N} / 27 \mathrm{~W}-7 \mathrm{~J} 4$ & 185 & 40 & $\mathrm{D}$ & 4N/27W-7J5 & 155 & 0 & $\mathrm{D}$ \\
\hline $4 \mathrm{~N} / 27 \mathrm{~W}-7 \mathrm{~J} 4$ & 195 & 0 & $\mathrm{D}$ & 4N/27W-7J5 & 165 & 100 & $\mathrm{D}$ \\
\hline 4N/27W-7J4 & 205 & 0 & $\mathrm{D}$ & 4N/27W-7J5 & 175 & 50 & $\mathrm{D}$ \\
\hline $4 \mathrm{~N} / 27 \mathrm{~W}-7 \mathrm{~J} 4$ & 215 & 0 & $\mathrm{D}$ & 4N/27W-7J5 & 185 & 40 & $\mathrm{D}$ \\
\hline $4 \mathrm{~N} / 27 \mathrm{~W}-7 \mathrm{~J} 4$ & 225 & 0 & $\mathrm{D}$ & $4 \mathrm{~N} / 27 \mathrm{~W}-7 \mathrm{~J} 5$ & 195 & 81.8 & $\mathrm{D}$ \\
\hline $4 \mathrm{~N} / 27 \mathrm{~W}-7 \mathrm{~J} 4$ & 235 & 0 & $\mathrm{D}$ & $4 \mathrm{~N} / 27 \mathrm{~W}-7 \mathrm{~J} 5$ & 205 & 55.6 & $\mathrm{D}$ \\
\hline $4 \mathrm{~N} / 27 \mathrm{~W}-7 \mathrm{~J} 4$ & 305 & 0 & $\mathrm{D}$ & $4 \mathrm{~N} / 27 \mathrm{~W}-7 \mathrm{~J} 5$ & 275 & 100 & $\mathrm{D}$ \\
\hline 4N/27W-7J4 & 315 & 30 & $\mathrm{D}$ & 4N/27W-7J5 & 285 & 100 & $\mathrm{D}$ \\
\hline $4 \mathrm{~N} / 27 \mathrm{~W}-7 \mathrm{~J} 4$ & 325 & 30 & $\mathrm{D}$ & $4 \mathrm{~N} / 27 \mathrm{~W}-7 \mathrm{~K} 2$ & 5 & 36.4 & $\mathrm{D}$ \\
\hline $4 \mathrm{~N} / 27 \mathrm{~W}-7 \mathrm{~J} 4$ & 335 & 0 & $\mathrm{D}$ & $4 \mathrm{~N} / 27 \mathrm{~W}-7 \mathrm{~K} 2$ & 15 & 0 & $\mathrm{D}$ \\
\hline $4 \mathrm{~N} / 27 \mathrm{~W}-7 \mathrm{~J} 4$ & 345 & 50 & $\mathrm{D}$ & $4 \mathrm{~N} / 27 \mathrm{~W}-7 \mathrm{~K} 2$ & 25 & 0 & $\mathrm{D}$ \\
\hline $4 \mathrm{~N} / 27 \mathrm{~W}-7 \mathrm{~J} 4$ & 355 & 33.3 & $\mathrm{D}$ & $4 \mathrm{~N} / 27 \mathrm{~W}-7 \mathrm{~K} 2$ & 35 & 60 & $\mathrm{D}$ \\
\hline $4 \mathrm{~N} / 27 \mathrm{~W}-7 \mathrm{~J} 4$ & 365 & 0 & $\mathrm{D}$ & $4 \mathrm{~N} / 27 \mathrm{~W}-7 \mathrm{~K} 2$ & 45 & 0 & $\mathrm{D}$ \\
\hline $4 \mathrm{~N} / 27 \mathrm{~W}-7 \mathrm{~J} 4$ & 375 & 40 & $\mathrm{D}$ & $4 \mathrm{~N} / 27 \mathrm{~W}-7 \mathrm{~K} 2$ & 55 & 0 & $\mathrm{D}$ \\
\hline $4 \mathrm{~N} / 27 \mathrm{~W}-7 \mathrm{~J} 4$ & 385 & 36.4 & $\mathrm{D}$ & $4 \mathrm{~N} / 27 \mathrm{~W}-7 \mathrm{~K} 2$ & 65 & 0 & $\mathrm{D}$ \\
\hline $4 \mathrm{~N} / 27 \mathrm{~W}-7 \mathrm{~J} 4$ & 395 & 100 & $\mathrm{D}$ & $4 \mathrm{~N} / 27 \mathrm{~W}-7 \mathrm{~K} 2$ & 75 & 0 & $\mathrm{D}$ \\
\hline $4 \mathrm{~N} / 27 \mathrm{~W}-7 \mathrm{~J} 4$ & 405 & 100 & $\mathrm{D}$ & $4 \mathrm{~N} / 27 \mathrm{~W}-7 \mathrm{~K} 2$ & 85 & 0 & $\mathrm{D}$ \\
\hline $4 \mathrm{~N} / 27 \mathrm{~W}-7 \mathrm{~J} 4$ & 415 & 100 & $\mathrm{D}$ & $4 \mathrm{~N} / 27 \mathrm{~W}-7 \mathrm{~K} 2$ & 95 & 0 & $\mathrm{D}$ \\
\hline
\end{tabular}


Table B1-1. Calculated percent-coarse intervals for all drillers' logs and e-logs used to define textural properties of the hydrogeologic framework model.-Continued

[Percent-coarse values are calculated for 10-foot intervals for the entire depth of each borehole. State well number is the state of California well identifier; interval midpoint depth is the midpoint of each 10-foot interval, in feet from top of borehole; PC is the percent-coarse value for each interval; log type defines whether the borehole is a drillers' lithology $\log (\mathrm{D})$ or a geophysical e-log (E).]

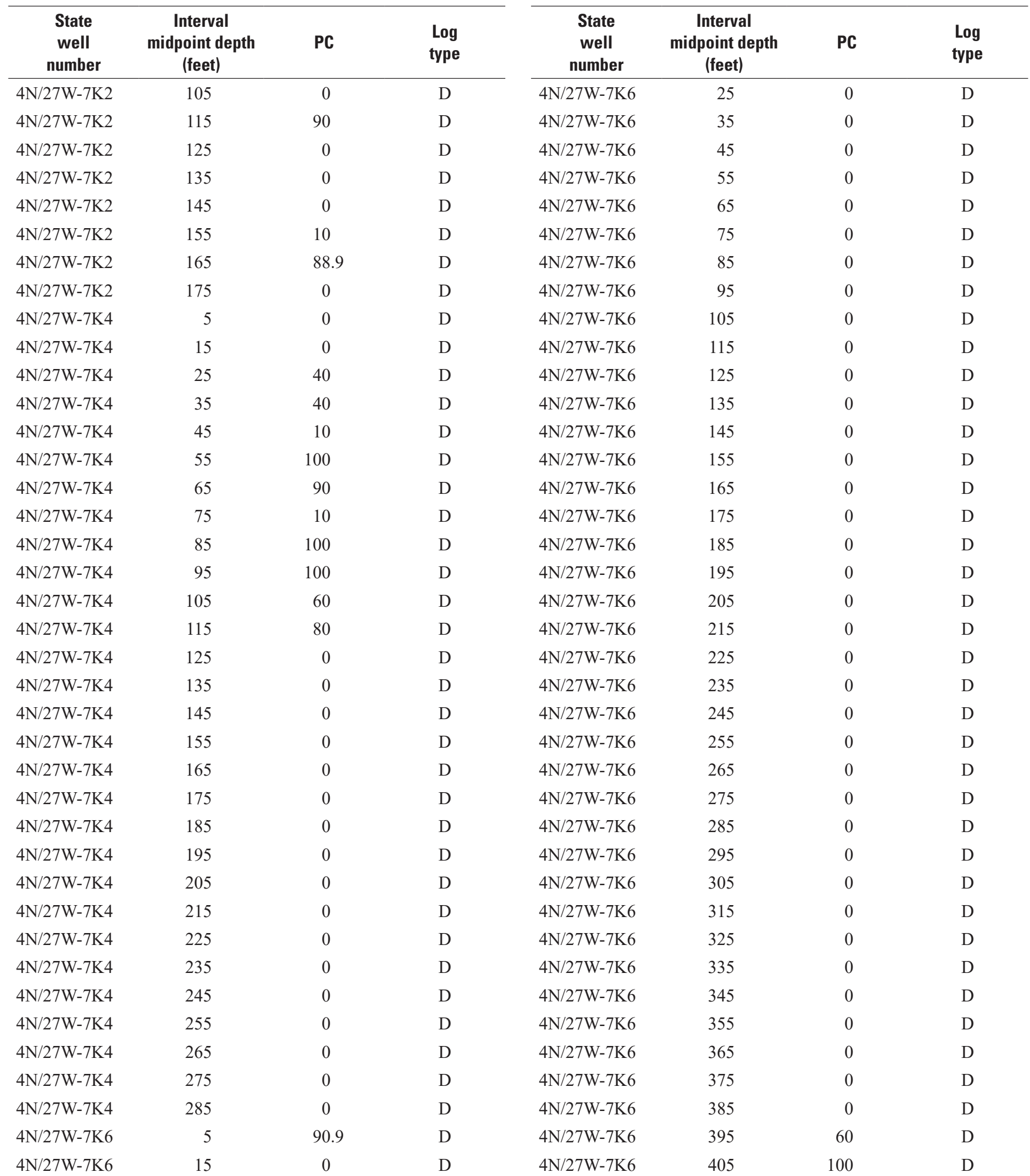


Table B1-1. Calculated percent-coarse intervals for all drillers' logs and e-logs used to define textural properties of the hydrogeologic framework model.-Continued

[Percent-coarse values are calculated for 10-foot intervals for the entire depth of each borehole. State well number is the state of California well identifier; interval midpoint depth is the midpoint of each 10-foot interval, in feet from top of borehole; PC is the percent-coarse value for each interval; log type defines whether the borehole is a drillers' lithology $\log (\mathrm{D})$ or a geophysical e-log (E).]

\begin{tabular}{|c|c|c|c|c|c|c|c|}
\hline $\begin{array}{c}\text { State } \\
\text { well } \\
\text { number }\end{array}$ & $\begin{array}{c}\text { Interval } \\
\text { midpoint depth } \\
\text { (feet) }\end{array}$ & PC & $\begin{array}{l}\text { Log } \\
\text { type }\end{array}$ & $\begin{array}{c}\text { State } \\
\text { well } \\
\text { number }\end{array}$ & $\begin{array}{c}\text { Interval } \\
\text { midpoint depth } \\
\text { (feet) }\end{array}$ & PC & $\begin{array}{l}\text { Log } \\
\text { type }\end{array}$ \\
\hline $4 \mathrm{~N} / 27 \mathrm{~W}-7 \mathrm{~K} 6$ & 415 & 100 & $\mathrm{D}$ & $4 \mathrm{~N} / 27 \mathrm{~W}-7 \mathrm{~K} 7$ & 285 & 0 & $\mathrm{D}$ \\
\hline 4N/27W-7K6 & 425 & 100 & $\mathrm{D}$ & $4 \mathrm{~N} / 27 \mathrm{~W}-7 \mathrm{~K} 7$ & 295 & 0 & $\mathrm{D}$ \\
\hline 4N/27W-7K6 & 435 & 100 & $\mathrm{D}$ & $4 \mathrm{~N} / 27 \mathrm{~W}-7 \mathrm{~K} 7$ & 305 & 0 & $\mathrm{D}$ \\
\hline 4N/27W-7K6 & 445 & 100 & $\mathrm{D}$ & $4 \mathrm{~N} / 27 \mathrm{~W}-7 \mathrm{~K} 7$ & 315 & 0 & $\mathrm{D}$ \\
\hline $4 \mathrm{~N} / 27 \mathrm{~W}-7 \mathrm{~K} 6$ & 465 & 100 & $\mathrm{D}$ & $4 \mathrm{~N} / 27 \mathrm{~W}-7 \mathrm{~K} 7$ & 335 & 0 & $\mathrm{D}$ \\
\hline 4N/27W-7K6 & 475 & 100 & $\mathrm{D}$ & $4 \mathrm{~N} / 27 \mathrm{~W}-7 \mathrm{~K} 7$ & 345 & 0 & $\mathrm{D}$ \\
\hline $4 \mathrm{~N} / 27 \mathrm{~W}-7 \mathrm{~K} 6$ & 485 & 100 & $\mathrm{D}$ & $4 \mathrm{~N} / 27 \mathrm{~W}-7 \mathrm{~K} 7$ & 355 & 0 & $\mathrm{D}$ \\
\hline 4N/27W-7K6 & 495 & 100 & $\mathrm{D}$ & $4 \mathrm{~N} / 27 \mathrm{~W}-7 \mathrm{~K} 7$ & 365 & 0 & $\mathrm{D}$ \\
\hline $4 \mathrm{~N} / 27 \mathrm{~W}-7 \mathrm{~K} 7$ & 15 & 100 & $\mathrm{D}$ & $4 \mathrm{~N} / 27 \mathrm{~W}-7 \mathrm{~K} 7$ & 405 & 0 & $\mathrm{D}$ \\
\hline $4 \mathrm{~N} / 27 \mathrm{~W}-7 \mathrm{~K} 7$ & 25 & 100 & $\mathrm{D}$ & $4 \mathrm{~N} / 27 \mathrm{~W}-7 \mathrm{~K} 7$ & 415 & 0 & $\mathrm{D}$ \\
\hline $4 \mathrm{~N} / 27 \mathrm{~W}-7 \mathrm{~K} 7$ & 35 & 0 & $\mathrm{D}$ & $4 \mathrm{~N} / 27 \mathrm{~W}-7 \mathrm{~K} 8$ & 5 & 27.3 & $\mathrm{D}$ \\
\hline $4 \mathrm{~N} / 27 \mathrm{~W}-7 \mathrm{~K} 7$ & 45 & 0 & $\mathrm{D}$ & $4 \mathrm{~N} / 27 \mathrm{~W}-7 \mathrm{~K} 8$ & 15 & 0 & $\mathrm{D}$ \\
\hline $4 \mathrm{~N} / 27 \mathrm{~W}-7 \mathrm{~K} 7$ & 55 & 0 & $\mathrm{D}$ & $4 \mathrm{~N} / 27 \mathrm{~W}-7 \mathrm{~K} 8$ & 25 & 0 & $\mathrm{D}$ \\
\hline $4 \mathrm{~N} / 27 \mathrm{~W}-7 \mathrm{~K} 7$ & 65 & 90 & $\mathrm{D}$ & $4 \mathrm{~N} / 27 \mathrm{~W}-7 \mathrm{~K} 8$ & 35 & 0 & $\mathrm{D}$ \\
\hline 4N/27W-7K7 & 75 & 100 & $\mathrm{D}$ & $4 \mathrm{~N} / 27 \mathrm{~W}-7 \mathrm{~K} 8$ & 45 & 60 & $\mathrm{D}$ \\
\hline $4 \mathrm{~N} / 27 \mathrm{~W}-7 \mathrm{~K} 7$ & 85 & 40 & $\mathrm{D}$ & $4 \mathrm{~N} / 27 \mathrm{~W}-7 \mathrm{~K} 8$ & 55 & 80 & $\mathrm{D}$ \\
\hline $4 \mathrm{~N} / 27 \mathrm{~W}-7 \mathrm{~K} 7$ & 95 & 0 & $\mathrm{D}$ & $4 \mathrm{~N} / 27 \mathrm{~W}-7 \mathrm{~K} 8$ & 65 & 0 & $\mathrm{D}$ \\
\hline $4 \mathrm{~N} / 27 \mathrm{~W}-7 \mathrm{~K} 7$ & 165 & 0 & $\mathrm{D}$ & $4 \mathrm{~N} / 27 \mathrm{~W}-7 \mathrm{~K} 8$ & 135 & 60 & $\mathrm{D}$ \\
\hline $4 \mathrm{~N} / 27 \mathrm{~W}-7 \mathrm{~K} 7$ & 175 & 0 & $\mathrm{D}$ & $4 \mathrm{~N} / 27 \mathrm{~W}-7 \mathrm{~K} 8$ & 145 & 100 & $\mathrm{D}$ \\
\hline $4 \mathrm{~N} / 27 \mathrm{~W}-7 \mathrm{~K} 7$ & 185 & 0 & $\mathrm{D}$ & $4 \mathrm{~N} / 27 \mathrm{~W}-7 \mathrm{~K} 8$ & 155 & 100 & $\mathrm{D}$ \\
\hline $4 \mathrm{~N} / 27 \mathrm{~W}-7 \mathrm{~K} 7$ & 195 & 0 & $\mathrm{D}$ & $4 \mathrm{~N} / 27 \mathrm{~W}-7 \mathrm{~K} 8$ & 165 & 90 & $\mathrm{D}$ \\
\hline $4 \mathrm{~N} / 27 \mathrm{~W}-7 \mathrm{~K} 7$ & 205 & 0 & $\mathrm{D}$ & $4 \mathrm{~N} / 27 \mathrm{~W}-7 \mathrm{~K} 8$ & 175 & 60 & $\mathrm{D}$ \\
\hline $4 \mathrm{~N} / 27 \mathrm{~W}-7 \mathrm{~K} 7$ & 215 & 0 & $\mathrm{D}$ & $4 \mathrm{~N} / 27 \mathrm{~W}-7 \mathrm{~K} 8$ & 185 & 70 & $\mathrm{D}$ \\
\hline $4 \mathrm{~N} / 27 \mathrm{~W}-7 \mathrm{~K} 7$ & 225 & 0 & $\mathrm{D}$ & $4 \mathrm{~N} / 27 \mathrm{~W}-7 \mathrm{~K} 8$ & 195 & 100 & $\mathrm{D}$ \\
\hline $4 \mathrm{~N} / 27 \mathrm{~W}-7 \mathrm{~K} 7$ & 235 & 0 & $\mathrm{D}$ & $4 \mathrm{~N} / 27 \mathrm{~W}-7 \mathrm{~K} 8$ & 205 & 100 & $\mathrm{D}$ \\
\hline $4 \mathrm{~N} / 27 \mathrm{~W}-7 \mathrm{~K} 7$ & 245 & 0 & $\mathrm{D}$ & $4 \mathrm{~N} / 27 \mathrm{~W}-7 \mathrm{~K} 8$ & 215 & 90.9 & $\mathrm{D}$ \\
\hline $4 \mathrm{~N} / 27 \mathrm{~W}-7 \mathrm{~K} 7$ & 255 & 0 & $\mathrm{D}$ & $4 \mathrm{~N} / 27 \mathrm{~W}-7 \mathrm{~K} 8$ & 225 & 90 & $\mathrm{D}$ \\
\hline $4 \mathrm{~N} / 27 \mathrm{~W}-7 \mathrm{~K} 7$ & 265 & 0 & $\mathrm{D}$ & $4 \mathrm{~N} / 27 \mathrm{~W}-7 \mathrm{~K} 8$ & 235 & 90 & $\mathrm{D}$ \\
\hline $4 \mathrm{~N} / 27 \mathrm{~W}-7 \mathrm{~K} 7$ & 275 & 0 & $\mathrm{D}$ & $4 \mathrm{~N} / 27 \mathrm{~W}-7 \mathrm{~K} 8$ & 245 & 100 & $\mathrm{D}$ \\
\hline
\end{tabular}


Table B1-1. Calculated percent-coarse intervals for all drillers' logs and e-logs used to define textural properties of the hydrogeologic framework model.-Continued

[Percent-coarse values are calculated for 10-foot intervals for the entire depth of each borehole. State well number is the state of California well identifier; interval midpoint depth is the midpoint of each 10-foot interval, in feet from top of borehole; PC is the percent-coarse value for each interval; log type defines whether the borehole is a drillers' lithology $\log (\mathrm{D})$ or a geophysical e-log (E).]

\begin{tabular}{|c|c|c|c|c|c|c|c|}
\hline $\begin{array}{c}\text { State } \\
\text { well } \\
\text { number }\end{array}$ & $\begin{array}{c}\text { Interval } \\
\text { midpoint depth } \\
\text { (feet) }\end{array}$ & PC & $\begin{array}{l}\text { Log } \\
\text { type }\end{array}$ & $\begin{array}{c}\text { State } \\
\text { well } \\
\text { number }\end{array}$ & $\begin{array}{c}\text { Interval } \\
\text { midpoint depth } \\
\text { (feet) }\end{array}$ & PC & $\begin{array}{l}\text { Log } \\
\text { type }\end{array}$ \\
\hline $4 \mathrm{~N} / 27 \mathrm{~W}-7 \mathrm{~K} 8$ & 255 & 30 & $\mathrm{D}$ & 4N/27W-7L4 & 75 & 60 & $\mathrm{D}$ \\
\hline $4 \mathrm{~N} / 27 \mathrm{~W}-7 \mathrm{~K} 8$ & 265 & 50 & $\mathrm{D}$ & 4N/27W-7L4 & 85 & 10 & $\mathrm{D}$ \\
\hline $4 \mathrm{~N} / 27 \mathrm{~W}-7 \mathrm{~K} 8$ & 275 & 40 & $\mathrm{D}$ & 4N/27W-7L4 & 95 & 0 & $\mathrm{D}$ \\
\hline $4 \mathrm{~N} / 27 \mathrm{~W}-7 \mathrm{~K} 8$ & 285 & 90 & $\mathrm{D}$ & 4N/27W-7L4 & 105 & 0 & $\mathrm{D}$ \\
\hline $4 \mathrm{~N} / 27 \mathrm{~W}-7 \mathrm{~K} 8$ & 305 & 30 & $\mathrm{D}$ & $4 \mathrm{~N} / 27 \mathrm{~W}-7 \mathrm{~L} 4$ & 125 & 50 & $\mathrm{D}$ \\
\hline $4 \mathrm{~N} / 27 \mathrm{~W}-7 \mathrm{~L} 1$ & 5 & 9.1 & $\mathrm{D}$ & $4 \mathrm{~N} / 27 \mathrm{~W}-7 \mathrm{~L} 4$ & 135 & 80 & $\mathrm{D}$ \\
\hline $4 \mathrm{~N} / 27 \mathrm{~W}-7 \mathrm{~L} 1$ & 15 & 0 & $\mathrm{D}$ & $4 \mathrm{~N} / 27 \mathrm{~W}-7 \mathrm{~L} 4$ & 145 & 60 & $\mathrm{D}$ \\
\hline 4N/27W-7L1 & 25 & 0 & $\mathrm{D}$ & 4N/27W-7L4 & 155 & 80 & $\mathrm{D}$ \\
\hline 4N/27W-7L1 & 65 & 0 & $\mathrm{D}$ & 4N/27W-7L7 & 35 & 30 & $\mathrm{D}$ \\
\hline 4N/27W-7L1 & 75 & 0 & $\mathrm{D}$ & 4N/27W-7L7 & 45 & 80 & $\mathrm{D}$ \\
\hline 4N/27W-7L1 & 85 & 0 & $\mathrm{D}$ & 4N/27W-7L7 & 55 & 100 & $\mathrm{D}$ \\
\hline 4N/27W-7L1 & 95 & 0 & $\mathrm{D}$ & 4N/27W-7L7 & 65 & 30 & $\mathrm{D}$ \\
\hline 4N/27W-7L1 & 105 & 0 & $\mathrm{D}$ & 4N/27W-7L7 & 75 & 50 & $\mathrm{D}$ \\
\hline 4N/27W-7L1 & 115 & 0 & $\mathrm{D}$ & $4 \mathrm{~N} / 27 \mathrm{~W}-7 \mathrm{~L} 7$ & 85 & 0 & $\mathrm{D}$ \\
\hline $4 \mathrm{~N} / 27 \mathrm{~W}-7 \mathrm{~L} 1$ & 125 & 0 & $\mathrm{D}$ & $4 \mathrm{~N} / 27 \mathrm{~W}-7 \mathrm{~L} 7$ & 95 & 70 & $\mathrm{D}$ \\
\hline $4 \mathrm{~N} / 27 \mathrm{~W}-7 \mathrm{~L} 1$ & 135 & 0 & $\mathrm{D}$ & 4N/27W-7L7 & 105 & 100 & $\mathrm{D}$ \\
\hline 4N/27W-7L3 & 5 & 72.7 & $\mathrm{D}$ & 4N/27W-7L7 & 115 & 100 & $\mathrm{D}$ \\
\hline 4N/27W-7L3 & 75 & 90 & $\mathrm{D}$ & $4 \mathrm{~N} / 27 \mathrm{~W}-7 \mathrm{~L} 10$ & 55 & 0 & $\mathrm{D}$ \\
\hline 4N/27W-7L3 & 85 & 0 & $\mathrm{D}$ & $4 \mathrm{~N} / 27 \mathrm{~W}-7 \mathrm{~L} 10$ & 65 & 0 & $\mathrm{D}$ \\
\hline 4N/27W-7L3 & 95 & 30 & $\mathrm{D}$ & $4 \mathrm{~N} / 27 \mathrm{~W}-7 \mathrm{~L} 10$ & 75 & 0 & $\mathrm{D}$ \\
\hline 4N/27W-7L3 & 105 & 40 & $\mathrm{D}$ & 4N/27W-7L10 & 85 & 0 & $\mathrm{D}$ \\
\hline $4 \mathrm{~N} / 27 \mathrm{~W}-7 \mathrm{~L} 3$ & 115 & 0 & $\mathrm{D}$ & 4N/27W-7L10 & 95 & 0 & $\mathrm{D}$ \\
\hline 4N/27W-7L4 & 5 & 100 & $\mathrm{D}$ & $4 \mathrm{~N} / 27 \mathrm{~W}-7 \mathrm{~L} 10$ & 105 & 0 & $\mathrm{D}$ \\
\hline 4N/27W-7L4 & 15 & 40 & $\mathrm{D}$ & $4 \mathrm{~N} / 27 \mathrm{~W}-7 \mathrm{~L} 10$ & 115 & 0 & $\mathrm{D}$ \\
\hline $4 \mathrm{~N} / 27 \mathrm{~W}-7 \mathrm{~L} 4$ & 25 & 10 & $\mathrm{D}$ & 4N/27W-7L10 & 125 & 0 & $\mathrm{D}$ \\
\hline $4 \mathrm{~N} / 27 \mathrm{~W}-7 \mathrm{~L} 4$ & 35 & 50 & $\mathrm{D}$ & $4 \mathrm{~N} / 27 \mathrm{~W}-7 \mathrm{~L} 10$ & 135 & 0 & $\mathrm{D}$ \\
\hline 4N/27W-7L4 & 45 & 50 & $\mathrm{D}$ & 4N/27W-7L10 & 145 & 0 & $\mathrm{D}$ \\
\hline 4N/27W-7L4 & 55 & 90 & $\mathrm{D}$ & $4 \mathrm{~N} / 27 \mathrm{~W}-7 \mathrm{~L} 10$ & 155 & 0 & $\mathrm{D}$ \\
\hline 4N/27W-7L4 & 65 & 30 & $\mathrm{D}$ & $4 \mathrm{~N} / 27 \mathrm{~W}-7 \mathrm{~L} 10$ & 165 & 0 & $\mathrm{D}$ \\
\hline
\end{tabular}


Table B1-1. Calculated percent-coarse intervals for all drillers' logs and e-logs used to define textural properties of the hydrogeologic framework model.-Continued

[Percent-coarse values are calculated for 10-foot intervals for the entire depth of each borehole. State well number is the state of California well identifier; interval midpoint depth is the midpoint of each 10-foot interval, in feet from top of borehole; PC is the percent-coarse value for each interval; log type defines whether the borehole is a drillers' lithology log (D) or a geophysical e-log (E).]

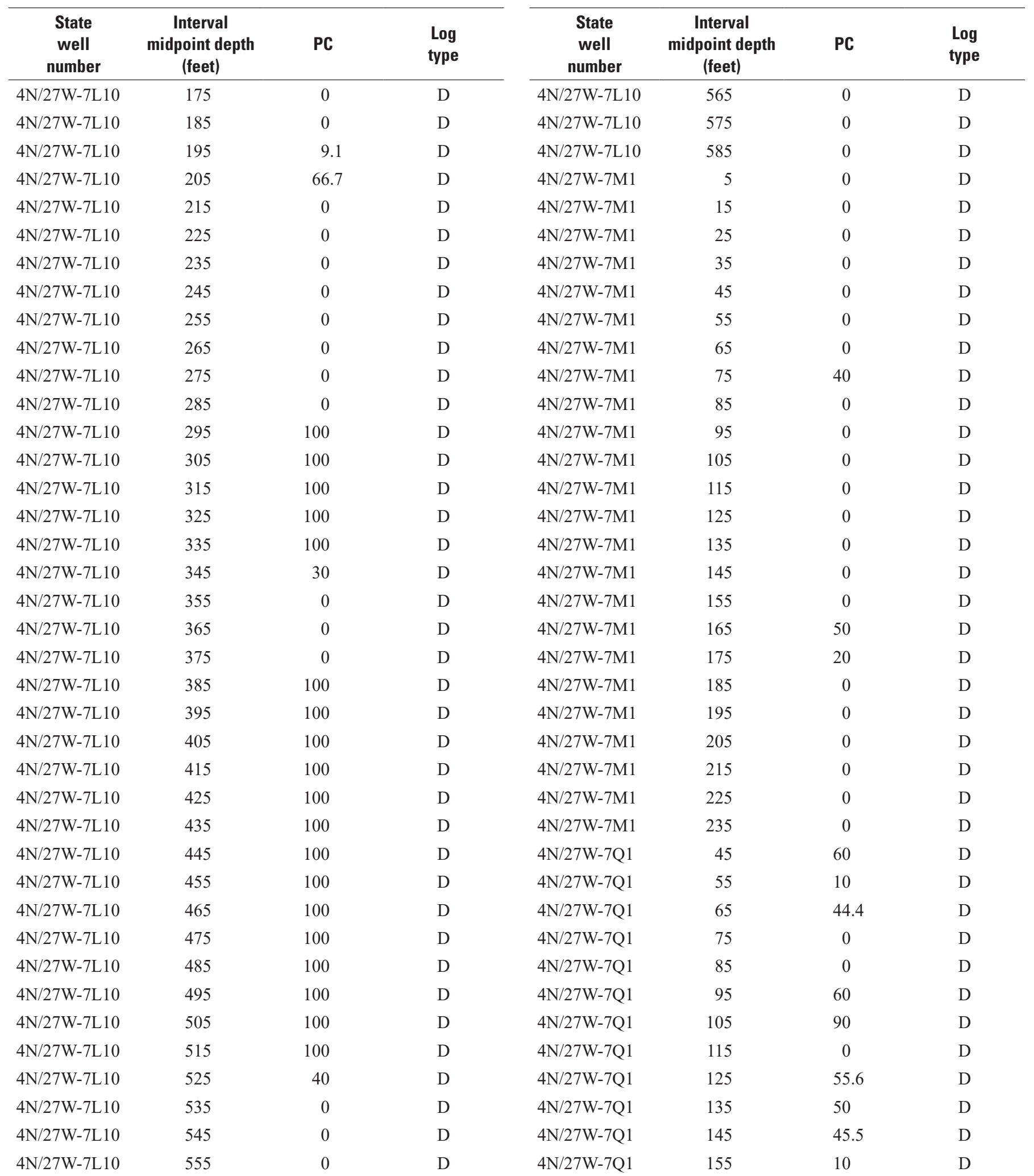


Table B1-1. Calculated percent-coarse intervals for all drillers' logs and e-logs used to define textural properties of the hydrogeologic framework model.-Continued

[Percent-coarse values are calculated for 10-foot intervals for the entire depth of each borehole. State well number is the state of California well identifier; interval midpoint depth is the midpoint of each 10-foot interval, in feet from top of borehole; PC is the percent-coarse value for each interval; log type defines whether the borehole is a drillers' lithology $\log (\mathrm{D})$ or a geophysical e-log (E).]

\begin{tabular}{|c|c|c|c|c|c|c|c|}
\hline $\begin{array}{c}\text { State } \\
\text { well } \\
\text { number }\end{array}$ & $\begin{array}{c}\text { Interval } \\
\text { midpoint depth } \\
\text { (feet) }\end{array}$ & PC & $\begin{array}{l}\text { Log } \\
\text { type }\end{array}$ & $\begin{array}{c}\text { State } \\
\text { well } \\
\text { number }\end{array}$ & $\begin{array}{c}\text { Interval } \\
\text { midpoint depth } \\
\text { (feet) }\end{array}$ & PC & $\begin{array}{l}\text { Log } \\
\text { type }\end{array}$ \\
\hline 4N/27W-7Q1 & 165 & 100 & $\mathrm{D}$ & 4N/27W-7Q3 & 195 & 72.7 & $\mathrm{D}$ \\
\hline 4N/27W-7Q1 & 175 & 100 & $\mathrm{D}$ & 4N/27W-7Q3 & 205 & 0 & $\mathrm{D}$ \\
\hline 4N/27W-7Q1 & 185 & 50 & $\mathrm{D}$ & 4N/27W-7Q3 & 215 & 54.5 & $\mathrm{D}$ \\
\hline 4N/27W-7Q1 & 195 & 0 & $\mathrm{D}$ & 4N/27W-7R3 & 45 & 0 & $\mathrm{D}$ \\
\hline 4N/27W-7Q2 & 15 & 100 & $\mathrm{D}$ & $4 \mathrm{~N} / 27 \mathrm{~W}-7 \mathrm{R} 3$ & 65 & 0 & $\mathrm{D}$ \\
\hline 4N/27W-7Q2 & 25 & 90 & $\mathrm{D}$ & $4 \mathrm{~N} / 27 \mathrm{~W}-7 \mathrm{R} 3$ & 75 & 9.1 & $\mathrm{D}$ \\
\hline 4N/27W-7Q2 & 35 & 90 & $\mathrm{D}$ & $4 \mathrm{~N} / 27 \mathrm{~W}-7 \mathrm{R} 3$ & 85 & 100 & $\mathrm{D}$ \\
\hline 4N/27W-7Q2 & 45 & 10 & $\mathrm{D}$ & $4 \mathrm{~N} / 27 \mathrm{~W}-7 \mathrm{R} 3$ & 95 & 100 & $\mathrm{D}$ \\
\hline 4N/27W-7Q2 & 85 & 100 & $\mathrm{D}$ & $4 \mathrm{~N} / 27 \mathrm{~W}-7 \mathrm{R} 3$ & 135 & 100 & $\mathrm{D}$ \\
\hline 4N/27W-7Q2 & 95 & 100 & $\mathrm{D}$ & $4 \mathrm{~N} / 27 \mathrm{~W}-7 \mathrm{R} 3$ & 145 & 100 & $\mathrm{D}$ \\
\hline 4N/27W-7Q2 & 105 & 50 & $\mathrm{D}$ & $4 \mathrm{~N} / 27 \mathrm{~W}-7 \mathrm{R} 3$ & 155 & 90 & $\mathrm{D}$ \\
\hline 4N/27W-7Q2 & 115 & 100 & $\mathrm{D}$ & 4N/27W-7R3 & 165 & 0 & $\mathrm{D}$ \\
\hline 4N/27W-7Q2 & 125 & 100 & $\mathrm{D}$ & $4 \mathrm{~N} / 27 \mathrm{~W}-7 \mathrm{R} 3$ & 175 & 100 & $\mathrm{D}$ \\
\hline 4N/27W-7Q2 & 135 & 100 & $\mathrm{D}$ & $4 \mathrm{~N} / 27 \mathrm{~W}-7 \mathrm{R} 3$ & 185 & 100 & $\mathrm{D}$ \\
\hline 4N/27W-7Q2 & 145 & 90.9 & $\mathrm{D}$ & $4 \mathrm{~N} / 27 \mathrm{~W}-7 \mathrm{R} 3$ & 195 & 90.9 & $\mathrm{D}$ \\
\hline 4N/27W-7Q2 & 155 & 0 & $\mathrm{D}$ & $4 \mathrm{~N} / 27 \mathrm{~W}-7 \mathrm{R} 3$ & 205 & 0 & $\mathrm{D}$ \\
\hline 4N/27W-7Q3 & 5 & 54.5 & $\mathrm{D}$ & 4N/27W-7R3 & 215 & 0 & $\mathrm{D}$ \\
\hline 4N/27W-7Q3 & 75 & 100 & $\mathrm{D}$ & $4 \mathrm{~N} / 27 \mathrm{~W}-8 \mathrm{E} 4$ & 55 & 100 & $\mathrm{D}$ \\
\hline 4N/27W-7Q3 & 85 & 100 & $\mathrm{D}$ & $4 \mathrm{~N} / 27 \mathrm{~W}-8 \mathrm{E} 4$ & 65 & 100 & $\mathrm{D}$ \\
\hline 4N/27W-7Q3 & 95 & 100 & $\mathrm{D}$ & $4 \mathrm{~N} / 27 \mathrm{~W}-8 \mathrm{E} 4$ & 75 & 100 & $\mathrm{D}$ \\
\hline 4N/27W-7Q3 & 105 & 100 & $\mathrm{D}$ & $4 \mathrm{~N} / 27 \mathrm{~W}-8 \mathrm{E} 4$ & 85 & 100 & $\mathrm{D}$ \\
\hline 4N/27W-7Q3 & 115 & 20 & $\mathrm{D}$ & $4 \mathrm{~N} / 27 \mathrm{~W}-8 \mathrm{E} 4$ & 95 & 100 & $\mathrm{D}$ \\
\hline 4N/27W-7Q3 & 125 & 100 & $\mathrm{D}$ & $4 \mathrm{~N} / 27 \mathrm{~W}-8 \mathrm{E} 4$ & 105 & 100 & $\mathrm{D}$ \\
\hline 4N/27W-7Q3 & 135 & 100 & $\mathrm{D}$ & $4 \mathrm{~N} / 27 \mathrm{~W}-8 \mathrm{E} 4$ & 115 & 90 & $\mathrm{D}$ \\
\hline 4N/27W-7Q3 & 145 & 100 & $\mathrm{D}$ & $4 \mathrm{~N} / 27 \mathrm{~W}-8 \mathrm{E} 4$ & 125 & 0 & $\mathrm{D}$ \\
\hline $4 \mathrm{~N} / 27 \mathrm{~W}-7 \mathrm{Q} 3$ & 155 & 100 & $\mathrm{D}$ & $4 \mathrm{~N} / 27 \mathrm{~W}-8 \mathrm{E} 4$ & 135 & 0 & $\mathrm{D}$ \\
\hline 4N/27W-7Q3 & 165 & 100 & $\mathrm{D}$ & $4 \mathrm{~N} / 27 \mathrm{~W}-8 \mathrm{E} 4$ & 145 & 0 & $\mathrm{D}$ \\
\hline 4N/27W-7Q3 & 175 & 100 & $\mathrm{D}$ & $4 \mathrm{~N} / 27 \mathrm{~W}-8 \mathrm{E} 4$ & 155 & 10 & $\mathrm{D}$ \\
\hline 4N/27W-7Q3 & 185 & 100 & $\mathrm{D}$ & $4 \mathrm{~N} / 27 \mathrm{~W}-8 \mathrm{E} 4$ & 165 & 100 & $\mathrm{D}$ \\
\hline
\end{tabular}


Table B1-1. Calculated percent-coarse intervals for all drillers' logs and e-logs used to define textural properties of the hydrogeologic framework model.-Continued

[Percent-coarse values are calculated for 10-foot intervals for the entire depth of each borehole. State well number is the state of California well identifier; interval midpoint depth is the midpoint of each 10-foot interval, in feet from top of borehole; PC is the percent-coarse value for each interval; log type defines whether the borehole is a drillers' lithology $\log (\mathrm{D})$ or a geophysical e-log (E).]

\begin{tabular}{|c|c|c|c|c|c|c|c|}
\hline $\begin{array}{c}\text { State } \\
\text { well } \\
\text { number }\end{array}$ & $\begin{array}{c}\text { Interval } \\
\text { midpoint depth } \\
\text { (feet) }\end{array}$ & PC & $\begin{array}{l}\text { Log } \\
\text { type }\end{array}$ & $\begin{array}{c}\text { State } \\
\text { well } \\
\text { number }\end{array}$ & $\begin{array}{c}\text { Interval } \\
\text { midpoint depth } \\
\text { (feet) }\end{array}$ & PC & $\begin{array}{l}\text { Log } \\
\text { type }\end{array}$ \\
\hline $4 \mathrm{~N} / 27 \mathrm{~W}-8 \mathrm{E} 4$ & 175 & 90 & $\mathrm{D}$ & $4 \mathrm{~N} / 27 \mathrm{~W}-8 \mathrm{E} 4$ & 565 & 0 & $\mathrm{D}$ \\
\hline $4 \mathrm{~N} / 27 \mathrm{~W}-8 \mathrm{E} 4$ & 185 & 0 & $\mathrm{D}$ & $4 \mathrm{~N} / 27 \mathrm{~W}-8 \mathrm{E} 4$ & 575 & 0 & $\mathrm{D}$ \\
\hline $4 \mathrm{~N} / 27 \mathrm{~W}-8 \mathrm{E} 4$ & 195 & 0 & $\mathrm{D}$ & $4 \mathrm{~N} / 27 \mathrm{~W}-8 \mathrm{E} 4$ & 585 & 0 & $\mathrm{D}$ \\
\hline $4 \mathrm{~N} / 27 \mathrm{~W}-8 \mathrm{E} 4$ & 205 & 0 & $\mathrm{D}$ & $4 \mathrm{~N} / 27 \mathrm{~W}-8 \mathrm{E} 4$ & 595 & 0 & $\mathrm{D}$ \\
\hline $4 \mathrm{~N} / 27 \mathrm{~W}-8 \mathrm{E} 4$ & 225 & 0 & $\mathrm{D}$ & $4 \mathrm{~N} / 27 \mathrm{~W}-8 \mathrm{~J} 1$ & 15 & 100 & $\mathrm{D}$ \\
\hline $4 \mathrm{~N} / 27 \mathrm{~W}-8 \mathrm{E} 4$ & 235 & 0 & $\mathrm{D}$ & 4N/27W-8J1 & 25 & 100 & $\mathrm{D}$ \\
\hline 4N/27W-8E4 & 245 & 0 & $\mathrm{D}$ & 4N/27W-8J1 & 35 & 100 & $\mathrm{D}$ \\
\hline 4N/27W-8E4 & 255 & 0 & $\mathrm{D}$ & $4 \mathrm{~N} / 27 \mathrm{~W}-8 \mathrm{~J} 1$ & 45 & 90 & $\mathrm{D}$ \\
\hline $4 \mathrm{~N} / 27 \mathrm{~W}-8 \mathrm{E} 4$ & 295 & 100 & $\mathrm{D}$ & 4N/27W-8J1 & 85 & 0 & $\mathrm{D}$ \\
\hline $4 \mathrm{~N} / 27 \mathrm{~W}-8 \mathrm{E} 4$ & 305 & 100 & $\mathrm{D}$ & $4 \mathrm{~N} / 27 \mathrm{~W}-8 \mathrm{~J} 1$ & 95 & 0 & $\mathrm{D}$ \\
\hline $4 \mathrm{~N} / 27 \mathrm{~W}-8 \mathrm{E} 4$ & 315 & 100 & $\mathrm{D}$ & $4 \mathrm{~N} / 27 \mathrm{~W}-8 \mathrm{~J} 1$ & 105 & 50 & $\mathrm{D}$ \\
\hline $4 \mathrm{~N} / 27 \mathrm{~W}-8 \mathrm{E} 4$ & 325 & 90 & $\mathrm{D}$ & 4N/27W-8J1 & 115 & 0 & $\mathrm{D}$ \\
\hline $4 \mathrm{~N} / 27 \mathrm{~W}-8 \mathrm{E} 4$ & 335 & 0 & $\mathrm{D}$ & 4N/27W-8J1 & 125 & 0 & $\mathrm{D}$ \\
\hline $4 \mathrm{~N} / 27 \mathrm{~W}-8 \mathrm{E} 4$ & 345 & 0 & $\mathrm{D}$ & $4 \mathrm{~N} / 27 \mathrm{~W}-8 \mathrm{~J} 1$ & 135 & 0 & $\mathrm{D}$ \\
\hline $4 \mathrm{~N} / 27 \mathrm{~W}-8 \mathrm{E} 4$ & 355 & 0 & $\mathrm{D}$ & 4N/27W-8J1 & 145 & 0 & $\mathrm{D}$ \\
\hline 4N/27W-8E4 & 365 & 0 & $\mathrm{D}$ & $4 \mathrm{~N} / 27 \mathrm{~W}-8 \mathrm{~J} 1$ & 155 & 0 & $\mathrm{D}$ \\
\hline $4 \mathrm{~N} / 27 \mathrm{~W}-8 \mathrm{E} 4$ & 375 & 0 & $\mathrm{D}$ & $4 \mathrm{~N} / 27 \mathrm{~W}-8 \mathrm{~J} 1$ & 165 & 0 & $\mathrm{D}$ \\
\hline $4 \mathrm{~N} / 27 \mathrm{~W}-8 \mathrm{E} 4$ & 445 & 100 & $\mathrm{D}$ & $4 \mathrm{~N} / 27 \mathrm{~W}-8 \mathrm{~J} 1$ & 235 & 40 & $\mathrm{D}$ \\
\hline $4 \mathrm{~N} / 27 \mathrm{~W}-8 \mathrm{E} 4$ & 455 & 100 & $\mathrm{D}$ & 4N/27W-8J1 & 245 & 0 & $\mathrm{D}$ \\
\hline 4N/27W-8E4 & 465 & 90 & $\mathrm{D}$ & 4N/27W-8J1 & 255 & 50 & $\mathrm{D}$ \\
\hline $4 \mathrm{~N} / 27 \mathrm{~W}-8 \mathrm{E} 4$ & 475 & 0 & $\mathrm{D}$ & $4 \mathrm{~N} / 27 \mathrm{~W}-8 \mathrm{~J} 1$ & 265 & 40 & $\mathrm{D}$ \\
\hline $4 \mathrm{~N} / 27 \mathrm{~W}-8 \mathrm{E} 4$ & 485 & 0 & $\mathrm{D}$ & $4 \mathrm{~N} / 27 \mathrm{~W}-8 \mathrm{~J} 1$ & 275 & 0 & $\mathrm{D}$ \\
\hline $4 \mathrm{~N} / 27 \mathrm{~W}-8 \mathrm{E} 4$ & 495 & 0 & $\mathrm{D}$ & $4 \mathrm{~N} / 27 \mathrm{~W}-8 \mathrm{~J} 1$ & 285 & 0 & $\mathrm{D}$ \\
\hline $4 \mathrm{~N} / 27 \mathrm{~W}-8 \mathrm{E} 4$ & 505 & 0 & $\mathrm{D}$ & $4 \mathrm{~N} / 27 \mathrm{~W}-8 \mathrm{~J} 1$ & 295 & 54.5 & $\mathrm{D}$ \\
\hline $4 \mathrm{~N} / 27 \mathrm{~W}-8 \mathrm{E} 4$ & 515 & 0 & $\mathrm{D}$ & $4 \mathrm{~N} / 27 \mathrm{~W}-8 \mathrm{~J} 1$ & 305 & 10 & $\mathrm{D}$ \\
\hline $4 \mathrm{~N} / 27 \mathrm{~W}-8 \mathrm{E} 4$ & 525 & 10 & $\mathrm{D}$ & $4 \mathrm{~N} / 27 \mathrm{~W}-8 \mathrm{~J} 1$ & 315 & 0 & $\mathrm{D}$ \\
\hline 4N/27W-8E4 & 535 & 100 & $\mathrm{D}$ & 4N/27W-8J1 & 325 & 40 & $\mathrm{D}$ \\
\hline $4 \mathrm{~N} / 27 \mathrm{~W}-8 \mathrm{E} 4$ & 545 & 100 & $\mathrm{D}$ & $4 \mathrm{~N} / 27 \mathrm{~W}-8 \mathrm{~J} 1$ & 335 & 30 & $\mathrm{D}$ \\
\hline 4N/27W-8E4 & 555 & 100 & $\mathrm{D}$ & $4 \mathrm{~N} / 27 \mathrm{~W}-8 \mathrm{~J} 1$ & 345 & 66.7 & $\mathrm{D}$ \\
\hline
\end{tabular}


Table B1-1. Calculated percent-coarse intervals for all drillers' logs and e-logs used to define textural properties of the hydrogeologic framework model.-Continued

[Percent-coarse values are calculated for 10-foot intervals for the entire depth of each borehole. State well number is the state of California well identifier; interval midpoint depth is the midpoint of each 10-foot interval, in feet from top of borehole; PC is the percent-coarse value for each interval; log type defines whether the borehole is a drillers' lithology $\log (\mathrm{D})$ or a geophysical e-log (E).]

\begin{tabular}{|c|c|c|c|c|c|c|c|}
\hline $\begin{array}{c}\text { State } \\
\text { well } \\
\text { number }\end{array}$ & $\begin{array}{c}\text { Interval } \\
\text { midpoint depth } \\
\text { (feet) }\end{array}$ & PC & $\begin{array}{l}\text { Log } \\
\text { type }\end{array}$ & $\begin{array}{c}\text { State } \\
\text { well } \\
\text { number }\end{array}$ & $\begin{array}{c}\text { Interval } \\
\text { midpoint depth } \\
\text { (feet) }\end{array}$ & PC & $\begin{array}{l}\text { Log } \\
\text { type }\end{array}$ \\
\hline $4 \mathrm{~N} / 27 \mathrm{~W}-8 \mathrm{~J} 1$ & 355 & 50 & $\mathrm{D}$ & 4N/27W-8L3 & 135 & 0 & $\mathrm{D}$ \\
\hline $4 \mathrm{~N} / 27 \mathrm{~W}-8 \mathrm{~J} 1$ & 365 & 0 & $\mathrm{D}$ & $4 \mathrm{~N} / 27 \mathrm{~W}-8 \mathrm{~L} 3$ & 145 & 0 & $\mathrm{D}$ \\
\hline $4 \mathrm{~N} / 27 \mathrm{~W}-8 \mathrm{~J} 1$ & 375 & 90 & $\mathrm{D}$ & $4 \mathrm{~N} / 27 \mathrm{~W}-8 \mathrm{~L} 3$ & 155 & 0 & $\mathrm{D}$ \\
\hline $4 \mathrm{~N} / 27 \mathrm{~W}-8 \mathrm{~J} 1$ & 385 & 100 & $\mathrm{D}$ & $4 \mathrm{~N} / 27 \mathrm{~W}-8 \mathrm{~L} 3$ & 165 & 0 & $\mathrm{D}$ \\
\hline $4 \mathrm{~N} / 27 \mathrm{~W}-8 \mathrm{~J} 1$ & 405 & 20 & $\mathrm{D}$ & $4 \mathrm{~N} / 27 \mathrm{~W}-8 \mathrm{~L} 3$ & 185 & 0 & $\mathrm{D}$ \\
\hline $4 \mathrm{~N} / 27 \mathrm{~W}-8 \mathrm{~J} 1$ & 415 & 77.8 & $\mathrm{D}$ & $4 \mathrm{~N} / 27 \mathrm{~W}-8 \mathrm{~L} 3$ & 195 & 0 & $\mathrm{D}$ \\
\hline $4 \mathrm{~N} / 27 \mathrm{~W}-8 \mathrm{~J} 1$ & 425 & 0 & $\mathrm{D}$ & $4 \mathrm{~N} / 27 \mathrm{~W}-8 \mathrm{~L} 3$ & 205 & 0 & $\mathrm{D}$ \\
\hline $4 \mathrm{~N} / 27 \mathrm{~W}-8 \mathrm{~J} 1$ & 435 & 0 & $\mathrm{D}$ & $4 \mathrm{~N} / 27 \mathrm{~W}-8 \mathrm{~L} 3$ & 215 & 0 & $\mathrm{D}$ \\
\hline 4N/27W-8J1 & 475 & 0 & $\mathrm{D}$ & $4 \mathrm{~N} / 27 \mathrm{~W}-8 \mathrm{~L} 3$ & 255 & 0 & $\mathrm{D}$ \\
\hline $4 \mathrm{~N} / 27 \mathrm{~W}-8 \mathrm{~J} 1$ & 485 & 0 & $\mathrm{D}$ & $4 \mathrm{~N} / 27 \mathrm{~W}-8 \mathrm{~L} 3$ & 265 & 0 & $\mathrm{D}$ \\
\hline 4N/27W-8J1 & 495 & 20 & $\mathrm{D}$ & $4 \mathrm{~N} / 27 \mathrm{~W}-8 \mathrm{~L} 3$ & 275 & 0 & $\mathrm{D}$ \\
\hline $4 \mathrm{~N} / 27 \mathrm{~W}-8 \mathrm{~J} 1$ & 505 & 20 & $\mathrm{D}$ & $4 \mathrm{~N} / 27 \mathrm{~W}-8 \mathrm{~L} 3$ & 285 & 0 & $\mathrm{D}$ \\
\hline $4 \mathrm{~N} / 27 \mathrm{~W}-8 \mathrm{~J} 1$ & 515 & 100 & $\mathrm{D}$ & $4 \mathrm{~N} / 27 \mathrm{~W}-8 \mathrm{~L} 3$ & 295 & 0 & $\mathrm{D}$ \\
\hline 4N/27W-8J1 & 525 & 100 & $\mathrm{D}$ & $4 \mathrm{~N} / 27 \mathrm{~W}-8 \mathrm{~L} 3$ & 305 & 0 & $\mathrm{D}$ \\
\hline $4 \mathrm{~N} / 27 \mathrm{~W}-8 \mathrm{~J} 1$ & 535 & 100 & $\mathrm{D}$ & $4 \mathrm{~N} / 27 \mathrm{~W}-8 \mathrm{~L} 3$ & 315 & 0 & $\mathrm{D}$ \\
\hline $4 \mathrm{~N} / 27 \mathrm{~W}-8 \mathrm{~J} 1$ & 545 & 100 & $\mathrm{D}$ & $4 \mathrm{~N} / 27 \mathrm{~W}-8 \mathrm{~L} 3$ & 325 & 0 & $\mathrm{D}$ \\
\hline $4 \mathrm{~N} / 27 \mathrm{~W}-8 \mathrm{~J} 1$ & 555 & 100 & $\mathrm{D}$ & $4 \mathrm{~N} / 27 \mathrm{~W}-8 \mathrm{~L} 3$ & 335 & 0 & $\mathrm{D}$ \\
\hline $4 \mathrm{~N} / 27 \mathrm{~W}-8 \mathrm{~J} 1$ & 625 & 0 & $\mathrm{D}$ & $4 \mathrm{~N} / 27 \mathrm{~W}-8 \mathrm{~L} 3$ & 405 & 0 & $\mathrm{D}$ \\
\hline $4 \mathrm{~N} / 27 \mathrm{~W}-8 \mathrm{~J} 1$ & 635 & 0 & $\mathrm{D}$ & $4 \mathrm{~N} / 27 \mathrm{~W}-8 \mathrm{~L} 3$ & 415 & 0 & $\mathrm{D}$ \\
\hline $4 \mathrm{~N} / 27 \mathrm{~W}-8 \mathrm{~J} 1$ & 645 & 100 & $\mathrm{D}$ & $4 \mathrm{~N} / 27 \mathrm{~W}-8 \mathrm{~L} 3$ & 425 & 0 & $\mathrm{D}$ \\
\hline 4N/27W-8J1 & 655 & 80 & $\mathrm{D}$ & $4 \mathrm{~N} / 27 \mathrm{~W}-8 \mathrm{~L} 3$ & 435 & 0 & $\mathrm{D}$ \\
\hline $4 \mathrm{~N} / 27 \mathrm{~W}-8 \mathrm{~J} 1$ & 665 & 50 & $\mathrm{D}$ & $4 \mathrm{~N} / 27 \mathrm{~W}-8 \mathrm{~L} 3$ & 445 & 0 & $\mathrm{D}$ \\
\hline $4 \mathrm{~N} / 27 \mathrm{~W}-8 \mathrm{~L} 3$ & 65 & 0 & $\mathrm{D}$ & $4 \mathrm{~N} / 27 \mathrm{~W}-8 \mathrm{~L} 3$ & 455 & 0 & $\mathrm{D}$ \\
\hline $4 \mathrm{~N} / 27 \mathrm{~W}-8 \mathrm{~L} 3$ & 75 & 0 & $\mathrm{D}$ & $4 \mathrm{~N} / 27 \mathrm{~W}-8 \mathrm{~L} 3$ & 465 & 0 & $\mathrm{D}$ \\
\hline $4 \mathrm{~N} / 27 \mathrm{~W}-8 \mathrm{~L} 3$ & 85 & 0 & $\mathrm{D}$ & $4 \mathrm{~N} / 27 \mathrm{~W}-8 \mathrm{~L} 3$ & 475 & 0 & $\mathrm{D}$ \\
\hline $4 \mathrm{~N} / 27 \mathrm{~W}-8 \mathrm{~L} 3$ & 95 & 0 & $\mathrm{D}$ & $4 \mathrm{~N} / 27 \mathrm{~W}-8 \mathrm{~L} 3$ & 485 & 0 & $\mathrm{D}$ \\
\hline 4N/27W-8L3 & 105 & 0 & $\mathrm{D}$ & $4 \mathrm{~N} / 27 \mathrm{~W}-8 \mathrm{~L} 3$ & 495 & 0 & $\mathrm{D}$ \\
\hline 4N/27W-8L3 & 115 & 0 & $\mathrm{D}$ & $4 \mathrm{~N} / 27 \mathrm{~W}-8 \mathrm{~L} 3$ & 505 & 0 & $\mathrm{D}$ \\
\hline 4N/27W-8L3 & 125 & 0 & $\mathrm{D}$ & $4 \mathrm{~N} / 27 \mathrm{~W}-8 \mathrm{~L} 3$ & 515 & 0 & $\mathrm{D}$ \\
\hline
\end{tabular}


Table B1-1. Calculated percent-coarse intervals for all drillers' logs and e-logs used to define textural properties of the hydrogeologic framework model.-Continued

[Percent-coarse values are calculated for 10-foot intervals for the entire depth of each borehole. State well number is the state of California well identifier; interval midpoint depth is the midpoint of each 10-foot interval, in feet from top of borehole; PC is the percent-coarse value for each interval; log type defines whether the borehole is a drillers' lithology log (D) or a geophysical e-log (E).]

\begin{tabular}{|c|c|c|c|c|c|c|c|}
\hline $\begin{array}{c}\text { State } \\
\text { well } \\
\text { number }\end{array}$ & $\begin{array}{c}\text { Interval } \\
\text { midpoint depth } \\
\text { (feet) }\end{array}$ & PC & $\begin{array}{l}\text { Log } \\
\text { type }\end{array}$ & $\begin{array}{c}\text { State } \\
\text { well } \\
\text { number }\end{array}$ & $\begin{array}{c}\text { Interval } \\
\text { midpoint depth } \\
\text { (feet) }\end{array}$ & PC & $\begin{array}{l}\text { Log } \\
\text { type }\end{array}$ \\
\hline 4N/27W-8L3 & 525 & 0 & $\mathrm{D}$ & $4 \mathrm{~N} / 27 \mathrm{~W}-8 \mathrm{M} 3$ & 145 & 0 & $\mathrm{D}$ \\
\hline 4N/27W-8L3 & 535 & 0 & $\mathrm{D}$ & $4 \mathrm{~N} / 27 \mathrm{~W}-8 \mathrm{M} 3$ & 155 & 0 & $\mathrm{D}$ \\
\hline 4N/27W-8L3 & 545 & 50 & $\mathrm{D}$ & $4 \mathrm{~N} / 27 \mathrm{~W}-8 \mathrm{M} 3$ & 165 & 0 & $\mathrm{D}$ \\
\hline 4N/27W-8L3 & 555 & 100 & $\mathrm{D}$ & $4 \mathrm{~N} / 27 \mathrm{~W}-8 \mathrm{M} 3$ & 175 & 0 & $\mathrm{D}$ \\
\hline $4 \mathrm{~N} / 27 \mathrm{~W}-8 \mathrm{~L} 3$ & 575 & 0 & $\mathrm{D}$ & $4 \mathrm{~N} / 27 \mathrm{~W}-8 \mathrm{R} 1$ & 5 & 100 & $\mathrm{D}$ \\
\hline 4N/27W-8L3 & 585 & 0 & $\mathrm{D}$ & $4 \mathrm{~N} / 27 \mathrm{~W}-8 \mathrm{R} 1$ & 15 & 100 & $\mathrm{D}$ \\
\hline $4 \mathrm{~N} / 27 \mathrm{~W}-8 \mathrm{~L} 3$ & 595 & 0 & $\mathrm{D}$ & $4 \mathrm{~N} / 27 \mathrm{~W}-8 \mathrm{R} 1$ & 25 & 50 & $\mathrm{D}$ \\
\hline 4N/27W-8L3 & 605 & 0 & $\mathrm{D}$ & $4 \mathrm{~N} / 27 \mathrm{~W}-8 \mathrm{R} 1$ & 35 & 0 & $\mathrm{D}$ \\
\hline $4 \mathrm{~N} / 27 \mathrm{~W}-8 \mathrm{M} 1$ & 15 & 10 & $\mathrm{D}$ & 4N/27W-8R1 & 75 & 100 & $\mathrm{D}$ \\
\hline $4 \mathrm{~N} / 27 \mathrm{~W}-8 \mathrm{M} 1$ & 25 & 0 & $\mathrm{D}$ & $4 \mathrm{~N} / 27 \mathrm{~W}-8 \mathrm{R} 1$ & 85 & 50 & $\mathrm{D}$ \\
\hline $4 \mathrm{~N} / 27 \mathrm{~W}-8 \mathrm{M} 1$ & 35 & 0 & $\mathrm{D}$ & 4N/27W-8R1 & 95 & 0 & $\mathrm{D}$ \\
\hline $4 \mathrm{~N} / 27 \mathrm{~W}-8 \mathrm{M} 1$ & 45 & 0 & $\mathrm{D}$ & $4 \mathrm{~N} / 27 \mathrm{~W}-8 \mathrm{R} 1$ & 105 & 0 & $\mathrm{D}$ \\
\hline $4 \mathrm{~N} / 27 \mathrm{~W}-8 \mathrm{M} 1$ & 55 & 0 & $\mathrm{D}$ & $4 \mathrm{~N} / 27 \mathrm{~W}-8 \mathrm{R} 1$ & 115 & 0 & $\mathrm{D}$ \\
\hline $4 \mathrm{~N} / 27 \mathrm{~W}-8 \mathrm{M} 1$ & 65 & 0 & $\mathrm{D}$ & $4 \mathrm{~N} / 27 \mathrm{~W}-8 \mathrm{R} 1$ & 125 & 0 & $\mathrm{D}$ \\
\hline $4 \mathrm{~N} / 27 \mathrm{~W}-8 \mathrm{M} 1$ & 75 & 0 & $\mathrm{D}$ & $4 \mathrm{~N} / 27 \mathrm{~W}-8 \mathrm{R} 1$ & 135 & 0 & $\mathrm{D}$ \\
\hline $4 \mathrm{~N} / 27 \mathrm{~W}-8 \mathrm{M} 1$ & 85 & 0 & $\mathrm{D}$ & $4 \mathrm{~N} / 27 \mathrm{~W}-8 \mathrm{R} 1$ & 145 & 0 & $\mathrm{D}$ \\
\hline $4 \mathrm{~N} / 27 \mathrm{~W}-8 \mathrm{M} 1$ & 95 & 0 & $\mathrm{D}$ & $4 \mathrm{~N} / 27 \mathrm{~W}-8 \mathrm{R} 1$ & 155 & 0 & $\mathrm{D}$ \\
\hline $4 \mathrm{~N} / 27 \mathrm{~W}-8 \mathrm{M} 3$ & 25 & 0 & $\mathrm{D}$ & $4 \mathrm{~N} / 27 \mathrm{~W}-8 \mathrm{R} 1$ & 225 & 90 & $\mathrm{D}$ \\
\hline $4 \mathrm{~N} / 27 \mathrm{~W}-8 \mathrm{M} 3$ & 35 & 0 & $\mathrm{D}$ & $4 \mathrm{~N} / 27 \mathrm{~W}-8 \mathrm{R} 1$ & 235 & 0 & $\mathrm{D}$ \\
\hline $4 \mathrm{~N} / 27 \mathrm{~W}-8 \mathrm{M} 3$ & 45 & 0 & $\mathrm{D}$ & $4 \mathrm{~N} / 27 \mathrm{~W}-8 \mathrm{R} 1$ & 245 & 0 & $\mathrm{D}$ \\
\hline $4 \mathrm{~N} / 27 \mathrm{~W}-8 \mathrm{M} 3$ & 55 & 0 & $\mathrm{D}$ & $4 \mathrm{~N} / 27 \mathrm{~W}-8 \mathrm{R} 1$ & 255 & 0 & $\mathrm{D}$ \\
\hline $4 \mathrm{~N} / 27 \mathrm{~W}-8 \mathrm{M} 3$ & 65 & 0 & $\mathrm{D}$ & 4N/27W-9Q1 & 5 & 0 & $\mathrm{D}$ \\
\hline $4 \mathrm{~N} / 27 \mathrm{~W}-8 \mathrm{M} 3$ & 75 & 0 & $\mathrm{D}$ & 4N/27W-9Q1 & 15 & 0 & $\mathrm{D}$ \\
\hline $4 \mathrm{~N} / 27 \mathrm{~W}-8 \mathrm{M} 3$ & 85 & 0 & $\mathrm{D}$ & 4N/27W-9Q1 & 25 & 0 & $\mathrm{D}$ \\
\hline $4 \mathrm{~N} / 27 \mathrm{~W}-8 \mathrm{M} 3$ & 95 & 0 & $\mathrm{D}$ & 4N/27W-9Q1 & 35 & 60 & $\mathrm{D}$ \\
\hline $4 \mathrm{~N} / 27 \mathrm{~W}-8 \mathrm{M} 3$ & 105 & 0 & $\mathrm{D}$ & 4N/27W-9Q1 & 45 & 100 & $\mathrm{D}$ \\
\hline $4 \mathrm{~N} / 27 \mathrm{~W}-8 \mathrm{M} 3$ & 115 & 0 & $\mathrm{D}$ & 4N/27W-9Q1 & 55 & 10 & $\mathrm{D}$ \\
\hline $4 \mathrm{~N} / 27 \mathrm{~W}-8 \mathrm{M} 3$ & 125 & 0 & $\mathrm{D}$ & 4N/27W-9Q1 & 65 & 0 & $\mathrm{D}$ \\
\hline $4 \mathrm{~N} / 27 \mathrm{~W}-8 \mathrm{M} 3$ & 135 & 0 & $\mathrm{D}$ & 4N/27W-9Q1 & 75 & 0 & $\mathrm{D}$ \\
\hline
\end{tabular}


Table B1-1. Calculated percent-coarse intervals for all drillers' logs and e-logs used to define textural properties of the hydrogeologic framework model.-Continued

[Percent-coarse values are calculated for 10-foot intervals for the entire depth of each borehole. State well number is the state of California well identifier; interval midpoint depth is the midpoint of each 10-foot interval, in feet from top of borehole; PC is the percent-coarse value for each interval; log type defines whether the borehole is a drillers' lithology $\log (\mathrm{D})$ or a geophysical e-log (E).]

\begin{tabular}{|c|c|c|c|c|c|c|c|}
\hline $\begin{array}{c}\text { State } \\
\text { well } \\
\text { number }\end{array}$ & $\begin{array}{c}\text { Interval } \\
\text { midpoint depth } \\
\text { (feet) }\end{array}$ & PC & $\begin{array}{l}\text { Log } \\
\text { type }\end{array}$ & $\begin{array}{c}\text { State } \\
\text { well } \\
\text { number }\end{array}$ & $\begin{array}{c}\text { Interval } \\
\text { midpoint depth } \\
\text { (feet) }\end{array}$ & PC & $\begin{array}{l}\text { Log } \\
\text { type }\end{array}$ \\
\hline 4N/27W-9Q1 & 85 & 0 & $\mathrm{D}$ & $4 \mathrm{~N} / 27 \mathrm{~W}-14 \mathrm{~K} 1$ & 215 & 0 & $\mathrm{D}$ \\
\hline 4N/27W-9Q1 & 95 & 0 & $\mathrm{D}$ & $4 \mathrm{~N} / 27 \mathrm{~W}-14 \mathrm{~K} 1$ & 225 & 0 & $\mathrm{D}$ \\
\hline 4N/27W-9Q1 & 105 & 0 & $\mathrm{D}$ & $4 \mathrm{~N} / 27 \mathrm{~W}-14 \mathrm{~K} 1$ & 235 & 0 & $\mathrm{D}$ \\
\hline 4N/27W-9Q1 & 115 & 0 & $\mathrm{D}$ & $4 \mathrm{~N} / 27 \mathrm{~W}-14 \mathrm{~K} 1$ & 245 & 10 & $\mathrm{D}$ \\
\hline 4N/27W-9Q1 & 135 & 0 & $\mathrm{D}$ & $4 \mathrm{~N} / 27 \mathrm{~W}-14 \mathrm{~K} 1$ & 265 & 100 & $\mathrm{D}$ \\
\hline 4N/27W-9Q1 & 145 & 0 & $\mathrm{D}$ & $4 \mathrm{~N} / 27 \mathrm{~W}-14 \mathrm{~K} 1$ & 275 & 100 & $\mathrm{D}$ \\
\hline 4N/27W-9Q1 & 155 & 0 & $\mathrm{D}$ & $4 \mathrm{~N} / 27 \mathrm{~W}-14 \mathrm{~K} 1$ & 285 & 100 & $\mathrm{D}$ \\
\hline 4N/27W-9Q1 & 165 & 0 & $\mathrm{D}$ & $4 \mathrm{~N} / 27 \mathrm{~W}-14 \mathrm{~K} 1$ & 295 & 100 & $\mathrm{D}$ \\
\hline 4N/27W-9Q1 & 205 & 0 & $\mathrm{D}$ & $4 \mathrm{~N} / 27 \mathrm{~W}-14 \mathrm{~K} 1$ & 335 & 100 & $\mathrm{D}$ \\
\hline 4N/27W-9Q1 & 215 & 0 & $\mathrm{D}$ & $4 \mathrm{~N} / 27 \mathrm{~W}-14 \mathrm{~K} 1$ & 345 & 100 & $\mathrm{D}$ \\
\hline 4N/27W-9Q1 & 225 & 0 & $\mathrm{D}$ & $4 \mathrm{~N} / 27 \mathrm{~W}-14 \mathrm{~K} 1$ & 355 & 100 & $\mathrm{D}$ \\
\hline 4N/27W-9Q1 & 235 & 0 & $\mathrm{D}$ & $4 \mathrm{~N} / 27 \mathrm{~W}-14 \mathrm{~K} 1$ & 365 & 100 & $\mathrm{D}$ \\
\hline 4N/27W-9Q1 & 245 & 0 & $\mathrm{D}$ & $4 \mathrm{~N} / 27 \mathrm{~W}-14 \mathrm{~K} 1$ & 375 & 100 & $\mathrm{D}$ \\
\hline 4N/27W-9Q1 & 255 & 0 & $\mathrm{D}$ & $4 \mathrm{~N} / 27 \mathrm{~W}-14 \mathrm{~K} 1$ & 385 & 100 & $\mathrm{D}$ \\
\hline $4 \mathrm{~N} / 27 \mathrm{~W}-14 \mathrm{~K} 1$ & 5 & 45.5 & $\mathrm{D}$ & $4 \mathrm{~N} / 27 \mathrm{~W}-14 \mathrm{~K} 1$ & 395 & 100 & $\mathrm{D}$ \\
\hline $4 \mathrm{~N} / 27 \mathrm{~W}-14 \mathrm{~K} 1$ & 15 & 0 & $\mathrm{D}$ & $4 \mathrm{~N} / 27 \mathrm{~W}-14 \mathrm{~K} 1$ & 405 & 100 & $\mathrm{D}$ \\
\hline $4 \mathrm{~N} / 27 \mathrm{~W}-14 \mathrm{~K} 1$ & 25 & 0 & $\mathrm{D}$ & $4 \mathrm{~N} / 27 \mathrm{~W}-14 \mathrm{~K} 1$ & 415 & 100 & $\mathrm{D}$ \\
\hline $4 \mathrm{~N} / 27 \mathrm{~W}-14 \mathrm{~K} 1$ & 95 & 0 & $\mathrm{D}$ & $4 \mathrm{~N} / 27 \mathrm{~W}-14 \mathrm{~K} 1$ & 485 & 100 & $\mathrm{D}$ \\
\hline $4 \mathrm{~N} / 27 \mathrm{~W}-14 \mathrm{~K} 1$ & 105 & 0 & $\mathrm{D}$ & $4 \mathrm{~N} / 27 \mathrm{~W}-14 \mathrm{~K} 1$ & 495 & 100 & $\mathrm{D}$ \\
\hline 4N/27W-14K1 & 115 & 0 & $\mathrm{D}$ & $4 \mathrm{~N} / 27 \mathrm{~W}-14 \mathrm{~K} 1$ & 505 & 100 & $\mathrm{D}$ \\
\hline 4N/27W-14K1 & 125 & 0 & $\mathrm{D}$ & $4 \mathrm{~N} / 27 \mathrm{~W}-14 \mathrm{~K} 1$ & 515 & 100 & $\mathrm{D}$ \\
\hline 4N/27W-14K1 & 135 & 0 & $\mathrm{D}$ & $4 \mathrm{~N} / 27 \mathrm{~W}-14 \mathrm{~K} 1$ & 525 & 100 & $\mathrm{D}$ \\
\hline $4 \mathrm{~N} / 27 \mathrm{~W}-14 \mathrm{~K} 1$ & 145 & 0 & $\mathrm{D}$ & $4 \mathrm{~N} / 27 \mathrm{~W}-14 \mathrm{~K} 1$ & 535 & 100 & $\mathrm{D}$ \\
\hline $4 \mathrm{~N} / 27 \mathrm{~W}-14 \mathrm{~K} 1$ & 155 & 0 & $\mathrm{D}$ & $4 \mathrm{~N} / 27 \mathrm{~W}-14 \mathrm{~K} 1$ & 545 & 100 & $\mathrm{D}$ \\
\hline $4 \mathrm{~N} / 27 \mathrm{~W}-14 \mathrm{~K} 1$ & 165 & 0 & $\mathrm{D}$ & $4 \mathrm{~N} / 27 \mathrm{~W}-14 \mathrm{~K} 1$ & 555 & 100 & $\mathrm{D}$ \\
\hline 4N/27W-14K1 & 175 & 0 & $\mathrm{D}$ & $4 \mathrm{~N} / 27 \mathrm{~W}-14 \mathrm{~K} 1$ & 565 & 0 & $\mathrm{D}$ \\
\hline $4 \mathrm{~N} / 27 \mathrm{~W}-14 \mathrm{~K} 1$ & 185 & 0 & $\mathrm{D}$ & $4 \mathrm{~N} / 27 \mathrm{~W}-14 \mathrm{~K} 1$ & 575 & 0 & $\mathrm{D}$ \\
\hline $4 \mathrm{~N} / 27 \mathrm{~W}-14 \mathrm{~K} 1$ & 195 & 0 & $\mathrm{D}$ & $4 \mathrm{~N} / 27 \mathrm{~W}-14 \mathrm{~K} 1$ & 585 & 0 & $\mathrm{D}$ \\
\hline $4 \mathrm{~N} / 27 \mathrm{~W}-14 \mathrm{~K} 1$ & 205 & 0 & $\mathrm{D}$ & $4 \mathrm{~N} / 27 \mathrm{~W}-14 \mathrm{~K} 1$ & 595 & 0 & $\mathrm{D}$ \\
\hline
\end{tabular}


Table B1-1. Calculated percent-coarse intervals for all drillers' logs and e-logs used to define textural properties of the hydrogeologic framework model.-Continued

[Percent-coarse values are calculated for 10-foot intervals for the entire depth of each borehole. State well number is the state of California well identifier; interval midpoint depth is the midpoint of each 10-foot interval, in feet from top of borehole; PC is the percent-coarse value for each interval; log type defines whether the borehole is a drillers' lithology $\log (\mathrm{D})$ or a geophysical e-log (E).]

\begin{tabular}{|c|c|c|c|c|c|c|c|}
\hline $\begin{array}{c}\text { State } \\
\text { well } \\
\text { number }\end{array}$ & $\begin{array}{c}\text { Interval } \\
\text { midpoint depth } \\
\text { (feet) }\end{array}$ & PC & $\begin{array}{l}\text { Log } \\
\text { type }\end{array}$ & $\begin{array}{c}\text { State } \\
\text { well } \\
\text { number }\end{array}$ & $\begin{array}{c}\text { Interval } \\
\text { midpoint depth } \\
\text { (feet) }\end{array}$ & PC & $\begin{array}{l}\text { Log } \\
\text { type }\end{array}$ \\
\hline $4 \mathrm{~N} / 27 \mathrm{~W}-14 \mathrm{~K} 1$ & 605 & 0 & $\mathrm{D}$ & $4 \mathrm{~N} / 27 \mathrm{~W}-14 \mathrm{~N} 1$ & 345 & 0 & $\mathrm{D}$ \\
\hline $4 \mathrm{~N} / 27 \mathrm{~W}-14 \mathrm{~K} 1$ & 615 & 0 & $\mathrm{D}$ & $4 \mathrm{~N} / 27 \mathrm{~W}-14 \mathrm{~N} 1$ & 355 & 0 & $\mathrm{D}$ \\
\hline $4 \mathrm{~N} / 27 \mathrm{~W}-14 \mathrm{~K} 1$ & 625 & 0 & $\mathrm{D}$ & $4 \mathrm{~N} / 27 \mathrm{~W}-14 \mathrm{~N} 1$ & 365 & 0 & $\mathrm{D}$ \\
\hline $4 \mathrm{~N} / 27 \mathrm{~W}-14 \mathrm{~K} 1$ & 635 & 0 & $\mathrm{D}$ & $4 \mathrm{~N} / 27 \mathrm{~W}-14 \mathrm{~N} 1$ & 375 & 0 & $\mathrm{D}$ \\
\hline $4 \mathrm{~N} / 27 \mathrm{~W}-14 \mathrm{~N} 1$ & 5 & 100 & $\mathrm{D}$ & $4 \mathrm{~N} / 27 \mathrm{~W}-14 \mathrm{~N} 1$ & 395 & 0 & $\mathrm{D}$ \\
\hline $4 \mathrm{~N} / 27 \mathrm{~W}-14 \mathrm{~N} 1$ & 15 & 50 & $\mathrm{D}$ & $4 \mathrm{~N} / 27 \mathrm{~W}-14 \mathrm{~N} 1$ & 405 & 0 & $\mathrm{D}$ \\
\hline $4 \mathrm{~N} / 27 \mathrm{~W}-14 \mathrm{~N} 1$ & 25 & 0 & $\mathrm{D}$ & $4 \mathrm{~N} / 27 \mathrm{~W}-14 \mathrm{~N} 1$ & 415 & 0 & $\mathrm{D}$ \\
\hline $4 \mathrm{~N} / 27 \mathrm{~W}-14 \mathrm{~N} 1$ & 35 & 0 & $\mathrm{D}$ & $4 \mathrm{~N} / 27 \mathrm{~W}-14 \mathrm{~N} 1$ & 425 & 0 & $\mathrm{D}$ \\
\hline $4 \mathrm{~N} / 27 \mathrm{~W}-14 \mathrm{~N} 1$ & 75 & 0 & $\mathrm{D}$ & $4 \mathrm{~N} / 27 \mathrm{~W}-14 \mathrm{~N} 1$ & 465 & 0 & $\mathrm{D}$ \\
\hline $4 \mathrm{~N} / 27 \mathrm{~W}-14 \mathrm{~N} 1$ & 85 & 0 & $\mathrm{D}$ & $4 \mathrm{~N} / 27 \mathrm{~W}-14 \mathrm{~N} 1$ & 475 & 0 & $\mathrm{D}$ \\
\hline $4 \mathrm{~N} / 27 \mathrm{~W}-14 \mathrm{~N} 1$ & 95 & 0 & $\mathrm{D}$ & $4 \mathrm{~N} / 27 \mathrm{~W}-14 \mathrm{~N} 1$ & 485 & 0 & $\mathrm{D}$ \\
\hline $4 \mathrm{~N} / 27 \mathrm{~W}-14 \mathrm{~N} 1$ & 105 & 0 & $\mathrm{D}$ & $4 \mathrm{~N} / 27 \mathrm{~W}-14 \mathrm{P} 2$ & 5 & 0 & $\mathrm{D}$ \\
\hline $4 \mathrm{~N} / 27 \mathrm{~W}-14 \mathrm{~N} 1$ & 115 & 0 & $\mathrm{D}$ & $4 \mathrm{~N} / 27 \mathrm{~W}-14 \mathrm{P} 2$ & 15 & 0 & $\mathrm{D}$ \\
\hline $4 \mathrm{~N} / 27 \mathrm{~W}-14 \mathrm{~N} 1$ & 125 & 0 & $\mathrm{D}$ & $4 \mathrm{~N} / 27 \mathrm{~W}-14 \mathrm{P} 2$ & 25 & 0 & $\mathrm{D}$ \\
\hline $4 \mathrm{~N} / 27 \mathrm{~W}-14 \mathrm{~N} 1$ & 135 & 10 & $\mathrm{D}$ & $4 \mathrm{~N} / 27 \mathrm{~W}-14 \mathrm{P} 2$ & 35 & 0 & $\mathrm{D}$ \\
\hline $4 \mathrm{~N} / 27 \mathrm{~W}-14 \mathrm{~N} 1$ & 145 & 100 & $\mathrm{D}$ & $4 \mathrm{~N} / 27 \mathrm{~W}-14 \mathrm{P} 2$ & 45 & 0 & $\mathrm{D}$ \\
\hline $4 \mathrm{~N} / 27 \mathrm{~W}-14 \mathrm{~N} 1$ & 155 & 90 & $\mathrm{D}$ & $4 \mathrm{~N} / 27 \mathrm{~W}-14 \mathrm{P} 2$ & 55 & 0 & $\mathrm{D}$ \\
\hline $4 \mathrm{~N} / 27 \mathrm{~W}-14 \mathrm{~N} 1$ & 225 & 0 & $\mathrm{D}$ & $4 \mathrm{~N} / 27 \mathrm{~W}-14 \mathrm{P} 2$ & 125 & 0 & $\mathrm{D}$ \\
\hline $4 \mathrm{~N} / 27 \mathrm{~W}-14 \mathrm{~N} 1$ & 235 & 0 & $\mathrm{D}$ & $4 \mathrm{~N} / 27 \mathrm{~W}-14 \mathrm{P} 2$ & 135 & 0 & $\mathrm{D}$ \\
\hline $4 \mathrm{~N} / 27 \mathrm{~W}-14 \mathrm{~N} 1$ & 245 & 0 & $\mathrm{D}$ & $4 \mathrm{~N} / 27 \mathrm{~W}-14 \mathrm{P} 2$ & 145 & 0 & $\mathrm{D}$ \\
\hline $4 \mathrm{~N} / 27 \mathrm{~W}-14 \mathrm{~N} 1$ & 255 & 0 & $\mathrm{D}$ & $4 \mathrm{~N} / 27 \mathrm{~W}-14 \mathrm{P} 2$ & 155 & 0 & $\mathrm{D}$ \\
\hline $4 \mathrm{~N} / 27 \mathrm{~W}-14 \mathrm{~N} 1$ & 265 & 0 & $\mathrm{D}$ & $4 \mathrm{~N} / 27 \mathrm{~W}-14 \mathrm{P} 2$ & 165 & 0 & $\mathrm{D}$ \\
\hline $4 \mathrm{~N} / 27 \mathrm{~W}-14 \mathrm{~N} 1$ & 275 & 50 & $\mathrm{D}$ & $4 \mathrm{~N} / 27 \mathrm{~W}-14 \mathrm{P} 2$ & 175 & 0 & $\mathrm{D}$ \\
\hline $4 \mathrm{~N} / 27 \mathrm{~W}-14 \mathrm{~N} 1$ & 285 & 20 & $\mathrm{D}$ & $4 \mathrm{~N} / 27 \mathrm{~W}-14 \mathrm{P} 2$ & 185 & 0 & $\mathrm{D}$ \\
\hline $4 \mathrm{~N} / 27 \mathrm{~W}-14 \mathrm{~N} 1$ & 295 & 0 & $\mathrm{D}$ & $4 \mathrm{~N} / 27 \mathrm{~W}-14 \mathrm{P} 2$ & 195 & 0 & $\mathrm{D}$ \\
\hline $4 \mathrm{~N} / 27 \mathrm{~W}-14 \mathrm{~N} 1$ & 305 & 0 & $\mathrm{D}$ & $4 \mathrm{~N} / 27 \mathrm{~W}-14 \mathrm{P} 2$ & 205 & 0 & $\mathrm{D}$ \\
\hline $4 \mathrm{~N} / 27 \mathrm{~W}-14 \mathrm{~N} 1$ & 315 & 0 & $\mathrm{D}$ & $4 \mathrm{~N} / 27 \mathrm{~W}-14 \mathrm{P} 2$ & 215 & 0 & $\mathrm{D}$ \\
\hline $4 \mathrm{~N} / 27 \mathrm{~W}-14 \mathrm{~N} 1$ & 325 & 0 & $\mathrm{D}$ & $4 \mathrm{~N} / 27 \mathrm{~W}-14 \mathrm{P} 2$ & 225 & 0 & $\mathrm{D}$ \\
\hline $4 \mathrm{~N} / 27 \mathrm{~W}-14 \mathrm{~N} 1$ & 335 & 0 & $\mathrm{D}$ & $4 \mathrm{~N} / 27 \mathrm{~W}-14 \mathrm{P} 2$ & 235 & 0 & $\mathrm{D}$ \\
\hline
\end{tabular}


Table B1-1. Calculated percent-coarse intervals for all drillers' logs and e-logs used to define textural properties of the hydrogeologic framework model.-Continued

[Percent-coarse values are calculated for 10-foot intervals for the entire depth of each borehole. State well number is the state of California well identifier; interval midpoint depth is the midpoint of each 10-foot interval, in feet from top of borehole; PC is the percent-coarse value for each interval; log type defines whether the borehole is a drillers' lithology $\log (\mathrm{D})$ or a geophysical e-log (E).]

\begin{tabular}{|c|c|c|c|c|c|c|c|}
\hline $\begin{array}{c}\text { State } \\
\text { well } \\
\text { number }\end{array}$ & $\begin{array}{c}\text { Interval } \\
\text { midpoint depth } \\
\text { (feet) }\end{array}$ & PC & $\begin{array}{l}\text { Log } \\
\text { type }\end{array}$ & $\begin{array}{c}\text { State } \\
\text { well } \\
\text { number }\end{array}$ & $\begin{array}{c}\text { Interval } \\
\text { midpoint depth } \\
\text { (feet) }\end{array}$ & PC & $\begin{array}{l}\text { Log } \\
\text { type }\end{array}$ \\
\hline $4 \mathrm{~N} / 27 \mathrm{~W}-14 \mathrm{P} 2$ & 245 & 0 & $\mathrm{D}$ & 4N/27W-14Q1 & 325 & 100 & $\mathrm{D}$ \\
\hline $4 \mathrm{~N} / 27 \mathrm{~W}-14 \mathrm{P} 2$ & 255 & 0 & $\mathrm{D}$ & 4N/27W-14Q1 & 335 & 100 & $\mathrm{D}$ \\
\hline $4 \mathrm{~N} / 27 \mathrm{~W}-14 \mathrm{P} 2$ & 265 & 0 & $\mathrm{D}$ & 4N/27W-14Q1 & 345 & 100 & $\mathrm{D}$ \\
\hline $4 \mathrm{~N} / 27 \mathrm{~W}-14 \mathrm{P} 2$ & 275 & 0 & $\mathrm{D}$ & 4N/27W-14Q1 & 355 & 100 & $\mathrm{D}$ \\
\hline $4 \mathrm{~N} / 27 \mathrm{~W}-14 \mathrm{P} 2$ & 295 & 0 & $\mathrm{D}$ & $4 \mathrm{~N} / 27 \mathrm{~W}-14 \mathrm{Q} 1$ & 375 & 100 & $\mathrm{D}$ \\
\hline $4 \mathrm{~N} / 27 \mathrm{~W}-14 \mathrm{P} 2$ & 305 & 0 & $\mathrm{D}$ & 4N/27W-14Q1 & 385 & 100 & $\mathrm{D}$ \\
\hline 4N/27W-14Q1 & 5 & 100 & $\mathrm{D}$ & 4N/27W-14Q1 & 395 & 100 & $\mathrm{D}$ \\
\hline 4N/27W-14Q1 & 15 & 100 & $\mathrm{D}$ & 4N/27W-14Q1 & 405 & 100 & $\mathrm{D}$ \\
\hline 4N/27W-14Q1 & 55 & 100 & $\mathrm{D}$ & 4N/27W-14Q1 & 445 & 100 & $\mathrm{D}$ \\
\hline 4N/27W-14Q1 & 65 & 100 & $\mathrm{D}$ & 4N/27W-14Q1 & 455 & 100 & $\mathrm{D}$ \\
\hline 4N/27W-14Q1 & 75 & 90.9 & $\mathrm{D}$ & 4N/27W-14Q1 & 465 & 100 & $\mathrm{D}$ \\
\hline 4N/27W-14Q1 & 85 & 0 & $\mathrm{D}$ & 4N/27W-14Q1 & 475 & 100 & $\mathrm{D}$ \\
\hline 4N/27W-14Q1 & 95 & 54.5 & $\mathrm{D}$ & $4 \mathrm{~N} / 27 \mathrm{~W}-14 \mathrm{Q} 1$ & 485 & 100 & $\mathrm{D}$ \\
\hline 4N/27W-14Q1 & 105 & 100 & $\mathrm{D}$ & 4N/27W-14Q1 & 495 & 100 & $\mathrm{D}$ \\
\hline 4N/27W-14Q1 & 115 & 100 & $\mathrm{D}$ & 4N/27W-14Q1 & 505 & 100 & $\mathrm{D}$ \\
\hline 4N/27W-14Q1 & 125 & 100 & $\mathrm{D}$ & 4N/27W-14Q1 & 515 & 100 & $\mathrm{D}$ \\
\hline 4N/27W-14Q1 & 135 & 0 & $\mathrm{D}$ & 4N/27W-14Q1 & 525 & 100 & $\mathrm{D}$ \\
\hline 4N/27W-14Q1 & 205 & 0 & $\mathrm{D}$ & 4N/27W-14Q1 & 595 & 100 & $\mathrm{D}$ \\
\hline 4N/27W-14Q1 & 215 & 0 & $\mathrm{D}$ & $4 \mathrm{~N} / 27 \mathrm{~W}-14 \mathrm{Q} 1$ & 605 & 100 & $\mathrm{D}$ \\
\hline 4N/27W-14Q1 & 225 & 0 & $\mathrm{D}$ & 4N/27W-14Q1 & 615 & 100 & $\mathrm{D}$ \\
\hline 4N/27W-14Q1 & 235 & 0 & $\mathrm{D}$ & $4 \mathrm{~N} / 27 \mathrm{~W}-14 \mathrm{Q} 1$ & 625 & 100 & $\mathrm{D}$ \\
\hline $4 \mathrm{~N} / 27 \mathrm{~W}-14 \mathrm{Q} 1$ & 245 & 0 & $\mathrm{D}$ & $4 \mathrm{~N} / 27 \mathrm{~W}-14 \mathrm{Q} 1$ & 635 & 100 & $\mathrm{D}$ \\
\hline 4N/27W-14Q1 & 255 & 0 & $\mathrm{D}$ & 4N/27W-14Q1 & 645 & 100 & $\mathrm{D}$ \\
\hline 4N/27W-14Q1 & 265 & 0 & $\mathrm{D}$ & 4N/27W-14Q1 & 655 & 100 & $\mathrm{D}$ \\
\hline 4N/27W-14Q1 & 275 & 0 & $\mathrm{D}$ & $4 \mathrm{~N} / 27 \mathrm{~W}-14 \mathrm{Q} 1$ & 665 & 100 & $\mathrm{D}$ \\
\hline 4N/27W-14Q1 & 285 & 100 & $\mathrm{D}$ & $4 \mathrm{~N} / 27 \mathrm{~W}-14 \mathrm{Q} 1$ & 675 & 100 & $\mathrm{D}$ \\
\hline 4N/27W-14Q1 & 295 & 100 & $\mathrm{D}$ & 4N/27W-14Q1 & 685 & 100 & $\mathrm{D}$ \\
\hline 4N/27W-14Q1 & 305 & 100 & $\mathrm{D}$ & $4 \mathrm{~N} / 27 \mathrm{~W}-15 \mathrm{E} 2$ & 5 & 0 & $\mathrm{D}$ \\
\hline 4N/27W-14Q1 & 315 & 100 & $\mathrm{D}$ & $4 \mathrm{~N} / 27 \mathrm{~W}-15 \mathrm{E} 2$ & 15 & 0 & $\mathrm{D}$ \\
\hline
\end{tabular}


Table B1-1. Calculated percent-coarse intervals for all drillers' logs and e-logs used to define textural properties of the hydrogeologic framework model.-Continued

[Percent-coarse values are calculated for 10-foot intervals for the entire depth of each borehole. State well number is the state of California well identifier; interval midpoint depth is the midpoint of each 10-foot interval, in feet from top of borehole; PC is the percent-coarse value for each interval; log type defines whether the borehole is a drillers' lithology log (D) or a geophysical e-log (E).]

\begin{tabular}{|c|c|c|c|c|c|c|c|}
\hline $\begin{array}{c}\text { State } \\
\text { well } \\
\text { number }\end{array}$ & $\begin{array}{c}\text { Interval } \\
\text { midpoint depth } \\
\text { (feet) }\end{array}$ & PC & $\begin{array}{l}\text { Log } \\
\text { type }\end{array}$ & $\begin{array}{c}\text { State } \\
\text { well } \\
\text { number }\end{array}$ & $\begin{array}{c}\text { Interval } \\
\text { midpoint depth } \\
\text { (feet) }\end{array}$ & PC & $\begin{array}{l}\text { Log } \\
\text { type }\end{array}$ \\
\hline $4 \mathrm{~N} / 27 \mathrm{~W}-15 \mathrm{E} 2$ & 25 & 0 & $\mathrm{D}$ & $4 \mathrm{~N} / 27 \mathrm{~W}-15 \mathrm{E} 2$ & 415 & 0 & $\mathrm{D}$ \\
\hline $4 \mathrm{~N} / 27 \mathrm{~W}-15 \mathrm{E} 2$ & 35 & 0 & $\mathrm{D}$ & $4 \mathrm{~N} / 27 \mathrm{~W}-15 \mathrm{E} 2$ & 425 & 54.5 & $\mathrm{D}$ \\
\hline $4 \mathrm{~N} / 27 \mathrm{~W}-15 \mathrm{E} 2$ & 45 & 10 & $\mathrm{D}$ & $4 \mathrm{~N} / 27 \mathrm{~W}-15 \mathrm{E} 2$ & 435 & 100 & $\mathrm{D}$ \\
\hline $4 \mathrm{~N} / 27 \mathrm{~W}-15 \mathrm{E} 2$ & 55 & 100 & $\mathrm{D}$ & $4 \mathrm{~N} / 27 \mathrm{~W}-15 \mathrm{E} 2$ & 445 & 100 & $\mathrm{D}$ \\
\hline 4N/27W-15E2 & 75 & 100 & $\mathrm{D}$ & 4N/27W-15E2 & 465 & 100 & $\mathrm{D}$ \\
\hline $4 \mathrm{~N} / 27 \mathrm{~W}-15 \mathrm{E} 2$ & 85 & 100 & $\mathrm{D}$ & $4 \mathrm{~N} / 27 \mathrm{~W}-15 \mathrm{E} 2$ & 475 & 90 & $\mathrm{D}$ \\
\hline $4 \mathrm{~N} / 27 \mathrm{~W}-15 \mathrm{E} 2$ & 95 & 90.9 & $\mathrm{D}$ & $4 \mathrm{~N} / 27 \mathrm{~W}-15 \mathrm{E} 2$ & 485 & 0 & $\mathrm{D}$ \\
\hline $4 \mathrm{~N} / 27 \mathrm{~W}-15 \mathrm{E} 2$ & 105 & 0 & $\mathrm{D}$ & $4 \mathrm{~N} / 27 \mathrm{~W}-15 \mathrm{E} 2$ & 495 & 0 & $\mathrm{D}$ \\
\hline $4 \mathrm{~N} / 27 \mathrm{~W}-15 \mathrm{E} 2$ & 145 & 0 & $\mathrm{D}$ & $4 \mathrm{~N} / 27 \mathrm{~W}-15 \mathrm{G} 1$ & 5 & 100 & $\mathrm{D}$ \\
\hline $4 \mathrm{~N} / 27 \mathrm{~W}-15 \mathrm{E} 2$ & 155 & 0 & $\mathrm{D}$ & $4 \mathrm{~N} / 27 \mathrm{~W}-15 \mathrm{G} 1$ & 15 & 100 & $\mathrm{D}$ \\
\hline $4 \mathrm{~N} / 27 \mathrm{~W}-15 \mathrm{E} 2$ & 165 & 0 & $\mathrm{D}$ & $4 \mathrm{~N} / 27 \mathrm{~W}-15 \mathrm{G} 1$ & 25 & 100 & $\mathrm{D}$ \\
\hline $4 \mathrm{~N} / 27 \mathrm{~W}-15 \mathrm{E} 2$ & 175 & 100 & $\mathrm{D}$ & $4 \mathrm{~N} / 27 \mathrm{~W}-15 \mathrm{G} 1$ & 35 & 90 & $\mathrm{D}$ \\
\hline 4N/27W-15E2 & 185 & 100 & $\mathrm{D}$ & $4 \mathrm{~N} / 27 \mathrm{~W}-15 \mathrm{G} 1$ & 45 & 0 & $\mathrm{D}$ \\
\hline $4 \mathrm{~N} / 27 \mathrm{~W}-15 \mathrm{E} 2$ & 195 & 100 & $\mathrm{D}$ & $4 \mathrm{~N} / 27 \mathrm{~W}-15 \mathrm{G} 1$ & 55 & 0 & $\mathrm{D}$ \\
\hline $4 \mathrm{~N} / 27 \mathrm{~W}-15 \mathrm{E} 2$ & 205 & 100 & $\mathrm{D}$ & $4 \mathrm{~N} / 27 \mathrm{~W}-15 \mathrm{G} 1$ & 65 & 0 & $\mathrm{D}$ \\
\hline $4 \mathrm{~N} / 27 \mathrm{~W}-15 \mathrm{E} 2$ & 215 & 100 & $\mathrm{D}$ & $4 \mathrm{~N} / 27 \mathrm{~W}-15 \mathrm{G} 1$ & 75 & 0 & $\mathrm{D}$ \\
\hline $4 \mathrm{~N} / 27 \mathrm{~W}-15 \mathrm{E} 2$ & 225 & 100 & $\mathrm{D}$ & $4 \mathrm{~N} / 27 \mathrm{~W}-15 \mathrm{G} 1$ & 85 & 0 & $\mathrm{D}$ \\
\hline $4 \mathrm{~N} / 27 \mathrm{~W}-15 \mathrm{E} 2$ & 295 & 100 & $\mathrm{D}$ & $4 \mathrm{~N} / 27 \mathrm{~W}-15 \mathrm{G} 1$ & 155 & 0 & $\mathrm{D}$ \\
\hline $4 \mathrm{~N} / 27 \mathrm{~W}-15 \mathrm{E} 2$ & 305 & 100 & $\mathrm{D}$ & $4 \mathrm{~N} / 27 \mathrm{~W}-15 \mathrm{G} 1$ & 165 & 0 & $\mathrm{D}$ \\
\hline $4 \mathrm{~N} / 27 \mathrm{~W}-15 \mathrm{E} 2$ & 315 & 100 & $\mathrm{D}$ & $4 \mathrm{~N} / 27 \mathrm{~W}-15 \mathrm{G} 1$ & 175 & 0 & $\mathrm{D}$ \\
\hline $4 \mathrm{~N} / 27 \mathrm{~W}-15 \mathrm{E} 2$ & 325 & 100 & $\mathrm{D}$ & $4 \mathrm{~N} / 27 \mathrm{~W}-15 \mathrm{G} 1$ & 185 & 0 & $\mathrm{D}$ \\
\hline $4 \mathrm{~N} / 27 \mathrm{~W}-15 \mathrm{E} 2$ & 335 & 100 & $\mathrm{D}$ & $4 \mathrm{~N} / 27 \mathrm{~W}-15 \mathrm{G} 1$ & 195 & 0 & $\mathrm{D}$ \\
\hline $4 \mathrm{~N} / 27 \mathrm{~W}-15 \mathrm{E} 2$ & 345 & 100 & $\mathrm{D}$ & $4 \mathrm{~N} / 27 \mathrm{~W}-15 \mathrm{G} 1$ & 205 & 0 & $\mathrm{D}$ \\
\hline 4N/27W-15E2 & 355 & 90 & $\mathrm{D}$ & $4 \mathrm{~N} / 27 \mathrm{~W}-15 \mathrm{G} 1$ & 215 & 100 & $\mathrm{D}$ \\
\hline $4 \mathrm{~N} / 27 \mathrm{~W}-15 \mathrm{E} 2$ & 365 & 0 & $\mathrm{D}$ & $4 \mathrm{~N} / 27 \mathrm{~W}-15 \mathrm{G} 1$ & 225 & 100 & $\mathrm{D}$ \\
\hline $4 \mathrm{~N} / 27 \mathrm{~W}-15 \mathrm{E} 2$ & 375 & 0 & $\mathrm{D}$ & $4 \mathrm{~N} / 27 \mathrm{~W}-15 \mathrm{G} 1$ & 235 & 100 & $\mathrm{D}$ \\
\hline $4 \mathrm{~N} / 27 \mathrm{~W}-15 \mathrm{E} 2$ & 385 & 0 & $\mathrm{D}$ & $4 \mathrm{~N} / 27 \mathrm{~W}-15 \mathrm{G} 1$ & 245 & 100 & $\mathrm{D}$ \\
\hline $4 \mathrm{~N} / 27 \mathrm{~W}-15 \mathrm{E} 2$ & 395 & 0 & $\mathrm{D}$ & $4 \mathrm{~N} / 27 \mathrm{~W}-15 \mathrm{G} 1$ & 255 & 100 & $\mathrm{D}$ \\
\hline $4 \mathrm{~N} / 27 \mathrm{~W}-15 \mathrm{E} 2$ & 405 & 0 & $\mathrm{D}$ & $4 \mathrm{~N} / 27 \mathrm{~W}-15 \mathrm{G} 1$ & 265 & 0 & $\mathrm{D}$ \\
\hline
\end{tabular}


Table B1-1. Calculated percent-coarse intervals for all drillers' logs and e-logs used to define textural properties of the hydrogeologic framework model.-Continued

[Percent-coarse values are calculated for 10-foot intervals for the entire depth of each borehole. State well number is the state of California well identifier; interval midpoint depth is the midpoint of each 10-foot interval, in feet from top of borehole; PC is the percent-coarse value for each interval; log type defines whether the borehole is a drillers' lithology $\log (\mathrm{D})$ or a geophysical e-log (E).]

\begin{tabular}{|c|c|c|c|c|c|c|c|}
\hline $\begin{array}{c}\text { State } \\
\text { well } \\
\text { number }\end{array}$ & $\begin{array}{c}\text { Interval } \\
\text { midpoint depth } \\
\text { (feet) }\end{array}$ & PC & $\begin{array}{l}\text { Log } \\
\text { type }\end{array}$ & $\begin{array}{c}\text { State } \\
\text { well } \\
\text { number }\end{array}$ & $\begin{array}{c}\text { Interval } \\
\text { midpoint depth } \\
\text { (feet) }\end{array}$ & PC & $\begin{array}{l}\text { Log } \\
\text { type }\end{array}$ \\
\hline $4 \mathrm{~N} / 27 \mathrm{~W}-15 \mathrm{G} 1$ & 275 & 0 & $\mathrm{D}$ & $4 \mathrm{~N} / 27 \mathrm{~W}-15 \mathrm{~J} 2$ & 355 & 0 & $\mathrm{D}$ \\
\hline 4N/27W-15G1 & 285 & 0 & $\mathrm{D}$ & $4 \mathrm{~N} / 27 \mathrm{~W}-15 \mathrm{~J} 2$ & 365 & 0 & $\mathrm{D}$ \\
\hline 4N/27W-15G1 & 295 & 100 & $\mathrm{D}$ & $4 \mathrm{~N} / 27 \mathrm{~W}-15 \mathrm{~J} 2$ & 375 & 0 & $\mathrm{D}$ \\
\hline $4 \mathrm{~N} / 27 \mathrm{~W}-15 \mathrm{G} 1$ & 305 & 90 & $\mathrm{D}$ & $4 \mathrm{~N} / 27 \mathrm{~W}-15 \mathrm{~J} 2$ & 385 & 0 & $\mathrm{D}$ \\
\hline $4 \mathrm{~N} / 27 \mathrm{~W}-15 \mathrm{~J} 2$ & 15 & 0 & $\mathrm{D}$ & $4 \mathrm{~N} / 27 \mathrm{~W}-15 \mathrm{~J} 2$ & 405 & 0 & $\mathrm{D}$ \\
\hline $4 \mathrm{~N} / 27 \mathrm{~W}-15 \mathrm{~J} 2$ & 25 & 0 & $\mathrm{D}$ & $4 \mathrm{~N} / 27 \mathrm{~W}-15 \mathrm{~J} 2$ & 415 & 0 & $\mathrm{D}$ \\
\hline $4 \mathrm{~N} / 27 \mathrm{~W}-15 \mathrm{~J} 2$ & 35 & 20 & $\mathrm{D}$ & $4 \mathrm{~N} / 27 \mathrm{~W}-15 \mathrm{~J} 2$ & 425 & 0 & $\mathrm{D}$ \\
\hline $4 \mathrm{~N} / 27 \mathrm{~W}-15 \mathrm{~J} 2$ & 45 & 0 & $\mathrm{D}$ & $4 \mathrm{~N} / 27 \mathrm{~W}-15 \mathrm{~J} 2$ & 435 & 30 & $\mathrm{D}$ \\
\hline $4 \mathrm{~N} / 27 \mathrm{~W}-15 \mathrm{~J} 2$ & 85 & 20 & $\mathrm{D}$ & $4 \mathrm{~N} / 27 \mathrm{~W}-15 \mathrm{~J} 2$ & 475 & 0 & $\mathrm{D}$ \\
\hline $4 \mathrm{~N} / 27 \mathrm{~W}-15 \mathrm{~J} 2$ & 95 & 90 & $\mathrm{D}$ & $4 \mathrm{~N} / 27 \mathrm{~W}-15 \mathrm{~J} 2$ & 485 & 0 & $\mathrm{D}$ \\
\hline $4 \mathrm{~N} / 27 \mathrm{~W}-15 \mathrm{~J} 2$ & 105 & 100 & $\mathrm{D}$ & $4 \mathrm{~N} / 27 \mathrm{~W}-15 \mathrm{~J} 2$ & 495 & 0 & $\mathrm{D}$ \\
\hline $4 \mathrm{~N} / 27 \mathrm{~W}-15 \mathrm{~J} 2$ & 115 & 50 & $\mathrm{D}$ & $4 \mathrm{~N} / 27 \mathrm{~W}-15 \mathrm{~J} 2$ & 505 & 0 & $\mathrm{D}$ \\
\hline $4 \mathrm{~N} / 27 \mathrm{~W}-15 \mathrm{~J} 2$ & 125 & 0 & $\mathrm{D}$ & $4 \mathrm{~N} / 27 \mathrm{~W}-15 \mathrm{~J} 2$ & 515 & 0 & $\mathrm{D}$ \\
\hline $4 \mathrm{~N} / 27 \mathrm{~W}-15 \mathrm{~J} 2$ & 135 & 0 & $\mathrm{D}$ & $4 \mathrm{~N} / 27 \mathrm{~W}-15 \mathrm{~J} 2$ & 525 & 50 & $\mathrm{D}$ \\
\hline $4 \mathrm{~N} / 27 \mathrm{~W}-15 \mathrm{~J} 2$ & 145 & 0 & $\mathrm{D}$ & $4 \mathrm{~N} / 27 \mathrm{~W}-15 \mathrm{~J} 2$ & 535 & 20 & $\mathrm{D}$ \\
\hline $4 \mathrm{~N} / 27 \mathrm{~W}-15 \mathrm{~J} 2$ & 155 & 0 & $\mathrm{D}$ & $4 \mathrm{~N} / 27 \mathrm{~W}-15 \mathrm{~J} 2$ & 545 & 0 & $\mathrm{D}$ \\
\hline $4 \mathrm{~N} / 27 \mathrm{~W}-15 \mathrm{~J} 2$ & 165 & 0 & $\mathrm{D}$ & $4 \mathrm{~N} / 27 \mathrm{~W}-15 \mathrm{~J} 2$ & 555 & 0 & $\mathrm{D}$ \\
\hline $4 \mathrm{~N} / 27 \mathrm{~W}-15 \mathrm{~J} 2$ & 235 & 0 & $\mathrm{D}$ & $4 \mathrm{~N} / 27 \mathrm{~W}-15 \mathrm{~J} 2$ & 625 & 0 & $\mathrm{D}$ \\
\hline $4 \mathrm{~N} / 27 \mathrm{~W}-15 \mathrm{~J} 2$ & 245 & 10 & $\mathrm{D}$ & $4 \mathrm{~N} / 27 \mathrm{~W}-15 \mathrm{Q} 1$ & 5 & 0 & $\mathrm{D}$ \\
\hline $4 \mathrm{~N} / 27 \mathrm{~W}-15 \mathrm{~J} 2$ & 255 & 100 & $\mathrm{D}$ & $4 \mathrm{~N} / 27 \mathrm{~W}-15 \mathrm{Q} 1$ & 15 & 0 & $\mathrm{D}$ \\
\hline $4 \mathrm{~N} / 27 \mathrm{~W}-15 \mathrm{~J} 2$ & 265 & 10 & $\mathrm{D}$ & 4N/27W-15Q1 & 25 & 0 & $\mathrm{D}$ \\
\hline $4 \mathrm{~N} / 27 \mathrm{~W}-15 \mathrm{~J} 2$ & 275 & 0 & $\mathrm{D}$ & 4N/27W-15Q1 & 35 & 0 & $\mathrm{D}$ \\
\hline $4 \mathrm{~N} / 27 \mathrm{~W}-15 \mathrm{~J} 2$ & 285 & 0 & $\mathrm{D}$ & $4 \mathrm{~N} / 27 \mathrm{~W}-15 \mathrm{Q} 1$ & 45 & 0 & $\mathrm{D}$ \\
\hline $4 \mathrm{~N} / 27 \mathrm{~W}-15 \mathrm{~J} 2$ & 295 & 0 & $\mathrm{D}$ & $4 \mathrm{~N} / 27 \mathrm{~W}-15 \mathrm{Q} 1$ & 55 & 0 & $\mathrm{D}$ \\
\hline $4 \mathrm{~N} / 27 \mathrm{~W}-15 \mathrm{~J} 2$ & 305 & 0 & $\mathrm{D}$ & $4 \mathrm{~N} / 27 \mathrm{~W}-15 \mathrm{Q} 1$ & 65 & 0 & $\mathrm{D}$ \\
\hline $4 \mathrm{~N} / 27 \mathrm{~W}-15 \mathrm{~J} 2$ & 315 & 0 & $\mathrm{D}$ & $4 \mathrm{~N} / 27 \mathrm{~W}-15 \mathrm{Q} 1$ & 75 & 0 & $\mathrm{D}$ \\
\hline $4 \mathrm{~N} / 27 \mathrm{~W}-15 \mathrm{~J} 2$ & 325 & 30 & $\mathrm{D}$ & 4N/27W-15Q1 & 85 & 40 & $\mathrm{D}$ \\
\hline $4 \mathrm{~N} / 27 \mathrm{~W}-15 \mathrm{~J} 2$ & 335 & 0 & $\mathrm{D}$ & $4 \mathrm{~N} / 27 \mathrm{~W}-15 \mathrm{Q} 1$ & 95 & 22.2 & $\mathrm{D}$ \\
\hline $4 \mathrm{~N} / 27 \mathrm{~W}-15 \mathrm{~J} 2$ & 345 & 0 & $\mathrm{D}$ & $4 \mathrm{~N} / 27 \mathrm{~W}-15 \mathrm{Q} 1$ & 105 & 63.6 & $\mathrm{D}$ \\
\hline
\end{tabular}


Table B1-1. Calculated percent-coarse intervals for all drillers' logs and e-logs used to define textural properties of the hydrogeologic framework model.-Continued

[Percent-coarse values are calculated for 10-foot intervals for the entire depth of each borehole. State well number is the state of California well identifier; interval midpoint depth is the midpoint of each 10-foot interval, in feet from top of borehole; PC is the percent-coarse value for each interval; log type defines whether the borehole is a drillers' lithology log (D) or a geophysical e-log (E).]

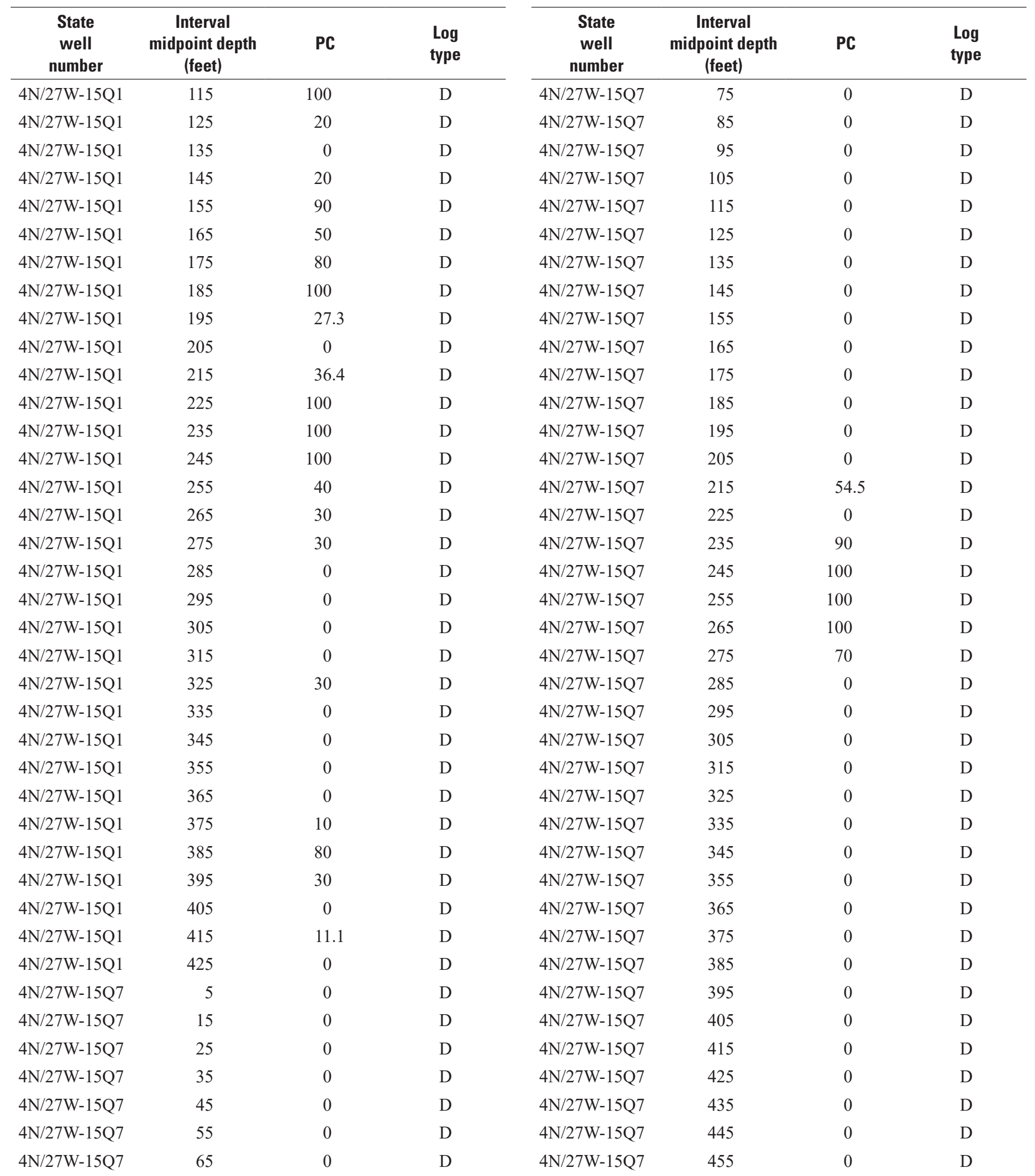


Table B1-1. Calculated percent-coarse intervals for all drillers' logs and e-logs used to define textural properties of the hydrogeologic framework model.-Continued

[Percent-coarse values are calculated for 10-foot intervals for the entire depth of each borehole. State well number is the state of California well identifier; interval midpoint depth is the midpoint of each 10-foot interval, in feet from top of borehole; PC is the percent-coarse value for each interval; log type defines whether the borehole is a drillers' lithology $\log (\mathrm{D})$ or a geophysical e-log (E).]

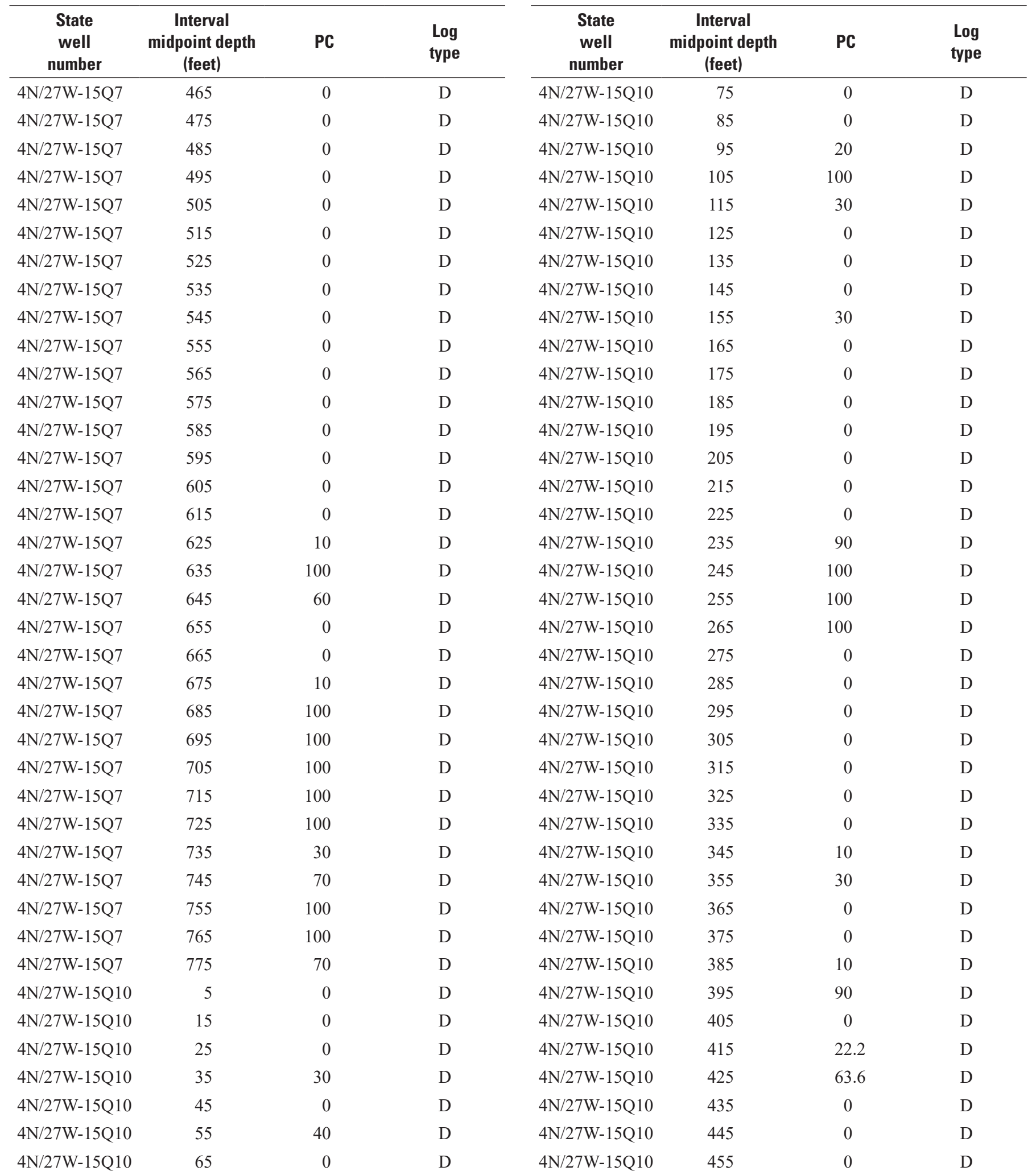


Table B1-1. Calculated percent-coarse intervals for all drillers' logs and e-logs used to define textural properties of the hydrogeologic framework model.-Continued

[Percent-coarse values are calculated for 10-foot intervals for the entire depth of each borehole. State well number is the state of California well identifier; interval midpoint depth is the midpoint of each 10-foot interval, in feet from top of borehole; PC is the percent-coarse value for each interval; log type defines whether the borehole is a drillers' lithology $\log (\mathrm{D})$ or a geophysical e-log (E).]

\begin{tabular}{|c|c|c|c|c|c|c|c|}
\hline $\begin{array}{l}\text { State } \\
\text { well } \\
\text { number }\end{array}$ & $\begin{array}{c}\text { Interval } \\
\text { midpoint depth } \\
\text { (feet) }\end{array}$ & PC & $\begin{array}{l}\text { Log } \\
\text { type }\end{array}$ & $\begin{array}{l}\text { State } \\
\text { well } \\
\text { number }\end{array}$ & $\begin{array}{c}\text { Interval } \\
\text { midpoint depth } \\
\text { (feet) }\end{array}$ & PC & $\begin{array}{l}\text { Log } \\
\text { type }\end{array}$ \\
\hline 4N/27W-15Q10 & 465 & 10 & $\mathrm{D}$ & 4N/27W-15R1 & 175 & 100 & $\mathrm{D}$ \\
\hline 4N/27W-15Q10 & 475 & 40 & $\mathrm{D}$ & 4N/27W-15R1 & 185 & 100 & $\mathrm{D}$ \\
\hline $4 \mathrm{~N} / 27 \mathrm{~W}-15 \mathrm{Q} 10$ & 485 & 0 & D & $4 \mathrm{~N} / 27 \mathrm{~W}-15 \mathrm{R} 1$ & 195 & 100 & $\mathrm{D}$ \\
\hline 4N/27W-15Q10 & 495 & 0 & $\mathrm{D}$ & $4 \mathrm{~N} / 27 \mathrm{~W}-15 \mathrm{R} 1$ & 205 & 100 & $\mathrm{D}$ \\
\hline 4N/27W-15Q10 & 505 & 0 & D & $4 \mathrm{~N} / 27 \mathrm{~W}-15 \mathrm{R} 1$ & 215 & 100 & $\mathrm{D}$ \\
\hline $4 \mathrm{~N} / 27 \mathrm{~W}-15 \mathrm{Q} 10$ & 515 & 0 & D & $4 \mathrm{~N} / 27 \mathrm{~W}-15 \mathrm{R} 1$ & 225 & 100 & $\mathrm{D}$ \\
\hline 4N/27W-15Q10 & 525 & 0 & D & $4 \mathrm{~N} / 27 \mathrm{~W}-15 \mathrm{R} 1$ & 235 & 100 & $\mathrm{D}$ \\
\hline 4N/27W-15Q10 & 535 & 0 & $\mathrm{D}$ & $4 \mathrm{~N} / 27 \mathrm{~W}-15 \mathrm{R} 1$ & 245 & 100 & $\mathrm{D}$ \\
\hline 4N/27W-15Q10 & 545 & 0 & $\mathrm{D}$ & $4 \mathrm{~N} / 27 \mathrm{~W}-15 \mathrm{R} 1$ & 255 & 100 & $\mathrm{D}$ \\
\hline $4 \mathrm{~N} / 27 \mathrm{~W}-15 \mathrm{Q} 10$ & 555 & 0 & D & $4 \mathrm{~N} / 27 \mathrm{~W}-15 \mathrm{R} 1$ & 265 & 100 & $\mathrm{D}$ \\
\hline 4N/27W-15Q10 & 565 & 0 & $\mathrm{D}$ & $4 \mathrm{~N} / 27 \mathrm{~W}-15 \mathrm{R} 1$ & 275 & 100 & $\mathrm{D}$ \\
\hline 4N/27W-15Q10 & 575 & 0 & $\mathrm{D}$ & $4 \mathrm{~N} / 27 \mathrm{~W}-15 \mathrm{R} 1$ & 285 & 100 & $\mathrm{D}$ \\
\hline 4N/27W-15Q10 & 585 & 0 & $\mathrm{D}$ & $4 \mathrm{~N} / 27 \mathrm{~W}-15 \mathrm{R} 1$ & 295 & 100 & $\mathrm{D}$ \\
\hline 4N/27W-15Q10 & 595 & 0 & $\mathrm{D}$ & $4 \mathrm{~N} / 27 \mathrm{~W}-15 \mathrm{R} 1$ & 305 & 100 & $\mathrm{D}$ \\
\hline 4N/27W-15Q10 & 605 & 0 & $\mathrm{D}$ & $4 \mathrm{~N} / 27 \mathrm{~W}-15 \mathrm{R} 1$ & 315 & 100 & $\mathrm{D}$ \\
\hline $4 \mathrm{~N} / 27 \mathrm{~W}-15 \mathrm{Q} 10$ & 615 & 30 & D & $4 \mathrm{~N} / 27 \mathrm{~W}-15 \mathrm{R} 1$ & 325 & 100 & $\mathrm{D}$ \\
\hline $4 \mathrm{~N} / 27 \mathrm{~W}-15 \mathrm{Q} 10$ & 625 & 100 & D & $4 \mathrm{~N} / 27 \mathrm{~W}-15 \mathrm{R} 1$ & 335 & 100 & $\mathrm{D}$ \\
\hline 4N/27W-15Q10 & 635 & 60 & $\mathrm{D}$ & $4 \mathrm{~N} / 27 \mathrm{~W}-15 \mathrm{R} 1$ & 345 & 100 & $\mathrm{D}$ \\
\hline 4N/27W-15Q10 & 645 & 100 & $\mathrm{D}$ & $4 \mathrm{~N} / 27 \mathrm{~W}-15 \mathrm{R} 1$ & 355 & 100 & $\mathrm{D}$ \\
\hline $4 \mathrm{~N} / 27 \mathrm{~W}-15 \mathrm{Q} 10$ & 655 & 40 & D & $4 \mathrm{~N} / 27 \mathrm{~W}-15 \mathrm{R} 1$ & 365 & 10 & $\mathrm{D}$ \\
\hline 4N/27W-15Q10 & 665 & 50 & $\mathrm{D}$ & $4 \mathrm{~N} / 27 \mathrm{~W}-15 \mathrm{R} 1$ & 375 & 0 & $\mathrm{D}$ \\
\hline $4 \mathrm{~N} / 27 \mathrm{~W}-15 \mathrm{Q} 10$ & 675 & 0 & D & $4 \mathrm{~N} / 27 \mathrm{~W}-15 \mathrm{R} 1$ & 385 & 0 & $\mathrm{D}$ \\
\hline $4 \mathrm{~N} / 27 \mathrm{~W}-15 \mathrm{R} 1$ & 5 & 100 & D & $4 \mathrm{~N} / 27 \mathrm{~W}-15 \mathrm{R} 1$ & 395 & 0 & $\mathrm{D}$ \\
\hline 4N/27W-15R1 & 15 & 100 & $\mathrm{D}$ & $4 \mathrm{~N} / 27 \mathrm{~W}-15 \mathrm{R} 1$ & 405 & 0 & $\mathrm{D}$ \\
\hline 4N/27W-15R1 & 25 & 100 & D & $4 \mathrm{~N} / 27 \mathrm{~W}-15 \mathrm{R} 1$ & 415 & 88.9 & $\mathrm{D}$ \\
\hline 4N/27W-15R1 & 35 & 100 & D & $4 \mathrm{~N} / 27 \mathrm{~W}-15 \mathrm{R} 1$ & 425 & 100 & $\mathrm{D}$ \\
\hline 4N/27W-15R1 & 45 & 100 & D & $4 \mathrm{~N} / 27 \mathrm{~W}-15 \mathrm{R} 1$ & 435 & 100 & $\mathrm{D}$ \\
\hline 4N/27W-15R1 & 55 & 100 & D & $4 \mathrm{~N} / 27 \mathrm{~W}-15 \mathrm{R} 1$ & 445 & 100 & $\mathrm{D}$ \\
\hline 4N/27W-15R1 & 65 & 100 & D & $4 \mathrm{~N} / 27 \mathrm{~W}-15 \mathrm{R} 1$ & 455 & 100 & $\mathrm{D}$ \\
\hline 4N/27W-15R1 & 75 & 100 & D & $4 \mathrm{~N} / 27 \mathrm{~W}-15 \mathrm{R} 1$ & 465 & 100 & $\mathrm{D}$ \\
\hline 4N/27W-15R1 & 85 & 100 & $\mathrm{D}$ & $4 \mathrm{~N} / 27 \mathrm{~W}-15 \mathrm{R} 1$ & 475 & 100 & $\mathrm{D}$ \\
\hline 4N/27W-15R1 & 95 & 100 & $\mathrm{D}$ & $4 \mathrm{~N} / 27 \mathrm{~W}-15 \mathrm{R} 1$ & 485 & 100 & $\mathrm{D}$ \\
\hline 4N/27W-15R1 & 105 & 100 & $\mathrm{D}$ & $4 \mathrm{~N} / 27 \mathrm{~W}-15 \mathrm{R} 1$ & 495 & 100 & $\mathrm{D}$ \\
\hline 4N/27W-15R1 & 115 & 100 & D & $4 \mathrm{~N} / 27 \mathrm{~W}-15 \mathrm{R} 1$ & 505 & 100 & $\mathrm{D}$ \\
\hline 4N/27W-15R1 & 125 & 100 & D & $4 \mathrm{~N} / 27 \mathrm{~W}-15 \mathrm{R} 1$ & 515 & 100 & $\mathrm{D}$ \\
\hline 4N/27W-15R1 & 135 & 100 & D & $4 \mathrm{~N} / 27 \mathrm{~W}-15 \mathrm{R} 1$ & 525 & 100 & $\mathrm{D}$ \\
\hline 4N/27W-15R1 & 145 & 100 & D & $4 \mathrm{~N} / 27 \mathrm{~W}-15 \mathrm{R} 1$ & 535 & 100 & $\mathrm{D}$ \\
\hline 4N/27W-15R1 & 155 & 100 & D & $4 \mathrm{~N} / 27 \mathrm{~W}-15 \mathrm{R} 1$ & 545 & 100 & $\mathrm{D}$ \\
\hline 4N/27W-15R1 & 165 & 100 & $\mathrm{D}$ & $4 \mathrm{~N} / 27 \mathrm{~W}-15 \mathrm{R} 1$ & 555 & 100 & $\mathrm{D}$ \\
\hline
\end{tabular}


Table B1-1. Calculated percent-coarse intervals for all drillers' logs and e-logs used to define textural properties of the hydrogeologic framework model.-Continued

[Percent-coarse values are calculated for 10-foot intervals for the entire depth of each borehole. State well number is the state of California well identifier; interval midpoint depth is the midpoint of each 10-foot interval, in feet from top of borehole; PC is the percent-coarse value for each interval; log type defines whether the borehole is a drillers' lithology $\log (\mathrm{D})$ or a geophysical e-log (E).]

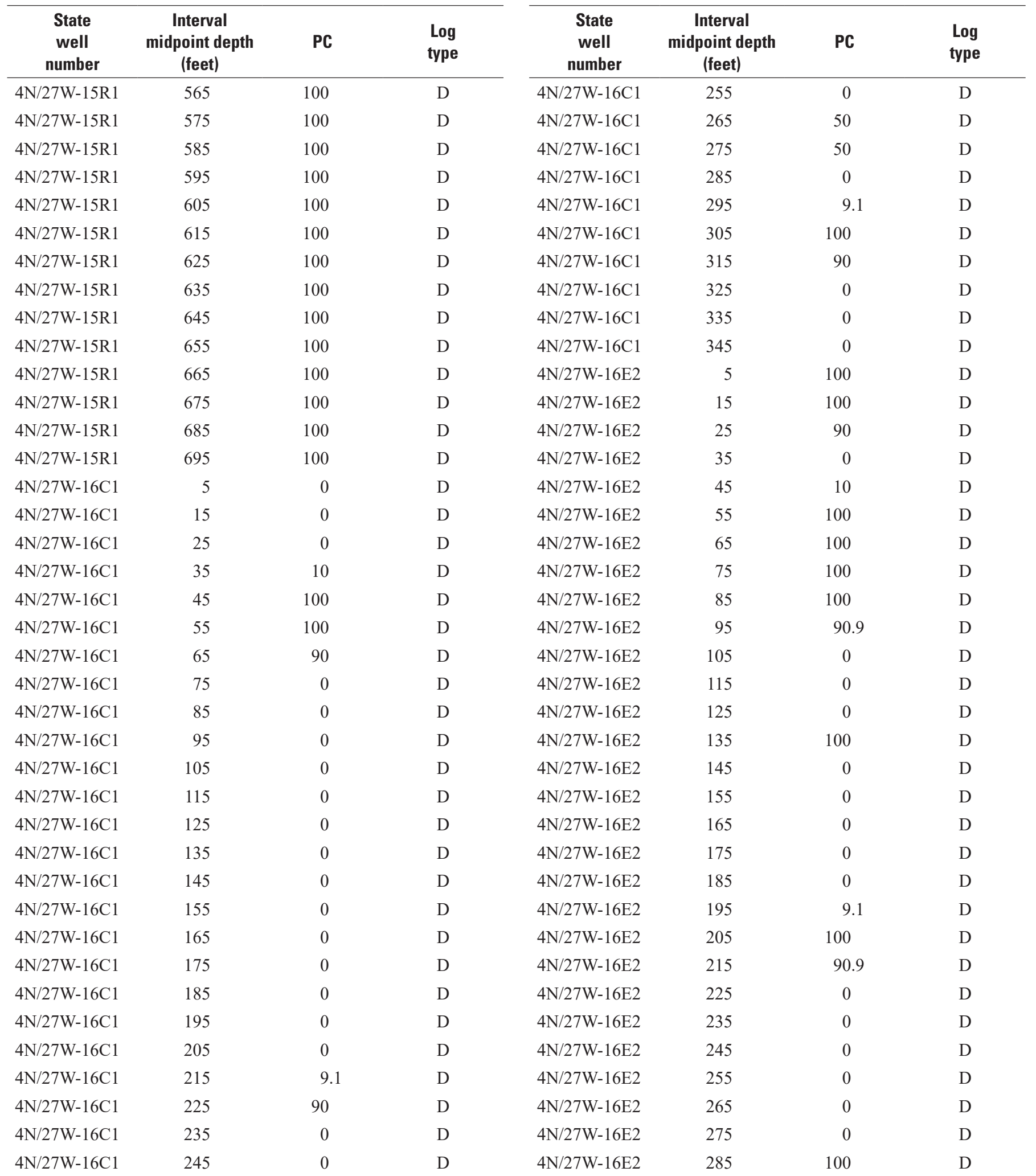


Table B1-1. Calculated percent-coarse intervals for all drillers' logs and e-logs used to define textural properties of the hydrogeologic framework model.-Continued

[Percent-coarse values are calculated for 10-foot intervals for the entire depth of each borehole. State well number is the state of California well identifier; interval midpoint depth is the midpoint of each 10-foot interval, in feet from top of borehole; PC is the percent-coarse value for each interval; log type defines whether the borehole is a drillers' lithology log (D) or a geophysical e-log (E).]

\begin{tabular}{|c|c|c|c|c|c|c|c|}
\hline $\begin{array}{c}\text { State } \\
\text { well } \\
\text { number }\end{array}$ & $\begin{array}{c}\text { Interval } \\
\text { midpoint depth } \\
\text { (feet) }\end{array}$ & PC & $\begin{array}{l}\text { Log } \\
\text { type }\end{array}$ & $\begin{array}{c}\text { State } \\
\text { well } \\
\text { number }\end{array}$ & $\begin{array}{c}\text { Interval } \\
\text { midpoint depth } \\
\text { (feet) }\end{array}$ & PC & $\begin{array}{l}\text { Log } \\
\text { type }\end{array}$ \\
\hline $4 \mathrm{~N} / 27 \mathrm{~W}-16 \mathrm{E} 2$ & 295 & 100 & $\mathrm{D}$ & $4 \mathrm{~N} / 27 \mathrm{~W}-16 \mathrm{M} 1$ & 255 & 0 & $\mathrm{D}$ \\
\hline $4 \mathrm{~N} / 27 \mathrm{~W}-16 \mathrm{E} 2$ & 305 & 100 & $\mathrm{D}$ & $4 \mathrm{~N} / 27 \mathrm{~W}-16 \mathrm{M} 1$ & 265 & 0 & $\mathrm{D}$ \\
\hline $4 \mathrm{~N} / 27 \mathrm{~W}-16 \mathrm{E} 2$ & 315 & 90 & $\mathrm{D}$ & $4 \mathrm{~N} / 27 \mathrm{~W}-16 \mathrm{M} 1$ & 275 & 0 & $\mathrm{D}$ \\
\hline $4 \mathrm{~N} / 27 \mathrm{~W}-16 \mathrm{E} 2$ & 325 & 10 & $\mathrm{D}$ & $4 \mathrm{~N} / 27 \mathrm{~W}-16 \mathrm{M} 1$ & 285 & 20 & $\mathrm{D}$ \\
\hline $4 \mathrm{~N} / 27 \mathrm{~W}-16 \mathrm{E} 2$ & 345 & 0 & $\mathrm{D}$ & $4 \mathrm{~N} / 27 \mathrm{~W}-16 \mathrm{M} 1$ & 305 & 0 & $\mathrm{D}$ \\
\hline $4 \mathrm{~N} / 27 \mathrm{~W}-16 \mathrm{E} 2$ & 355 & 0 & $\mathrm{D}$ & $4 \mathrm{~N} / 27 \mathrm{~W}-16 \mathrm{M} 1$ & 315 & 0 & $\mathrm{D}$ \\
\hline $4 \mathrm{~N} / 27 \mathrm{~W}-16 \mathrm{E} 2$ & 365 & 0 & $\mathrm{D}$ & $4 \mathrm{~N} / 27 \mathrm{~W}-16 \mathrm{M} 1$ & 325 & 0 & $\mathrm{D}$ \\
\hline $4 \mathrm{~N} / 27 \mathrm{~W}-16 \mathrm{E} 2$ & 375 & 0 & $\mathrm{D}$ & $4 \mathrm{~N} / 27 \mathrm{~W}-16 \mathrm{M} 1$ & 335 & 0 & $\mathrm{D}$ \\
\hline $4 \mathrm{~N} / 27 \mathrm{~W}-16 \mathrm{E} 2$ & 415 & 0 & $\mathrm{D}$ & $4 \mathrm{~N} / 27 \mathrm{~W}-16 \mathrm{P} 1$ & 15 & 100 & $\mathrm{D}$ \\
\hline $4 \mathrm{~N} / 27 \mathrm{~W}-16 \mathrm{E} 2$ & 425 & 0 & $\mathrm{D}$ & $4 \mathrm{~N} / 27 \mathrm{~W}-16 \mathrm{P} 1$ & 25 & 70 & $\mathrm{D}$ \\
\hline $4 \mathrm{~N} / 27 \mathrm{~W}-16 \mathrm{M} 1$ & 5 & 100 & $\mathrm{D}$ & $4 \mathrm{~N} / 27 \mathrm{~W}-16 \mathrm{P} 1$ & 35 & 0 & $\mathrm{D}$ \\
\hline 4N/27W-16M1 & 15 & 50 & $\mathrm{D}$ & $4 \mathrm{~N} / 27 \mathrm{~W}-16 \mathrm{P} 1$ & 45 & 0 & $\mathrm{D}$ \\
\hline $4 \mathrm{~N} / 27 \mathrm{~W}-16 \mathrm{M} 1$ & 25 & 30 & $\mathrm{D}$ & $4 \mathrm{~N} / 27 \mathrm{~W}-16 \mathrm{P} 1$ & 55 & 0 & $\mathrm{D}$ \\
\hline $4 \mathrm{~N} / 27 \mathrm{~W}-16 \mathrm{M} 1$ & 35 & 0 & $\mathrm{D}$ & $4 \mathrm{~N} / 27 \mathrm{~W}-16 \mathrm{P} 1$ & 65 & 0 & $\mathrm{D}$ \\
\hline $4 \mathrm{~N} / 27 \mathrm{~W}-16 \mathrm{M} 1$ & 45 & 0 & $\mathrm{D}$ & $4 \mathrm{~N} / 27 \mathrm{~W}-16 \mathrm{P} 1$ & 75 & 54.5 & $\mathrm{D}$ \\
\hline $4 \mathrm{~N} / 27 \mathrm{~W}-16 \mathrm{M} 1$ & 55 & 0 & $\mathrm{D}$ & $4 \mathrm{~N} / 27 \mathrm{~W}-16 \mathrm{P} 1$ & 85 & 0 & $\mathrm{D}$ \\
\hline $4 \mathrm{~N} / 27 \mathrm{~W}-16 \mathrm{M} 1$ & 65 & 0 & $\mathrm{D}$ & $4 \mathrm{~N} / 27 \mathrm{~W}-16 \mathrm{P} 1$ & 95 & 0 & $\mathrm{D}$ \\
\hline $4 \mathrm{~N} / 27 \mathrm{~W}-16 \mathrm{M} 1$ & 135 & 0 & $\mathrm{D}$ & $4 \mathrm{~N} / 27 \mathrm{~W}-16 \mathrm{P} 1$ & 165 & 0 & $\mathrm{D}$ \\
\hline $4 \mathrm{~N} / 27 \mathrm{~W}-16 \mathrm{M} 1$ & 145 & 0 & $\mathrm{D}$ & $4 \mathrm{~N} / 27 \mathrm{~W}-16 \mathrm{P} 1$ & 175 & 0 & $\mathrm{D}$ \\
\hline $4 \mathrm{~N} / 27 \mathrm{~W}-16 \mathrm{M} 1$ & 155 & 30 & $\mathrm{D}$ & $4 \mathrm{~N} / 27 \mathrm{~W}-16 \mathrm{P} 1$ & 185 & 40 & $\mathrm{D}$ \\
\hline $4 \mathrm{~N} / 27 \mathrm{~W}-16 \mathrm{M} 1$ & 165 & 0 & $\mathrm{D}$ & $4 \mathrm{~N} / 27 \mathrm{~W}-16 \mathrm{P} 1$ & 195 & 36.4 & $\mathrm{D}$ \\
\hline $4 \mathrm{~N} / 27 \mathrm{~W}-16 \mathrm{M} 1$ & 175 & 0 & $\mathrm{D}$ & $4 \mathrm{~N} / 27 \mathrm{~W}-16 \mathrm{P} 1$ & 205 & 0 & $\mathrm{D}$ \\
\hline $4 \mathrm{~N} / 27 \mathrm{~W}-16 \mathrm{M} 1$ & 185 & 0 & $\mathrm{D}$ & $4 \mathrm{~N} / 27 \mathrm{~W}-16 \mathrm{P} 1$ & 215 & 0 & $\mathrm{D}$ \\
\hline $4 \mathrm{~N} / 27 \mathrm{~W}-16 \mathrm{M} 1$ & 195 & 0 & $\mathrm{D}$ & $4 \mathrm{~N} / 27 \mathrm{~W}-16 \mathrm{P} 1$ & 225 & 40 & $\mathrm{D}$ \\
\hline $4 \mathrm{~N} / 27 \mathrm{~W}-16 \mathrm{M} 1$ & 205 & 0 & $\mathrm{D}$ & $4 \mathrm{~N} / 27 \mathrm{~W}-16 \mathrm{P} 1$ & 235 & 0 & $\mathrm{D}$ \\
\hline $4 \mathrm{~N} / 27 \mathrm{~W}-16 \mathrm{M} 1$ & 215 & 36.4 & $\mathrm{D}$ & $4 \mathrm{~N} / 27 \mathrm{~W}-16 \mathrm{P} 1$ & 245 & 0 & $\mathrm{D}$ \\
\hline $4 \mathrm{~N} / 27 \mathrm{~W}-16 \mathrm{M} 1$ & 225 & 0 & $\mathrm{D}$ & $4 \mathrm{~N} / 27 \mathrm{~W}-16 \mathrm{P} 1$ & 255 & 0 & $\mathrm{D}$ \\
\hline $4 \mathrm{~N} / 27 \mathrm{~W}-16 \mathrm{M} 1$ & 235 & 0 & $\mathrm{D}$ & $4 \mathrm{~N} / 27 \mathrm{~W}-16 \mathrm{P} 1$ & 265 & 0 & $\mathrm{D}$ \\
\hline $4 \mathrm{~N} / 27 \mathrm{~W}-16 \mathrm{M} 1$ & 245 & 0 & $\mathrm{D}$ & $4 \mathrm{~N} / 27 \mathrm{~W}-16 \mathrm{P} 1$ & 275 & 0 & $\mathrm{D}$ \\
\hline
\end{tabular}


Table B1-1. Calculated percent-coarse intervals for all drillers' logs and e-logs used to define textural properties of the hydrogeologic framework model.-Continued

[Percent-coarse values are calculated for 10-foot intervals for the entire depth of each borehole. State well number is the state of California well identifier; interval midpoint depth is the midpoint of each 10-foot interval, in feet from top of borehole; PC is the percent-coarse value for each interval; log type defines whether the borehole is a drillers' lithology $\log (\mathrm{D})$ or a geophysical e-log (E).]

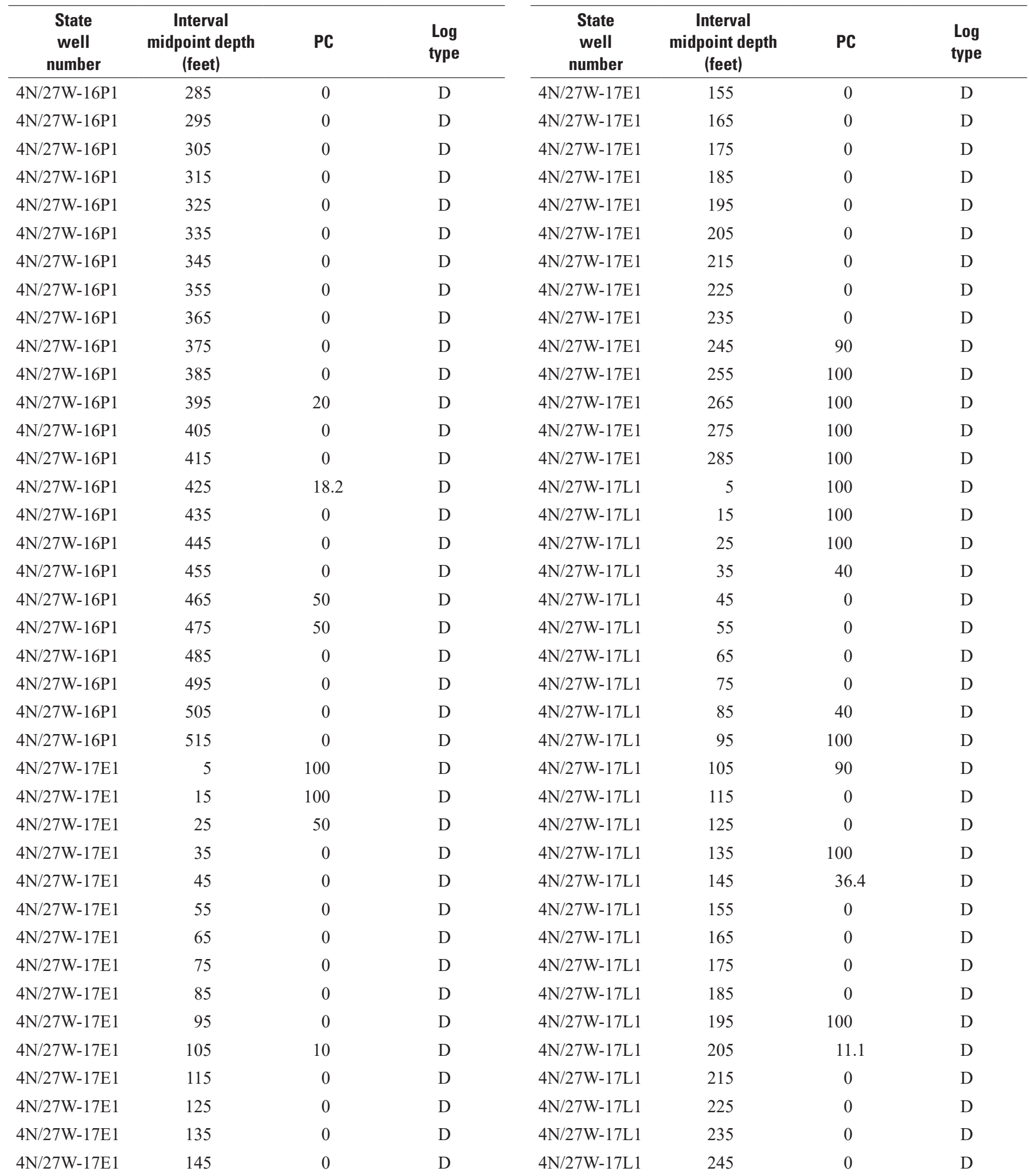


Table B1-1. Calculated percent-coarse intervals for all drillers' logs and e-logs used to define textural properties of the hydrogeologic framework model.-Continued

[Percent-coarse values are calculated for 10-foot intervals for the entire depth of each borehole. State well number is the state of California well identifier; interval midpoint depth is the midpoint of each 10-foot interval, in feet from top of borehole; PC is the percent-coarse value for each interval; log type defines whether the borehole is a drillers' lithology log (D) or a geophysical e-log (E).]

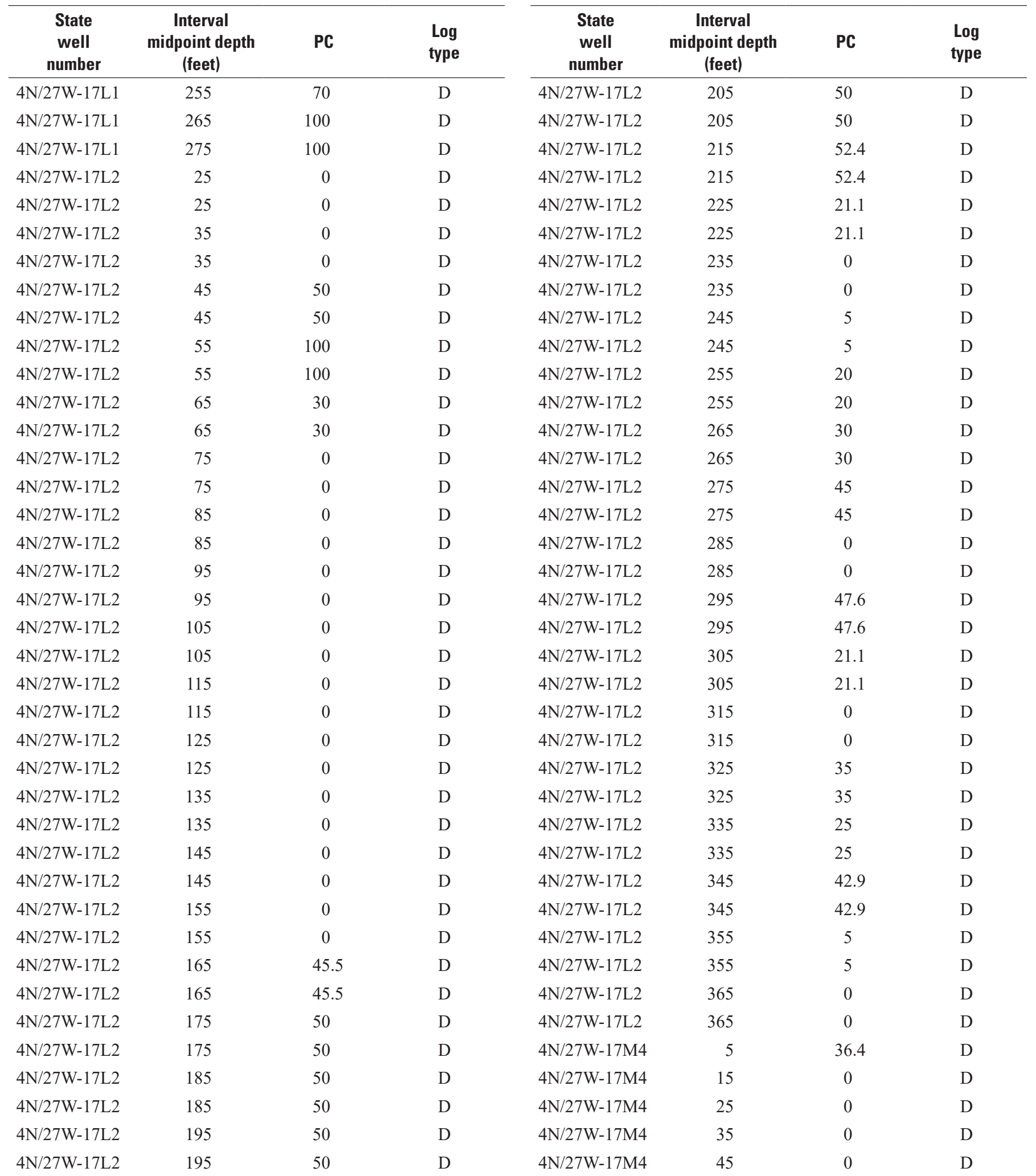


Table B1-1. Calculated percent-coarse intervals for all drillers' logs and e-logs used to define textural properties of the hydrogeologic framework model.-Continued

[Percent-coarse values are calculated for 10-foot intervals for the entire depth of each borehole. State well number is the state of California well identifier; interval midpoint depth is the midpoint of each 10-foot interval, in feet from top of borehole; PC is the percent-coarse value for each interval; log type defines whether the borehole is a drillers' lithology $\log (\mathrm{D})$ or a geophysical e-log (E).]

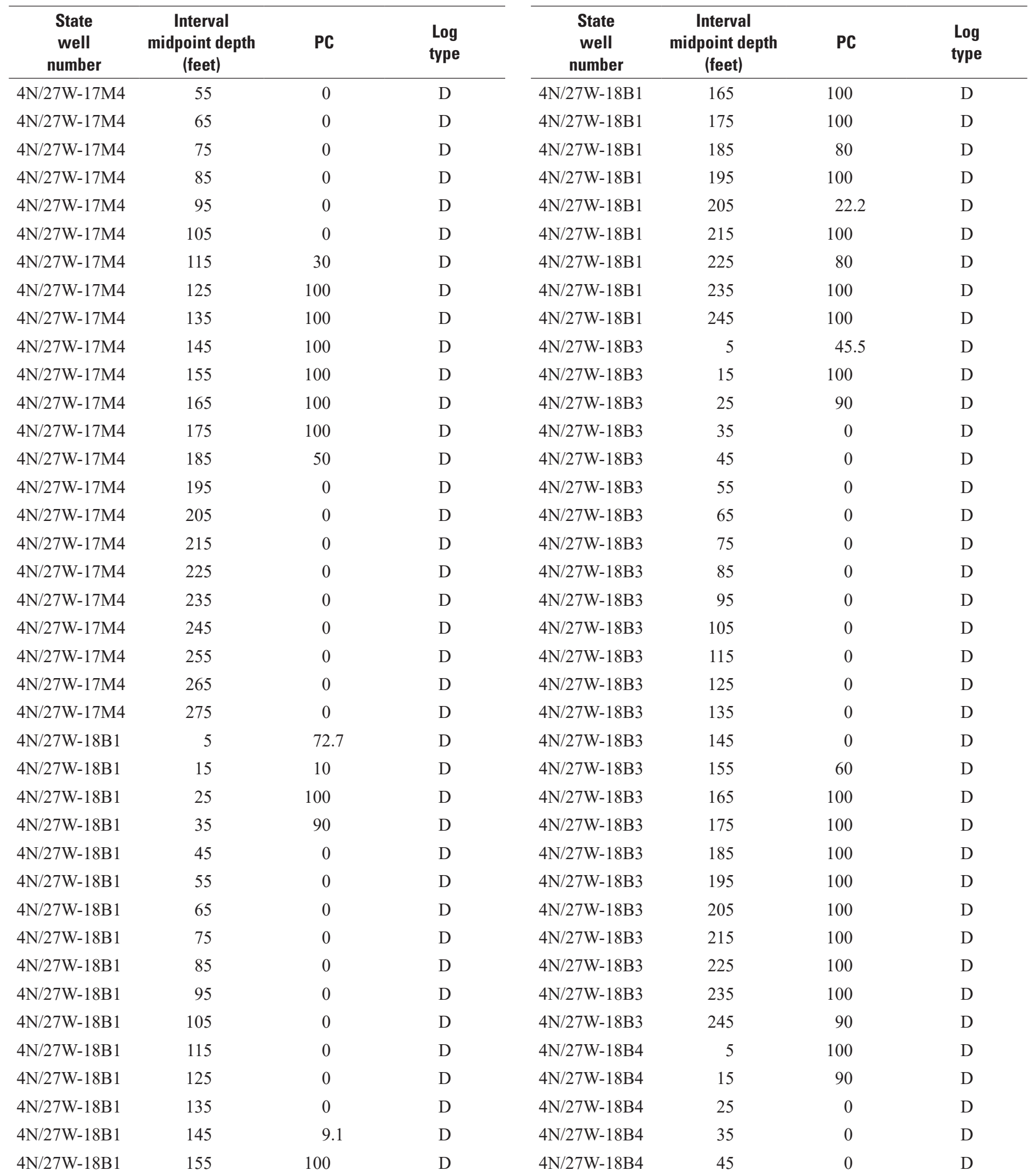


Table B1-1. Calculated percent-coarse intervals for all drillers' logs and e-logs used to define textural properties of the hydrogeologic framework model.-Continued

[Percent-coarse values are calculated for 10-foot intervals for the entire depth of each borehole. State well number is the state of California well identifier; interval midpoint depth is the midpoint of each 10-foot interval, in feet from top of borehole; PC is the percent-coarse value for each interval; log type defines whether the borehole is a drillers' lithology $\log (\mathrm{D})$ or a geophysical e-log (E).]

\begin{tabular}{|c|c|c|c|c|c|c|c|}
\hline $\begin{array}{c}\text { State } \\
\text { well } \\
\text { number }\end{array}$ & $\begin{array}{c}\text { Interval } \\
\text { midpoint depth } \\
\text { (feet) }\end{array}$ & PC & $\begin{array}{l}\text { Log } \\
\text { type }\end{array}$ & $\begin{array}{c}\text { State } \\
\text { well } \\
\text { number }\end{array}$ & $\begin{array}{c}\text { Interval } \\
\text { midpoint depth } \\
\text { (feet) }\end{array}$ & PC & $\begin{array}{l}\text { Log } \\
\text { type }\end{array}$ \\
\hline $4 \mathrm{~N} / 27 \mathrm{~W}-18 \mathrm{~B} 4$ & 55 & 0 & $\mathrm{D}$ & $4 \mathrm{~N} / 27 \mathrm{~W}-18 \mathrm{G} 1$ & 55 & 0 & $\mathrm{D}$ \\
\hline $4 \mathrm{~N} / 27 \mathrm{~W}-18 \mathrm{~B} 4$ & 65 & 0 & $\mathrm{D}$ & $4 \mathrm{~N} / 27 \mathrm{~W}-18 \mathrm{G} 1$ & 65 & 55.6 & $\mathrm{D}$ \\
\hline $4 \mathrm{~N} / 27 \mathrm{~W}-18 \mathrm{~B} 4$ & 75 & 0 & $\mathrm{D}$ & $4 \mathrm{~N} / 27 \mathrm{~W}-18 \mathrm{G} 1$ & 75 & 9.1 & $\mathrm{D}$ \\
\hline $4 \mathrm{~N} / 27 \mathrm{~W}-18 \mathrm{~B} 4$ & 85 & 0 & $\mathrm{D}$ & $4 \mathrm{~N} / 27 \mathrm{~W}-18 \mathrm{G} 1$ & 85 & 0 & $\mathrm{D}$ \\
\hline $4 \mathrm{~N} / 27 \mathrm{~W}-18 \mathrm{~B} 4$ & 105 & 100 & $\mathrm{D}$ & $4 \mathrm{~N} / 27 \mathrm{~W}-18 \mathrm{G} 1$ & 105 & 0 & $\mathrm{D}$ \\
\hline $4 \mathrm{~N} / 27 \mathrm{~W}-18 \mathrm{~B} 4$ & 115 & 100 & $\mathrm{D}$ & $4 \mathrm{~N} / 27 \mathrm{~W}-18 \mathrm{G} 1$ & 115 & 0 & $\mathrm{D}$ \\
\hline $4 \mathrm{~N} / 27 \mathrm{~W}-18 \mathrm{~B} 4$ & 125 & 100 & $\mathrm{D}$ & $4 \mathrm{~N} / 27 \mathrm{~W}-18 \mathrm{G} 1$ & 125 & 22.2 & $\mathrm{D}$ \\
\hline $4 \mathrm{~N} / 27 \mathrm{~W}-18 \mathrm{~B} 4$ & 135 & 100 & $\mathrm{D}$ & $4 \mathrm{~N} / 27 \mathrm{~W}-18 \mathrm{G} 1$ & 135 & 100 & $\mathrm{D}$ \\
\hline $4 \mathrm{~N} / 27 \mathrm{~W}-18 \mathrm{~B} 4$ & 175 & 100 & $\mathrm{D}$ & $4 \mathrm{~N} / 27 \mathrm{~W}-18 \mathrm{H} 1$ & 15 & 10 & $\mathrm{D}$ \\
\hline $4 \mathrm{~N} / 27 \mathrm{~W}-18 \mathrm{~B} 4$ & 185 & 100 & $\mathrm{D}$ & $4 \mathrm{~N} / 27 \mathrm{~W}-18 \mathrm{H} 1$ & 25 & 0 & $\mathrm{D}$ \\
\hline $4 \mathrm{~N} / 27 \mathrm{~W}-18 \mathrm{~B} 4$ & 195 & 90.9 & $\mathrm{D}$ & $4 \mathrm{~N} / 27 \mathrm{~W}-18 \mathrm{H} 1$ & 35 & 0 & $\mathrm{D}$ \\
\hline $4 \mathrm{~N} / 27 \mathrm{~W}-18 \mathrm{~B} 4$ & 205 & 0 & $\mathrm{D}$ & $4 \mathrm{~N} / 27 \mathrm{~W}-18 \mathrm{H} 1$ & 45 & 0 & $\mathrm{D}$ \\
\hline $4 \mathrm{~N} / 27 \mathrm{~W}-18 \mathrm{~B} 4$ & 215 & 0 & $\mathrm{D}$ & $4 \mathrm{~N} / 27 \mathrm{~W}-18 \mathrm{H} 1$ & 55 & 0 & $\mathrm{D}$ \\
\hline $4 \mathrm{~N} / 27 \mathrm{~W}-18 \mathrm{~B} 4$ & 225 & 0 & $\mathrm{D}$ & $4 \mathrm{~N} / 27 \mathrm{~W}-18 \mathrm{H} 1$ & 65 & 33.3 & $\mathrm{D}$ \\
\hline $4 \mathrm{~N} / 27 \mathrm{~W}-18 \mathrm{~B} 4$ & 235 & 0 & $\mathrm{D}$ & $4 \mathrm{~N} / 27 \mathrm{~W}-18 \mathrm{H} 1$ & 75 & 9.1 & $\mathrm{D}$ \\
\hline $4 \mathrm{~N} / 27 \mathrm{~W}-18 \mathrm{~B} 4$ & 245 & 0 & $\mathrm{D}$ & $4 \mathrm{~N} / 27 \mathrm{~W}-18 \mathrm{H} 1$ & 85 & 30 & $\mathrm{D}$ \\
\hline $4 \mathrm{~N} / 27 \mathrm{~W}-18 \mathrm{C} 1$ & 25 & 0 & $\mathrm{D}$ & $4 \mathrm{~N} / 27 \mathrm{~W}-18 \mathrm{H} 1$ & 95 & 0 & $\mathrm{D}$ \\
\hline $4 \mathrm{~N} / 27 \mathrm{~W}-18 \mathrm{C} 1$ & 95 & 100 & $\mathrm{D}$ & $4 \mathrm{~N} / 27 \mathrm{~W}-18 \mathrm{H} 2$ & 5 & 0 & $\mathrm{D}$ \\
\hline $4 \mathrm{~N} / 27 \mathrm{~W}-18 \mathrm{C} 1$ & 105 & 100 & $\mathrm{D}$ & $4 \mathrm{~N} / 27 \mathrm{~W}-18 \mathrm{H} 2$ & 15 & 0 & $\mathrm{D}$ \\
\hline $4 \mathrm{~N} / 27 \mathrm{~W}-18 \mathrm{C} 1$ & 115 & 100 & $\mathrm{D}$ & $4 \mathrm{~N} / 27 \mathrm{~W}-18 \mathrm{H} 2$ & 25 & 0 & $\mathrm{D}$ \\
\hline $4 \mathrm{~N} / 27 \mathrm{~W}-18 \mathrm{C} 1$ & 125 & 100 & $\mathrm{D}$ & $4 \mathrm{~N} / 27 \mathrm{~W}-18 \mathrm{H} 2$ & 35 & 0 & $\mathrm{D}$ \\
\hline $4 \mathrm{~N} / 27 \mathrm{~W}-18 \mathrm{C} 1$ & 135 & 100 & $\mathrm{D}$ & $4 \mathrm{~N} / 27 \mathrm{~W}-18 \mathrm{H} 2$ & 45 & 60 & $\mathrm{D}$ \\
\hline $4 \mathrm{~N} / 27 \mathrm{~W}-18 \mathrm{C} 1$ & 145 & 100 & $\mathrm{D}$ & $4 \mathrm{~N} / 27 \mathrm{~W}-18 \mathrm{H} 2$ & 55 & 100 & $\mathrm{D}$ \\
\hline $4 \mathrm{~N} / 27 \mathrm{~W}-18 \mathrm{C} 1$ & 155 & 100 & $\mathrm{D}$ & $4 \mathrm{~N} / 27 \mathrm{~W}-18 \mathrm{H} 2$ & 65 & 100 & $\mathrm{D}$ \\
\hline 4N/27W-18G1 & 5 & 0 & $\mathrm{D}$ & $4 \mathrm{~N} / 27 \mathrm{~W}-18 \mathrm{H} 2$ & 75 & 100 & $\mathrm{D}$ \\
\hline $4 \mathrm{~N} / 27 \mathrm{~W}-18 \mathrm{G} 1$ & 15 & 0 & $\mathrm{D}$ & $4 \mathrm{~N} / 27 \mathrm{~W}-18 \mathrm{H} 2$ & 85 & 100 & $\mathrm{D}$ \\
\hline $4 \mathrm{~N} / 27 \mathrm{~W}-18 \mathrm{G} 1$ & 25 & 0 & $\mathrm{D}$ & $4 \mathrm{~N} / 27 \mathrm{~W}-18 \mathrm{H} 2$ & 95 & 100 & $\mathrm{D}$ \\
\hline $4 \mathrm{~N} / 27 \mathrm{~W}-18 \mathrm{G} 1$ & 35 & 0 & $\mathrm{D}$ & $4 \mathrm{~N} / 27 \mathrm{~W}-18 \mathrm{H} 2$ & 105 & 100 & $\mathrm{D}$ \\
\hline $4 \mathrm{~N} / 27 \mathrm{~W}-18 \mathrm{G} 1$ & 45 & 0 & $\mathrm{D}$ & $4 \mathrm{~N} / 27 \mathrm{~W}-18 \mathrm{H} 2$ & 115 & 90 & $\mathrm{D}$ \\
\hline
\end{tabular}


Table B1-1. Calculated percent-coarse intervals for all drillers' logs and e-logs used to define textural properties of the hydrogeologic framework model.-Continued

[Percent-coarse values are calculated for 10-foot intervals for the entire depth of each borehole. State well number is the state of California well identifier; interval midpoint depth is the midpoint of each 10-foot interval, in feet from top of borehole; PC is the percent-coarse value for each interval; log type defines whether the borehole is a drillers' lithology $\log (\mathrm{D})$ or a geophysical e-log (E).]

\begin{tabular}{|c|c|c|c|c|c|c|c|}
\hline $\begin{array}{c}\text { State } \\
\text { well } \\
\text { number }\end{array}$ & $\begin{array}{c}\text { Interval } \\
\text { midpoint depth } \\
\text { (feet) }\end{array}$ & PC & $\begin{array}{l}\text { Log } \\
\text { type }\end{array}$ & $\begin{array}{c}\text { State } \\
\text { well } \\
\text { number }\end{array}$ & $\begin{array}{c}\text { Interval } \\
\text { midpoint depth } \\
\text { (feet) }\end{array}$ & PC & $\begin{array}{l}\text { Log } \\
\text { type }\end{array}$ \\
\hline $4 \mathrm{~N} / 27 \mathrm{~W}-18 \mathrm{H} 2$ & 125 & 0 & $\mathrm{D}$ & $4 \mathrm{~N} / 27 \mathrm{~W}-18 \mathrm{~L} 1$ & 55 & 0 & $\mathrm{D}$ \\
\hline $4 \mathrm{~N} / 27 \mathrm{~W}-18 \mathrm{H} 2$ & 135 & 0 & $\mathrm{D}$ & $4 \mathrm{~N} / 27 \mathrm{~W}-18 \mathrm{~L} 1$ & 65 & 0 & $\mathrm{D}$ \\
\hline $4 \mathrm{~N} / 27 \mathrm{~W}-18 \mathrm{H} 2$ & 145 & 9.1 & $\mathrm{D}$ & $4 \mathrm{~N} / 27 \mathrm{~W}-18 \mathrm{~L} 1$ & 75 & 0 & $\mathrm{D}$ \\
\hline $4 \mathrm{~N} / 27 \mathrm{~W}-18 \mathrm{H} 2$ & 155 & 100 & $\mathrm{D}$ & $4 \mathrm{~N} / 27 \mathrm{~W}-18 \mathrm{~L} 1$ & 85 & 44.4 & $\mathrm{D}$ \\
\hline 4N/27W-18J1 & 5 & 0 & $\mathrm{D}$ & 4N/27W-18L1 & 105 & 100 & $\mathrm{D}$ \\
\hline $4 \mathrm{~N} / 27 \mathrm{~W}-18 \mathrm{~J} 1$ & 15 & 10 & $\mathrm{D}$ & $4 \mathrm{~N} / 27 \mathrm{~W}-18 \mathrm{~L} 1$ & 115 & 100 & $\mathrm{D}$ \\
\hline 4N/27W-18J1 & 25 & 100 & $\mathrm{D}$ & $4 \mathrm{~N} / 27 \mathrm{~W}-18 \mathrm{~L} 1$ & 125 & 100 & $\mathrm{D}$ \\
\hline $4 \mathrm{~N} / 27 \mathrm{~W}-18 \mathrm{~J} 1$ & 35 & 100 & $\mathrm{D}$ & $4 \mathrm{~N} / 27 \mathrm{~W}-18 \mathrm{~L} 1$ & 135 & 60 & $\mathrm{D}$ \\
\hline 4N/27W-18J1 & 75 & 10 & $\mathrm{D}$ & 4N/27W-18L1 & 175 & 10 & $\mathrm{D}$ \\
\hline $4 \mathrm{~N} / 27 \mathrm{~W}-18 \mathrm{~J} 1$ & 85 & 100 & $\mathrm{D}$ & $4 \mathrm{~N} / 27 \mathrm{~W}-18 \mathrm{~L} 1$ & 185 & 100 & $\mathrm{D}$ \\
\hline 4N/27W-18J1 & 95 & 100 & $\mathrm{D}$ & $4 \mathrm{~N} / 27 \mathrm{~W}-18 \mathrm{~L} 1$ & 195 & 0 & $\mathrm{D}$ \\
\hline 4N/27W-18J1 & 105 & 100 & $\mathrm{D}$ & $4 \mathrm{~N} / 27 \mathrm{~W}-18 \mathrm{~L} 1$ & 205 & 55.6 & $\mathrm{D}$ \\
\hline $4 \mathrm{~N} / 27 \mathrm{~W}-18 \mathrm{~J} 1$ & 115 & 80 & $\mathrm{D}$ & $4 \mathrm{~N} / 27 \mathrm{~W}-18 \mathrm{~L} 1$ & 215 & 100 & $\mathrm{D}$ \\
\hline $4 \mathrm{~N} / 27 \mathrm{~W}-18 \mathrm{~J} 1$ & 125 & 0 & $\mathrm{D}$ & $4 \mathrm{~N} / 27 \mathrm{~W}-18 \mathrm{~L} 1$ & 225 & 100 & $\mathrm{D}$ \\
\hline $4 \mathrm{~N} / 27 \mathrm{~W}-18 \mathrm{~J} 1$ & 135 & 100 & $\mathrm{D}$ & $4 \mathrm{~N} / 27 \mathrm{~W}-18 \mathrm{~L} 1$ & 235 & 60 & $\mathrm{D}$ \\
\hline 4N/27W-18J1 & 145 & 90 & $\mathrm{D}$ & $4 \mathrm{~N} / 27 \mathrm{~W}-18 \mathrm{P} 2$ & 5 & 0 & $\mathrm{D}$ \\
\hline 4N/27W-18J1 & 155 & 0 & $\mathrm{D}$ & $4 \mathrm{~N} / 27 \mathrm{~W}-18 \mathrm{P} 2$ & 15 & 0 & $\mathrm{D}$ \\
\hline $4 \mathrm{~N} / 27 \mathrm{~W}-18 \mathrm{~J} 1$ & 225 & 0 & $\mathrm{D}$ & $4 \mathrm{~N} / 27 \mathrm{~W}-18 \mathrm{P} 2$ & 85 & 0 & $\mathrm{D}$ \\
\hline $4 \mathrm{~N} / 27 \mathrm{~W}-18 \mathrm{~J} 1$ & 235 & 0 & $\mathrm{D}$ & $4 \mathrm{~N} / 27 \mathrm{~W}-18 \mathrm{P} 2$ & 95 & 81.8 & $\mathrm{D}$ \\
\hline $4 \mathrm{~N} / 27 \mathrm{~W}-18 \mathrm{~J} 1$ & 245 & 0 & $\mathrm{D}$ & $4 \mathrm{~N} / 27 \mathrm{~W}-18 \mathrm{P} 2$ & 105 & 0 & $\mathrm{D}$ \\
\hline $4 \mathrm{~N} / 27 \mathrm{~W}-18 \mathrm{~J} 1$ & 255 & 0 & $\mathrm{D}$ & $4 \mathrm{~N} / 27 \mathrm{~W}-18 \mathrm{P} 2$ & 115 & 90 & $\mathrm{D}$ \\
\hline $4 \mathrm{~N} / 27 \mathrm{~W}-18 \mathrm{~J} 1$ & 265 & 0 & $\mathrm{D}$ & $4 \mathrm{~N} / 27 \mathrm{~W}-18 \mathrm{P} 2$ & 125 & 100 & $\mathrm{D}$ \\
\hline $4 \mathrm{~N} / 27 \mathrm{~W}-18 \mathrm{~J} 1$ & 275 & 0 & $\mathrm{D}$ & $4 \mathrm{~N} / 27 \mathrm{~W}-18 \mathrm{P} 2$ & 135 & 100 & $\mathrm{D}$ \\
\hline $4 \mathrm{~N} / 27 \mathrm{~W}-18 \mathrm{~J} 1$ & 285 & 80 & $\mathrm{D}$ & $4 \mathrm{~N} / 27 \mathrm{~W}-18 \mathrm{P} 2$ & 145 & 100 & $\mathrm{D}$ \\
\hline $4 \mathrm{~N} / 27 \mathrm{~W}-18 \mathrm{~L} 1$ & 5 & 72.7 & $\mathrm{D}$ & $4 \mathrm{~N} / 27 \mathrm{~W}-18 \mathrm{P} 2$ & 155 & 50 & $\mathrm{D}$ \\
\hline $4 \mathrm{~N} / 27 \mathrm{~W}-18 \mathrm{~L} 1$ & 15 & 0 & $\mathrm{D}$ & $4 \mathrm{~N} / 27 \mathrm{~W}-18 \mathrm{P} 2$ & 165 & 11.1 & $\mathrm{D}$ \\
\hline 4N/27W-18L1 & 25 & 0 & $\mathrm{D}$ & $4 \mathrm{~N} / 27 \mathrm{~W}-18 \mathrm{P} 2$ & 175 & 0 & $\mathrm{D}$ \\
\hline 4N/27W-18L1 & 35 & 0 & $\mathrm{D}$ & $4 \mathrm{~N} / 27 \mathrm{~W}-18 \mathrm{P} 2$ & 185 & 0 & $\mathrm{D}$ \\
\hline $4 \mathrm{~N} / 27 \mathrm{~W}-18 \mathrm{~L} 1$ & 45 & 30 & $\mathrm{D}$ & $4 \mathrm{~N} / 27 \mathrm{~W}-18 \mathrm{Q} 2$ & 5 & 100 & $\mathrm{D}$ \\
\hline
\end{tabular}


Table B1-1. Calculated percent-coarse intervals for all drillers' logs and e-logs used to define textural properties of the hydrogeologic framework model.-Continued

[Percent-coarse values are calculated for 10-foot intervals for the entire depth of each borehole. State well number is the state of California well identifier; interval midpoint depth is the midpoint of each 10-foot interval, in feet from top of borehole; PC is the percent-coarse value for each interval; log type defines whether the borehole is a drillers' lithology log (D) or a geophysical e-log (E).]

\begin{tabular}{|c|c|c|c|c|c|c|c|}
\hline $\begin{array}{c}\text { State } \\
\text { well } \\
\text { number }\end{array}$ & $\begin{array}{c}\text { Interval } \\
\text { midpoint depth } \\
\text { (feet) }\end{array}$ & PC & $\begin{array}{l}\text { Log } \\
\text { type }\end{array}$ & $\begin{array}{c}\text { State } \\
\text { well } \\
\text { number }\end{array}$ & $\begin{array}{c}\text { Interval } \\
\text { midpoint depth } \\
\text { (feet) }\end{array}$ & PC & $\begin{array}{l}\text { Log } \\
\text { type }\end{array}$ \\
\hline 4N/27W-18Q2 & 15 & 100 & $\mathrm{D}$ & $4 \mathrm{~N} / 27 \mathrm{~W}-18 \mathrm{R} 1$ & 225 & 0 & $\mathrm{D}$ \\
\hline 4N/27W-18Q2 & 25 & 100 & $\mathrm{D}$ & $4 \mathrm{~N} / 27 \mathrm{~W}-18 \mathrm{R} 1$ & 235 & 0 & $\mathrm{D}$ \\
\hline 4N/27W-18Q2 & 35 & 90 & $\mathrm{D}$ & $4 \mathrm{~N} / 27 \mathrm{~W}-19 \mathrm{~A} 1$ & 5 & 100 & $\mathrm{D}$ \\
\hline 4N/27W-18Q2 & 45 & 0 & $\mathrm{D}$ & $4 \mathrm{~N} / 27 \mathrm{~W}-19 \mathrm{~A} 1$ & 15 & 100 & $\mathrm{D}$ \\
\hline 4N/27W-18Q2 & 65 & 100 & $\mathrm{D}$ & 4N/27W-19A1 & 35 & 100 & $\mathrm{D}$ \\
\hline 4N/27W-18Q2 & 75 & 100 & $\mathrm{D}$ & $4 \mathrm{~N} / 27 \mathrm{~W}-19 \mathrm{~A} 1$ & 45 & 100 & $\mathrm{D}$ \\
\hline 4N/27W-18Q2 & 85 & 100 & $\mathrm{D}$ & $4 \mathrm{~N} / 27 \mathrm{~W}-19 \mathrm{~A} 1$ & 55 & 100 & $\mathrm{D}$ \\
\hline 4N/27W-18Q2 & 95 & 100 & $\mathrm{D}$ & $4 \mathrm{~N} / 27 \mathrm{~W}-19 \mathrm{~A} 1$ & 65 & 100 & $\mathrm{D}$ \\
\hline 4N/27W-18Q2 & 135 & 100 & $\mathrm{D}$ & $4 \mathrm{~N} / 27 \mathrm{~W}-19 \mathrm{~A} 1$ & 105 & 0 & $\mathrm{D}$ \\
\hline 4N/27W-18Q2 & 145 & 100 & $\mathrm{D}$ & $4 \mathrm{~N} / 27 \mathrm{~W}-19 \mathrm{~A} 1$ & 115 & 0 & $\mathrm{D}$ \\
\hline $4 \mathrm{~N} / 27 \mathrm{~W}-18 \mathrm{Q} 2$ & 155 & 100 & $\mathrm{D}$ & $4 \mathrm{~N} / 27 \mathrm{~W}-19 \mathrm{~A} 1$ & 125 & 0 & $\mathrm{D}$ \\
\hline 4N/27W-18Q2 & 165 & 100 & $\mathrm{D}$ & $4 \mathrm{~N} / 27 \mathrm{~W}-19 \mathrm{~A} 1$ & 135 & 0 & $\mathrm{D}$ \\
\hline 4N/27W-18Q2 & 175 & 100 & $\mathrm{D}$ & 4N/27W-19A1 & 145 & 0 & $\mathrm{D}$ \\
\hline $4 \mathrm{~N} / 27 \mathrm{~W}-18 \mathrm{R} 1$ & 5 & 100 & $\mathrm{D}$ & $4 \mathrm{~N} / 27 \mathrm{~W}-19 \mathrm{~A} 1$ & 155 & 10 & $\mathrm{D}$ \\
\hline $4 \mathrm{~N} / 27 \mathrm{~W}-18 \mathrm{R} 1$ & 15 & 100 & $\mathrm{D}$ & $4 \mathrm{~N} / 27 \mathrm{~W}-19 \mathrm{~A} 1$ & 165 & 0 & $\mathrm{D}$ \\
\hline $4 \mathrm{~N} / 27 \mathrm{~W}-18 \mathrm{R} 1$ & 25 & 100 & $\mathrm{D}$ & $4 \mathrm{~N} / 27 \mathrm{~W}-19 \mathrm{~A} 1$ & 175 & 0 & $\mathrm{D}$ \\
\hline $4 \mathrm{~N} / 27 \mathrm{~W}-18 \mathrm{R} 1$ & 35 & 100 & $\mathrm{D}$ & $4 \mathrm{~N} / 27 \mathrm{~W}-19 \mathrm{~A} 1$ & 185 & 0 & $\mathrm{D}$ \\
\hline $4 \mathrm{~N} / 27 \mathrm{~W}-18 \mathrm{R} 1$ & 105 & 100 & $\mathrm{D}$ & $4 \mathrm{~N} / 27 \mathrm{~W}-19 \mathrm{~A} 1$ & 255 & 0 & $\mathrm{D}$ \\
\hline 4N/27W-18R1 & 115 & 100 & $\mathrm{D}$ & $4 \mathrm{~N} / 27 \mathrm{~W}-19 \mathrm{~A} 1$ & 265 & 0 & $\mathrm{D}$ \\
\hline $4 \mathrm{~N} / 27 \mathrm{~W}-18 \mathrm{R} 1$ & 125 & 100 & $\mathrm{D}$ & $4 \mathrm{~N} / 27 \mathrm{~W}-19 \mathrm{~A} 1$ & 275 & 0 & $\mathrm{D}$ \\
\hline 4N/27W-18R1 & 135 & 100 & $\mathrm{D}$ & $4 \mathrm{~N} / 27 \mathrm{~W}-19 \mathrm{~A} 1$ & 285 & 0 & $\mathrm{D}$ \\
\hline $4 \mathrm{~N} / 27 \mathrm{~W}-18 \mathrm{R} 1$ & 145 & 100 & $\mathrm{D}$ & $4 \mathrm{~N} / 27 \mathrm{~W}-19 \mathrm{~A} 1$ & 295 & 0 & $\mathrm{D}$ \\
\hline $4 \mathrm{~N} / 27 \mathrm{~W}-18 \mathrm{R} 1$ & 155 & 40 & $\mathrm{D}$ & $4 \mathrm{~N} / 27 \mathrm{~W}-19 \mathrm{~A} 1$ & 305 & 0 & $\mathrm{D}$ \\
\hline $4 \mathrm{~N} / 27 \mathrm{~W}-18 \mathrm{R} 1$ & 165 & 0 & $\mathrm{D}$ & $4 \mathrm{~N} / 27 \mathrm{~W}-19 \mathrm{~A} 1$ & 315 & 0 & $\mathrm{D}$ \\
\hline $4 \mathrm{~N} / 27 \mathrm{~W}-18 \mathrm{R} 1$ & 175 & 0 & $\mathrm{D}$ & $4 \mathrm{~N} / 27 \mathrm{~W}-19 \mathrm{~A} 1$ & 325 & 0 & $\mathrm{D}$ \\
\hline $4 \mathrm{~N} / 27 \mathrm{~W}-18 \mathrm{R} 1$ & 185 & 0 & $\mathrm{D}$ & $4 \mathrm{~N} / 27 \mathrm{~W}-19 \mathrm{~A} 1$ & 335 & 0 & $\mathrm{D}$ \\
\hline $4 \mathrm{~N} / 27 \mathrm{~W}-18 \mathrm{R} 1$ & 195 & 0 & $\mathrm{D}$ & $4 \mathrm{~N} / 27 \mathrm{~W}-19 \mathrm{~A} 1$ & 345 & 0 & $\mathrm{D}$ \\
\hline $4 \mathrm{~N} / 27 \mathrm{~W}-18 \mathrm{R} 1$ & 205 & 0 & $\mathrm{D}$ & $4 \mathrm{~N} / 27 \mathrm{~W}-21 \mathrm{~B} 1$ & 5 & 100 & $\mathrm{D}$ \\
\hline $4 \mathrm{~N} / 27 \mathrm{~W}-18 \mathrm{R} 1$ & 215 & 0 & $\mathrm{D}$ & $4 \mathrm{~N} / 27 \mathrm{~W}-21 \mathrm{~B} 1$ & 15 & 100 & $\mathrm{D}$ \\
\hline
\end{tabular}


Table B1-1. Calculated percent-coarse intervals for all drillers' logs and e-logs used to define textural properties of the hydrogeologic framework model.-Continued

[Percent-coarse values are calculated for 10-foot intervals for the entire depth of each borehole. State well number is the state of California well identifier; interval midpoint depth is the midpoint of each 10-foot interval, in feet from top of borehole; PC is the percent-coarse value for each interval; log type defines whether the borehole is a drillers' lithology $\log (\mathrm{D})$ or a geophysical e-log (E).]

\begin{tabular}{|c|c|c|c|c|c|c|c|}
\hline $\begin{array}{c}\text { State } \\
\text { well } \\
\text { number }\end{array}$ & $\begin{array}{c}\text { Interval } \\
\text { midpoint depth } \\
\text { (feet) }\end{array}$ & PC & $\begin{array}{l}\text { Log } \\
\text { type }\end{array}$ & $\begin{array}{c}\text { State } \\
\text { well } \\
\text { number }\end{array}$ & $\begin{array}{c}\text { Interval } \\
\text { midpoint depth } \\
\text { (feet) }\end{array}$ & PC & $\begin{array}{l}\text { Log } \\
\text { type }\end{array}$ \\
\hline $4 \mathrm{~N} / 27 \mathrm{~W}-21 \mathrm{~B} 1$ & 25 & 0 & $\mathrm{D}$ & $4 \mathrm{~N} / 27 \mathrm{~W}-21 \mathrm{~B} 1$ & 415 & 100 & $\mathrm{D}$ \\
\hline $4 \mathrm{~N} / 27 \mathrm{~W}-21 \mathrm{~B} 1$ & 35 & 0 & $\mathrm{D}$ & $4 \mathrm{~N} / 27 \mathrm{~W}-21 \mathrm{~B} 1$ & 425 & 100 & $\mathrm{D}$ \\
\hline $4 \mathrm{~N} / 27 \mathrm{~W}-21 \mathrm{~B} 1$ & 45 & 0 & $\mathrm{D}$ & $4 \mathrm{~N} / 27 \mathrm{~W}-21 \mathrm{~B} 1$ & 435 & 100 & $\mathrm{D}$ \\
\hline $4 \mathrm{~N} / 27 \mathrm{~W}-21 \mathrm{~B} 1$ & 55 & 0 & $\mathrm{D}$ & $4 \mathrm{~N} / 27 \mathrm{~W}-21 \mathrm{~B} 1$ & 445 & 100 & $\mathrm{D}$ \\
\hline $4 \mathrm{~N} / 27 \mathrm{~W}-21 \mathrm{~B} 1$ & 75 & 0 & $\mathrm{D}$ & $4 \mathrm{~N} / 27 \mathrm{~W}-21 \mathrm{~B} 2$ & 15 & 0 & $\mathrm{D}$ \\
\hline $4 \mathrm{~N} / 27 \mathrm{~W}-21 \mathrm{~B} 1$ & 85 & 0 & $\mathrm{D}$ & $4 \mathrm{~N} / 27 \mathrm{~W}-21 \mathrm{~B} 2$ & 25 & 0 & $\mathrm{D}$ \\
\hline $4 \mathrm{~N} / 27 \mathrm{~W}-21 \mathrm{~B} 1$ & 95 & 0 & $\mathrm{D}$ & $4 \mathrm{~N} / 27 \mathrm{~W}-21 \mathrm{~B} 2$ & 35 & 0 & $\mathrm{D}$ \\
\hline $4 \mathrm{~N} / 27 \mathrm{~W}-21 \mathrm{~B} 1$ & 105 & 70 & $\mathrm{D}$ & $4 \mathrm{~N} / 27 \mathrm{~W}-21 \mathrm{~B} 2$ & 45 & 0 & $\mathrm{D}$ \\
\hline $4 \mathrm{~N} / 27 \mathrm{~W}-21 \mathrm{~B} 1$ & 145 & 0 & $\mathrm{D}$ & $4 \mathrm{~N} / 27 \mathrm{~W}-21 \mathrm{~B} 2$ & 85 & 0 & $\mathrm{D}$ \\
\hline $4 \mathrm{~N} / 27 \mathrm{~W}-21 \mathrm{~B} 1$ & 155 & 0 & $\mathrm{D}$ & $4 \mathrm{~N} / 27 \mathrm{~W}-21 \mathrm{~B} 2$ & 95 & 0 & $\mathrm{D}$ \\
\hline $4 \mathrm{~N} / 27 \mathrm{~W}-21 \mathrm{~B} 1$ & 165 & 0 & $\mathrm{D}$ & $4 \mathrm{~N} / 27 \mathrm{~W}-21 \mathrm{~B} 2$ & 105 & 0 & $\mathrm{D}$ \\
\hline $4 \mathrm{~N} / 27 \mathrm{~W}-21 \mathrm{~B} 1$ & 175 & 30 & $\mathrm{D}$ & $4 \mathrm{~N} / 27 \mathrm{~W}-21 \mathrm{~B} 2$ & 115 & 0 & $\mathrm{D}$ \\
\hline $4 \mathrm{~N} / 27 \mathrm{~W}-21 \mathrm{~B} 1$ & 185 & 0 & $\mathrm{D}$ & $4 \mathrm{~N} / 27 \mathrm{~W}-21 \mathrm{~B} 2$ & 125 & 0 & $\mathrm{D}$ \\
\hline $4 \mathrm{~N} / 27 \mathrm{~W}-21 \mathrm{~B} 1$ & 195 & 0 & $\mathrm{D}$ & $4 \mathrm{~N} / 27 \mathrm{~W}-21 \mathrm{~B} 2$ & 135 & 0 & $\mathrm{D}$ \\
\hline $4 \mathrm{~N} / 27 \mathrm{~W}-21 \mathrm{~B} 1$ & 205 & 0 & $\mathrm{D}$ & $4 \mathrm{~N} / 27 \mathrm{~W}-21 \mathrm{~B} 2$ & 145 & 0 & $\mathrm{D}$ \\
\hline $4 \mathrm{~N} / 27 \mathrm{~W}-21 \mathrm{~B} 1$ & 215 & 100 & $\mathrm{D}$ & $4 \mathrm{~N} / 27 \mathrm{~W}-21 \mathrm{~B} 2$ & 155 & 0 & $\mathrm{D}$ \\
\hline $4 \mathrm{~N} / 27 \mathrm{~W}-21 \mathrm{~B} 1$ & 225 & 20 & $\mathrm{D}$ & $4 \mathrm{~N} / 27 \mathrm{~W}-21 \mathrm{~B} 2$ & 165 & 44.4 & $\mathrm{D}$ \\
\hline $4 \mathrm{~N} / 27 \mathrm{~W}-21 \mathrm{~B} 1$ & 295 & 0 & $\mathrm{D}$ & $4 \mathrm{~N} / 27 \mathrm{~W}-21 \mathrm{~B} 2$ & 235 & 0 & $\mathrm{D}$ \\
\hline $4 \mathrm{~N} / 27 \mathrm{~W}-21 \mathrm{~B} 1$ & 305 & 50 & $\mathrm{D}$ & $4 \mathrm{~N} / 27 \mathrm{~W}-21 \mathrm{~B} 2$ & 245 & 0 & $\mathrm{D}$ \\
\hline $4 \mathrm{~N} / 27 \mathrm{~W}-21 \mathrm{~B} 1$ & 315 & 100 & $\mathrm{D}$ & $4 \mathrm{~N} / 27 \mathrm{~W}-21 \mathrm{~B} 2$ & 255 & 0 & $\mathrm{D}$ \\
\hline $4 \mathrm{~N} / 27 \mathrm{~W}-21 \mathrm{~B} 1$ & 325 & 100 & $\mathrm{D}$ & $4 \mathrm{~N} / 27 \mathrm{~W}-21 \mathrm{~B} 2$ & 265 & 40 & $\mathrm{D}$ \\
\hline $4 \mathrm{~N} / 27 \mathrm{~W}-21 \mathrm{~B} 1$ & 335 & 100 & $\mathrm{D}$ & $4 \mathrm{~N} / 27 \mathrm{~W}-21 \mathrm{~B} 2$ & 275 & 0 & $\mathrm{D}$ \\
\hline $4 \mathrm{~N} / 27 \mathrm{~W}-21 \mathrm{~B} 1$ & 345 & 100 & $\mathrm{D}$ & $4 \mathrm{~N} / 27 \mathrm{~W}-21 \mathrm{~B} 2$ & 285 & 0 & $\mathrm{D}$ \\
\hline $4 \mathrm{~N} / 27 \mathrm{~W}-21 \mathrm{~B} 1$ & 355 & 100 & $\mathrm{D}$ & $4 \mathrm{~N} / 27 \mathrm{~W}-21 \mathrm{~B} 2$ & 295 & 0 & $\mathrm{D}$ \\
\hline $4 \mathrm{~N} / 27 \mathrm{~W}-21 \mathrm{~B} 1$ & 365 & 100 & $\mathrm{D}$ & $4 \mathrm{~N} / 27 \mathrm{~W}-21 \mathrm{~B} 2$ & 305 & 0 & $\mathrm{D}$ \\
\hline $4 \mathrm{~N} / 27 \mathrm{~W}-21 \mathrm{~B} 1$ & 375 & 100 & $\mathrm{D}$ & $4 \mathrm{~N} / 27 \mathrm{~W}-21 \mathrm{~B} 2$ & 315 & 0 & $\mathrm{D}$ \\
\hline $4 \mathrm{~N} / 27 \mathrm{~W}-21 \mathrm{~B} 1$ & 385 & 100 & $\mathrm{D}$ & $4 \mathrm{~N} / 27 \mathrm{~W}-21 \mathrm{~B} 2$ & 325 & 0 & $\mathrm{D}$ \\
\hline $4 \mathrm{~N} / 27 \mathrm{~W}-21 \mathrm{~B} 1$ & 395 & 100 & $\mathrm{D}$ & $4 \mathrm{~N} / 27 \mathrm{~W}-21 \mathrm{~B} 2$ & 335 & 0 & $\mathrm{D}$ \\
\hline $4 \mathrm{~N} / 27 \mathrm{~W}-21 \mathrm{~B} 1$ & 405 & 100 & $\mathrm{D}$ & $4 \mathrm{~N} / 27 \mathrm{~W}-21 \mathrm{~B} 2$ & 345 & 0 & $\mathrm{D}$ \\
\hline
\end{tabular}


Table B1-1. Calculated percent-coarse intervals for all drillers' logs and e-logs used to define textural properties of the hydrogeologic framework model.-Continued

[Percent-coarse values are calculated for 10-foot intervals for the entire depth of each borehole. State well number is the state of California well identifier; interval midpoint depth is the midpoint of each 10-foot interval, in feet from top of borehole; PC is the percent-coarse value for each interval; log type defines whether the borehole is a drillers' lithology $\log (\mathrm{D})$ or a geophysical e-log (E).]

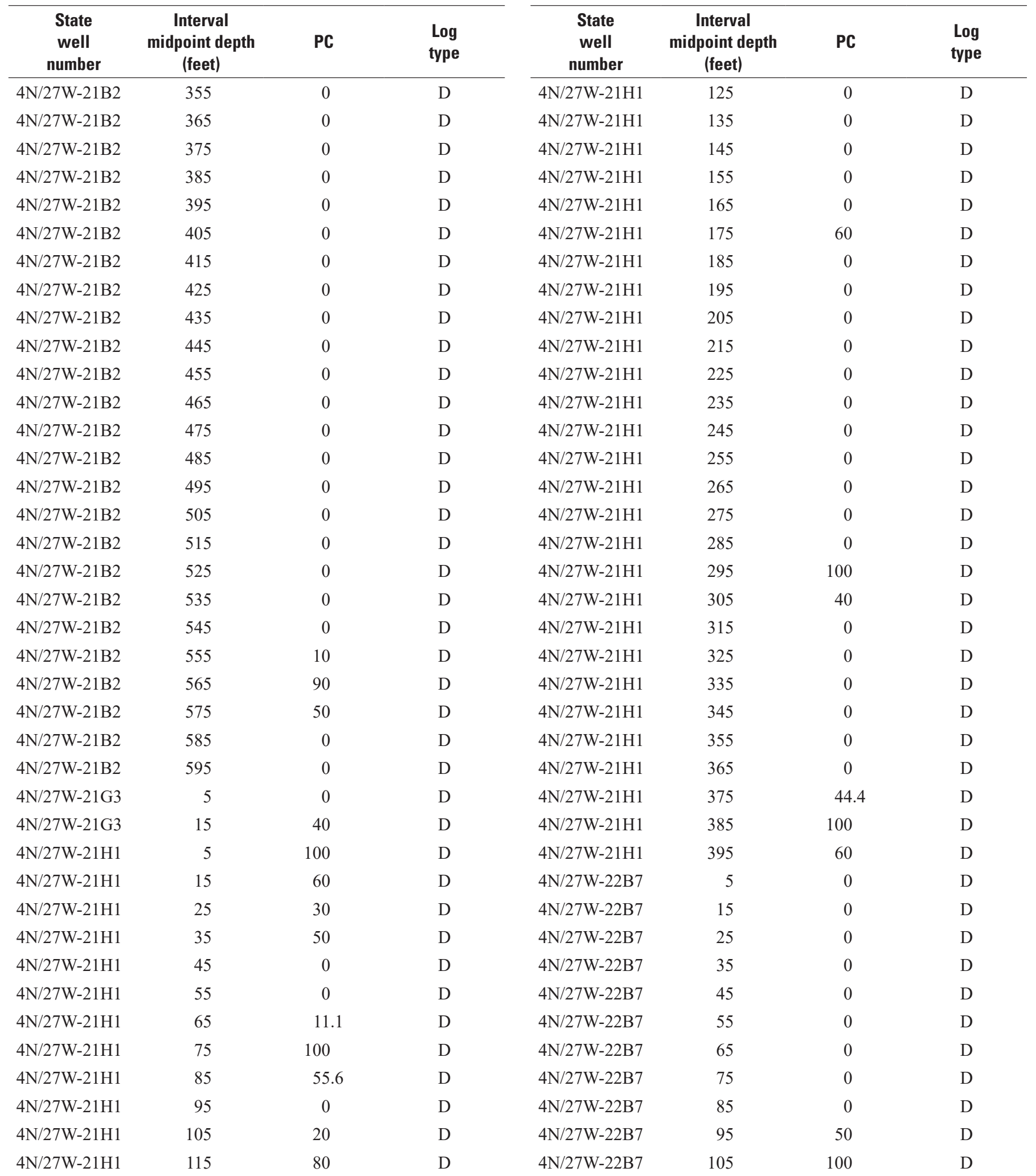


Table B1-1. Calculated percent-coarse intervals for all drillers' logs and e-logs used to define textural properties of the hydrogeologic framework model.-Continued

[Percent-coarse values are calculated for 10-foot intervals for the entire depth of each borehole. State well number is the state of California well identifier; interval midpoint depth is the midpoint of each 10-foot interval, in feet from top of borehole; PC is the percent-coarse value for each interval; log type defines whether the borehole is a drillers' lithology log (D) or a geophysical e-log (E).]

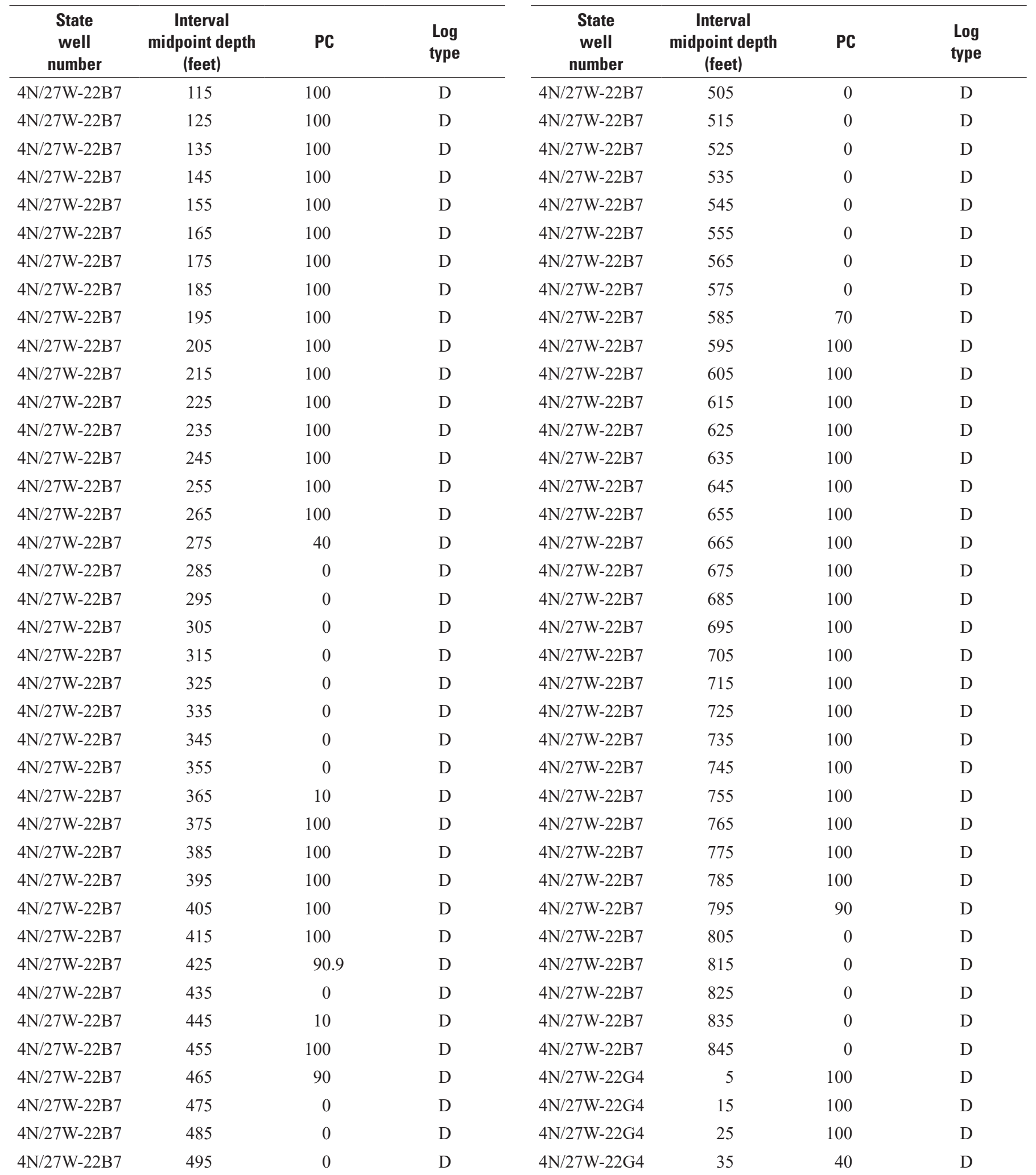


Table B1-1. Calculated percent-coarse intervals for all drillers' logs and e-logs used to define textural properties of the hydrogeologic framework model.-Continued

[Percent-coarse values are calculated for 10-foot intervals for the entire depth of each borehole. State well number is the state of California well identifier; interval midpoint depth is the midpoint of each 10-foot interval, in feet from top of borehole; PC is the percent-coarse value for each interval; log type defines whether the borehole is a drillers' lithology $\log (\mathrm{D})$ or a geophysical e-log (E).]

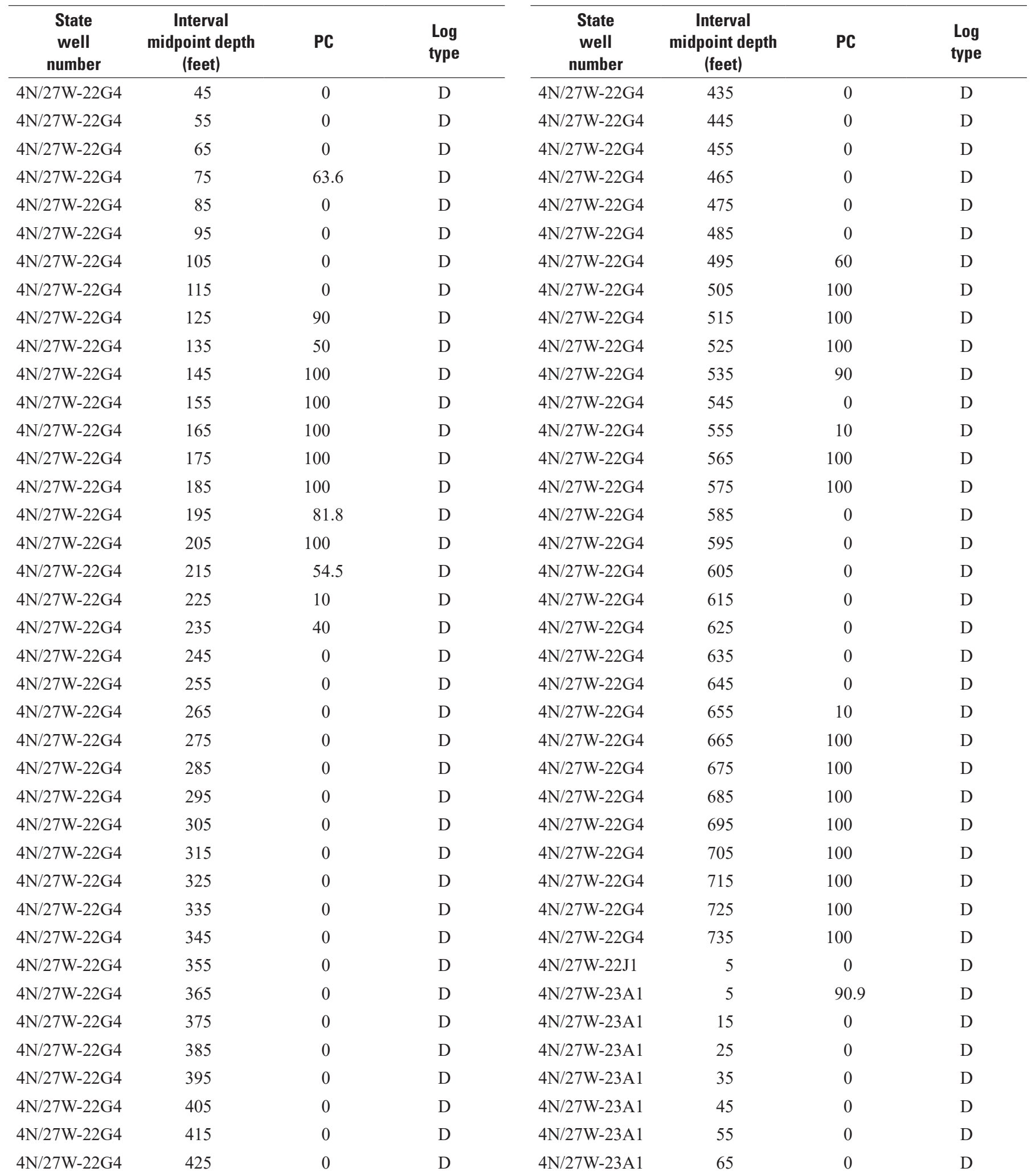


Table B1-1. Calculated percent-coarse intervals for all drillers' logs and e-logs used to define textural properties of the hydrogeologic framework model.-Continued

[Percent-coarse values are calculated for 10-foot intervals for the entire depth of each borehole. State well number is the state of California well identifier; interval midpoint depth is the midpoint of each 10-foot interval, in feet from top of borehole; PC is the percent-coarse value for each interval; log type defines whether the borehole is a drillers' lithology $\log (\mathrm{D})$ or a geophysical e-log (E).]

\begin{tabular}{|c|c|c|c|c|c|c|c|}
\hline $\begin{array}{c}\text { State } \\
\text { well } \\
\text { number }\end{array}$ & $\begin{array}{c}\text { Interval } \\
\text { midpoint depth } \\
\text { (feet) }\end{array}$ & PC & $\begin{array}{l}\text { Log } \\
\text { type }\end{array}$ & $\begin{array}{c}\text { State } \\
\text { well } \\
\text { number }\end{array}$ & $\begin{array}{c}\text { Interval } \\
\text { midpoint depth } \\
\text { (feet) }\end{array}$ & PC & $\begin{array}{l}\text { Log } \\
\text { type }\end{array}$ \\
\hline $4 \mathrm{~N} / 27 \mathrm{~W}-23 \mathrm{~A} 1$ & 75 & 0 & $\mathrm{D}$ & $4 \mathrm{~N} / 27 \mathrm{~W}-23 \mathrm{~A} 2$ & 145 & 0 & $\mathrm{D}$ \\
\hline $4 \mathrm{~N} / 27 \mathrm{~W}-23 \mathrm{~A} 1$ & 85 & 0 & $\mathrm{D}$ & $4 \mathrm{~N} / 27 \mathrm{~W}-23 \mathrm{~A} 2$ & 155 & 0 & $\mathrm{D}$ \\
\hline $4 \mathrm{~N} / 27 \mathrm{~W}-23 \mathrm{~A} 1$ & 95 & 0 & $\mathrm{D}$ & $4 \mathrm{~N} / 27 \mathrm{~W}-23 \mathrm{~A} 2$ & 165 & 0 & $\mathrm{D}$ \\
\hline $4 \mathrm{~N} / 27 \mathrm{~W}-23 \mathrm{~A} 1$ & 105 & 0 & $\mathrm{D}$ & $4 \mathrm{~N} / 27 \mathrm{~W}-23 \mathrm{~A} 2$ & 175 & 0 & $\mathrm{D}$ \\
\hline $4 \mathrm{~N} / 27 \mathrm{~W}-23 \mathrm{~A} 1$ & 125 & 0 & $\mathrm{D}$ & $4 \mathrm{~N} / 27 \mathrm{~W}-23 \mathrm{~A} 2$ & 195 & 100 & $\mathrm{D}$ \\
\hline $4 \mathrm{~N} / 27 \mathrm{~W}-23 \mathrm{~A} 1$ & 135 & 0 & $\mathrm{D}$ & $4 \mathrm{~N} / 27 \mathrm{~W}-23 \mathrm{~A} 2$ & 205 & 100 & $\mathrm{D}$ \\
\hline $4 \mathrm{~N} / 27 \mathrm{~W}-23 \mathrm{~A} 1$ & 145 & 0 & $\mathrm{D}$ & $4 \mathrm{~N} / 27 \mathrm{~W}-23 \mathrm{~A} 2$ & 215 & 100 & $\mathrm{D}$ \\
\hline $4 \mathrm{~N} / 27 \mathrm{~W}-23 \mathrm{~A} 1$ & 155 & 0 & $\mathrm{D}$ & $4 \mathrm{~N} / 27 \mathrm{~W}-23 \mathrm{~A} 2$ & 225 & 100 & $\mathrm{D}$ \\
\hline $4 \mathrm{~N} / 27 \mathrm{~W}-23 \mathrm{~A} 1$ & 195 & 0 & $\mathrm{D}$ & $4 \mathrm{~N} / 27 \mathrm{~W}-23 \mathrm{~A} 2$ & 265 & 40 & $\mathrm{D}$ \\
\hline $4 \mathrm{~N} / 27 \mathrm{~W}-23 \mathrm{~A} 1$ & 205 & 0 & $\mathrm{D}$ & $4 \mathrm{~N} / 27 \mathrm{~W}-23 \mathrm{~A} 2$ & 275 & 30 & $\mathrm{D}$ \\
\hline $4 \mathrm{~N} / 27 \mathrm{~W}-23 \mathrm{~A} 1$ & 215 & 0 & $\mathrm{D}$ & $4 \mathrm{~N} / 27 \mathrm{~W}-23 \mathrm{~A} 2$ & 285 & 100 & $\mathrm{D}$ \\
\hline $4 \mathrm{~N} / 27 \mathrm{~W}-23 \mathrm{~A} 1$ & 225 & 0 & $\mathrm{D}$ & $4 \mathrm{~N} / 27 \mathrm{~W}-23 \mathrm{~A} 2$ & 295 & 100 & $\mathrm{D}$ \\
\hline $4 \mathrm{~N} / 27 \mathrm{~W}-23 \mathrm{~A} 1$ & 235 & 0 & $\mathrm{D}$ & $4 \mathrm{~N} / 27 \mathrm{~W}-23 \mathrm{~F} 4$ & 5 & 0 & $\mathrm{D}$ \\
\hline $4 \mathrm{~N} / 27 \mathrm{~W}-23 \mathrm{~A} 1$ & 245 & 0 & $\mathrm{D}$ & $4 \mathrm{~N} / 27 \mathrm{~W}-23 \mathrm{~F} 4$ & 15 & 0 & $\mathrm{D}$ \\
\hline $4 \mathrm{~N} / 27 \mathrm{~W}-23 \mathrm{~A} 1$ & 255 & 80 & $\mathrm{D}$ & $4 \mathrm{~N} / 27 \mathrm{~W}-23 \mathrm{~F} 4$ & 25 & 0 & $\mathrm{D}$ \\
\hline $4 \mathrm{~N} / 27 \mathrm{~W}-23 \mathrm{~A} 1$ & 265 & 100 & $\mathrm{D}$ & $4 \mathrm{~N} / 27 \mathrm{~W}-23 \mathrm{~F} 4$ & 35 & 0 & $\mathrm{D}$ \\
\hline $4 \mathrm{~N} / 27 \mathrm{~W}-23 \mathrm{~A} 1$ & 275 & 100 & $\mathrm{D}$ & $4 \mathrm{~N} / 27 \mathrm{~W}-23 \mathrm{~F} 4$ & 45 & 0 & $\mathrm{D}$ \\
\hline $4 \mathrm{~N} / 27 \mathrm{~W}-23 \mathrm{~A} 2$ & 25 & 0 & $\mathrm{D}$ & $4 \mathrm{~N} / 27 \mathrm{~W}-23 \mathrm{~F} 4$ & 115 & 0 & $\mathrm{D}$ \\
\hline $4 \mathrm{~N} / 27 \mathrm{~W}-23 \mathrm{~A} 2$ & 35 & 0 & $\mathrm{D}$ & $4 \mathrm{~N} / 27 \mathrm{~W}-23 \mathrm{~F} 4$ & 125 & 0 & $\mathrm{D}$ \\
\hline $4 \mathrm{~N} / 27 \mathrm{~W}-23 \mathrm{~A} 2$ & 45 & 0 & $\mathrm{D}$ & $4 \mathrm{~N} / 27 \mathrm{~W}-23 \mathrm{~F} 4$ & 135 & 0 & $\mathrm{D}$ \\
\hline $4 \mathrm{~N} / 27 \mathrm{~W}-23 \mathrm{~A} 2$ & 55 & 0 & $\mathrm{D}$ & $4 \mathrm{~N} / 27 \mathrm{~W}-23 \mathrm{~F} 4$ & 145 & 10 & $\mathrm{D}$ \\
\hline $4 \mathrm{~N} / 27 \mathrm{~W}-23 \mathrm{~A} 2$ & 65 & 0 & $\mathrm{D}$ & $4 \mathrm{~N} / 27 \mathrm{~W}-23 \mathrm{~F} 4$ & 155 & 100 & $\mathrm{D}$ \\
\hline $4 \mathrm{~N} / 27 \mathrm{~W}-23 \mathrm{~A} 2$ & 75 & 0 & $\mathrm{D}$ & $4 \mathrm{~N} / 27 \mathrm{~W}-23 \mathrm{~F} 4$ & 165 & 100 & $\mathrm{D}$ \\
\hline $4 \mathrm{~N} / 27 \mathrm{~W}-23 \mathrm{~A} 2$ & 85 & 0 & $\mathrm{D}$ & $4 \mathrm{~N} / 27 \mathrm{~W}-23 \mathrm{~F} 4$ & 175 & 100 & $\mathrm{D}$ \\
\hline $4 \mathrm{~N} / 27 \mathrm{~W}-23 \mathrm{~A} 2$ & 95 & 0 & $\mathrm{D}$ & $4 \mathrm{~N} / 27 \mathrm{~W}-23 \mathrm{~F} 4$ & 185 & 40 & $\mathrm{D}$ \\
\hline $4 \mathrm{~N} / 27 \mathrm{~W}-23 \mathrm{~A} 2$ & 105 & 90.9 & $\mathrm{D}$ & $4 \mathrm{~N} / 27 \mathrm{~W}-23 \mathrm{~F} 4$ & 195 & 0 & $\mathrm{D}$ \\
\hline $4 \mathrm{~N} / 27 \mathrm{~W}-23 \mathrm{~A} 2$ & 115 & 100 & $\mathrm{D}$ & $4 \mathrm{~N} / 27 \mathrm{~W}-23 \mathrm{~F} 4$ & 205 & 0 & $\mathrm{D}$ \\
\hline $4 \mathrm{~N} / 27 \mathrm{~W}-23 \mathrm{~A} 2$ & 125 & 30 & $\mathrm{D}$ & $4 \mathrm{~N} / 27 \mathrm{~W}-23 \mathrm{~F} 4$ & 215 & 0 & $\mathrm{D}$ \\
\hline $4 \mathrm{~N} / 27 \mathrm{~W}-23 \mathrm{~A} 2$ & 135 & 0 & $\mathrm{D}$ & $4 \mathrm{~N} / 27 \mathrm{~W}-23 \mathrm{~F} 4$ & 225 & 0 & $\mathrm{D}$ \\
\hline
\end{tabular}


Table B1-1. Calculated percent-coarse intervals for all drillers' logs and e-logs used to define textural properties of the hydrogeologic framework model.-Continued

[Percent-coarse values are calculated for 10-foot intervals for the entire depth of each borehole. State well number is the state of California well identifier; interval midpoint depth is the midpoint of each 10-foot interval, in feet from top of borehole; PC is the percent-coarse value for each interval; log type defines whether the borehole is a drillers' lithology $\log (\mathrm{D})$ or a geophysical e-log (E).]

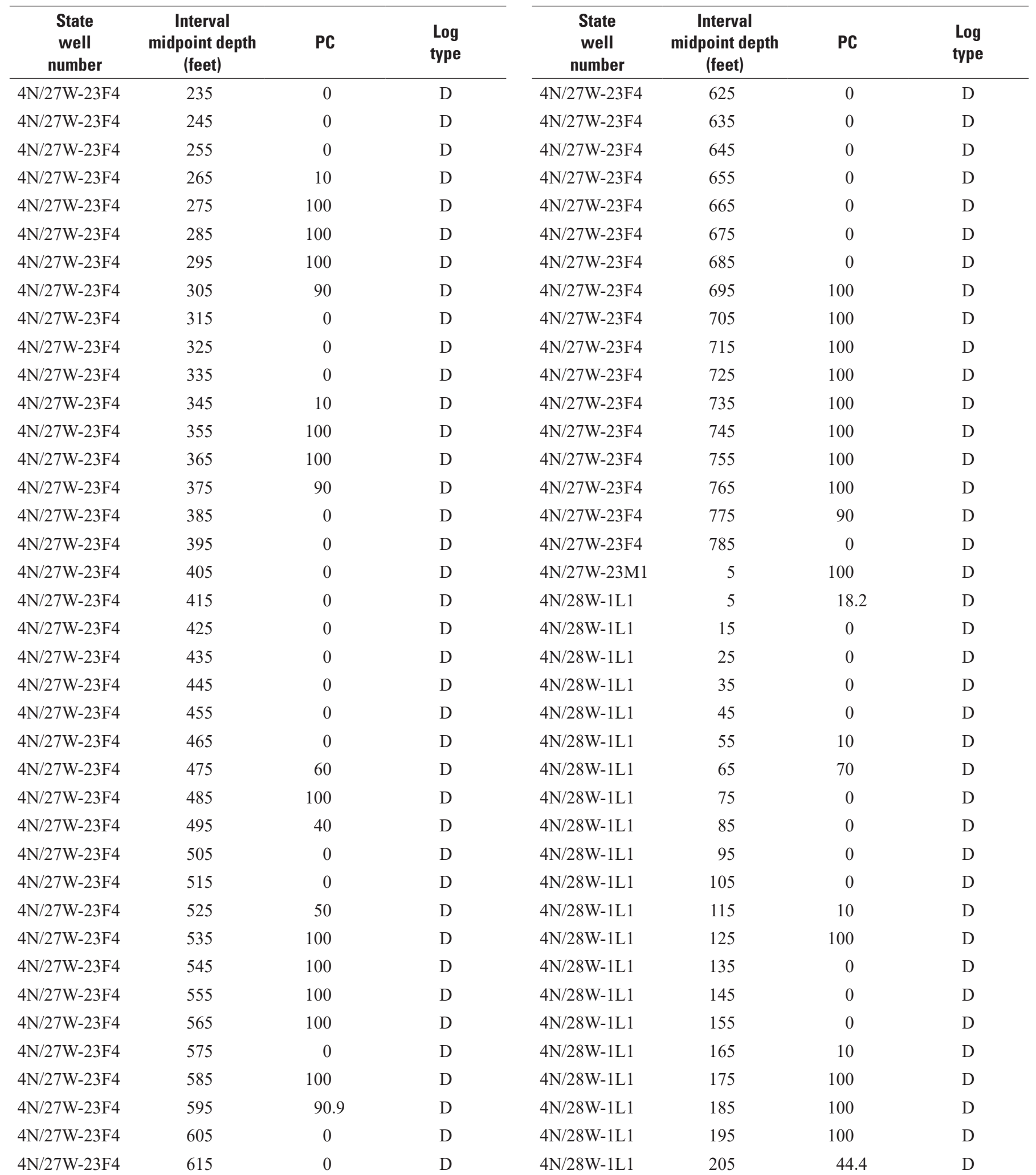


Table B1-1. Calculated percent-coarse intervals for all drillers' logs and e-logs used to define textural properties of the hydrogeologic framework model.-Continued

[Percent-coarse values are calculated for 10-foot intervals for the entire depth of each borehole. State well number is the state of California well identifier; interval midpoint depth is the midpoint of each 10-foot interval, in feet from top of borehole; PC is the percent-coarse value for each interval; log type defines whether the borehole is a drillers' lithology $\log (\mathrm{D})$ or a geophysical e-log (E).]

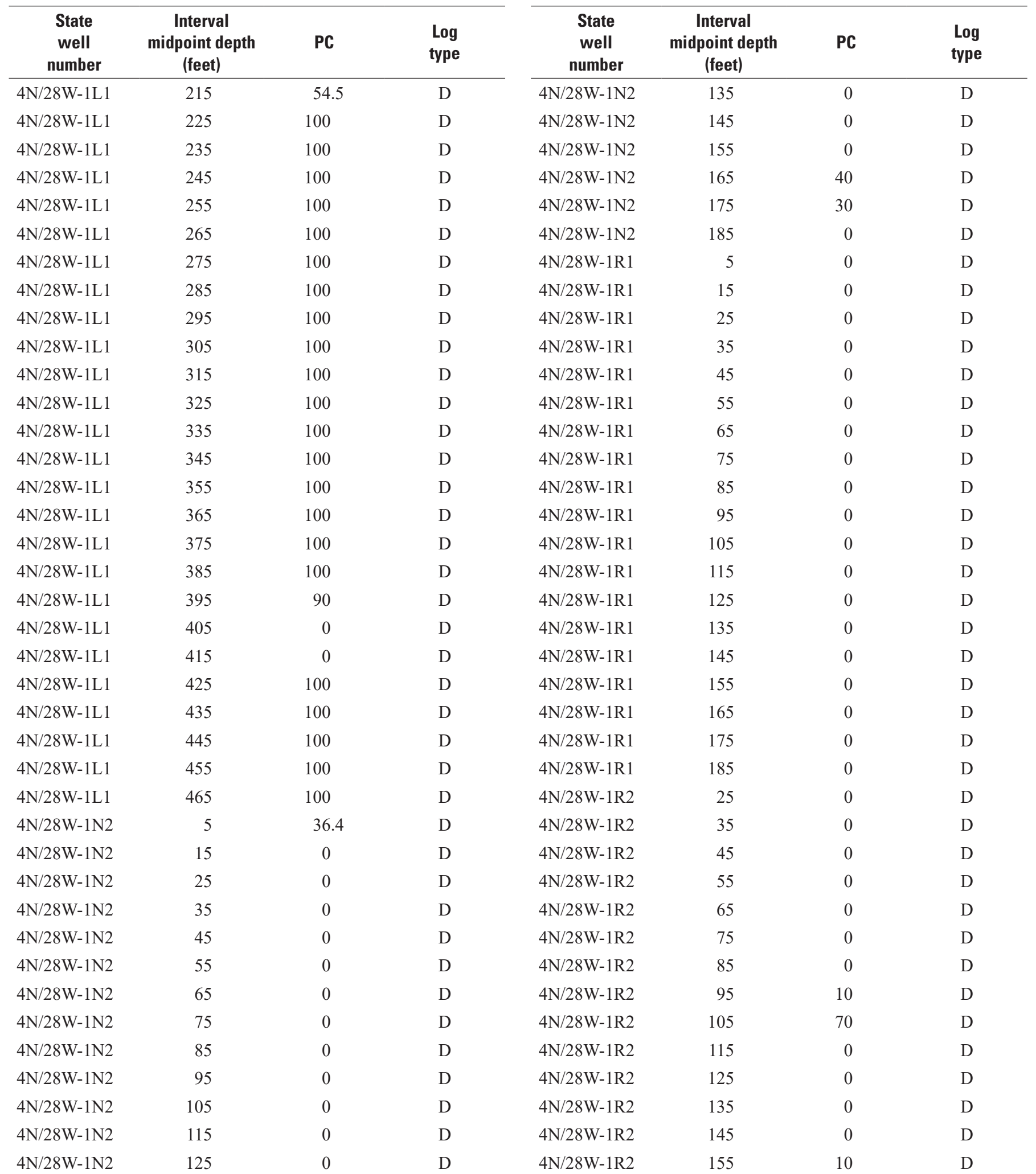


Table B1-1. Calculated percent-coarse intervals for all drillers' logs and e-logs used to define textural properties of the hydrogeologic framework model.-Continued

[Percent-coarse values are calculated for 10-foot intervals for the entire depth of each borehole. State well number is the state of California well identifier; interval midpoint depth is the midpoint of each 10-foot interval, in feet from top of borehole; PC is the percent-coarse value for each interval; log type defines whether the borehole is a drillers' lithology $\log (\mathrm{D})$ or a geophysical e-log (E).]

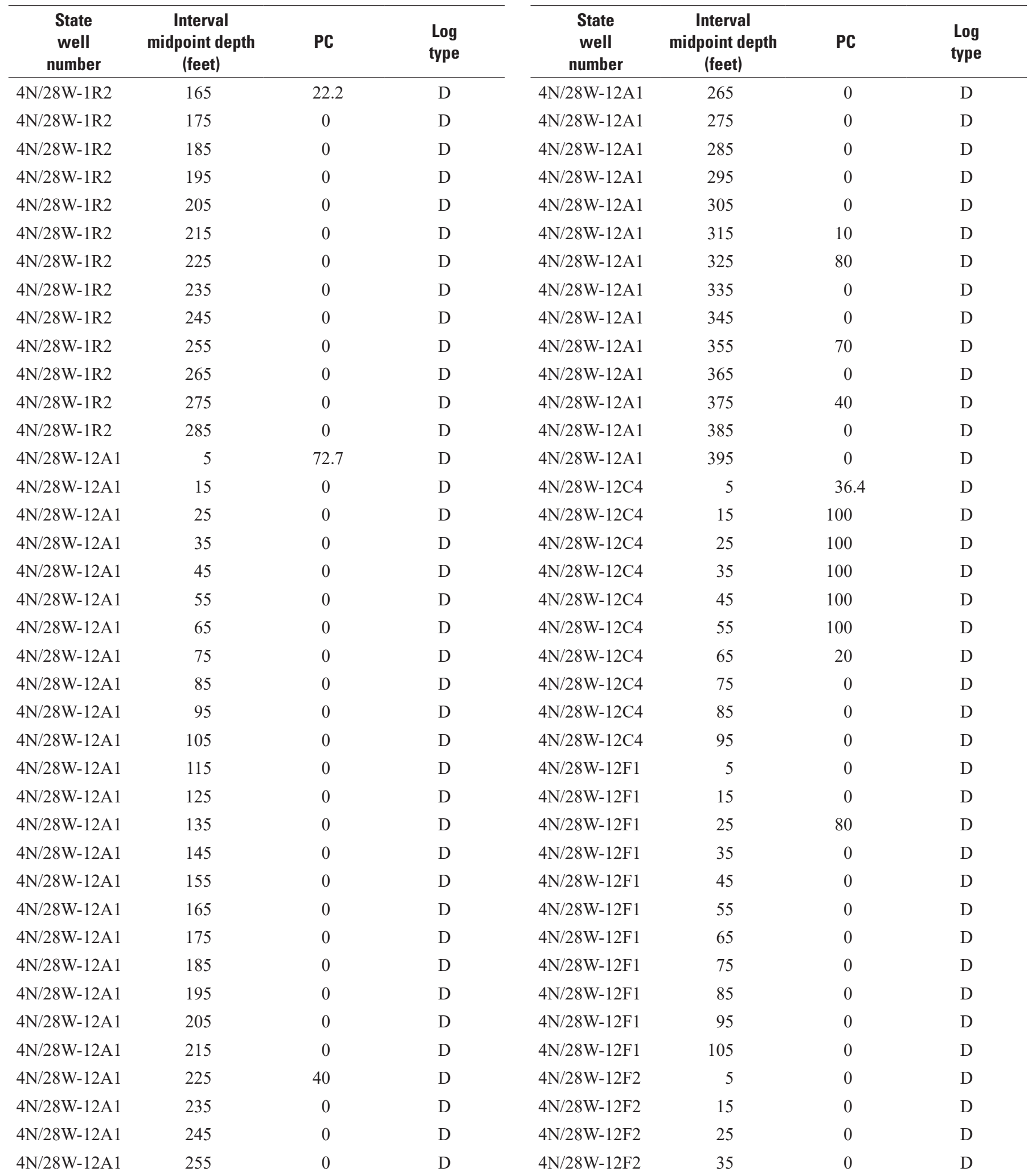


Table B1-1. Calculated percent-coarse intervals for all drillers' logs and e-logs used to define textural properties of the hydrogeologic framework model.-Continued

[Percent-coarse values are calculated for 10-foot intervals for the entire depth of each borehole. State well number is the state of California well identifier; interval midpoint depth is the midpoint of each 10-foot interval, in feet from top of borehole; PC is the percent-coarse value for each interval; log type defines whether the borehole is a drillers' lithology $\log (\mathrm{D})$ or a geophysical e-log (E).]

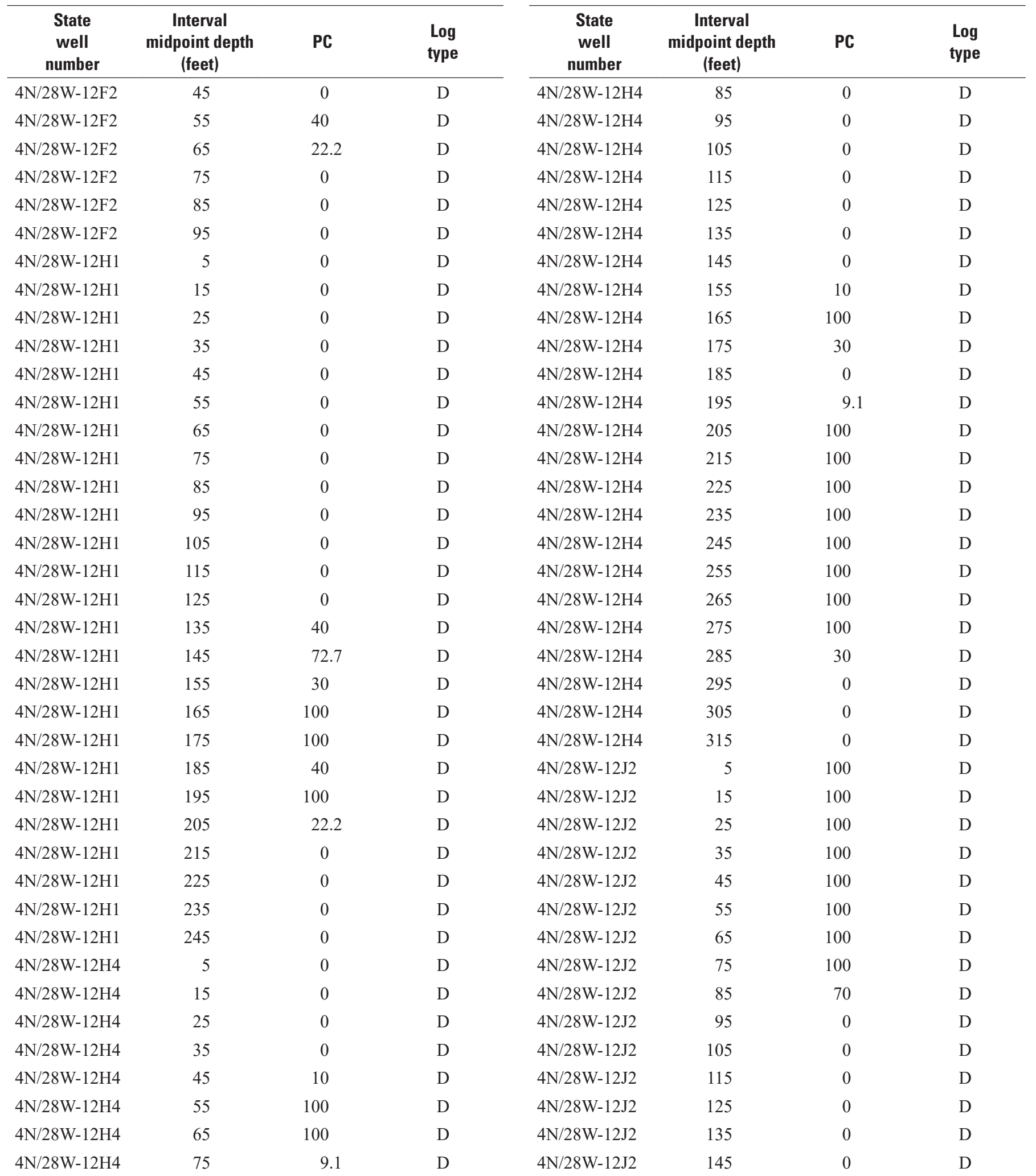


Table B1-1. Calculated percent-coarse intervals for all drillers' logs and e-logs used to define textural properties of the hydrogeologic framework model.-Continued

[Percent-coarse values are calculated for 10-foot intervals for the entire depth of each borehole. State well number is the state of California well identifier; interval midpoint depth is the midpoint of each 10-foot interval, in feet from top of borehole; PC is the percent-coarse value for each interval; log type defines whether the borehole is a drillers' lithology $\log (\mathrm{D})$ or a geophysical e-log (E).]

\begin{tabular}{|c|c|c|c|c|c|c|c|}
\hline $\begin{array}{c}\text { State } \\
\text { well } \\
\text { number }\end{array}$ & $\begin{array}{c}\text { Interval } \\
\text { midpoint depth } \\
\text { (feet) }\end{array}$ & PC & $\begin{array}{l}\text { Log } \\
\text { type }\end{array}$ & $\begin{array}{c}\text { State } \\
\text { well } \\
\text { number }\end{array}$ & $\begin{array}{c}\text { Interval } \\
\text { midpoint depth } \\
\text { (feet) }\end{array}$ & PC & $\begin{array}{l}\text { Log } \\
\text { type }\end{array}$ \\
\hline $4 \mathrm{~N} / 28 \mathrm{~W}-12 \mathrm{~J} 2$ & 155 & 10 & $\mathrm{D}$ & $4 \mathrm{~N} / 28 \mathrm{~W}-12 \mathrm{~K} 1$ & 245 & 60 & $\mathrm{D}$ \\
\hline $4 \mathrm{~N} / 28 \mathrm{~W}-12 \mathrm{~J} 2$ & 165 & 100 & $\mathrm{D}$ & $4 \mathrm{~N} / 28 \mathrm{~W}-12 \mathrm{~K} 1$ & 255 & 10 & $\mathrm{D}$ \\
\hline $4 \mathrm{~N} / 28 \mathrm{~W}-12 \mathrm{~J} 2$ & 175 & 100 & $\mathrm{D}$ & $4 \mathrm{~N} / 28 \mathrm{~W}-12 \mathrm{~K} 1$ & 265 & 55.6 & $\mathrm{D}$ \\
\hline $4 \mathrm{~N} / 28 \mathrm{~W}-12 \mathrm{~J} 2$ & 185 & 100 & $\mathrm{D}$ & $4 \mathrm{~N} / 28 \mathrm{~W}-12 \mathrm{~K} 1$ & 275 & 30 & $\mathrm{D}$ \\
\hline $4 \mathrm{~N} / 28 \mathrm{~W}-12 \mathrm{~J} 2$ & 205 & 100 & $\mathrm{D}$ & $4 \mathrm{~N} / 28 \mathrm{~W}-12 \mathrm{~K} 2$ & 5 & 100 & $\mathrm{D}$ \\
\hline $4 \mathrm{~N} / 28 \mathrm{~W}-12 \mathrm{~J} 2$ & 215 & 100 & $\mathrm{D}$ & $4 \mathrm{~N} / 28 \mathrm{~W}-12 \mathrm{~K} 2$ & 15 & 50 & $\mathrm{D}$ \\
\hline $4 \mathrm{~N} / 28 \mathrm{~W}-12 \mathrm{~J} 2$ & 225 & 100 & $\mathrm{D}$ & $4 \mathrm{~N} / 28 \mathrm{~W}-12 \mathrm{~K} 2$ & 25 & 0 & $\mathrm{D}$ \\
\hline $4 \mathrm{~N} / 28 \mathrm{~W}-12 \mathrm{~J} 2$ & 235 & 100 & $\mathrm{D}$ & $4 \mathrm{~N} / 28 \mathrm{~W}-12 \mathrm{~K} 2$ & 35 & 0 & $\mathrm{D}$ \\
\hline $4 \mathrm{~N} / 28 \mathrm{~W}-12 \mathrm{~J} 2$ & 275 & 100 & $\mathrm{D}$ & $4 \mathrm{~N} / 28 \mathrm{~W}-12 \mathrm{~K} 2$ & 75 & 0 & $\mathrm{D}$ \\
\hline $4 \mathrm{~N} / 28 \mathrm{~W}-12 \mathrm{~J} 2$ & 285 & 100 & $\mathrm{D}$ & $4 \mathrm{~N} / 28 \mathrm{~W}-12 \mathrm{~K} 2$ & 85 & 0 & $\mathrm{D}$ \\
\hline $4 \mathrm{~N} / 28 \mathrm{~W}-12 \mathrm{~J} 2$ & 295 & 100 & $\mathrm{D}$ & $4 \mathrm{~N} / 28 \mathrm{~W}-12 \mathrm{~K} 2$ & 95 & 0 & $\mathrm{D}$ \\
\hline $4 \mathrm{~N} / 28 \mathrm{~W}-12 \mathrm{~K} 1$ & 5 & 90.9 & $\mathrm{D}$ & $4 \mathrm{~N} / 28 \mathrm{~W}-12 \mathrm{~K} 2$ & 105 & 0 & $\mathrm{D}$ \\
\hline $4 \mathrm{~N} / 28 \mathrm{~W}-12 \mathrm{~K} 1$ & 15 & 0 & $\mathrm{D}$ & $4 \mathrm{~N} / 28 \mathrm{~W}-12 \mathrm{~K} 2$ & 115 & 0 & $\mathrm{D}$ \\
\hline $4 \mathrm{~N} / 28 \mathrm{~W}-12 \mathrm{~K} 1$ & 25 & 90 & $\mathrm{D}$ & $4 \mathrm{~N} / 28 \mathrm{~W}-12 \mathrm{~K} 2$ & 125 & 0 & $\mathrm{D}$ \\
\hline $4 \mathrm{~N} / 28 \mathrm{~W}-12 \mathrm{~K} 1$ & 35 & 100 & $\mathrm{D}$ & $4 \mathrm{~N} / 28 \mathrm{~W}-12 \mathrm{~K} 2$ & 135 & 0 & $\mathrm{D}$ \\
\hline $4 \mathrm{~N} / 28 \mathrm{~W}-12 \mathrm{~K} 1$ & 45 & 100 & $\mathrm{D}$ & $4 \mathrm{~N} / 28 \mathrm{~W}-12 \mathrm{~K} 2$ & 145 & 0 & $\mathrm{D}$ \\
\hline $4 \mathrm{~N} / 28 \mathrm{~W}-12 \mathrm{~K} 1$ & 55 & 100 & $\mathrm{D}$ & $4 \mathrm{~N} / 28 \mathrm{~W}-12 \mathrm{~K} 2$ & 155 & 0 & $\mathrm{D}$ \\
\hline $4 \mathrm{~N} / 28 \mathrm{~W}-12 \mathrm{~K} 1$ & 125 & 55.6 & $\mathrm{D}$ & $4 \mathrm{~N} / 28 \mathrm{~W}-12 \mathrm{~K} 2$ & 225 & 0 & $\mathrm{D}$ \\
\hline $4 \mathrm{~N} / 28 \mathrm{~W}-12 \mathrm{~K} 1$ & 135 & 100 & $\mathrm{D}$ & $4 \mathrm{~N} / 28 \mathrm{~W}-12 \mathrm{~K} 2$ & 235 & 0 & $\mathrm{D}$ \\
\hline $4 \mathrm{~N} / 28 \mathrm{~W}-12 \mathrm{~K} 1$ & 145 & 90.9 & $\mathrm{D}$ & $4 \mathrm{~N} / 28 \mathrm{~W}-12 \mathrm{~K} 2$ & 245 & 0 & $\mathrm{D}$ \\
\hline $4 \mathrm{~N} / 28 \mathrm{~W}-12 \mathrm{~K} 1$ & 155 & 0 & $\mathrm{D}$ & $4 \mathrm{~N} / 28 \mathrm{~W}-12 \mathrm{~K} 2$ & 255 & 0 & $\mathrm{D}$ \\
\hline $4 \mathrm{~N} / 28 \mathrm{~W}-12 \mathrm{~K} 1$ & 165 & 0 & $\mathrm{D}$ & $4 \mathrm{~N} / 28 \mathrm{~W}-12 \mathrm{~K} 2$ & 265 & 0 & $\mathrm{D}$ \\
\hline $4 \mathrm{~N} / 28 \mathrm{~W}-12 \mathrm{~K} 1$ & 175 & 0 & $\mathrm{D}$ & $4 \mathrm{~N} / 28 \mathrm{~W}-12 \mathrm{~K} 2$ & 275 & 0 & $\mathrm{D}$ \\
\hline $4 \mathrm{~N} / 28 \mathrm{~W}-12 \mathrm{~K} 1$ & 185 & 80 & $\mathrm{D}$ & $4 \mathrm{~N} / 28 \mathrm{~W}-12 \mathrm{~K} 2$ & 285 & 0 & $\mathrm{D}$ \\
\hline $4 \mathrm{~N} / 28 \mathrm{~W}-12 \mathrm{~K} 1$ & 195 & 0 & $\mathrm{D}$ & $4 \mathrm{~N} / 28 \mathrm{~W}-12 \mathrm{~K} 3$ & 5 & 0 & $\mathrm{D}$ \\
\hline $4 \mathrm{~N} / 28 \mathrm{~W}-12 \mathrm{~K} 1$ & 205 & 0 & $\mathrm{D}$ & $4 \mathrm{~N} / 28 \mathrm{~W}-12 \mathrm{~K} 3$ & 15 & 0 & $\mathrm{D}$ \\
\hline $4 \mathrm{~N} / 28 \mathrm{~W}-12 \mathrm{~K} 1$ & 215 & 0 & $\mathrm{D}$ & $4 \mathrm{~N} / 28 \mathrm{~W}-12 \mathrm{~K} 3$ & 25 & 0 & $\mathrm{D}$ \\
\hline $4 \mathrm{~N} / 28 \mathrm{~W}-12 \mathrm{~K} 1$ & 225 & 0 & $\mathrm{D}$ & $4 \mathrm{~N} / 28 \mathrm{~W}-12 \mathrm{~K} 3$ & 35 & 0 & $\mathrm{D}$ \\
\hline $4 \mathrm{~N} / 28 \mathrm{~W}-12 \mathrm{~K} 1$ & 235 & 10 & $\mathrm{D}$ & $4 \mathrm{~N} / 28 \mathrm{~W}-12 \mathrm{~K} 3$ & 45 & 0 & $\mathrm{D}$ \\
\hline
\end{tabular}


Table B1-1. Calculated percent-coarse intervals for all drillers' logs and e-logs used to define textural properties of the hydrogeologic framework model.-Continued

[Percent-coarse values are calculated for 10-foot intervals for the entire depth of each borehole. State well number is the state of California well identifier; interval midpoint depth is the midpoint of each 10-foot interval, in feet from top of borehole; PC is the percent-coarse value for each interval; log type defines whether the borehole is a drillers' lithology $\log (\mathrm{D})$ or a geophysical e-log (E).]

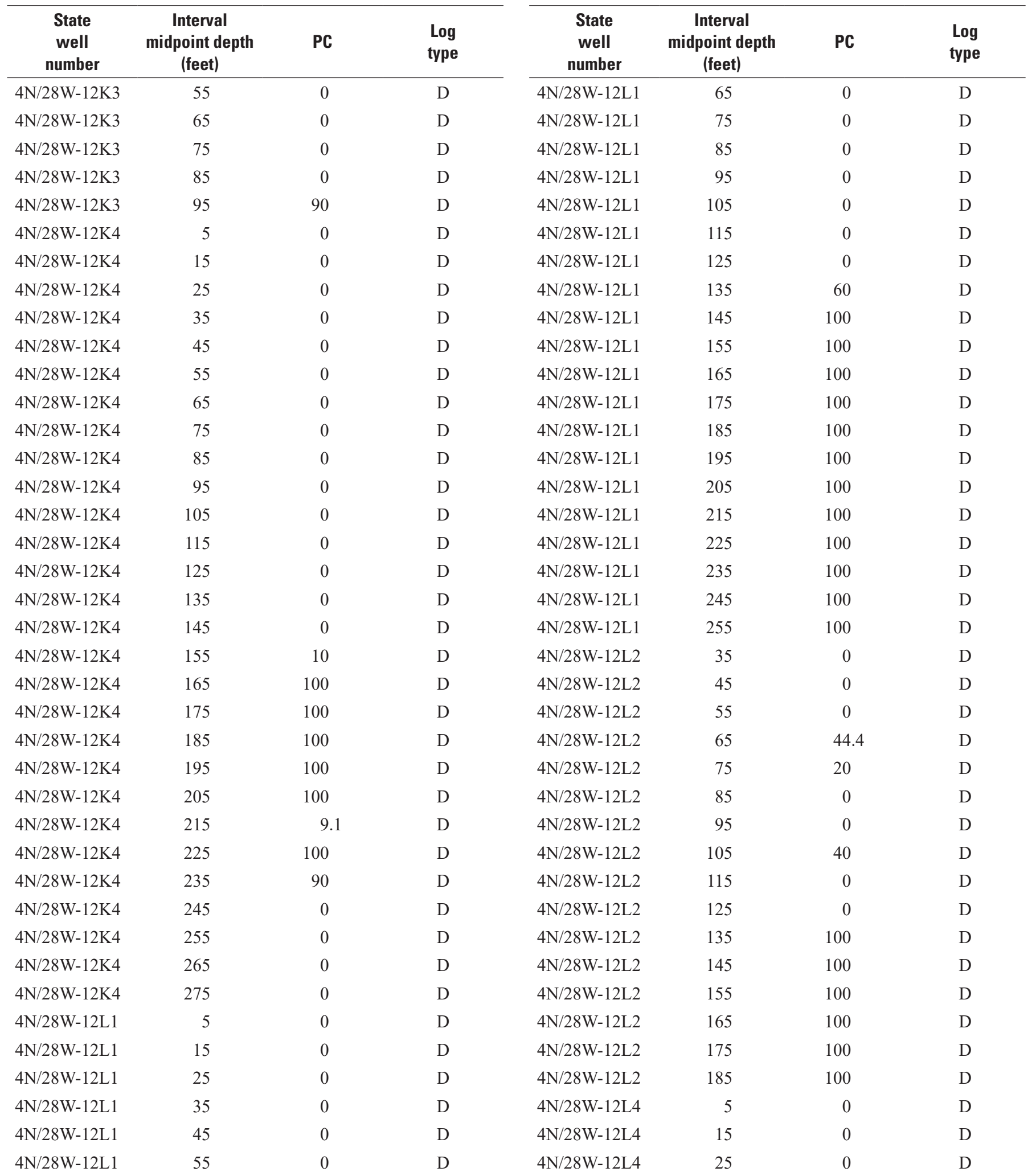


Table B1-1. Calculated percent-coarse intervals for all drillers' logs and e-logs used to define textural properties of the hydrogeologic framework model.-Continued

[Percent-coarse values are calculated for 10-foot intervals for the entire depth of each borehole. State well number is the state of California well identifier; interval midpoint depth is the midpoint of each 10-foot interval, in feet from top of borehole; PC is the percent-coarse value for each interval; log type defines whether the borehole is a drillers' lithology $\log (\mathrm{D})$ or a geophysical e-log (E).]

\begin{tabular}{|c|c|c|c|c|c|c|c|}
\hline $\begin{array}{c}\text { State } \\
\text { well } \\
\text { number }\end{array}$ & $\begin{array}{c}\text { Interval } \\
\text { midpoint depth } \\
\text { (feet) }\end{array}$ & PC & $\begin{array}{l}\text { Log } \\
\text { type }\end{array}$ & $\begin{array}{c}\text { State } \\
\text { well } \\
\text { number }\end{array}$ & $\begin{array}{c}\text { Interval } \\
\text { midpoint depth } \\
\text { (feet) }\end{array}$ & PC & $\begin{array}{l}\text { Log } \\
\text { type }\end{array}$ \\
\hline $4 \mathrm{~N} / 28 \mathrm{~W}-12 \mathrm{~L} 4$ & 35 & 0 & $\mathrm{D}$ & $4 \mathrm{~N} / 28 \mathrm{~W}-12 \mathrm{~L} 5$ & 25 & 0 & $\mathrm{D}$ \\
\hline $4 \mathrm{~N} / 28 \mathrm{~W}-12 \mathrm{~L} 4$ & 45 & 0 & $\mathrm{D}$ & $4 \mathrm{~N} / 28 \mathrm{~W}-12 \mathrm{~L} 5$ & 35 & 0 & $\mathrm{D}$ \\
\hline $4 \mathrm{~N} / 28 \mathrm{~W}-12 \mathrm{~L} 4$ & 55 & 0 & $\mathrm{D}$ & $4 \mathrm{~N} / 28 \mathrm{~W}-12 \mathrm{~L} 5$ & 45 & 0 & $\mathrm{D}$ \\
\hline $4 \mathrm{~N} / 28 \mathrm{~W}-12 \mathrm{~L} 4$ & 65 & 0 & $\mathrm{D}$ & 4N/28W-12L5 & 55 & 0 & $\mathrm{D}$ \\
\hline $4 \mathrm{~N} / 28 \mathrm{~W}-12 \mathrm{~L} 4$ & 85 & 0 & $\mathrm{D}$ & $4 \mathrm{~N} / 28 \mathrm{~W}-12 \mathrm{~L} 5$ & 75 & 0 & $\mathrm{D}$ \\
\hline 4N/28W-12L4 & 95 & 0 & $\mathrm{D}$ & $4 \mathrm{~N} / 28 \mathrm{~W}-12 \mathrm{~L} 5$ & 85 & 0 & $\mathrm{D}$ \\
\hline $4 \mathrm{~N} / 28 \mathrm{~W}-12 \mathrm{~L} 4$ & 105 & 0 & $\mathrm{D}$ & $4 \mathrm{~N} / 28 \mathrm{~W}-12 \mathrm{~L} 5$ & 95 & 0 & $\mathrm{D}$ \\
\hline $4 \mathrm{~N} / 28 \mathrm{~W}-12 \mathrm{~L} 4$ & 115 & 0 & $\mathrm{D}$ & $4 \mathrm{~N} / 28 \mathrm{~W}-12 \mathrm{~L} 5$ & 105 & 0 & $\mathrm{D}$ \\
\hline $4 \mathrm{~N} / 28 \mathrm{~W}-12 \mathrm{~L} 4$ & 155 & 0 & $\mathrm{D}$ & $4 \mathrm{~N} / 28 \mathrm{~W}-12 \mathrm{~L} 5$ & 145 & 100 & $\mathrm{D}$ \\
\hline $4 \mathrm{~N} / 28 \mathrm{~W}-12 \mathrm{~L} 4$ & 165 & 0 & $\mathrm{D}$ & $4 \mathrm{~N} / 28 \mathrm{~W}-12 \mathrm{~L} 5$ & 155 & 100 & $\mathrm{D}$ \\
\hline $4 \mathrm{~N} / 28 \mathrm{~W}-12 \mathrm{~L} 4$ & 175 & 0 & $\mathrm{D}$ & $4 \mathrm{~N} / 28 \mathrm{~W}-12 \mathrm{~L} 5$ & 165 & 100 & $\mathrm{D}$ \\
\hline 4N/28W-12L4 & 185 & 0 & $\mathrm{D}$ & $4 \mathrm{~N} / 28 \mathrm{~W}-12 \mathrm{~L} 5$ & 175 & 100 & $\mathrm{D}$ \\
\hline $4 \mathrm{~N} / 28 \mathrm{~W}-12 \mathrm{~L} 4$ & 195 & 0 & $\mathrm{D}$ & $4 \mathrm{~N} / 28 \mathrm{~W}-12 \mathrm{~L} 5$ & 185 & 100 & $\mathrm{D}$ \\
\hline $4 \mathrm{~N} / 28 \mathrm{~W}-12 \mathrm{~L} 4$ & 205 & 0 & $\mathrm{D}$ & $4 \mathrm{~N} / 28 \mathrm{~W}-12 \mathrm{~L} 5$ & 195 & 100 & $\mathrm{D}$ \\
\hline $4 \mathrm{~N} / 28 \mathrm{~W}-12 \mathrm{~L} 4$ & 215 & 9.1 & $\mathrm{D}$ & 4N/28W-12L5 & 205 & 100 & $\mathrm{D}$ \\
\hline $4 \mathrm{~N} / 28 \mathrm{~W}-12 \mathrm{~L} 4$ & 225 & 10 & $\mathrm{D}$ & $4 \mathrm{~N} / 28 \mathrm{~W}-12 \mathrm{~L} 5$ & 215 & 100 & $\mathrm{D}$ \\
\hline $4 \mathrm{~N} / 28 \mathrm{~W}-12 \mathrm{~L} 4$ & 235 & 0 & $\mathrm{D}$ & $4 \mathrm{~N} / 28 \mathrm{~W}-12 \mathrm{~L} 5$ & 225 & 90 & $\mathrm{D}$ \\
\hline 4N/28W-12L4 & 305 & 0 & $\mathrm{D}$ & $4 \mathrm{~N} / 28 \mathrm{~W}-12 \mathrm{R} 1$ & 55 & 40 & $\mathrm{D}$ \\
\hline $4 \mathrm{~N} / 28 \mathrm{~W}-12 \mathrm{~L} 4$ & 315 & 0 & $\mathrm{D}$ & $4 \mathrm{~N} / 28 \mathrm{~W}-12 \mathrm{R} 1$ & 65 & 0 & $\mathrm{D}$ \\
\hline $4 \mathrm{~N} / 28 \mathrm{~W}-12 \mathrm{~L} 4$ & 325 & 0 & $\mathrm{D}$ & $4 \mathrm{~N} / 28 \mathrm{~W}-12 \mathrm{R} 1$ & 75 & 0 & $\mathrm{D}$ \\
\hline 4N/28W-12L4 & 335 & 90 & $\mathrm{D}$ & $4 \mathrm{~N} / 28 \mathrm{~W}-12 \mathrm{R} 1$ & 85 & 0 & $\mathrm{D}$ \\
\hline $4 \mathrm{~N} / 28 \mathrm{~W}-12 \mathrm{~L} 4$ & 345 & 40 & $\mathrm{D}$ & $4 \mathrm{~N} / 28 \mathrm{~W}-12 \mathrm{R} 1$ & 95 & 0 & $\mathrm{D}$ \\
\hline 4N/28W-12L4 & 355 & 0 & $\mathrm{D}$ & $4 \mathrm{~N} / 28 \mathrm{~W}-12 \mathrm{R} 1$ & 105 & 80 & $\mathrm{D}$ \\
\hline $4 \mathrm{~N} / 28 \mathrm{~W}-12 \mathrm{~L} 4$ & 365 & 80 & $\mathrm{D}$ & $4 \mathrm{~N} / 28 \mathrm{~W}-12 \mathrm{R} 1$ & 115 & 60 & $\mathrm{D}$ \\
\hline 4N/28W-12L4 & 375 & 66.7 & $\mathrm{D}$ & $4 \mathrm{~N} / 28 \mathrm{~W}-12 \mathrm{R} 1$ & 125 & 0 & $\mathrm{D}$ \\
\hline $4 \mathrm{~N} / 28 \mathrm{~W}-12 \mathrm{~L} 4$ & 385 & 0 & $\mathrm{D}$ & $4 \mathrm{~N} / 28 \mathrm{~W}-12 \mathrm{R} 1$ & 135 & 0 & $\mathrm{D}$ \\
\hline $4 \mathrm{~N} / 28 \mathrm{~W}-12 \mathrm{~L} 4$ & 395 & 0 & $\mathrm{D}$ & $4 \mathrm{~N} / 28 \mathrm{~W}-12 \mathrm{R} 1$ & 145 & 0 & $\mathrm{D}$ \\
\hline $4 \mathrm{~N} / 28 \mathrm{~W}-12 \mathrm{~L} 5$ & 5 & 72.7 & $\mathrm{D}$ & $4 \mathrm{~N} / 28 \mathrm{~W}-12 \mathrm{R} 1$ & 155 & 0 & $\mathrm{D}$ \\
\hline $4 \mathrm{~N} / 28 \mathrm{~W}-12 \mathrm{~L} 5$ & 15 & 0 & $\mathrm{D}$ & $4 \mathrm{~N} / 28 \mathrm{~W}-12 \mathrm{R} 1$ & 165 & 0 & $\mathrm{D}$ \\
\hline
\end{tabular}


Table B1-1. Calculated percent-coarse intervals for all drillers' logs and e-logs used to define textural properties of the hydrogeologic framework model.-Continued

[Percent-coarse values are calculated for 10-foot intervals for the entire depth of each borehole. State well number is the state of California well identifier; interval midpoint depth is the midpoint of each 10-foot interval, in feet from top of borehole; PC is the percent-coarse value for each interval; log type defines whether the borehole is a drillers' lithology $\log (\mathrm{D})$ or a geophysical e-log (E).]

\begin{tabular}{|c|c|c|c|c|c|c|c|}
\hline $\begin{array}{c}\text { State } \\
\text { well } \\
\text { number }\end{array}$ & $\begin{array}{c}\text { Interval } \\
\text { midpoint depth } \\
\text { (feet) }\end{array}$ & PC & $\begin{array}{l}\text { Log } \\
\text { type }\end{array}$ & $\begin{array}{c}\text { State } \\
\text { well } \\
\text { number }\end{array}$ & $\begin{array}{c}\text { Interval } \\
\text { midpoint depth } \\
\text { (feet) }\end{array}$ & PC & $\begin{array}{l}\text { Log } \\
\text { type }\end{array}$ \\
\hline $4 \mathrm{~N} / 28 \mathrm{~W}-12 \mathrm{R} 1$ & 175 & 0 & $\mathrm{D}$ & $4 \mathrm{~N} / 28 \mathrm{~W}-12 \mathrm{R} 2$ & 205 & 55.6 & $\mathrm{D}$ \\
\hline $4 \mathrm{~N} / 28 \mathrm{~W}-12 \mathrm{R} 1$ & 185 & 0 & $\mathrm{D}$ & $4 \mathrm{~N} / 28 \mathrm{~W}-12 \mathrm{R} 2$ & 215 & 100 & $\mathrm{D}$ \\
\hline $4 \mathrm{~N} / 28 \mathrm{~W}-12 \mathrm{R} 1$ & 195 & 0 & $\mathrm{D}$ & $4 \mathrm{~N} / 28 \mathrm{~W}-12 \mathrm{R} 2$ & 225 & 100 & $\mathrm{D}$ \\
\hline $4 \mathrm{~N} / 28 \mathrm{~W}-12 \mathrm{R} 1$ & 205 & 55.6 & $\mathrm{D}$ & $4 \mathrm{~N} / 28 \mathrm{~W}-12 \mathrm{R} 2$ & 235 & 100 & $\mathrm{D}$ \\
\hline $4 \mathrm{~N} / 28 \mathrm{~W}-12 \mathrm{R} 1$ & 225 & 40 & $\mathrm{D}$ & $4 \mathrm{~N} / 28 \mathrm{~W}-12 \mathrm{R} 2$ & 255 & 100 & $\mathrm{D}$ \\
\hline $4 \mathrm{~N} / 28 \mathrm{~W}-12 \mathrm{R} 1$ & 235 & 0 & $\mathrm{D}$ & $4 \mathrm{~N} / 28 \mathrm{~W}-12 \mathrm{R} 2$ & 265 & 100 & $\mathrm{D}$ \\
\hline $4 \mathrm{~N} / 28 \mathrm{~W}-12 \mathrm{R} 1$ & 245 & 100 & $\mathrm{D}$ & $4 \mathrm{~N} / 28 \mathrm{~W}-12 \mathrm{R} 2$ & 275 & 0 & $\mathrm{D}$ \\
\hline $4 \mathrm{~N} / 28 \mathrm{~W}-12 \mathrm{R} 1$ & 255 & 100 & $\mathrm{D}$ & $4 \mathrm{~N} / 28 \mathrm{~W}-12 \mathrm{R} 2$ & 285 & 90 & $\mathrm{D}$ \\
\hline $4 \mathrm{~N} / 28 \mathrm{~W}-12 \mathrm{R} 1$ & 295 & 63.6 & $\mathrm{D}$ & $4 \mathrm{~N} / 28 \mathrm{~W}-12 \mathrm{R} 2$ & 325 & 100 & $\mathrm{D}$ \\
\hline $4 \mathrm{~N} / 28 \mathrm{~W}-12 \mathrm{R} 1$ & 305 & 0 & $\mathrm{D}$ & 4N/27W-8M6 & 45 & 0 & $\mathrm{E}$ \\
\hline $4 \mathrm{~N} / 28 \mathrm{~W}-12 \mathrm{R} 1$ & 315 & 0 & $\mathrm{D}$ & 4N/27W-8M6 & 55 & 0 & $\mathrm{E}$ \\
\hline $4 \mathrm{~N} / 28 \mathrm{~W}-12 \mathrm{R} 1$ & 325 & 0 & $\mathrm{D}$ & 4N/27W-8M6 & 65 & 0 & $\mathrm{E}$ \\
\hline 4N/28W-12R1 & 335 & 0 & $\mathrm{D}$ & 4N/27W-8M6 & 75 & 0 & $\mathrm{E}$ \\
\hline $4 \mathrm{~N} / 28 \mathrm{~W}-12 \mathrm{R} 1$ & 345 & 0 & $\mathrm{D}$ & 4N/27W-8M6 & 85 & 0 & $\mathrm{E}$ \\
\hline $4 \mathrm{~N} / 28 \mathrm{~W}-12 \mathrm{R} 1$ & 355 & 0 & $\mathrm{D}$ & 4N/27W-8M6 & 95 & 0 & $\mathrm{E}$ \\
\hline $4 \mathrm{~N} / 28 \mathrm{~W}-12 \mathrm{R} 2$ & 5 & 100 & $\mathrm{D}$ & 4N/27W-8M6 & 105 & 0 & $\mathrm{E}$ \\
\hline $4 \mathrm{~N} / 28 \mathrm{~W}-12 \mathrm{R} 2$ & 15 & 100 & $\mathrm{D}$ & 4N/27W-8M6 & 115 & 0 & $\mathrm{E}$ \\
\hline $4 \mathrm{~N} / 28 \mathrm{~W}-12 \mathrm{R} 2$ & 85 & 50 & $\mathrm{D}$ & 4N/27W-8M6 & 185 & 0 & $\mathrm{E}$ \\
\hline $4 \mathrm{~N} / 28 \mathrm{~W}-12 \mathrm{R} 2$ & 95 & 100 & $\mathrm{D}$ & $4 \mathrm{~N} / 27 \mathrm{~W}-8 \mathrm{M} 6$ & 195 & 0 & $\mathrm{E}$ \\
\hline $4 \mathrm{~N} / 28 \mathrm{~W}-12 \mathrm{R} 2$ & 105 & 40 & $\mathrm{D}$ & 4N/27W-8M6 & 205 & 0 & $\mathrm{E}$ \\
\hline $4 \mathrm{~N} / 28 \mathrm{~W}-12 \mathrm{R} 2$ & 115 & 0 & $\mathrm{D}$ & 4N/27W-8M6 & 215 & 0 & $\mathrm{E}$ \\
\hline $4 \mathrm{~N} / 28 \mathrm{~W}-12 \mathrm{R} 2$ & 125 & 0 & $\mathrm{D}$ & 4N/27W-8M6 & 225 & 0 & $\mathrm{E}$ \\
\hline $4 \mathrm{~N} / 28 \mathrm{~W}-12 \mathrm{R} 2$ & 135 & 0 & $\mathrm{D}$ & 4N/27W-8M6 & 235 & 0 & $\mathrm{E}$ \\
\hline $4 \mathrm{~N} / 28 \mathrm{~W}-12 \mathrm{R} 2$ & 145 & 0 & $\mathrm{D}$ & 4N/27W-8M6 & 245 & 20 & $\mathrm{E}$ \\
\hline $4 \mathrm{~N} / 28 \mathrm{~W}-12 \mathrm{R} 2$ & 155 & 0 & $\mathrm{D}$ & 4N/27W-8M6 & 255 & 0 & $\mathrm{E}$ \\
\hline $4 \mathrm{~N} / 28 \mathrm{~W}-12 \mathrm{R} 2$ & 165 & 0 & $\mathrm{D}$ & 4N/27W-8M6 & 265 & 70 & $\mathrm{E}$ \\
\hline $4 \mathrm{~N} / 28 \mathrm{~W}-12 \mathrm{R} 2$ & 175 & 0 & $\mathrm{D}$ & 4N/27W-8M6 & 275 & 90 & $\mathrm{E}$ \\
\hline $4 \mathrm{~N} / 28 \mathrm{~W}-12 \mathrm{R} 2$ & 185 & 0 & $\mathrm{D}$ & 4N/27W-8M6 & 285 & 0 & $\mathrm{E}$ \\
\hline $4 \mathrm{~N} / 28 \mathrm{~W}-12 \mathrm{R} 2$ & 195 & 0 & $\mathrm{D}$ & $4 \mathrm{~N} / 27 \mathrm{~W}-8 \mathrm{M} 6$ & 295 & 0 & $\mathrm{E}$ \\
\hline
\end{tabular}


Table B1-1. Calculated percent-coarse intervals for all drillers' logs and e-logs used to define textural properties of the hydrogeologic framework model.-Continued

[Percent-coarse values are calculated for 10-foot intervals for the entire depth of each borehole. State well number is the state of California well identifier; interval midpoint depth is the midpoint of each 10-foot interval, in feet from top of borehole; PC is the percent-coarse value for each interval; log type defines whether the borehole is a drillers' lithology log (D) or a geophysical e-log (E).]

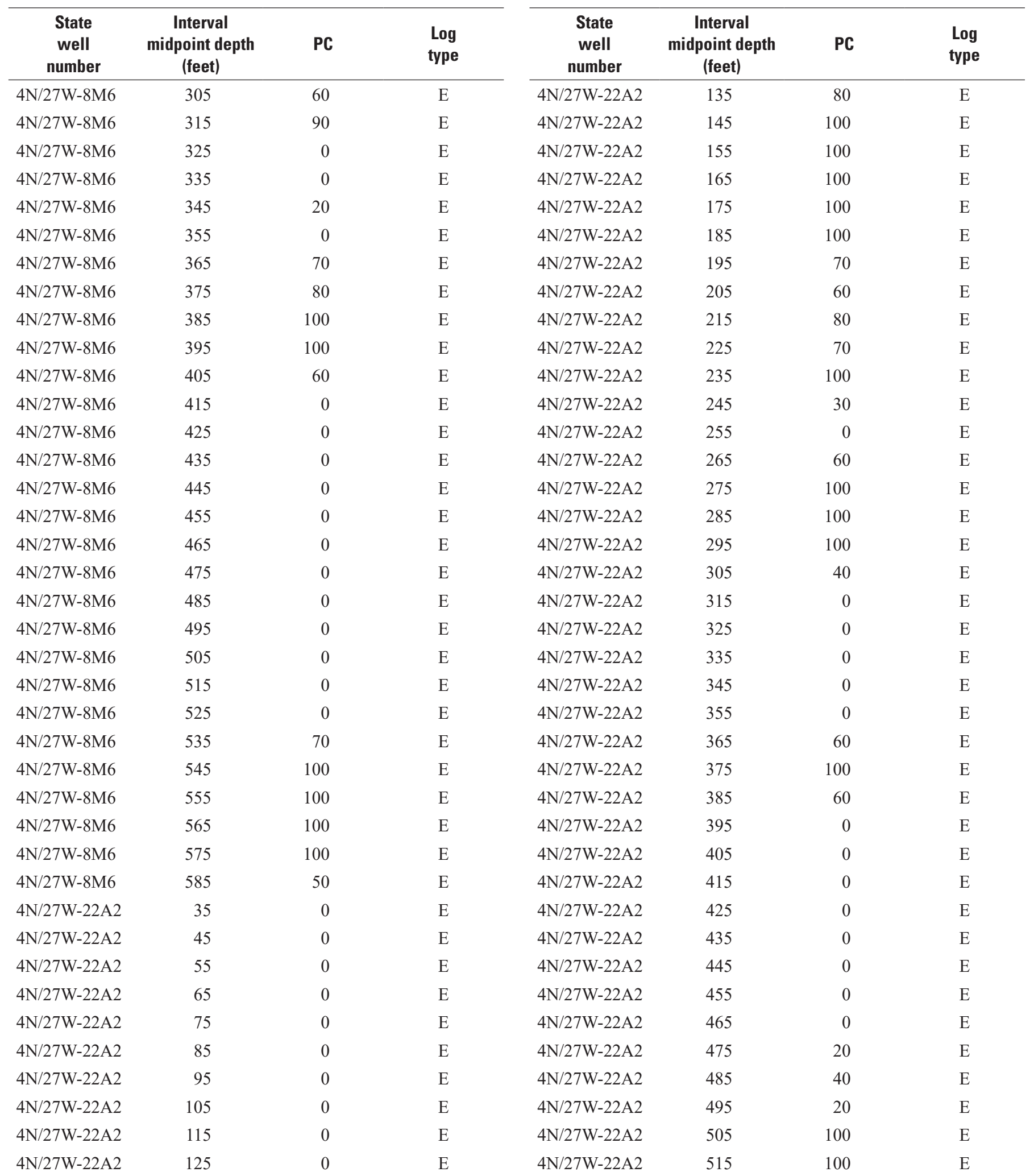


Table B1-1. Calculated percent-coarse intervals for all drillers' logs and e-logs used to define textural properties of the hydrogeologic framework model.-Continued

[Percent-coarse values are calculated for 10-foot intervals for the entire depth of each borehole. State well number is the state of California well identifier; interval midpoint depth is the midpoint of each 10-foot interval, in feet from top of borehole; PC is the percent-coarse value for each interval; log type defines whether the borehole is a drillers' lithology $\log (\mathrm{D})$ or a geophysical e-log (E).]

\begin{tabular}{|c|c|c|c|c|c|c|c|}
\hline $\begin{array}{c}\text { State } \\
\text { well } \\
\text { number }\end{array}$ & $\begin{array}{c}\text { Interval } \\
\text { midpoint depth } \\
\text { (feet) }\end{array}$ & PC & $\begin{array}{l}\text { Log } \\
\text { type }\end{array}$ & $\begin{array}{c}\text { State } \\
\text { well } \\
\text { number }\end{array}$ & $\begin{array}{c}\text { Interval } \\
\text { midpoint depth } \\
\text { (feet) }\end{array}$ & PC & $\begin{array}{l}\text { Log } \\
\text { type }\end{array}$ \\
\hline $4 \mathrm{~N} / 27 \mathrm{~W}-22 \mathrm{~A} 2$ & 525 & 100 & $\mathrm{E}$ & $4 \mathrm{~N} / 27 \mathrm{~W}-18 \mathrm{Q} 4$ & 235 & 0 & $\mathrm{E}$ \\
\hline $4 \mathrm{~N} / 27 \mathrm{~W}-22 \mathrm{~A} 2$ & 535 & 100 & $\mathrm{E}$ & $4 \mathrm{~N} / 28 \mathrm{~W}-12 \mathrm{~L} 6$ & 35 & 0 & $\mathrm{E}$ \\
\hline $4 \mathrm{~N} / 27 \mathrm{~W}-22 \mathrm{~A} 2$ & 545 & 10 & $\mathrm{E}$ & $4 \mathrm{~N} / 28 \mathrm{~W}-12 \mathrm{~L} 6$ & 45 & 0 & $\mathrm{E}$ \\
\hline $4 \mathrm{~N} / 27 \mathrm{~W}-22 \mathrm{~A} 2$ & 555 & 0 & $\mathrm{E}$ & $4 \mathrm{~N} / 28 \mathrm{~W}-12 \mathrm{~L} 6$ & 55 & 0 & $\mathrm{E}$ \\
\hline $4 \mathrm{~N} / 27 \mathrm{~W}-22 \mathrm{~A} 2$ & 575 & 100 & $\mathrm{E}$ & 4N/28W-12L6 & 75 & 0 & $\mathrm{E}$ \\
\hline $4 \mathrm{~N} / 27 \mathrm{~W}-22 \mathrm{~A} 2$ & 585 & 40 & $\mathrm{E}$ & $4 \mathrm{~N} / 28 \mathrm{~W}-12 \mathrm{~L} 6$ & 85 & 0 & $\mathrm{E}$ \\
\hline $4 \mathrm{~N} / 27 \mathrm{~W}-22 \mathrm{~A} 2$ & 595 & 0 & $\mathrm{E}$ & $4 \mathrm{~N} / 28 \mathrm{~W}-12 \mathrm{~L} 6$ & 95 & 0 & $\mathrm{E}$ \\
\hline $4 \mathrm{~N} / 27 \mathrm{~W}-22 \mathrm{~A} 2$ & 605 & 0 & $\mathrm{E}$ & 4N/28W-12L6 & 105 & 0 & $\mathrm{E}$ \\
\hline $4 \mathrm{~N} / 27 \mathrm{~W}-22 \mathrm{~A} 2$ & 645 & 0 & $\mathrm{E}$ & 4N/28W-12L6 & 145 & 0 & $\mathrm{E}$ \\
\hline $4 \mathrm{~N} / 27 \mathrm{~W}-22 \mathrm{~A} 2$ & 655 & 0 & $\mathrm{E}$ & 4N/28W-12L6 & 155 & 0 & $\mathrm{E}$ \\
\hline $4 \mathrm{~N} / 27 \mathrm{~W}-22 \mathrm{~A} 2$ & 665 & 100 & $\mathrm{E}$ & $4 \mathrm{~N} / 28 \mathrm{~W}-12 \mathrm{~L} 6$ & 165 & 0 & $\mathrm{E}$ \\
\hline $4 \mathrm{~N} / 27 \mathrm{~W}-22 \mathrm{~A} 2$ & 675 & 100 & $\mathrm{E}$ & 4N/28W-12L6 & 175 & 40 & $\mathrm{E}$ \\
\hline $4 \mathrm{~N} / 27 \mathrm{~W}-22 \mathrm{~A} 2$ & 685 & 100 & $\mathrm{E}$ & $4 \mathrm{~N} / 28 \mathrm{~W}-12 \mathrm{~L} 6$ & 185 & 90 & $\mathrm{E}$ \\
\hline $4 \mathrm{~N} / 27 \mathrm{~W}-22 \mathrm{~A} 2$ & 695 & 100 & $\mathrm{E}$ & 4N/28W-12L6 & 195 & 0 & $\mathrm{E}$ \\
\hline 4N/27W-18Q4 & 25 & 0 & $\mathrm{E}$ & $4 \mathrm{~N} / 28 \mathrm{~W}-12 \mathrm{~L} 6$ & 205 & 0 & $\mathrm{E}$ \\
\hline 4N/27W-18Q4 & 35 & 0 & $\mathrm{E}$ & $4 \mathrm{~N} / 28 \mathrm{~W}-12 \mathrm{~L} 6$ & 215 & 0 & $\mathrm{E}$ \\
\hline 4N/27W-18Q4 & 45 & 0 & $\mathrm{E}$ & 4N/28W-12L6 & 225 & 0 & $\mathrm{E}$ \\
\hline 4N/27W-18Q4 & 115 & 80 & $\mathrm{E}$ & $4 \mathrm{~N} / 28 \mathrm{~W}-12 \mathrm{~L} 6$ & 295 & 100 & $\mathrm{E}$ \\
\hline 4N/27W-18Q4 & 125 & 0 & $\mathrm{E}$ & 4N/28W-12L6 & 305 & 100 & $\mathrm{E}$ \\
\hline 4N/27W-18Q4 & 135 & 0 & $\mathrm{E}$ & 4N/28W-12L6 & 315 & 80 & $\mathrm{E}$ \\
\hline 4N/27W-18Q4 & 145 & 0 & $\mathrm{E}$ & 4N/28W-12L6 & 325 & 0 & $\mathrm{E}$ \\
\hline 4N/27W-18Q4 & 155 & 0 & $\mathrm{E}$ & 4N/28W-12L6 & 335 & 30 & $\mathrm{E}$ \\
\hline 4N/27W-18Q4 & 165 & 0 & $\mathrm{E}$ & 4N/28W-12L6 & 345 & 100 & $\mathrm{E}$ \\
\hline 4N/27W-18Q4 & 175 & 0 & $\mathrm{E}$ & 4N/28W-12L6 & 355 & 100 & $\mathrm{E}$ \\
\hline $4 \mathrm{~N} / 27 \mathrm{~W}-18 \mathrm{Q} 4$ & 185 & 0 & $\mathrm{E}$ & $4 \mathrm{~N} / 28 \mathrm{~W}-12 \mathrm{~L} 6$ & 365 & 100 & $\mathrm{E}$ \\
\hline 4N/27W-18Q4 & 195 & 0 & $\mathrm{E}$ & 4N/28W-12L6 & 375 & 70 & $\mathrm{E}$ \\
\hline 4N/27W-18Q4 & 205 & 0 & $\mathrm{E}$ & 4N/28W-12L6 & 385 & 0 & $\mathrm{E}$ \\
\hline 4N/27W-18Q4 & 215 & 0 & $\mathrm{E}$ & $4 \mathrm{~N} / 27 \mathrm{~W}-24 \mathrm{D} 2$ & 55 & 0 & $\mathrm{E}$ \\
\hline $4 \mathrm{~N} / 27 \mathrm{~W}-18 \mathrm{Q} 4$ & 225 & 0 & $\mathrm{E}$ & $4 \mathrm{~N} / 27 \mathrm{~W}-24 \mathrm{D} 2$ & 65 & 0 & $\mathrm{E}$ \\
\hline
\end{tabular}


Table B1-1. Calculated percent-coarse intervals for all drillers' logs and e-logs used to define textural properties of the hydrogeologic framework model.-Continued

[Percent-coarse values are calculated for 10-foot intervals for the entire depth of each borehole. State well number is the state of California well identifier; interval midpoint depth is the midpoint of each 10-foot interval, in feet from top of borehole; PC is the percent-coarse value for each interval; log type defines whether the borehole is a drillers' lithology log (D) or a geophysical e-log (E).]

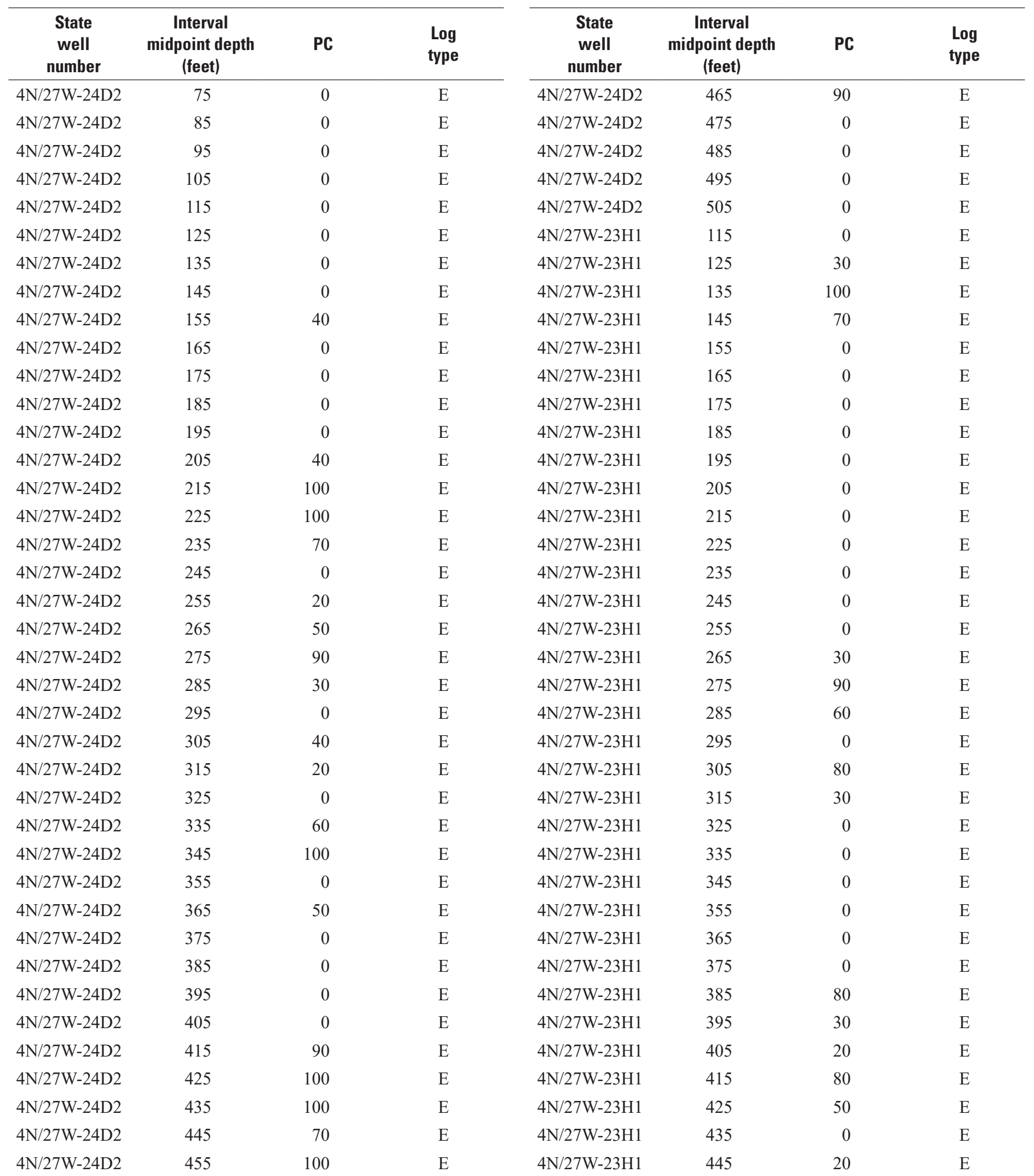


Table B1-1. Calculated percent-coarse intervals for all drillers' logs and e-logs used to define textural properties of the hydrogeologic framework model.-Continued

[Percent-coarse values are calculated for 10-foot intervals for the entire depth of each borehole. State well number is the state of California well identifier; interval midpoint depth is the midpoint of each 10-foot interval, in feet from top of borehole; PC is the percent-coarse value for each interval; log type defines whether the borehole is a drillers' lithology $\log (\mathrm{D})$ or a geophysical e-log (E).]

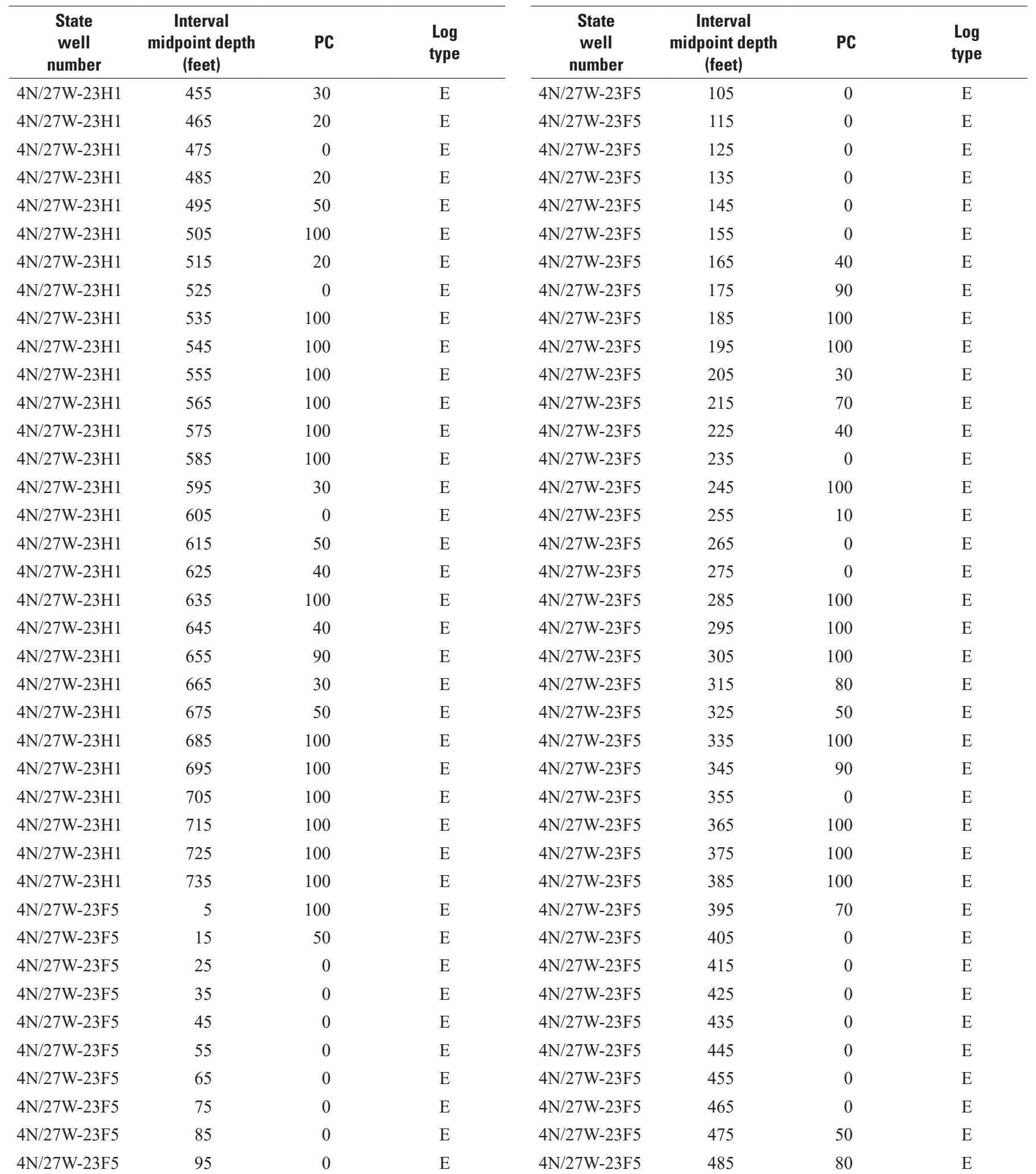


Table B1-1. Calculated percent-coarse intervals for all drillers' logs and e-logs used to define textural properties of the hydrogeologic framework model.-Continued

[Percent-coarse values are calculated for 10-foot intervals for the entire depth of each borehole. State well number is the state of California well identifier; interval midpoint depth is the midpoint of each 10-foot interval, in feet from top of borehole; PC is the percent-coarse value for each interval; log type defines whether the borehole is a drillers' lithology log (D) or a geophysical e-log (E).]

\begin{tabular}{|c|c|c|c|c|c|c|c|}
\hline $\begin{array}{c}\text { State } \\
\text { well } \\
\text { number }\end{array}$ & $\begin{array}{c}\text { Interval } \\
\text { midpoint depth } \\
\text { (feet) }\end{array}$ & PC & $\begin{array}{l}\text { Log } \\
\text { type }\end{array}$ & $\begin{array}{c}\text { State } \\
\text { well } \\
\text { number }\end{array}$ & $\begin{array}{c}\text { Interval } \\
\text { midpoint depth } \\
\text { (feet) }\end{array}$ & PC & $\begin{array}{l}\text { Log } \\
\text { type }\end{array}$ \\
\hline $4 \mathrm{~N} / 27 \mathrm{~W}-23 \mathrm{~F} 5$ & 495 & 100 & $\mathrm{E}$ & $4 \mathrm{~N} / 27 \mathrm{~W}-23 \mathrm{E} 6$ & 125 & 0 & $\mathrm{E}$ \\
\hline $4 \mathrm{~N} / 27 \mathrm{~W}-23 \mathrm{~F} 5$ & 505 & 70 & $\mathrm{E}$ & $4 \mathrm{~N} / 27 \mathrm{~W}-23 \mathrm{E} 6$ & 135 & 80 & $\mathrm{E}$ \\
\hline $4 \mathrm{~N} / 27 \mathrm{~W}-23 \mathrm{~F} 5$ & 515 & 80 & $\mathrm{E}$ & $4 \mathrm{~N} / 27 \mathrm{~W}-23 \mathrm{E} 6$ & 145 & 100 & $\mathrm{E}$ \\
\hline $4 \mathrm{~N} / 27 \mathrm{~W}-23 \mathrm{~F} 5$ & 525 & 60 & $\mathrm{E}$ & 4N/27W-23E6 & 155 & 100 & $\mathrm{E}$ \\
\hline 4N/27W-23F5 & 545 & 100 & $\mathrm{E}$ & 4N/27W-23E6 & 175 & 0 & $\mathrm{E}$ \\
\hline $4 \mathrm{~N} / 27 \mathrm{~W}-23 \mathrm{~F} 5$ & 555 & 100 & $\mathrm{E}$ & 4N/27W-23E6 & 185 & 0 & $\mathrm{E}$ \\
\hline $4 \mathrm{~N} / 27 \mathrm{~W}-23 \mathrm{~F} 5$ & 565 & 100 & $\mathrm{E}$ & $4 \mathrm{~N} / 27 \mathrm{~W}-23 \mathrm{E} 6$ & 195 & 0 & $\mathrm{E}$ \\
\hline $4 \mathrm{~N} / 27 \mathrm{~W}-23 \mathrm{~F} 5$ & 575 & 100 & $\mathrm{E}$ & $4 \mathrm{~N} / 27 \mathrm{~W}-23 \mathrm{E} 6$ & 205 & 0 & $\mathrm{E}$ \\
\hline $4 \mathrm{~N} / 27 \mathrm{~W}-23 \mathrm{~F} 5$ & 615 & 100 & $\mathrm{E}$ & $4 \mathrm{~N} / 27 \mathrm{~W}-23 \mathrm{E} 6$ & 245 & 0 & $\mathrm{E}$ \\
\hline $4 \mathrm{~N} / 27 \mathrm{~W}-23 \mathrm{~F} 5$ & 625 & 40 & $\mathrm{E}$ & $4 \mathrm{~N} / 27 \mathrm{~W}-23 \mathrm{E} 6$ & 255 & 90 & $\mathrm{E}$ \\
\hline $4 \mathrm{~N} / 27 \mathrm{~W}-23 \mathrm{~F} 5$ & 635 & 0 & $\mathrm{E}$ & $4 \mathrm{~N} / 27 \mathrm{~W}-23 \mathrm{E} 6$ & 265 & 100 & $\mathrm{E}$ \\
\hline $4 \mathrm{~N} / 27 \mathrm{~W}-23 \mathrm{~F} 5$ & 645 & 10 & $\mathrm{E}$ & 4N/27W-23E6 & 275 & 100 & $\mathrm{E}$ \\
\hline 4N/27W-23F5 & 655 & 60 & $\mathrm{E}$ & 4N/27W-23E6 & 285 & 20 & $\mathrm{E}$ \\
\hline $4 \mathrm{~N} / 27 \mathrm{~W}-23 \mathrm{~F} 5$ & 665 & 100 & $\mathrm{E}$ & $4 \mathrm{~N} / 27 \mathrm{~W}-23 \mathrm{E} 6$ & 295 & 40 & $\mathrm{E}$ \\
\hline $4 \mathrm{~N} / 27 \mathrm{~W}-23 \mathrm{~F} 5$ & 675 & 100 & $\mathrm{E}$ & 4N/27W-23E6 & 305 & 70 & $\mathrm{E}$ \\
\hline $4 \mathrm{~N} / 27 \mathrm{~W}-23 \mathrm{~F} 5$ & 685 & 100 & $\mathrm{E}$ & $4 \mathrm{~N} / 27 \mathrm{~W}-23 \mathrm{E} 6$ & 315 & 100 & $\mathrm{E}$ \\
\hline $4 \mathrm{~N} / 27 \mathrm{~W}-23 \mathrm{~F} 5$ & 695 & 100 & $\mathrm{E}$ & $4 \mathrm{~N} / 27 \mathrm{~W}-23 \mathrm{E} 6$ & 325 & 70 & $\mathrm{E}$ \\
\hline $4 \mathrm{~N} / 27 \mathrm{~W}-23 \mathrm{~F} 5$ & 765 & 100 & $\mathrm{E}$ & 4N/27W-23E6 & 395 & 0 & $\mathrm{E}$ \\
\hline $4 \mathrm{~N} / 27 \mathrm{~W}-23 \mathrm{~F} 5$ & 775 & 100 & $\mathrm{E}$ & 4N/27W-23E6 & 405 & 0 & $\mathrm{E}$ \\
\hline $4 \mathrm{~N} / 27 \mathrm{~W}-23 \mathrm{~F} 5$ & 785 & 100 & $\mathrm{E}$ & $4 \mathrm{~N} / 27 \mathrm{~W}-23 \mathrm{E} 6$ & 415 & 60 & $\mathrm{E}$ \\
\hline 4N/27W-23E6 & 35 & 100 & $\mathrm{E}$ & 4N/27W-23E6 & 425 & 0 & $\mathrm{E}$ \\
\hline $4 \mathrm{~N} / 27 \mathrm{~W}-23 \mathrm{E} 6$ & 45 & 60 & $\mathrm{E}$ & $4 \mathrm{~N} / 27 \mathrm{~W}-23 \mathrm{E} 6$ & 435 & 0 & $\mathrm{E}$ \\
\hline $4 \mathrm{~N} / 27 \mathrm{~W}-23 \mathrm{E} 6$ & 55 & 20 & $\mathrm{E}$ & 4N/27W-23E6 & 445 & 60 & $\mathrm{E}$ \\
\hline $4 \mathrm{~N} / 27 \mathrm{~W}-23 \mathrm{E} 6$ & 65 & 10 & $\mathrm{E}$ & 4N/27W-23E6 & 455 & 0 & $\mathrm{E}$ \\
\hline 4N/27W-23E6 & 75 & 100 & $\mathrm{E}$ & $4 \mathrm{~N} / 27 \mathrm{~W}-23 \mathrm{E} 6$ & 465 & 0 & $\mathrm{E}$ \\
\hline 4N/27W-23E6 & 85 & 100 & $\mathrm{E}$ & 4N/27W-23E6 & 475 & 0 & $\mathrm{E}$ \\
\hline $4 \mathrm{~N} / 27 \mathrm{~W}-23 \mathrm{E} 6$ & 95 & 100 & $\mathrm{E}$ & $4 \mathrm{~N} / 27 \mathrm{~W}-23 \mathrm{E} 6$ & 485 & 0 & $\mathrm{E}$ \\
\hline $4 \mathrm{~N} / 27 \mathrm{~W}-23 \mathrm{E} 6$ & 105 & 70 & $\mathrm{E}$ & $4 \mathrm{~N} / 27 \mathrm{~W}-23 \mathrm{E} 6$ & 495 & 0 & $\mathrm{E}$ \\
\hline 4N/27W-23E6 & 115 & 100 & $\mathrm{E}$ & $4 \mathrm{~N} / 27 \mathrm{~W}-23 \mathrm{E} 6$ & 505 & 100 & $\mathrm{E}$ \\
\hline
\end{tabular}


Table B1-1. Calculated percent-coarse intervals for all drillers' logs and e-logs used to define textural properties of the hydrogeologic framework model.-Continued

[Percent-coarse values are calculated for 10-foot intervals for the entire depth of each borehole. State well number is the state of California well identifier; interval midpoint depth is the midpoint of each 10-foot interval, in feet from top of borehole; PC is the percent-coarse value for each interval; log type defines whether the borehole is a drillers' lithology $\log (\mathrm{D})$ or a geophysical e-log (E).]

\begin{tabular}{|c|c|c|c|c|c|c|c|}
\hline $\begin{array}{c}\text { State } \\
\text { well } \\
\text { number }\end{array}$ & $\begin{array}{c}\text { Interval } \\
\text { midpoint depth } \\
\text { (feet) }\end{array}$ & PC & $\begin{array}{l}\text { Log } \\
\text { type }\end{array}$ & $\begin{array}{c}\text { State } \\
\text { well } \\
\text { number }\end{array}$ & $\begin{array}{c}\text { Interval } \\
\text { midpoint depth } \\
\text { (feet) }\end{array}$ & PC & $\begin{array}{l}\text { Log } \\
\text { type }\end{array}$ \\
\hline 4N/27W-23E6 & 515 & 100 & $\mathrm{E}$ & $4 \mathrm{~N} / 27 \mathrm{~W}-23 \mathrm{E} 1$ & 215 & 10 & $\mathrm{E}$ \\
\hline 4N/27W-23E6 & 525 & 100 & $\mathrm{E}$ & $4 \mathrm{~N} / 27 \mathrm{~W}-23 \mathrm{E} 1$ & 225 & 100 & $\mathrm{E}$ \\
\hline 4N/27W-23E6 & 535 & 0 & $\mathrm{E}$ & $4 \mathrm{~N} / 27 \mathrm{~W}-23 \mathrm{E} 1$ & 235 & 20 & $\mathrm{E}$ \\
\hline $4 \mathrm{~N} / 27 \mathrm{~W}-23 \mathrm{E} 6$ & 545 & 0 & $\mathrm{E}$ & $4 \mathrm{~N} / 27 \mathrm{~W}-23 \mathrm{E} 1$ & 245 & 0 & $\mathrm{E}$ \\
\hline 4N/27W-23E6 & 565 & 60 & $\mathrm{E}$ & $4 \mathrm{~N} / 27 \mathrm{~W}-23 \mathrm{E} 1$ & 265 & 100 & $\mathrm{E}$ \\
\hline 4N/27W-23E6 & 575 & 0 & $\mathrm{E}$ & $4 \mathrm{~N} / 27 \mathrm{~W}-23 \mathrm{E} 1$ & 275 & 100 & $\mathrm{E}$ \\
\hline 4N/27W-23E6 & 585 & 0 & $\mathrm{E}$ & $4 \mathrm{~N} / 27 \mathrm{~W}-23 \mathrm{E} 1$ & 285 & 50 & $\mathrm{E}$ \\
\hline 4N/27W-23E6 & 595 & 0 & $\mathrm{E}$ & $4 \mathrm{~N} / 27 \mathrm{~W}-23 \mathrm{E} 1$ & 295 & 0 & $\mathrm{E}$ \\
\hline 4N/27W-23E6 & 635 & 0 & $\mathrm{E}$ & $4 \mathrm{~N} / 27 \mathrm{~W}-23 \mathrm{E} 1$ & 335 & 70 & $\mathrm{E}$ \\
\hline $4 \mathrm{~N} / 27 \mathrm{~W}-23 \mathrm{E} 6$ & 645 & 30 & $\mathrm{E}$ & $4 \mathrm{~N} / 27 \mathrm{~W}-23 \mathrm{E} 1$ & 345 & 100 & $\mathrm{E}$ \\
\hline 4N/27W-23E6 & 655 & 100 & $\mathrm{E}$ & $4 \mathrm{~N} / 27 \mathrm{~W}-23 \mathrm{E} 1$ & 355 & 100 & $\mathrm{E}$ \\
\hline $4 \mathrm{~N} / 27 \mathrm{~W}-23 \mathrm{E} 6$ & 665 & 100 & $\mathrm{E}$ & $4 \mathrm{~N} / 27 \mathrm{~W}-23 \mathrm{E} 1$ & 365 & 100 & $\mathrm{E}$ \\
\hline 4N/27W-23E6 & 675 & 40 & $\mathrm{E}$ & $4 \mathrm{~N} / 27 \mathrm{~W}-23 \mathrm{E} 1$ & 375 & 70 & $\mathrm{E}$ \\
\hline $4 \mathrm{~N} / 27 \mathrm{~W}-23 \mathrm{E} 6$ & 685 & 0 & $\mathrm{E}$ & $4 \mathrm{~N} / 27 \mathrm{~W}-23 \mathrm{E} 1$ & 385 & 0 & $\mathrm{E}$ \\
\hline 4N/27W-23E6 & 695 & 0 & $\mathrm{E}$ & $4 \mathrm{~N} / 27 \mathrm{~W}-23 \mathrm{E} 1$ & 395 & 0 & $\mathrm{E}$ \\
\hline 4N/27W-23E6 & 705 & 50 & $\mathrm{E}$ & $4 \mathrm{~N} / 27 \mathrm{~W}-23 \mathrm{E} 1$ & 405 & 0 & $\mathrm{E}$ \\
\hline 4N/27W-23E6 & 715 & 0 & $\mathrm{E}$ & $4 \mathrm{~N} / 27 \mathrm{~W}-23 \mathrm{E} 1$ & 415 & 0 & $\mathrm{E}$ \\
\hline $4 \mathrm{~N} / 27 \mathrm{~W}-23 \mathrm{E} 1$ & 95 & 50 & $\mathrm{E}$ & $4 \mathrm{~N} / 27 \mathrm{~W}-23 \mathrm{E} 1$ & 485 & 0 & $\mathrm{E}$ \\
\hline $4 \mathrm{~N} / 27 \mathrm{~W}-23 \mathrm{E} 1$ & 105 & 0 & $\mathrm{E}$ & $4 \mathrm{~N} / 27 \mathrm{~W}-23 \mathrm{E} 1$ & 495 & 0 & $\mathrm{E}$ \\
\hline $4 \mathrm{~N} / 27 \mathrm{~W}-23 \mathrm{E} 1$ & 115 & 0 & $\mathrm{E}$ & $4 \mathrm{~N} / 27 \mathrm{~W}-23 \mathrm{E} 1$ & 505 & 50 & $\mathrm{E}$ \\
\hline $4 \mathrm{~N} / 27 \mathrm{~W}-23 \mathrm{E} 1$ & 125 & 0 & $\mathrm{E}$ & $4 \mathrm{~N} / 27 \mathrm{~W}-23 \mathrm{E} 1$ & 515 & 100 & $\mathrm{E}$ \\
\hline $4 \mathrm{~N} / 27 \mathrm{~W}-23 \mathrm{E} 1$ & 135 & 100 & $\mathrm{E}$ & $4 \mathrm{~N} / 27 \mathrm{~W}-23 \mathrm{E} 1$ & 525 & 20 & $\mathrm{E}$ \\
\hline $4 \mathrm{~N} / 27 \mathrm{~W}-23 \mathrm{E} 1$ & 145 & 100 & $\mathrm{E}$ & $4 \mathrm{~N} / 27 \mathrm{~W}-23 \mathrm{E} 1$ & 535 & 0 & $\mathrm{E}$ \\
\hline $4 \mathrm{~N} / 27 \mathrm{~W}-23 \mathrm{E} 1$ & 155 & 100 & $\mathrm{E}$ & $4 \mathrm{~N} / 27 \mathrm{~W}-23 \mathrm{E} 1$ & 545 & 70 & $\mathrm{E}$ \\
\hline $4 \mathrm{~N} / 27 \mathrm{~W}-23 \mathrm{E} 1$ & 165 & 100 & $\mathrm{E}$ & $4 \mathrm{~N} / 27 \mathrm{~W}-23 \mathrm{E} 1$ & 555 & 30 & $\mathrm{E}$ \\
\hline $4 \mathrm{~N} / 27 \mathrm{~W}-23 \mathrm{E} 1$ & 175 & 80 & $\mathrm{E}$ & $4 \mathrm{~N} / 27 \mathrm{~W}-23 \mathrm{E} 1$ & 565 & 0 & $\mathrm{E}$ \\
\hline 4N/27W-23E1 & 185 & 0 & $\mathrm{E}$ & $4 \mathrm{~N} / 27 \mathrm{~W}-23 \mathrm{E} 1$ & 575 & 0 & $\mathrm{E}$ \\
\hline $4 \mathrm{~N} / 27 \mathrm{~W}-23 \mathrm{E} 1$ & 195 & 0 & $\mathrm{E}$ & $4 \mathrm{~N} / 27 \mathrm{~W}-23 \mathrm{E} 1$ & 585 & 50 & $\mathrm{E}$ \\
\hline 4N/27W-23E1 & 205 & 0 & $\mathrm{E}$ & $4 \mathrm{~N} / 27 \mathrm{~W}-23 \mathrm{E} 1$ & 595 & 100 & $\mathrm{E}$ \\
\hline
\end{tabular}


Table B1-1. Calculated percent-coarse intervals for all drillers' logs and e-logs used to define textural properties of the hydrogeologic framework model.-Continued

[Percent-coarse values are calculated for 10-foot intervals for the entire depth of each borehole. State well number is the state of California well identifier; interval midpoint depth is the midpoint of each 10-foot interval, in feet from top of borehole; PC is the percent-coarse value for each interval; log type defines whether the borehole is a drillers' lithology $\log (\mathrm{D})$ or a geophysical e-log (E).]

\begin{tabular}{|c|c|c|c|c|c|c|c|}
\hline $\begin{array}{c}\text { State } \\
\text { well } \\
\text { number }\end{array}$ & $\begin{array}{c}\text { Interval } \\
\text { midpoint depth } \\
\text { (feet) }\end{array}$ & PC & $\begin{array}{l}\text { Log } \\
\text { type }\end{array}$ & $\begin{array}{c}\text { State } \\
\text { well } \\
\text { number }\end{array}$ & $\begin{array}{c}\text { Interval } \\
\text { midpoint depth } \\
\text { (feet) }\end{array}$ & PC & $\begin{array}{l}\text { Log } \\
\text { type }\end{array}$ \\
\hline $4 \mathrm{~N} / 27 \mathrm{~W}-23 \mathrm{E} 1$ & 605 & 30 & $\mathrm{E}$ & $4 \mathrm{~N} / 27 \mathrm{~W}-23 \mathrm{D} 1$ & 175 & 30 & $\mathrm{E}$ \\
\hline $4 \mathrm{~N} / 27 \mathrm{~W}-23 \mathrm{E} 1$ & 615 & 0 & $\mathrm{E}$ & $4 \mathrm{~N} / 27 \mathrm{~W}-23 \mathrm{D} 1$ & 185 & 80 & $\mathrm{E}$ \\
\hline $4 \mathrm{~N} / 27 \mathrm{~W}-23 \mathrm{E} 1$ & 625 & 20 & $\mathrm{E}$ & $4 \mathrm{~N} / 27 \mathrm{~W}-23 \mathrm{D} 1$ & 195 & 0 & $\mathrm{E}$ \\
\hline 4N/27W-23E1 & 635 & 100 & $\mathrm{E}$ & $4 \mathrm{~N} / 27 \mathrm{~W}-23 \mathrm{D} 1$ & 205 & 0 & $\mathrm{E}$ \\
\hline 4N/27W-23E1 & 655 & 0 & $\mathrm{E}$ & $4 \mathrm{~N} / 27 \mathrm{~W}-23 \mathrm{D} 1$ & 225 & 60 & $\mathrm{E}$ \\
\hline $4 \mathrm{~N} / 27 \mathrm{~W}-23 \mathrm{E} 1$ & 665 & 0 & $\mathrm{E}$ & $4 \mathrm{~N} / 27 \mathrm{~W}-23 \mathrm{D} 1$ & 235 & 0 & $\mathrm{E}$ \\
\hline $4 \mathrm{~N} / 27 \mathrm{~W}-23 \mathrm{E} 1$ & 675 & 0 & $\mathrm{E}$ & $4 \mathrm{~N} / 27 \mathrm{~W}-23 \mathrm{D} 1$ & 245 & 40 & $\mathrm{E}$ \\
\hline $4 \mathrm{~N} / 27 \mathrm{~W}-23 \mathrm{E} 1$ & 685 & 0 & $\mathrm{E}$ & $4 \mathrm{~N} / 27 \mathrm{~W}-23 \mathrm{D} 1$ & 255 & 100 & $\mathrm{E}$ \\
\hline $4 \mathrm{~N} / 27 \mathrm{~W}-23 \mathrm{E} 1$ & 725 & 0 & $\mathrm{E}$ & $4 \mathrm{~N} / 27 \mathrm{~W}-23 \mathrm{D} 1$ & 295 & 60 & $\mathrm{E}$ \\
\hline $4 \mathrm{~N} / 27 \mathrm{~W}-23 \mathrm{E} 1$ & 735 & 0 & $\mathrm{E}$ & $4 \mathrm{~N} / 27 \mathrm{~W}-23 \mathrm{D} 1$ & 305 & 0 & $\mathrm{E}$ \\
\hline $4 \mathrm{~N} / 27 \mathrm{~W}-23 \mathrm{E} 1$ & 745 & 0 & $\mathrm{E}$ & $4 \mathrm{~N} / 27 \mathrm{~W}-23 \mathrm{D} 1$ & 315 & 0 & $\mathrm{E}$ \\
\hline $4 \mathrm{~N} / 27 \mathrm{~W}-23 \mathrm{E} 1$ & 755 & 0 & $\mathrm{E}$ & $4 \mathrm{~N} / 27 \mathrm{~W}-23 \mathrm{D} 1$ & 325 & 100 & $\mathrm{E}$ \\
\hline 4N/27W-23E1 & 765 & 0 & $\mathrm{E}$ & $4 \mathrm{~N} / 27 \mathrm{~W}-23 \mathrm{D} 1$ & 335 & 100 & $\mathrm{E}$ \\
\hline $4 \mathrm{~N} / 27 \mathrm{~W}-23 \mathrm{E} 1$ & 775 & 100 & $\mathrm{E}$ & $4 \mathrm{~N} / 27 \mathrm{~W}-23 \mathrm{D} 1$ & 345 & 100 & $\mathrm{E}$ \\
\hline $4 \mathrm{~N} / 27 \mathrm{~W}-23 \mathrm{E} 1$ & 785 & 80 & $\mathrm{E}$ & $4 \mathrm{~N} / 27 \mathrm{~W}-23 \mathrm{D} 1$ & 355 & 100 & $\mathrm{E}$ \\
\hline $4 \mathrm{~N} / 27 \mathrm{~W}-23 \mathrm{E} 1$ & 795 & 0 & $\mathrm{E}$ & $4 \mathrm{~N} / 27 \mathrm{~W}-23 \mathrm{D} 1$ & 365 & 50 & $\mathrm{E}$ \\
\hline $4 \mathrm{~N} / 27 \mathrm{~W}-23 \mathrm{E} 1$ & 805 & 10 & $\mathrm{E}$ & $4 \mathrm{~N} / 27 \mathrm{~W}-23 \mathrm{D} 1$ & 375 & 0 & $\mathrm{E}$ \\
\hline $4 \mathrm{~N} / 27 \mathrm{~W}-23 \mathrm{D} 1$ & 55 & 0 & $\mathrm{E}$ & $4 \mathrm{~N} / 27 \mathrm{~W}-22 \mathrm{~B} 2$ & 45 & 0 & $\mathrm{E}$ \\
\hline 4N/27W-23D1 & 65 & 0 & $\mathrm{E}$ & $4 \mathrm{~N} / 27 \mathrm{~W}-22 \mathrm{~B} 2$ & 55 & 0 & $\mathrm{E}$ \\
\hline $4 \mathrm{~N} / 27 \mathrm{~W}-23 \mathrm{D} 1$ & 75 & 0 & $\mathrm{E}$ & $4 \mathrm{~N} / 27 \mathrm{~W}-22 \mathrm{~B} 2$ & 65 & 0 & $\mathrm{E}$ \\
\hline 4N/27W-23D1 & 85 & 0 & $\mathrm{E}$ & $4 \mathrm{~N} / 27 \mathrm{~W}-22 \mathrm{~B} 2$ & 75 & 0 & $\mathrm{E}$ \\
\hline $4 \mathrm{~N} / 27 \mathrm{~W}-23 \mathrm{D} 1$ & 95 & 80 & $\mathrm{E}$ & $4 \mathrm{~N} / 27 \mathrm{~W}-22 \mathrm{~B} 2$ & 85 & 0 & $\mathrm{E}$ \\
\hline 4N/27W-23D1 & 105 & 50 & $\mathrm{E}$ & $4 \mathrm{~N} / 27 \mathrm{~W}-22 \mathrm{~B} 2$ & 95 & 40 & $\mathrm{E}$ \\
\hline $4 \mathrm{~N} / 27 \mathrm{~W}-23 \mathrm{D} 1$ & 115 & 20 & $\mathrm{E}$ & $4 \mathrm{~N} / 27 \mathrm{~W}-22 \mathrm{~B} 2$ & 105 & 30 & $\mathrm{E}$ \\
\hline $4 \mathrm{~N} / 27 \mathrm{~W}-23 \mathrm{D} 1$ & 125 & 100 & $\mathrm{E}$ & $4 \mathrm{~N} / 27 \mathrm{~W}-22 \mathrm{~B} 2$ & 115 & 30 & $\mathrm{E}$ \\
\hline $4 \mathrm{~N} / 27 \mathrm{~W}-23 \mathrm{D} 1$ & 135 & 100 & $\mathrm{E}$ & $4 \mathrm{~N} / 27 \mathrm{~W}-22 \mathrm{~B} 2$ & 125 & 30 & $\mathrm{E}$ \\
\hline $4 \mathrm{~N} / 27 \mathrm{~W}-23 \mathrm{D} 1$ & 145 & 90 & $\mathrm{E}$ & $4 \mathrm{~N} / 27 \mathrm{~W}-22 \mathrm{~B} 2$ & 135 & 40 & $\mathrm{E}$ \\
\hline $4 \mathrm{~N} / 27 \mathrm{~W}-23 \mathrm{D} 1$ & 155 & 0 & $\mathrm{E}$ & $4 \mathrm{~N} / 27 \mathrm{~W}-22 \mathrm{~B} 2$ & 145 & 0 & $\mathrm{E}$ \\
\hline $4 \mathrm{~N} / 27 \mathrm{~W}-23 \mathrm{D} 1$ & 165 & 0 & $\mathrm{E}$ & $4 \mathrm{~N} / 27 \mathrm{~W}-22 \mathrm{~B} 2$ & 155 & 0 & $\mathrm{E}$ \\
\hline
\end{tabular}


Table B1-1. Calculated percent-coarse intervals for all drillers' logs and e-logs used to define textural properties of the hydrogeologic framework model.-Continued

[Percent-coarse values are calculated for 10-foot intervals for the entire depth of each borehole. State well number is the state of California well identifier; interval midpoint depth is the midpoint of each 10-foot interval, in feet from top of borehole; PC is the percent-coarse value for each interval; log type defines whether the borehole is a drillers' lithology $\log (\mathrm{D})$ or a geophysical e-log (E).]

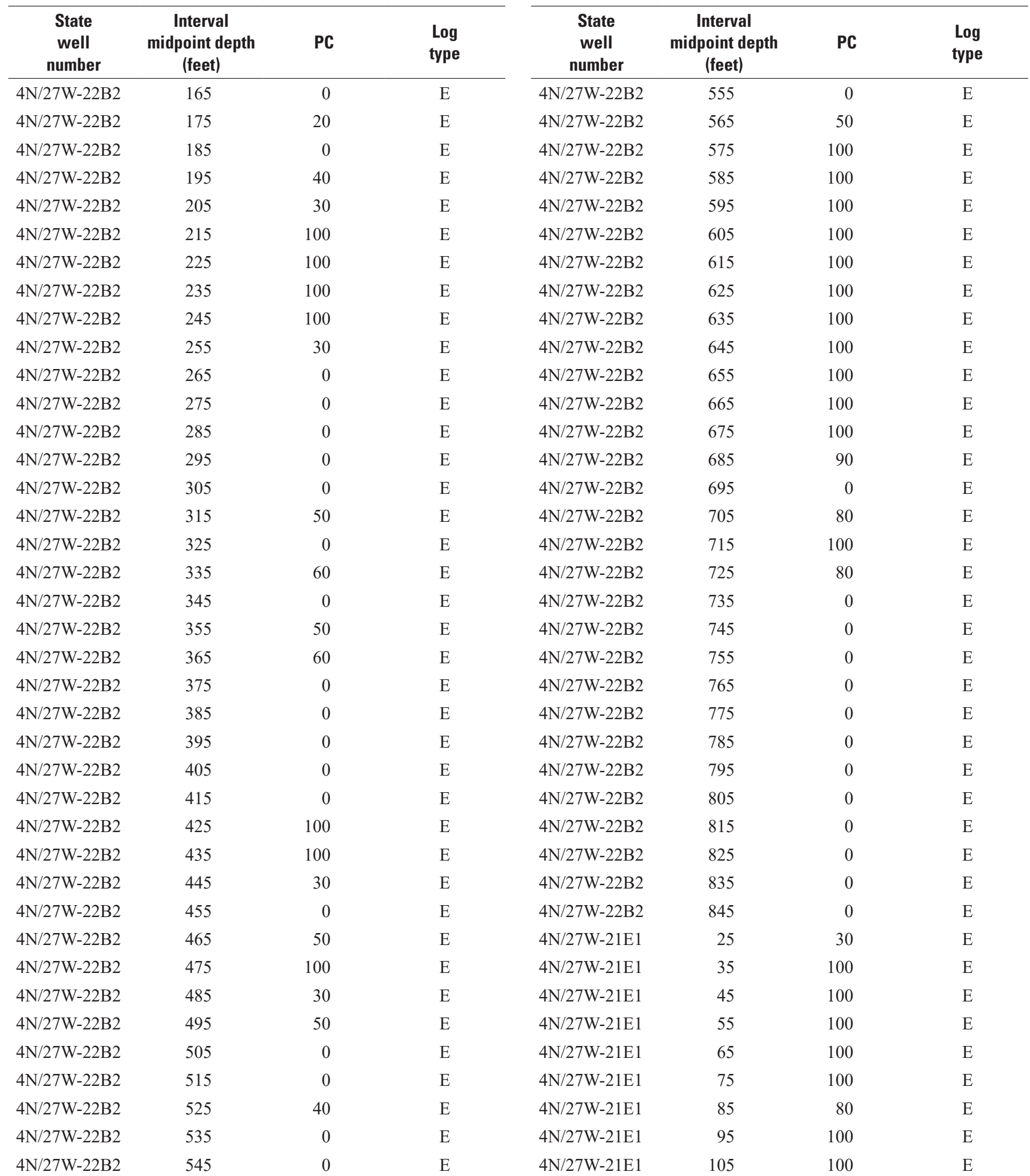


Table B1-1. Calculated percent-coarse intervals for all drillers' logs and e-logs used to define textural properties of the hydrogeologic framework model.-Continued

[Percent-coarse values are calculated for 10-foot intervals for the entire depth of each borehole. State well number is the state of California well identifier; interval midpoint depth is the midpoint of each 10-foot interval, in feet from top of borehole; PC is the percent-coarse value for each interval; log type defines whether the borehole is a drillers' lithology $\log (\mathrm{D})$ or a geophysical e-log (E).]

\begin{tabular}{|c|c|c|c|c|c|c|c|}
\hline $\begin{array}{c}\text { State } \\
\text { well } \\
\text { number }\end{array}$ & $\begin{array}{c}\text { Interval } \\
\text { midpoint depth } \\
\text { (feet) }\end{array}$ & PC & $\begin{array}{l}\text { Log } \\
\text { type }\end{array}$ & $\begin{array}{c}\text { State } \\
\text { well } \\
\text { number }\end{array}$ & $\begin{array}{c}\text { Interval } \\
\text { midpoint depth } \\
\text { (feet) }\end{array}$ & PC & $\begin{array}{l}\text { Log } \\
\text { type }\end{array}$ \\
\hline $4 \mathrm{~N} / 27 \mathrm{~W}-21 \mathrm{E} 1$ & 115 & 100 & $\mathrm{E}$ & 4N/27W2-E1 & 335 & 10 & $\mathrm{E}$ \\
\hline $4 \mathrm{~N} / 27 \mathrm{~W}-21 \mathrm{E} 1$ & 125 & 100 & $\mathrm{E}$ & 4N/27W2-E1 & 345 & 0 & $\mathrm{E}$ \\
\hline $4 \mathrm{~N} / 27 \mathrm{~W}-21 \mathrm{E} 1$ & 135 & 100 & $\mathrm{E}$ & $4 \mathrm{~N} / 27 \mathrm{~W}-18 \mathrm{~B} 5$ & 25 & 60 & $\mathrm{E}$ \\
\hline $4 \mathrm{~N} / 27 \mathrm{~W}-21 \mathrm{E} 1$ & 145 & 100 & $\mathrm{E}$ & $4 \mathrm{~N} / 27 \mathrm{~W}-18 \mathrm{~B} 5$ & 35 & 0 & $\mathrm{E}$ \\
\hline $4 \mathrm{~N} / 27 \mathrm{~W}-21 \mathrm{E} 1$ & 165 & 100 & $\mathrm{E}$ & $4 \mathrm{~N} / 27 \mathrm{~W}-18 \mathrm{~B} 5$ & 55 & 30 & $\mathrm{E}$ \\
\hline $4 \mathrm{~N} / 27 \mathrm{~W}-21 \mathrm{E} 1$ & 175 & 70 & $\mathrm{E}$ & $4 \mathrm{~N} / 27 \mathrm{~W}-18 \mathrm{~B} 5$ & 65 & 40 & $\mathrm{E}$ \\
\hline $4 \mathrm{~N} / 27 \mathrm{~W}-21 \mathrm{E} 1$ & 185 & 80 & $\mathrm{E}$ & $4 \mathrm{~N} / 27 \mathrm{~W}-18 \mathrm{~B} 5$ & 75 & 80 & $\mathrm{E}$ \\
\hline $4 \mathrm{~N} / 27 \mathrm{~W}-21 \mathrm{E} 1$ & 195 & 80 & $\mathrm{E}$ & $4 \mathrm{~N} / 27 \mathrm{~W}-18 \mathrm{~B} 5$ & 85 & 20 & $\mathrm{E}$ \\
\hline $4 \mathrm{~N} / 27 \mathrm{~W}-21 \mathrm{E} 1$ & 235 & 0 & $\mathrm{E}$ & $4 \mathrm{~N} / 27 \mathrm{~W}-17 \mathrm{M} 1$ & 45 & 0 & $\mathrm{E}$ \\
\hline 4N/27W2-E1 & 75 & 0 & $\mathrm{E}$ & $4 \mathrm{~N} / 27 \mathrm{~W}-17 \mathrm{M} 1$ & 55 & 20 & $\mathrm{E}$ \\
\hline 4N/27W2-E1 & 85 & 0 & $\mathrm{E}$ & $4 \mathrm{~N} / 27 \mathrm{~W}-17 \mathrm{M} 1$ & 65 & 0 & $\mathrm{E}$ \\
\hline 4N/27W2-E1 & 95 & 0 & $\mathrm{E}$ & 4N/27W-17M1 & 75 & 0 & $\mathrm{E}$ \\
\hline 4N/27W2-E1 & 105 & 0 & $\mathrm{E}$ & $4 \mathrm{~N} / 27 \mathrm{~W}-17 \mathrm{M} 1$ & 85 & 70 & $\mathrm{E}$ \\
\hline 4N/27W2-E1 & 115 & 0 & $\mathrm{E}$ & $4 \mathrm{~N} / 27 \mathrm{~W}-17 \mathrm{M} 1$ & 95 & 100 & $\mathrm{E}$ \\
\hline 4N/27W2-E1 & 125 & 0 & $\mathrm{E}$ & $4 \mathrm{~N} / 27 \mathrm{~W}-17 \mathrm{M} 1$ & 105 & 100 & $\mathrm{E}$ \\
\hline 4N/27W2-E1 & 135 & 0 & $\mathrm{E}$ & $4 \mathrm{~N} / 27 \mathrm{~W}-17 \mathrm{M} 1$ & 115 & 100 & $\mathrm{E}$ \\
\hline 4N/27W2-E1 & 145 & 0 & $\mathrm{E}$ & $4 \mathrm{~N} / 27 \mathrm{~W}-17 \mathrm{M} 1$ & 125 & 100 & $\mathrm{E}$ \\
\hline 4N/27W2-E1 & 215 & 0 & $\mathrm{E}$ & $4 \mathrm{~N} / 27 \mathrm{~W}-17 \mathrm{M} 1$ & 195 & 100 & $\mathrm{E}$ \\
\hline 4N/27W2-E1 & 225 & 20 & $\mathrm{E}$ & 4N/27W-17M1 & 205 & 100 & $\mathrm{E}$ \\
\hline 4N/27W2-E1 & 235 & 100 & $\mathrm{E}$ & $4 \mathrm{~N} / 27 \mathrm{~W}-17 \mathrm{M} 1$ & 215 & 100 & $\mathrm{E}$ \\
\hline 4N/27W2-E1 & 245 & 100 & $\mathrm{E}$ & 4N/27W-17M1 & 225 & 100 & $\mathrm{E}$ \\
\hline 4N/27W2-E1 & 255 & 100 & $\mathrm{E}$ & $4 \mathrm{~N} / 27 \mathrm{~W}-17 \mathrm{M} 1$ & 235 & 100 & $\mathrm{E}$ \\
\hline $4 \mathrm{~N} / 27 \mathrm{~W} 2-\mathrm{E} 1$ & 265 & 100 & $\mathrm{E}$ & 4N/27W-17M1 & 245 & 100 & $\mathrm{E}$ \\
\hline 4N/27W2-E1 & 275 & 60 & $\mathrm{E}$ & $4 \mathrm{~N} / 27 \mathrm{~W}-17 \mathrm{M} 1$ & 255 & 20 & $\mathrm{E}$ \\
\hline $4 \mathrm{~N} / 27 \mathrm{~W} 2-\mathrm{E} 1$ & 285 & 60 & $\mathrm{E}$ & 4N/27W-17M1 & 265 & 50 & $\mathrm{E}$ \\
\hline 4N/27W2-E1 & 295 & 100 & $\mathrm{E}$ & $4 \mathrm{~N} / 27 \mathrm{~W}-17 \mathrm{M} 1$ & 275 & 100 & $\mathrm{E}$ \\
\hline 4N/27W2-E1 & 305 & 100 & $\mathrm{E}$ & $4 \mathrm{~N} / 27 \mathrm{~W}-17 \mathrm{~J} 1$ & 25 & 87.5 & $\mathrm{E}$ \\
\hline 4N/27W2-E1 & 315 & 100 & $\mathrm{E}$ & $4 \mathrm{~N} / 27 \mathrm{~W}-17 \mathrm{~J} 1$ & 35 & 60 & $\mathrm{E}$ \\
\hline 4N/27W2-E1 & 325 & 100 & $\mathrm{E}$ & $4 \mathrm{~N} / 27 \mathrm{~W}-17 \mathrm{~J} 1$ & 45 & 50 & $\mathrm{E}$ \\
\hline
\end{tabular}


Table B1-1. Calculated percent-coarse intervals for all drillers' logs and e-logs used to define textural properties of the hydrogeologic framework model.-Continued

[Percent-coarse values are calculated for 10-foot intervals for the entire depth of each borehole. State well number is the state of California well identifier; interval midpoint depth is the midpoint of each 10-foot interval, in feet from top of borehole; PC is the percent-coarse value for each interval; log type defines whether the borehole is a drillers' lithology $\log (\mathrm{D})$ or a geophysical e-log (E).]

\begin{tabular}{|c|c|c|c|c|c|c|c|}
\hline $\begin{array}{c}\text { State } \\
\text { well } \\
\text { number }\end{array}$ & $\begin{array}{c}\text { Interval } \\
\text { midpoint depth } \\
\text { (feet) }\end{array}$ & PC & $\begin{array}{l}\text { Log } \\
\text { type }\end{array}$ & $\begin{array}{c}\text { State } \\
\text { well } \\
\text { number }\end{array}$ & $\begin{array}{c}\text { Interval } \\
\text { midpoint depth } \\
\text { (feet) }\end{array}$ & PC & $\begin{array}{l}\text { Log } \\
\text { type }\end{array}$ \\
\hline $4 \mathrm{~N} / 27 \mathrm{~W}-17 \mathrm{~J} 1$ & 55 & 70 & $\mathrm{E}$ & $4 \mathrm{~N} / 27 \mathrm{~W}-17 \mathrm{H} 1$ & 115 & 40 & $\mathrm{E}$ \\
\hline $4 \mathrm{~N} / 27 \mathrm{~W}-17 \mathrm{~J} 1$ & 65 & 0 & $\mathrm{E}$ & $4 \mathrm{~N} / 27 \mathrm{~W}-17 \mathrm{H} 1$ & 125 & 0 & $\mathrm{E}$ \\
\hline $4 \mathrm{~N} / 27 \mathrm{~W}-17 \mathrm{~J} 1$ & 75 & 20 & $\mathrm{E}$ & $4 \mathrm{~N} / 27 \mathrm{~W}-17 \mathrm{H} 1$ & 135 & 0 & $\mathrm{E}$ \\
\hline $4 \mathrm{~N} / 27 \mathrm{~W}-17 \mathrm{~J} 1$ & 85 & 100 & $\mathrm{E}$ & $4 \mathrm{~N} / 27 \mathrm{~W}-17 \mathrm{H} 1$ & 145 & 90 & $\mathrm{E}$ \\
\hline $4 \mathrm{~N} / 27 \mathrm{~W}-17 \mathrm{~J} 1$ & 105 & 0 & $\mathrm{E}$ & $4 \mathrm{~N} / 27 \mathrm{~W}-17 \mathrm{H} 1$ & 165 & 80 & $\mathrm{E}$ \\
\hline $4 \mathrm{~N} / 27 \mathrm{~W}-17 \mathrm{~J} 1$ & 115 & 0 & $\mathrm{E}$ & $4 \mathrm{~N} / 27 \mathrm{~W}-17 \mathrm{H} 1$ & 175 & 70 & $\mathrm{E}$ \\
\hline $4 \mathrm{~N} / 27 \mathrm{~W}-17 \mathrm{~J} 1$ & 125 & 0 & $\mathrm{E}$ & $4 \mathrm{~N} / 27 \mathrm{~W}-17 \mathrm{H} 1$ & 185 & 0 & $\mathrm{E}$ \\
\hline $4 \mathrm{~N} / 27 \mathrm{~W}-17 \mathrm{~J} 1$ & 135 & 0 & $\mathrm{E}$ & $4 \mathrm{~N} / 27 \mathrm{~W}-17 \mathrm{H} 1$ & 195 & 70 & $\mathrm{E}$ \\
\hline 4N/27W-17J1 & 175 & 0 & $\mathrm{E}$ & $4 \mathrm{~N} / 27 \mathrm{~W}-17 \mathrm{H} 1$ & 235 & 60 & $\mathrm{E}$ \\
\hline $4 \mathrm{~N} / 27 \mathrm{~W}-17 \mathrm{~J} 1$ & 185 & 0 & $\mathrm{E}$ & $4 \mathrm{~N} / 27 \mathrm{~W}-17 \mathrm{H} 1$ & 245 & 100 & $\mathrm{E}$ \\
\hline $4 \mathrm{~N} / 27 \mathrm{~W}-17 \mathrm{~J} 1$ & 195 & 30 & $\mathrm{E}$ & $4 \mathrm{~N} / 27 \mathrm{~W}-17 \mathrm{H} 1$ & 255 & 100 & $\mathrm{E}$ \\
\hline $4 \mathrm{~N} / 27 \mathrm{~W}-17 \mathrm{~J} 1$ & 205 & 90 & $\mathrm{E}$ & $4 \mathrm{~N} / 27 \mathrm{~W}-17 \mathrm{H} 1$ & 265 & 100 & $\mathrm{E}$ \\
\hline $4 \mathrm{~N} / 27 \mathrm{~W}-17 \mathrm{~J} 1$ & 215 & 30 & $\mathrm{E}$ & $4 \mathrm{~N} / 27 \mathrm{~W}-17 \mathrm{H} 1$ & 275 & 100 & $\mathrm{E}$ \\
\hline $4 \mathrm{~N} / 27 \mathrm{~W}-17 \mathrm{~J} 1$ & 225 & 100 & $\mathrm{E}$ & $4 \mathrm{~N} / 27 \mathrm{~W}-17 \mathrm{H} 1$ & 285 & 100 & $\mathrm{E}$ \\
\hline 4N/27W-17J1 & 235 & 100 & $\mathrm{E}$ & $4 \mathrm{~N} / 27 \mathrm{~W}-17 \mathrm{H} 1$ & 295 & 50 & $\mathrm{E}$ \\
\hline $4 \mathrm{~N} / 27 \mathrm{~W}-17 \mathrm{~J} 1$ & 245 & 0 & $\mathrm{E}$ & $4 \mathrm{~N} / 27 \mathrm{~W}-17 \mathrm{H} 1$ & 305 & 10 & $\mathrm{E}$ \\
\hline $4 \mathrm{~N} / 27 \mathrm{~W}-17 \mathrm{~J} 1$ & 255 & 30 & $\mathrm{E}$ & $4 \mathrm{~N} / 27 \mathrm{~W}-17 \mathrm{H} 1$ & 315 & 50 & $\mathrm{E}$ \\
\hline $4 \mathrm{~N} / 27 \mathrm{~W}-17 \mathrm{~J} 1$ & 325 & 0 & $\mathrm{E}$ & 4N/27W-16R1 & 115 & 100 & $\mathrm{E}$ \\
\hline $4 \mathrm{~N} / 27 \mathrm{~W}-17 \mathrm{~J} 1$ & 335 & 0 & $\mathrm{E}$ & 4N/27W-16R1 & 125 & 100 & $\mathrm{E}$ \\
\hline $4 \mathrm{~N} / 27 \mathrm{~W}-17 \mathrm{~J} 1$ & 345 & 0 & $\mathrm{E}$ & $4 \mathrm{~N} / 27 \mathrm{~W}-16 \mathrm{R} 1$ & 135 & 100 & $\mathrm{E}$ \\
\hline $4 \mathrm{~N} / 27 \mathrm{~W}-17 \mathrm{~J} 1$ & 355 & 0 & $\mathrm{E}$ & $4 \mathrm{~N} / 27 \mathrm{~W}-16 \mathrm{R} 1$ & 145 & 100 & $\mathrm{E}$ \\
\hline 4N/27W-17J1 & 365 & 0 & $\mathrm{E}$ & $4 \mathrm{~N} / 27 \mathrm{~W}-16 \mathrm{R} 1$ & 155 & 100 & $\mathrm{E}$ \\
\hline $4 \mathrm{~N} / 27 \mathrm{~W}-17 \mathrm{H} 1$ & 45 & 70 & $\mathrm{E}$ & 4N/27W-16R1 & 165 & 100 & $\mathrm{E}$ \\
\hline $4 \mathrm{~N} / 27 \mathrm{~W}-17 \mathrm{H} 1$ & 55 & 30 & $\mathrm{E}$ & $4 \mathrm{~N} / 27 \mathrm{~W}-16 \mathrm{R} 1$ & 175 & 100 & $\mathrm{E}$ \\
\hline $4 \mathrm{~N} / 27 \mathrm{~W}-17 \mathrm{H} 1$ & 65 & 0 & $\mathrm{E}$ & $4 \mathrm{~N} / 27 \mathrm{~W}-16 \mathrm{R} 1$ & 185 & 100 & $\mathrm{E}$ \\
\hline $4 \mathrm{~N} / 27 \mathrm{~W}-17 \mathrm{H} 1$ & 75 & 20 & $\mathrm{E}$ & $4 \mathrm{~N} / 27 \mathrm{~W}-16 \mathrm{R} 1$ & 195 & 100 & $\mathrm{E}$ \\
\hline $4 \mathrm{~N} / 27 \mathrm{~W}-17 \mathrm{H} 1$ & 85 & 0 & $\mathrm{E}$ & $4 \mathrm{~N} / 27 \mathrm{~W}-16 \mathrm{R} 1$ & 205 & 100 & $\mathrm{E}$ \\
\hline 4N/27W-17H1 & 95 & 50 & $\mathrm{E}$ & $4 \mathrm{~N} / 27 \mathrm{~W}-16 \mathrm{R} 1$ & 215 & 100 & $\mathrm{E}$ \\
\hline $4 \mathrm{~N} / 27 \mathrm{~W}-17 \mathrm{H} 1$ & 105 & 0 & $\mathrm{E}$ & 4N/27W-16R1 & 225 & 100 & $\mathrm{E}$ \\
\hline
\end{tabular}


Table B1-1. Calculated percent-coarse intervals for all drillers' logs and e-logs used to define textural properties of the hydrogeologic framework model.-Continued

[Percent-coarse values are calculated for 10-foot intervals for the entire depth of each borehole. State well number is the state of California well identifier; interval midpoint depth is the midpoint of each 10-foot interval, in feet from top of borehole; PC is the percent-coarse value for each interval; log type defines whether the borehole is a drillers' lithology $\log (\mathrm{D})$ or a geophysical e-log (E).]

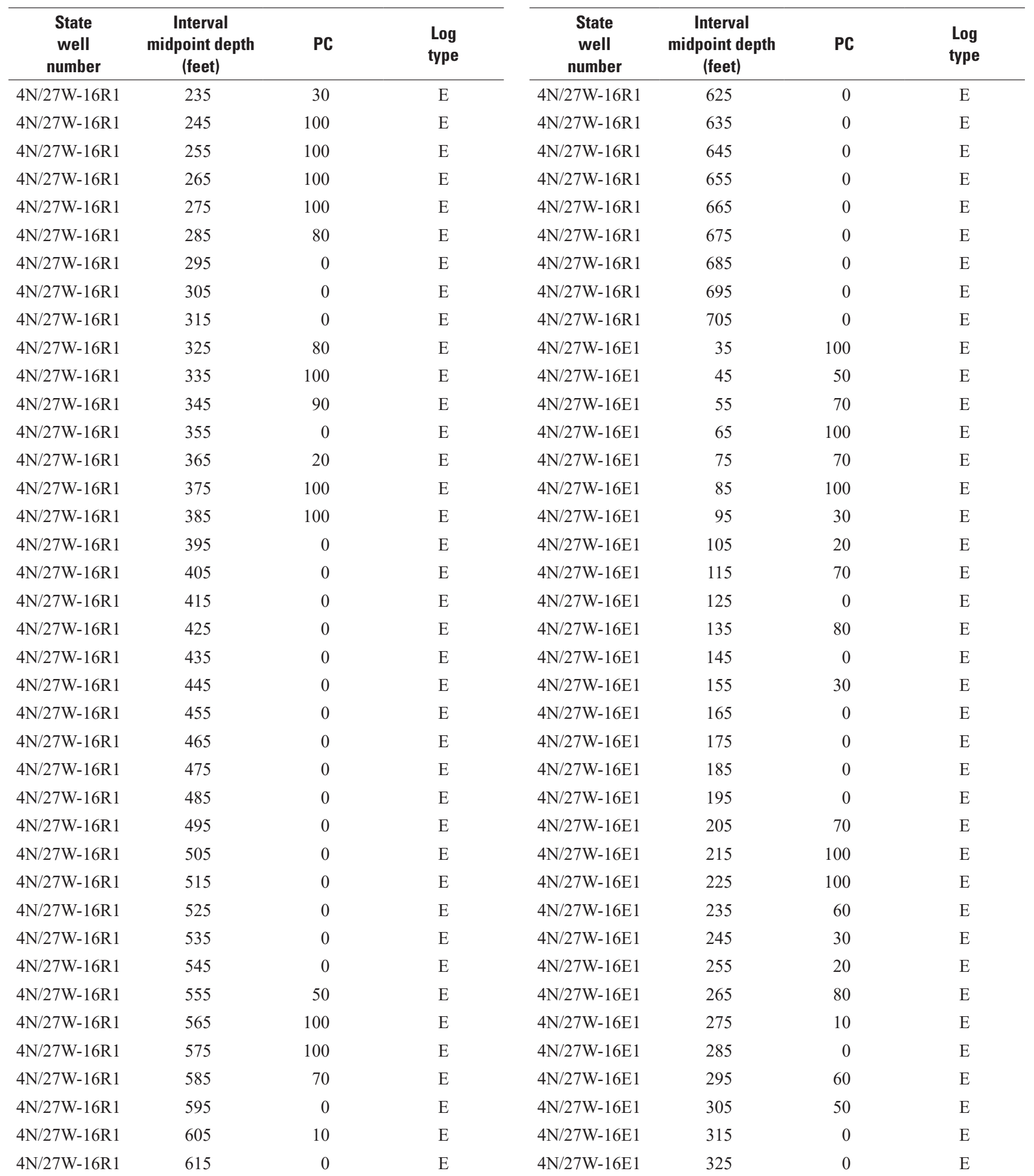


Table B1-1. Calculated percent-coarse intervals for all drillers' logs and e-logs used to define textural properties of the hydrogeologic framework model.-Continued

[Percent-coarse values are calculated for 10-foot intervals for the entire depth of each borehole. State well number is the state of California well identifier; interval midpoint depth is the midpoint of each 10-foot interval, in feet from top of borehole; PC is the percent-coarse value for each interval; log type defines whether the borehole is a drillers' lithology $\log (\mathrm{D})$ or a geophysical e-log (E).]

\begin{tabular}{|c|c|c|c|c|c|c|c|}
\hline $\begin{array}{c}\text { State } \\
\text { well } \\
\text { number }\end{array}$ & $\begin{array}{c}\text { Interval } \\
\text { midpoint depth } \\
\text { (feet) }\end{array}$ & PC & $\begin{array}{l}\text { Log } \\
\text { type }\end{array}$ & $\begin{array}{c}\text { State } \\
\text { well } \\
\text { number }\end{array}$ & $\begin{array}{c}\text { Interval } \\
\text { midpoint depth } \\
\text { (feet) }\end{array}$ & PC & $\begin{array}{l}\text { Log } \\
\text { type }\end{array}$ \\
\hline $4 \mathrm{~N} / 27 \mathrm{~W}-16 \mathrm{E} 1$ & 335 & 80 & $\mathrm{E}$ & $4 \mathrm{~N} / 27 \mathrm{~W}-15 \mathrm{Q} 9$ & 305 & 0 & $\mathrm{E}$ \\
\hline $4 \mathrm{~N} / 27 \mathrm{~W}-16 \mathrm{E} 1$ & 345 & 100 & $\mathrm{E}$ & 4N/27W-15Q9 & 315 & 0 & $\mathrm{E}$ \\
\hline $4 \mathrm{~N} / 27 \mathrm{~W}-16 \mathrm{E} 1$ & 355 & 100 & $\mathrm{E}$ & 4N/27W-15Q9 & 325 & 20 & $\mathrm{E}$ \\
\hline $4 \mathrm{~N} / 27 \mathrm{~W}-16 \mathrm{E} 1$ & 365 & 100 & $\mathrm{E}$ & 4N/27W-15Q9 & 335 & 0 & $\mathrm{E}$ \\
\hline $4 \mathrm{~N} / 27 \mathrm{~W}-16 \mathrm{E} 1$ & 385 & 100 & $\mathrm{E}$ & 4N/27W-15Q9 & 355 & 0 & $\mathrm{E}$ \\
\hline $4 \mathrm{~N} / 27 \mathrm{~W}-16 \mathrm{E} 1$ & 395 & 100 & $\mathrm{E}$ & $4 \mathrm{~N} / 27 \mathrm{~W}-15 \mathrm{Q} 9$ & 365 & 70 & $\mathrm{E}$ \\
\hline $4 \mathrm{~N} / 27 \mathrm{~W}-16 \mathrm{E} 1$ & 405 & 100 & $\mathrm{E}$ & 4N/27W-15Q9 & 375 & 80 & $\mathrm{E}$ \\
\hline $4 \mathrm{~N} / 27 \mathrm{~W}-16 \mathrm{E} 1$ & 415 & 100 & $\mathrm{E}$ & $4 \mathrm{~N} / 27 \mathrm{~W}-15 \mathrm{Q} 9$ & 385 & 100 & $\mathrm{E}$ \\
\hline 4N/27W-15Q9 & 35 & 0 & $\mathrm{E}$ & 4N/27W-15Q9 & 425 & 10 & $\mathrm{E}$ \\
\hline 4N/27W-15Q9 & 45 & 0 & $\mathrm{E}$ & $4 \mathrm{~N} / 27 \mathrm{~W}-15 \mathrm{Q} 9$ & 435 & 0 & $\mathrm{E}$ \\
\hline 4N/27W-15Q9 & 55 & 0 & $\mathrm{E}$ & 4N/27W-15Q9 & 445 & 0 & $\mathrm{E}$ \\
\hline 4N/27W-15Q9 & 65 & 40 & $\mathrm{E}$ & 4N/27W-15Q9 & 455 & 0 & $\mathrm{E}$ \\
\hline 4N/27W-15Q9 & 75 & 0 & $\mathrm{E}$ & 4N/27W-15Q9 & 465 & 0 & $\mathrm{E}$ \\
\hline 4N/27W-15Q9 & 85 & 0 & $\mathrm{E}$ & 4N/27W-15Q9 & 475 & 0 & $\mathrm{E}$ \\
\hline 4N/27W-15Q9 & 95 & 0 & $\mathrm{E}$ & $4 \mathrm{~N} / 27 \mathrm{~W}-15 \mathrm{Q} 9$ & 485 & 0 & $\mathrm{E}$ \\
\hline 4N/27W-15Q9 & 105 & 0 & $\mathrm{E}$ & 4N/27W-15Q9 & 495 & 100 & $\mathrm{E}$ \\
\hline 4N/27W-15Q9 & 115 & 0 & $\mathrm{E}$ & 4N/27W-15Q9 & 505 & 20 & $\mathrm{E}$ \\
\hline 4N/27W-15Q9 & 185 & 0 & $\mathrm{E}$ & $4 \mathrm{~N} / 27 \mathrm{~W}-15 \mathrm{Q} 9$ & 575 & 0 & $\mathrm{E}$ \\
\hline 4N/27W-15Q9 & 195 & 70 & $\mathrm{E}$ & 4N/27W-15Q9 & 585 & 30 & $\mathrm{E}$ \\
\hline 4N/27W-15Q9 & 205 & 20 & $\mathrm{E}$ & $4 \mathrm{~N} / 27 \mathrm{~W}-15 \mathrm{Q} 9$ & 595 & 100 & $\mathrm{E}$ \\
\hline 4N/27W-15Q9 & 215 & 40 & $\mathrm{E}$ & 4N/27W-15Q9 & 605 & 0 & $\mathrm{E}$ \\
\hline 4N/27W-15Q9 & 225 & 20 & $\mathrm{E}$ & $4 \mathrm{~N} / 27 \mathrm{~W}-15 \mathrm{Q} 9$ & 615 & 0 & $\mathrm{E}$ \\
\hline 4N/27W-15Q9 & 235 & 100 & $\mathrm{E}$ & 4N/27W-15Q9 & 625 & 20 & $\mathrm{E}$ \\
\hline 4N/27W-15Q9 & 245 & 100 & $\mathrm{E}$ & $4 \mathrm{~N} / 27 \mathrm{~W}-15 \mathrm{Q} 9$ & 635 & 0 & $\mathrm{E}$ \\
\hline 4N/27W-15Q9 & 255 & 100 & $\mathrm{E}$ & $4 \mathrm{~N} / 27 \mathrm{~W}-15 \mathrm{Q} 9$ & 645 & 100 & $\mathrm{E}$ \\
\hline 4N/27W-15Q9 & 265 & 80 & $\mathrm{E}$ & $4 \mathrm{~N} / 27 \mathrm{~W}-15 \mathrm{Q} 9$ & 655 & 100 & $\mathrm{E}$ \\
\hline 4N/27W-15Q9 & 275 & 0 & $\mathrm{E}$ & 4N/27W-15Q9 & 665 & 70 & $\mathrm{E}$ \\
\hline 4N/27W-15Q9 & 285 & 20 & $\mathrm{E}$ & $4 \mathrm{~N} / 27 \mathrm{~W}-15 \mathrm{Q} 9$ & 675 & 0 & $\mathrm{E}$ \\
\hline 4N/27W-15Q9 & 295 & 0 & $\mathrm{E}$ & 4N/27W-15Q9 & 685 & 0 & $\mathrm{E}$ \\
\hline
\end{tabular}


Table B1-1. Calculated percent-coarse intervals for all drillers' logs and e-logs used to define textural properties of the hydrogeologic framework model.-Continued

[Percent-coarse values are calculated for 10-foot intervals for the entire depth of each borehole. State well number is the state of California well identifier; interval midpoint depth is the midpoint of each 10-foot interval, in feet from top of borehole; PC is the percent-coarse value for each interval; log type defines whether the borehole is a drillers' lithology $\log (\mathrm{D})$ or a geophysical e-log (E).]

\begin{tabular}{|c|c|c|c|c|c|c|c|}
\hline $\begin{array}{c}\text { State } \\
\text { well } \\
\text { number }\end{array}$ & $\begin{array}{c}\text { Interval } \\
\text { midpoint depth } \\
\text { (feet) }\end{array}$ & PC & $\begin{array}{l}\text { Log } \\
\text { type }\end{array}$ & $\begin{array}{c}\text { State } \\
\text { well } \\
\text { number }\end{array}$ & $\begin{array}{c}\text { Interval } \\
\text { midpoint depth } \\
\text { (feet) }\end{array}$ & PC & $\begin{array}{l}\text { Log } \\
\text { type }\end{array}$ \\
\hline 4N/27W-15Q9 & 695 & 20 & $\mathrm{E}$ & $4 \mathrm{~N} / 27 \mathrm{~W}-15 \mathrm{~K} 1$ & 235 & 20 & $\mathrm{E}$ \\
\hline 4N/27W-15Q9 & 705 & 60 & $\mathrm{E}$ & $4 \mathrm{~N} / 27 \mathrm{~W}-15 \mathrm{~K} 1$ & 245 & 0 & $\mathrm{E}$ \\
\hline $4 \mathrm{~N} / 27 \mathrm{~W}-15 \mathrm{Q} 8$ & 45 & 0 & $\mathrm{E}$ & $4 \mathrm{~N} / 27 \mathrm{~W}-15 \mathrm{~K} 1$ & 255 & 0 & $\mathrm{E}$ \\
\hline $4 \mathrm{~N} / 27 \mathrm{~W}-15 \mathrm{Q} 8$ & 55 & 0 & $\mathrm{E}$ & $4 \mathrm{~N} / 27 \mathrm{~W}-15 \mathrm{~K} 1$ & 265 & 0 & $\mathrm{E}$ \\
\hline 4N/27W-15Q8 & 75 & 100 & $\mathrm{E}$ & $4 \mathrm{~N} / 27 \mathrm{~W}-15 \mathrm{~K} 1$ & 285 & 0 & $\mathrm{E}$ \\
\hline 4N/27W-15Q8 & 85 & 100 & $\mathrm{E}$ & $4 \mathrm{~N} / 27 \mathrm{~W}-15 \mathrm{~K} 1$ & 295 & 10 & $\mathrm{E}$ \\
\hline $4 \mathrm{~N} / 27 \mathrm{~W}-15 \mathrm{Q} 8$ & 95 & 0 & $\mathrm{E}$ & $4 \mathrm{~N} / 27 \mathrm{~W}-15 \mathrm{~K} 1$ & 305 & 70 & $\mathrm{E}$ \\
\hline $4 \mathrm{~N} / 27 \mathrm{~W}-15 \mathrm{Q} 8$ & 105 & 0 & $\mathrm{E}$ & $4 \mathrm{~N} / 27 \mathrm{~W}-15 \mathrm{~K} 1$ & 315 & 100 & $\mathrm{E}$ \\
\hline $4 \mathrm{~N} / 27 \mathrm{~W}-15 \mathrm{Q} 8$ & 145 & 0 & $\mathrm{E}$ & $4 \mathrm{~N} / 27 \mathrm{~W}-15 \mathrm{~K} 1$ & 355 & 30 & $\mathrm{E}$ \\
\hline $4 \mathrm{~N} / 27 \mathrm{~W}-15 \mathrm{Q} 8$ & 155 & 10 & $\mathrm{E}$ & $4 \mathrm{~N} / 27 \mathrm{~W}-15 \mathrm{~K} 1$ & 365 & 0 & $\mathrm{E}$ \\
\hline $4 \mathrm{~N} / 27 \mathrm{~W}-15 \mathrm{Q} 8$ & 165 & 30 & $\mathrm{E}$ & $4 \mathrm{~N} / 27 \mathrm{~W}-15 \mathrm{~K} 1$ & 375 & 0 & $\mathrm{E}$ \\
\hline 4N/27W-15Q8 & 175 & 0 & $\mathrm{E}$ & $4 \mathrm{~N} / 27 \mathrm{~W}-15 \mathrm{~K} 1$ & 385 & 0 & $\mathrm{E}$ \\
\hline 4N/27W-15Q8 & 185 & 0 & $\mathrm{E}$ & $4 \mathrm{~N} / 27 \mathrm{~W}-15 \mathrm{~K} 1$ & 395 & 0 & $\mathrm{E}$ \\
\hline $4 \mathrm{~N} / 27 \mathrm{~W}-15 \mathrm{Q} 8$ & 195 & 0 & $\mathrm{E}$ & $4 \mathrm{~N} / 27 \mathrm{~W}-15 \mathrm{~K} 1$ & 405 & 0 & $\mathrm{E}$ \\
\hline $4 \mathrm{~N} / 27 \mathrm{~W}-15 \mathrm{Q} 8$ & 205 & 0 & $\mathrm{E}$ & $4 \mathrm{~N} / 27 \mathrm{~W}-15 \mathrm{~K} 1$ & 415 & 0 & $\mathrm{E}$ \\
\hline $4 \mathrm{~N} / 27 \mathrm{~W}-15 \mathrm{Q} 8$ & 215 & 90 & $\mathrm{E}$ & $4 \mathrm{~N} / 27 \mathrm{~W}-15 \mathrm{~K} 1$ & 425 & 60 & $\mathrm{E}$ \\
\hline $4 \mathrm{~N} / 27 \mathrm{~W}-15 \mathrm{Q} 8$ & 225 & 100 & $\mathrm{E}$ & $4 \mathrm{~N} / 27 \mathrm{~W}-15 \mathrm{~K} 1$ & 435 & 100 & $\mathrm{E}$ \\
\hline $4 \mathrm{~N} / 27 \mathrm{~W}-15 \mathrm{~K} 1$ & 115 & 100 & $\mathrm{E}$ & $4 \mathrm{~N} / 27 \mathrm{~W}-15 \mathrm{~J} 1$ & 45 & 0 & $\mathrm{E}$ \\
\hline $4 \mathrm{~N} / 27 \mathrm{~W}-15 \mathrm{~K} 1$ & 125 & 100 & $\mathrm{E}$ & $4 \mathrm{~N} / 27 \mathrm{~W}-15 \mathrm{~J} 1$ & 55 & 30 & $\mathrm{E}$ \\
\hline $4 \mathrm{~N} / 27 \mathrm{~W}-15 \mathrm{~K} 1$ & 135 & 100 & $\mathrm{E}$ & $4 \mathrm{~N} / 27 \mathrm{~W}-15 \mathrm{~J} 1$ & 65 & 40 & $\mathrm{E}$ \\
\hline $4 \mathrm{~N} / 27 \mathrm{~W}-15 \mathrm{~K} 1$ & 145 & 90 & $\mathrm{E}$ & $4 \mathrm{~N} / 27 \mathrm{~W}-15 \mathrm{~J} 1$ & 75 & 0 & $\mathrm{E}$ \\
\hline $4 \mathrm{~N} / 27 \mathrm{~W}-15 \mathrm{~K} 1$ & 155 & 0 & $\mathrm{E}$ & $4 \mathrm{~N} / 27 \mathrm{~W}-15 \mathrm{~J} 1$ & 85 & 0 & $\mathrm{E}$ \\
\hline $4 \mathrm{~N} / 27 \mathrm{~W}-15 \mathrm{~K} 1$ & 165 & 0 & $\mathrm{E}$ & $4 \mathrm{~N} / 27 \mathrm{~W}-15 \mathrm{~J} 1$ & 95 & 0 & $\mathrm{E}$ \\
\hline $4 \mathrm{~N} / 27 \mathrm{~W}-15 \mathrm{~K} 1$ & 175 & 90 & $\mathrm{E}$ & $4 \mathrm{~N} / 27 \mathrm{~W}-15 \mathrm{~J} 1$ & 105 & 100 & $\mathrm{E}$ \\
\hline $4 \mathrm{~N} / 27 \mathrm{~W}-15 \mathrm{~K} 1$ & 185 & 50 & $\mathrm{E}$ & $4 \mathrm{~N} / 27 \mathrm{~W}-15 \mathrm{~J} 1$ & 115 & 0 & $\mathrm{E}$ \\
\hline $4 \mathrm{~N} / 27 \mathrm{~W}-15 \mathrm{~K} 1$ & 195 & 0 & $\mathrm{E}$ & $4 \mathrm{~N} / 27 \mathrm{~W}-15 \mathrm{~J} 1$ & 125 & 0 & $\mathrm{E}$ \\
\hline $4 \mathrm{~N} / 27 \mathrm{~W}-15 \mathrm{~K} 1$ & 205 & 60 & $\mathrm{E}$ & $4 \mathrm{~N} / 27 \mathrm{~W}-15 \mathrm{~J} 1$ & 135 & 0 & $\mathrm{E}$ \\
\hline $4 \mathrm{~N} / 27 \mathrm{~W}-15 \mathrm{~K} 1$ & 215 & 90 & $\mathrm{E}$ & $4 \mathrm{~N} / 27 \mathrm{~W}-15 \mathrm{~J} 1$ & 145 & 10 & $\mathrm{E}$ \\
\hline $4 \mathrm{~N} / 27 \mathrm{~W}-15 \mathrm{~K} 1$ & 225 & 100 & $\mathrm{E}$ & $4 \mathrm{~N} / 27 \mathrm{~W}-15 \mathrm{~J} 1$ & 155 & 40 & $\mathrm{E}$ \\
\hline
\end{tabular}


Table B1-1. Calculated percent-coarse intervals for all drillers' logs and e-logs used to define textural properties of the hydrogeologic framework model.-Continued

[Percent-coarse values are calculated for 10-foot intervals for the entire depth of each borehole. State well number is the state of California well identifier; interval midpoint depth is the midpoint of each 10-foot interval, in feet from top of borehole; PC is the percent-coarse value for each interval; log type defines whether the borehole is a drillers' lithology $\log (\mathrm{D})$ or a geophysical e-log (E).]

\begin{tabular}{|c|c|c|c|c|c|c|c|}
\hline $\begin{array}{c}\text { State } \\
\text { well } \\
\text { number }\end{array}$ & $\begin{array}{c}\text { Interval } \\
\text { midpoint depth } \\
\text { (feet) }\end{array}$ & PC & $\begin{array}{l}\text { Log } \\
\text { type }\end{array}$ & $\begin{array}{c}\text { State } \\
\text { well } \\
\text { number }\end{array}$ & $\begin{array}{c}\text { Interval } \\
\text { midpoint depth } \\
\text { (feet) }\end{array}$ & PC & $\begin{array}{l}\text { Log } \\
\text { type }\end{array}$ \\
\hline $4 \mathrm{~N} / 27 \mathrm{~W}-15 \mathrm{~J} 1$ & 165 & 80 & $\mathrm{E}$ & $4 \mathrm{~N} / 27 \mathrm{~W}-15 \mathrm{~J} 1$ & 555 & 80 & $\mathrm{E}$ \\
\hline $4 \mathrm{~N} / 27 \mathrm{~W}-15 \mathrm{~J} 1$ & 175 & 0 & $\mathrm{E}$ & $4 \mathrm{~N} / 27 \mathrm{~W}-15 \mathrm{~J} 1$ & 565 & 60 & $\mathrm{E}$ \\
\hline $4 \mathrm{~N} / 27 \mathrm{~W}-15 \mathrm{~J} 1$ & 185 & 30 & $\mathrm{E}$ & $4 \mathrm{~N} / 27 \mathrm{~W}-15 \mathrm{~J} 1$ & 575 & 0 & $\mathrm{E}$ \\
\hline $4 \mathrm{~N} / 27 \mathrm{~W}-15 \mathrm{~J} 1$ & 195 & 100 & $\mathrm{E}$ & $4 \mathrm{~N} / 27 \mathrm{~W}-15 \mathrm{~J} 1$ & 585 & 80 & $\mathrm{E}$ \\
\hline $4 \mathrm{~N} / 27 \mathrm{~W}-15 \mathrm{~J} 1$ & 215 & 0 & $\mathrm{E}$ & $4 \mathrm{~N} / 27 \mathrm{~W}-15 \mathrm{~J} 1$ & 605 & 0 & $\mathrm{E}$ \\
\hline $4 \mathrm{~N} / 27 \mathrm{~W}-15 \mathrm{~J} 1$ & 225 & 0 & $\mathrm{E}$ & $4 \mathrm{~N} / 27 \mathrm{~W}-15 \mathrm{~J} 1$ & 615 & 0 & $\mathrm{E}$ \\
\hline $4 \mathrm{~N} / 27 \mathrm{~W}-15 \mathrm{~J} 1$ & 235 & 0 & $\mathrm{E}$ & $4 \mathrm{~N} / 27 \mathrm{~W}-15 \mathrm{~J} 1$ & 625 & 0 & $\mathrm{E}$ \\
\hline $4 \mathrm{~N} / 27 \mathrm{~W}-15 \mathrm{~J} 1$ & 245 & 0 & $\mathrm{E}$ & $4 \mathrm{~N} / 27 \mathrm{~W}-15 \mathrm{E} 1$ & 75 & 100 & $\mathrm{E}$ \\
\hline $4 \mathrm{~N} / 27 \mathrm{~W}-15 \mathrm{~J} 1$ & 285 & 60 & $\mathrm{E}$ & $4 \mathrm{~N} / 27 \mathrm{~W}-15 \mathrm{E} 1$ & 115 & 100 & $\mathrm{E}$ \\
\hline $4 \mathrm{~N} / 27 \mathrm{~W}-15 \mathrm{~J} 1$ & 295 & 0 & $\mathrm{E}$ & $4 \mathrm{~N} / 27 \mathrm{~W}-15 \mathrm{E} 1$ & 125 & 100 & $\mathrm{E}$ \\
\hline $4 \mathrm{~N} / 27 \mathrm{~W}-15 \mathrm{~J} 1$ & 305 & 0 & $\mathrm{E}$ & $4 \mathrm{~N} / 27 \mathrm{~W}-15 \mathrm{E} 1$ & 135 & 100 & $\mathrm{E}$ \\
\hline $4 \mathrm{~N} / 27 \mathrm{~W}-15 \mathrm{~J} 1$ & 315 & 0 & $\mathrm{E}$ & $4 \mathrm{~N} / 27 \mathrm{~W}-15 \mathrm{E} 1$ & 145 & 40 & $\mathrm{E}$ \\
\hline $4 \mathrm{~N} / 27 \mathrm{~W}-15 \mathrm{~J} 1$ & 325 & 30 & $\mathrm{E}$ & $4 \mathrm{~N} / 27 \mathrm{~W}-15 \mathrm{E} 1$ & 155 & 0 & $\mathrm{E}$ \\
\hline $4 \mathrm{~N} / 27 \mathrm{~W}-15 \mathrm{~J} 1$ & 335 & 0 & $\mathrm{E}$ & $4 \mathrm{~N} / 27 \mathrm{~W}-15 \mathrm{E} 1$ & 165 & 0 & $\mathrm{E}$ \\
\hline $4 \mathrm{~N} / 27 \mathrm{~W}-15 \mathrm{~J} 1$ & 345 & 0 & $\mathrm{E}$ & $4 \mathrm{~N} / 27 \mathrm{~W}-15 \mathrm{E} 1$ & 175 & 80 & $\mathrm{E}$ \\
\hline $4 \mathrm{~N} / 27 \mathrm{~W}-15 \mathrm{~J} 1$ & 355 & 0 & $\mathrm{E}$ & $4 \mathrm{~N} / 27 \mathrm{~W}-15 \mathrm{E} 1$ & 185 & 20 & $\mathrm{E}$ \\
\hline $4 \mathrm{~N} / 27 \mathrm{~W}-15 \mathrm{~J} 1$ & 365 & 40 & $\mathrm{E}$ & $4 \mathrm{~N} / 27 \mathrm{~W}-15 \mathrm{E} 1$ & 195 & 10 & $\mathrm{E}$ \\
\hline $4 \mathrm{~N} / 27 \mathrm{~W}-15 \mathrm{~J} 1$ & 435 & 0 & $\mathrm{E}$ & $4 \mathrm{~N} / 27 \mathrm{~W}-15 \mathrm{E} 1$ & 265 & 0 & $\mathrm{E}$ \\
\hline $4 \mathrm{~N} / 27 \mathrm{~W}-15 \mathrm{~J} 1$ & 445 & 20 & $\mathrm{E}$ & $4 \mathrm{~N} / 27 \mathrm{~W}-15 \mathrm{E} 1$ & 275 & 50 & $\mathrm{E}$ \\
\hline $4 \mathrm{~N} / 27 \mathrm{~W}-15 \mathrm{~J} 1$ & 455 & 0 & $\mathrm{E}$ & $4 \mathrm{~N} / 27 \mathrm{~W}-15 \mathrm{E} 1$ & 285 & 80 & $\mathrm{E}$ \\
\hline $4 \mathrm{~N} / 27 \mathrm{~W}-15 \mathrm{~J} 1$ & 465 & 30 & $\mathrm{E}$ & $4 \mathrm{~N} / 27 \mathrm{~W}-15 \mathrm{E} 1$ & 295 & 0 & $\mathrm{E}$ \\
\hline $4 \mathrm{~N} / 27 \mathrm{~W}-15 \mathrm{~J} 1$ & 475 & 70 & $\mathrm{E}$ & $4 \mathrm{~N} / 27 \mathrm{~W}-15 \mathrm{E} 1$ & 305 & 0 & $\mathrm{E}$ \\
\hline $4 \mathrm{~N} / 27 \mathrm{~W}-15 \mathrm{~J} 1$ & 485 & 0 & $\mathrm{E}$ & $4 \mathrm{~N} / 27 \mathrm{~W}-15 \mathrm{E} 1$ & 315 & 0 & $\mathrm{E}$ \\
\hline $4 \mathrm{~N} / 27 \mathrm{~W}-15 \mathrm{~J} 1$ & 495 & 0 & $\mathrm{E}$ & $4 \mathrm{~N} / 27 \mathrm{~W}-15 \mathrm{E} 1$ & 325 & 0 & $\mathrm{E}$ \\
\hline $4 \mathrm{~N} / 27 \mathrm{~W}-15 \mathrm{~J} 1$ & 505 & 20 & $\mathrm{E}$ & $4 \mathrm{~N} / 27 \mathrm{~W}-15 \mathrm{E} 1$ & 335 & 0 & $\mathrm{E}$ \\
\hline $4 \mathrm{~N} / 27 \mathrm{~W}-15 \mathrm{~J} 1$ & 515 & 100 & $\mathrm{E}$ & $4 \mathrm{~N} / 27 \mathrm{~W}-15 \mathrm{E} 1$ & 345 & 40 & $\mathrm{E}$ \\
\hline $4 \mathrm{~N} / 27 \mathrm{~W}-15 \mathrm{~J} 1$ & 525 & 30 & $\mathrm{E}$ & $4 \mathrm{~N} / 27 \mathrm{~W}-15 \mathrm{E} 1$ & 355 & 0 & $\mathrm{E}$ \\
\hline $4 \mathrm{~N} / 27 \mathrm{~W}-15 \mathrm{~J} 1$ & 535 & 100 & $\mathrm{E}$ & $4 \mathrm{~N} / 27 \mathrm{~W}-15 \mathrm{E} 1$ & 365 & 80 & $\mathrm{E}$ \\
\hline 4N/27W-15J1 & 545 & 100 & $\mathrm{E}$ & $4 \mathrm{~N} / 27 \mathrm{~W}-15 \mathrm{E} 1$ & 375 & 0 & $\mathrm{E}$ \\
\hline
\end{tabular}


Table B1-1. Calculated percent-coarse intervals for all drillers' logs and e-logs used to define textural properties of the hydrogeologic framework model.-Continued

[Percent-coarse values are calculated for 10-foot intervals for the entire depth of each borehole. State well number is the state of California well identifier; interval midpoint depth is the midpoint of each 10-foot interval, in feet from top of borehole; PC is the percent-coarse value for each interval; log type defines whether the borehole is a drillers' lithology $\log (\mathrm{D})$ or a geophysical e-log (E).]

\begin{tabular}{|c|c|c|c|c|c|c|c|}
\hline $\begin{array}{c}\text { State } \\
\text { well } \\
\text { number }\end{array}$ & $\begin{array}{c}\text { Interval } \\
\text { midpoint depth } \\
\text { (feet) }\end{array}$ & PC & $\begin{array}{l}\text { Log } \\
\text { type }\end{array}$ & $\begin{array}{c}\text { State } \\
\text { well } \\
\text { number }\end{array}$ & $\begin{array}{c}\text { Interval } \\
\text { midpoint depth } \\
\text { (feet) }\end{array}$ & PC & $\begin{array}{l}\text { Log } \\
\text { type }\end{array}$ \\
\hline $4 \mathrm{~N} / 27 \mathrm{~W}-15 \mathrm{E} 1$ & 385 & 10 & $\mathrm{E}$ & $4 \mathrm{~N} / 27 \mathrm{~W}-14 \mathrm{R} 1$ & 275 & 0 & $\mathrm{E}$ \\
\hline $4 \mathrm{~N} / 27 \mathrm{~W}-15 \mathrm{E} 1$ & 395 & 30 & $\mathrm{E}$ & $4 \mathrm{~N} / 27 \mathrm{~W}-14 \mathrm{R} 1$ & 285 & 20 & $\mathrm{E}$ \\
\hline $4 \mathrm{~N} / 27 \mathrm{~W}-15 \mathrm{E} 1$ & 405 & 0 & $\mathrm{E}$ & $4 \mathrm{~N} / 27 \mathrm{~W}-14 \mathrm{R} 1$ & 295 & 50 & $\mathrm{E}$ \\
\hline $4 \mathrm{~N} / 27 \mathrm{~W}-15 \mathrm{E} 1$ & 415 & 30 & $\mathrm{E}$ & 4N/27W-14R1 & 305 & 20 & $\mathrm{E}$ \\
\hline 4N/27W-15E1 & 435 & 100 & $\mathrm{E}$ & 4N/27W-14R1 & 325 & 30 & $\mathrm{E}$ \\
\hline $4 \mathrm{~N} / 27 \mathrm{~W}-15 \mathrm{E} 1$ & 445 & 30 & $\mathrm{E}$ & $4 \mathrm{~N} / 27 \mathrm{~W}-14 \mathrm{R} 1$ & 335 & 60 & $\mathrm{E}$ \\
\hline $4 \mathrm{~N} / 27 \mathrm{~W}-15 \mathrm{E} 1$ & 455 & 100 & $\mathrm{E}$ & $4 \mathrm{~N} / 27 \mathrm{~W}-14 \mathrm{R} 1$ & 345 & 100 & $\mathrm{E}$ \\
\hline $4 \mathrm{~N} / 27 \mathrm{~W}-15 \mathrm{E} 1$ & 465 & 100 & $\mathrm{E}$ & $4 \mathrm{~N} / 27 \mathrm{~W}-14 \mathrm{R} 1$ & 355 & 20 & $\mathrm{E}$ \\
\hline $4 \mathrm{~N} / 27 \mathrm{~W}-15 \mathrm{E} 1$ & 505 & 0 & $\mathrm{E}$ & $4 \mathrm{~N} / 27 \mathrm{~W}-14 \mathrm{R} 1$ & 395 & 0 & $\mathrm{E}$ \\
\hline $4 \mathrm{~N} / 27 \mathrm{~W}-15 \mathrm{E} 1$ & 515 & 0 & $\mathrm{E}$ & $4 \mathrm{~N} / 27 \mathrm{~W}-14 \mathrm{R} 1$ & 405 & 0 & $\mathrm{E}$ \\
\hline $4 \mathrm{~N} / 27 \mathrm{~W}-14 \mathrm{R} 1$ & 25 & 0 & $\mathrm{E}$ & $4 \mathrm{~N} / 27 \mathrm{~W}-14 \mathrm{R} 1$ & 415 & 0 & $\mathrm{E}$ \\
\hline $4 \mathrm{~N} / 27 \mathrm{~W}-14 \mathrm{R} 1$ & 35 & 40 & $\mathrm{E}$ & $4 \mathrm{~N} / 27 \mathrm{~W}-14 \mathrm{R} 1$ & 425 & 0 & $\mathrm{E}$ \\
\hline $4 \mathrm{~N} / 27 \mathrm{~W}-14 \mathrm{R} 1$ & 45 & 0 & $\mathrm{E}$ & 4N/27W-14R1 & 435 & 100 & $\mathrm{E}$ \\
\hline $4 \mathrm{~N} / 27 \mathrm{~W}-14 \mathrm{R} 1$ & 55 & 0 & $\mathrm{E}$ & $4 \mathrm{~N} / 27 \mathrm{~W}-14 \mathrm{R} 1$ & 445 & 10 & $\mathrm{E}$ \\
\hline $4 \mathrm{~N} / 27 \mathrm{~W}-14 \mathrm{R} 1$ & 65 & 0 & $\mathrm{E}$ & $4 \mathrm{~N} / 27 \mathrm{~W}-14 \mathrm{R} 1$ & 455 & 20 & $\mathrm{E}$ \\
\hline $4 \mathrm{~N} / 27 \mathrm{~W}-14 \mathrm{R} 1$ & 75 & 0 & $\mathrm{E}$ & $4 \mathrm{~N} / 27 \mathrm{~W}-14 \mathrm{R} 1$ & 465 & 0 & $\mathrm{E}$ \\
\hline $4 \mathrm{~N} / 27 \mathrm{~W}-14 \mathrm{R} 1$ & 85 & 40 & $\mathrm{E}$ & $4 \mathrm{~N} / 27 \mathrm{~W}-14 \mathrm{R} 1$ & 475 & 20 & $\mathrm{E}$ \\
\hline $4 \mathrm{~N} / 27 \mathrm{~W}-14 \mathrm{R} 1$ & 155 & 0 & $\mathrm{E}$ & $4 \mathrm{~N} / 27 \mathrm{~W}-14 \mathrm{R} 1$ & 545 & 10 & $\mathrm{E}$ \\
\hline $4 \mathrm{~N} / 27 \mathrm{~W}-14 \mathrm{R} 1$ & 165 & 0 & $\mathrm{E}$ & $4 \mathrm{~N} / 27 \mathrm{~W}-14 \mathrm{R} 1$ & 555 & 70 & $\mathrm{E}$ \\
\hline $4 \mathrm{~N} / 27 \mathrm{~W}-14 \mathrm{R} 1$ & 175 & 0 & $\mathrm{E}$ & $4 \mathrm{~N} / 27 \mathrm{~W}-14 \mathrm{R} 1$ & 565 & 100 & $\mathrm{E}$ \\
\hline 4N/27W-14R1 & 185 & 0 & $\mathrm{E}$ & $4 \mathrm{~N} / 27 \mathrm{~W}-14 \mathrm{R} 1$ & 575 & 60 & $\mathrm{E}$ \\
\hline $4 \mathrm{~N} / 27 \mathrm{~W}-14 \mathrm{R} 1$ & 195 & 0 & $\mathrm{E}$ & $4 \mathrm{~N} / 27 \mathrm{~W}-14 \mathrm{R} 1$ & 585 & 50 & $\mathrm{E}$ \\
\hline $4 \mathrm{~N} / 27 \mathrm{~W}-14 \mathrm{R} 1$ & 205 & 0 & $\mathrm{E}$ & $4 \mathrm{~N} / 27 \mathrm{~W}-14 \mathrm{R} 1$ & 595 & 100 & $\mathrm{E}$ \\
\hline 4N/27W-14R1 & 215 & 0 & $\mathrm{E}$ & 4N/27W-14R1 & 605 & 100 & $\mathrm{E}$ \\
\hline $4 \mathrm{~N} / 27 \mathrm{~W}-14 \mathrm{R} 1$ & 225 & 0 & $\mathrm{E}$ & $4 \mathrm{~N} / 27 \mathrm{~W}-14 \mathrm{R} 1$ & 615 & 100 & $\mathrm{E}$ \\
\hline $4 \mathrm{~N} / 27 \mathrm{~W}-14 \mathrm{R} 1$ & 235 & 0 & $\mathrm{E}$ & $4 \mathrm{~N} / 27 \mathrm{~W}-14 \mathrm{R} 1$ & 625 & 90 & $\mathrm{E}$ \\
\hline $4 \mathrm{~N} / 27 \mathrm{~W}-14 \mathrm{R} 1$ & 245 & 0 & $\mathrm{E}$ & $4 \mathrm{~N} / 27 \mathrm{~W}-14 \mathrm{R} 1$ & 635 & 70 & $\mathrm{E}$ \\
\hline $4 \mathrm{~N} / 27 \mathrm{~W}-14 \mathrm{R} 1$ & 255 & 60 & $\mathrm{E}$ & $4 \mathrm{~N} / 27 \mathrm{~W}-14 \mathrm{R} 1$ & 645 & 100 & $\mathrm{E}$ \\
\hline 4N/27W-14R1 & 265 & 0 & $\mathrm{E}$ & $4 \mathrm{~N} / 27 \mathrm{~W}-14 \mathrm{R} 1$ & 655 & 100 & $\mathrm{E}$ \\
\hline
\end{tabular}


Table B1-1. Calculated percent-coarse intervals for all drillers' logs and e-logs used to define textural properties of the hydrogeologic framework model.-Continued

[Percent-coarse values are calculated for 10-foot intervals for the entire depth of each borehole. State well number is the state of California well identifier; interval midpoint depth is the midpoint of each 10-foot interval, in feet from top of borehole; PC is the percent-coarse value for each interval; log type defines whether the borehole is a drillers' lithology $\log (\mathrm{D})$ or a geophysical e-log (E).]

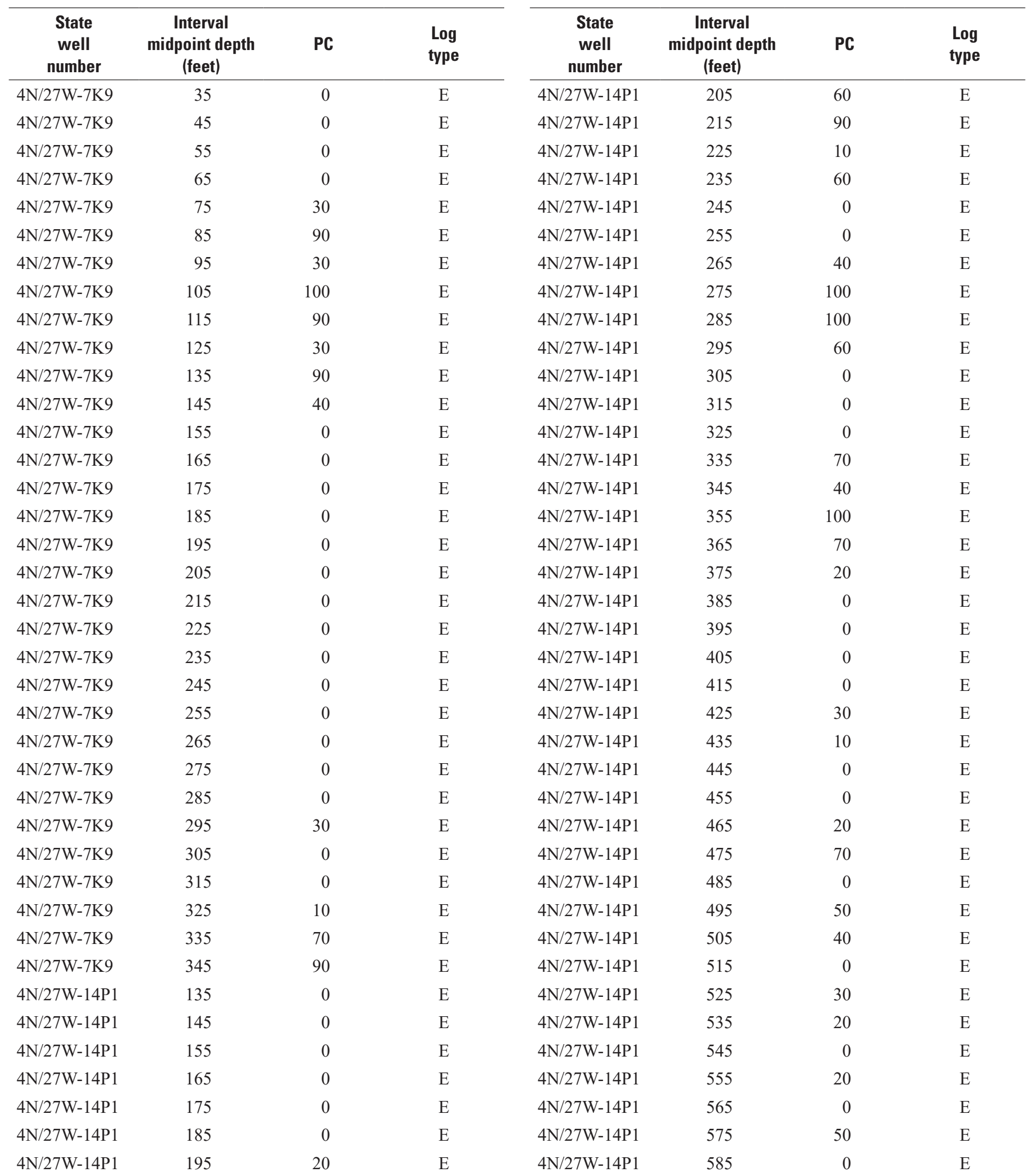


Table B1-1. Calculated percent-coarse intervals for all drillers' logs and e-logs used to define textural properties of the hydrogeologic framework model.-Continued

[Percent-coarse values are calculated for 10-foot intervals for the entire depth of each borehole. State well number is the state of California well identifier; interval midpoint depth is the midpoint of each 10-foot interval, in feet from top of borehole; PC is the percent-coarse value for each interval; log type defines whether the borehole is a drillers' lithology log (D) or a geophysical e-log (E).]

\begin{tabular}{|c|c|c|c|c|c|c|c|}
\hline $\begin{array}{c}\text { State } \\
\text { well } \\
\text { number }\end{array}$ & $\begin{array}{c}\text { Interval } \\
\text { midpoint depth } \\
\text { (feet) }\end{array}$ & PC & $\begin{array}{l}\text { Log } \\
\text { type }\end{array}$ & $\begin{array}{c}\text { State } \\
\text { well } \\
\text { number }\end{array}$ & $\begin{array}{c}\text { Interval } \\
\text { midpoint depth } \\
\text { (feet) }\end{array}$ & PC & $\begin{array}{l}\text { Log } \\
\text { type }\end{array}$ \\
\hline $4 \mathrm{~N} / 27 \mathrm{~W}-14 \mathrm{P} 1$ & 595 & 0 & $\mathrm{E}$ & $4 \mathrm{~N} / 27 \mathrm{~W}-14 \mathrm{~K} 2$ & 265 & 20 & $\mathrm{E}$ \\
\hline $4 \mathrm{~N} / 27 \mathrm{~W}-14 \mathrm{P} 1$ & 605 & 0 & $\mathrm{E}$ & $4 \mathrm{~N} / 27 \mathrm{~W}-14 \mathrm{~K} 2$ & 275 & 100 & $\mathrm{E}$ \\
\hline $4 \mathrm{~N} / 27 \mathrm{~W}-14 \mathrm{P} 1$ & 615 & 50 & $\mathrm{E}$ & $4 \mathrm{~N} / 27 \mathrm{~W}-14 \mathrm{~K} 2$ & 285 & 100 & $\mathrm{E}$ \\
\hline $4 \mathrm{~N} / 27 \mathrm{~W}-14 \mathrm{P} 1$ & 625 & 70 & $\mathrm{E}$ & $4 \mathrm{~N} / 27 \mathrm{~W}-14 \mathrm{~K} 2$ & 295 & 100 & $\mathrm{E}$ \\
\hline $4 \mathrm{~N} / 27 \mathrm{~W}-14 \mathrm{P} 1$ & 645 & 30 & $\mathrm{E}$ & $4 \mathrm{~N} / 27 \mathrm{~W}-14 \mathrm{~K} 2$ & 315 & 30 & $\mathrm{E}$ \\
\hline $4 \mathrm{~N} / 27 \mathrm{~W}-14 \mathrm{P} 1$ & 655 & 20 & $\mathrm{E}$ & $4 \mathrm{~N} / 27 \mathrm{~W}-14 \mathrm{~K} 2$ & 325 & 0 & $\mathrm{E}$ \\
\hline $4 \mathrm{~N} / 27 \mathrm{~W}-14 \mathrm{P} 1$ & 665 & 0 & $\mathrm{E}$ & $4 \mathrm{~N} / 27 \mathrm{~W}-14 \mathrm{~K} 2$ & 335 & 0 & $\mathrm{E}$ \\
\hline $4 \mathrm{~N} / 27 \mathrm{~W}-14 \mathrm{P} 1$ & 675 & 100 & $\mathrm{E}$ & $4 \mathrm{~N} / 27 \mathrm{~W}-14 \mathrm{~K} 2$ & 345 & 20 & $\mathrm{E}$ \\
\hline $4 \mathrm{~N} / 27 \mathrm{~W}-14 \mathrm{P} 1$ & 715 & 100 & $\mathrm{E}$ & $4 \mathrm{~N} / 27 \mathrm{~W}-14 \mathrm{~K} 2$ & 385 & 0 & $\mathrm{E}$ \\
\hline $4 \mathrm{~N} / 27 \mathrm{~W}-14 \mathrm{P} 1$ & 725 & 100 & $\mathrm{E}$ & $4 \mathrm{~N} / 27 \mathrm{~W}-14 \mathrm{~K} 2$ & 395 & 0 & $\mathrm{E}$ \\
\hline $4 \mathrm{~N} / 27 \mathrm{~W}-14 \mathrm{P} 1$ & 735 & 100 & $\mathrm{E}$ & $4 \mathrm{~N} / 27 \mathrm{~W}-14 \mathrm{~K} 2$ & 405 & 0 & $\mathrm{E}$ \\
\hline $4 \mathrm{~N} / 27 \mathrm{~W}-14 \mathrm{P} 1$ & 745 & 100 & $\mathrm{E}$ & $4 \mathrm{~N} / 27 \mathrm{~W}-14 \mathrm{~K} 2$ & 415 & 0 & $\mathrm{E}$ \\
\hline $4 \mathrm{~N} / 27 \mathrm{~W}-14 \mathrm{P} 1$ & 755 & 100 & $\mathrm{E}$ & $4 \mathrm{~N} / 27 \mathrm{~W}-14 \mathrm{~K} 2$ & 425 & 0 & $\mathrm{E}$ \\
\hline $4 \mathrm{~N} / 27 \mathrm{~W}-14 \mathrm{P} 1$ & 765 & 70 & $\mathrm{E}$ & $4 \mathrm{~N} / 27 \mathrm{~W}-14 \mathrm{~K} 2$ & 435 & 0 & $\mathrm{E}$ \\
\hline $4 \mathrm{~N} / 27 \mathrm{~W}-14 \mathrm{P} 1$ & 775 & 30 & $\mathrm{E}$ & 4N/27W-9M1 & 25 & 50 & $\mathrm{E}$ \\
\hline $4 \mathrm{~N} / 27 \mathrm{~W}-14 \mathrm{~K} 2$ & 65 & 0 & $\mathrm{E}$ & 4N/27W-9M1 & 35 & 30 & $\mathrm{E}$ \\
\hline $4 \mathrm{~N} / 27 \mathrm{~W}-14 \mathrm{~K} 2$ & 75 & 80 & $\mathrm{E}$ & 4N/27W-9M1 & 45 & 80 & $\mathrm{E}$ \\
\hline $4 \mathrm{~N} / 27 \mathrm{~W}-14 \mathrm{~K} 2$ & 145 & 0 & $\mathrm{E}$ & 4N/27W-9M1 & 115 & 0 & $\mathrm{E}$ \\
\hline $4 \mathrm{~N} / 27 \mathrm{~W}-14 \mathrm{~K} 2$ & 155 & 0 & $\mathrm{E}$ & 4N/27W-9M1 & 125 & 0 & $\mathrm{E}$ \\
\hline $4 \mathrm{~N} / 27 \mathrm{~W}-14 \mathrm{~K} 2$ & 165 & 0 & $\mathrm{E}$ & 4N/27W-9M1 & 135 & 0 & $\mathrm{E}$ \\
\hline $4 \mathrm{~N} / 27 \mathrm{~W}-14 \mathrm{~K} 2$ & 175 & 0 & $\mathrm{E}$ & 4N/27W-9M1 & 145 & 0 & $\mathrm{E}$ \\
\hline $4 \mathrm{~N} / 27 \mathrm{~W}-14 \mathrm{~K} 2$ & 185 & 0 & $\mathrm{E}$ & 4N/27W-9M1 & 155 & 0 & $\mathrm{E}$ \\
\hline $4 \mathrm{~N} / 27 \mathrm{~W}-14 \mathrm{~K} 2$ & 195 & 0 & $\mathrm{E}$ & 4N/27W-9M1 & 165 & 0 & $\mathrm{E}$ \\
\hline $4 \mathrm{~N} / 27 \mathrm{~W}-14 \mathrm{~K} 2$ & 205 & 0 & $\mathrm{E}$ & 4N/27W-9M1 & 175 & 0 & $\mathrm{E}$ \\
\hline $4 \mathrm{~N} / 27 \mathrm{~W}-14 \mathrm{~K} 2$ & 215 & 0 & $\mathrm{E}$ & $4 \mathrm{~N} / 27 \mathrm{~W}-8 \mathrm{R} 2$ & 25 & 0 & $\mathrm{E}$ \\
\hline $4 \mathrm{~N} / 27 \mathrm{~W}-14 \mathrm{~K} 2$ & 225 & 0 & $\mathrm{E}$ & $4 \mathrm{~N} / 27 \mathrm{~W}-8 \mathrm{R} 2$ & 35 & 0 & $\mathrm{E}$ \\
\hline $4 \mathrm{~N} / 27 \mathrm{~W}-14 \mathrm{~K} 2$ & 235 & 0 & $\mathrm{E}$ & $4 \mathrm{~N} / 27 \mathrm{~W}-8 \mathrm{R} 2$ & 45 & 40 & $\mathrm{E}$ \\
\hline $4 \mathrm{~N} / 27 \mathrm{~W}-14 \mathrm{~K} 2$ & 245 & 0 & $\mathrm{E}$ & $4 \mathrm{~N} / 27 \mathrm{~W}-8 \mathrm{R} 2$ & 55 & 100 & $\mathrm{E}$ \\
\hline $4 \mathrm{~N} / 27 \mathrm{~W}-14 \mathrm{~K} 2$ & 255 & 0 & $\mathrm{E}$ & $4 \mathrm{~N} / 27 \mathrm{~W}-8 \mathrm{R} 2$ & 65 & 40 & $\mathrm{E}$ \\
\hline
\end{tabular}


Table B1-1. Calculated percent-coarse intervals for all drillers' logs and e-logs used to define textural properties of the hydrogeologic framework model.-Continued

[Percent-coarse values are calculated for 10-foot intervals for the entire depth of each borehole. State well number is the state of California well identifier; interval midpoint depth is the midpoint of each 10-foot interval, in feet from top of borehole; PC is the percent-coarse value for each interval; log type defines whether the borehole is a drillers' lithology $\log (\mathrm{D})$ or a geophysical e-log (E).]

\begin{tabular}{|c|c|c|c|c|c|c|c|}
\hline $\begin{array}{c}\text { State } \\
\text { well } \\
\text { number }\end{array}$ & $\begin{array}{c}\text { Interval } \\
\text { midpoint depth } \\
\text { (feet) }\end{array}$ & PC & $\begin{array}{l}\text { Log } \\
\text { type }\end{array}$ & $\begin{array}{c}\text { State } \\
\text { well } \\
\text { number }\end{array}$ & $\begin{array}{c}\text { Interval } \\
\text { midpoint depth } \\
\text { (feet) }\end{array}$ & PC & $\begin{array}{l}\text { Log } \\
\text { type }\end{array}$ \\
\hline 4N/27W-8R2 & 75 & 90 & $\mathrm{E}$ & $4 \mathrm{~N} / 27 \mathrm{~W}-8 \mathrm{E} 1$ & 295 & 50 & $\mathrm{E}$ \\
\hline $4 \mathrm{~N} / 27 \mathrm{~W}-8 \mathrm{R} 2$ & 85 & 60 & $\mathrm{E}$ & $4 \mathrm{~N} / 27 \mathrm{~W}-8 \mathrm{E} 1$ & 305 & 100 & $\mathrm{E}$ \\
\hline 4N/27W-8R2 & 95 & 0 & $\mathrm{E}$ & $4 \mathrm{~N} / 27 \mathrm{~W}-8 \mathrm{E} 1$ & 315 & 100 & $\mathrm{E}$ \\
\hline $4 \mathrm{~N} / 27 \mathrm{~W}-8 \mathrm{R} 2$ & 105 & 0 & $\mathrm{E}$ & $4 \mathrm{~N} / 27 \mathrm{~W}-8 \mathrm{E} 1$ & 325 & 80 & $\mathrm{E}$ \\
\hline $4 \mathrm{~N} / 27 \mathrm{~W}-8 \mathrm{R} 2$ & 125 & 0 & $\mathrm{E}$ & 4N/27W-8E1 & 345 & 10 & $\mathrm{E}$ \\
\hline $4 \mathrm{~N} / 27 \mathrm{~W}-8 \mathrm{R} 2$ & 135 & 0 & $\mathrm{E}$ & $4 \mathrm{~N} / 27 \mathrm{~W}-8 \mathrm{E} 1$ & 355 & 20 & $\mathrm{E}$ \\
\hline 4N/27W-8R2 & 145 & 40 & $\mathrm{E}$ & $4 \mathrm{~N} / 27 \mathrm{~W}-8 \mathrm{E} 1$ & 365 & 20 & $\mathrm{E}$ \\
\hline 4N/27W-8R2 & 155 & 100 & $\mathrm{E}$ & $4 \mathrm{~N} / 27 \mathrm{~W}-8 \mathrm{E} 1$ & 375 & 50 & $\mathrm{E}$ \\
\hline 4N/27W-8R2 & 195 & 20 & $\mathrm{E}$ & $4 \mathrm{~N} / 27 \mathrm{~W}-8 \mathrm{E} 1$ & 415 & 0 & $\mathrm{E}$ \\
\hline 4N/27W-8R2 & 205 & 0 & $\mathrm{E}$ & $4 \mathrm{~N} / 27 \mathrm{~W}-8 \mathrm{E} 1$ & 425 & 0 & $\mathrm{E}$ \\
\hline $4 \mathrm{~N} / 27 \mathrm{~W}-8 \mathrm{R} 2$ & 215 & 70 & $\mathrm{E}$ & $4 \mathrm{~N} / 27 \mathrm{~W}-8 \mathrm{E} 1$ & 435 & 90 & $\mathrm{E}$ \\
\hline $4 \mathrm{~N} / 27 \mathrm{~W}-8 \mathrm{R} 2$ & 225 & 0 & $\mathrm{E}$ & $4 \mathrm{~N} / 27 \mathrm{~W}-8 \mathrm{E} 1$ & 445 & 100 & $\mathrm{E}$ \\
\hline $4 \mathrm{~N} / 27 \mathrm{~W}-8 \mathrm{R} 2$ & 235 & 30 & $\mathrm{E}$ & 4N/27W-8E1 & 455 & 90 & $\mathrm{E}$ \\
\hline $4 \mathrm{~N} / 27 \mathrm{~W}-8 \mathrm{R} 2$ & 245 & 0 & $\mathrm{E}$ & $4 \mathrm{~N} / 27 \mathrm{~W}-8 \mathrm{E} 1$ & 465 & 0 & $\mathrm{E}$ \\
\hline $4 \mathrm{~N} / 27 \mathrm{~W}-8 \mathrm{E} 1$ & 85 & 71.4 & $\mathrm{E}$ & $4 \mathrm{~N} / 27 \mathrm{~W}-8 \mathrm{E} 1$ & 475 & 0 & $\mathrm{E}$ \\
\hline $4 \mathrm{~N} / 27 \mathrm{~W}-8 \mathrm{E} 1$ & 95 & 0 & $\mathrm{E}$ & $4 \mathrm{~N} / 27 \mathrm{~W}-8 \mathrm{E} 1$ & 485 & 0 & $\mathrm{E}$ \\
\hline $4 \mathrm{~N} / 27 \mathrm{~W}-8 \mathrm{E} 1$ & 105 & 0 & $\mathrm{E}$ & $4 \mathrm{~N} / 27 \mathrm{~W}-8 \mathrm{E} 1$ & 495 & 0 & $\mathrm{E}$ \\
\hline $4 \mathrm{~N} / 27 \mathrm{~W}-8 \mathrm{E} 1$ & 175 & 0 & $\mathrm{E}$ & $4 \mathrm{~N} / 27 \mathrm{~W}-8 \mathrm{E} 1$ & 565 & 30 & $\mathrm{E}$ \\
\hline $4 \mathrm{~N} / 27 \mathrm{~W}-8 \mathrm{E} 1$ & 185 & 0 & $\mathrm{E}$ & $4 \mathrm{~N} / 27 \mathrm{~W}-8 \mathrm{E} 1$ & 575 & 0 & $\mathrm{E}$ \\
\hline $4 \mathrm{~N} / 27 \mathrm{~W}-8 \mathrm{E} 1$ & 195 & 0 & $\mathrm{E}$ & $4 \mathrm{~N} / 27 \mathrm{~W}-8 \mathrm{E} 1$ & 585 & 0 & $\mathrm{E}$ \\
\hline $4 \mathrm{~N} / 27 \mathrm{~W}-8 \mathrm{E} 1$ & 205 & 0 & $\mathrm{E}$ & $4 \mathrm{~N} / 27 \mathrm{~W}-8 \mathrm{E} 1$ & 595 & 0 & $\mathrm{E}$ \\
\hline $4 \mathrm{~N} / 27 \mathrm{~W}-8 \mathrm{E} 1$ & 215 & 0 & $\mathrm{E}$ & $4 \mathrm{~N} / 27 \mathrm{~W}-8 \mathrm{E} 1$ & 605 & 0 & $\mathrm{E}$ \\
\hline $4 \mathrm{~N} / 27 \mathrm{~W}-8 \mathrm{E} 1$ & 225 & 0 & $\mathrm{E}$ & 4N/27W-6Q12 & 75 & 42.9 & $\mathrm{E}$ \\
\hline $4 \mathrm{~N} / 27 \mathrm{~W}-8 \mathrm{E} 1$ & 235 & 0 & $\mathrm{E}$ & 4N/27W-6Q12 & 85 & 100 & $\mathrm{E}$ \\
\hline $4 \mathrm{~N} / 27 \mathrm{~W}-8 \mathrm{E} 1$ & 245 & 10 & $\mathrm{E}$ & $4 \mathrm{~N} / 27 \mathrm{~W}-6 \mathrm{Q} 12$ & 95 & 60 & $\mathrm{E}$ \\
\hline $4 \mathrm{~N} / 27 \mathrm{~W}-8 \mathrm{E} 1$ & 255 & 80 & $\mathrm{E}$ & 4N/27W-6Q12 & 105 & 70 & $\mathrm{E}$ \\
\hline 4N/27W-8E1 & 265 & 30 & $\mathrm{E}$ & 4N/27W-6Q12 & 115 & 100 & $\mathrm{E}$ \\
\hline $4 \mathrm{~N} / 27 \mathrm{~W}-8 \mathrm{E} 1$ & 275 & 0 & $\mathrm{E}$ & 4N/27W-6Q12 & 125 & 70 & $\mathrm{E}$ \\
\hline $4 \mathrm{~N} / 27 \mathrm{~W}-8 \mathrm{E} 1$ & 285 & 40 & $\mathrm{E}$ & 4N/27W-6Q12 & 135 & 0 & $\mathrm{E}$ \\
\hline
\end{tabular}


Table B1-1. Calculated percent-coarse intervals for all drillers' logs and e-logs used to define textural properties of the hydrogeologic framework model.-Continued

[Percent-coarse values are calculated for 10-foot intervals for the entire depth of each borehole. State well number is the state of California well identifier; interval midpoint depth is the midpoint of each 10-foot interval, in feet from top of borehole; PC is the percent-coarse value for each interval; log type defines whether the borehole is a drillers' lithology $\log (\mathrm{D})$ or a geophysical e-log (E).]

\begin{tabular}{|c|c|c|c|c|c|c|c|}
\hline $\begin{array}{c}\text { State } \\
\text { well } \\
\text { number }\end{array}$ & $\begin{array}{c}\text { Interval } \\
\text { midpoint depth } \\
\text { (feet) }\end{array}$ & PC & $\begin{array}{l}\text { Log } \\
\text { type }\end{array}$ & $\begin{array}{c}\text { State } \\
\text { well } \\
\text { number }\end{array}$ & $\begin{array}{c}\text { Interval } \\
\text { midpoint depth } \\
\text { (feet) }\end{array}$ & PC & $\begin{array}{l}\text { Log } \\
\text { type }\end{array}$ \\
\hline 4N/27W-6Q12 & 145 & 0 & $\mathrm{E}$ & $4 \mathrm{~N} / 28 \mathrm{~W}-12 \mathrm{H} 3$ & 45 & 0 & $\mathrm{E}$ \\
\hline 4N/27W-6Q12 & 155 & 0 & $\mathrm{E}$ & $4 \mathrm{~N} / 28 \mathrm{~W}-12 \mathrm{H} 3$ & 55 & 20 & $\mathrm{E}$ \\
\hline 4N/27W-6Q12 & 165 & 0 & $\mathrm{E}$ & $4 \mathrm{~N} / 28 \mathrm{~W}-12 \mathrm{H} 3$ & 65 & 0 & $\mathrm{E}$ \\
\hline 4N/27W-6Q12 & 175 & 0 & $\mathrm{E}$ & $4 \mathrm{~N} / 28 \mathrm{~W}-12 \mathrm{H} 3$ & 75 & 30 & $\mathrm{E}$ \\
\hline 4N/27W-6Q12 & 195 & 0 & $\mathrm{E}$ & $4 \mathrm{~N} / 28 \mathrm{~W}-12 \mathrm{H} 3$ & 95 & 60 & $\mathrm{E}$ \\
\hline 4N/27W-6Q12 & 205 & 0 & $\mathrm{E}$ & $4 \mathrm{~N} / 28 \mathrm{~W}-12 \mathrm{H} 3$ & 105 & 50 & $\mathrm{E}$ \\
\hline 4N/27W-6Q12 & 215 & 50 & $\mathrm{E}$ & $4 \mathrm{~N} / 28 \mathrm{~W}-12 \mathrm{H} 3$ & 115 & 50 & $\mathrm{E}$ \\
\hline 4N/27W-6Q12 & 225 & 100 & $\mathrm{E}$ & $4 \mathrm{~N} / 28 \mathrm{~W}-12 \mathrm{H} 3$ & 125 & 0 & $\mathrm{E}$ \\
\hline 4N/27W-6Q12 & 265 & 0 & $\mathrm{E}$ & $4 \mathrm{~N} / 28 \mathrm{~W}-12 \mathrm{H} 3$ & 165 & 100 & $\mathrm{E}$ \\
\hline 4N/27W-6Q12 & 275 & 60 & $\mathrm{E}$ & $4 \mathrm{~N} / 28 \mathrm{~W}-12 \mathrm{H} 3$ & 175 & 50 & $\mathrm{E}$ \\
\hline 4N/27W-6Q12 & 285 & 100 & $\mathrm{E}$ & $4 \mathrm{~N} / 28 \mathrm{~W}-12 \mathrm{H} 3$ & 185 & 30 & $\mathrm{E}$ \\
\hline 4N/27W-6Q12 & 295 & 100 & $\mathrm{E}$ & $4 \mathrm{~N} / 28 \mathrm{~W}-12 \mathrm{H} 3$ & 195 & 60 & $\mathrm{E}$ \\
\hline 4N/27W-6Q12 & 305 & 0 & $\mathrm{E}$ & $4 \mathrm{~N} / 28 \mathrm{~W}-12 \mathrm{H} 3$ & 205 & 0 & $\mathrm{E}$ \\
\hline $4 \mathrm{~N} / 27 \mathrm{~W}-6 \mathrm{Q} 12$ & 315 & 0 & $\mathrm{E}$ & $4 \mathrm{~N} / 28 \mathrm{~W}-12 \mathrm{H} 3$ & 215 & 20 & $\mathrm{E}$ \\
\hline $4 \mathrm{~N} / 27 \mathrm{~W}-5 \mathrm{P} 1$ & 15 & 44.4 & $\mathrm{E}$ & $4 \mathrm{~N} / 28 \mathrm{~W}-12 \mathrm{H} 3$ & 225 & 60 & $\mathrm{E}$ \\
\hline $4 \mathrm{~N} / 27 \mathrm{~W}-5 \mathrm{P} 1$ & 25 & 10 & $\mathrm{E}$ & $4 \mathrm{~N} / 28 \mathrm{~W}-12 \mathrm{H} 3$ & 235 & 30 & $\mathrm{E}$ \\
\hline $4 \mathrm{~N} / 27 \mathrm{~W}-5 \mathrm{P} 1$ & 35 & 0 & $\mathrm{E}$ & $4 \mathrm{~N} / 28 \mathrm{~W}-12 \mathrm{H} 3$ & 245 & 0 & $\mathrm{E}$ \\
\hline $4 \mathrm{~N} / 27 \mathrm{~W}-5 \mathrm{P} 1$ & 105 & 10 & $\mathrm{E}$ & $4 \mathrm{~N} / 27 \mathrm{~W}-1 \mathrm{R} 1$ & 95 & 70 & $\mathrm{E}$ \\
\hline $4 \mathrm{~N} / 27 \mathrm{~W}-5 \mathrm{P} 1$ & 115 & 100 & $\mathrm{E}$ & $4 \mathrm{~N} / 27 \mathrm{~W}-1 \mathrm{R} 1$ & 105 & 100 & $\mathrm{E}$ \\
\hline $4 \mathrm{~N} / 27 \mathrm{~W}-5 \mathrm{P} 1$ & 125 & 100 & $\mathrm{E}$ & $4 \mathrm{~N} / 27 \mathrm{~W}-1 \mathrm{R} 1$ & 115 & 50 & $\mathrm{E}$ \\
\hline $4 \mathrm{~N} / 27 \mathrm{~W}-5 \mathrm{P} 1$ & 135 & 50 & $\mathrm{E}$ & $4 \mathrm{~N} / 27 \mathrm{~W}-1 \mathrm{R} 1$ & 125 & 50 & $\mathrm{E}$ \\
\hline $4 \mathrm{~N} / 27 \mathrm{~W}-5 \mathrm{P} 1$ & 145 & 10 & $\mathrm{E}$ & $4 \mathrm{~N} / 27 \mathrm{~W}-1 \mathrm{R} 1$ & 135 & 50 & $\mathrm{E}$ \\
\hline $4 \mathrm{~N} / 27 \mathrm{~W}-5 \mathrm{P} 1$ & 155 & 100 & $\mathrm{E}$ & $4 \mathrm{~N} / 27 \mathrm{~W}-1 \mathrm{R} 1$ & 145 & 40 & $\mathrm{E}$ \\
\hline $4 \mathrm{~N} / 27 \mathrm{~W}-5 \mathrm{P} 1$ & 165 & 80 & $\mathrm{E}$ & $4 \mathrm{~N} / 27 \mathrm{~W}-1 \mathrm{R} 1$ & 155 & 100 & $\mathrm{E}$ \\
\hline 4N/27W-5P1 & 175 & 10 & $\mathrm{E}$ & $4 \mathrm{~N} / 27 \mathrm{~W}-1 \mathrm{R} 1$ & 165 & 40 & $\mathrm{E}$ \\
\hline $4 \mathrm{~N} / 28 \mathrm{~W}-12 \mathrm{H} 3$ & 5 & 0 & $\mathrm{E}$ & $4 \mathrm{~N} / 27 \mathrm{~W}-1 \mathrm{R} 1$ & 175 & 80 & $\mathrm{E}$ \\
\hline $4 \mathrm{~N} / 28 \mathrm{~W}-12 \mathrm{H} 3$ & 15 & 0 & $\mathrm{E}$ & $4 \mathrm{~N} / 27 \mathrm{~W}-1 \mathrm{R} 1$ & 185 & 0 & $\mathrm{E}$ \\
\hline $4 \mathrm{~N} / 28 \mathrm{~W}-12 \mathrm{H} 3$ & 25 & 0 & $\mathrm{E}$ & $4 \mathrm{~N} / 27 \mathrm{~W}-1 \mathrm{R} 1$ & 195 & 0 & $\mathrm{E}$ \\
\hline $4 \mathrm{~N} / 28 \mathrm{~W}-12 \mathrm{H} 3$ & 35 & 0 & $\mathrm{E}$ & $4 \mathrm{~N} / 27 \mathrm{~W}-1 \mathrm{R} 1$ & 205 & 0 & $\mathrm{E}$ \\
\hline
\end{tabular}


Table B1-1. Calculated percent-coarse intervals for all drillers' logs and e-logs used to define textural properties of the hydrogeologic framework model._Continued

[Percent-coarse values are calculated for 10-foot intervals for the entire depth of each borehole. State well number is the state of California well identifier; interval midpoint depth is the midpoint of each 10-foot interval, in feet from top of borehole; PC is the percent-coarse value for each interval; log type defines whether the borehole is a drillers' lithology $\log (\mathrm{D})$ or a geophysical e-log (E).]

\begin{tabular}{|c|c|c|c|c|c|c|c|}
\hline $\begin{array}{c}\text { State } \\
\text { well } \\
\text { number }\end{array}$ & $\begin{array}{c}\text { Interval } \\
\text { midpoint depth } \\
\text { (feet) }\end{array}$ & PC & $\begin{array}{l}\text { Log } \\
\text { type }\end{array}$ & $\begin{array}{c}\text { State } \\
\text { well } \\
\text { number }\end{array}$ & $\begin{array}{c}\text { Interval } \\
\text { midpoint depth } \\
\text { (feet) }\end{array}$ & PC & $\begin{array}{l}\text { Log } \\
\text { type }\end{array}$ \\
\hline 4N/27W-1R1 & 215 & 0 & $\mathrm{E}$ & $4 \mathrm{~N} / 27 \mathrm{~W}-22 \mathrm{C} 1$ & 195 & 100 & $\mathrm{E}$ \\
\hline 4N/27W-1R1 & 225 & 0 & $\mathrm{E}$ & $4 \mathrm{~N} / 27 \mathrm{~W}-22 \mathrm{C} 1$ & 205 & 100 & $\mathrm{E}$ \\
\hline $4 \mathrm{~N} / 27 \mathrm{~W}-1 \mathrm{R} 1$ & 235 & 0 & $\mathrm{E}$ & $4 \mathrm{~N} / 27 \mathrm{~W}-22 \mathrm{C} 1$ & 215 & 80 & $\mathrm{E}$ \\
\hline 4N/27W-1R1 & 245 & 0 & $\mathrm{E}$ & $4 \mathrm{~N} / 27 \mathrm{~W}-22 \mathrm{C} 1$ & 225 & 0 & $\mathrm{E}$ \\
\hline 4N/27W-1R1 & 265 & 0 & $\mathrm{E}$ & $4 \mathrm{~N} / 27 \mathrm{~W}-22 \mathrm{C} 1$ & 245 & 0 & $\mathrm{E}$ \\
\hline 4N/27W-1R1 & 275 & 0 & $\mathrm{E}$ & $4 \mathrm{~N} / 27 \mathrm{~W}-22 \mathrm{C} 1$ & 255 & 40 & $\mathrm{E}$ \\
\hline $4 \mathrm{~N} / 27 \mathrm{~W}-1 \mathrm{R} 1$ & 285 & 0 & $\mathrm{E}$ & $4 \mathrm{~N} / 27 \mathrm{~W}-22 \mathrm{C} 1$ & 265 & 0 & $\mathrm{E}$ \\
\hline 4N/27W-7Q5 & 25 & 0 & $\mathrm{E}$ & $4 \mathrm{~N} / 27 \mathrm{~W}-22 \mathrm{C} 1$ & 275 & 0 & $\mathrm{E}$ \\
\hline 4N/27W-7Q5 & 65 & 60 & $\mathrm{E}$ & $4 \mathrm{~N} / 27 \mathrm{~W}-22 \mathrm{C} 1$ & 315 & 60 & $\mathrm{E}$ \\
\hline 4N/27W-7Q5 & 75 & 0 & $\mathrm{E}$ & $4 \mathrm{~N} / 27 \mathrm{~W}-22 \mathrm{C} 1$ & 325 & 0 & $\mathrm{E}$ \\
\hline 4N/27W-7Q5 & 85 & 20 & $\mathrm{E}$ & $4 \mathrm{~N} / 27 \mathrm{~W}-22 \mathrm{C} 1$ & 335 & 0 & $\mathrm{E}$ \\
\hline 4N/27W-7Q5 & 95 & 0 & $\mathrm{E}$ & $4 \mathrm{~N} / 27 \mathrm{~W}-22 \mathrm{C} 1$ & 345 & 0 & $\mathrm{E}$ \\
\hline 4N/27W-7Q5 & 105 & 0 & $\mathrm{E}$ & $4 \mathrm{~N} / 27 \mathrm{~W}-22 \mathrm{C} 1$ & 355 & 40 & $\mathrm{E}$ \\
\hline 4N/27W-7Q5 & 115 & 0 & $\mathrm{E}$ & $4 \mathrm{~N} / 27 \mathrm{~W}-22 \mathrm{C} 1$ & 365 & 0 & $\mathrm{E}$ \\
\hline 4N/27W-7Q5 & 125 & 0 & $\mathrm{E}$ & $4 \mathrm{~N} / 27 \mathrm{~W}-22 \mathrm{C} 1$ & 375 & 20 & $\mathrm{E}$ \\
\hline 4N/27W-7Q5 & 135 & 0 & $\mathrm{E}$ & $4 \mathrm{~N} / 27 \mathrm{~W}-22 \mathrm{C} 1$ & 385 & 0 & $\mathrm{E}$ \\
\hline 4N/27W-7Q5 & 145 & 0 & $\mathrm{E}$ & $4 \mathrm{~N} / 27 \mathrm{~W}-22 \mathrm{C} 1$ & 395 & 0 & $\mathrm{E}$ \\
\hline $4 \mathrm{~N} / 27 \mathrm{~W}-22 \mathrm{C} 1$ & 75 & 0 & $\mathrm{E}$ & $4 \mathrm{~N} / 27 \mathrm{~W}-22 \mathrm{C} 1$ & 465 & 0 & $\mathrm{E}$ \\
\hline $4 \mathrm{~N} / 27 \mathrm{~W}-22 \mathrm{C} 1$ & 85 & 10 & $\mathrm{E}$ & $4 \mathrm{~N} / 27 \mathrm{~W}-22 \mathrm{C} 1$ & 475 & 0 & $\mathrm{E}$ \\
\hline $4 \mathrm{~N} / 27 \mathrm{~W}-22 \mathrm{C} 1$ & 95 & 90 & $\mathrm{E}$ & $4 \mathrm{~N} / 27 \mathrm{~W}-22 \mathrm{C} 1$ & 485 & 0 & $\mathrm{E}$ \\
\hline $4 \mathrm{~N} / 27 \mathrm{~W}-22 \mathrm{C} 1$ & 105 & 40 & $\mathrm{E}$ & $4 \mathrm{~N} / 27 \mathrm{~W}-22 \mathrm{C} 1$ & 495 & 0 & $\mathrm{E}$ \\
\hline $4 \mathrm{~N} / 27 \mathrm{~W}-22 \mathrm{C} 1$ & 115 & 20 & $\mathrm{E}$ & $4 \mathrm{~N} / 27 \mathrm{~W}-22 \mathrm{C} 1$ & 505 & 20 & $\mathrm{E}$ \\
\hline $4 \mathrm{~N} / 27 \mathrm{~W}-22 \mathrm{C} 1$ & 125 & 100 & $\mathrm{E}$ & $4 \mathrm{~N} / 27 \mathrm{~W}-22 \mathrm{C} 1$ & 515 & 10 & $\mathrm{E}$ \\
\hline $4 \mathrm{~N} / 27 \mathrm{~W}-22 \mathrm{C} 1$ & 135 & 100 & $\mathrm{E}$ & $4 \mathrm{~N} / 27 \mathrm{~W}-22 \mathrm{C} 1$ & 525 & 0 & $\mathrm{E}$ \\
\hline $4 \mathrm{~N} / 27 \mathrm{~W}-22 \mathrm{C} 1$ & 145 & 90 & $\mathrm{E}$ & $4 \mathrm{~N} / 27 \mathrm{~W}-22 \mathrm{C} 1$ & 535 & 80 & $\mathrm{E}$ \\
\hline $4 \mathrm{~N} / 27 \mathrm{~W}-22 \mathrm{C} 1$ & 155 & 100 & $\mathrm{E}$ & $4 \mathrm{~N} / 27 \mathrm{~W}-22 \mathrm{C} 1$ & 545 & 100 & $\mathrm{E}$ \\
\hline $4 \mathrm{~N} / 27 \mathrm{~W}-22 \mathrm{C} 1$ & 165 & 90 & $\mathrm{E}$ & $4 \mathrm{~N} / 27 \mathrm{~W}-22 \mathrm{C} 1$ & 555 & 100 & $\mathrm{E}$ \\
\hline $4 \mathrm{~N} / 27 \mathrm{~W}-22 \mathrm{C} 1$ & 175 & 40 & $\mathrm{E}$ & $4 \mathrm{~N} / 27 \mathrm{~W}-22 \mathrm{C} 1$ & 565 & 60 & $\mathrm{E}$ \\
\hline $4 \mathrm{~N} / 27 \mathrm{~W}-22 \mathrm{C} 1$ & 185 & 100 & $\mathrm{E}$ & $4 \mathrm{~N} / 27 \mathrm{~W}-22 \mathrm{C} 1$ & 575 & 80 & $\mathrm{E}$ \\
\hline
\end{tabular}


Table B1-1. Calculated percent-coarse intervals for all drillers' logs and e-logs used to define textural properties of the hydrogeologic framework model.-Continued

[Percent-coarse values are calculated for 10-foot intervals for the entire depth of each borehole. State well number is the state of California well identifier; interval midpoint depth is the midpoint of each 10-foot interval, in feet from top of borehole; PC is the percent-coarse value for each interval; log type defines whether the borehole is a drillers' lithology $\log (\mathrm{D})$ or a geophysical e-log (E).]

\begin{tabular}{|c|c|c|c|c|c|c|c|}
\hline $\begin{array}{c}\text { State } \\
\text { well } \\
\text { number }\end{array}$ & $\begin{array}{c}\text { Interval } \\
\text { midpoint depth } \\
\text { (feet) }\end{array}$ & PC & $\begin{array}{l}\text { Log } \\
\text { type }\end{array}$ & $\begin{array}{c}\text { State } \\
\text { well } \\
\text { number }\end{array}$ & $\begin{array}{c}\text { Interval } \\
\text { midpoint depth } \\
\text { (feet) }\end{array}$ & PC & $\begin{array}{l}\text { Log } \\
\text { type }\end{array}$ \\
\hline $4 \mathrm{~N} / 27 \mathrm{~W}-22 \mathrm{C} 1$ & 585 & 100 & $\mathrm{E}$ & $4 \mathrm{~N} / 27 \mathrm{~W}-22 \mathrm{~B} 6$ & 245 & 50 & $\mathrm{E}$ \\
\hline $4 \mathrm{~N} / 27 \mathrm{~W}-22 \mathrm{C} 1$ & 595 & 100 & $\mathrm{E}$ & $4 \mathrm{~N} / 27 \mathrm{~W}-22 \mathrm{~B} 6$ & 255 & 0 & $\mathrm{E}$ \\
\hline $4 \mathrm{~N} / 27 \mathrm{~W}-22 \mathrm{C} 1$ & 605 & 100 & $\mathrm{E}$ & $4 \mathrm{~N} / 27 \mathrm{~W}-22 \mathrm{~B} 6$ & 265 & 0 & $\mathrm{E}$ \\
\hline $4 \mathrm{~N} / 27 \mathrm{~W}-22 \mathrm{C} 1$ & 615 & 90 & $\mathrm{E}$ & $4 \mathrm{~N} / 27 \mathrm{~W}-22 \mathrm{~B} 6$ & 275 & 0 & $\mathrm{E}$ \\
\hline $4 \mathrm{~N} / 27 \mathrm{~W}-22 \mathrm{C} 1$ & 635 & 100 & $\mathrm{E}$ & $4 \mathrm{~N} / 27 \mathrm{~W}-22 \mathrm{~B} 6$ & 295 & 0 & $\mathrm{E}$ \\
\hline $4 \mathrm{~N} / 27 \mathrm{~W}-22 \mathrm{C} 1$ & 645 & 100 & $\mathrm{E}$ & $4 \mathrm{~N} / 27 \mathrm{~W}-22 \mathrm{~B} 6$ & 305 & 0 & $\mathrm{E}$ \\
\hline $4 \mathrm{~N} / 27 \mathrm{~W}-22 \mathrm{C} 1$ & 655 & 100 & $\mathrm{E}$ & $4 \mathrm{~N} / 27 \mathrm{~W}-22 \mathrm{~B} 6$ & 315 & 0 & $\mathrm{E}$ \\
\hline $4 \mathrm{~N} / 27 \mathrm{~W}-22 \mathrm{C} 1$ & 665 & 60 & $\mathrm{E}$ & $4 \mathrm{~N} / 27 \mathrm{~W}-22 \mathrm{~B} 6$ & 325 & 0 & $\mathrm{E}$ \\
\hline $4 \mathrm{~N} / 27 \mathrm{~W}-22 \mathrm{C} 1$ & 705 & 0 & $\mathrm{E}$ & $4 \mathrm{~N} / 27 \mathrm{~W}-22 \mathrm{~B} 6$ & 365 & 0 & $\mathrm{E}$ \\
\hline $4 \mathrm{~N} / 27 \mathrm{~W}-22 \mathrm{C} 1$ & 715 & 0 & $\mathrm{E}$ & $4 \mathrm{~N} / 27 \mathrm{~W}-22 \mathrm{~B} 6$ & 375 & 0 & $\mathrm{E}$ \\
\hline $4 \mathrm{~N} / 27 \mathrm{~W}-22 \mathrm{C} 1$ & 725 & 0 & $\mathrm{E}$ & $4 \mathrm{~N} / 27 \mathrm{~W}-22 \mathrm{~B} 6$ & 385 & 0 & $\mathrm{E}$ \\
\hline $4 \mathrm{~N} / 27 \mathrm{~W}-22 \mathrm{C} 1$ & 735 & 0 & $\mathrm{E}$ & $4 \mathrm{~N} / 27 \mathrm{~W}-22 \mathrm{~B} 6$ & 395 & 0 & $\mathrm{E}$ \\
\hline $4 \mathrm{~N} / 27 \mathrm{~W}-22 \mathrm{C} 1$ & 745 & 0 & $\mathrm{E}$ & $4 \mathrm{~N} / 27 \mathrm{~W}-22 \mathrm{~B} 6$ & 405 & 0 & $\mathrm{E}$ \\
\hline $4 \mathrm{~N} / 27 \mathrm{~W}-22 \mathrm{C} 1$ & 755 & 0 & $\mathrm{E}$ & $4 \mathrm{~N} / 27 \mathrm{~W}-22 \mathrm{~B} 6$ & 415 & 90 & $\mathrm{E}$ \\
\hline $4 \mathrm{~N} / 27 \mathrm{~W}-22 \mathrm{C} 1$ & 765 & 0 & $\mathrm{E}$ & $4 \mathrm{~N} / 27 \mathrm{~W}-22 \mathrm{~B} 6$ & 425 & 100 & $\mathrm{E}$ \\
\hline $4 \mathrm{~N} / 27 \mathrm{~W}-22 \mathrm{C} 1$ & 775 & 0 & $\mathrm{E}$ & $4 \mathrm{~N} / 27 \mathrm{~W}-22 \mathrm{~B} 6$ & 435 & 40 & $\mathrm{E}$ \\
\hline $4 \mathrm{~N} / 27 \mathrm{~W}-22 \mathrm{~B} 6$ & 55 & 0 & $\mathrm{E}$ & $4 \mathrm{~N} / 27 \mathrm{~W}-22 \mathrm{~B} 6$ & 445 & 0 & $\mathrm{E}$ \\
\hline $4 \mathrm{~N} / 27 \mathrm{~W}-22 \mathrm{~B} 6$ & 125 & 0 & $\mathrm{E}$ & $4 \mathrm{~N} / 27 \mathrm{~W}-22 \mathrm{~B} 6$ & 515 & 40 & $\mathrm{E}$ \\
\hline 4N/27W-22B6 & 135 & 0 & $\mathrm{E}$ & $4 \mathrm{~N} / 27 \mathrm{~W}-22 \mathrm{~B} 6$ & 525 & 0 & $\mathrm{E}$ \\
\hline $4 \mathrm{~N} / 27 \mathrm{~W}-22 \mathrm{~B} 6$ & 145 & 0 & $\mathrm{E}$ & $4 \mathrm{~N} / 27 \mathrm{~W}-22 \mathrm{~B} 6$ & 535 & 0 & $\mathrm{E}$ \\
\hline $4 \mathrm{~N} / 27 \mathrm{~W}-22 \mathrm{~B} 6$ & 155 & 0 & $\mathrm{E}$ & $4 \mathrm{~N} / 27 \mathrm{~W}-22 \mathrm{~B} 6$ & 545 & 0 & $\mathrm{E}$ \\
\hline $4 \mathrm{~N} / 27 \mathrm{~W}-22 \mathrm{~B} 6$ & 165 & 0 & $\mathrm{E}$ & $4 \mathrm{~N} / 27 \mathrm{~W}-22 \mathrm{~B} 6$ & 555 & 30 & $\mathrm{E}$ \\
\hline $4 \mathrm{~N} / 27 \mathrm{~W}-22 \mathrm{~B} 6$ & 175 & 20 & $\mathrm{E}$ & $4 \mathrm{~N} / 27 \mathrm{~W}-22 \mathrm{~B} 6$ & 565 & 100 & $\mathrm{E}$ \\
\hline $4 \mathrm{~N} / 27 \mathrm{~W}-22 \mathrm{~B} 6$ & 185 & 0 & $\mathrm{E}$ & $4 \mathrm{~N} / 27 \mathrm{~W}-22 \mathrm{~B} 6$ & 575 & 100 & $\mathrm{E}$ \\
\hline $4 \mathrm{~N} / 27 \mathrm{~W}-22 \mathrm{~B} 6$ & 195 & 0 & $\mathrm{E}$ & $4 \mathrm{~N} / 27 \mathrm{~W}-22 \mathrm{~B} 6$ & 585 & 50 & $\mathrm{E}$ \\
\hline $4 \mathrm{~N} / 27 \mathrm{~W}-22 \mathrm{~B} 6$ & 205 & 40 & $\mathrm{E}$ & $4 \mathrm{~N} / 27 \mathrm{~W}-22 \mathrm{~B} 6$ & 595 & 40 & $\mathrm{E}$ \\
\hline $4 \mathrm{~N} / 27 \mathrm{~W}-22 \mathrm{~B} 6$ & 215 & 100 & $\mathrm{E}$ & $4 \mathrm{~N} / 27 \mathrm{~W}-22 \mathrm{~B} 6$ & 605 & 100 & $\mathrm{E}$ \\
\hline $4 \mathrm{~N} / 27 \mathrm{~W}-22 \mathrm{~B} 6$ & 225 & 100 & $\mathrm{E}$ & $4 \mathrm{~N} / 27 \mathrm{~W}-22 \mathrm{~B} 6$ & 615 & 100 & $\mathrm{E}$ \\
\hline $4 \mathrm{~N} / 27 \mathrm{~W}-22 \mathrm{~B} 6$ & 235 & 100 & $\mathrm{E}$ & $4 \mathrm{~N} / 27 \mathrm{~W}-22 \mathrm{~B} 6$ & 625 & 80 & $\mathrm{E}$ \\
\hline
\end{tabular}


Table B1-1. Calculated percent-coarse intervals for all drillers' logs and e-logs used to define textural properties of the hydrogeologic framework model.-Continued

[Percent-coarse values are calculated for 10-foot intervals for the entire depth of each borehole. State well number is the state of California well identifier; interval midpoint depth is the midpoint of each 10-foot interval, in feet from top of borehole; PC is the percent-coarse value for each interval; log type defines whether the borehole is a drillers' lithology $\log (\mathrm{D})$ or a geophysical e-log (E).]

\begin{tabular}{|c|c|c|c|c|c|c|c|}
\hline $\begin{array}{l}\text { State } \\
\text { well } \\
\text { number }\end{array}$ & $\begin{array}{c}\text { Interval } \\
\text { midpoint depth } \\
\text { (feet) }\end{array}$ & PC & $\begin{array}{l}\text { Log } \\
\text { type }\end{array}$ & $\begin{array}{l}\text { State } \\
\text { well } \\
\text { number }\end{array}$ & $\begin{array}{c}\text { Interval } \\
\text { midpoint depth } \\
\text { (feet) }\end{array}$ & PC & $\begin{array}{l}\text { Log } \\
\text { type }\end{array}$ \\
\hline $4 \mathrm{~N} / 27 \mathrm{~W}-22 \mathrm{~B} 6$ & 635 & 70 & E & 4N/27W-8L2 & 335 & 100 & $\mathrm{E}$ \\
\hline $4 \mathrm{~N} / 27 \mathrm{~W}-22 \mathrm{~B} 6$ & 645 & 100 & E & 4N/27W-8L2 & 345 & 10 & E \\
\hline $4 \mathrm{~N} / 27 \mathrm{~W}-22 \mathrm{~B} 6$ & 655 & 100 & E & 4N/27W-8L2 & 355 & 0 & E \\
\hline $4 \mathrm{~N} / 27 \mathrm{~W}-22 \mathrm{~B} 6$ & 665 & 100 & E & $4 \mathrm{~N} / 27 \mathrm{~W}-8 \mathrm{~L} 2$ & 365 & 0 & E \\
\hline $4 \mathrm{~N} / 27 \mathrm{~W}-22 \mathrm{~B} 6$ & 675 & 100 & E & 4N/27W-8L2 & 375 & 0 & E \\
\hline $4 \mathrm{~N} / 27 \mathrm{~W}-22 \mathrm{~B} 6$ & 685 & 30 & E & $4 \mathrm{~N} / 27 \mathrm{~W}-8 \mathrm{~L} 2$ & 385 & 0 & E \\
\hline 4N/27W-8L2 & 85 & 100 & E & 4N/27W-8L2 & 395 & 0 & E \\
\hline $4 \mathrm{~N} / 27 \mathrm{~W}-8 \mathrm{~L} 2$ & 95 & 100 & E & $4 \mathrm{~N} / 27 \mathrm{~W}-8 \mathrm{~L} 2$ & 405 & 70 & E \\
\hline 4N/27W-8L2 & 105 & 100 & E & 4N/27W-8L2 & 415 & 100 & E \\
\hline 4N/27W-8L2 & 115 & 80 & E & $4 \mathrm{~N} / 27 \mathrm{~W}-8 \mathrm{~L} 2$ & 425 & 40 & $\mathrm{E}$ \\
\hline 4N/27W-8L2 & 125 & 100 & E & $4 \mathrm{~N} / 27 \mathrm{~W}-8 \mathrm{~L} 2$ & 435 & 0 & E \\
\hline 4N/27W-8L2 & 135 & 20 & E & $4 \mathrm{~N} / 27 \mathrm{~W}-8 \mathrm{~L} 2$ & 445 & 0 & E \\
\hline 4N/27W-8L2 & 145 & 60 & E & $4 \mathrm{~N} / 27 \mathrm{~W}-8 \mathrm{~L} 2$ & 455 & 0 & E \\
\hline $4 \mathrm{~N} / 27 \mathrm{~W}-8 \mathrm{~L} 2$ & 155 & 100 & E & $4 \mathrm{~N} / 27 \mathrm{~W}-8 \mathrm{~L} 2$ & 465 & 10 & E \\
\hline 4N/27W-8L2 & 165 & 100 & E & $4 \mathrm{~N} / 27 \mathrm{~W}-8 \mathrm{~L} 2$ & 475 & 100 & E \\
\hline 4N/27W-8L2 & 175 & 100 & E & 4N/27W-8L2 & 485 & 100 & E \\
\hline $4 \mathrm{~N} / 27 \mathrm{~W}-8 \mathrm{~L} 2$ & 185 & 100 & E & 4N/27W-8L2 & 495 & 100 & E \\
\hline 4N/27W-8L2 & 195 & 100 & E & 4N/27W-8L2 & 505 & 80 & E \\
\hline $4 \mathrm{~N} / 27 \mathrm{~W}-8 \mathrm{~L} 2$ & 205 & 100 & E & $4 \mathrm{~N} / 27 \mathrm{~W}-8 \mathrm{~L} 2$ & 515 & 0 & E \\
\hline 4N/27W-8L2 & 215 & 40 & E & $4 \mathrm{~N} / 27 \mathrm{~W}-8 \mathrm{~L} 2$ & 525 & 60 & E \\
\hline 4N/27W-8L2 & 225 & 0 & E & $4 \mathrm{~N} / 27 \mathrm{~W}-8 \mathrm{~L} 2$ & 535 & 100 & E \\
\hline $4 \mathrm{~N} / 27 \mathrm{~W}-8 \mathrm{~L} 2$ & 235 & 0 & E & $4 \mathrm{~N} / 27 \mathrm{~W}-8 \mathrm{~L} 2$ & 545 & 100 & E \\
\hline 4N/27W-8L2 & 245 & 0 & E & $4 \mathrm{~N} / 27 \mathrm{~W}-8 \mathrm{~L} 2$ & 555 & 60 & E \\
\hline 4N/27W-8L2 & 255 & 0 & E & $4 \mathrm{~N} / 27 \mathrm{~W}-8 \mathrm{~L} 2$ & 565 & 100 & E \\
\hline $4 \mathrm{~N} / 27 \mathrm{~W}-8 \mathrm{~L} 2$ & 265 & 50 & E & $4 \mathrm{~N} / 27 \mathrm{~W}-8 \mathrm{~L} 2$ & 575 & 100 & E \\
\hline 4N/27W-8L2 & 275 & 100 & E & $4 \mathrm{~N} / 27 \mathrm{~W}-8 \mathrm{~L} 2$ & 585 & 100 & E \\
\hline $4 \mathrm{~N} / 27 \mathrm{~W}-8 \mathrm{~L} 2$ & 285 & 100 & E & 4N/27W-8L2 & 595 & 100 & E \\
\hline $4 \mathrm{~N} / 27 \mathrm{~W}-8 \mathrm{~L} 2$ & 295 & 100 & E & 4N/27W-8L2 & 605 & 100 & E \\
\hline 4N/27W-8L2 & 305 & 100 & E & 4N/27W-8L2 & 615 & 100 & E \\
\hline 4N/27W-8L2 & 315 & 100 & E & 4N/27W-8L2 & 625 & 70 & E \\
\hline 4N/27W-8L2 & 325 & 100 & E & 4N/27W-8L2 & 635 & 0 & $\mathrm{E}$ \\
\hline
\end{tabular}





\title{
Chapter C: Numerical Model of Groundwater Flow and Solute Transport
}

\author{
By Scott R. Paulinski
}

\section{Introduction}

The city of Santa Barbara is interested in simulating seawater intrusion under various water-management scenarios, estimating the sustainability of the groundwater basins, and determining optimal strategies to manage the groundwater basins. The existing U.S. Geological Survey (USGS) Santa Barbara groundwater-flow model by Freckleton and others (1998) simulates flow in the Santa Barbara and Foothill groundwater basins from 1978 to 1992 . To simulate seawater intrusion into the Santa Barbara groundwater basin and evaluate various management strategies for the Santa Barbara groundwater basin, updates to the existing model were necessary, including the simulation of salinity transport and of variable-density flow and an extension of the timeframe of the model. A new model was developed, the Santa Barbara Flow and Transport Model (SBFTM), to simulate variable-density flow and transport from 1929 through 2013. The SBFTM was then used to determine optimal solutions for various watermanagement scenarios and estimate the sustainability of the groundwater basins.

\section{Groundwater Model Development}

A three-dimensional groundwater-flow and solutetransport model of the Santa Barbara and Foothill groundwater basins was developed using SEAWAT (Langevin and others, 2008). SEAWAT is a groundwater-flow and solute-transport simulation code that is based on MODFLOW-2000 (Harbaugh and others, 2000) and MT3DMS (Zheng and Wang, 1999; Zheng, 2006). Two versions of the SBFTM were constructed: an initial-condition model and a simulation-period transient model. The initial-condition model is a long-term transient model that simulates flow and solute-transport conditions during a period of lesser anthropogenic influences leading up to the start of the simulation-period transient model. A steady-state simulation would not suffice for determining these initial conditions, because the solute-transport computations require transience to determine concentration distribution. The simulation-period transient model simulates flow and transport conditions from 1929 through 2013. The flow and transport models use the SEAWAT packages listed in table 1.

The SBFTM was constructed in three steps. First, a hydrogeologic framework model (HFM) was constructed based on previous studies and available hydrologic, geologic, and geophysical data for the basin. As described in chapter B, the HFM has two parts: a geometric model that describes the extent and thickness of the hydrogeologic units and a textural model that defines the properties of these units. Next, this HFM was partitioned into the layers and cells of the groundwater-flow model. Finally, the available hydrologic observation and stress data were compiled and used to construct and calibrate the SBFTM.

\section{Spatial and Temporal Discretization}

The SBFTM is spatially discretized on a grid with 58 rows, 152 columns, and 56 layers (fig. 1) with 113,072 active cells. The model grid was rotated by 25 degrees clockwise from true north to align with the dominant direction of flow in Storage Unit I. The horizontal discretization is 250 by 250 feet (ft), and the vertical discretization is spatially variable; most model cells are approximately $20 \mathrm{ft}$ thick. The coordinates of the four corners for the model grid are presented in table 2 . The 56 model layers were necessary to replicate the horizontal variability in constituent transport reasonably and follow the contours of the land surface. They do not represent specific geologic layers, hydrogeologic units, or water-bearing units. Properties from the various hydrogeologic units and textural distribution in the HFM were used to populate the flow and transport model.

To simulate predevelopment conditions (no stresses), groundwater flow and solute transport were simulated for 10,000 years with 10 -year time steps to allow the flow and concentration fields to equilibrate to the specified initial and boundary conditions. No pumpage data were available prior to 1929 , and it was assumed that pumping prior to $1929 \mathrm{had}$ a minor effect on the study area hydrology; therefore, it was assumed that 1929 represented predevelopment conditions. 
Table 1. SEAWAT Version 4 packages and processes used with the Santa Barbara flow and transport model, Santa Barbara, California.

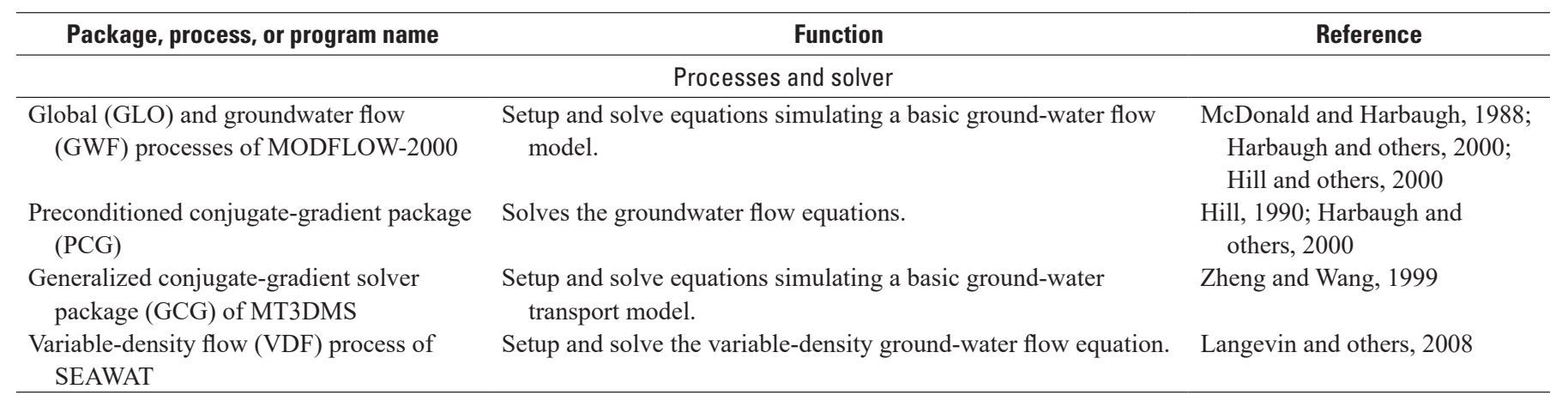

\section{SEAWAT}

Global file

List file

(n)

Name file (name)
Output control option (OC)

\section{Files}

Controls the capabilities of SEAWAT (MODFLOW-2000 and MT3DMS) utilized during a simulation.

Used to output head, drawdown, and budget information for specified time periods.

Output file for information that applied to the model simulation Harbaugh and others, 2000 as a whole.

Output file for allocation information, values used by the GWF process, and calculated results such as head, drawdown, and the water budget.

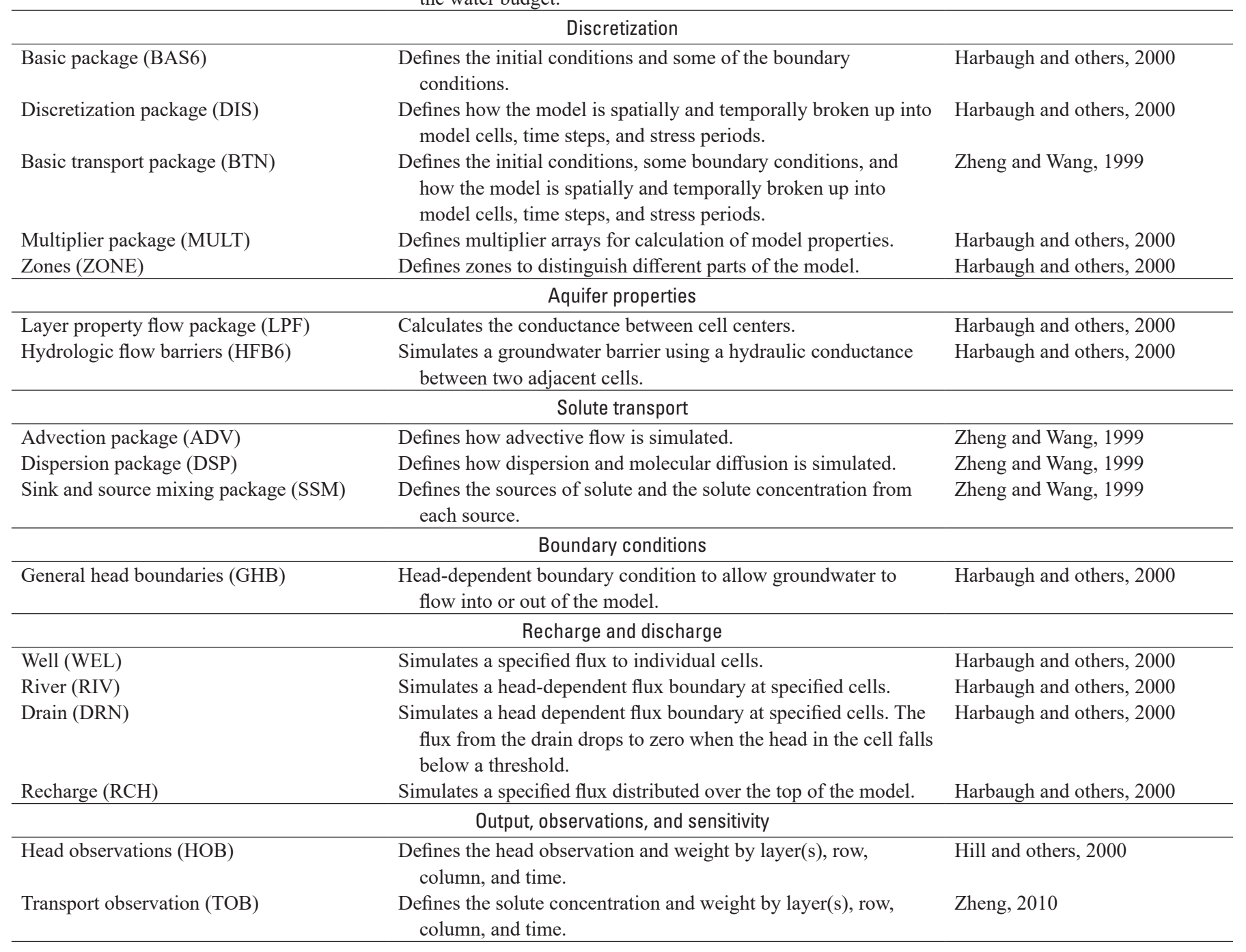

Harbaugh and others, 2000

Harbaugh and others, 2000

Harbaugh and others, 2000

to

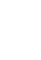




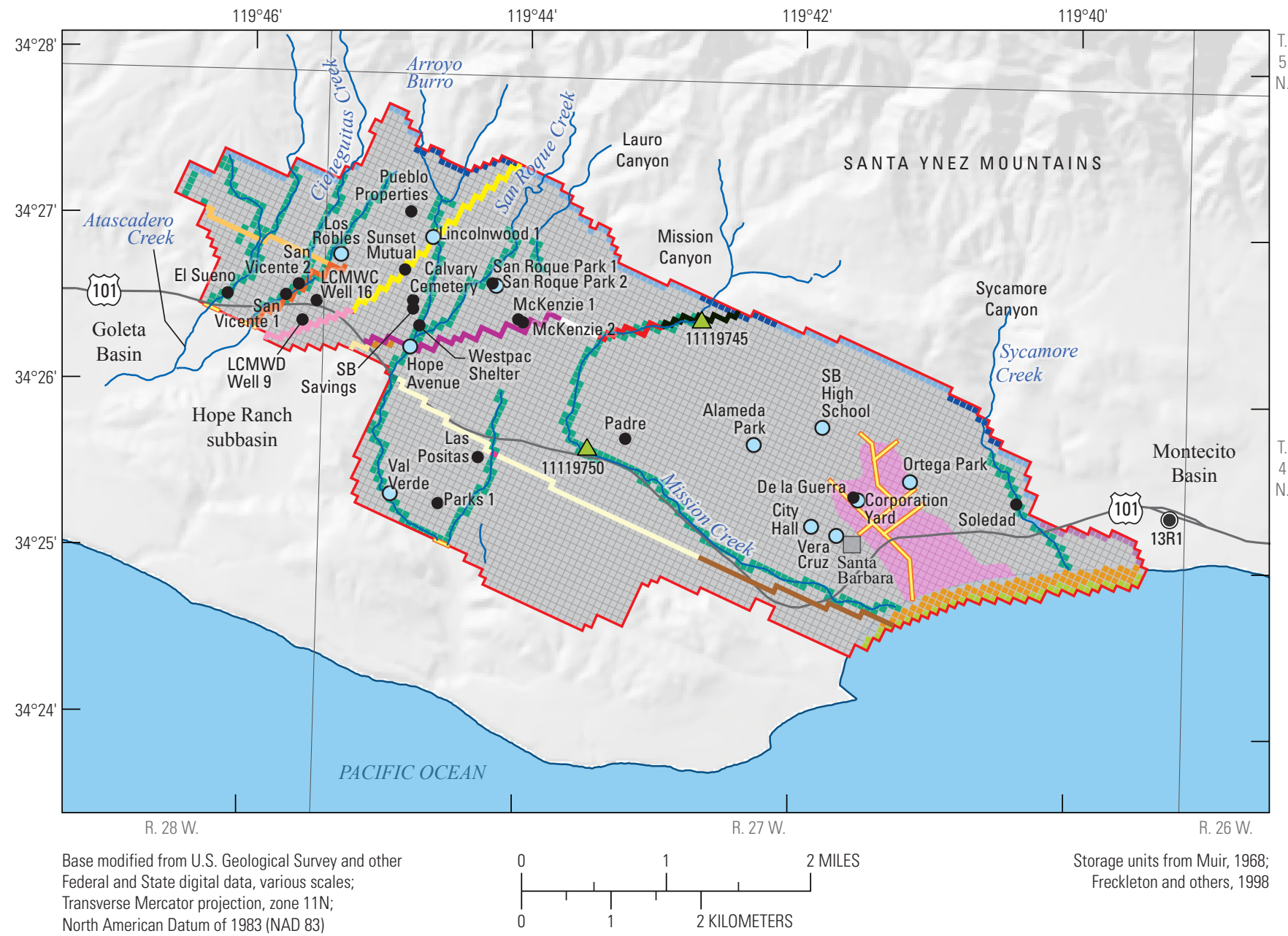

\section{EXPLANATION}

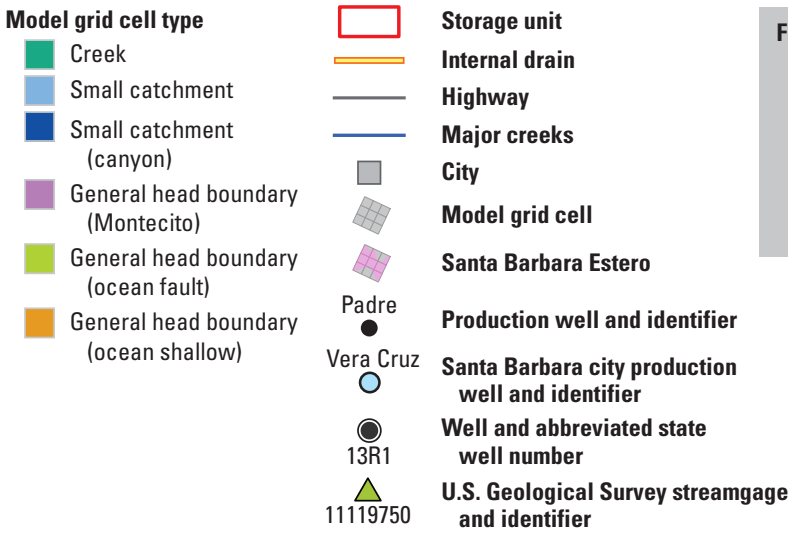

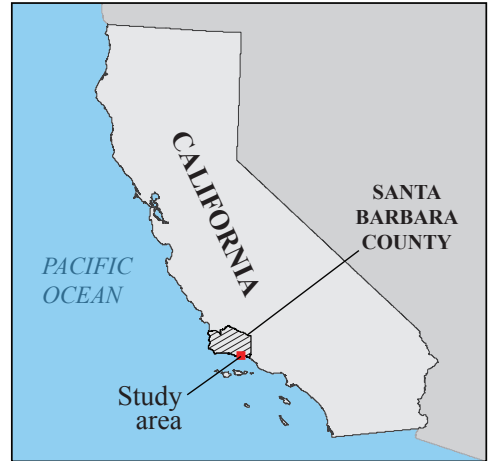

Figure 1. Distribution of flow barriers, general-head boundaries, drains, small-catchment recharge, and creek recharge in the Santa Barbara flow and transport model, Santa Barbara, California. 
Table 2. Coordinates of the Santa Barbara Flow and Transport Model grid, Santa Barbara, California.

[NAD 1983, North American Datum of 1983; UTM, Universal Transverse Mercator]

\begin{tabular}{lcccc}
\hline $\begin{array}{c}\text { Corner of } \\
\text { model grid }\end{array}$ & $\begin{array}{c}\text { Model } \\
\text { coordinates } \\
\text { X } \\
\text { (column) }\end{array}$ & $\begin{array}{c}\text { Model } \\
\text { coordinates } \\
\text { Y } \\
\text { (row) }\end{array}$ & $\begin{array}{c}\text { NAD 1983 } \\
\text { UTM zone } \\
\text { 11N X } \\
\text { (meters) }\end{array}$ & $\begin{array}{c}\text { NAD 1983 } \\
\text { UTM zone } \\
\text { 11N Y } \\
\text { (meters) }\end{array}$ \\
\hline Northeast & 1 & 1 & 245,775 & $3,817,516$ \\
Northwest & 1 & 58 & 243,907 & $3,813,510$ \\
Southeast & 152 & 1 & 256,272 & $3,812,621$ \\
Southwest & 152 & 58 & 254,405 & $3,808,615$ \\
\hline
\end{tabular}

The transient simulation represented 1929-2013 conditions; however, the primary focus was 1972-2013 because of superior data availability (in terms of reported stresses and measured data). The transient SBFTM was temporally discretized into 43 1-year stress periods from 1929 to 1971 and an additional 504 1-month stress periods from 1972 to 2013. One-year stress periods were used from 1929 to 1971 because of limited pumpage data during these years. One-month stress periods were chosen from 1972 to 2013 to simulate monthly and seasonal changes in pumpage and recharge.

\section{Boundary Conditions}

Model boundary conditions mathematically define the interaction of the SBFTM domain with hydrologic conditions outside of the SBFTM boundaries. For the groundwater-flow model, two types of boundary conditions were used: head dependent and specified flux.

Head-dependent boundaries include creeks, internal drains, and general-head boundaries (fig. 1). Creeks are discussed in the "Simulated Groundwater Recharge" section.

As described in chapter A, groundwater can leave Storage Unit III laterally as underflow at Arroyo Burro across the Lavigia fault or at Atascadero and Cieneguitas Creeks across the Modoc fault from Foothill to Goleta groundwater basin. Following Freckleton and others (1998), drain boundaries were used to simulate flow across the Lavigia and Modoc faults (figs. 1, B-1). Drains were also used to simulate groundwater flow into the Santa Barbara Estero.

The general-head boundary (GHB) package was used to simulate flow across the offshore fault along the coastal boundary (ocean-fault and shallow-ocean GHBs) and simulate flow between the Santa Ynez Mountains and Storage Unit I near the coast (Montecito GHB). The SBFTM extends laterally $500-900 \mathrm{ft}$ to the offshore fault on the eastern edge of Storage Unit I. The ocean-fault and shallow-ocean GHBs simulate flow between the Pacific Ocean and the groundwater system. The ocean-fault GHB is on the vertical face of the southeastern boundary with the Pacific Ocean, and the shallow-ocean GHB is on the top model layer that extends throughout the offshore model area. The head at the ocean-fault GHB remains constant at sea level throughout the simulation. When using GHB in SEAWAT, the GHB seawater heads are set at the measured or assumed elevation and SEAWAT adjusts the head to address the greater density of seawater. The shallow-ocean GHB has a constant head at sea level, and a single conductance parameter is used.

The Montecito GHB simulates flow between the mountains and Storage Unit I and is on the southeastern part of the northeast boundary of the study area. It has varying head values based on observation data from well 4N/27W13R1 (fig. 1).

Specified-flux boundaries also include no-flow boundaries. No-flow boundary conditions were used to simulate the lateral model boundary where not simulated by head-dependent boundaries or recharge. In addition, the base of the model, at the contact with a relatively low-permeability shale (see chapter B), was simulated as a no-flow boundary.

For the solute-transport model, chloride concentrations are associated with the flow boundaries. Appropriate chloride concentrations were specified at the general-head and specified-flux boundaries for any inflowing water (for example, a standard seawater value of 19,000 milligrams per liter at the ocean boundary).

\section{Simulated Groundwater Recharge}

Sources of inflow to the groundwater system include small-catchment recharge, leakage from creeks, underflow from neighboring basins, areal recharge, and septic-tank effluent. Small-catchment recharge was simulated on the north-northeast edge of the SBFTM (fig. 2) by injecting water into model layers 1 and 2 in the highlighted areas shown in figure 2. The fluxes were estimated as explained in the "Model Calibration Approach" section of this chapter. Small-catchment recharge segments were simulated along the north-northeast boundary of the SBFTM by using the well (WEL) package to inject water at a constant rate into the model cells bordering the foothills (fig. 2). Small-catchment recharge segments were aligned with the canyons to simulate the recharge rates from different canyons.

Creeks were simulated using the river (RIV) package (Harbaugh and others, 2000). The RIV package was used because the constituent-transport option in SEAWAT does not support other MODFLOW stream-routing packages (Langevin and others, 2008). Simulated creeks include Sycamore, Mission, San Roque, Arroyo Burro, Cieneguitas, and Atascadero creeks (fig. 1). Stage, creek-bed conductance, and creek-bed elevation are specified for the RIV package. The creek-bed elevations were based on a 10-m digitalelevation model of the study area. The RIV package allows fluxes to enter or leave the groundwater system as a function of creek stage and groundwater level. If the creek stage is higher than the groundwater level, then creek water recharges the groundwater system. If the creek stage is lower than the groundwater level, then groundwater discharges to the creek. 


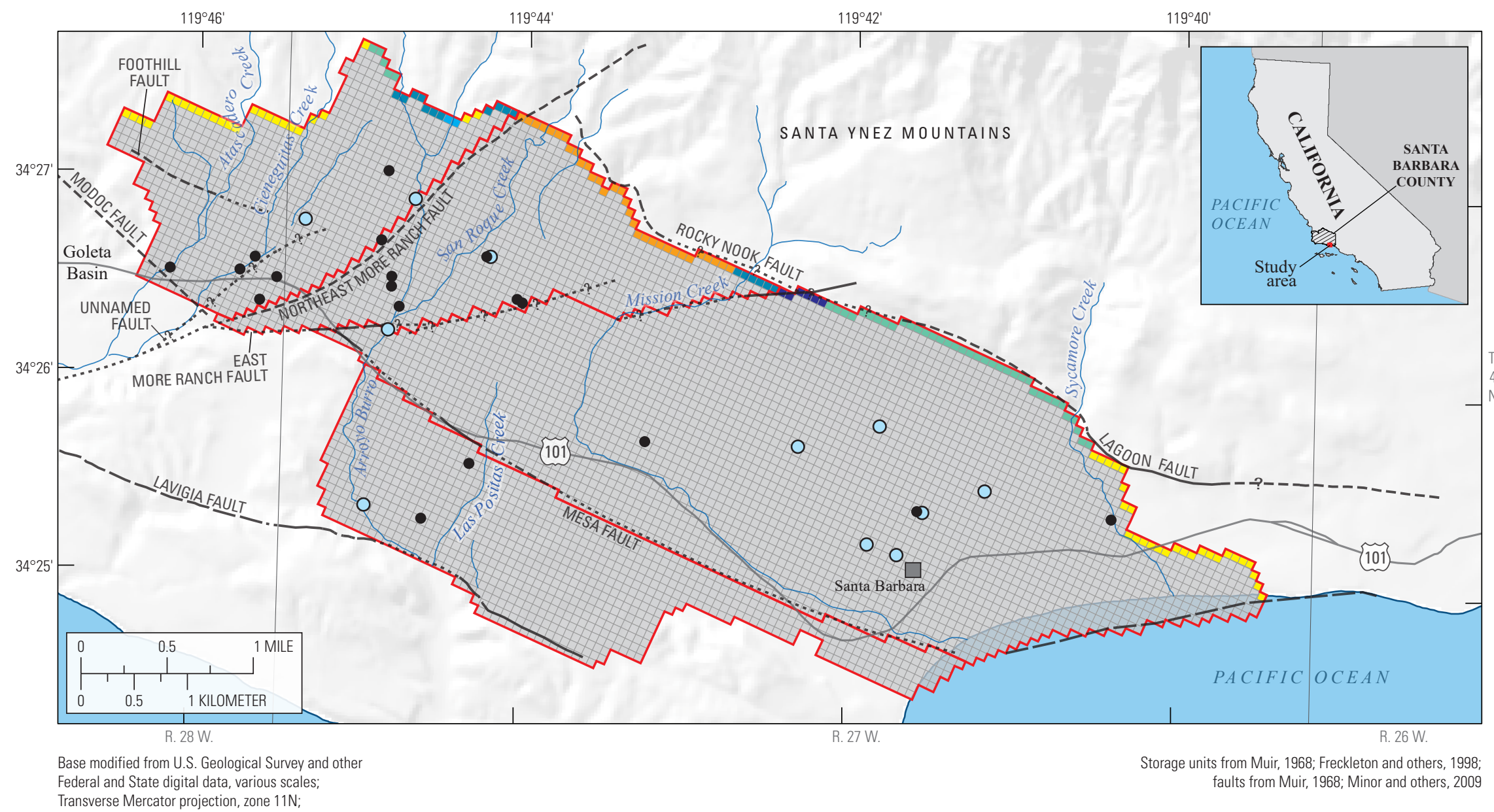

Federal and State digital data, various scales;
Transverse Mercator projection, zone $11 \mathrm{~N}$;

North American Datum of 1983 (NAD 83)

\section{EXPLANATION}

\begin{tabular}{|c|c|c|c|c|c|}
\hline \multirow{2}{*}{\multicolumn{2}{|c|}{$\begin{array}{l}\text { Average small catchment recharge, } \\
\text { in cubic feet per day }\end{array}$}} & Faults & & & \multirow{3}{*}{$\begin{array}{l}\text { Storage units } \\
\text { Highway }\end{array}$} \\
\hline & & ---- & Existence certain, location inferred & & \\
\hline 0 & $>500$ to 1,000 & $--?--$ & Existence questionable, location inferred & & \\
\hline$>0$ to 100 & $>1,000$ to 3,000 & ........ & Existence certain, location concealed & $\square$ & City \\
\hline$>100$ to 200 & $>3,000$ to 5,653 & $\cdots ? \cdots$ & Existence questionable, location concealed & $\bullet$ & Production well \\
\hline$>200$ to 500 & & - & $\begin{array}{l}\text { Existence certain, location accurate } \\
\text { Existence certain, location approximate }\end{array}$ & O & $\begin{array}{l}\text { Santa Barbara city } \\
\text { production well }\end{array}$ \\
\hline
\end{tabular}

Figure 2. Rates of small-catchment recharge for the Santa Barbara flow and transport model, Santa Barbara, California. 
The creeks were defined by multiple segments. Creek stage and creek-bed conductance parameters were allowed to vary during model calibration among creek segments in the model area. Creek stage for all the creek segments was initially estimated using the average of the reported Mission Creek stage data (11119745, Mission Creek at Rocky Nook Park) from 1997 to 2013 (U.S. Geological Survey, 2013). Creek stage was assumed to be constant throughout the simulation period. Final values of creek-stage and creek-bed conductance for each creek segment were both determined by parameter estimation.

Areal recharge represents direct infiltration to the groundwater system throughout the active model domain and is simulated using the recharge ( $\mathrm{RCH}$ ) package (Harbaugh and others, 2000). Areal recharge was estimated on the basis of a percentage of monthly precipitation data from the Parameterelevation Regressions on Independent Slopes Model (PRISM; Daly and Bryant, 2013), soil permeability (U.S. Department of Agriculture, 2008), and parcel land-use data (California Department of Water Resources, 1996). PRISM uses climate stations and topography to estimate precipitation amounts on a $2.5 \mathrm{mi}$ (4-kilometer) grid throughout the United States. A land-use map was used to define paved, partially paved, and unpaved areas. Soil maps were used to define areas of high, medium, and low permeability and impermeable soils. Based on these classifications of land use and soil, four infiltration zones were identified that range from low (infiltration zone 1) to high infiltration (infiltration zone 4; fig. $3 A$ ). Infiltration zone 1 represents areas with low-permeability soils that are partially paved. Infiltration zone 2 represents areas with lower to moderate-permeability soils that are minimally to moderately paved. Infiltration zone 3 represents areas with moderate to high permeability soils that are minimally to moderately paved. Infiltration zone 4 represents areas with high-permeability soils that are unpaved. Paved areas and areas with impermeable soils were not included in infiltration zones $1-4$, and no infiltration was assigned to these areas. Each infiltration zone was assigned a percentage of infiltration for precipitation, which was determined by parameter estimation. Each topmost active model cell in an infiltration zone was assigned a monthly precipitation rate, and the total recharge rate in a given model cell was determined by multiplying the monthly precipitation rate for that cell by the infiltration zone's infiltration percentage.

Septic-tank recharge was simulated using the $\mathrm{RCH}$ package and addressed by defining a fifth infiltration zone (fig. $3 B$ ). Data for parcels with septic systems were provided by the Santa Barbara County Clerk-Recorder-Assessor's Mapping Division. Septic recharge was applied to all top-most model cells containing parcels where there was septic-system usage.

The map of parcels with septic systems was overlaid onto the model grid, showing 255 acres in the model area with septic systems. Note that this value is greater than the area of the parcels that the septic systems occupy (about 156 acres) because if a parcel overlaid part of a model cell, the entire model cell was included in the area. The parcel map also indicated there were 195 single-family residences (SFR), 16 multi-unit dwellings (MUD) with 2-4 units, one rest home, one restaurant, and one retail store with septic systems. As described in chapter A, assuming 2.5 residents per SFR (U.S. Census Bureau, 2017), 10 residents per MUD, and a septic-effluent rate of 90 gallons per day per person yields a total septic flow rate of about 65 acre-feet per year (acre-ft/yr). Further assuming the model-grid area of 255 acres, yields a flux rate of 7.0E-04 feet per day (ft/d). The initial septic flux rate was assumed to equal $3.3 \mathrm{E}-03 \mathrm{ft} / \mathrm{d}$, and the final septic flux rate was determined by parameter estimation.

\section{Simulated Groundwater Discharge}

Groundwater outflow is primarily from groundwater pumping, underflow, discharge to streams and drains, and evapotranspiration. With the exception of available measurements of pumpage for public supply, flow rates for these components of groundwater outflow were relatively unknown and were simulated as part of this study.

Groundwater pumpage was simulated using the WEL package (Harbaugh and others, 2000). A total of 29 production wells are simulated in the SBFTM (fig. 1). Well location, construction, and production data were obtained from the USGS National Water Information System (NWIS) database and the city's records (Kelley Dyer, City of Santa Barbara, personal commun., 2013). Pumpage data from individual wells were used to estimate pumpage on an annual basis from 1929 to 1971 and on a monthly basis from 1972 to 2013. Total groundwater production in the Santa Barbara and Foothill groundwater basins was highly variable between 1972 and 2008 (fig. A-8). Total groundwater pumpage varied from less than $500 \mathrm{acre}-\mathrm{ft} / \mathrm{yr}$ during typical years to more than 3,000 acre-ft/yr during dry years. The percentage of pumpage for a single production well extracted from each model cell the well perforates was calculated using equation 1 : 


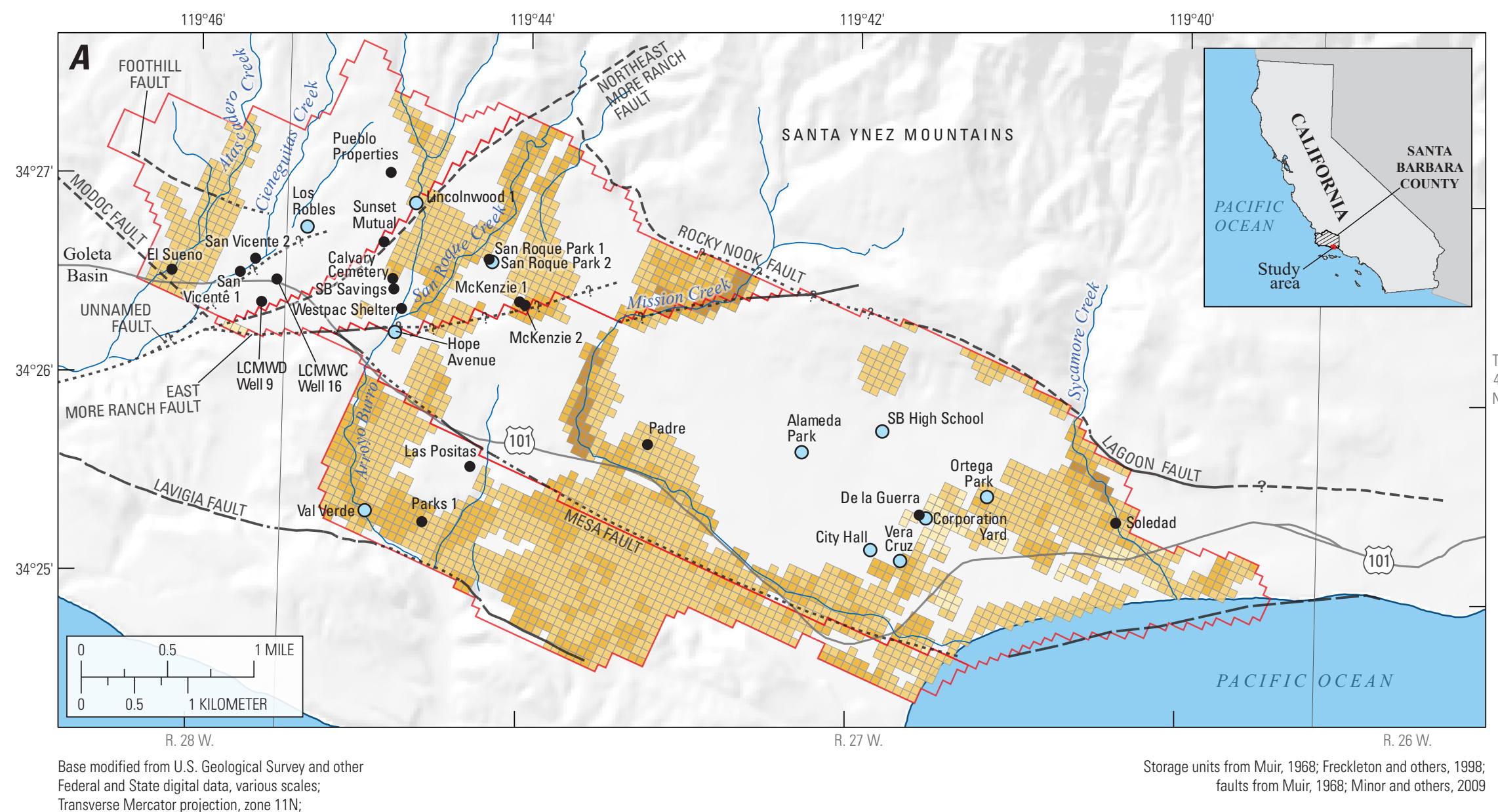

Federal and State digital data, various scales;
Transverse Mercator projection, zone 11N;

North American Datum of 1983 (NAD 83)

\section{EXPLANATION}

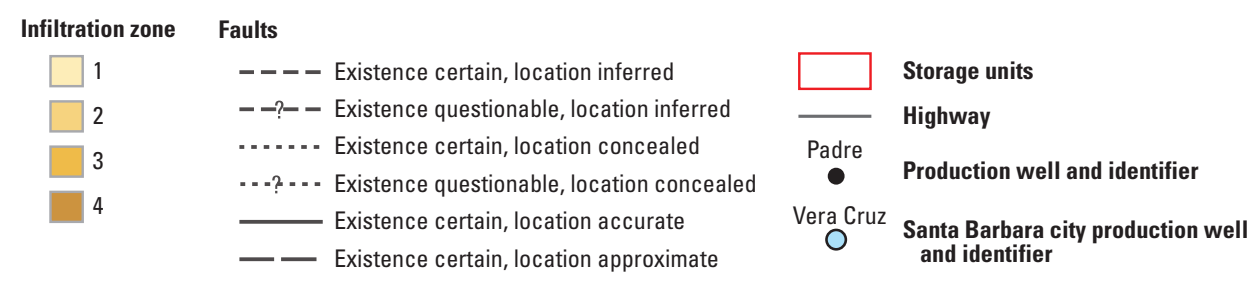

Figure 3. Areas of potential groundwater recharge for the Santa Barbara flow and transport model, Santa Barbara, California: $A$, infiltration zones and $B$, septic-recharge parcels. 


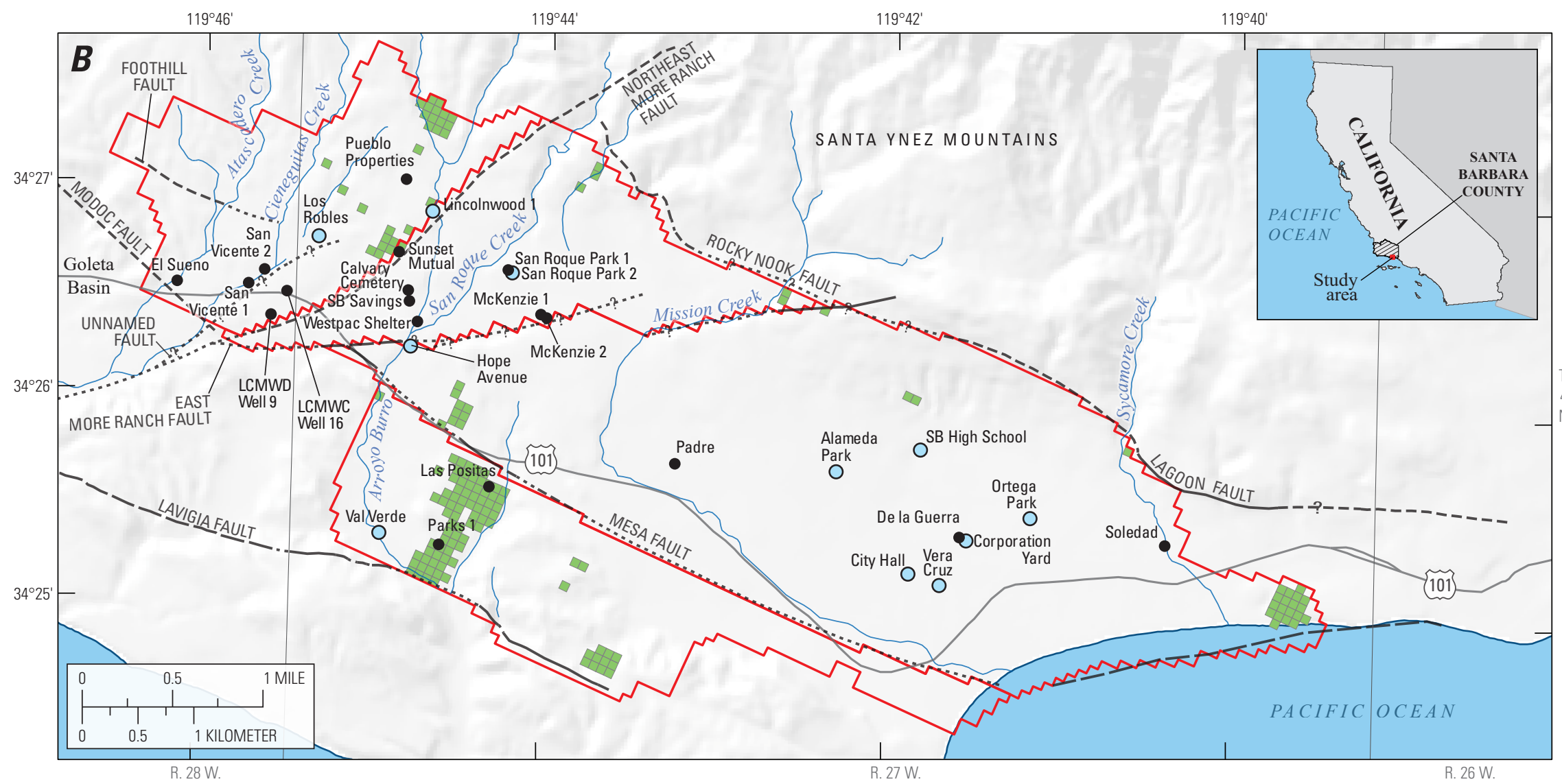

Base modified from U.S. Geological Survey and other

Storage units from Muir, 1968; Freckleton and others, 1998:

Federal and State digital data, various scales,

faults from Muir, 1968; Minor and others, 2009

Transverse Mercator projection, zone 11N

EXPLANATION

\section{Faults}

- - - Existence certain, location inferred

- -?- - Existence questionable, location inferred

......... Existence certain, location concealed

...? ... Existence questionable, location concealed

- Existence certain, location accurate

- - Existence certain, location approximate

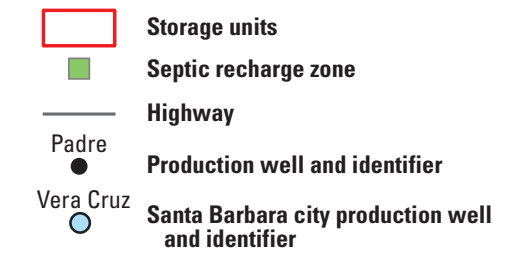

Figure 3. - Continued 


$$
\begin{aligned}
P c t_{\text {cell }}= & \left(H G L F_{\text {zone }} \times H K_{\text {cell }} \times S L_{\text {cell }}\right) / \\
& \left(\sum_{i=1}^{n}\left(H G L F_{i} \times H K_{i} \times S L_{i}\right)\right)
\end{aligned}
$$

where

$$
\begin{aligned}
& P c t_{\text {cell }} \quad \text { is the percentage of pumpage extracted from a } \\
& \text { given model cell, } \\
& H G L F_{\text {zone }} \quad \text { is a parameter assigned to each hydrogeologic } \\
& \text { zone (that is, the shallow zone, upper } \\
& \text { producing zone, and so on, as described in } \\
& \text { chapter A) that contains the model cell, } \\
& H K_{\text {cell }} \text { is the hydraulic conductivity at the well in a } \\
& \text { given model cell, } \\
& S L_{\text {cell }} \quad \text { is the length of the perforated interval in a } \\
& \text { given model cell, } \\
& H G L F_{i} \quad \text { is a multiplier for model cell } i \text { (allowed } \\
& \text { to range from } 1 \mathrm{E}-5 \text { to } 10 \text { in Storage } \\
& \text { Unit I and from } 0.1 \text { to } 10 \text { in Foothill and } \\
& \text { Storage Unit III) that allows fine tuning } \\
& \text { of the vertical pumping distribution (this } \\
& \text { estimate was updated iteratively during } \\
& \text { calibration as the hydraulic conductivity } \\
& \text { was estimated), } \\
& H K_{i} \quad \text { is the hydraulic conductivity at the well for } \\
& \text { model cell } i \text {, } \\
& S L_{i} \quad \text { is the length of the perforated interval for } \\
& \text { model cell } i \text {, } \\
& n \quad \text { is the total number of cells connected to the } \\
& \text { perforated interval of the well. }
\end{aligned}
$$

Basin outflows through creek channels and the city's drain system are simulated using the drain (DRN) package (Harbaugh and others, 2000). Outflow from the Foothill to the Goleta groundwater basin across the Modoc fault was assumed to be mostly along the Atascadero and Cieneguitas Creek deposits, and outflow from Storage Unit III across the Lavigia fault was assumed to be mostly along the Arroyo Burro and Las Positas Creek deposits (fig. 1). Drain head values along these creeks were initially assigned based on nearby well observations; final drain head values were determined by parameter estimation. The manmade drains installed by the city in Storage Unit I to drain the Estero were simulated based on Freckleton and others (1998). Drain head values for manmade drains installed by the city were defined by the drain elevation. Drain conductance values for creeks and the city's drain system were both determined by model calibration.

\section{Aquifer Properties}

Aquifer properties used in the simulation were horizontal and vertical hydraulic conductivity, specific storage, conductance across horizontal-flow barriers, and the constituent transport parameters porosity, dispersivity, and diffusion. Percentage of coarse-grained sediment from the texture model was used to define hydraulic conductivity and specific storage. The locations of the horizontal-flow barriers were determined by fault locations reported by Minor and others (2009). Porosity and dispersivity were estimated by parameter estimation and allowed to vary within reasonable limits.

\section{Model Parameterization}

To aid in the calibration of the hydraulic properties and boundary conditions in the model, the four groundwater subbasins were divided into regions, subregions, and areas (figs. 4-6). The subbasins were divided into five regions (fig. 4); each region was assigned different hydraulic conductivity ranges. The regions were divided into 14 subregions (fig. 5), which were used to improve the representation of different depositional environments and were based on the geography and geology of the study area, including proximity to stream channels, mountains, and the coast. Each subregion was assigned different multipliers, which were estimated during model calibration. Finally, the model domain was divided into coastal and inland areas (fig. 6), where each area was assigned layer-wise multipliers, which were estimated during model calibration. The coastal area (fig. 6) was parameterized separately from the rest of Storage Unit I because some shallow, low-permeability areas are limited to the flatter areas of the coastal plain; therefore, the coastal and inland areas have different layer-wise multipliers. For example, assume a hydraulic conductivity value for region $i$, in subregion $j$, in the coastal area; therefore, the hydraulic conductivity (HK) value for layer $k$ is as follows:

$$
H K_{k}=H K_{i} \times m_{j} \times m_{c k}
$$

where

$$
\begin{array}{cl}
m_{j} & \text { is the multiplier for subregion } j, \text { and } \\
m_{c k} & \text { is the coastal multiplier for layer } k .
\end{array}
$$

\section{Hydrogeologic Units}

The Santa Barbara and Foothill groundwater basins contain two main high-permeability hydrogeologic units: the upper and lower producing zones (fig. 7). Near the coast, Storage Unit I markedly thickens and contains the additional middle producing zone that does not extend inland into the other subbasins, as discussed in chapters A and B (fig. A-6). As stated earlier, the SBFTM simulates the transport of chloride due to seawater intrusion in Storage Unit I, which requires a relatively high level of model detail; therefore, the hydrogeologic units were defined in greater detail for Storage Unit I than for Storage Unit III and the Foothill subbasins. 


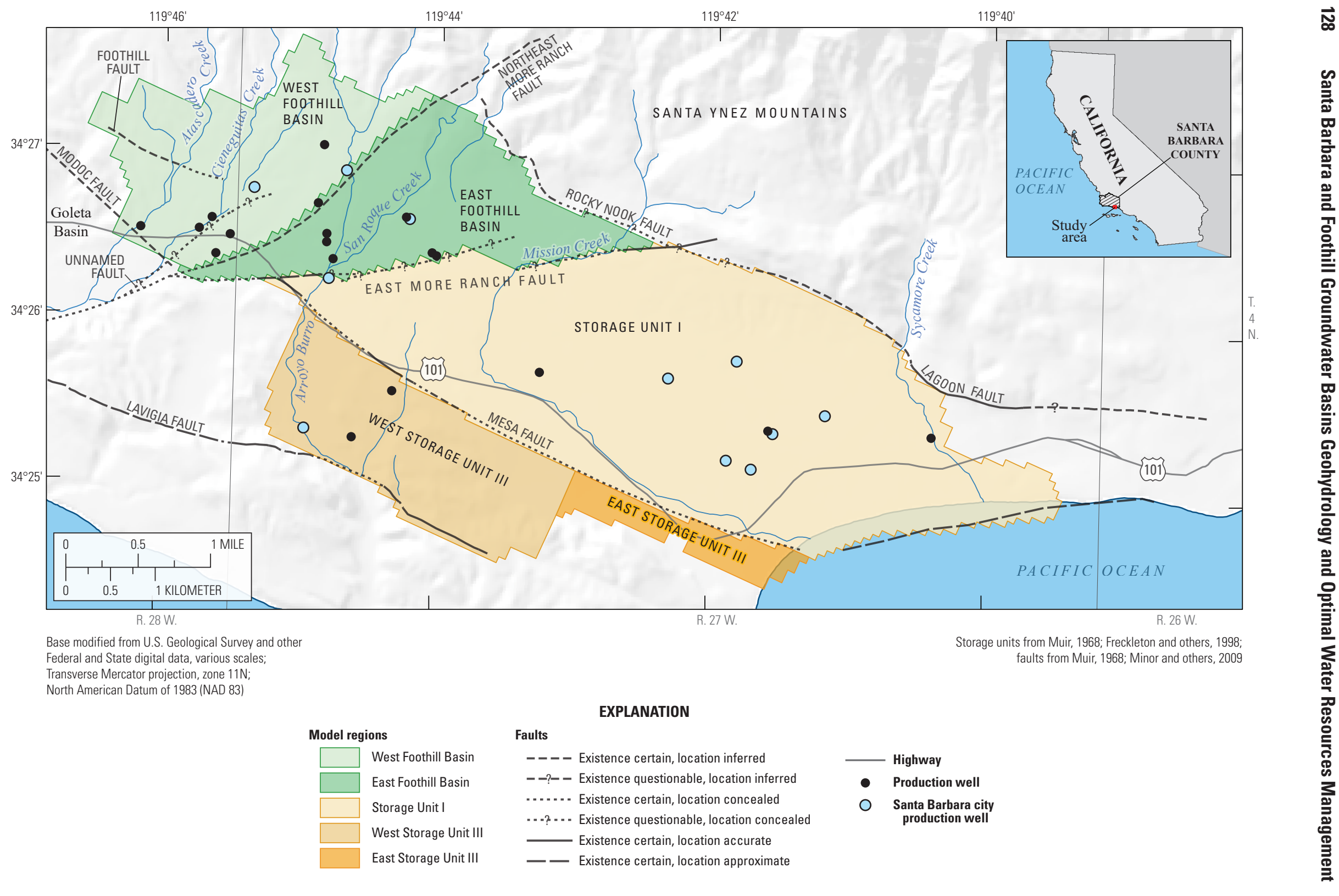

Figure 4. Five regions used in the Santa Barbara flow and transport model, Santa Barbara, California. 


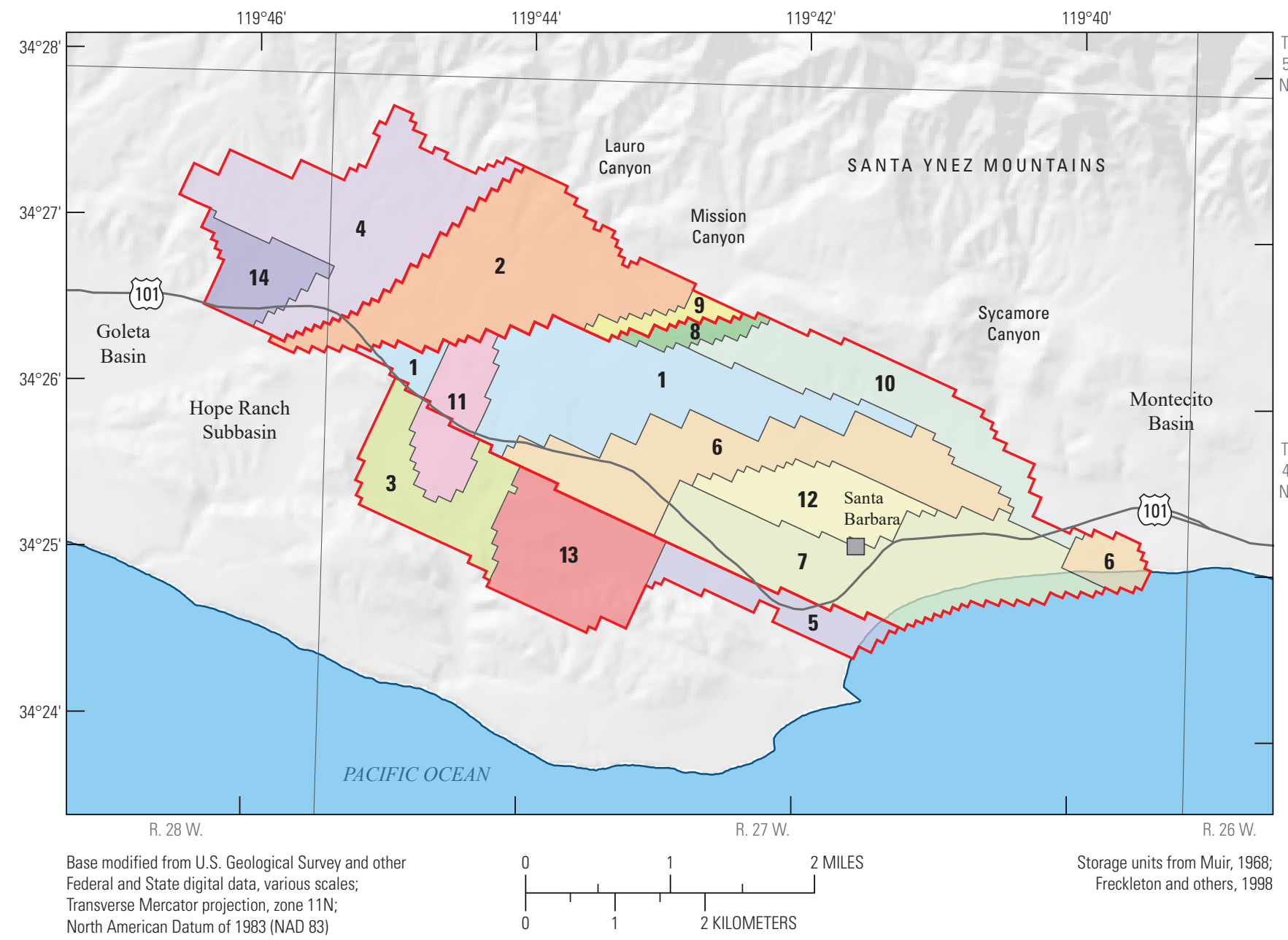

EXPLANATION
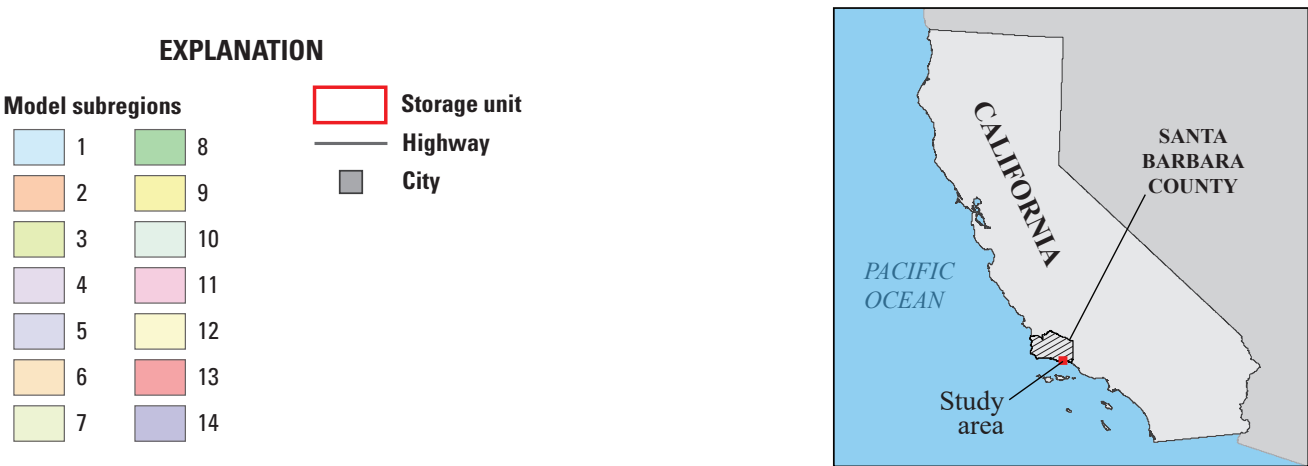

Figure 5. The 14 subregions used in the Santa Barbara flow and transport model, Santa Barbara, California. 


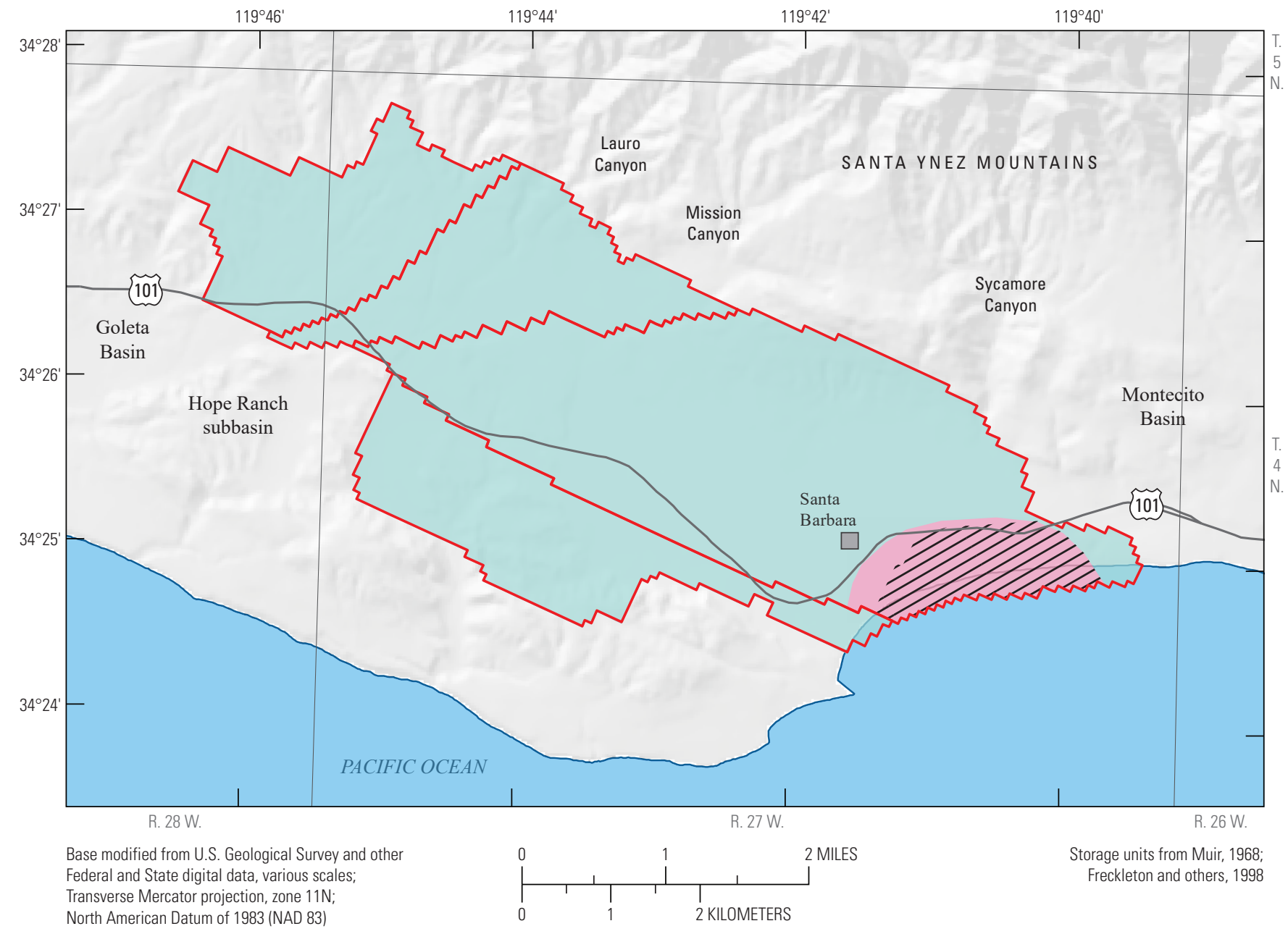

EXPLANATION
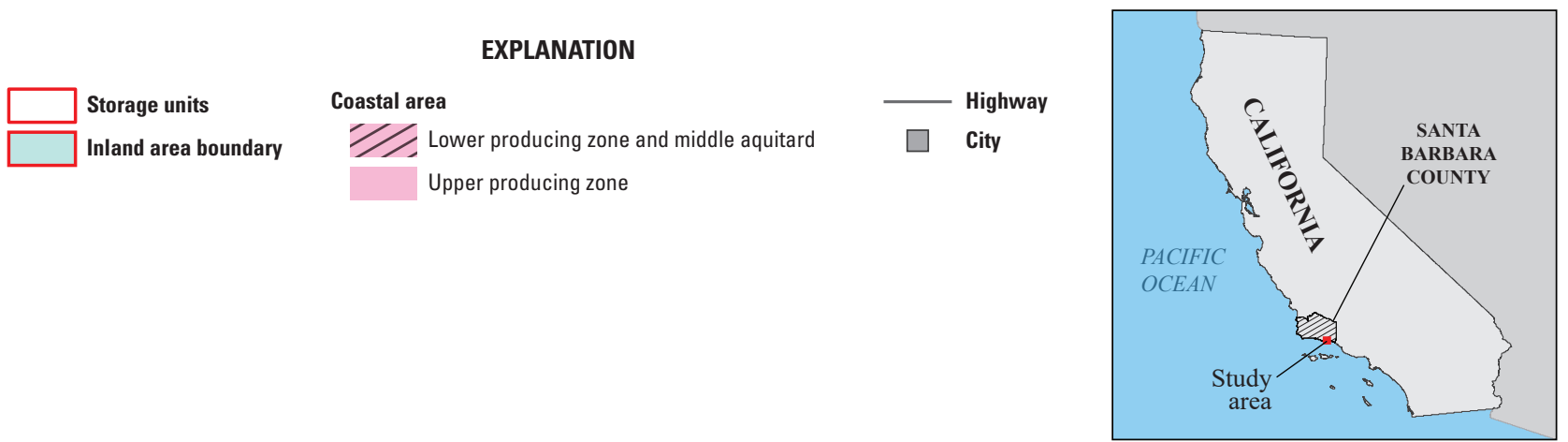

Figure 6. The two areas used in the Santa Barbara flow and transport model, Santa Barbara, California. 


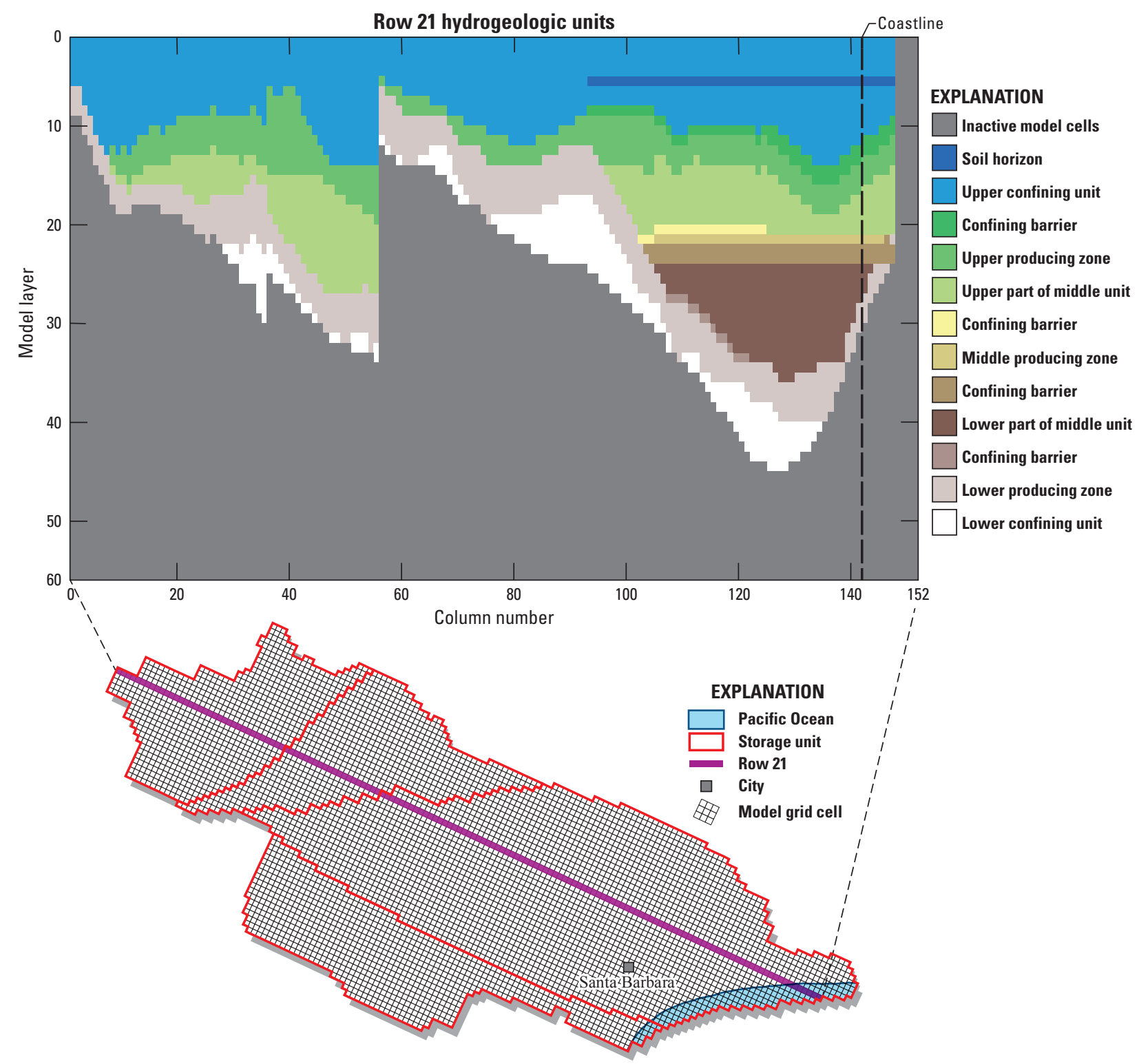

Figure 7. Hydrogeologic units defined for the Santa Barbara flow and transport model, Santa Barbara, California.

\section{Hydraulic and Transport Properties}

Hydraulic properties include hydraulic conductivity of the aquifer; conductance of faults, GHBs, and drains; creekbed conductance; and aquifer storage. Transport properties include porosity, dispersivity, and diffusion.

\section{Hydraulic Conductivity}

Hydraulic conductivity was estimated using the sediment texture defined in the HFM (chapter B) by regions, subregions, areas, and creek channels. The hydraulic conductivity for each groundwater-model cell was calculated using the percentage of coarse-grained material from the HFM (see chapter B). The calculation of hydraulic conductivity for the horizontal and vertical directions was done using the power mean. Using this mean, the hydraulic conductivity of the textural end members, representing the coarsest and finest materials, was used in conjunction with the percentage of coarse-grained material in each cell to calculate the overall hydraulic conductivity in each direction. Different lithologic end-member hydraulic conductivities were used for each region (table 3). Initial horizontal hydraulic conductivities were calculated using arithmetic averaging, and initial vertical hydraulic conductivities were calculated using a power approximating the geometric mean. These initial texture-based horizontal and vertical hydraulic-conductivity values were calculated using equations 3 and 4, respectively: 


$$
\begin{gathered}
H K_{\text {texture }}=P C \times K_{c x}+P F \times K_{f x} \\
V K_{\text {texture }}=\left({K_{c k}}^{-1} \times P C+{K_{f x}}^{-1} \times P F\right)^{-1}
\end{gathered}
$$

where

$$
\begin{gathered}
K_{c x} \quad \begin{array}{l}
\text { is the coarse hydraulic-conductivity end } \\
\text { member; }
\end{array} \\
K_{f x} \quad \text { is the fine hydraulic-conductivity end } \\
\text { member; } \\
P C \quad \text { is the percentage of coarse sediment in a } \\
\text { given model cell, expressed as a fraction; } \\
\text { and } \\
P F \quad \text { is the percentage of fine sediment in a given } \\
\text { model cell, expressed as a fraction. }
\end{gathered}
$$

The texture-based hydraulic-conductivity values were adjusted during model calibration by multipliers defined for each subregion, area, and hydrogeologic unit. The horizontal and vertical hydraulic-conductivity values for specific subregion, area, and hydrogeologic unit combinations were defined by equations 5 and 6 :

$$
\begin{gathered}
H K_{\text {srl }}=H K_{\text {texture }} \times H K S R M \times H K L M \\
V K_{\text {srl }}=V K_{\text {texture }} \times V K S R M \times V K L M
\end{gathered}
$$

where

$H K S R M$ and $V K S R M$ are multipliers for the horizontal and vertical hydraulic conductivity, respectively, applied to each subregion, and

HKLM and VKLM are the horizontal and vertical hydraulic conductivity multipliers, respectively, applied to each hydrogeologic unit. Note that HKLM and VKLM are also functions of area (coastal or inland).

Relatively coarse creek beds are thought to exist in the upper Mission and Sycamore Creeks that were not captured in the textural model because of a lack of borehole data for the creek beds. To simulate the coarse-grained material in these creek beds, a scalar multiplier was applied to the HK and VK in all model layers in creek-infiltration zones. This implicitly assumes that the creek channel has not moved over time and that the coarse-grained material extends vertically beneath the creek bed to the water table.

\section{Conductance Properties}

Conductance properties affect the flow across faults, GHBs, and drains. Flow across creek beds is affected by creek-bed conductance and stage; these are discussed in the following section.

On the basis of previous work and water-level offsets, the Mesa, East More Ranch, Northeast More Ranch, unnamed, and Foothill faults are thought to restrict groundwater flow (fig. 8). Accordingly, these faults are simulated using the horizontal-flow barrier (HFB) package (Hsieh and Freckleton, 1993). An HFB is a low-permeability feature that is used to restrict flow across the simulated barrier with a specified hydraulic-characteristic term. The lower the hydraulic characteristic, the greater the effect of the barrier. The East More Ranch, Mesa, Northeast More Ranch, and Foothill faults were divided into segments, and a separate hydrauliccharacteristic parameter was estimated for each segment. The unnamed fault and the Foothill fault each have a single hydraulic-characteristic value for the entire length of the fault. In order to simulate increased flow across faults intersected by coarse fluvial deposits, separate hydraulic-characteristic parameters were assigned to the upper model cells at the intersection of faults and major creeks.

The ocean-fault GHB was simulated using seven conductance parameters defined by hydrogeologic zone: one for each of the three producing zones and four for the zones between, above, and below the producing zones. The shallowocean GHB was assigned a single conductance parameter. The Montecito GHB was assigned three conductance parameters, one for the middle producing zone, one for all model layers above it, and one for all model layers below it.

As was previously described, there are two general sets of drains: the Santa Barbara city drains and the creek-underflow drains for Arroyo Burro, Atascadero, and Cienguitas Creeks. One conductance parameter was used for the Santa Barbara city drain, and two conductance parameters were used for each creek underflow drain - one parameter for the upper layers and one for the lower layers.

\section{Creek Properties}

There are two properties that govern whether and how much a creek gains or loses water: creek-bed conductance and creek stage. Model cells of the stream type (fig. 1) were divided into segments along most creeks, and each segment was assigned a separate value for creek-bed conductance (table 3). In addition, each creek segment was assigned a parameter for the stage of the creek, which was fixed for the entire simulation. 
Table 3. Calibrated groundwater flow and solute transport model parameter values, Santa Barbara flow and transport model, Santa Barbara, California.

[/day, per day; ft, foot; ft², square foot; GHB, general-head boundary package; HK, horizontal hydraulic conductivity; Rd., road; St., street; VK, vertical hydraulic conductivity; - , not applicable]

\begin{tabular}{|c|c|c|c|c|c|c|c|c|}
\hline $\begin{array}{l}\text { Parameter } \\
\text { name }\end{array}$ & Group & Subgroup & Description & $\begin{array}{l}\text { Calibrated } \\
\text { value }\end{array}$ & $\begin{array}{l}\text { Lower } \\
\text { bound }\end{array}$ & $\begin{array}{l}\text { Upper } \\
\text { bound }\end{array}$ & $\begin{array}{l}\text { Preliminary } \\
\text { value }\end{array}$ & Units \\
\hline ab_t_t & streamk & $\begin{array}{l}\text { Creek conductance adjustment } \\
\text { parameter }\end{array}$ & Las Positas Creek & $3.91 \mathrm{E}-04$ & $1.08 \mathrm{E}-04$ & $1.08 \mathrm{E}+04$ & $1.23 \mathrm{E}+00$ & $\mathrm{ft}^{2} /$ day \\
\hline ab_w_t & streamk & $\begin{array}{l}\text { Creek conductance adjustment } \\
\text { parameter }\end{array}$ & $\begin{array}{l}\text { Arroyo Burro Creek between Northridge and San } \\
\text { Roque Creek junctions }\end{array}$ & $4.57 \mathrm{E}-05$ & $1.08 \mathrm{E}-05$ & $1.08 \mathrm{E}+04$ & $3.33 \mathrm{E}+02$ & $\mathrm{ft}^{2} /$ day \\
\hline ab_we_t & streamk & $\begin{array}{l}\text { Creek conductance adjustment } \\
\text { parameter }\end{array}$ & Northridge Creek & $5.37 \mathrm{E}+01$ & $1.08 \mathrm{E}-04$ & $1.08 \mathrm{E}+04$ & $6.39 \mathrm{E}+01$ & $\mathrm{ft}^{2} /$ day \\
\hline ab_ww_t & streamk & $\begin{array}{l}\text { Creek conductance adjustment } \\
\text { parameter }\end{array}$ & $\begin{array}{l}\text { Upstream Arroyo Burro Creek upstream of } \\
\text { Northridge Creek junction }\end{array}$ & 4.73E+01 & $1.08 \mathrm{E}-04$ & $1.08 \mathrm{E}+04$ & $1.11 \mathrm{E}+02$ & $\mathrm{ft}^{2} /$ day \\
\hline abcynrchmt & $\mathrm{rch}$ & Small catchment recharge multiplier & Small catchment recharge Arroyo Burro Creek area & $2.20 \mathrm{E}+00$ & $1.00 \mathrm{E}-05$ & $2.50 \mathrm{E}+00$ & $5.59 \mathrm{E}-02$ & - \\
\hline arroyo_br_t & streamk & $\begin{array}{l}\text { Creek conductance adjustment } \\
\text { parameter }\end{array}$ & $\begin{array}{l}\text { Arroyo Burro Creek from San Roque Creek to about } \\
0.7 \text { miles south of San Roque Creek junction }\end{array}$ & $1.54 \mathrm{E}-03$ & $1.08 \mathrm{E}-04$ & $1.08 \mathrm{E}+04$ & $9.61 \mathrm{E}+01$ & $\mathrm{ft}^{2} /$ day \\
\hline arroyobrs_t & streamk & $\begin{array}{l}\text { Creek conductance adjustment } \\
\text { parameter }\end{array}$ & $\begin{array}{l}\text { Downstream portion of Arroy Burro Creak to edge } \\
\text { of model }\end{array}$ & $3.56 \mathrm{E}-01$ & $1.08 \mathrm{E}-04$ & $1.08 \mathrm{E}+04$ & $3.56 \mathrm{E}-01$ & $\mathrm{ft}^{2} /$ day \\
\hline arryob_s & rivers & Creek stage adjustment parameter & $\begin{array}{l}\text { Upstream Arroyo Burro Creek upstream of } \\
\text { Northridge Creek junction }\end{array}$ & 4.64E-01 & $3.28 \mathrm{E}-06$ & $3.28 \mathrm{E}+00$ & $1.38 \mathrm{E}-04$ & $\mathrm{ft}$ \\
\hline arryoc_s & rivers & Creek stage adjustment parameter & $\begin{array}{l}\text { Arroyo Burro Creek between Northridge and San } \\
\text { Roque Creek junctions }\end{array}$ & 4.64E-01 & $3.28 \mathrm{E}-06$ & $3.28 \mathrm{E}+00$ & $1.38 \mathrm{E}-04$ & $\mathrm{ft}$ \\
\hline arryod_s & rivers & Creek stage adjustment parameter & $\begin{array}{l}\text { Arroyo Burro Creek from San Roque Creek to about } \\
0.7 \text { miles south of San Roque Creek junction }\end{array}$ & 4.64E-01 & $3.28 \mathrm{E}-06$ & $3.28 \mathrm{E}+00$ & $1.38 \mathrm{E}-04$ & $\mathrm{ft}$ \\
\hline arryol_s & rivers & Creek stage adjustment parameter & $\begin{array}{l}\text { Downstream portion of Arroy Burro Creak to edge } \\
\text { of model }\end{array}$ & $4.64 \mathrm{E}-01$ & $3.28 \mathrm{E}-06$ & $3.28 \mathrm{E}+00$ & $1.38 \mathrm{E}-04$ & $\mathrm{ft}$ \\
\hline atas_e_t & streamk & $\begin{array}{l}\text { Creek conductance adjustment } \\
\text { parameter }\end{array}$ & $\begin{array}{l}\text { Upstream Atascadero Creek upstream of Via } \\
\text { Chaparral Creek junction }\end{array}$ & $3.04 \mathrm{E}+01$ & $1.08 \mathrm{E}-04$ & $1.08 \mathrm{E}+04$ & $1.12 \mathrm{E}+00$ & $\mathrm{ft}^{2} /$ day \\
\hline atas_t_t & streamk & $\begin{array}{l}\text { Creek conductance adjustment } \\
\text { parameter }\end{array}$ & La Paloma Creek & $2.51 \mathrm{E}-05$ & $1.08 \mathrm{E}-05$ & $1.08 \mathrm{E}+04$ & $6.43 \mathrm{E}+01$ & $\mathrm{ft}^{2} /$ day \\
\hline atas_w_t & streamk & $\begin{array}{l}\text { Creek conductance adjustment } \\
\text { parameter }\end{array}$ & Via Chaparral Creek & $2.67 \mathrm{E}-05$ & $1.08 \mathrm{E}-05$ & $1.08 \mathrm{E}+04$ & $2.20 \mathrm{E}+00$ & $\mathrm{ft}^{2} /$ day \\
\hline atasca_s & rivers & Creek stage adjustment parameter & $\begin{array}{l}\text { Upstream Atascadero Creek upstream of Via } \\
\text { Chaparral Creek junction }\end{array}$ & $4.61 \mathrm{E}-01$ & $3.28 \mathrm{E}-06$ & $4.92 \mathrm{E}+00$ & $1.87 \mathrm{E}-01$ & $\mathrm{ft}$ \\
\hline atascadero_t & streamk & $\begin{array}{l}\text { Creek conductance adjustment } \\
\text { parameter }\end{array}$ & $\begin{array}{l}\text { Atascadero Creek downstream of Via Chaparral } \\
\text { Creek junction }\end{array}$ & $8.11 \mathrm{E}+00$ & $1.08 \mathrm{E}-04$ & $1.08 \mathrm{E}+04$ & $1.12 \mathrm{E}+00$ & $\mathrm{ft}^{2} /$ day \\
\hline atascb_s & rivers & Creek stage adjustment parameter & $\begin{array}{l}\text { Atascadero Creek downstream of Via Chaparral } \\
\text { Creek junction }\end{array}$ & $4.60 \mathrm{E}-01$ & $3.28 \mathrm{E}-06$ & $3.28 \mathrm{E}+00$ & $6.22 \mathrm{E}-02$ & $\mathrm{ft}$ \\
\hline atasel & streamk & $\begin{array}{l}\text { Creek conductance adjustment } \\
\text { parameter }\end{array}$ & $\begin{array}{l}\text { Where Atascadero and Via Chaparral Creek closely } \\
\text { parallel one another up to junction }\end{array}$ & $9.73 \mathrm{E}+00$ & $1.08 \mathrm{E}-04$ & $1.08 \mathrm{E}+04$ & $1.15 \mathrm{E}-04$ & $\mathrm{ft}^{2} /$ day \\
\hline
\end{tabular}


Table 3. Calibrated groundwater flow and solute transport model parameter values, Santa Barbara flow and transport model, Santa Barbara, California.-Continued

[/day, per day; ft, foot; ft² , square foot; GHB, general-head boundary package; HK, horizontal hydraulic conductivity; Rd., road; St., street; VK, vertical hydraulic conductivity; - , not applicable]

\begin{tabular}{|c|c|c|c|c|c|c|c|c|}
\hline $\begin{array}{l}\text { Parameter } \\
\text { name }\end{array}$ & Group & Subgroup & Description & $\begin{array}{l}\text { Calibrated } \\
\text { value }\end{array}$ & $\begin{array}{l}\text { Lower } \\
\text { bound }\end{array}$ & $\begin{array}{l}\text { Upper } \\
\text { bound }\end{array}$ & $\begin{array}{l}\text { Preliminary } \\
\text { value }\end{array}$ & Units \\
\hline cien_e_t & streamk & $\begin{array}{l}\text { Creek conductance adjustment } \\
\text { parameter }\end{array}$ & Eastern fork of Cieneguitas Creek & $6.06 \mathrm{E}+00$ & $1.08 \mathrm{E}-04$ & $1.08 \mathrm{E}+04$ & $1.14 \mathrm{E}+00$ & $\mathrm{ft}^{2} / \mathrm{day}$ \\
\hline cien_w_t & streamk & $\begin{array}{l}\text { Creek conductance adjustment } \\
\text { parameter }\end{array}$ & Western fork of Cieneguitas Creek & $5.50 \mathrm{E}+01$ & $1.08 \mathrm{E}-04$ & $1.08 \mathrm{E}+04$ & $2.64 \mathrm{E}+01$ & $\mathrm{ft}^{2} /$ day \\
\hline cienee_s & rivers & Creek stage adjustment parameter & Eastern fork of Cieneguitas Creek & $4.82 \mathrm{E}-01$ & $3.28 \mathrm{E}-06$ & $3.28 \mathrm{E}+00$ & $1.87 \mathrm{E}-01$ & $\mathrm{ft}$ \\
\hline cieneguita_t & streamk & $\begin{array}{l}\text { Creek conductance adjustment } \\
\text { parameter }\end{array}$ & Cieneguitas Creek downstream of fork & $3.94 \mathrm{E}-05$ & $1.08 \mathrm{E}-05$ & $1.08 \mathrm{E}+04$ & $1.24 \mathrm{E}-01$ & $\mathrm{ft}^{2} /$ day \\
\hline cienel_s & rivers & Creek stage adjustment parameter & Cieneguitas Creek downstream of fork & 4.82E-01 & $3.28 \mathrm{E}-06$ & $3.28 \mathrm{E}+00$ & $3.94 \mathrm{E}-02$ & $\mathrm{ft}$ \\
\hline cieneu_s & rivers & Creek stage adjustment parameter & Western fork of Cieneguitas Creek & $4.82 \mathrm{E}-01$ & $3.28 \mathrm{E}-06$ & $3.28 \mathrm{E}+00$ & $3.94 \mathrm{E}-02$ & $\mathrm{ft}$ \\
\hline disp & dispersion & Longitudinal dispersivity & Shallow & $6.56 \mathrm{E}+00$ & $4.00 \mathrm{E}-02$ & $2.50 \mathrm{E}+02$ & $2.80 \mathrm{E}+03$ & $\mathrm{ft}$ \\
\hline dispdp & dispersion & Longitudinal dispersivity & Deep & $4.92 \mathrm{E}+01$ & $4.00 \mathrm{E}-02$ & $2.50 \mathrm{E}+02$ & $6.56 \mathrm{E}+00$ & $\mathrm{ft}$ \\
\hline draincond & otherk & Drain conductance & City drain conductance & $3.70 \mathrm{E}+02$ & $1.08 \mathrm{E}-02$ & $1.08 \mathrm{E}+04$ & $2.73 \mathrm{E}+01$ & $\mathrm{ft}^{2} /$ day \\
\hline drainfha & otherk & Drain conductance & Atascadero Creek drain conductance shallow & $1.27 \mathrm{E}+00$ & $1.08 \mathrm{E}-04$ & $1.08 \mathrm{E}+04$ & $1.31 \mathrm{E}-03$ & $\mathrm{ft}^{2} /$ day \\
\hline drainfhadp & otherk & Drain conductance & Atascadero Creek drain conductance deep & $1.56 \mathrm{E}+01$ & $1.08 \mathrm{E}-02$ & $1.08 \mathrm{E}+04$ & $3.11 \mathrm{E}+00$ & $\mathrm{ft}^{2} /$ day \\
\hline drainfhc & otherk & Drain conductance & Cieneguitas Creek drain conductance shallow & $5.57 \mathrm{E}-01$ & $1.08 \mathrm{E}-04$ & $1.08 \mathrm{E}+04$ & $1.31 \mathrm{E}-04$ & $\mathrm{ft}^{2} / \mathrm{day}$ \\
\hline drainfhcdp & otherk & Drain conductance & Cieneguitas Creek drain conductance deep & $5.84 \mathrm{E}+00$ & $1.08 \mathrm{E}-02$ & $1.08 \mathrm{E}+04$ & $3.08 \mathrm{E}-01$ & $\mathrm{ft}^{2} / \mathrm{day}$ \\
\hline drainsu3 & otherk & Drain conductance & Arroyo Burro Creek drain conductance & $2.47 \mathrm{E}+01$ & $1.08 \mathrm{E}-02$ & $1.08 \mathrm{E}+04$ & $2.41 \mathrm{E}+02$ & $\mathrm{ft}^{2} / \mathrm{day}$ \\
\hline e_more_1 & faultk & Fault conductance & $\begin{array}{l}\text { East More Ranch fault between western edge of } \\
\text { model and } 101 \text { freeway }\end{array}$ & $7.49 \mathrm{E}-08$ & $1.00 \mathrm{E}-08$ & $1.00 \mathrm{E}+00$ & $2.23 \mathrm{E}-04$ & /day \\
\hline e_more_2 & faultk & Fault conductance & $\begin{array}{l}\text { East More Ranch fault between } 101 \text { freeway and } \\
\text { State Street }\end{array}$ & $5.21 \mathrm{E}-08$ & $1.00 \mathrm{E}-08$ & $1.00 \mathrm{E}+02$ & $1.42 \mathrm{E}-04$ & /day \\
\hline e_more_3 & faultk & Fault conductance & $\begin{array}{l}\text { East More Ranch fault between State Street and } \\
\text { Mission Creek }\end{array}$ & $1.13 \mathrm{E}-08$ & $1.00 \mathrm{E}-08$ & $1.00 \mathrm{E}+00$ & $5.39 \mathrm{E}-05$ & /day \\
\hline e_more_4a & faultk & Fault conductance & $\begin{array}{l}\text { East More Ranch fault following Mission Creek } \\
\text { northeast of the Mission }\end{array}$ & $8.61 \mathrm{E}-05$ & $1.00 \mathrm{E}-07$ & $1.00 \mathrm{E}+00$ & $1.56 \mathrm{E}-05$ & /day \\
\hline e_more_4b & faultk & Fault conductance & $\begin{array}{l}\text { East More Ranch fault following Mission Creek } \\
\text { west of the Mission }\end{array}$ & $2.64 \mathrm{E}-06$ & $1.00 \mathrm{E}-07$ & $1.00 \mathrm{E}+00$ & $1.56 \mathrm{E}-05$ & /day \\
\hline e_more_wr & faultk & Fault conductance & East More Ranch fault at Arroyo Burro Creek & $1.00 \mathrm{E}+01$ & $1.00 \mathrm{E}-07$ & $1.00 \mathrm{E}+01$ & $1.00 \mathrm{E}+01$ & /day \\
\hline fhdrainhd & other_hd & Drain head & Atascadero and Cieneguitas Creek drain head & $-1.32 \mathrm{E}-02$ & $-6.55 \mathrm{E}+01$ & $1.64 \mathrm{E}+02$ & $-1.32 \mathrm{E}-02$ & $\mathrm{ft}$ \\
\hline foothillrd & faultk & Fault conductance & Foothill fault & $1.04 \mathrm{E}-04$ & $1.00 \mathrm{E}-07$ & $1.00 \mathrm{E}+00$ & $2.63 \mathrm{E}-05$ & /day \\
\hline htransd & dispersion & Horizontal transverse dispersivity & Horizontal shallow & $1.64 \mathrm{E}-02$ & $1.64 \mathrm{E}-02$ & $1.64 \mathrm{E}+00$ & $2.76 \mathrm{E}-01$ & $\mathrm{ft}$ \\
\hline htransddp & dispersion & Horizontal transverse dispersivity & Horizontal deep & $1.64 \mathrm{E}+00$ & $1.64 \mathrm{E}-01$ & $1.64 \mathrm{E}+01$ & $1.64 \mathrm{E}+00$ & $\mathrm{ft}$ \\
\hline
\end{tabular}


Table 3. Calibrated groundwater flow and solute transport model parameter values, Santa Barbara flow and transport model, Santa Barbara, California.-Continued

[/day, per day; ft, foot; $\mathrm{ft}^{2}$, square foot; GHB, general-head boundary package; HK, horizontal hydraulic conductivity; Rd., road; St., street; VK, vertical hydraulic conductivity; —, not applicable]

\begin{tabular}{|c|c|c|c|c|c|c|c|c|}
\hline $\begin{array}{l}\text { Parameter } \\
\text { name }\end{array}$ & Group & Subgroup & Description & $\begin{array}{c}\text { Calibrated } \\
\text { value }\end{array}$ & $\begin{array}{l}\text { Lower } \\
\text { bound }\end{array}$ & $\begin{array}{l}\text { Upper } \\
\text { bound }\end{array}$ & $\begin{array}{l}\text { Preliminary } \\
\text { value }\end{array}$ & Units \\
\hline ic_pmp_mac & $\mathrm{rch}$ & $\begin{array}{l}\text { Early } 20 \text { th century pumping } \\
\text { multiplier }\end{array}$ & Early 20th century pumping multiplier & $2.00 \mathrm{E}-01$ & $1.00 \mathrm{E}-02$ & $8.00 \mathrm{E}-01$ & $8.00 \mathrm{E}-01$ & - \\
\hline $\mathrm{kc1}$ & aquiferk & $\mathrm{HK} / \mathrm{VK}$ end member & Coarse end member for Storage Unit I & $8.58 \mathrm{E}+00$ & $3.28 \mathrm{E}-02$ & $3.28 \mathrm{E}+03$ & $6.62 \mathrm{E}+00$ & $\mathrm{ft} /$ day \\
\hline $\mathrm{kc} 2$ & aquiferk & $\mathrm{HK} / \mathrm{VK}$ end member & Coarse end member for East Foothill Subbasin & $3.97 \mathrm{E}+00$ & $3.28 \mathrm{E}-02$ & $3.28 \mathrm{E}+03$ & $3.67 \mathrm{E}+02$ & $\mathrm{ft} /$ day \\
\hline $\mathrm{kc} 3$ & aquiferk & $\mathrm{HK} / \mathrm{VK}$ end member & Coarse end member for Storage Unit III West & $3.44 \mathrm{E}+00$ & $3.28 \mathrm{E}-02$ & $3.28 \mathrm{E}+03$ & $5.76 \mathrm{E}+00$ & $\mathrm{ft} / \mathrm{day}$ \\
\hline $\mathrm{kc} 4$ & aquiferk & $\mathrm{HK} / \mathrm{VK}$ end member & Coarse end member for West Foothill Subbasin & $6.61 \mathrm{E}+00$ & $3.28 \mathrm{E}-02$ & $3.28 \mathrm{E}+03$ & $7.34 \mathrm{E}+01$ & $\mathrm{ft} /$ day \\
\hline $\mathrm{kc} 5$ & aquiferk & $\mathrm{HK} / \mathrm{VK}$ end member & Coarse end member for Storage Unit III East & $2.62 \mathrm{E}-01$ & $3.28 \mathrm{E}-02$ & $3.28 \mathrm{E}+03$ & $3.51 \mathrm{E}+01$ & $\mathrm{ft} /$ day \\
\hline $\mathrm{kfl}$ & aquiferk & $\mathrm{HK} / \mathrm{VK}$ end member & Fine end member for Storage Unit I & $2.33 \mathrm{E}-02$ & $3.28 \mathrm{E}-05$ & $3.28 \mathrm{E}+00$ & $1.86 \mathrm{E}-03$ & $\mathrm{ft} /$ day \\
\hline $\mathrm{kf2}$ & aquiferk & $\mathrm{HK} / \mathrm{VK}$ end member & Fine end member for East Foothill Subbasin & $3.85 \mathrm{E}-03$ & $3.28 \mathrm{E}-05$ & $3.28 \mathrm{E}+00$ & $3.12 \mathrm{E}-03$ & $\mathrm{ft} /$ day \\
\hline $\mathrm{kf3}$ & aquiferk & $\mathrm{HK} / \mathrm{VK}$ end member & Fine end member for Storage Unit III West & $2.86 \mathrm{E}-03$ & $3.28 \mathrm{E}-05$ & $3.28 \mathrm{E}+00$ & $2.67 \mathrm{E}-03$ & $\mathrm{ft} / \mathrm{day}$ \\
\hline kf4 & aquiferk & $\mathrm{HK} / \mathrm{VK}$ end member & Fine end member for West Foothill Subbasin & $1.68 \mathrm{E}-02$ & $3.28 \mathrm{E}-05$ & $3.28 \mathrm{E}+00$ & $1.64 \mathrm{E}-03$ & $\mathrm{ft} /$ day \\
\hline kf5 & aquiferk & $\mathrm{HK} / \mathrm{VK}$ end member & Fine end member for Storage Unit III East & $1.47 \mathrm{E}-03$ & $3.28 \mathrm{E}-05$ & $3.28 \mathrm{E}+00$ & $4.90 \mathrm{E}-03$ & $\mathrm{ft} /$ day \\
\hline lapalo_s & rivers & Creek stage adjustment parameter & La Paloma Creek & 4.72E-01 & $3.28 \mathrm{E}-06$ & $3.28 \mathrm{E}+00$ & $6.23 \mathrm{E}-02$ & $\mathrm{ft}$ \\
\hline laspos_s & rivers & Creek stage adjustment parameter & Las Positas Creek & $4.60 \mathrm{E}-01$ & $3.28 \mathrm{E}-06$ & $3.28 \mathrm{E}+00$ & $1.48 \mathrm{E}-01$ & $\mathrm{ft}$ \\
\hline lauro_t & streamk & $\begin{array}{l}\text { Creek conductance adjustment } \\
\text { parameter }\end{array}$ & Lauro Canyon Creek & $5.26 \mathrm{E}-04$ & $1.08 \mathrm{E}-04$ & $1.08 \mathrm{E}+04$ & $6.64 \mathrm{E}+01$ & $\mathrm{ft}^{2} /$ day \\
\hline lauroc_s & rivers & Creek stage adjustment parameter & Lauro Canyon Creek & 4.85E-01 & $3.28 \mathrm{E}-06$ & $3.28 \mathrm{E}+00$ & $3.38 \mathrm{E}-02$ & $\mathrm{ft}$ \\
\hline mc_1_4_t & streamk & $\begin{array}{l}\text { Creek conductance adjustment } \\
\text { parameter }\end{array}$ & $\begin{array}{l}\text { Upstream segment of Mission Creek near northern } \\
\text { boundary of model to just north of the Mission }\end{array}$ & $2.44 \mathrm{E}+02$ & $1.08 \mathrm{E}-02$ & $1.08 \mathrm{E}+03$ & $5.48 \mathrm{E}+02$ & $\mathrm{ft}^{2} /$ day \\
\hline mc_4_6_t & streamk & $\begin{array}{l}\text { Creek conductance adjustment } \\
\text { parameter }\end{array}$ & Mission Creek just north of the Mission to State St. & $1.72 \mathrm{E}+02$ & $1.08 \mathrm{E}-02$ & $1.08 \mathrm{E}+04$ & $2.73 \mathrm{E}+02$ & $\mathrm{ft}^{2} /$ day \\
\hline mc_6_7_t & streamk & $\begin{array}{l}\text { Creek conductance adjustment } \\
\text { parameter }\end{array}$ & $\begin{array}{l}\text { Mission Creek from State St. to where it runs } \\
\text { parallel to } 101 \text { freeway }\end{array}$ & $6.43 \mathrm{E}+01$ & $1.08 \mathrm{E}-02$ & $1.08 \mathrm{E}+04$ & $3.12 \mathrm{E}+00$ & $\mathrm{ft}^{2} /$ day \\
\hline mc_7_8_t & streamk & $\begin{array}{l}\text { Creek conductance adjustment } \\
\text { parameter }\end{array}$ & $\begin{array}{l}\text { Mission Creek parallel to } 101 \text { freeway upstream of } \\
\text { Micheltorena St. }\end{array}$ & $1.98 \mathrm{E}+00$ & $1.08 \mathrm{E}-05$ & $1.08 \mathrm{E}+04$ & $5.84 \mathrm{E}+01$ & $\mathrm{ft}^{2} /$ day \\
\hline mc_8_11_t & streamk & $\begin{array}{l}\text { Creek conductance adjustment } \\
\text { parameter }\end{array}$ & $\begin{array}{l}\text { Mission Creek parallel to } 101 \text { freeway downstream } \\
\text { of Micheltorena St. }\end{array}$ & $2.15 \mathrm{E}+02$ & $1.08 \mathrm{E}-02$ & $1.08 \mathrm{E}+04$ & $9.37 \mathrm{E}+00$ & $\mathrm{ft}^{2} /$ day \\
\hline mcynftrmlt & $\mathrm{rch}$ & Small catchment recharge multiplier & $\begin{array}{l}\text { Small catchment recharge Mission Canyon area } \\
\text { Storage Unit I }\end{array}$ & $6.51 \mathrm{E}+00$ & $1.00 \mathrm{E}-05$ & $4.00 \mathrm{E}+01$ & $1.56 \mathrm{E}+01$ & - \\
\hline mcynrchmlt & $\mathrm{rch}$ & Small catchment recharge multiplier & $\begin{array}{l}\text { Small catchment recharge Mission Canyon area } \\
\text { Foothill subbasin }\end{array}$ & $1.26 \mathrm{E}+01$ & $1.00 \mathrm{E}-05$ & $4.00 \mathrm{E}+01$ & $2.06 \mathrm{E}+01$ & - \\
\hline mesa & faultk & Fault conductance & Mesa fault west of Anapamu St. & $2.08 \mathrm{E}-05$ & $1.00 \mathrm{E}-07$ & $1.00 \mathrm{E}+00$ & $6.08 \mathrm{E}-05$ & /day \\
\hline mesa_ab & faultk & Fault conductance & Mesa fault at Arroyo Burro Creek & $2.25 \mathrm{E}+00$ & $1.00 \mathrm{E}-07$ & $5.00 \mathrm{E}+00$ & $2.25 \mathrm{E}+00$ & /day \\
\hline
\end{tabular}


Table 3. Calibrated groundwater flow and solute transport model parameter values, Santa Barbara flow and transport model, Santa Barbara, California.-Continued

[/day, per day; ft, foot; $\mathrm{ft}^{2}$, square foot; GHB, general-head boundary package; HK, horizontal hydraulic conductivity; Rd., road; St., street; VK, vertical hydraulic conductivity; - , not applicable]

\begin{tabular}{|c|c|c|c|c|c|c|c|c|}
\hline $\begin{array}{l}\text { Parameter } \\
\text { name }\end{array}$ & Group & Subgroup & Description & $\begin{array}{l}\text { Calibrated } \\
\text { value }\end{array}$ & $\begin{array}{l}\text { Lower } \\
\text { bound }\end{array}$ & $\begin{array}{l}\text { Upper } \\
\text { bound }\end{array}$ & $\begin{array}{l}\text { Preliminary } \\
\text { value }\end{array}$ & Units \\
\hline mesa_lp & faultk & Fault conductance & Mesa fault at Las Positas Creek & $3.05 \mathrm{E}-01$ & $1.00 \mathrm{E}-07$ & $5.00 \mathrm{E}+00$ & $3.05 \mathrm{E}-01$ & /day \\
\hline mesal & faultk & Fault conductance & Mesa fault east of Anapamu St. & $5.08 \mathrm{E}-04$ & $1.00 \mathrm{E}-07$ & $1.00 \mathrm{E}+00$ & $5.08 \mathrm{E}-04$ & /day \\
\hline missinit & rivers & Creek stage adjustment parameter & $\begin{array}{l}\text { Base Creek water level above ground surface for all } \\
\text { creeks }\end{array}$ & 4.93E-01 & $3.28 \mathrm{E}-01$ & $6.56 \mathrm{E}+00$ & $2.65 \mathrm{E}-01$ & $\mathrm{ft}$ \\
\hline missnb_s & rivers & Creek stage adjustment parameter & $\begin{array}{l}\text { Upstream segment of Mission Creek near northern } \\
\text { boundary of model to just north of the Mission }\end{array}$ & $3.28 \mathrm{E}-05$ & $3.28 \mathrm{E}-06$ & $1.64 \mathrm{E}+00$ & $2.56 \mathrm{E}-05$ & $\mathrm{ft}$ \\
\hline missnc_s & rivers & Creek stage adjustment parameter & Mission Creek just north of the Mission to State St. & $2.53 \mathrm{E}-02$ & $3.28 \mathrm{E}-06$ & $1.64 \mathrm{E}+00$ & $2.56 \mathrm{E}-05$ & $\mathrm{ft}$ \\
\hline missnd_s & rivers & Creek stage adjustment parameter & $\begin{array}{l}\text { Mission Creek from State St. to where it runs } \\
\text { parallel to } 101 \text { freeway }\end{array}$ & $2.53 \mathrm{E}-02$ & $3.28 \mathrm{E}-06$ & $1.64 \mathrm{E}+00$ & $2.56 \mathrm{E}-05$ & $\mathrm{ft}$ \\
\hline missne_s & rivers & Creek stage adjustment parameter & $\begin{array}{l}\text { Mission Creek parallel to } 101 \text { freeway upstream of } \\
\text { Micheltorena St. }\end{array}$ & $2.53 \mathrm{E}-02$ & $3.28 \mathrm{E}-06$ & $1.64 \mathrm{E}+00$ & $2.56 \mathrm{E}-05$ & $\mathrm{ft}$ \\
\hline missnf_s & rivers & Creek stage adjustment parameter & $\begin{array}{l}\text { Mission Creek parallel to } 101 \text { freeway downstream } \\
\text { of Micheltorena St. }\end{array}$ & $2.53 \mathrm{E}-02$ & $3.28 \mathrm{E}-06$ & $1.64 \mathrm{E}+00$ & $2.56 \mathrm{E}-05$ & $\mathrm{ft}$ \\
\hline montecito & otherk & General head boundary conductance & Montecito GHB conductance shallow & $1.61 \mathrm{E}+01$ & $1.08 \mathrm{E}-05$ & $1.08 \mathrm{E}+04$ & $1.49 \mathrm{E}+02$ & $\mathrm{ft}^{2} /$ day \\
\hline montecitol & otherk & General head boundary conductance & Montecito GHB conductance deep & $1.61 \mathrm{E}+01$ & $1.08 \mathrm{E}-05$ & $1.08 \mathrm{E}+04$ & $1.49 \mathrm{E}+02$ & $\mathrm{ft}^{2} /$ day \\
\hline montecitom & otherk & General head boundary conductance & Montecito GHB conductance middle producing zone & $1.08 \mathrm{E}-02$ & $1.08 \mathrm{E}-05$ & $1.08 \mathrm{E}+04$ & $1.49 \mathrm{E}+02$ & $\mathrm{ft}^{2} /$ day \\
\hline $\mathrm{mpc}$ & fact & Mobile porosity end member & Shallow coarse & $1.00 \mathrm{E}-01$ & $5.00 \mathrm{E}-02$ & $3.50 \mathrm{E}-01$ & $3.50 \mathrm{E}-01$ & - \\
\hline mpclower & fact & Mobile porosity end member & Deep coarse & $7.00 \mathrm{E}-02$ & $5.00 \mathrm{E}-02$ & $3.50 \mathrm{E}-01$ & $7.00 \mathrm{E}-02$ & - \\
\hline mpcmid & fact & Mobile porosity end member & Middle coarse & $3.50 \mathrm{E}-01$ & $5.00 \mathrm{E}-02$ & $3.50 \mathrm{E}-01$ & $2.00 \mathrm{E}-01$ & - \\
\hline $\mathrm{mpf}$ & fact & Mobile porosity end member & Shallow fine & $5.00 \mathrm{E}-02$ & $1.00 \mathrm{E}-03$ & $1.50 \mathrm{E}-01$ & $5.78 \mathrm{E}-02$ & - \\
\hline mpflower & fact & Mobile porosity end member & Deep fine & $4.00 \mathrm{E}-02$ & $1.00 \mathrm{E}-03$ & $1.50 \mathrm{E}-01$ & $4.00 \mathrm{E}-02$ & - \\
\hline mpfmid & fact & Mobile porosity end member & Middle fine & $2.00 \mathrm{E}-01$ & $1.00 \mathrm{E}-03$ & $1.50 \mathrm{E}-01$ & $1.00 \mathrm{E}-01$ & - \\
\hline mtnrchmult & $\mathrm{rch}$ & Small catchment recharge multiplier & Small catchment recharge multiplier & $1.00 \mathrm{E}-05$ & $1.00 \mathrm{E}-05$ & $7.50 \mathrm{E}-01$ & $1.07 \mathrm{E}-01$ & - \\
\hline n_more & faultk & Fault conductance & North More Ranch fault & $1.12 \mathrm{E}-05$ & $1.00 \mathrm{E}-07$ & $1.00 \mathrm{E}+00$ & $4.71 \mathrm{E}-04$ & /day \\
\hline ne_more & faultk & Fault conductance & Northeast More Ranch fault north of Calle Real Rd. & $7.59 \mathrm{E}-04$ & $1.00 \mathrm{E}-07$ & $1.00 \mathrm{E}+00$ & $2.24 \mathrm{E}-04$ & /day \\
\hline ne_mores & faultk & Fault conductance & Northeast More Ranch fault south of Calle Real Rd. & $2.16 \mathrm{E}-04$ & $1.00 \mathrm{E}-07$ & $1.00 \mathrm{E}+00$ & $1.16 \mathrm{E}-04$ & /day \\
\hline norrb_s & rivers & Creek stage adjustment parameter & Northridge Creek & $4.87 \mathrm{E}-01$ & $3.28 \mathrm{E}-06$ & $4.92 \mathrm{E}+00$ & $2.26 \mathrm{E}-02$ & $\mathrm{ft}$ \\
\hline oceancond1 & otherk & General head boundary conductance & $\begin{array}{l}\text { Ocean GHB conductance upper confining layer } \\
\text { across offshore fault }\end{array}$ & $2.34 \mathrm{E}-04$ & $1.08 \mathrm{E}-05$ & $1.08 \mathrm{E}+04$ & $2.41 \mathrm{E}+03$ & $\mathrm{ft}^{2} /$ day \\
\hline oceancond2 & otherk & General head boundary conductance & $\begin{array}{l}\text { Ocean GHB conductance upper producing zone } \\
\text { across offshore fault }\end{array}$ & $1.14 \mathrm{E}-02$ & $1.08 \mathrm{E}-05$ & $1.08 \mathrm{E}+04$ & $2.17 \mathrm{E}+03$ & $\mathrm{ft}^{2} /$ day \\
\hline oceancond3 & otherk & General head boundary conductance & $\begin{array}{l}\text { Ocean GHB conductance middle confining layer } \\
\text { below middle producing zone across offshore fault }\end{array}$ & $1.63 \mathrm{E}-01$ & $1.08 \mathrm{E}-05$ & $1.08 \mathrm{E}+04$ & $9.95 \mathrm{E}-01$ & $\mathrm{ft}^{2} /$ day \\
\hline
\end{tabular}


Table 3. Calibrated groundwater flow and solute transport model parameter values, Santa Barbara flow and transport model, Santa Barbara, California._-Continued

[/day, per day; ft, foot; ft², square foot; GHB, general-head boundary package; HK, horizontal hydraulic conductivity; Rd., road; St., street; VK, vertical hydraulic conductivity; —, not applicable]

\begin{tabular}{|c|c|c|c|c|c|c|c|c|}
\hline $\begin{array}{l}\text { Parameter } \\
\text { name }\end{array}$ & Group & Subgroup & Description & $\begin{array}{l}\text { Calibrated } \\
\text { value }\end{array}$ & $\begin{array}{l}\text { Lower } \\
\text { bound }\end{array}$ & $\begin{array}{l}\text { Upper } \\
\text { bound }\end{array}$ & $\begin{array}{l}\text { Preliminary } \\
\text { value }\end{array}$ & Units \\
\hline oceancond4 & otherk & General head boundary conductance & $\begin{array}{l}\text { Ocean GHB conductance lower producing zone } \\
\text { across offshore fault }\end{array}$ & $1.94 \mathrm{E}+00$ & $1.08 \mathrm{E}-02$ & $1.08 \mathrm{E}+04$ & $8.00 \mathrm{E}-01$ & $\mathrm{ft}^{2} /$ day \\
\hline oceancond5 & otherk & General head boundary conductance & $\begin{array}{l}\text { Ocean GHB conductance lower confining layer } \\
\text { across offshore fault }\end{array}$ & $3.94 \mathrm{E}-05$ & $1.08 \mathrm{E}-05$ & $1.08 \mathrm{E}+04$ & 4.41E-05 & $\mathrm{ft}^{2} /$ day \\
\hline oceancond6 & otherk & General head boundary conductance & $\begin{array}{l}\text { Ocean GHB conductance middle producing zone } \\
\text { across offshore fault }\end{array}$ & $3.23 \mathrm{E}+02$ & $1.08 \mathrm{E}-03$ & $1.08 \mathrm{E}+04$ & $1.01 \mathrm{E}+00$ & $\mathrm{ft}^{2} /$ day \\
\hline oceancond7 & otherk & General head boundary conductance & $\begin{array}{l}\text { Ocean GHB conductance shallow zone across } \\
\text { offshore fault }\end{array}$ & $1.08 \mathrm{E}-01$ & $1.08 \mathrm{E}-03$ & $1.08 \mathrm{E}+04$ & $5.47 \mathrm{E}+02$ & $\mathrm{ft}^{2} /$ day \\
\hline oceanconds & otherk & General head boundary conductance & Ocean GHB conductance offshore ocean to ground & $1.55 \mathrm{E}+01$ & $1.08 \mathrm{E}-03$ & $1.08 \mathrm{E}+04$ & $1.62 \mathrm{E}+01$ & $\mathrm{ft}^{2} /$ day \\
\hline pcrivfac & aquiferk & HK/VK multiplier & $\begin{array}{l}\text { Multiplier for } \mathrm{HK} / \mathrm{VK} \text { at upper Mission Creek river } \\
\text { bed }\end{array}$ & $3.65 \mathrm{E}+01$ & $1.00 \mathrm{E}-01$ & $1.00 \mathrm{E}+02$ & $4.94 \mathrm{E}+01$ & - \\
\hline pow & fact & VK power function for end members & Power function exponent & -1.0 & -1.0 & $-1.00 \mathrm{E}-02$ & - & - \\
\hline rainrchlabs & $\mathrm{rch}$ & Areal recharge parameter & Rain recharge for infiltration zone 1 & $7.22 \mathrm{E}-05$ & $3.28 \mathrm{E}-05$ & $3.28 \mathrm{E}+00$ & $7.61 \mathrm{E}-03$ & $\mathrm{ft} /$ day \\
\hline rainrch2add & $\mathrm{rch}$ & Areal recharge parameter & Additional rain recharge for infiltration zone 2 & $3.28 \mathrm{E}-03$ & $3.28 \mathrm{E}-03$ & $3.28 \mathrm{E}+00$ & $1.93 \mathrm{E}-02$ & $\mathrm{ft} /$ day \\
\hline rainrch3add & $\mathrm{rch}$ & Areal recharge parameter & Additional rain recharge for infiltration zone 3 & $3.30 \mathrm{E}-03$ & $3.28 \mathrm{E}-03$ & $3.28 \mathrm{E}+00$ & $7.89 \mathrm{E}-03$ & $\mathrm{ft} /$ day \\
\hline rainrch4add & $\mathrm{rch}$ & Areal recharge parameter & Additional rain recharge for infiltration zone 4 & $1.03 \mathrm{E}-02$ & $3.28 \mathrm{E}-03$ & $3.28 \mathrm{E}+00$ & $1.08 \mathrm{E}+00$ & $\mathrm{ft} /$ day \\
\hline sanroque_t & streamk & $\begin{array}{l}\text { Creek conductance adjustment } \\
\text { parameter }\end{array}$ & San Roque Creek & $1.01 \mathrm{E}-03$ & $1.08 \mathrm{E}-04$ & $1.08 \mathrm{E}+04$ & $4.30 \mathrm{E}+01$ & $\mathrm{ft}^{2} /$ day \\
\hline sanrql_s & rivers & Creek stage adjustment parameter & San Roque Creek & $2.04 \mathrm{E}-01$ & $3.28 \mathrm{E}-06$ & $4.92 \mathrm{E}+00$ & $5.39 \mathrm{E}-02$ & $\mathrm{ft}$ \\
\hline scynrchmlt & $\mathrm{rch}$ & Small catchment recharge multiplier & Small catchment recharge Sycamore Canyon area & $1.41 \mathrm{E}+00$ & $1.00 \mathrm{E}-05$ & $2.00 \mathrm{E}+01$ & $5.42 \mathrm{E}+00$ & - \\
\hline septicrchabs & $\mathrm{rch}$ & Septic recharge parameter & Septic recharge & $7.58 \mathrm{E}-04$ & $3.28 \mathrm{E}-10$ & $8.20 \mathrm{E}-03$ & $3.28 \mathrm{E}-03$ & $\mathrm{ft} / \mathrm{day}$ \\
\hline $\operatorname{sscstcs}$ & ss & Specific storage end member & Specific storage coarse end member coastal area & $1.05 \mathrm{E}-05$ & $10.00 \mathrm{E}-07$ & $10.00 \mathrm{E}-04$ & $1.21 \mathrm{E}-05$ & $/ \mathrm{ft}$ \\
\hline sscstfn & ss & Specific storage end member & Specific storage fine end member coastal area & $2.40 \mathrm{E}-04$ & $10.00 \mathrm{E}-07$ & $10.00 \mathrm{E}-02$ & $3.46 \mathrm{E}-04$ & $/ \mathrm{ft}$ \\
\hline sszon10cs & ss & Specific storage end member & Specific storage coarse end member subregion 10 & $1.91 \mathrm{E}-06$ & $10.00 \mathrm{E}-07$ & $10.00 \mathrm{E}-04$ & $10.00 \mathrm{E}-07$ & $/ \mathrm{ft}$ \\
\hline sszon10fn & ss & Specific storage end member & Specific storage fine end member subregion 10 & $3.79 \mathrm{E}-05$ & $10.00 \mathrm{E}-07$ & $10.00 \mathrm{E}-02$ & $3.95 \mathrm{E}-05$ & $/ \mathrm{ft}$ \\
\hline sszonlacs & ss & Specific storage end member & $\begin{array}{l}\text { Specific storage coarse end member subregions } 1,8 \text {, } \\
\text { and } 11\end{array}$ & $7.38 \mathrm{E}-07$ & $10.00 \mathrm{E}-07$ & $10.00 \mathrm{E}-04$ & $1.96 \mathrm{E}-06$ & $/ \mathrm{ft}$ \\
\hline sszon1afn & ss & Specific storage end member & $\begin{array}{l}\text { Specific storage fine end member subregions } 1,8 \text {, } \\
\text { and } 11\end{array}$ & $7.76 \mathrm{E}-06$ & $10.00 \mathrm{E}-07$ & $10.00 \mathrm{E}-02$ & $4.69 \mathrm{E}-06$ & $/ \mathrm{ft}$ \\
\hline sszonlbcs & ss & Specific storage end member & Specific storage coarse end member subregion 6 & $2.51 \mathrm{E}-06$ & $10.00 \mathrm{E}-07$ & $10.00 \mathrm{E}-04$ & $1.30 \mathrm{E}-06$ & $/ \mathrm{ft}$ \\
\hline sszon1bfn & ss & Specific storage end member & Specific storage fine end member subregion 6 & $2.91 \mathrm{E}-05$ & $10.00 \mathrm{E}-07$ & $10.00 \mathrm{E}-02$ & $1.49 \mathrm{E}-05$ & $/ \mathrm{ft}$ \\
\hline sszon1ces & ss & Specific storage end member & Specific storage coarse end member subregion 7 & $3.05 \mathrm{E}-07$ & $3.05 \mathrm{E}-07$ & $10.00 \mathrm{E}-04$ & $3.05 \mathrm{E}-07$ & $/ \mathrm{ft}$ \\
\hline sszon $1 \mathrm{cfn}$ & ss & Specific storage end member & Specific storage fine end member subregion 7 & $10.00 \mathrm{E}-07$ & $10.00 \mathrm{E}-07$ & $10.00 \mathrm{E}-02$ & $1.02 \mathrm{E}-06$ & $/ \mathrm{ft}$ \\
\hline
\end{tabular}


Table 3. Calibrated groundwater flow and solute transport model parameter values, Santa Barbara flow and transport model, Santa Barbara, California.-Continued

[/day, per day; ft, foot; $\mathrm{ft}^{2}$, square foot; GHB, general-head boundary package; HK, horizontal hydraulic conductivity; Rd., road; St., street; VK, vertical hydraulic conductivity; —, not applicable]

\begin{tabular}{|c|c|c|c|c|c|c|c|c|}
\hline $\begin{array}{l}\text { Parameter } \\
\text { name }\end{array}$ & Group & Subgroup & Description & $\begin{array}{l}\text { Calibrated } \\
\text { value }\end{array}$ & $\begin{array}{l}\text { Lower } \\
\text { bound }\end{array}$ & $\begin{array}{l}\text { Upper } \\
\text { bound }\end{array}$ & $\begin{array}{l}\text { Preliminary } \\
\text { value }\end{array}$ & Units \\
\hline sszon $2 \mathrm{cs}$ & ss & Specific storage end member & $\begin{array}{l}\text { Specific storage coarse end member subregions } 2 \\
\text { and } 9\end{array}$ & 4.19E-07 & $3.05 \mathrm{E}-07$ & $10.00 \mathrm{E}-04$ & $2.00 \mathrm{E}-05$ & $/ \mathrm{ft}$ \\
\hline sszon $2 \mathrm{fn}$ & ss & Specific storage end member & Specific storage fine end member subregions 2 and 9 & $2.57 \mathrm{E}-05$ & $10.00 \mathrm{E}-07$ & $10.00 \mathrm{E}-02$ & $5.91 \mathrm{E}-04$ & $/ \mathrm{ft}$ \\
\hline sszon $3 \mathrm{cs}$ & ss & Specific storage end member & $\begin{array}{l}\text { Specific storage coarse end member subregions } 3 \\
\text { and } 13\end{array}$ & $3.90 \mathrm{E}-07$ & $3.90 \mathrm{E}-07$ & $10.00 \mathrm{E}-04$ & $4.23 \mathrm{E}-07$ & $/ \mathrm{ft}$ \\
\hline sszon3fn & ss & Specific storage end member & $\begin{array}{l}\text { Specific storage fine end member subregions } 3 \text { and } \\
13\end{array}$ & $1.01 \mathrm{E}-04$ & $10.00 \mathrm{E}-07$ & $10.00 \mathrm{E}-02$ & $1.05 \mathrm{E}-04$ & $/ \mathrm{ft}$ \\
\hline sszon $4 \mathrm{cs}$ & ss & Specific storage end member & $\begin{array}{l}\text { Specific storage coarse end member subregions } 4 \\
\text { and } 14\end{array}$ & $2.35 \mathrm{E}-05$ & $10.00 \mathrm{E}-07$ & $10.00 \mathrm{E}-04$ & $1.36 \mathrm{E}-06$ & $/ \mathrm{ft}$ \\
\hline sszon4fn & ss & Specific storage end member & $\begin{array}{l}\text { Specific storage fine end member subregions } 4 \text { and } \\
14\end{array}$ & $1.29 \mathrm{E}-04$ & $10.00 \mathrm{E}-07$ & $10.00 \mathrm{E}-02$ & $3.19 \mathrm{E}-05$ & $/ \mathrm{ft}$ \\
\hline sszon $5 \mathrm{cs}$ & ss & Specific storage end member & Specific storage coarse end member subregion 5 & $10.00 \mathrm{E}-07$ & $10.00 \mathrm{E}-07$ & $10.00 \mathrm{E}-04$ & $10.00 \mathrm{E}-07$ & $/ \mathrm{ft}$ \\
\hline sszon5fn & ss & Specific storage end member & Specific storage fine end member subregion 5 & $2.52 \mathrm{E}-05$ & $10.00 \mathrm{E}-07$ & $10.00 \mathrm{E}-02$ & $2.49 \mathrm{E}-05$ & $/ \mathrm{ft}$ \\
\hline sszonpmpcs & ss & Specific storage end member & Specific storage coarse end member subregion 12 & $1.60 \mathrm{E}-06$ & $3.90 \mathrm{E}-07$ & $10.00 \mathrm{E}-04$ & $9.87 \mathrm{E}-06$ & $/ \mathrm{ft}$ \\
\hline sszonpmpfn & Ss & Specific storage end member & Specific storage fine end member subregion 12 & $1.69 \mathrm{E}-05$ & $10.00 \mathrm{E}-07$ & $10.00 \mathrm{E}-04$ & $4.67 \mathrm{E}-05$ & $/ \mathrm{ft}$ \\
\hline strcondinit & streamk & $\begin{array}{l}\text { Creek conductance adjustment } \\
\text { parameter }\end{array}$ & Base Creek conductance for all creeks & $8.19 \mathrm{E}+00$ & $1.08 \mathrm{E}-01$ & $1.08 \mathrm{E}+02$ & $2.15 \mathrm{E}-01$ & $\mathrm{ft}^{2} /$ day \\
\hline sufrlhk & aquiferk & HK/VK regional multiplier & Storage Unit Four lower HK & 1 & 0.001 & 100 & 1 & - \\
\hline sufrlvk & aquiferk & HK/VK regional multiplier & Storage Unit Four lower VK & 0.001 & 0.001 & 100 & 0.001 & - \\
\hline sufrrchmlt & $\mathrm{rch}$ & Small catchment recharge multiplier & $\begin{array}{l}\text { Small catchment recharge multiplier West Foothill } \\
\text { Subbasin }\end{array}$ & $3.03 \mathrm{E}-01$ & $1.00 \mathrm{E}-05$ & $2.50 \mathrm{E}+00$ & $2.03 \mathrm{E}-01$ & - \\
\hline suoneadt & aquiferk & HK/VK regional/layer multiplier & Subregion 1 lower confining layer & $1.00 \mathrm{E}+03$ & $1.00 \mathrm{E}-03$ & $1.00 \mathrm{E}+05$ & $1.00 \mathrm{E}+03$ & - \\
\hline suoneahkm & aquiferk & HK/VK regional multiplier & Subregion $1 \mathrm{HK}$ & $3.41 \mathrm{E}+00$ & $1.00 \mathrm{E}-03$ & $1.00 \mathrm{E}+01$ & $8.00 \mathrm{E}-01$ & - \\
\hline suonealp & aquiferk & HK/VK regional/layer multiplier & Subregion 1 lower producing zone & $2.00 \mathrm{E}+00$ & $1.00 \mathrm{E}-03$ & $1.00 \mathrm{E}+01$ & $2.00 \mathrm{E}+00$ & - \\
\hline suoneamp & aquiferk & HK/VK regional/layer multiplier & Subregion 1 middle producing zone & $1.00 \mathrm{E}+00$ & $1.00 \mathrm{E}-03$ & $1.00 \mathrm{E}+01$ & $1.00 \mathrm{E}+00$ & - \\
\hline suoneaup & aquiferk & HK/VK regional/layer multiplier & Subregion 1 upper producing zone & $1.00 \mathrm{E}+00$ & $1.00 \mathrm{E}-03$ & $1.00 \mathrm{E}+01$ & $1.00 \mathrm{E}+00$ & - \\
\hline suoneauth & aquiferk & HK/VK regional/layer multiplier & Subregion 1 upper confining layer HK & $1.00 \mathrm{E}-02$ & $1.00 \mathrm{E}-03$ & $1.00 \mathrm{E}+02$ & $1.00 \mathrm{E}-02$ & - \\
\hline suoneautv & aquiferk & HK/VK regional/layer multiplier & Subregion 1 upper confining layer VK & $1.00 \mathrm{E}+00$ & $1.00 \mathrm{E}-03$ & $1.00 \mathrm{E}+02$ & $5.00 \mathrm{E}+01$ & - \\
\hline suoneavkm & aquiferk & $\mathrm{HK} / \mathrm{VK}$ regional multiplier & Subregion $1 \mathrm{VK}$ & $9.91 \mathrm{E}-01$ & $1.00 \mathrm{E}-03$ & $1.00 \mathrm{E}+01$ & $9.99 \mathrm{E}-01$ & - \\
\hline suonebdt & aquiferk & HK/VK regional/layer multiplier & Subregion 6 lower confining layer & $1.00 \mathrm{E}+03$ & $1.00 \mathrm{E}-03$ & $1.00 \mathrm{E}+05$ & $1.00 \mathrm{E}+03$ & - \\
\hline suonebhkm & aquiferk & HK/VK regional multiplier & Subregion $6 \mathrm{HK}$ & $9.52 \mathrm{E}-01$ & $1.00 \mathrm{E}-03$ & $1.00 \mathrm{E}+01$ & $1.38 \mathrm{E}+00$ & - \\
\hline suoneblp & aquiferk & HK/VK regional/layer multiplier & Subregion 6 lower producing zone & $8.00 \mathrm{E}-01$ & $1.00 \mathrm{E}-03$ & $1.00 \mathrm{E}+01$ & $1.00 \mathrm{E}+00$ & - \\
\hline suonebmp & aquiferk & HK/VK regional/layer multiplier & Subregion 6 middle producing zone & $1.00 \mathrm{E}+00$ & $1.00 \mathrm{E}-03$ & $1.00 \mathrm{E}+01$ & $1.00 \mathrm{E}+00$ & - \\
\hline
\end{tabular}


Table 3. Calibrated groundwater flow and solute transport model parameter values, Santa Barbara flow and transport model, Santa Barbara, California.-Continued

[/day, per day; ft, foot; $\mathrm{ft}^{2}$, square foot; GHB, general-head boundary package; HK, horizontal hydraulic conductivity; Rd., road; St., street; VK, vertical hydraulic conductivity; —, not applicable]

\begin{tabular}{|c|c|c|c|c|c|c|c|c|}
\hline $\begin{array}{c}\text { Parameter } \\
\text { name }\end{array}$ & Group & Subgroup & Description & $\begin{array}{c}\text { Calibrated } \\
\text { value }\end{array}$ & $\begin{array}{l}\text { Lower } \\
\text { bound }\end{array}$ & $\begin{array}{l}\text { Upper } \\
\text { bound }\end{array}$ & $\begin{array}{c}\text { Preliminary } \\
\text { value }\end{array}$ & Units \\
\hline suonebup & aquiferk & $\mathrm{HK} / \mathrm{VK}$ regional/layer multiplier & Subregion 6 upper producing zone & $3.00 \mathrm{E}-01$ & $1.00 \mathrm{E}-03$ & $1.00 \mathrm{E}+01$ & $3.00 \mathrm{E}-01$ & - \\
\hline suonebuth & aquiferk & $\mathrm{HK} / \mathrm{VK}$ regional/layer multiplier & Subregion 6 upper confining layer HK & $1.00 \mathrm{E}+00$ & $1.00 \mathrm{E}-03$ & $1.00 \mathrm{E}+02$ & $1.00 \mathrm{E}+00$ & - \\
\hline suonebutv & aquiferk & HK/VK regional/layer multiplier & Subregion 6 upper confining layer VK & $1.00 \mathrm{E}+00$ & $1.00 \mathrm{E}-03$ & $1.00 \mathrm{E}+02$ & $1.00 \mathrm{E}+00$ & - \\
\hline suonebvkm & aquiferk & HK/VK regional multiplier & Subregion $6 \mathrm{VK}$ & $1.08 \mathrm{E}+00$ & $1.00 \mathrm{E}-03$ & $1.00 \mathrm{E}+01$ & $1.11 \mathrm{E}+00$ & - \\
\hline suonecdt & aquiferk & HK/VK regional/layer multiplier & Subregion 7 lower confining layer & $1.00 \mathrm{E}+03$ & $1.00 \mathrm{E}-03$ & $1.00 \mathrm{E}+05$ & $1.00 \mathrm{E}+03$ & - \\
\hline suonechkm & aquiferk & HK/VK regional multiplier & Subregion $7 \mathrm{HK}$ & $1.29 \mathrm{E}+00$ & $1.00 \mathrm{E}-03$ & $1.00 \mathrm{E}+01$ & $1.50 \mathrm{E}+00$ & - \\
\hline suoneclp & aquiferk & HK/VK regional/layer multiplier & Subregion 7 lower producing zone & $6.00 \mathrm{E}-02$ & $1.00 \mathrm{E}-03$ & $1.00 \mathrm{E}+01$ & $1.00 \mathrm{E}-01$ & - \\
\hline suonecmp & aquiferk & HK/VK regional/layer multiplier & Subregion 7 middle producing zone & $1.20 \mathrm{E}-01$ & $1.00 \mathrm{E}-03$ & $1.00 \mathrm{E}+01$ & $1.00 \mathrm{E}-01$ & - \\
\hline suonecup & aquiferk & HK/VK regional/layer multiplier & Subregion 7 upper producing zone & $1.60 \mathrm{E}+00$ & $1.00 \mathrm{E}-03$ & $1.00 \mathrm{E}+01$ & $1.60 \mathrm{E}+00$ & - \\
\hline suonecuth & aquiferk & HK/VK regional/layer multiplier & Subregion 7 upper confining layer HK & $1.00 \mathrm{E}+00$ & $1.00 \mathrm{E}-03$ & $1.00 \mathrm{E}+02$ & $1.00 \mathrm{E}+00$ & - \\
\hline suonecutv & aquiferk & HK/VK regional/layer multiplier & Subregion 7 upper confining layer VK & $1.00 \mathrm{E}+00$ & $1.00 \mathrm{E}-03$ & $1.00 \mathrm{E}+02$ & $1.00 \mathrm{E}+00$ & - \\
\hline suonecvkm & aquiferk & HK/VK regional multiplier & Subregion $7 \mathrm{VK}$ & $1.35 \mathrm{E}+00$ & $1.00 \mathrm{E}-03$ & $1.00 \mathrm{E}+01$ & $1.27 \mathrm{E}+00$ & - \\
\hline suonemchk & aquiferk & HK/VK regional multiplier & Subregion $8 \mathrm{HK}$ & $6.71 \mathrm{E}+00$ & $1.00 \mathrm{E}-03$ & $1.00 \mathrm{E}+02$ & $4.68 \mathrm{E}+00$ & - \\
\hline suonemcvk & aquiferk & HK/VK regional multiplier & Subregion $8 \mathrm{VK}$ & $6.07 \mathrm{E}+00$ & $1.00 \mathrm{E}-03$ & $1.00 \mathrm{E}+02$ & $4.87 \mathrm{E}+00$ & - \\
\hline suonemtlp & aquiferk & $\mathrm{HK} / \mathrm{VK}$ regional/layer multiplier & Subregion 8 lower producing zone & $1.00 \mathrm{E}+00$ & $1.00 \mathrm{E}-03$ & $1.00 \mathrm{E}+01$ & $1.00 \mathrm{E}-01$ & - \\
\hline suonenehk & aquiferk & HK/VK regional multiplier & Subregion $6 \mathrm{HK}$ & $1.11 \mathrm{E}+00$ & $1.00 \mathrm{E}-03$ & $1.00 \mathrm{E}+02$ & $1.40 \mathrm{E}+00$ & - \\
\hline suonenevk & aquiferk & HK/VK regional multiplier & Subregion $6 \mathrm{VK}$ & $9.16 \mathrm{E}+00$ & $1.00 \mathrm{E}-03$ & $1.00 \mathrm{E}+02$ & $1.04 \mathrm{E}+01$ & - \\
\hline suonenwhk & aquiferk & HK/VK regional multiplier & Subregion $10 \mathrm{HK}$ & $1.00 \mathrm{E}-01$ & $1.00 \mathrm{E}-03$ & $1.00 \mathrm{E}+02$ & $1.00 \mathrm{E}-01$ & - \\
\hline suonenwvk & aquiferk & HK/VK regional multiplier & Subregion $10 \mathrm{VK}$ & $1.00 \mathrm{E}-01$ & $1.00 \mathrm{E}-03$ & $1.00 \mathrm{E}+02$ & $1.00 \mathrm{E}-01$ & - \\
\hline suonepdt & aquiferk & HK/VK regional/layer multiplier & Subregion 12 lower confining layer & $1.00 \mathrm{E}+03$ & $1.00 \mathrm{E}-03$ & $1.00 \mathrm{E}+05$ & $1.00 \mathrm{E}+03$ & - \\
\hline suoneplp & aquiferk & HK/VK regional/layer multiplier & Subregion 12 lower producing zone & $1.40 \mathrm{E}+00$ & $1.00 \mathrm{E}-03$ & $1.00 \mathrm{E}+01$ & $1.40 \mathrm{E}+00$ & - \\
\hline suonepmp & aquiferk & $\mathrm{HK} / \mathrm{VK}$ regional/layer multiplier & Subregion 12 middle producing zone & $2.00 \mathrm{E}+00$ & $1.00 \mathrm{E}-03$ & $1.00 \mathrm{E}+01$ & $1.40 \mathrm{E}+00$ & - \\
\hline suonepumphk & aquiferk & HK/VK regional multiplier & Subregion $12 \mathrm{HK}$ & $8.99 \mathrm{E}+00$ & $1.00 \mathrm{E}-03$ & $1.00 \mathrm{E}+01$ & $8.99 \mathrm{E}+00$ & - \\
\hline suonepumpvk & aquiferk & HK/VK regional multiplier & Subregion $12 \mathrm{VK}$ & $1.08 \mathrm{E}+00$ & $1.00 \mathrm{E}-03$ & $1.00 \mathrm{E}+01$ & $1.08 \mathrm{E}+00$ & - \\
\hline suonepup & aquiferk & $\mathrm{HK} / \mathrm{VK}$ regional/layer multiplier & Subregion 12 upper producing zone & $1.00 \mathrm{E}+00$ & $1.00 \mathrm{E}-03$ & $1.00 \mathrm{E}+01$ & $1.00 \mathrm{E}+00$ & - \\
\hline suoneputh & aquiferk & $\mathrm{HK} / \mathrm{VK}$ regional/layer multiplier & Subregion 12 upper confining layer HK & $1.00 \mathrm{E}+00$ & $1.00 \mathrm{E}-03$ & $1.00 \mathrm{E}+02$ & $1.00 \mathrm{E}+00$ & - \\
\hline suoneputv & aquiferk & HK/VK regional/layer multiplier & Subregion 12 upper confining layer VK & $1.00 \mathrm{E}+00$ & $1.00 \mathrm{E}-03$ & $1.00 \mathrm{E}+02$ & $1.00 \mathrm{E}+00$ & - \\
\hline suonerchmlt & $\operatorname{rch}$ & Small catchment recharge multiplier & Small catchment recharge multiplier Storage Unit I & $9.03 \mathrm{E}-01$ & $1.00 \mathrm{E}-05$ & $2.50 \mathrm{E}+00$ & $1.99 \mathrm{E}+00$ & - \\
\hline suonezegt & $\mathrm{rch}$ & Layer pumping adjustment factor & $\begin{array}{l}\text { Storage Unit I pumping adjustment factor middle } \\
\text { confining layer below middle producing zone }\end{array}$ & $1.00 \mathrm{E}-04$ & $1.00 \mathrm{E}-04$ & $1.00 \mathrm{E}+01$ & $2.20 \mathrm{E}-04$ & - \\
\hline suonezetn & $\mathrm{rch}$ & Layer pumping adjustment factor & $\begin{array}{l}\text { Storage Unit I pumping adjustment factor confining } \\
\text { layer immediately above lower producing zone }\end{array}$ & $1.00 \mathrm{E}-04$ & $1.00 \mathrm{E}-04$ & $1.00 \mathrm{E}+01$ & $1.00 \mathrm{E}-04$ & - \\
\hline
\end{tabular}


Table 3. Calibrated groundwater flow and solute transport model parameter values, Santa Barbara flow and transport model, Santa Barbara, California.-Continued

[/day, per day; ft, foot; $\mathrm{ft}^{2}$, square foot; GHB, general-head boundary package; HK, horizontal hydraulic conductivity; Rd., road; St., street; VK, vertical hydraulic conductivity; - , not applicable]

\begin{tabular}{|c|c|c|c|c|c|c|c|c|}
\hline $\begin{array}{l}\text { Parameter } \\
\text { name }\end{array}$ & Group & Subgroup & Description & $\begin{array}{l}\text { Calibrated } \\
\text { value }\end{array}$ & $\begin{array}{l}\text { Lower } \\
\text { bound }\end{array}$ & $\begin{array}{l}\text { Upper } \\
\text { bound }\end{array}$ & $\begin{array}{l}\text { Preliminary } \\
\text { value }\end{array}$ & Units \\
\hline suonezfive & $\mathrm{rch}$ & Layer pumping adjustment factor & $\begin{array}{l}\text { Storage Unit I pumping adjustment factor lower } \\
\text { confining layer }\end{array}$ & $1.00 \mathrm{E}-04$ & $1.00 \mathrm{E}-04$ & $1.00 \mathrm{E}+01$ & $1.00 \mathrm{E}-04$ & - \\
\hline suonezfour & $\mathrm{rch}$ & Layer pumping adjustment factor & $\begin{array}{l}\text { Storage Unit I pumping adjustment factor lower } \\
\text { producing zone }\end{array}$ & $3.99 \mathrm{E}+00$ & $1.00 \mathrm{E}-04$ & $1.00 \mathrm{E}+01$ & $4.09 \mathrm{E}+00$ & - \\
\hline suonezhdrd & $\mathrm{rch}$ & Layer pumping adjustment factor & $\begin{array}{l}\text { Storage Unit I pumping adjustment factor confining } \\
\text { layer immediately above upper producing zone }\end{array}$ & $1.00 \mathrm{E}-04$ & $1.00 \mathrm{E}-04$ & $1.00 \mathrm{E}+01$ & $1.00 \mathrm{E}-04$ & - \\
\hline suoneznine & $\mathrm{rch}$ & Layer pumping adjustment factor & $\begin{array}{l}\text { Storage Unit I pumping adjustment factor soil } \\
\text { horizon confining layer }\end{array}$ & $1.00 \mathrm{E}-04$ & $1.00 \mathrm{E}-04$ & $1.00 \mathrm{E}+01$ & $1.00 \mathrm{E}-04$ & - \\
\hline suonezone & $\mathrm{rch}$ & Layer pumping adjustment factor & $\begin{array}{l}\text { Storage Unit I pumping adjustment factor upper } \\
\text { confining layer }\end{array}$ & $1.00 \mathrm{E}-05$ & $1.00 \mathrm{E}-05$ & $1.00 \mathrm{E}+01$ & $1.00 \mathrm{E}-05$ & - \\
\hline suonezsix & $\mathrm{rch}$ & Layer pumping adjustment factor & $\begin{array}{l}\text { Storage Unit I pumping adjustment factor middle } \\
\text { producing zone }\end{array}$ & $4.13 \mathrm{E}+00$ & $1.00 \mathrm{E}-02$ & $1.00 \mathrm{E}+01$ & $1.10 \mathrm{E}+00$ & - \\
\hline suonezsvn & $\mathrm{rch}$ & Layer pumping adjustment factor & $\begin{array}{l}\text { Storage Unit I pumping adjustment factor confining } \\
\text { layer immediately below middle producing zone }\end{array}$ & $1.00 \mathrm{E}-04$ & $1.00 \mathrm{E}-04$ & $1.00 \mathrm{E}+01$ & $1.00 \mathrm{E}-04$ & - \\
\hline suonezthree & $\mathrm{rch}$ & Layer pumping adjustment factor & $\begin{array}{l}\text { Storage Unit I pumping adjustment factor middle } \\
\text { confining layer above middle producing zone }\end{array}$ & $1.00 \mathrm{E}-01$ & $1.00 \mathrm{E}-04$ & $1.00 \mathrm{E}+01$ & $1.00 \mathrm{E}-04$ & - \\
\hline suoneztwo & $\mathrm{rch}$ & Layer pumping adjustment factor & $\begin{array}{l}\text { Storage Unit I pumping adjustment factor upper } \\
\text { producing zone }\end{array}$ & $3.25 \mathrm{E}+00$ & $1.00 \mathrm{E}-02$ & $1.00 \mathrm{E}+01$ & $2.10 \mathrm{E}+00$ & - \\
\hline suthehk & aquiferk & HK/VK regional multiplier & Subregion $13 \mathrm{HK}$ & $1.00 \mathrm{E}-01$ & $1.00 \mathrm{E}-03$ & $1.00 \mathrm{E}+02$ & $1.00 \mathrm{E}-01$ & - \\
\hline suthevk & aquiferk & HK/VK regional multiplier & Subregion $13 \mathrm{VK}$ & $1.00 \mathrm{E}-01$ & $1.00 \mathrm{E}-03$ & $1.00 \mathrm{E}+02$ & $1.00 \mathrm{E}-01$ & - \\
\hline suttfzfive & $\mathrm{rch}$ & Layer pumping adjustment factor & $\begin{array}{l}\text { Storage Unit III and Foothill Basin pumping } \\
\text { adjustment factor lower confining layer }\end{array}$ & $1.00 \mathrm{E}+00$ & $1.00 \mathrm{E}-01$ & $1.00 \mathrm{E}+01$ & $4.38 \mathrm{E}-01$ & - \\
\hline suttfzfour & rch & Layer pumping adjustment factor & $\begin{array}{l}\text { Storage Unit III and Foothill Basin pumping } \\
\text { adjustment factor lower producing zone }\end{array}$ & $1.10 \mathrm{E}+01$ & $1.00 \mathrm{E}-01$ & $1.00 \mathrm{E}+01$ & $2.01 \mathrm{E}+00$ & - \\
\hline suttfzone & $\mathrm{rch}$ & Layer pumping adjustment factor & $\begin{array}{l}\text { Storage Unit III and Foothill Basin pumping } \\
\text { adjustment factor upper confining layer }\end{array}$ & $6.04 \mathrm{E}-01$ & $1.00 \mathrm{E}-01$ & $1.00 \mathrm{E}+01$ & $4.70 \mathrm{E}-01$ & - \\
\hline suttfzthree & $\mathrm{rch}$ & Layer pumping adjustment factor & $\begin{array}{l}\text { Storage Unit III and Foothill Basin pumping } \\
\text { adjustment factor middle confining layer }\end{array}$ & $1.00 \mathrm{E}+00$ & $1.00 \mathrm{E}-01$ & $1.00 \mathrm{E}+01$ & $4.05 \mathrm{E}-01$ & - \\
\hline suttfztwo & $\mathrm{rch}$ & Layer pumping adjustment factor & $\begin{array}{l}\text { Storage Unit III and Foothill Basin pumping } \\
\text { adjustment factor upper producing zone }\end{array}$ & $3.02 \mathrm{E}+00$ & $1.00 \mathrm{E}-01$ & $1.00 \mathrm{E}+01$ & $1.05 \mathrm{E}+00$ & - \\
\hline sutwolp & aquiferk & HK/VK regional/layer multiplier & Subregion 2 lower producing zone & $1.00 \mathrm{E}+00$ & $1.00 \mathrm{E}-03$ & $1.00 \mathrm{E}+01$ & $1.00 \mathrm{E}+00$ & - \\
\hline sutwomchk & aquiferk & HK/VK regional multiplier & Subregion $9 \mathrm{HK}$ & $2.08 \mathrm{E}+01$ & $1.00 \mathrm{E}-03$ & $1.00 \mathrm{E}+02$ & $2.96 \mathrm{E}+01$ & - \\
\hline sutwomcvk & aquiferk & HK/VK regional multiplier & Subregion $9 \mathrm{VK}$ & $2.13 \mathrm{E}+00$ & $1.00 \mathrm{E}-03$ & $1.00 \mathrm{E}+02$ & $3.04 \mathrm{E}+00$ & - \\
\hline sutwouth & aquiferk & HK/VK regional/layer multiplier & Subregion 2 upper confining layer HK & $1.00 \mathrm{E}-03$ & $1.00 \mathrm{E}-03$ & $1.00 \mathrm{E}+02$ & $1.00 \mathrm{E}-03$ & - \\
\hline sutwoutv & aquiferk & HK/VK regional/layer multiplier & Subregion 2 lower confining layer VK & $2.00 \mathrm{E}+00$ & $1.00 \mathrm{E}-03$ & $1.00 \mathrm{E}+02$ & $2.00 \mathrm{E}+00$ & - \\
\hline
\end{tabular}


Table 3. Calibrated groundwater flow and solute transport model parameter values, Santa Barbara flow and transport model, Santa Barbara, California._-Continued

[/day, per day; ft, foot; $\mathrm{ft}^{2}$, square foot; GHB, general-head boundary package; HK, horizontal hydraulic conductivity; Rd., road; St., street; VK, vertical hydraulic conductivity; —, not applicable]

\begin{tabular}{|c|c|c|c|c|c|c|c|c|}
\hline $\begin{array}{l}\text { Parameter } \\
\text { name }\end{array}$ & Group & Subgroup & Description & $\begin{array}{l}\text { Calibrated } \\
\text { value }\end{array}$ & $\begin{array}{l}\text { Lower } \\
\text { bound }\end{array}$ & $\begin{array}{l}\text { Upper } \\
\text { bound }\end{array}$ & $\begin{array}{l}\text { Preliminary } \\
\text { value }\end{array}$ & Units \\
\hline sycacl_s & rivers & Creek stage adjustment parameter & Sycamore Canyon Creek & $4.61 \mathrm{E}-01$ & $3.28 \mathrm{E}-06$ & $3.28 \mathrm{E}+00$ & $6.07 \mathrm{E}-02$ & $\mathrm{ft}$ \\
\hline sycamore_t & streamk & $\begin{array}{l}\text { Creek conductance adjustment } \\
\text { parameter }\end{array}$ & Sycamore Canyon Creek & $1.07 \mathrm{E}+00$ & $1.00 \mathrm{E}-05$ & $1.00 \mathrm{E}+03$ & $8.97 \mathrm{E}-01$ & - \\
\hline sycoast & ss & Specific yield simulated value & Specific yield coastal area & $1.60 \mathrm{E}-01$ & $5.00 \mathrm{E}-02$ & $3.50 \mathrm{E}-01$ & $1.95 \mathrm{E}-01$ & - \\
\hline syzon 10 & ss & Specific yield simulated value & Specific yield subregion 10 & $1.79 \mathrm{E}-01$ & $5.00 \mathrm{E}-02$ & $3.50 \mathrm{E}-01$ & $1.97 \mathrm{E}-01$ & - \\
\hline syzon1a & ss & Specific yield simulated value & Specific yield subregions 1,8 , and 11 & $4.18 \mathrm{E}-02$ & $1.00 \mathrm{E}-02$ & $3.50 \mathrm{E}-01$ & $1.02 \mathrm{E}-01$ & - \\
\hline syzon $1 b$ & ss & Specific yield simulated value & Specific yield subregion 6 and 12 & $5.69 \mathrm{E}-02$ & $5.00 \mathrm{E}-02$ & $3.50 \mathrm{E}-01$ & $1.64 \mathrm{E}-01$ & - \\
\hline syzon1c & ss & Specific yield simulated value & Specific yield subregion 7 & $8.12 \mathrm{E}-02$ & $5.00 \mathrm{E}-02$ & $3.50 \mathrm{E}-01$ & $5.63 \mathrm{E}-02$ & - \\
\hline syzon2 & ss & Specific yield simulated value & Specific yield subregions 2 and 9 & $1.07 \mathrm{E}-02$ & $1.00 \mathrm{E}-02$ & $3.50 \mathrm{E}-01$ & $1.72 \mathrm{E}-02$ & - \\
\hline syzon3 & ss & Specific yield simulated value & Specific yield subregions 3 and 13 & $1.10 \mathrm{E}-01$ & $1.00 \mathrm{E}-02$ & $3.50 \mathrm{E}-01$ & $1.18 \mathrm{E}-02$ & - \\
\hline syzon4 & ss & Specific yield simulated value & Specific yield subregions 4 and 14 & $2.71 \mathrm{E}-02$ & $5.00 \mathrm{E}-03$ & $3.50 \mathrm{E}-01$ & $3.05 \mathrm{E}-02$ & - \\
\hline syzon5 & Ss & Specific yield simulated value & Specific yield subregion 5 & $1.43 \mathrm{E}-01$ & $5.00 \mathrm{E}-02$ & $3.50 \mathrm{E}-01$ & $1.46 \mathrm{E}-01$ & - \\
\hline viacha_s & rivers & Creek stage adjustment parameter & Via Chaparral Creek & $4.75 \mathrm{E}-01$ & $3.28 \mathrm{E}-06$ & $3.28 \mathrm{E}+00$ & $3.86 \mathrm{E}-02$ & $\mathrm{ft}$ \\
\hline vtransd & dispersion & Vertical transverse dispersivity & Vertical shallow & $3.28 \mathrm{E}-04$ & $1.64 \mathrm{E}-04$ & $1.64 \mathrm{E}-01$ & $2.98 \mathrm{E}-02$ & $\mathrm{ft}$ \\
\hline vtransddp & dispersion & Vertical transverse dispersivity & Vertical deep & $1.64 \mathrm{E}-01$ & $1.64 \mathrm{E}-04$ & $1.64 \mathrm{E}-01$ & $3.28 \mathrm{E}-02$ & $\mathrm{ft}$ \\
\hline zoneegtym & aquiferk & HK/VK layer multiplier & $\begin{array}{l}\text { Storage Unit I coastal confining layer between } \\
\text { middle and lower producing zone HK }\end{array}$ & $4.83 \mathrm{E}-01$ & $1.00 \mathrm{E}-01$ & $1.00 \mathrm{E}+02$ & $2.41 \mathrm{E}+00$ & - \\
\hline zoneegtyvm & aquiferk & HK/VK layer multiplier & $\begin{array}{l}\text { Storage Unit I coastal confining layer between } \\
\text { middle and lower producing zone VK }\end{array}$ & $5.00 \mathrm{E}-02$ & $1.00 \mathrm{E}-05$ & $1.00 \mathrm{E}+01$ & $1.69 \mathrm{E}+00$ & - \\
\hline zoneeightm & aquiferk & HK/VK layer multiplier & $\begin{array}{l}\text { Storage Unit I non-coastal confining layer between } \\
\text { middle and lower producing zone HK }\end{array}$ & $5.05 \mathrm{E}-01$ & $1.00 \mathrm{E}-05$ & $1.00 \mathrm{E}+01$ & $5.36 \mathrm{E}-01$ & - \\
\hline zoneeightvm & aquiferk & HK/VK layer multiplier & $\begin{array}{l}\text { Storage Unit I non-coastal confining layer between } \\
\text { middle and lower producing zone VK }\end{array}$ & $4.98 \mathrm{E}-01$ & $1.00 \mathrm{E}-05$ & $1.00 \mathrm{E}+00$ & $4.90 \mathrm{E}-01$ & - \\
\hline zoneetnm & aquiferk & HK/VK layer multiplier & $\begin{array}{l}\text { Storage Unit I bottom of confining layer between } \\
\text { middle and lower producing zone HK }\end{array}$ & $7.82 \mathrm{E}-04$ & $1.00 \mathrm{E}-06$ & $1.00 \mathrm{E}+01$ & $8.25 \mathrm{E}-05$ & - \\
\hline zoneetnvm & aquiferk & HK/VK layer multiplier & $\begin{array}{l}\text { Storage Unit I bottom of confining layer between } \\
\text { middle and lower producing zone VK }\end{array}$ & $7.82 \mathrm{E}-04$ & $1.00 \mathrm{E}-06$ & $1.00 \mathrm{E}+01$ & $7.82 \mathrm{E}-04$ & - \\
\hline zonefiveam & aquiferk & HK/VK layer multiplier & $\begin{array}{l}\text { Foothill basin and Storage Unit III lower confining } \\
\text { layer below lower producing zone HK }\end{array}$ & $1.08 \mathrm{E}-01$ & $1.00 \mathrm{E}-03$ & $1.00 \mathrm{E}+01$ & $4.21 \mathrm{E}-01$ & - \\
\hline zonefivem & aquiferk & HK/VK layer multiplier & $\begin{array}{l}\text { Storage Unit I lower confining layer below lower } \\
\text { producing zone HK }\end{array}$ & $1.00 \mathrm{E}-05$ & $1.00 \mathrm{E}-05$ & $1.00 \mathrm{E}+00$ & $2.16 \mathrm{E}-01$ & - \\
\hline zonefivevm & aquiferk & HK/VK layer multiplier & $\begin{array}{l}\text { Storage Unit I lower confining layer below lower } \\
\text { producing zone VK }\end{array}$ & $1.00 \mathrm{E}-05$ & $1.00 \mathrm{E}-05$ & $1.00 \mathrm{E}+00$ & $1.00 \mathrm{E}-05$ & - \\
\hline
\end{tabular}
producing zone VK 
Table 3. Calibrated groundwater flow and solute transport model parameter values, Santa Barbara flow and transport model, Santa Barbara, California.-Continued

[/day, per day; ft, foot; $\mathrm{ft}^{2}$, square foot; GHB, general-head boundary package; HK, horizontal hydraulic conductivity; Rd., road; St., street; VK, vertical hydraulic conductivity; - , not applicable]

\begin{tabular}{|c|c|c|c|c|c|c|c|c|}
\hline $\begin{array}{l}\text { Parameter } \\
\text { name }\end{array}$ & Group & Subgroup & Description & $\begin{array}{l}\text { Calibrated } \\
\text { value }\end{array}$ & $\begin{array}{l}\text { Lower } \\
\text { bound }\end{array}$ & $\begin{array}{l}\text { Upper } \\
\text { bound }\end{array}$ & $\begin{array}{l}\text { Preliminary } \\
\text { value }\end{array}$ & Units \\
\hline zonefouram & aquiferk & HK/VK layer multiplier & $\begin{array}{l}\text { Foothill basin and Storage Unit III lower producing } \\
\text { zone HK }\end{array}$ & $3.89 \mathrm{E}+00$ & $1.00 \mathrm{E}+00$ & $1.00 \mathrm{E}+01$ & $2.72 \mathrm{E}+00$ & - \\
\hline zonefourm & aquiferk & HK/VK layer multiplier & Storage Unit I non-coastal lower producing zone HK & $1.00 \mathrm{E}+00$ & $1.00 \mathrm{E}+00$ & $1.00 \mathrm{E}+01$ & $1.20 \mathrm{E}+00$ & - \\
\hline zonefrsu2 & aquiferk & HK/VK layer multiplier & Foothill basin lower producing zone HK & $8.00 \mathrm{E}-02$ & $1.00 \mathrm{E}-05$ & $1.00 \mathrm{E}+01$ & $1.20 \mathrm{E}+00$ & - \\
\hline zonefrtym & aquiferk & HK/VK layer multiplier & Storage Unit I coastal lower producing zone HK & $1.70 \mathrm{E}-01$ & $1.00 \mathrm{E}+00$ & $1.00 \mathrm{E}+02$ & $1.62 \mathrm{E}+01$ & - \\
\hline zonefrtyvm & aquiferk & HK/VK layer multiplier & Storage Unit I coastal lower producing zone VK & $3.08 \mathrm{E}-02$ & $1.00 \mathrm{E}-02$ & $1.00 \mathrm{E}+02$ & $1.02 \mathrm{E}+01$ & - \\
\hline zoneninem & aquiferk & HK/VK layer multiplier & $\begin{array}{l}\text { Storage Unit I non-coastal upper confining layer soil } \\
\text { surface HK }\end{array}$ & $7.50 \mathrm{E}-02$ & $1.00 \mathrm{E}-05$ & $1.00 \mathrm{E}+00$ & $7.77 \mathrm{E}-03$ & - \\
\hline zoneninevm & aquiferk & HK/VK layer multiplier & $\begin{array}{l}\text { Storage Unit I non-coastal upper confining layer soil } \\
\text { surface VK }\end{array}$ & $7.50 \mathrm{E}-02$ & $1.00 \mathrm{E}-05$ & $1.00 \mathrm{E}+00$ & $7.50 \mathrm{E}-02$ & - \\
\hline zonenintym & aquiferk & HK/VK layer multiplier & $\begin{array}{l}\text { Storage Unit I coastal upper confining layer soil } \\
\text { surface HK }\end{array}$ & $7.50 \mathrm{E}-02$ & $1.00 \mathrm{E}-05$ & $1.00 \mathrm{E}+00$ & $7.50 \mathrm{E}-02$ & - \\
\hline zonenintyvm & aquiferk & HK/VK layer multiplier & $\begin{array}{l}\text { Storage Unit I coastal upper confining layer soil } \\
\text { surface VK }\end{array}$ & $7.50 \mathrm{E}-02$ & $1.00 \mathrm{E}-05$ & $1.00 \mathrm{E}+00$ & $7.50 \mathrm{E}-02$ & - \\
\hline zoneohdrm & aquiferk & HK/VK layer multiplier & Storage Unit I bottom of upper confining layer HK & $6.53 \mathrm{E}-04$ & $1.00 \mathrm{E}-04$ & $1.00 \mathrm{E}+01$ & $7.45 \mathrm{E}-04$ & - \\
\hline zoneohdrom & aquiferk & HK/VK layer multiplier & Upper confining layer at Mission Creek HK & $1.00 \mathrm{E}+01$ & $1.00 \mathrm{E}-04$ & $1.00 \mathrm{E}+01$ & $1.00 \mathrm{E}+01$ & - \\
\hline zoneoneam & aquiferk & HK/VK layer multiplier & $\begin{array}{l}\text { Foothill Basin and Storage Unit III upper confining } \\
\text { layer HK }\end{array}$ & $2.71 \mathrm{E}+00$ & $1.00 \mathrm{E}-03$ & $5.00 \mathrm{E}+00$ & $2.00 \mathrm{E}-01$ & - \\
\hline zoneonem & aquiferk & HK/VK layer multiplier & Storage Unit I upper confining layer HK & $7.41 \mathrm{E}-01$ & $1.00 \mathrm{E}-03$ & $1.00 \mathrm{E}+00$ & $1.05 \mathrm{E}-01$ & - \\
\hline zoneonevm & aquiferk & HK/VK layer multiplier & Storage Unit I upper confining layer VK & $7.02 \mathrm{E}-01$ & $1.00 \mathrm{E}-05$ & $1.00 \mathrm{E}+00$ & $1.31 \mathrm{E}-01$ & - \\
\hline zoneothsm & aquiferk & HK/VK layer multiplier & $\begin{array}{l}\text { Storage Unit I coastal bottom of upper confining } \\
\text { layer HK }\end{array}$ & $9.76 \mathrm{E}-04$ & $1.00 \mathrm{E}-04$ & $1.00 \mathrm{E}+01$ & $1.07 \mathrm{E}-02$ & - \\
\hline zonesevenm & aquiferk & HK/VK layer multiplier & $\begin{array}{l}\text { Storage Unit I non-coastal confining layer directly } \\
\text { below middle producing zone HK }\end{array}$ & $5.34 \mathrm{E}-05$ & $1.00 \mathrm{E}-06$ & $1.00 \mathrm{E}+01$ & $7.31 \mathrm{E}-03$ & - \\
\hline zonesevenvm & aquiferk & HK/VK layer multiplier & $\begin{array}{l}\text { Storage Unit I non-coastal confining layer directly } \\
\text { below middle producing zone VK }\end{array}$ & $5.34 \mathrm{E}-05$ & $1.00 \mathrm{E}-06$ & $1.00 \mathrm{E}+01$ & $5.34 \mathrm{E}-05$ & - \\
\hline zonesixm & aquiferk & HK/VK layer multiplier & $\begin{array}{l}\text { Storage Unit I non-coastal middle producing } \\
\text { zone HK }\end{array}$ & $8.17 \mathrm{E}-01$ & $1.00 \mathrm{E}-01$ & $1.00 \mathrm{E}+01$ & $1.26 \mathrm{E}+00$ & - \\
\hline zonesixvm & aquiferk & HK/VK layer multiplier & $\begin{array}{l}\text { Storage Unit I non-coastal middle producing } \\
\text { zone VK }\end{array}$ & $8.17 \mathrm{E}-01$ & $1.00 \mathrm{E}-01$ & $1.00 \mathrm{E}+01$ & $8.17 \mathrm{E}-01$ & - \\
\hline zonesvtym & aquiferk & HK/VK layer multiplier & $\begin{array}{l}\text { Storage Unit I coastal confining layer directly below } \\
\text { middle producing zone HK }\end{array}$ & $1.09 \mathrm{E}-01$ & $1.00 \mathrm{E}-06$ & $1.00 \mathrm{E}+02$ & $1.09 \mathrm{E}-02$ & - \\
\hline zonesvtyvm & aquiferk & HK/VK layer multiplier & $\begin{array}{l}\text { Storage Unit I coastal confining layer directly below } \\
\text { middle producing zone VK }\end{array}$ & $3.52 \mathrm{E}-04$ & $1.00 \mathrm{E}-06$ & $1.10 \mathrm{E}+01$ & $3.52 \mathrm{E}-04$ & - \\
\hline
\end{tabular}
middle producing zone VK 
Table 3. Calibrated groundwater flow and solute transport model parameter values, Santa Barbara flow and transport model, Santa Barbara, California._-Continued

[/day, per day; ft, foot; ft², square foot; GHB, general-head boundary package; HK, horizontal hydraulic conductivity; Rd., road; St., street; VK, vertical hydraulic conductivity; -, not applicable]

\begin{tabular}{|c|c|c|c|c|c|c|c|c|}
\hline $\begin{array}{l}\text { Parameter } \\
\text { name }\end{array}$ & Group & Subgroup & Description & $\begin{array}{l}\text { Calibrated } \\
\text { value }\end{array}$ & $\begin{array}{l}\text { Lower } \\
\text { bound }\end{array}$ & $\begin{array}{l}\text { Upper } \\
\text { bound }\end{array}$ & $\begin{array}{l}\text { Preliminary } \\
\text { value }\end{array}$ & Units \\
\hline zonesxhdm & aquiferk & HK/VK layer multiplier & Storage Unit I coastal middle producing zone HK & $1.00 \mathrm{E}+00$ & $1.00 \mathrm{E}-02$ & $1.00 \mathrm{E}+01$ & $1.62 \mathrm{E}+00$ & - \\
\hline zonesxhdvm & aquiferk & $\mathrm{HK} / \mathrm{VK}$ layer multiplier & Storage Unit I coastal middle producing zone VK & $1.00 \mathrm{E}-05$ & $1.00 \mathrm{E}-06$ & $1.00 \mathrm{E}+01$ & $1.00 \mathrm{E}-04$ & - \\
\hline zonesxtym & aquiferk & HK/VK layer multiplier & $\begin{array}{l}\text { Storage Unit I western edge of middle producing } \\
\text { zone HK }\end{array}$ & $1.00 \mathrm{E}-02$ & $1.00 \mathrm{E}-03$ & $1.00 \mathrm{E}+01$ & $1.00 \mathrm{E}-02$ & - \\
\hline zonesxtyom & aquiferk & HK/VK layer multiplier & $\begin{array}{l}\text { Storage Unit I eastern edge of middle producing } \\
\text { zone HK }\end{array}$ & $1.00 \mathrm{E}-05$ & $1.00 \mathrm{E}-06$ & $1.00 \mathrm{E}+01$ & $1.00 \mathrm{E}-05$ & - \\
\hline zonesxtyvm & aquiferk & HK/VK layer multiplier & $\begin{array}{l}\text { Storage Unit I western edge of middle producing } \\
\text { zone VK }\end{array}$ & $1.00 \mathrm{E}-02$ & $1.00 \mathrm{E}-03$ & $1.00 \mathrm{E}+01$ & $1.00 \mathrm{E}-02$ & - \\
\hline zonetenm & aquiferk & HK/VK layer multiplier & $\begin{array}{l}\text { Storage Unit I coastal upper zone above upper } \\
\text { producing zone HK }\end{array}$ & $4.74 \mathrm{E}+00$ & $1.00 \mathrm{E}-01$ & $1.00 \mathrm{E}+01$ & $4.99 \mathrm{E}+00$ & - \\
\hline zonetenvm & aquiferk & HK/VK layer multiplier & $\begin{array}{l}\text { Storage Unit I coastal upper zone above upper } \\
\text { producing zone VK }\end{array}$ & $4.55 \mathrm{E}+00$ & $1.00 \mathrm{E}-01$ & $1.00 \mathrm{E}+01$ & $4.31 \mathrm{E}+00$ & - \\
\hline zonethd & aquiferk & HK/VK layer multiplier & $\begin{array}{l}\text { Storage Unit I non-coastal confining layer directly } \\
\text { above middle producing zone VK }\end{array}$ & $1.00 \mathrm{E}-01$ & $1.00 \mathrm{E}-05$ & $1.00 \mathrm{E}+00$ & $5.00 \mathrm{E}-03$ & - \\
\hline zonethreeam & aquiferk & HK/VK layer multiplier & $\begin{array}{l}\text { Foothill Basin and Storage Unit III middle confining } \\
\text { layer HK }\end{array}$ & $3.00 \mathrm{E}-01$ & $1.00 \mathrm{E}-05$ & $1.00 \mathrm{E}+00$ & $7.49 \mathrm{E}-01$ & - \\
\hline zonethreem & aquiferk & HK/VK layer multiplier & $\begin{array}{l}\text { Storage Unit I non-coastal middle zone above middle } \\
\text { producing zone HK }\end{array}$ & $3.01 \mathrm{E}-01$ & $1.00 \mathrm{E}-03$ & $1.00 \mathrm{E}+00$ & $1.10 \mathrm{E}-01$ & - \\
\hline zonethreevm & aquiferk & HK/VK layer multiplier & $\begin{array}{l}\text { Storage Unit I non-coastal middle zone above middle } \\
\text { producing zone VK }\end{array}$ & $3.01 \mathrm{E}-01$ & $1.00 \mathrm{E}-03$ & $1.00 \mathrm{E}+00$ & $3.01 \mathrm{E}-01$ & - \\
\hline zonethrsu2 & aquiferk & HK/VK layer multiplier & Foothill Basin middle confining layer HK & $6.33 \mathrm{E}-03$ & $1.00 \mathrm{E}-05$ & $1.00 \mathrm{E}+00$ & $1.33 \mathrm{E}-02$ & - \\
\hline zonethtym & aquiferk & HK/VK layer multiplier & $\begin{array}{l}\text { Storage Unit I coastal middle zone above middle } \\
\text { producing zone HK }\end{array}$ & $5.74 \mathrm{E}-02$ & $1.00 \mathrm{E}-06$ & $1.00 \mathrm{E}+01$ & $4.38 \mathrm{E}-01$ & - \\
\hline zonethtyvm & aquiferk & HK/VK layer multiplier & $\begin{array}{l}\text { Storage Unit I coastal middle zone above middle } \\
\text { producing zone VK }\end{array}$ & $2.74 \mathrm{E}-02$ & $1.00 \mathrm{E}-06$ & $1.00 \mathrm{E}+01$ & $1.07 \mathrm{E}-06$ & - \\
\hline zonetwoam & aquiferk & HK/VK layer multiplier & $\begin{array}{l}\text { Foothill Basin and Storage Unit III upper producing } \\
\text { zone HK }\end{array}$ & $5.45 \mathrm{E}+00$ & $1.00 \mathrm{E}+00$ & $1.00 \mathrm{E}+01$ & $2.76 \mathrm{E}+00$ & - \\
\hline zonetwom & aquiferk & HK/VK layer multiplier & Storage Unit I non-coastal upper producing zone HK & $1.30 \mathrm{E}+00$ & $8.50 \mathrm{E}-01$ & $1.00 \mathrm{E}+01$ & $1.37 \mathrm{E}+00$ & - \\
\hline zonetwosu2 & aquiferk & HK/VK layer multiplier & Foothill Basin upper producing zone HK & $5.00 \mathrm{E}+00$ & $1.00 \mathrm{E}-05$ & $1.00 \mathrm{E}+01$ & $1.37 \mathrm{E}+00$ & - \\
\hline
\end{tabular}




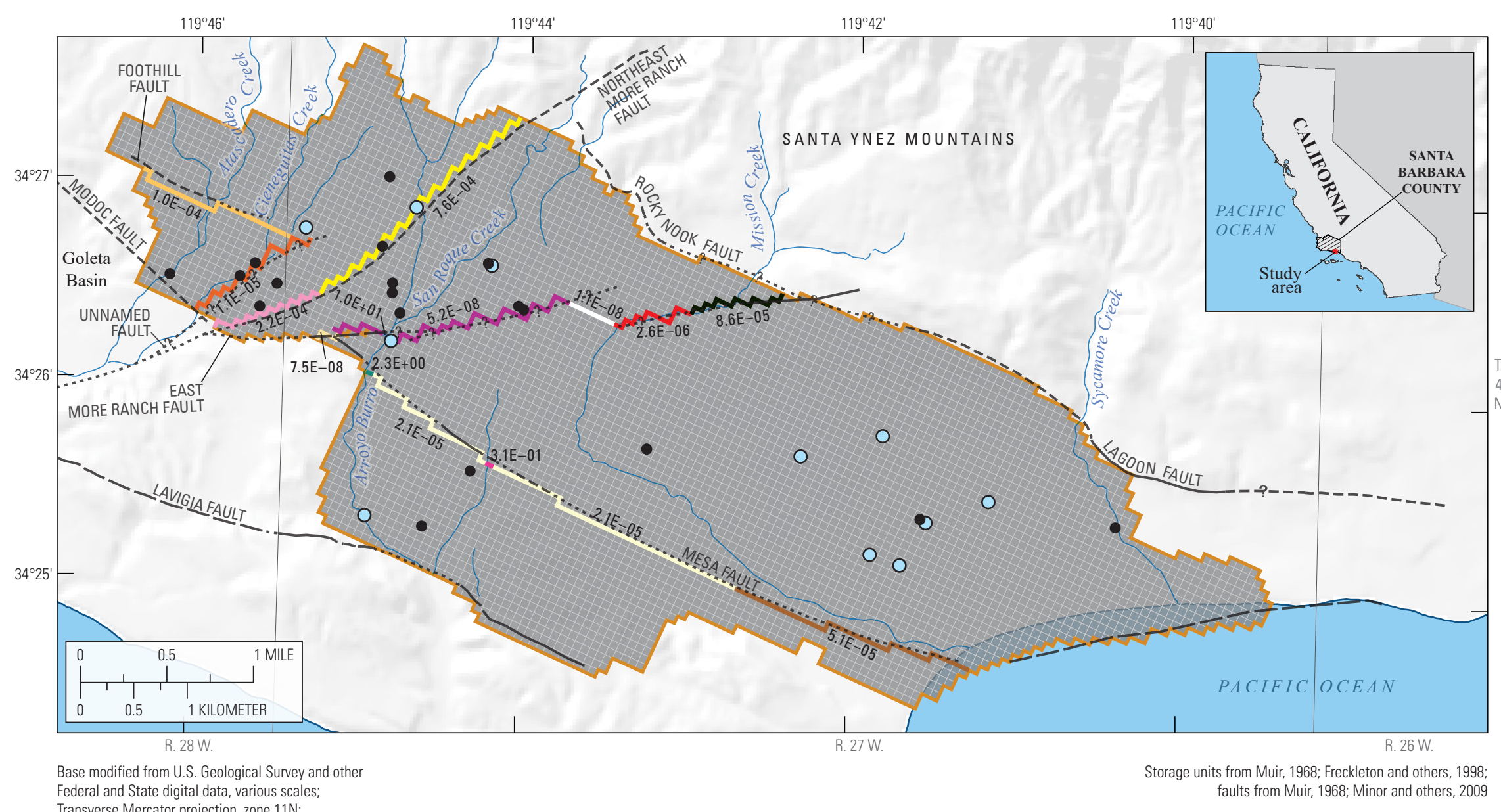

Federal and State digital data, various scales:

rransverse Mercator projection, zone 11N:

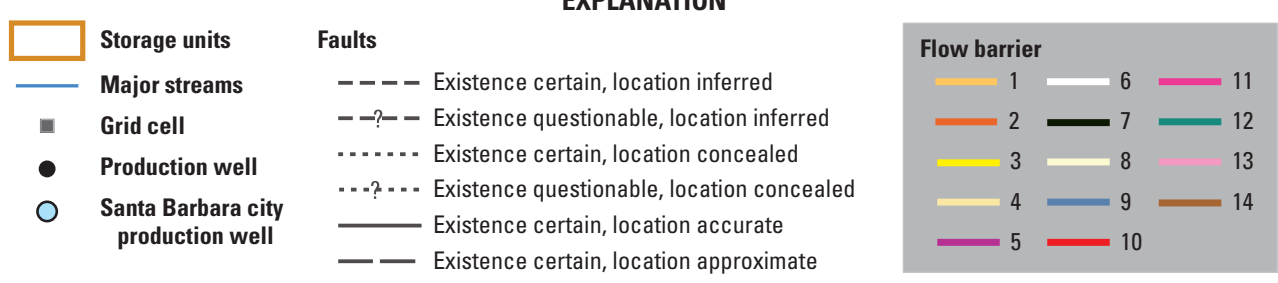

Figure 8. Calibrated horizontal-flow-barrier hydraulic-characteristic values (per day), Santa Barbara flow and transport model, Santa Barbara, California. 


\section{Storage Properties}

Specific storage (SS) was estimated for each subregion and area using the textural model. The percentage of coarseand fine-grained material in each groundwater-model cell from the HFM (see chapter B) was used to define specificstorage values. Specific storage was calculated using weighted averaging, as shown in equation 7 :

$$
S S_{\text {texture }}=P C \times S S_{c x}+P F \times S S_{f x}
$$

where

$P C$ and $P F \quad$ are the percentage of coarse and fine sediment in a given model percentage, expressed as a fraction, of course and fine sediment, respectively, in a given model cell, and

$S S_{c x}$ and $S S_{f x} \quad$ are the coarse and fine specific-storage end members, respectively, by subregion.

To simulate unconfined conditions, the specific-storage values of the uppermost layer of the SBFTM were estimated separately. The specific-storage values for each cell in the topmost layer were defined by specific yield $(S Y)$ divided by the thickness of the model cell and were not based on the HFM. A separate specific-yield value was estimated for each subregion (see parameter group "ss" in table 3).

\section{Solute-Transport Properties}

Each source of recharge in the SBFTM was assigned a chloride concentration to represent the salinity. Precipitation, small-catchment recharge, stream recharge, septic recharge, and flow across the Montecito GHB were considered freshwater recharge and were assigned a chloride concentration of 100 milligrams per liter $(\mathrm{mg} / \mathrm{L})$, which is typical of fresh water (Goldman and Horne, 1983). Recharge across the ocean GHB was considered to be seawater and assigned a chloride concentration representative of seawater: $19,000 \mathrm{mg} / \mathrm{L}$ (Langevin and others, 2008).

Three additional aquifer properties are needed to simulate solute transport: porosity, dispersivity, and diffusion. Porosity values $(\mathrm{P})$ for each model cell were approximated using the percentage of coarse and fine sediments in that model cell. Different textural end-member porosity values $\left(\mathrm{P}_{c x}\right.$ and $\left.\mathrm{P}_{\mathrm{fx}}\right)$ were used for the lower producing zone, the middle producing zone, and the rest of the SBFTM. Similar to storage values, porosity was calculated using weighted averages (equation 7).

The three components of dispersivity (longitudinal, horizontal transverse, and vertical transverse) were defined for two dispersivity zones: an upper dispersivity zone consisting of model layers 1-27 and a lower dispersivity zone consisting of model layers $28-56$. The upper dispersivity zone includes the upper and middle producing zones, which, on the basis of numerical testing, had different transport properties than the lower producing zone. The lower dispersivity zone included the deeper part of the middle zone and the lower producing zone. The dispersivity values were estimated separately by trial-and-error for the upper and lower dispersivity zones. The final, calibrated dispersivity values are presented in table 3 . Diffusion (representing molecular effects) was defined as a constant value, $9.30 \mathrm{E}-04 \mathrm{ft}^{2} / \mathrm{d}$, typical of alluvial sediments throughout the SBFTM area (Freeze and Cherry, 1979).

\section{Model Calibration Approach}

The SBFTM was calibrated in two parts: predevelopment and transient. Model calibration of the predevelopment and transient models was done in sequence and simultaneously using measured data described in the next section. As mentioned previously, to simulate predevelopment conditions (no stresses), groundwater flow and solute transport were simulated for 10,000 years to allow the flow and concentration fields to equilibrate to specified initial and boundary conditions in 10-year time steps. Calibration of the predevelopment conditions was accomplished by varying small-catchment recharge, creek-bed conductance and stage, areal recharge, drain conductance, general-head boundary conductance, hydraulic conductivity, flow-barrier conductance, porosity, dispersivity, and diffusion. As mentioned earlier, the transient simulation represents the period from 1929 to 2013; however, the focus of the transient calibration was the period from 1972 to 2013. In addition to the predevelopment parameters listed above, specific storage, specific yield, the vertical distribution of pumpage, and septic recharge were also varied.

Calibration of the SBFTM was accomplished using a combination of automated and trial-and-error methods. Initially, PEST (Doherty, 2010) was used for automated calibration of the flow and transport parameters. In addition, PEST was used to assess the sensitivities of parameters used in the parameter-estimation process. During the preliminary calibration process, it was found that PEST did not estimate the transport parameters well; this could be related to the highly nonlinear nature of the density-dependent groundwater flow and transport problem. Therefore, automated calibration was primarily used to calibrate the groundwater-flow component of the SBFTM and trial-and-error calibration was used to calibrate the solute-transport component of the SBFTM. Bounds on model parameters were set to keep parameters within a realistic range of values.

Hydraulic properties were checked for unrealistic values during calibration. Groundwater budgets were regularly examined during the calibration process, and budget components were compared with previously published results (for example, Freckleton and others, 1998). 


\section{Measured Data}

Five types of observation data were used during model calibration: water levels, changes in water levels (drawdowns), chloride concentrations, changes in chloride concentrations (figs. 8, 9), and published infiltration rates along segments of Mission Creek. The SBFTM was calibrated to 24,884 waterlevel observations from 75 wells and to 1,064 chlorideconcentration observations from 52 wells. Water-level observations were used to compute changes in water levels for each stress period for each well. Similarly, chlorideconcentration observations were used to calculate the changes in chloride concentrations. Water-level observations, computed water-level changes, chloride-concentration observations, and computed chloride-concentration changes were compared with simulated results. Infiltration rates along segments of Mission Creek estimated by McFadden and others (1987) also were used in the calibration process (fig. 9).

\section{Selection of Well Data}

Water-level and chloride data were reviewed for consistency and accuracy before use as model-observation data. Data points that were inconsistent with other data points or trends and appeared to be in error were removed, as were data collected from wells at or very near a production well and recorded while the production well was active. In addition, water-level data from wells perforated in the shallow zone (a perched aquifer) were not included because the SBFTM was not designed to simulate a perched aquifer. Likewise, chloride data were reviewed with water-level data to determine if and when well casings had corroded to the point of failure. Chloride and water-level data that were thought to be influenced by failed well casings were excluded; for example, water levels from wells 4N/27W-09Q1 and 4N/27W-09M1 (fig. 9) were not used.

\section{Water-Level Data}

For model calibration, water-level data were needed that were representative of the main aquifer conditions and distributed spatially and temporally throughout the simulated subbasins - vertically through the upper, middle, and lower producing zones and temporally during the key transient calibration period (1972-2013). Water-level data were distributed at 75 geographic locations throughout Storage Unit I, Storage Unit III, and East and West Foothill subbasins (fig. 9). Multiple-completion wells recording groundwater levels at multiple depths were present at 22 of the 75 geographic well locations. Water-level data from these wells included the entire main simulation period from 1972 to 2013; however, water-level data prior to 1975 were available for only a few of these wells.

\section{Chloride-Concentration Data}

To calibrate the SBHTM for seawater intrusion, chlorideconcentration data measured in Storage Unit I were used. Specifically, data were available from between the coast and the City Hall, Vera Cruz, Corporation Yard, De la Guerra, and Ortega Park production wells in the upper, middle, and lower producing zones (fig. 10). Chloride data from eight key well clusters recorded chloride concentrations at various depths. Four of the clusters (4N/27W-23F02-04, 4N/27W-23F05-08, $4 \mathrm{~N} / 27 \mathrm{~W}-23 \mathrm{E} 01-04$, and 4N/27W-23E05-06) are near the coast; two of the clusters (4N/27W-22A02-05 and 4N/27W22G02-04) are between the production wells and the coast, about 0.5 miles (mi) from the coast and $0.25 \mathrm{mi}$ from the production wells. The last two clusters (4N/27W-22B02-05 and 4N/27W-22B08-11) are near the Vera Cruz production well (fig. 10).

The SBFTM was not designed to simulate the flow of chlorides from the underlying bedrock to the lower producing zone. As stated in chapter A, higher chloride concentrations in wells 4N/27W-22B04, 05, and 08 were likely due to pumping from the Vera Cruz production well drawing saline water from the underlying shale. The SBFTM was, therefore, not calibrated to these particular measured chloride concentrations.

\section{Model Parameters}

A total of 254 parameters were used in the SBFTM to define horizontal and vertical hydraulic conductivity; specific storage; specific yield; the pumping distribution of individual wells by layer; the conductance of horizontal-flow barriers, general-head boundaries, drains, and creeks; stream stage; drain elevation; areal and septic recharge infiltration rates; dispersivity; and porosity. In general, all 254 parameters were estimated; however, some were fixed during the course of calibration.

Table 3 contains the calibrated model-parameter values used in the SBFTM. Parameters in table 3 are grouped into eight types: hydraulic conductivity, storage, conductance, head or stage, pumpage distribution, recharge, porosity, and dispersivity.

Parameters affecting the hydraulic and transport properties and the vertical distribution of pumping were described earlier. In addition to these parameters, those affecting small-catchment recharge, areal recharge, and early pumpage were estimated.

As discussed in the "Simulated Groundwater Recharge" section, small-catchment recharge was divided into seven segments based on topography (fig. 2) and was estimated by scaling parameters for each small-catchment segment along the northern model border with the Santa Ynez Mountains (table 3). Recharge was determined by parameter estimation, and the initial recharge amounts were based on Freckleton and others (1998). The initial recharge amounts were modified using a segment multiplier, which was determined by parameter estimation. 


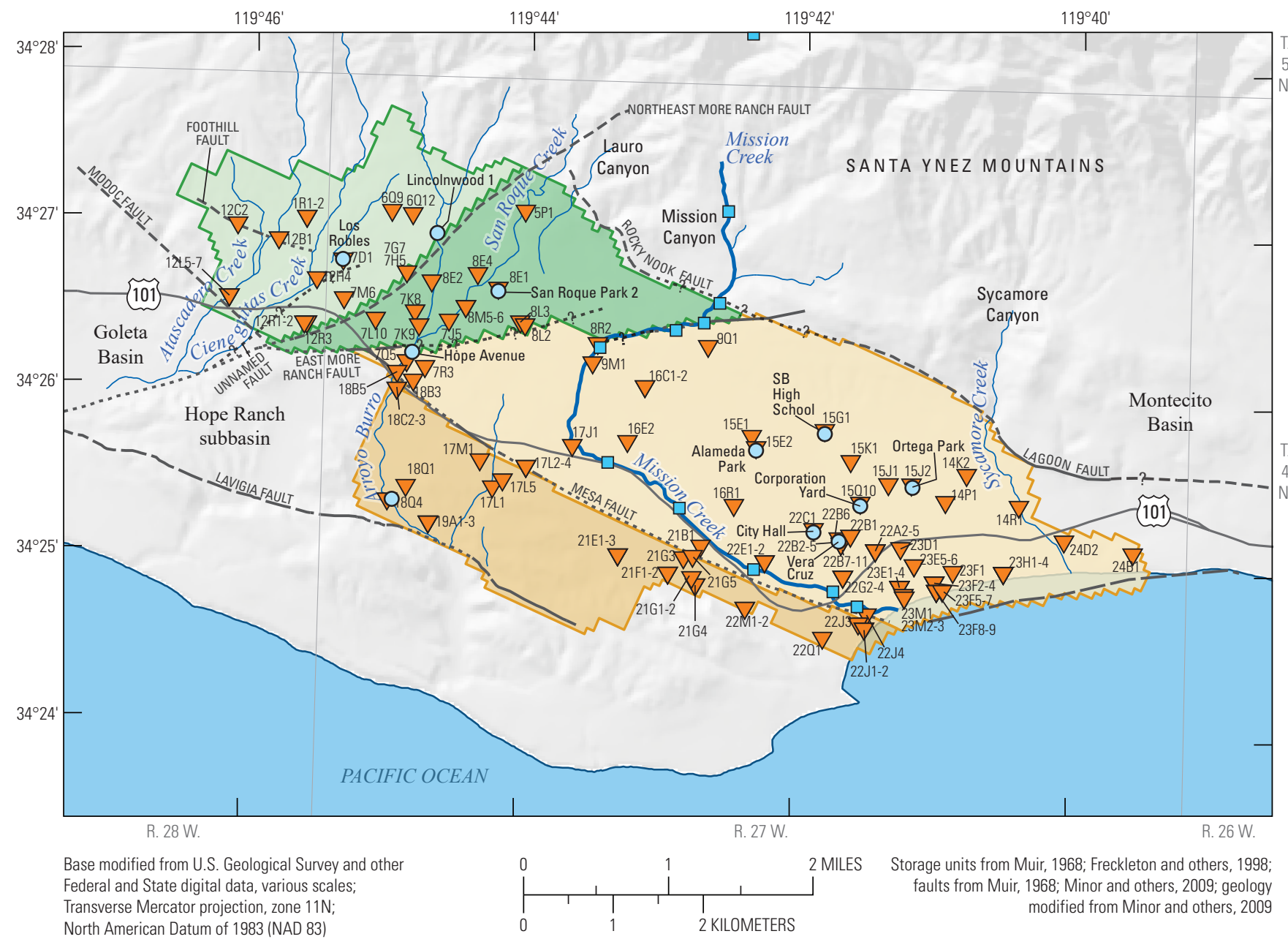

EXPLANATION
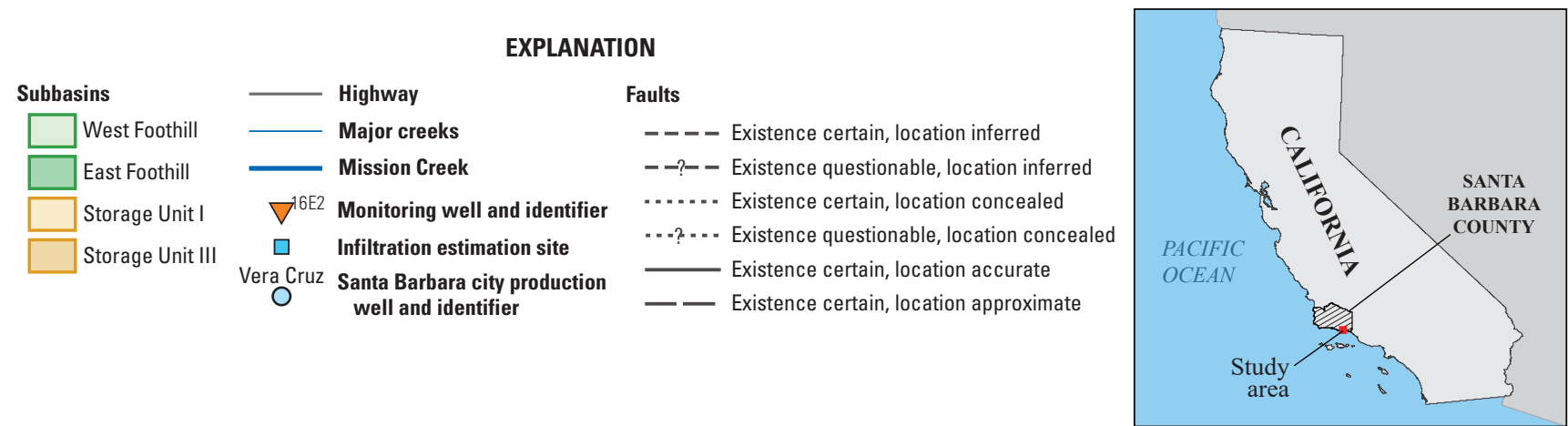

Figure 9. Monitoring wells with available water-level data and Mission Creek segments where infiltration rates were estimated by McFadden and others (1987), Santa Barbara and Foothill groundwater basins, Santa Barbara, California. 


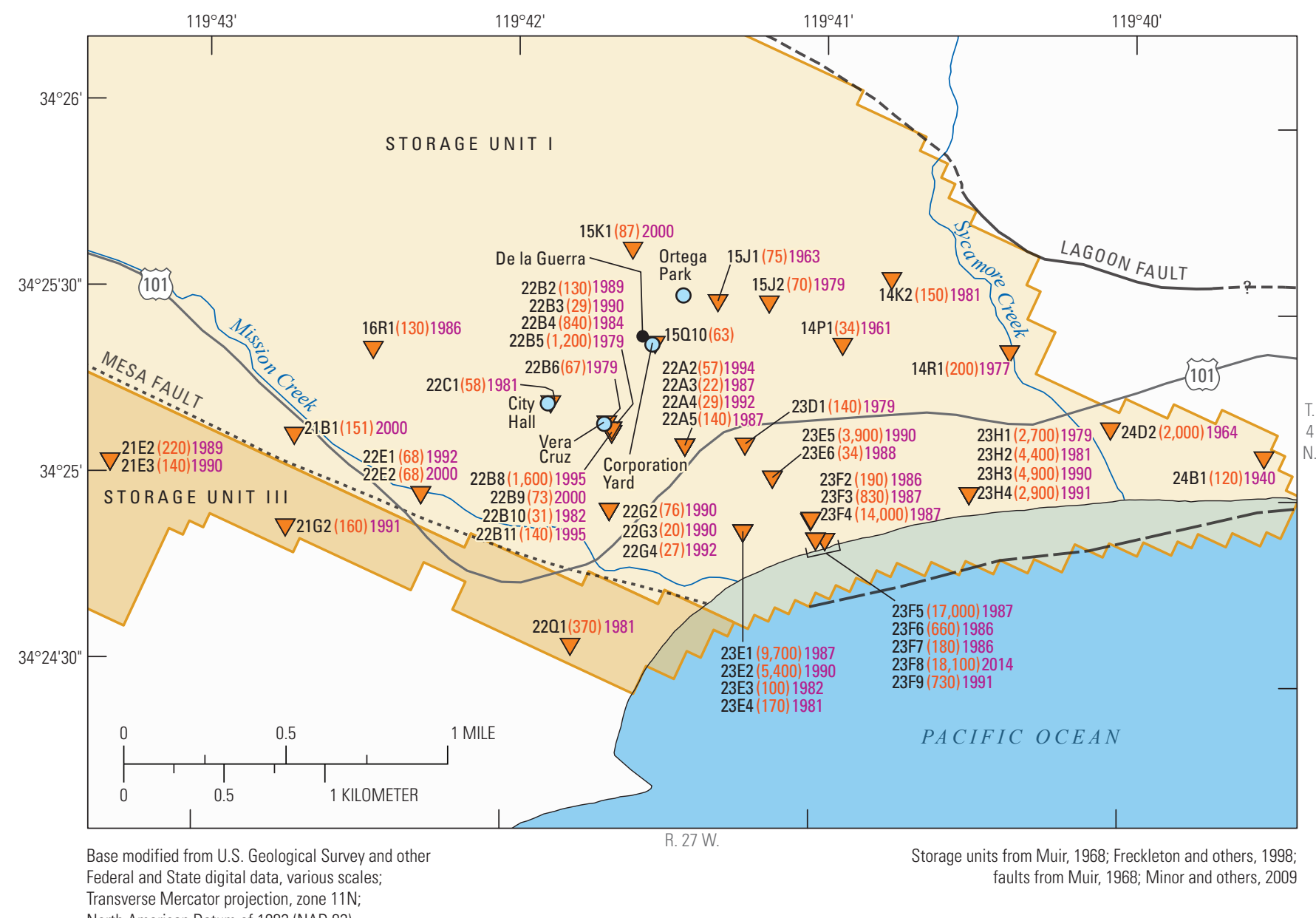

Transverse Mercator projection, zone $11 \mathrm{~N}$;

North American Datum of 1983 (NAD 83)

\section{EXPLANATION}

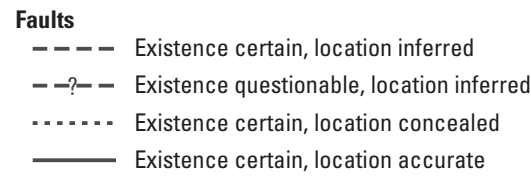

Subbasins

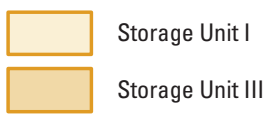

Highway

21E2(220)1989

$\nabla \quad$ Monitoring well and identifier-

Number in parentheses is peak chloride concentration in milligram per liter and purple number indicates year

measurement was made

De la Guerra

- Production well and identifier

Vera Cruz Santa Barbara city production

$\mathrm{w}$ well and identifier

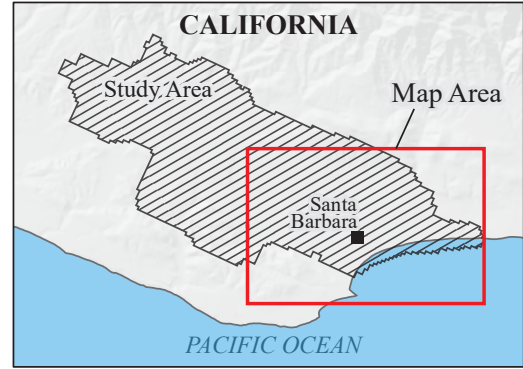

PACIFIC OCEAN

Figure 10. Monitoring wells with chloride concentration data near the coast of the city of Santa Barbara, California. 
Areal recharge infiltration rates for four infiltration zones were simulated using four parameters that control the infiltration percentage of precipitation in each zone. The range for the calibrated areal recharge parameters was 7.22E-05$1.69 \mathrm{E}-02 \mathrm{ft} / \mathrm{d}$ (table 3 ). Note that in table 3 , the recharge flux rates for infiltration zones 2-4 are cumulative. For example, the recharge flux rate for infiltration zone 4 equals rainrchlabs +rainrch2add+rainrch3add+rainrch4add.

Septic recharge was simulated using a single multiplier as a parameter. The calibrated septic recharge parameter was $7.58 \mathrm{E}-04 \mathrm{ft} / \mathrm{d}$ (table 3 ), which equals a total flow rate of about $71 \mathrm{acre}-\mathrm{ft} / \mathrm{yr}$. This flow rate is greater than the previously calculated septic recharge of 65 acre-ft/yr; however, this value did not include the unknown recharge from the rest home, restaurant, or retail store. Therefore, 71 acre-ft/yr was considered to be a reasonable estimate.

Before 1971, the pumpage data were incomplete. Total model-area pumpage was often known, but the amount of pumpage from each production well could not always be deduced. One of these cases was from 1964 to 1971, when only the McKenzie \#1 and De La Guerra production wells were in operation. A single parameter was used to distribute pumpage between the McKenzie \#1 and De La Guerra wells during these years. The parameter was constrained such that the total pumpage between these two wells equaled the known total pumpage.

\section{Sensitivity Analysis}

PEST calculates an objective function value based on the sum of squared differences between measured and simulated results (Doherty, 2010). The sensitivity process in PEST calculates the change of the objective function as each parameter is varied. The sensitivity of the simulated results to a parameter is determined by the amount the objective function changes when that parameter is varied incrementally; the more the objective function changes, the more sensitive the objective function is to that parameter. Composite scaled sensitivity (CSS) values were used to show relative sensitivity; the definition and derivation are described in Doherty (2010). Note that the solute-transport parameters resulting from trialand-error calibration were included in the sensitivity analysis.

The sensitivity analysis indicated that, in general, the SBFTM is sensitive to recharge-related parameters (fig. 11A). Specifically, the SBFTM is most sensitive to parameters controlling the amount of small-catchment recharge (parameter mtnrchmult) and areal recharge (parameter rainrch1abs; fig. 11A). The SBFTM is also sensitive to parameters controlling stream-recharge rates and pumping distribution.
The CSS values for simulated hydraulic head and drawdowns are presented in figures $11 B$ and $C$, respectively. The hydraulic heads and drawdowns were sensitive to the same parameters (for example, small catchment, areal, and creeks); however, the drawdown CSS values were less than the hydraulic head CSS values. Because drawdown is a quantity expressing change (difference in initial and final head) and hydraulic head is an absolute quantity, it is not surprising that drawdown was less sensitive to the parameterization of the model.

The CSS values for simulated chloride concentrations and changes in simulated chloride concentrations are presented in figures $11 D$ and $E$, respectively. The chloride concentrations and changes in concentrations were sensitive to the same parameters (for example, recharge-related and pumpage-distribution parameters), and the three most sensitive parameters for each had very similar CSS values. For the less sensitive parameters, however, the CSS values for the chloride concentrations were greater than those for the change in chloride concentrations, for the same reason as was explained in the previous paragraph for hydraulic head and drawdown.

Of the solute-transport parameters, simulated chloride concentrations and changes in chloride concentrations were most sensitive to porosity (figs. $11 D, E$ ). Specifically, the simulated values were more sensitive to the porosity of coarse-grained sediments (parameters mpclower and mpc in figs. $11 D, E$ ) than to that of fine sediment (parameter $\mathrm{mpf}$ ) because pumping in the coarse-grained layers draws seawater landward. Dispersivity parameters were generally less sensitive than the porosity parameters; deep longitudinal dispersivity (parameter dispdp) was the most sensitive dispersivity parameter, with a CSS value of 1.39 (fig. 11D).

Figure $11 F$ presents the CSS values for the simulated values overall and for four of the simulated variables or their derivatives: hydraulic head, change in hydraulic head, chloride concentration, and change in chloride concentration; sensitivity scores were grouped by parameter type (horizontal axis). It is misleading to compare the CSS values within a parameter group because the CSS values are functions of the simulated values. Simulated chloride concentrations were as high as $19,000 \mathrm{mg} / \mathrm{L}$, whereas simulated hydraulic head values might be in the hundreds; therefore, the CSS values for chloride were greater than those for hydraulic head. For comparative purposes, the CSS values in a parameter group were divided by the greatest value in order to normalize the CSS values (fig. 12). The most sensitive parameter groups were fault conductance, creek stage, creek conductance, and specific storage related (fig. 12). The least sensitive parameter groups were transport related (dispersion and porosity; fig. 12). 

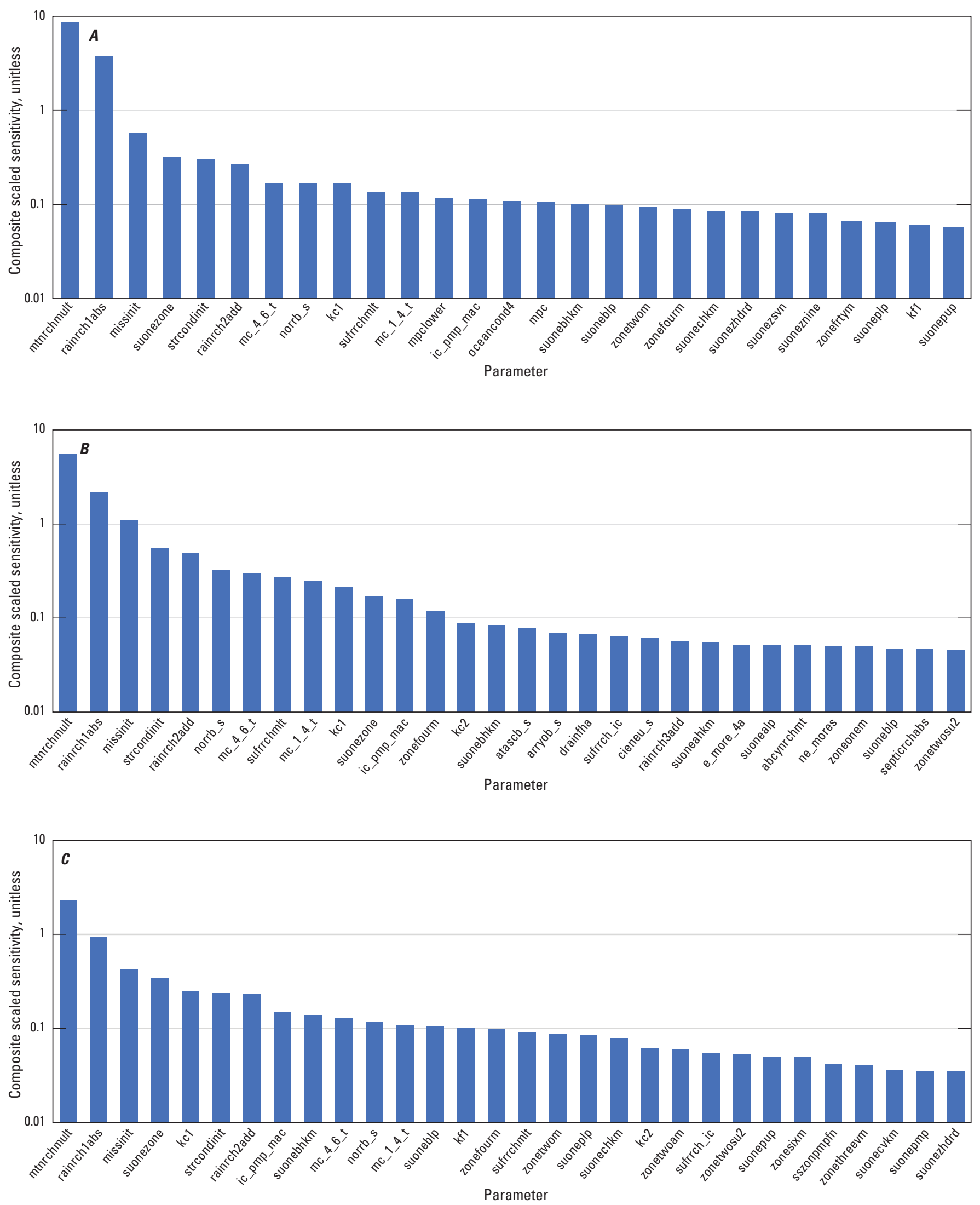

Figure 11. Composite scaled sensitivity values for the Santa Barbara flow and transport model, Santa Barbara, California: $A$, all simulated values; $B$, hydraulic head; $C$, drawdown; $D$, chloride concentration; $E$, change in chloride concentration; and $F$, grouped by parameter type. 

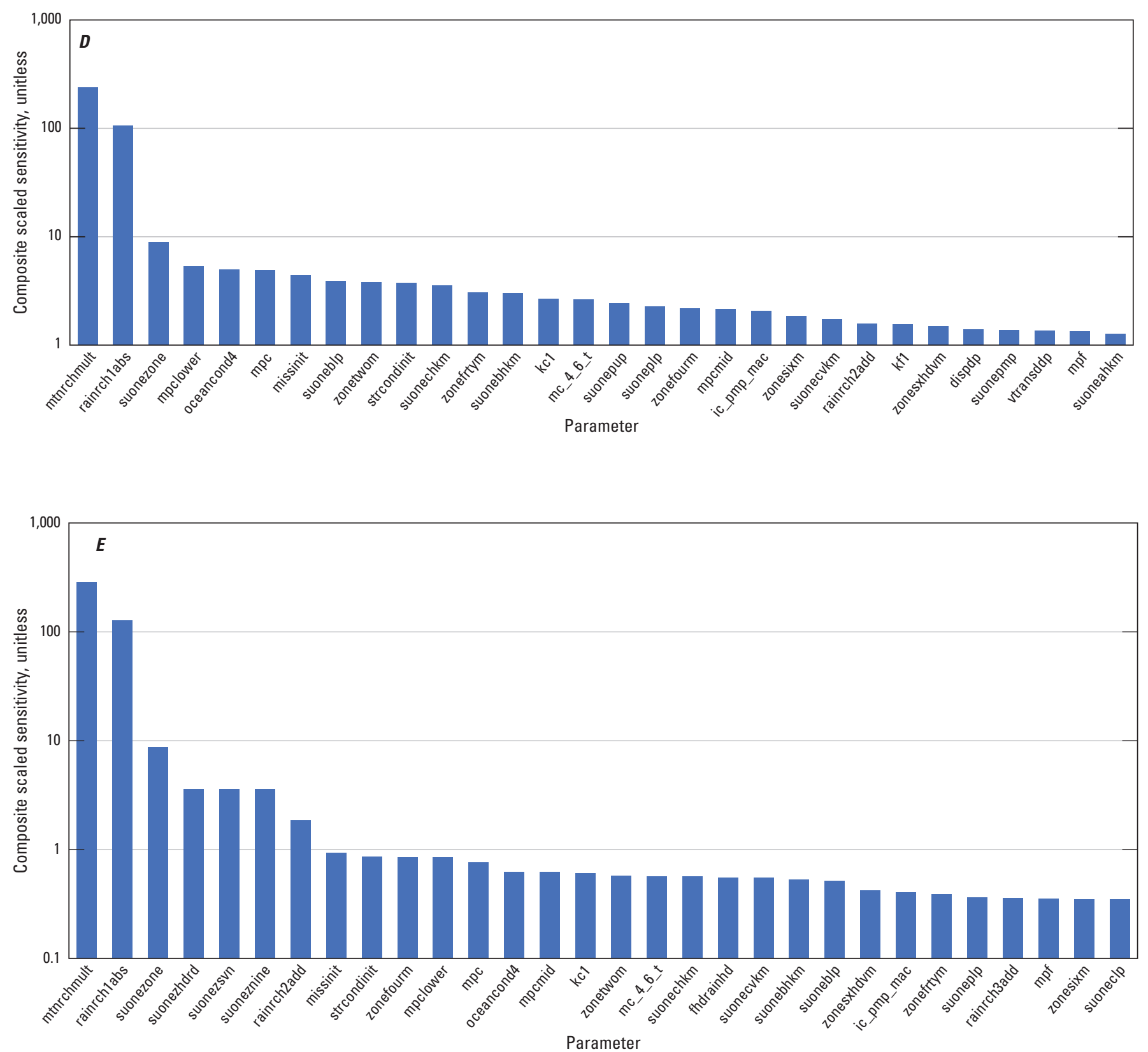

Figure 11. - Continued 


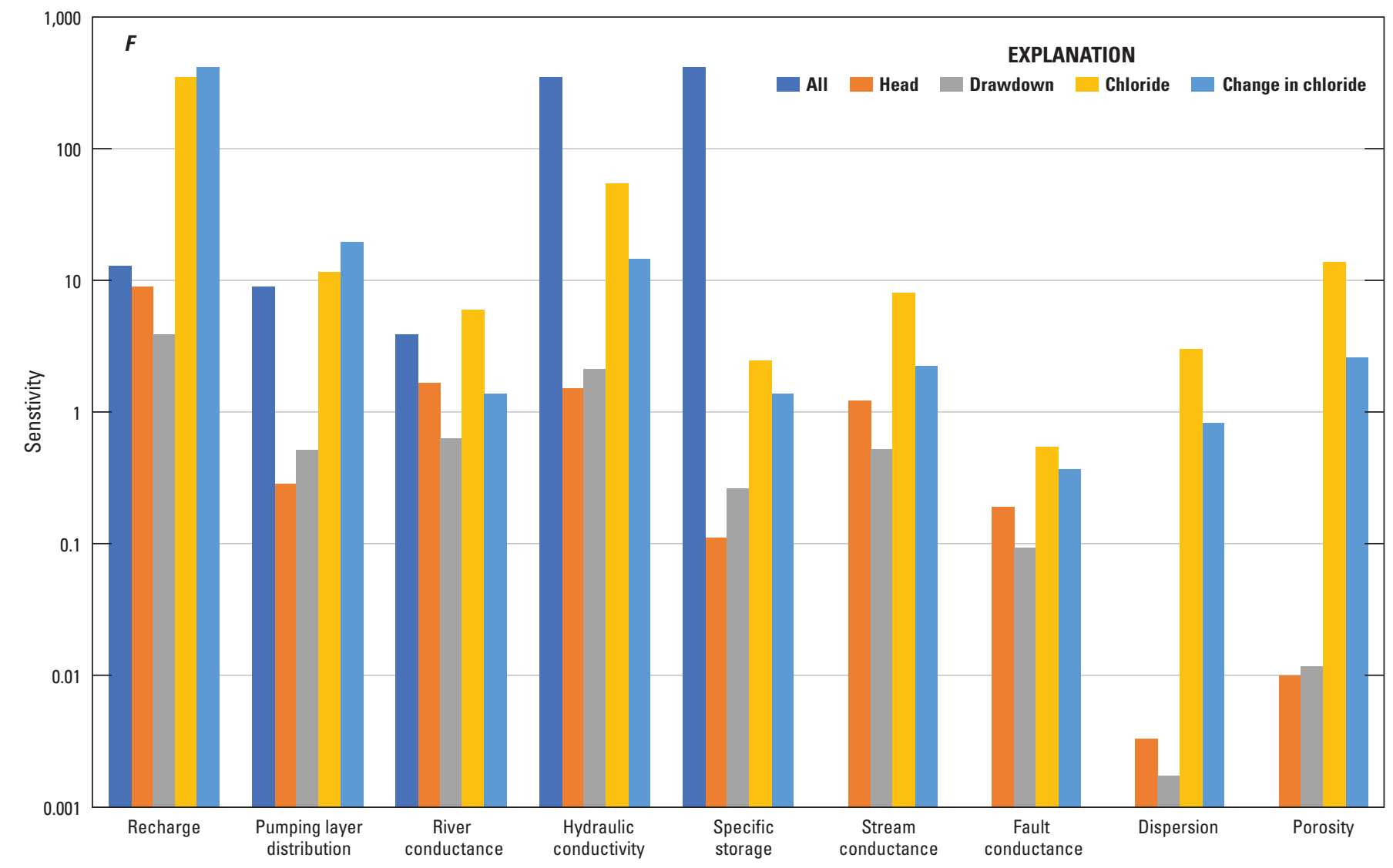

Parameter group

Figure 11. - Continued

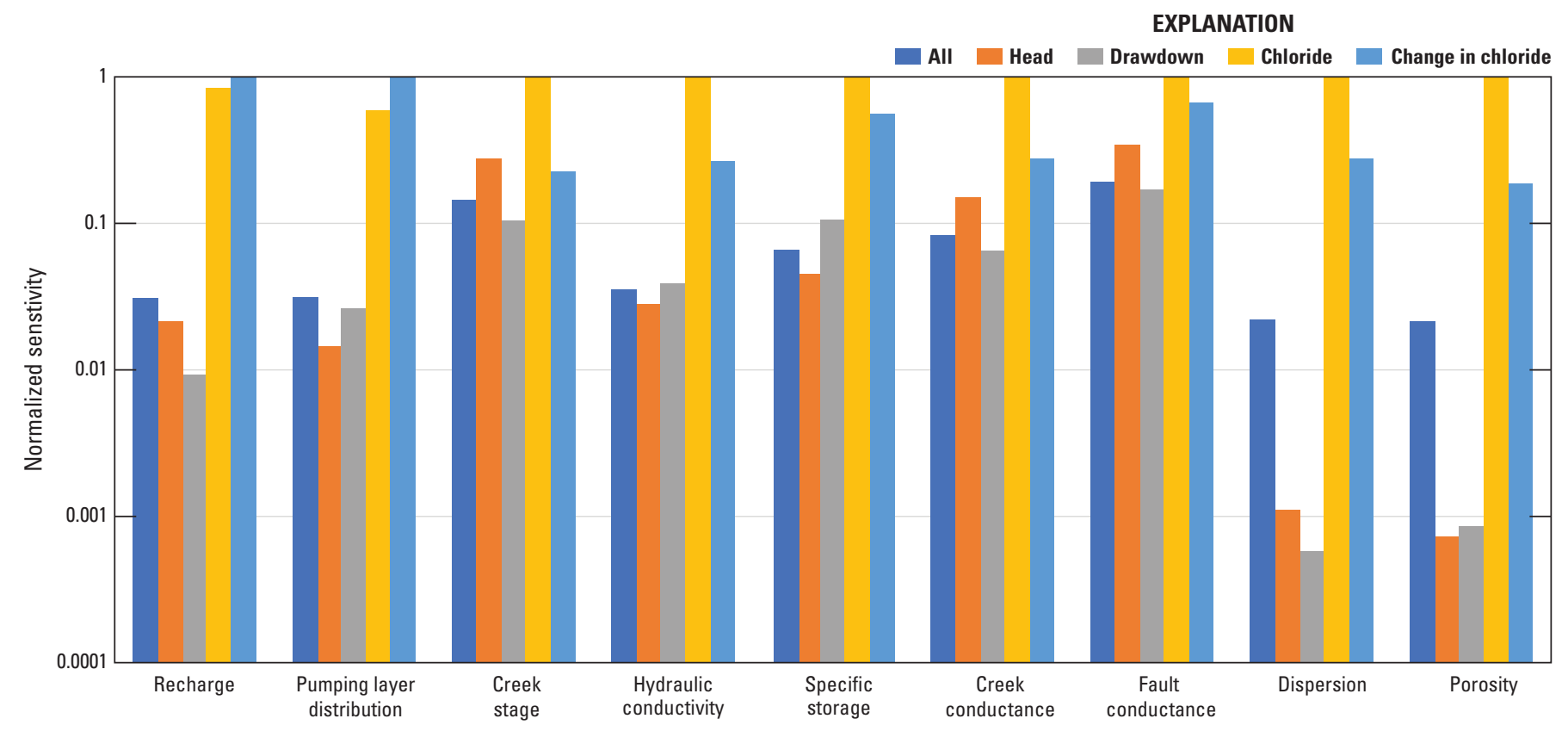

Parameter group

Figure 12. Normalized composite scaled sensitivity values, grouped by parameter types for all simulated values, hydraulic head, drawdown, chloride concentration, and change in chloride concentration, Santa Barbara flow and transport model, Santa Barbara, California. 


\section{Simulation Results}

Hydraulic-conductivity and storage values were not estimated directly through parameter estimation. Values for these flow parameters were calculated using the extent of the parameter range and multipliers. A brief discussion regarding these parameters follows.

The majority of calibrated hydraulic conductivities ranged from about $10^{2}$ to about $10^{-5} \mathrm{ft} / \mathrm{d}$ (fig. 13). Horizontal hydraulic conductivities had a median value of $1.02 \mathrm{ft} / \mathrm{d}$ and were concentrated over a narrow range; more than 74 percent were between 0.5 and $50.0 \mathrm{ft} / \mathrm{d}$. Vertical hydraulic conductivities had a median value of $0.02 \mathrm{ft} / \mathrm{d}$ and were also concentrated over a narrow range; more than 56 percent were between 0.005 and $0.5 \mathrm{ft} / \mathrm{d}$. The median anisotropy ratio of vertical to horizontal hydraulic conductivity was 0.03 .

Land subsidence has not been detected in the Santa Barbara area. If the estimated storage values indicate land subsidence, therefore, the estimated storage values are not reasonable (Devin L. Galloway, U.S. Geological Survey, written commun., 2015). The SBFTM results were used to estimate land-surface subsidence due to compaction on the basis of water-level drawdowns and specific-storage values. Subsidence was calculated to verify that the model calibrated specific-storage values were realistic for the Santa Barbara groundwater basin, where no land-surface subsidence has been detected. Compaction $(\Delta b)$ was calculated using equation 8 (Devin L. Galloway, U.S. Geological Survey, written commun, 2015):

$$
\Delta b=-S_{s k} \times b \times \Delta h
$$

where

$$
\begin{aligned}
S_{s k} & \text { is skeletal specific storage, } \\
b & \text { is layer thickness, and } \\
\Delta h & \text { is drawdown in head. }
\end{aligned}
$$

Equation 8 was used to estimate compaction in areas of the SBFTM where drawdowns due to pumping were large. Maximum estimated compaction was approximately 1 inch during the simulation period. Land-surface subsidence has not been detected in the study area; however, an inch of compaction might or might not be detected by geodetic surveys. Therefore, the subsidence estimates based on SBFTM parameter values and simulation results were consistent with no detected subsidence.

\section{Groundwater Budget}

Figure 14 shows the simulated annual hydrologic budgets for the entire model area and the subbasins for two different simulation periods. In general, the budgets for 1929 to 1971 are based on annual stress periods and the budgets for 1972 to 2013 are based on monthly stress periods. The SBFTM hydrologic budget for the entire model area from
1929 to 1971 is shown in figure $14 A$, and the budget for the entire model area from 1972 to 2013 is shown in figure $14 B$. Simulated recharge was mostly from small catchments and creeks; there was only a small amount of areal recharge, and recharge across the offshore fault was limited to times of heavy pumping (figs. 14A, $B$ ). Small-catchment recharge was the primary source of recharge during wetter periods, when there is little pumping; net creek recharge was relatively low because of higher groundwater levels, which resulted in discharge from the groundwater system to the creeks (figs. 14A, B). During times of relatively high pumping rates, usually during dry years, creek leakage was the primary source of recharge. Note that it was assumed that the creek stages (that is, the depth of the creek above the creek bed) were always greater than zero; therefore, the creeks are a constant source of water in the model.

Between 1929 and 1971 discharge due to pumping varied greatly (fig. 14A). Pumping rates generally were higher during drier periods and lower during wetter periods (fig. 14A). There was a net loss in storage from 1929 to 1946 because of a small amount of pumping (fig. 15). Pumpage in the Santa Barbara and Foothill basins increased after 1947, particularly during dry periods (fig. 14A). From 1949 to 1952, heavy pumpage resulted in a loss of about 9,200 acre-ft from storage (fig. 15). Pumpage decreased between 1953 and 1960, resulting in about 2,900 acre-ft of storage being recovered. Higher pumpage resumed from 1961 to 1971, resulting in an additional loss of 8,300 acre-ft from storage.

A general decrease in pumpage from 1972 to 1983 in comparison with the previous eight years (figs. 14A, B) resulted in the recovery of about 6,200 acre-ft of storage, which was then lost because of heavy pumping between 1984 and 1990 (figs. 14B, 15). From 1991 to 2006, pumpage was greatly reduced in general, leading to the recovery of over 10,800 acre-ft of storage. Pumping increased generally from 2007 to 2013, resulting in a loss of over 2,900 acre-ft of storage. Overall, groundwater pumping resulted in a net loss of about 11,400 acre-ft of groundwater storage between 1929 and 2013 (fig. 15).

From 1929 to 1971 , the majority of water entering the SBFTM was from creek and small catchment recharge, and the majority of the water leaving the SBFTM was from pumping, discharge to creeks (baseflow), and drains (table $4 A$ ). In addition, about 37 percent of the total pumpage was loss from storage. From 1972 to 2013, the majority of water entering the SBFTM was from creek and small catchment recharge, and the majority of the water leaving the SBFTM was from pumping, discharge to creeks (baseflow), and drains (table $4 B$ ). In addition, the reduction in pumpage during this period resulted in about 7,800 acre-ft of water being added to storage. During July 1990, a month when approximately 705 acre-ft of groundwater was pumped in the study area, pumpage was much greater than all sources of recharge combined, and about 382 acre-ft of water was removed from storage; therefore, storage accounted for about 54 percent of groundwater pumpage (table $4 C$ ). 

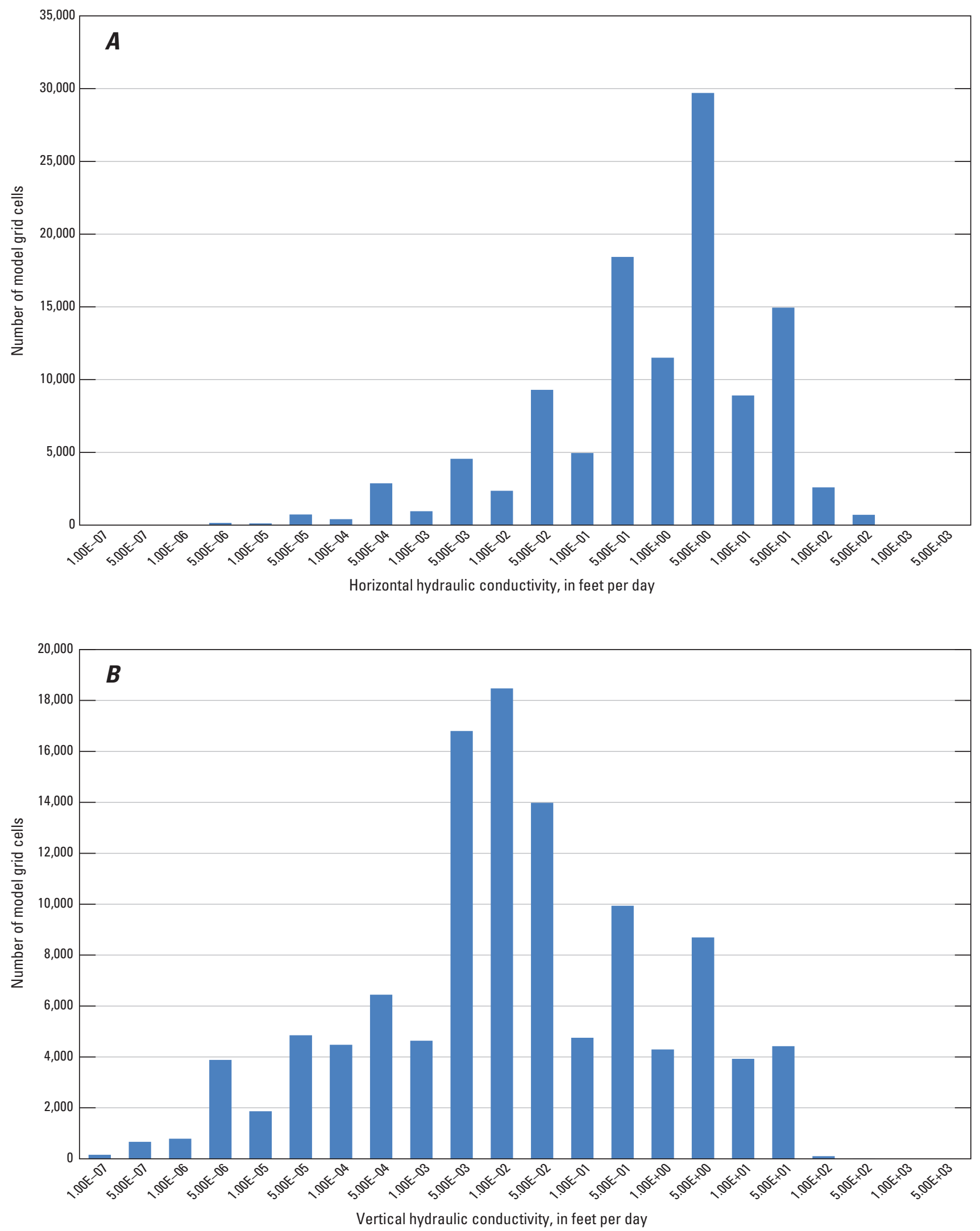

Figure 13. Distribution of hydraulic conductivity values used in the Santa Barbara flow and transport model, Santa Barbara, California: $A$, horizontal, and $B$, vertical. 

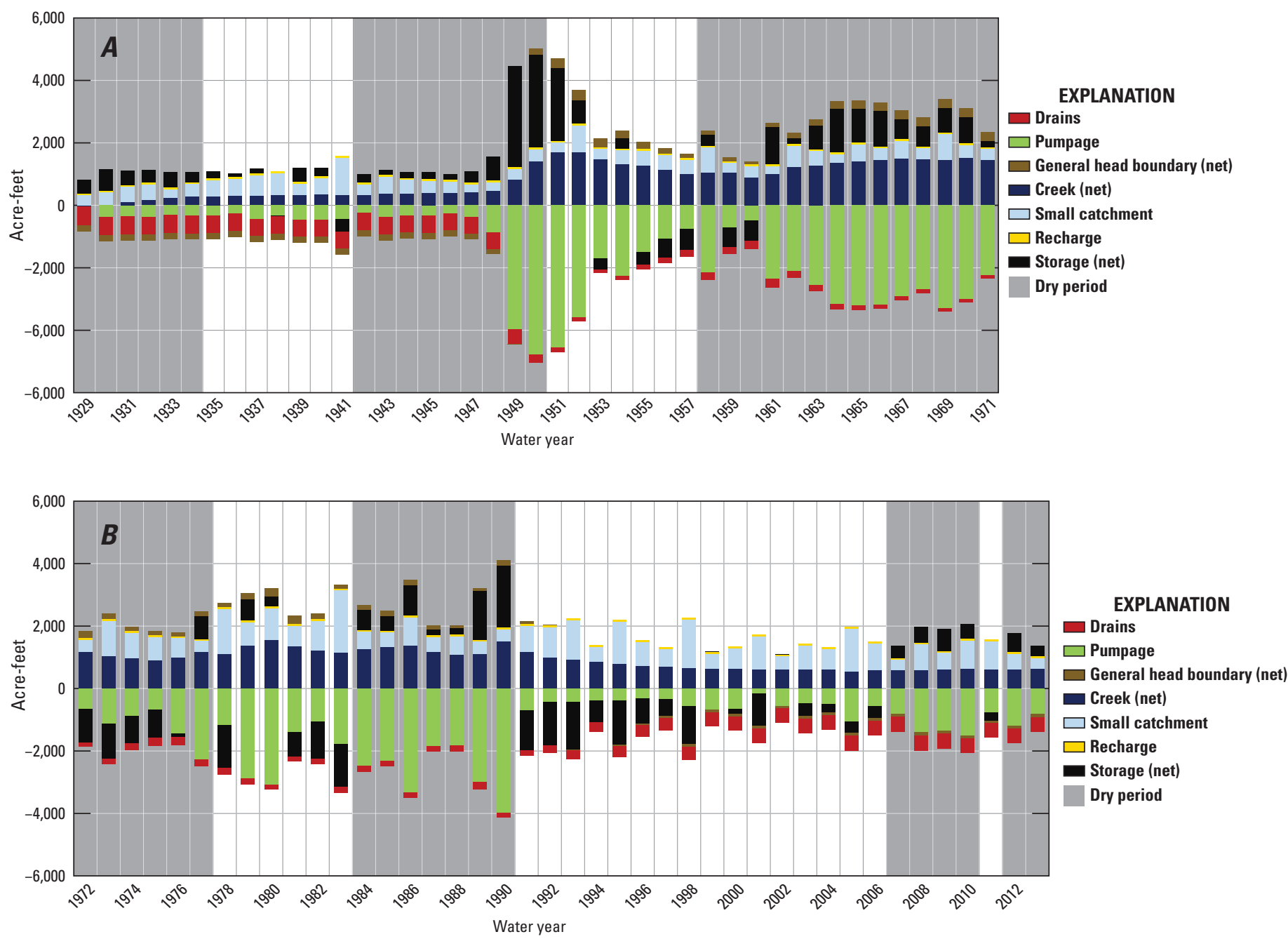

Figure 14. Simulated annual hydrologic budget for two simulation periods, Santa Barbara flow and transport model, Santa Barbara, California: $A$, entire model area, 1929-71; $B$, entire model area, 1972-2013; $C$, Storage Unit I, 1929-71; $D$, Storage Unit I, 1972-2013; E, Storage Unit III, 1929-71; F, Storage Unit III, 1972-2013; G, East Foothill subbasin, 1929-71; H, East Foothill subbasin, 1972-2013; I, West Foothill subbasin, 1929-71; and J, West Foothill subbasin, 1972-2013. 

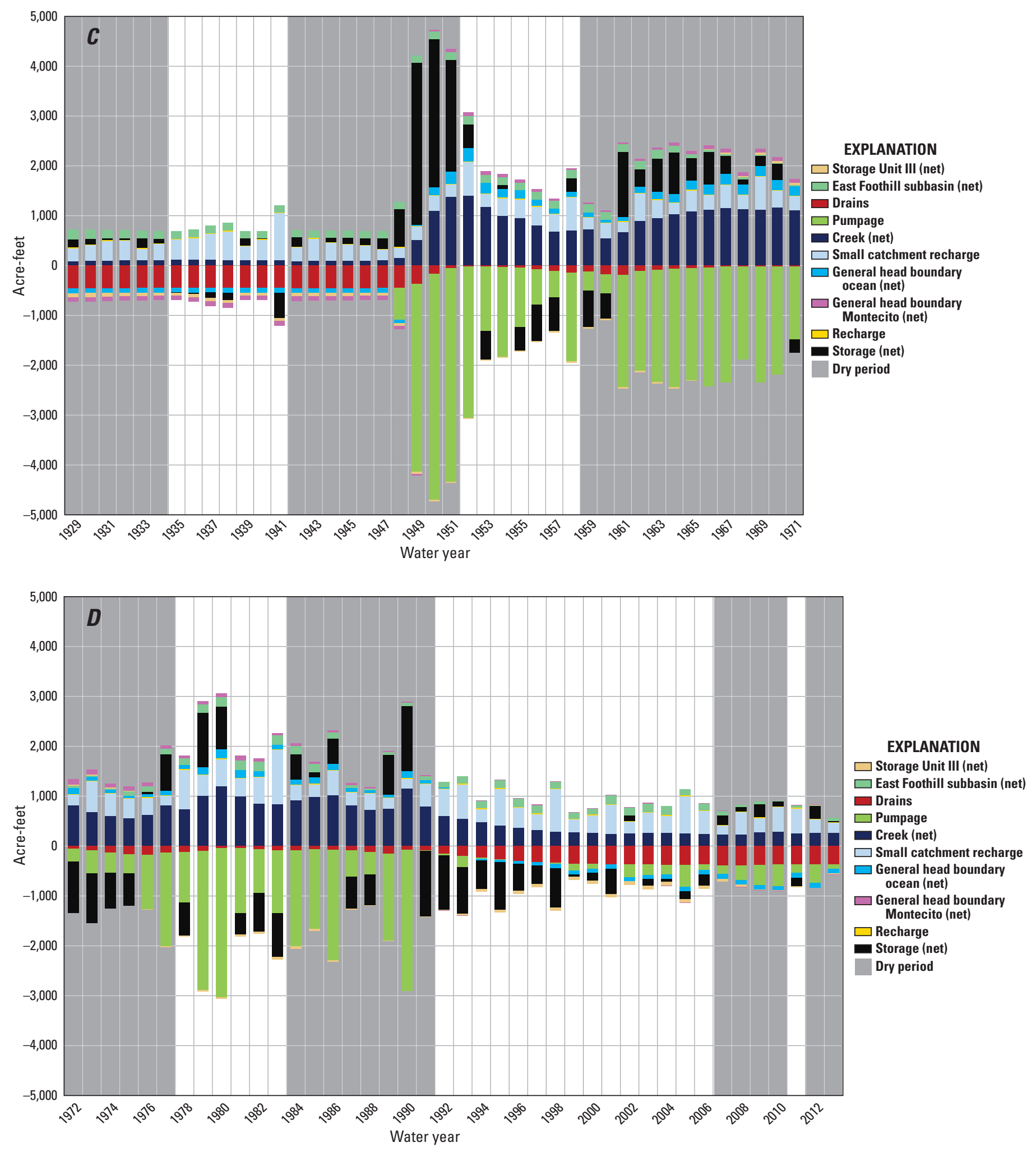

Figure 14. - Continued 

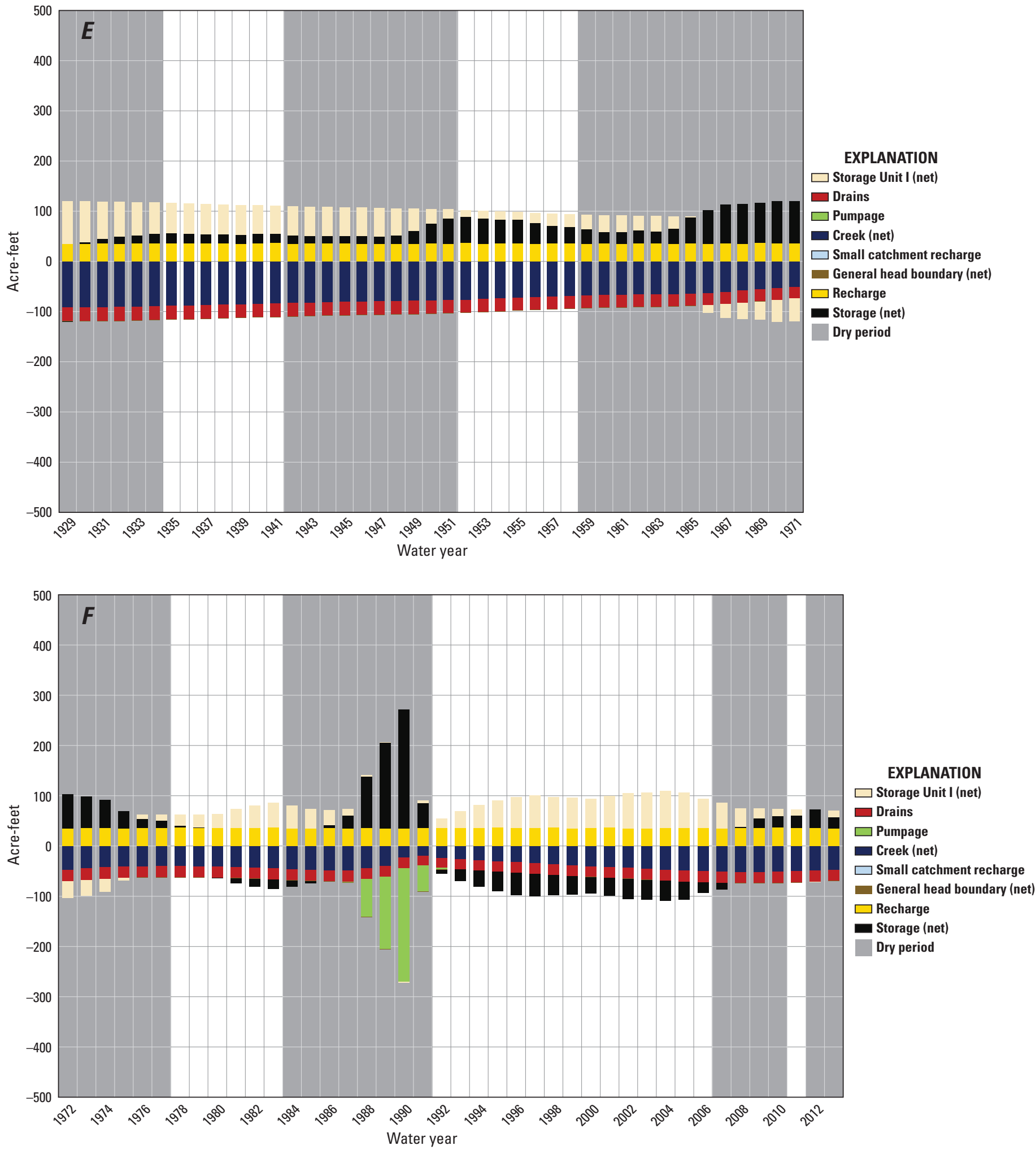

Figure 14. -Continued 

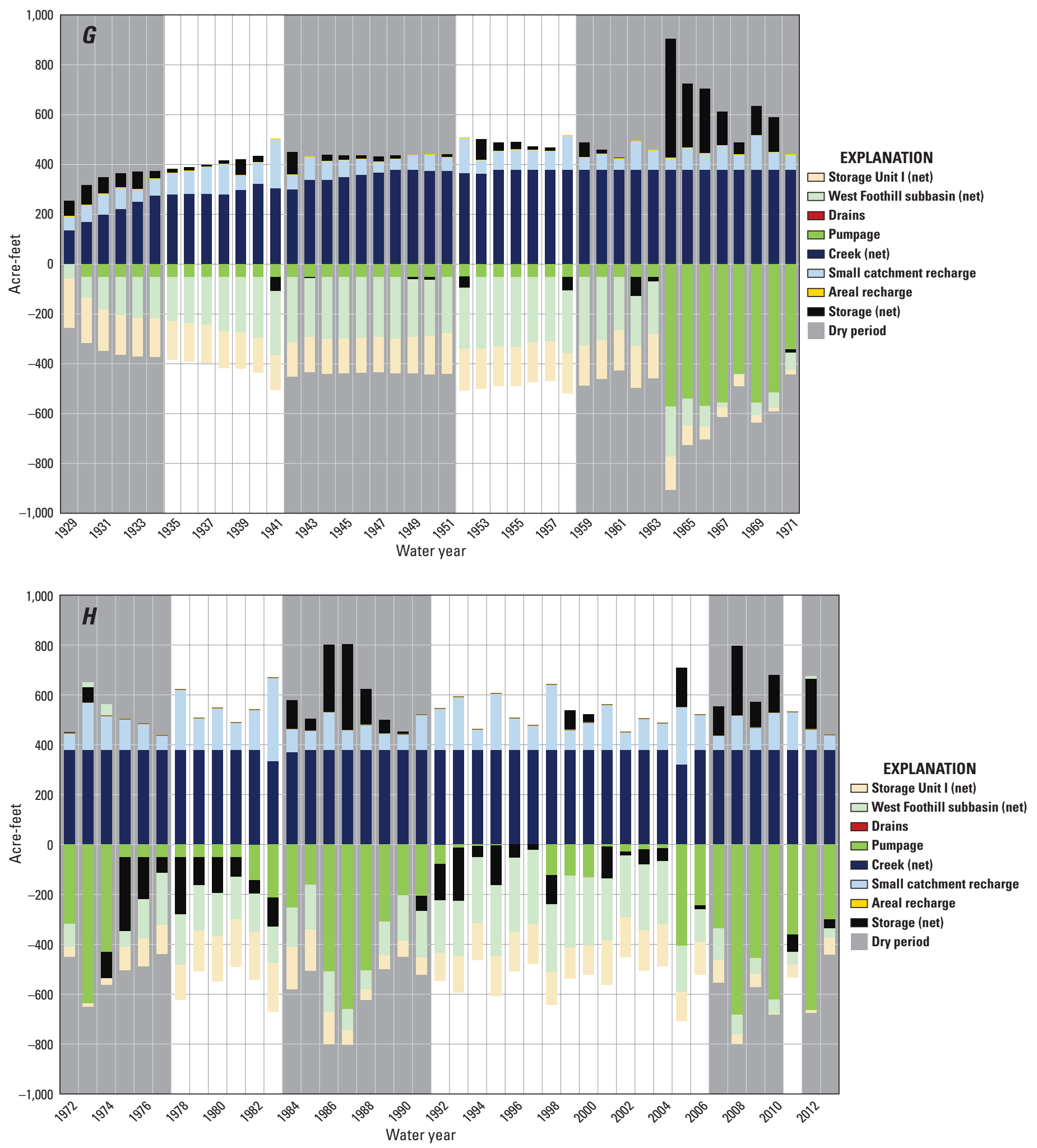

Figure 14. - Continued 

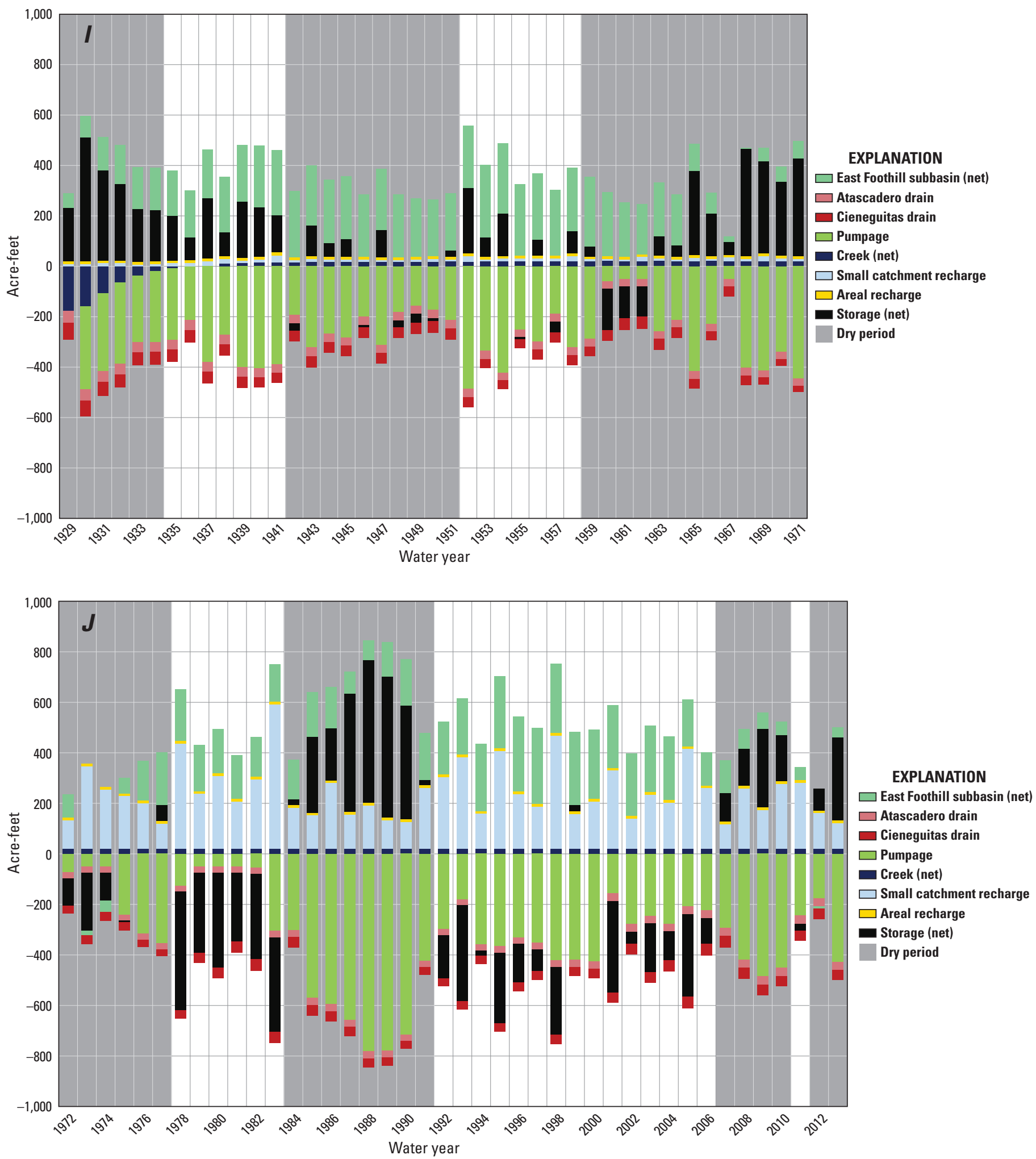

Figure 14. - Continued 


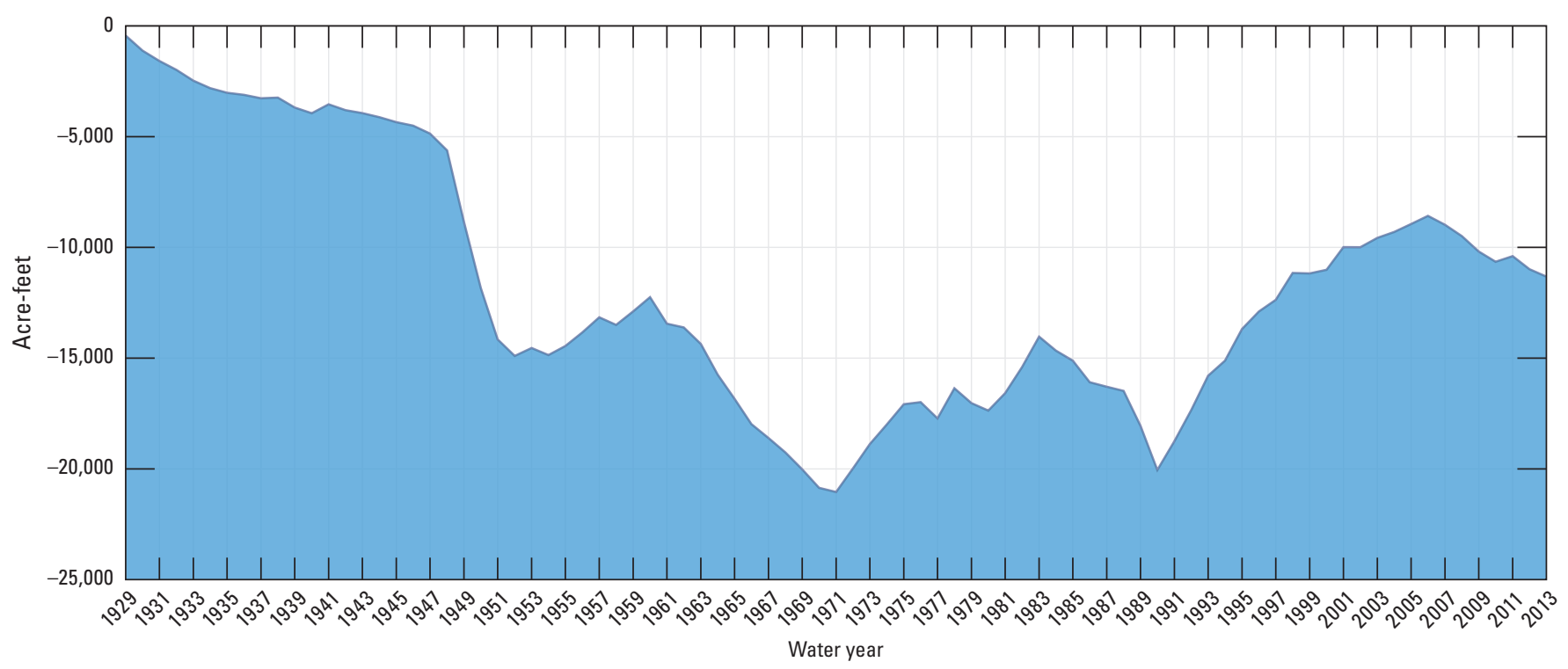

Figure 15. Simulated cumulative change in groundwater storage, 1929-2013, Santa Barbara flow and transport model, Santa Barbara, California.

Table 4A. Simulated cumulative groundwater budget, 1929-71, Santa Barbara flow and transport model, Santa Barbara, California.

\begin{tabular}{lrrrrrr}
\hline & \multicolumn{4}{c}{ Total volume of water, in acre-feet, 1929-71 } \\
\cline { 2 - 7 } & Storage & Wells & Drains & Recharge & $\begin{array}{c}\text { General-head } \\
\text { boundary }\end{array}$ & $\begin{array}{c}\text { Small } \\
\text { Creek }\end{array}$ \\
\hline In & 65,018 & 0 & 0 & 5,485 & 10,353 & 111,215 \\
Out & 22,527 & 131,311 & 31,357 & 0 & 8,230 & 39,363 \\
Change & 42,491 & $-131,311$ & $-31,357$ & 5,485 & 2,123 & 71,852 \\
\hline
\end{tabular}

Mass balance $42,491-131,311-31,357+5,485+2,123+71,852+40,528=-189$

Table 4B. Simulated cumulative groundwater budget, 1972-2013, Santa Barbara flow and transport model, Santa Barbara, California.

\begin{tabular}{|c|c|c|c|c|c|c|c|}
\hline & \multicolumn{7}{|c|}{ Total volume of water, in acre-feet, 1972-2013 } \\
\hline & Storage & Wells & Drains & Recharge & $\begin{array}{c}\text { General-head } \\
\text { boundary }\end{array}$ & Creek & $\begin{array}{c}\text { Small } \\
\text { catchment }\end{array}$ \\
\hline Out & 85,537 & 106,307 & 26,833 & 0 & 4,615 & 30,456 & 0 \\
\hline Change & $-16,858$ & $-106,307$ & $-26,833$ & 5,320 & 2,567 & 76,307 & 65,761 \\
\hline
\end{tabular}

Table 4C. Simulated water budget, July 1990, Santa Barbara flow and transport model, Santa Barbara, California.

\begin{tabular}{|c|c|c|c|c|c|c|c|}
\hline & \multicolumn{7}{|c|}{ Total volume of water, in acre-feet, July 1990} \\
\hline & Storage & Wells & Drains & Recharge & $\begin{array}{c}\text { General-head } \\
\text { boundary }\end{array}$ & Creek & $\begin{array}{c}\text { Small } \\
\text { catchment }\end{array}$ \\
\hline Out & 18 & 705 & 25 & 0 & 3 & 16 & 0 \\
\hline Change & 382 & -705 & -25 & 10 & 29 & 262 & 47 \\
\hline
\end{tabular}

Mass balance $382-705-25+10+29+262+47=-1$ 
The SBFTM hydrologic budget from 1929 to 1971 by subbasin is shown in figures $14 C, E, G$, and $I$. The SBFTM hydrologic budget from 1972 to 2013 by subbasin is shown in figures $14 D, F, H$, and $J$. The hydrologic budget for Storage Unit $I$ is shown in figures $14 C$ and $D$. The primary sources of recharge were small catchment recharge and net inflow from creeks, although during times of high pumping (for example, 1950 in fig. 14C) water removed from groundwater storage was the primary source of water to the groundwater system. In addition to pumping, the primary groundwater discharges were drains and water added to storage.

The hydrologic budget for Storage Unit III is shown in figures $14 E$ and $F$ (note the much lower values than Storage Unit I). There were three sources of recharge to Storage Unit III: inflow from Storage Unit I, net inflow from groundwater storage, and surface recharge (precipitation), but during times of low inflow from Storage Unit I or outflow to Storage Unit I, water removed from groundwater storage was the primary source of water to the groundwater system (fig. 14E). The primary discharge sinks were net creek discharge and outflow from the subbasin (drains).

The hydrologic budget for East Foothill subbasin is shown in figures $14 G$ and $H$. The primary sources of recharge were net creek inflow and small catchment recharge. The primary discharge sinks were pumpage (after 1964) and net discharge to Storage Unit I and West Foothill subbasin.

The hydrologic budget for West Foothill subbasin is shown in figures $14 I$ and $J$. The primary sources of recharge were net inflow from East Foothill subbasin, inflow from groundwater storage, and small catchment recharge. The primary discharge sinks were pumpage, groundwater added to storage, and outflow from the subbasin (drains).

\section{Simulated Groundwater Flow and Movement}

In order to examine changes in hydraulic conditions related to pumpage, the simulated hydraulic heads for initial conditions (1929) and July 1990 were examined for each producing zone. July 1990 was the end of an extended period of high pumping in Storage Unit I and moderate pumping in the Foothill groundwater basin.

In the upper producing zone, the simulated initialcondition hydraulic heads indicated that groundwater in the Foothill groundwater basin generally flowed from the Mission and Lauro Canyons west and southwest toward the southwest corner of the basin and across the Modoc fault to the Goleta groundwater basin (fig. 16A). Groundwater also flowed to the Santa Barbara groundwater basin by moving across the East More Ranch fault at Arroyo Burro and, to a limited extent, across other parts of the fault as well. In Storage Unit I, groundwater generally flowed east from the East More Ranch fault toward the Pacific Ocean (fig. 16A). Note that the simulated hydraulic heads near the ocean in Storage Unit I were higher than sea level; therefore, with the exception of density effects, seawater would not intrude under these hydraulic conditions. In Storage Unit III, groundwater generally flowed to the southeast (fig. 16A).
The middle producing zone only exists in Storage Unit I. The contours of simulated initial-condition hydraulic head indicated that groundwater flowed east toward the Pacific Ocean (fig. 16B).

In the lower producing zone, the simulated initialcondition hydraulic heads indicate that groundwater in the Foothill groundwater basin generally flowed from the Mission and Lauro Canyons west and southwest toward the southwest corner of the basin (fig. 16C). Unlike the upper producing zone, the lower producing zone was not simulated as connecting hydraulically to the Goleta groundwater basin; therefore, groundwater did not cross into the Goleta groundwater basin, as it did in the upper producing zone. In Storage Unit I, groundwater generally flowed east from the East More Ranch fault toward the Pacific Ocean (fig. 16C). In Storage Unit III, groundwater generally flowed to the southeast, except where it deflects more southward along creek channels (fig. 16C).

Contours of simulated July 1990 hydraulic heads for the upper, middle, and lower producing zones are shown in figure 17 . In general, the simulated July 1990 hydraulic heads were much lower than the initial conditions as a result of groundwater pumping. There was a simulated groundwater low in all three producing zones in Storage Unit I near the Ortega Park production well (fig. 17). In addition, there was a groundwater low in the lower producing zone only in the West Foothill subbasin near the Los Robles production well (fig. 17C). Simulated July 1990 hydraulic heads in all three producing zones were below sea level in the coastal area of Storage Unit I; therefore, seawater intrusion in the producing zones was possible.

In the upper producing zone, the simulated July 1990 hydraulic heads indicated that groundwater in the Foothill groundwater basin generally flowed from the Mission and Lauro Canyons west and southwest toward the southwest corner of the basin and across the Modoc fault to the Goleta groundwater basin (fig. 17A). In Storage Unit I, the simulated July 1990 hydraulic heads indicated that groundwater flowed east toward the groundwater low near the Ortega Park production well (fig. 17A). In Storage Unit III, the simulated July 1990 hydraulic heads indicated that groundwater flowed south toward a groundwater low near the Val Verde production well (fig. 17A).

Again, the middle producing zone only exists in Storage Unit I. The simulated results indicated that groundwater flowed radially toward the groundwater low near the Ortega Park production well (fig. 17B).

In the lower producing zone, the simulated July 1990 hydraulic heads indicated that groundwater in the West Foothill subbasin flowed radially toward the groundwater low near the Los Robles production well (fig. 17C). In the East Foothill subbasin, groundwater flowed west and southwest. In Storage Unit I, groundwater flowed east from the East More Ranch fault, then radially toward the groundwater low (fig. 17C). In Storage Unit III, groundwater generally flowed south toward a low near the Val Verde production well (fig. 17C). 


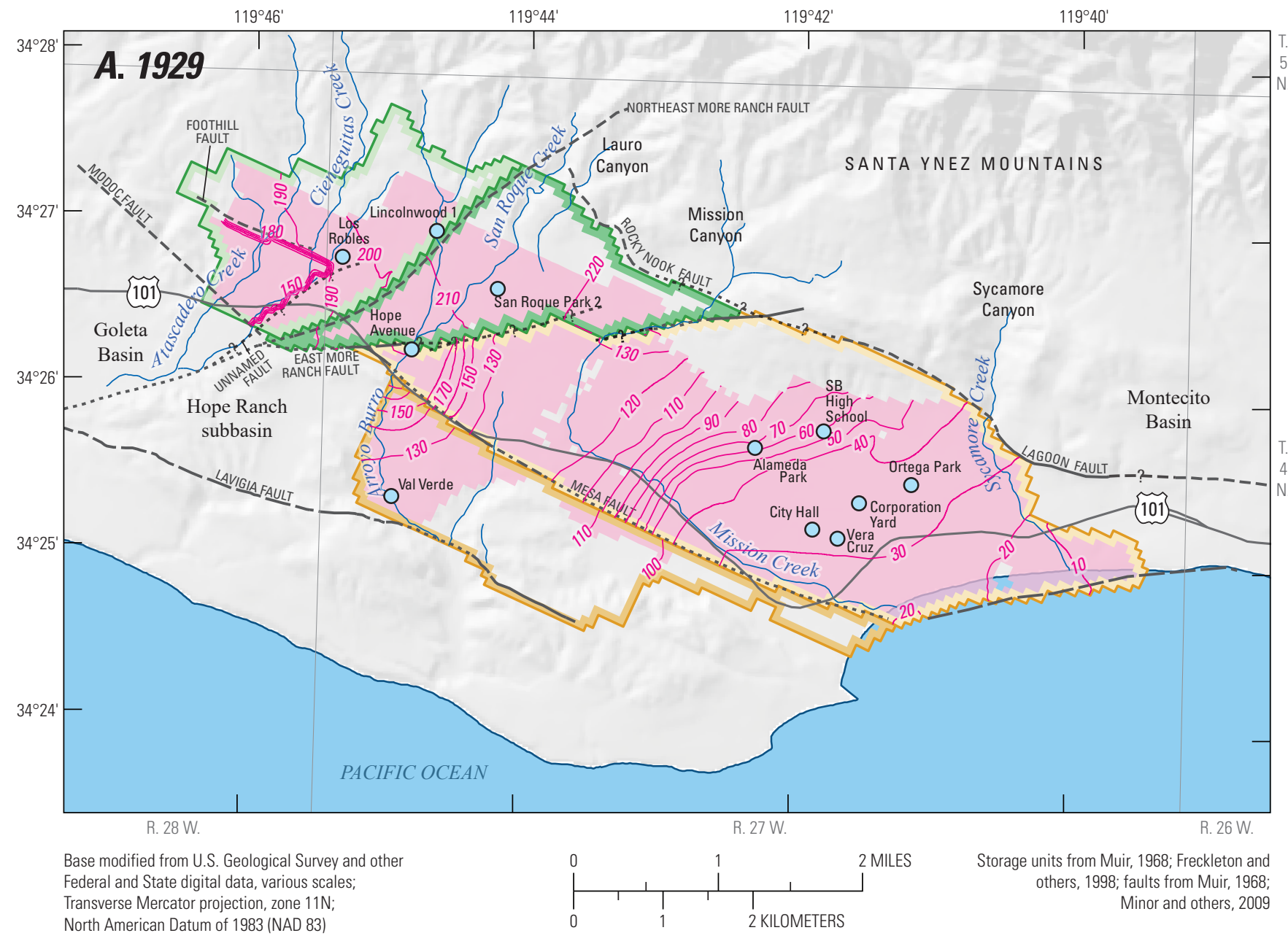

EXPLANATION

\begin{tabular}{|c|c|}
\hline & $\begin{array}{l}\text { Upper producin } \\
\text { zone }\end{array}$ \\
\hline \multicolumn{2}{|c|}{ Subbasins } \\
\hline & West Foothill \\
\hline 0 & East Foothill \\
\hline & Storage Unit I \\
\hline 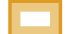 & Storage Unit III \\
\hline
\end{tabular}

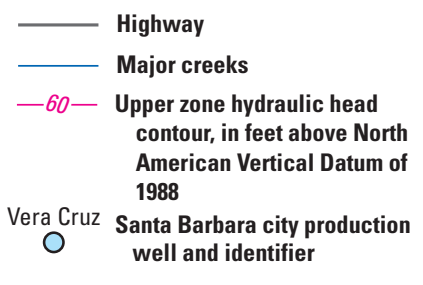

Faults

- - - Existence certain, location inferred

- -? - Existence questionable, location inferred

........ Existence certain, location concealed

...? ... Existence questionable, location concealed

_- Existence certain, location accurate

- Existence certain, location approximate

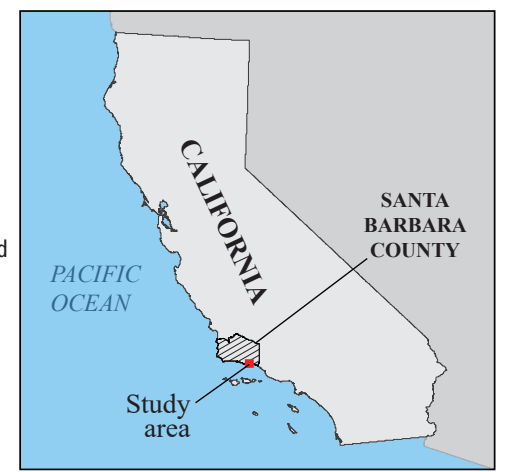

Figure 16. Contours of simulated initial-condition (average 1929) hydraulic head for producing zones of the Santa Barbara groundwater basin, California: $A$, upper; $B$, middle; and $C$, lower. 

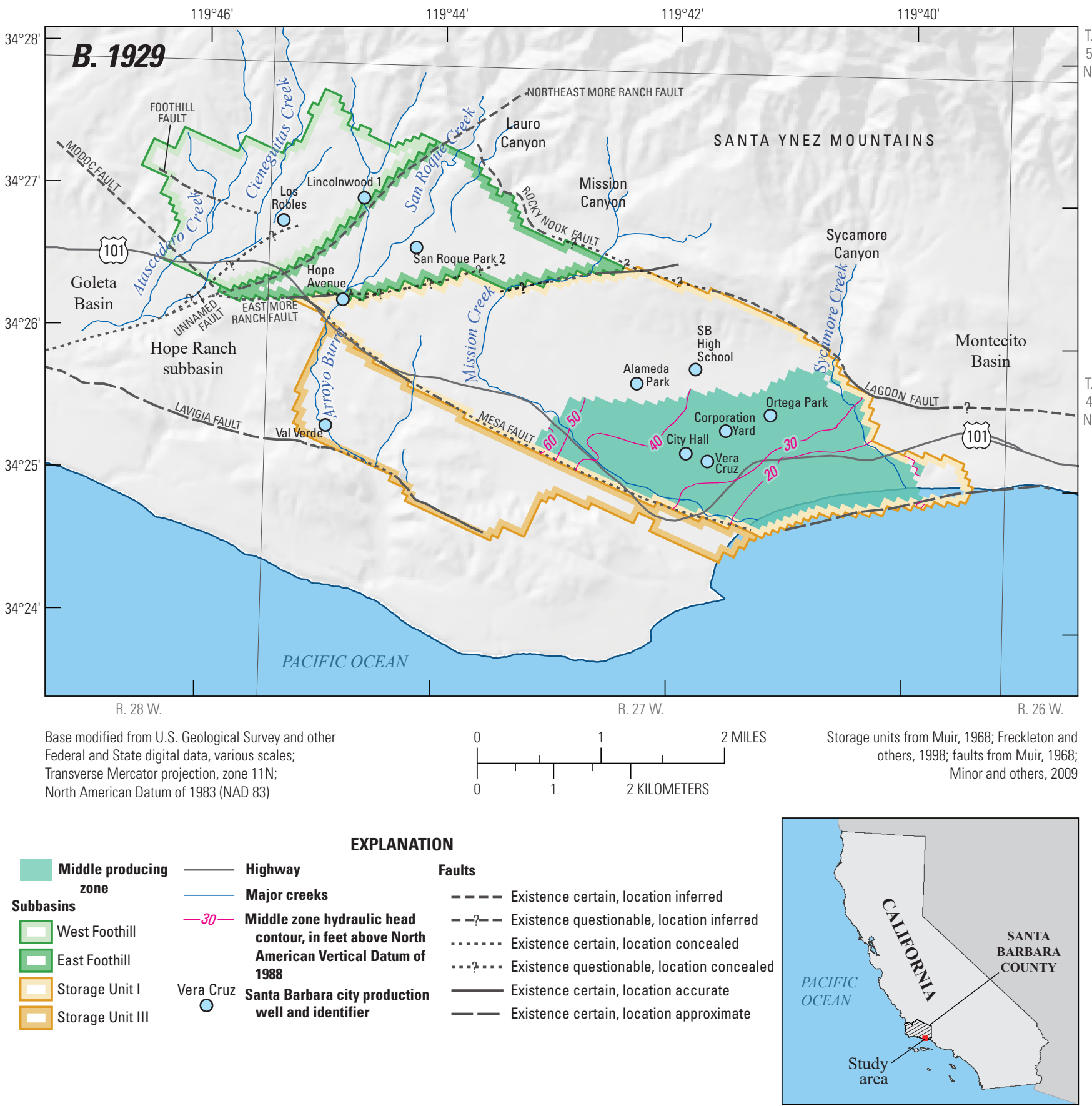

Figure 16. - Continued 


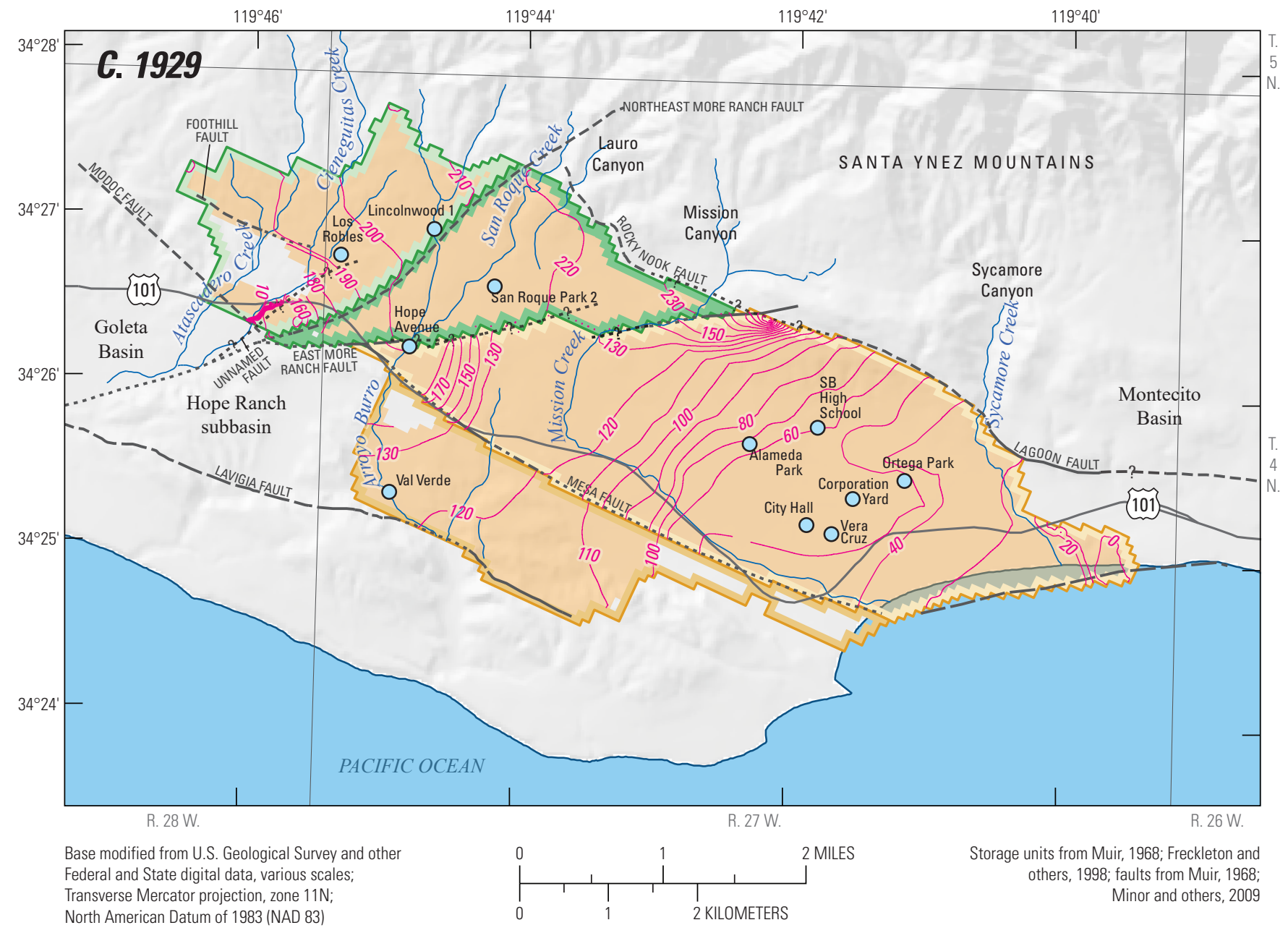

rcator projection, zone 111

\section{EXPLANATION}

\begin{tabular}{|c|c|}
\hline & $\begin{array}{l}\text { Lower producin } \\
\text { zone }\end{array}$ \\
\hline \multicolumn{2}{|c|}{ Subbasins } \\
\hline & West Foothill \\
\hline & East Foothill \\
\hline & Storage Unit I \\
\hline & Storage Unit II \\
\hline
\end{tabular}

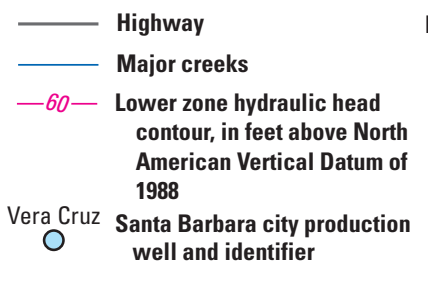

Faults

- - Existence certain, location inferred

- -? - Existence questionable, location inferred

....... Existence certain, location concealed

...? ... Existence questionable, location concealed

_- Existence certain, location accurate

—— Existence certain, location approximate

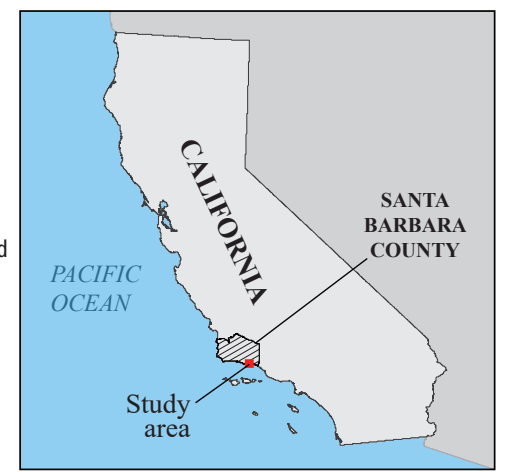

Figure 16. —Continued 


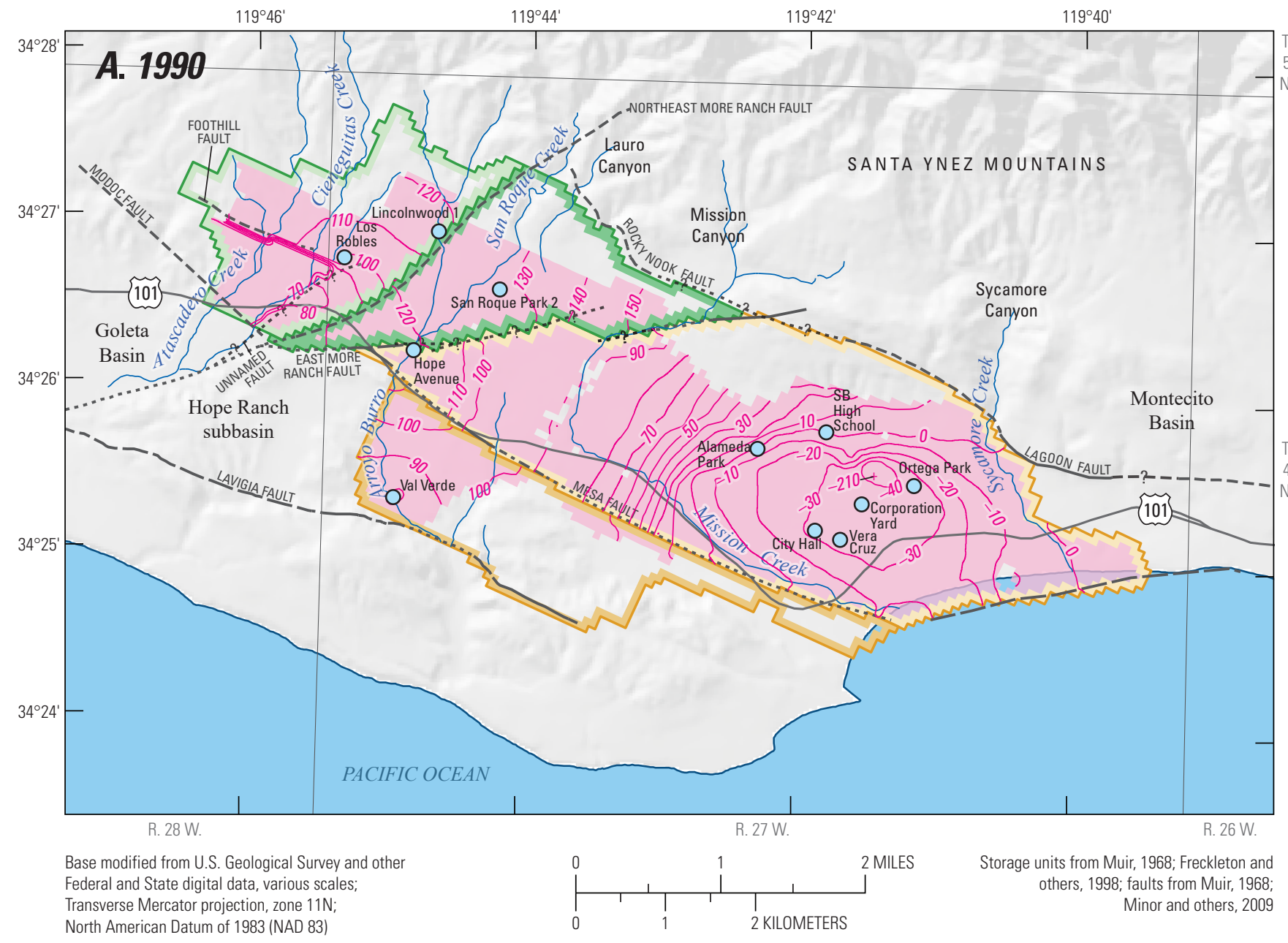

EXPLANATION

\begin{tabular}{|c|c|}
\hline & $\begin{array}{l}\text { Upper producin } \\
\text { zone }\end{array}$ \\
\hline \multicolumn{2}{|c|}{ Subbasins } \\
\hline & West Foothill \\
\hline $\mathrm{E}$ & East Foothill \\
\hline & Storage Unit I \\
\hline S & Storage Unit III \\
\hline
\end{tabular}

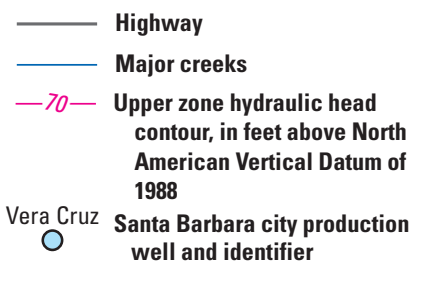

Faults

- - - Existence certain, location inferred

- -? - Existence questionable, location inferred

........ Existence certain, location concealed

...? ... Existence questionable, location concealed

_- Existence certain, location accurate

_- Existence certain, location approximate

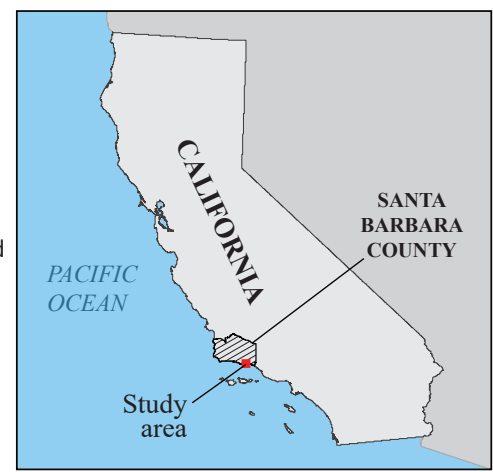

Figure 17. Contours of simulated July 1990 hydraulic head for the producing zones of the Santa Barbara groundwater basin, California: $A$, upper; $B$, middle; and $C$, lower. 


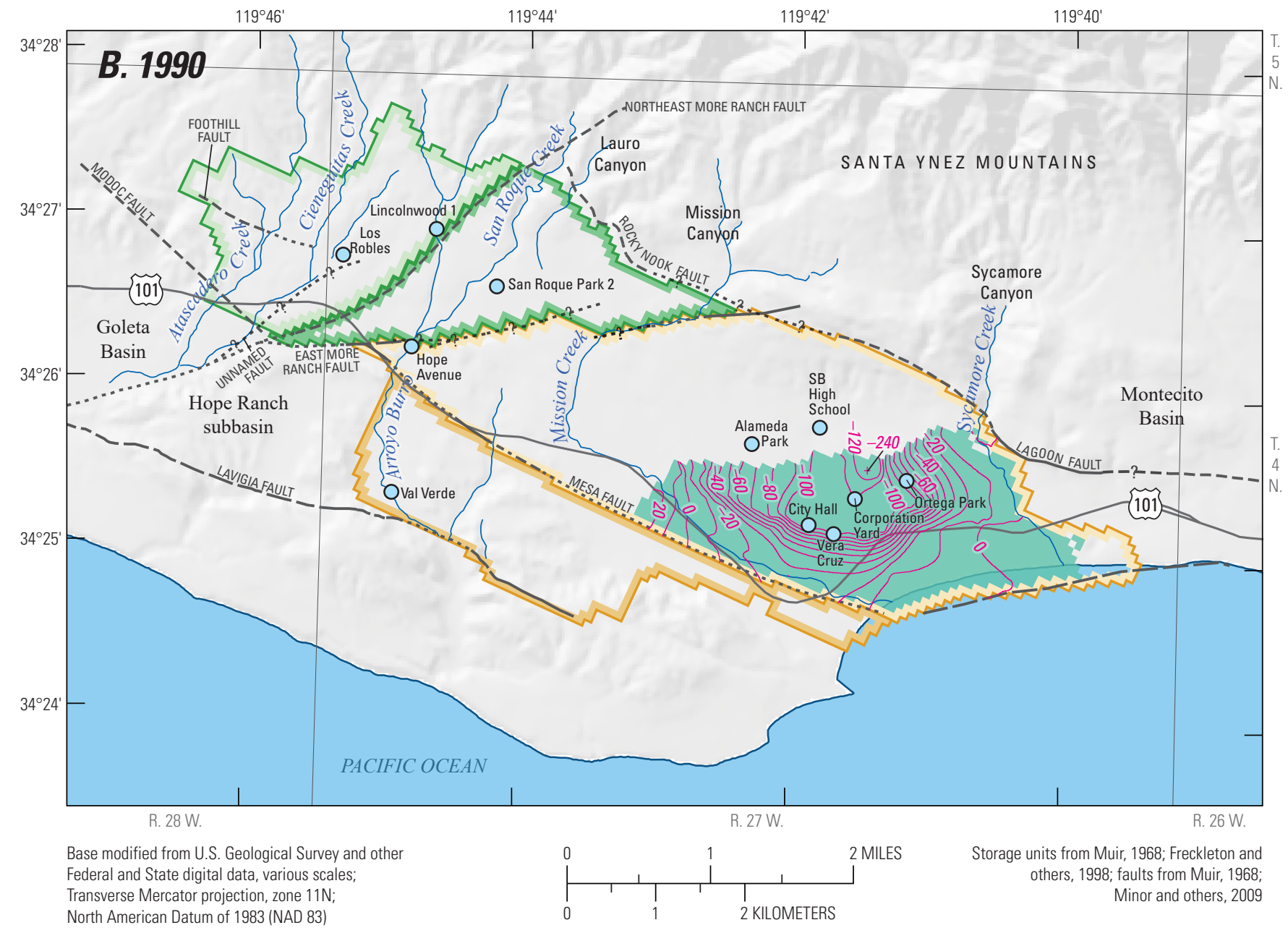

(None $11 \mathrm{~N}$

\section{EXPLANATION}

\begin{tabular}{|c|c|}
\hline & $\begin{array}{l}\text { Middle produci } \\
\text { zone }\end{array}$ \\
\hline \multicolumn{2}{|c|}{ Subbasins } \\
\hline & West Foothill \\
\hline$\square$ & East Foothill \\
\hline & Storage Unit I \\
\hline$\square$ & Storage Unit III \\
\hline
\end{tabular}

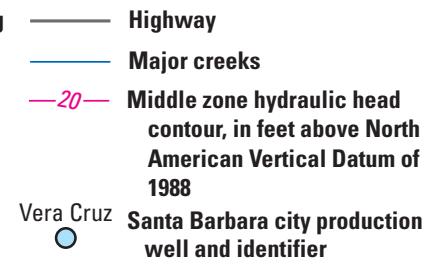

Faults

- - Existence certain, location inferred

- -? - Existence questionable, location inferred

....... Existence certain, location concealed

...? ... Existence questionable, location concealed

- Existence certain, location accurate

—— Existence certain, location approximate

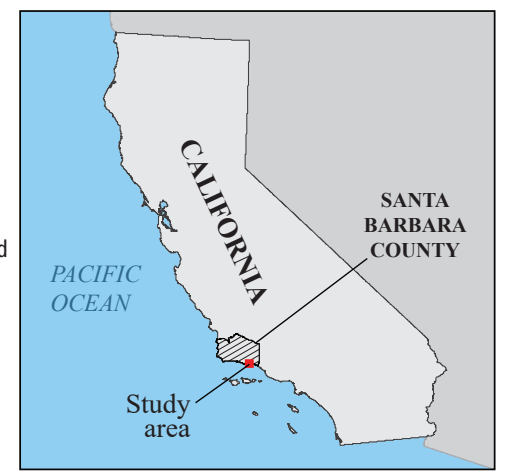

Figure 17. - Continued 


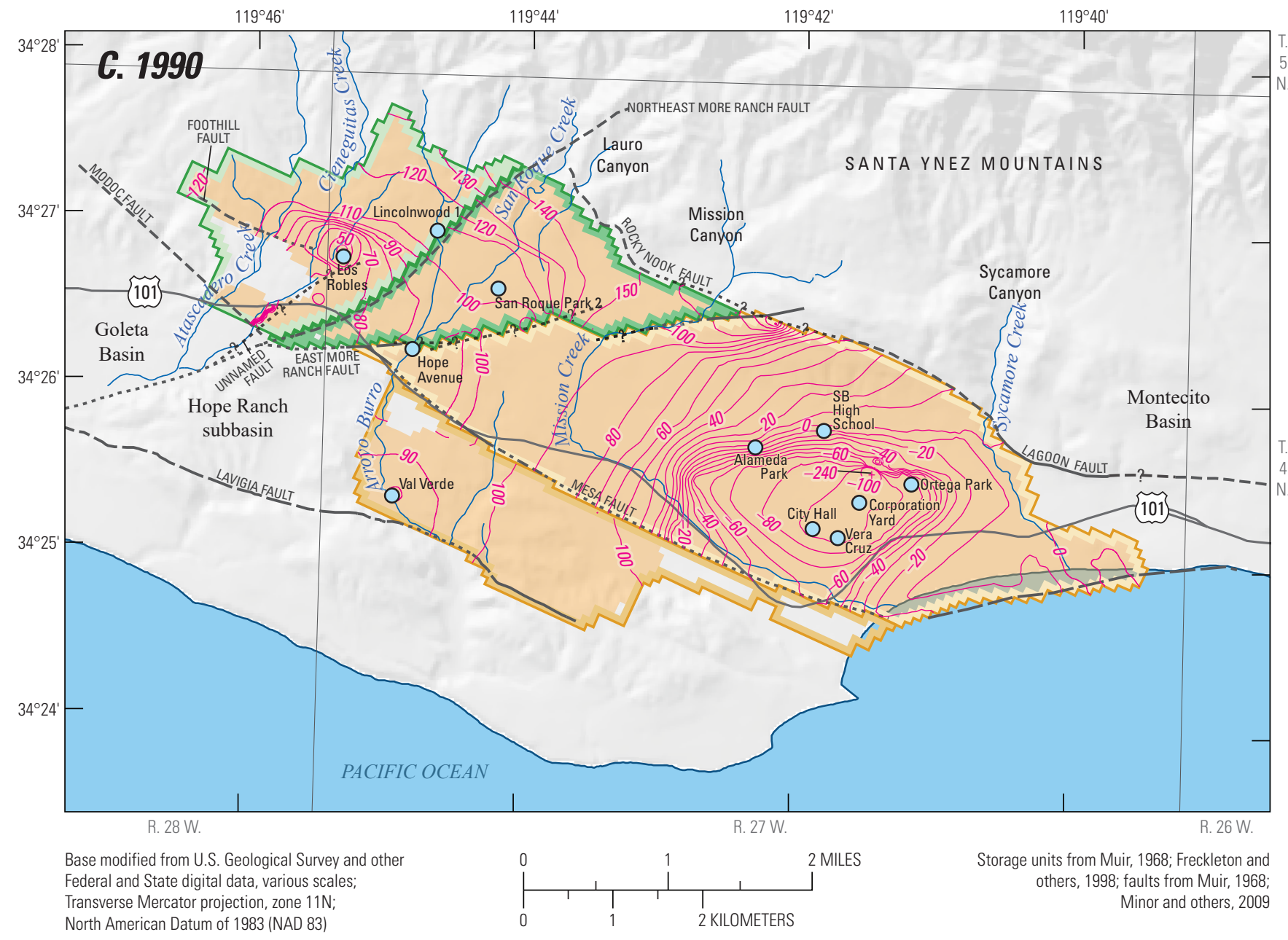

Federal and State digital data, various scales;

North American Datum of 1983 (NAD 83)

\section{EXPLANATION}

\begin{tabular}{|c|c|}
\hline & $\begin{array}{l}\text { Lower producin } \\
\text { zone }\end{array}$ \\
\hline \multicolumn{2}{|c|}{ Subbasins } \\
\hline & West Foothill \\
\hline 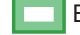 & East Foothill \\
\hline & Storage Unit I \\
\hline & Storage Unit III \\
\hline
\end{tabular}

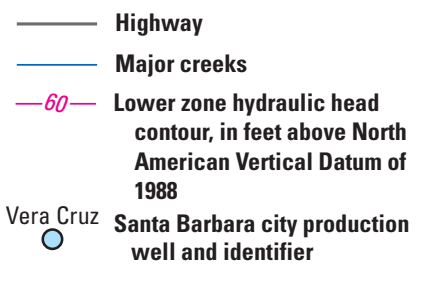

Faults

- - - - Existence certain, location inferred

- -?- - Existence questionable, location inferred

........ Existence certain, location concealed

........ Existence questionable, location concealed

_- Existence certain, location accurate

- - Existence certain, location approximate

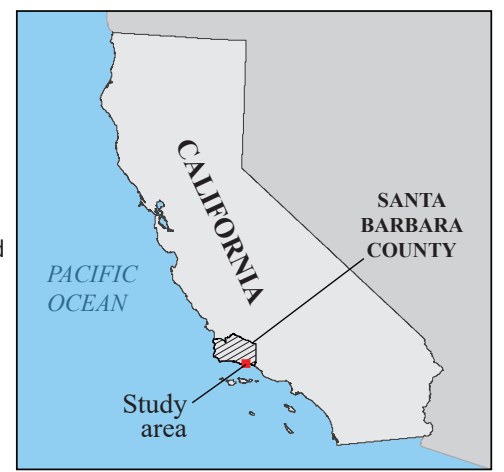

Figure 17. - Continued 
In order to examine changes in hydraulic conditions related to pumpage, the simulated hydraulic-head drawdown between initial conditions (1929) and July 1990 was plotted and is shown in figure 18. There were pumping depressions in all three producing zones. In the upper, middle, and lower producing zones, there was a pumping depression near the Ortega Park production well in Storage Unit I (figs. 18A-C). In the lower producing zone, there was an additional pumping depression in the West Foothill subbasin near the Los Robles production well (fig. 18C).
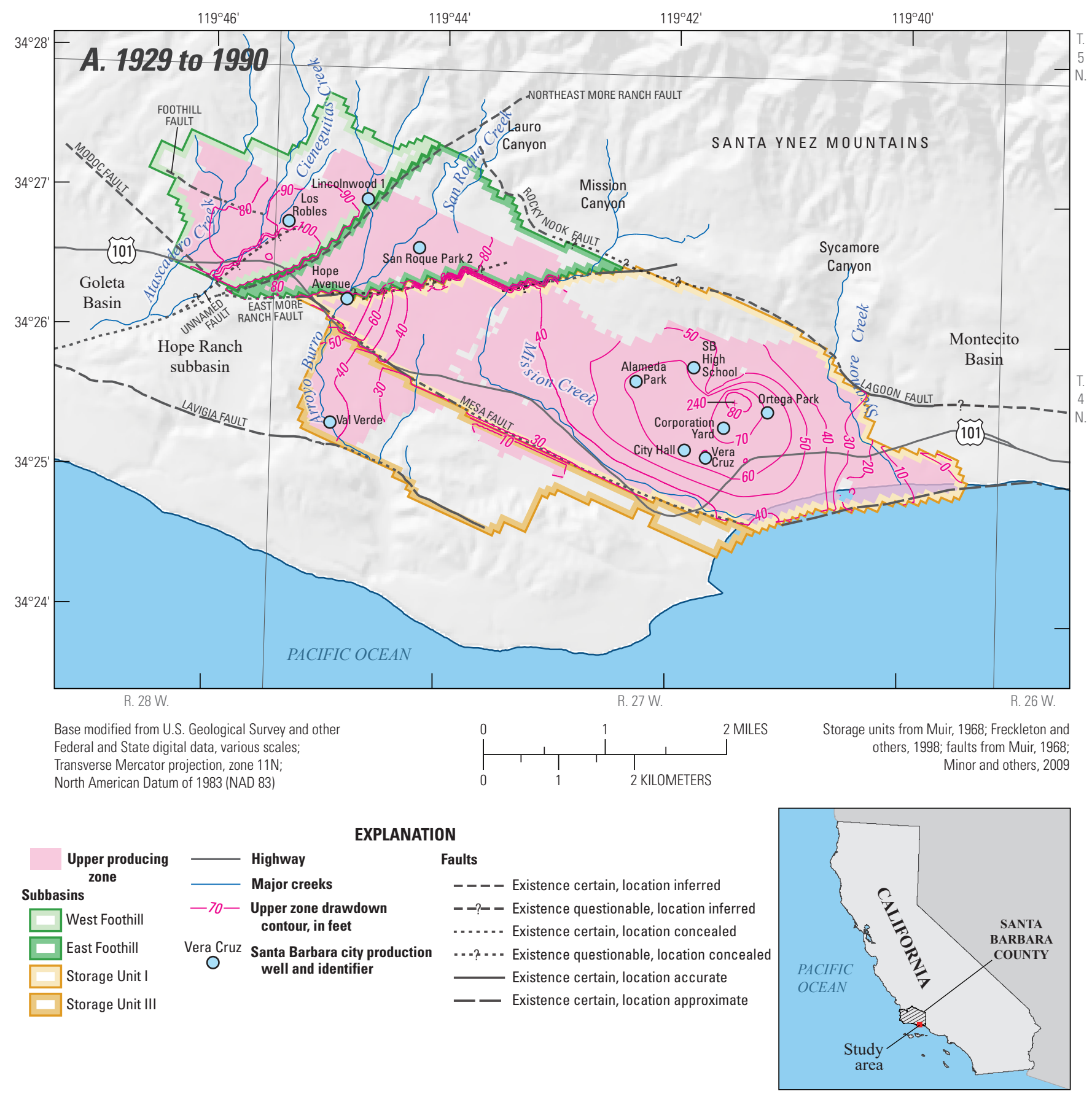

Figure 18. Contours of simulated drawdown between average 1929 and July 1990 hydraulic heads for the producing zones of the Santa Barbara groundwater basin, California: $A$, upper; $B$, middle; and $C$, lower. 

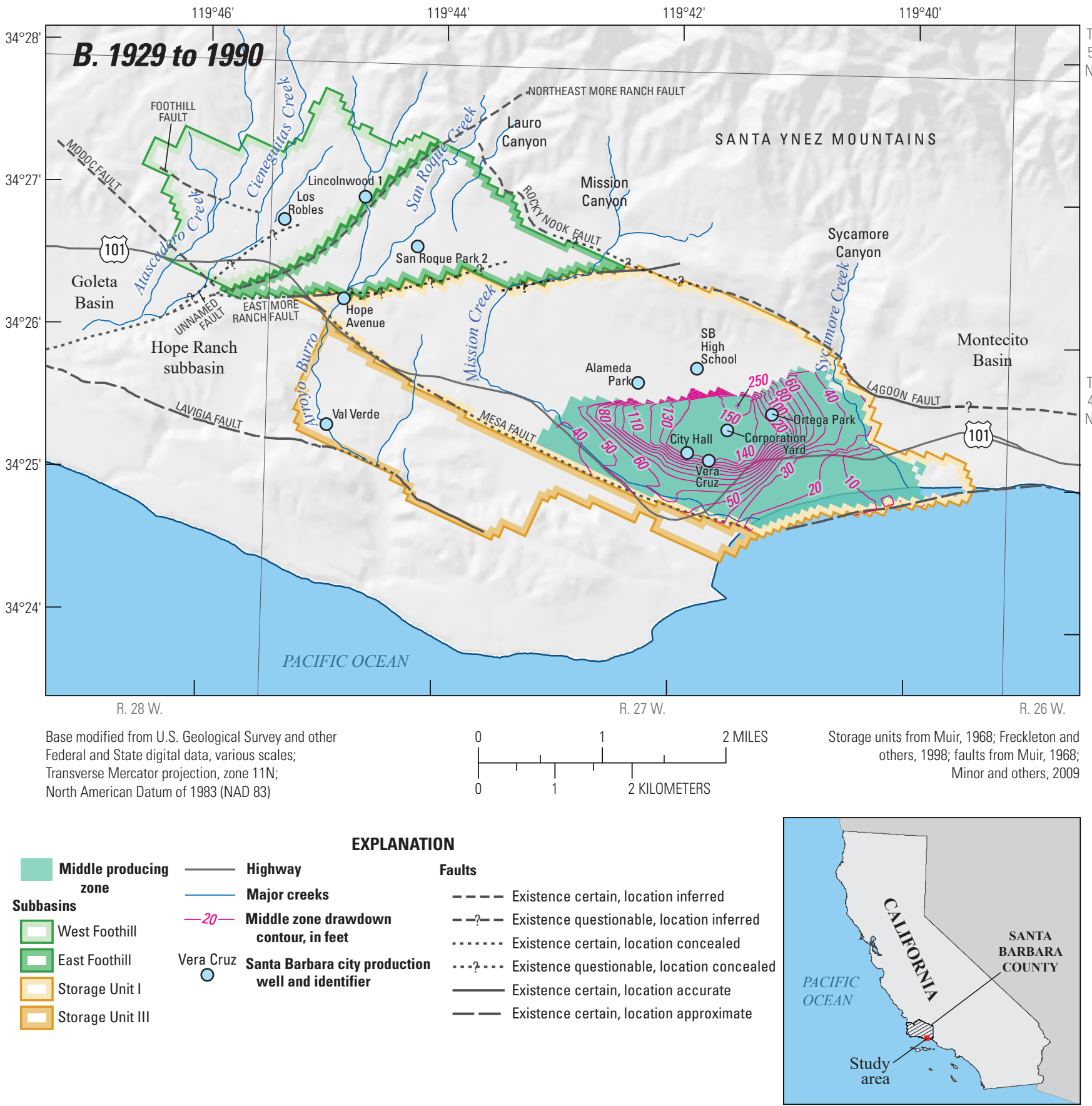

Figure 18. - Continued 

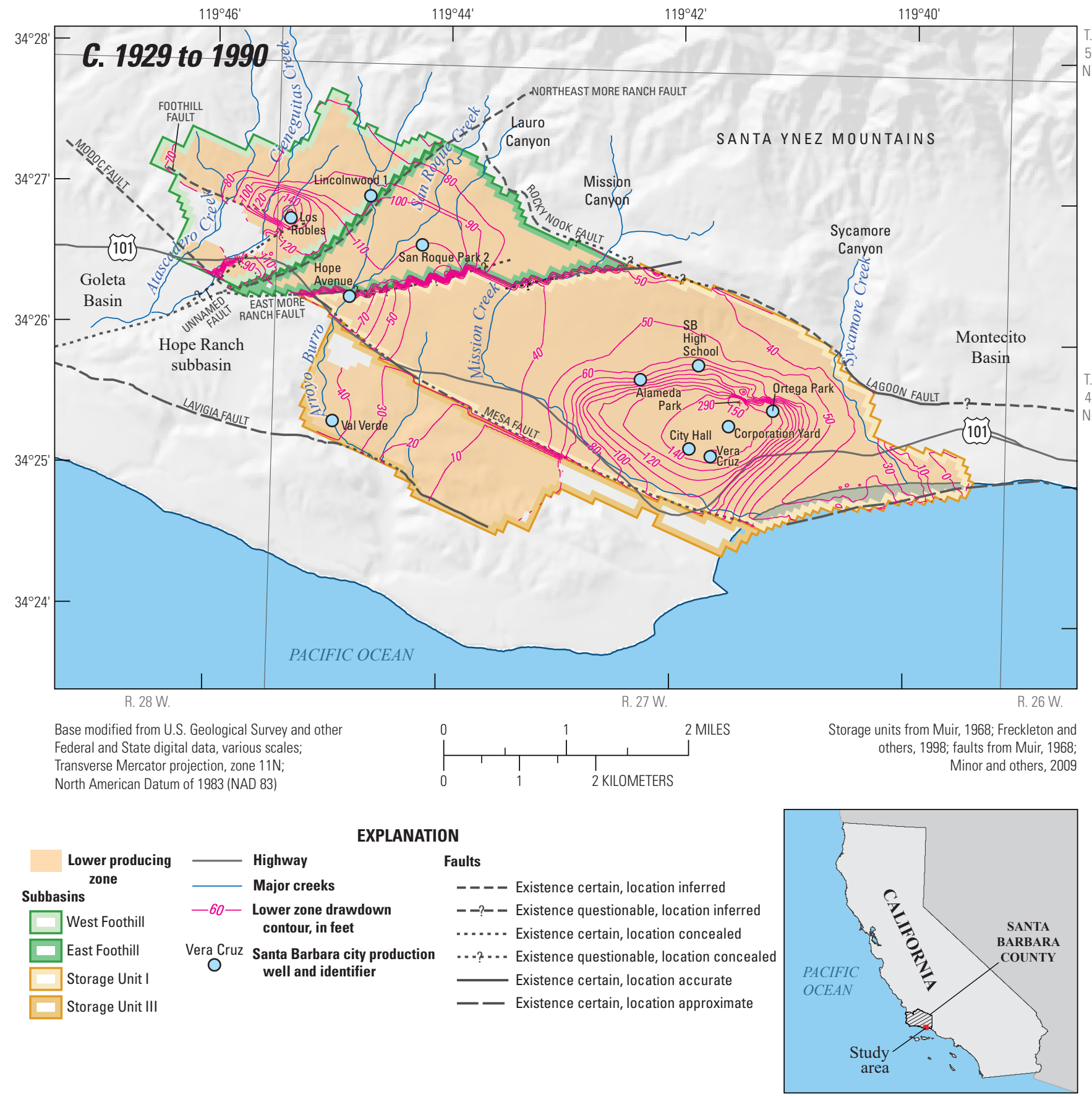

Figure 18. -Continued

\section{Simulated Hydrographs}

Several wells were selected as representative of conditions, and changes in conditions, in the four subbasins. The simulated hydraulic heads and corresponding measured water levels at selected wells in West Foothill subbasin,
East Foothill subbasin, Storage Unit III, and Storage Unit I are shown in figures $19 A-D$, respectively. To show vertical hydraulic gradients, where possible, water levels are shown in each of the producing zones. Each of the subbasins are discussed in following sections. 


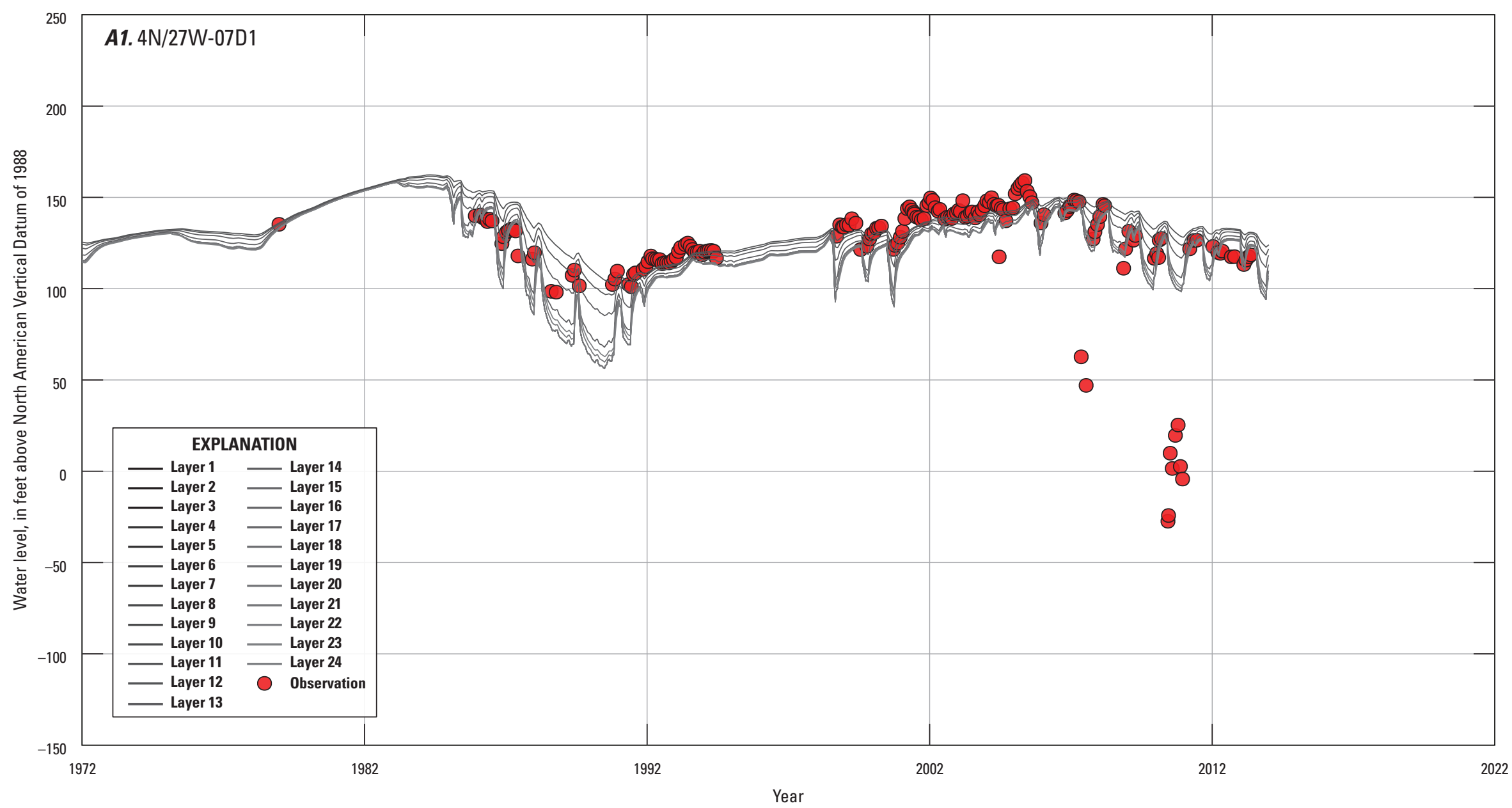

Figure 19. Simulated hydraulic heads and measured water levels for selected wells in the Santa Barbara groundwater basin, California, by subbasin: $A$, West Foothill subbasin; $B$, East Foothill subbasin; $C$, Storage Unit III; and $D$, Storage Unit I. 


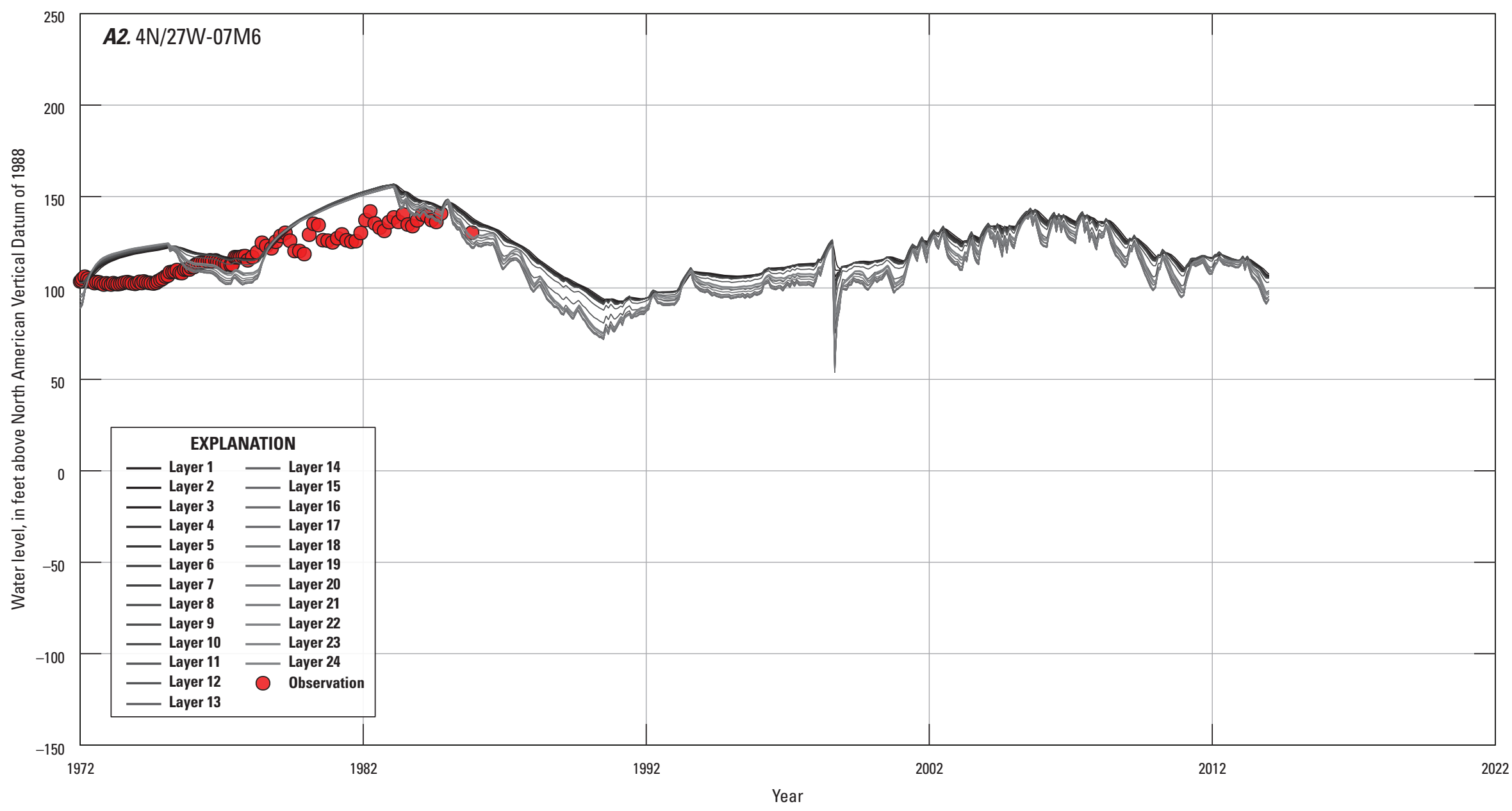

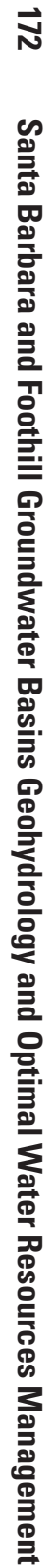

Figure 19. - Continued 


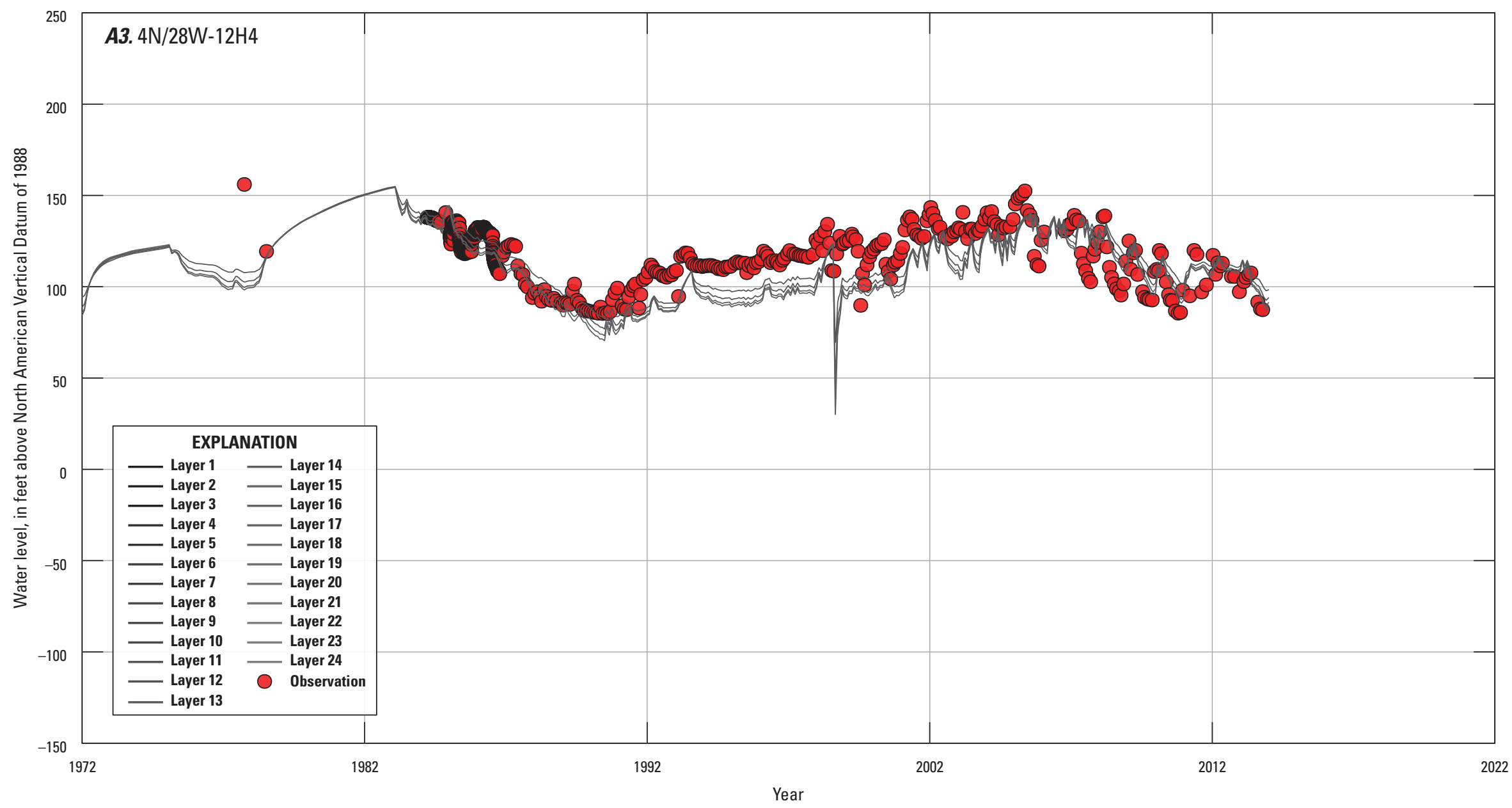

Figure 19. - Continued 


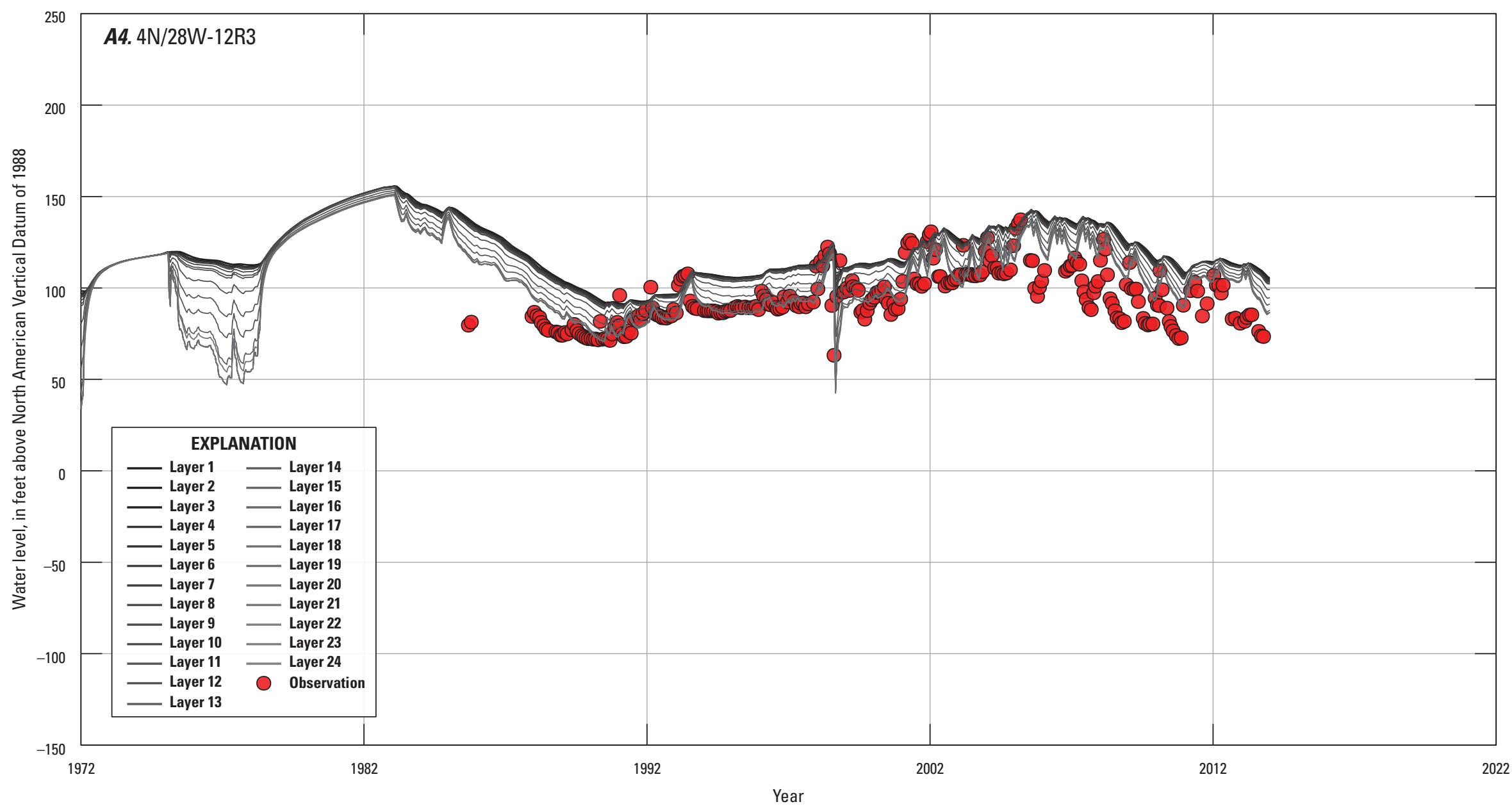

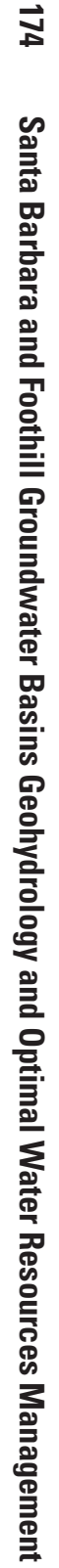

Figure 19. - Continued 


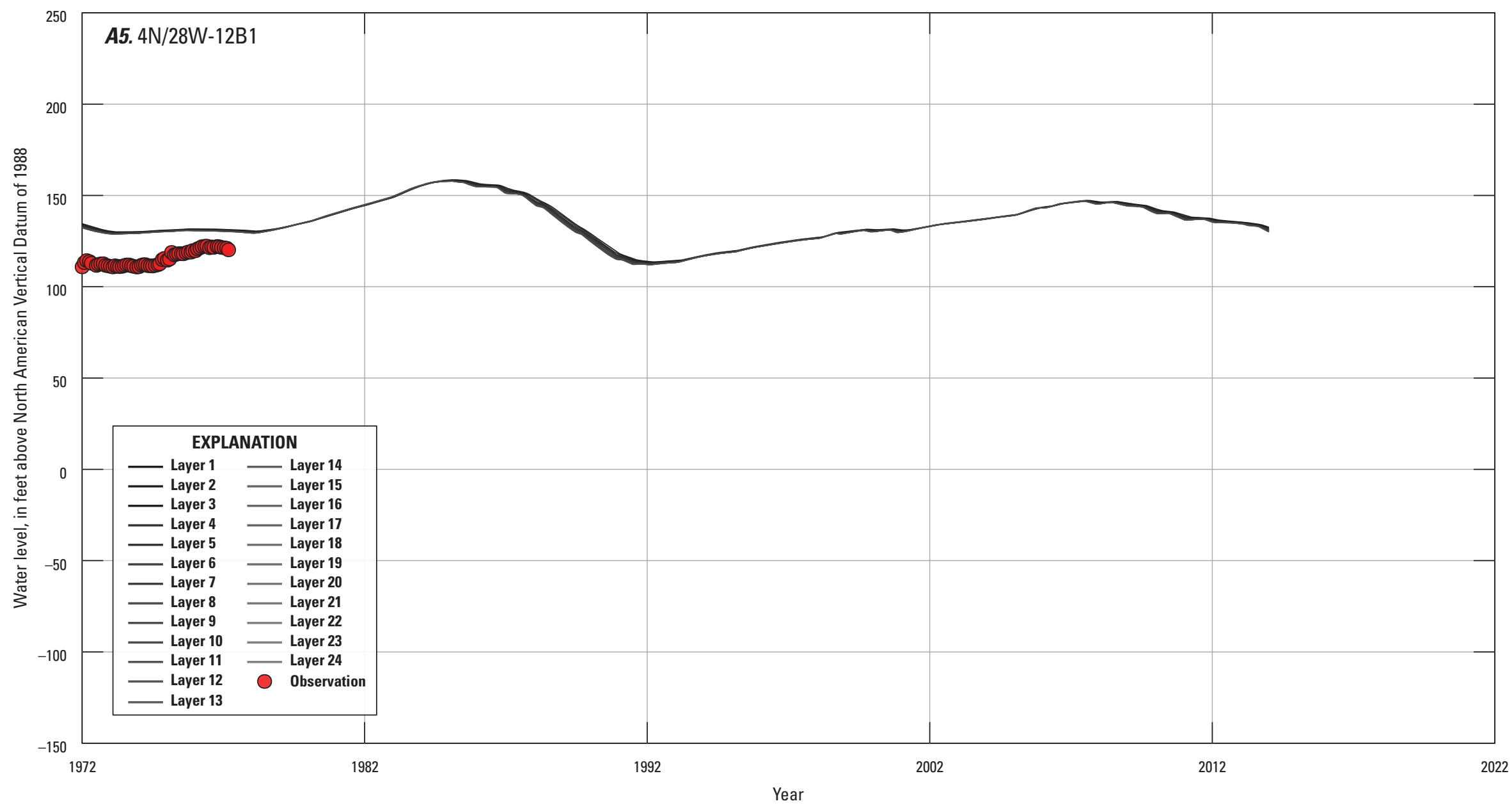

Figure 19. - Continued 


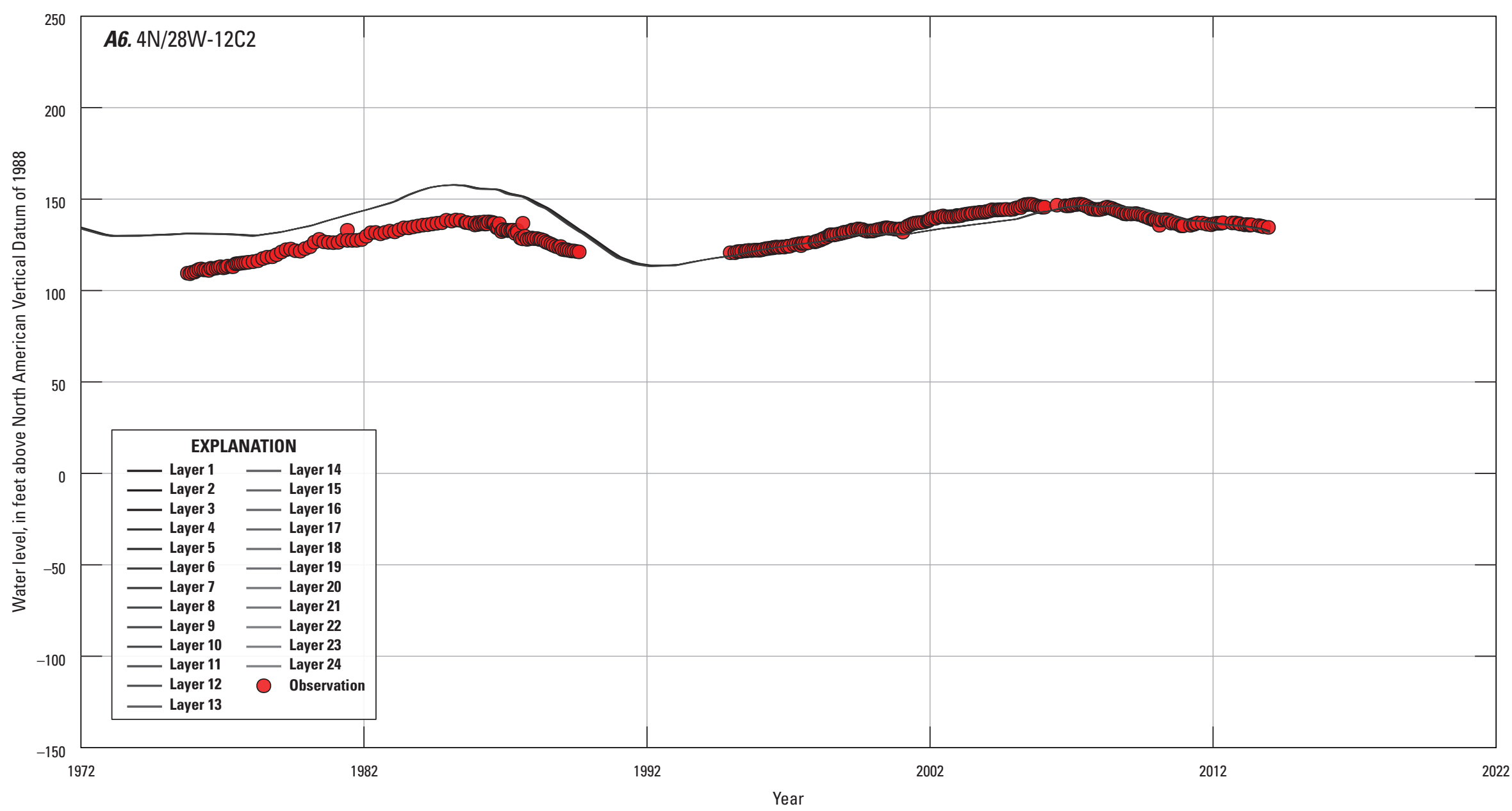

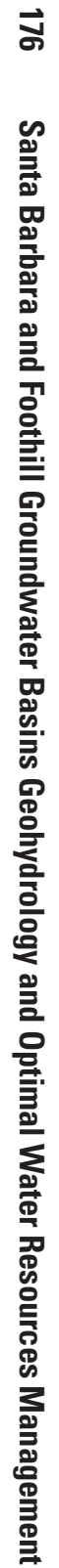

Figure 19. - Continued 


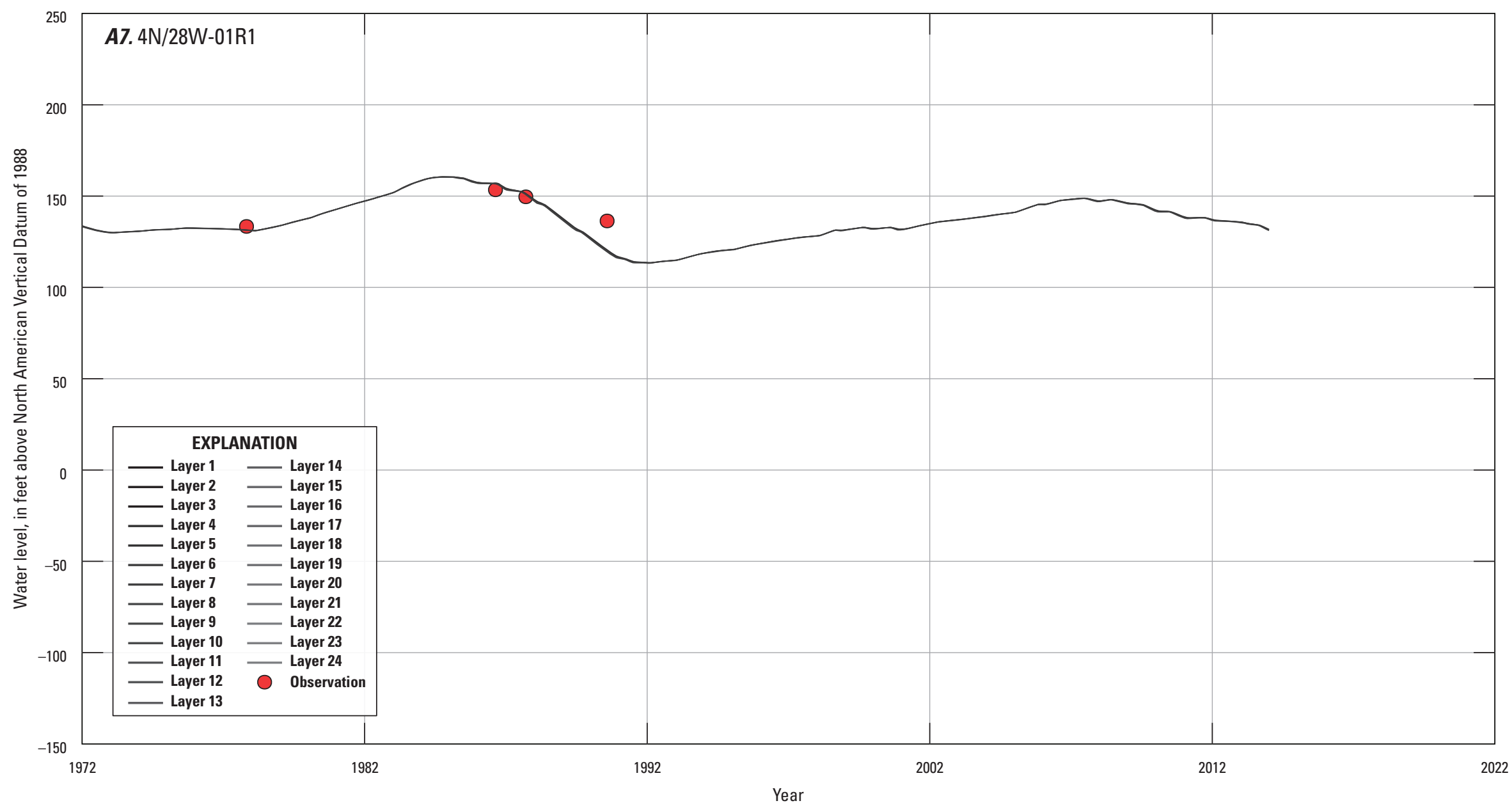

Figure 19. - Continued 


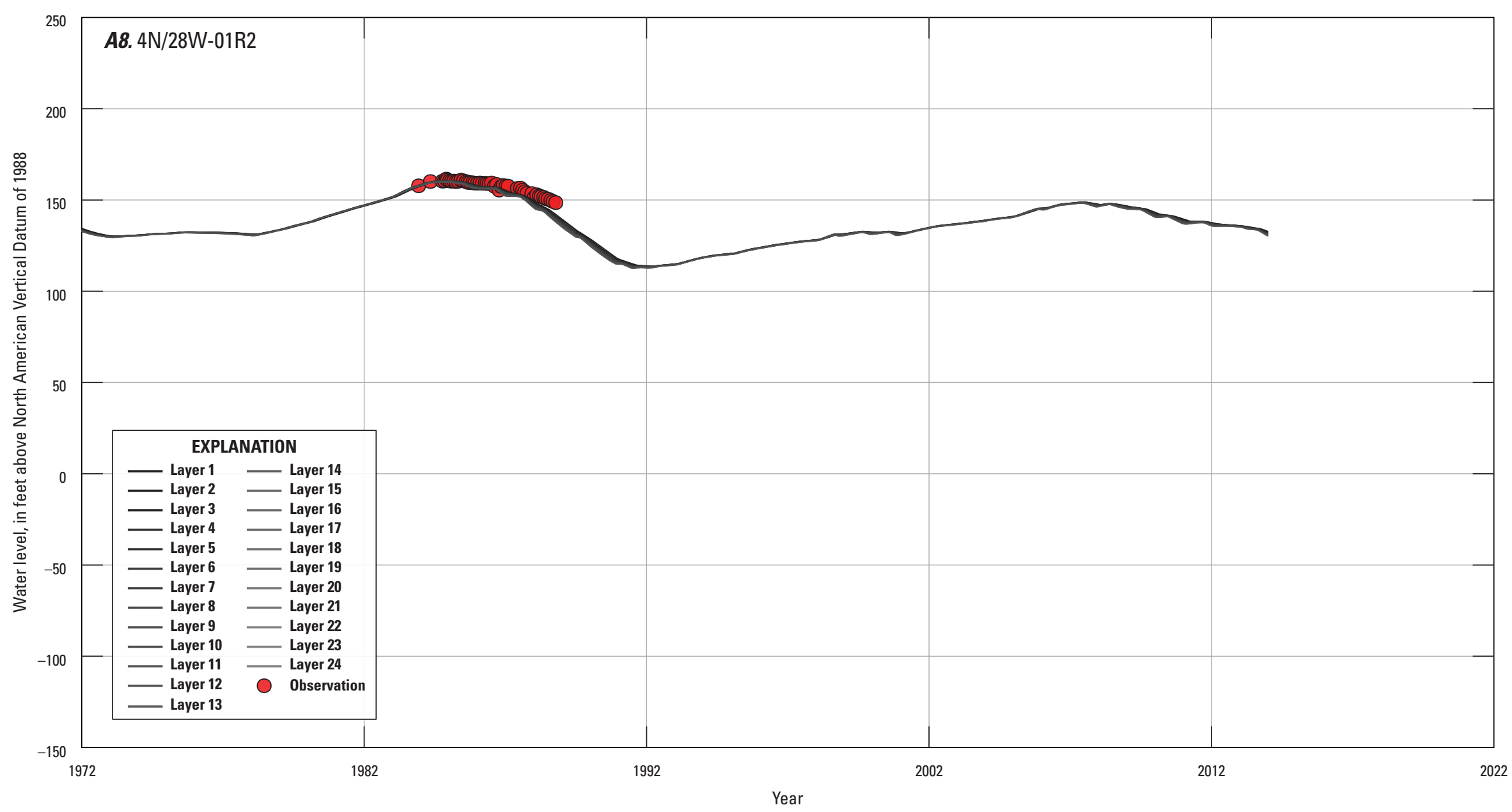

Figure 19. - Continued 


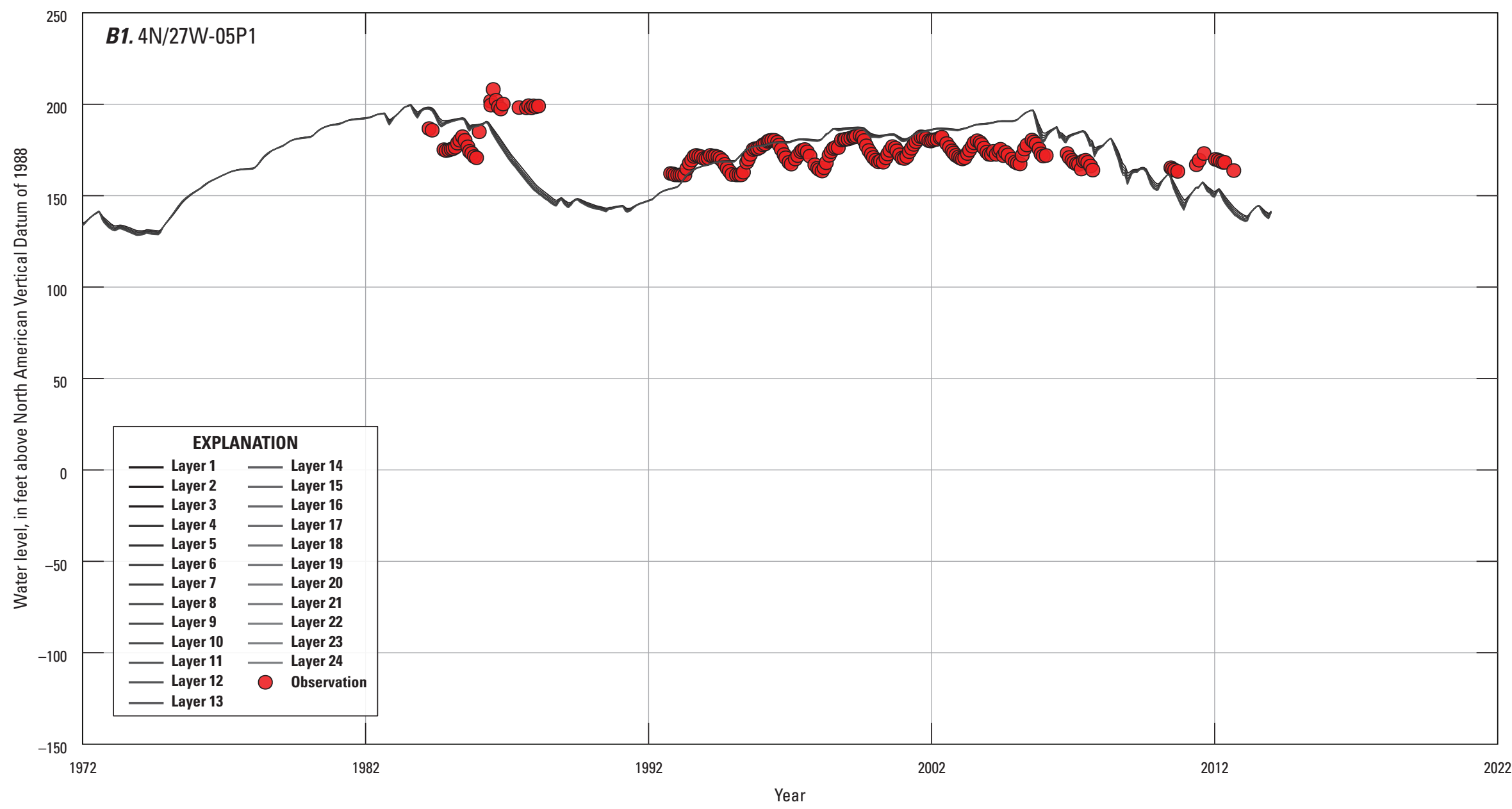

Figure 19. - Continued 


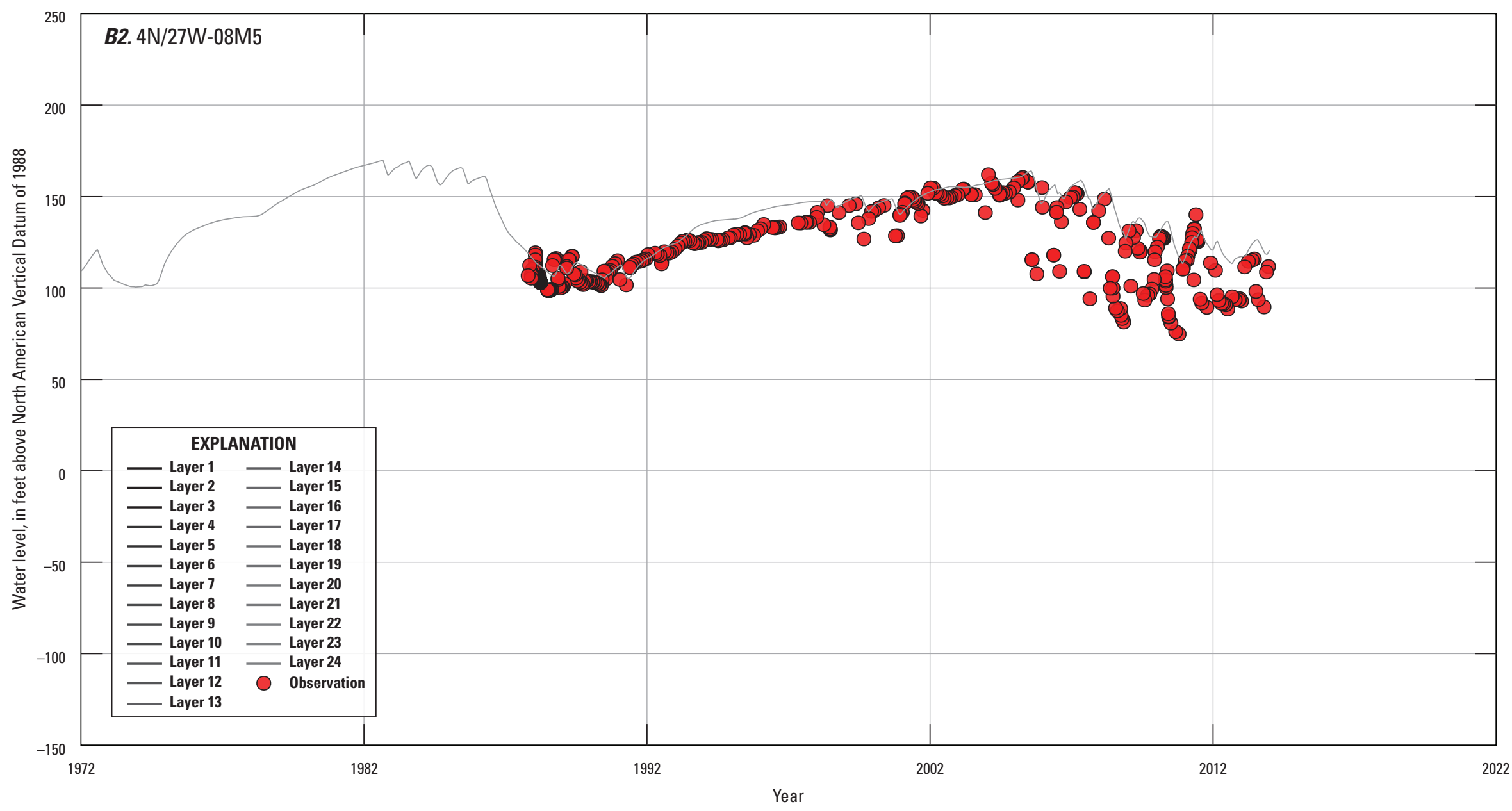

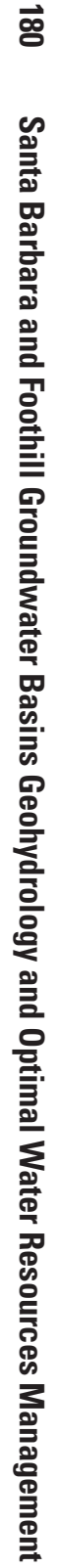

Figure 19. - Continued 


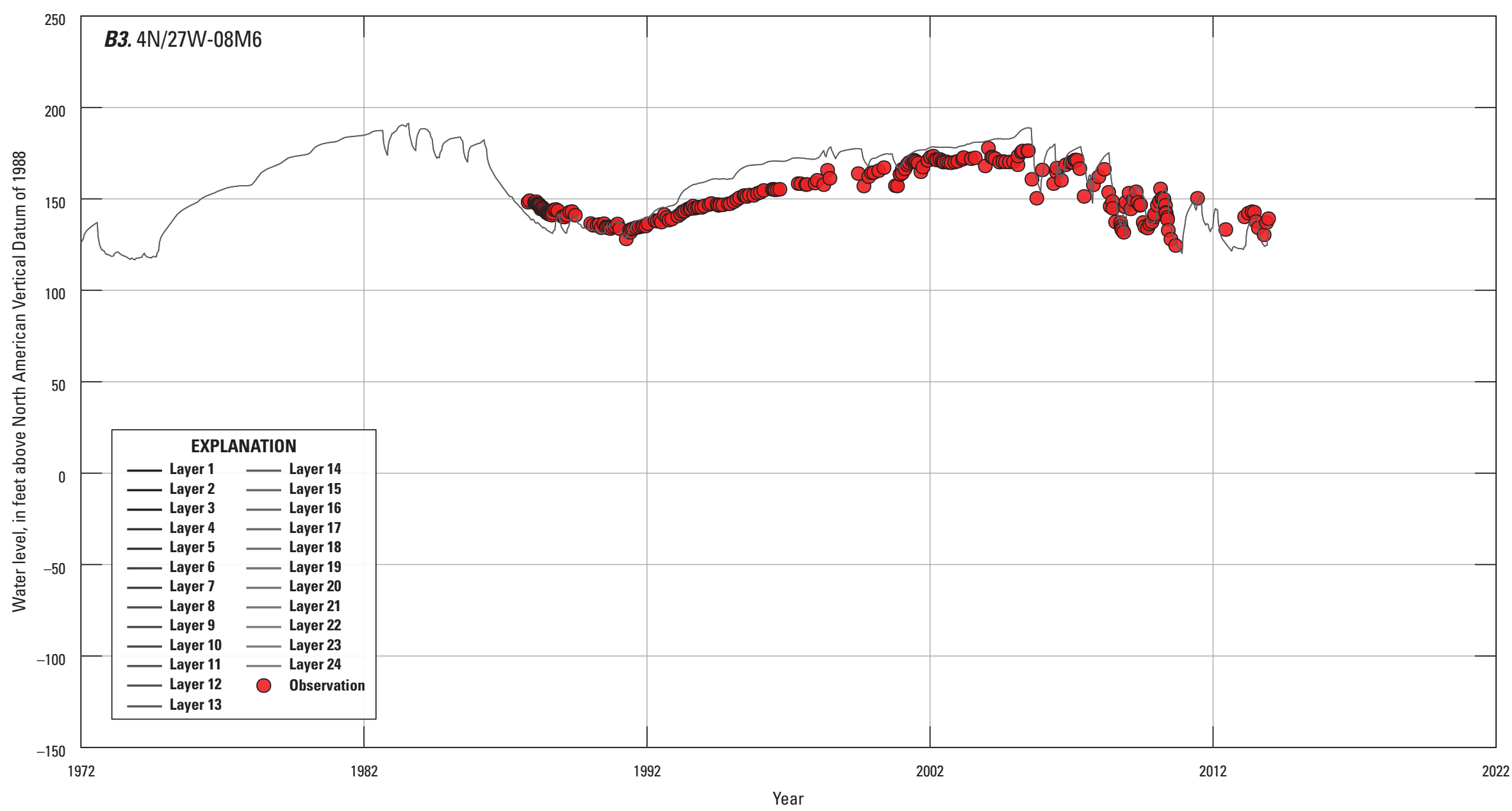

Figure 19. - Continued 


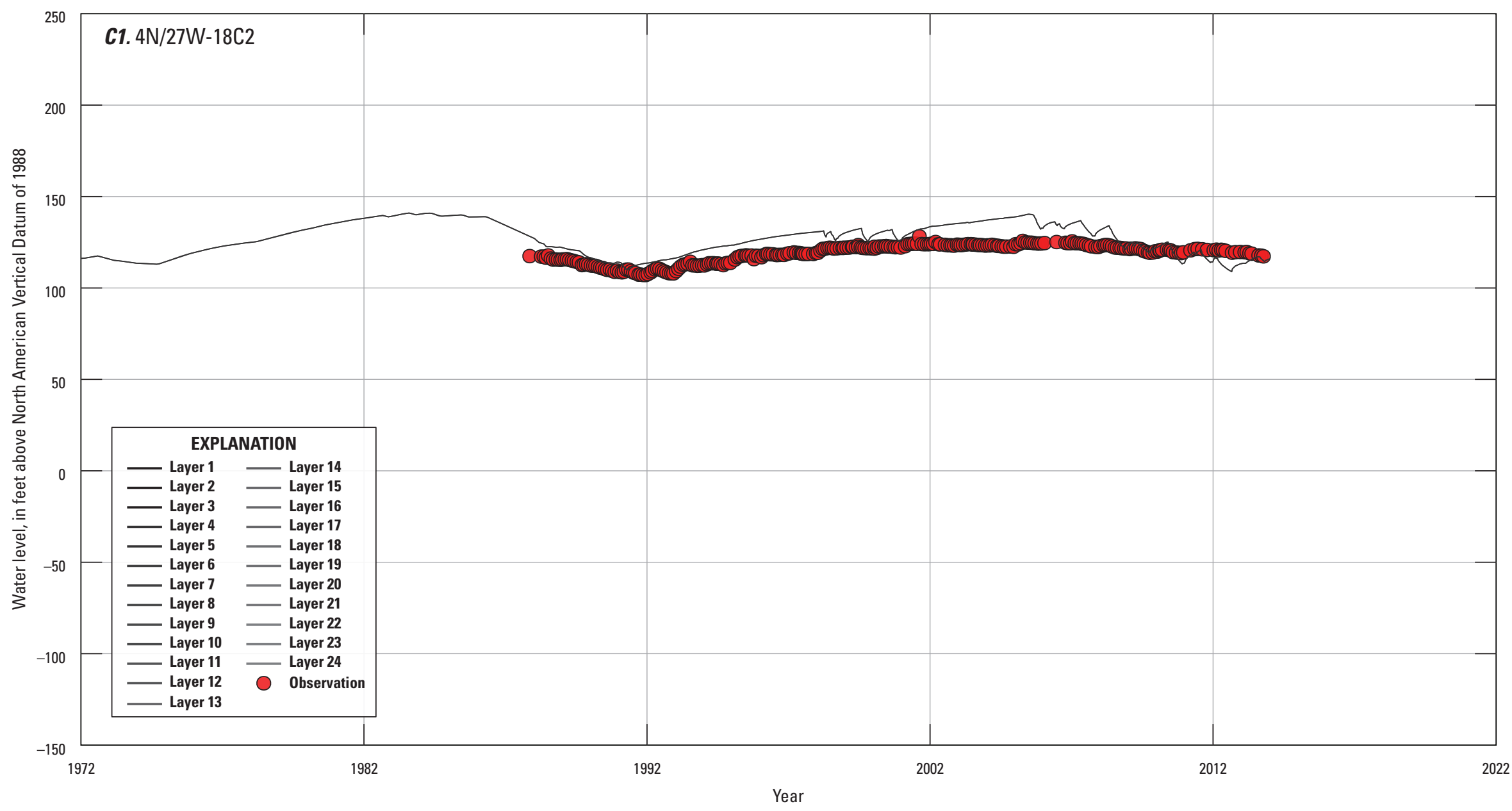

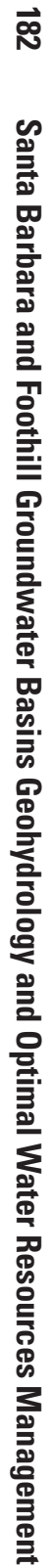

Figure 19. - Continued 


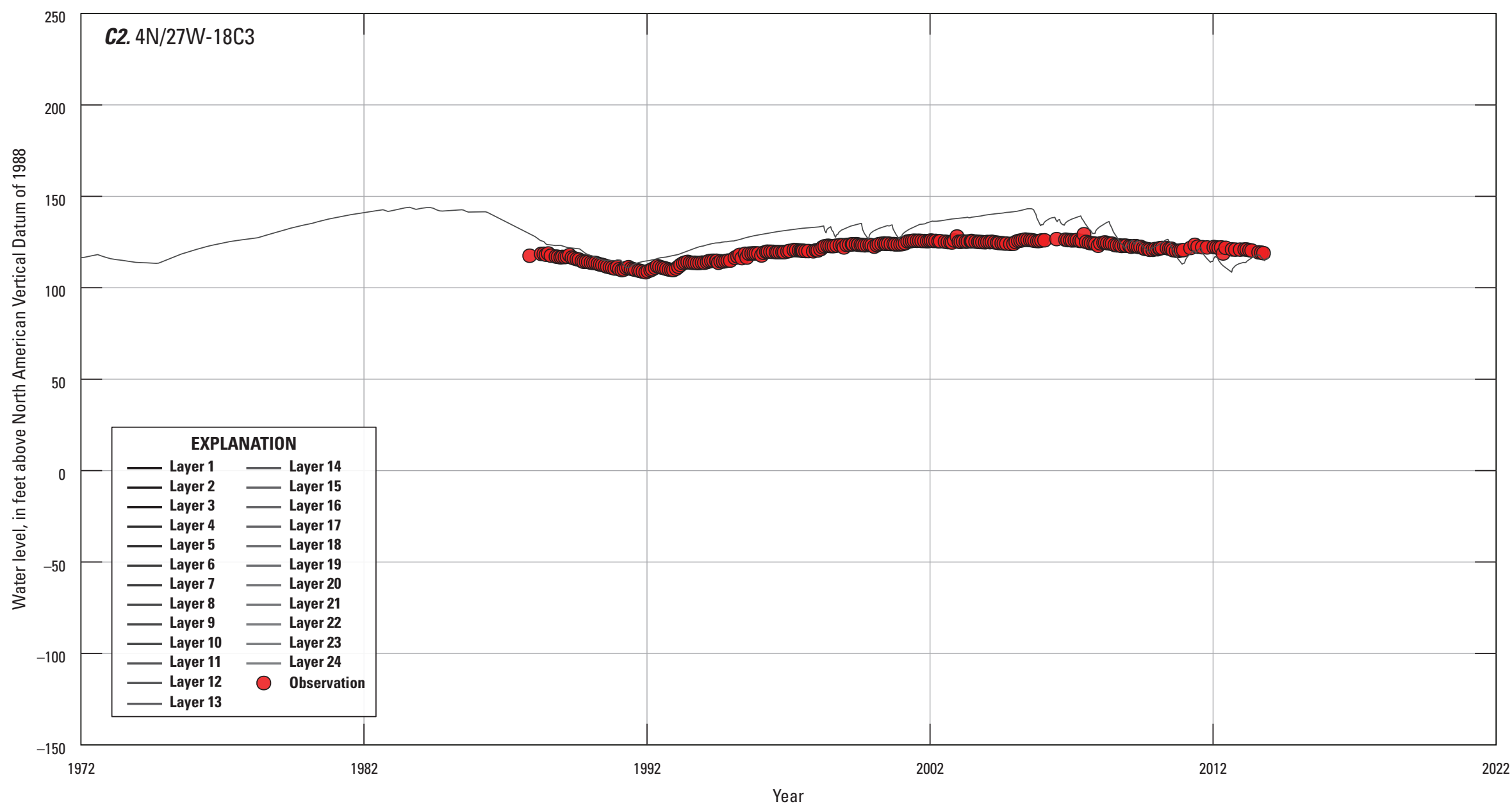

Figure 19. - Continued 


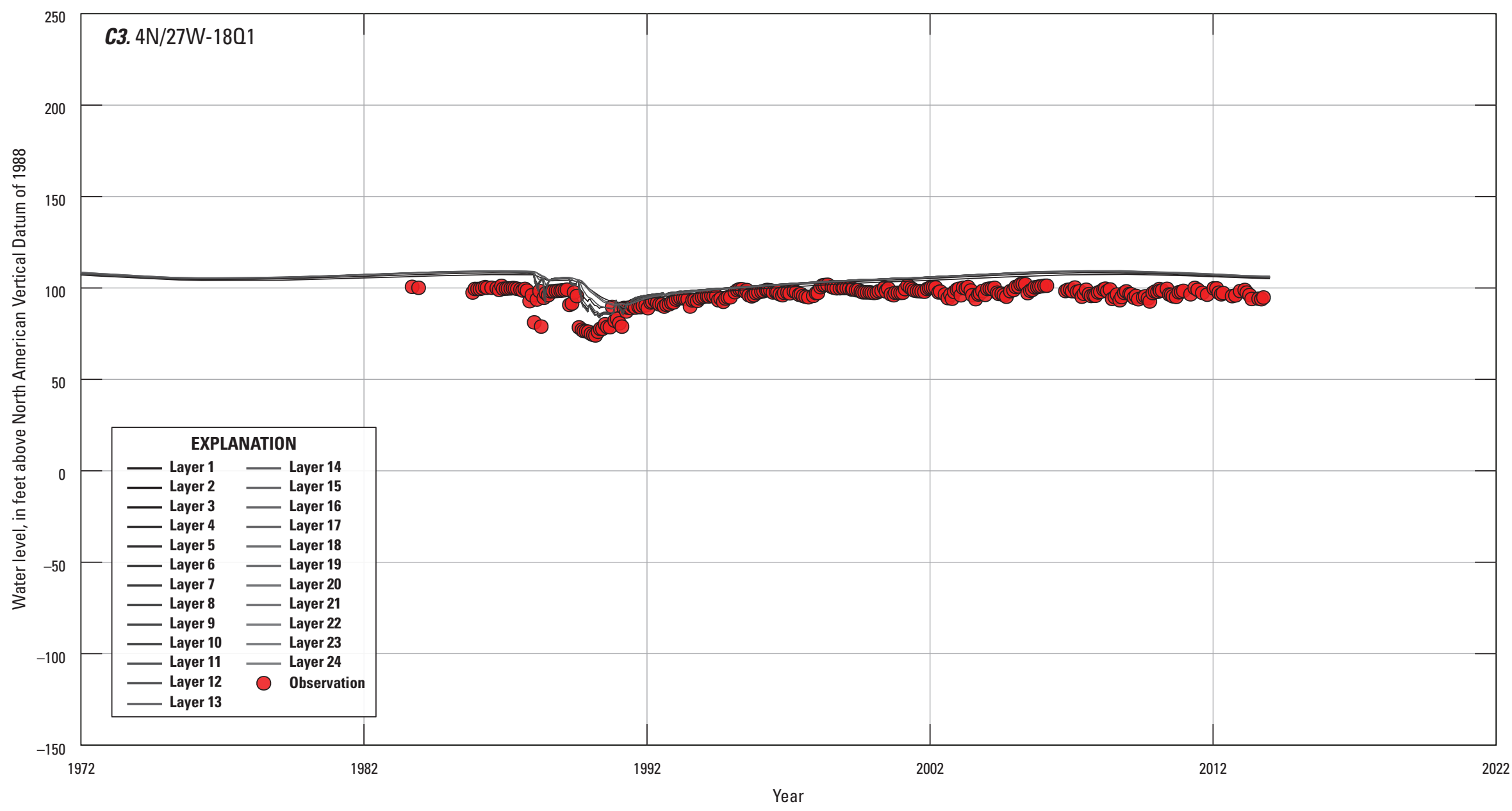

Figure 19. - Continued 


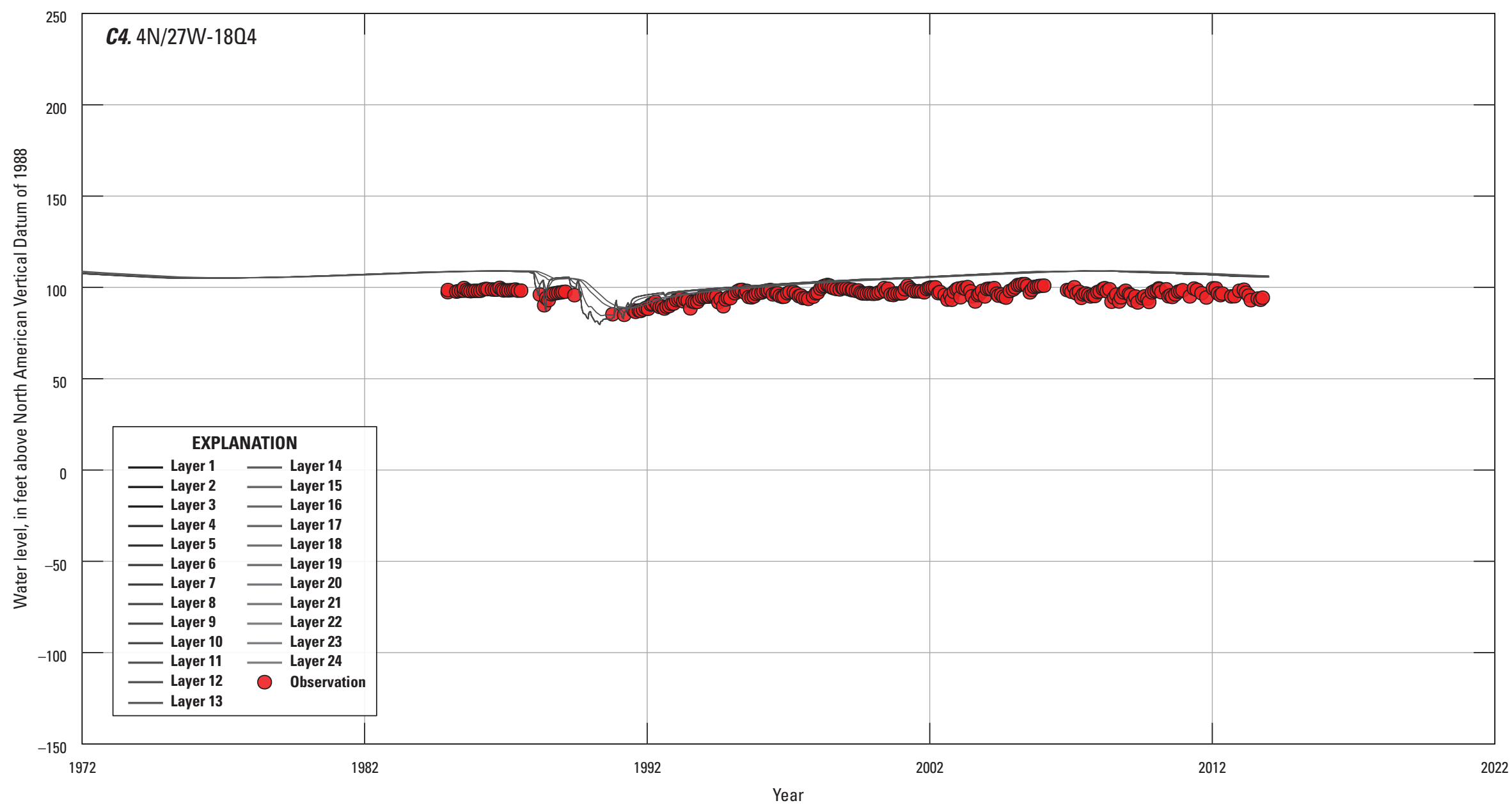

Figure 19. - Continued 


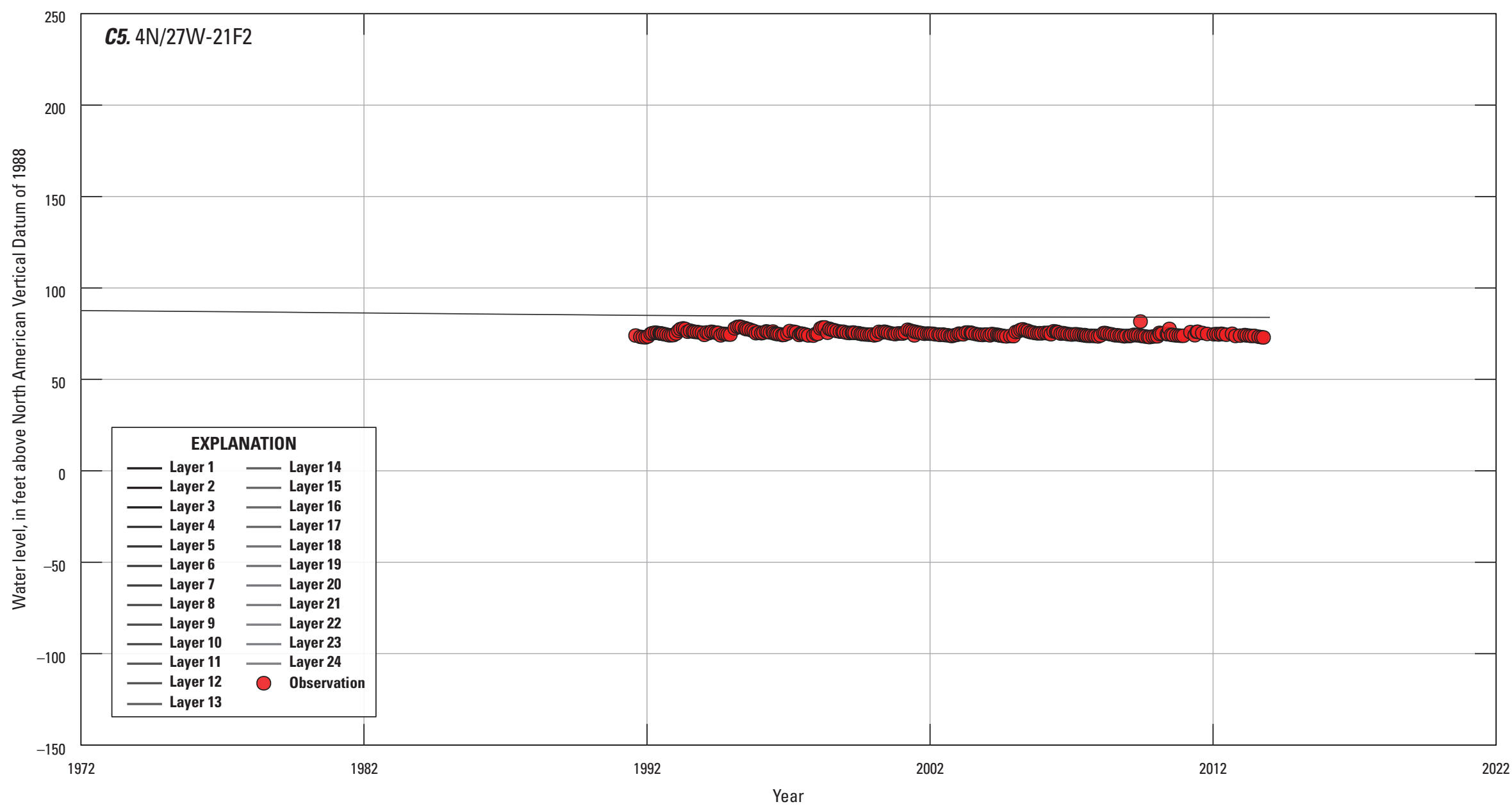

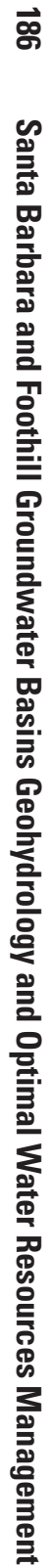

Figure 19. - Continued 


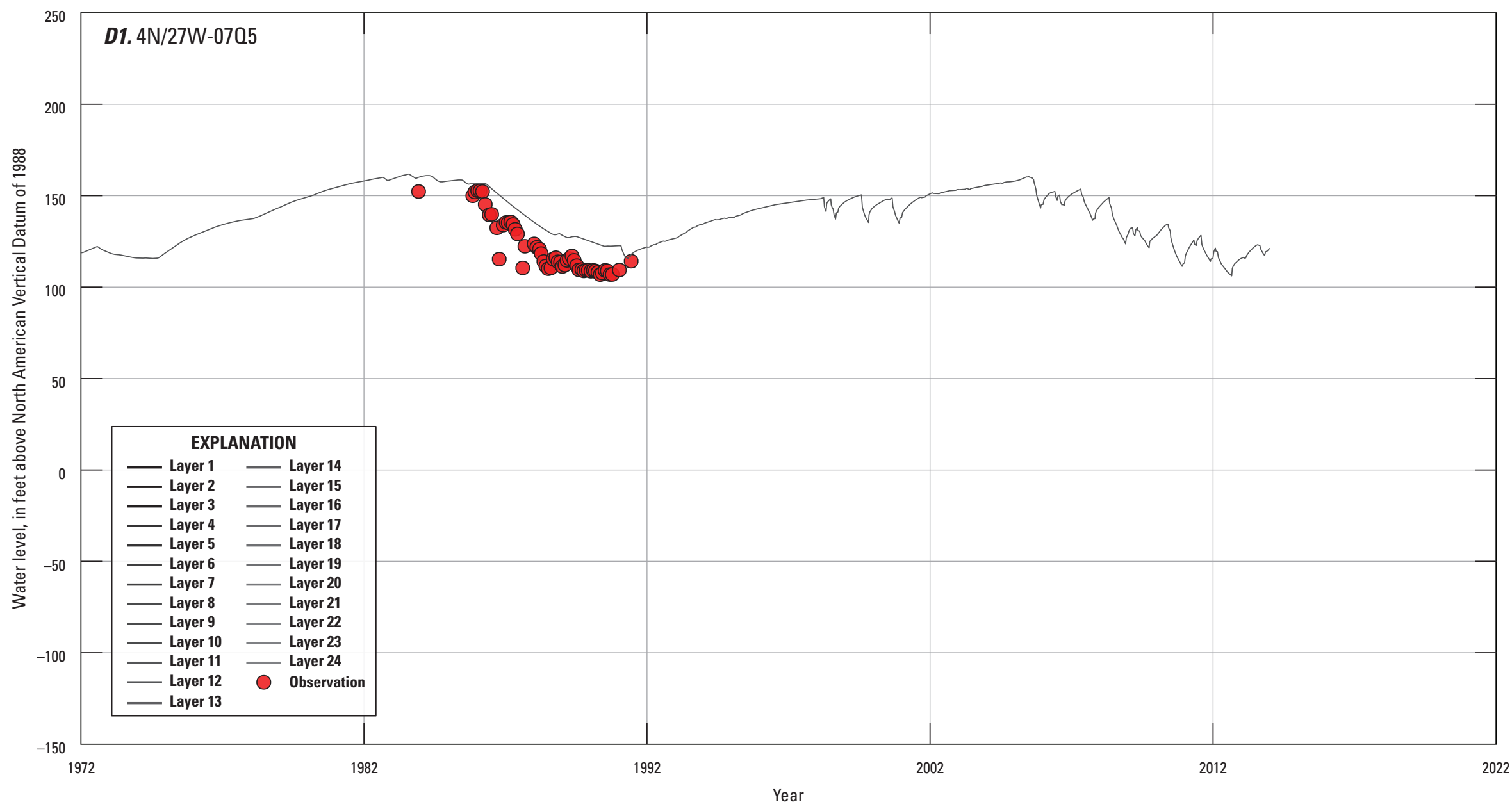

Figure 19. - Continued 


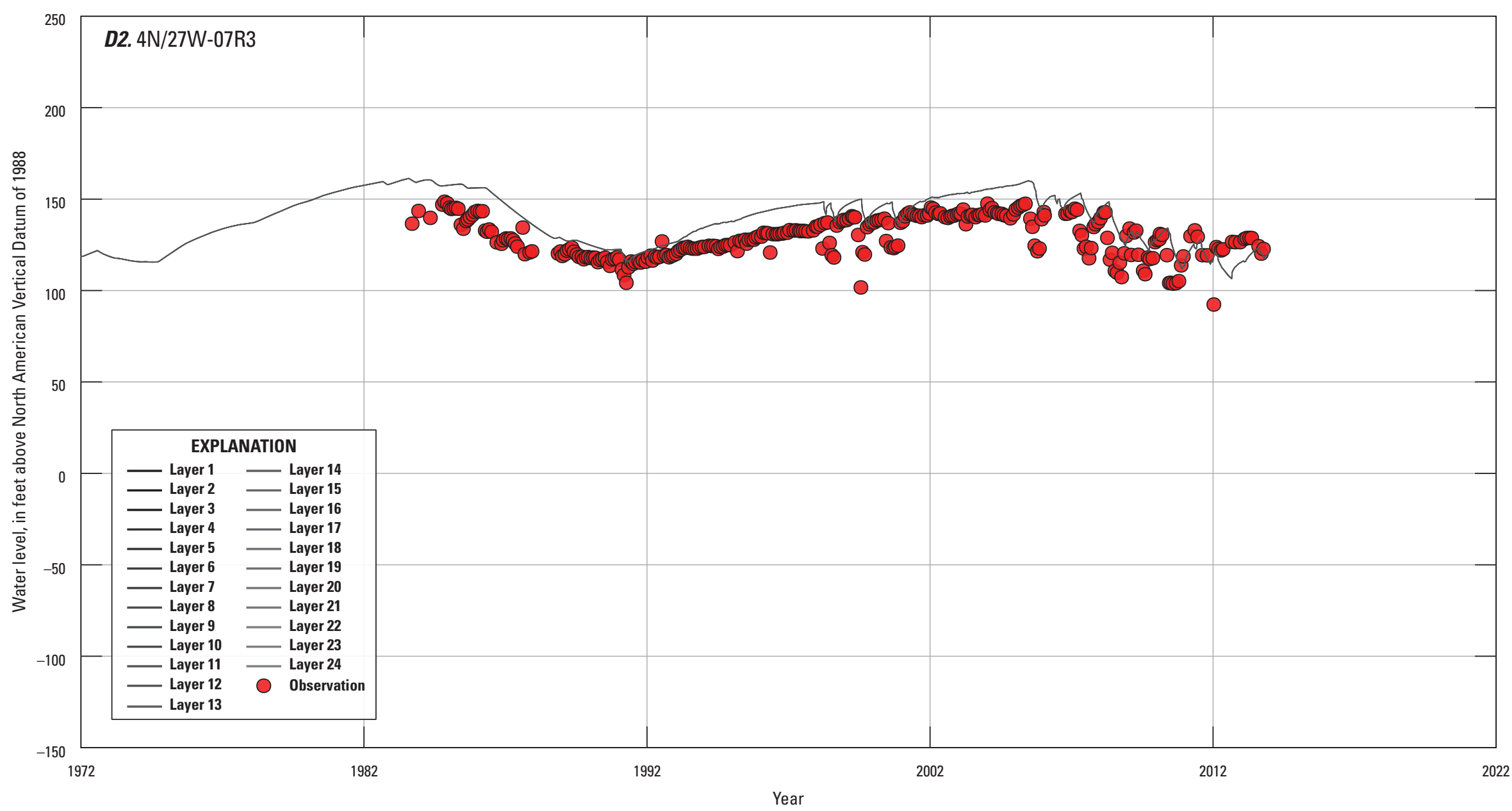

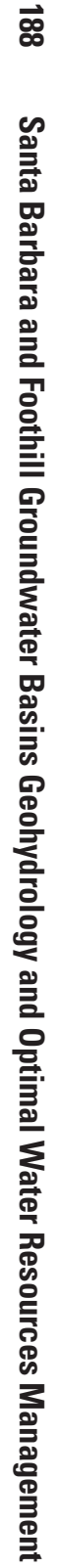

Figure 19. - Continued 


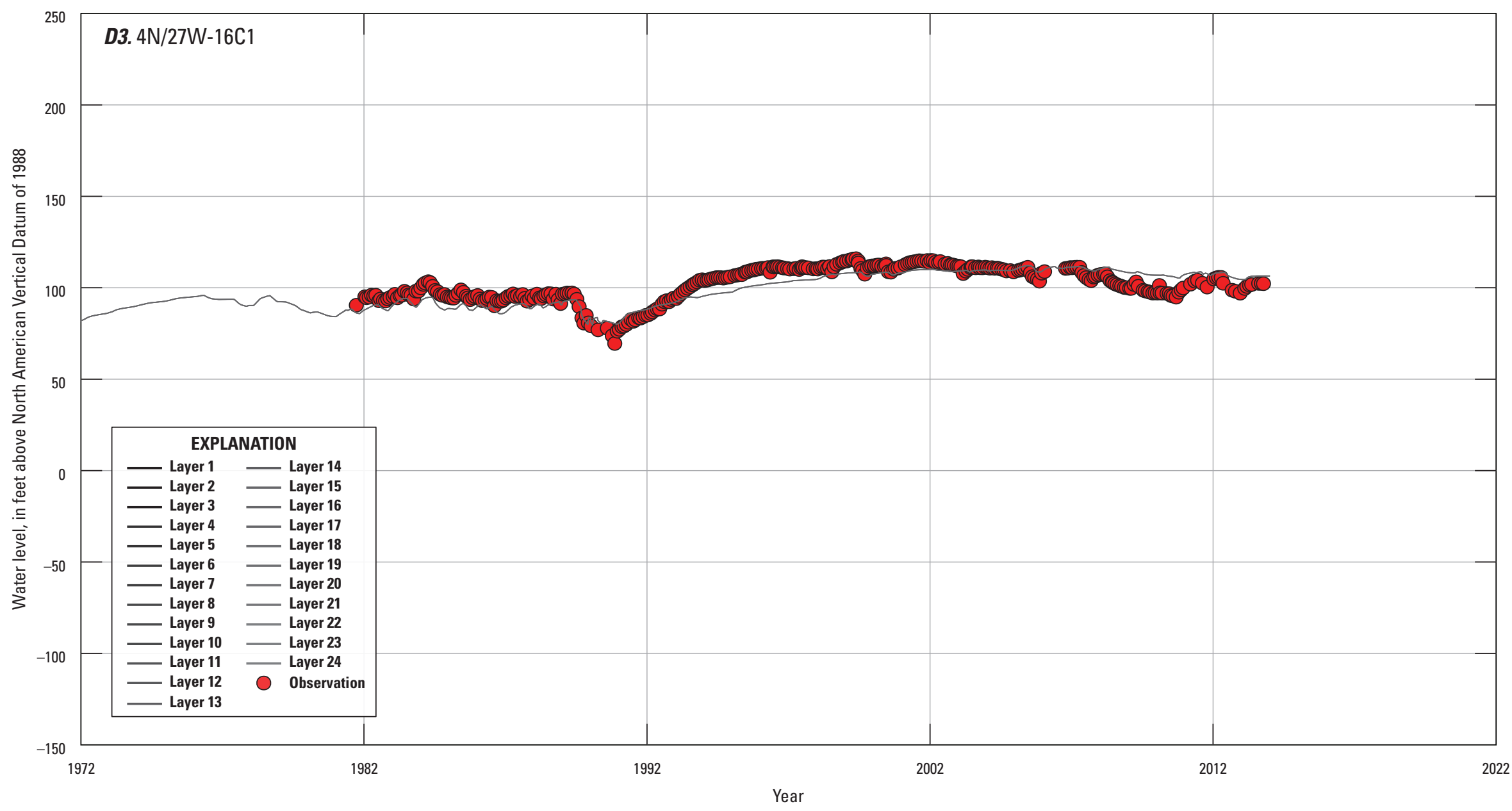

Figure 19. - Continued 


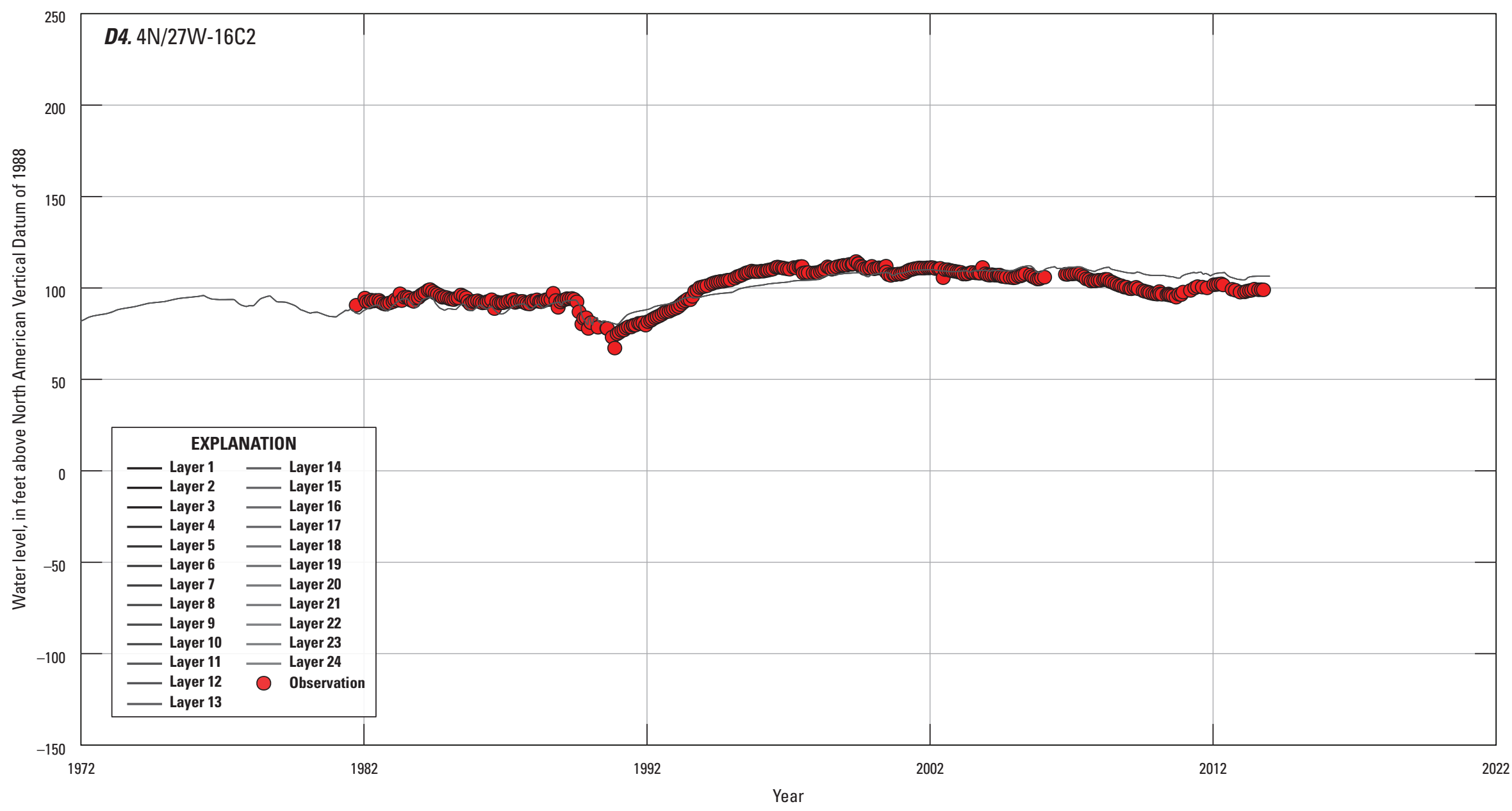

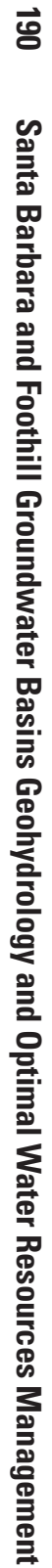

Figure 19. - Continued 


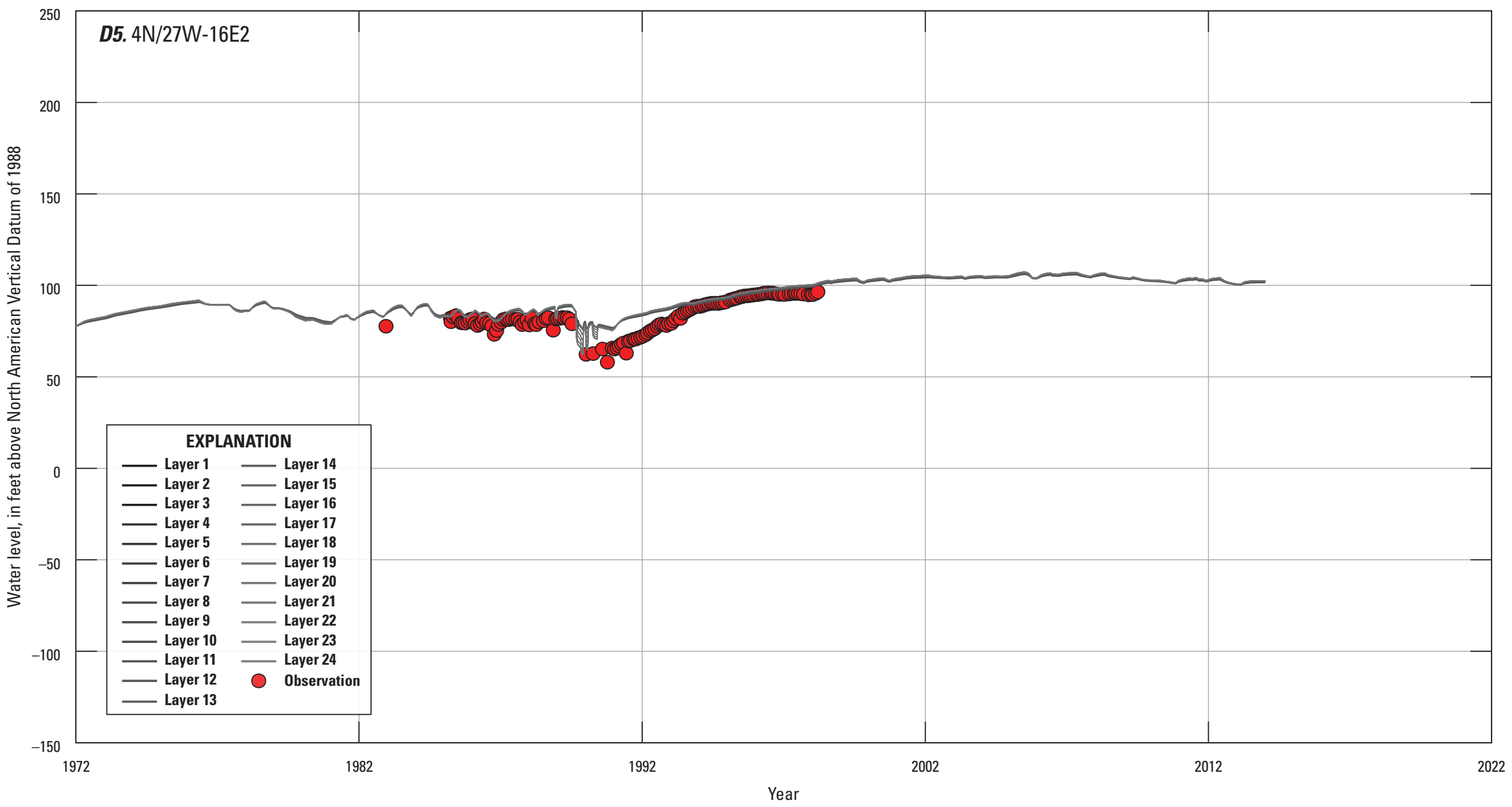

Figure 19. - Continued

옿

$\vec{\varphi}$ 


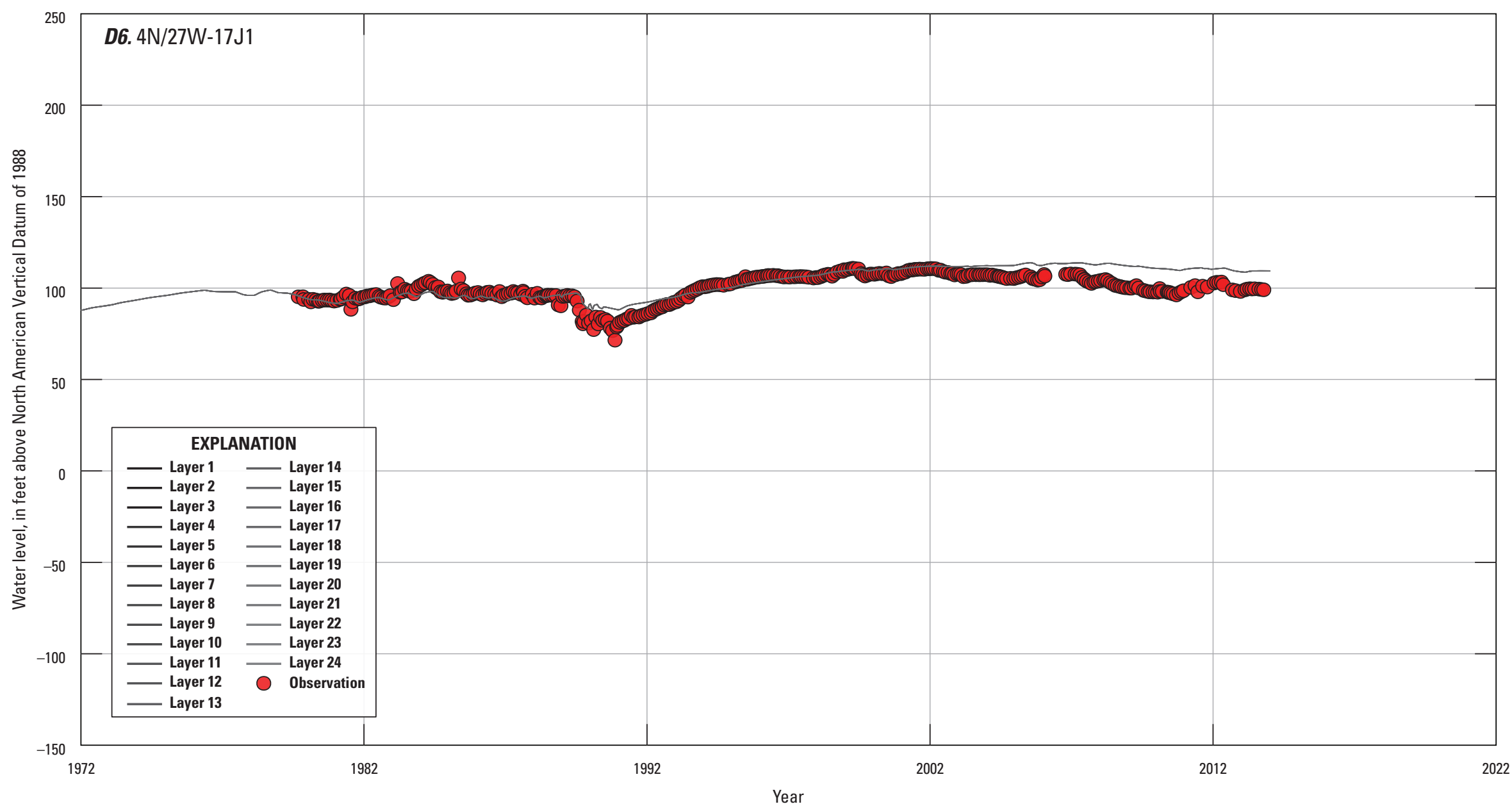

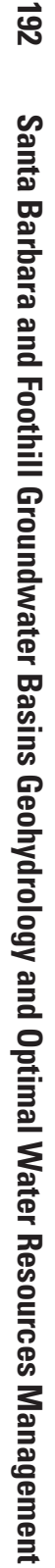

Figure 19. - Continued 


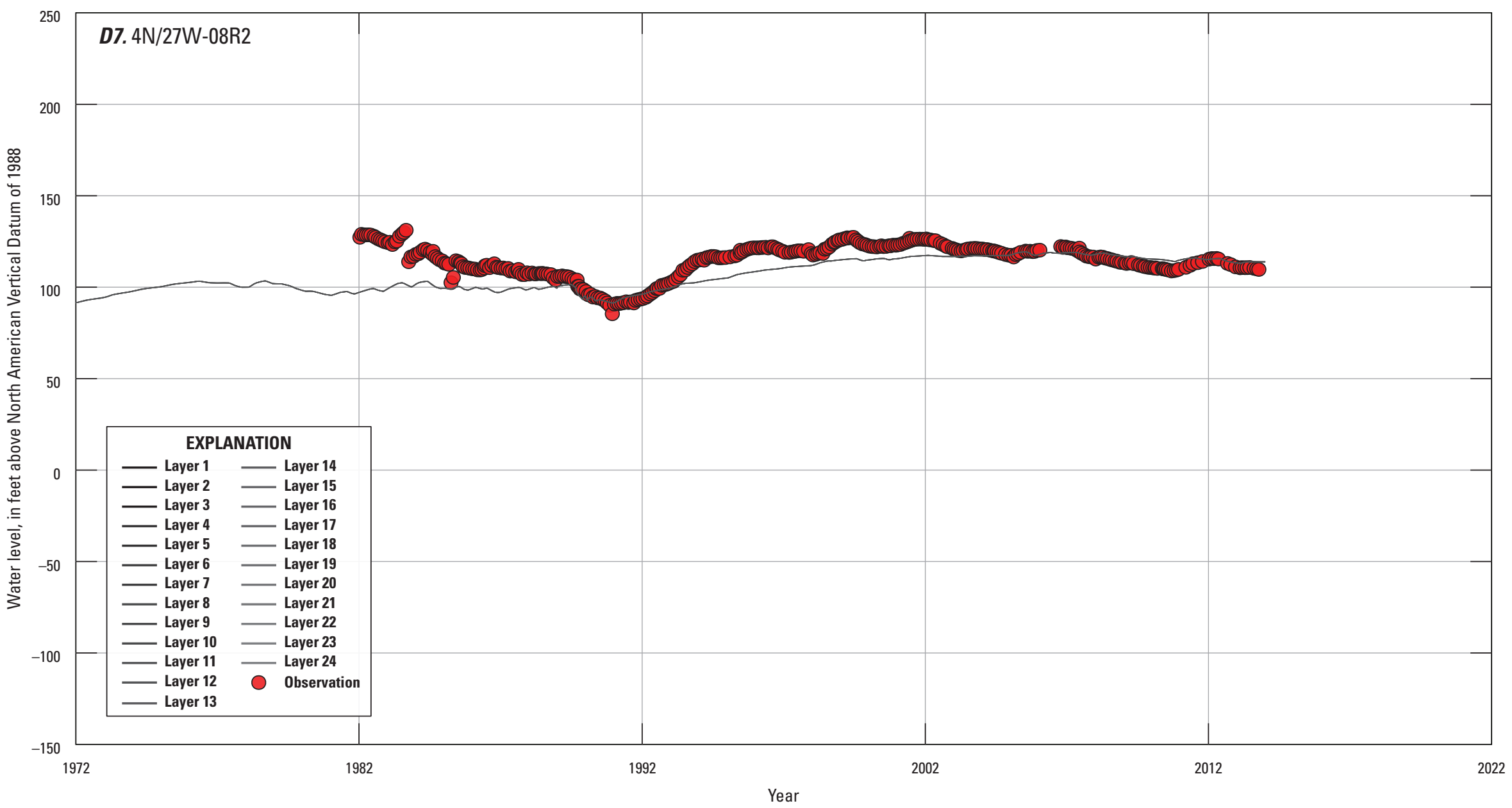

Figure 19. - Continued

હ 


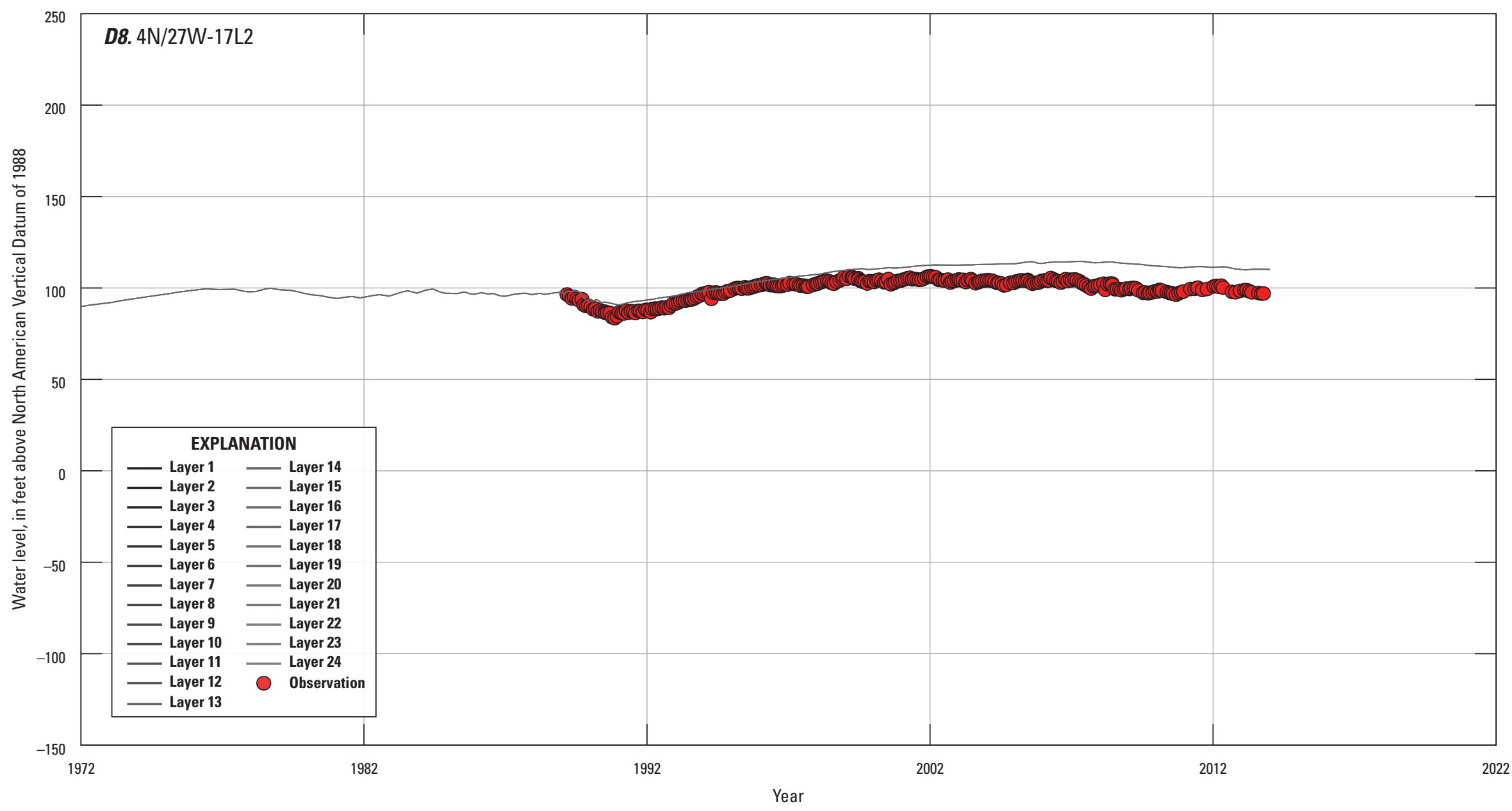

Figure 19. - Continued 


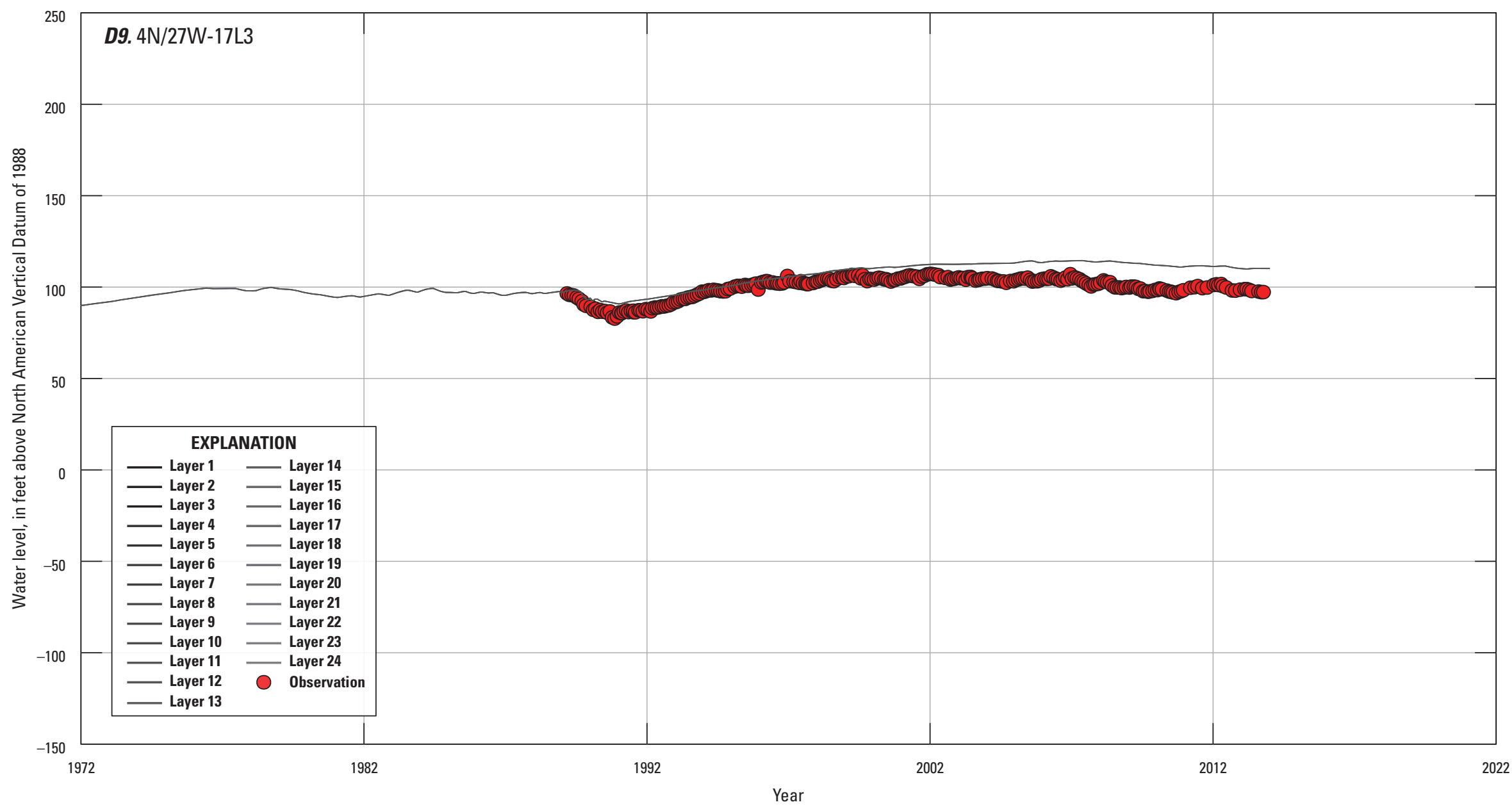

Figure 19. - Continued 


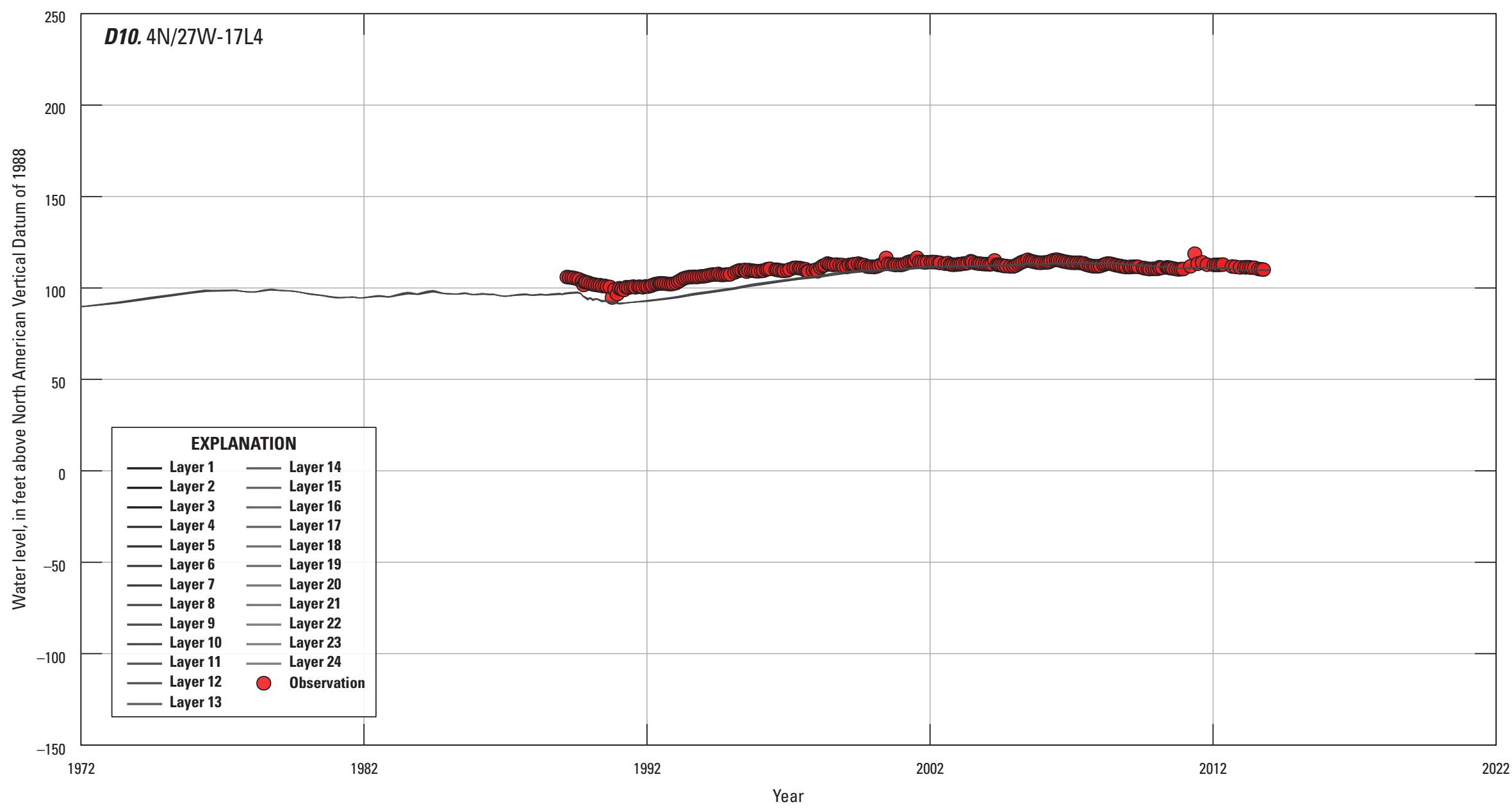

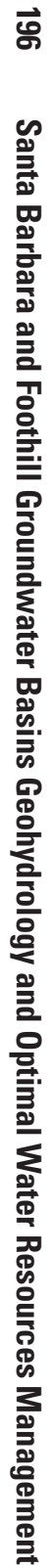




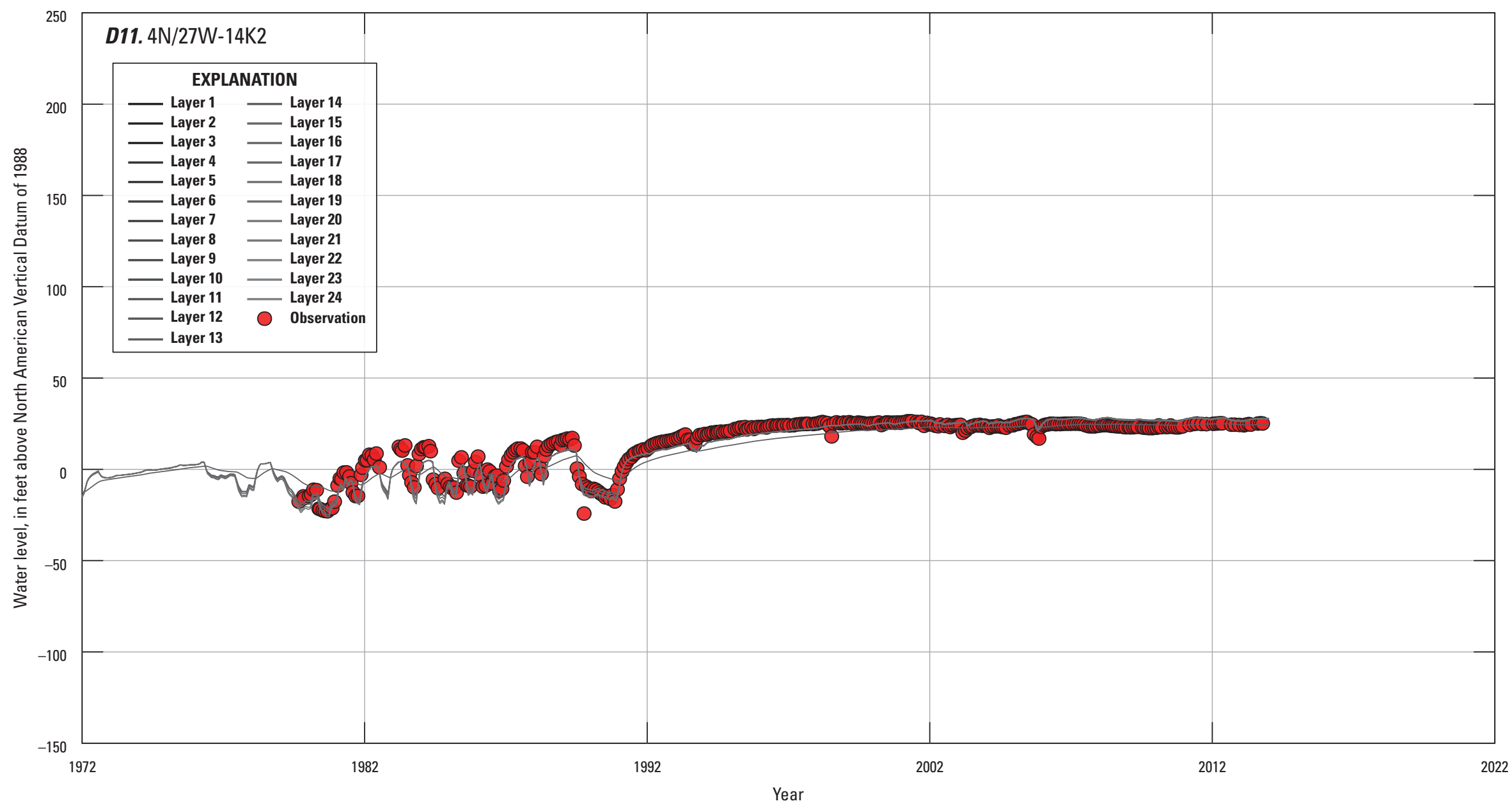

Figure 19. - Continued 


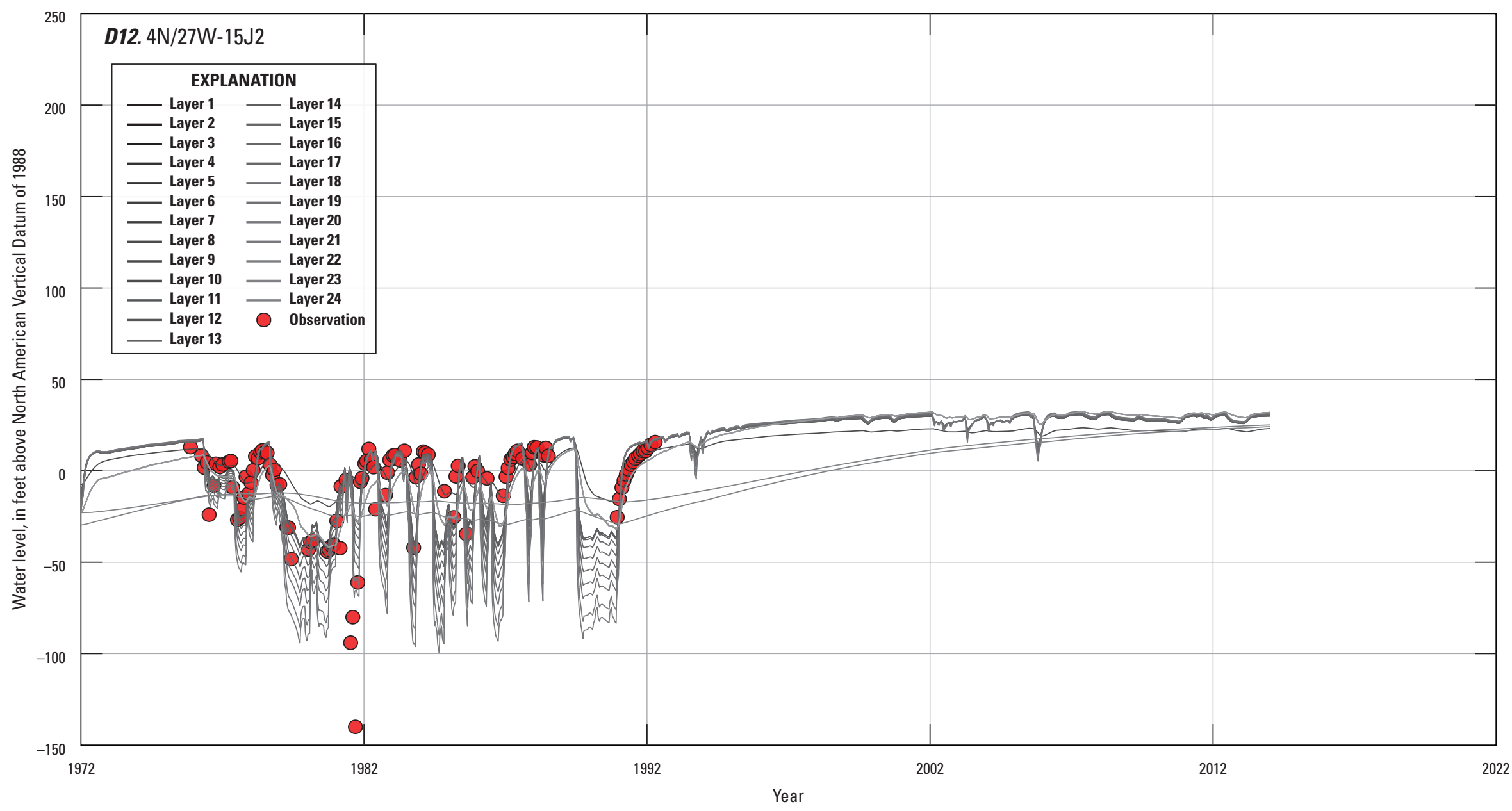

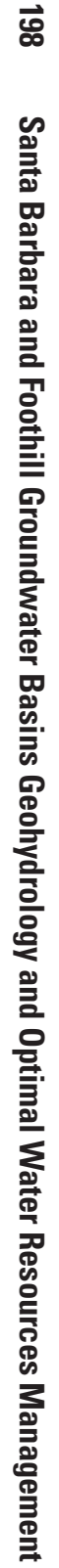

Figure 19. - Continued 


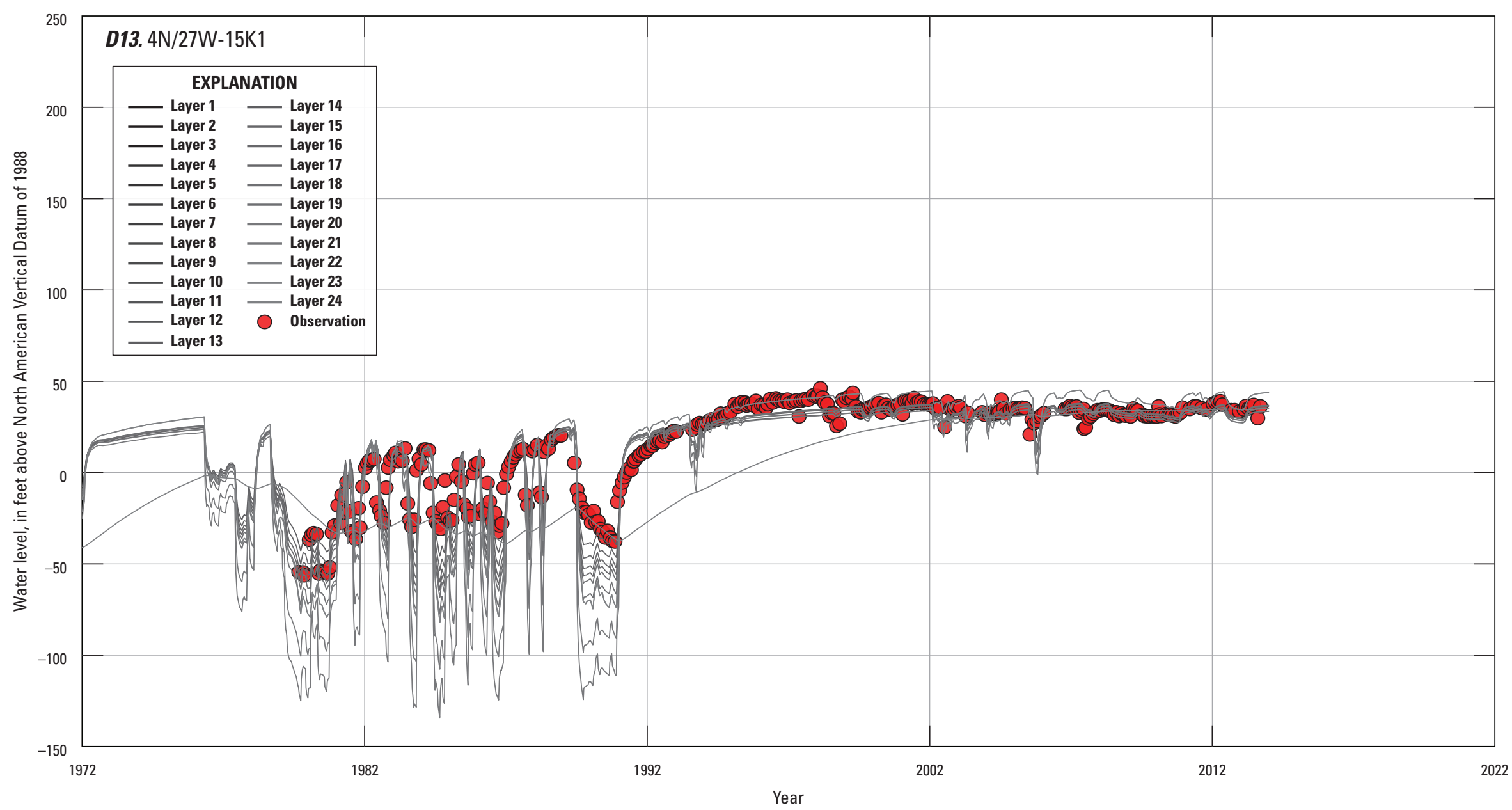

Figure 19. - Continued 


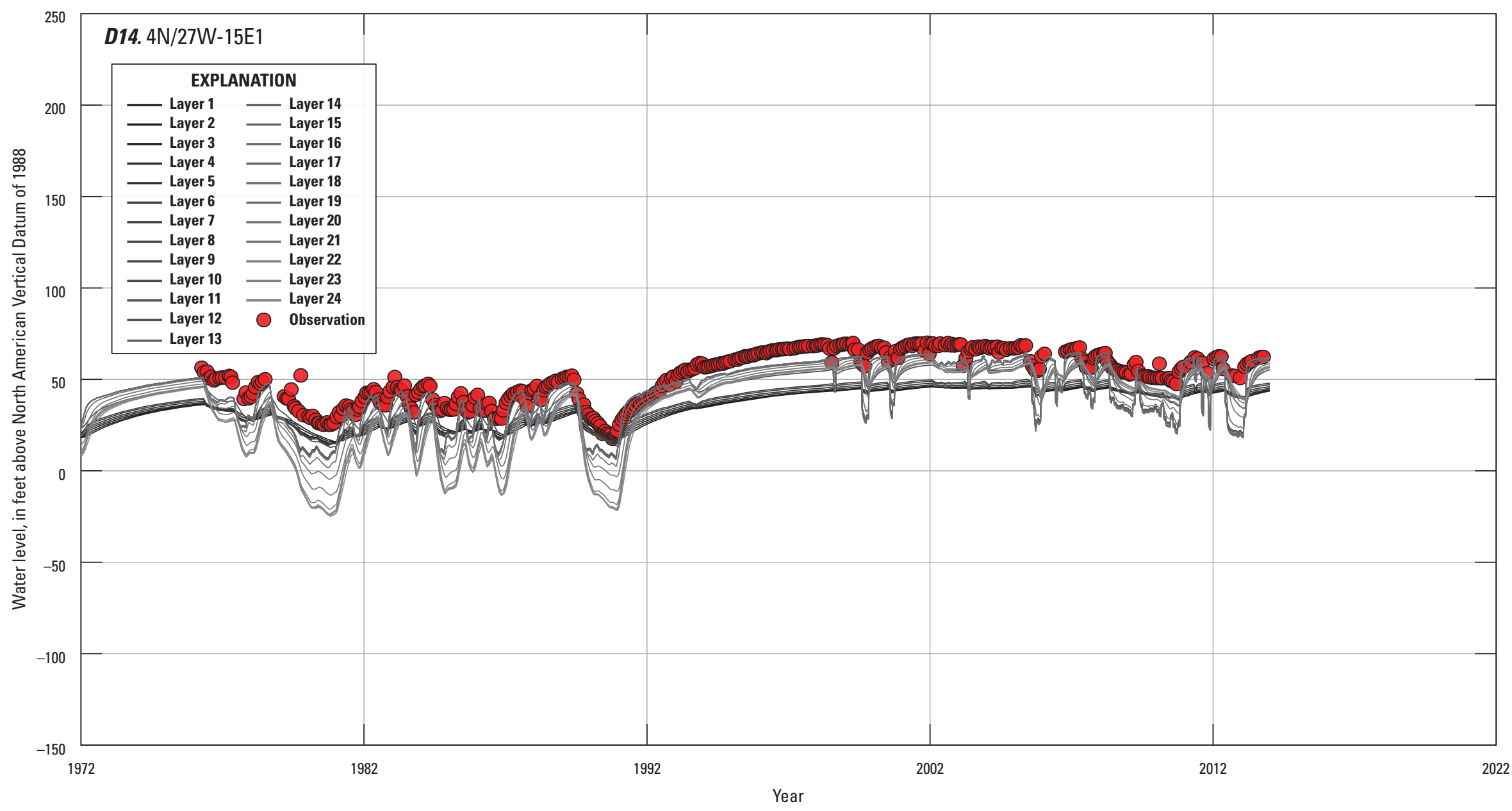

Figure 19. - Continued 


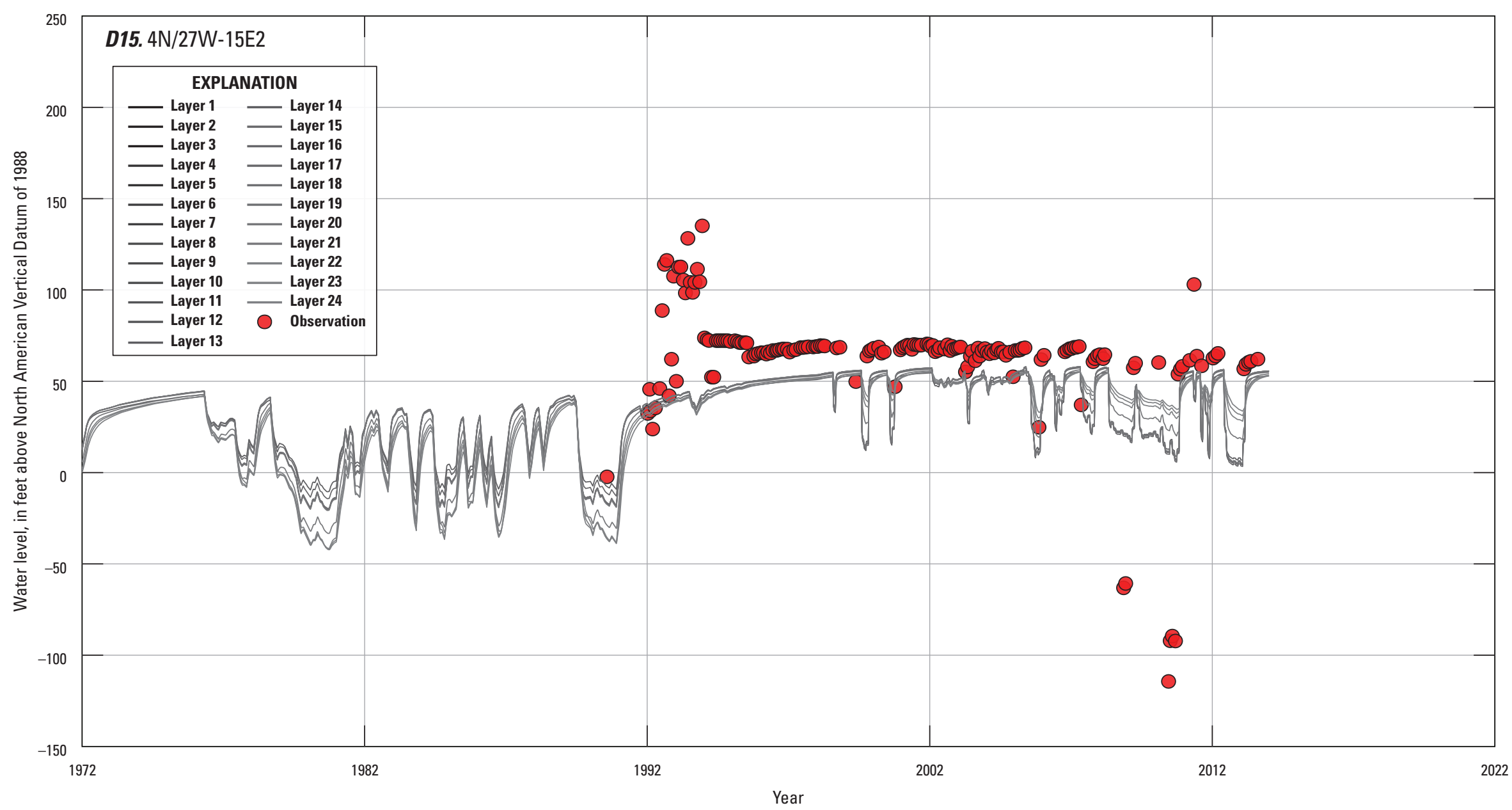

Figure 19. - Continued 


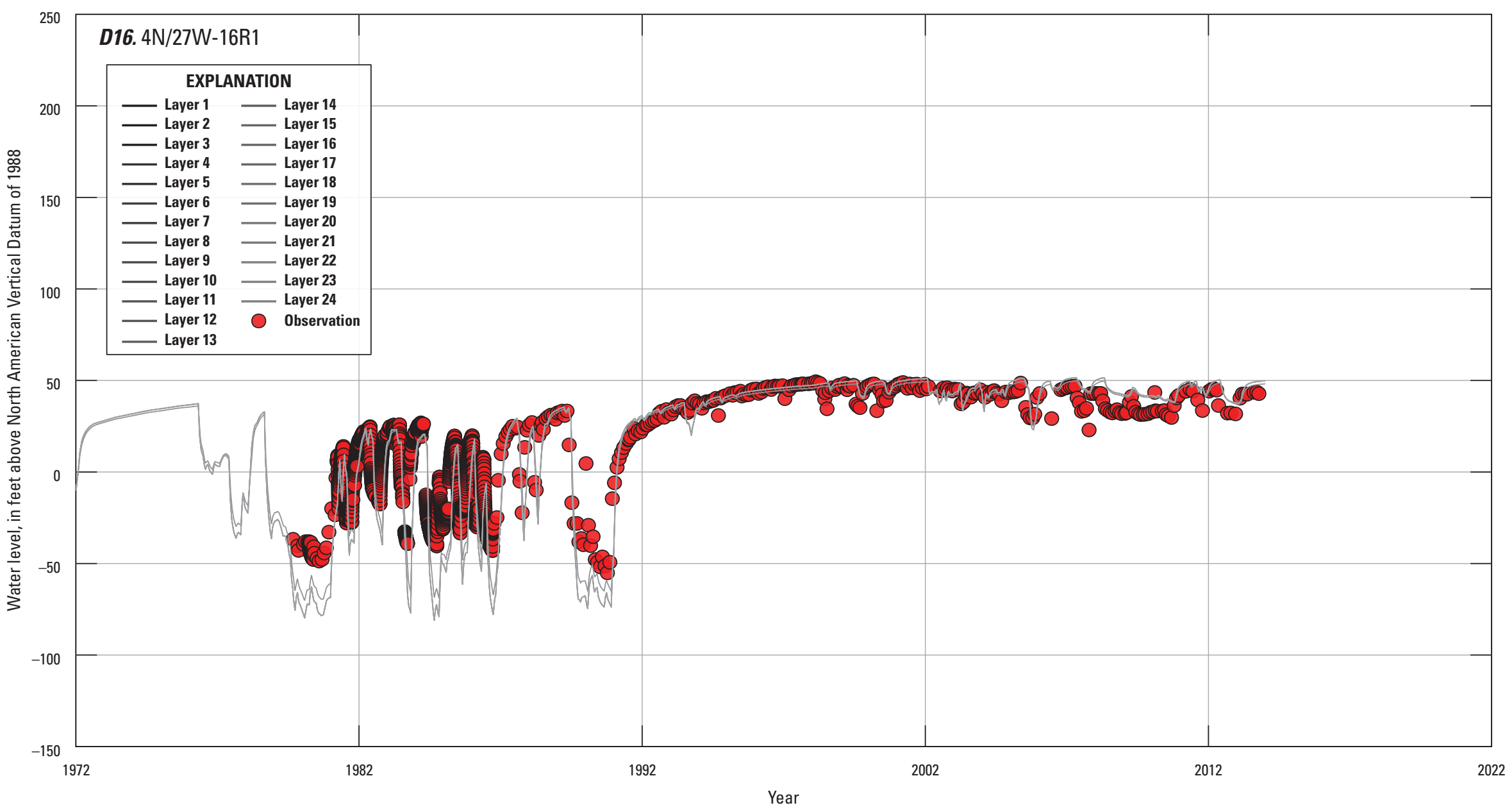

ิㅡำ

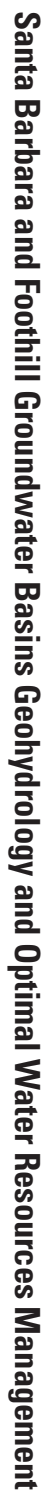




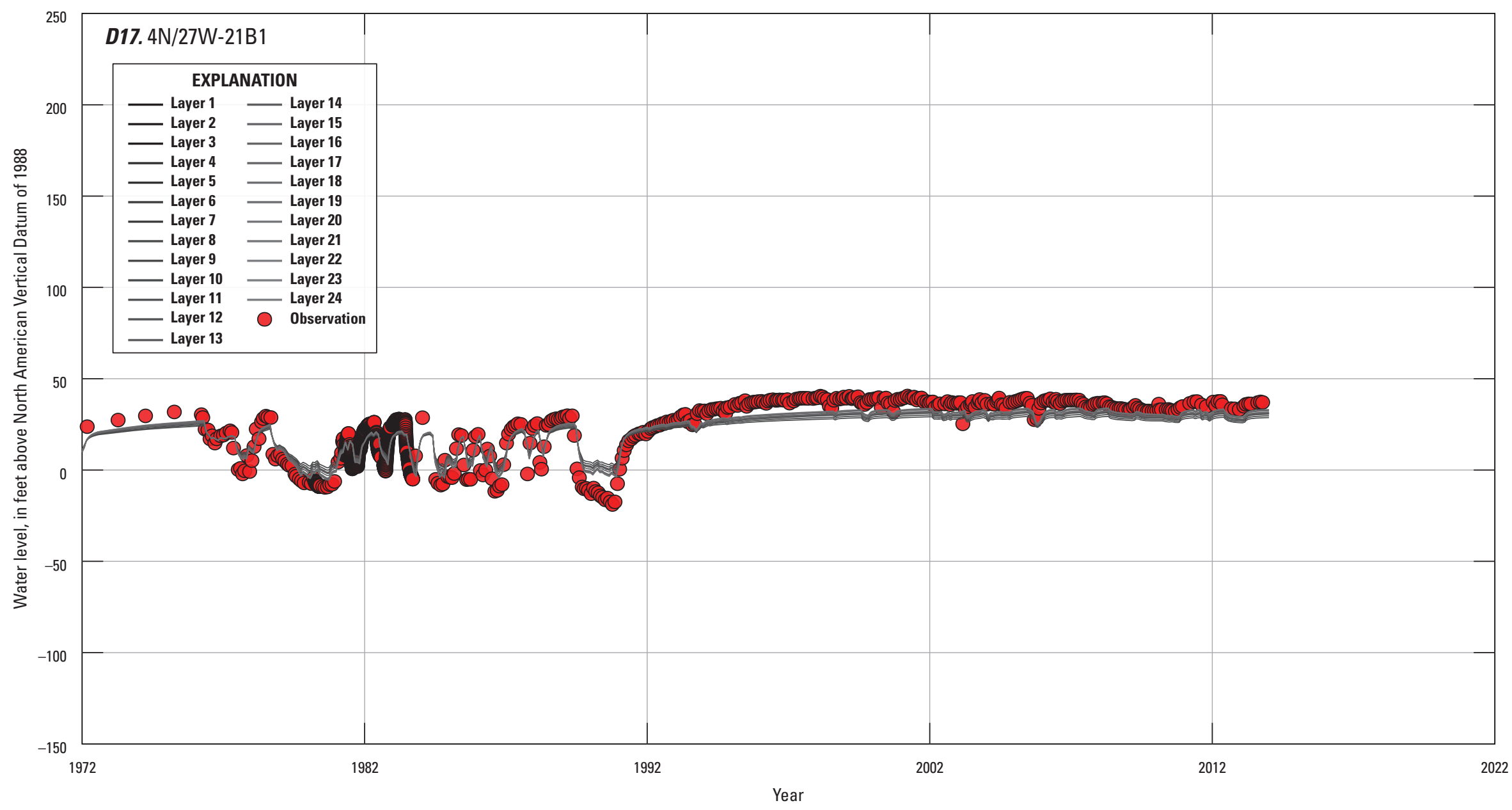

Figure 19. - Continued 


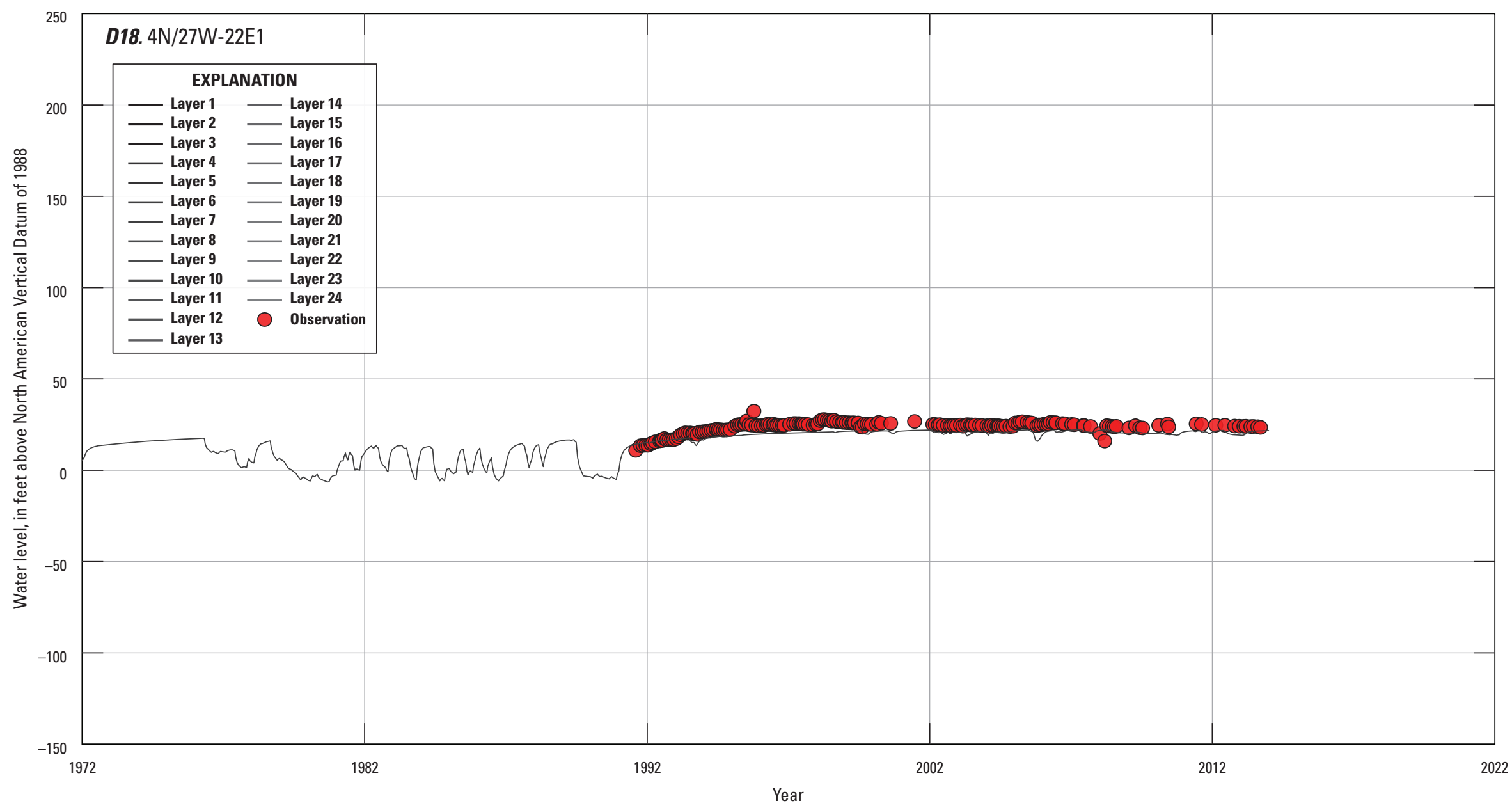

Figure 19. - Continued 


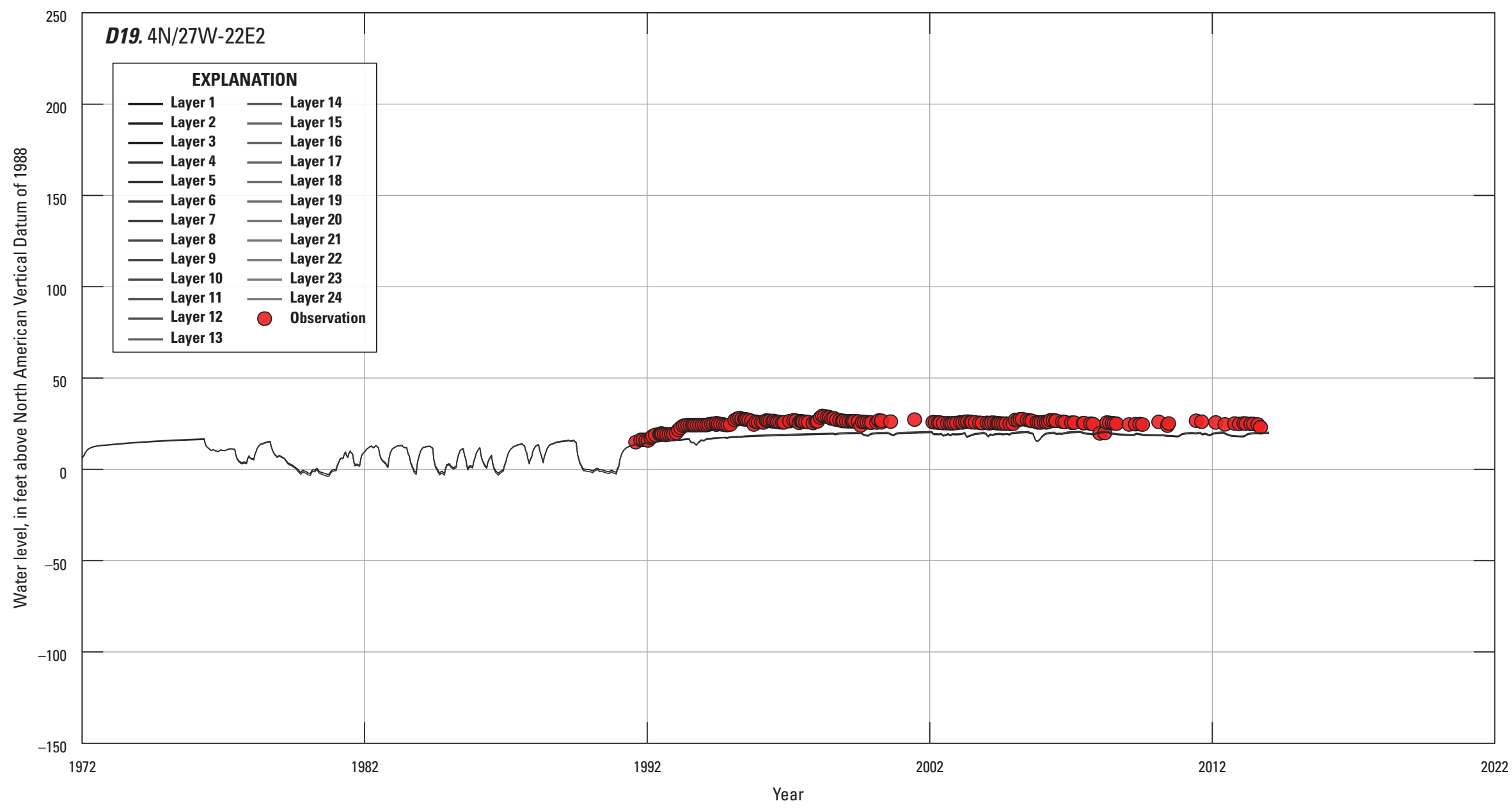

Figure 19. - Continued 


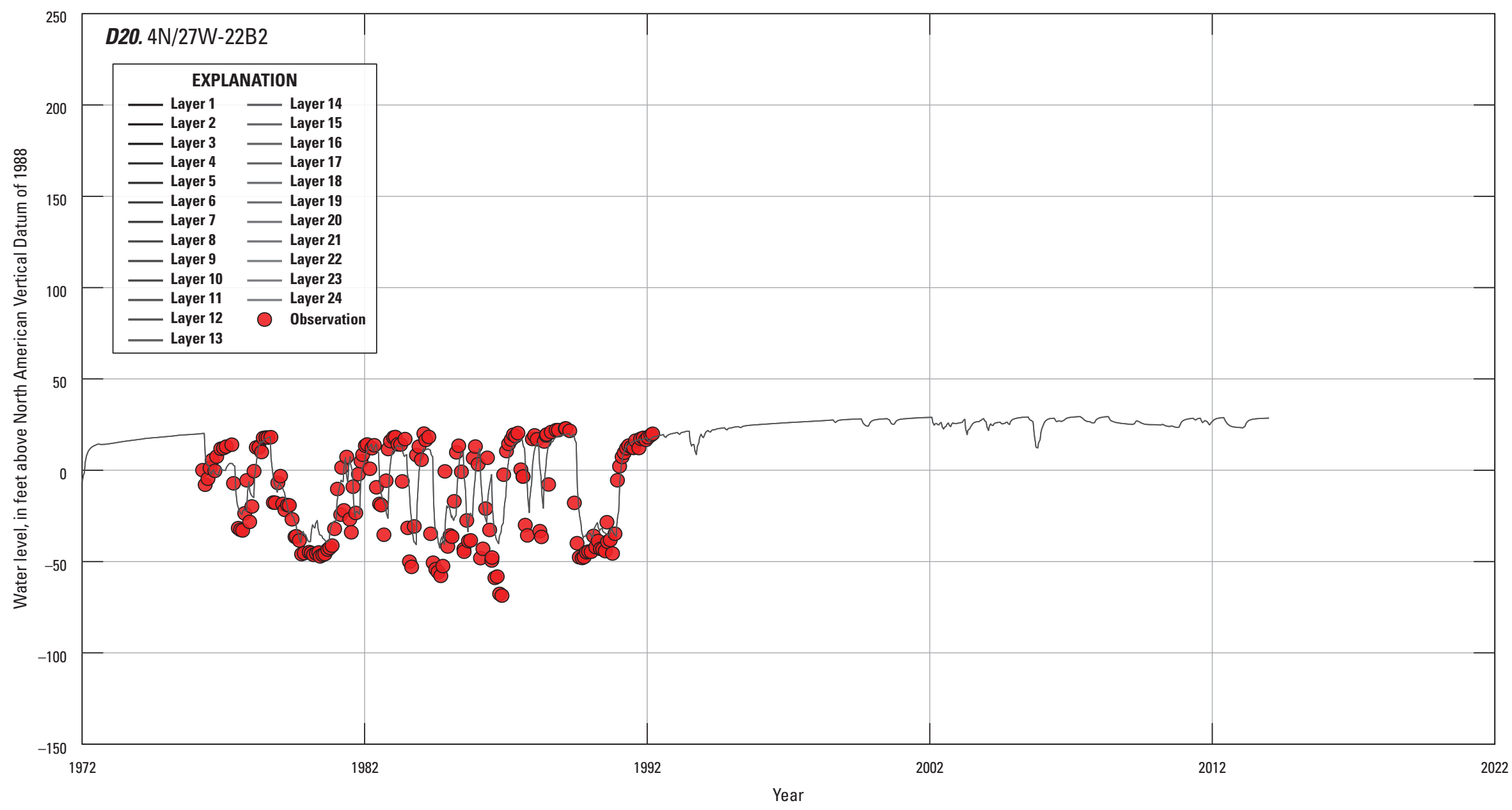

Figure 19. - Continued 


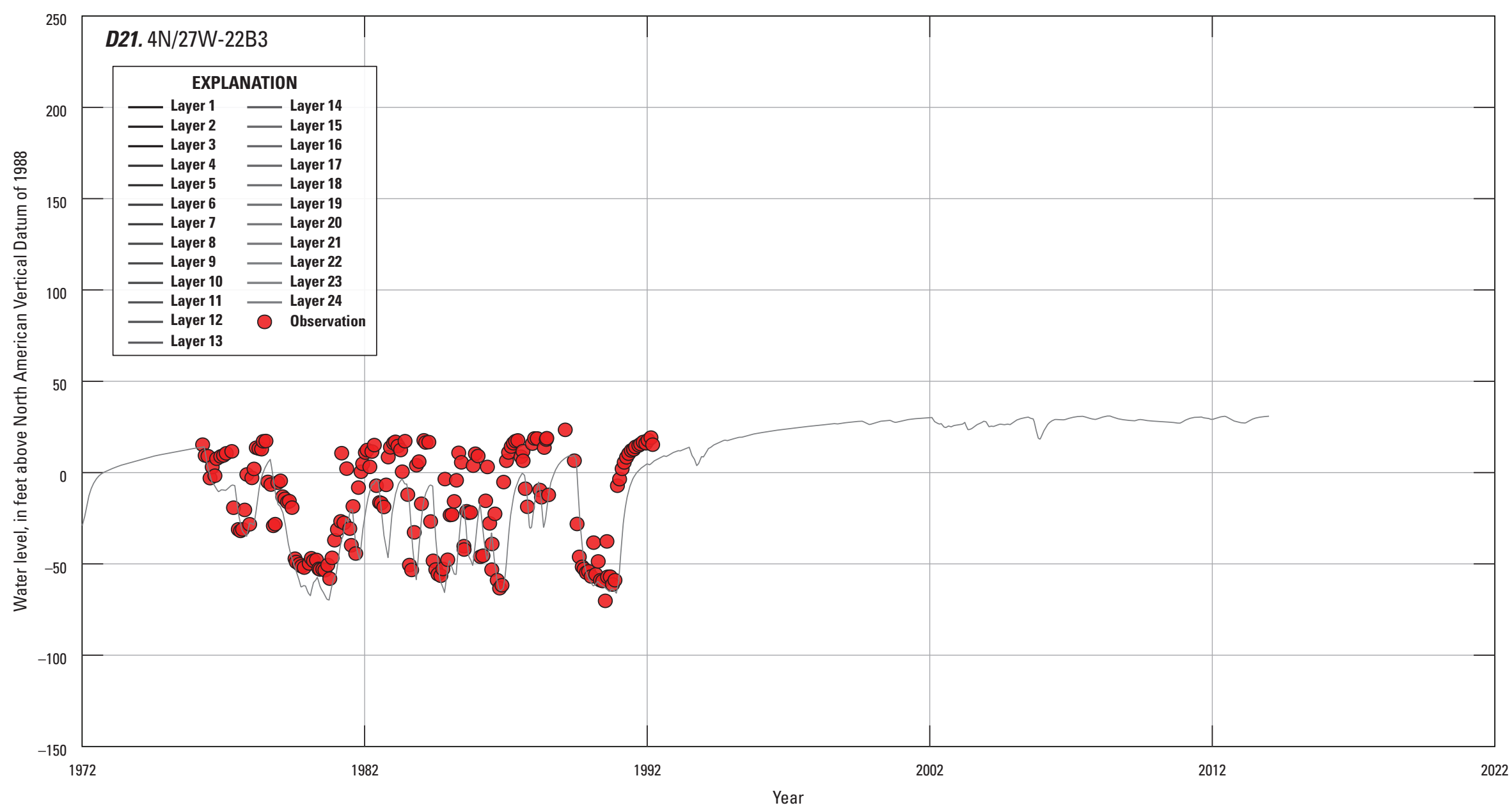

Figure 19. - Continued 


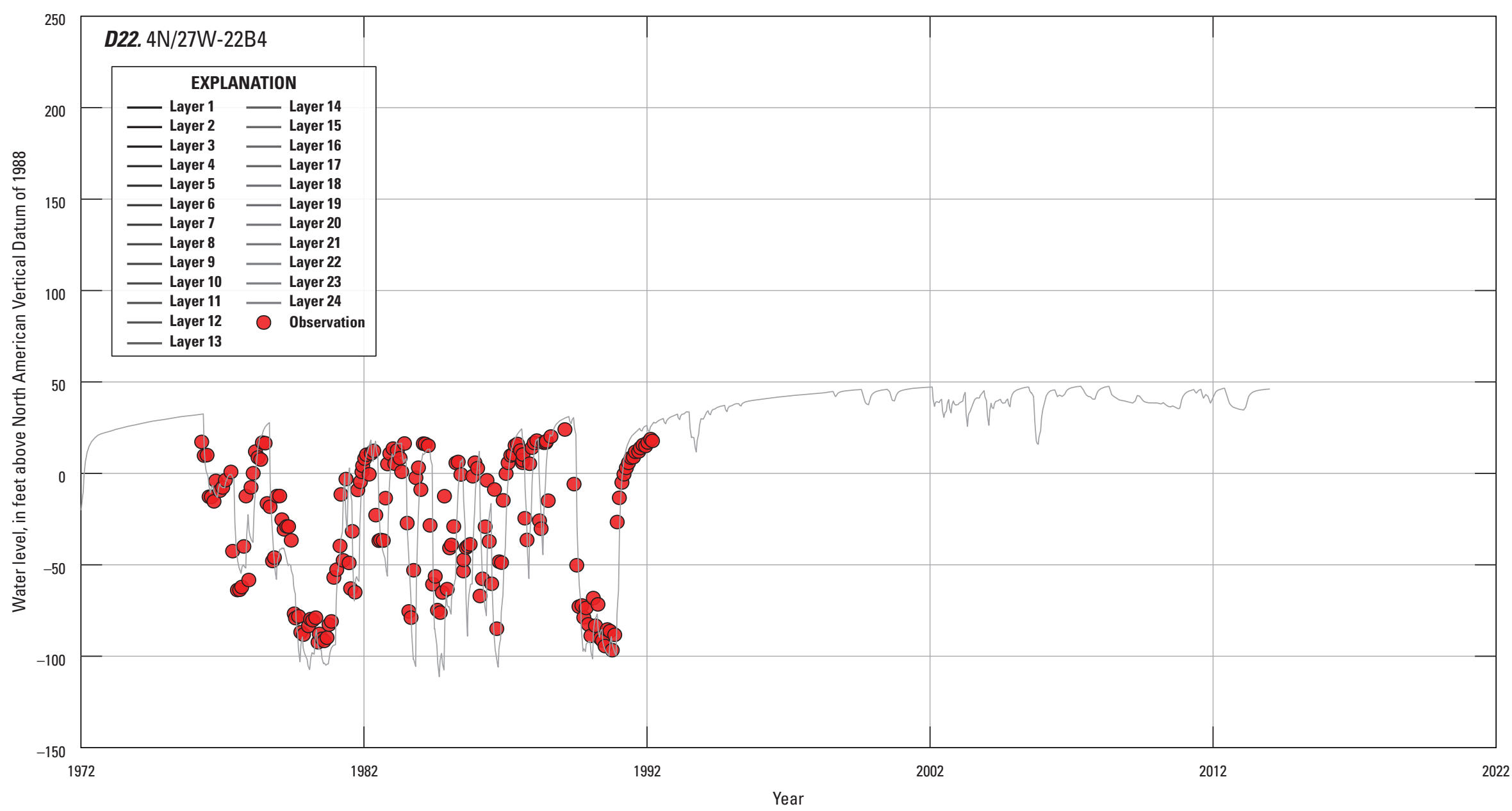

Figure 19. - Continued 


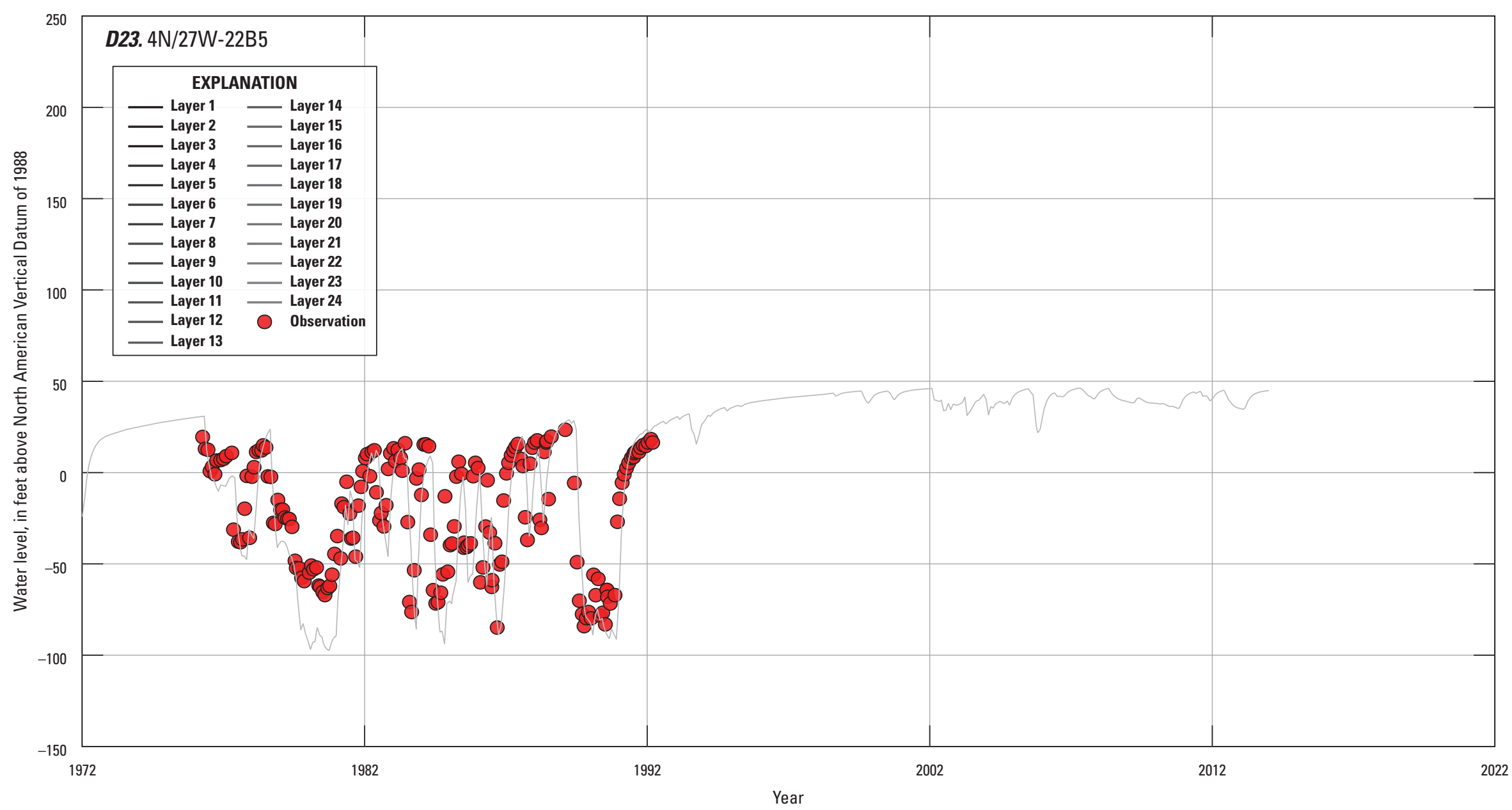

Figure 19. - Continued 


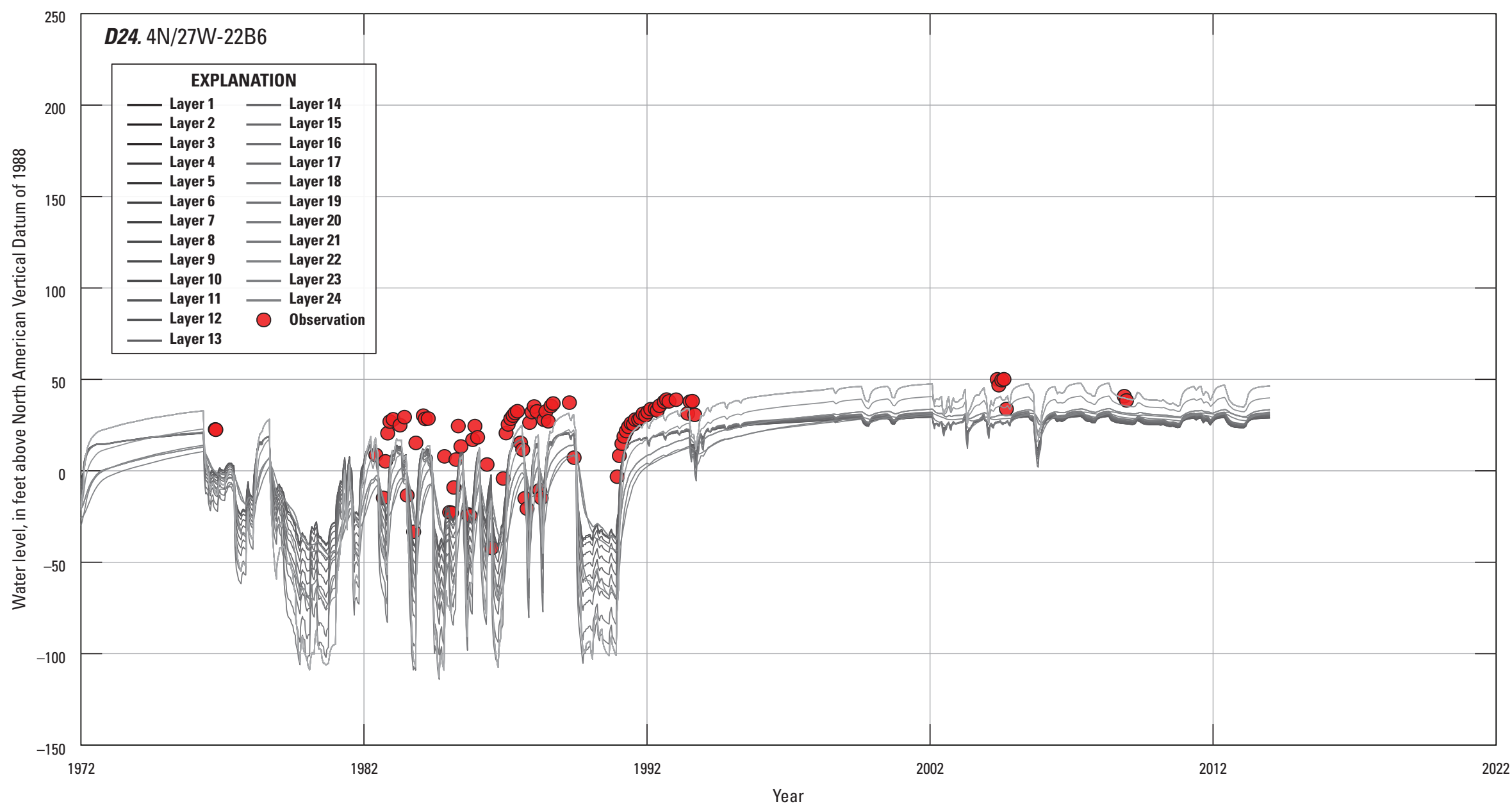

Figure 19. - Continued 


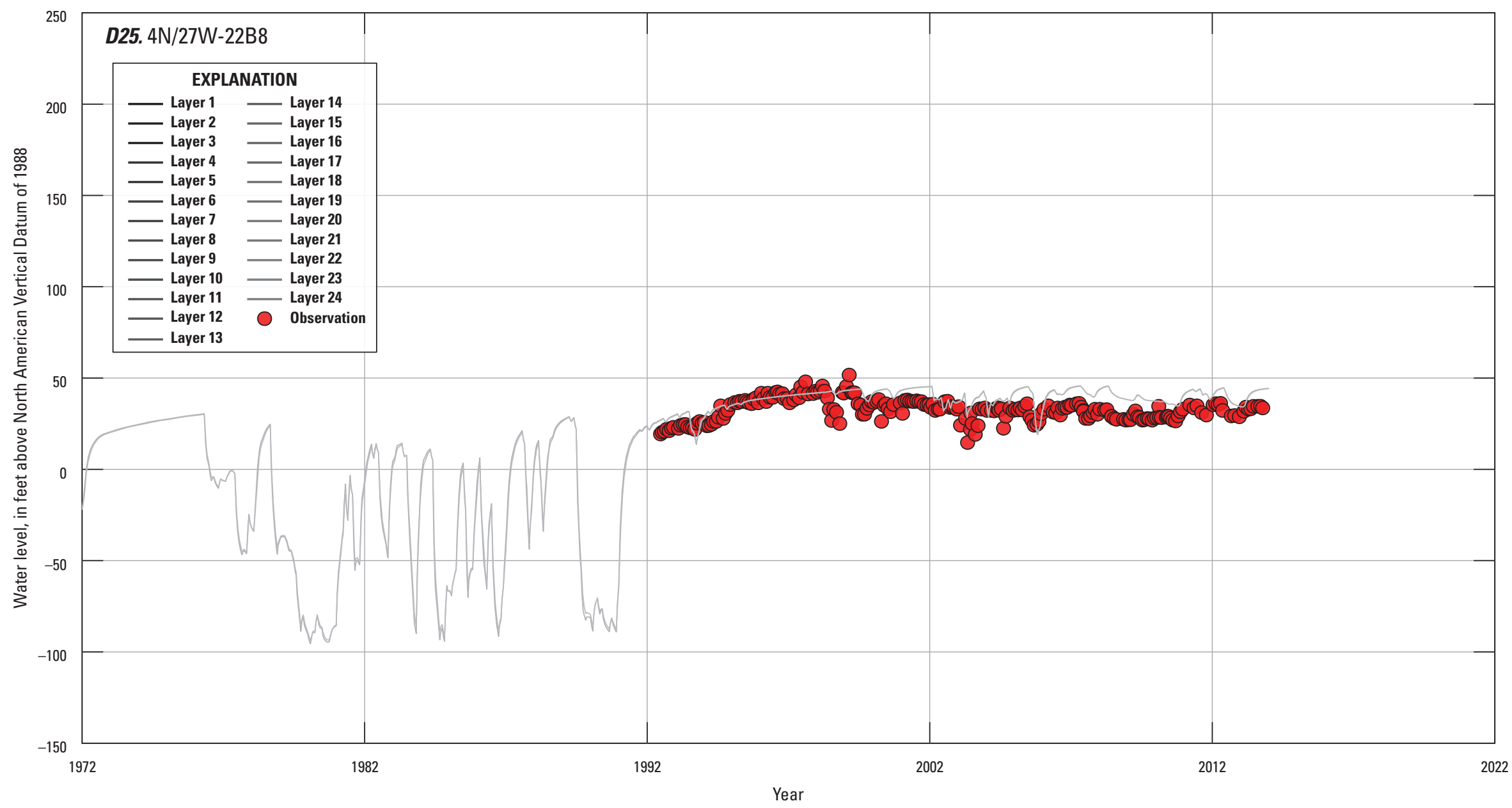

Figure 19. - Continued 


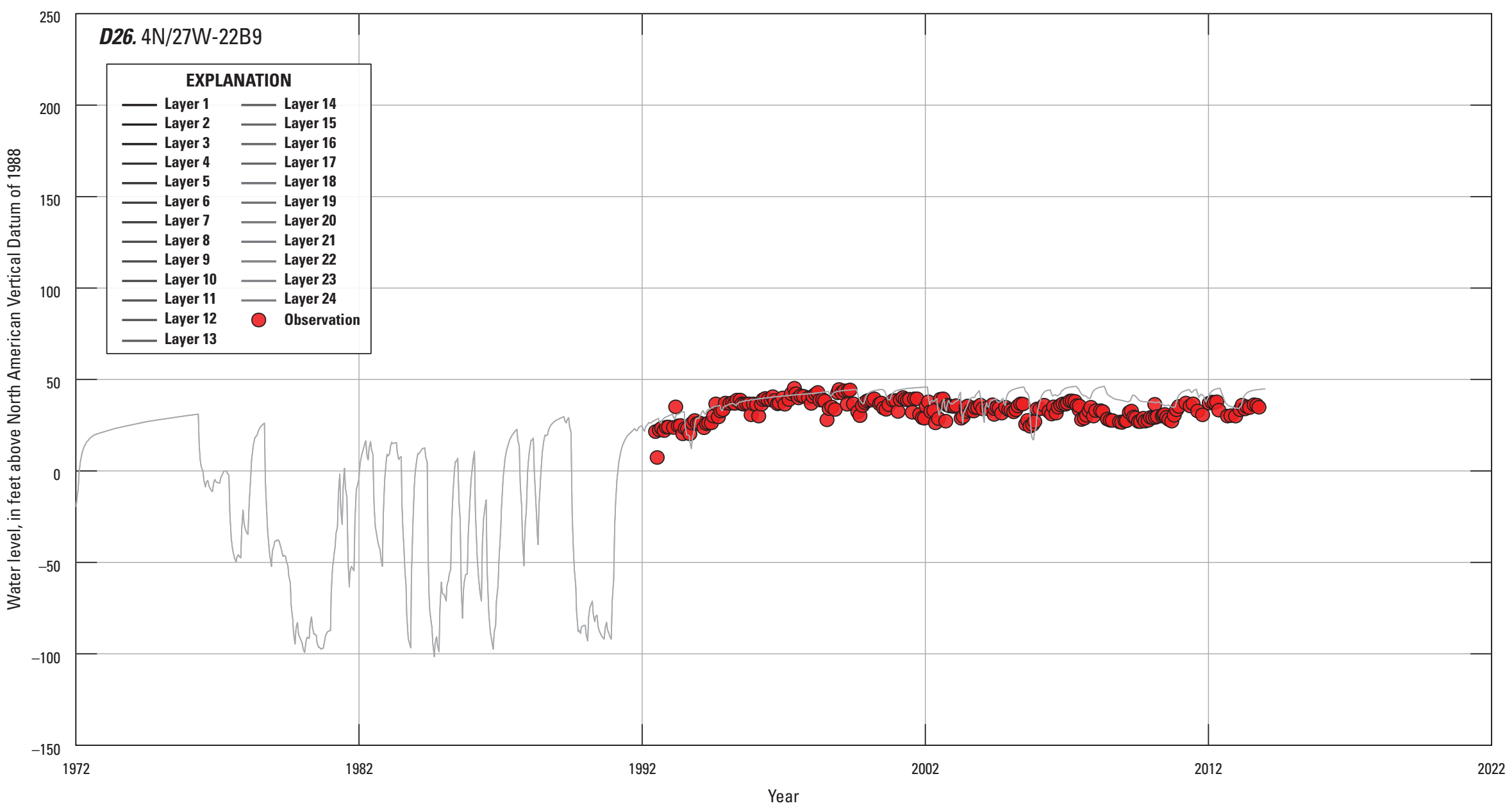

궁

Figure 19. - Continued 


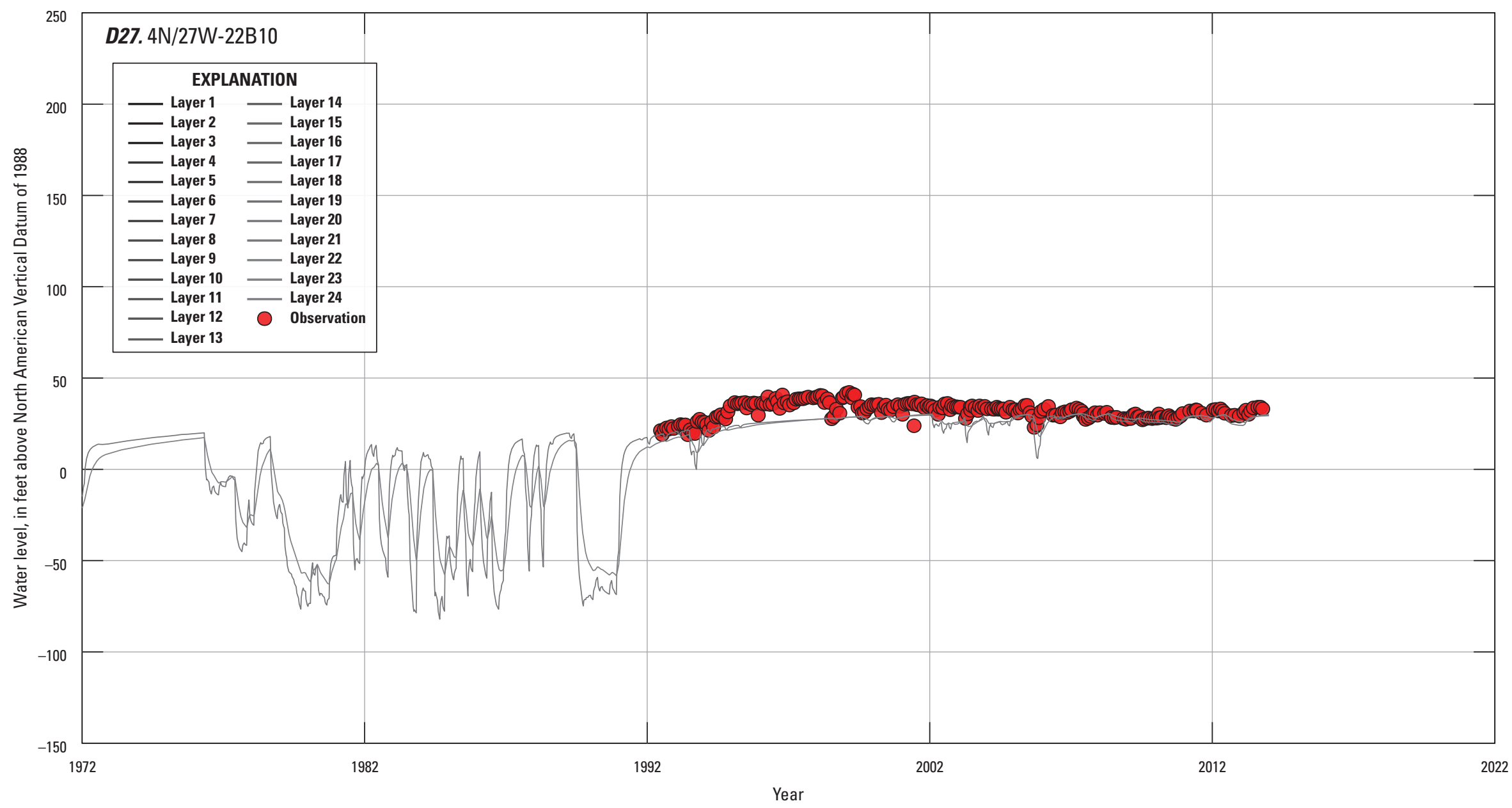

Figure 19. - Continued 


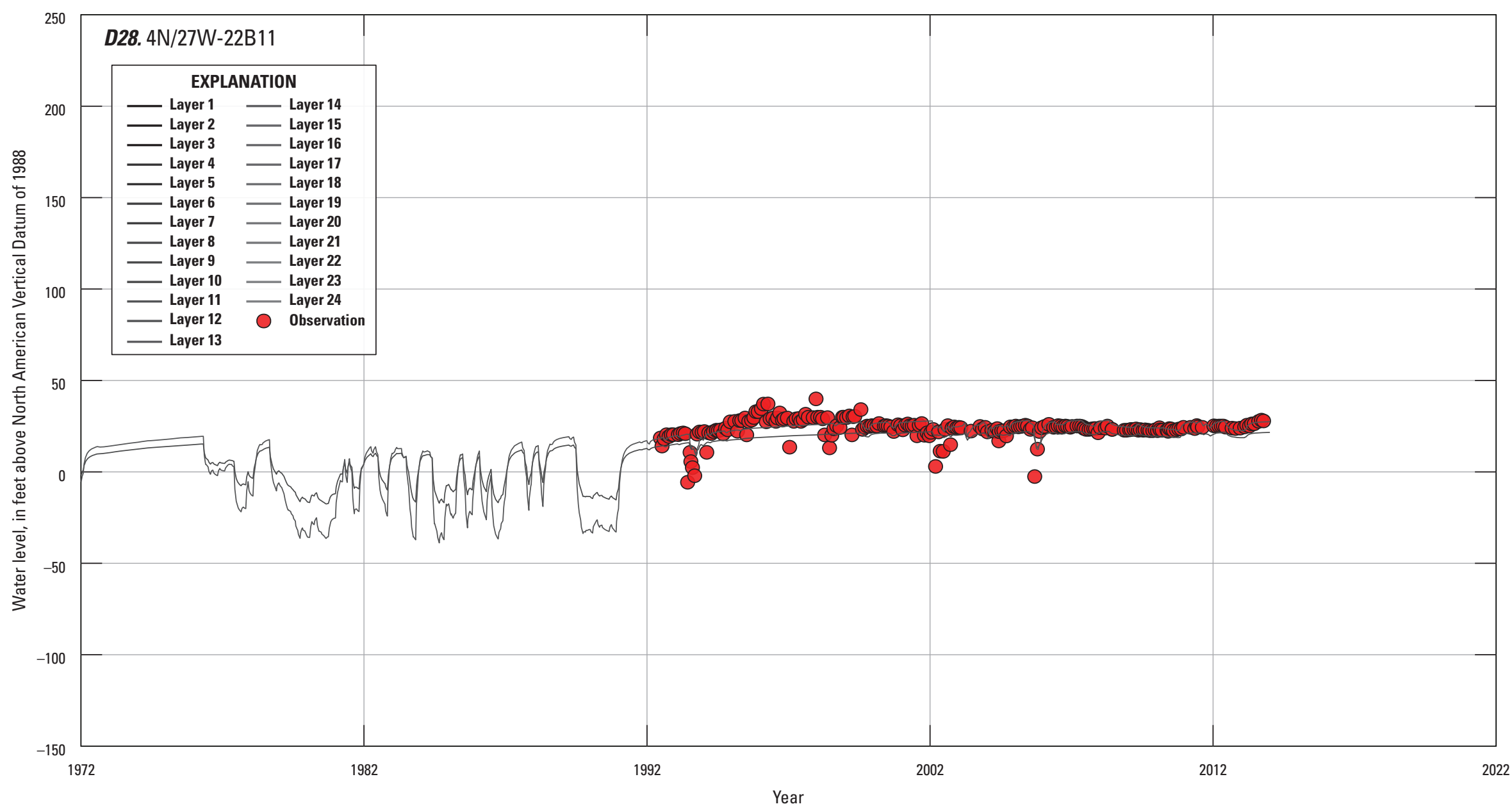

Figure 19. - Continued 


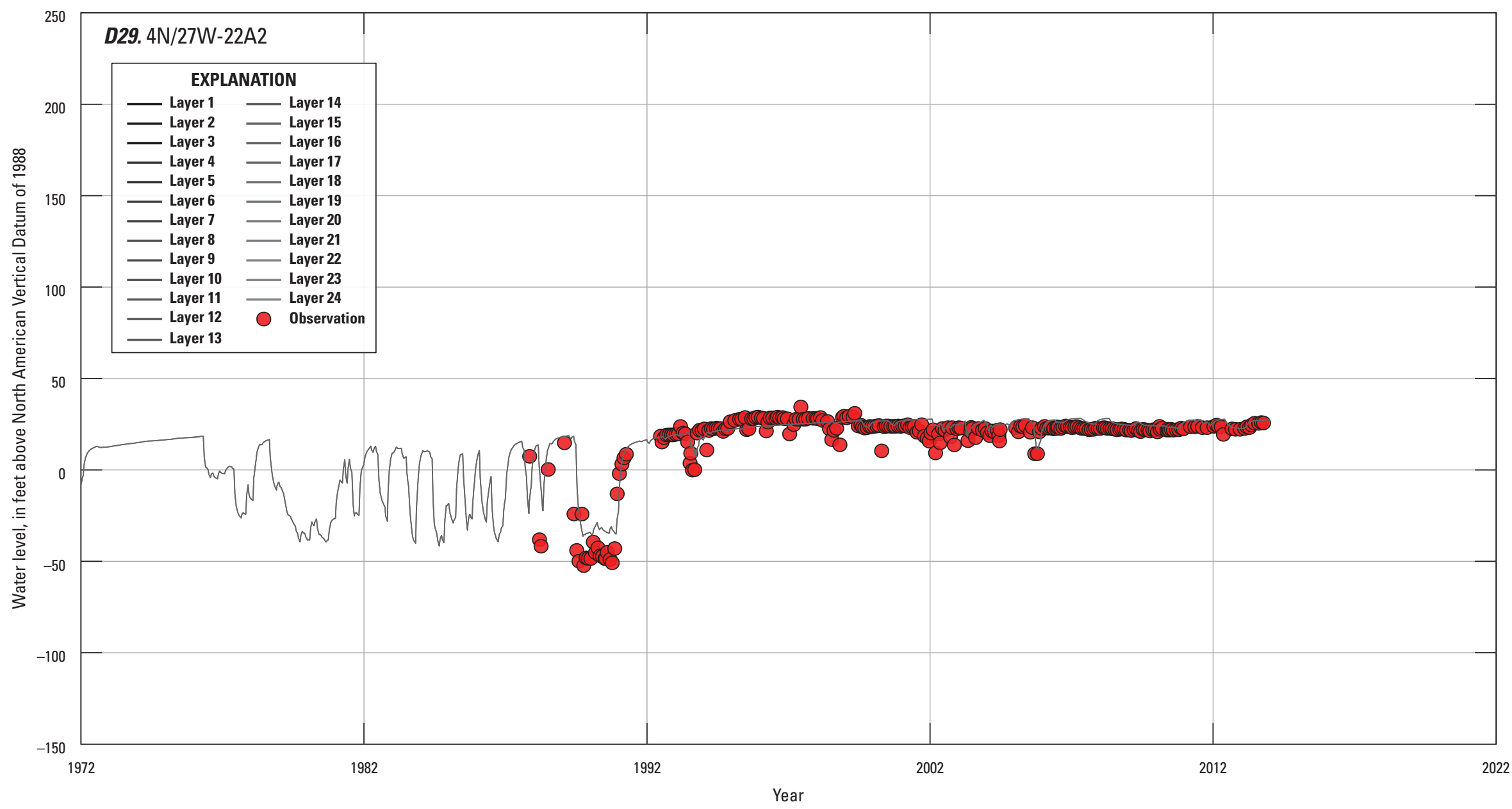

Figure 19. - Continued 


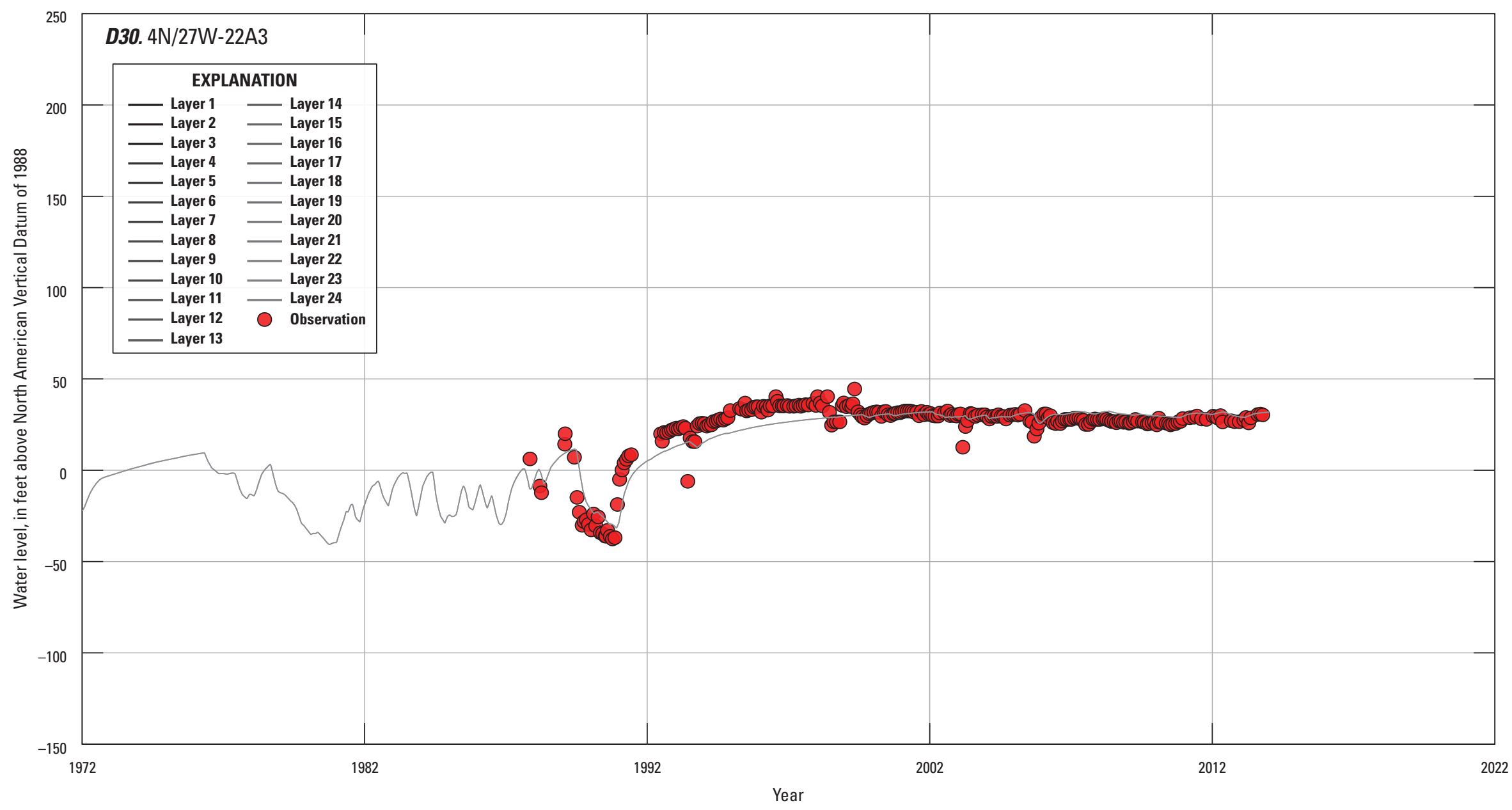

을

Figure 19. - Continued 


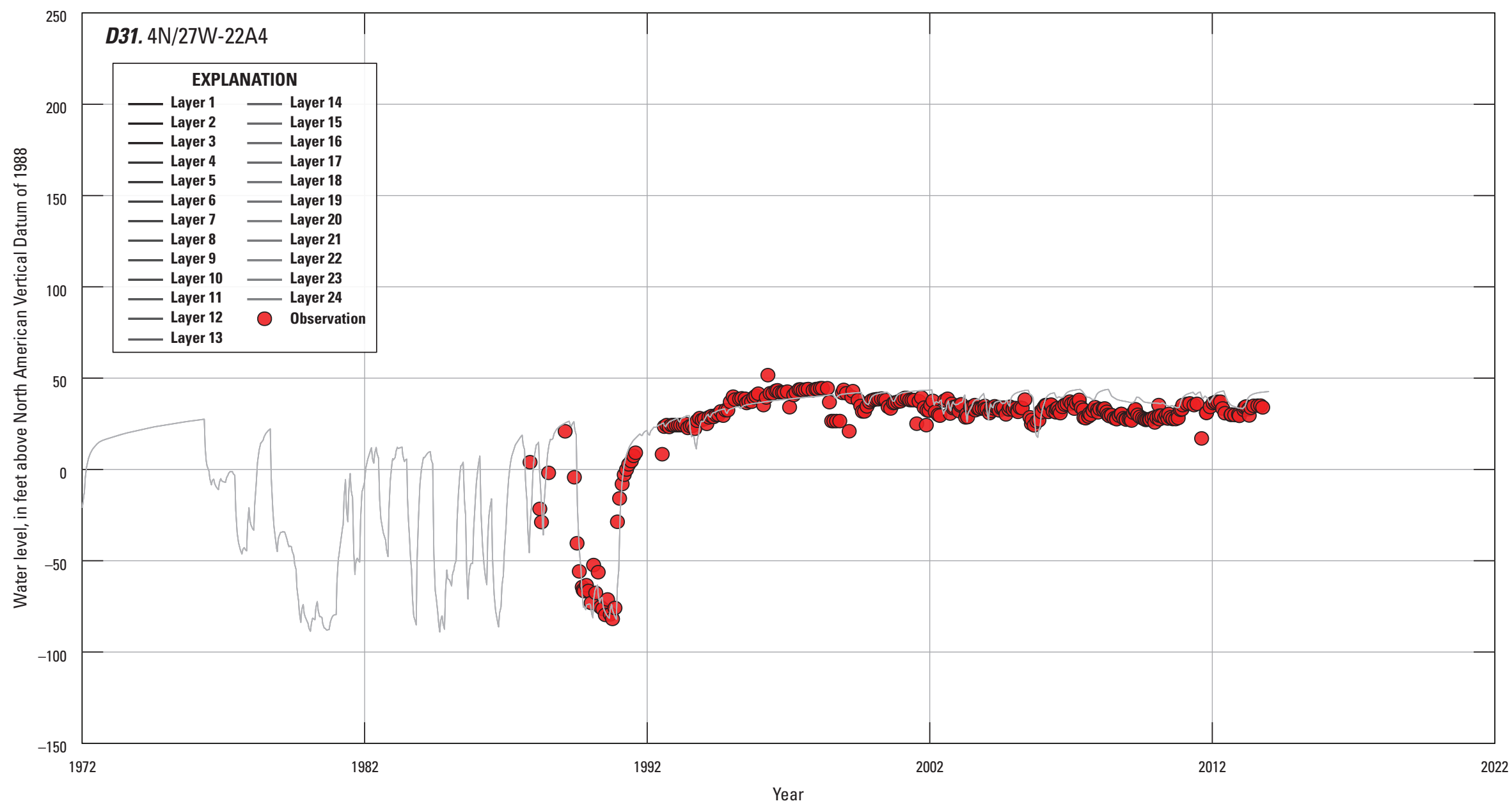

Figure 19. - Continued 


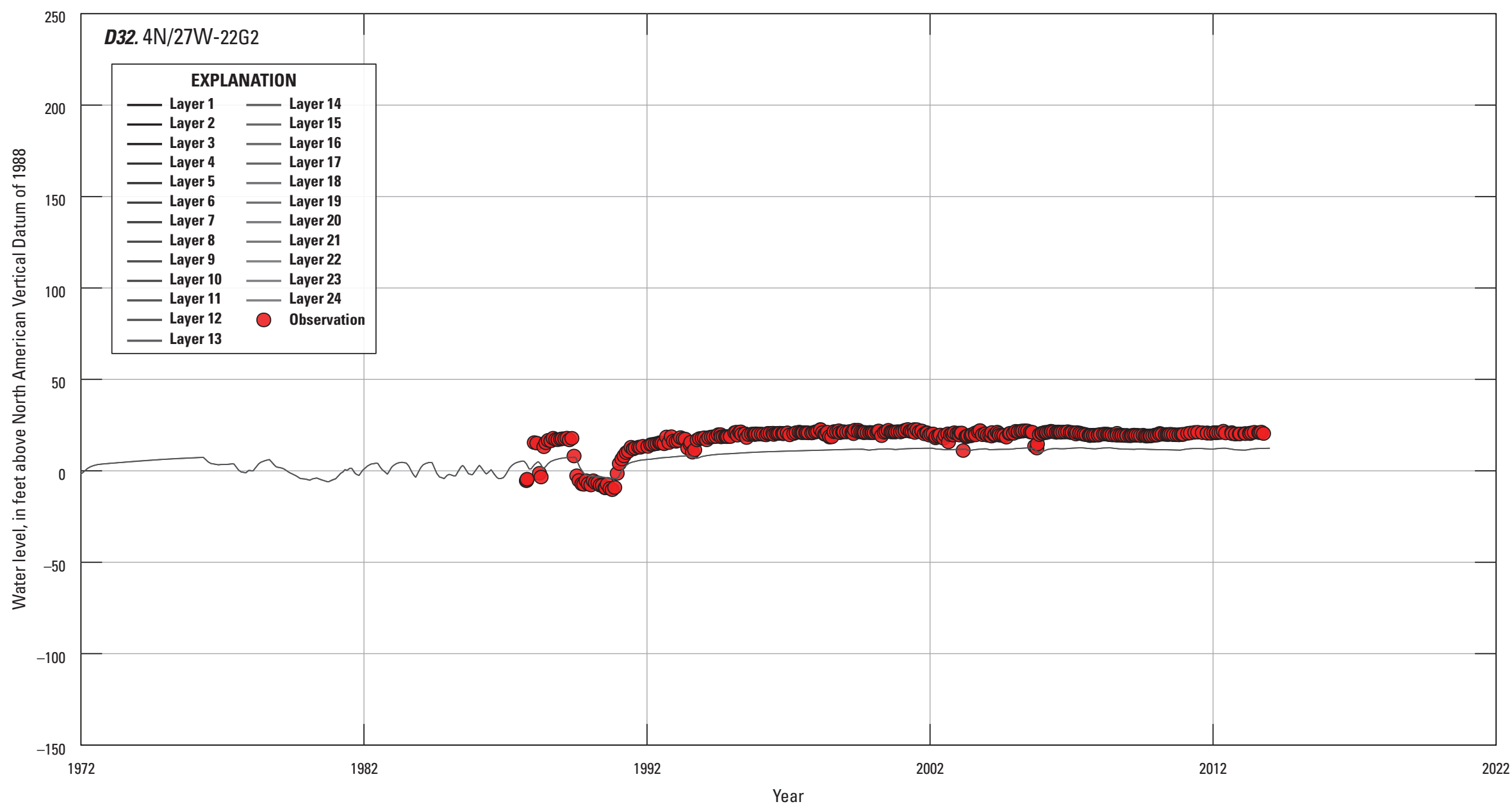

Figure 19. - Continued 


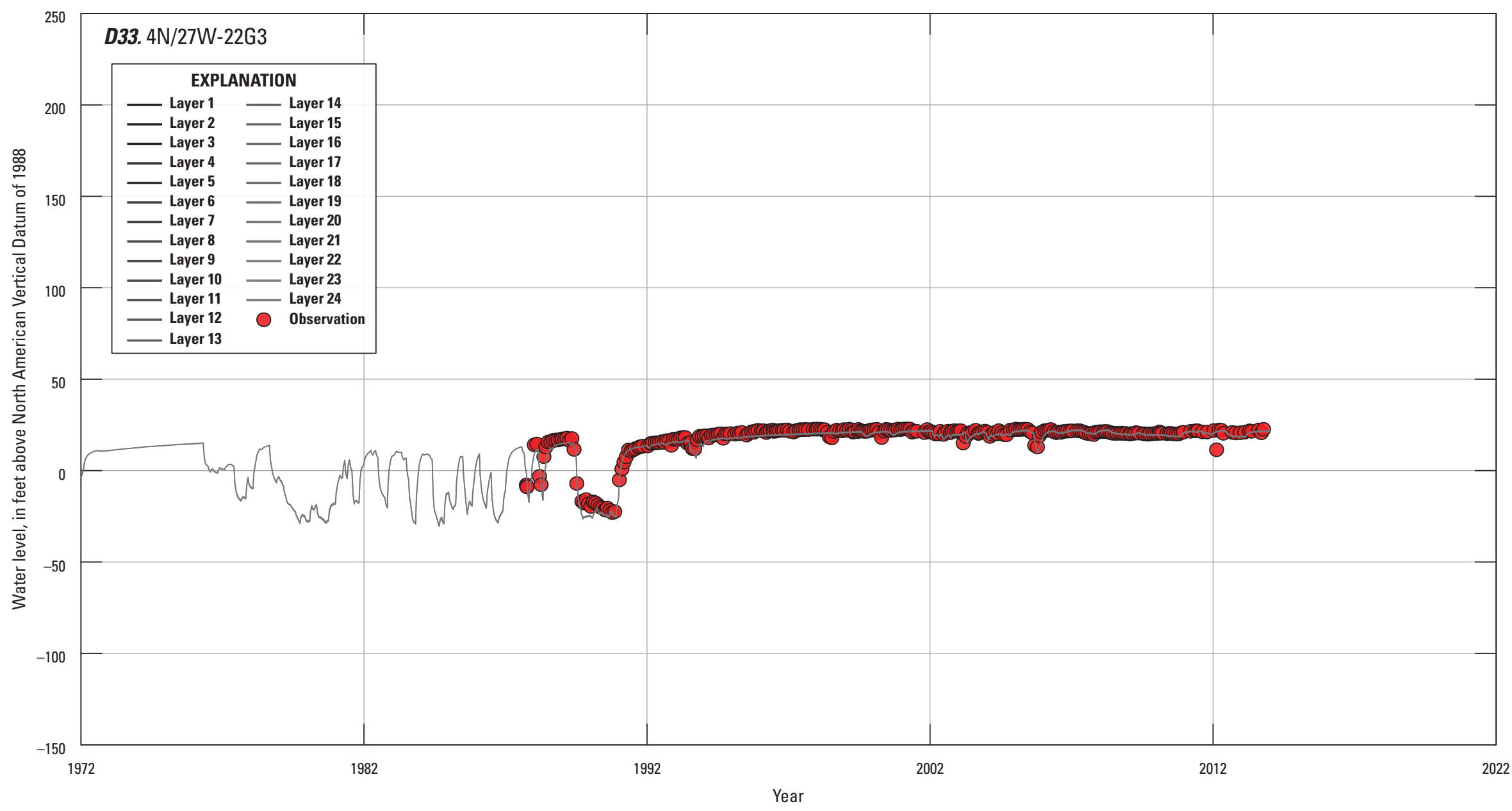

Figure 19. - Continued 


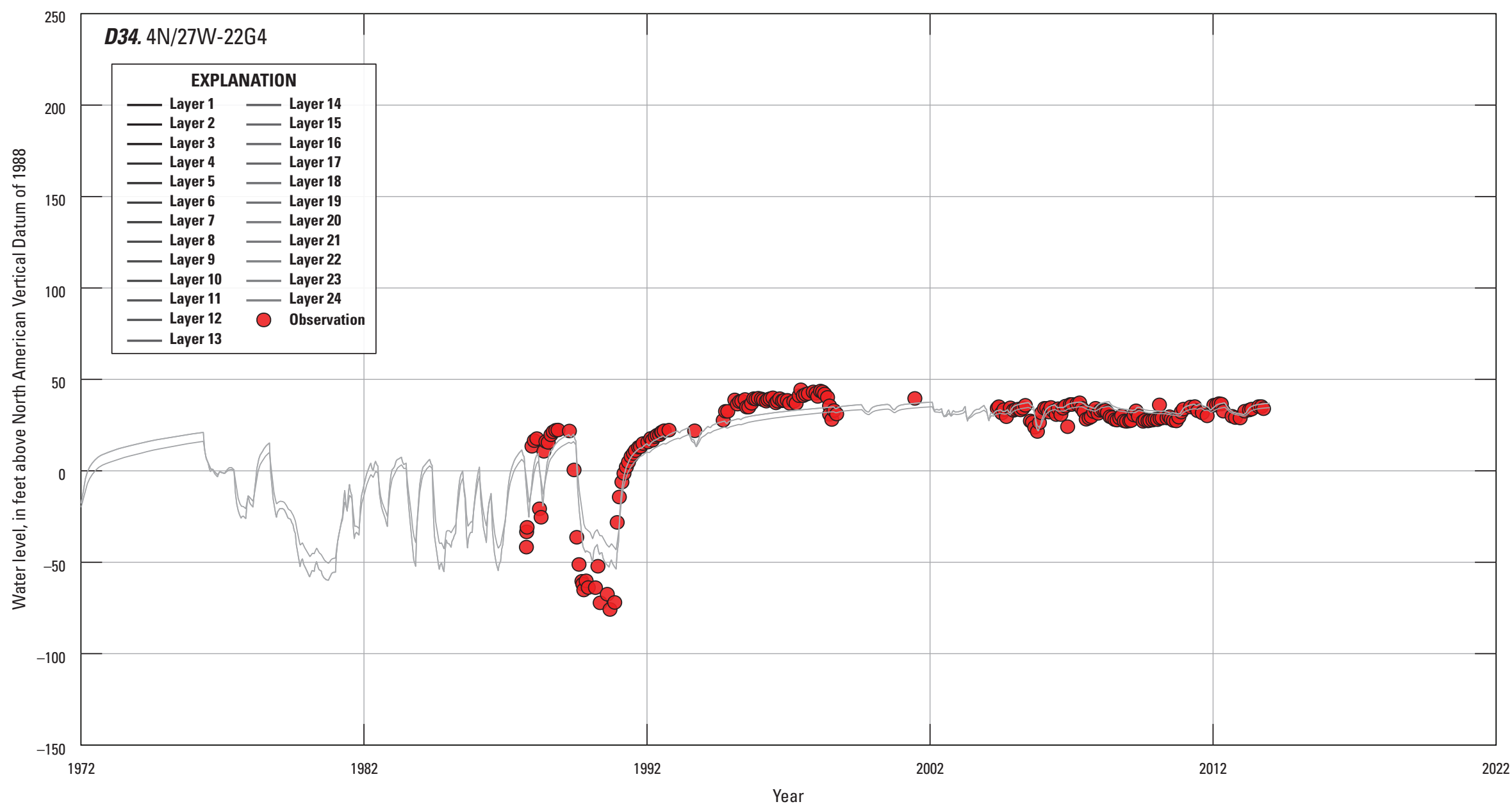

Figure 19. - Continued 


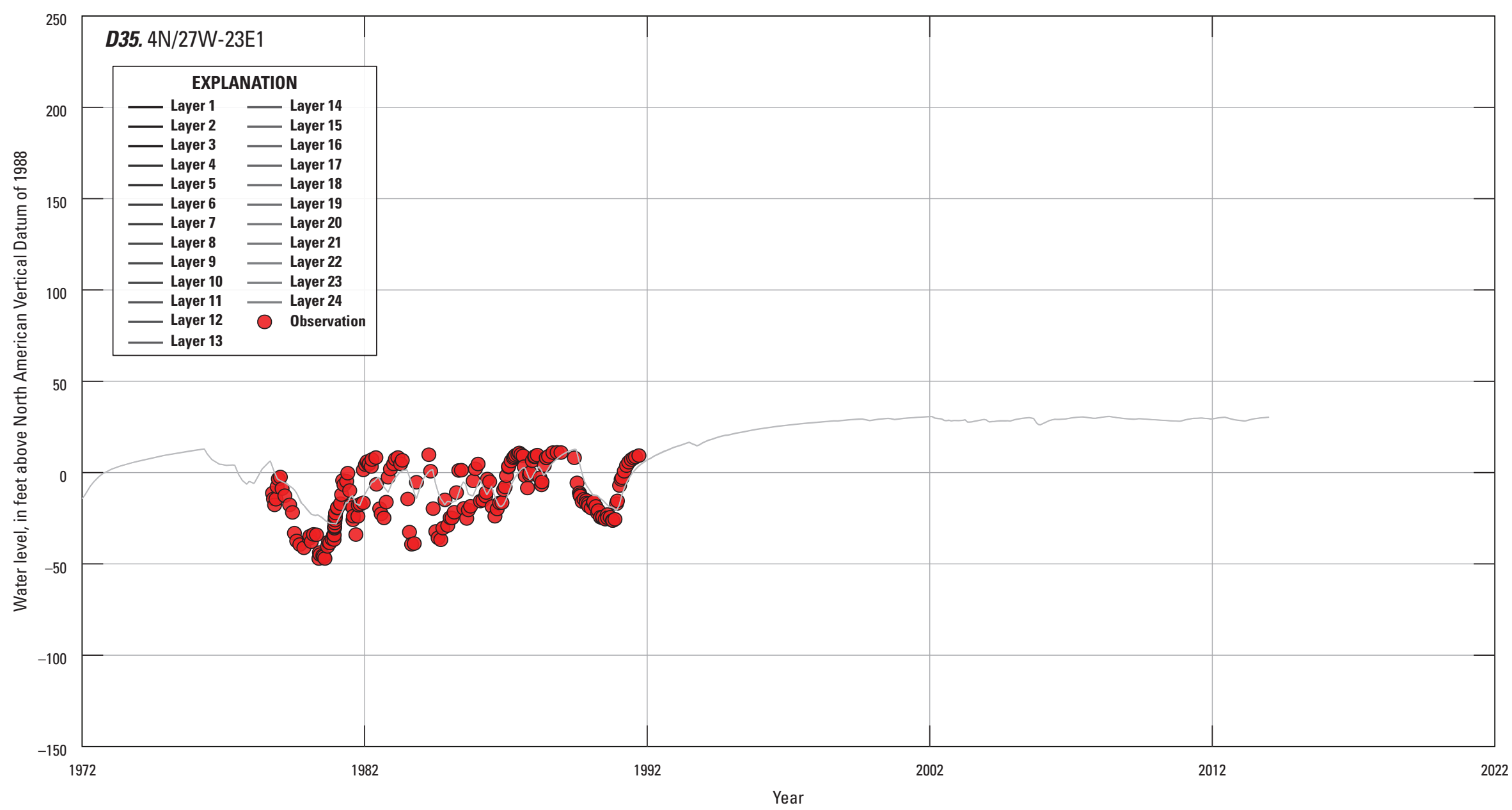

Figure 19. - Continued 


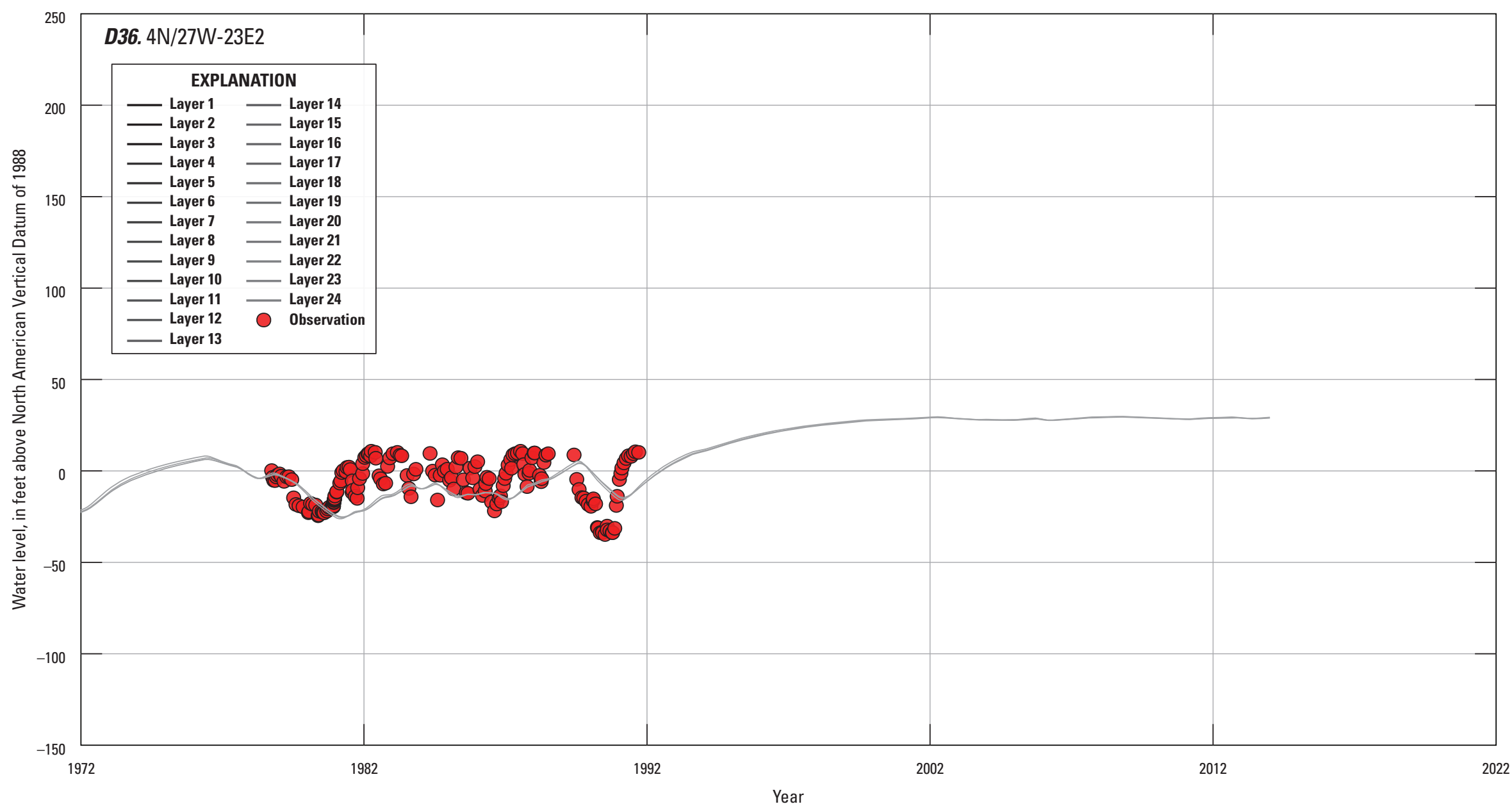

Figure 19. - Continued 


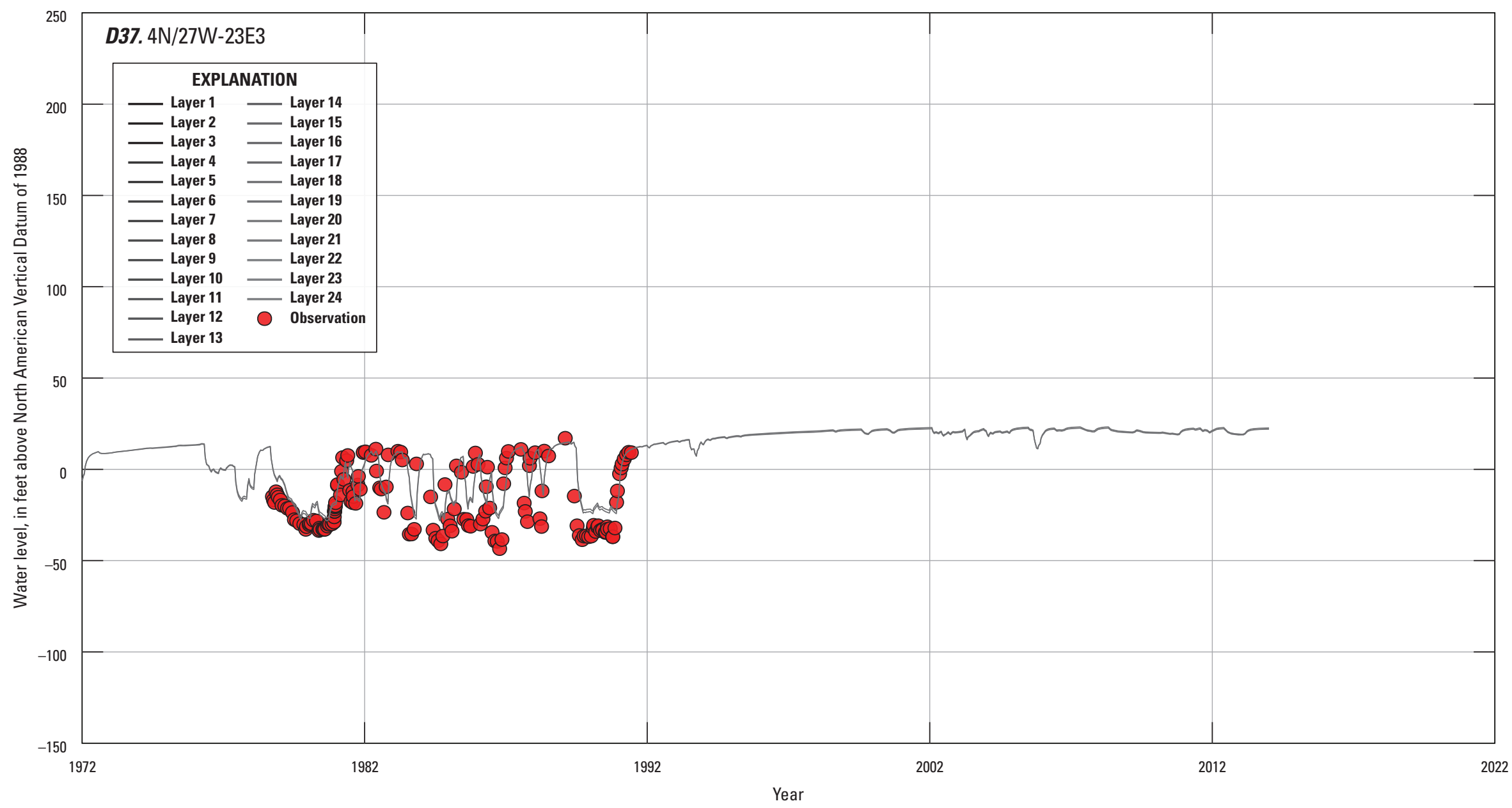

Figure 19. - Continued 


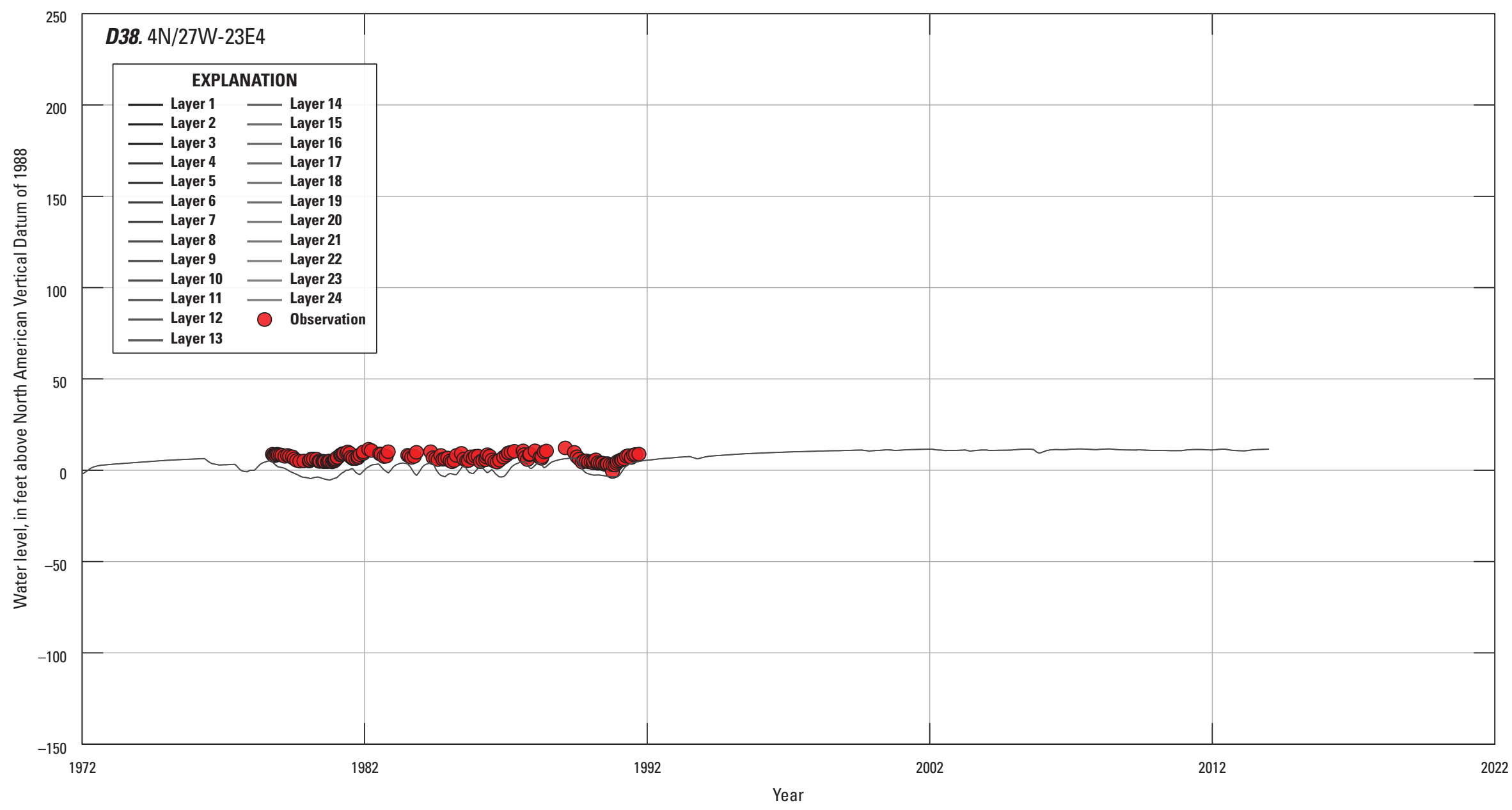

Figure 19. - Continued 


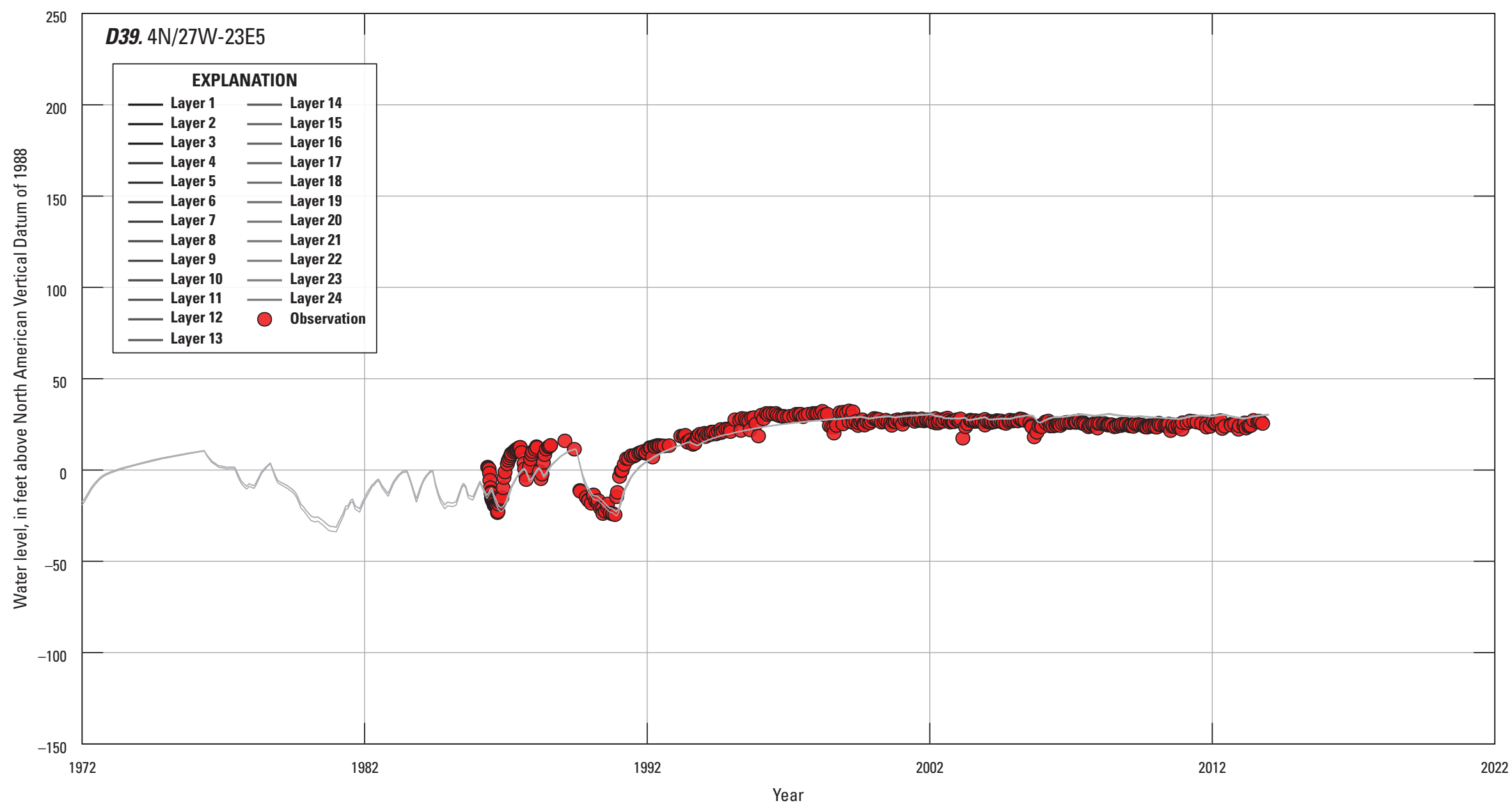

Figure 19. - Continued 


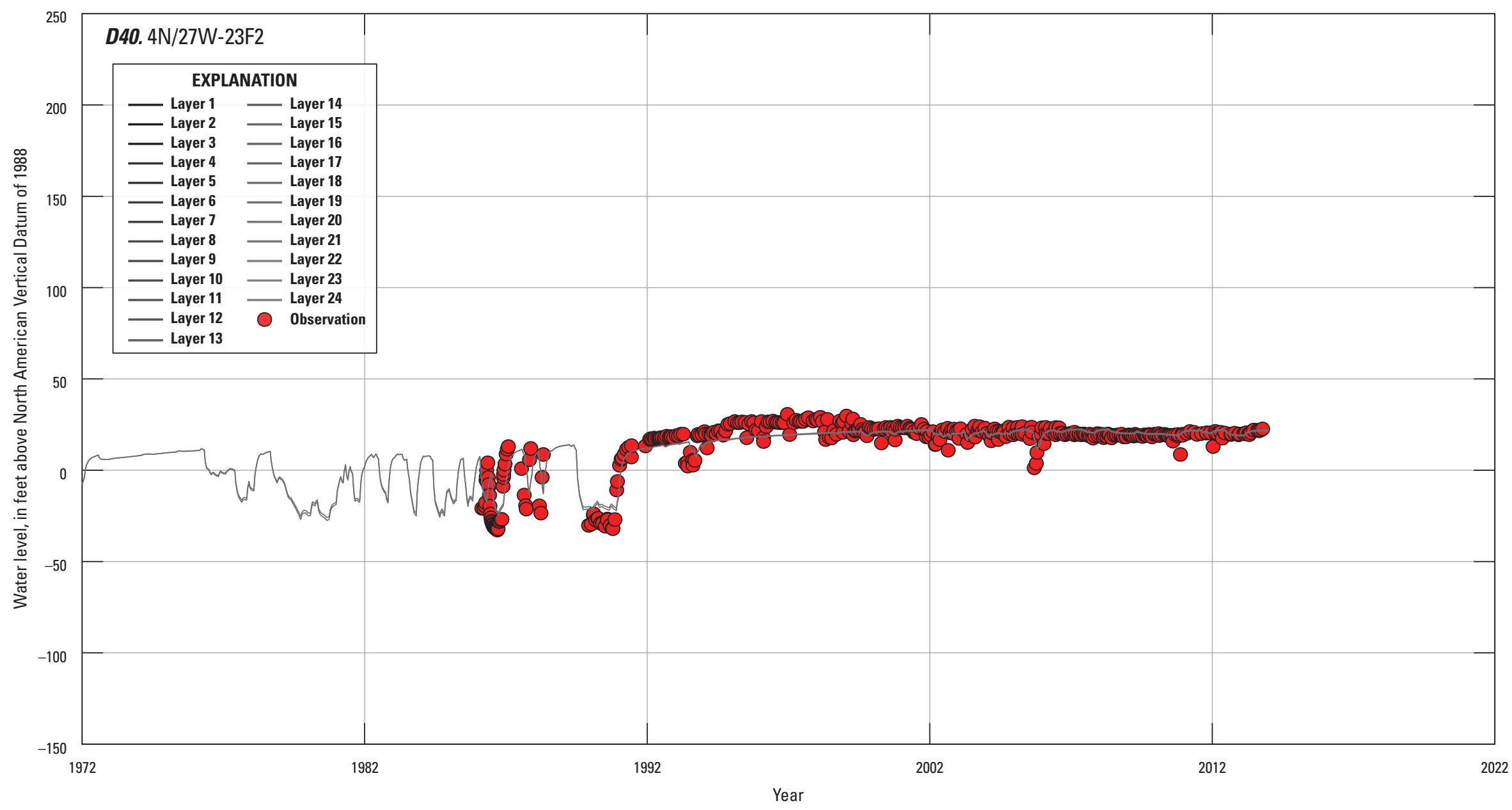

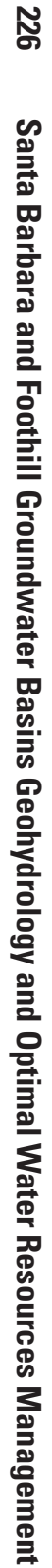

Figure 19. - Continued 


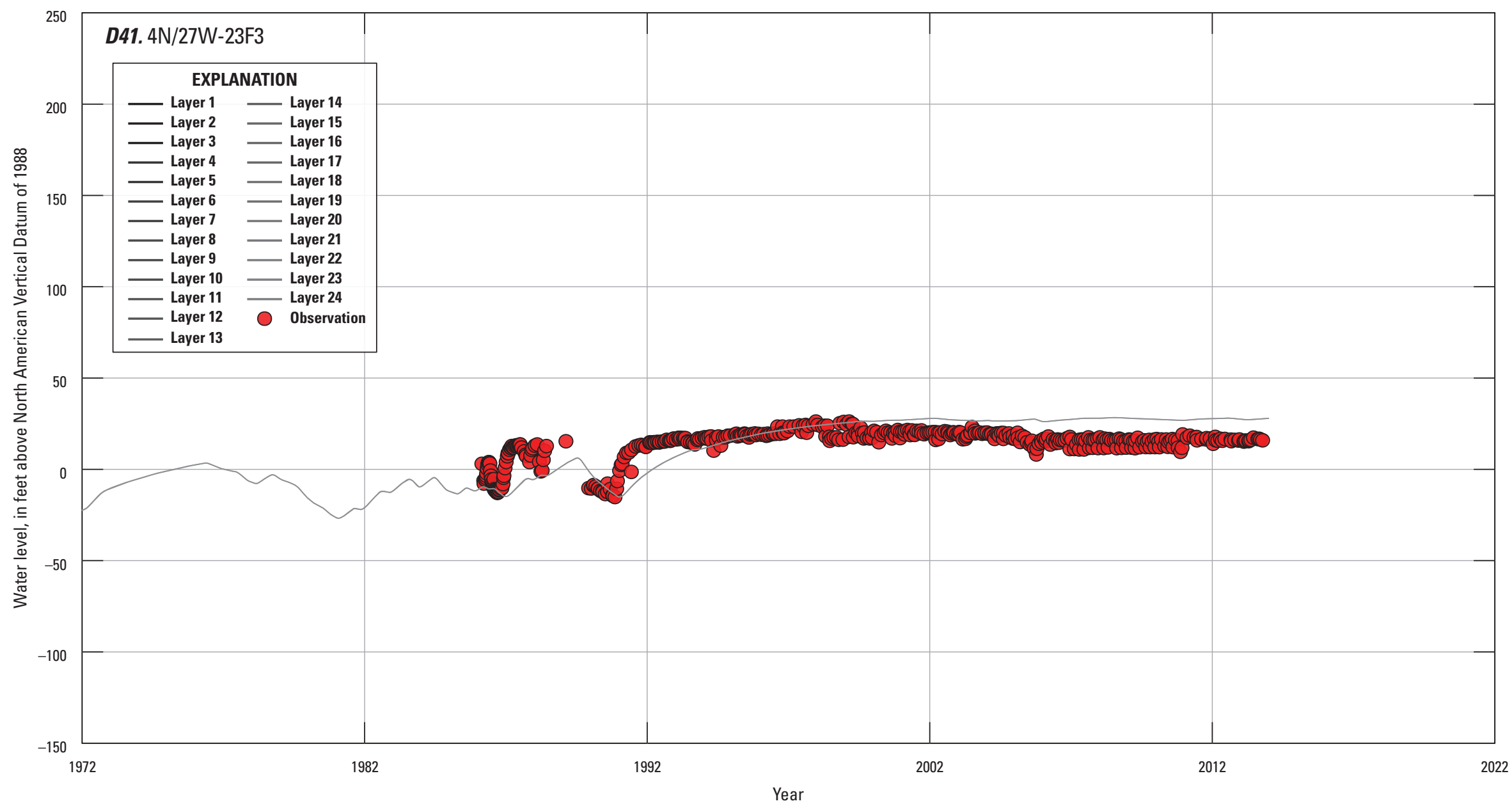

Figure 19. - Continued 


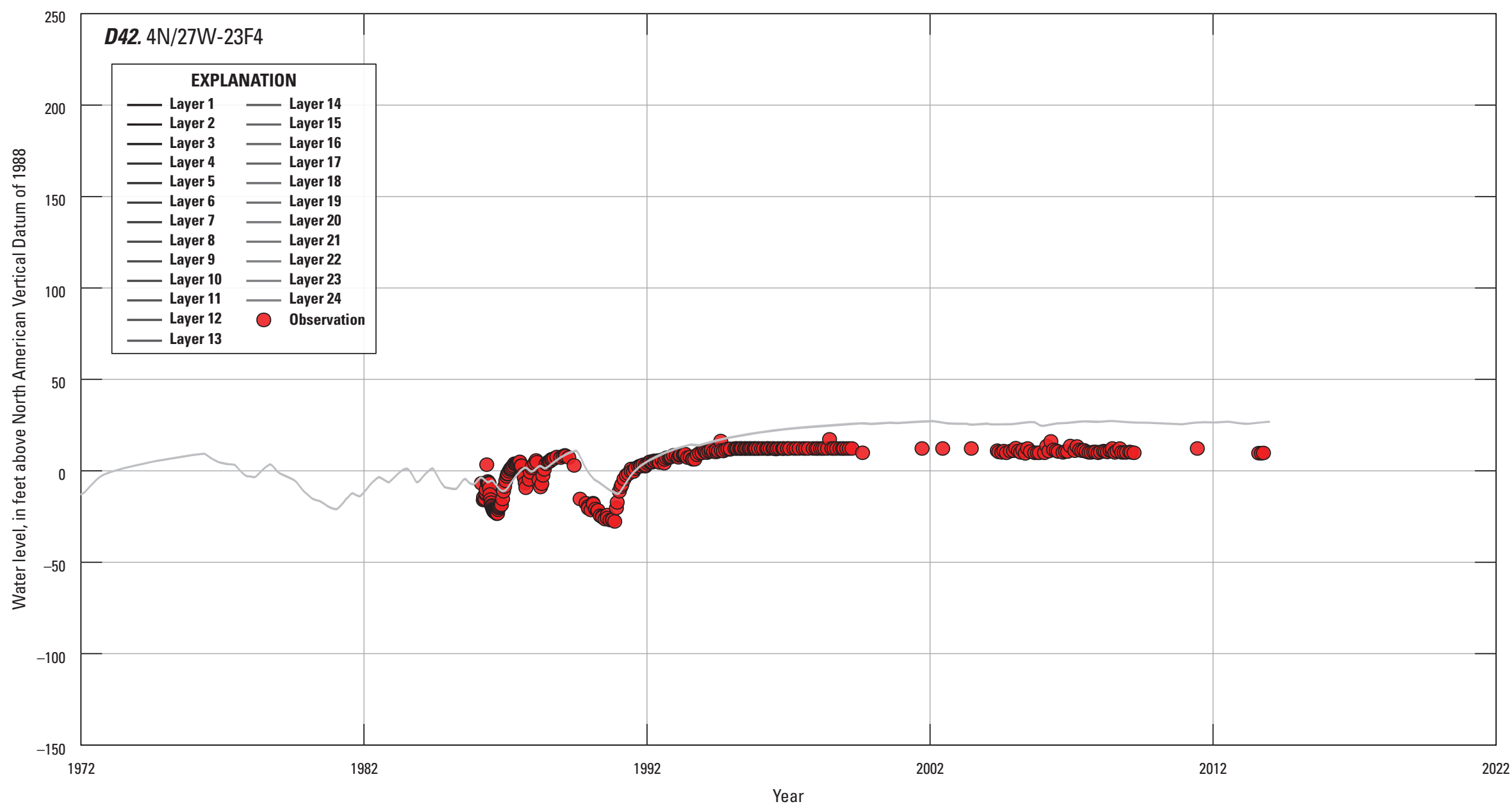

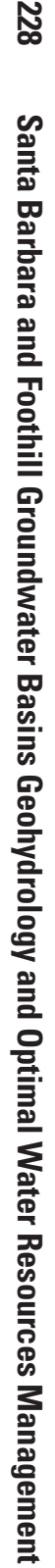

Figure 19. - Continued 


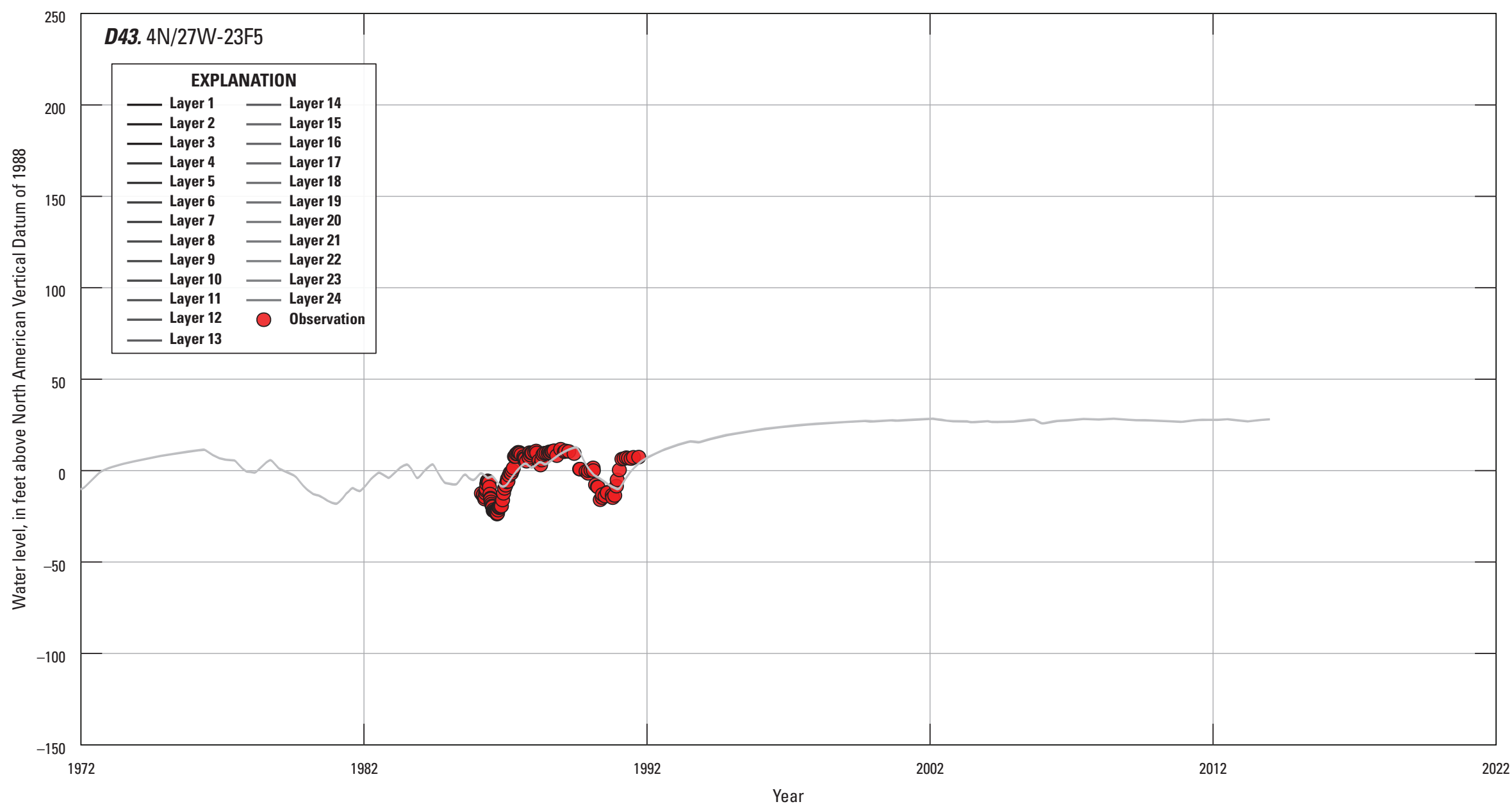

Figure 19. - Continued 


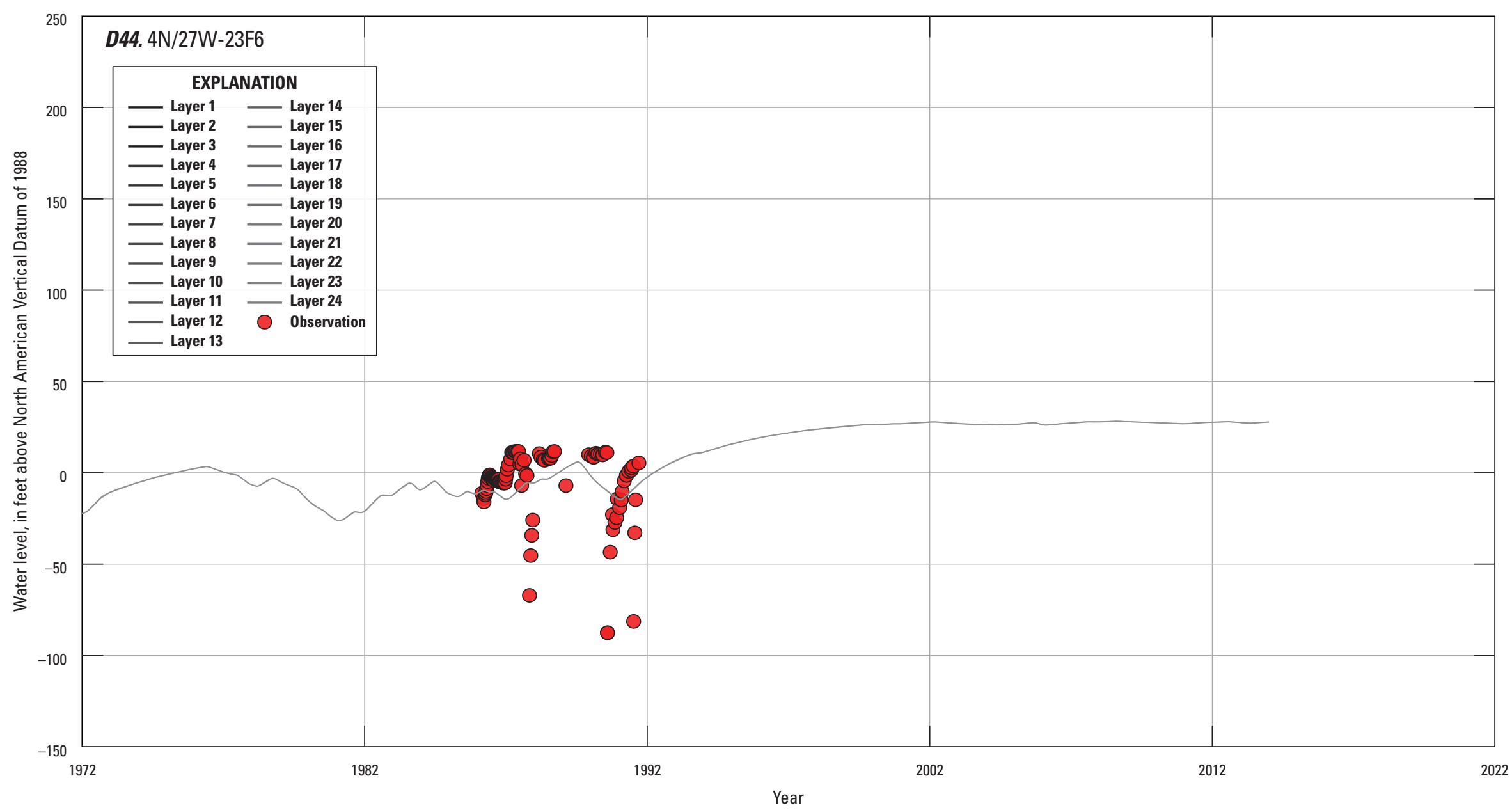

Figure 19. - Continued 


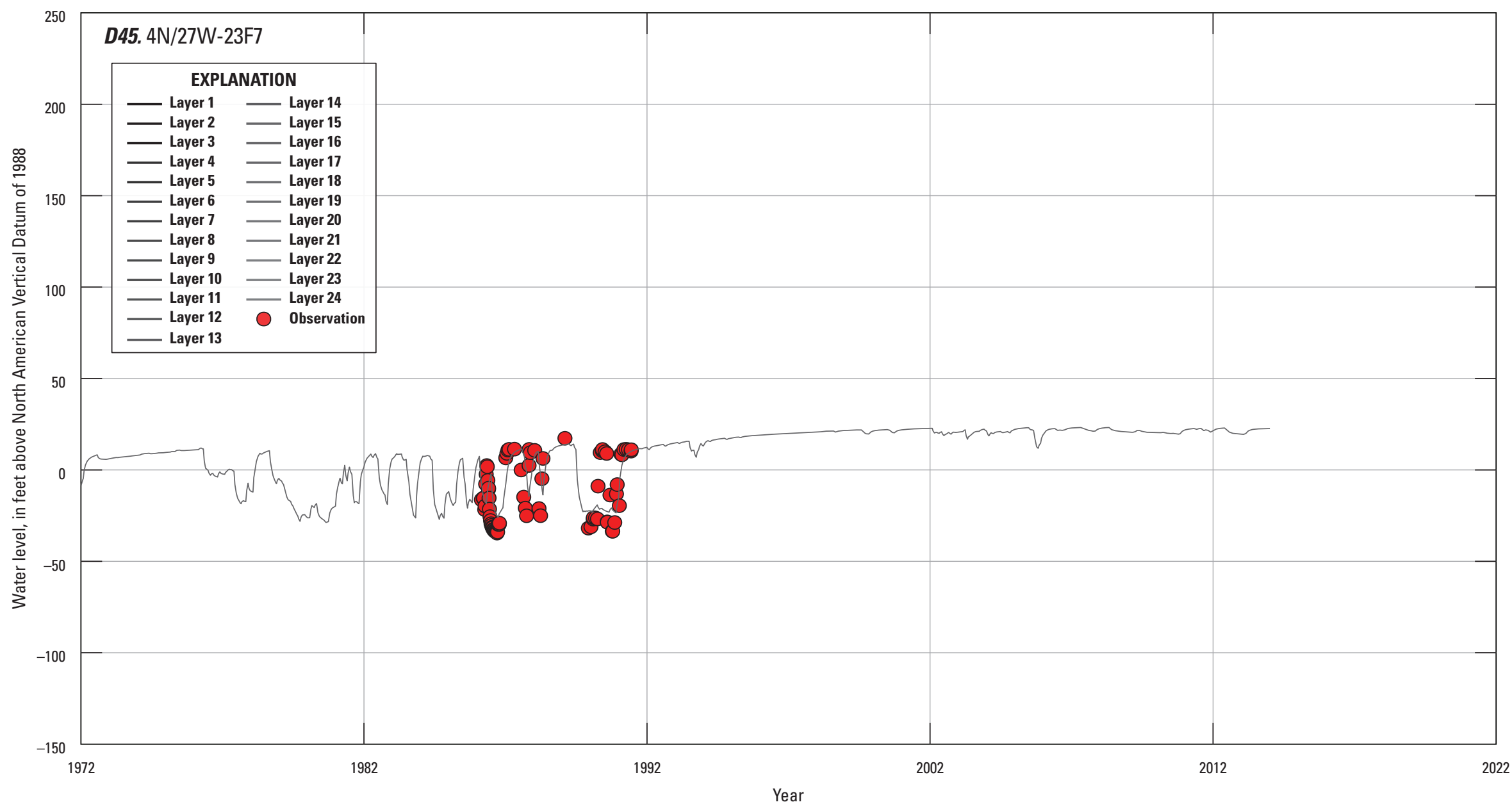

Figure 19. - Continued 


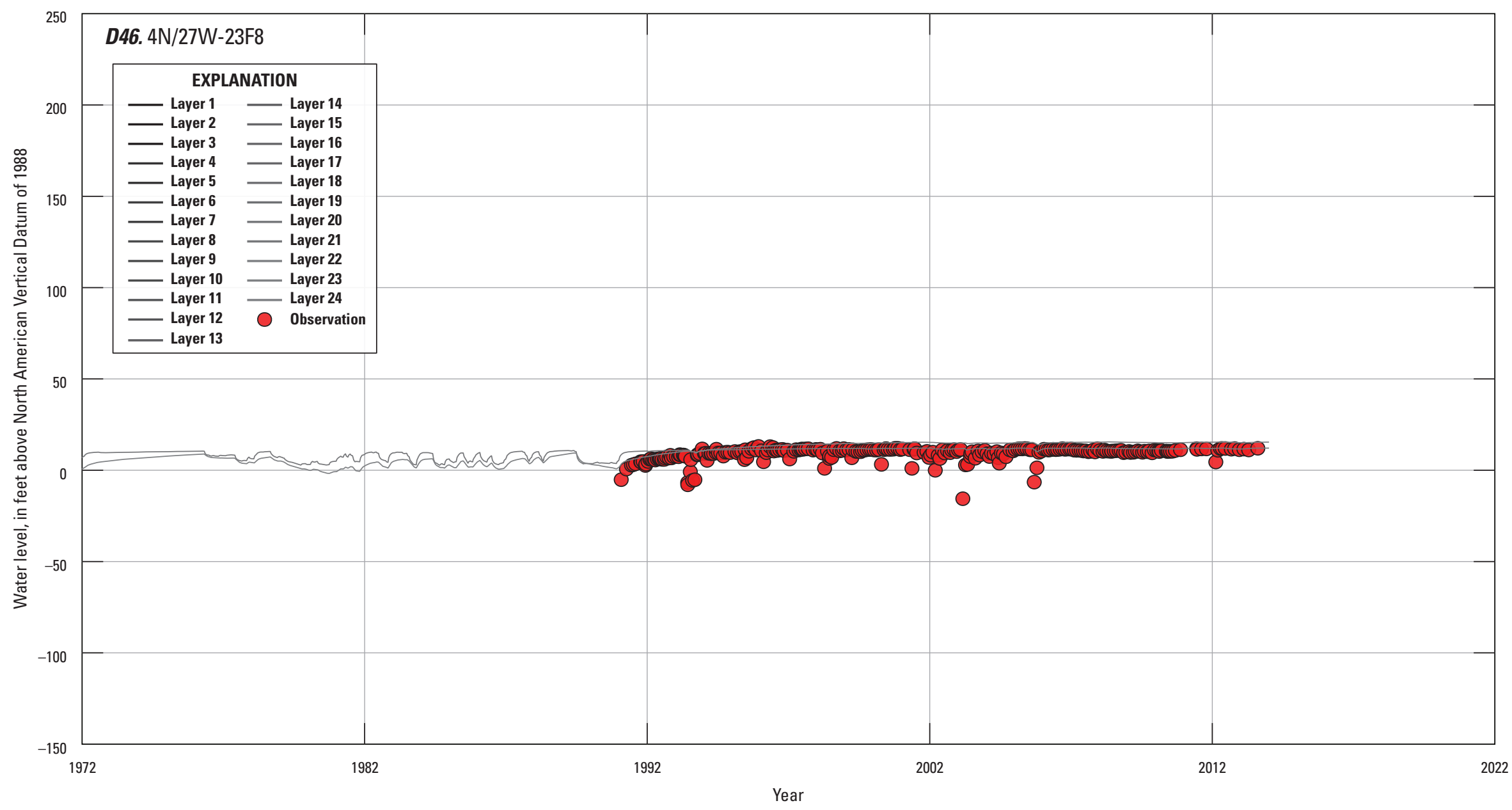

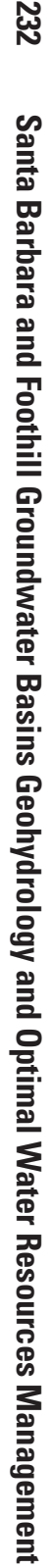

Figure 19. - Continued 


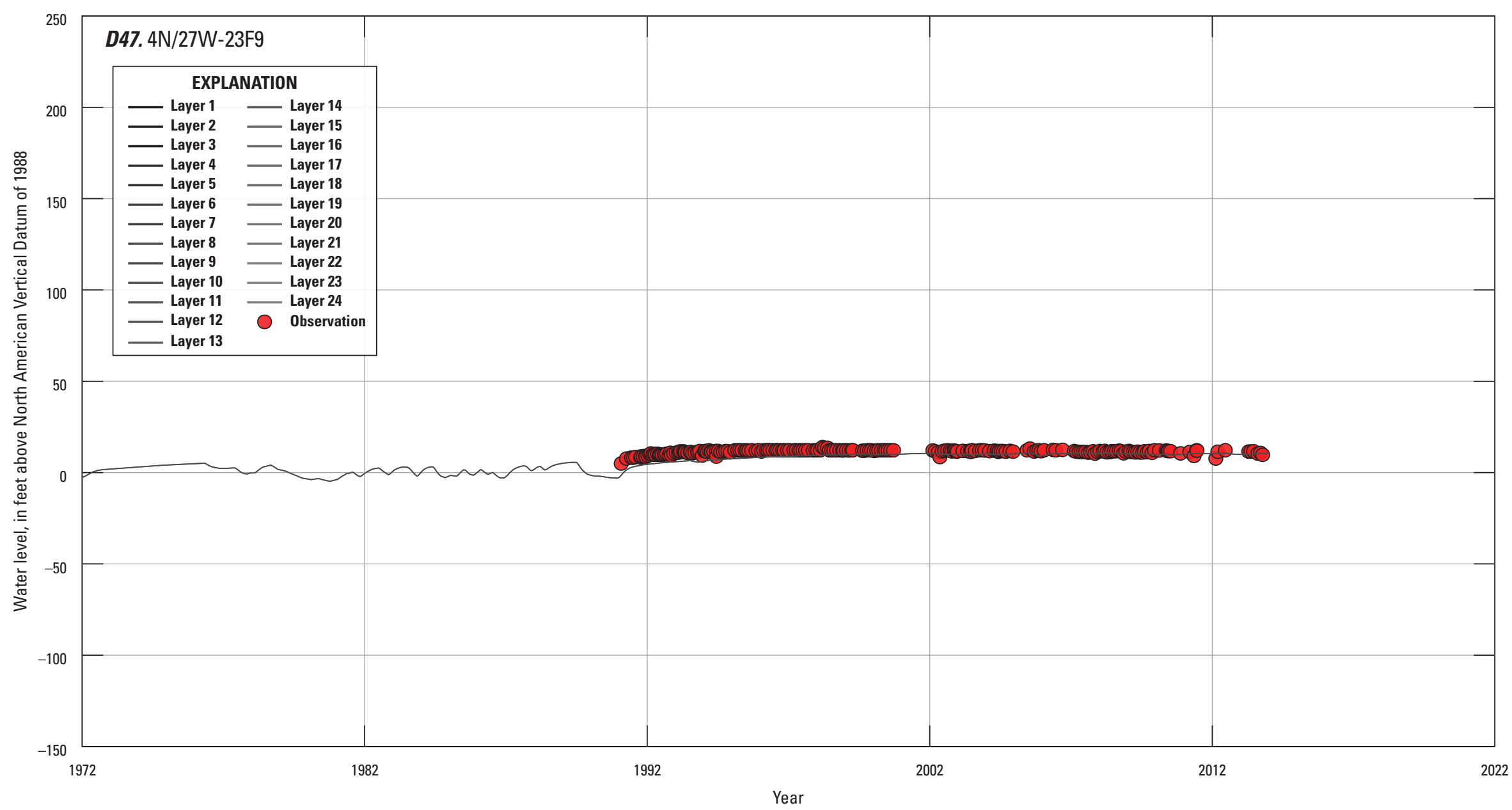

Figure 19. - Continued 


\section{West Foothill Subbasin}

West Foothill subbasin simulated hydrographs showed a reasonable fit to measured data, with most hydrographs matching water levels as well as changes in water levels through time (fig. 19A). As would be expected, water-level changes were strongly influenced by the production wells in this region. Wells 4N/27W-07D1, 4N/27W-07M6, 4N/28W$12 \mathrm{H} 4$, and $4 \mathrm{~N} / 28 \mathrm{~W}-12 \mathrm{R} 3$ are near the major production wells in West Foothill subbasin, including Los Robles and La Cumbre Mutual Water Company (LCM) wells \#9 and \#16 (figs. 9, 19A). Of the four wells, the fit of simulated to measured water levels was worst for well 4N/27W-07M6 (fig. 19A). From 1975 to 1977, the simulated hydraulic heads in well 4N/27W-07M6 declined because of pumping from the LCM \#9 production well; however, measured water levels did not decline during this time. Wells 4N/27W-07M6 and LCM $\# 9$ are both perforated in the upper and lower producing zones. Additionally, wells LCM \#9 and 4N/27W-07M6 are both in a narrow zone between the Northeast More Ranch fault and the unnamed fault, less than a third of a mile from each other (fig. 9). With no known barrier separating well 4N/27W-07M6 from LCM \#9, this well proved difficult to calibrate. This indicated that groundwater flow is restricted between these two wells in some way that is not addressed by the conceptual model.

Wells 4N/28W-12B1, 4N/28W-12C2, and 4N/28W01R1-2 are on the western side of the West Foothill subbasin (fig. 9). The nearest production well is Los Robles, which is slightly down gradient from these wells. In general, measured and simulated changes in head were very similar for these wells (fig. 19A). Simulated hydraulic heads and measured water levels were a reasonable match for wells $4 \mathrm{~N} / 28 \mathrm{~W}$ 01R1-2; however, water levels for wells 4N/28W-12B1 and 4N/28W-12C2 were overestimated (fig. 19A). Matching water levels in wells $4 \mathrm{~N} / 28 \mathrm{~W}-01 \mathrm{R} 1-2$ and $4 \mathrm{~N} / 28 \mathrm{~W}-12 \mathrm{C} 2$ proved to be difficult because these wells are both perforated in the upper producing zone, are less than $0.5 \mathrm{mi}$ apart, but had a water-level difference of approximately $20 \mathrm{ft}$, with lower water levels in well $4 \mathrm{~N} / 28 \mathrm{~W}-12 \mathrm{C} 2$. No outflow exists on the west side of well $4 \mathrm{~N} / 28 \mathrm{~W}-12 \mathrm{C} 2$ large enough to drive this water-level difference between the wells. In addition, even though it is closer to the nearest production well, water-levels fluctuated less in well 4N/28W-01R1 than in well 4N/28W$12 \mathrm{C} 2$. This indicates that wells $4 \mathrm{~N} / 28 \mathrm{~W}-01 \mathrm{R} 1-2$ have a weak hydraulic connection to the south. This could be explained by a local decrease in sediment coarseness or layer thickness around wells 4N/28W-01R1-2 not captured in the HFM.

\section{East Foothill Subbasin}

In general, the hydrographs from wells (4N/27W-05P1 and 4N/27W-08M5-6), in the East Foothill subbasin, showed simulated trends in hydraulic head matching those measured (fig. 19B). One exception is well $4 \mathrm{~N} / 27 \mathrm{~W}-05 \mathrm{P} 1$, in the northern part of the basin, because the seasonal variability in simulated hydraulic heads did not match the measured data; the simulated results were less variable than measured data. The lack of variability in the simulated results could be associated with unknown pumping near the well or be caused by the manner in which creeks were simulated. There could be unknown or unreported pumping that, if included, could affect the simulated results. Perhaps more importantly, San Roque Creek is less than $100 \mathrm{ft}$ from well $4 \mathrm{~N} / 27 \mathrm{~W}-05 \mathrm{P} 1$, and the creeks in the model area are simulated using constant-stage streams in the RIV package; therefore, seasonal variability of the stream stages, which likely affect water levels in these wells, were not addressed.

Monitoring wells 4N/27W-08M5-6 are in the middle of the East Foothill subbasin, approximately 1,400 ft southwest of the San Roque Park production wells and approximately 2,100 ft northwest of the McKenzie Park production wells (fig. 9). Wells 4N/27W-08M5-6 are perforated in the lower and upper producing zones, respectively. The simulated hydraulic heads and measured water levels for well 4N/27W08M5 were very similar in terms of magnitude and trend (fig. 19B). Simulated hydraulic heads and measured water levels in well 4N/27W-08M6 followed a similar pattern, with simulated heads having slightly more drawdown and recovery than the measured data. The declines in simulated hydraulic heads and measured water levels in wells 4N/27W-08M5-6 corresponded to increased pumping in the East Foothill subbasin from 1984 to 1991 and from 2007 to 2013, and the period of increasing water levels during the 1990s and early 2000s corresponded to a time of relatively little pumping (fig. A-8). In addition to being consistent with the measured water levels at these wells, the simulated vertical gradient between these wells was consistent with the measured data.

\section{Storage Unit III}

In general, simulated hydraulic heads matched measured water levels well for the west side of Storage Unit III, but there were greater errors in simulated values on the east side (fig. 19C). Simulated hydraulic heads for wells in the west side of Storage Unit III, including wells 4N/27W-18C2-3 and 4N/27W-18Q1 and 18Q4, matched measured water levels during periods of high pumping, such as 1988-93 and 2010-13, fairly well. However, simulated heads were consistently higher than measured water levels during periods of low pumping, such as 1994-2008. Toward the east side of Storage Unit III, simulated hydraulic heads poorly matched measured water levels and seasonal changes in water levels (for example, well 4N/27W-21F2; fig. 19C). This is difficult to see given the hydrograph's scale, but the simulated seasonal fluctuations were under simulated. The eastern side of Storage Unit III consists of a thin layer of alluvium with underlying shale, which is bounded by the Mesa fault to the north and a mesa to the south with elevations up to $450 \mathrm{ft}$. The thin layer of alluvium could have low transmissivity values, allowing runoff from the mesa to the south to seasonally influence water levels in this area. Because runoff from the mesa is not simulated in the SBFTM, this could explain discrepancies between measured and simulated heads in this area. 


\section{Storage Unit I}

Simulated hydrographs for selected wells in the western part of Storage Unit I (4N/27W-07Q5, 4N/27W07R3, 4N/27W-16C1-2, 4N/27W-16E2, 4N/27W-17J1, 4N/27W-08R2, and 4N/27W-17L2-4; fig. 9) were generally in agreement with the measured data in terms of values and trends. Wells 4N/27W-07Q5 and 4N/27W-07R3 are in the westernmost corner of Storage Unit I (fig. 9), and the simulated hydraulic heads matched measured water levels fairly well in terms of value and trend (fig. 19D). To the east, simulated hydraulic heads and measured water levels near Mission Creek fit reasonably (for example, wells 4N/27W16C1-2, 4N/27W-16E2, and 4N/27W-17J1). Well 4N/27W08R2 is close to where Mission Creek runs along the East More Ranch fault (fig. 9). Measured water levels from this well showed influences from both Foothill subbasins and Storage Unit I. This indicated that well 4N/27W-08R2 could either pass through the East More Ranch fault at depth or is in the East More Ranch fault zone. Wells 4N/27W-17L2-4 are close to the Mesa fault (fig. 9). Simulated hydraulic heads and measured water levels fit reasonably for the shallow well 4N/27W-17L4, which was perforated above the upper producing zone, but did not fit as well for the deeper wells 4N/27W-17L2 and 4N/27W-17L3, which were perforated in the upper and lower producing zones, respectively (fig. 19D). Measured water levels in wells 4N/27W-17L2 and 4N/27W-17L3 indicated an influence from Storage Unit III, where water levels were relatively low during the post-1991 recovery. This indicated the well cluster could cross the Mesa fault at depth.

Simulated hydrographs for selected wells in the eastern part of Storage Unit I (4N/27W-14K2, 4N/27W-15J2, 4N/27W-15K1, 4N/27W-15E1-2, 4N/27W-16R1, 4N/27W21B1, and 4N/27W-22E1-2), which are near the main Storage Unit I production wells (fig. 9), generally matched measured water levels. Wells 4N/27W-14K2 and 4N/27W-15J2 are east of the main production wells and are perforated in the upper producing zone and in the upper and middle producing zones, respectively. The simulated hydraulic heads and drawdown matched the measured data in both wells (fig. 19D). Well $4 \mathrm{~N} / 27 \mathrm{~W}-15 \mathrm{~K} 1$ is just north of the main production wells and is perforated in the lower producing zone (fig. 9). Simulated hydraulic heads and measured water levels were similar, but simulated drawdowns were slightly greater than measured drawdowns (fig. 19D). Wells 4N/27W-15E1-2, northwest of the main production wells (fig. 9), are perforated in the upper and lower producing zones and in the lower producing zone, respectively. In general, for both of these wells, simulated hydraulic heads were less than measured water levels, but amounts of drawdown were similar (fig. 19D). The measured water levels from 15E2 were much more variable in the early 1990s and 2010s than the simulated hydraulic heads (fig. 19D), however. West of the main production wells, well $4 \mathrm{~N} / 27 \mathrm{~W}-16 \mathrm{R} 1$ is perforated in the lower producing zone, well
$4 \mathrm{~N} / 27 \mathrm{~W}-21 \mathrm{~B} 1$ is perforated in the upper producing zone, and wells $4 \mathrm{~N} / 27 \mathrm{~W}-22 \mathrm{E} 1-2$ are both perforated in the upper aquitard. Simulated hydraulic heads and measured water levels matched fairly well, although simulated drawdown was greater than measured during the pumping events between 1980 and 1990 for well 4N/27W-16R1, and it was less than measured in well 4N/27W-21B1 (fig. 19D). For wells 4N/27W-21B1 and $4 \mathrm{~N} / 27 \mathrm{~W}-22 \mathrm{E} 2$, simulated heads were less than measured water levels during the recovery period following 1991 (fig. 19D).

In addition to the wells in the eastern part of Storage Unit I, there are a number of monitoring-well clusters between the production wells and the coastline (for example, 4N/27W-22B2-6, 4N/27W-22B8-11, 4N/27W-22A2-4, 4N/27W-22G2-4, 4N/27W-23E1-4, 4N/27W-23E5, 4N/27W-23F2-4, and 4N/27W-23F5-9; fig. 9). Monitoring wells $4 \mathrm{~N} / 27 \mathrm{~W}-22 \mathrm{~B} 2-6$ and 22B8-11 are within $300 \mathrm{ft}$ of the Vera Cruz well, one of the main production wells in the Santa Barbara groundwater basin (fig. 9). Wells 22B2-6 are perforated in the upper producing zone, middle zone, lower producing zone, lower aquitard, and the three producing zones, respectively, and wells $22 \mathrm{~B} 8-11$ are perforated in the lower aquitard, lower producing zone, middle producing zone, and upper producing zone, respectively. Water-level data were collected at wells 22B2-6 primarily during mid1970 s to the early 1990s, whereas data were collected at wells 22B8-11 primarily during the early 1990 s to 2013 Overall, simulated hydraulic heads and measured water levels matched fairly well for these wells, except for wells 22B3 and 22B6 (fig. 19D). Monitoring well 4N/27W-22B3 was assumed to be perforated in the middle zone, which, with the exception of the middle producing zone, is generally composed of fine-grained material; therefore, the simulated response of the hydraulic heads to pumping was less variable than that shown by the measured data. Monitoring well 4N/27W-22B6 is perforated throughout all three producing zones in an area where there was a large vertical gradient between these zones. The lower simulated hydraulic heads for this well could, therefore, be partially due to the manner in which SBFTM averages the heads in each layer to produce a representative head value for the monitoring well. The direction of the vertical gradient was simulated accurately in wells 4N/27W-22B9 and 22B11, with highest heads in the lower producing zone (22B9) and lowest heads in the upper producing zone (22B11). The simulated vertical gradient between the lower and middle producing zones was slightly exaggerated; simulated hydraulic heads in the lower producing zone were slightly higher than measured during the recovery period from 1991 to 2013 (fig. 19D).

Monitoring wells 4N/27W-22A2-4 and 4N/27W-22G2-4 are about $0.25 \mathrm{mi}$ southeast and south, respectively, from the Vera Cruz production well (fig. 9). Both sets of monitoring wells are between the main production wells and the coast and are about $0.5 \mathrm{mi}$ from the coast. Monitoring wells $4 \mathrm{~N} / 27 \mathrm{~W}$ $22 \mathrm{~A} 2-4$ are perforated in the upper producing zone, middle producing zone, and lower producing zone, respectively. 
Monitoring wells 4N/27W-22G2-4 are perforated in the upper aquitard, middle producing and lower producing zones, respectively. Simulated hydraulic heads, drawdowns, and vertical gradients were all in agreement with the measured data for both sets of monitoring wells (fig. 19D). In both wells, simulated and measured drawdowns were greater at depth, and the vertical difference in simulated hydraulic head and measured water levels from the lower to the upper zones was about $15 \mathrm{ft}$ during the post-1991 recovery years.

Monitoring wells 4N/27W-23E1-4, 4N/27W-23E5-6, 4N/27W-23F2-4, and 4N/27W-23F5-9 are within $1.0 \mathrm{mi}$ southeast of the Vera Cruz production well (fig. 9). Simulated hydraulic heads, drawdowns, and vertical differences in simulated hydraulic heads were similar to measured data for wells 4N/27W-23E1-5 and 4N/27W-23F2-9. Measured and simulated drawdowns for the upper, middle, and lower producing zones were all similar, with much smaller drawdowns in the upper aquitard layer (4N/27W-23E4).

\section{Water-Level Data Fit}

A simple method to assess overall model fit is to plot the simulated hydraulic heads against the measured waterlevel (fig. 20). For a perfect fit, all points should show a 1:1 relation (fall on the 1:1 diagonal line). The root mean square error (RMSE) between measured water-level elevations and simulated hydraulic heads was $14.7 \mathrm{ft}$. Given the scale of the SBFTM, simulated hydraulic heads reasonably matched measured water-level elevations. The average residual was $-2.7 \mathrm{ft}$ and the standard deviation was $14.5 \mathrm{ft}$. The average residual indicates a slight bias towards underpredicting measured water-level elevations. The residuals ranged from -156.4 to $159.5 \mathrm{ft}$; these extremes possibly represent errors in the databases, measurements that represent pumping conditions, or seasonal variations beyond the ability of the model to simulate. More than 54 percent of the simulated hydraulic heads were within $8 \mathrm{ft}$ of measured water-level elevations, more than 80 percent were within $16 \mathrm{ft}$, and more than 95 percent were within $30 \mathrm{ft}$.

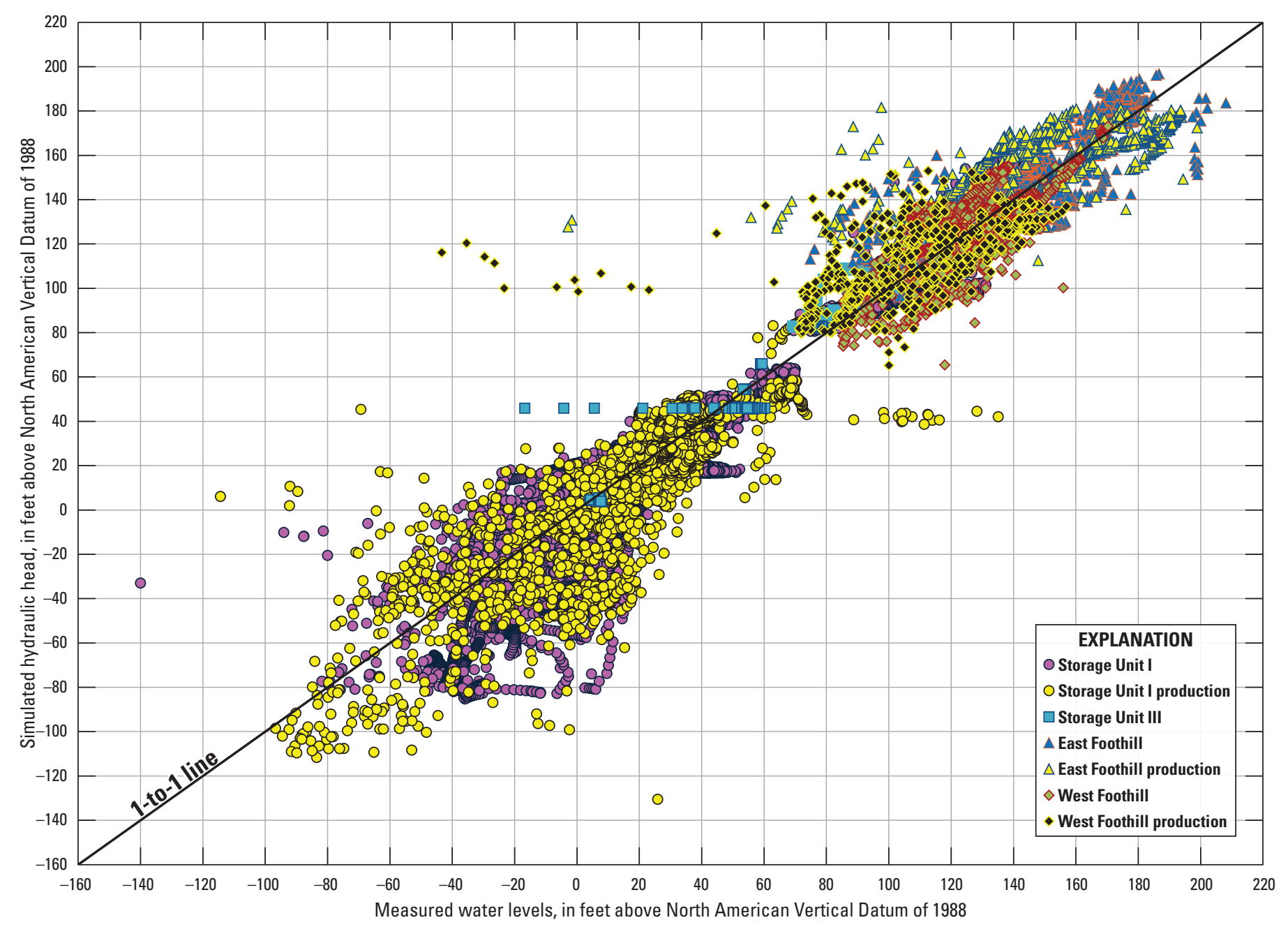

Figure 20. Simulated hydraulic heads compared with measured water levels by subbasin and well type for the Santa Barbara flow and transport model, Santa Barbara, California. 
Figure 20 indicates that, overall, the SBFTM matched water levels above sea level (NAVD88) fairly well; however, it did not match water levels below sea level as well, as indicated by the greater spread in the plot of negative (below sea level) water levels. Simulated hydraulic heads in the Foothill subbasins, Storage Unit III, and the upper part of Storage Unit I, which were consistently above sea level, matched measured water levels fairly well. Simulated hydraulic heads and measured water levels in Storage Unit I that were below sea level did not match well during heavy pumping events. There were two primary factors contributing to the water-level discrepancy at these times. First, much of the measured data were from production wells or monitoring wells very close to production wells, which could result in discrepancies between the measured and simulated results (fig. 20). Water levels have a large spatial variation near pumping wells, and the SBFTM's cell dimensions are substantially greater than the well diameters; therefore, the accuracy of simulated water levels very near or in active production wells is limited. Second, recovery of water levels near the main production wells is rapid. Slight deviations between simulated and measured recovery rates lead to very large, short-term discrepancies in head. This overestimation of low water level elevations could result in underestimation of seawater intrusion should seawater reach the production wells.

\section{Simulated Chloride Concentrations and Model Data Fit}

Contours of simulated chloride concentrations for January 1972 and July 1990 are shown in figure 21. The chloride contours for January 1972 show conditions prior to seawater intruding during the 1980s and early 1990s, whereas the chloride contours for July 1990 show conditions near the peak of the historical groundwater pumpage and seawater intrusion. In general, the simulated chloride concentrations for January 1972 show limited seawater intrusion in the upper, middle, and lower producing zones; the greatest seawater intrusion occurred in the middle producing zone (figs. 21 A, C, $E)$. Simulated chloride concentrations in the upper producing zone were less than $2,000 \mathrm{mg} / \mathrm{L}$ in all onshore model cells (fig. 21A). Simulated chloride concentrations were greater than $2,000 \mathrm{mg} / \mathrm{L}$ in the middle producing zone as far as $1,600 \mathrm{ft}$ inland and about 2,300 ft from the Vera Cruz production well (fig. 21C). Simulated chloride concentrations were greater than $2,000 \mathrm{mg} / \mathrm{L}$ in the lower producing zone as far as $1,000 \mathrm{ft}$ inland and about 2,640 ft from the Vera Cruz production well (fig. 21E).

Between January 1972 and July 1990, the simulated chloride plume moved toward the production wells in response to increased pumpage from the main production wells in Storage Unit I in all three producing zones. In the upper producing zone, the simulated $1,500 \mathrm{mg} / \mathrm{L}$ contour advanced about $790 \mathrm{ft}$ toward the Vera Cruz production well and about $550 \mathrm{ft}$ toward the Corporation Yard production well (figs. 21A, B). Simulated chloride concentrations were higher in July 1990 in the middle producing zone; the 1,500 mg/L contour moved beyond U.S. Route 101 in some areas to about $0.5 \mathrm{mi}$ inland (figs. $21 C, D$ ). The $1,500 \mathrm{mg} / \mathrm{L}$ contour advanced toward the Vera Cruz and Corporation Yard wells, about 200 and $880 \mathrm{ft}$, respectively. In the lower producing zone, the $1,500 \mathrm{mg} / \mathrm{L}$ contour advanced toward the Vera Cruz and Corporation Yard wells about 540 and $610 \mathrm{ft}$, respectively (figs. 21E, F).

Figure 22 shows simulated and measured chloride breakthrough curves for selected wells in Storage Unit I where the coastal monitoring wells are on the seaward side of U.S. Route 101 and the inland monitoring wells are on the inland side U.S. Route 101. Coastal wells 4N/27W-23E1, $4 \mathrm{~N} / 27 \mathrm{~W}-23 \mathrm{E} 5$, and $4 \mathrm{~N} / 27 \mathrm{~W}-23 \mathrm{~F} 4$ are perforated in the lower producing zone. The trend of increasing measured chloride concentrations from the late 1970 s to the early 1990 s was reasonably well simulated in these wells (fig. 22A). The trend of decreasing measured chloride concentrations in these wells from the early 1990s through 2014 was steeper than that indicated by simulated chloride concentrations, although simulated chloride concentrations decreased during this period.

Well $4 \mathrm{~N} / 27 \mathrm{~W}-23 \mathrm{~F} 8$ is near the coast and is perforated in the middle producing zone. The simulated and measured chloride concentrations for this well generally were between 15,000 and 18,000 mg/L. There were some notably lower measurements that are thought to be outliers or erroneous data. Simulated concentrations were close to measured, although measured concentrations appeared to decline slowly or not at all from 1990 to 2014, whereas simulated concentrations continuously declined during this period (fig. 22A).

Measured chloride concentrations in wells perforated in the upper producing zone (4N/27W-23E3, 4N/27W-23F2, $4 \mathrm{~N} / 27 \mathrm{~W}-23 \mathrm{~F} 7$ ) increased only slightly during the $1980 \mathrm{~s}$; however, the simulated chloride concentrations increased more than measured, such that simulated concentrations in wells $24 \mathrm{~N} / 27 \mathrm{~W}-23 \mathrm{E} 3$ and $4 \mathrm{~N} / 27 \mathrm{~W}-23 \mathrm{~F} 2$ increased to about $1,500 \mathrm{mg} / \mathrm{L}$ by 1990 , compared to a maximum measured concentrations of 20 and $30 \mathrm{mg} / \mathrm{L}$, respectively (fig. 22A).

The simulated chloride concentrations were in agreement with the measured data for wells perforated in the upper aquitard (4N/27W-23E4 and 4N/27W-23F9) and middle zone (4N/27W-23E6 and 4N/27W-23F6), where the chloride concentrations increased minimally during the $1980 \mathrm{~s}$ (fig. 22A). 

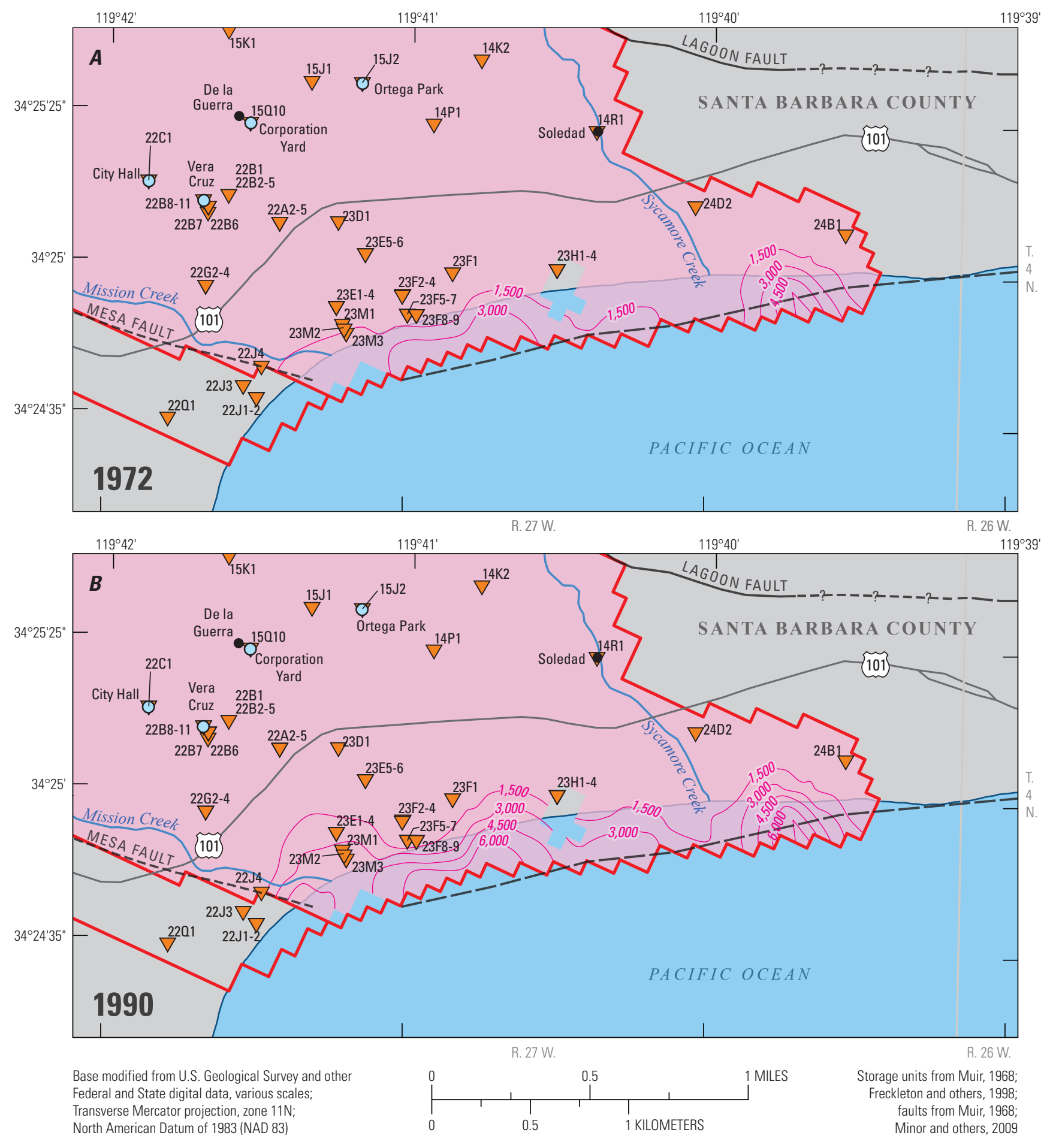

North American D

EXPLANATION
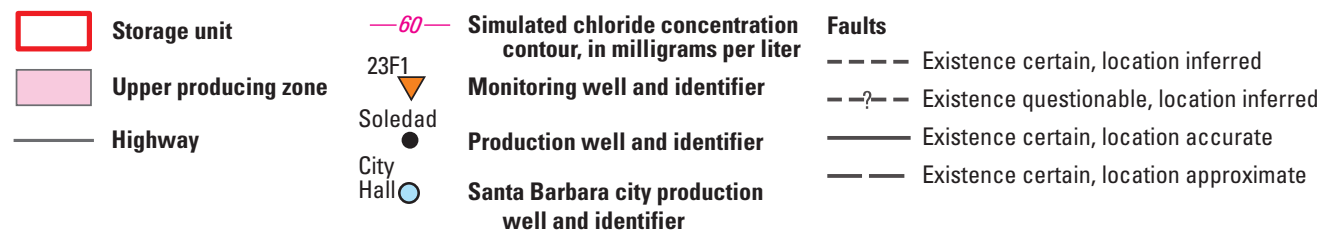

Figure 21. Simulated chloride concentration contours for different years and producing zones for the Santa Barbara groundwater basin, California: $A$, upper, January 1972; $B$, upper, July 1990; $C$, middle, January 1972; $D$, middle, July 1990; $E$, lower, January 1972; and F, lower, July 1990. 

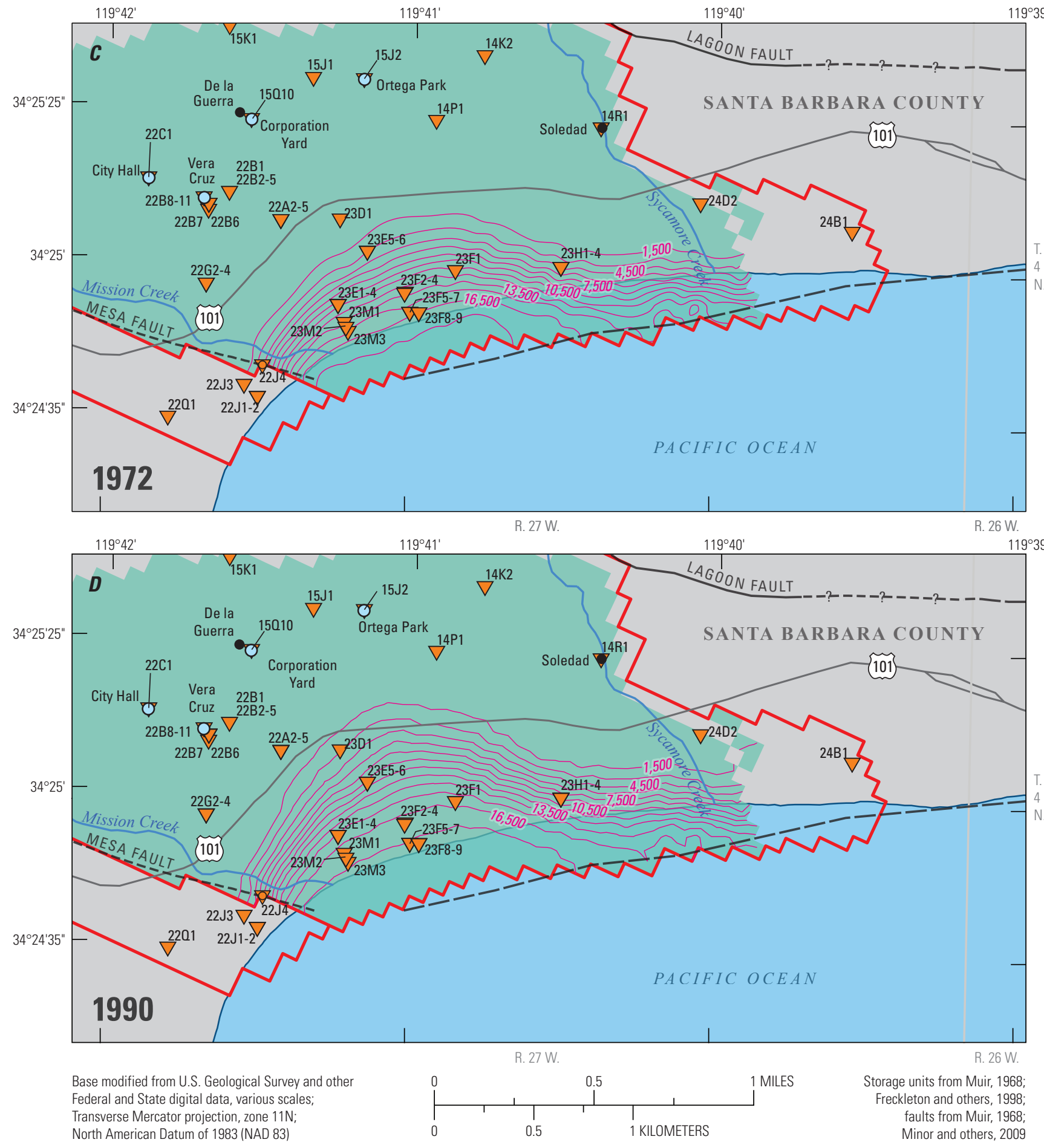

\section{EXPLANATION}

$\begin{array}{ll}\square & \text { Storage unit } \\ \square & \text { Middle producing zone } \\ & \text { Highway }\end{array}$

Highway

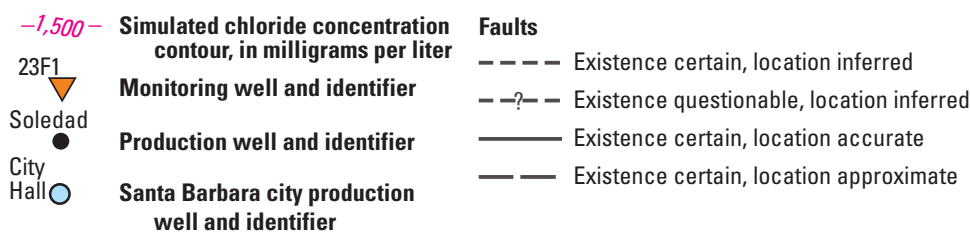

Figure 21. - Continued 

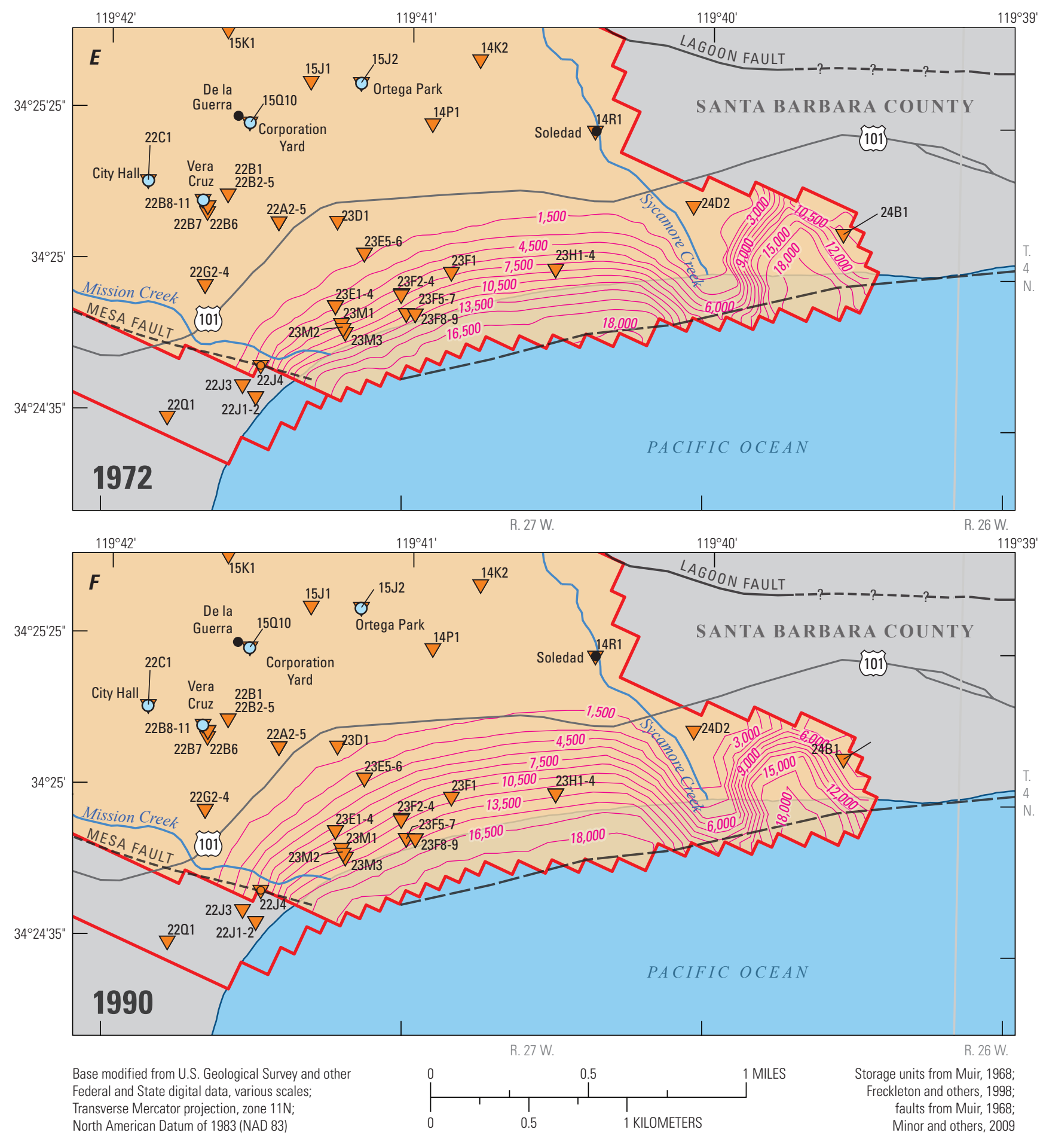

North American Da

EXPLANATION

\begin{tabular}{ll}
\hline & Storage unit \\
\hline & Lower producing zone \\
& Highway
\end{tabular}

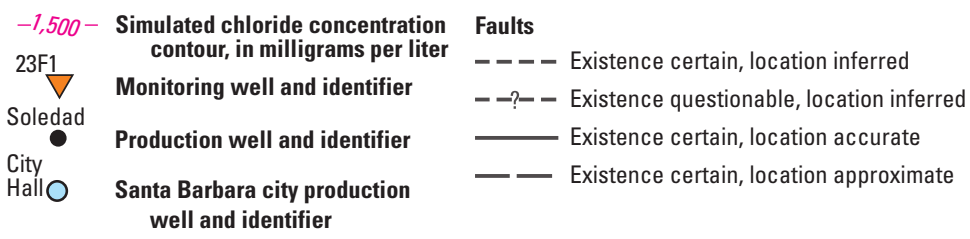

Figure 21. - Continued 
$\boldsymbol{A}$
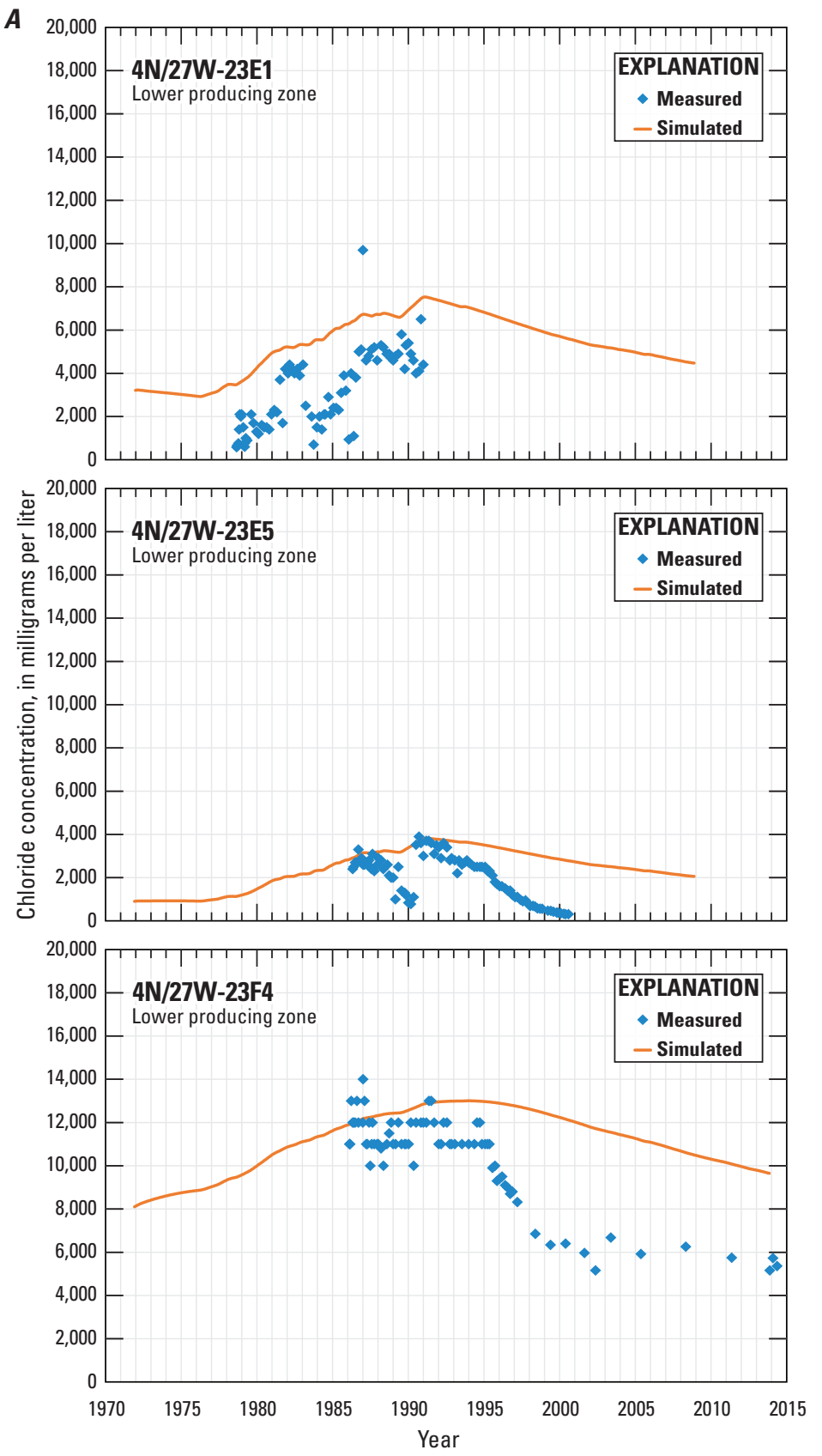
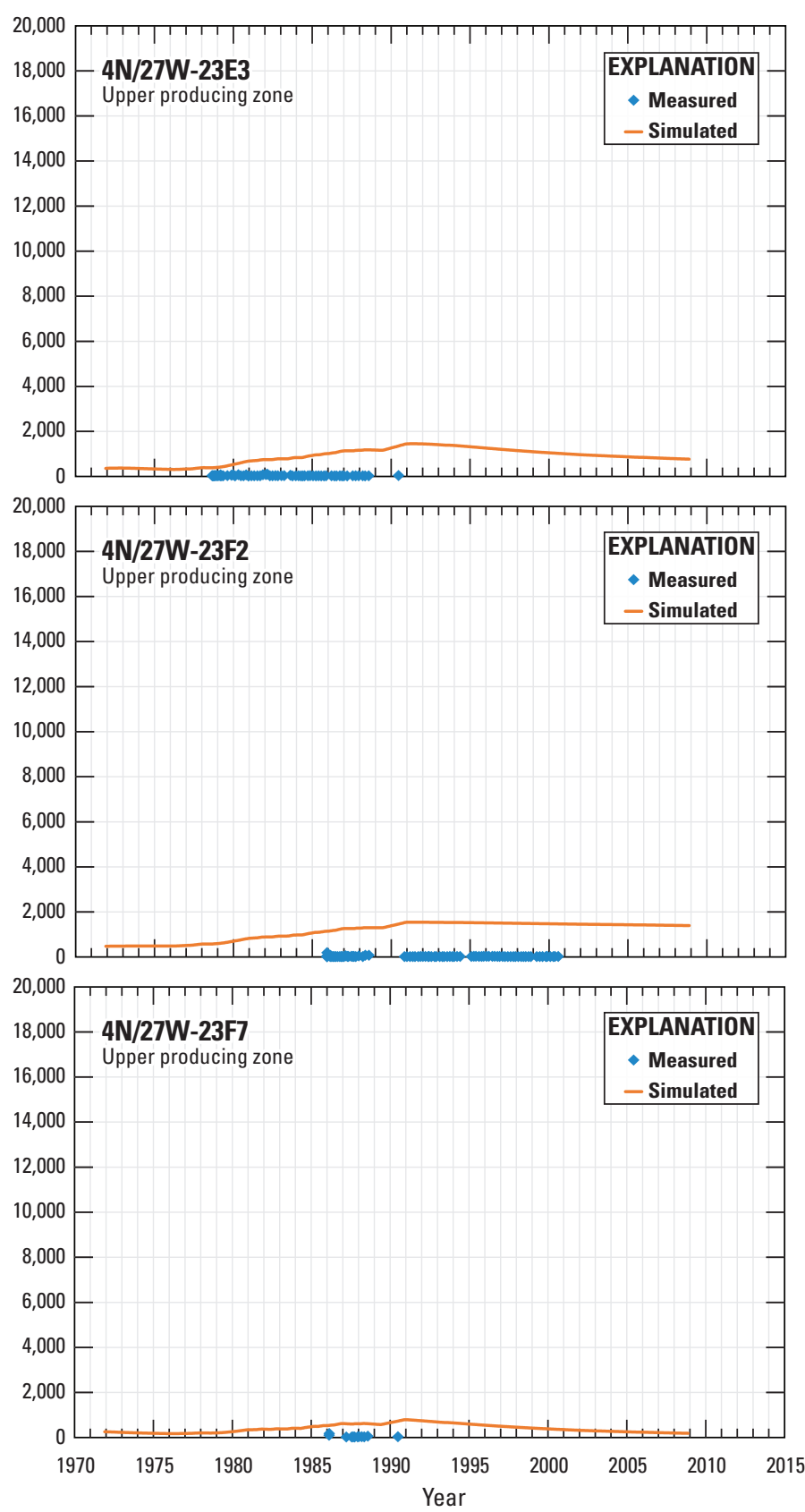

Figure 22. Simulated and measured chloride breakthrough curves for selected wells in Storage Unit I, Santa Barbara groundwater basin, California: $A$, coastal monitoring wells, and $B$, inland monitoring wells. 
$\boldsymbol{A}$
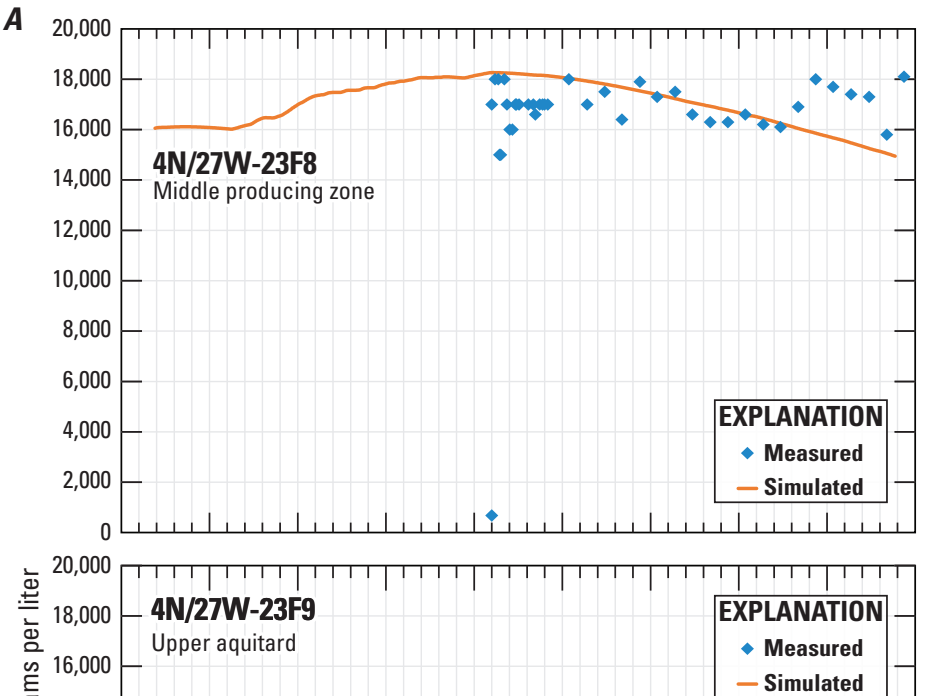

흔 14,000 -

言 12,000

ᄃ 10,000 -

$8,000-$

6,000 -

4,000

2,000

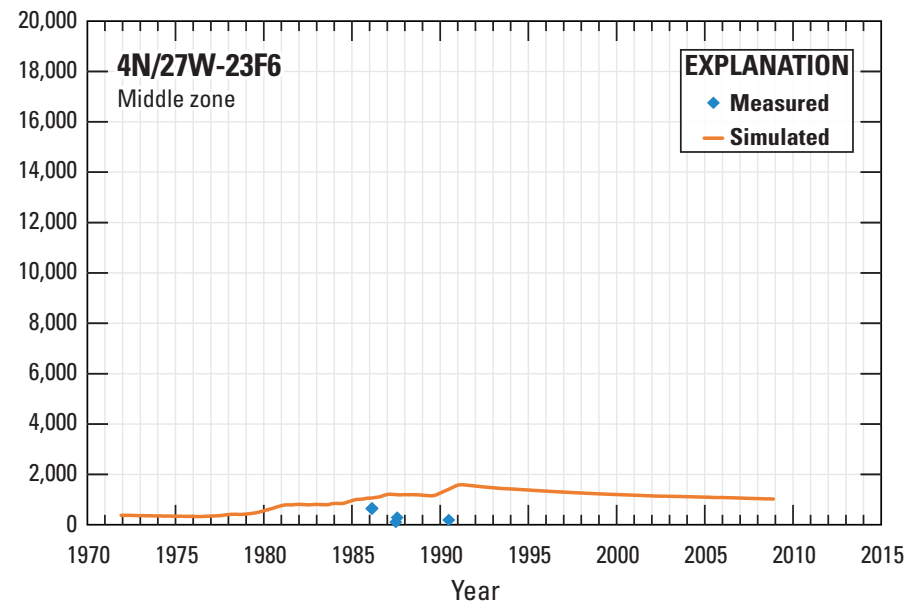

Figure 22. - Continued
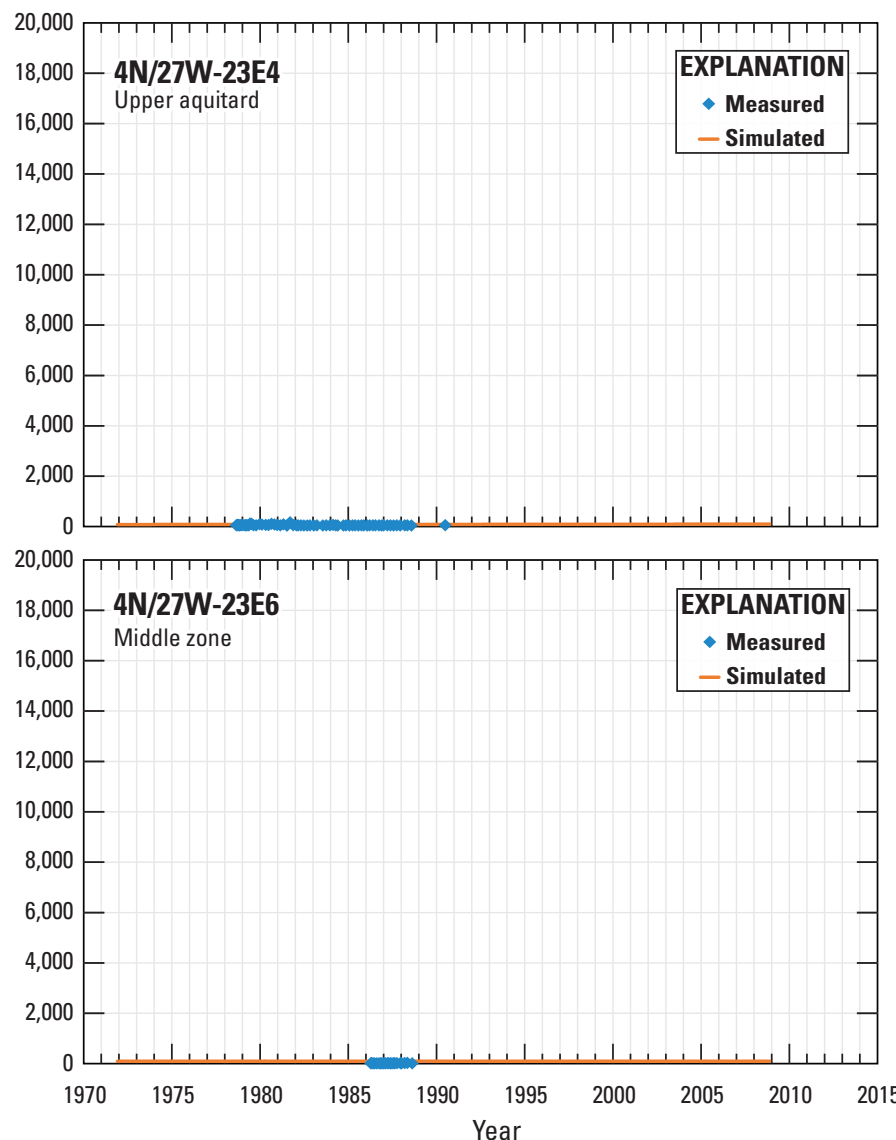
B
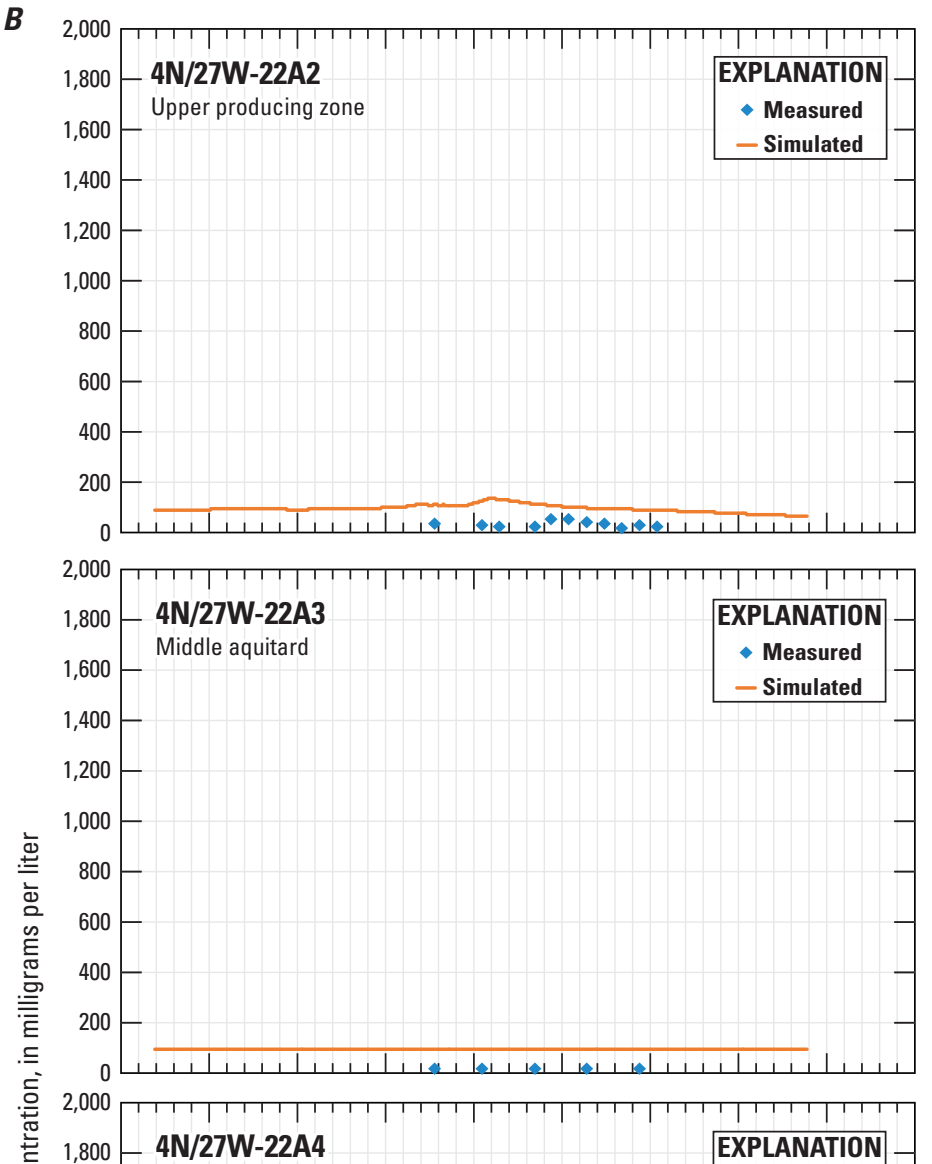

옹

은

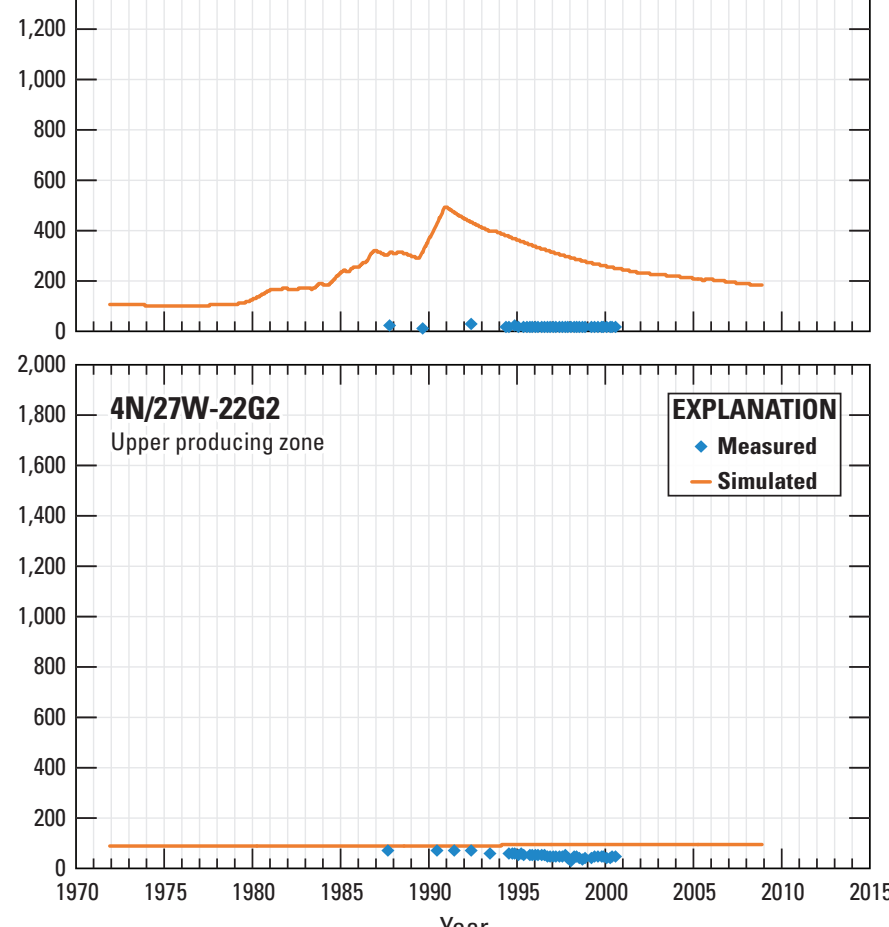

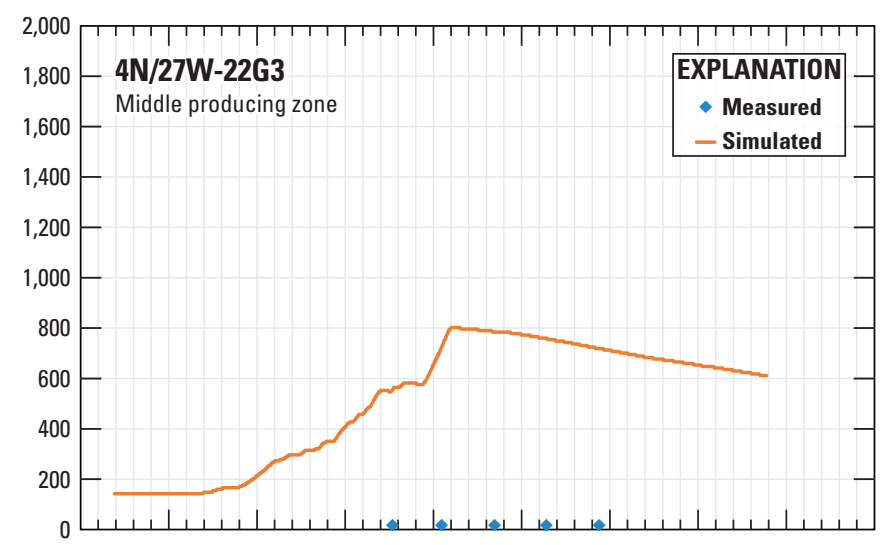
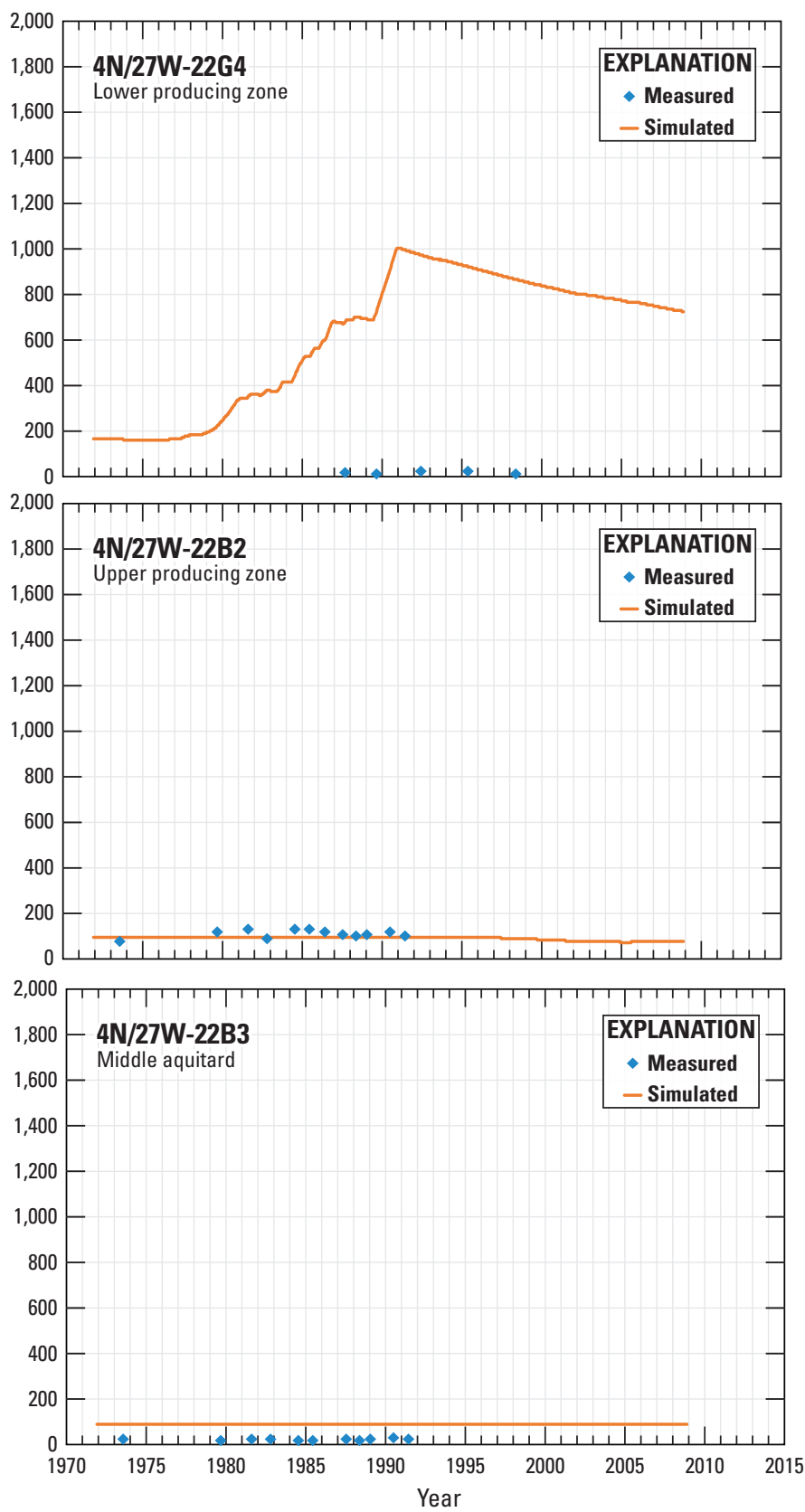

Figure 22. - Continued 
B
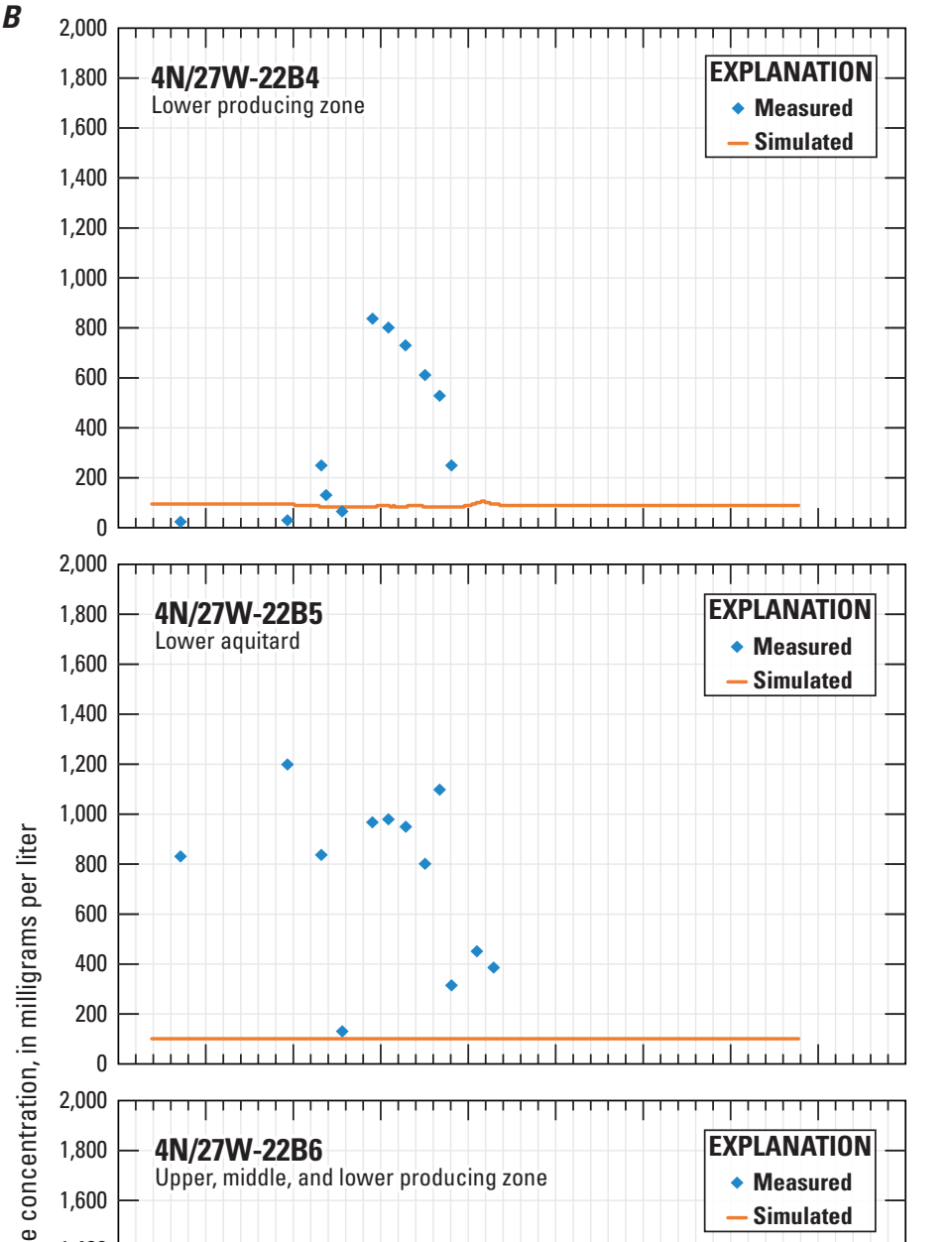

은
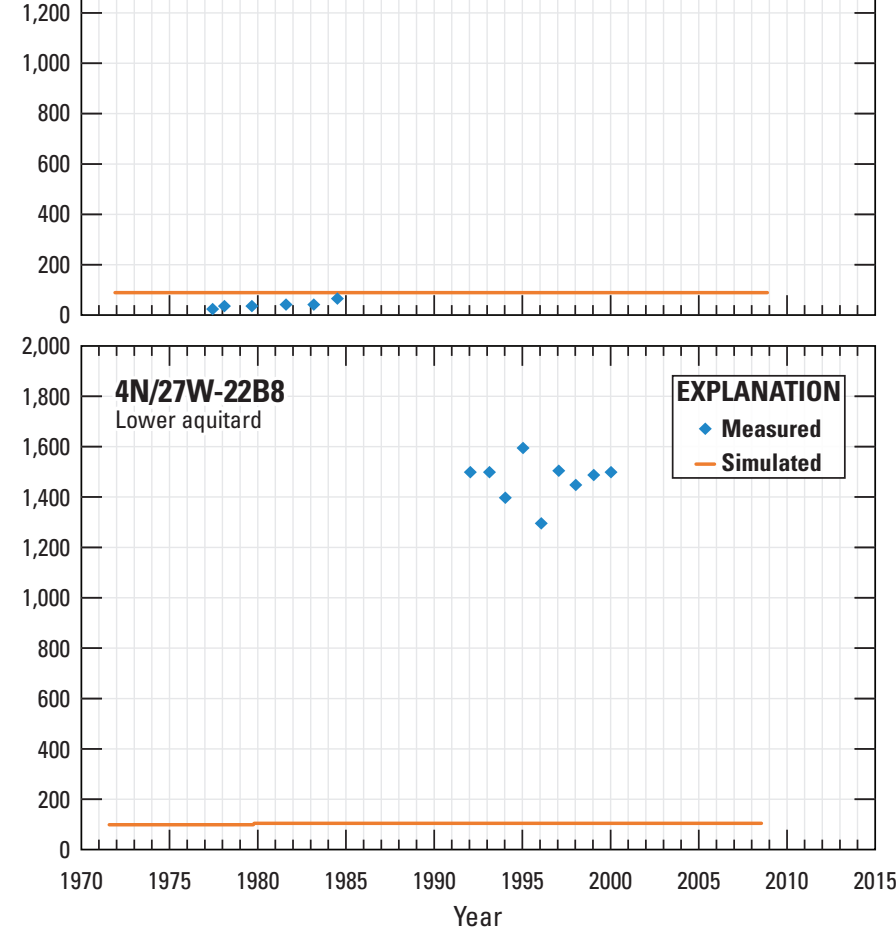

Figure 22. - Continued
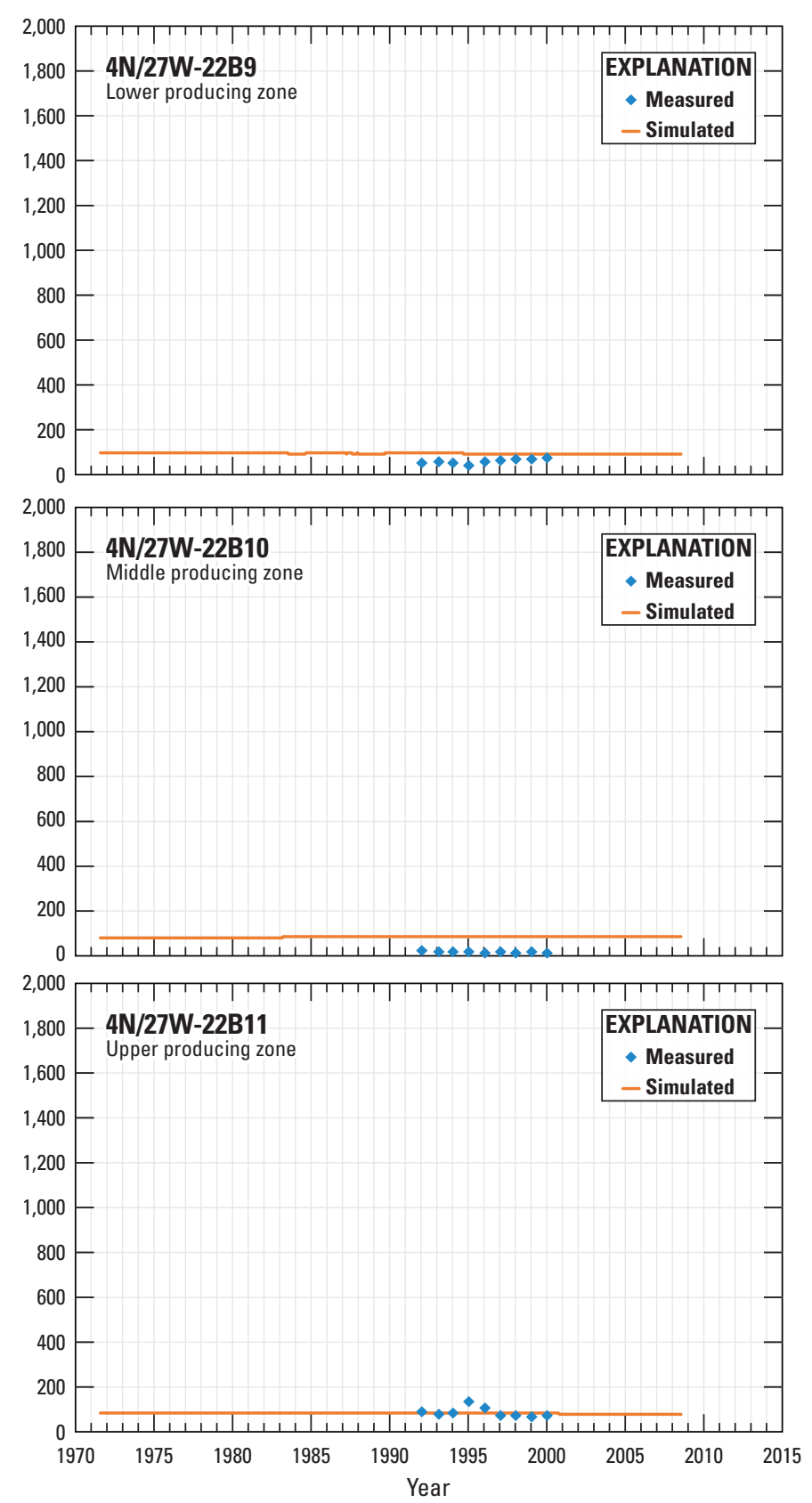
For conditions farther inland, monitoring wells 4N/27W22A2-4 and 4N/27W22G2-4 are about $0.25 \mathrm{mi}$ from the Vera Cruz production well and $0.5 \mathrm{mi}$ from the coast (fig. 10). Measured chloride concentrations in these wells were consistently low, ranging from 10 to $60 \mathrm{mg} / \mathrm{L}$. Simulated concentrations in wells $4 \mathrm{~N} / 27 \mathrm{~W} 22 \mathrm{~A} 2-3$ (perforated in the upper producing zone and middle aquitard, respectively) were also consistently low; however, the simulated concentrations for well 22A4 (perforated in the lower producing zone) were greater that measured (fig. 22B). Simulated concentrations in wells 4N/27W22G2-4 increased in the middle and lower producing zones (22G3 and 4, respectively) during and after the $1980 \mathrm{~s}$, peaking at about $1,000 \mathrm{mg} / \mathrm{L}$. Simulated chloride concentrations in the upper producing zone (well 4N/27W22G2) were consistently low, at about $90 \mathrm{mg} / \mathrm{L}$.

Monitoring wells 4N/27W-22B2-6 and 4N/27W22B8-11 are the farthest inland sites used for monitoring chloride; they are near the Vera Cruz production well (fig. 10). In general, the measured and simulated chloride concentrations were generally low for these wells. Measured and simulated concentrations were less than $100 \mathrm{mg} / \mathrm{L}$ for the wells perforated above the lower producing zone. Wells $4 \mathrm{~N} / 27 \mathrm{~W} 22 \mathrm{~B} 4,5$, and 8 , are perforated in the lower producing zone or the lower aquitard and have measured chloride concentrations as high as $1,600 \mathrm{mg} / \mathrm{L}$; however, simulated chloride concentrations for these wells were substantially lower, at about $100 \mathrm{mg} / \mathrm{L}$ (fig. 22B). Recall that barium and boron concentrations in excess of the concentration of the two constituents in ocean water indicated that seawater intrusion was not the source of the elevated chloride concentrations in the deeper Vera Cruz wells (Martin, 1984). Because the SBFTM only simulates elevated chloride concentrations from seawater intrusion, it was not expected to match these chloride concentrations.

A simple method of assessing overall model fit is to plot the simulated chloride concentration against the measured chloride concentration (fig. 23). In a perfect fit, all points show a 1:1 relation (fall on the 1:1 diagonal line). The RMSE between measured and simulated chloride concentration was $1,586.5 \mathrm{mg} / \mathrm{L}$. Given the scale of the SBFTM, simulated chloride concentration reasonably matched measured chloride concentration. The average residual was $-426.0 \mathrm{mg} / \mathrm{L}$, and the standard deviation was $1,529.0 \mathrm{mg} / \mathrm{L}$. The average residual indicated a bias toward underpredicting the measured chloride concentrations. The residuals ranged from $-6,449.0$ to $4,889.4 \mathrm{mg} / \mathrm{L}$; these extremes possibly represent errors in simulating the movement of the seawater intrusion front due to limitations of the model. More than 50 percent of the simulated chloride concentrations were within $600 \mathrm{mg} / \mathrm{L}$ of measured chloride concentration, more than 81 percent were within $1,900 \mathrm{mg} / \mathrm{L}$, and more than 94 percent were within $3,600 \mathrm{mg} / \mathrm{L}$.

As stated previously, the underestimation of low water levels at the production wells could lead to an underestimation of seawater intrusion. However, there are a number of other assumptions and discrepancies in the model that could lead to an overestimation of seawater intrusion. A comparison of simulated and measured chloride concentrations indicated that the SBFTM matched them fairly well both at high and low concentrations, but tended to slightly overestimate chloride concentrations, especially at lower concentrations (fig. 23). The slight overestimation of low chloride concentrations was related to the SBFTM slightly overestimating the advance of the chloride front during periods of heavy pumping and underestimating the retreat of the chloride front during periods of low pumping. Some of the chloride data from wells $4 \mathrm{~N} / 27 \mathrm{~W}-23 \mathrm{~F} 4$, and $23 \mathrm{~F} 8$ were rounded to the nearest 1,000 $\mathrm{mg} / \mathrm{L}$ at chloride concentrations above $10,000 \mathrm{mg} / \mathrm{L}$. As a result, discrete jumps in measured chloride values can be seen in figure 23 for many of the measured chloride concentrations above $10,000 \mathrm{mg} / \mathrm{L}$. 


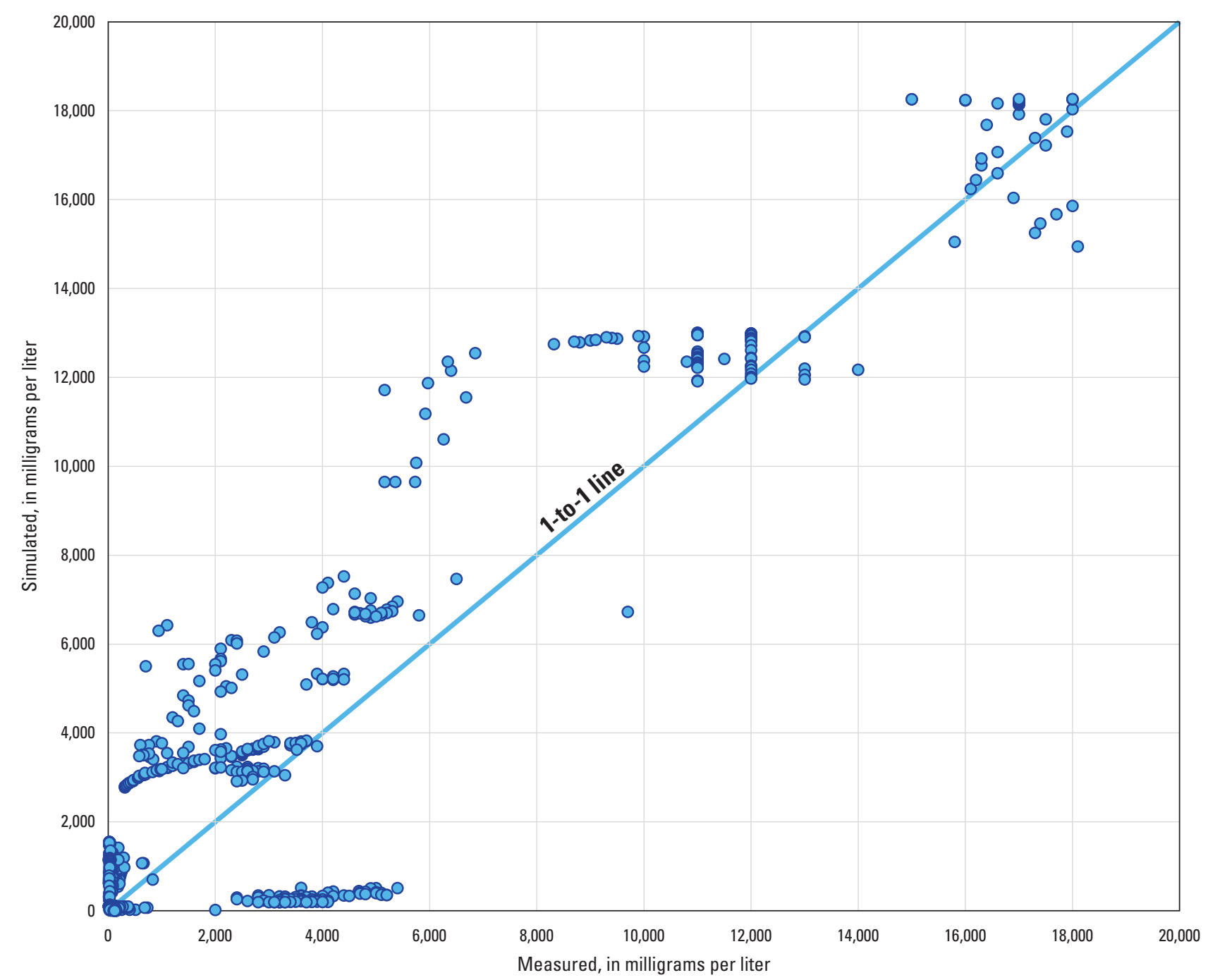

Figure 23. Simulated chloride concentrations compared with measured chloride concentrations for selected monitoring wells in Storage Unit I, Santa Barbara groundwater basin, California.

\section{Model Limitations and Appropriate Use}

A groundwater-flow and solute-transport model is a valuable tool for testing the conceptualization of the groundwater-flow system and for predicting the response of the system to changes in aquifer stresses. However, a model is only an approximation of the actual aquifer system and is not expected to exactly mimic the system being simulated. The model relies on estimates of aquifer properties and stresses, which have some degree of uncertainty.

Water levels and chloride concentrations simulated by the model are average values for the volume represented by each model cell. Simulated heads can vary considerably from measured water levels because of well location, depth, and construction. For example, wells may be perforated over a depth represented by more than one model layer, whereas corresponding measured water levels could represent an unknown composite of the hydraulic head across this perforated interval. The size of the model cells and the length of the stress periods of the model used in this work were appropriate when considering the resolution of available data and the regional scale of the simulation, however. Because model uncertainty increases substantially as the size of the area of interest decreases, the model generally is not appropriate for addressing detailed, local-scale problems. However, the regional model presented in this work can be used in the development of boundary conditions for other refined, local-scale groundwater models. 
The SBFTM assumes constant stage for each creek in the model area. Streams in the model area are known to vary in stage. These seasonal fluctuations in stage, which could influence groundwater flow, are not simulated by the SBFTM. The SBFTM was constructed using a 56-layer model grid, with cell thickness for most of the model set to $20 \mathrm{ft}$. The model grid follows land surface, which sometimes differs from the path of groundwater flow in Storage Unit I, where the lower producing zone crosses multiple model layers as it increases in depth toward the coast. As the lower producing zone crosses model layers, the horizontal model connections between the lower producing zone and the surrounding confining layer can lead to additional flow between the producing zone and the aquitard. Layers in the SBFTM were all treated as confined; therefore, model layers could not go dry, and the SBFTM might overestimate groundwater flow near the water table during times when the water table is relatively low.

The bottom of the SBFTM was assumed to be a no-flow boundary, which does not allow flow or transport between the deep zone (bottom of the model) and the underlying bedrock. Previous studies have indicated that the underlying shale contains water with high chloride concentrations and that this water is a source of high chlorides in some wells perforated in the deep and lower producing zones. Therefore, it is a limitation in some parts of the model domain that the SBFTM does not simulate the transport of chlorides from the underlying shale to the deep zone and underestimates chloride concentrations in the deep zone and some areas of the lower producing zone.

The pumpage data available in the study area before 1971 were incomplete. Overall, pumpage from the major production wells in the study area was often known, but the amount of pumpage from each production well could not always be determined. When the distribution of pumpage could not be determined from data, the simulated pumpage distribution was approximated by parameter estimation, and might not reflect the actual distribution of pumpage; therefore, the early time responses to pumping might not be accurate. In addition, agricultural pumpage was not estimated for the model.

The amount of underflow from adjacent uplands (smallcatchment recharge) was unknown and was determined by parameter estimation; therefore, the model might not accurately simulate the total underflow from adjacent uplands. Small-catchment recharge zones were aligned with the canyons to simulate separate recharge rates from different canyons. The model only simulates variations in smallcatchment recharge on a zone-by-zone basis, and the model does not simulate variations on a smaller scale. That is, the model averages variations in small-catchment recharge that might exist in each zone and applies recharge homogenously across the zone, which could affect model accuracy.

The middle zone consists of fine-grained deposits interspersed with occasional coarse-grained water-bearing deposits. The coarse-grained water-bearing deposits in the middle zone vary in thickness and some could be substantially less than $20 \mathrm{ft}$ thick, the approximate thickness of SBFTM model cells. The transport of chlorides could take a circuitous path through thin connecting lenses of coarse-grained deposits. The 20-ft-thick cells of the SBFTM can only approximate transport along such a small, circuitous path. Transport of chlorides through thin connecting lenses can lead to concentrating chlorides primarily in the connecting lenses and not in the rest of the model cell. Circuitous paths across connected coarse-grained lenses can lead to longer transport times because the chlorides must travel a greater distance. Therefore, the model discretization of $20 \mathrm{ft}$-thick cells might limit the model's ability to accurately predict transport time and concentrations of chlorides if interconnected coarsegrained lenses exist on a smaller scale.

Despite the uncertainty and these limitations, the SBFTM provides a conservative estimate of the amount of pumping permissible before seawater intrusion becomes an issue, because the SBFTM slightly overestimates the amount of seawater intrusion and slightly underestimates the dilution rate of seawater after the intrusion event. These simulation errors are conservative in terms of, for example, engineering solutions for seawater intrusion. That is, any engineering solution based on the simulated results would be designed for higher chloride concentrations than what might be found in reality, which would result in a conservative design.

In addition, the SBFTM can be used to estimate the effects of water-resource management strategies both on water levels and on seawater intrusion, assuming current and future climate scenarios. In addition, it can be coupled with an optimization algorithm to identify water-resource management strategies that optimize a given objective (or objectives) subject to a set of constraints. The SBFTM has been coupled with a multi-objective evolutionary algorithm, and the results are presented in chapter D.

\section{Summary and Conclusions}

In order to simulate seawater intrusion under various water-management scenarios, estimate the sustainability of the Santa Barbara and Foothill groundwater basins, and determine optimal strategies to manage the groundwater basins, a three-dimensional, density-dependent groundwaterflow and solute-transport model (SBFTM) of the groundwater basins was developed. The simulation time series used annual stress periods from 1929 to 1971 and monthly stress periods from 1972 to 2013 . The SBFTM uses 250 -by-250 ft cells and was divided into 56 layers to simulate flow in the upper, middle, and lower producing zones and the surrounding zones. The SBFTM was calibrated using 24,884 water-level observations, 1,064 chloride-concentration observations, and 254 parameters. The parameters represent hydraulic parameters (hydraulic conductivity, storage, and others), transport parameters (dispersivity, porosity, and others), conductance properties (fault, general-head boundary, and drains), creek properties (bed conductance and stage), and recharge or discharge parameters. 
Parameter sensitivities varied over about two orders of magnitude for the 30 most sensitive parameters. The 30 most sensitive parameters are of varying types, including those controlling small-catchment recharge, pumping, horizontal and vertical hydraulic conductivity, porosity, and flow between the SBFTM and the ocean. The SBFTM was most sensitive to parameters controlling recharge, pumping, and hydraulic conductivity. The sensitivity of recharge and pumping parameters was most likely amplified by faults limiting flow out of Storage Unit I, making recharge and pumping the main mechanisms of inflow and outflow in Storage Unit I.

Analyses of the simulated hydrologic budget indicated that the sources of simulated recharge were primarily small catchments and creeks. Small catchments were the primary source of recharge during wetter periods, when pumping was limited. Creek leakage was the primary source of recharge during dry years. It was assumed that the creek stages are always greater than zero; therefore, the creeks are a constant source of water to the model.

The primary groundwater sink was pumping. Between 1929 and 2013, pumpage varied greatly; generally, pumping rates were higher during drier periods, and pumping rates were lower during wetter periods. Overall, groundwater pumping resulted in a net loss of about 11,400 acre-feet (acre-ft) of groundwater storage between 1929 and 2013.

Overall, the SBFTM replicated the natural groundwaterflow direction from the Sycamore, Mission, Lauro, and Aliso Canyons southwest through the Foothill groundwater basin and southeast through the Santa Barbara groundwater basin. In addition, the model replicated the change in direction of groundwater flow from northwest-to-southeast to a radial path toward the pumping center in the southeastern part of Storage Unit I caused by pumping during the late 1980s and early 1990s. The simulated radial-flow pattern extended to the offshore fault, which reversed the direction of flow across the fault, resulting in the simulation of seawater intrusion into the middle and lower producing zones.

Overall, the SBFTM simulated the areal distribution of seawater intrusion and the rising limb of chloride breakthrough curves well. The SBFTM simulated the areal distribution and response of chloride to pumpage. Seawater intrusion was overestimated during the 1990s, however. Regarding the chloride breakthrough curves, the SBFTM generally simulated the rising limbs of the measured breakthrough curves; however, the falling limbs were overestimated. This was caused by a combination of the SBFTM overestimating the advance of the chloride front during periods of heavy pumping and underestimating the retreat of the chloride front during periods of low pumping.

Overall, the SBFTM replicated the measured hydrographs. Measured values and trends were replicated as well as vertical gradients. The SBFTM simulates seawater intrusion but slightly overestimates the amount of seawater intrusion and slightly underestimates the dilution rate of seawater after the intrusion event.

\section{References Cited}

California Department of Water Resources, 1996, 1996 South central coast land use survey data: Sacramento, Calif., State of California, digital map accessed on July 12, 2011, at http://www.water.ca.gov/landwateruse/lusrvymain.cfm, currently available at https://www.water.ca.gov/Programs/ Water-Use-And-Efficiency/Land-And-Water-Use/LandUse-Surveys.

Daly, C., and Bryant, K., 2013, The PRISM climate and weather system-An introduction: Prism Climate Group, 5 p., accessed January 21, 2015, at http://www.prism. oregonstate.edu/documents/PRISM_history_jun2013.pdf.

Doherty, J.E., 2010, PEST-Model-independent parameter estimation, user manual (5th ed.), addendum to the PEST manual: Watermark Numerical Computing, $336 \mathrm{p}$.

Freckleton, J.R., Martin, P., and Nishikawa, T., 1998, Geohydrology of Storage Unit III and a combined flow model of the Santa Barbara and Foothill ground-water basins, Santa Barbara County, California: U.S. Geological Survey Water-Resources Investigations Report 97-4121, 80 p., http://pubs.er.usgs.gov/publication/wri974121.

Freeze, R.A., and Cherry J.A., 1979, Groundwater: Englewood Cliffs, N.J., Prentice-Hall, 604 p., http://hydrogeologistswithoutborders.org/wordpress/1979toc/.

Goldman, C.R., and Horne, A.J., 1983, Limnology: New York, McGraw-Hill Book Co., 464 p.

Harbaugh, A.W., Banta, E.R., Hill, M.C., and McDonald, M.G., 2000, MODFLOW-2000, the U.S. Geological Survey modular ground-water modelUser guide to modularization concepts and the groundwater flow process: U.S. Geological Survey Open-File Report 2000-92, 121 p., https://pubs.er.usgs.gov/publication/ofr200092.

Hill, M.C., 1990, Preconditioned conjugate-gradient 2 (PCG2), a computer program for solving ground-water flow equation: U.S. Geological Survey Water-Resources Investigations Report 90-4048, 43 p., https://pubs.er.usgs.gov/publication/wri904048.

Hsieh, P.A., and Freckleton, J.R., 1993, Documentation of a computer program to simulate horizontal-flow barriers using the U.S. Geological Survey's modular three-dimensional finite-difference ground-water flow model: U.S. Geological Survey Open-File Report 92-477, 32 p., https://pubs.er.usgs.gov/publication/ofr92477. 
Langevin, C.D., Thorne, D.T., Jr., Dausman, A.M., Sukop, M.C., and Guo, W., 2008, SEAWAT version 4: A computer program for simulation of multi-species solute and heat transport: U.S. Geological Survey Techniques and Methods book 6, chap. A22, 39 p., https://pubs.er.usgs.gov/publication/tm6A22.

Martin, P., 1984, Ground-water monitoring at Santa Barbara, California; Phase 2-Effects of pumping on water levels and on water quality in the Santa Barbara ground-water basin: U.S. Geological Survey Water Supply Paper 2197, 31 p., https://pubs.er.usgs.gov/publication/wsp2197.

McDonald, M.G., and Harbaugh, A.W., 1988, A modular three-dimensional finite-difference ground-water flow model: U.S. Geological Survey Techniques of WaterResouces Investigations book 6, chap. A1, 586 p., https://pubs.er.usgs.gov/publication/twri06A1.

McFadden, M.C., Polinoski, K.G., and Martin, P.M., 1991, Measurement of streamflow gains and losses on Mission Creek at Santa Barbara, California; July and September 1987: U.S. Geological Survey Water-Resources Investigations Report 91-4002, 15 p., https://pubs.er.usgs.gov/publication/wri914002.

Minor, S.A., Kellogg, K.S., Stanley, R.G., Gurrola, L.D., Keller, E.A., and Brandt, T.R., 2009, Geologic map of the Santa Barbara coastal plain area, Santa Barbara County, California: U.S. Geological Survey Scientific Investigations Map 3001, scale 1:25,000, 1 sheet, pamphlet, 38 p., https://pubs.er.usgs.gov/publication/sim3001.

Muir, K.S., 1968, Ground-water reconnaissance of the Santa Barbara-Montecito area, Santa Barbara County, California: U.S. Geological Survey Water Supply Paper 1859-A, 28 p., https://pubs.er.usgs.gov/publication/wsp1859A.

U.S. Census Bureau, 2017, QuickFacts from the U.S. Census Bureau, Santa Barbara city, California, accessed September 2017 at https://www.census.gov/quickfacts/fact/ table/santabarbaracitycalifornia/HSD310215.

U.S. Department of Agriculture, 2008, Natural Resources Conservation Service, Soil Survey Geographic (SSURGO) database for Santa Barbara County, California, south coastal part: National Resources Conservation Service, accessed on July 12, 2011, at https://www.nrcs.usda.gov/wps/portal/nrcs/ surveylist/soils/survey/state/?stateId=CA.

U.S. Geological Survey, 2013, USGS 11119745 Mission C A Rocky Nook Park A Santa Barbara CA: U.S. Geological Survey National Water Information System database, accessed November 2013, at https://waterdata.usgs.gov/ nwis/uv?site_no=11119745.
Zheng, C., 2006, MT3DMS v5.2 supplemental user's guide: Tuscaloosa, Ala., University of Alabama, Department of Geological Sciences, 41 p., http://inside.mines.edu/ epoeter/583/14/discussion/ mt3dms_v5_supplemental.pdf.

Zheng, C., 2010, MT3DMS v.5.3 supplemental user's guide: Tuscaloosa, Ala., University of Alabama, Department of Geological Sciences, 51 p., https://hydro.geo.ua.edu/mt3d/ mt3dms_v5_supplemental.pdf.

Zheng, C., and Wang, P.P., 1999, MT3DMS—A modular three-dimensional multispecies transport model for simulation of advection, dispersion, and chemical reactions of contaminants in groundwater systems; Documentation and user's guide: Tuscaloosa, Ala., University of Alabama, Contract Report SERDP-99-1, 221 p., https://hydro.geo.ua.edu/mt3d/mt3dmanual.pdf. 



\title{
Chapter D: Multi-Objective Simulation-Optimization Model
}

\author{
By Zachary P. Stanko and Tracy Nishikawa
}

\section{Introduction}

The city of Santa Barbara is interested in identifying management strategies that maximize the available groundwater resources while limiting drawdown and any associated seawater intrusion. In order to identify the best strategies, the U.S. Geological Survey (USGS) and the city cooperatively developed a simulation-optimization model, which uses the Santa Barbara flow and solute-transport model (SBFTM), described in chapter $\mathrm{C}$, to derive optimal management strategies and estimate maximum pumping rates for a range of potential future climatic conditions. The SBFTM was used to develop a collection of predictive simulations that can be used to produce optimal pumping schedules and optimal pumping rates for each climatic condition. These simulations have different management horizons (time periods over which pumpage is optimized), initial groundwater levels, chloride concentrations, and climatic conditions. Overall, five management-optimization scenarios were developed in a multi-objective framework to obtain optimal pumping rates for all of the city's managed wells that maximize withdrawals, while minimizing drawdown and seawater intrusion.

This chapter describes the groundwater-management problem, reviews the multi-objective framework and optimization methods used to approximate optimal solutions for multiple competing objectives, defines the selected future-management scenarios, and presents the resulting solutions that optimizes pumping under the constraints and objectives of each of the scenarios. The simulated scenarios have management horizons ranging from 2 to 10 years; initial conditions from historically low to relatively high-water levels; and climatic conditions ranging from typical of the recent past to those of a 10-year drought.

Following the availability of imported water from the California State Water Project (SWP) in 1997 and the construction of a desalination plant, Nishikawa (1998) used a groundwater-flow model of the Santa Barbara groundwater basin to optimize pumping rates. Using a flow-only simulation, that is, the transport of chloride and computation of its concentrations were not simulated, the optimization was formulated as a linear-programming (LP) problem with a single minimize-cost objective function. Mild nonlinearities in the groundwater model, due to simulation of drains, were resolved with a simple iterative method. A 5-year management horizon was used, which corresponded to the city's design drought of calendar year 1947-51. The decision variables - or the choices involved in making management decisions, in this case associated with the water sources available for use-were the monthly pumpage from groundwater wells, imported water from the SWP, as well as water supply available from local surface water and desalination. Constraints included groundwater levels, pumping-well capacity, and water demand. Results of the 1998 study showed that a minimumcost solution could be met by maximizing the lowest cost water source (surface water, when available) and continuously pumping the inland wells. The 1998 study also showed that desalinated water (the highest cost water source) was not used during the design drought of 1947-51. Note that the 1998 study was based on rules and regulations for surface water in effect at the time; since then, a Biological Opinion for the Cachuma Project (Cachuma Operation and Maintenance Board, 2017) has been issued and is under reconsultation by the Bureau of Reclamation and National Marine Fisheries Service.

\section{Literature Review}

Optimization methods have been widely used for waterresources management. A general overview of optimization methods will first be presented followed by a discussion of their applications to water-resources management. 


\section{Optimization Methods}

A variety of techniques can be used to formulate and solve optimization problems. For the purposes of this study, there are two general techniques for solving large-scale (characterized by a large number of decision variables) optimization problems: mathematical programming and global search. Strict mathematical programming techniques can guarantee convergence to a local optimum but require that the underlying functions have certain inherent features (for example, continuity, differentiability, and convexity) to find a solution. Mathematical programming techniques require differentiability, because gradient information is required; this is computationally intensive. For additional information regarding mathematical programming techniques, see Nocedal and Wright (2006).

Global-search techniques can find or approximate a global optimum without gradient information; instead, a randomized search strategy is used. A global search is not associated with the features of the underlying mathematics. The functional relationships between inputs and outputs of an embedded or linked simulation model are not important for the global-search optimization techniques; therefore, problems that are highly nonlinear, discontinuous, or not differentiable are solvable. In addition, running simulations external to the optimization allows the use of parallel computing to conduct the global search in a computationally efficient manner (McKinney and Lin, 1994). For additional information regarding global-search techniques, see Coello and others (2007).

Frequently, an optimization problem has more than one objective. Different project goals can conflict with each other. These goals could make an optimization problem infeasible if incorporated as constraints, so alternatively, they can be posed as separate objectives. Given complex relationships among competing objectives, a multi-objective optimization problem can be formulated to find compromise solutions. Instead of a unique solution, a multi-objective optimization produces a set of solutions from which a decision maker can choose the solution that most aligns with the most important needs at the time. No single solution in the set can be better than other solutions in the set with respect to all objectives; this concept is called Pareto optimality (Coello and others, 2007). A Pareto-optimal solution is a feasible solution that cannot improve any of the objectives without worsening at least one other objective. To illustrate this concept of Pareto-optimality, figure 1 demonstrates a Pareto-optimal solution for a generic two-objective problem. For more detailed discussion of multiobjective optimization, see Coello and others (2007).

Evolutionary algorithms (EA) are a class of globalsearch techniques that can work with any problem formulation regardless of the nonlinearities and extent of complexity related to the embedded simulation model. These are a class of methods that treat the solutions of the optimization problem

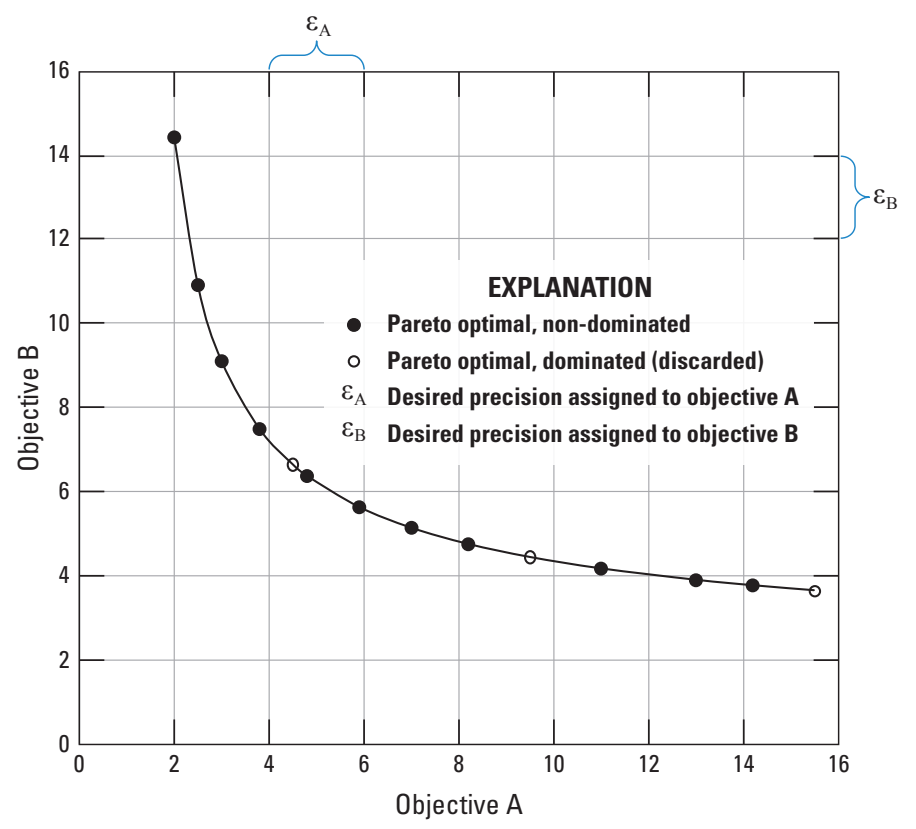

Figure 1. Concepts of Pareto-optimality are shown with two generic objectives, which are assumed to be minimization; no single point is optimal because a decrease in one objective causes an increase in the other, so any point on the curve is considered Pareto-optimal (Kollat and Reed, 2006).

as individuals in a population that are manipulated by a set of operators (mutation, combination, and selection) to evolve toward an optimal solution. The objective function of an EA is viewed as a measure of fitness for each individual solution in the population, and each iteration of selecting solutions from a population, and operating on them, is termed a generation (Coello and others, 2007). A genetic algorithm (GA) is a type of EA that primarily utilizes mutation and crossover combination to operate on variables of individual solutions that are encoded as genes on a chromosome. The concept of 'survival of the fittest' is used in GAs to rank solutions; the objective criteria are evaluated as a measure of fitness. For additional information regarding GAs and their genetic operators, the reader is referred to Coello and others (2007).

Use of EAs has been successful in a wide range of problem types, especially multi-objective optimization. A major advantage when using an EA is the ability to randomly search a large solution space in an intelligent and efficient manner without being overly susceptible to stopping prematurely in a sub-optimal region (local optima). The major disadvantage is that an EA can be slow to produce nearly optimal solutions if the problem dimensions are too high. For multi-objective problems, multi-objective evolutionary algorithms (MOEAs) have been shown to efficiently search large-scale problems with up to six objectives (Kasprzyk and others, 2009). 


\section{Groundwater-Management Models}

Groundwater-flow and solute-transport models are often linked with optimization methods to solve groundwater-management problems. This linkage is the basis of a simulation-optimization model, which in the context of groundwater modeling, is also called a groundwater-management problem. When the simulation model is differentiable, or a gradient can be approximated, mathematical programming can be used. Examples of mathematical programming techniques that have been linked to groundwater models include linear programming (Molz and Bell, 1977; Lefkoff and Gorelick, 1986), nonlinear programming (Ahlfeld and others, 1988; Wang and Ahlfeld, 1994; McKinney and Lin, 1995; Das and Datta, 2000), successive linear programming (Nishikawa, 1998; Ahlfeld and Baro-Montes, 2008), and dynamic programming (Willis, 1979; Chang and others, 1992; Culver and Shoemaker, 1992, 1997). Many additional simulation-optimization review papers summarize the applications of these methods (for example, Gorelick, 1983; Yeh, 1992; Wagner, 1995; Ahlfeld and Mulligan, 2000; Mayer and others, 2002).

For groundwater-management problems specifically addressing seawater-intrusion issues, the simple approach, which constrains freshwater hydraulic heads along the coast to be above sea level, only works if the aquifer is relatively thin (Abarca and others, 2006). Singh (2014) reviews a range of simulation-optimization techniques for seawater-intrusion problems, including applications of GAs and multi-objective formulations. When the underlying flow and transport simulation model is complex, GAs are often applied (Qahman and others, 2005). Studies often use some type of EA to find solutions to single-objective seawater-intrusion problems (Qahman and others, 2005; Abd-Elhamid and Javadi, 2011). Various multi-objective optimization techniques also have been applied to seawater-intrusion problems (Cieniawski and others, 1995; Gordon and others, 2001). Dhar and Datta (2009) present an application of a GA to a multi-objective optimization of a seawater-intrusion model, where the two conflicting objectives were to (1) maximize total pumpage from all production wells and (2) to minimize pumpage from barrier wells.

Because EAs are particularly well-suited for multiobjective optimizations, they have been applied to waterresource problems (Sreekanth and Datta, 2015). Nicklow and others (2010) reviewed some applications of MOEAs to water-resource problems and specifically addressed numerous studies that use MOEAs to overcome some of the inherent difficulties of optimizations involving groundwater models. In a recent comparison among MOEAs, 10 algorithms were tested on several water-resource applications (Reed and others, 2013). The proprietary MOEA algorithm developed by Hadka and Reed (2013), referred to as Borg, consistently outperformed the others on the basis of various metrics used to evaluate algorithm effectiveness (the quality of solutions attained) and efficiency (the time it takes to attain these solutions). A study that assessed the performance of several MOEAs on a management plan for a contaminated lake, considering robust decision-making criteria and environmental-pollution constraints, also found Borg to surpass other algorithms in nearly all metrics (Ward and others, 2015).

After consulting with city personnel, it was determined that a multi-objective optimization technique was appropriate to address the city's water-resource management problem. Four objectives were identified as important goals of the study: maximize pumpage, minimize seawater intrusion, minimize total drawdown, and minimize the maximum drawdown. Given the complex nonlinear SEAWAT model and the number of decision variables, an MOEA was chosen instead of a mathematical-programming technique. The main advantages of using an MOEA for this study are that a gradient approximation is not required and that a high degree of parallelization is possible.

For this study, Borg was used to solve the multi-objective optimization using the SEAWAT groundwater-flow and solute-transport model. Borg was chosen as the optimization algorithm for this study for several reasons: (1) it is very efficient in automatically sorting, evolving, and retaining solutions; (2) it can be used in parallel to efficiently make use of multiple processors on multiple computers; (3) the algorithm is self-tuning, so there are no search or operation parameters to determine prior to using the algorithm; and (4) it is designed to effectively approximate Pareto-optimal solutions (Pareto curve) for multiple competing objectives. The first three reasons can be lumped together as features that allow the algorithm to proceed toward the optimal solution(s) at the fastest possible rate. The fourth reason (an approximate Pareto curve) can allow water managers to make the best possible decision in accordance with their preferences; for the purposes of this study the approximate Pareto-optimal solutions will be referred to as Pareto-optimal solutions. Once the Pareto curve is produced, a variety of decisions can be made with knowledge of the potential sacrifices or tradeoffs without any additional model runs. The alternative approach, to weigh each objective in advance, is very limiting and obscures possible solutions if those weights do not accurately represent future preferences. In Kasprzyk and others (2015), a comparison of the two approaches illustrates the benefits of a full multi-objective formulation. 


\section{Solution Space Discretization}

In a continuous solution space, there are an infinite number of Pareto-optimal solutions. In order to choose from among the infinite number of solutions, a precision is assigned to each objective, defined by an epsilon value ( $\varepsilon$ value) that represents its favorability. Setting this $\varepsilon$ value imposes a grid on the solution space to prevent searching for too many solutions that have similar objective values (fig. 1). Lesser values of $\varepsilon$ with respect to the objective values themselves places more emphasis on solutions closer to the absolute minimum of a single objective. Greater values of $\varepsilon$ with respect to the objective values themselves indicate that fewer trade-off solutions are desired with respect to an objective and an overall minimization of this objective is less important. Lesser values of $\varepsilon$ allow for more trade-off analysis in the optimal solutions, but can also retain many solutions that differ only slightly. For example, a large $\varepsilon_{A}$ (for objective A) represents less preference for maximizing A. Additionally, a lesser $\varepsilon_{B}$ accepts more solutions in the full range of the objective B values. Each $\varepsilon$ can be thought of as the weight of the objective (relative to its magnitude) on the overall decision making. The rectangular domain defined by $\varepsilon_{A}, \varepsilon_{B}$ is termed the $\varepsilon$ neighborhood, in which there is only one solution that is non-dominated, or optimal in the Pareto sense. For example, in figure 1 only one solution in each grid block is retained as Pareto-optimal (solid points). By defining the grid with $\varepsilon_{A}$ and $\varepsilon_{B}$, the solid points dominate the hollow points as determined by the point's distance to the origin (Kollat and Reed, 2006). For additional information regarding $\varepsilon$ dominance, the reader is referred to Hadka and Reed (2013).

For this report, additional $\varepsilon$ values were chosen postoptimization to highlight optimization results; they were not chosen intentionally to reflect objective-function preference. If a water manager wishes to change the objective-function preference, a different set of $\varepsilon$ values would be used and, potentially, the highlighted solutions would be different. This change in $\varepsilon$ values would not require any additional model runs, however; all necessary information was generated in the original optimization runs.

\section{Problem Formulation}

As stated previously, the simulation-optimization problem was formulated as a multi-objective programming problem. In general, the objectives were to maximize pumpage and to minimize seawater intrusion and drawdown in production wells. The drawdown objective was formulated as two separate objective functions: minimize total drawdown to address an overall decline in water levels across all basins and minimize the maximum drawdown to address water-level declines around individual wells. In general, the constraints included pump-capacity limits, chloride drinking-water standards, and water-level limits. The decision variables were quarterly pumpage by well. The managed wells for all scenarios included the following city wells: Alameda Park, City Hall, Corporation Yard, Hope Avenue, Lincolnwood, Los Robles, Ortega Park, San Roque Park (2), Santa Barbara High School, Val Verde, and Vera Cruz (fig. 2). The Lincolnwood well, although not managed by the city at the time of this study, was included as a surrogate for a future city well that could be added in the Foothill groundwater basin. Note that background and unmanaged pumpage were not included because the focus of this study was the management of the city's wells. In addition, during times when the city's wells were pumped, most pumpage was from the city wells. The general formulation can be summarized as follows:

Objective A: Pumpage

$$
\operatorname{minimize} \sum_{i=1}^{n q t r} \sum_{j=1}^{n p w} q_{i j}
$$

Objective B: Seawater intrusion

$$
\operatorname{minimize} \sum_{i=1}^{n c o} \sum_{k=1}^{n c w} c_{i k}
$$

Objective $\mathrm{C}_{\mathrm{t}}$ : Total drawdown

$$
\operatorname{minimize} \sum_{i=1}^{n y r} \sum_{j=1}^{n p w}\left(h_{i j}-H_{j, 0}\right)
$$

Objective $\mathrm{C}_{\mathrm{m}}$ : Maximum drawdown

$$
\text { minimize } \max _{\substack{1 \leq i \leq n y r \\ 1 \leq j \leq n p w}}\left(h_{i j}-H_{j, 0}\right)
$$

subject to:

Constraint 1

$$
\left(250 \frac{m g}{L}-S_{i j}\right) \geq 0 \forall i=1, \ldots, \text { nper } ; j=1, \ldots, n p w
$$

Constraint 2

$$
\left(q_{i j}-Q_{j, \max }\right) \geq 0 \forall i=1, \ldots, n q t r ; j=1, \ldots, n p w
$$

Constraint 3

$$
\sum_{j \in \Omega} q_{i j} \geq Q_{\text {oTP }} \forall i=1, \ldots, n q t r
$$

Constraint 4

$$
h_{i j}-H_{j, \min } \geq 0 \forall i=1, \ldots, n y r ; j=1, \ldots, n p w
$$


where

\begin{tabular}{|c|c|}
\hline$q_{i j}$ & is the pumpage rate (negative out of aquifer) \\
\hline$c_{i k}$ & $\begin{array}{l}\text { for pumping well } j \text { at time } i \text {, } \\
\text { is the concentration for chloride monitoring } \\
\text { well } k \text { at time } i \text {, }\end{array}$ \\
\hline$h_{i j}$ & $\begin{array}{l}\text { is the weighted average hydraulic head for } \\
\text { pumping well } j \text { at time } i \text {, }\end{array}$ \\
\hline$H_{j, 0}$ & $\begin{array}{l}\text { is the initial hydraulic head for pumping } \\
\text { well } j \text {, }\end{array}$ \\
\hline$H_{j, \text { min }}$ & $\begin{array}{l}\text { is the minimum acceptable hydraulic head for } \\
\text { pumping well } j \text {, }\end{array}$ \\
\hline$S_{i j}$ & $\begin{array}{l}\text { is the weighted average solute concentration } \\
\text { for pumping well } j \text { at time } i \text {, }\end{array}$ \\
\hline$Q_{j, \max }$ & $\begin{array}{l}\text { is the maximum pumpage rate for pumping } \\
\text { well } j,\end{array}$ \\
\hline nqtr & is the total number of quarters, \\
\hline nco & $\begin{array}{l}\text { is the total number of concentration } \\
\text { observations, }\end{array}$ \\
\hline$n y r$ & is the total number of years, \\
\hline$n p w$ & is the total number of pumping wells, \\
\hline$n c w$ & $\begin{array}{l}\text { is the total number of chloride monitoring } \\
\text { wells, }\end{array}$ \\
\hline$Q_{\text {OTP }}$ & $\begin{array}{l}\text { is the minimum flow to the Ortega treatment } \\
\text { plant, and }\end{array}$ \\
\hline$\Omega$ & $\begin{array}{l}\text { is the set of all wells that feed the Ortega } \\
\text { plant (Alameda Park, City Hall, SBHS, } \\
\text { Corporation Yard, Ortega Park, Vera Cruz) }\end{array}$ \\
\hline
\end{tabular}

Objective A maximizes the total pumpage for the management horizon (pumping out of the aquifer is negative in the model; therefore, the minimization used in the aforementioned formulation). Objective B minimizes the sum of chloride concentrations at 14 monitoring sites (wells 4N/27W-22A2, -22A4, -22B9, -22B10, -22B11, $-22 \mathrm{G} 3,-22 \mathrm{G} 4,-23 \mathrm{E} 3,-23 \mathrm{E} 5,-23 \mathrm{~F} 2,-23 \mathrm{~F} 4,-23 \mathrm{~F} 5,-23 \mathrm{~F} 7$ and -23F8; fig. 2) for all stress periods in the simulation. These monitoring sites represented existing monitoring locations near the leading edge of the observed seawater intrusion. For the purposes of this study, the sum of chloride concentrations is called "seawater intrusion"; therefore, objective B minimizes seawater intrusion. Objective $\mathrm{C}_{\mathrm{t}}$ minimizes the sum of any drawdown below the initial hydraulic head in all city production wells during all stress periods (fig. 2). Although the intentions of objectives A and B are straightforward, the intent of objective $\mathrm{C}_{\mathrm{t}}$ could be to control the loss of production and storage capacity and to control seawater intrusion. Objective $\mathrm{C}_{\mathrm{m}}$ ensures that drawdowns are not too large in wells that might not directly affect seawater intrusion, such as those in the Foothill groundwater basin. For objectives $\mathrm{C}_{\mathrm{t}}$ and $\mathrm{C}_{\mathrm{m}}$, weighted average heads were calculated using the percentage of total pumping coming from each layer as the weights.

Four constraints were defined initially in this problem formulation. Constraint 1 was a water-quality constraint. The chloride concentrations in water pumped from city production wells must remain below the water-quality standards for chloride concentrations. In this case, the secondary drinkingwater standard for chloride of 250 milligrams per liter $(\mathrm{mg} / \mathrm{L})$, as defined by the U.S. Environmental Protection Agency, was used (U.S. Environmental Protection Agency, 2017). Concentrations were calculated as a weighted average of concentrations in each model layer penetrated by the well screen using the same weights that were used to calculate the average heads; the constraint must be honored in all layers at all times. Constraint 2 was a well-capacity constraint; a well could not pump at a rate greater than the capacity of its pump (table 1). Constraint 3 maintained a minimum flow from the Alameda, City Hall, Santa Barbara High School (SBHS), Corporation Yard, Ortega, and Vera Cruz wells to the city-operated Ortega treatment plant (fig. 2). Constraint 4 ensured that the water levels (weighted average heads) at each production well were maintained above a safe operating level (table 1).

\section{Optimization Scenarios}

To exploit the information that can be generated by the calibrated simulation model, five optimization scenarios were developed in consultation with city of Santa Barbara personnel. These scenarios allow water managers to evaluate a range of optimal solutions, given various states of the system (water levels and chloride concentrations) and potential climatic conditions. These scenarios are described in table 2 . Three scenarios $(1,2$, and 5$)$ consisted of a multiobjective formulation to allow for variations in management preferences. The other two scenarios (3 and 4) were designed to examine the results of the optimizations with respect to sustainable groundwater yield and basin response to changes in pumping.

In general, initial conditions (water level or chloride concentration) can greatly affect the optimal solutions. The solutions are also affected by the amount of precipitation that recharges the basin, and this input is highly uncertain; therefore, a range of possible climatic conditions was evaluated. In this study, precipitation was used as a surrogate for climate; for example, the surrogate for a dry climatic condition was low precipitation. Additionally, because optimizing exact pumping rates for every well in every month for 10 years creates a problem so large it is nearly unsolvable in a realistic timeframe, aggregation techniques were used to reduce the number of management actions to be optimized (decision variables). For this study, monthly stress periods were grouped into 3-month quarters (January-March, AprilJune, and so on) to simplify the problem.

The five optimization scenarios were characterized by their problem formulation (objectives, constraints, and decision variables), initial conditions, management horizon, and climatic conditions. The details of the five scenarios are summarized in table 2. 


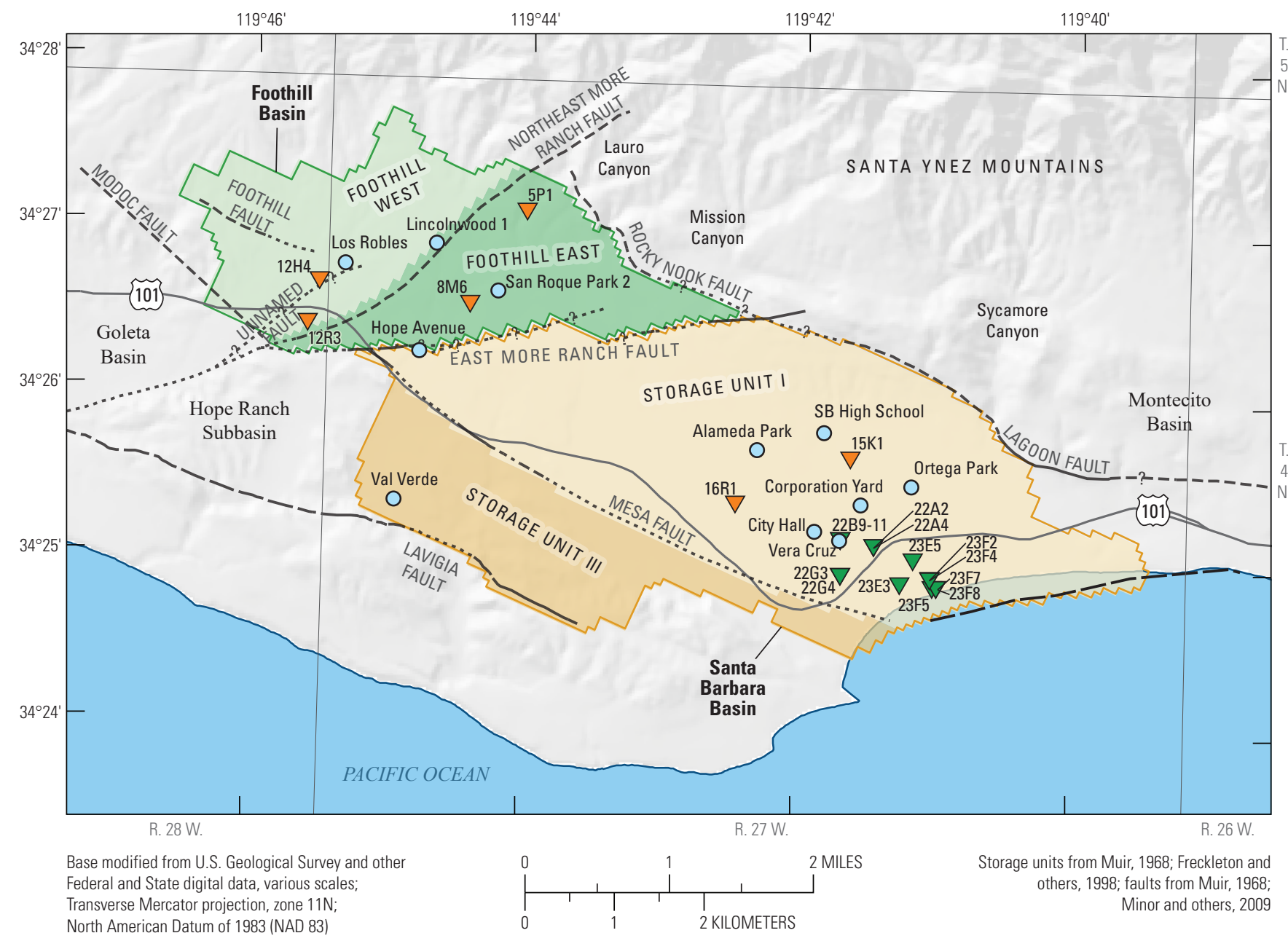

EXPLANATION
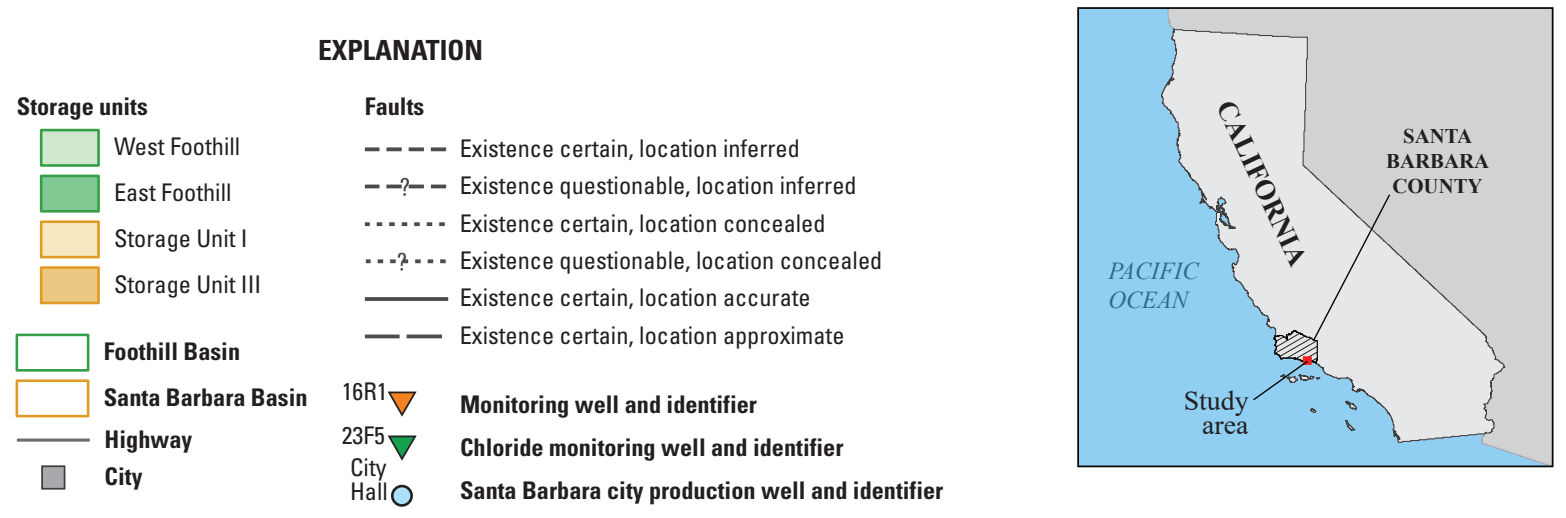

Figure 2. Locations of selected production wells, monitoring wells, and chloride monitoring wells, Santa Barbara and Foothill groundwater basins, Santa Barbara, California. 
Table 1. Maximum pumping capacities for city of Santa Barbara production wells, Santa Barbara, California.

[cfd, cubic feet per day; ft, feet; gpm, gallons per minute; m, meters]

\begin{tabular}{|c|c|c|c|c|c|c|c|c|c|}
\hline & & & & & & & & & \\
\hline & & & Minimun & Minimum & & & & Minimum & linimum \\
\hline Well name & GPM & CFD & $\begin{array}{c}\text { head } \\
\text { (m) }\end{array}$ & $\begin{array}{c}\text { head } \\
\text { (ft) }\end{array}$ & Well name & GPM & CFD & $\begin{array}{c}\text { head } \\
(\mathrm{m})\end{array}$ & $\begin{array}{l}\text { head } \\
\text { (ft) }\end{array}$ \\
\hline Alameda Park & 9,507 & $1,830,225$ & -33.5 & -110 & Ortega Park & 11,175 & $2,151,337$ & -63.4 & -208 \\
\hline City Hall & 10,695 & $2,058,930$ & -73.2 & -240 & San Roque Park (2) & 16,762 & $3,226,909$ & -17.4 & -57 \\
\hline Corporation Yard & 8,558 & $1,647,529$ & -39.3 & -129 & Santa Barbara High School & 9,152 & $1,761,882$ & -42.7 & -140 \\
\hline Hope Avenue & 5,767 & $1,110,225$ & -0.9 & -3 & Val Verde & 10,902 & $2,098,781$ & -27.4 & -90 \\
\hline Lincolnwood 1 & 10,902 & $2,098,781$ & 25.0 & 82 & Vera Cruz & 15,214 & $2,928,898$ & -74.7 & -245 \\
\hline Los Robles & 5,887 & $1,133,326$ & -9.1 & -30 & & & & & \\
\hline
\end{tabular}

Table 2. A summary of the optimization scenarios, Santa Barbara multi-objective management model, Santa Barbara, California.

[The total number of decision variables is included as a gauge for problem size. Abbreviation: N/A, not applicable]

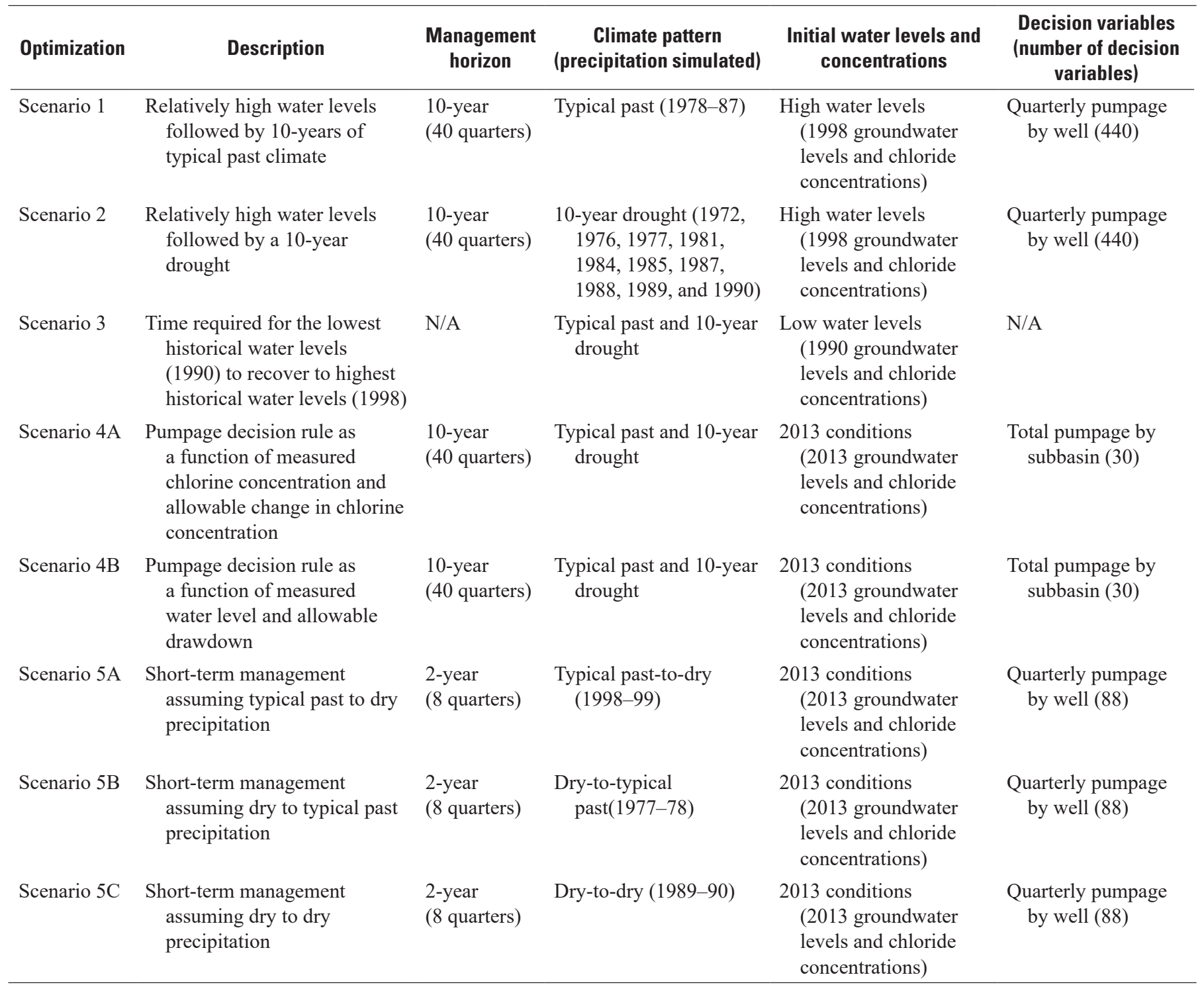




\section{Scenario 1}

Scenario 1 identifies Pareto-optimal pumping schedules based on the assumptions of relatively high initial groundwater levels, followed by 10 years of typical climatic conditions. To represent high initial groundwater levels, those from 1998 (fig. 3), which corresponded to the end of a 6-year period of low pumpage (see fig. A-8), were chosen. Therefore, simulated hydraulic heads and chloride concentrations from 1998 in the calibrated model were used as initial conditions. The scenario used a 10-year management horizon characterized by typical precipitation (1978-87; fig. A-2). The optimization formulation was the same as the general formulation described previously.

\section{Scenario 2}

Scenario 2 identifies optimal pumping schedules based on the assumptions of relatively high initial groundwater levels, followed by a 10 -year drought. As in scenario 1 , relatively high water levels and associated chloride concentrations were used as initial conditions (1998 water levels and chloride concentrations). However, this 10 -year management horizon was characterized by a very dry precipitation record. Figure A-2 shows the annual precipitation in Santa Barbara measured at the Santa Barbara County building and the cumulative departure from the mean. The dry precipitation record used for this scenario was an aggregate of 10 dry years from the calibration period (1972, 1976, 1977, 1981, 1984, 1985, 1987, 1988, 1989, and 1990; fig. A-2) replicated chronologically to simulate an extreme drought during the 10 -year management horizon. For comparison, the average annual precipitation for the simulated drought was $11.4 \mathrm{in}$., and the average annual precipitation for the drought of 2012 16 was 10.2 in. Like scenario 1, the objectives and constraints for this scenario were the same as the general formulation.

\section{Scenario 3}

In contrast to the other scenarios, scenario 3 simulated the time required for the lowest historical water levels (1990) to recover to the highest historical water levels (1998; fig. 3). Hence, the initial conditions were based on 1990 simulated hydraulic heads and chloride concentrations. Most of the groundwater from wells in the Storage Unit I subbasin is treated at the Ortega groundwater treatment plant prior to distribution. For modeling purposes, the minimum operational flowrate to the treatment plant was assumed to be 300 gallons per minute (gal/min), or 484 acre-feet per year (acre-ft/yr; Kelley Dyer, Water Resources Supervisor, Public Works Department, Santa Barbara, Calif., written commun., 2014). Typical and dry climatic conditions were used over 10 years to assess the sensitivity of basin recovery to precipitation, assuming the minimal pumping from Storage Unit I.

\section{Scenario 4}

Scenario 4 was designed to produce tools that can help water managers make decisions regarding groundwater pumpage in response to newly collected water-level or chloride field data. For this study, these tools will be called "decision rules," which are defined as formulas or principles used to choose a course of action in response to any given input of data.

The resulting decision-rule curves show the optimal pumping rates for a chosen well for a range of water levels and chloride concentrations. The 10-year typical period in scenario 1 and the 10-year drought period in scenario 2 were used for the 10-year management horizon. Two approaches were used to reflect how potential chloride concentration and drawdowns could affect the total optimal pumpage. Each approach can be used to identify a Pareto-optimal solution that reflects specific conditions (measured chloride concentrations or drawdown) and the resulting pumpage that satisfies management goals. Once a decision about total pumpage is made, the pumping schedule by well can be obtained from the optimization results from scenarios 1 or 2 , depending on the climatic condition. Whenever new measurements are taken, optimal pumping schedules can be updated without re-running the simulationoptimization model.

\section{Scenario 4A}

In scenario 4A, the first step was to assume a measured chloride concentration at selected coastal monitoring wells. The next step was to search the Pareto-optimal solutions for simulated chloride concentrations equal to the assumed measured value at each selected well. An optimal total pumpage for Storage Unit I for the following year and associated change in chloride concentration were recorded and plotted for each simulated chloride concentration in the selected well. This rule-curve can be referenced to new chloride measurements to retrieve an optimal pumpage volume.

\section{Scenario 4B}

In scenario 4B, the first step was to store the maximum annual drawdown for each production well in the Foothill groundwater basin. The next step was to store the associated total pumpage for Storage Unit I and Foothill groundwater basin for those 10 years. The total optimal pumpage for Storage Unit I and Foothill groundwater basin was presented for each simulated maximum drawdown value.

\section{Scenario 5}

In scenario 5, a suite of potential, future pumping estimates was evaluated relative to a short-term climatic condition defined by a 2-year precipitation record. The management horizon was reduced to 2 years, and the initial conditions replicated those from the end of the calibration period (2013 water levels and chloride concentrations). 


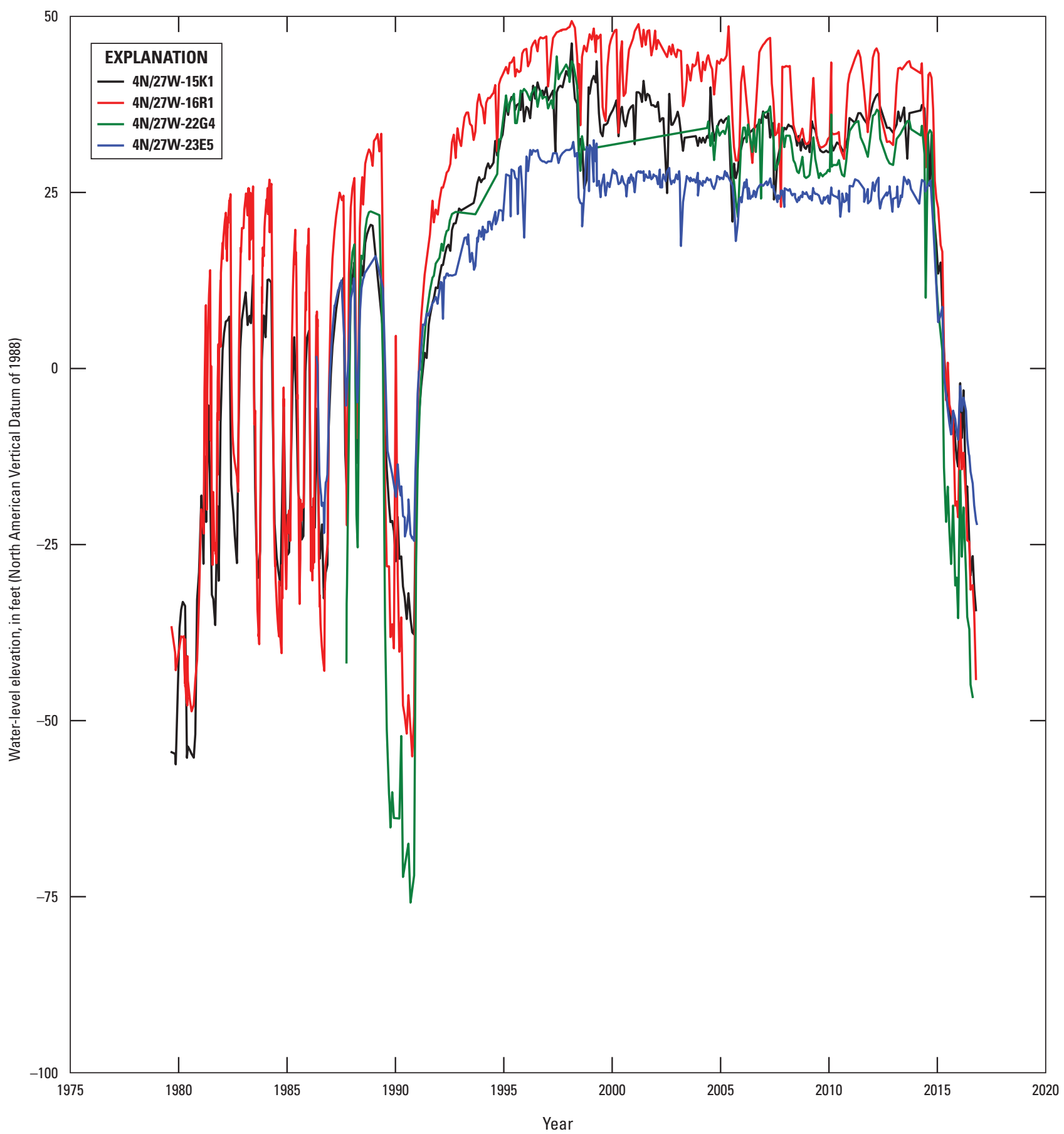

Figure 3. Hydrograph showing measured water levels for wells 4N/27W-15K1, 4N/27W-16R1, 4N/27W-22G4, and 4N/27W-23E5 in Storage Unit I, Santa Barbara groundwater basin, Santa Barbara, California. 


\section{Simulation-Optimization Results}

In order to determine the best possible approximation of the true Pareto-optimal solution set, each scenario was formulated and run until minimum improvement was observed. For more information regarding MOEA convergence criteria, the reader is referred to Ward and others (2015). The $\varepsilon$ values for all scenarios were $\varepsilon_{A}=1,000, \varepsilon_{B}=10, \varepsilon_{C t}=10$, and $\varepsilon_{C m}=1$ for objectives A, $\mathrm{B}, \mathrm{C}_{\mathrm{t}}$, and $\mathrm{C}_{\mathrm{m}}$, respectively. Scenarios 1 and 2 were run for 90,000 function evaluations each (a function evaluation is defined as one forward run of the simulation model), after which, changes in the pumpage objective were less than 1 percent of total pumpage. Scenario 5 optimizations were run for 80,000 function evaluations, or to the point where improvements to all objectives were nearly stagnant.

Results for each scenario are presented and discussed separately. The values of each objective are converted to units that allow for practical interpretation. Objective A, pumpage, is presented as the sum of all quarterly pumping volumes (acre-ft) divided by the simulation time for an average pumping rate in acre-feet per year (acre-ft/yr). Objective B, seawater intrusion, is presented as formulated, that is, the sum of concentrations $(\mathrm{mg} / \mathrm{L})$ in all the model cells that make up the chloride-monitoring well and over all observation times. Objective $\mathrm{C}_{\mathrm{t}}$ is shown as total drawdown in feet; objective $\mathrm{C}_{\mathrm{m}}$ is the maximum drawdown in feet.

Because many Pareto-optimal solutions are generated, a few must be selected for further consideration and discussion purposes. Two strategies were employed to select optimal pumping solutions. First, the $\varepsilon$-dominance criterion was used to sort the solutions according to preference in each objective. This was done in the Borg MOEA with $\varepsilon$ values, defined previously. Second, after filtering by $\varepsilon$ dominance, Paretooptimal pumpage rates by well (schedules) were selected from the remaining Pareto set according to the following convention:

Schedule $\{\mathrm{x}\}_{\mathrm{A}}$ maximize objective A-pumpage

Schedule $\{\mathrm{x}\}_{\mathrm{B}}$ minimize objective B-seawater intrusion

Schedule $\{\mathrm{x}\}_{\mathrm{Ct}}$ minimize objective $\mathrm{C}_{\mathrm{t}}$-total drawdown

Schedule $\{\mathrm{x}\}_{\mathrm{Cm}}$ minimize objective $\mathrm{C}_{\mathrm{m}}$-maximum drawdown

Schedule $\{\mathrm{x}\}_{\mathrm{D}}$ best overall compromise among the four objectives

Schedule $\{\mathrm{x}\}_{\mathrm{E}}$ best compromise among objectives $\mathrm{B}, \mathrm{C}_{\mathrm{t}}$, and $\mathrm{C}_{\mathrm{m}}$ where

$\{\mathrm{x}\} \quad$ is the scenario number.

For example, schedule $1_{\mathrm{A}}$ is the solution that minimized objective A (or maximized pumpage) for scenario 1.

Schedule $\{\mathrm{x}\}_{\mathrm{E}}$ can be considered the best compromise among the environmental mitigation objectives (that is, seawater intrusion and both drawdown objectives).

Selection of the pumping schedules was intended to simplify the presentation of overall Pareto-optimal results. The complete Pareto-optimal solution set is given as hundreds of different pumping schedules for each scenario. Each of these schedules is optimal in the sense that no other solution can improve any of the objective values without worsening at least one other objective value. Selection of the pumping schedule from the solution set can be made according to the needs of the time. For example, given a projected water demand, the Pareto-optimal schedule that most closely matches the demand with pumpage might be chosen. It is important to point out that this schedule might not be one of the example schedules examined in this report. If minimizing seawater intrusion was paramount to all other objectives, however, then schedule $\{x\}_{B}$ pumpage would be a good guide for the available basin yield.

Although identifying schedules $\{\mathrm{x}\}_{\mathrm{A}},\{\mathrm{x}\}_{\mathrm{B}}$, and $\{\mathrm{x}\}_{\mathrm{C}}$ is straightforward - the best performing solution in each of the objectives - all possible pumping schedules need to be ranked to identify schedules $\{x\}_{D}$ and $\{x\}_{E}$. Schedules $\{\mathrm{x}\}_{\mathrm{D}}$ and $\{\mathrm{x}\}_{\mathrm{E}}$ were identified by using scaled objective scores (SOS) for each Pareto-optimal solution. The use of SOS is a normalization that maps each objective value to the common interval $[0,1]$ to more easily compare the ranking of each schedule in each objective. It is calculated as follows:

$$
S O S=\left(1-\left|\frac{o b j-\min }{\max -\min }\right|\right)
$$

where

$$
\begin{aligned}
& \text { obj is the objective-function value of a given } \\
& \text { pumping schedule, } \\
& \min \text { is the minimum value of the objective, } \\
& \max \text { is the maximum value of the objective, and } \\
& \| \text { is the absolute value. }
\end{aligned}
$$

Because all objectives were formulated as minimizations, a score of 1 is the best possible value obtained in the Paretooptimal set for that objective, and 0 is the worst possible value. Using the SOS, schedule $\{\mathrm{x}\}_{\mathrm{D}}$ is defined as the largest sum of scores for all four objectives, and schedule $\{x\}_{\mathrm{E}}$ is the largest sum of scores for objectives $\mathrm{B}, \mathrm{C}_{\mathrm{t}}$, and $\mathrm{C}_{\mathrm{m}}$. 


\section{Scenario 1}

With relatively high-water levels as the initial conditions and typical precipitation used during a 10-year management horizon, this optimization produced the best possible yield for the basin under favorable conditions. The city can choose a pumping schedule according to the tradeoffs among the four objectives that meet the needs at that time. Figure 4 shows the 912 Pareto-optimal solutions for scenario 1, with pumping schedules $1_{\mathrm{A}}$ through $1_{\mathrm{E}}$ highlighted for clarity. Schedules with little seawater intrusion varied between about 26,000 and 24,200 acre-ft of pumpage (fig. 4). The schedules with the least seawater intrusion around schedule $1_{B}$ had more total drawdown and greater maximum drawdown than those that allowed a modest amount of seawater intrusion at a similar total pumpage. The scenario 1 results also indicated that about 31,300 acre-ft was the maximum yield for the basin during a 10 -year period under typical conditions. All scenario 1 results assumed the simulated recharge pattern reasonably represented that of the precipitation record from 1978 to 1987.

The results indicated that the total chloride concentration (objective $\mathrm{B}$ ) and total drawdown (objective $\mathrm{C}_{\mathrm{t}}$ ) were sensitive to total pumpage (objective A) in different ways (fig. 4). One might expect that chloride concentration and drawdown would simply increase with pumpage; however, because the spatial distribution of pumpage varies, this is not always the case. For example, the increased pumpage could be from the Foothill groundwater basin, where drawdown has little effect on seawater intrusion. Schedules $1_{B}$ and $1_{C t}$ both had more pumpage than schedule $1_{\mathrm{Cm}}$, but less seawater intrusion (fig. 4); at the same time, schedule $1_{B}$ had moderate overall drawdown, yet schedules $1_{\mathrm{Ct}}, 1_{\mathrm{Cm}}, 1_{\mathrm{D}}$, and $1_{\mathrm{E}}$ had an overall increase in water levels (negative drawdown). Tradeoffs were complex, and relationships among various objectives were not immediately apparent; however, overall, more pumpage resulted in more drawdown and more seawater intrusion. Schedules with similar total drawdown (for example, $300 \mathrm{ft}$, fig. 4) can result in a wide range of seawater intrusion.

Conversely, schedules with similar pumpage have a relatively narrow range of seawater intrusion.

Table 3 presents the selected Pareto-optimal results for the total pumpage, total chloride concentration, total drawdown, and maximum drawdown for schedules $1_{\mathrm{A}}-1_{\mathrm{E}}$. Schedules $1_{\mathrm{A}}, 1_{\mathrm{B}}, 1_{\mathrm{Ct}}$ and $1_{\mathrm{Cm}}$ resulted in the greatest objective values for objectives $\mathrm{A}, \mathrm{B}, \mathrm{C}_{\mathrm{t}}$ and $\mathrm{C}_{\mathrm{m}}(31,300$ acre-ft, $2.74 \mathrm{E} 7 \mathrm{mg} / \mathrm{L},-790 \mathrm{ft}$, and $41 \mathrm{ft}$, respectively). Schedules $1_{B}$ through $1_{\mathrm{E}}$ resulted in similar total pumpage values - a range of 900 acre-ft after 10 years. The best overall compromise solution, schedule $1_{D}$, represented the largest SOS for the four objectives. In this case, schedule $1_{D}$ resulted in less total pumpage than schedules $1_{\mathrm{A}}, 1_{\mathrm{B}}, 1_{\mathrm{Ct}, \text { or }} 1_{\mathrm{Cm}}$, but more pumpage than schedule $1_{\mathrm{E}}$. Schedule $1_{\mathrm{E}}$ was the best compromise among the environmental mitigation objectives and resulted in the least amount of pumpage (table 3).

Figure 5 presents the SOS for each schedule in table 3, illustrating the relative tradeoffs on a common scale. The extreme solutions (schedules $1_{\mathrm{A}}, 1_{\mathrm{B}}, 1_{\mathrm{Ct}}$ and $1_{\mathrm{Cm}}$ ) all exhibited poor performance in at least one objective, relative to the highest attainable value (a score of 1 ). Schedule $1_{B}$ showed that a small decrease in overall seawater intrusion resulted in a large increase in total drawdown and maximum drawdown when compared to Schedules $1_{\mathrm{C}}$ through $1_{\mathrm{E}}$. The compromise solutions (schedules $1_{\mathrm{D}}$ and $1_{\mathrm{E}}$ ) showed a better balance among the environmental objectives (B and $\mathrm{C}$ ) than the extreme solutions. Schedules $1_{\mathrm{Ct}}$ and $1_{\mathrm{D}}$, appeared to be similar, but showed that for a small decrease in pumpage, better scores for objectives $B$ and $C_{m}$ were attained. Furthermore, schedule $1_{E}$ showed that high SOS (greater than 0.8) for all three environmental objectives was possible if pumpage could be reduced to about 25,000 acre-ft (table 3), but this would also result in lower water levels throughout the basin and a larger maximum drawdown compared to scenario $1_{D}$ (slightly worse objective $\mathrm{C}_{t}$ and $\mathrm{C}_{\mathrm{m}}$ scores; fig. 5).

The selected Pareto-optimal pumping schedules for the Storage Unit I and Foothill groundwater basin are shown in figure 6, and the pumpage values by individual well for Schedule $1_{D}$ are presented in appendix $\mathrm{D}-1$. These pumping schedules present a range of Pareto-optimal operations under typical rainfall. All schedules had relatively high pumpage during the first three quarters of year 10 because there is no year 11 as far as the optimization is concerned, implying that the state of the basin after year 10 is of no concern to the model beyond meeting the constraints. This is discussed in greater detail in the "Limitations" section of this chapter. Also, pumpage was usually large in the first quarter of each schedule, which indicates that the initial conditions were not violating any constraints and the system could be heavily pumped for one quarter, if less pumpage was imposed in the following quarters. It is also apparent from figure 6 that schedule $1_{\mathrm{A}}$ did not necessarily result in more pumpage in each quarter. In fact, in some quarters $(8,12$, and 15 , for example), schedule $1_{\mathrm{A}}$ (fig. $6 A$ ) had less pumpage from Storage Unit 1 than schedule $1_{E}$ (fig. $6 F$ ), and in some other quarters $(21,25$, and 33 , for example) schedule $1_{A}$ had less pumpage from the Foothill groundwater basin than schedule $1_{D}$ (fig. $6 E$ ). Among all the schedules, the general timing of the large pumpage and small pumpage quarters was highly variable.

In summary, the optimal annual pumping for schedule $1_{\mathrm{D}}$ ranged from 1,829 to 3,418 acre- $\mathrm{ft}$ and averaged $2,558 \mathrm{acre}-\mathrm{ft} / \mathrm{yr}$ for the 10 -year period. Of this amount, the annual city pumpage in the Foothill basin ranged from 535 to 862 acre- $\mathrm{ft}$ and averaged 746 acre- $\mathrm{ft} / \mathrm{yr}$; and the annual city pumpage in Storage Unit I ranged from 925 to 2,457 acre-ft and averaged 1,620 acre-ft/yr. Although not presented in figure 6, the annual city pumpage in Storage Unit III ranged from 128 to 239 acre-ft and averaged 191 acre-ft/yr.

Figure 7 shows the contours of simulated hydraulic head in the upper, middle, and lower producing zones after 10 years of pumping, assuming schedule $1_{D}$ pumpage. A cone of depression formed around the wells in the three producing zones of Storage Unit I (fig. 7). In addition, there was a depression in the lower producing zone around the Hope Avenue well (fig. 7C), which could reflect the proximity of the well to the East More Ranch fault. An additional cone of depression was evident in the lower producing zone of the Foothill groundwater basin around the Los Robles well (fig. 7C). 


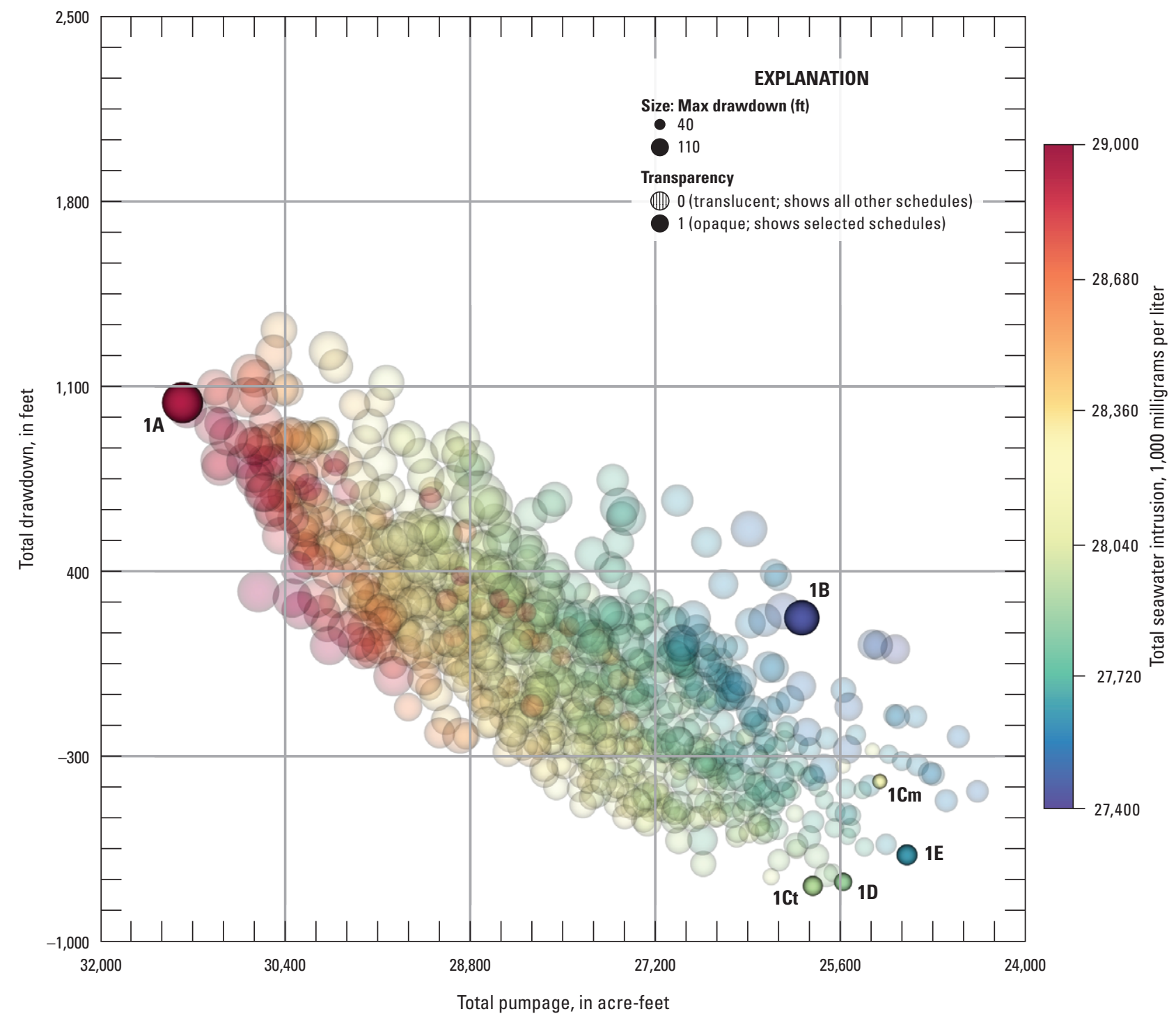

Figure 4. Selected Pareto-optimal solutions for scenario 1, Santa Barbara multi-objective management model, Santa Barbara, California.

Table 3. Values for the four objectives for selected Pareto-optimal pumping schedules, scenario 1, Santa Barbara multi-objective management model, Santa Barbara, California.

[Objective A is also shown divided by storage unit. Abbreviations: acre-ft, acre-feet; ft, feet; mg/L, milligrams per liter]

\begin{tabular}{lccccccr}
\hline Schedule & $\begin{array}{c}\text { Objective A } \\
\text { total pumpage } \\
\text { (acre-ft) }\end{array}$ & $\begin{array}{c}\text { Objective B } \\
\text { seawater intrusion } \\
(\mathbf{1 , 0 0 0} \mathbf{~ m g / L )}\end{array}$ & $\begin{array}{c}\text { Objective Ct } \\
\text { total drawdown } \\
\text { (ft) }\end{array}$ & $\begin{array}{c}\text { Objective Cm } \\
\text { maximum drawdown } \\
\text { (ft) }\end{array}$ & $\begin{array}{c}\text { Storage Unit I } \\
\text { pumpage } \\
\text { (acre-ft) }\end{array}$ & $\begin{array}{c}\text { Foothill } \\
\text { pumpage } \\
\text { (acre-ft) }\end{array}$ & $\begin{array}{c}\text { Storage Unit III } \\
\text { pumpage } \\
\text { (acre-ft) }\end{array}$ \\
\hline $1_{\mathrm{A}}$ & 31,300 & 28,900 & 1,040 & 74 & 20,100 & 9,200 & 2,000 \\
$1_{\mathrm{B}}$ & 25,900 & 27,400 & 230 & 67 & 15,100 & 8,800 & 2,100 \\
$1_{\mathrm{Ct}}$ & 25,800 & 27,900 & -790 & 48 & 16,400 & 7,600 & 1,900 \\
$1_{\mathrm{Cm}}$ & 25,300 & 28,200 & -390 & 41 & 16,300 & 7,300 & 1,600 \\
$1_{\mathrm{D}}$ & 25,600 & 27,900 & -770 & 46 & 16,200 & 7,500 & 1,900 \\
$1_{\mathrm{E}}$ & 25,000 & 27,600 & -670 & 49 & 15,500 & 7,600 & 1,900 \\
\hline
\end{tabular}




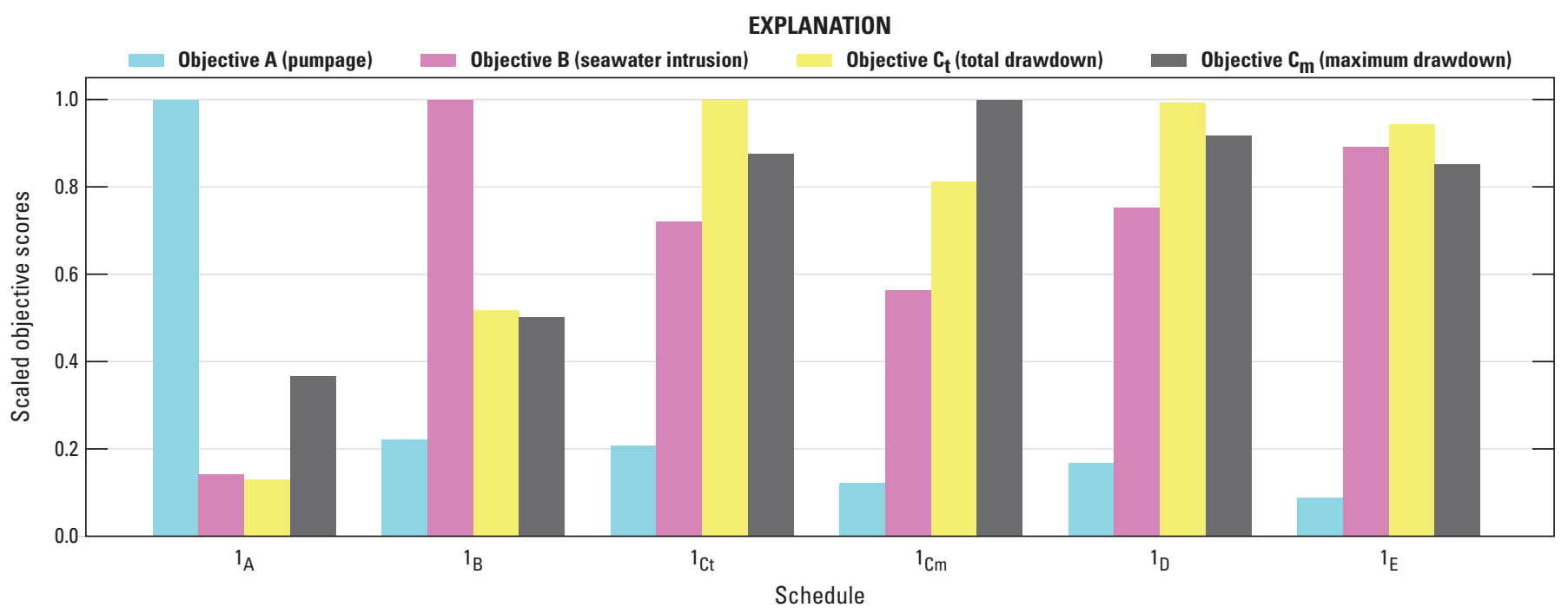

Figure 5. Scaled objective scores for schedules $1_{A}$ through $1_{E^{\prime}}$ Santa Barbara multi-objective management model, Santa Barbara, California.

Figure 8 shows a vertical section of simulated chloride concentration and contours of simulated chloride concentrations for the middle and lower producing zones, assuming schedule $1_{D}$ pumpage. Some of the monitoring wells near the coast that were used to establish the seawaterintrusion objective are also shown in the vertical section. Wells $4 \mathrm{~N} / 27 \mathrm{~W}-22 \mathrm{~A} 2$ and $23 \mathrm{~F} 2$ are perforated in the upper producing zone. Well 22A2 was near the leading edge of seawater intrusion in the upper producing zone, whereas well $23 \mathrm{~F} 2$ is perforated near the top of the middle producing zone (fig. 8A). The mean simulated chloride concentrations at wells $22 \mathrm{~A} 2$ and $23 \mathrm{~F} 2$ at the end of the 10 -year simulation were 130 and $1,900 \mathrm{mg} / \mathrm{L}$, respectively. The simulated results for well 22A2 indicated that pumping from the City Hall or Vera Cruz wells could induce an upward migration of seawater from the top of the middle producing zone (fig. $8 A$ ). Wells $4 \mathrm{~N} / 27 \mathrm{~W}-22 \mathrm{~A} 4$ and $23 \mathrm{~F} 4$ are perforated in the lower producing zone. Well 22A4 was near the leading edge of the seawater intrusion in the lower producing zone, whereas well $23 \mathrm{~F} 4$ was near the centroid of the lower seawater plume (fig. 8) and its simulated chloride concentration was about 70-percent seawater. At the end of the 10-year simulation, the mean simulated chloride concentrations at wells 22A4 and 23F4 were 870 and $13,400 \mathrm{mg} / \mathrm{L}$, respectively.
Simulated chloride breakthrough curves for the coastal monitoring wells, assuming schedule $1_{D}$ pumpage, are shown in figure 9. Higher chloride concentrations in the lower producing zone (wells 23F4 and 23F5) and the middle producing zone (well 23F8) are apparent. Overall, the rate of change in chloride concentration was greater in the lower producing zone than in the other two zones. For example, the rate of increase in chloride concentrations in well 22G4, which is perforated in the lower producing zone, was higher than that of its companion well in the middle producing zone (well 22G3). The largest increase in chloride concentration was in well 23E5, which is perforated in the lower producing zone; the simulated chloride concentration increased from about $2,700 \mathrm{mg} / \mathrm{L}$ to about $4,200 \mathrm{mg} / \mathrm{L}$ (fig. 9).

For Schedule $1_{\mathrm{D}}$, the largest simulated chloride concentration at any production well was about $158 \mathrm{mg} / \mathrm{L}$ in the middle producing zone of the Corporation Yard well. This estimate of concentration is only for a single model layer in the entire perforated interval; therefore, the actual chloride concentration of extracted water would be likely be less because it would be mixed with less-saline water from other depths. 

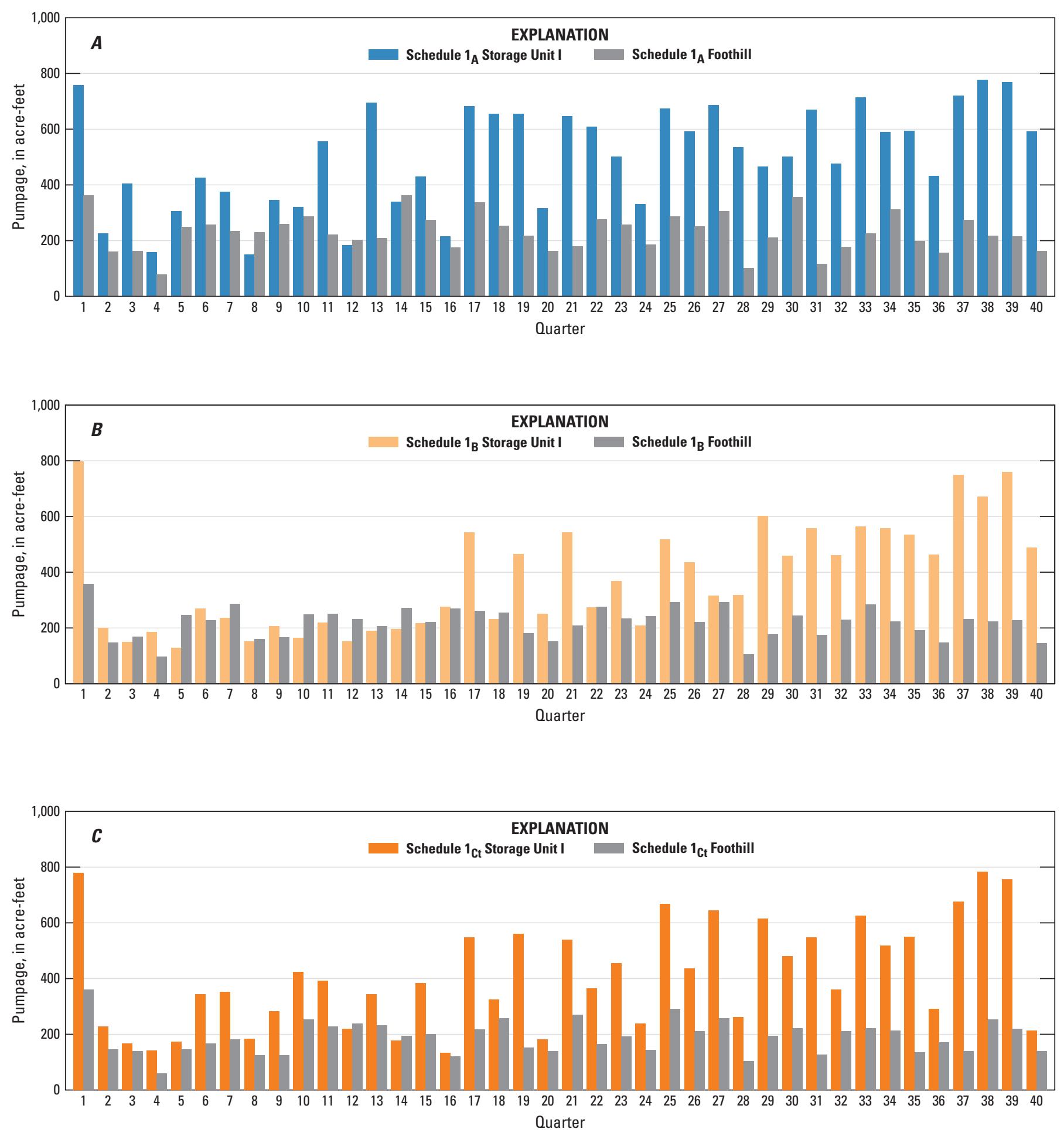

Figure 6. Quarterly pumpage for Storage Unit I and Foothill groundwater basin, Santa Barbara multi-objective management model, Santa Barbara, California, for $A$, schedule $1_{\mathrm{A}^{\prime}} B$, schedule $1_{\mathrm{B}^{\prime}} C$, schedule $1_{\mathrm{Ct}} ; D$, schedule $1_{\mathrm{Cm}^{\prime}} E$, schedule $1_{\mathrm{D}^{\prime}}$, and $F$, schedule $1_{\mathrm{E}^{\prime}}$. 

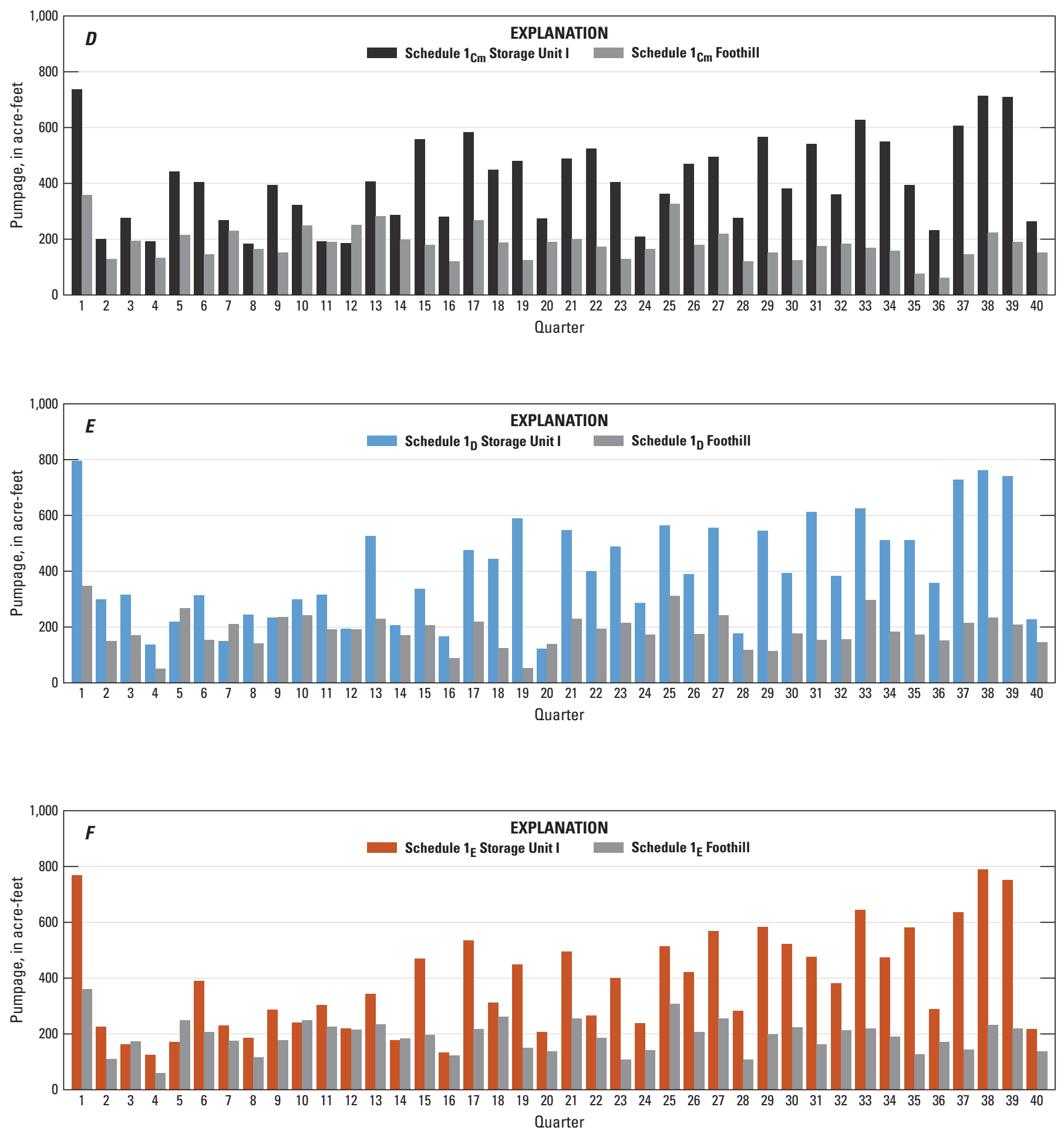

Figure 6. - Continued 


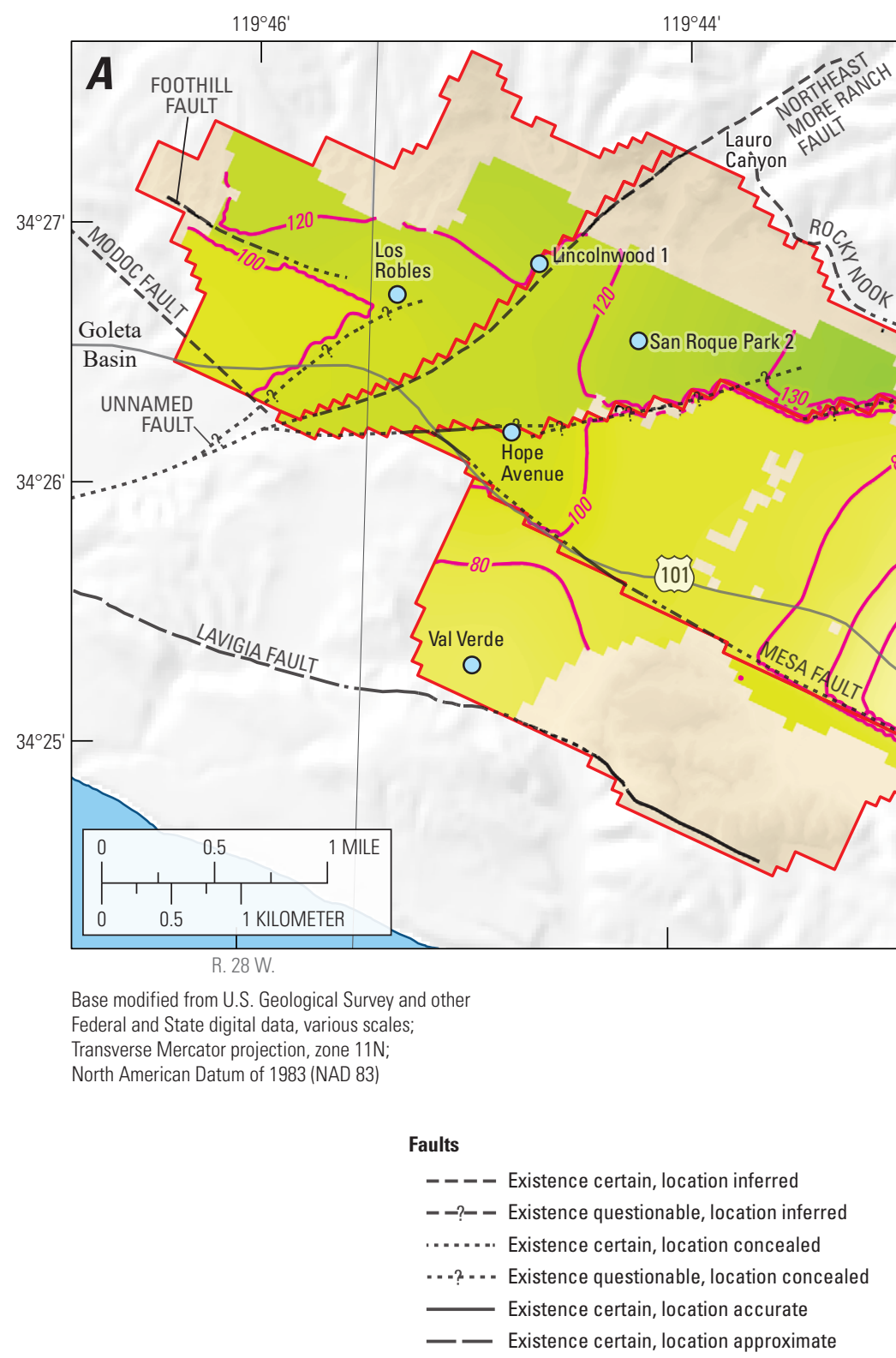

\section{SANTA YNEZ MOUNTAINS}

Mission FAULT
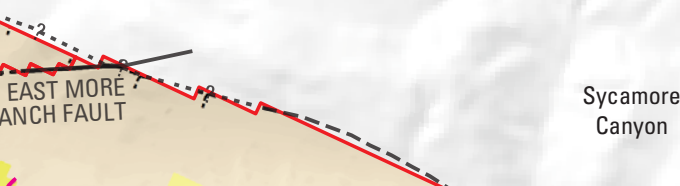

$119^{\circ} 40^{\prime}$

Figure 7. Contours of simulated hydraulic head after 10 years for schedule $1_{D^{\prime}}$ Santa Barbara multi-objective management model, Santa Barbara, California: $A$, upper producing zone; $B$, middle producing zone; and $C$, lower producing zone. 


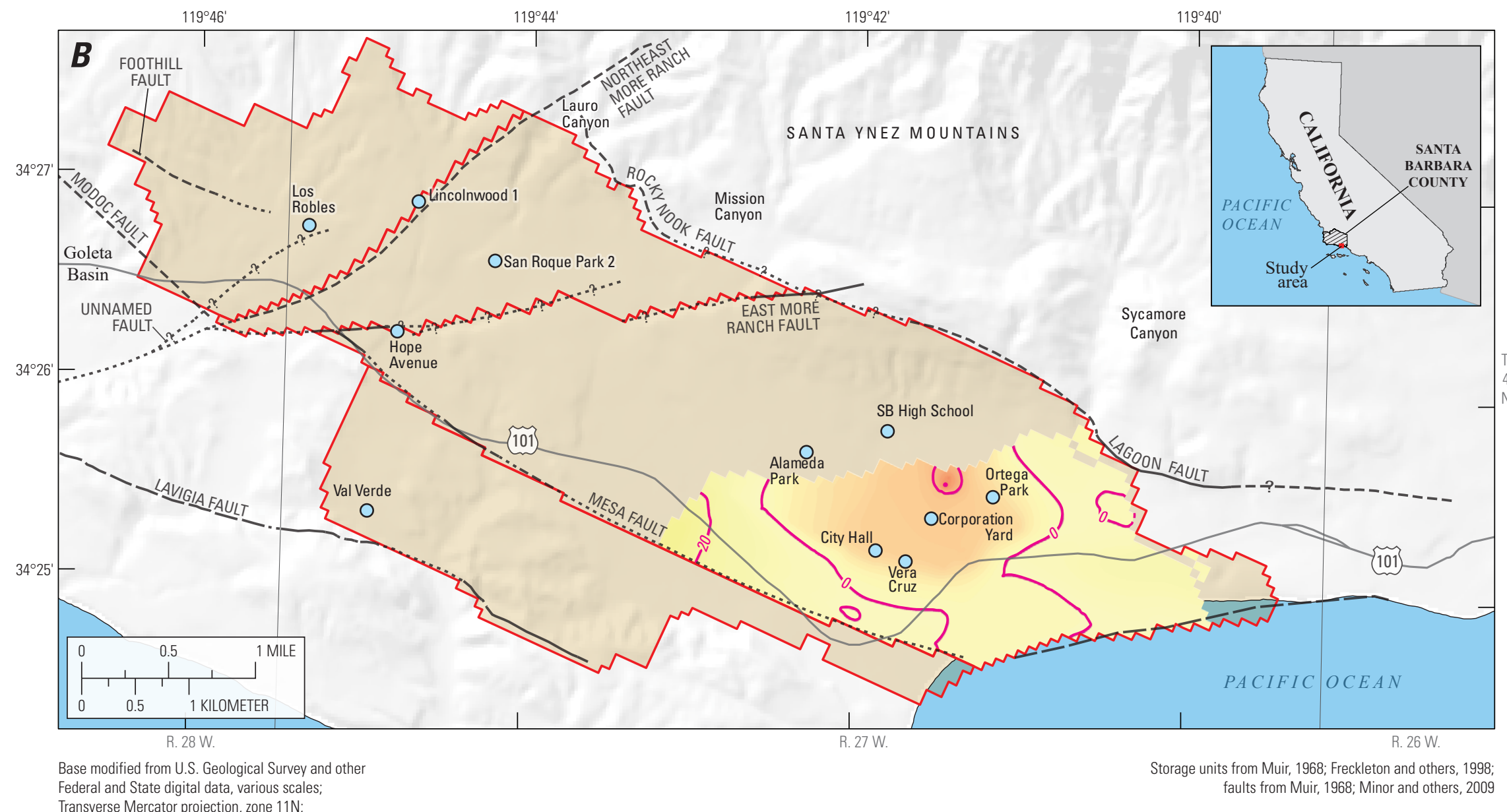

Federal and State digital data, various scales;
Transverse Mercator projection, zone 11N:

North American Datum of 1983 (NAD 83)

\section{Faults}

- - - - Existence certain, location inferred

- -? - Existence questionable, location inferred

........ Existence certain, location concealed

... . .... Existence questionable, location concealed

Existence certain, location accurate

- - Existence certain, location approximate

\section{EXPLANATION}

$\square$ Storage units

-20- Simulated hydraulic head contours, in feet above North American Vertical

Sera Cruz Santa Barbara city production well and identifier

Simulated hydraulic head,
in feet above NAVD 88
High
\begin{tabular}{c|c} 
& 250 \\
& 200 \\
-150 \\
-100 \\
-50 \\
-0 \\
-50 \\
-100 \\
-150
\end{tabular}

Figure 7. - Continued 


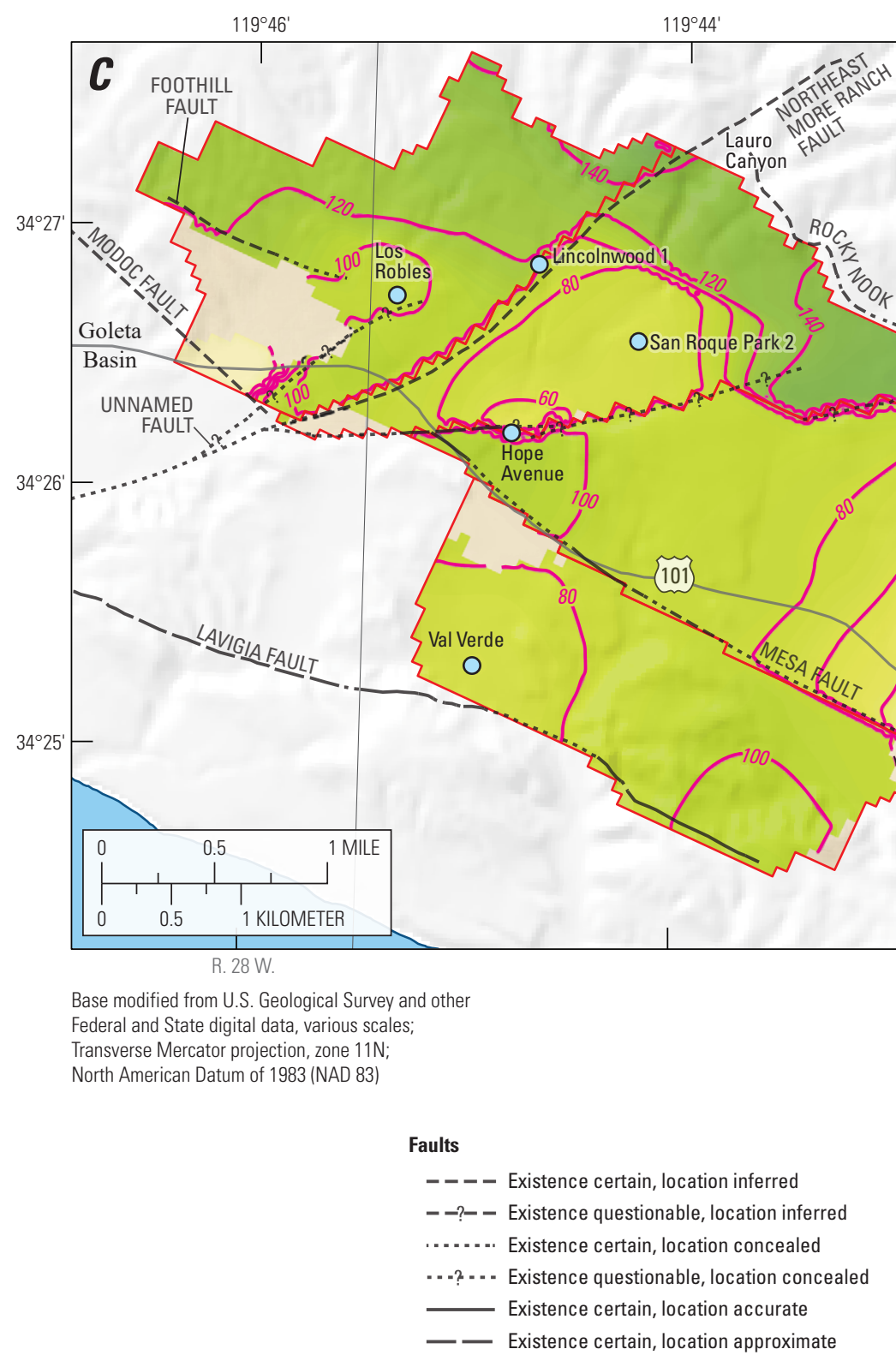

\section{SANTA YNEZ MOUNTAINS}

Mission Canyon EAST MORE

Figure 7. - Continued

\section{EXPLANATION}

Storage units

-20- Simulated hydraulic head contours, in feet above North American Vertical Datum of 1988 (NAVD 88)

Vera Cruz Santa Barbara city production well and identifier

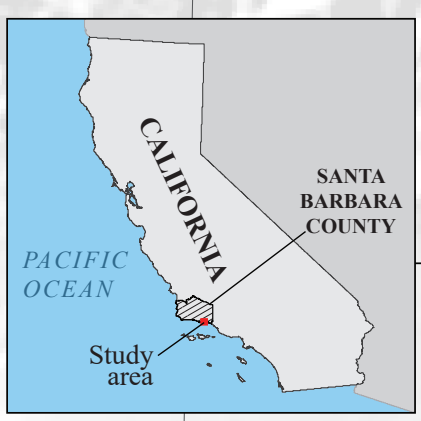

Sycamore Canyon 


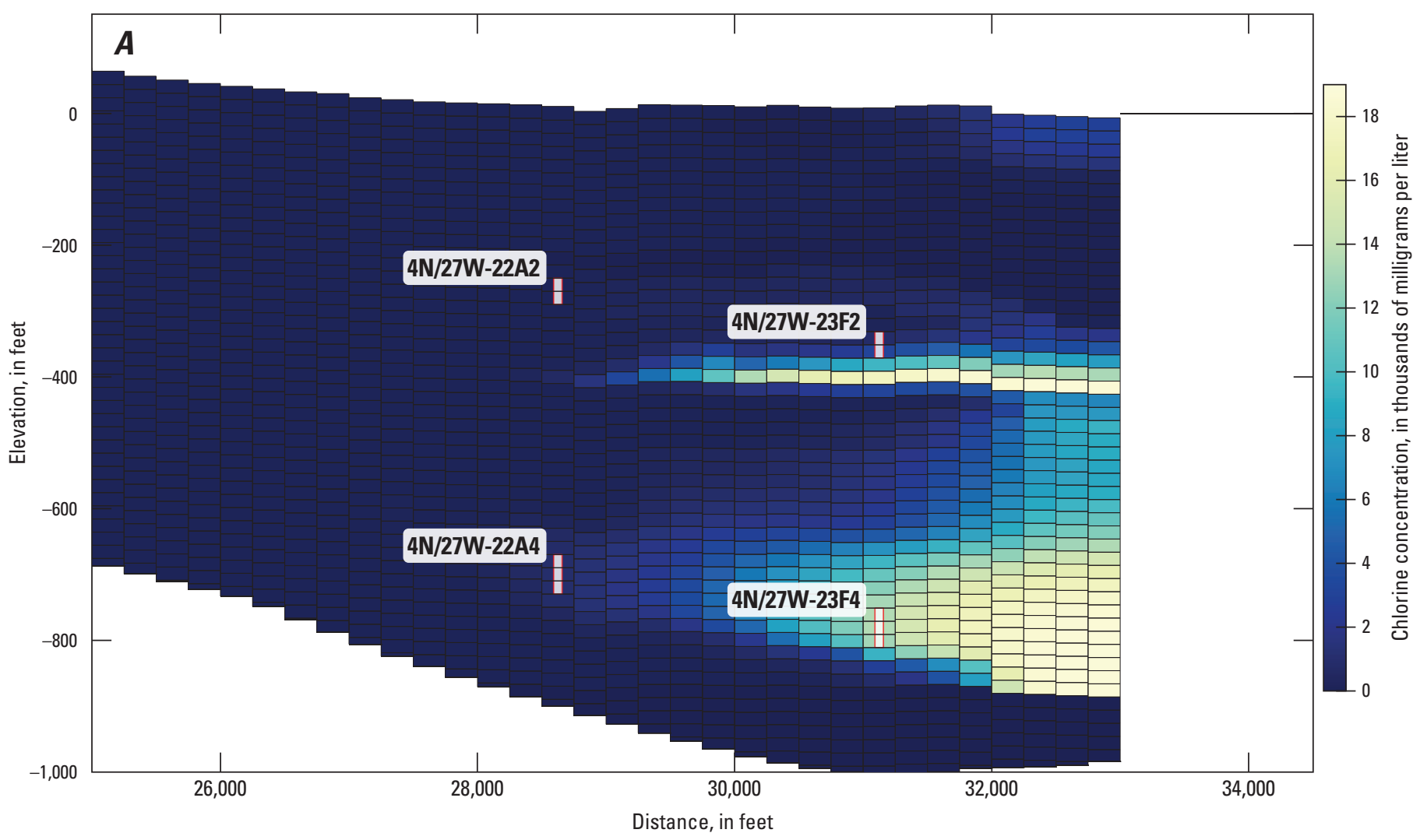

Figure 8. Simulated year-10 chloride concentration distribution for Storage Unit I, schedule $1_{D}$, Santa Barbara multi-objective management model, Santa Barbara, California: $A$, section along model row 30; $B$, plan view of the middle producing zone; and $C$, plan view of the lower producing zone. 

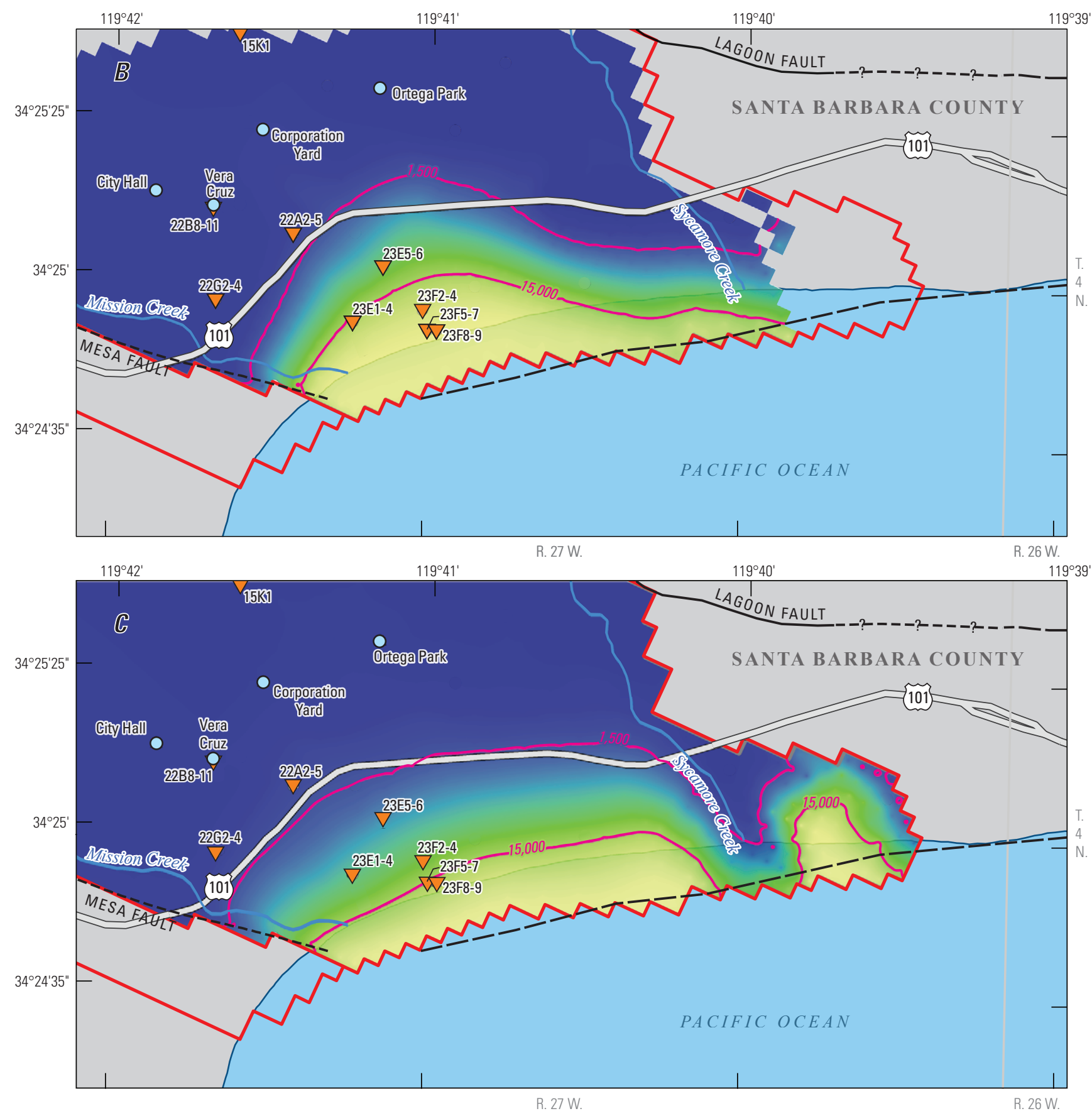

Base modified from U.S. Geological Survey and other Federal and State digital data, various scales; Transverse Mercator projection, zone 11N; North American Datum of 1983 (NAD 83)

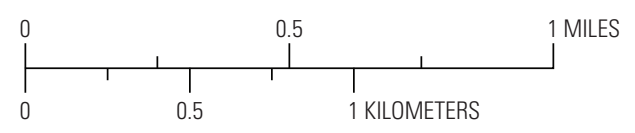

Storage units from Muir, 1968; Freckleton and others, 1998; faults from Muir, 1968 Minor and others, 2009

\section{EXPLANATION}

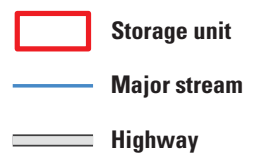

Simulated chloride concentration, in milligrams per liter

- - - Existence certain, location inferred

- -? - Existence questionable, location inferred Existence certain, location accurate _- Existence certain, location approximate

Figure 8. - Continued 

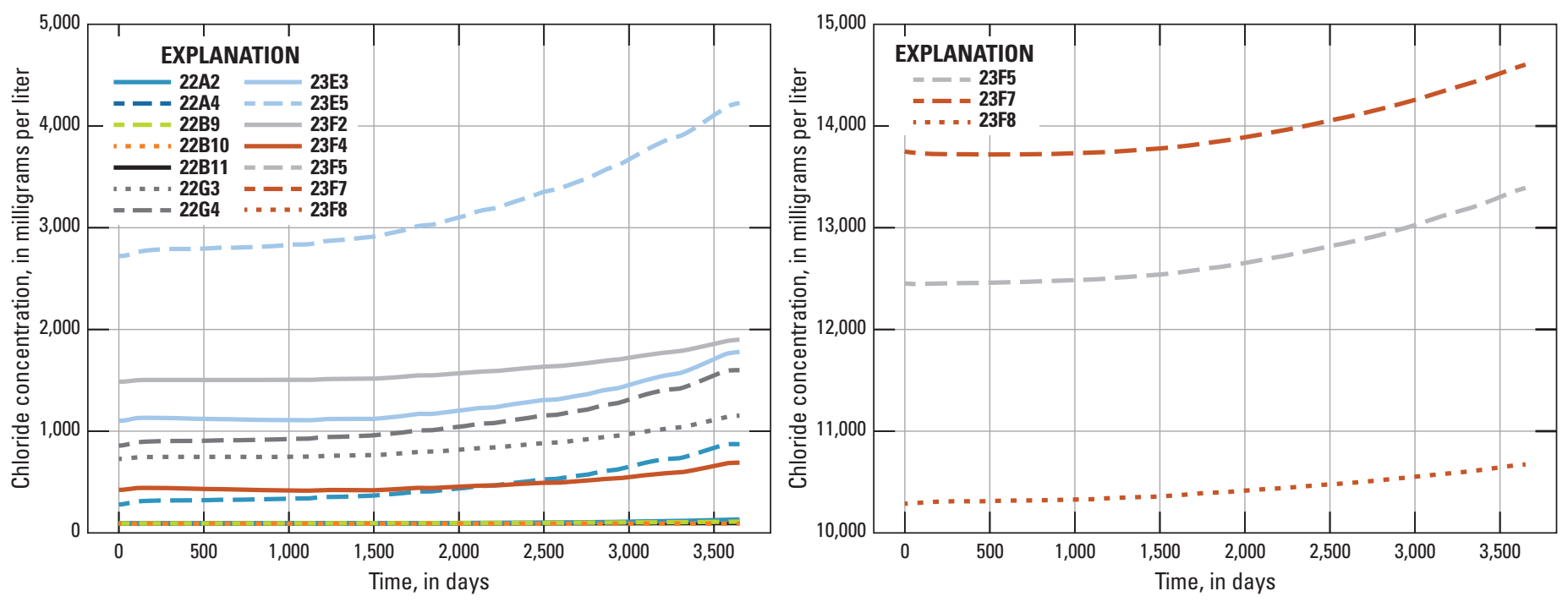

Figure 9. Simulated chloride breakthrough curves for selected monitoring wells for schedule $1_{D^{\prime}}$ Santa Barbara multi-objective management model, Santa Barbara, California.

\section{Scenario 2}

Using relatively high-water levels for the initial conditions and dry climatic conditions during a 10-year management horizon, this optimization scenario produces the best possible yield of the basin under a simulated drought. The city can decide on a pumping schedule by choosing the tradeoff among the four objectives that meet their needs best at the time. Figure 10 shows the 629 Pareto-optimal solutions for scenario 2. A range of low seawater-intrusion values was only feasible for much larger drawdowns when compared to scenario 1 (fig. 4). Schedules $2_{\mathrm{A}}, 2_{\mathrm{B}}, 2_{\mathrm{Ct}}$ and $2_{\mathrm{Cm}}$ represented the extreme solutions that achieved the lowest possible objective value (with respect to a minimization) for objectives $\mathrm{A}, \mathrm{B}, \mathrm{C}_{\mathrm{t}}$, and $\mathrm{C}_{\mathrm{m}}$, respectively (fig. 10). The scenario 2 tradeoffs differed from those of scenario 1 by having a smaller range of feasible values for objectives $\mathrm{A}$ and $\mathrm{B}$, yet a larger range for objectives $\mathrm{C}_{t}$ and $\mathrm{C}_{\mathrm{m}}$. For scenario 2, schedules $2_{\mathrm{Ct}}$, $2_{\mathrm{D}}$, and $2_{\mathrm{E}}$ were equivalent. Also notable is that there were no Pareto-optimal solutions for scenario 2 that resulted in negative total drawdown, as was found for scenario 1 . In general, total pumpage and seawater intrusion were similar for scenarios 1 and 2; however, total drawdowns were larger in all schedules of scenario 2 . All scenario 2 results assumed the simulated recharge pattern reasonably represents that of the dry precipitation record for a simulated 10-year drought.
Selected Pareto-optimal results are presented in table 4, and the associated SOS are shown in figure 11. The total pumpage, total chloride concentration, total drawdown, and maximum drawdown are shown for schedules $2_{\mathrm{A}}-2_{\mathrm{E}}$ in table 4. For scenario 2, the best overall compromise solution (schedule $2_{\mathrm{D}}$ ) and the best compromise between seawater intrusion and both drawdown objectives (schedule $2_{\mathrm{E}}$ ) were identical to those for schedule $2_{\mathrm{Ct}}$ and are, therefore, not presented separately in table 4 . Schedules $2_{\mathrm{A}}, 2_{\mathrm{B}}, 2_{\mathrm{Ct}}$, and $2_{\mathrm{Cm}}$ resulted in the greatest values for objectives $\mathrm{A}, \mathrm{B}, \mathrm{C}_{\mathrm{t}}$, and $\mathrm{C}_{\mathrm{m}}$ $(30,000$ acre-ft, $2.75 \mathrm{E} 7 \mathrm{mg} / \mathrm{L}, 500 \mathrm{ft}$, and $54 \mathrm{ft}$, respectively, table 4). Achieving an SOS greater than 0.8 (fig. 11) for both drawdown objectives was possible for schedule $2_{\mathrm{Ct}}$ at a total pumpage of about 26,200 acre-ft (table 4). Pumpage was greater for schedule $2_{\mathrm{Ct}}$ than $2_{\mathrm{Cm}}$, but total drawdown was less and maximum drawdown was greater.

The tradeoffs for scenario 2 also showed that the lowest values of seawater intrusion were not attainable without a substantial drop in the SOS for the other three objectives (schedule $2_{\mathrm{B}}$ compared to schedules $2_{\mathrm{Ct}}$ and $2_{\mathrm{Cm}}$ in fig. 11). Seawater intrusion increased for schedule $2_{\mathrm{Ct}}$ and $2_{\mathrm{Cm}}$ because minimizing either drawdown objective, much of which occurred in the Foothill groundwater basin, led to more pumpage in Storage Unit I. Similar to scenario 1, the SOS for objective $\mathrm{B}$ was very low for the schedule $2_{\mathrm{A}}$ solution (fig. 11), making it an unlikely candidate for a management plan, but still valuable for its maximal dry-climate yield. 


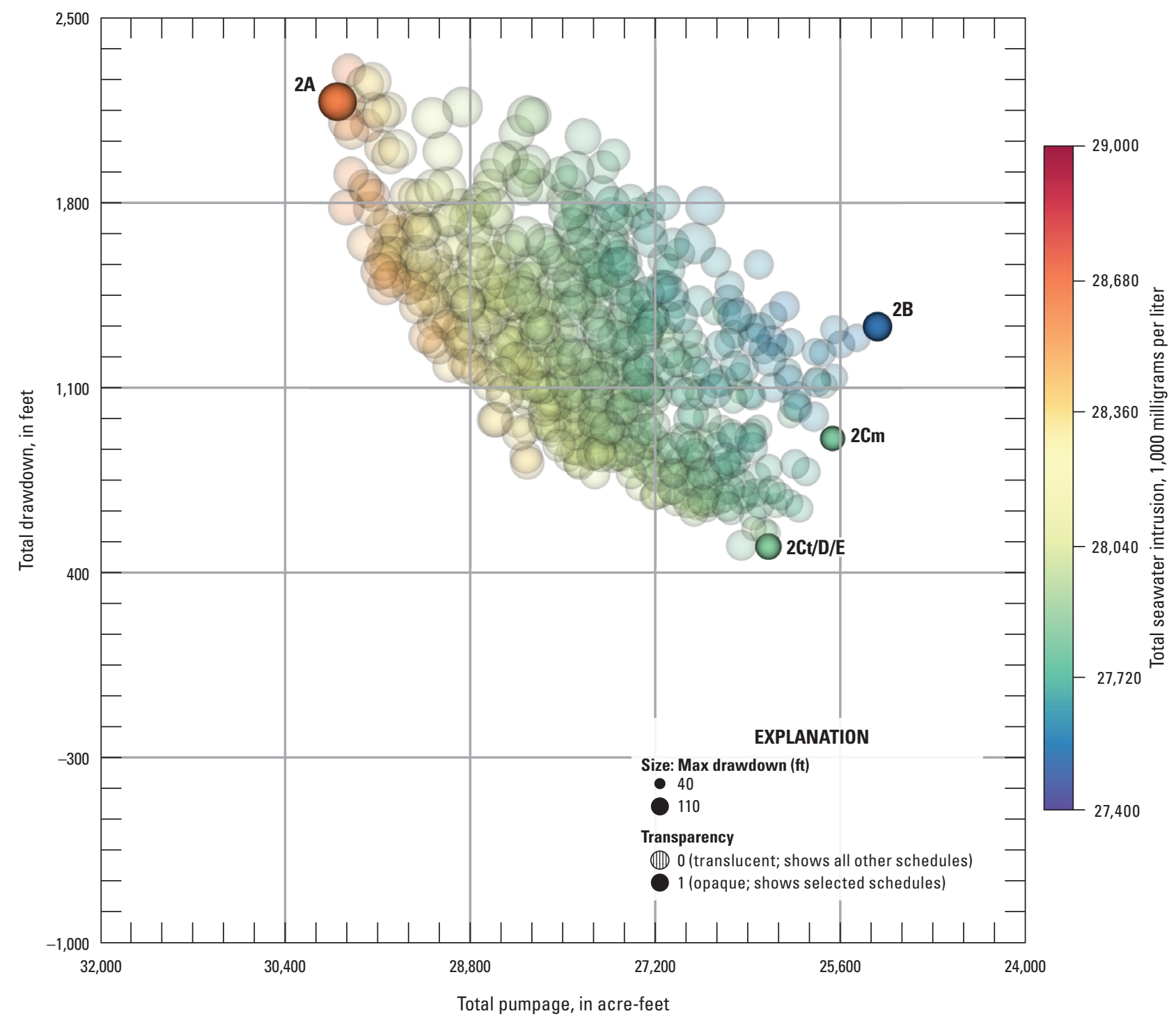

Figure 10. Selected Pareto-optimal solutions for scenario 2, Santa Barbara multi-objective management model, Santa Barbara, California.

Table 4. Values for the four objectives for selected Pareto-optimal pumping schedules, scenario 2, Santa Barbara multi-objective management model, Santa Barbara, California.

[Schedules $2_{\mathrm{D}}$ and $2_{\mathrm{E}}$ results are identical to Schedule $2_{\mathrm{Ct}}$. Objective A is also shown divided by storage unit. Abbreviations: acre-ft, acre-feet; ft, feet; $\mathrm{mg} / \mathrm{L}$, milligrams per liter]

\begin{tabular}{lccccccr}
\hline Schedule & $\begin{array}{c}\text { Objective A } \\
\text { total pumpage } \\
\text { (acre-ft) }\end{array}$ & $\begin{array}{c}\text { Objective B } \\
\text { seawater intrusion } \\
(\mathbf{1 , 0 0 0} \mathbf{~ m g / L )}\end{array}$ & $\begin{array}{c}\text { Objective Ct } \\
\text { total drawdown } \\
\text { (ft) }\end{array}$ & $\begin{array}{c}\text { Objective } \mathbf{C m} \\
\text { maximum drawdown } \\
\text { (ft) }\end{array}$ & $\begin{array}{c}\text { Storage Unit I } \\
\text { pumpage } \\
\text { (acre-ft) }\end{array}$ & $\begin{array}{c}\text { Foothill } \\
\text { pumpage } \\
\text { (acre-ft) }\end{array}$ & $\begin{array}{c}\text { Storage Unit III } \\
\text { pumpage } \\
\text { (acre-ft) }\end{array}$ \\
\hline $2_{\mathrm{A}}$ & 30,000 & 28,600 & 2,180 & 71 & 18,900 & 9,100 & 2,000 \\
$2_{\mathrm{B}}$ & 25,300 & 27,500 & 1,330 & 60 & 14,900 & 8,500 & 1,900 \\
$2_{\mathrm{Ct}, \mathrm{D}, \mathrm{E}}$ & 26,200 & 27,800 & 500 & 56 & 16,100 & 8,100 & 2,000 \\
$2_{\mathrm{Cm}}$ & 25,700 & 27,800 & 910 & 54 & 15,600 & 8,100 & 2,000 \\
\hline
\end{tabular}




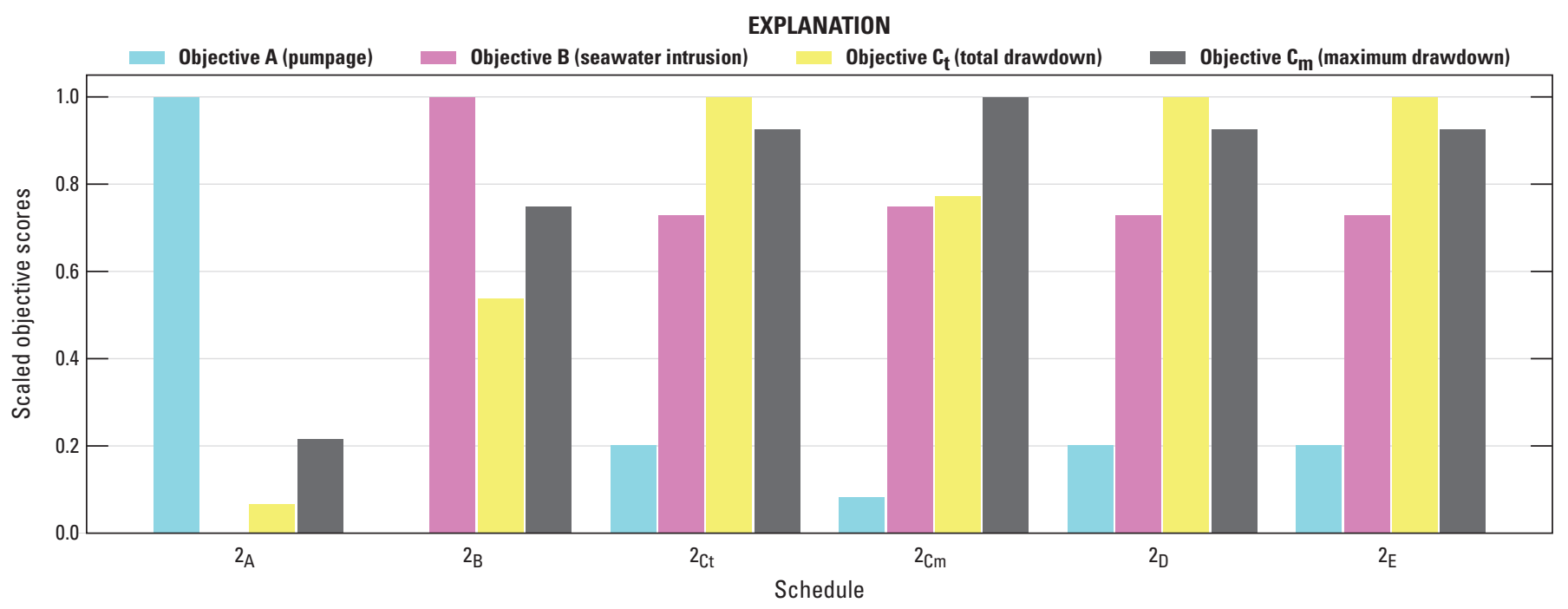

Figure 11. Scaled objective scores for schedules $2_{A^{\prime}}, 2_{B^{\prime}}, 2_{\mathrm{Ct}^{\prime}} 2_{\mathrm{Cm}^{\prime}} 2_{\mathrm{D}^{\prime}}$ and $2_{\mathrm{E}^{\prime}}$, Santa Barbara multi-objective management model, Santa Barbara, California.

The selected Pareto-optimal pumping schedules for the Storage Unit I subbasin and Foothill groundwater basin are shown in figure 12, and the pumpage by individual well for Schedule 2 is presented in appendix D-2. The bars in figure 12 are divided into values of total pumping in Storage Unit I and Foothill groundwater basin. The nuances of these pumping schedules were similar to those of scenario 1 in terms of large initial pumpage in the first quarter, followed by a period of low pumpage, and then increased pumpage in the latter quarters of the 10-year management horizon. With few exceptions, the pumpage for schedule $2_{\mathrm{A}}$ was generally larger in all quarters than for the other schedules. The temporal distribution of recharge was not considered, although it could also dictate which quarters receive more pumpage than others, depending on the assumed climatic conditions. An analysis of annual precipitation and the resulting pumpage, seawater intrusion, and drawdown was included as part of scenario 4 .

In summary, the results for optimal annual pumping for schedule $2_{\mathrm{D}}$ ranged from 1,617 to 3,463 acre-ft and averaged $2,623 \mathrm{acre}-\mathrm{ft} / \mathrm{yr}$ for the 10 -year period. Of this amount, the annual city pumpage from the Foothill groundwater basin ranged from 571 to 962 acre-ft and averaged 813 acre-ft/yr; the annual city pumpage from Storage Unit I ranged from 700 to 2,441 acre-ft and averaged 1,609 acre-ft/yr. Although not presented in figure 12, the annual city pumpage from Storage Unit III ranged from 169 to 251 acre-ft and averaged $201 \mathrm{acre}-\mathrm{ft} / \mathrm{yr}$. Figure 13 shows the contours of simulated hydraulic head for the upper, middle, and lower producing zones after 10 years of pumping for schedule $2_{D}$ (compromise schedule). A cone of depression formed around the Storage Unit I wells in the three producing zones (fig. 13). In addition, a cone of depression developed in the lower producing zone around the Hope Avenue well along the East More Ranch fault (fig. 13C). Additional depressions formed around the Val Verde well in Storage Unit III for the upper (fig. 13A) and lower (fig. 13C) producing zones.
Figure 14 shows a vertical section of chloride concentration and simulated chloride concentrations for the middle and lower producing zones, assuming schedule $2_{\mathrm{D}}$ pumpage. The mean simulated chloride concentrations at wells $22 \mathrm{~A} 2$ and $23 \mathrm{~F} 2$ were 137 and $1,938 \mathrm{mg} / \mathrm{L}$, respectively, at the end of the 10-year simulation. For the lower producing zones, the mean simulated chloride concentrations at wells 22A4 and 23F4 were 917 and 13,405 mg/L, respectively, at the end of the 10-year simulation. In the middle producing zone, the maximum simulated chloride concentration directly below well 22A2 was about $600 \mathrm{mg} / \mathrm{L}$.

Simulated chloride breakthrough curves for the coastal monitoring wells, assuming schedule $2_{\mathrm{D}}$ pumpage, showed trends similar to those for scenario 1 (fig. 15). The chloride concentration increase was still greatest in well 23E5, but the reduced pumpage in this schedule, compared to schedule $1_{D}$, resulted in a higher final concentration $(4,304 \mathrm{mg} / \mathrm{L}$ compared to $4,227 \mathrm{mg} / \mathrm{L}$ ). Similarly, all other wells that showed seawater intrusion increased to a slightly higher concentration compared to schedule $1_{D}$.

The results of scenario 2 showed that optimization of well pumpage identifies management schedules that mitigate seawater intrusion in a drier climatic condition. With less rainfall, there is less available groundwater, and the yield, considering the chloride and drawdown tradeoffs, is lower. Less pumping generally results in less seawater intrusion. Because the complex dynamics among the four objectives depend strongly on climate, choosing an optimal schedule from the Pareto curve that produces the desired pumpage results in a clear tradeoff between drawdown and seawater intrusion. In other words, if demand can be reduced when anticipating a drier climatic condition, an effective pumping schedule is available to meet any feasible drawdown and chloride specification without needing to re-run the model. 

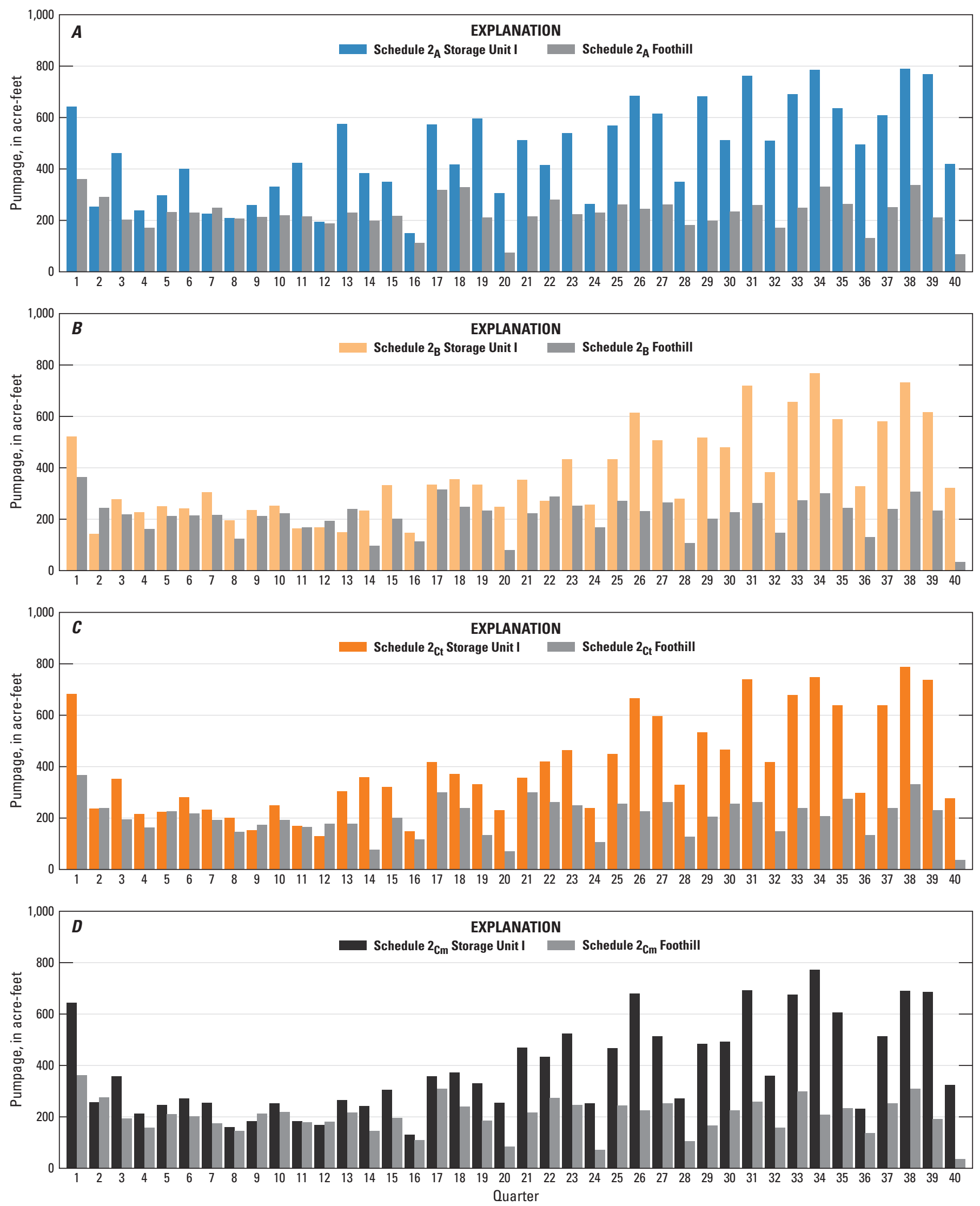

Figure 12. Scenario 2 quarterly pumpage for Storage Unit I and Foothill groundwater basin, Santa Barbara multi-objective management model, Santa Barbara, California, for $A$, schedule $2_{\mathrm{A}^{\prime}} ; B$, schedule $2_{\mathrm{B}^{\prime}} C$, schedules $2_{\mathrm{Ct}^{\prime}} 2_{\mathrm{D}^{\prime}}$, and $2_{\mathrm{E}^{\prime}}$ and $D$, schedule $2_{\mathrm{Cm}}$. 


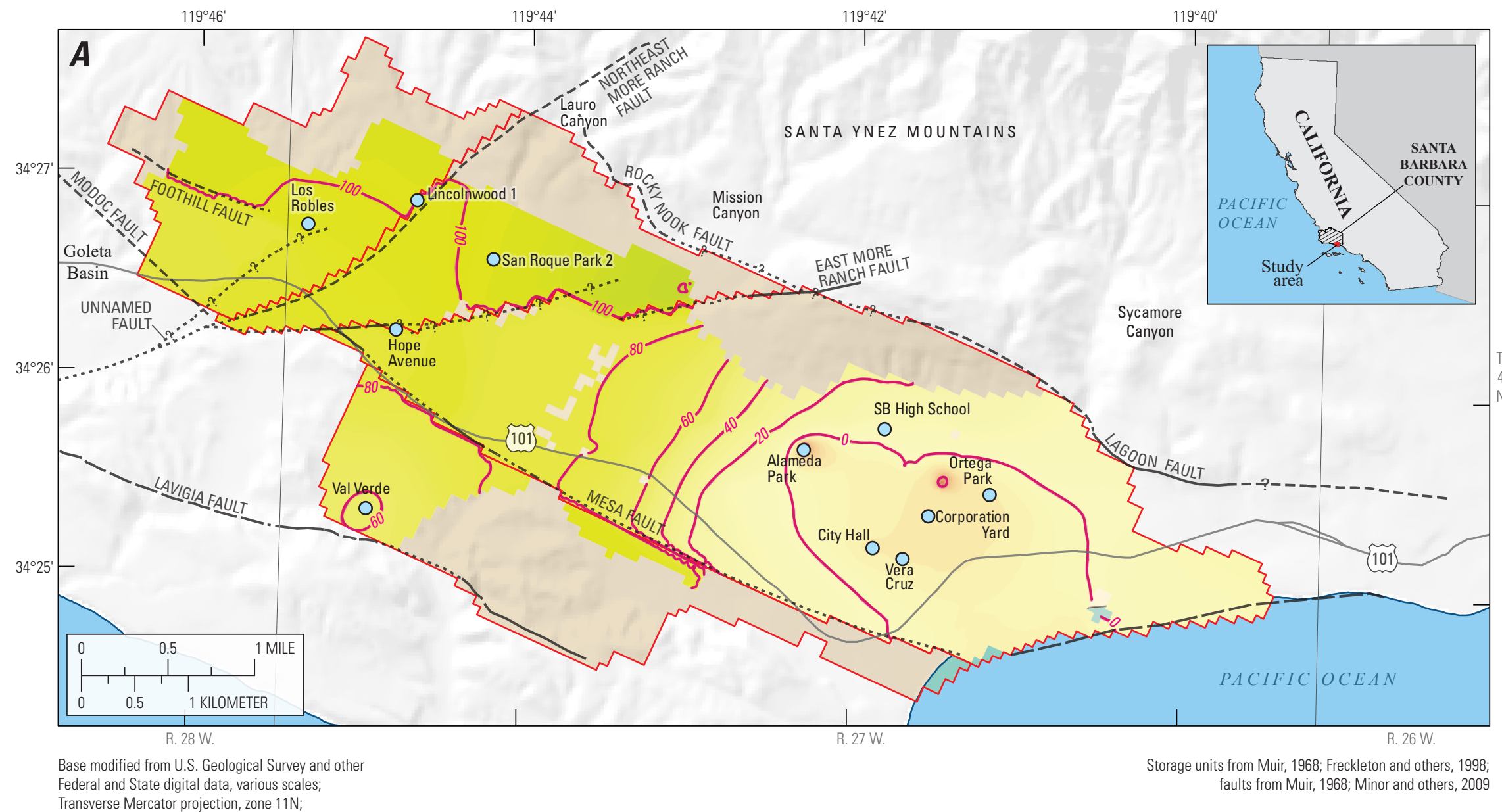

Federal and State digital data, various scales;
Transverse Mercator projection, zone $11 \mathrm{~N}$;

North American Datum of 1983 (NAD 83)

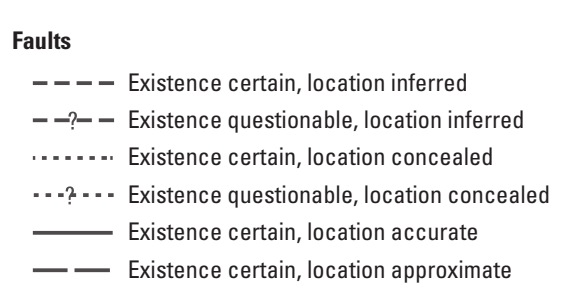

EXPLANATION

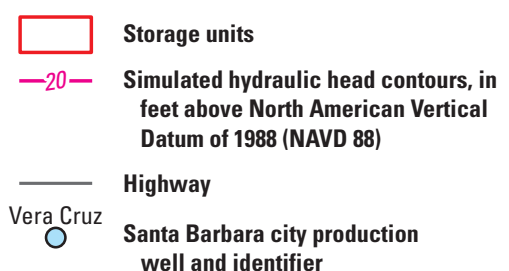

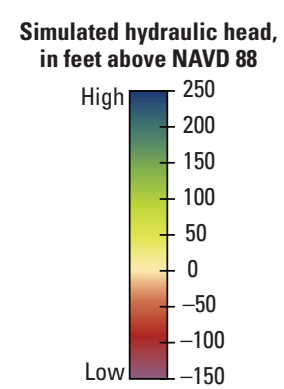

Figure 13. Contours of simulated hydraulic head after 10 years for scenario 2, schedule 2 , Santa Barbara multi-objective management model, Santa Barbara, California: $A$, upper producing zone; $B$, middle producing zone; and $C$, lower producing zone. 


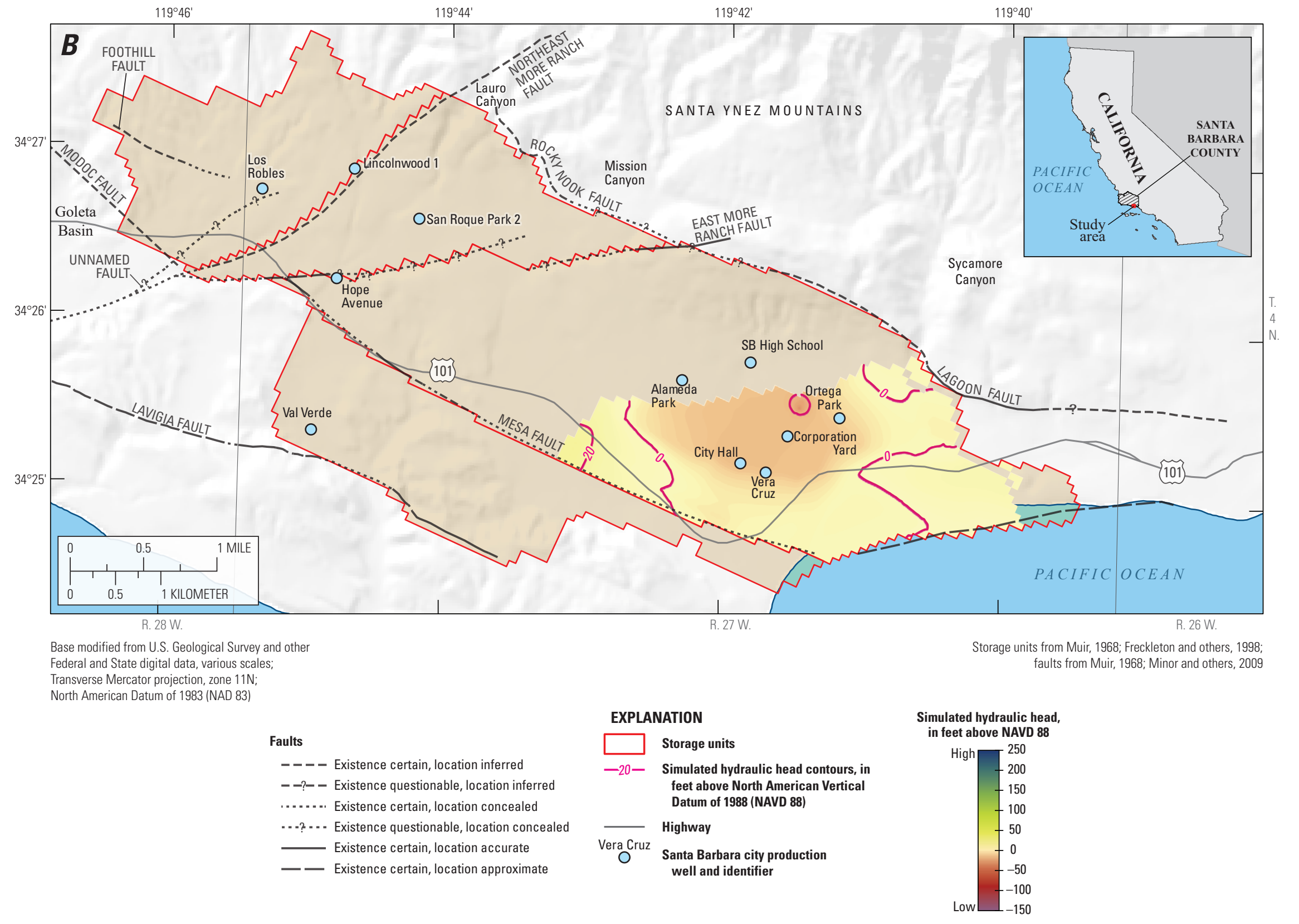

Figure 13. - Continued 


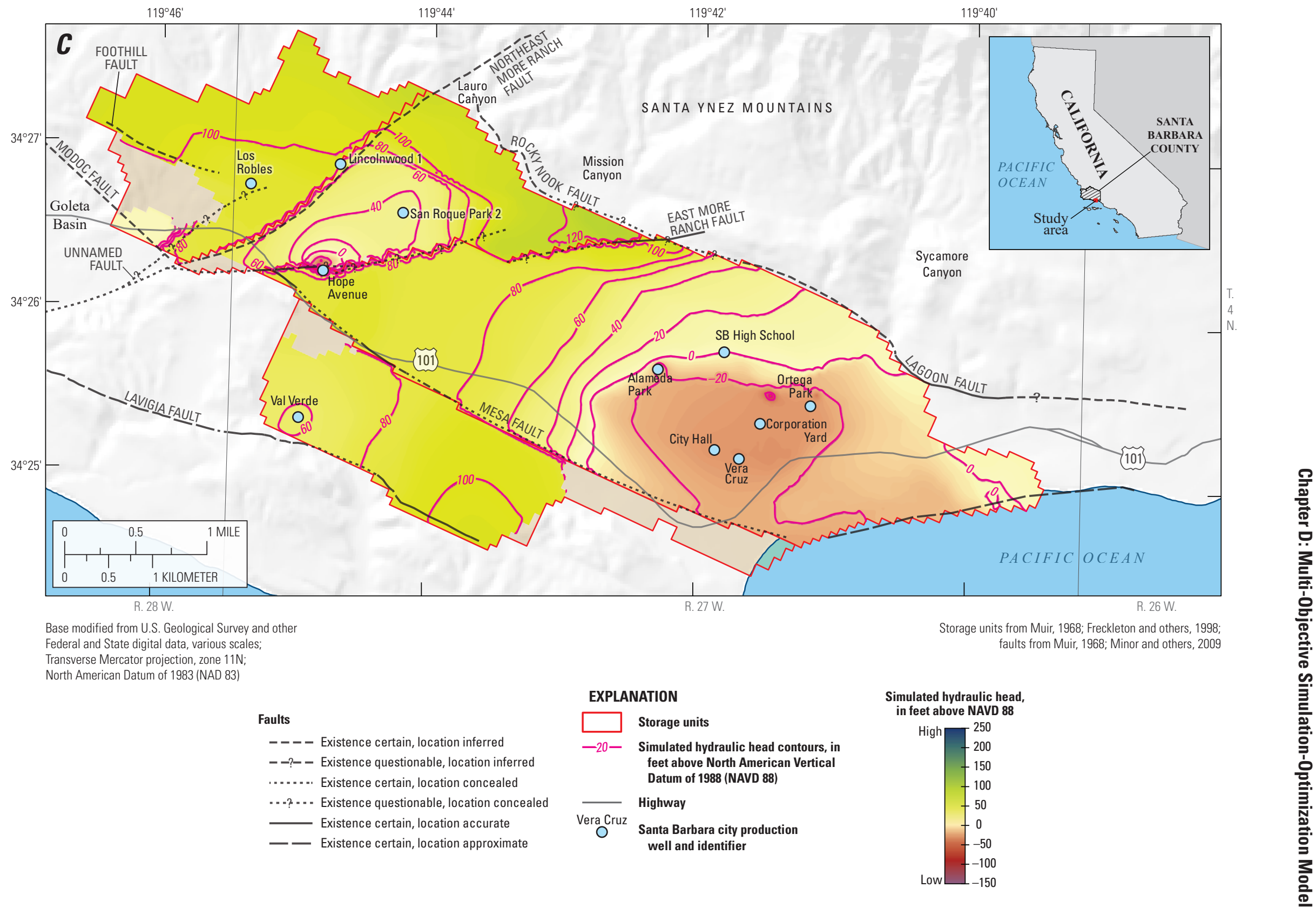

Figure 13. - Continued 


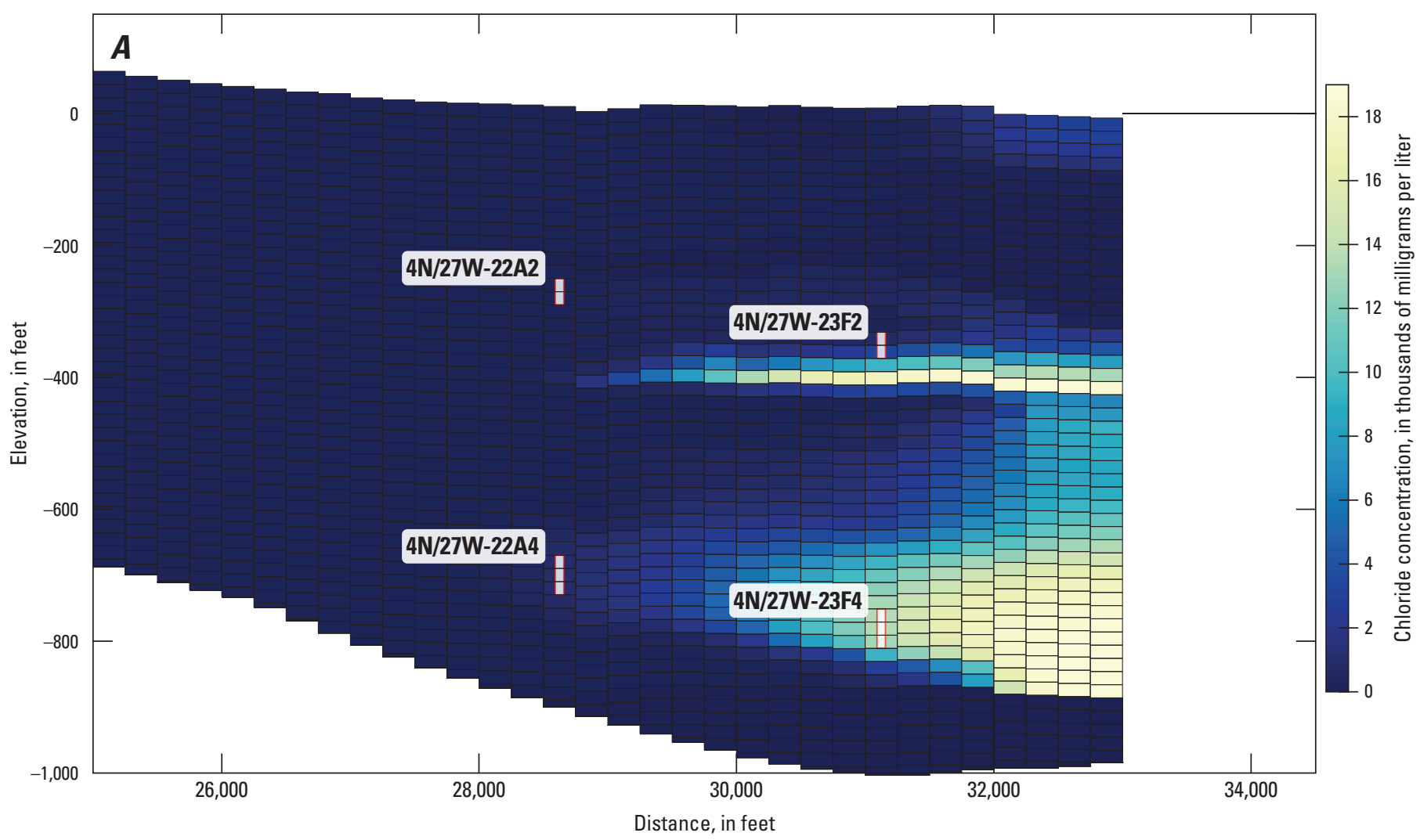

Figure 14. Simulated year-10 Storage Unit I chloride concentration distribution for schedule $2_{D^{\prime}}$ Santa Barbara multi-objective management model, Santa Barbara, California: $A$, section along model row $30 ; B$, plan view of middle producing zone; and $C$, plan view of the lower producing zone. 

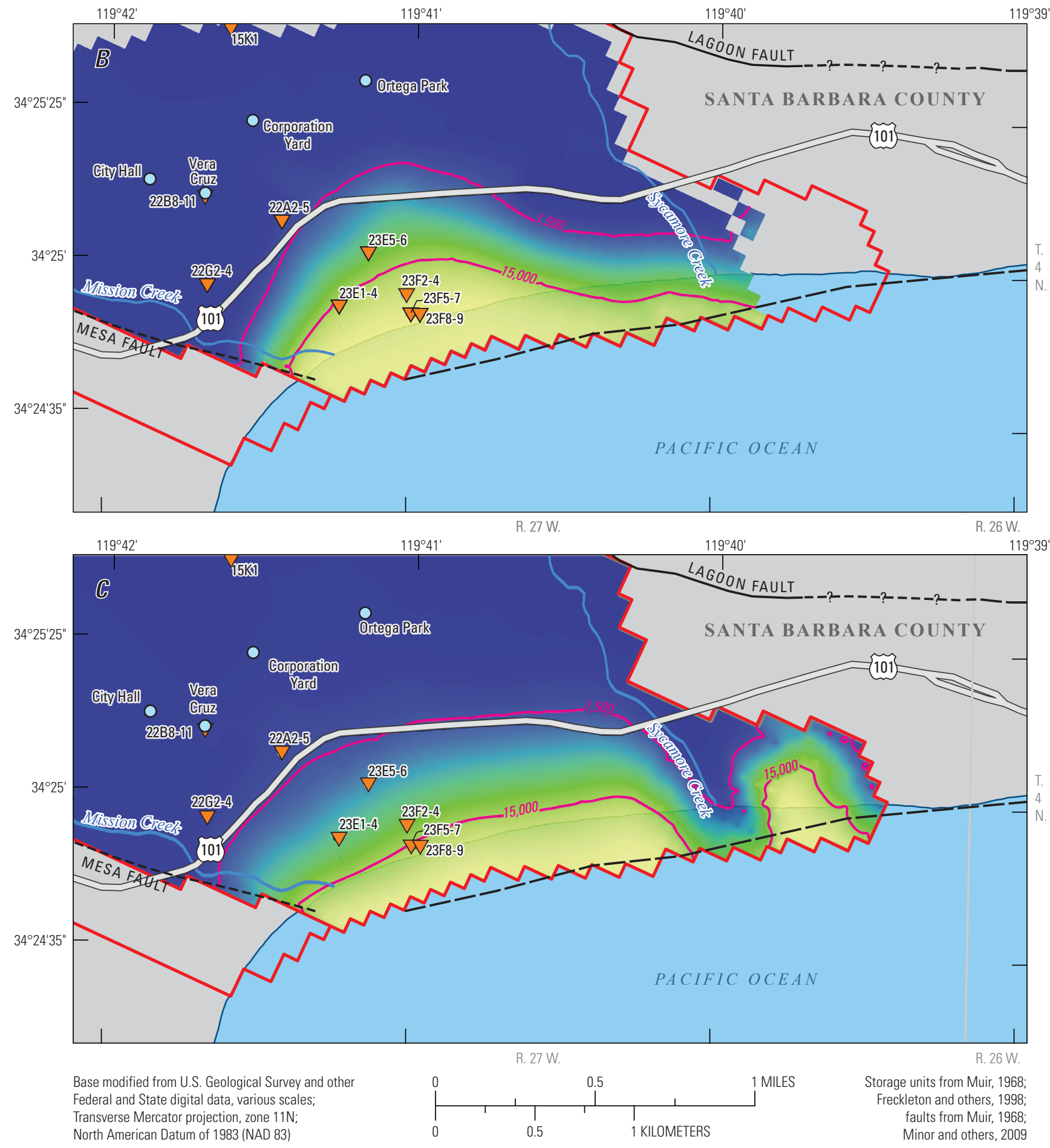

EXPLANATION

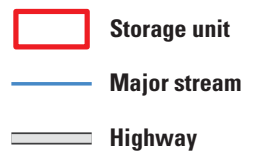

\section{Faults}

- - - Existence certain, location inferred

- -? - Existence questionable, location inferred

Existence certain, location accurate

_- Existence certain, location approximate
Simulated chloride concentration, in milligrams per liter High 19,000 ta Barbara city production well and identifier 

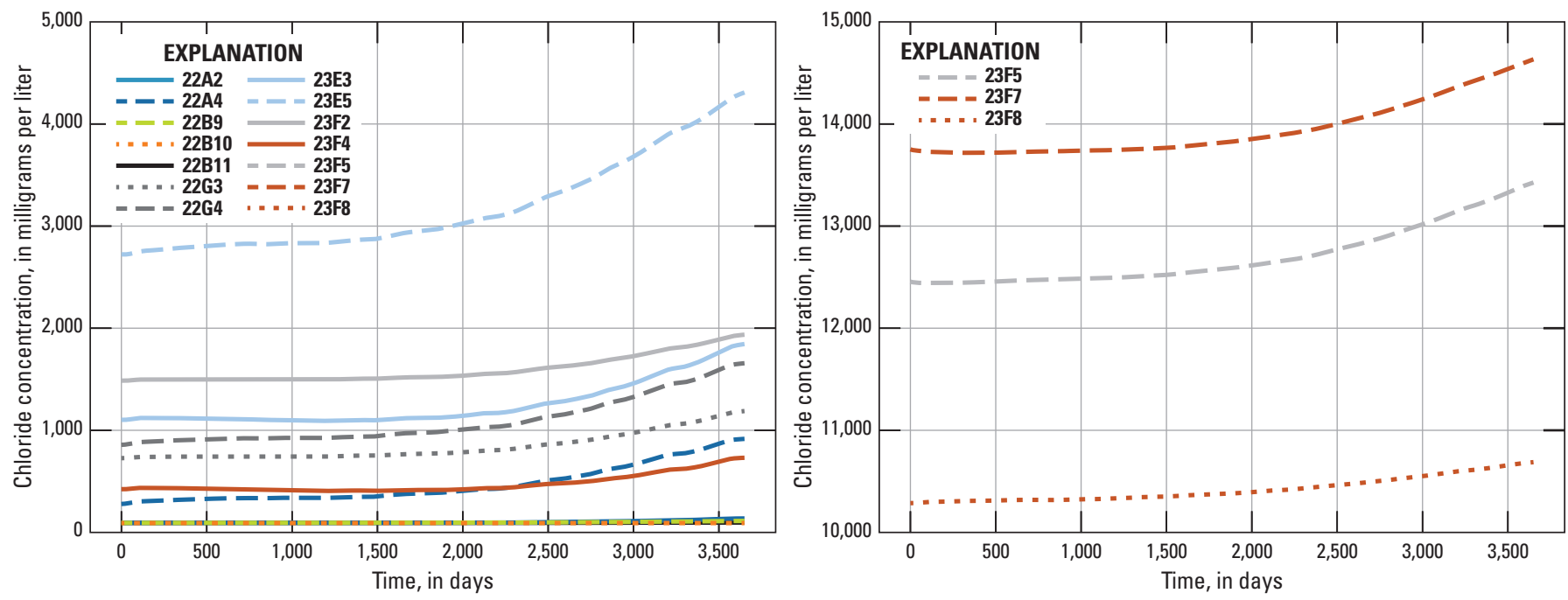

Figure 15. Simulated chloride breakthrough curves for selected monitoring wells for schedule $2_{0}$, Santa Barbara multi-objective management model, Santa Barbara, California.

\section{Scenario 3}

Scenario 3 assumed 484 acre-ft/yr was pumped from the six production wells in Storage Unit I (based on assumed minimum flowrates for Ortega groundwater treatment-plant operations); there was no pumpage from Foothill groundwater basin or Storage Unit III. The pumpage was assigned to each well in Storage Unit I by scaling the quarterly pumpage for schedule $1_{D}$ (typical) and schedule $2_{D}$ (dry), such that the total annual pumpage was 484 acre-ft. See appendix D-3 for the quarterly pumpage by well. This scenario simulated minimal initial water levels (1990), the 10-year typical precipitation used in scenario 1, and the 10-year drought used in scenario 2 .

Figure $16 A-B$ shows drawdown hydrographs with respect to 1998 water levels for four monitoring wells in Storage Unit I (4N/27W-15K1, -16R1, -22G4, and -23E5), assuming typical and dry climatic conditions, respectively. Instead of using initial conditions to calculate drawdown, the 1998 high water level for each well was used as the reference point; therefore, as drawdown approached zero, the simulated hydraulic heads approached 1998 water levels. For both climatic conditions, the simulated hydraulic heads recovered quickly in the first year, after which the hydraulic heads recovered more slowly. For the typical climatic condition, only well 16R1 recovered to 1998 levels after 10 years; for the dry climatic condition, none of the wells recovered to 1998 conditions (as indicated by $0 \mathrm{ft}$ of drawdown in fig. 16). These results indicated that Storage Unit I would not be able to completely recover to 1998 water levels within 10 years under a typical or dry climatic condition while constantly extracting $484 \mathrm{acre}-\mathrm{ft} / \mathrm{yr}$ from Storage Unit I; however, water levels appeared to stabilize as they approached about $0-5 \mathrm{ft}$ below 1998 levels for a typical climatic condition and about 3-8 ft below 1998 levels for a dry climatic condition.

Figure $16 C-D$ shows drawdown hydrographs for four monitoring wells in the Foothill groundwater basin (4N/27W12R3, $-12 \mathrm{H} 4,-5 \mathrm{P} 1$, and $-8 \mathrm{M} 6$ ), assuming typical and dry climatic conditions, respectively. As with the Storage Unit I drawdown hydrographs, zero drawdown equaled the simulated water levels from 1998. For the Foothill groundwater basin, initial water levels from 1990 were not as far below 1998 levels as those for Storage Unit I. With no pumping simulated in the Foothill groundwater basin, there was a smooth recovery to water levels between about 30 and $70 \mathrm{ft}$ above 1998 levels for a typical climatic condition (fig 16C) and to between about 20 and $60 \mathrm{ft}$ above 1998 levels for a dry climatic condition (fig 16D). 

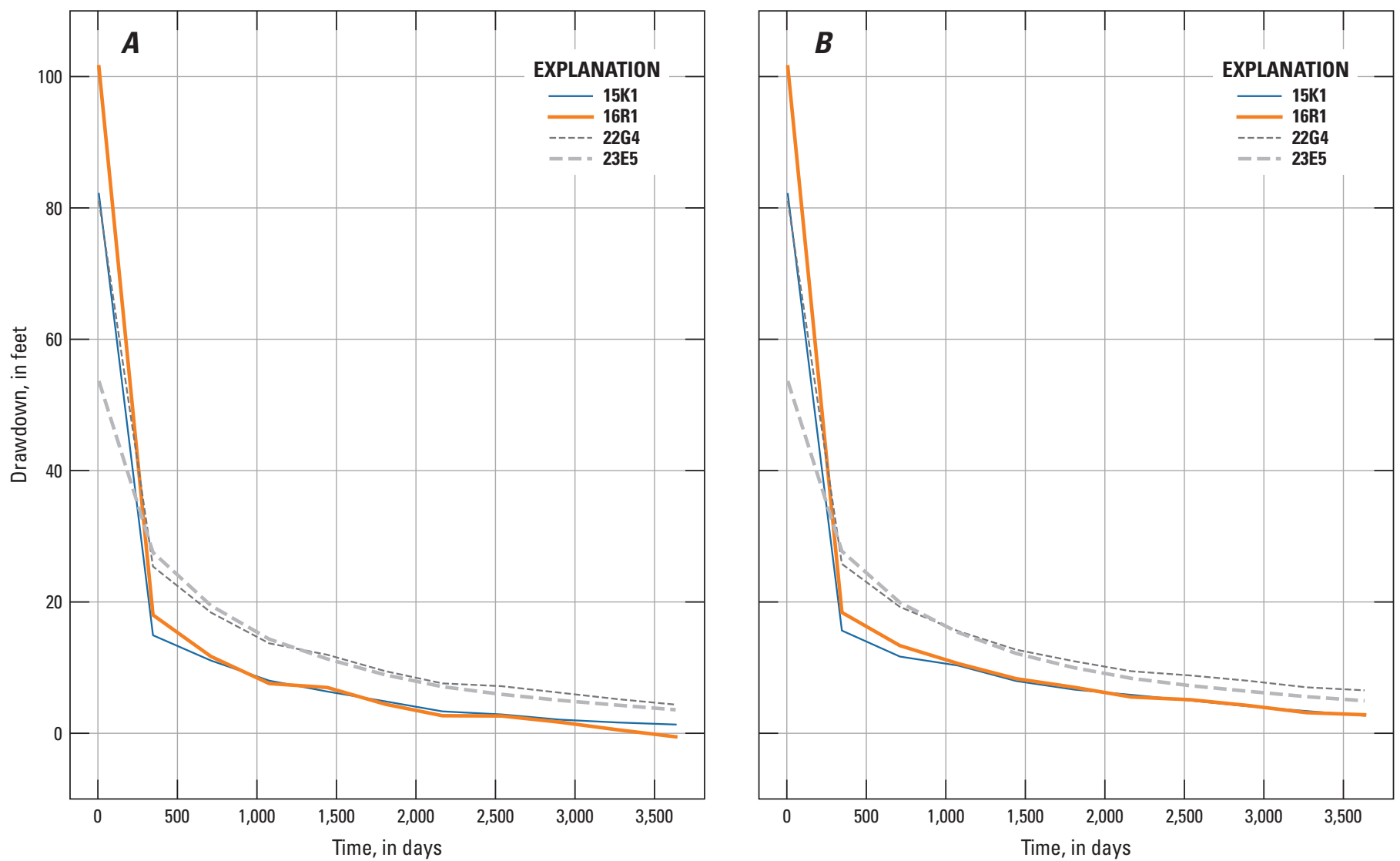

Figure 16. Simulated drawdowns with respect to 1998 water levels for selected wells and total pumpage equaling 484 acre-feet per year (acre-ft/yr), scenario 3, Santa Barbara multi-objective management model, Santa Barbara, California, for $A$, Storage Unit I (SU1) with a 10-year typical climatic condition; $B$, Storage Unit I with a 10-year dry climatic condition; $C$, the Foothill groundwater basin with a 10-year typical climatic condition; and $D$, the Foothill groundwater basin with a 10-year dry climatic condition. 

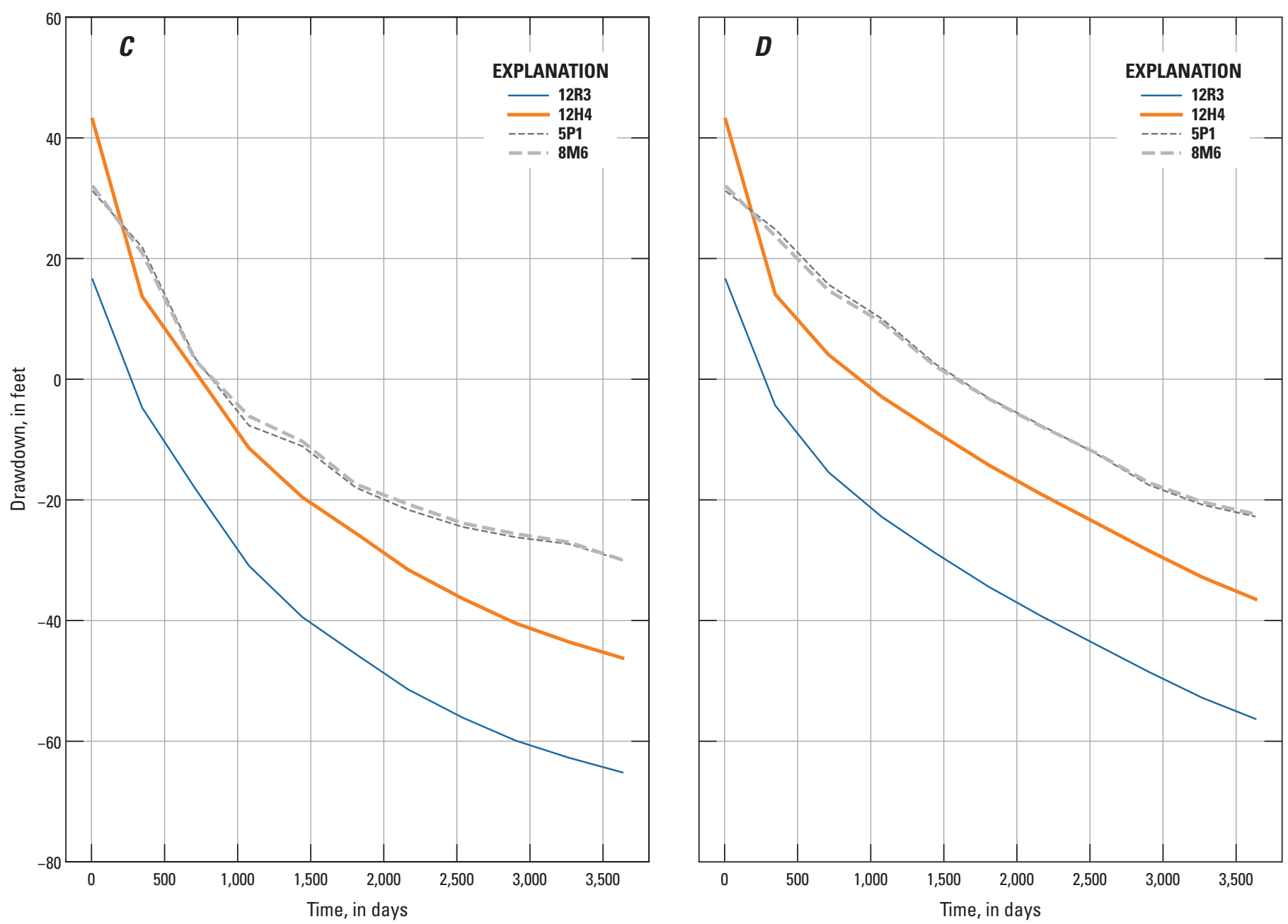

Figure 16. - Continued

Figure 17 shows contours of simulated year-10 drawdowns with respect to 1998 water levels for the upper, middle, and lower producing zones for scenario 3 , assuming typical conditions. Note that the hydrographs in figure 16 are for a single model cell in the well's perforated interval, and the contours in figure 17 are based on a depth-averaged, simulated hydraulic head in each producing zone. The hydrographs and contours, therefore, are not directly comparable. In the upper producing zone, drawdowns ranged from 0 to $10 \mathrm{ft}$ throughout most of Storage Unit I and ranged from -20 to $-60 \mathrm{ft}$ in the Foothill groundwater basin (fig. 17A). In the middle producing zone, drawdowns ranged from 0 to $10 \mathrm{ft}$ in Storage Unit I (fig. 17B). In the lower producing zone, the drawdown was more than $0 \mathrm{ft}$ and less than $20 \mathrm{ft}$ throughout most of
Storage Unit I and ranged from -20 to $-80 \mathrm{ft}$ in the Foothill groundwater basin (fig. 17C). The recovery would likely have been greater in all the producing zones if not for the pumping to supply the Ortega treatment plant.

Figure 18 shows contours of the initial (1990) chloride distribution for the middle and lower producing zones (fig. 18A) and the simulated year-10 chloride concentrations for the middle and lower producing zones for scenario 3 , assuming typical conditions (fig. 18B). The simulated results indicated the extent of seawater intrusion after 10 years of pumping 484 acre-ft/yr from Storage Unit I. The changes in the $15,000-\mathrm{mg} / \mathrm{L}$ contours show seawater intrusion was retreating near the coast both for the middle and lower producing zones (fig. 18B). 


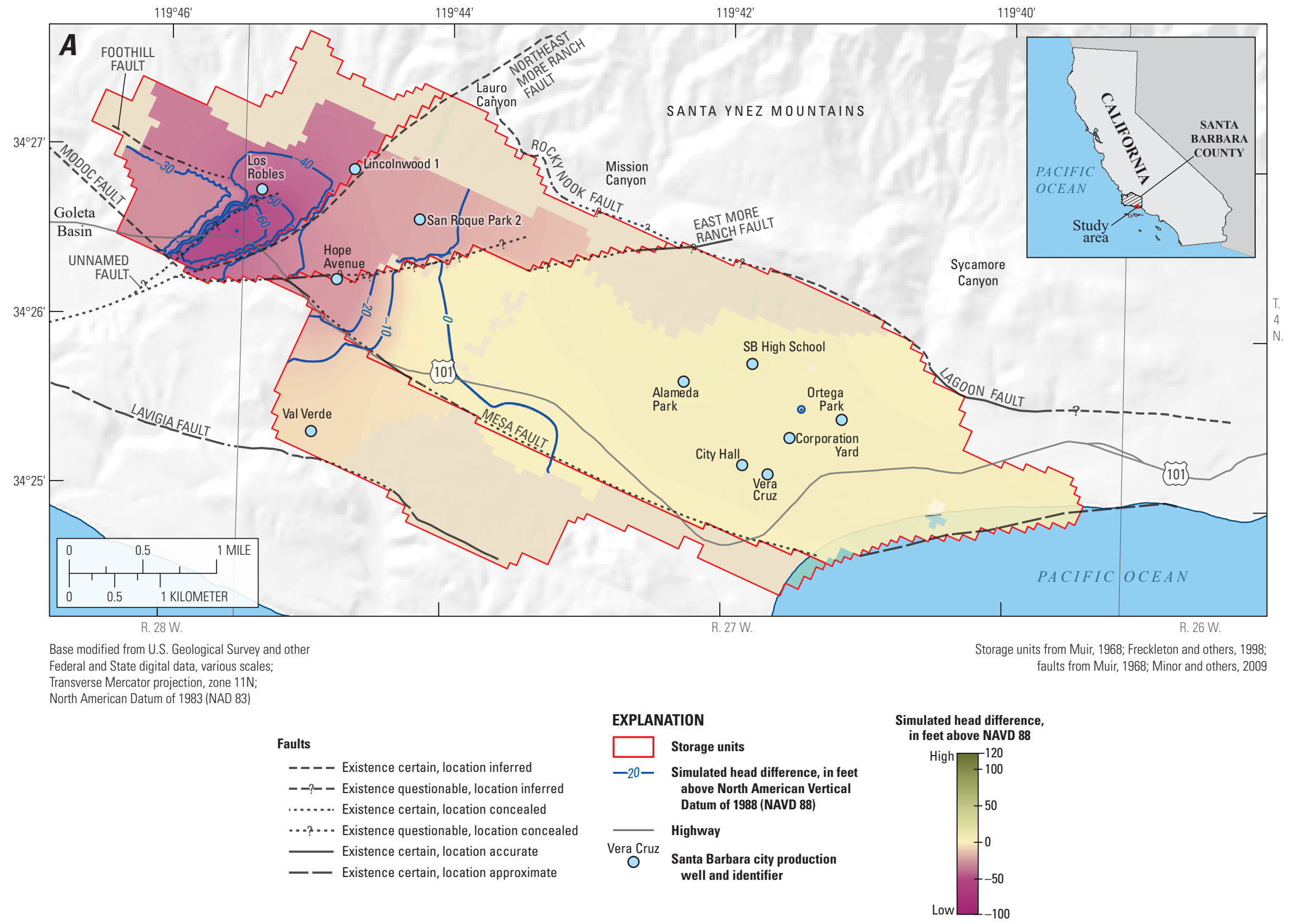

Figure 17. Contours of simulated year-10 drawdowns with respect to 1998 water levels, assuming typical conditions, scenario 3, Santa Barbara multi-objective management model, Santa Barbara, California, for the $A$, upper producing zone; $B$, middle producing zone; and $C$, lower producing zone. 


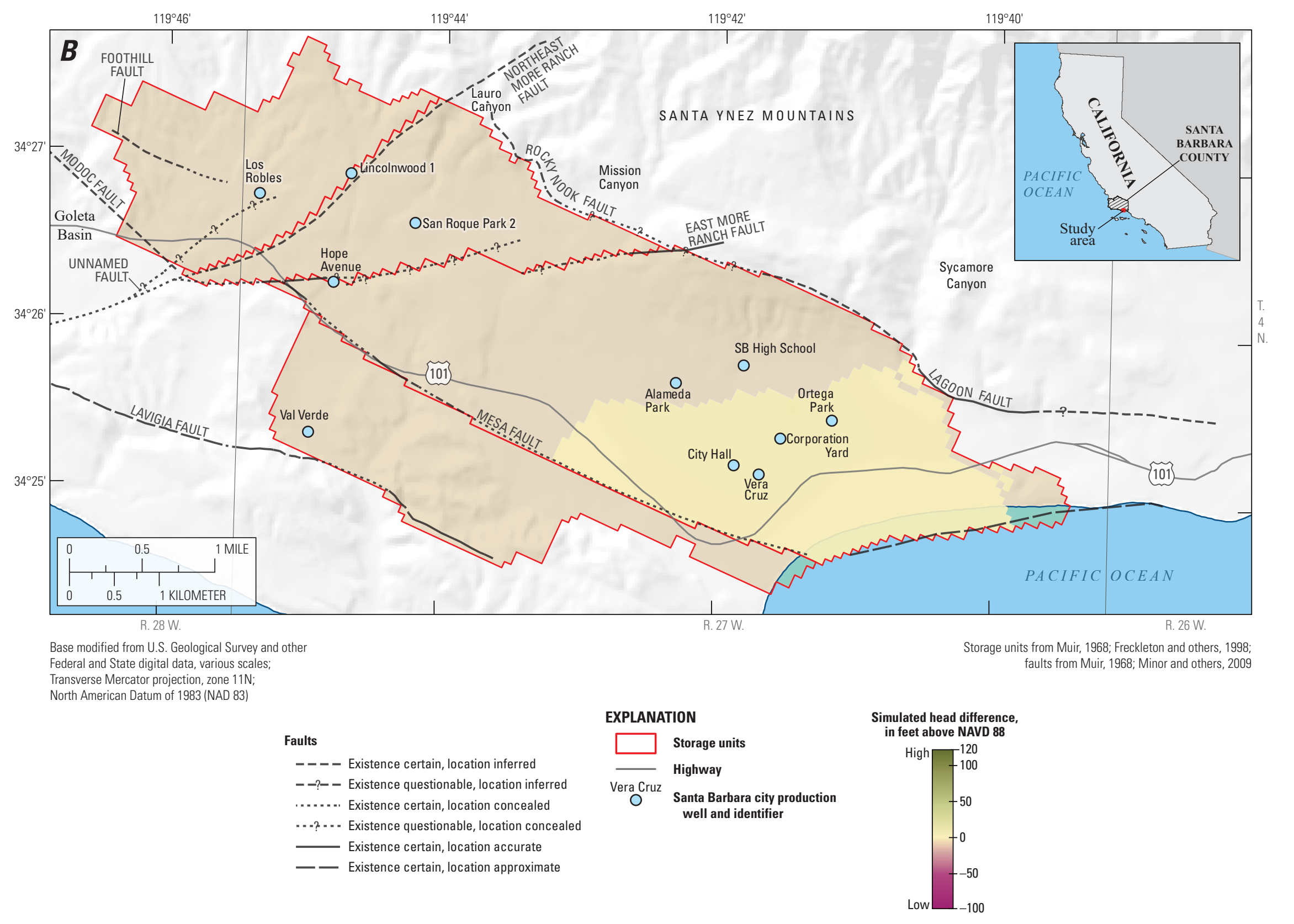

Figure 17. - Continued 


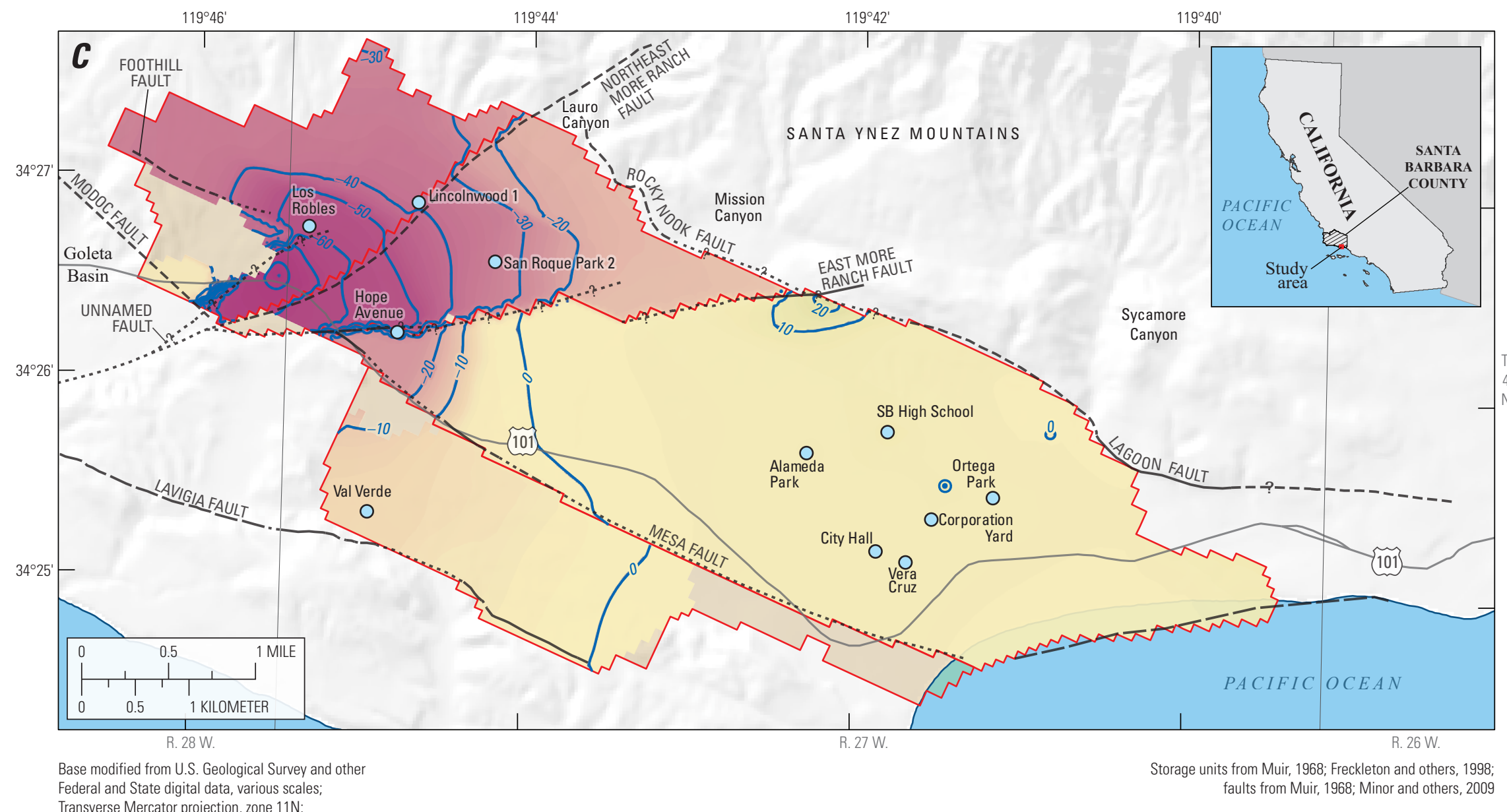

Federal and State digital data, various scales;
Transverse Mercator projection, zone 11N;

North American Datum of 1983 (NAD 83)

\section{Faults}

- - - - Existence certain, location inferred

- -?- - Existence questionable, location inferred

........ Existence certain, location concealed

...? .... Existence questionable, location concealed

Existence certain, location accurate

- - Existence certain, location approximate

\section{EXPLANATION}

$\square$ Storage units

-20- Simulated head difference, in feet above North American Vertical AVD 88)

Highway

Vera Cruz Santa Barbara city production well and identifier

\section{Simulated head difference,}

in feet abover

High $T^{120}$

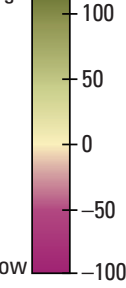

Figure 17. - Continued 

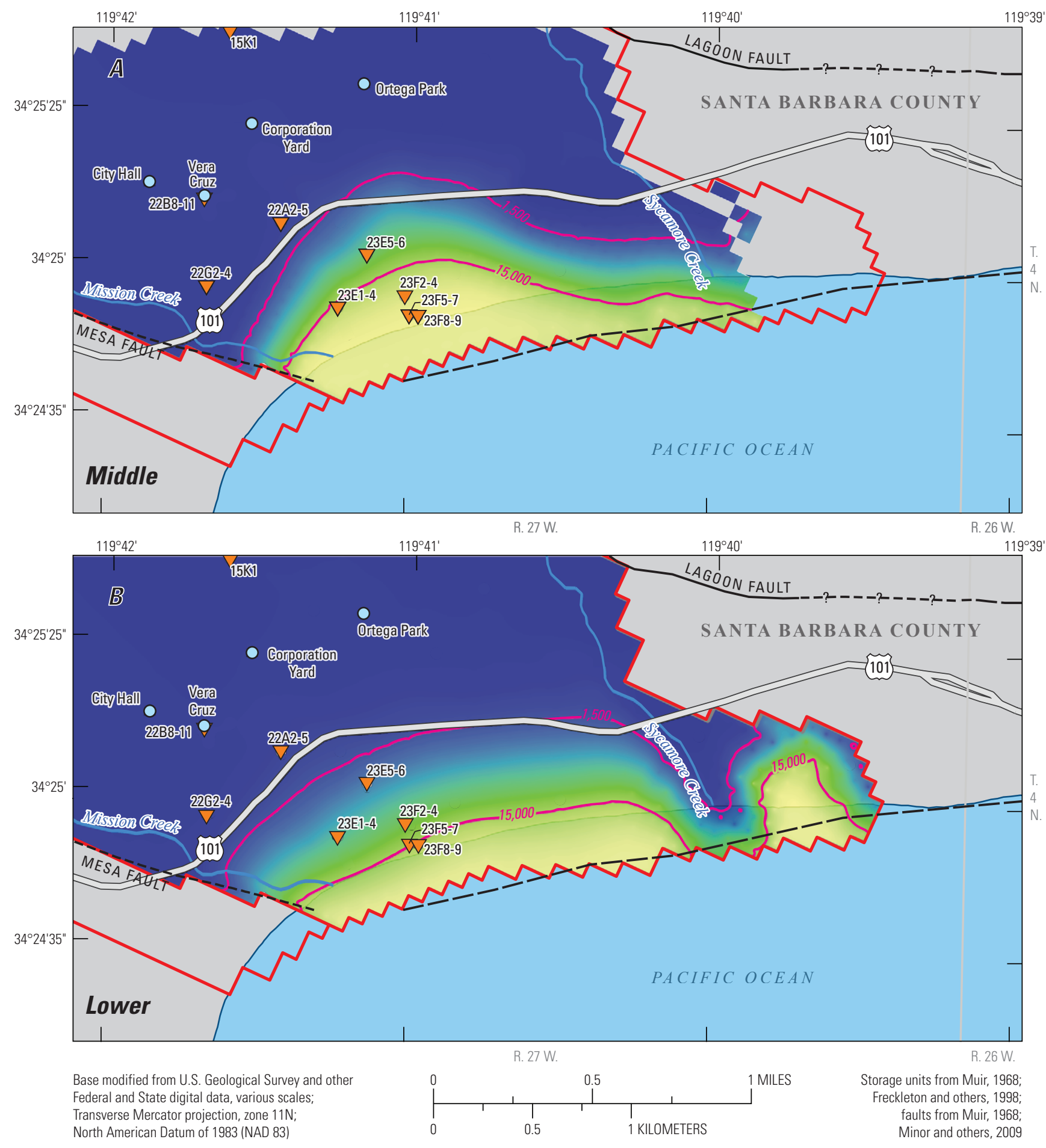

North American Datum of 1983 (NAD 83)

\section{EXPLANATION}

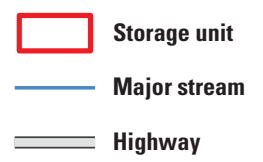

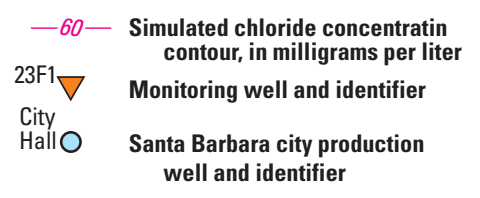
Faults
- - - Existence certain, location inferred
- -?- - Existence questionable, location inferred Existence certain, location accurate
_ _ Existence certain, location approximate

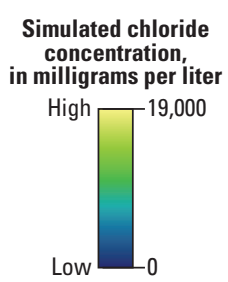

Simulated chloride milligrams per liter

Figure 18. Contours, assuming a typical climatic condition, scenario 3, Santa Barbara multi-objective management model, Santa
Barbara, California, of $A$, initial (1990) chloride concentrations in the middle producing zone; $B$, initial (1990) chloride concentrations in the lower producing zone; $C$, simulated year-10 chloride concentrations in the middle producing zone; and $D$, simulated year-10 chloride concentrations in the lower producing zone. 

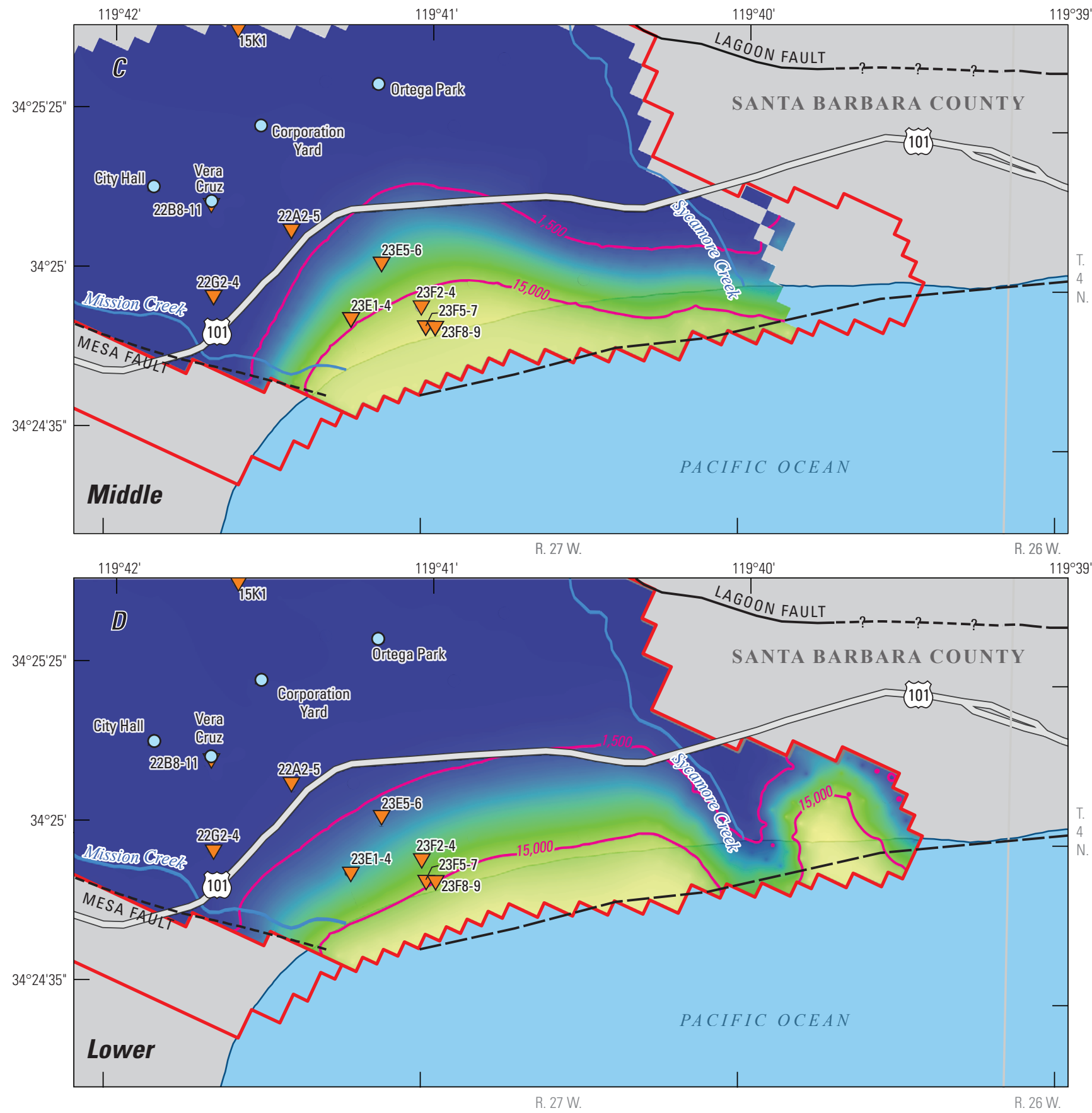

Base modified from U.S. Geological Survey and other Federal and State digital data, various scales; Transverse Mercator projection, zone 11N; North American Datum of 1983 (NAD 83)

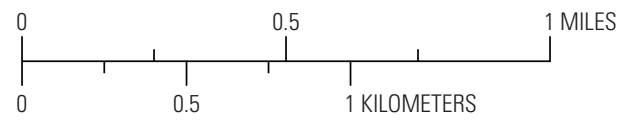

EXPLANATION

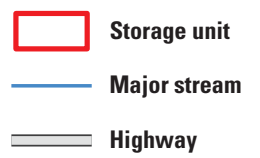
Faults

onitoring well and identifier

Santa Barbara city production well and identifier
- - - Existence certain, location inferred

- -? - Existence questionable, location inferred Existence certain, location accurate Existence certain, location approximate
Storage units from Muir, 1968 Freckleton and others, 1998; faults from Muir, 1968 Minor and others, 2009

Simulated chloride concentration, in milligrams per lite High $\square$ 19,000

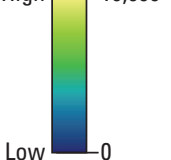

Figure 18. - Continued 
Figure 19 shows contours of simulated year-10 drawdown with respect to 1998 water levels for the upper, middle, and lower producing zones for scenario 3 , assuming dry conditions. In the upper producing zone, drawdowns ranged from 0 to $10 \mathrm{ft}$ throughout most of Storage Unit I and ranged from -20 to $-60 \mathrm{ft}$ in the Foothill groundwater basin (fig. 19A), yet were slightly greater than the simulated drawdowns for a typical climatic condition (fig. 17A). In the middle producing zone, drawdowns ranged from 0 to $10 \mathrm{ft}$ in Storage Unit I (fig. 19B) and were similar to those of a typical climatic condition (fig. 17B). In the lower producing zone, drawdowns ranged from more than $0 \mathrm{ft}$ to less than $70 \mathrm{ft}$ throughout most of Storage Unit I and ranged from 0 to $-70 \mathrm{ft}$ in the Foothill groundwater basin (fig. 19C). The simulated drawdown in the northern part of Storage Unit I (fig. 19C) could be the result of less natural recharge associated with the dry climatic condition. In general, the water-level recovery for the upper zone, assuming dry conditions, was similar to that for the typical climatic condition, yet water-level contours were slightly farther inland, indicative of less recharge; for the lower zone under dry conditions, water-level recovery was less than that for the typical climate throughout both subbasins.

Figure 20 shows contours of simulated year-10 chloride concentrations for the middle and lower producing zones for scenario 3, assuming dry conditions. The changes in the $15,000-\mathrm{mg} / \mathrm{L}$ contours show seawater intrusion was retreating near the coast, particularly in the middle producing zone (fig. 20). In general, there was little difference in the chloride contours between dry and typical climatic conditions, indicating that climate did not substantially affect seawater intrusion under the same pumpage schedule.

\section{Scenario 4}

The two cases in scenario 4 were designed to produce decision rules to support management decisions based on the optimal results. The initial conditions for scenario 4 were designed to emulate recent conditions; therefore, simulated 2013 hydraulic heads and chloride concentrations are used as initial conditions. The goal of scenario $4 \mathrm{~A}$ was to provide a decision rule for groundwater pumpage as a function of allowable change in measured chloride concentration in selected coastal monitoring wells. For example, a water manager would choose an allowable maximum increase in chloride concentration for the year according to the measured concentration in a given well; the result of the decision rule would be the optimal total pumpage for Storage Unit I for the allowable maximum chloride increase in the following year. The goal of scenario $4 \mathrm{~B}$ was to provide a decision rule for groundwater pumpage as a function of desired maximum drawdown in selected wells in the Foothill groundwater basin. For example, a water manager would choose an allowable maximum drawdown in a given well for 10 years of pumping. The result of the decision rule would be the optimal total 10-year pumpage for Storage Unit I and Foothill groundwater basin.

In this scenario, 10-year simulations were made using the Pareto-optimal pumping schedules for the typical (scenario 1) and dry (scenario 2) climatic conditions. Recall that scenario 1 had 912 Pareto-optimal solutions (fig. 4), and scenario 2 had 629 Pareto-optimal solutions (fig. 10). The epsilon values were adjusted such that a reasonably large number of Pareto-optimal pumping schedules were identified - in this case, about 100 such pumping schedules were identified for each scenarioand such that the schedules represented the full range of objective values. Scenario 1 had 127 Pareto-optimal pumping schedules using $\varepsilon_{\mathrm{A}}, \varepsilon_{\mathrm{B}}, \varepsilon_{\mathrm{Ct}}$, and $\varepsilon_{\mathrm{Cm}}$ values of 500, 20, 200, and 5, respectively (fig. 21A). Scenario 2 had 97 Pareto-optimal pumping schedules using $\varepsilon_{\mathrm{A}}, \varepsilon_{\mathrm{B}}, \varepsilon_{\mathrm{Ct}}$, and $\varepsilon_{\mathrm{Cm}}$ values of 500, 20, 50 , and 5, respectively (fig. $21 B$ ). Figures $21 A$ and $B$ represent subsets of the entire Pareto-optimal solutions as defined by these $\varepsilon$ values. The decision-rule curves for scenarios $4 \mathrm{~A}$ and 4B were produced from the subsets of Pareto-optimal results for scenarios 1 and 2 by running a 10-year simulation for each solution in the subsets and searching for specific levels of chloride concentration or maximum drawdown in selected monitoring or production wells.

\section{Scenario $4 \mathrm{~A}$}

For this scenario, the decision-rule curves were generated from the 10-year simulation results using the following steps:

1. Assume measured chloride concentrations at selected monitoring sites.

2. Search the scenario 1 and 2 Pareto-optimal pumping schedules for simulated chloride concentrations that equal the assumed measured chloride concentration at each monitoring site.

3. If the simulated value equals the assumed measured concentration, then the total pumpage in Storage Unit I for the following year and the associated change in chloride concentration are saved.

Figure 22 shows example decision-rule curves for two monitoring wells (4N/27W-23E3 and 23E5) assuming typical and dry climatic conditions. Assume that $800 \mathrm{mg} / \mathrm{L}$ and 2,000 $\mathrm{mg} / \mathrm{L}$ chloride concentrations were measured in wells 23E3 and 23E5, respectively. For well 23E3, assuming typical climatic conditions and an allowable increase in measured chloride concentration of $76 \mathrm{mg} / \mathrm{L}$, the Paretooptimal results indicated that during the year following the time when $800 \mathrm{mg} / \mathrm{L}$ was measured, about 1,937 acre-ft of water was pumped from Storage Unit I. This results in one point on the rule curve. Each red circle in figure 22 represents a similar analysis for every point on the Pareto front, assuming a concentration of $800 \mathrm{mg} / \mathrm{L}$ was reached at well 23E3. A threshold of 2,000 mg/L was applied to well 23E5, and similar analyses were performed (fig. 22). 


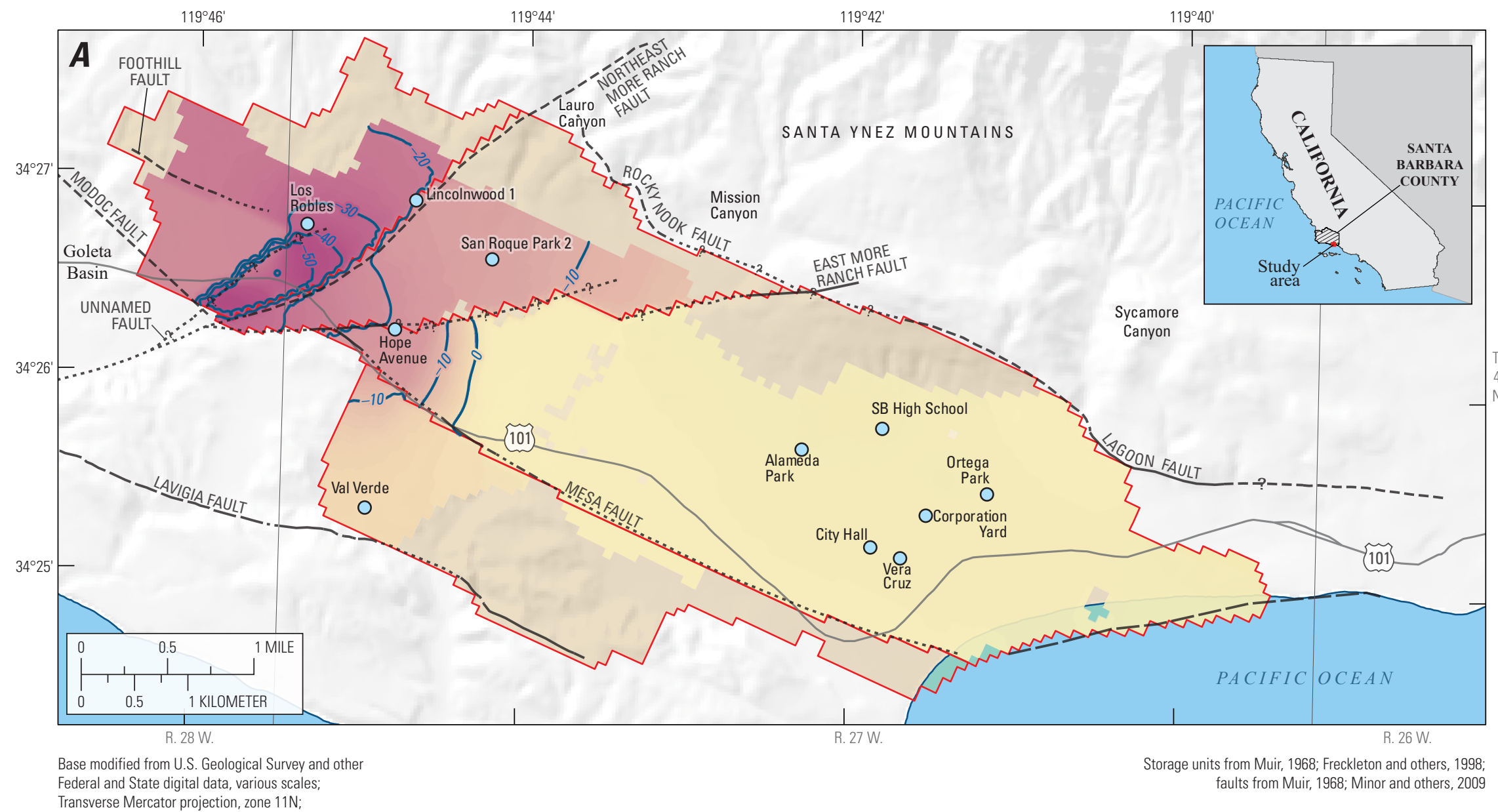

Federal and State digital data, various scales;
Transverse Mercator projection, zone $11 \mathrm{~N}$;

North American Datum of 1983 (NAD 83)

\section{Faults}

- - - - Existence certain, location inferred

- -? - Existence questionable, location inferred

........ Existence certain, location concealed

...? .... Existence questionable, location concealed

__ Existence certain, location accurate

- - Existence certain, location approximate
EXPLANATION

$\square$ Storage units

-20- Simulated head difference, in feet above North American Vetic (NAVD 88)

Highway

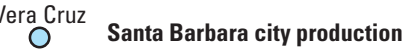
well and identifier

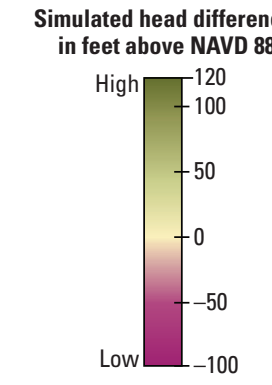

Figure 19. Contours of simulated year-10 drawdown with respect to 1998 water levels, assuming dry climatic condition, scenario 3, Santa Barbara multi-objective management model, Santa Barbara, California, for the $A$, upper producing zone, $B$, middle producing zone, and $C$, lower producing zone. 


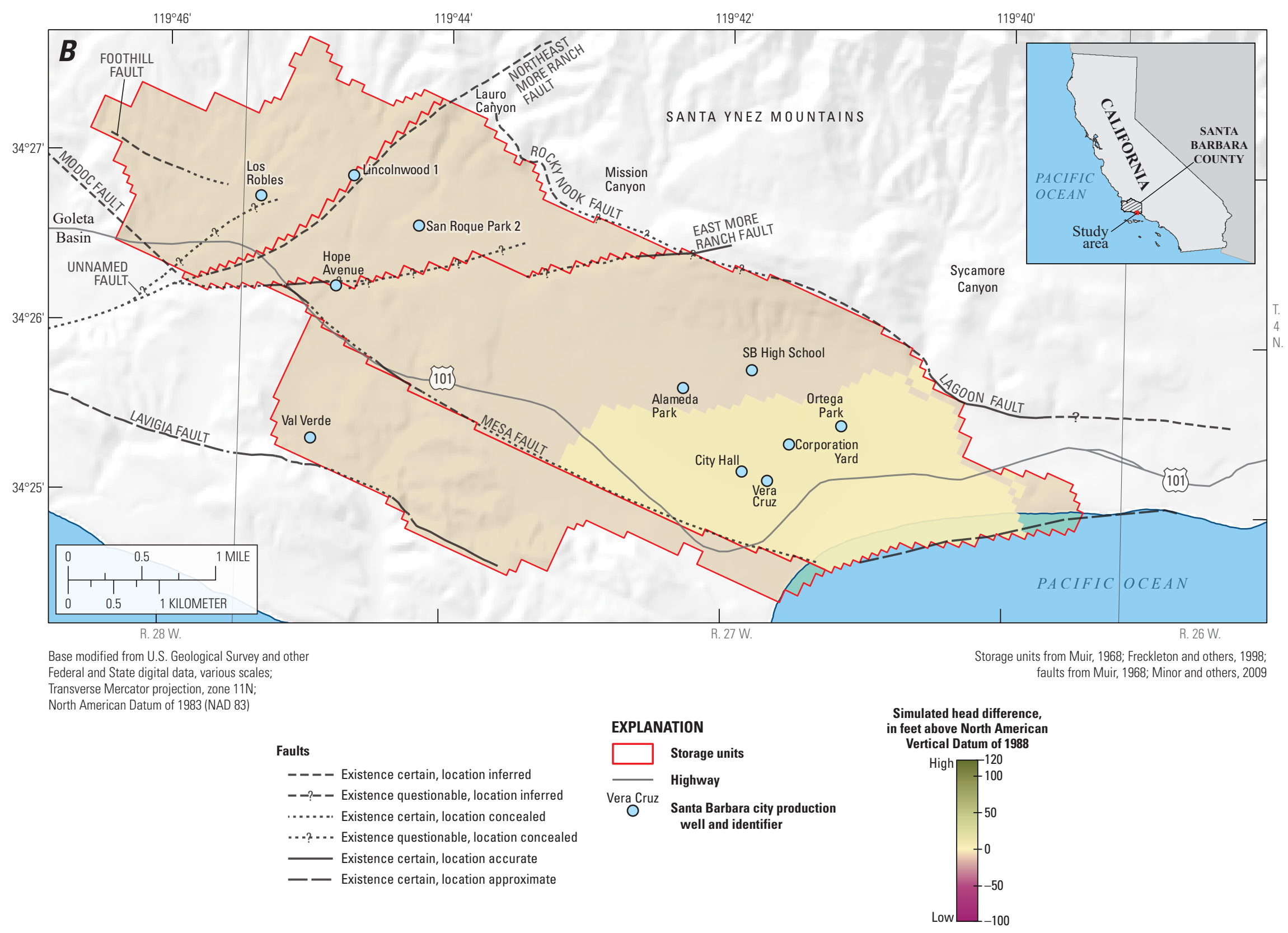

Figure 19. - Continued 


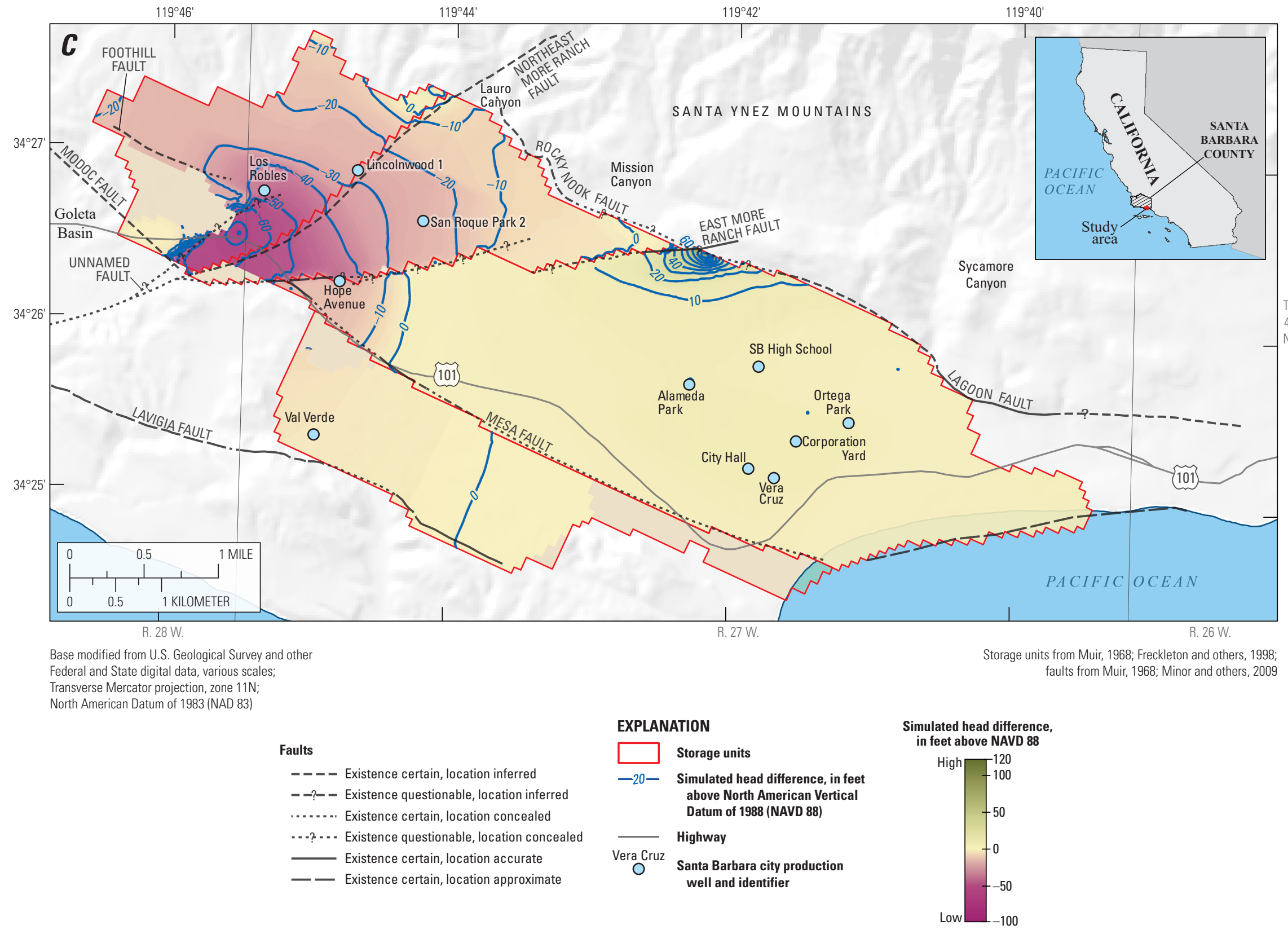

Figure 19. - Continued 

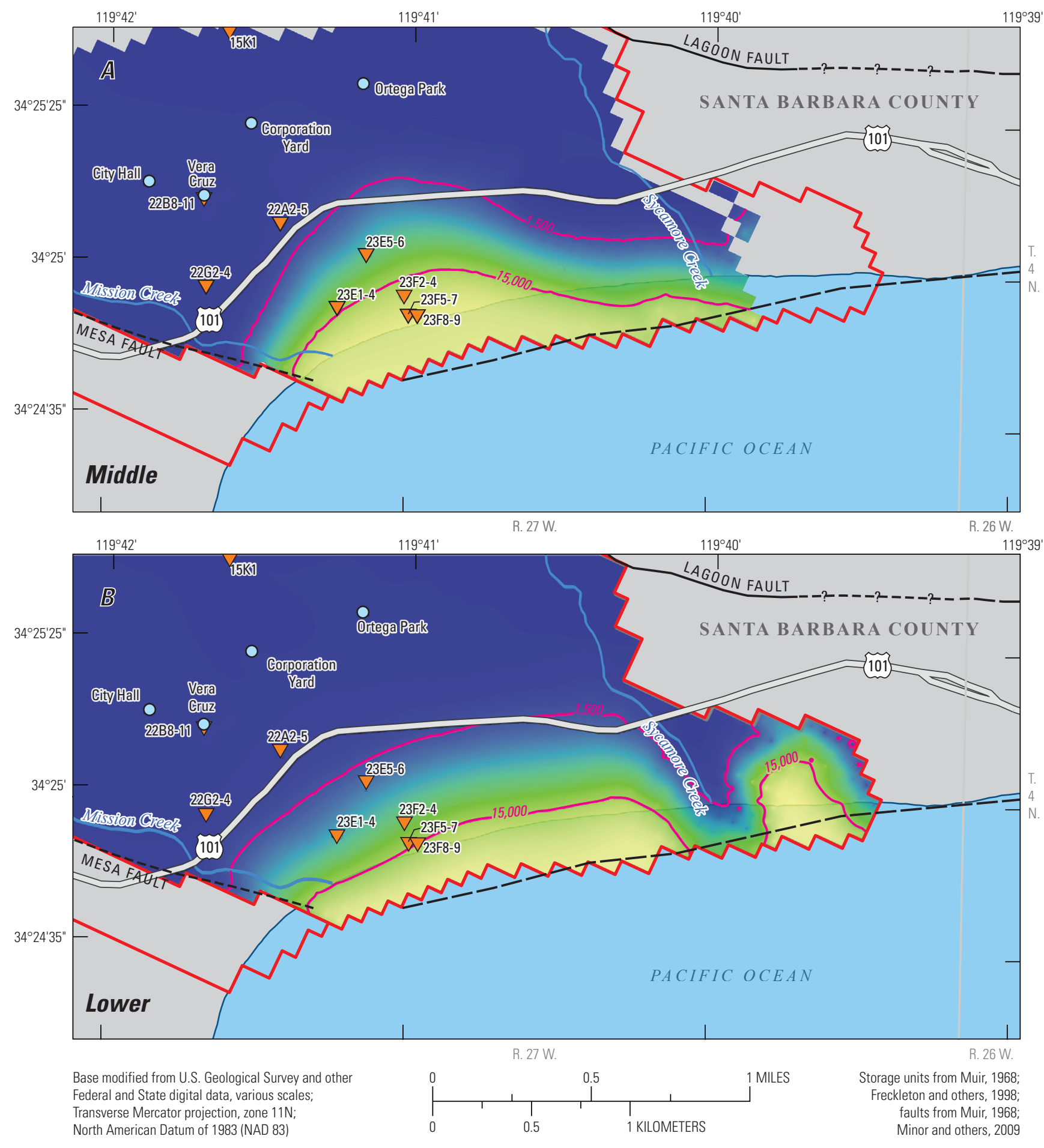

North American Datum of 1983 (NAD 83)

\section{EXPLANATION}

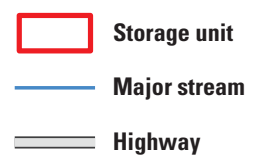

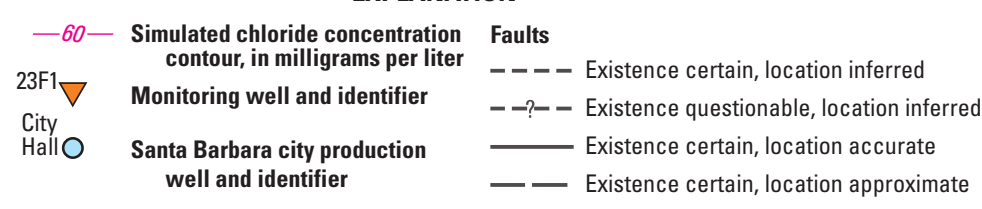

Simulated chloride concentration, in milligrams per liter High 19,000

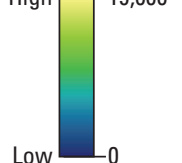

Figure 20. Contours; assuming a dry climatic condition, scenario 3, Santa Barbara multi-objective management model, Santa Barbara, California, of simulated year-10 chloride concentrations for $A$, the middle producing zone, and $B$, the lower producing zone. 


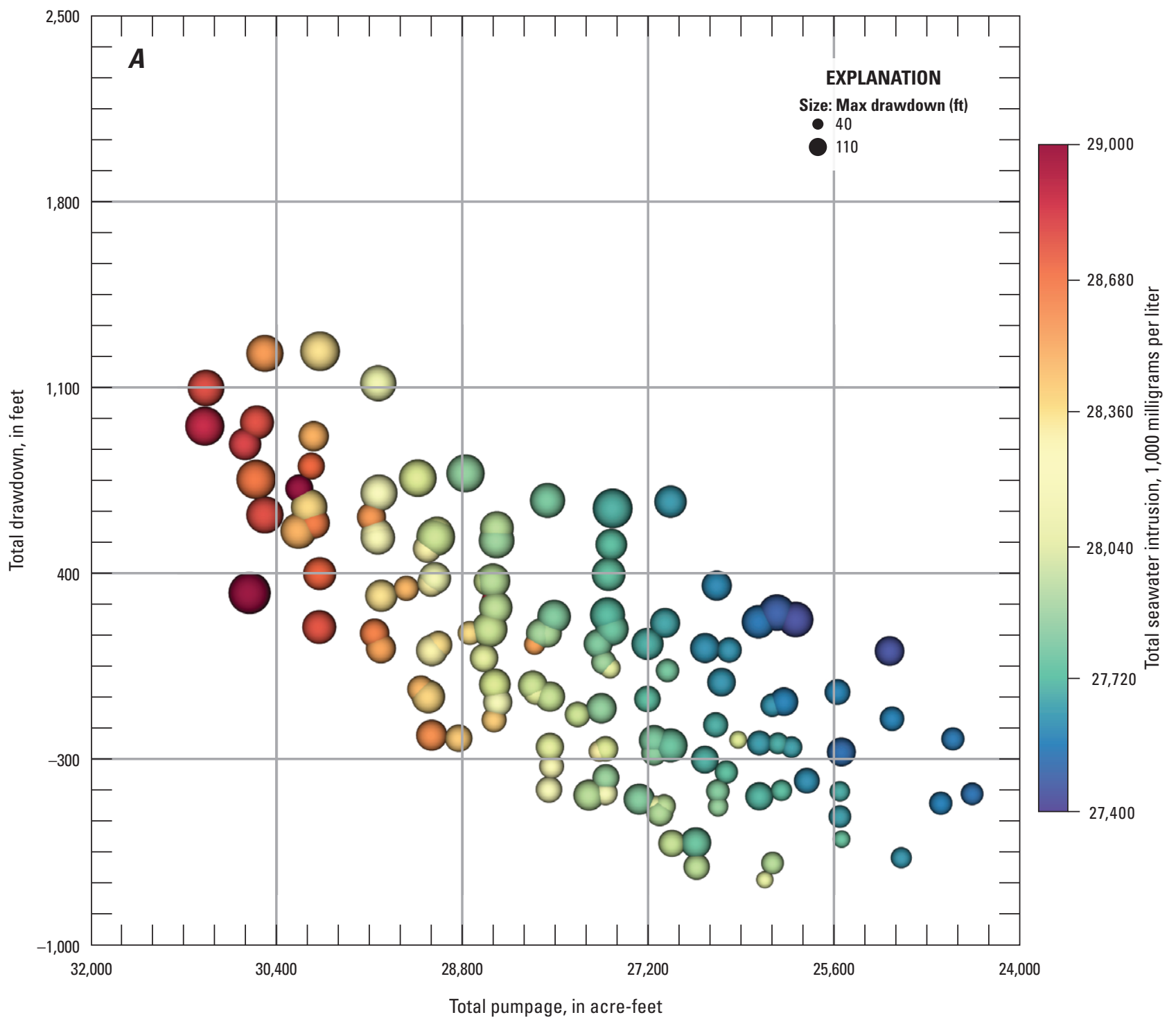

Figure 21. Subsets of Pareto-optimal solutions, Santa Barbara multi-objective management model, Santa Barbara, California, for $A$, scenario $4 \mathrm{~A}$, and $B$, scenario $4 \mathrm{~B}$. 


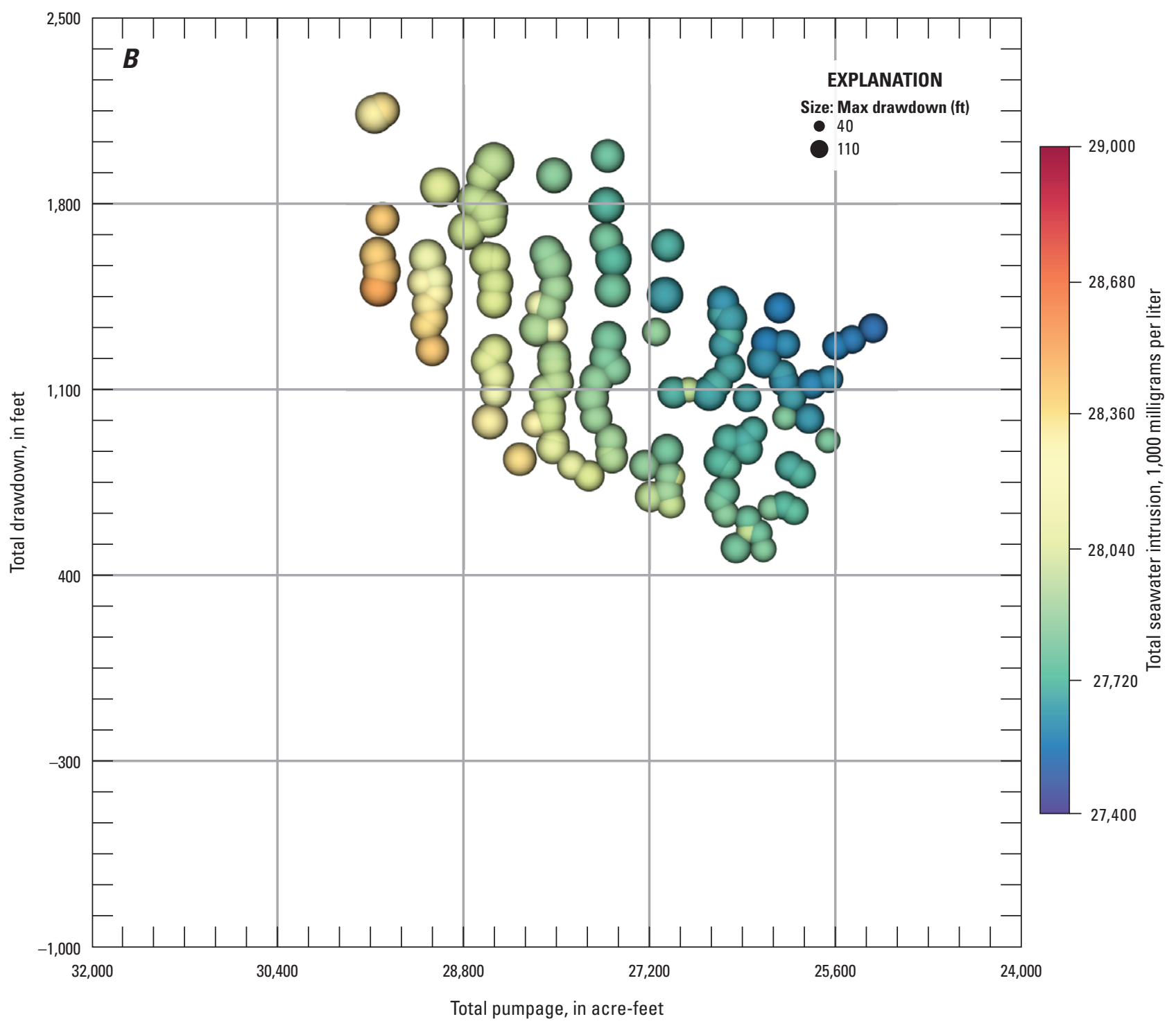

Figure 21. - Continued 

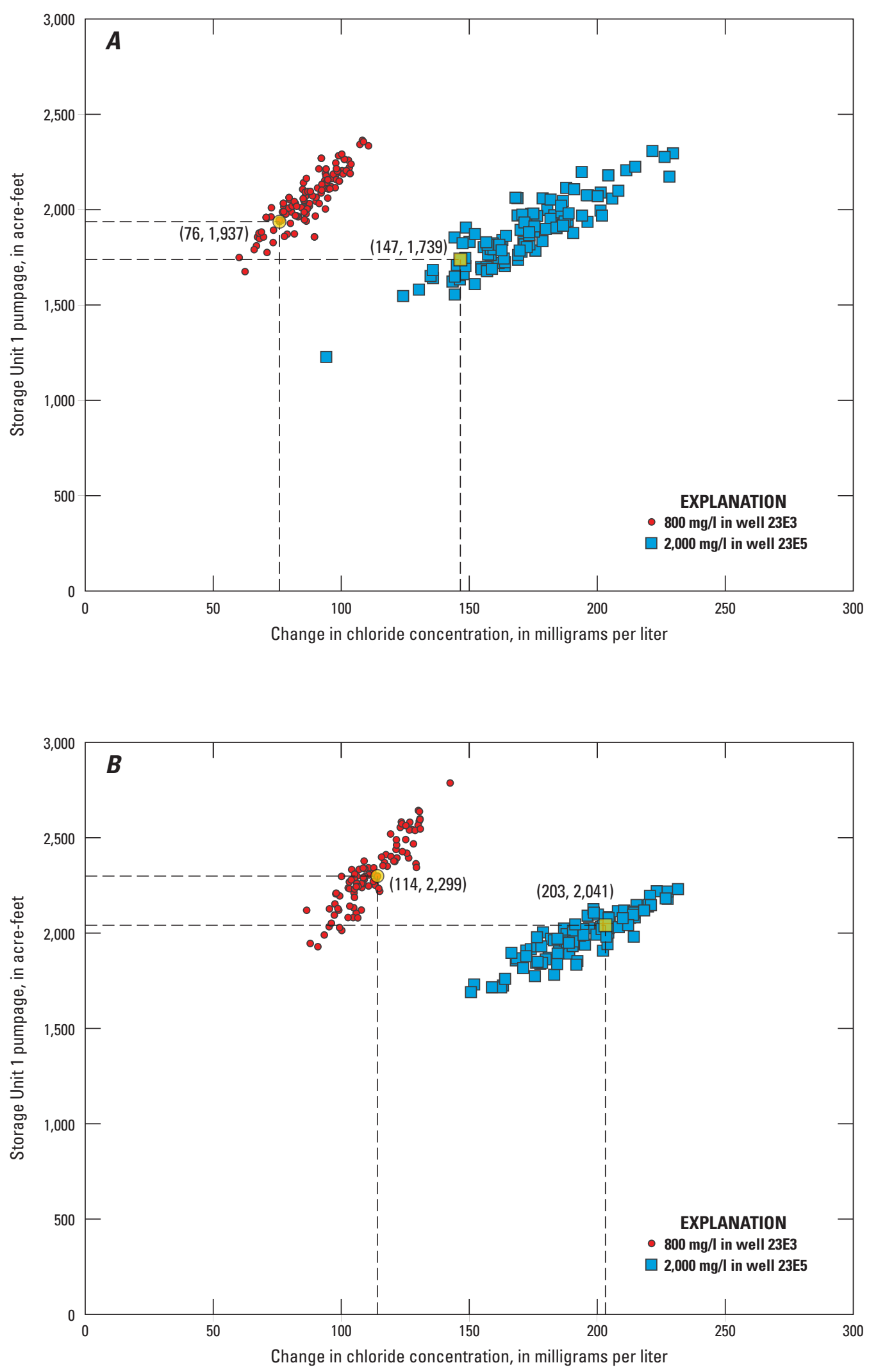

Figure 22. Example decision-rule curves for wells 4N/27W-23E3 and 4N/27W-23E5 used to assess total pumpage from Storage Unit I on the basis of observed chloride concentrations in selected monitoring wells, Santa Barbara multi-objective management model, Santa Barbara, California. The pumpage and change in chloride concentration are for the year following the measured level. $A$, scenario 1 (typical climatic condition), and $B$, scenario 2 (dry climatic condition). The highlighted dot and square represent schedules $1_{D}$ and $2_{D}$ pumpage for scenarios 1 and 2 , respectively. 
As an example of interpreting the decision rules, consider the decision rule for well 23E3, assuming a dry climatic condition (fig. 22B) and that a chloride concentration of $800 \mathrm{mg} / \mathrm{L}$ was measured in the well. Further assume that an increase of about $114 \mathrm{mg} / \mathrm{L}$ was deemed allowable for this well during the year following when the measurement was taken, then the total Pareto-optimal pumpage from Storage Unit I would be about 2,299 acre-ft. Note that the decision rule was only valid for points shown in figure 22; it would not be appropriate to interpolate between points for a given well.

An alternative approach to interpreting the decision rules is to identify a desired total pumpage and then determine the resulting change in chloride concentration. For example, again consider well 23E3, assuming a dry climatic condition (fig. 22B) and that a chloride concentration of $800 \mathrm{mg} / \mathrm{L}$ was measured in the well. Further assume that the city desired to pump a total of about 2,300 acre-ft in a year. Pumping 2,300 acre-ft increased chloride concentrations by about $100 \mathrm{mg} / \mathrm{L}$ to $114 \mathrm{mg} / \mathrm{L}$ (fig. 22B). The differences in chloride concentrations resulted from differing pumping patterns. The city could choose the pumping pattern with the least change in chloride concentration or by other criteria. For points clustered near each other, the specific pumping schedules associated with each point near the desired pumpage value would have to be inspected and compared in order to make a decision about which schedule to choose.

\section{Scenario 4B}

For this scenario, decision-rule curves were created to assess the effect of allowable drawdown in Foothill groundwater basin wells on potential management decisions. Note that decision-rule curves are not presented for Storage Unit I wells, because it was determined that seawater intrusion was a higher priority in this basin than drawdown. The decision-rule curves were generated from the 10-year simulation results using the following steps:

1. The maximum annual drawdown for each production well in Foothill groundwater basin for 10 years of pumping was stored.

2. The associated total pumpage for Storage Unit I and Foothill groundwater basin for those 10 years was stored separately.

The Los Robles well decision rules for Foothill groundwater basin pumpage under typical and dry climatic conditions are shown in figures $23 A$ and $23 B$, respectively. The Los Robles well decision rules for pumpage in Storage Unit I, under typical and dry climatic conditions, are shown in figures $23 C$ and $23 D$, respectively. The decision rules for all production wells in Storage Unit I and the Foothill groundwater basin are presented in appendix D-4.

As an example of interpreting the decision rules, consider the decision rule for the Los Robles well, assuming a typical climatic condition (fig. 23A). Further assume that a maximum drawdown of $22 \mathrm{ft}$ was desired for a 10 -year management horizon. The decision rule indicated that the total pumpage from the city wells in Foothill groundwater basin was about 7,461 acre-ft for the 10-year period.

Consider the total pumpage for Storage Unit I, assuming a typical climatic condition, as indicated by the decision rule for the Los Robles well (fig. 23C). Further assume that a maximum drawdown of $22 \mathrm{ft}$ was desired during a 10-year management horizon. The decision rule indicates that the total pumpage from the city wells in Storage Unit I was about 16,203 acre- $\mathrm{ft}$ for the 10-year period.

Many of the decision rules for pumpage in Storage Unit I as a function of maximum, annual drawdown at wells in the Foothill groundwater basin either were very disperse (for example, the Hope Avenue, Lincolnwood 1, and San Roque Park (2) wells in appendix D-4) or were slightly nonlinear (for example, the Los Robles well). This could indicate that drawdown in the Foothill groundwater basin is not a strong function of the total pumpage for Storage Unit I, probably because Storage Unit I and Foothill groundwater basin are hydraulically disconnected by the East More Ranch fault.

\section{Scenario 5}

Scenario 5 investigated the short-term (2-year) effects of extreme climatic conditions on the optimal pumping schedules. Specifically, three cases were investigated: typicalto-dry (scenario 5A, 1998-99), dry-to-typical (scenario 5B, 1977-78), and dry-to-dry (scenario 5C, 1989-90) periods. The Pareto-optimal solutions and pumping schedules for the three scenario- 5 cases are shown in figure 24 and presented in tables 5-7. Overall, the Pareto curves were similar for scenarios $5 \mathrm{~A}$ and $5 \mathrm{~B}$, but shifted toward less drawdown and toward less pumpage for scenario $5 \mathrm{C}$. In general, all the pumping schedules resulted in water-level recovery for all the scenarios, except for schedules $5 \mathrm{~A}_{\mathrm{A}}, 5 \mathrm{~B}_{\mathrm{A}}$, and $5 \mathrm{~B}_{\mathrm{B}}$, which resulted in water-level declines. In addition, schedule $5 \mathrm{~A}_{B}$ resulted in a water-level recovery, whereas for the other two climatic conditions, schedule $\mathrm{B}$ resulted in a waterlevel decline (scenario $5 \mathrm{~B}_{\mathrm{B}}$ ) or no change in water level (scenario $5 \mathrm{C}_{\mathrm{B}}$ ).

The results for all three scenario- 5 cases indicated that schedule A pumpage resulted in similar seawater intrusion, but large differences in drawdown; schedules B and C pumpage varied in their yield more than the other schedules for all the scenarios; and the schedule D pumpage for all the scenarios results in similarly large increases in water levels. With no regard for future availability beyond the 2-year horizon, the total pumpage for schedules $\mathrm{A}$ and $\mathrm{D}$ were similar for scenarios 5A and 5B (tables 5 and 6); however, the total schedule $5 \mathrm{~A}_{\mathrm{Cm}}$ pumpage (about 6,800 acre- $\mathrm{ft}$, table 5) was nearly 1,000 acre-ft greater than the pumpage of Schedule $5 \mathrm{~B}_{\mathrm{Cm}}$ (about 5,800 acre- $\mathrm{ft}$, table 6), indicating that pumpage was sensitive to the climate in year two when minimizing the maximum drawdown. Conversely, pumpage with respect to total drawdowns appeared more sensitive to the climate in year one, because the yield for schedules $5 \mathrm{~B}_{\mathrm{Ct}}$ and $5 \mathrm{C}_{\mathrm{Ct}}$ (year one was dry) were similar and that of schedule $5 \mathrm{~A}_{\mathrm{Ct}}$ was larger. When minimizing seawater intrusion, pumpage was largest in scenario $5 \mathrm{C}_{\mathrm{B}}(5,900$ acre-ft; table 7$)$ and smallest in scenario $5 \mathrm{~A}_{\mathrm{B}}(5,000$ acre-ft; table 5$)$. 

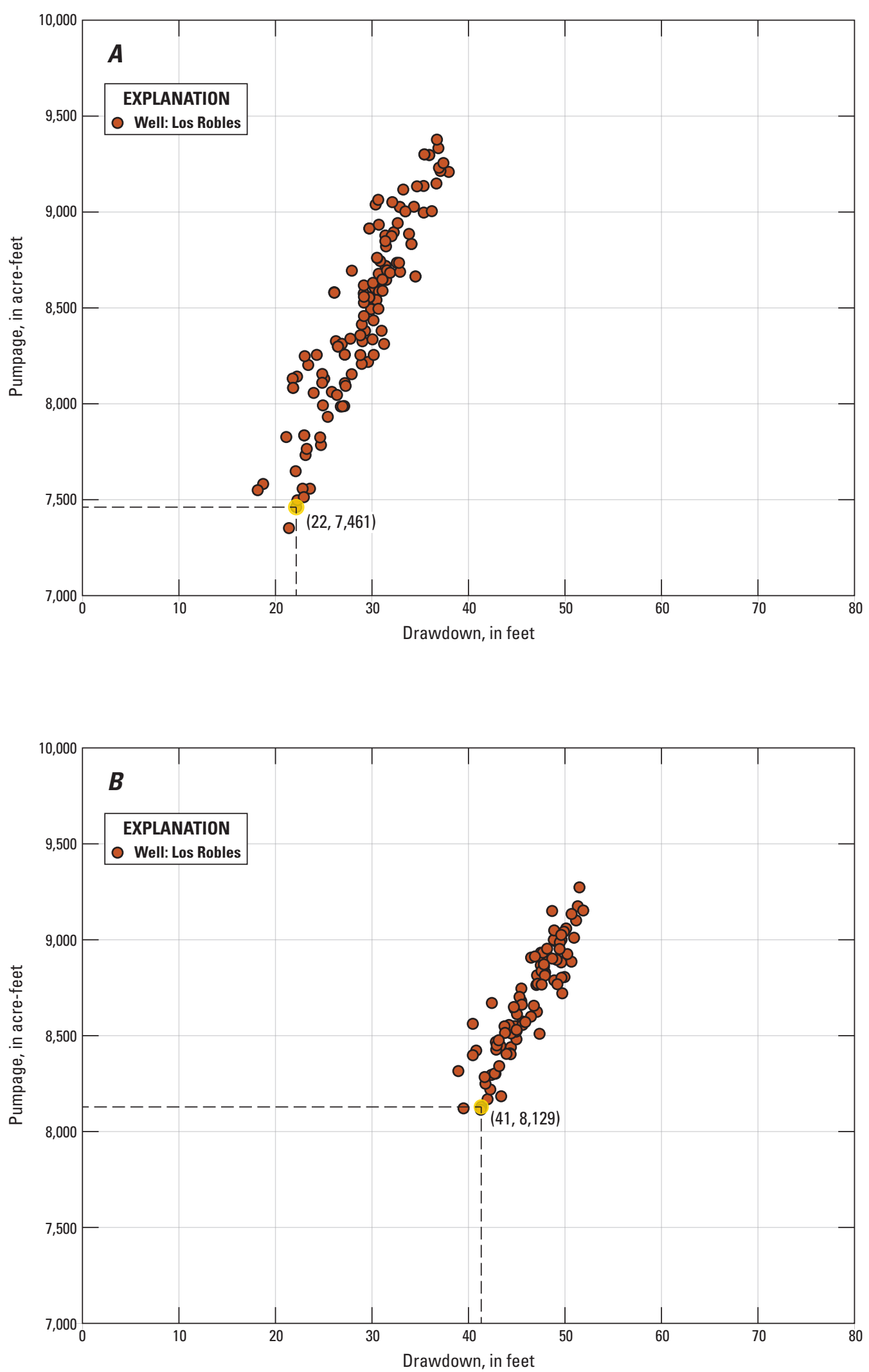

Figure 23. Example decision-rule curves for the Los Robles well, Santa Barbara multi-objective management model, Santa Barbara, California, for $A$, total 10-year pumpage Foothill groundwater basin scenario $1 ; B$, total 10-year pumpage Foothill groundwater basin scenario 2; $C$, for total 10-year pumpage Storage Unit I scenario 1; and $D$, for total 10-year pumpage, Storage Unit I scenario 2. 

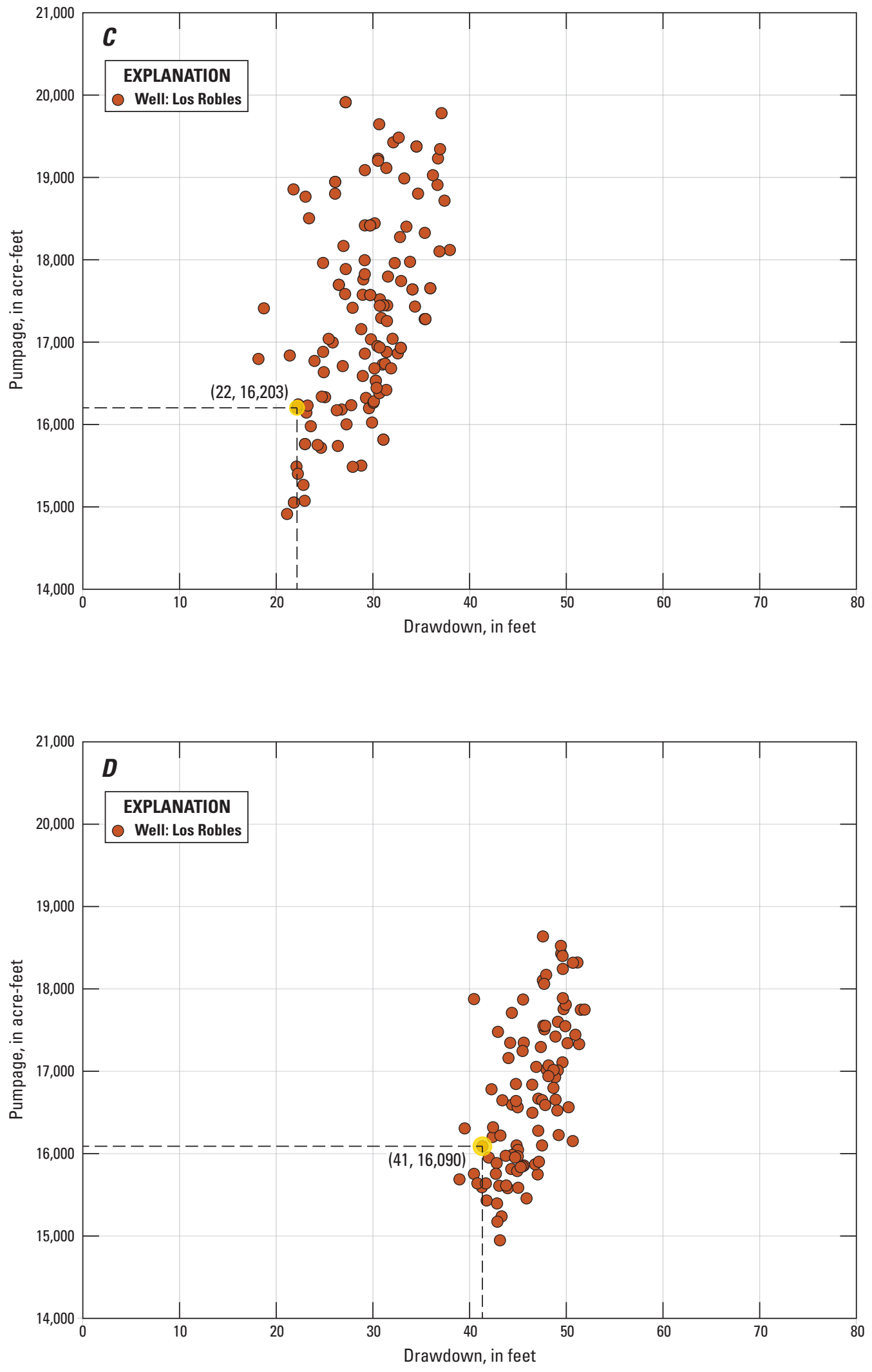

Figure 23. - Continued 


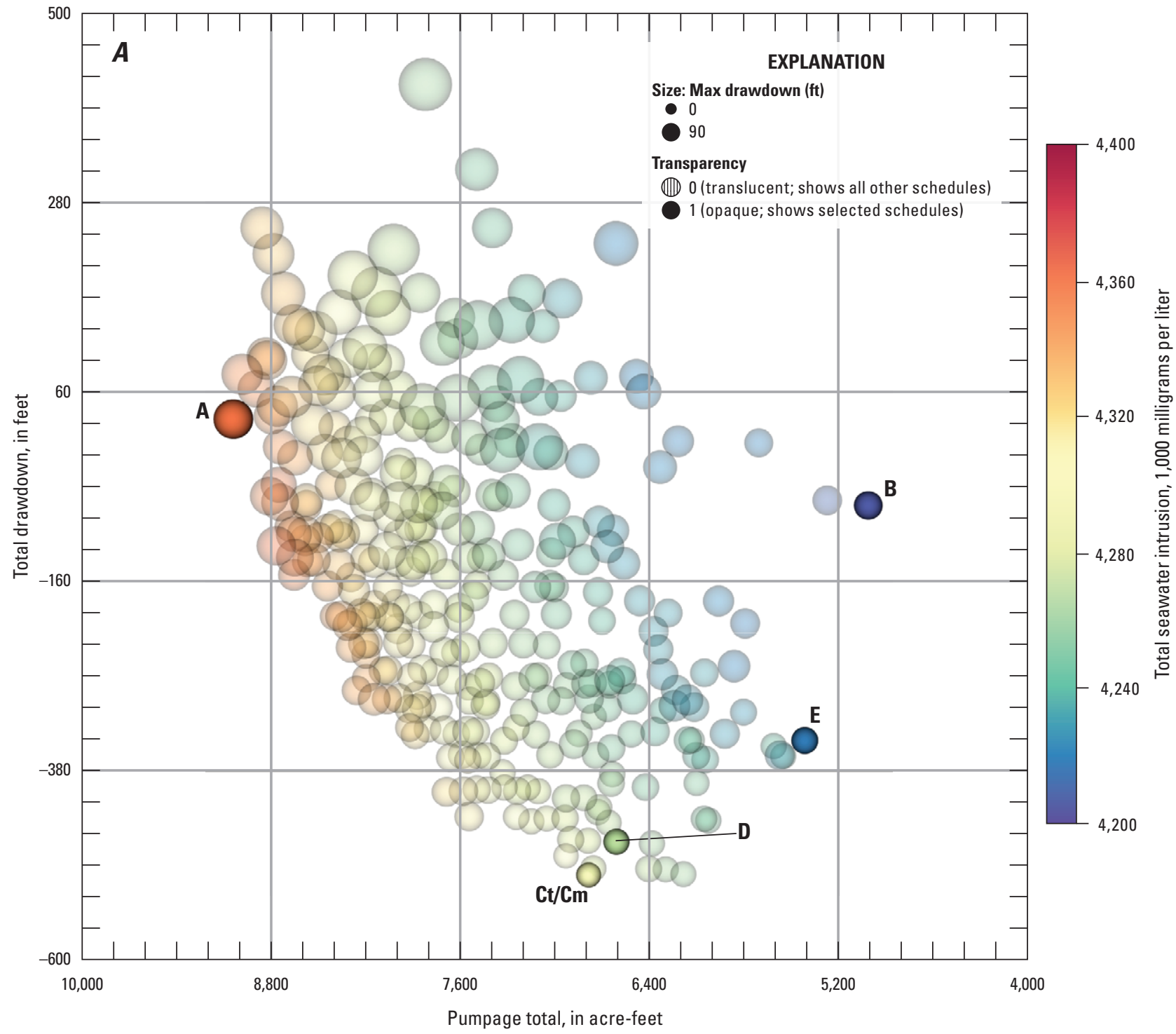

Figure 24. Selected Pareto-optimal solutions relative to seawater intrusion, maximum drawdown, total pumpage, and total drawdown, Santa Barbara multi-objective management model, Santa Barbara, California, for $A$, scenario 5A (typical-dry); $B$, scenario 5B (drytypical); and C, scenario 5C (dry-dry). 


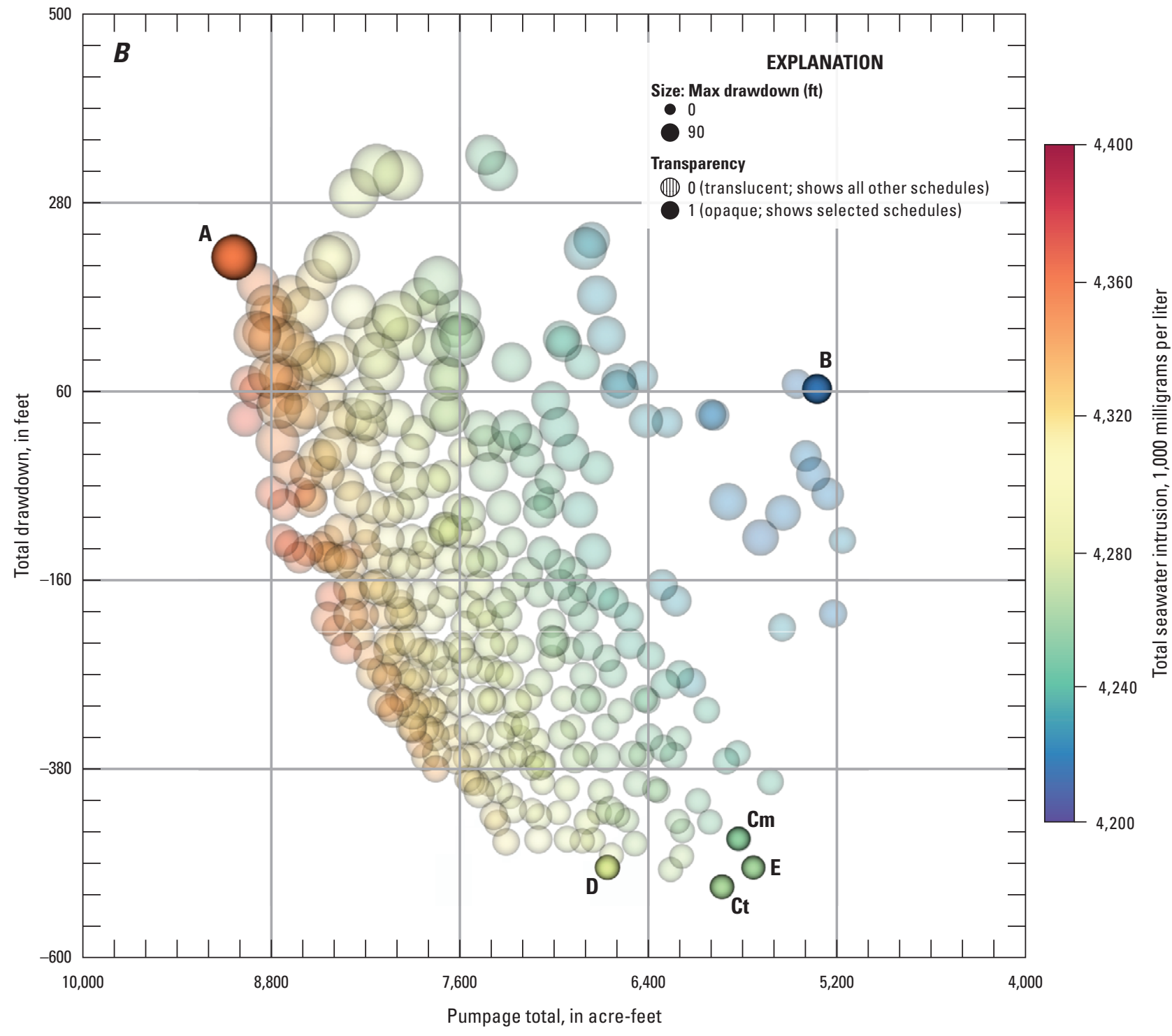

Figure 24. - Continued 


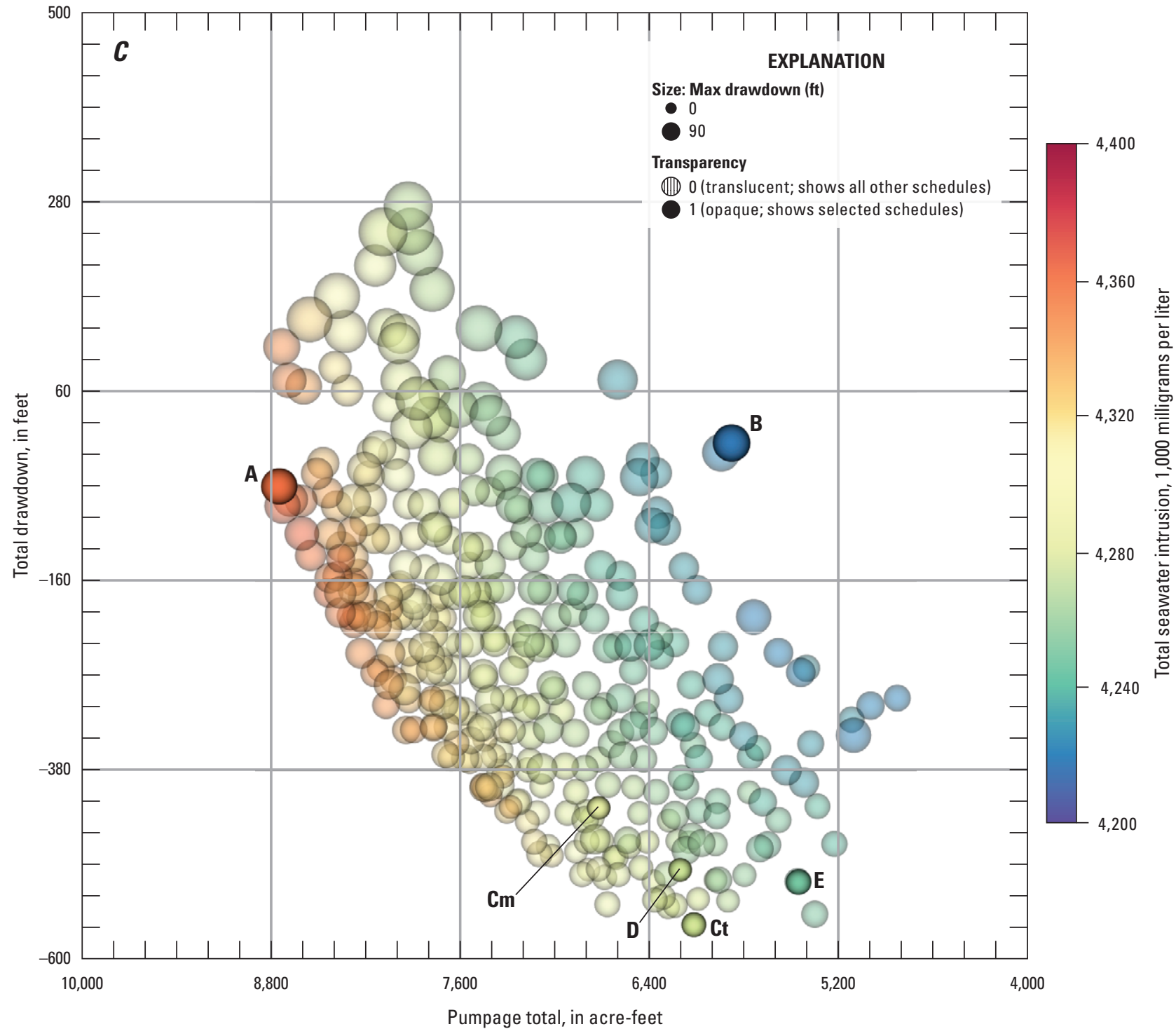

Figure 24. - Continued 
Table 5. Objective values for scenario 5A, Santa Barbara multi-objective management model, Santa Barbara, California. [acre-ft, acre-feet; ft, feet; mg/L, milligrams per liter]

\begin{tabular}{lcccc}
\hline Schedule & $\begin{array}{c}\text { Objective } \mathbf{A} \\
\text { total pumpage } \\
\text { (acre-ft) }\end{array}$ & $\begin{array}{c}\text { Objective } \mathbf{B} \\
\text { seawater intrusion } \\
(\mathbf{1 , 0 0 0} \mathbf{~ m g / L )}\end{array}$ & $\begin{array}{c}\text { Objective } \mathbf{C t} \\
\text { total drawdown } \\
\text { (ft) }\end{array}$ & $\begin{array}{c}\text { Objective Cm } \\
\text { maximum drawdown } \\
\text { (ft) }\end{array}$ \\
\hline $5 \mathrm{~A}_{\mathrm{A}}$ & 9,000 & 4,357 & 30 & 47 \\
$5 \mathrm{~A}_{\mathrm{B}}$ & 5,000 & 4,205 & -70 & 19 \\
$5 \mathrm{~A}_{\mathrm{Ct}}$ & 6,800 & 4,299 & -500 & 10 \\
$5 \mathrm{~A}_{\mathrm{Cm}}$ & 6,800 & 4,299 & -500 & 10 \\
$5 \mathrm{~A}_{\mathrm{D}}$ & 6,600 & 4,266 & -460 & 12 \\
$5 \mathrm{~A}_{\mathrm{E}}$ & 5,400 & 4,216 & -340 & 14 \\
\hline
\end{tabular}

Table 6. Objective values for scenario $5 B$, Santa Barbara multi-objective management model, Santa Barbara, California. [acre-ft, acre-feet; ft, feet; mg/L, milligrams per liter]

\begin{tabular}{lcccc}
\hline Schedule & $\begin{array}{c}\text { Objective } \mathbf{A} \\
\text { total pumpage } \\
\text { (acre-ft) }\end{array}$ & $\begin{array}{c}\text { Objective B } \\
\text { seawater intrusion } \\
(\mathbf{1 , 0 0 0 ~} \mathbf{~ m g} / \mathbf{L})\end{array}$ & $\begin{array}{c}\text { Objective } \mathbf{C t} \\
\text { total drawdown } \\
\text { (ft) }\end{array}$ & $\begin{array}{c}\text { Objective Cm } \\
\text { maximum drawdown } \\
\text { (ft) }\end{array}$ \\
\hline $5 \mathrm{~B}_{\mathrm{A}}$ & 9,000 & 4,355 & 220 & 62 \\
$5 \mathrm{~B}_{\mathrm{B}}$ & 5,300 & 4,214 & 60 & 22 \\
$5 \mathrm{~B}_{\mathrm{Ct}}$ & 5,900 & 4,263 & -520 & 9 \\
$5 \mathrm{~B}_{\mathrm{Cm}}$ & 5,800 & 4,254 & -460 & 7 \\
$5 \mathrm{~B}_{\mathrm{D}}$ & 6,700 & 4,279 & -490 & 10 \\
$5 \mathrm{~B}_{\mathrm{E}}$ & 5,700 & 4,260 & -490 & 7 \\
\hline
\end{tabular}

Table 7. Objective values for scenario 5C, Santa Barbara multi-objective management model, Santa Barbara, California. [acre-ft, acre-feet; ft, feet; mg/L, milligrams per liter]

\begin{tabular}{lcccc}
\hline Schedule & $\begin{array}{c}\text { Objective A } \\
\text { total pumpage } \\
\text { (acre-ft) }\end{array}$ & $\begin{array}{c}\text { Objective B } \\
\text { seawater intrusion } \\
(\mathbf{1 , 0 0 0 ~} \mathbf{~ m} / \mathbf{L})\end{array}$ & $\begin{array}{c}\text { Objective } \mathbf{C t} \\
\text { total drawdown } \\
\text { (ft) }\end{array}$ & $\begin{array}{c}\text { Objective } \mathbf{C m} \\
\text { maximum drawdown } \\
\text { (ft) }\end{array}$ \\
\hline $5 \mathrm{C}_{\mathrm{A}}$ & 8,700 & 4,358 & -50 & 38 \\
$5 \mathrm{C}_{\mathrm{B}}$ & 5,900 & 4,215 & 0 & 41 \\
$5 \mathrm{C}_{\mathrm{Ct}}$ & 6,100 & 4,275 & -560 & 9 \\
$5 \mathrm{C}_{\mathrm{Cm}}$ & 6,700 & 4,289 & -420 & 6 \\
$5 \mathrm{C}_{\mathrm{D}}$ & 6,200 & 4,273 & -490 & 6 \\
$5 \mathrm{C}_{\mathrm{E}}$ & 5,500 & 4,246 & -510 & 11 \\
\hline
\end{tabular}


For comparison among the three scenario- 5 cases, schedule $\mathrm{D}$, the best compromise among all four objectives, was selected from each case. The contours of simulated hydraulic heads for year two for the three cases are shown in figures 25-27 for the upper, middle, and lower producing zones, respectively (figure parts $A-C$ ). In general, the simulated contours for each producing zone were similar in form for scenarios $5 \mathrm{~A}-\mathrm{C}$, with the lowest water levels for all producing zones in Storage Unit I (figs. 25-27). The simulated hydraulic heads in Storage Unit I, however, were slightly lower for scenario 5B than for scenarios $5 \mathrm{~A}$ and $5 \mathrm{C}$. The differences were most obvious in the upper producing zone (figs. 25A, 26A, and 27A) of Storage Unit I and the lower producing zone of the Foothill groundwater basin.

The contours of simulated chloride concentrations at the end of year two for the three cases are shown in figure 28 for the middle and lower aquifers. The distribution of chloride was similar among the scenarios; however, the seawater intruded slightly farther inland for scenario $5 \mathrm{~B}$ than for scenarios $5 \mathrm{~A}$ and $5 \mathrm{C}$.

The total quarterly pumpage by storage unit for schedules A (maximize pumping) and B (minimize seawater intrusion) of scenario 5 is presented in figure 29. In general, when maximizing the pumpage, a greater percentage of the total pumpage was extracted from Storage Unit I. However, when minimizing seawater intrusion, a greater percentage of the total pumpage was sometimes from the Foothill groundwater basin in quarters 2,3 , and 4 (see, for example, quarter 3 and 4 in fig. 29A).

For scenario 5, the SOS illustrated different tradeoff dynamics depending on the climatic condition. Figure 30 shows the SOS for the six pumping schedules of scenarios $5 \mathrm{~A}-\mathrm{C}$. The tradeoff dynamics for schedule $\mathrm{D}$ were similar for all three climatic conditions $\left(5 A_{D}, 5 B_{D}\right.$, and $\left.5 C_{D}\right)$. Schedule $E$ shows the relative tradeoff in pumpage for each climatic condition when prioritizing the three environmental objectives compared to an equal preference for minimizing all objectives in schedule D.

For scenario 5A (typical-to-dry), the extreme solutions for maximizing pumpage and minimizing seawater intrusion showed substantial tradeoffs: the best score for objective A $\left(5 \mathrm{~A}_{\mathrm{A}}\right)$ resulted in the worst score for objective $\mathrm{B}$, and the best score for objective $B\left(5 A_{B}\right)$ resulted in the worst score for objective $\mathrm{A}$, indicating that maximizing pumpage resulted in maximum seawater intrusion, and minimal seawater intrusion resulted in minimal pumpage (fig. 30A). The solution for minimizing total drawdown, schedule $5 \mathrm{~A}_{\mathrm{Ct}}$ was the same as $5 \mathrm{~A}_{\mathrm{Cm}}$ for this climatic condition, indicating that the solution that resulted in the minimal total drawdown also resulted in the minimal maximum drawdown (fig. 30A). Comparing the compromise solutions $\left(5 \mathrm{~A}_{\mathrm{D}}\right.$ and $\left.5 \mathrm{~A}_{\mathrm{E}}\right)$ showed that the objective $\mathrm{B}$ score for schedule $5 \mathrm{~A}_{\mathrm{E}}$ increased as its objective $\mathrm{A}, \mathrm{C}_{\mathrm{t}}$, and $\mathrm{C}_{\mathrm{m}}$ scores decreased, indicating that to improve the seawater-intrusion results from schedule $\mathrm{D}$, one must accept slightly worse drawdown results.

In scenario 5B (dry-to-typical), the maximum pumpage solution (schedule $5 \mathrm{~B}_{\mathrm{A}}$ ) had scores below 0.3 for all other objectives; however, none were zero, indicating that there were worse solutions for each objective that also resulted in less pumpage (fig. 30B). Similarly, the minimal seawaterintrusion solution (schedule $5 \mathrm{~B}_{\mathrm{B}}$ ) did not result in the least pumpage, although the score was less than 0.1. Both solutions for the drawdown objectives $\left(5 \mathrm{~B}_{\mathrm{Ct}}\right.$ and $5 \mathrm{~B}_{\mathrm{Cm}}$, fig. $\left.30 B\right)$ showed a larger tradeoff for objective $\mathrm{A}$ (scores below 0.2) than objective B (scores between 0.6 and 0.8 ). To achieve the minimal total drawdown (schedule $5 \mathrm{~B}_{\mathrm{CC}}$ ), there was a slightly larger tradeoff in objective $\mathrm{B}$ and a slightly smaller tradeoff in objective A compared to the minimum maximum drawdown (schedule $5 \mathrm{~B}_{\mathrm{Cm}}$ ), indicating that the solution that minimized the maximum drawdown resulted in less seawater intrusion and less pumpage than schedule $5 \mathrm{~B}_{\mathrm{Ct}}$. The compromise solutions (schedules $5 \mathrm{~B}_{\mathrm{D}}$ and $5 \mathrm{~B}_{\mathrm{E}}$ ) resulted in scores greater than 0.9 for the drawdown objectives with scores less than 0.7 for objective $\mathrm{B}$ and less than 0.4 for objective A. Between schedules $5 \mathrm{~B}_{\mathrm{D}}$ and $5 \mathrm{~B}_{\mathrm{E}}$, a small tradeoff in maximum drawdown resulted in more pumpage, but more seawater intrusion as well.

For the extreme solutions in the dry-to-dry climate of scenario $5 \mathrm{C}$, schedule $5 \mathrm{C}_{\mathrm{A}}$ had a score near zero for objective $\mathrm{B}$, yet the minimum seawater-intrusion solution (schedule $5 \mathrm{C}_{\mathrm{B}}$ ) had a score greater than 0.2 for objective $\mathrm{A}$ (fig. 30C). These scores indicated that there were few solutions that maximized pumpage that resulted in more seawater intrusion (schedule $5 \mathrm{C}_{\mathrm{A}}$ ), whereas there were relatively more solutions that increased seawater intrusion, but had less pumpage (schedule $5 \mathrm{C}_{\mathrm{B}}$ ). For the drawdown objectives, the results indicated that Schedule $5 \mathrm{C}_{\mathrm{Cm}}$ allowed more pumpage than schedule $5 \mathrm{C}_{\mathrm{Ct}}$, but with more seawater intrusion and total drawdown. For the compromise solutions (schedules $5 \mathrm{C}_{\mathrm{D}}$ and $5 \mathrm{C}_{\mathrm{E}}$ ), the results indicated that schedule $5 \mathrm{C}_{\mathrm{E}}$ allowed less seawater intrusion than schedule $5 \mathrm{C}_{\mathrm{D}}$ with less pumpage, slightly less total drawdown, and a slightly greater maximum drawdown.

The simulated breakthrough curves for scenarios $5 \mathrm{~A}-\mathrm{C}$, assuming schedule-D pumpage, are shown in figure 31. Much like the results presented in tables 5-7, the flat breakthrough curves indicated that the chloride concentrations were fairly insensitive to pumping schedule, except for well 23E5, which had the largest increase in concentration (fig. 31). The increase in simulated chloride concentrations in well 23E5 for schedule $5 \mathrm{~B}_{\mathrm{D}}$ (fig. $31 \mathrm{~B}$ ) reflected the greater total pumpage for that scenario than for schedules $5 \mathrm{~A}_{\mathrm{D}}$ and $5 \mathrm{C}_{\mathrm{D}}$ (tables 5-7). The chloride breakthroughs for scenario 5B (fig. $31 B$ ) were noticeably different than the other two climatic conditions in the second year, where typical precipitation was simulated. 


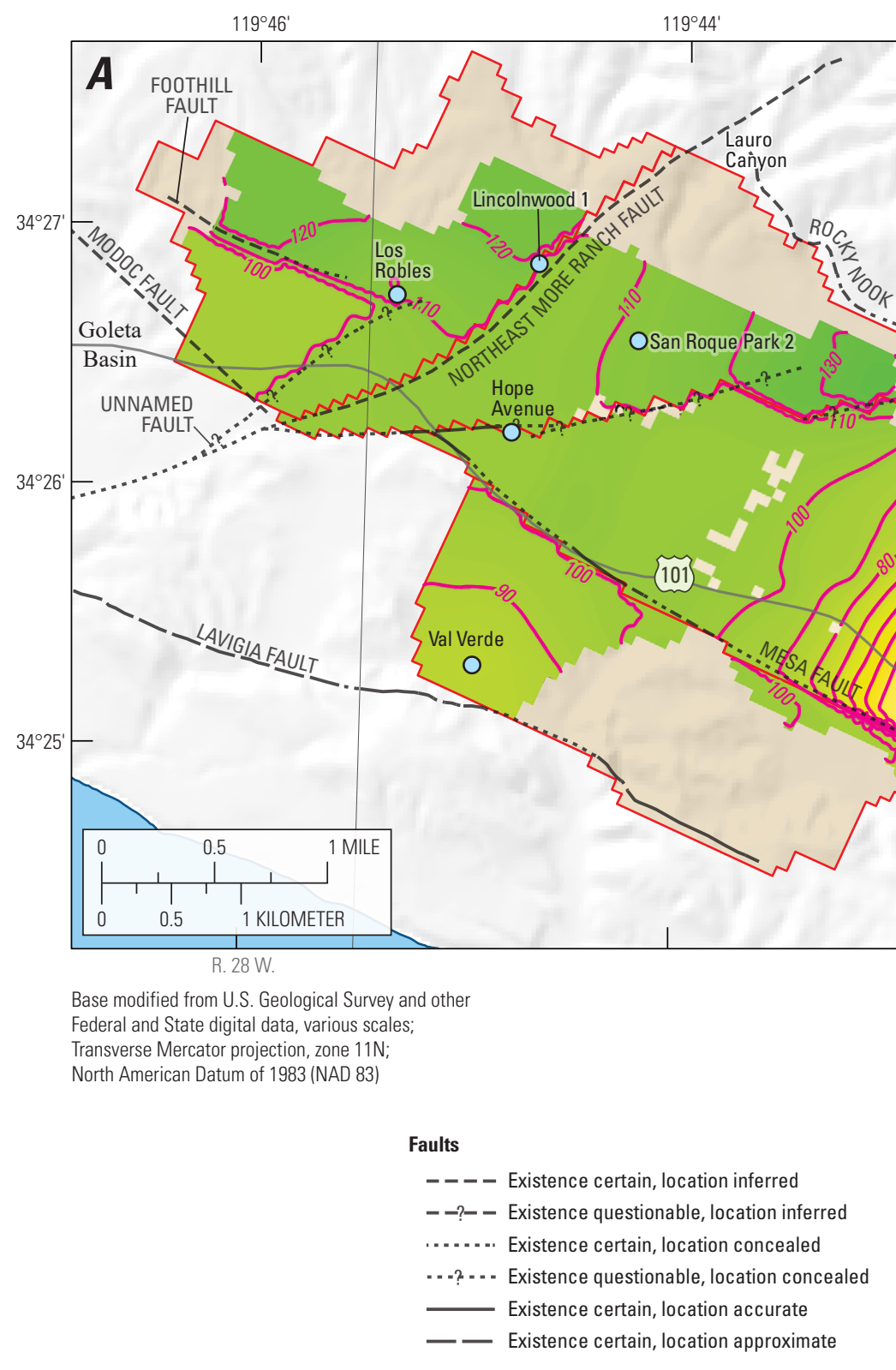

\section{SANTA YNEZ MOUNTAINS}

\section{Mission}

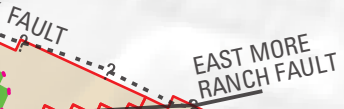

EAST MORE
RANCHFAUL

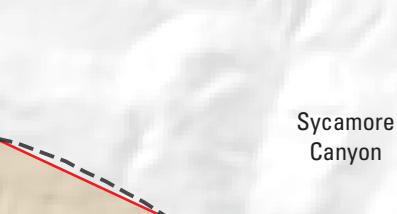

$19^{\circ} 40^{\prime}$

Figure 25. Contours of simulated hydraulic heads for year two, Santa Barbara multi-objective management model, Santa Barbara, California, for pumping schedule $5 A_{0}$ : $A$, upper producing zone; $B$, middle producing zone; and $C$, lower producing zone. 


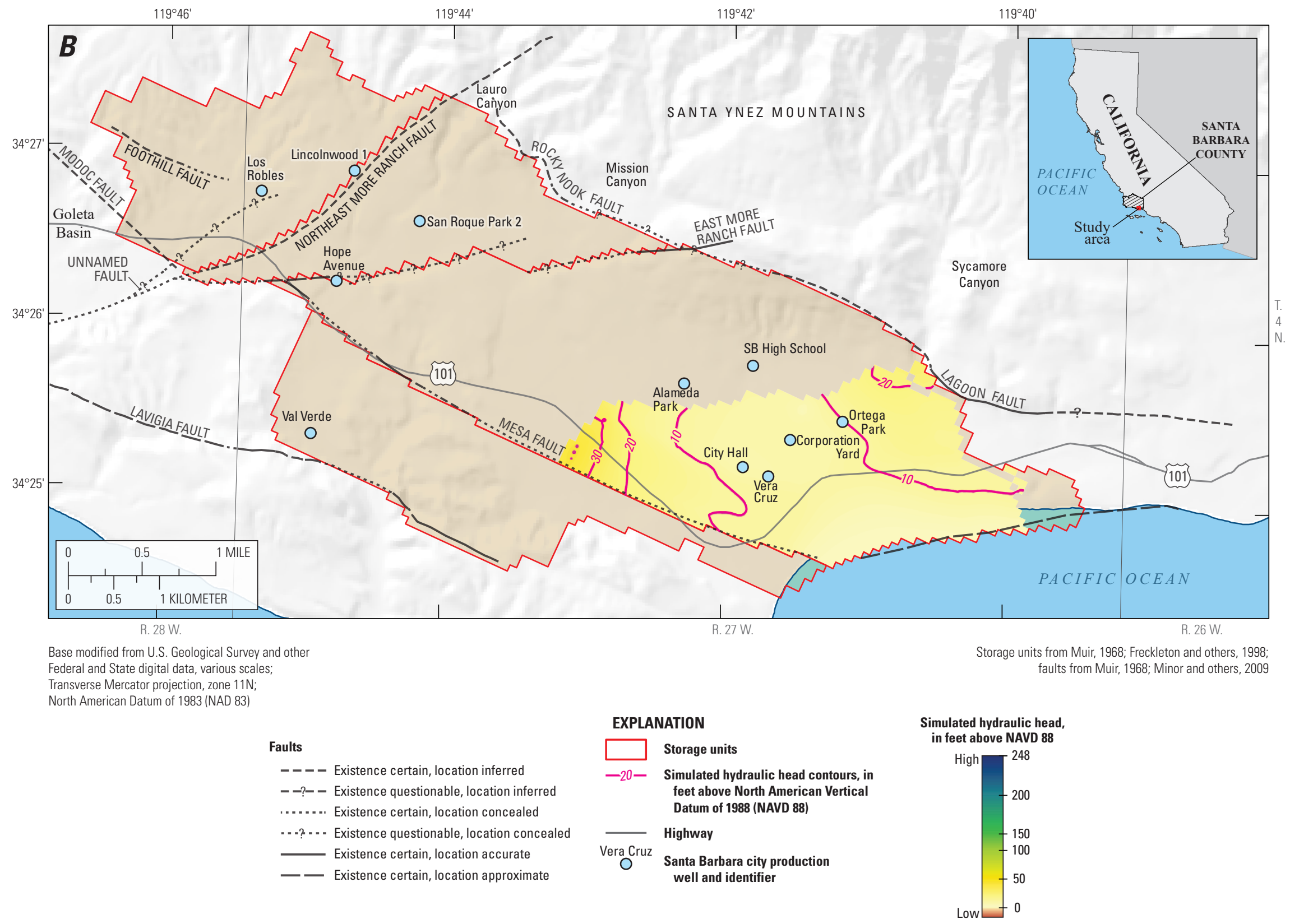

Figure 25. - Continued 


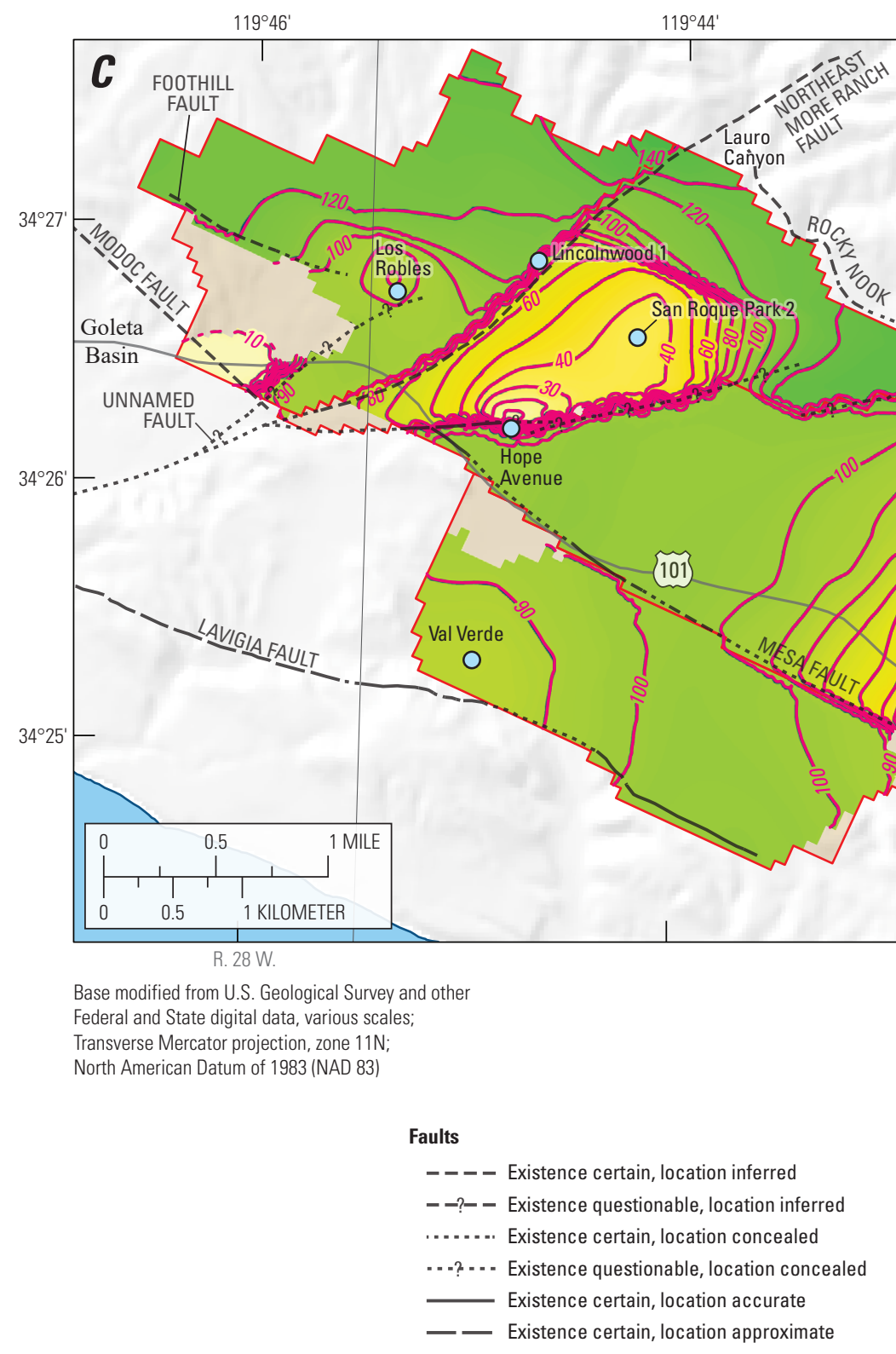

$119^{\circ} 42^{\prime}$

$119^{\circ} 40^{\prime}$

SANTA YNEZ MOUNTAINS

Mission

FAULT

EAST MORE
RANCHFAULT

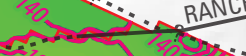

3
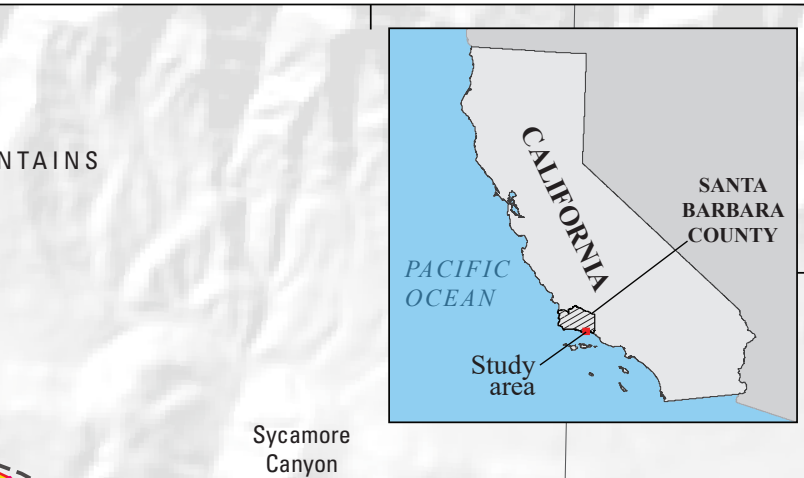

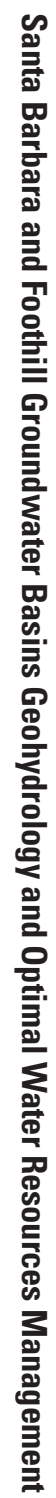

Figure 25. - Continued

EXPLANATION

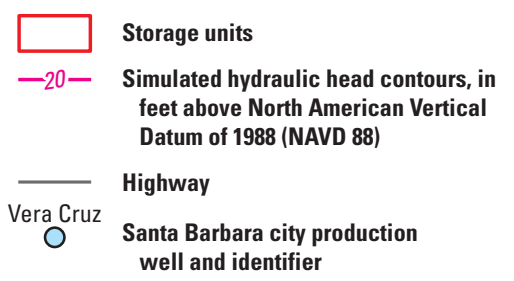

in feet above NAVD 88

High 248

-200
-150
-100
-50
-0

well and identifier

PACIFIC OCEAN

Simulated hydraulic head,

Storage units from Muir, 1968; Freckleton and others, 1998; faults from Muir, 1968; Minor and others, 2009 


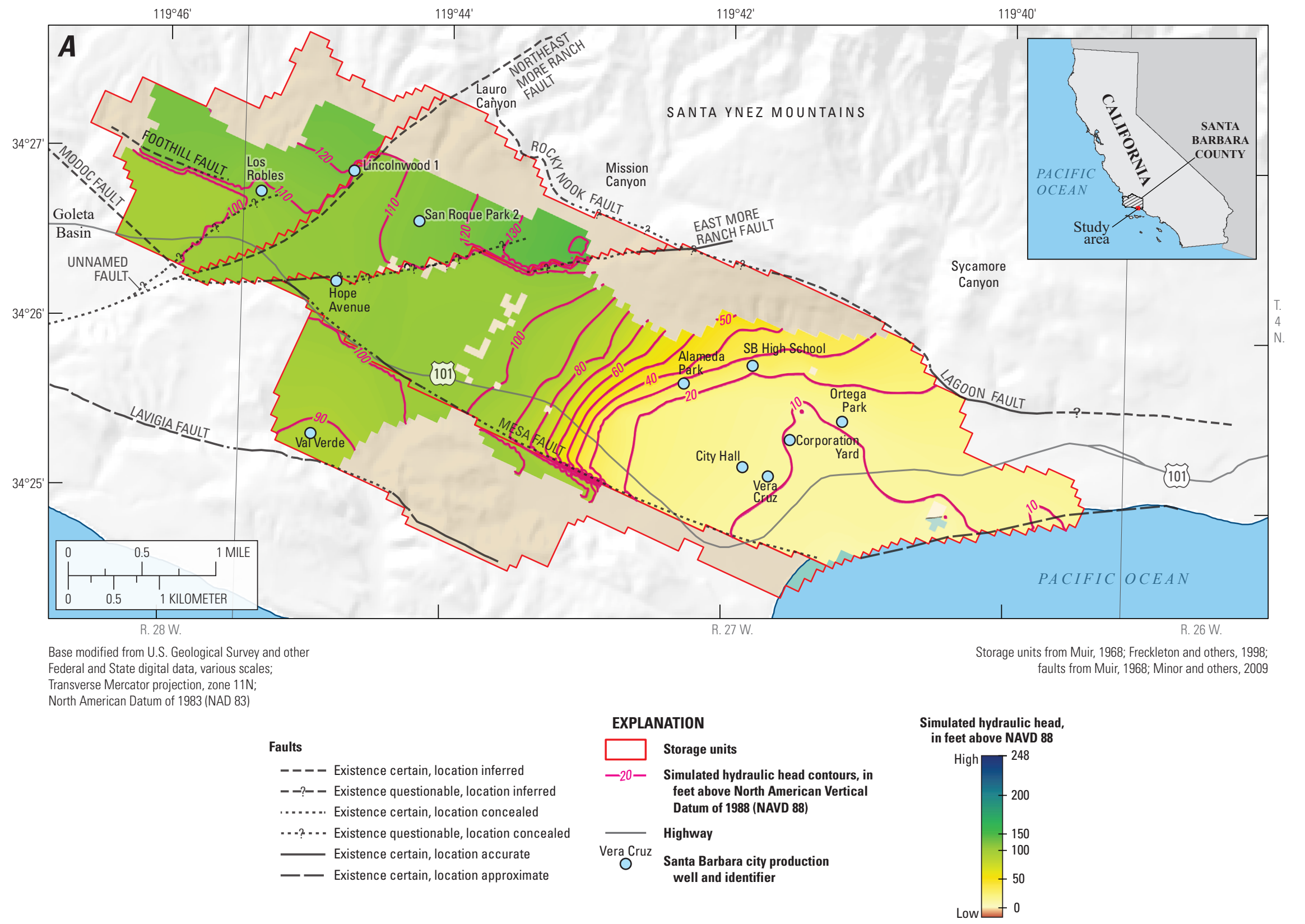

Figure 26. Contours of simulated hydraulic heads for year two, Santa Barbara multi-objective management model, Santa Barbara, California, for pumping schedule $5 \mathrm{~B}_{0}$ : $A$, upper producing zone; $B$, middle producing zone; and $C$, lower producing zone. 


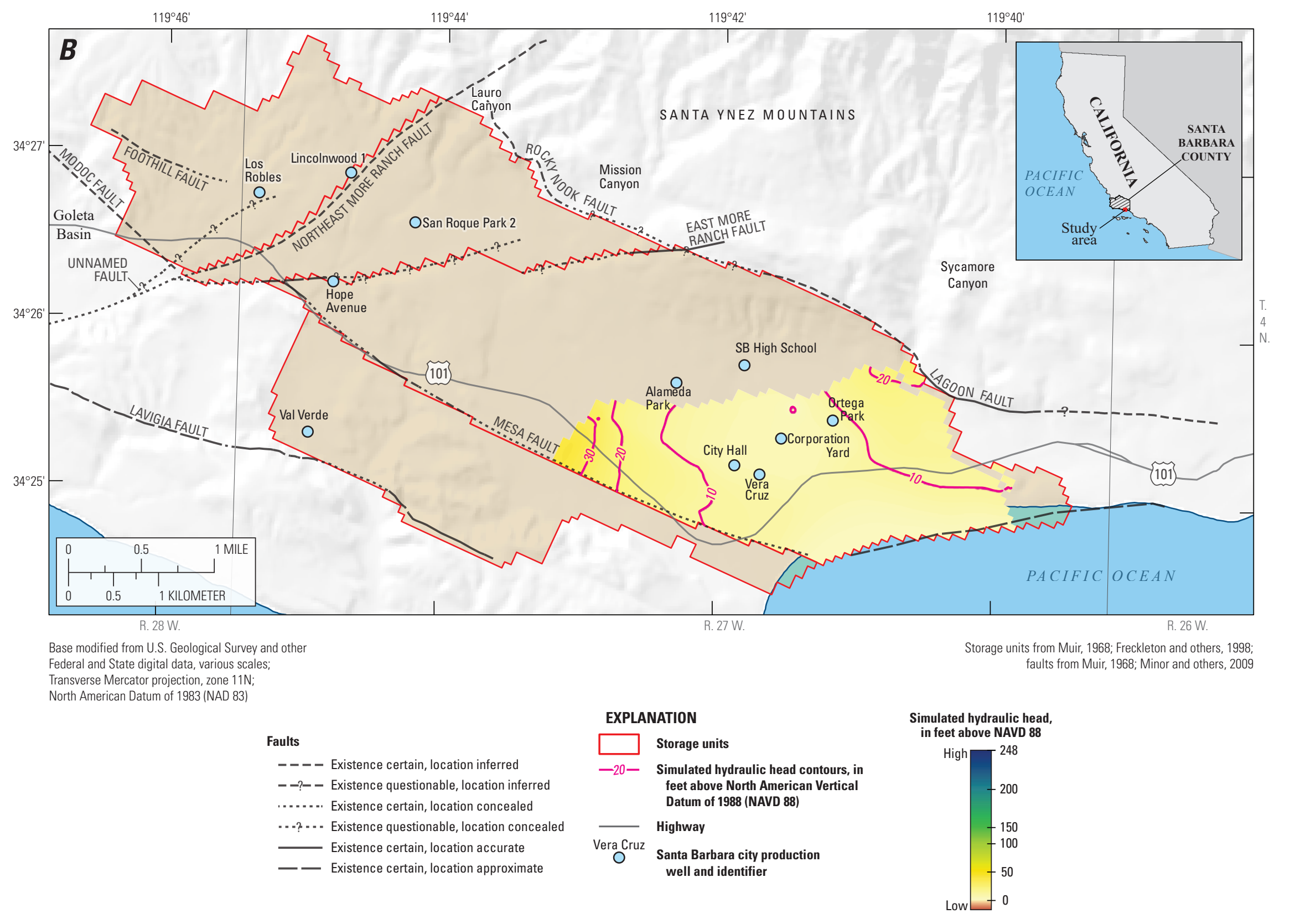

Figure 26. - Continued 


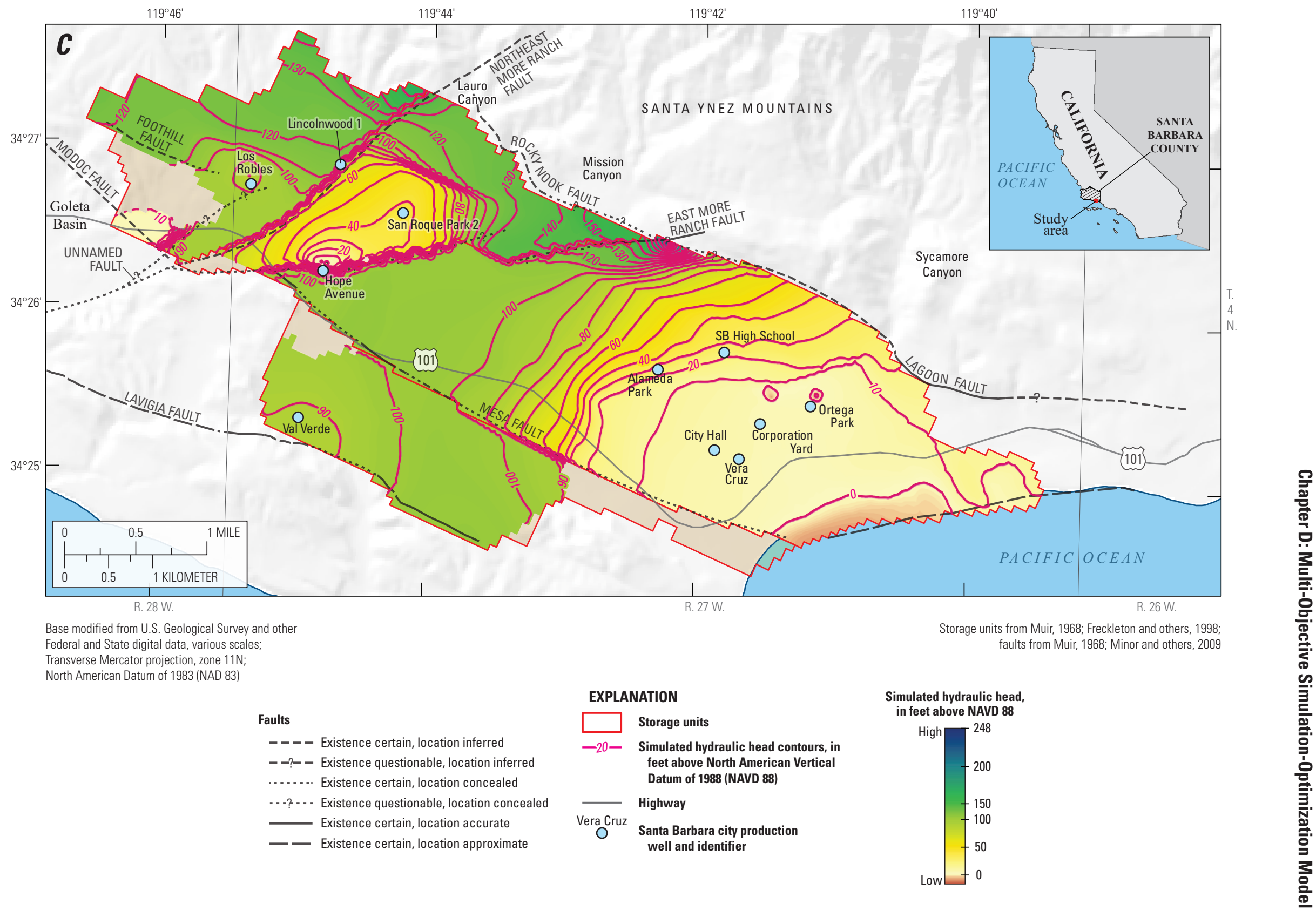

Figure 26. - Continued 


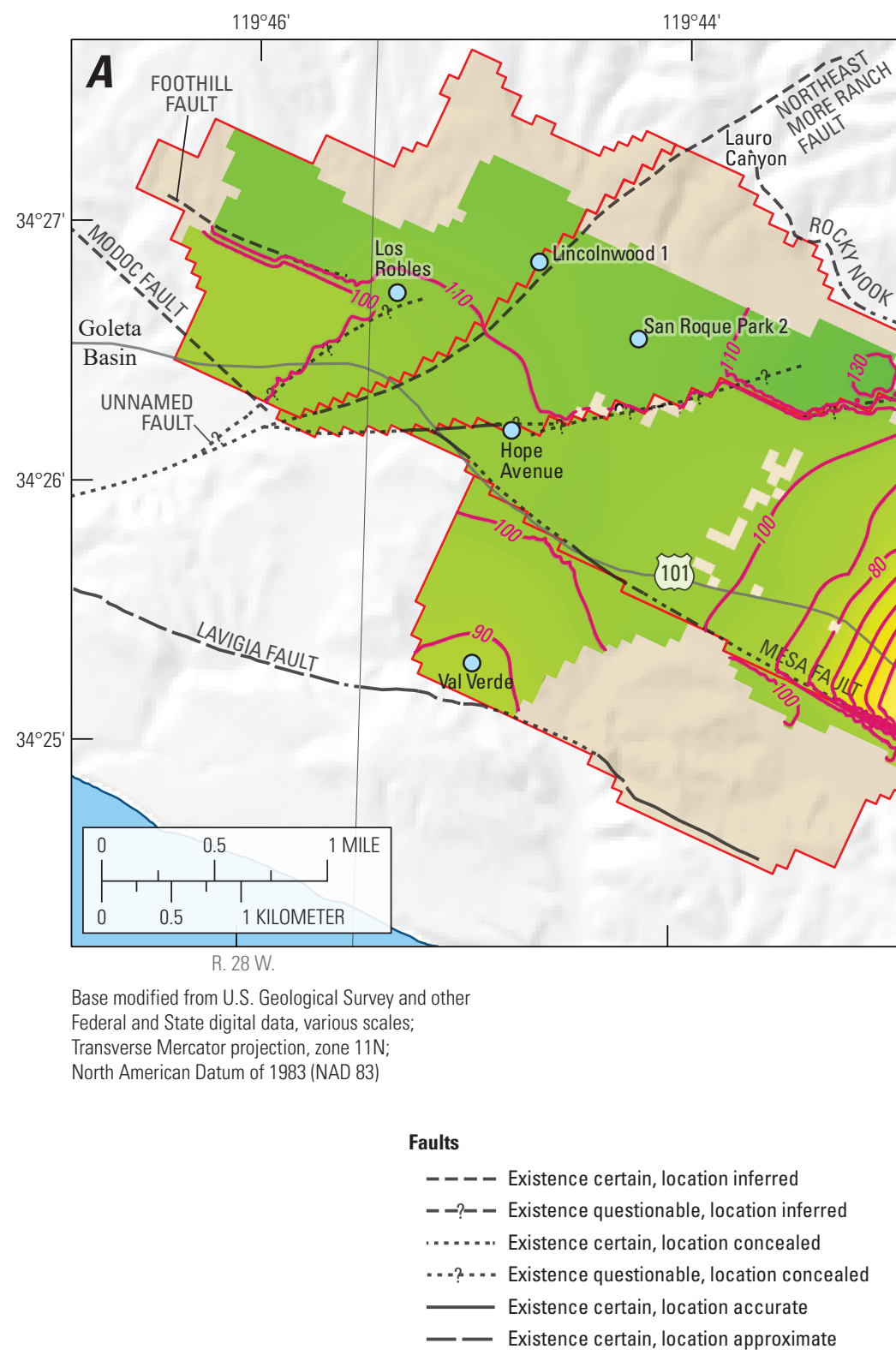

\section{SANTA YNEZ MOUNTAINS}

\section{Mission} FAULT

-..2. EASTMORE

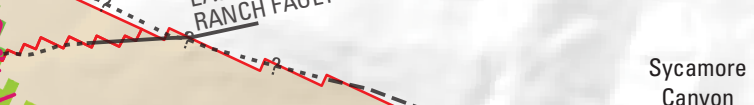

Figure 27. Contours of simulated hydraulic heads for year two, Santa Barbara multi-objective management model, Santa Barbara, California, for pumping schedule 5C : $A$, upper producing zone; $B$, middle producing zone; and $C$, lower producing zone. 


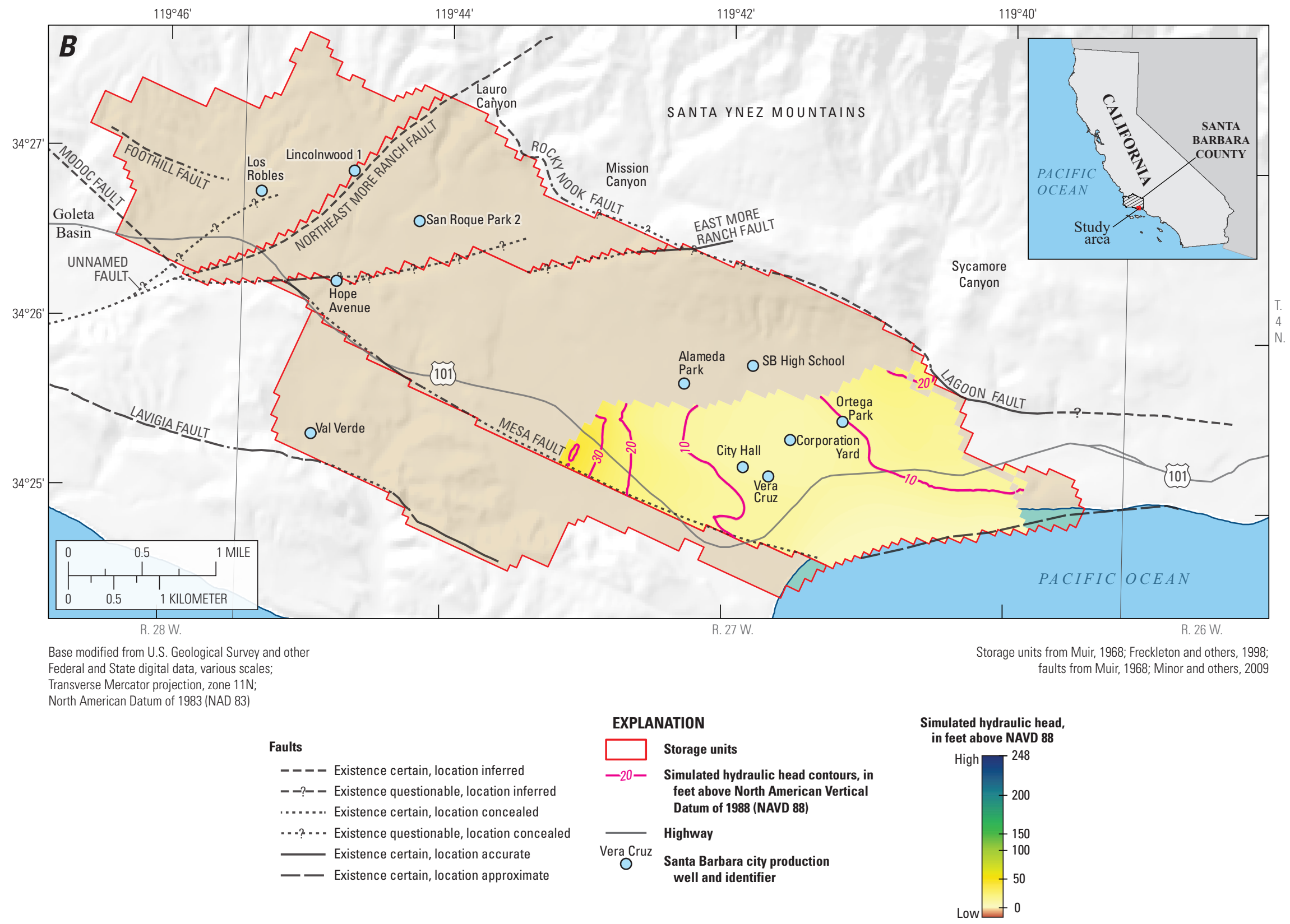

Figure 27. - Continued 


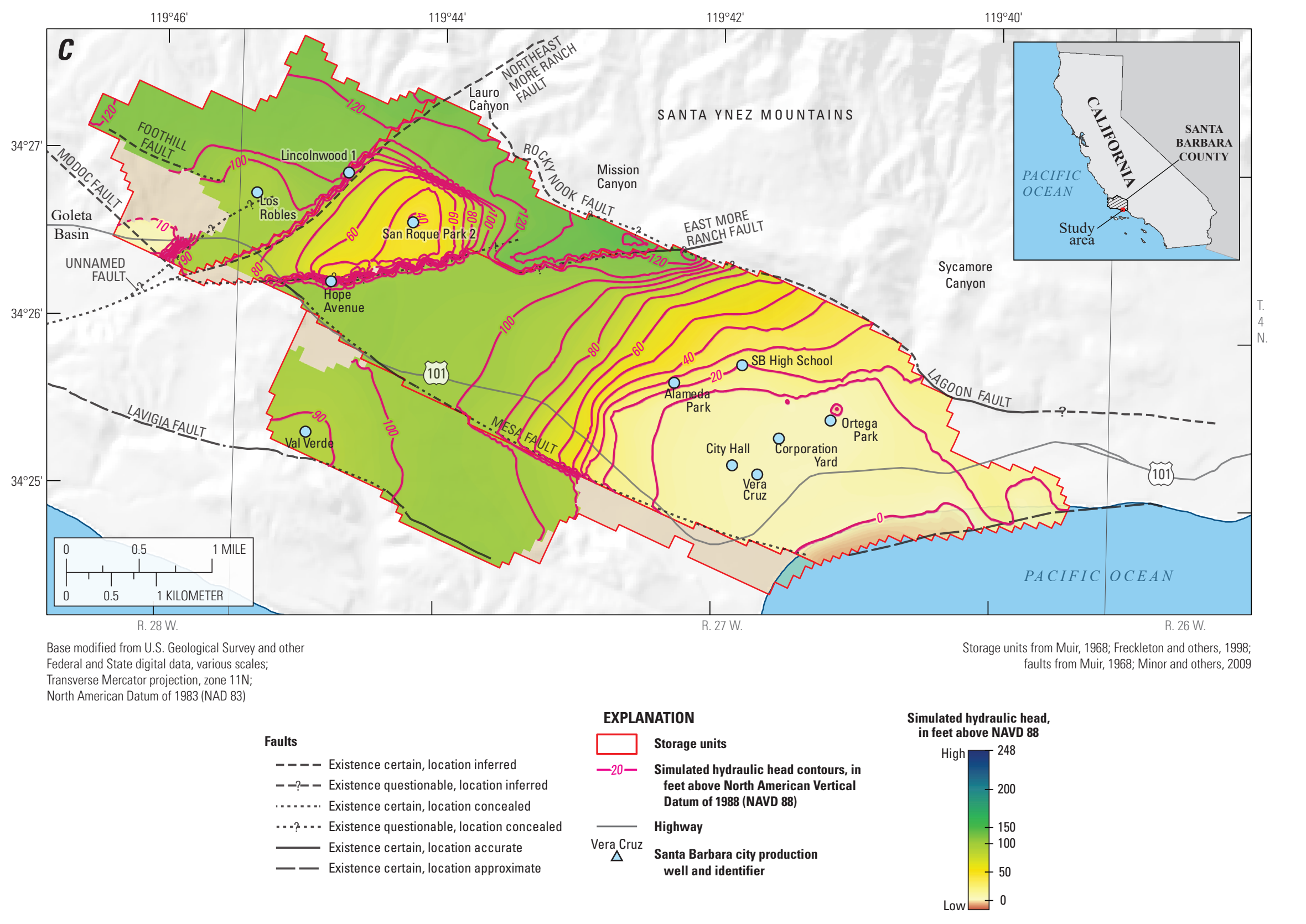

Figure 27. - Continued 

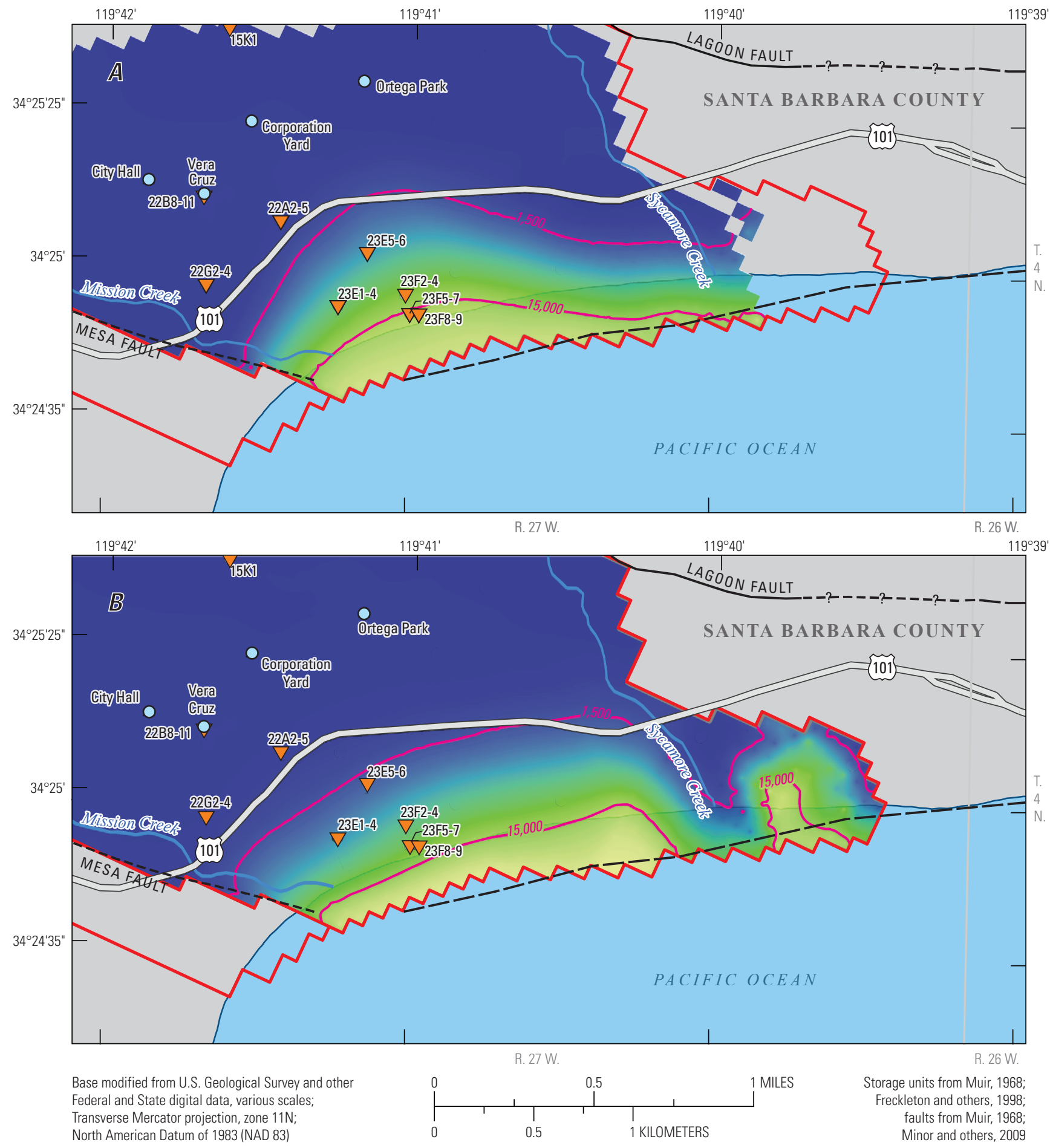
North American Datum of 1983 (NAD 83)

\section{EXPLANATION}

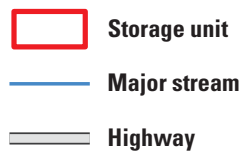

Simulated chloride concentration, in milligrams per liter High

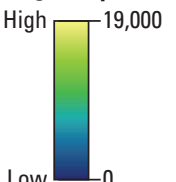

Figure 28. Contours of simulated chloride concentrations for year two, Santa Barbara multi-objective management model, Santa Barbara, California for pumping schedule: $A, 5 \mathrm{~A}_{0}$ in the middle producing zone; $B, 5 \mathrm{~A}_{0}$ in the lower producing zone; $C, 5 \mathrm{~B}_{0}$ in the middle producing zone; $D, 5 \mathrm{~B}_{0}$ in the lower producing zone; $E, 5 C_{D}$ in the middle producing zone; and $F, 5 C_{D}$ in the lower producing zone. 

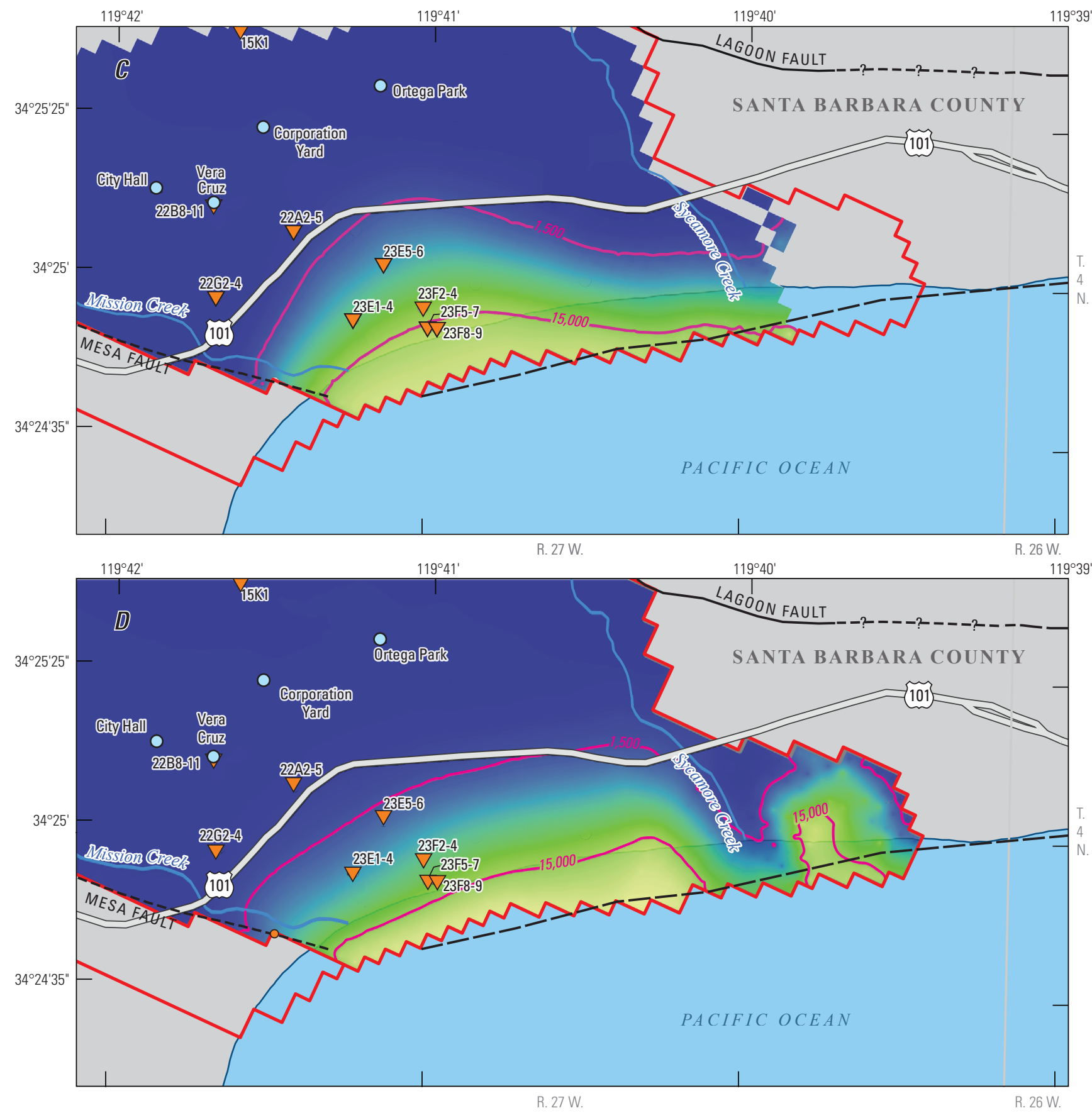

Base modified from U.S. Geological Survey and other Federal and State digital data, various scales; Transverse Mercator projection, zone 11N; North American Datum of 1983 (NAD 83)

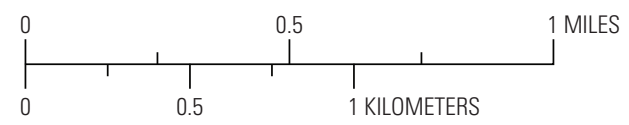

Storage units from Muir, 1968 Freckleton and others, 1998 faults from Muir, 1968 Minor and others, 2009

EXPLANATION

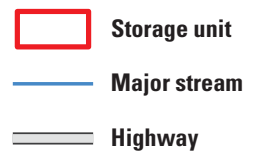

Simulated chloride concentration, in milligrams per liter

- - - - Existence certain, location inferred

- -? - Existence questionable, location inferred Existence certain, location accurate —— Existence certain, location approximate

Figure 28. - Continued 

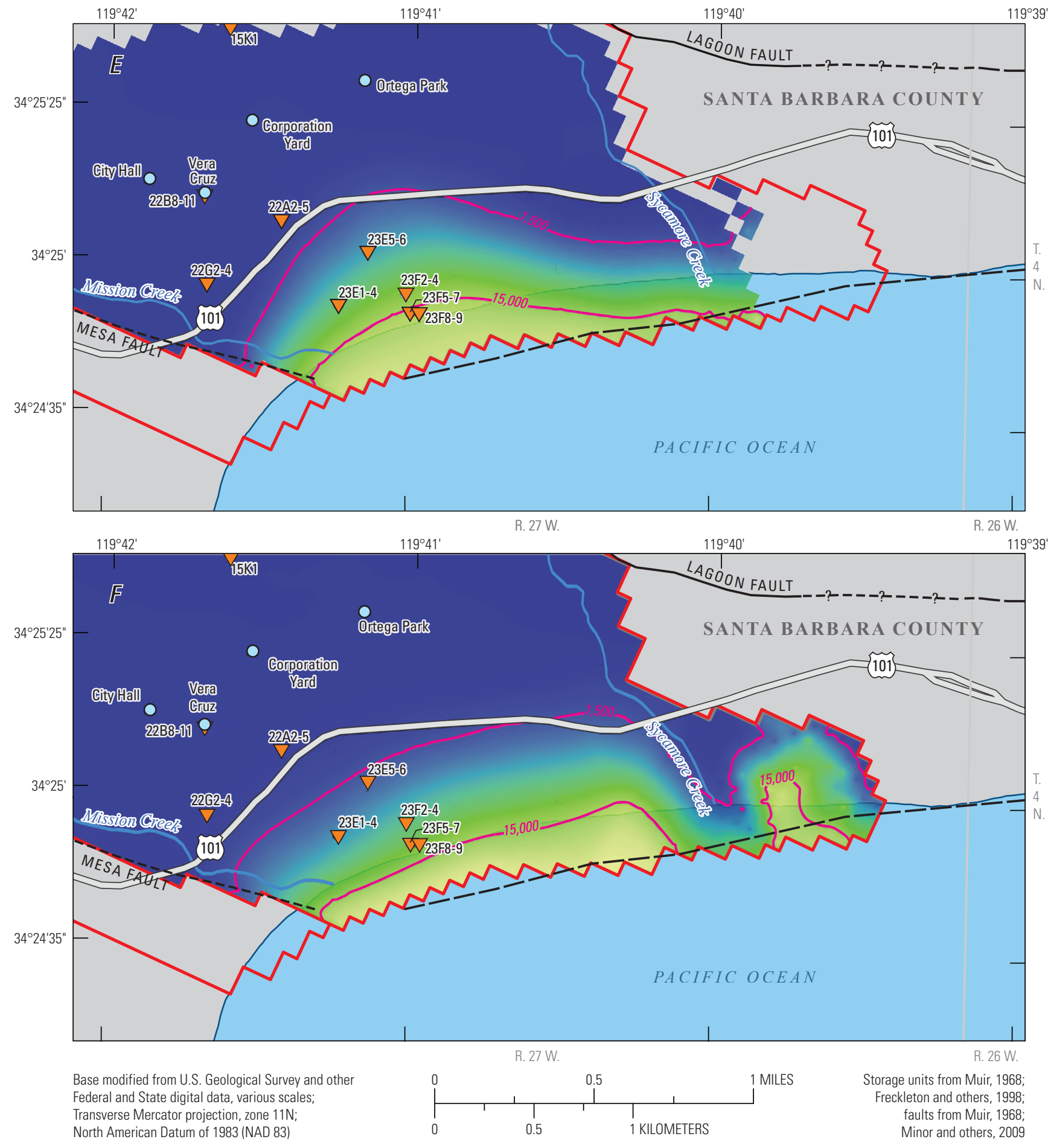
North American Datum of 1983 (NAD 83

EXPLANATION

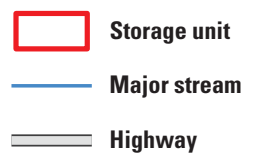

Simulated chloride concentration, in milligrams per liter High 19,000 - -? - Existence questionable, location inferred Existence certain, location accurate —— Existence certain, location approximate 

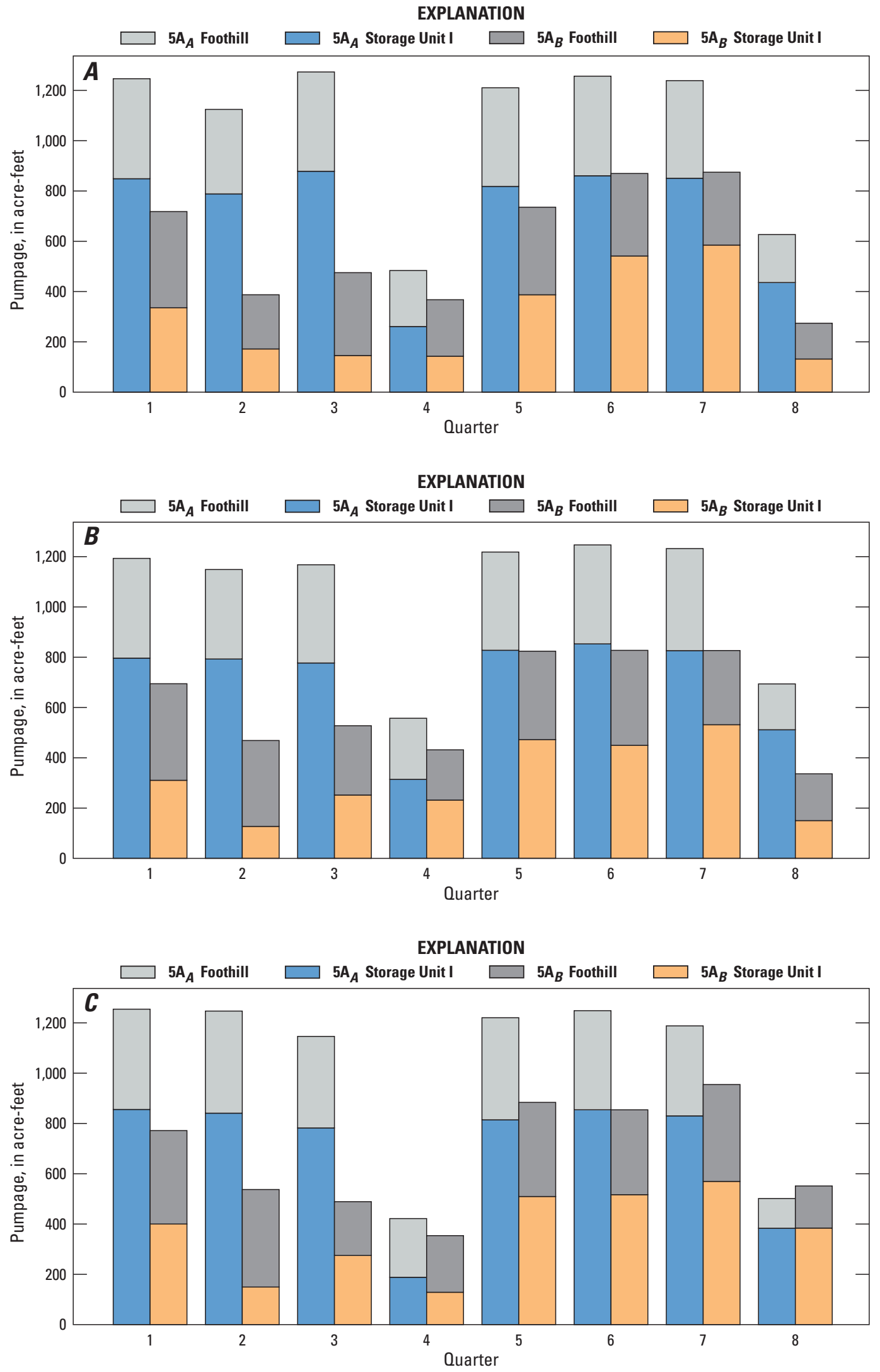

Figure 29. Pumping schedules $A$ and $B$ (maximum pumpage and minimum seawater intrusion, respectively), Storage Unit I and Foothill groundwater basin, Santa Barbara multi-objective management model, Santa Barbara, California, for scenarios $A, 5 \mathrm{~A} ; B, 5 \mathrm{~B}$; and $C, 5 \mathrm{C}$. 

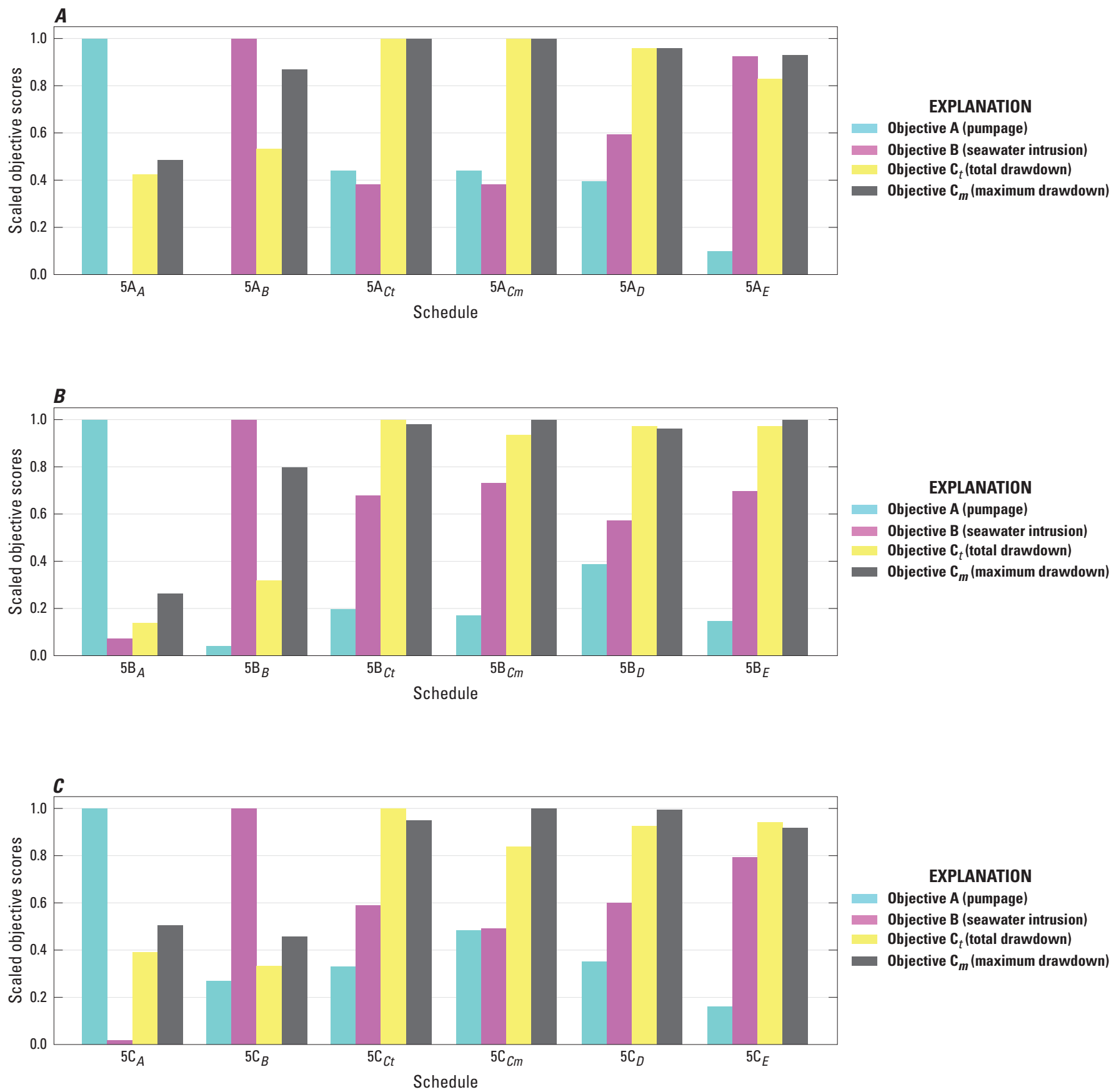

Figure 30. Scaled objective scores by scenario 5 pumping schedule used in the Santa Barbara multi-objective management model, Santa Barbara, California: $A, 5 \mathrm{~A} ; B, 5 \mathrm{~B}$; and $C, 5 \mathrm{C}$. 

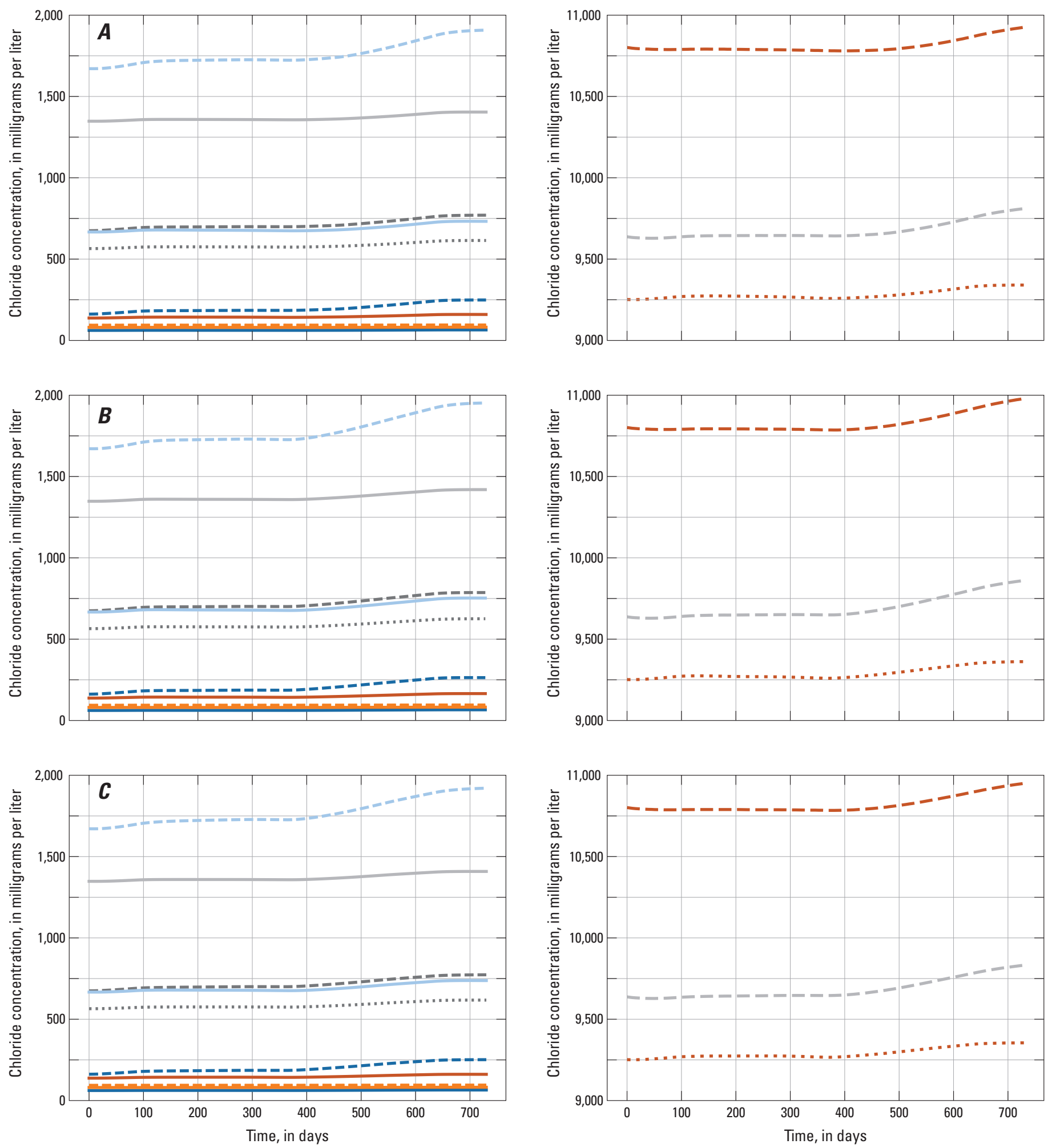

EXPLANATION

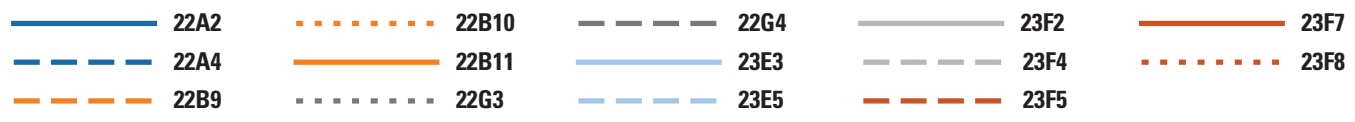

Figure 31. Breakthrough curves of simulated chloride concentrations for selected monitoring wells, Santa Barbara multi-objective management model, Santa Barbara, California, for $A$, schedule $5 A_{D} ; B$, schedule $5 B_{D}$; and $C$, schedule $5 C_{D}$. 


\section{Limitations}

The simulation-optimization model described in this chapter has limitations because the underlying SEAWAT model is an approximation of reality, future precipitation is uncertain, and the optimization technique cannot guarantee a global optimum. The limitations of the simulation model are discussed in chapter $\mathrm{C}$ and include uncertainties in the stresses, hydraulic parameters, and measured data and various simplifying assumptions made during the modeling process. The implications of these simulation-model limitations for the optimization results were not examined and were beyond the scope of the study. The assumed climatic conditions were intended to span a large range of precipitation, which accounts for some uncertainty in rainfall data, but the optimization necessarily assumes the simulation is an adequate representation of the natural system. Given the inherent limitations of the simulation and the necessary simplifications in the problem formulation - for example, aggregation of monthly pumping into quarterly pumping - the optimization problem is one for which a global solution can only be approximated.

Undoubtedly, future precipitation could vary and not match the values used in any of the scenarios; therefore, the Pareto-optimal results only represent an empirical range of pumpage given specific climatic patterns. Although the Pareto-optimal pumping schedules can be adjusted (by scaling all values by a constant fraction) to meet demand, the corresponding system states cannot be scaled accordingly. It is safe to assume, however, that if a chosen Pareto-optimal pumping schedule produced acceptable seawater intrusion and drawdowns, scaling down the pumpage would not increase these effects to unacceptable levels. It must also be assumed that no additional stress to the system is incurred during the simulation period. Pareto-optimal results might no longer be optimal if an additional well (unaccounted for in the model) began pumping.

Although Borg was selected as the optimization algorithm partly because of its superior performance avoiding common EA perils (such as search stagnation in local optima), a global optimum can never be assured, even given a large number of simulation runs. If the optimization were allowed to run infinitely, slightly different Pareto-optimal solutions could be produced. The overall approximate Pareto fronts produced by Borg were monitored, and once no differences were observed for several generations of the algorithm, the optimization was determined to be complete. Exact values (as opposed to general magnitudes and trends) for schedules on the Pareto front are not ultimate answers to any problem. Instead, they represent what is feasible and optimal with respect to the problem formulation and the simulation model.

An additional limitation involves utilizing the optimal results for the entire management horizon. The last few stress periods of any scenario can show increased pumpage strictly because there was no further restriction on system state (water levels and chloride concentrations) beyond the end of the simulation (final condition). In other words, in a 10 -year simulation, there is no year 11 ; therefore, pumpage can increase in year 10 until all of the constraints become binding. This can leave the system in a poor state moving forward, and seawater intrusion, for example, could continue increasing. The rates of seawater intrusion or water-level declines were not considered. Additionally, the first year of the optimal schedule can be considerably influenced by the initial condition. To account for changes to and uncertainty in the initial condition, the first year of the pumping schedules could also be discarded. Consequently, the middle 8 years of any 10-year optimization result is more reliable for planning and management guidance. Similar limitations are present in the 2-year simulations of scenario 5; however, it is difficult to determine the effects of the initial and final conditions because of the short management horizon.

\section{Summary and Conclusions}

The city of Santa Barbara is interested in identifying management strategies that maximize the available groundwater resources, while limiting seawater intrusion and groundwater drawdown. In order to identify the best management strategies, the U.S. Geological Survey in cooperation with the city of Santa Barbara undertook a simulation-optimization study that used the Santa Barbara flow and solute-transport model, described in chapter $\mathrm{C}$ of this report, to obtain optimal management strategies and estimate maximum pumping rates for a range of potential future climatic conditions.

The simulation-optimization problem was formulated as a multi-objective optimization problem and was solved using the Borg multi-objective evolutionary algorithm (MOEA). Borg was chosen as the optimization algorithm for this study for several reasons: (1) it is very computationally efficient; (2) it can run in parallel; (3) it requires little user input; and (4) it can solve for multiple competing objectives. The first three points allow the algorithm to proceed toward the optimal solutions at the fastest possible rate. The fourth point is advantageous for large, complex optimization problems because it is difficult to formulate the optimization problem in a way that produces only one optimal solution.

Results for each optimization are presented in Paretooptimal surfaces in four objective dimensions: objective A sought to maximize total pumpage; objective $\mathrm{B}$ sought to minimize seawater intrusion; objective $\mathrm{C}_{t}$ sought to minimize total drawdown; and objective $\mathrm{C}_{\mathrm{m}}$ sought to minimize the maximum drawdown. The decision variables were quarterly pumpage for 11 production wells. Strict optimization constraints were pumping capacities for each well implemented as variable bounds. Additional constraints were drinking-water-quality standards for chloride concentration at production wells, minimum-head specifications, and minimum flow to the Ortega groundwater-treatment plant. 
Five scenarios were designed to assess the optimization results with respect to an uncertain future climatic condition. Scenario 1 was an optimization of a 10 -year simulation assuming a typical climatic condition. Scenario 2 was an optimization of the same 10-year simulation model as scenario 1 but assumed a dry climatic condition to replicate a 10-year drought. Scenario 3 was designed to determine the potential for aquifer recovery if pumpage was limited to the minimum flow required to supply the Ortega groundwatertreatment plant. Scenario 4 was presented to create decisionrule curves from the database of scenario- 1 and scenario-2 optimization results. These decision-rule curves allow water managers to adapt pumping schedules on the basis of chloride concentrations or drawdown measurements at select monitoring wells. Scenario 5 was an optimization of a 2-year simulation under three different climatic conditions: typicalto-dry (scenario 5A), dry-to-typical (scenario 5B), and dry-todry (scenario 5C). Scenario 5 demonstrated the sensitivity of Pareto-optimal results to short-term climatic conditions.

For each Pareto optimal solution in scenarios 1, 2, and 5, a pumping schedule, a set of chloride breakthrough curves, and water-level contours were produced. Six pumping schedules (A, B, $\mathrm{C}_{\mathrm{t}}, \mathrm{C}_{\mathrm{m}}, \mathrm{D}$, and $\left.\mathrm{E}\right)$ were identified in each Pareto-optimal set to illustrate the tradeoffs among the four objectives. The goals of pumping schedules A-E were to maximize pumpage; minimize seawater intrusion; minimize total drawdown; minimize maximum drawdown; achieve the best overall compromise among the four objectives; and achieve the best compromise among objectives $\mathrm{B}, \mathrm{C}_{\mathrm{t}}$, and $\mathrm{C}_{\mathrm{m}}$.

The results of scenarios 1 and 2 presented the tradeoffs among the four conflicting objectives for a 10 -year horizon. The maximum yield of scenario 1 (schedule $1_{\mathrm{A}}$ ) was 31,300 acre-feet (acre-ft); that of scenario 2 (schedule 2 ) was 30,000 acre-ft. These schedules are not necessarily preferable, but identify the extent to which the basins can be pumped. The best compromises among all objectives for the two climatic conditions were schedules $1_{D}$ and $2_{D}$. Schedule $1_{\mathrm{D}}$ represents optimal pumping schedules, assuming typical climatic conditions, and resulted in an average yield of 2,558 acre-feet per year (acre-ft/yr), ranging from 1,829 to 3,418 acre-ft/yr. Schedule 2 represents optimal pumping schedules, assuming dry climatic conditions, and resulted in an average yield of 2,623 acre-ft/yr, ranging from 1,617 to 3,463 acre-ft/yr. Schedule $2_{\mathrm{D}}$ resulted in more pumpage and less seawater intrusion than schedule $1_{D}$; however, schedule $2_{D}$ resulted in much larger drawdowns as well.

Scenario 3 showed the potential for aquifer recovery from 1990 water levels to 1998 water levels under minimal pumpage and either typical or dry climatic conditions during a 10 -year simulation. Aquifer recovery was illustrated by hydrographs that showed drawdown relative to 1998 water levels. There were minor differences in the recovery between the typical and dry climatic conditions. The drawdown at selected Storage Unit I wells stabilized between about 0 and 5 feet (ft) with a typical climatic condition and about 3 to $8 \mathrm{ft}$ for the dry climatic condition. Therefore, despite minimal pumpage, the water levels did not recover to 1998 levels after 10 years of typical or dry climatic condition. Also, there was little difference between the extents of simulated seawater intrusion after 10 years for these climatic conditions. This scenario demonstrated that simulated seawater intrusion was not sensitive to recharge, even with pumpage substantially decreased.

The scenario-4 results produced decision-rule curves that can be used to help make management decisions without needing to run a simulation. The optimizations of scenarios 1 and 2 produced a set of feasible pumping schedules from which a subset was selected for further analysis. The rule curves were produced as a reference for when additional field measurements are taken of water levels and chloride concentrations. In the case of a measured concentration, a curve was produced that showed simulated results that matched that concentration and the associated Pareto-optimal pumpage in Storage Unit I and the ensuing seawater intrusion. For the case of measured water levels, each decision-rule curve showed a subset of Pareto-optimal pumpage, separated by storage unit, for each production well. Combined, these curves provide an approach to adapt production management as measurements are taken in the future. In other words, total pumpage by storage unit can be adjusted to future conditions, and the associated Pareto-optimal pumping schedule can be derived from these results.

For scenario 5, the Pareto optimal results illustrated the effect of short-term precipitation patterns on preferred pumping schedules. In general, the Pareto optimal results for Scenario 5A (typical-to-dry) and 5B (dry-to-typical) showed a greater range of pumpage and drawdown than scenario 5C (dry-to-dry). The solution for the objective of minimal seawater intrusion (schedule $\mathrm{B}$ ) for the typical-todry simulation (schedule $5 \mathrm{~A}_{\mathrm{B}}$ ) had substantially less total drawdown (in fact, simulated hydraulic heads increased for schedule $5 \mathrm{~A}_{\mathrm{B}}$ ) than schedules $5 \mathrm{~B}_{\mathrm{B}}$ and $5 \mathrm{C}_{\mathrm{B}}$. For the minimal maximum-drawdown solution (schedule $\mathrm{C}_{\mathrm{m}}$ ), there was much less pumpage in the dry-to-typical climatic condition (schedule $5 \mathrm{~B}_{\mathrm{Cm}}$ ) than in the other two climatic conditions (schedules $5 \mathrm{~A}_{\mathrm{Cm}}$ and $5 \mathrm{C}_{\mathrm{Cm}}$ ). The best compromise solution (schedule $\mathrm{D}$ ) in the typical-to-dry climatic condition (schedule $5 \mathrm{~A}_{\mathrm{D}}$ ) also had less simulated seawater intrusion than schedules $5 \mathrm{~B}_{\mathrm{D}}$ and $5 \mathrm{C}_{\mathrm{D}}$. The simulated hydraulic heads were similar for each producing zone in each climatic condition; any differences were most notable in the lower producing zone of the Foothill groundwater basin and the upper producing zone of Storage Unit I. The quarterly pumping schedules by well showed the increase in Foothill groundwater basin pumpage for minimal seawater-intrusion solutions. The tradeoffs among all three objectives were more pronounced in the scenario-5B results. Very little difference was noticeable in the chloride breakthrough curves for all three climatic conditions, however. 
The Borg MOEA offers the most efficient way of collecting simulation results by using Pareto-optimality and searching the decision-variable space in a logical manner. Typically, unanticipated climatic conditions or changing management preferences would require additional simulations and, possibly, an additional study altogether. In this study, the Pareto-optimal results for two climatic conditions (typical and dry) provide the city with a collection of simulation results to help make decisions without additional model simulations.

The Pareto-optimal results for each scenario can be used for assessing the differences between scenarios or for assessing the tradeoffs among the four competing objectives. The differences between scenario- 1 and scenario- 2 results showed the effect on basin yield, when simulating typical precipitation, and indicated that it was beneficial to simulate a drought. In scenario 2, the complex dynamics among the four objectives depended strongly on climate and choosing an optimal schedule from the Pareto curve that produced the desired pumpage results in a clear tradeoff between drawdown and seawater intrusion.

Further conclusions can be drawn from scenarios 1 and 2 by investigating scenarios 3 and 4 . Considering scenario- 3 results, the best-case scenario (10-year typical precipitation), assuming minimal pumpage, was a return to water levels about $0-10 \mathrm{ft}$ lower than those in 1998 . The basin responded quickly when pumping was reduced, but the rate of the response was greatly diminished after a few years. At that point, drawdown stabilized at about 0-10 ft less than 1998 levels with continued pumping of 484 acre-ft/yr from Storage Unit I. The scenario-4 results provide a way to mitigate further seawater intrusion or excessive drawdown. As data are collected, regardless of the pumping schedule that is implemented, management actions can be adapted to prevent unnecessary losses in yield, increases in chlorides, or decreases in water levels.

Lastly, the scenario-5 results were also useful for assessing the consequences of assuming typical or dry precipitation for 2-year climatic conditions. Objective B values did not vary over a wide range among the pumping schedules of all scenarios, meaning seawater intrusion was relatively insensitive to a 2-year precipitation pattern. Results showed that optimal pumpage was more sensitive to climate in year one when minimizing total drawdown, and conversely, optimal pumpage was more sensitive to climate in year two when minimizing the maximum drawdown. The maximum pumpage in each scenario (schedule A) was distributed more to Storage Unit I than the Foothill groundwater basin in all quarters. For the minimal seawater-intrusion pumpage (schedule B), there were some quarters in which a larger portion of pumpage came from the Foothill groundwater basin than from Storage Unit I. The dry-to-dry precipitation pattern of scenario $5 \mathrm{C}$ generally represented conservative estimates of optimal pumpage, seawater intrusion, and drawdown. These conservative pumping schedules from drier climate simulations ensured the short-term annual yield of the basin would not jeopardize future yield if precipitation remained low.

\section{References Cited}

Abarca, E., Vázquez-Suñé, E., Carrera, J., Capino, B., Gámez, D., and Batlle, F., 2006, Optimal design of measures to correct seawater intrusion: Water Resources Research, v. 42, no. 9, p. 1-14.

Abd-Elhamid, H.F., and Javadi, A.A., 2011, A cost-effective method to control seawater intrusion in coastal aquifers: Water Resources Management, v. 25, no. 11, p. 2755-2780.

Ahlfeld, D.P., and Baro-Montes, G., 2008, Solving unconfined groundwater flow management problems with successive linear programming: Journal of Water Resources Planning and Management, v. 134, no. 5, p. 404-412.

Ahlfeld, D.P., and Mulligan, A.E., 2000, Optimal management of flow in groundwater systems: San Diego, California, Academic, $185 \mathrm{p}$.

Ahlfeld, D.P., Mulvey, J.M., Pinder, G.F., and Wood, E.F., 1988, Contaminated groundwater remediation design using simulation, optimization, and sensitivity theory; 1 . Model development: Water Resources Research, v. 24, no. 3, p. 431-441.

Cachuma Operation and Maintenance Board, 2017, National Marine Fisheries Service biological opinion: accessed October 2017, at http://fmp.cachuma-board.org/nmfs.htm.

Chang, L.C., Shoemaker, C.A., and Liu, P.L.F., 1992, Optimal time-varying pumping rates for groundwater remediation; Application of a constrained optimal control algorithm: Water Resources Research, v. 28, no. 12, p. 3157-3171.

Cieniawski, S.E., Eheart, J.W., and Ranjithan, S., 1995, Using genetic algorithms to solve a multiobjective groundwater monitoring problem: Water Resources Research, v. 31, no. 2, p. 399-409.

Coello, C.A.C., Lamont, G.B., and Van Veldhuizen, D.A., 2007, Evolutionary algorithms for solving multi-objective problems (2d ed.), Genetic and Evolutionary Computation Series: Boston, Mass., Springer, $800 \mathrm{p}$.

Culver, T.B., and Shoemaker, C.A., 1992, Dynamic optimal control for groundwater remediation with flexible management periods: Water Resources Research, v. 28, no. 3, p. 629-641.

Culver, T.B., and Shoemaker, C.A., 1997, Dynamic optimal ground-water reclamation with treatment capital costs: Journal of Water Resources Planning and Management, v. 123 , no. 1 , p. 23-29.

Das, A., and Datta, B., 2000, Optimization based solution of density dependent seawater intrusion in coastal aquifers: Journal of Hydrologic Engineering, v. 5, no. 1, p. 82-89.

Dhar, A., and Datta, B., 2009, Saltwater intrusion management of coastal aquifers; I : linked simulation-optimization: Journal of Hydrologic Engineering, v. 14, no. 12, p. 1263 1272 . 
Freckleton, J.R., Martin, P., and Nishikawa, T., 1998, Geohydrology of Storage Unit III and a combined flow model of the Santa Barbara and Foothill ground-water basins, Santa Barbara County, California: U.S. Geological Survey Water-Resources Investigations Report 97-4121, 80 p., http://pubs.er.usgs.gov/publication/wri974121.

Gordon, E., Shamir, U., and Bensabat, J., 2001, Optimal extraction of water from regional aquifer under salinization: Journal of Water Resources Planning and Management, v. 127 , no. 2 , p. $71-77$.

Gorelick, S.M., 1983, Review of distributed parameter groundwater management modeling methods: Water Resources Research, v. 19, no. 2, p. 305-319.

Hadka, D.M., and Reed, P.M., 2013, Borg; An auto-adaptive many-objective evolutionary computing framework: Evolutionary Computation, v. 1, no. 2, p. 231-259, http://doi.org/10.1162/EVCO_a_00075.

Kasprzyk, J.R., Reed, P.M., Kirsch, B.R., and Characklis, G.W., 2009, Managing population and drought risks using manyobjective water portfolio planning under uncertainty: Water Resources Research, v. 45, no. 12, 18 p., doi:10.1029/2009WR008121.

Kasprzyk, J.R., Reed, P.M., and Hadka, D.M., 2015, Battling Arrow's Paradox to discover robust water management alternatives: Journal of Water Resources Planning and Management, v. 142, no. 2, 04015053 , http://doi.org/10.1061/(ASCE)WR.1943-5452.0000572.

Kollat, J.B., and Reed, P.M., 2006, Comparing state-ofthe-art evolutionary multi-objective algorithms for long-term groundwater monitoring design: Advances in Water Resources, v. 29, no. 6, p. 792-807, doi:10.1016/j. advwatres.2005.07.010.

Lefkoff, L.J., and Gorelick, S.M., 1986, Design and cost analysis of rapid aquifer restoration systems using flow simulation and quadratic programming: Ground Water, v. 24, no. 6, p. 777-790.

Mayer, A.S., Kelley, C.T., and Miller, C.T., 2002, Optimal design for problems involving flow and transport phenomena in saturated subsurface systems: Advances in Water Resource, v. 25, no. 8-12, p. 1233-1256.

McKinney, D.C., and Lin, M.D., 1994, Genetic algorithm solution of groundwater management models: Water Resources Research, v. 30, no. 6, p. 1897-1906.

McKinney, D.C., and Lin, M.D., 1995, Approximate mixedinteger nonlinear programming methods for optimal aquifer remediation design: Water Resources Research, v. 31, no. 3, p. 731-740.

Minor, S.A., Kellogg, K.S., Stanley, R.G., Gurrola, L.D., Keller, E.A., and Brandt, T.R., 2009, Geologic map of the Santa Barbara coastal plain area, Santa Barbara County, California: U.S. Geological Survey Scientific Investigations Map 3001, scale 1:25,000, 1 sheet, pamphlet, 38 p., https://pubs.er.usgs.gov/publication/sim3001.

Muir, K.S., 1968, Ground-water reconnaissance of the Santa Barbara-Montecito area, Santa Barbara County, California: U.S. Geological Survey Water Supply Paper 1859-A, 28 p., https://pubs.er.usgs.gov/publication/wsp1859A.
Molz, F.J., and Bell, L.C., 1977, Head gradient control in aquifers used for fluid storage: Water Resources Research, v. 13, no. 4, p. 795-798.

Nicklow, J., Reed, P.M., Savic, D., Dessalegne, T., Harrell, L., Chan-Hilton, A., Karamouz, M., Minsker, B., Ostfeld, A., Singh, A., Zechman, E., and ASCE Task Committee on Evolutionary Computation in Environmental and Water Resources Engineering, 2010, State of the art for genetic algorithms and beyond in water resources planning and management: Journal of Water Resources Planning and Management, v. 136, no. 4, p. 412-432.

Nishikawa, T., 1998, A water-resources optimization model for Santa Barbara, California: ASCE Journal of Water Resources Planning and Management, v. 124, no. 5, p. 252-263.

Nocedal, J., and Wright, S.J., 2006, Numerical optimization: New York, Springer Science and Business Media, 664 p.

Qahman, K., Larabi, A., Ouazar, D., Naji, A., and Cheng, A.H.-D., 2005, Optimal and sustainable extraction of groundwater in coastal aquifers: Stochastic Environmental Research and Risk Assessment, v. 19, no. 2, p. 99-110.

Reed, P.M., Hadka, D.M., Herman, J.D., Kasprzyk, J.R., and Kollat, J.B., 2013, Evolutionary multiobjective optimization in water resources; The past, present, and future: Advances in Water Resources, 51, p. 438-456, http://doi.org/10.1016/j.advwatres.2012.01.005.

Singh, A., 2014, Optimization modelling for seawater intrusion management: Journal of Hydrology, v. 508, p. 43-52.

Sreekanth, J., and Datta, B., 2015, Review; Simulationoptimization models for the management and monitoring of coastal aquifers: Hydrogeology Journal, v. 23, no. 6, p. $1155-1166$.

U.S. Environmental Protection Agency, 2017, Secondary drinking water standards; Guidance for nuisance chemicals: accessed on January 11, 2017, at https://www.epa.gov/ dwstandardsregulations/secondary-drinking-water-standardsguidance-nuisance-chemicals.

Wagner, B.J., 1995, Recent advances in simulation-optimization groundwater management modeling: Review in Geophysics, v. 33 , p. $1021-1028$.

Wang, W., and Ahlfeld, D.P., 1994, Optimal groundwater remediation with well locations as a decision variable; Model development: Water Resources Research, v. 30, no. 5, p. 1605-1618.

Ward, V.L., Singh, R., Reed, P.M., and Keller, K., 2015, Confronting tipping points; Can multi-objective evolutionary algorithms discover pollution control tradeoffs given environmental thresholds?: Environmental Modelling and Software, v. 73, p. 27-43, http://doi.org/10.1016/j.envsoft.2015.07.020.

Willis, R.L., 1979, A planning model for the management of groundwater quality: Water Resources Research, v. 15, no. 6, p. 1305-1312.

Yeh, W.W.-G., 1992, Systems analysis in groundwater planning and management: Journal of Water Resources Planning and Management, v. 118, no. 1, p. 224-237. 


\section{Appendix D-1: Schedule 1D Pumpage, by Well, Santa Barbara Multi-Objective Management Model, Santa Barbara, California}




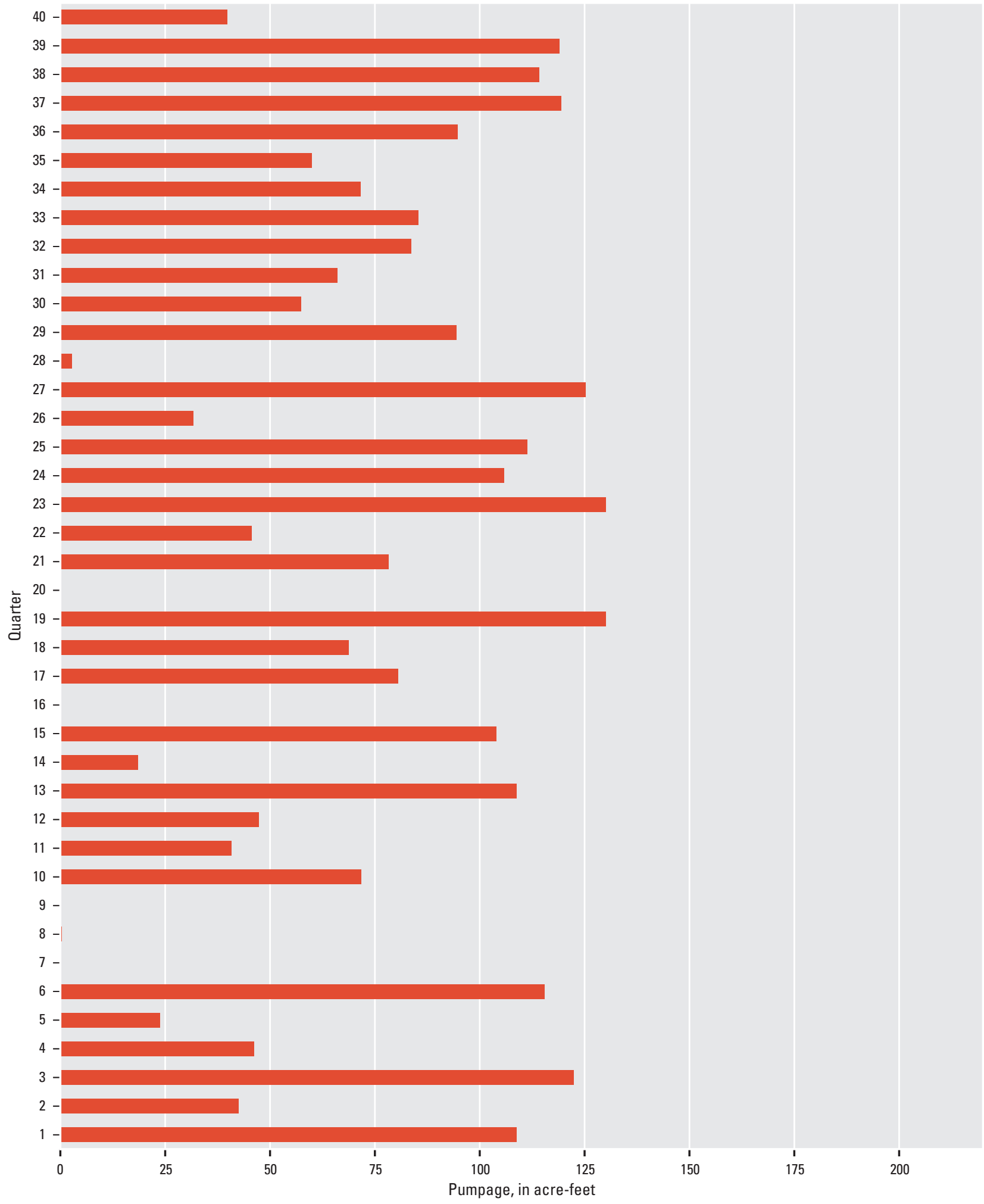

Figure D1-1. Optimal quarterly pumpage for the Alameda Park well, schedule $1_{D^{\prime}}$ Santa Barbara, California. 


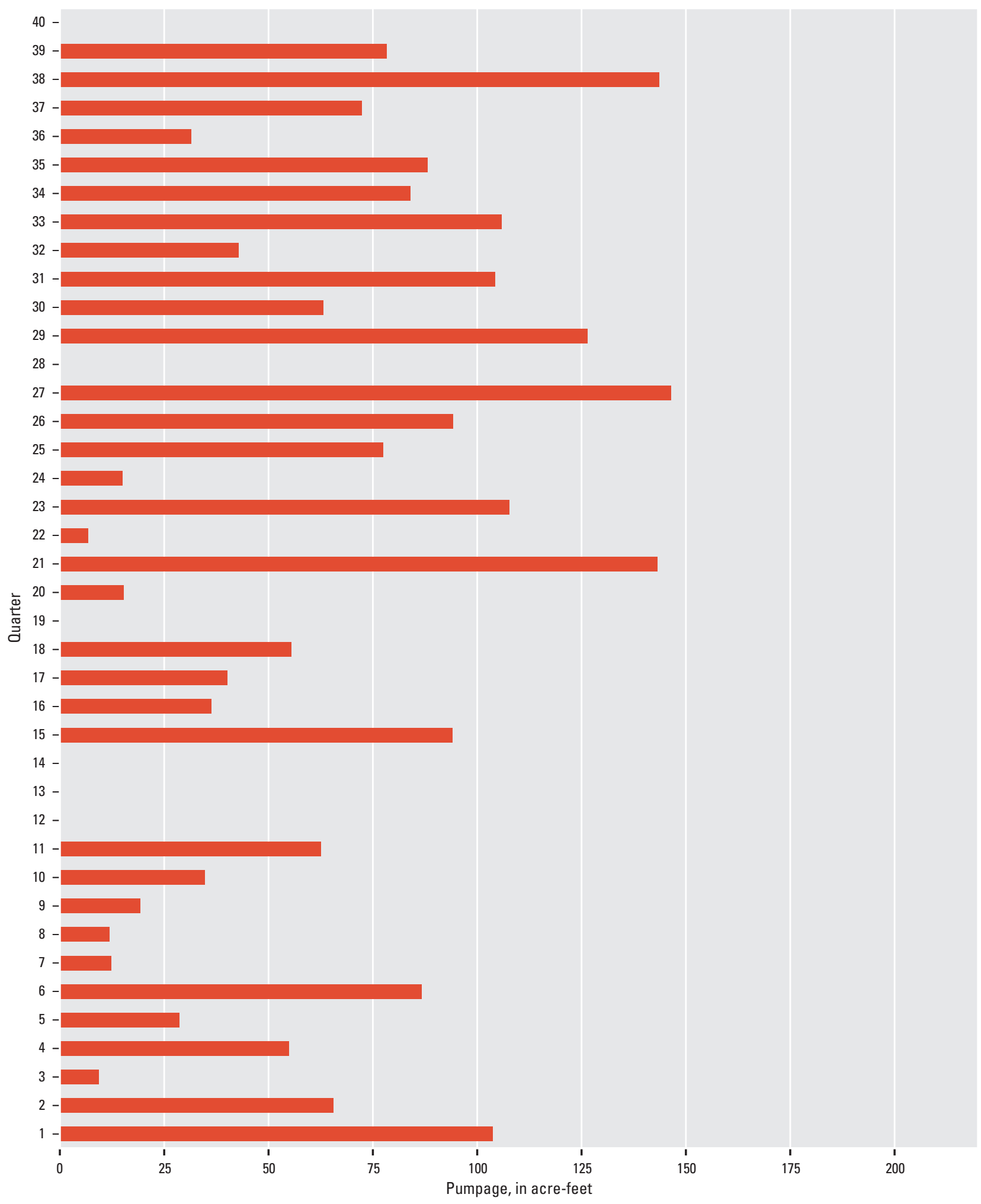

Figure D1-2. Optimal quarterly pumpage for the City Hall well, schedule $1_{\mathrm{D}^{\prime}}$ Santa Barbara, California. 


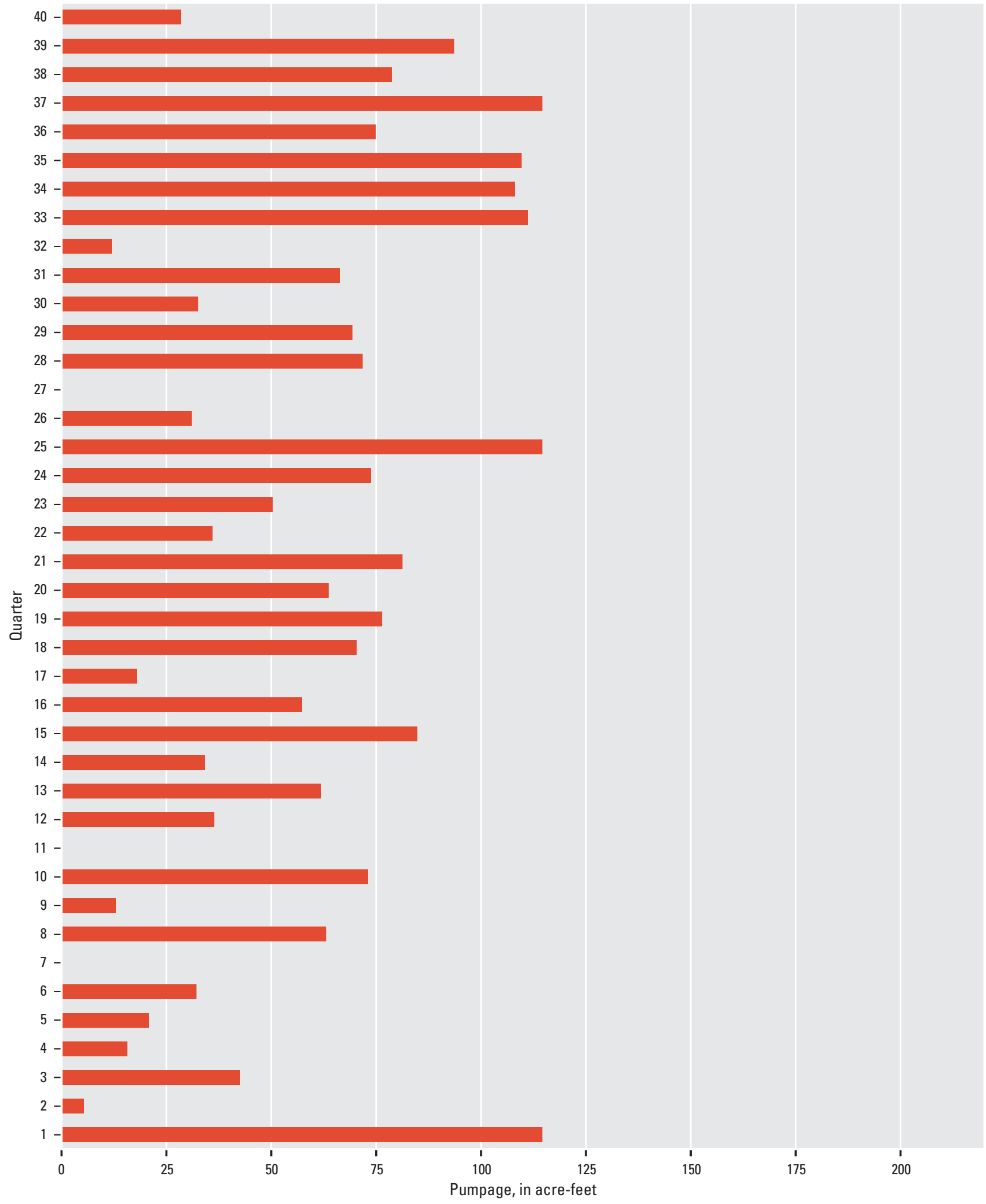

Figure D1-3. Optimal quarterly pumpage for the Corporation Yard well, schedule $1_{D^{\prime}}$ Santa Barbara, California. 


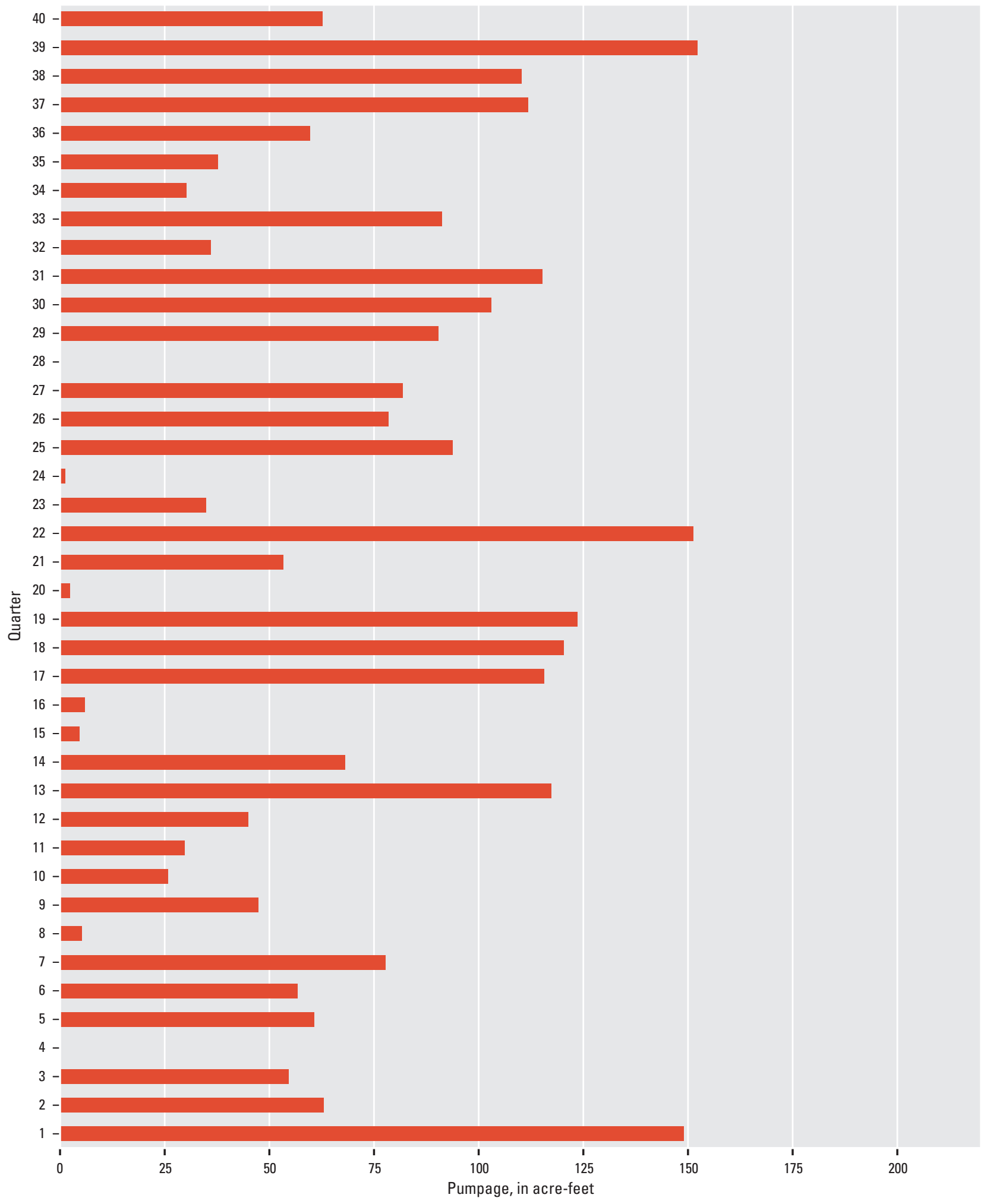

Figure D1-4. Optimal quarterly pumpage for the Ortega Park well, schedule $1_{D^{\prime}}$ Santa Barbara, California. 


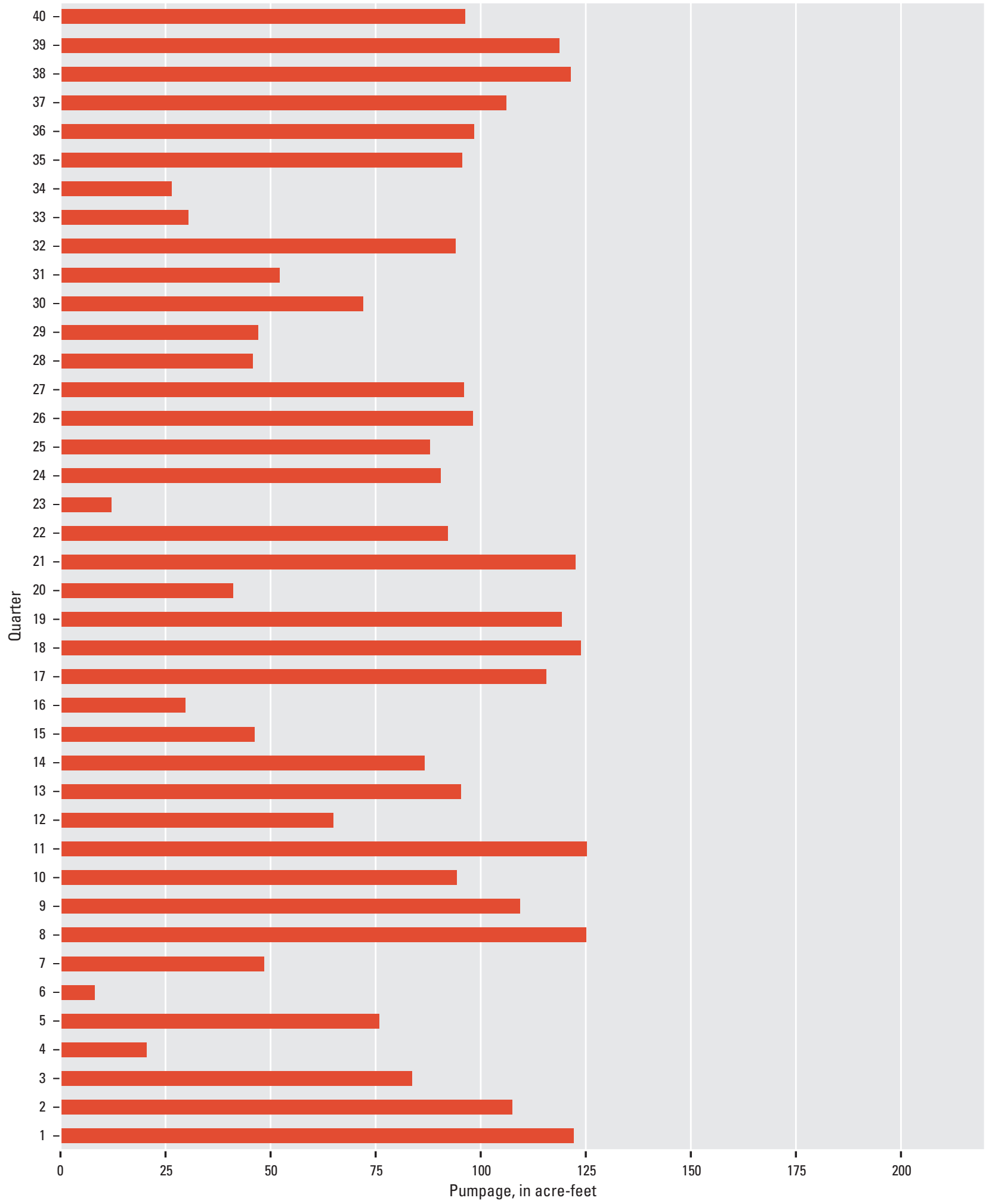

Figure D1-5. Optimal quarterly pumpage for the Santa Barbara High School well, schedule $1_{D^{\prime}}$ Santa Barbara, California. 


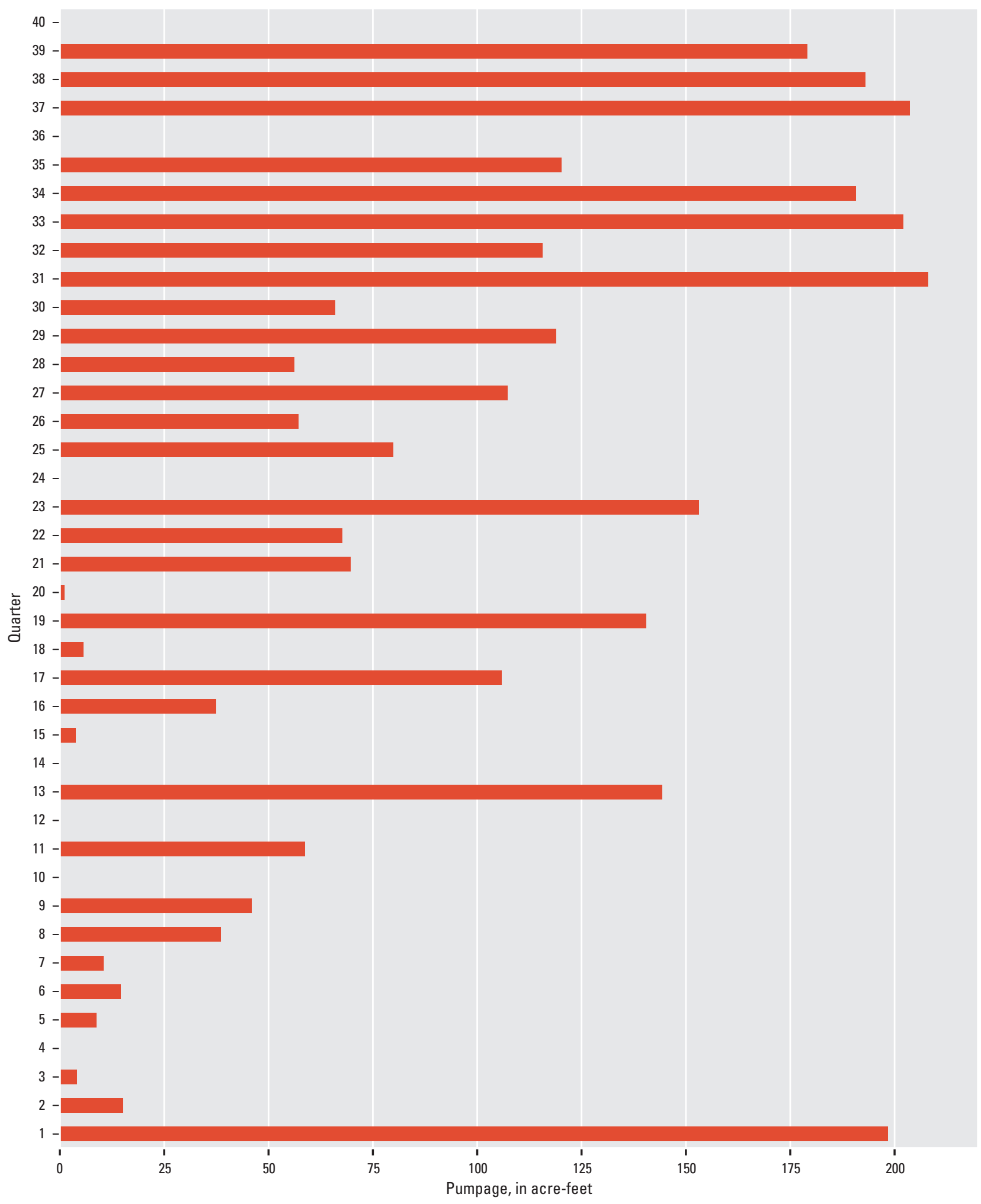

Figure D1-6. Optimal quarterly pumpage for the Vera Cruz well, schedule $1_{D^{\prime}}$ Santa Barbara, California. 


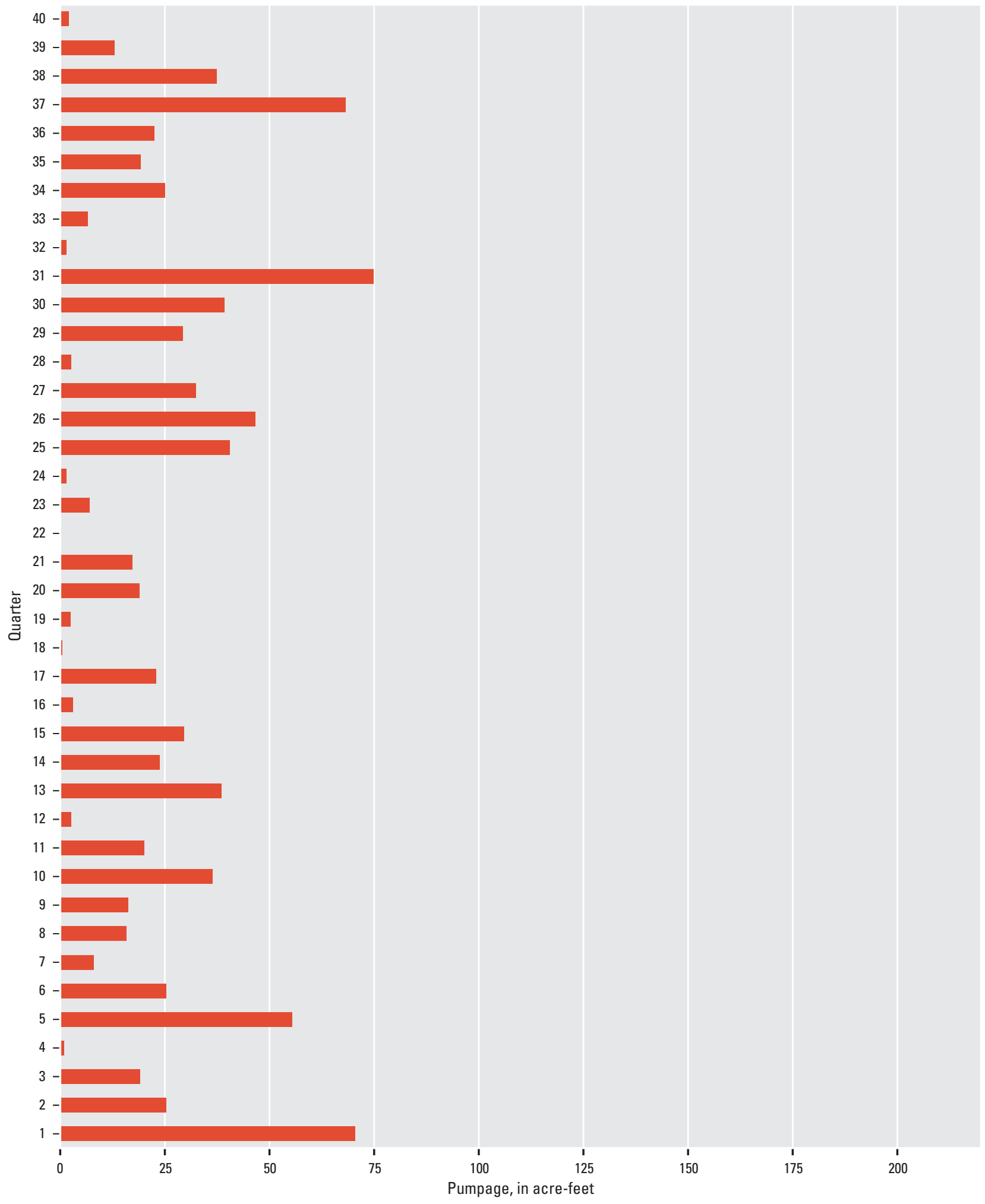

Figure D1-7. Optimal quarterly pumpage for the Hope Avenue well, schedule $1_{D^{\prime}}$ Santa Barbara, California. 


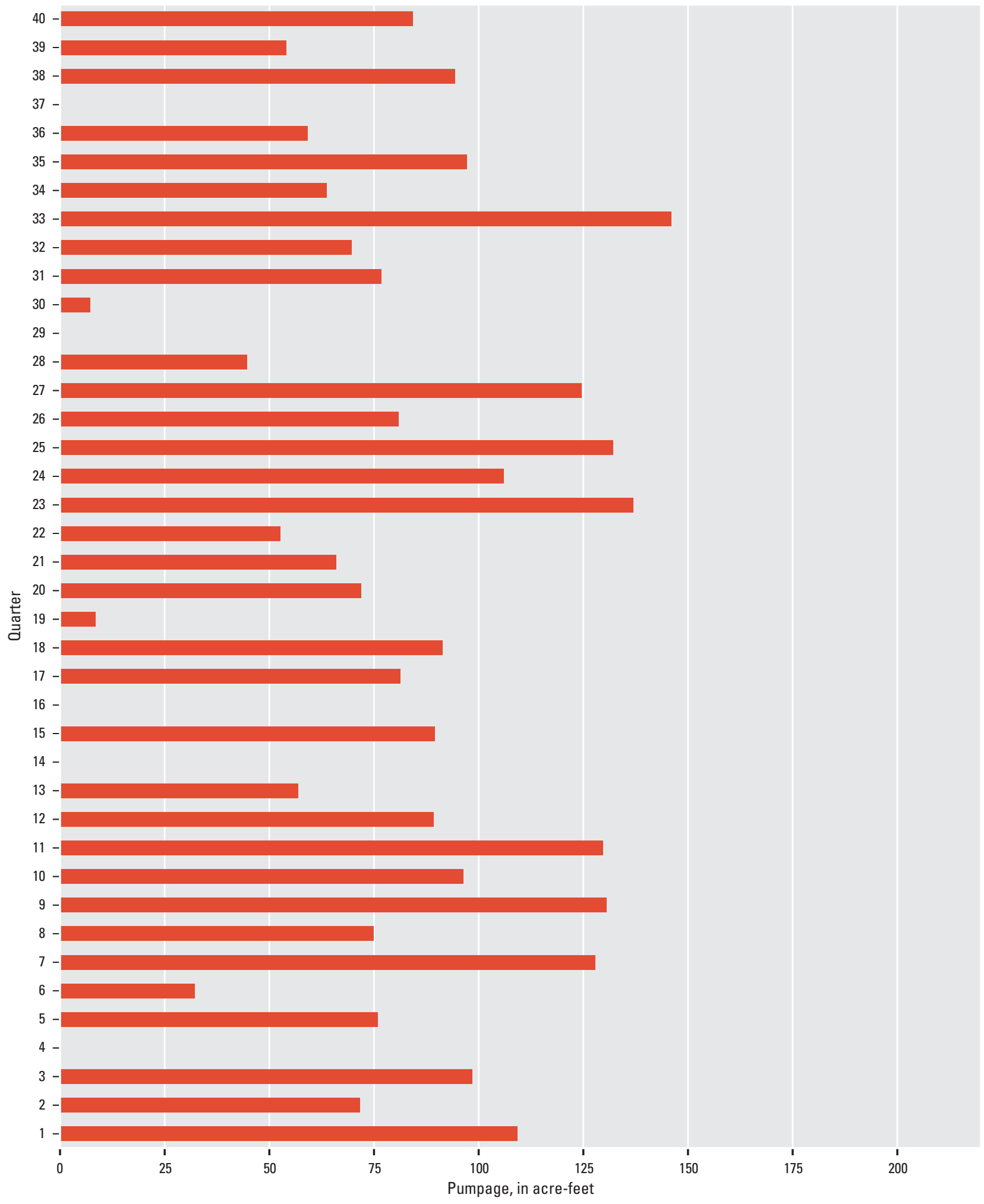

Figure D1-8. Optimal quarterly pumpage for the Lincolnwood 1 well, schedule $1_{D^{\prime}}$ Santa Barbara, California. 


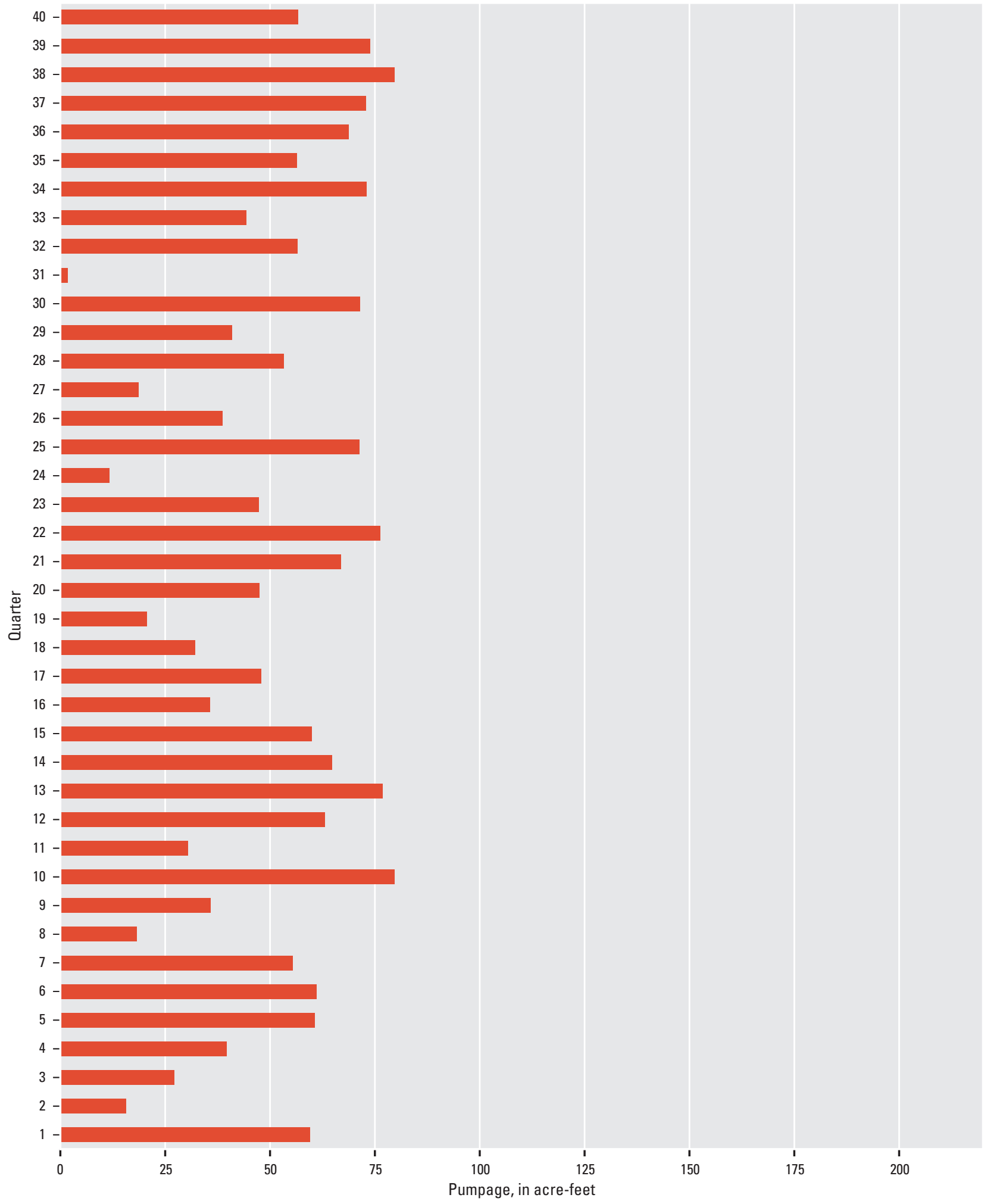

Figure D1-9. Optimal quarterly pumpage for the Los Robles well, schedule $1_{\mathrm{D}^{\prime}}$ Santa Barbara, California. 


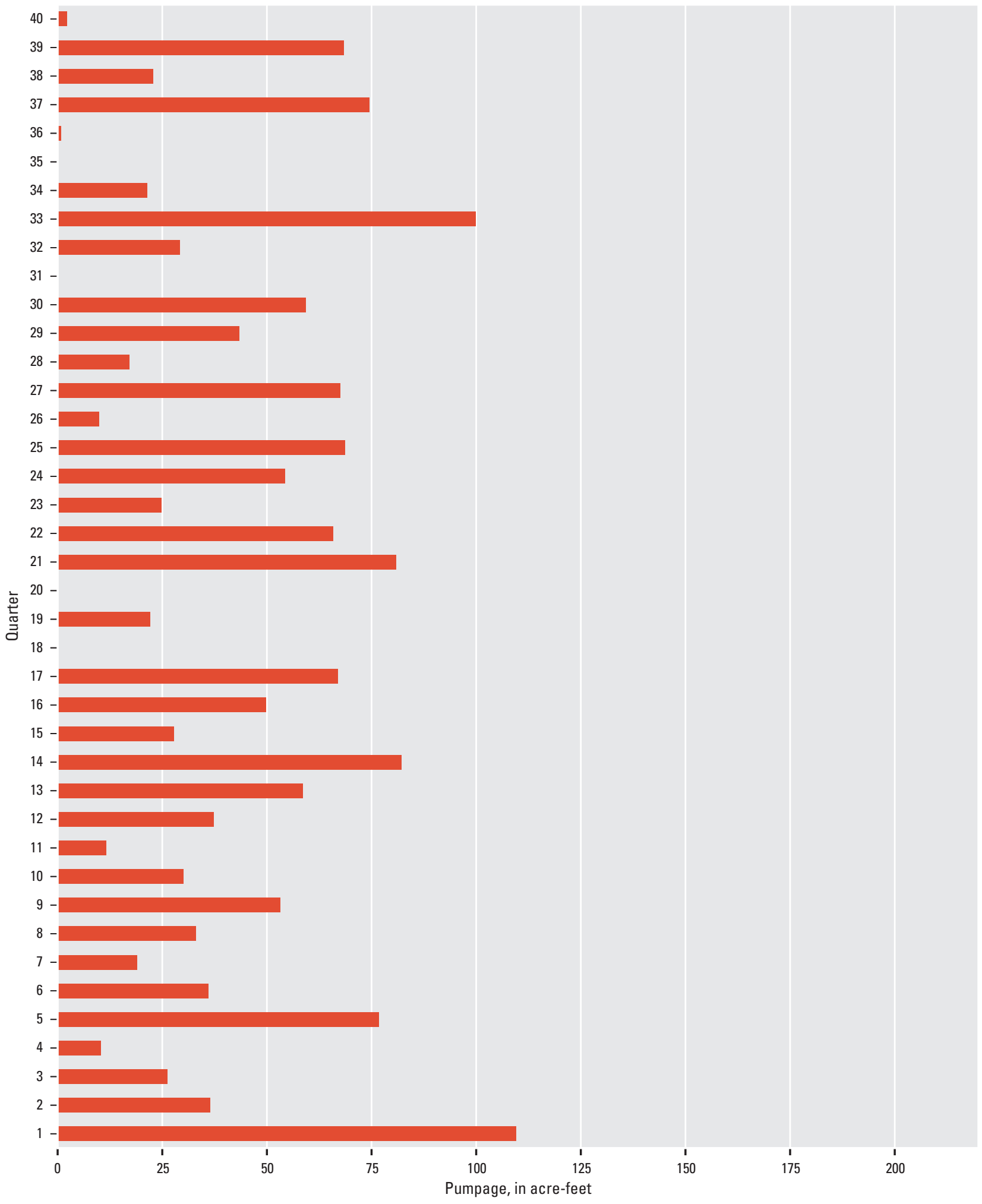

Figure D1-10. Optimal quarterly pumpage for the San Roque Park 2 well, schedule $1_{D^{\prime}}$ Santa Barbara, California. 


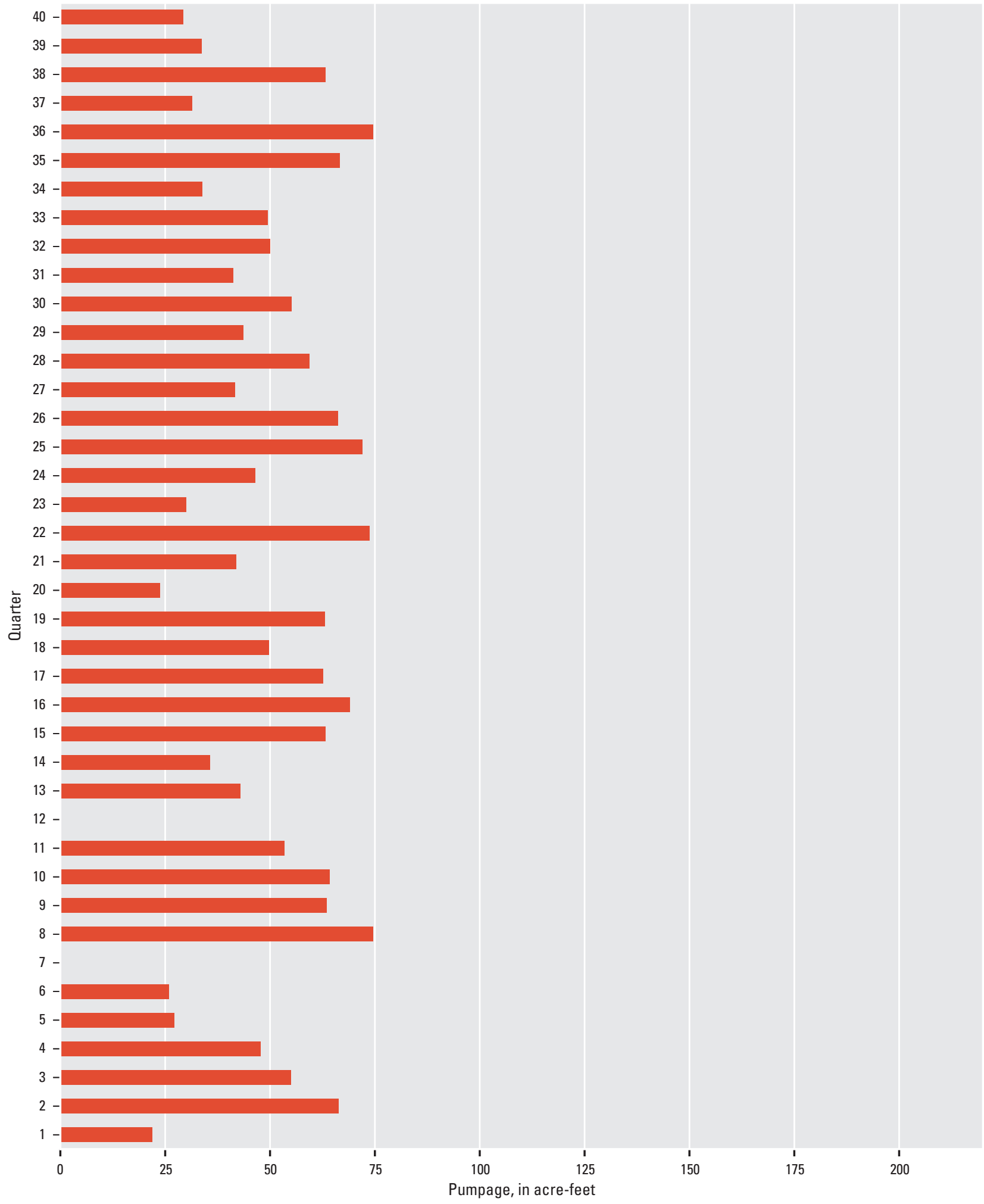

Figure D1-11. Optimal quarterly pumpage for the Val Verde well, schedule $1_{D^{\prime}}$ Santa Barbara, California. 


\section{Appendix D-2: Schedule 2D Pumpage, by Well, Santa Barbara Multi-Objective Management Model, Santa Barbara, California}




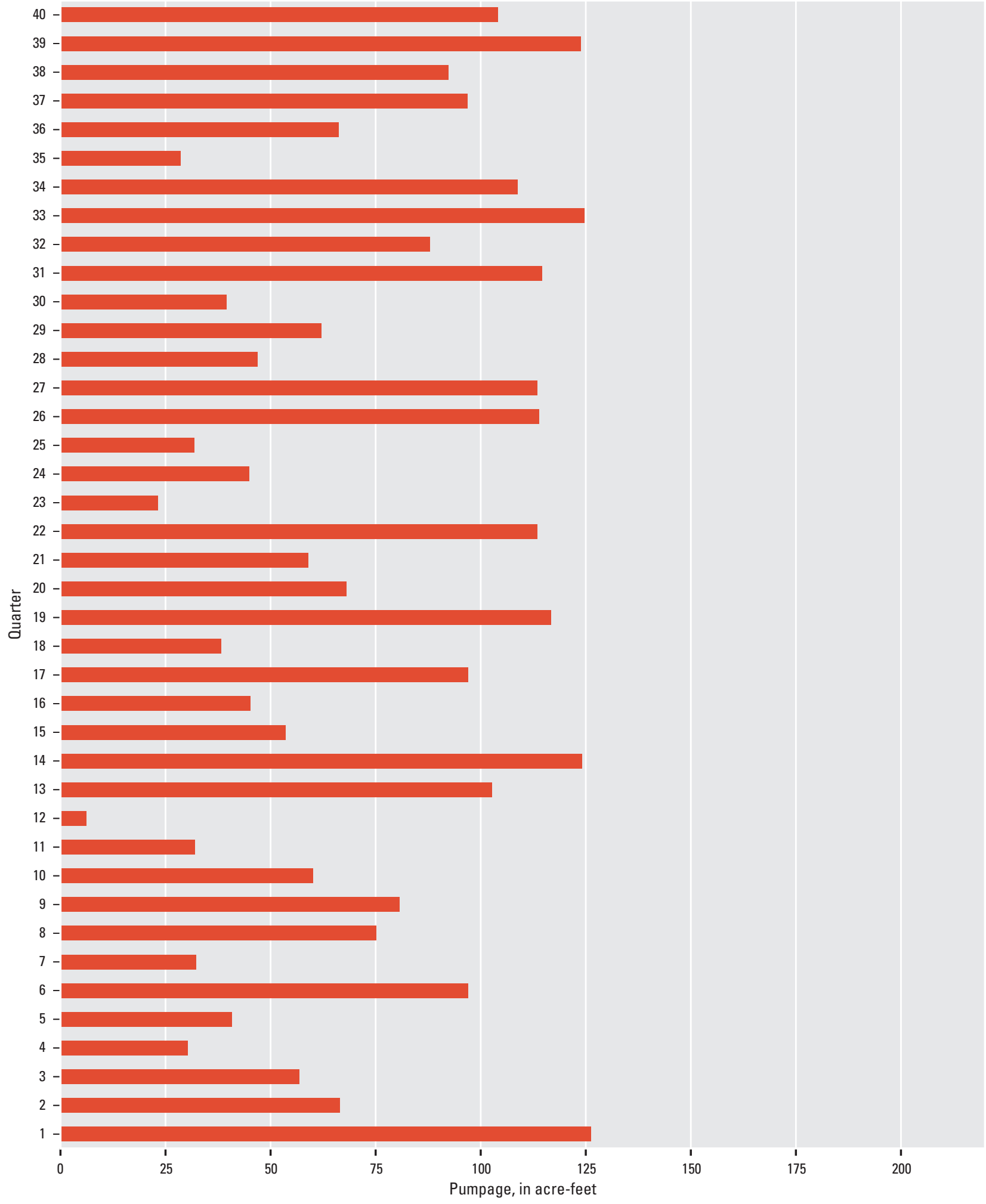

Figure D2-1. Optimal quarterly pumpage for the Alameda Park well, schedule ${ }_{\mathrm{D}^{\prime}}$ Santa Barbara, California. 


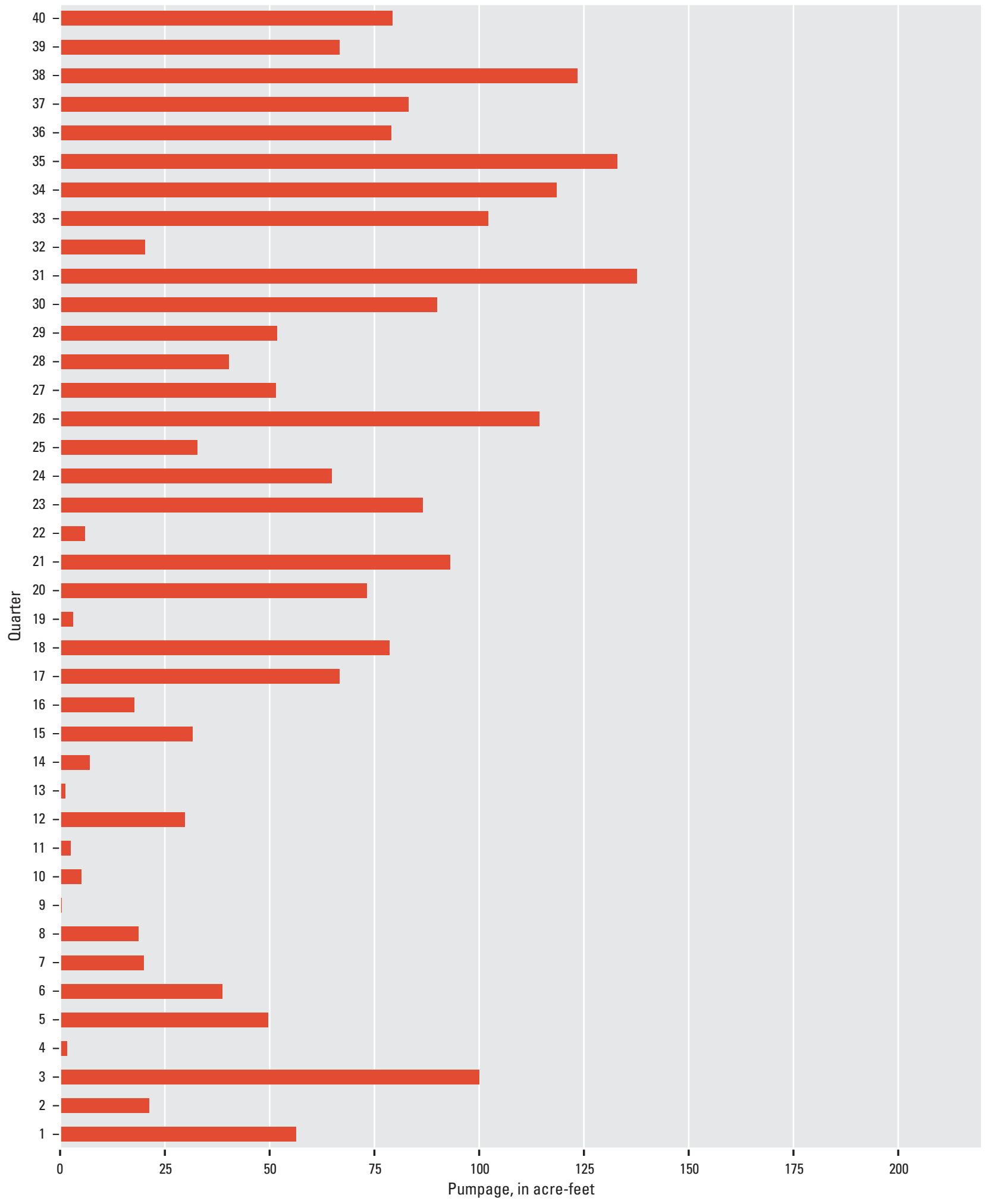

Figure D2-2. Optimal quarterly pumpage for the City Hall well, schedule $2{ }_{\mathrm{D}^{\prime}}$ Santa Barbara, California. 


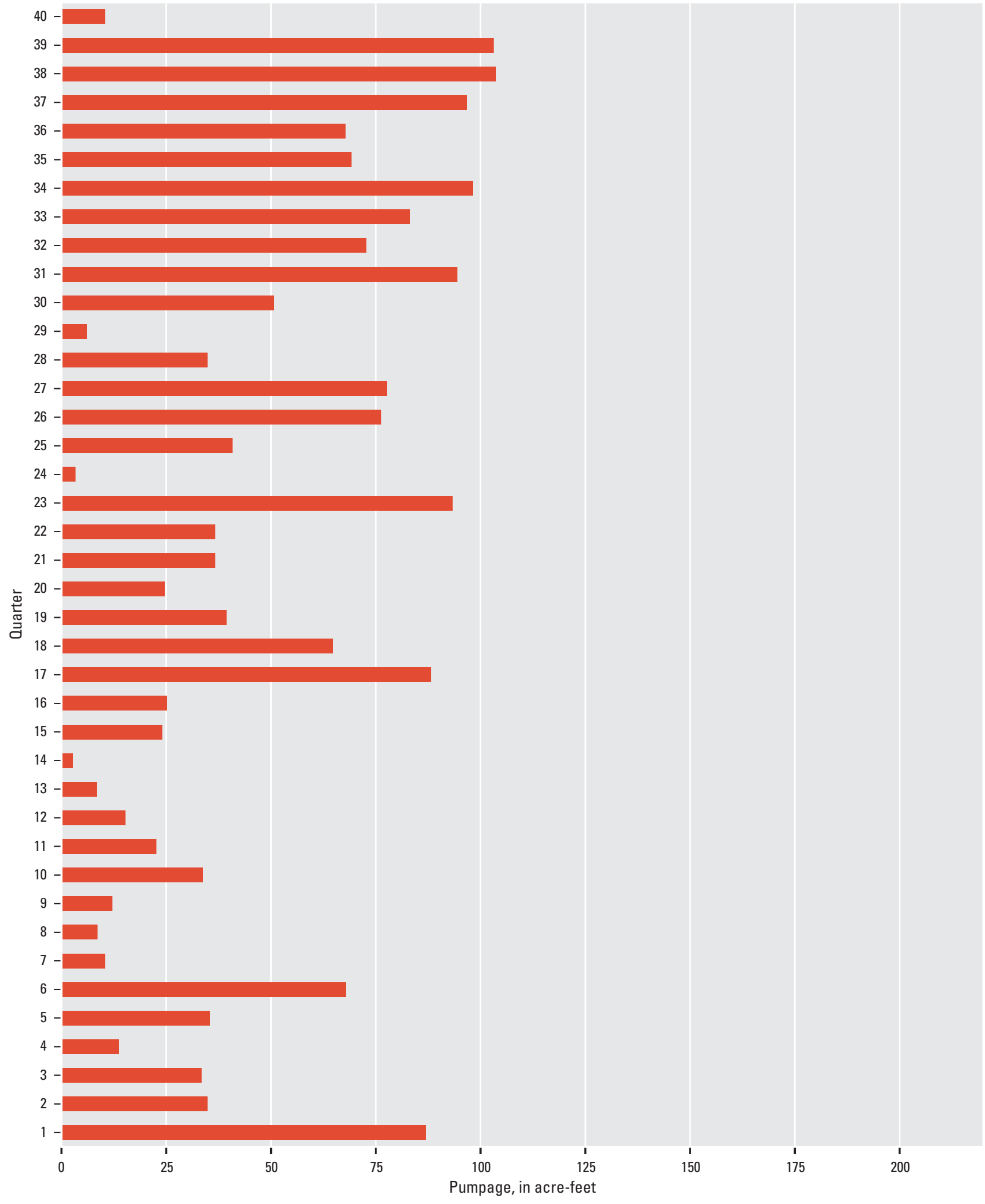

Figure D2-3. Optimal quarterly pumpage for the Corporation Yard well, schedule 2 ${ }_{\mathrm{D}^{\prime}}$ Santa Barbara, California. 


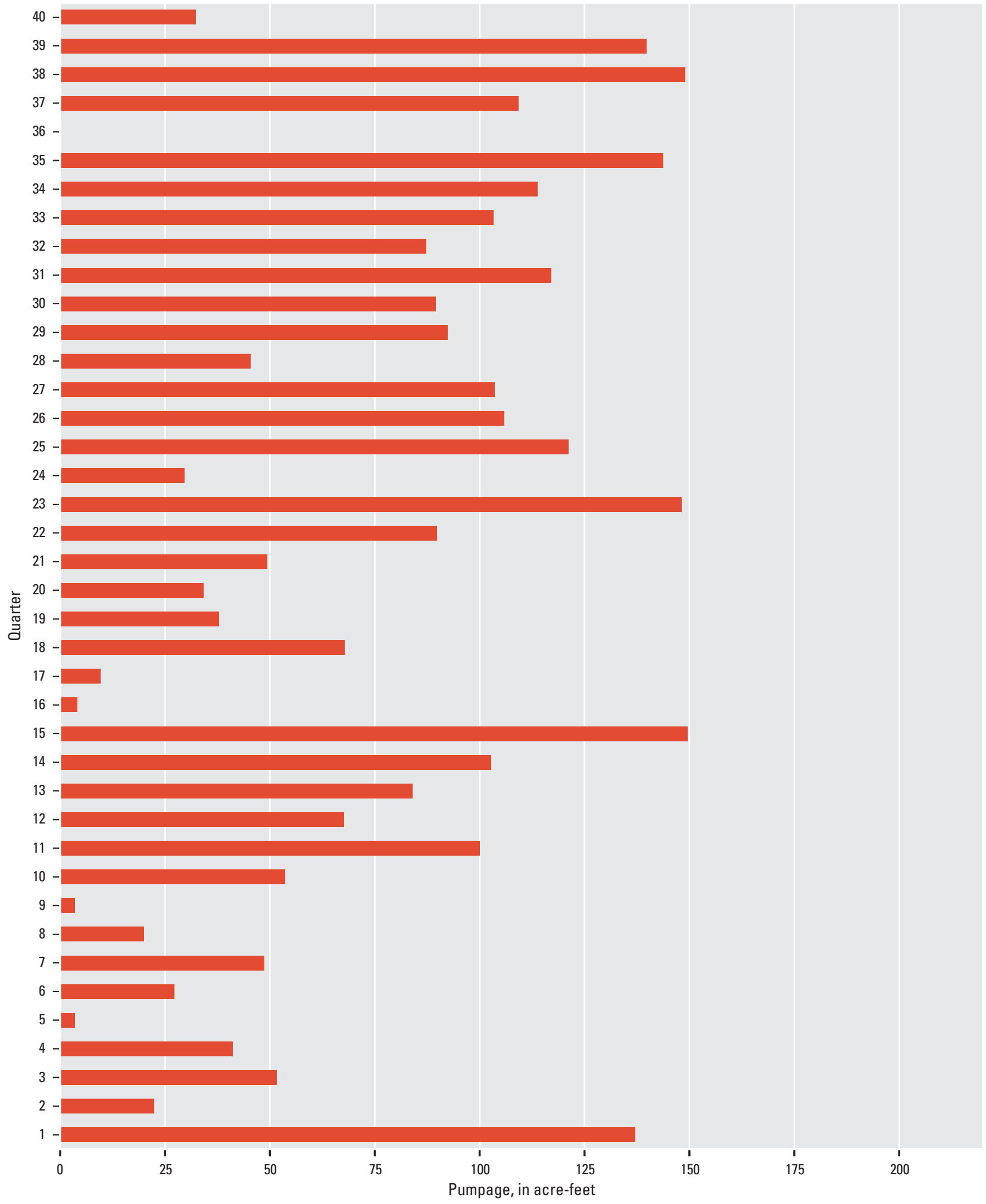

Figure D2-4. Optimal quarterly pumpage for the Ortega Park well, schedule ${ }_{\mathrm{D}^{\prime}}$ Santa Barbara, California. 


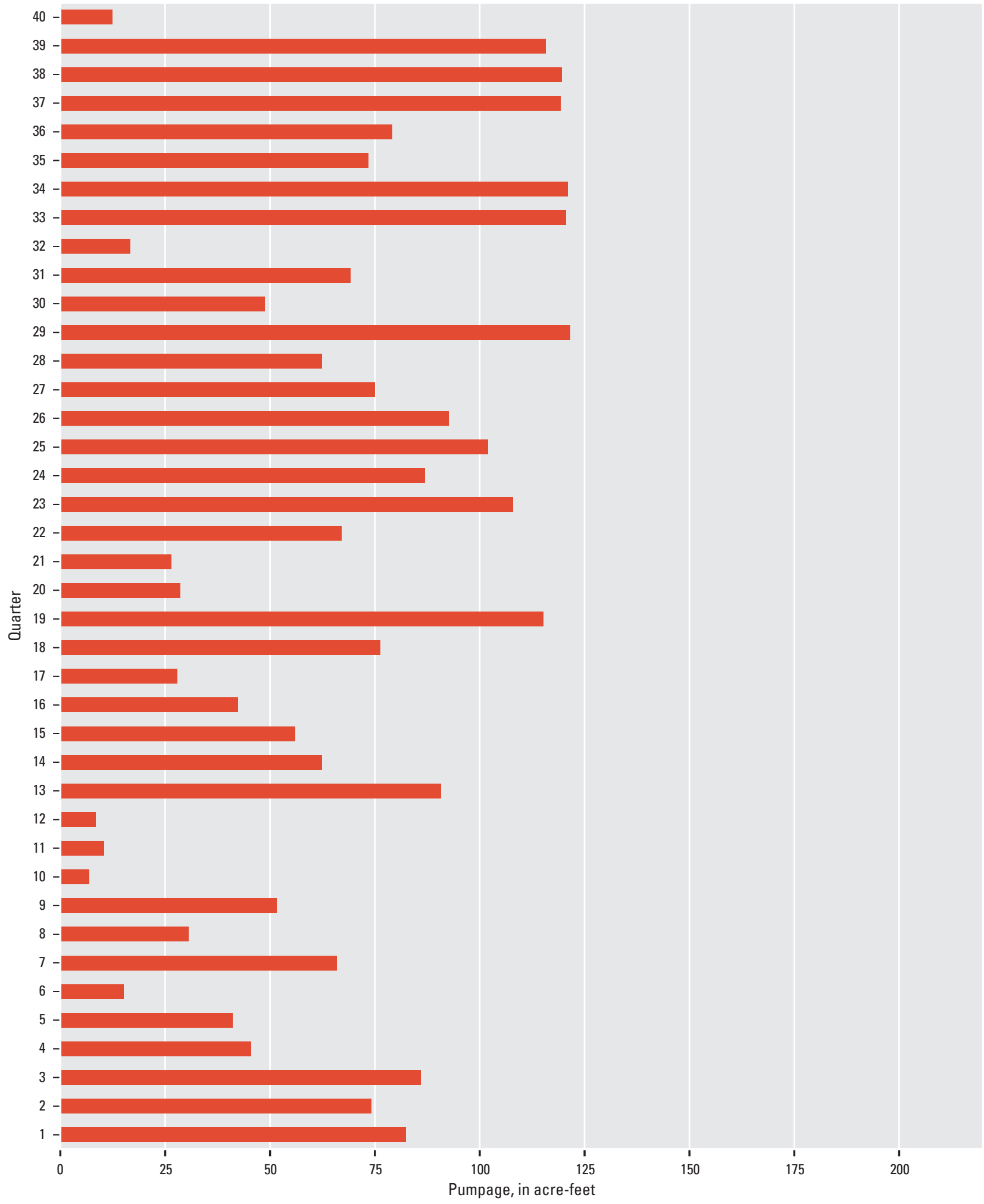

Figure D2-5. Optimal quarterly pumpage for the Santa Barbara High School well, schedule 2 ${ }_{\mathrm{D}^{\prime}}$ Santa Barbara, California. 


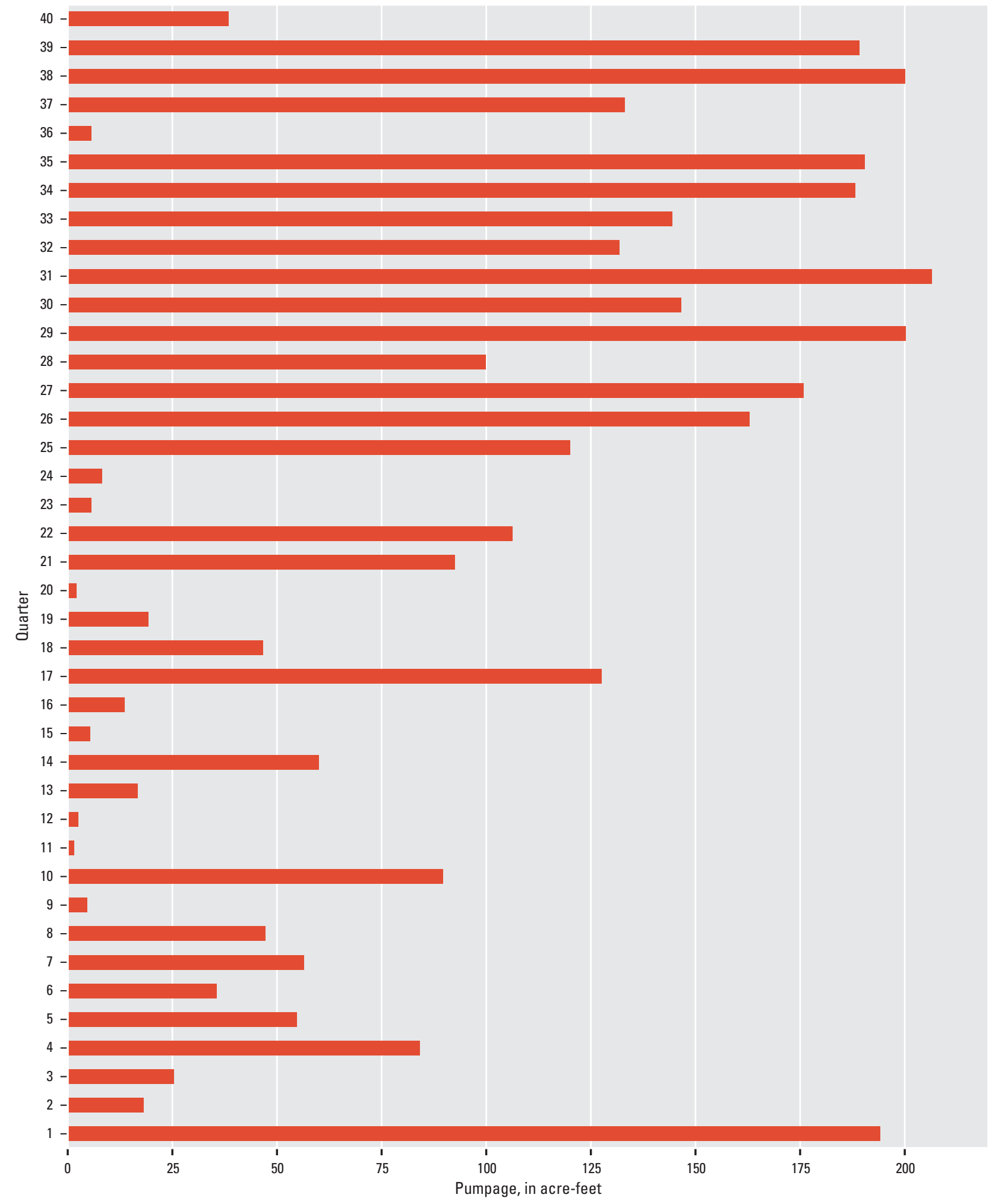

Figure D2-6. Optimal quarterly pumpage for the Vera Cruz well, schedule 2 ${ }_{\mathrm{D}}$ Santa Barbara, California. 


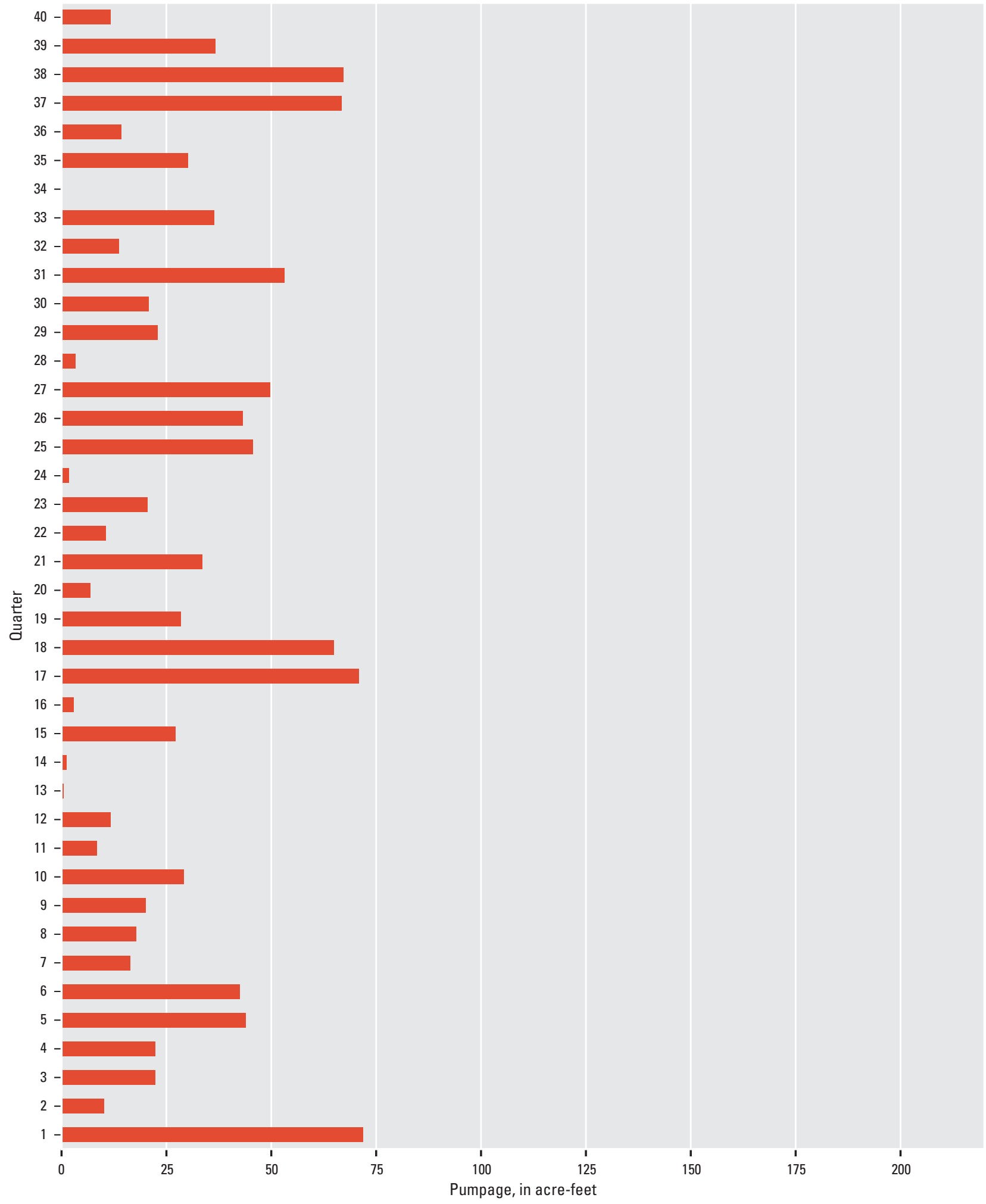

Figure D2-7. Optimal quarterly pumpage for the Hope Avenue well, schedule ${ }_{\mathrm{D}^{\prime}}$ Santa Barbara, California. 


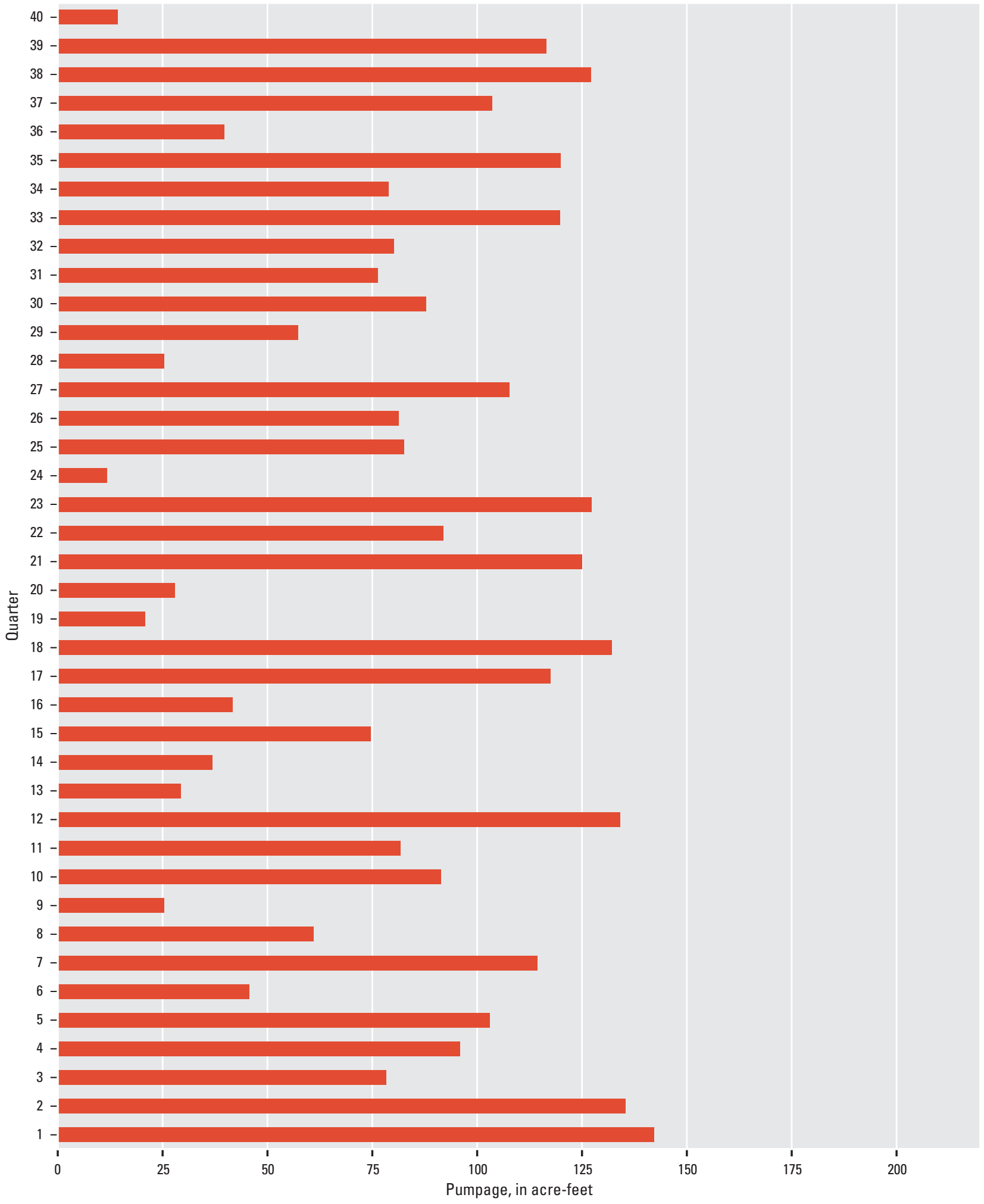

Figure D2-8. Optimal quarterly pumpage for the Lincolnwood 1 well, schedule ${ }_{\mathrm{D}^{\prime}}$ Santa Barbara, California. 


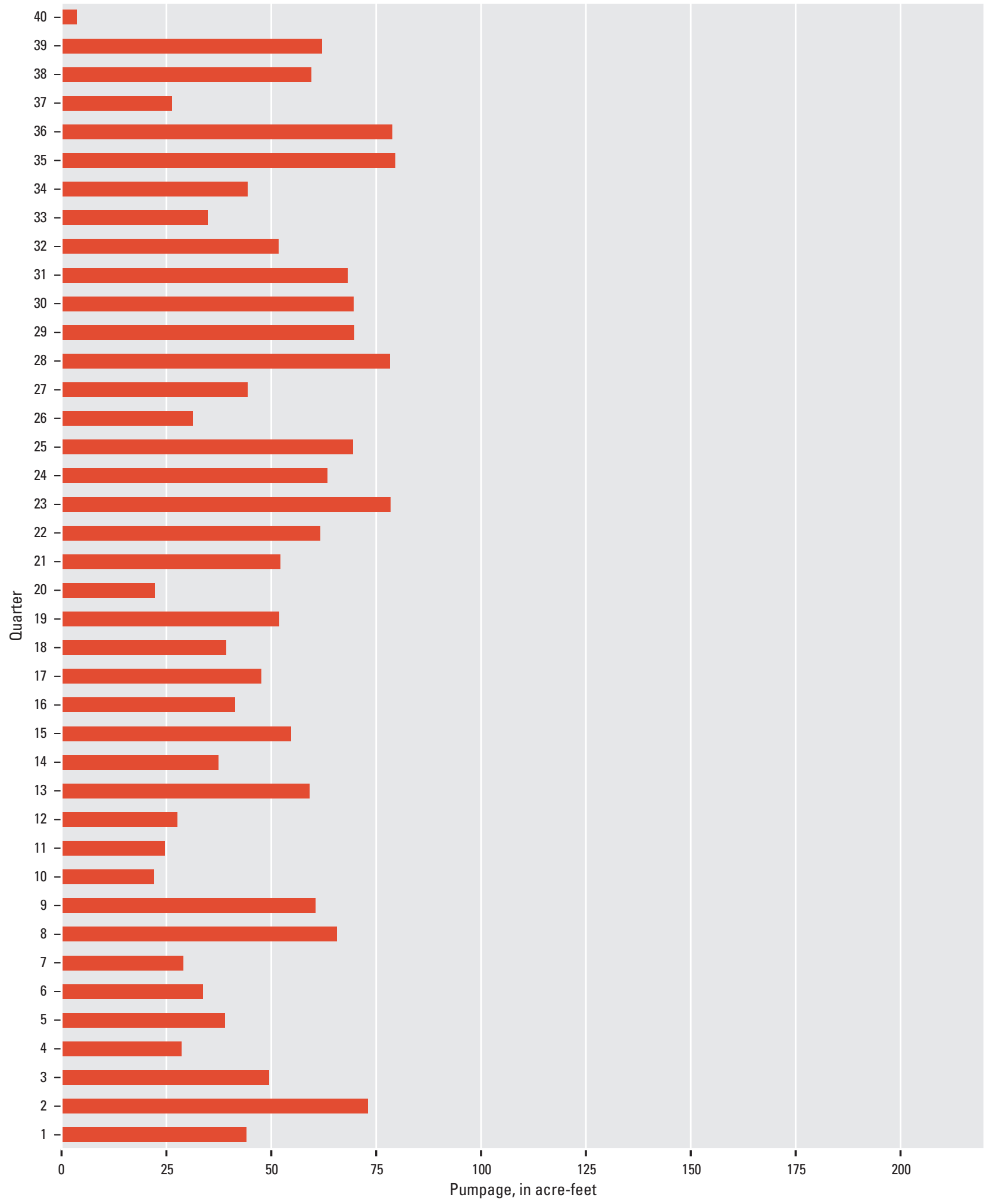

Figure D2-9. Optimal quarterly pumpage for the Los Robles well, schedule ${ }_{\mathrm{D}^{\prime}}$ Santa Barbara, California. 


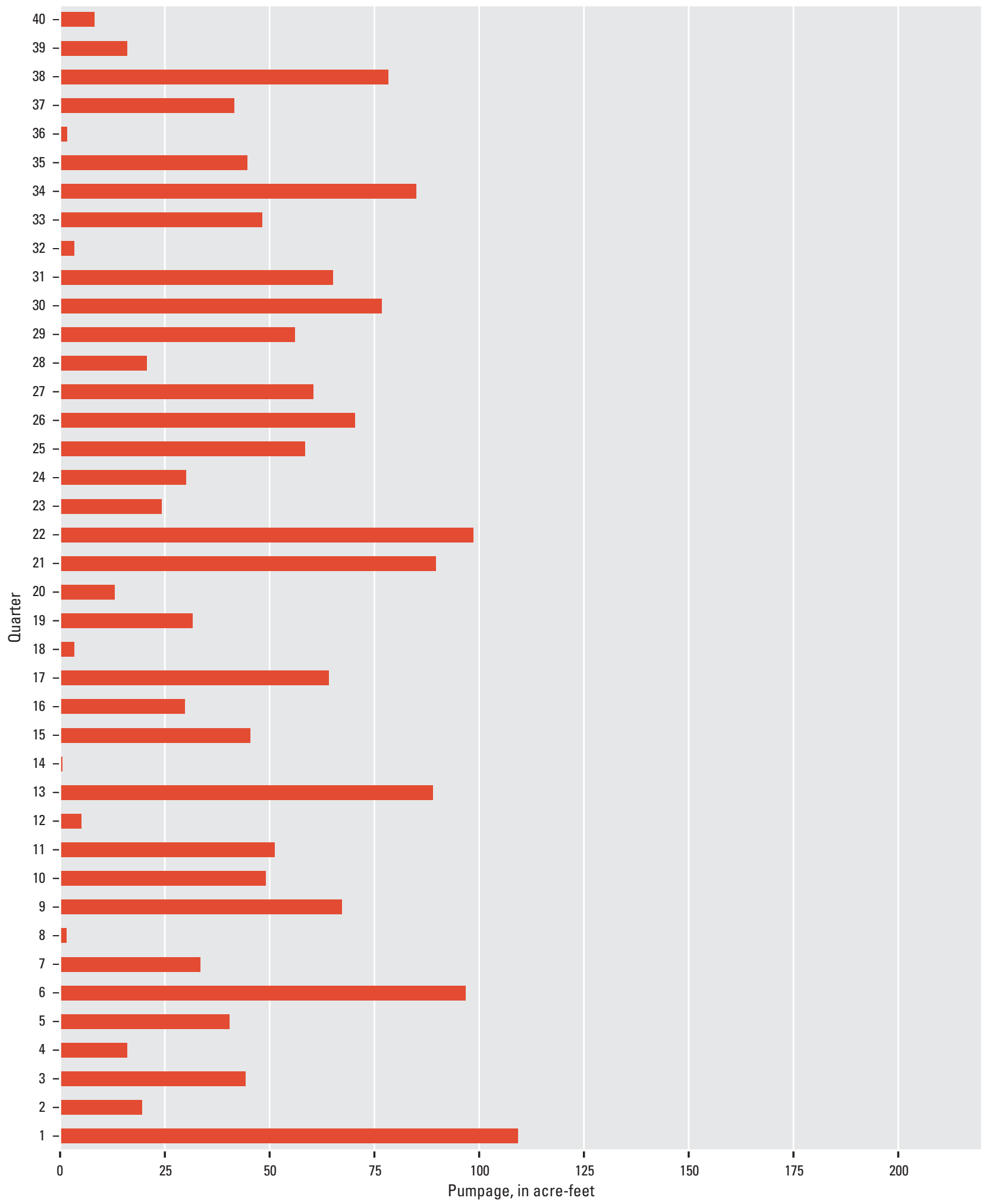

Figure D2-10. Optimal quarterly pumpage for the San Roque Park 2 well, schedule $2_{0^{\prime}}$ Santa Barbara, California. 


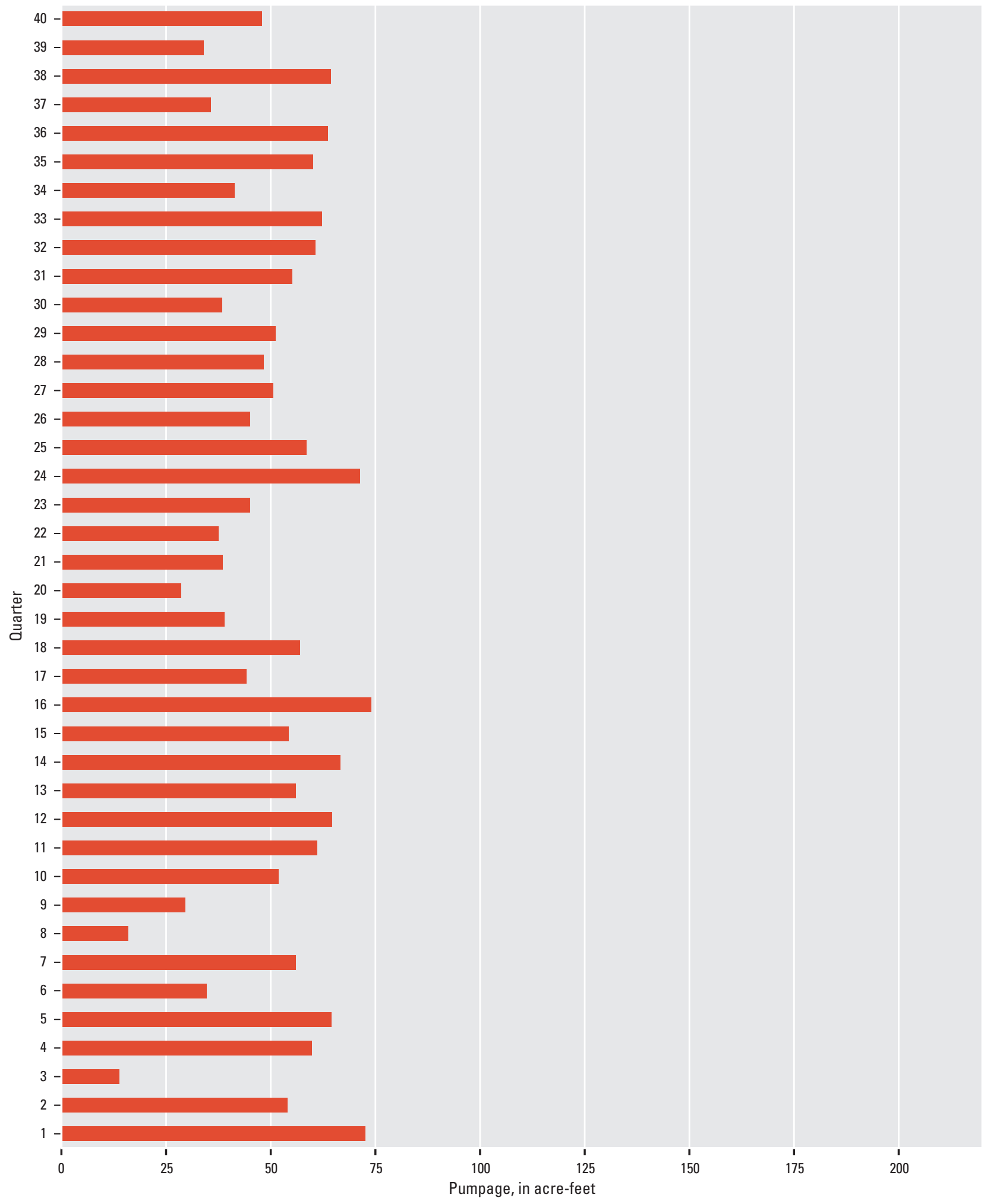

Figure D2-11. Optimal quarterly pumpage for the Val Verde well, schedule ${ }_{\mathrm{D}^{\prime}}$ Santa Barbara, California. 


\section{Appendix D-3: Scenario 3 Pumpage, by Well, Santa Barbara Multi-Objective Management Model, Santa Barbara, California}




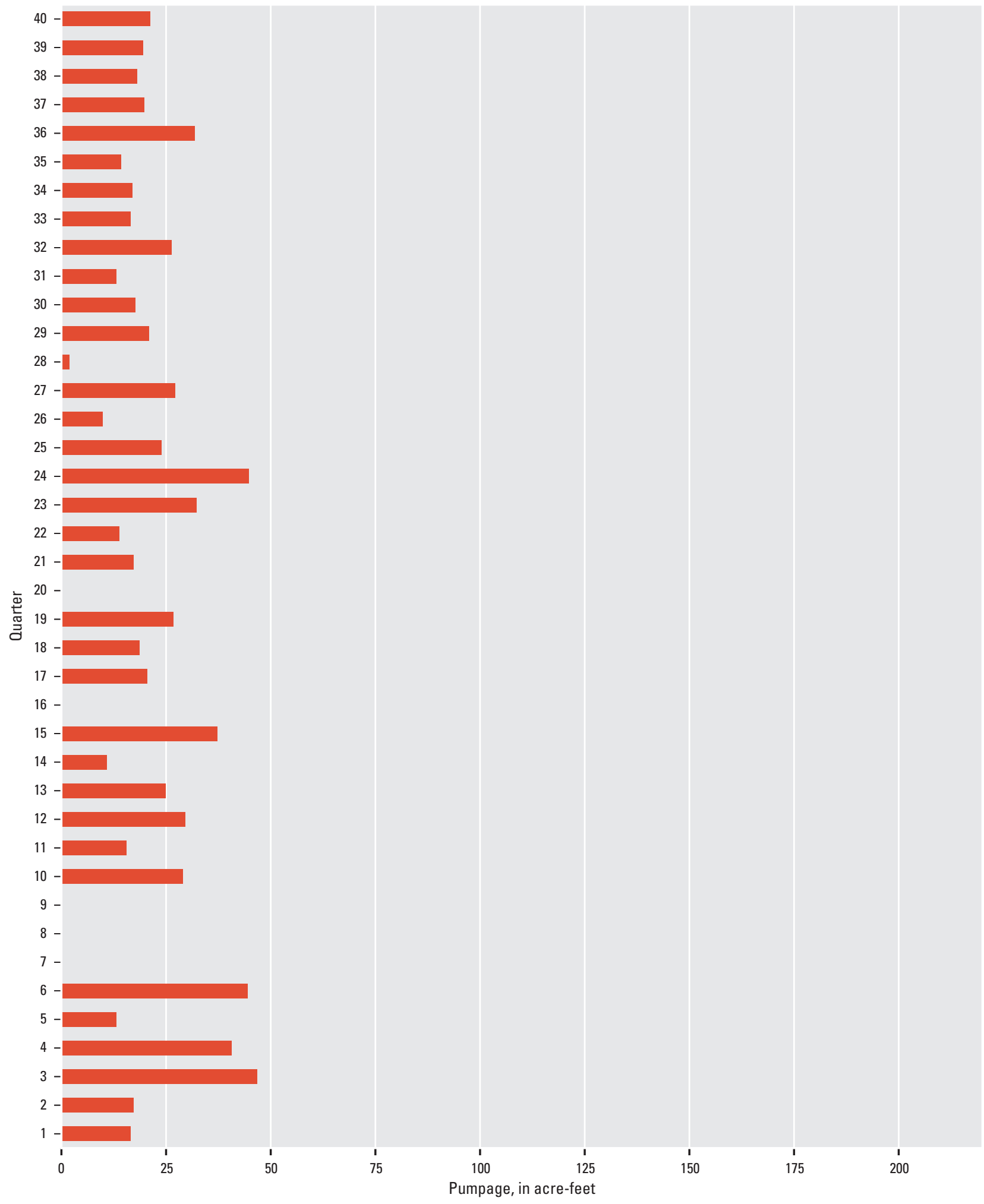

Figure D3-1. Quarterly pumpage (scaled from schedule $1_{0}$ to meet the demand of the Ortega water treatment plant) for the Alameda Park well, scenario 3, typical conditions, Santa Barbara, California. 


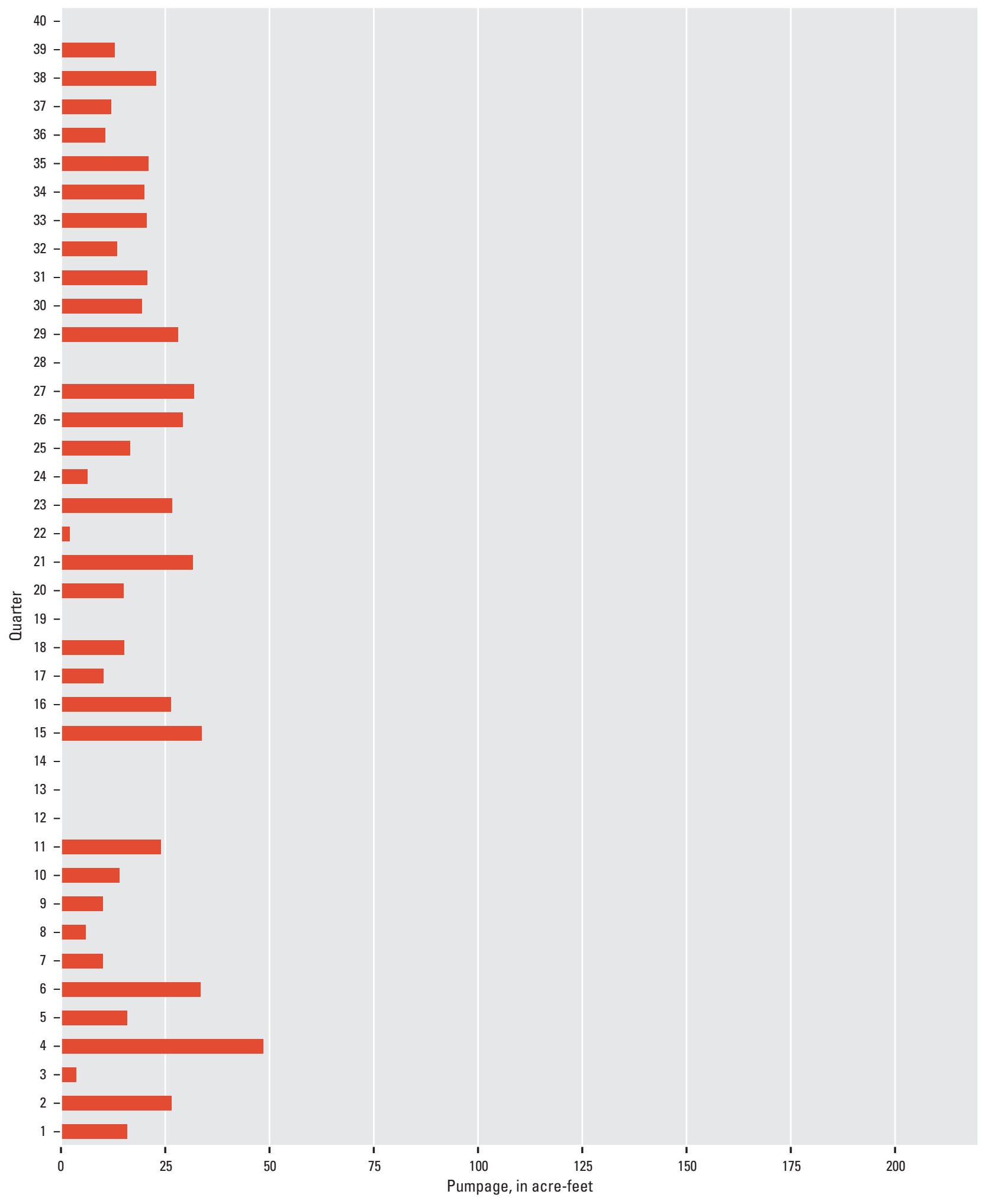

Figure D3-2. Quarterly pumpage (scaled from schedule $1_{D}$ to meet the demand of the Ortega water treatment plant) for the City Hall well, scenario 3, typical conditions, Santa Barbara, California. 


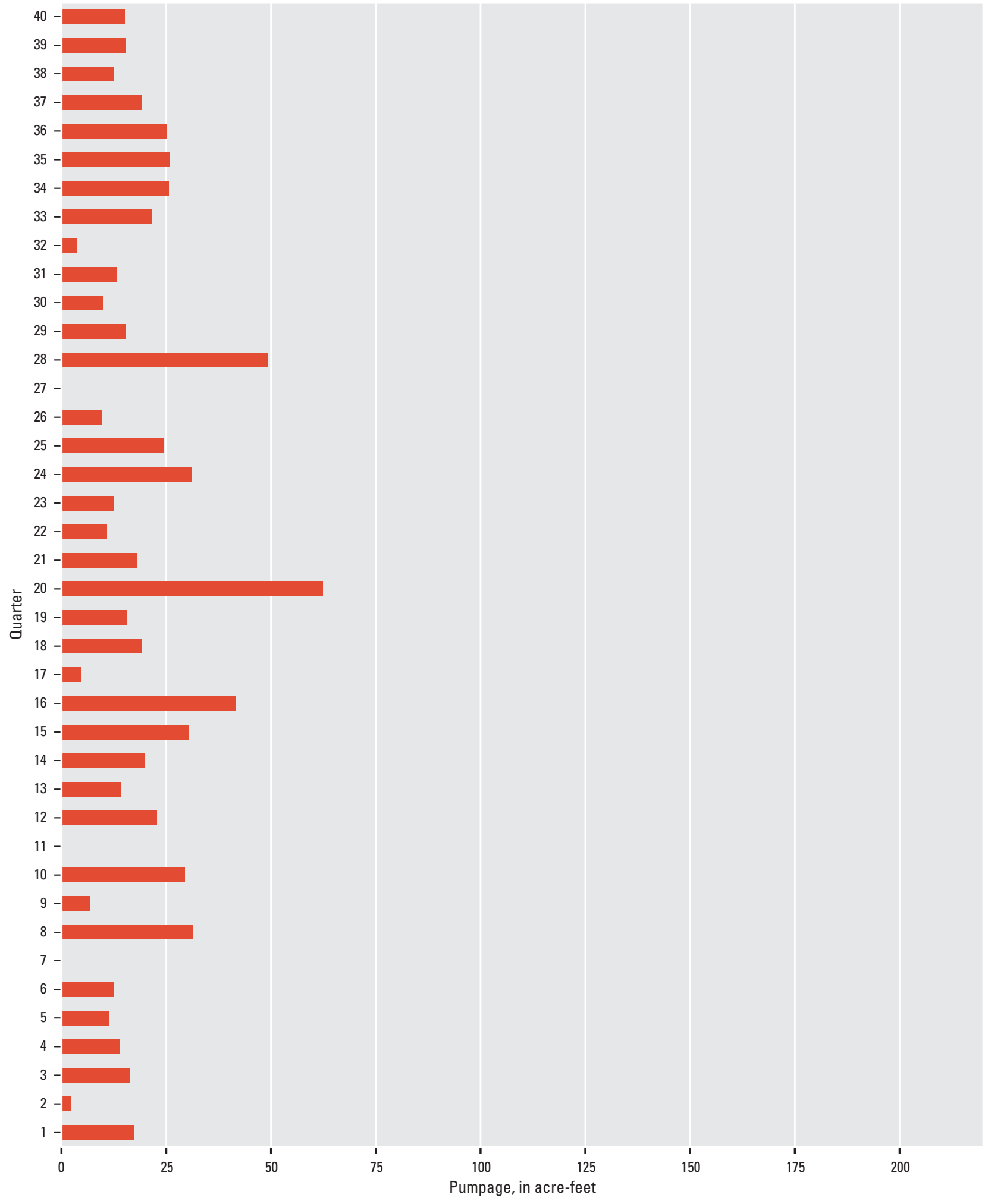

Figure D3-3. Quarterly pumpage (scaled from schedule $1_{0}$ to meet the demand of the Ortega water treatment plant) for the Corporation Yard well, scenario 3, typical conditions, Santa Barbara, California. 


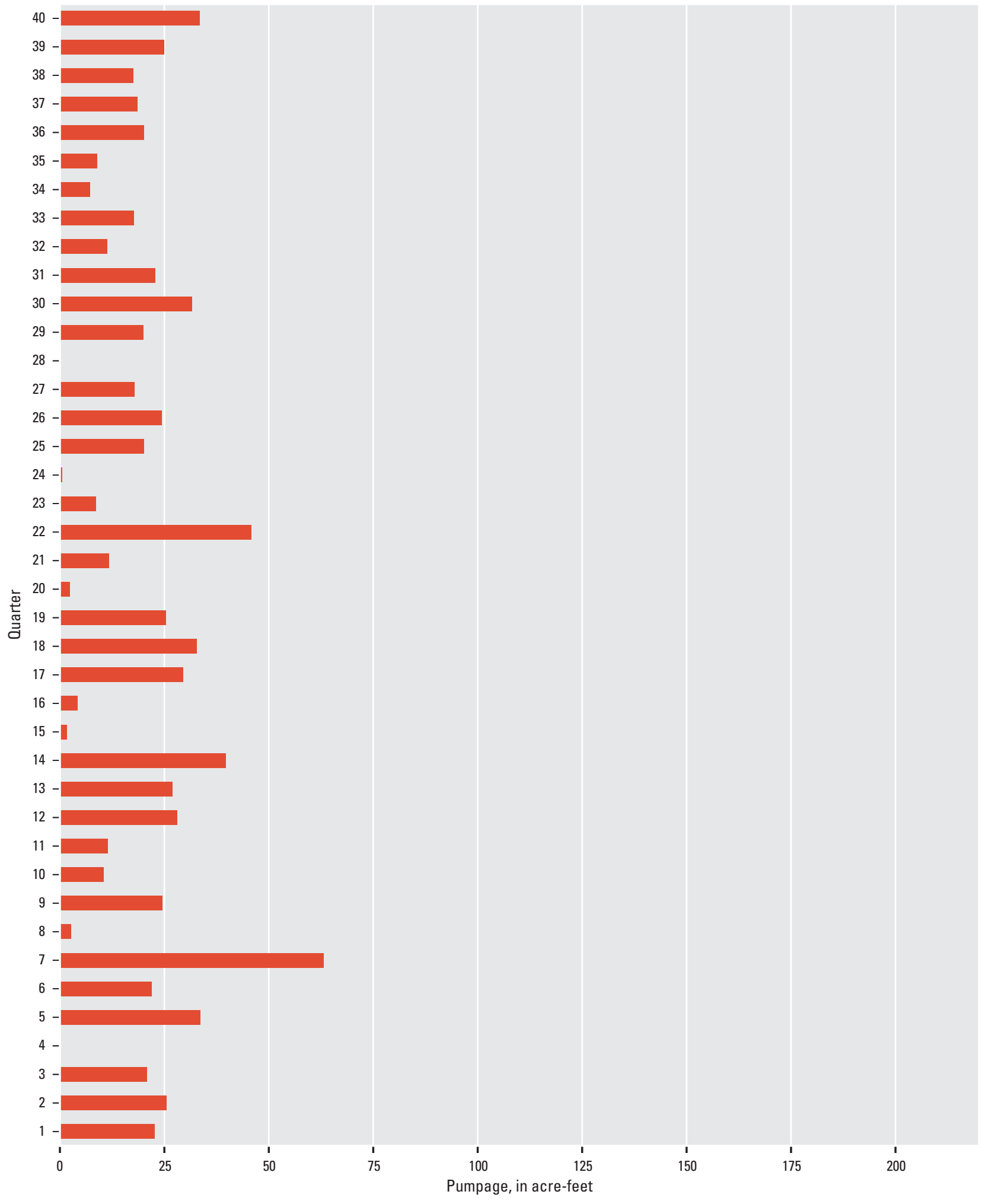

Figure D3-4. Quarterly pumpage (scaled from schedule $1_{D}$ to meet the demand of the Ortega water treatment plant) for the Ortega Park well, scenario 3, typical conditions, Santa Barbara, California. 


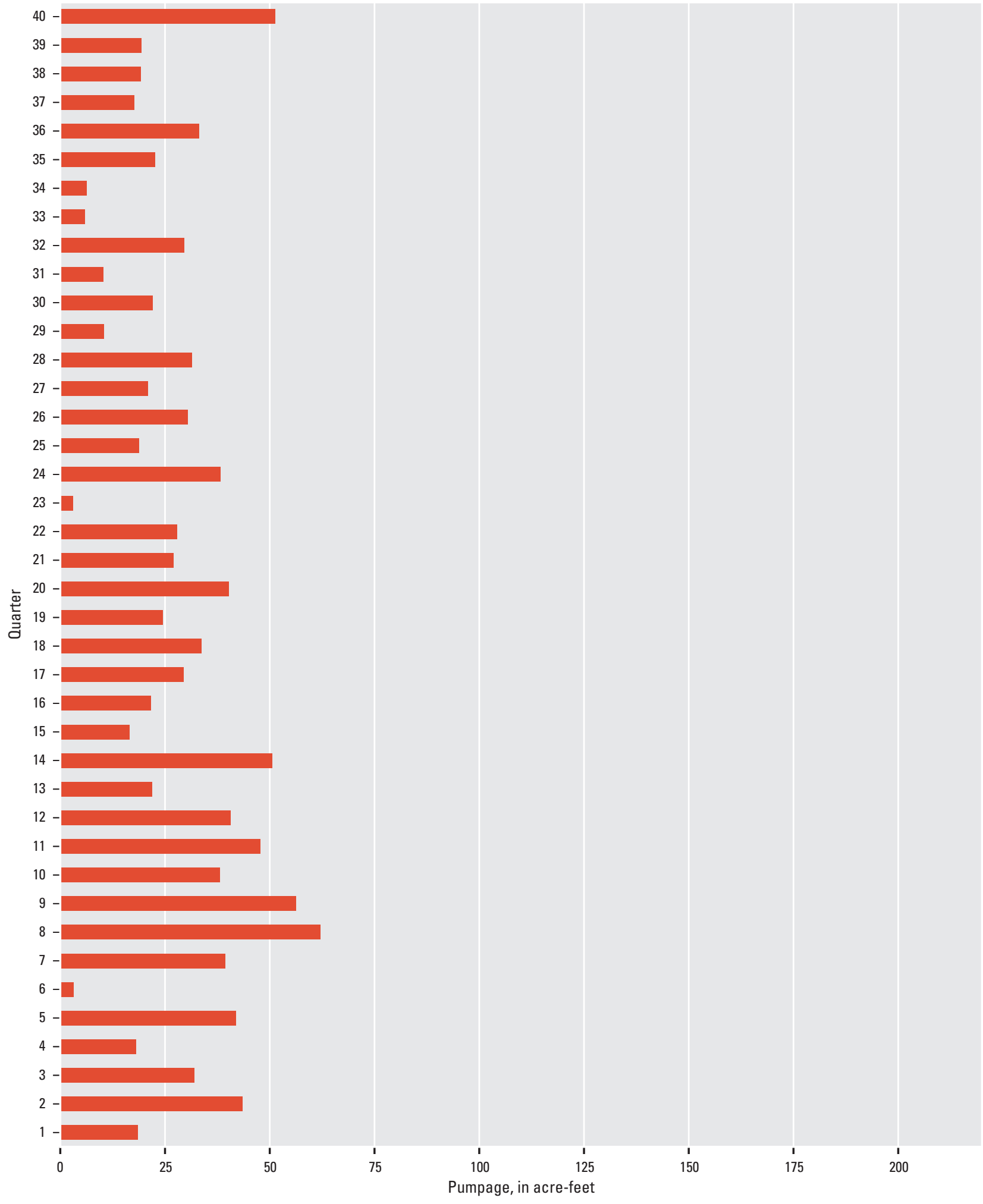

Figure D3-5. Quarterly pumpage (scaled from schedule $1_{D}$ to meet the demand of the Ortega water treatment plant) for the Santa Barbara High School well, scenario 3, typical conditions, Santa Barbara, California. 


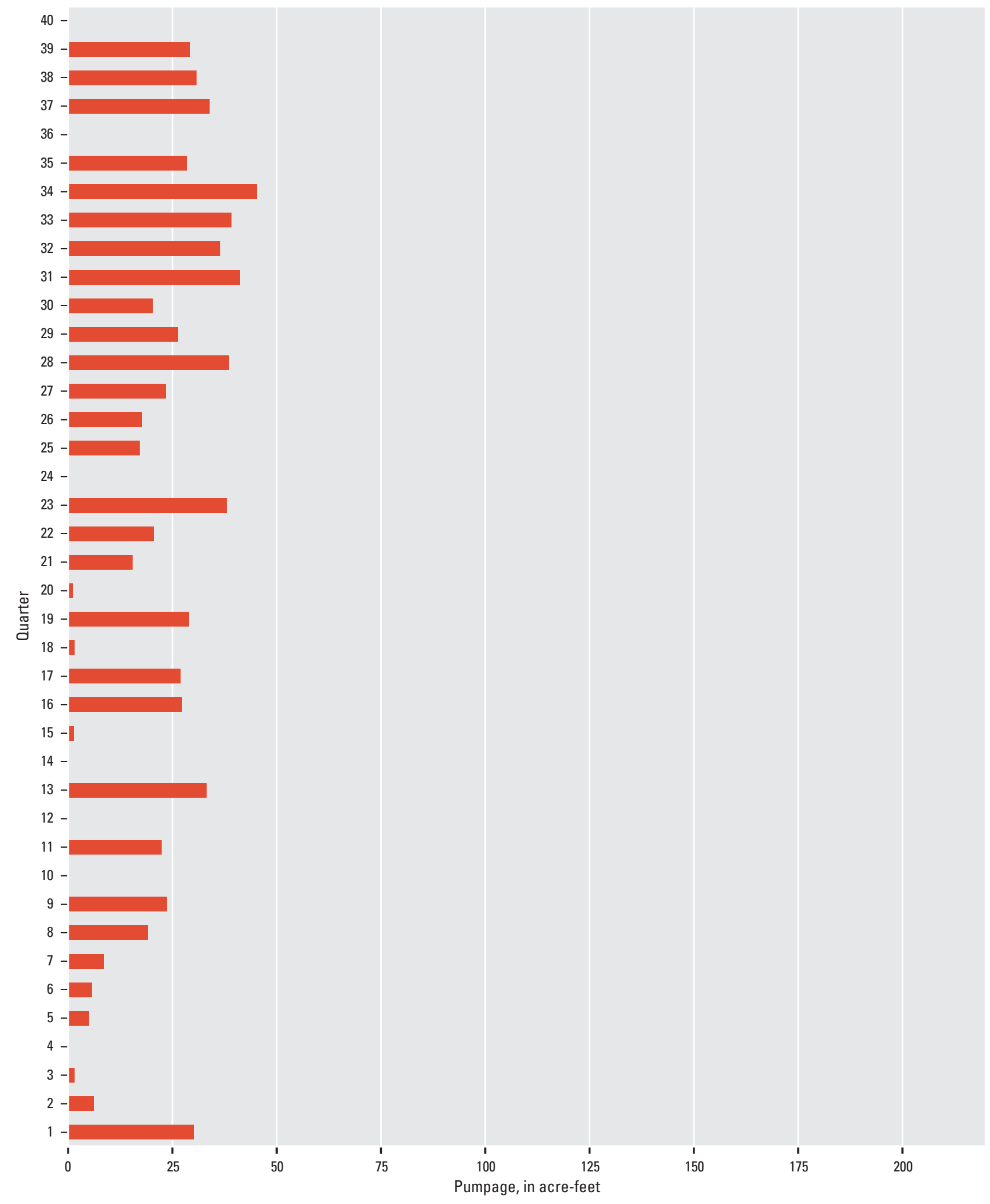

Figure D3-6. Quarterly pumpage (scaled from schedule $1_{D}$ to meet the demand of the Ortega water treatment plant) for the Vera Cruz well, scenario 3, typical conditions, Santa Barbara, California. 


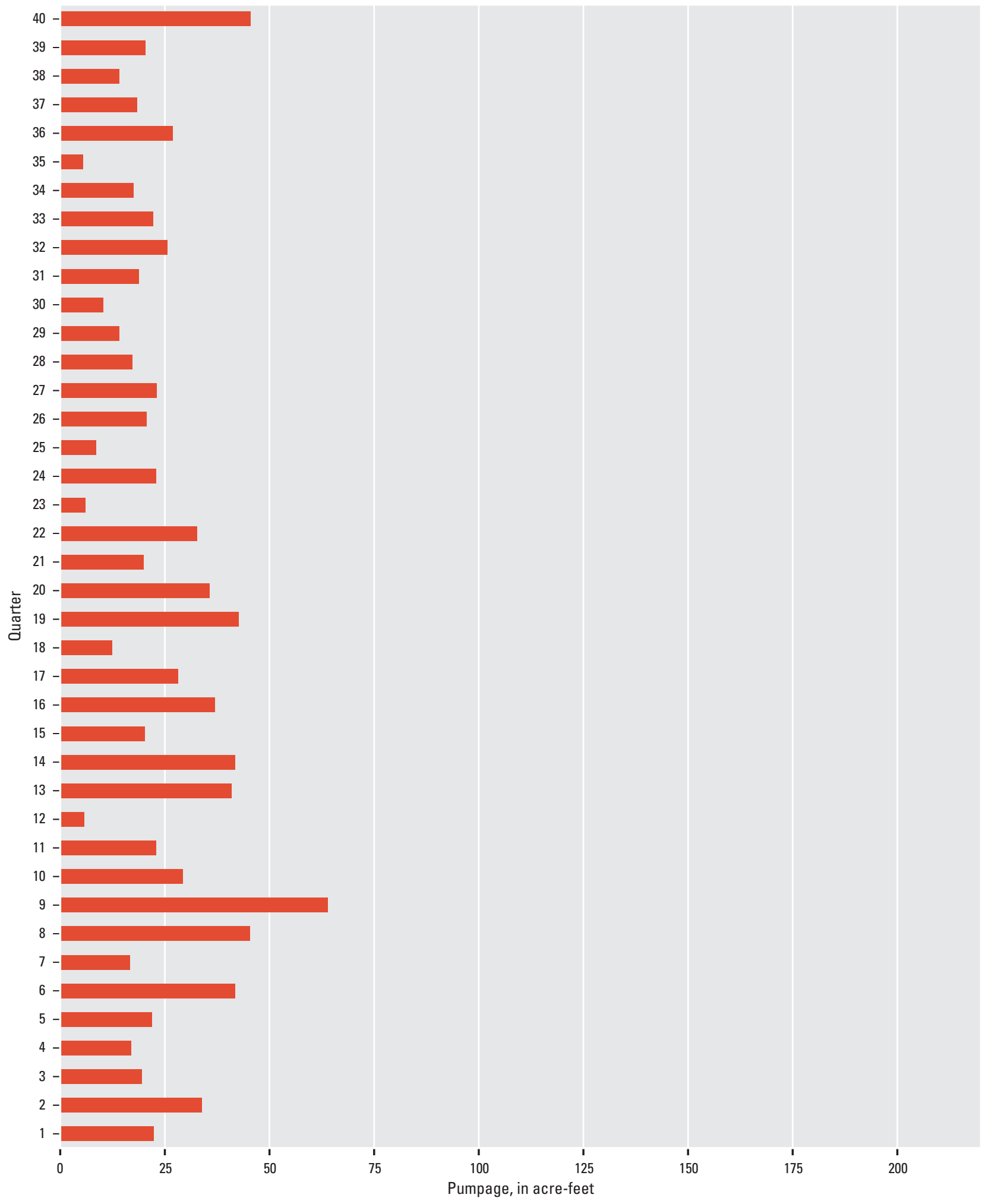

Figure D3-7. Quarterly pumpage (scaled from schedule $2_{0}$ to meet the demand of the Ortega water treatment plant) for the Alameda Park well, scenario 3, drought conditions, Santa Barbara, California. 


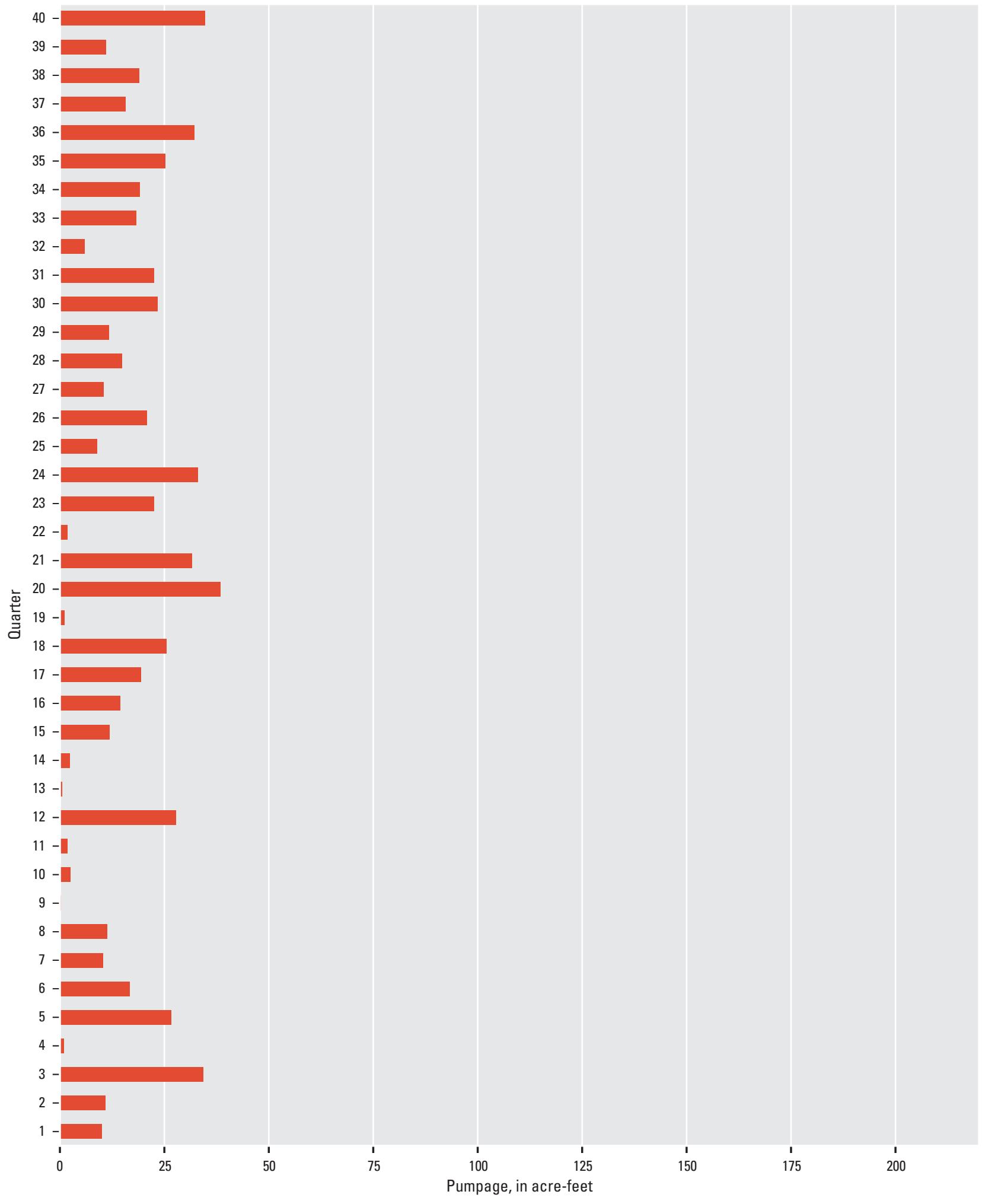

Figure D3-8. Quarterly pumpage (scaled from schedule $2_{D}$ to meet the demand of the Ortega water treatment plant) for the City Hall well, scenario 3, drought conditions, Santa Barbara, California. 


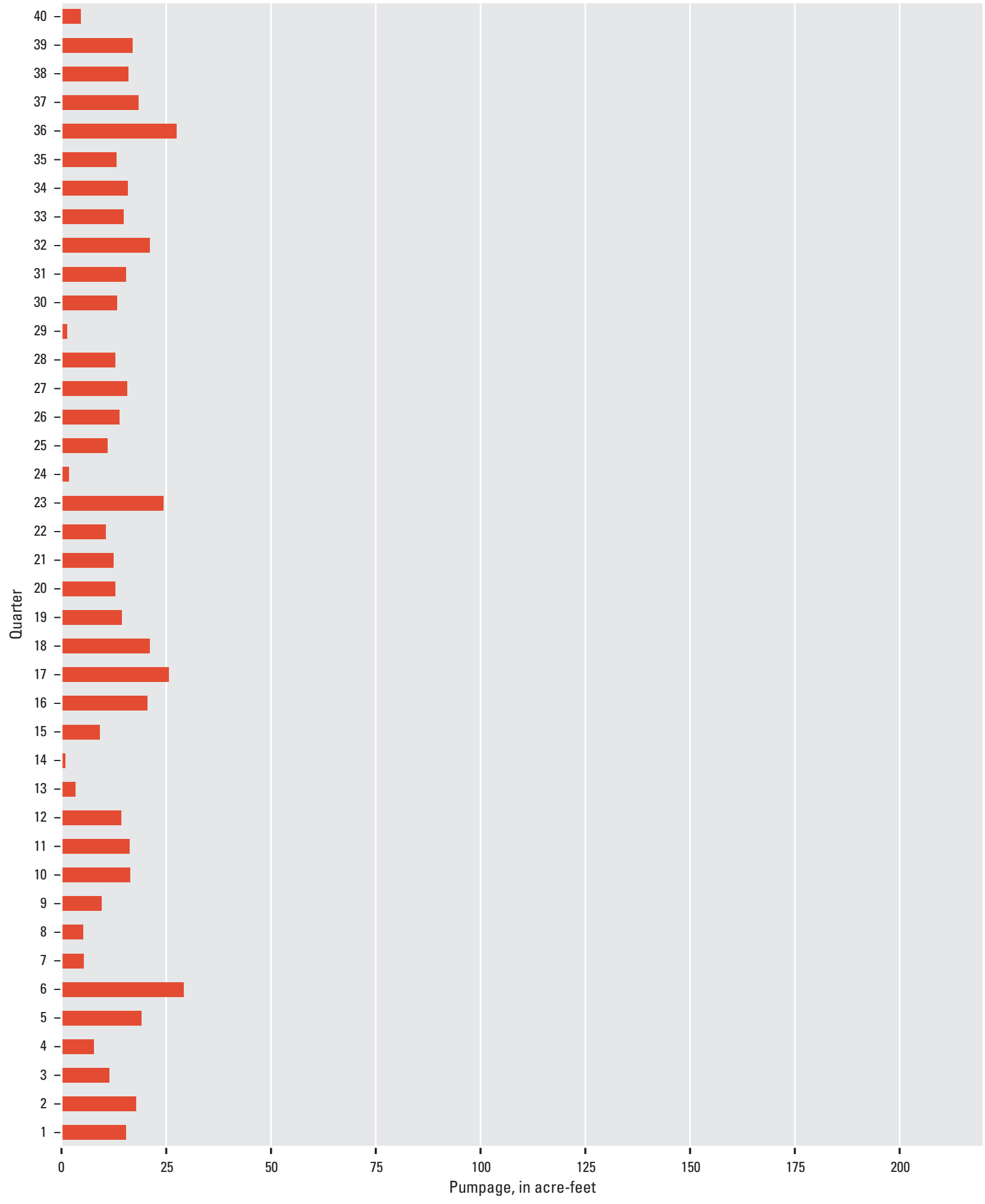

Figure D3-9. Quarterly pumpage (scaled from schedule $2_{D}$ to meet the demand of the Ortega water treatment plant) for the Corporation Yard well, scenario 3, drought conditions, Santa Barbara, California. 


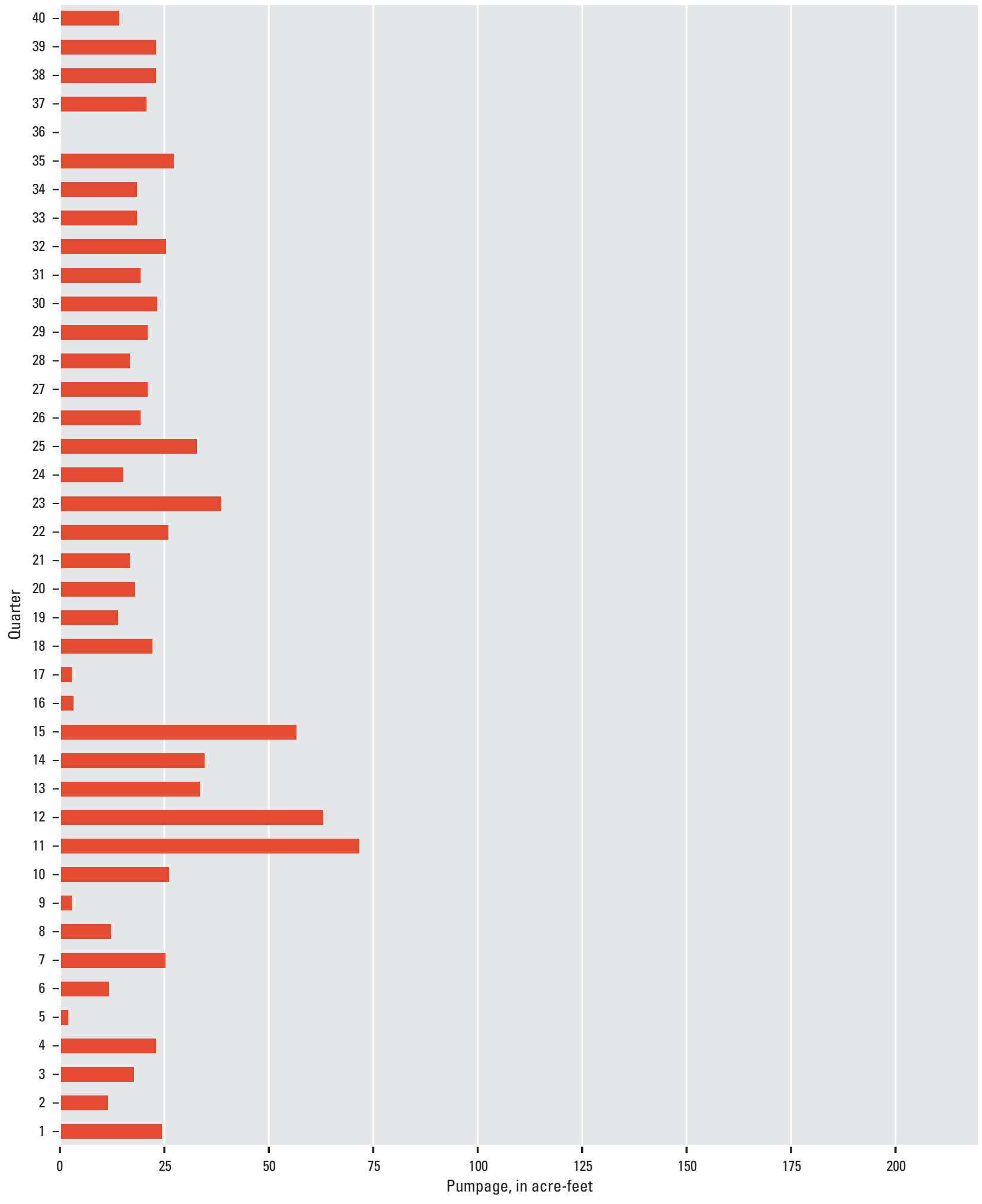

Figure D3-10. Quarterly pumpage (scaled from schedule 2 to meet the demand of the Ortega water treatment plant) for the Ortega Park well, scenario 3, drought conditions, Santa Barbara, California. 


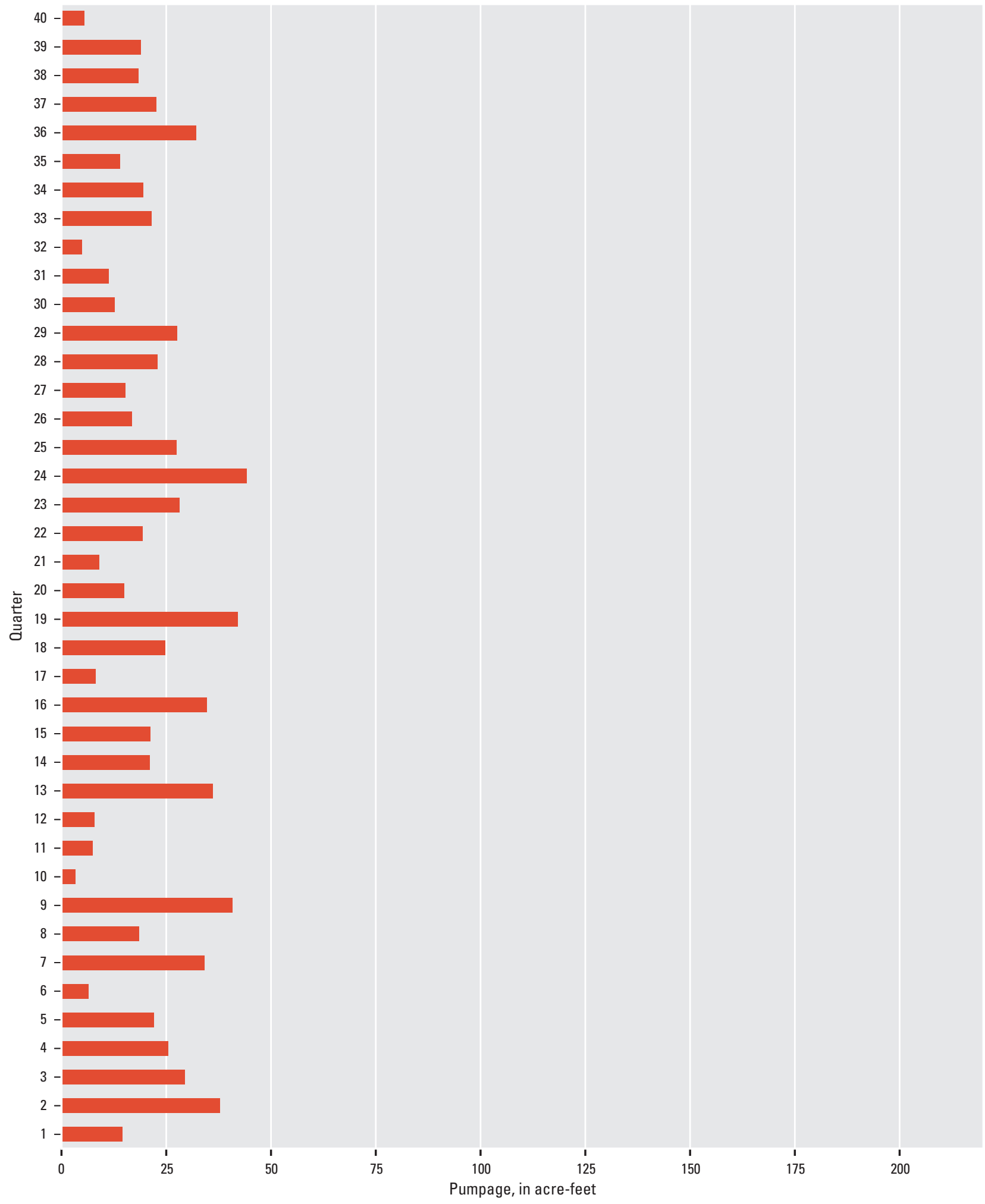

Figure D3-11. Quarterly pumpage (scaled from schedule $2_{D}$ to meet the demand of the Ortega water treatment plant) for the Santa Barbara High School well, scenario 3, drought conditions, Santa Barbara, California. 


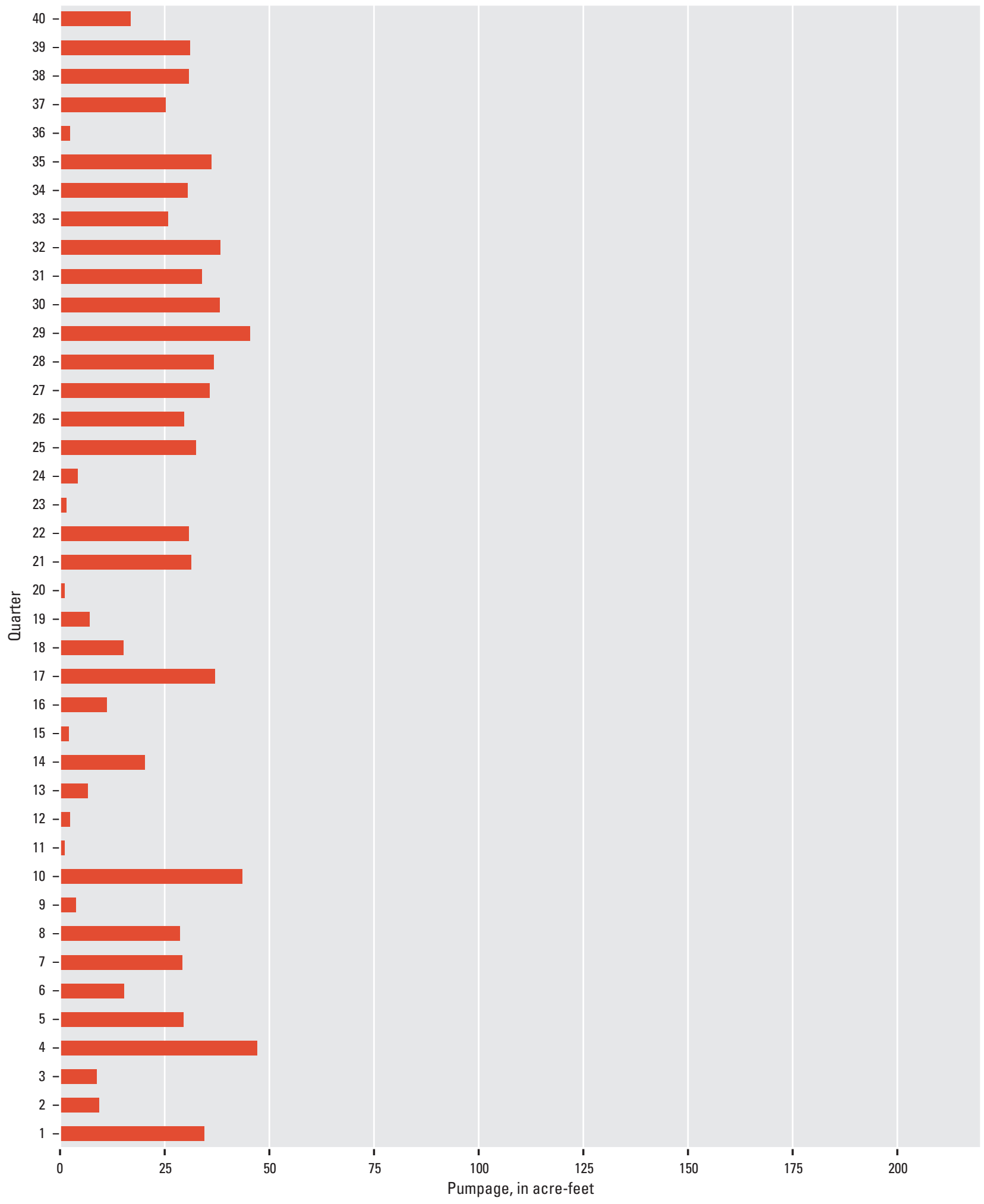

Figure D3-12. Quarterly pumpage (scaled from schedule $2_{0}$ to meet the demand of the Ortega water treatment plant) for the Vera Cruz well, scenario 3, drought conditions, Santa Barbara, California. 
360 Santa Barbara and Foothill Groundwater Basins Geohydrology and Optimal Water Resources Management

\section{Appendix D-4: Scenario 4B Decision Rules, Santa Barbara Multi-Objective Management Model, Santa Barbara, California}



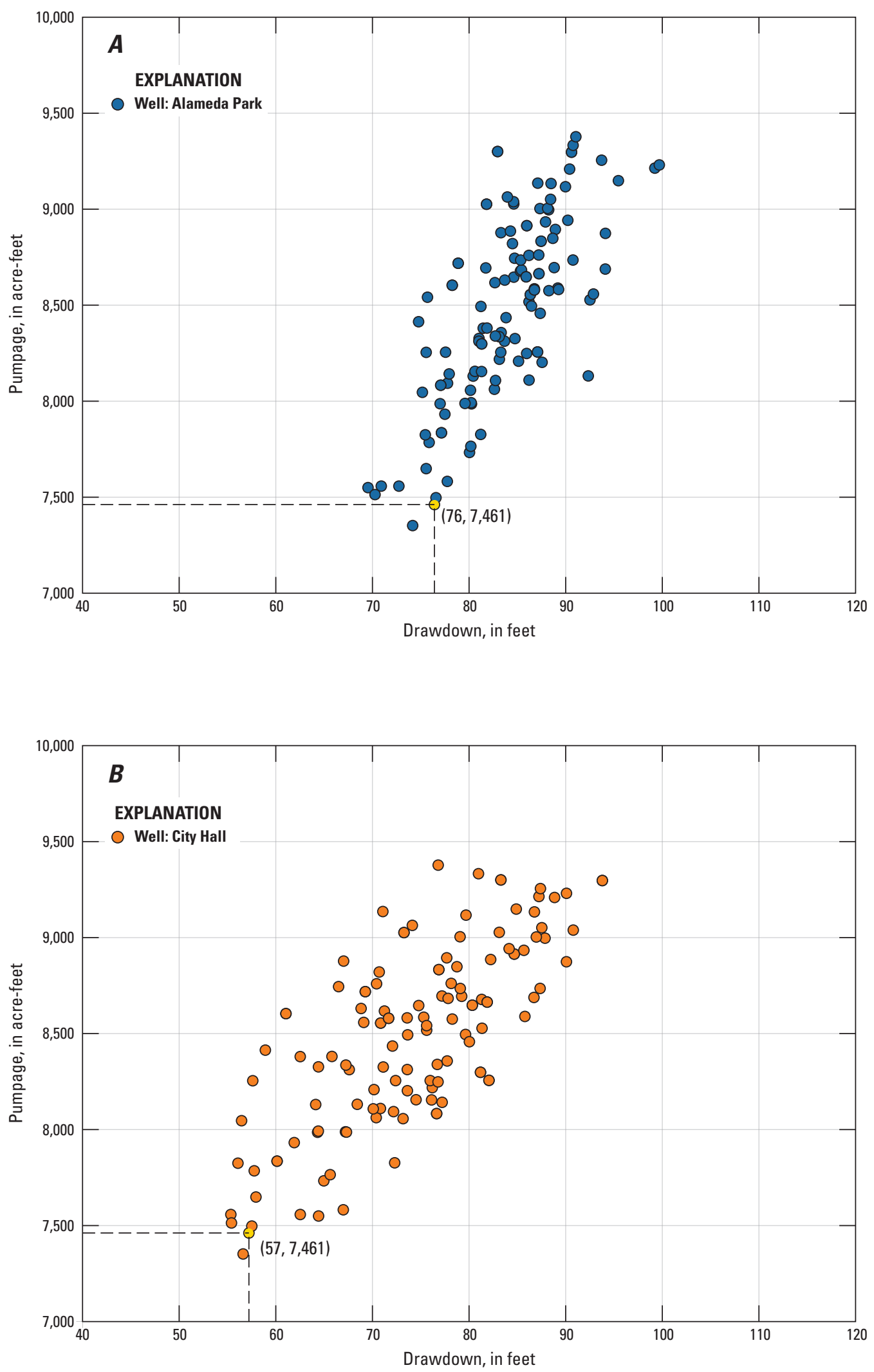

Figure D4-1. Decision rules for total Foothill groundwater basin pumpage as a function of maximum drawdown at the city of Santa Barbara production wells assuming scenario 1 (typical) precipitation, Santa Barbara groundwater basin, Santa Barbara, California (the yellow dot represents the total schedule $1_{D}$ pumpage for Foothill groundwater basin), $A$, Alameda Park; $B$, City Hall; $C$, Corporation Yard; $D$, Hope Avenue; E, Lincolnwood 1; F, Los Robles; G, Ortega Park; H, San Roque Park 2; I, Santa Barbara High School; J, Val Verde; and $K$, Vera Cruz. 

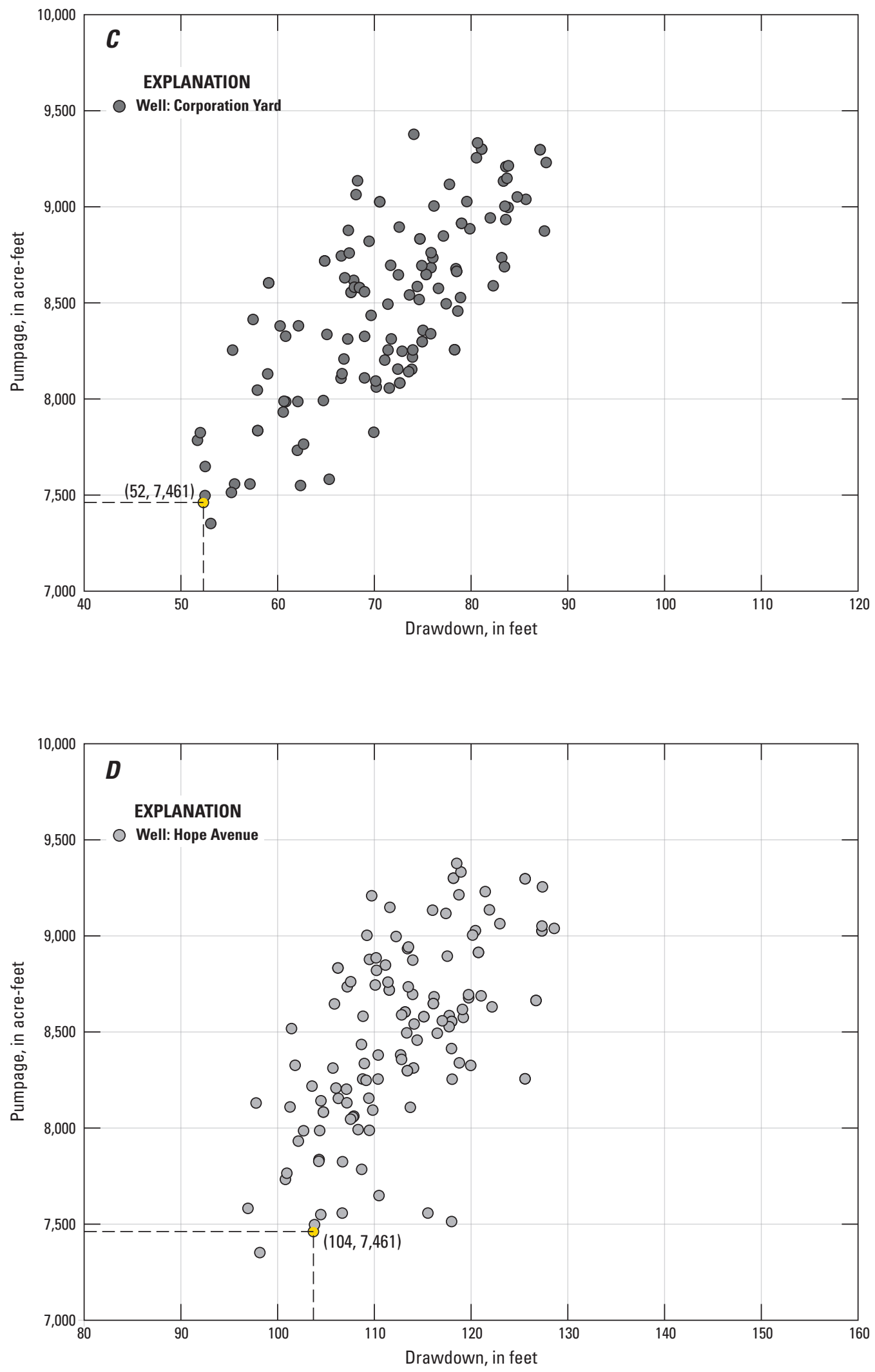

Figure D4-1. - Continued 

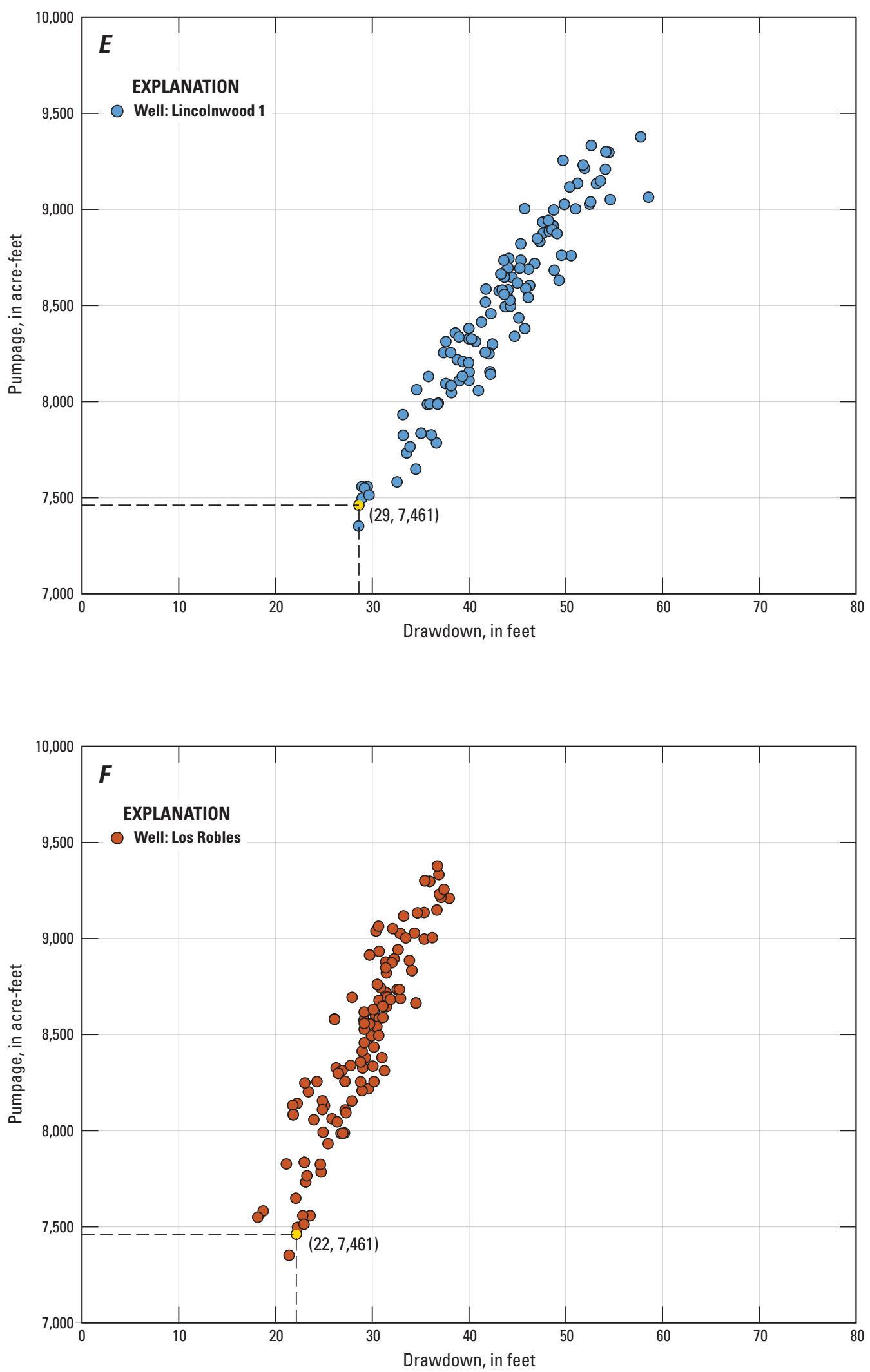

Figure D4-1. - Continued 

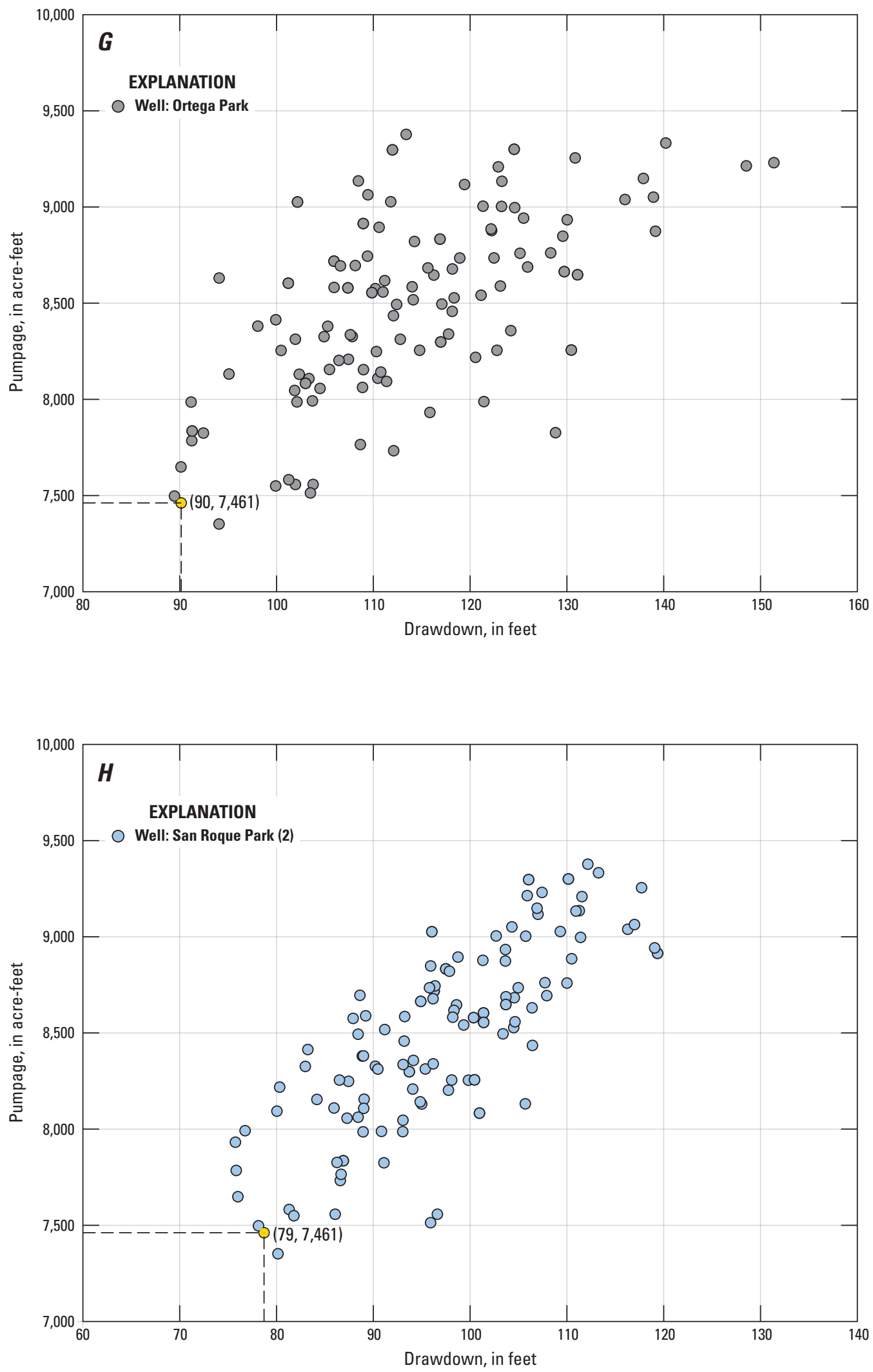

Figure D4-1. - Continued 

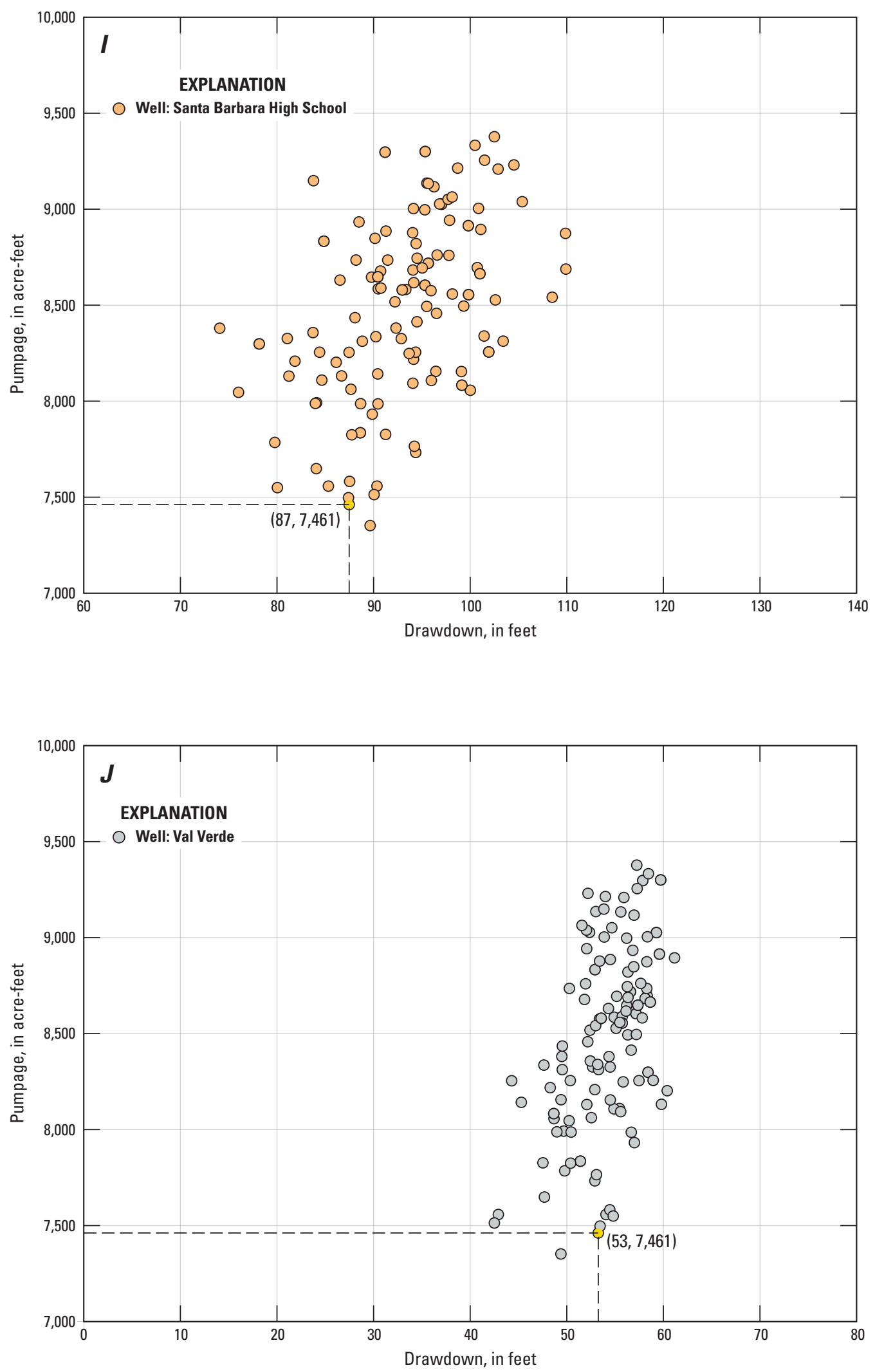

Figure D4-1. - Continued 
366 Santa Barbara and Foothill Groundwater Basins Geohydrology and Optimal Water Resources Management

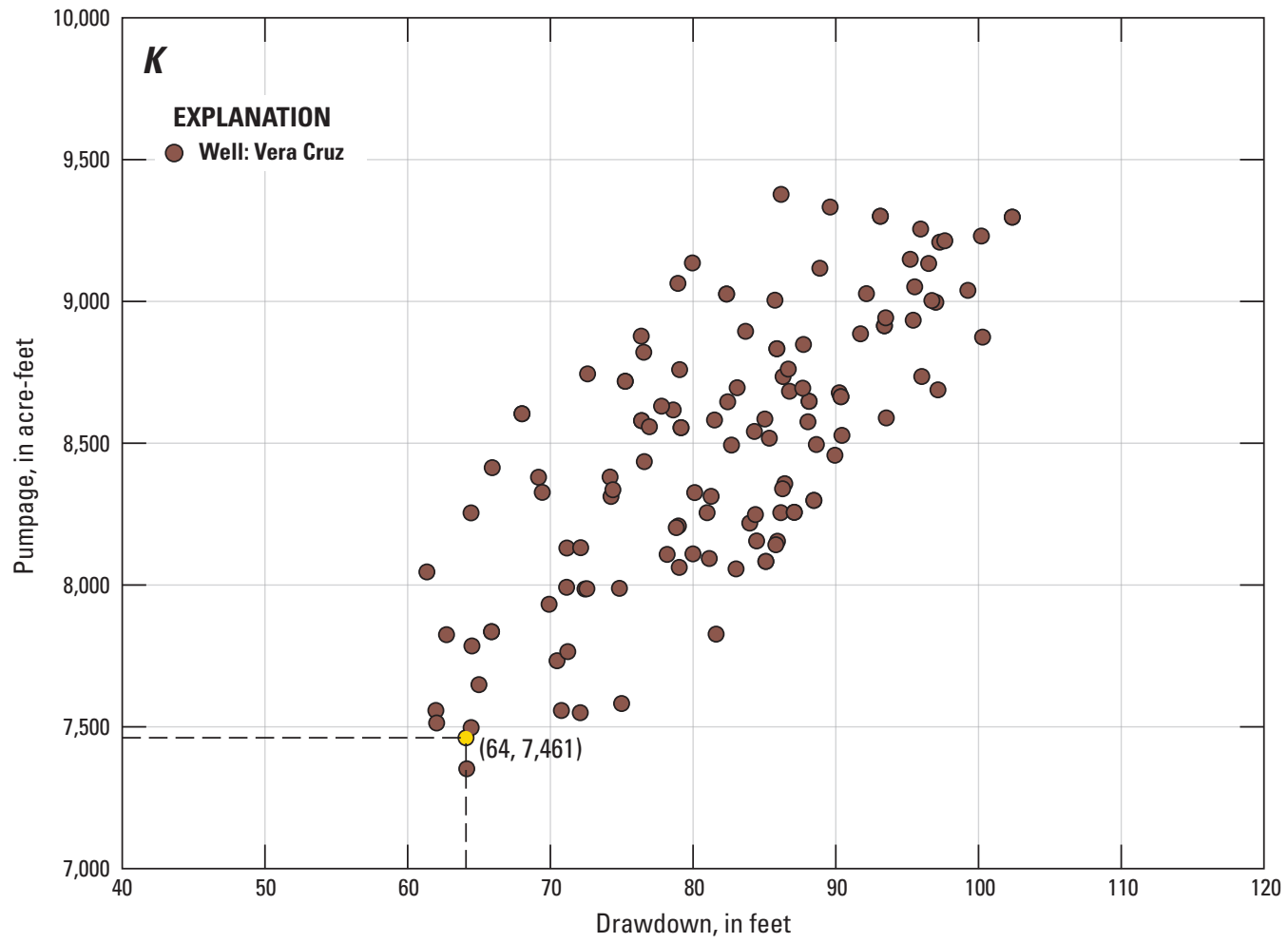

Figure D4-1. - Continued 

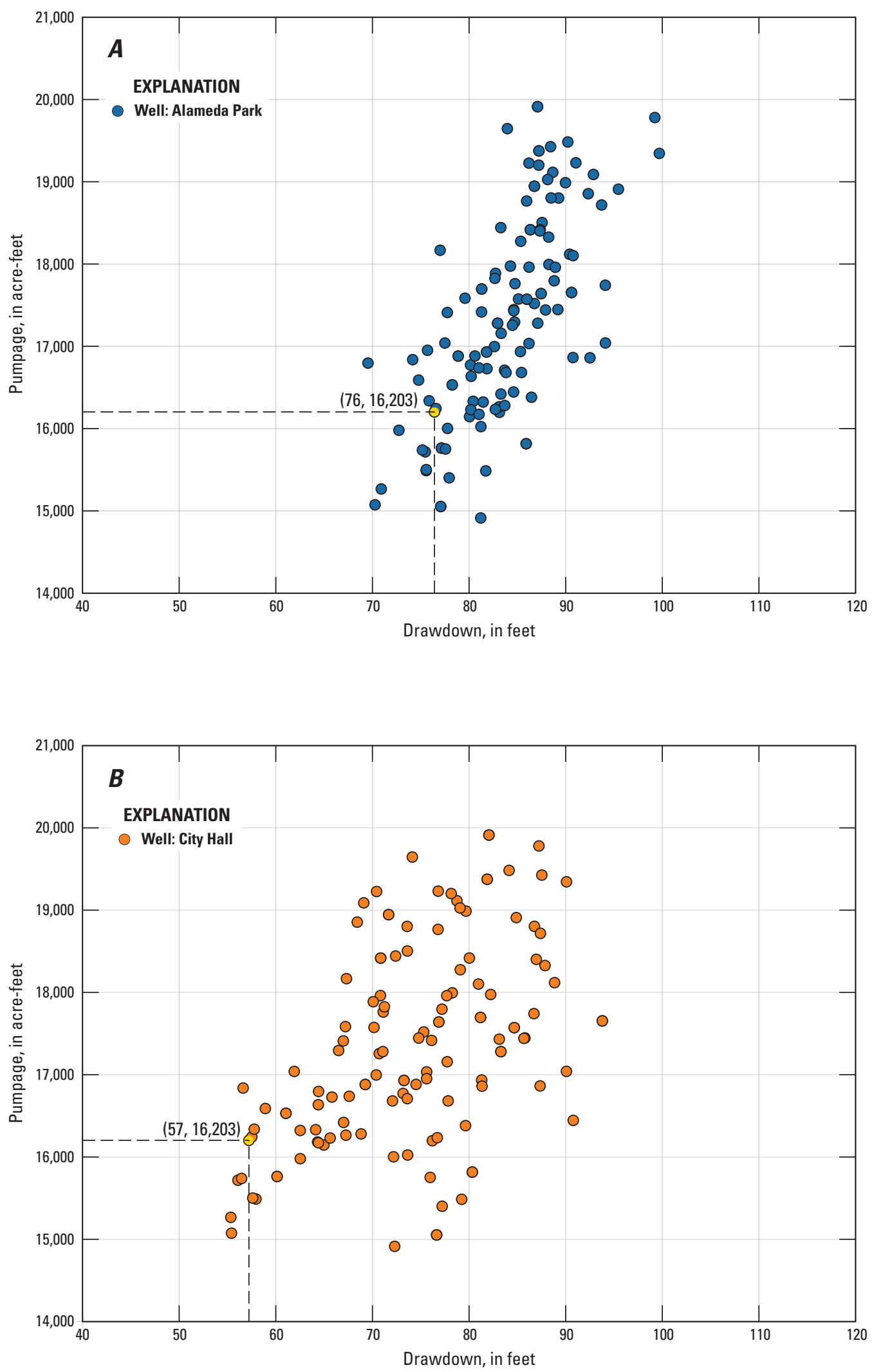

Figure D4-2. Decision rules for total Storage Unit I pumpage as a function of maximum drawdown at the city of Santa Barbara production wells assuming scenario 1 (typical) precipitation, Santa Barbara groundwater basin, Santa Barbara, California (the yellow dot represents the total schedule $1_{D}$ pumpage for Storage Unit I), $A$, Alameda Park; $B$, City Hall; $C$, Corporation Yard; $D$, Hope Avenue; E, Lincolnwood 1; F, Los Robles; G, Ortega Park; H, San Roque Park 2; I, Santa Barbara High School; J, Val Verde; and K, Vera Cruz. 

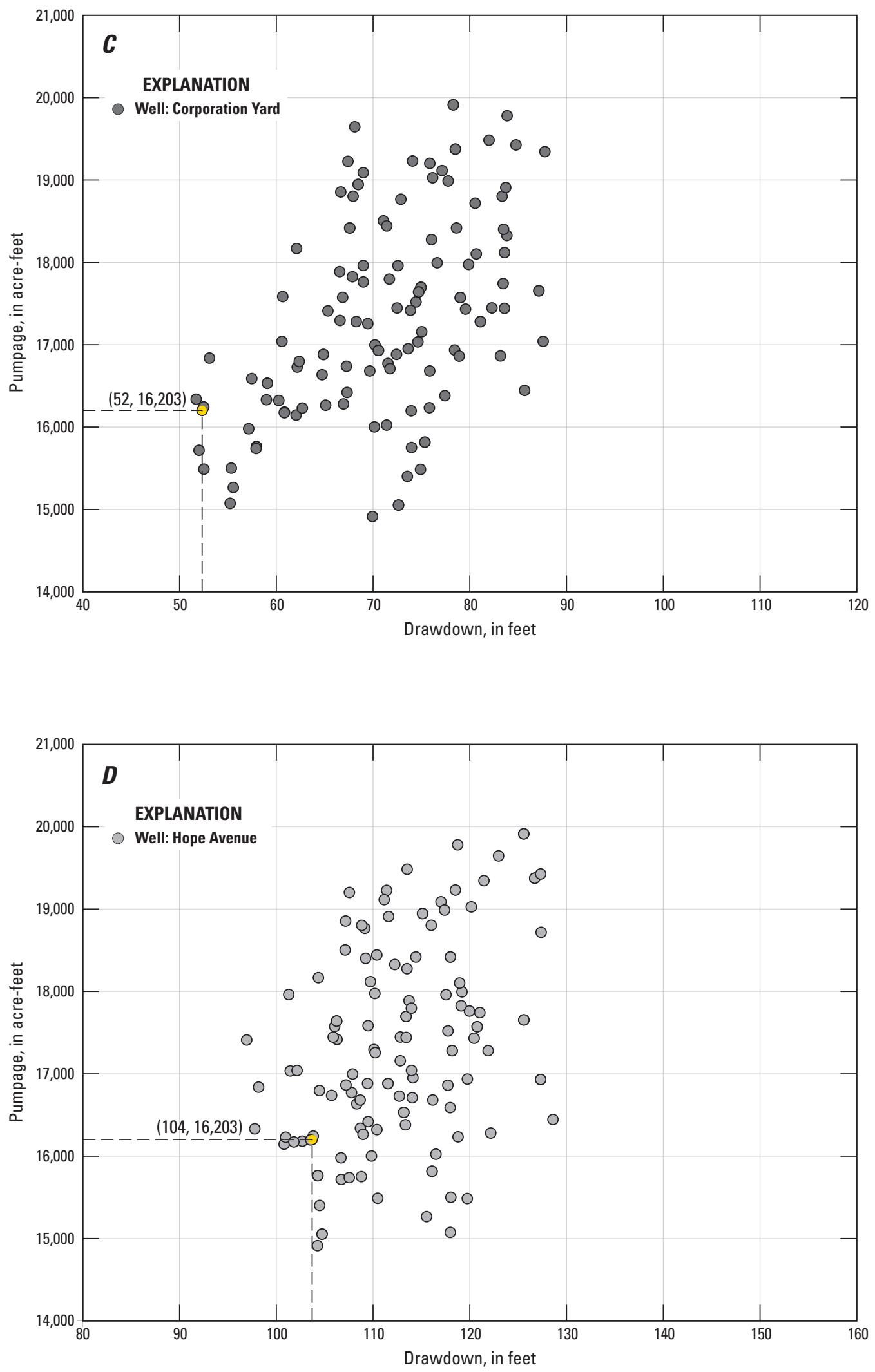

Figure D4-2. - Continued 

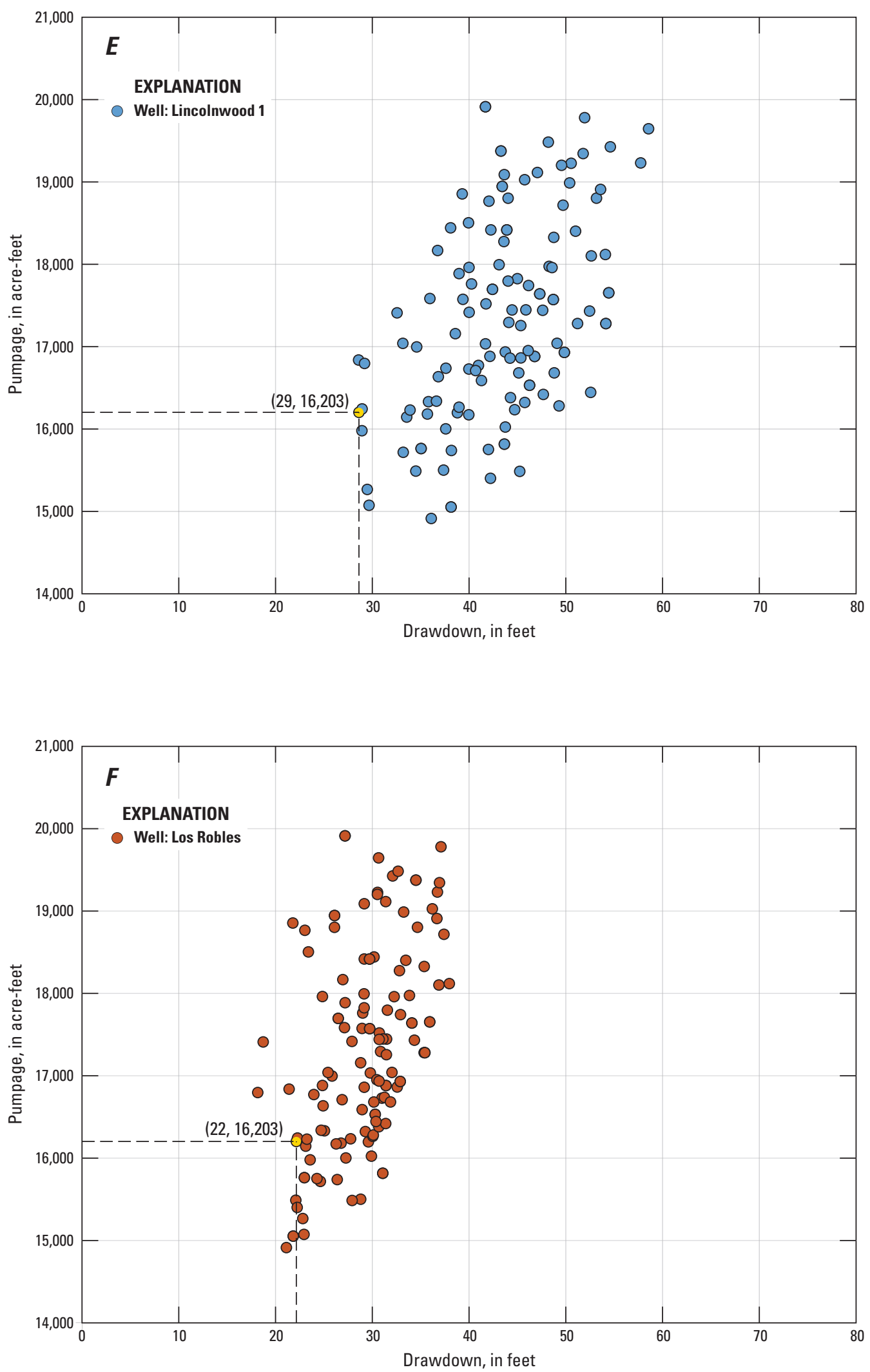

Figure D4-2. - Continued 

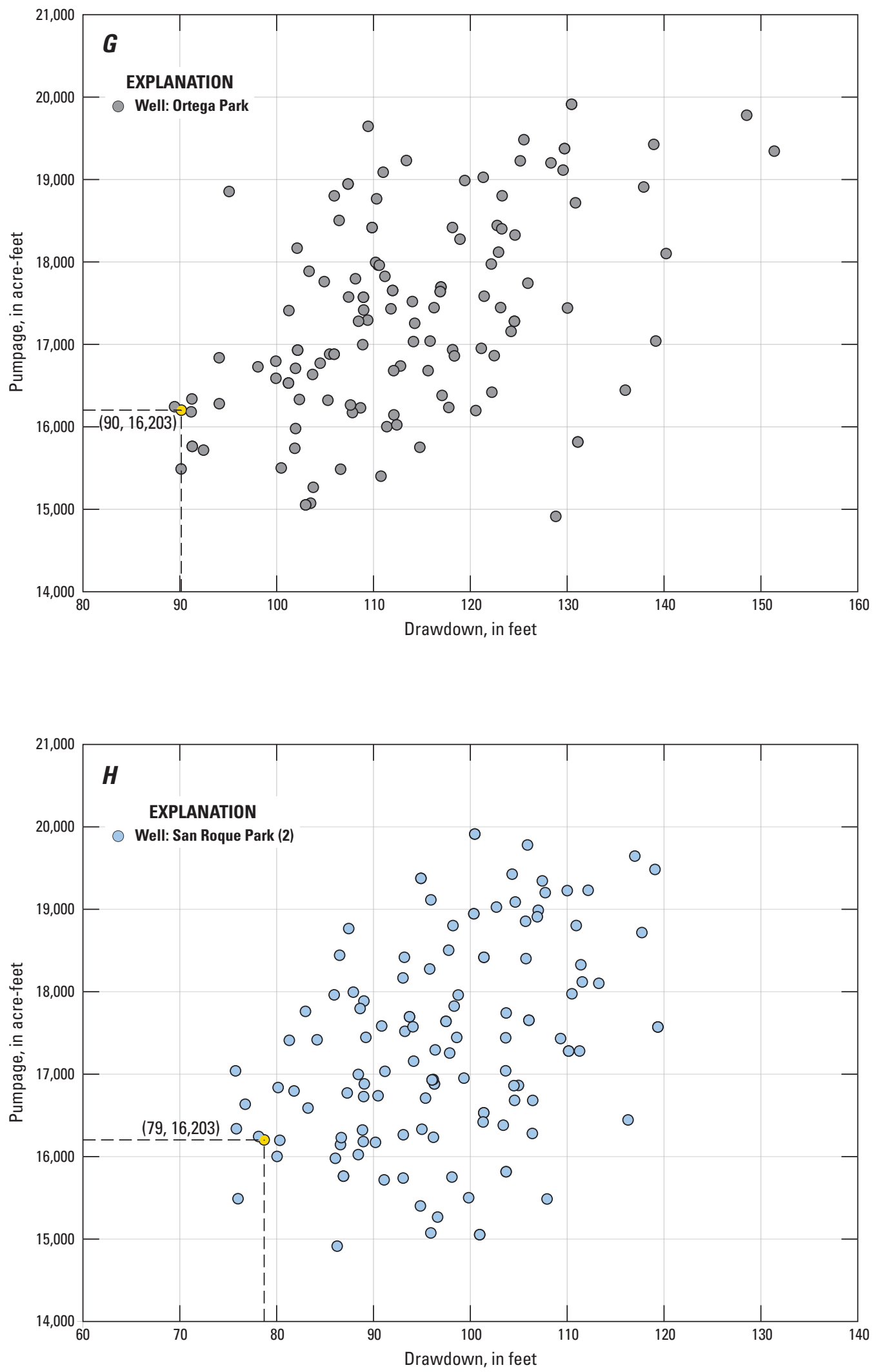

Figure D4-2. - Continued 

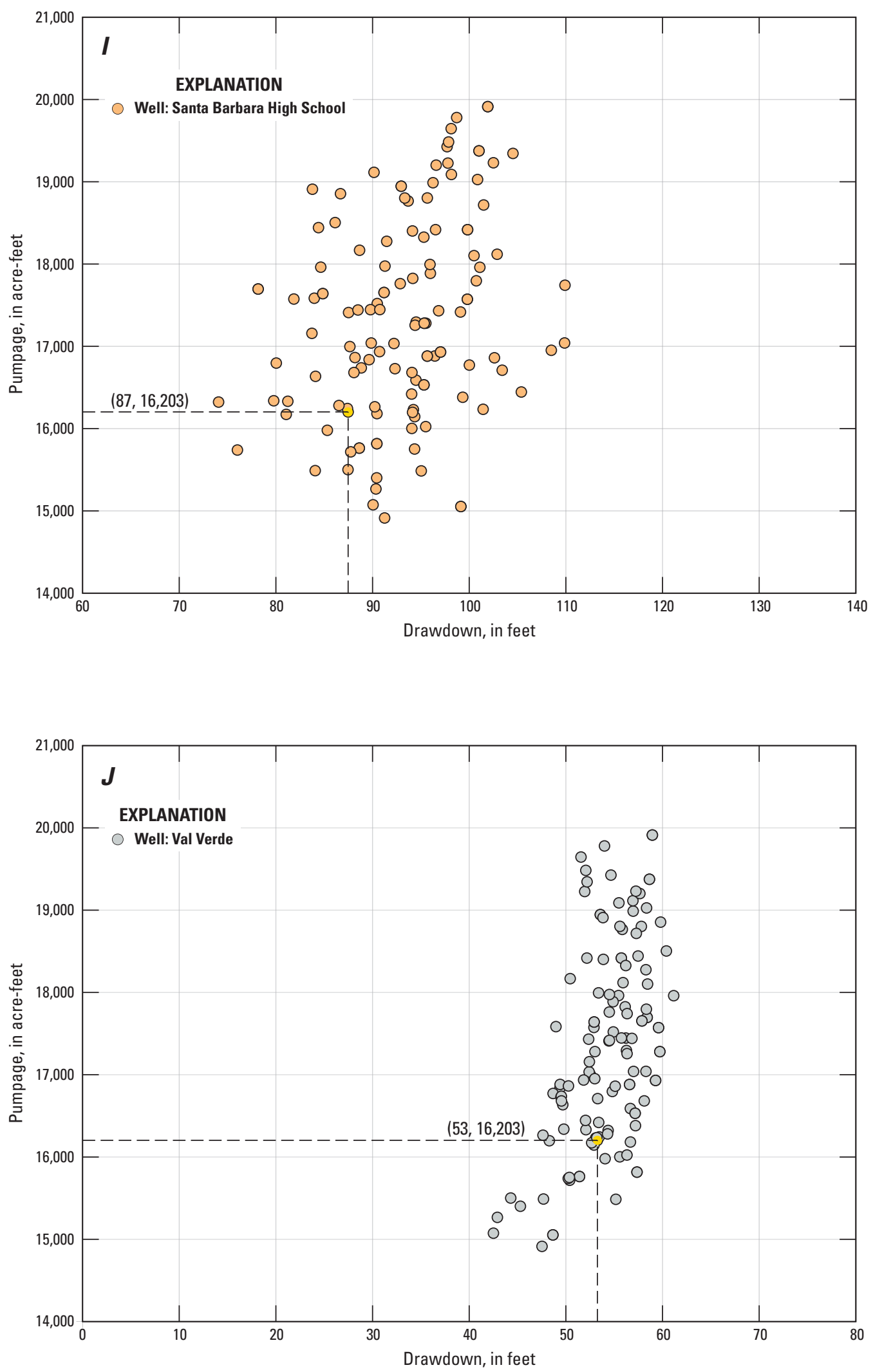

Figure D4-2. - Continued 
372 Santa Barbara and Foothill Groundwater Basins Geohydrology and Optimal Water Resources Management

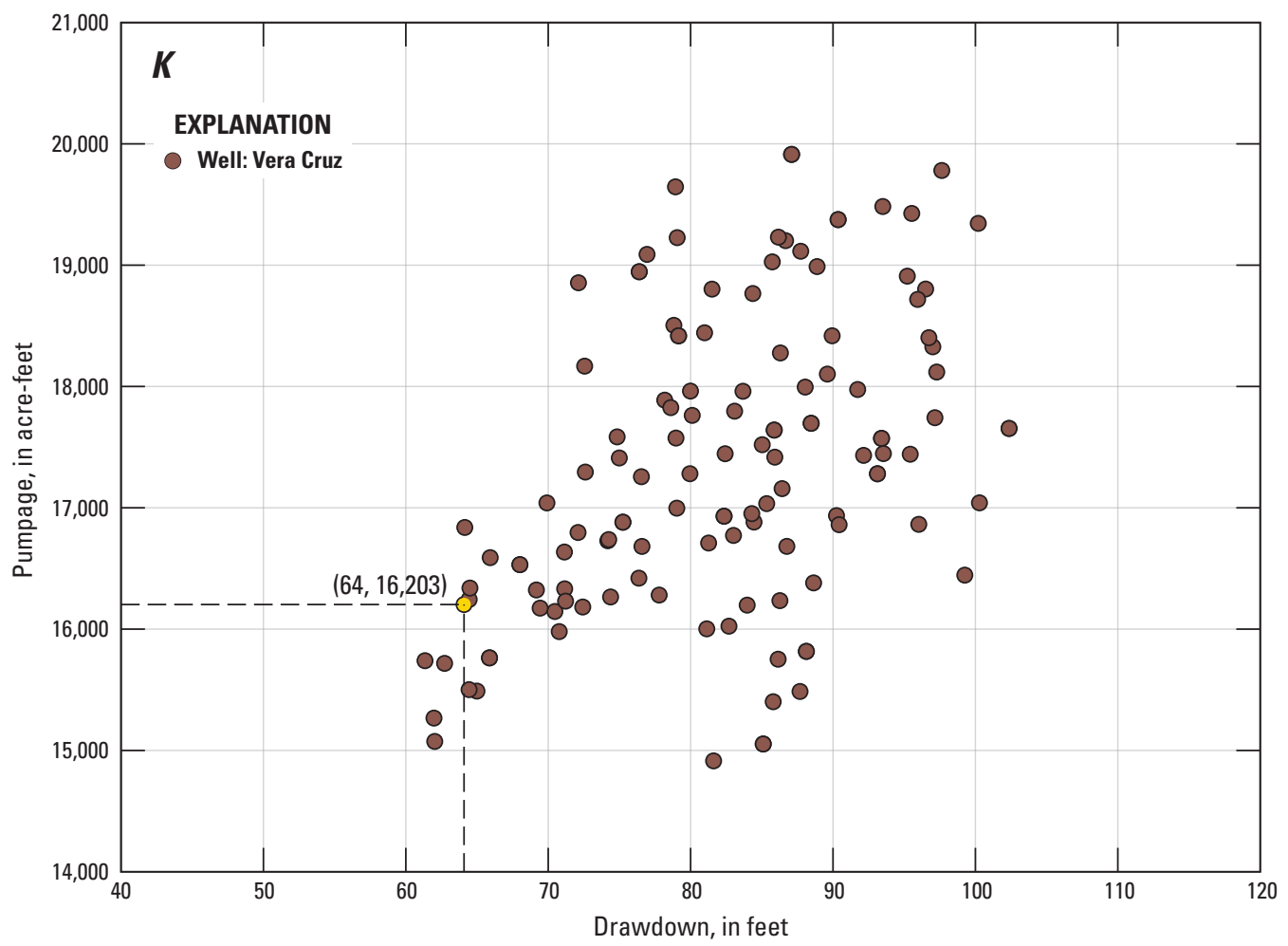

Figure D4-2. - Continued 

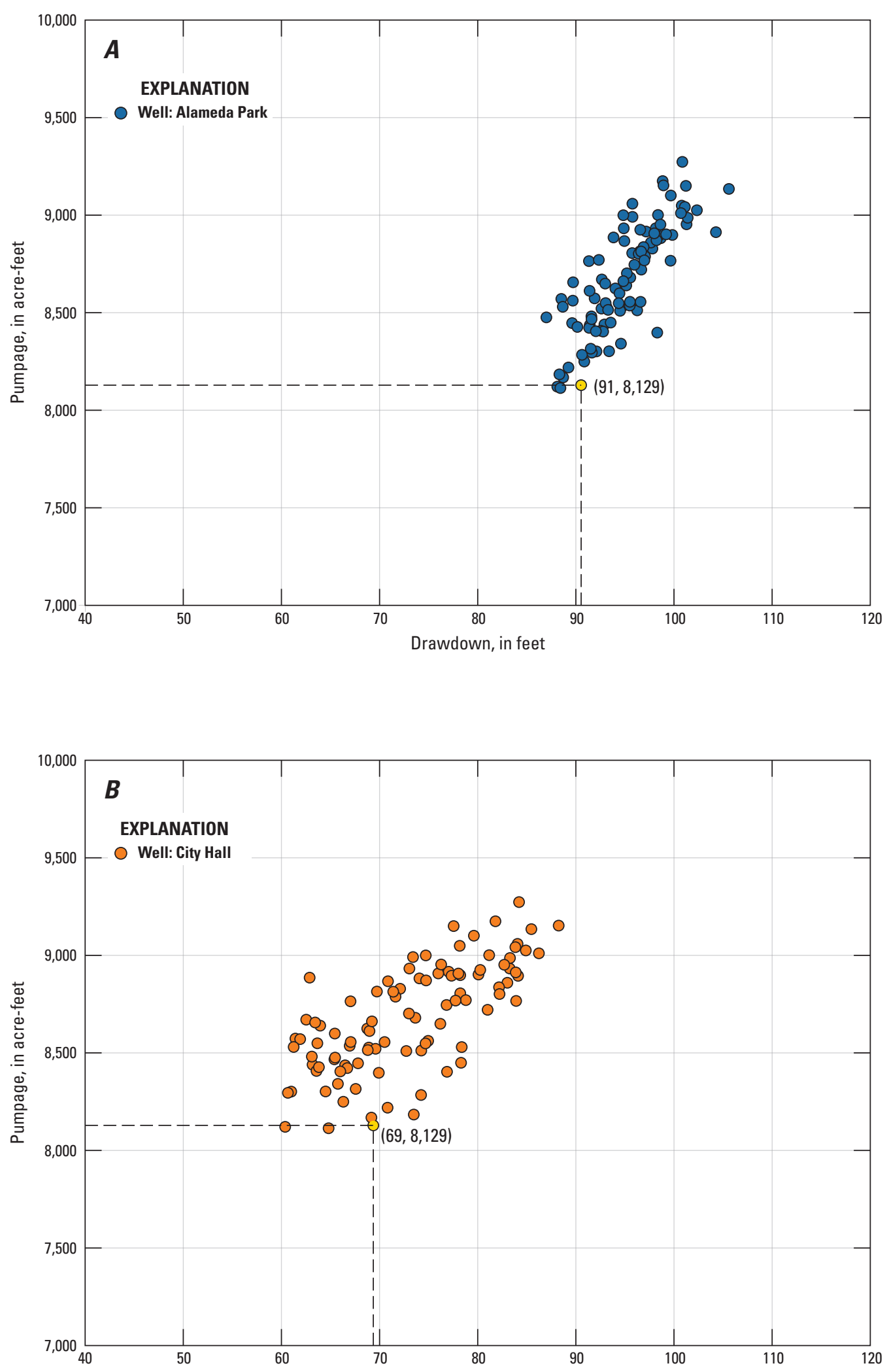

Figure D4-3. Decision rules for total Foothill groundwater basin pumpage as a function of maximum drawdown at the city of Santa Barbara production wells assuming scenario 2 (dry) precipitation, Santa Barbara groundwater basin, Santa Barbara, California (the yellow dot represents the total schedule 2 ${ }_{0}$ pumpage for Foothill groundwater basin), $A$, Alameda Park; $B$, City Hall; C, Corporation Yard; $D$, Hope Avenue; E, Lincolnwood 1; F, Los Robles; G, Ortega Park; H, San Roque Park 2; I, Santa Barbara High School; J, Val Verde; and $K$, Vera Cruz. 

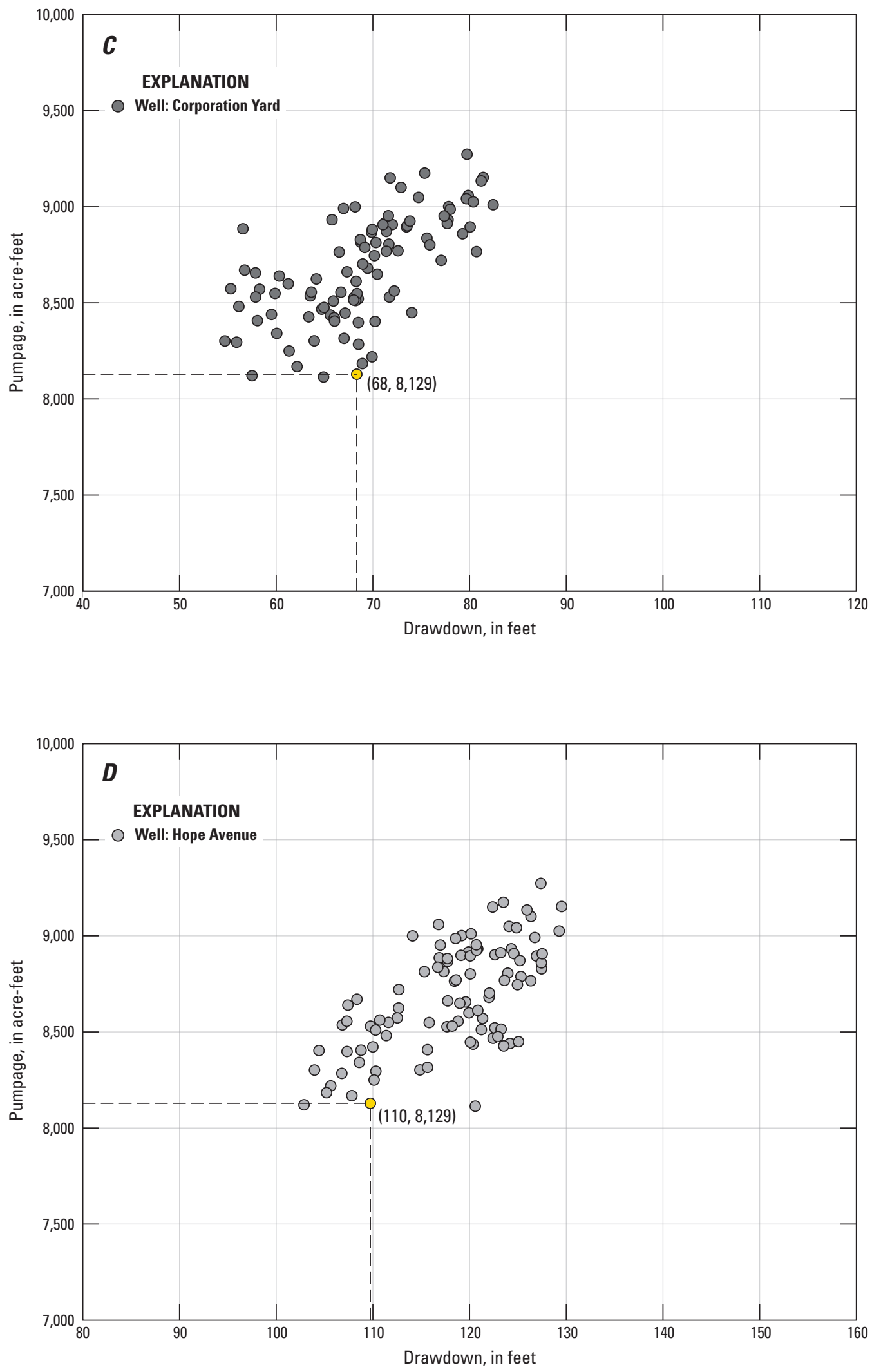

Figure D4-3. - Continued 

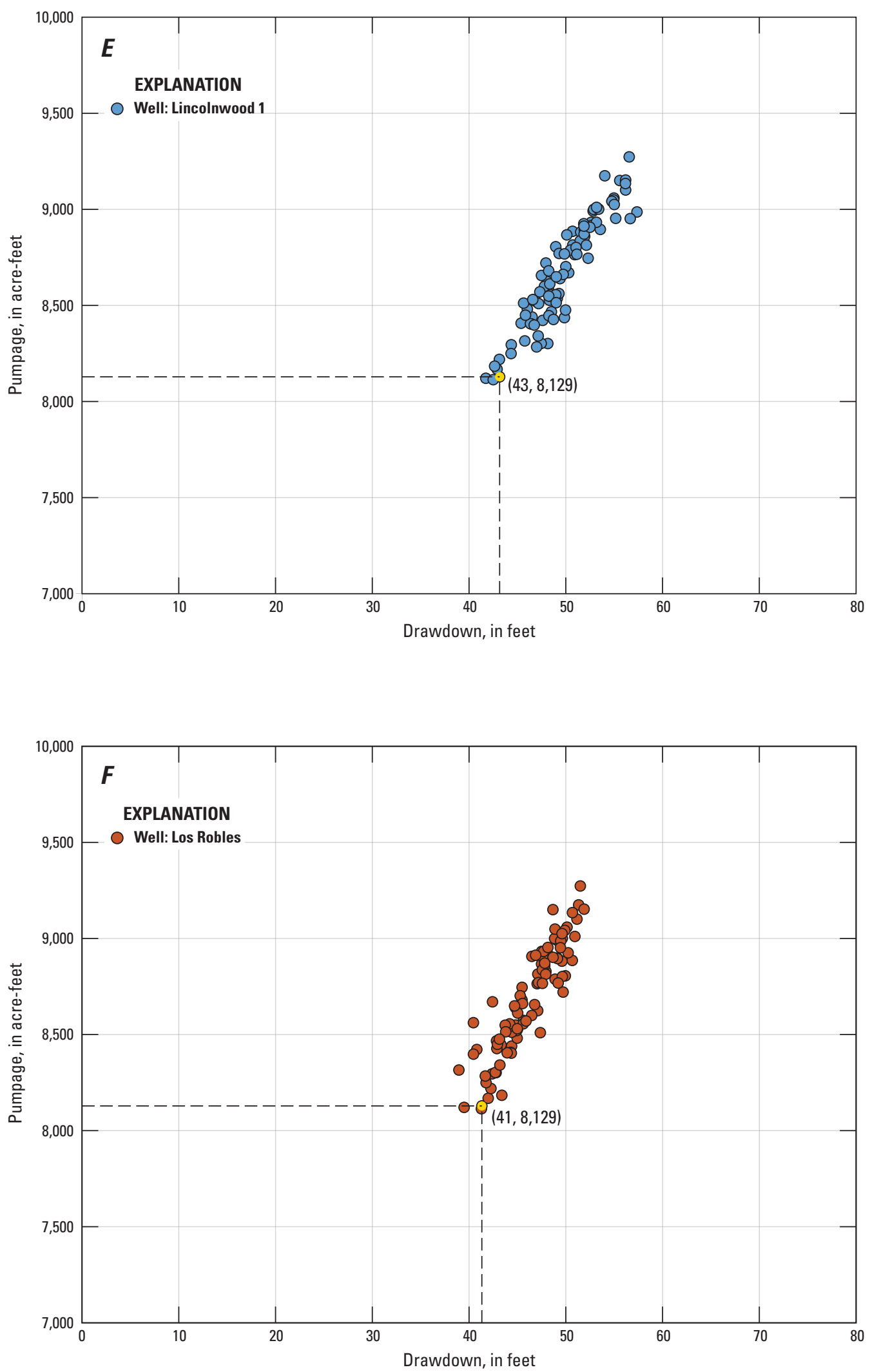

Figure D4-3. - Continued 

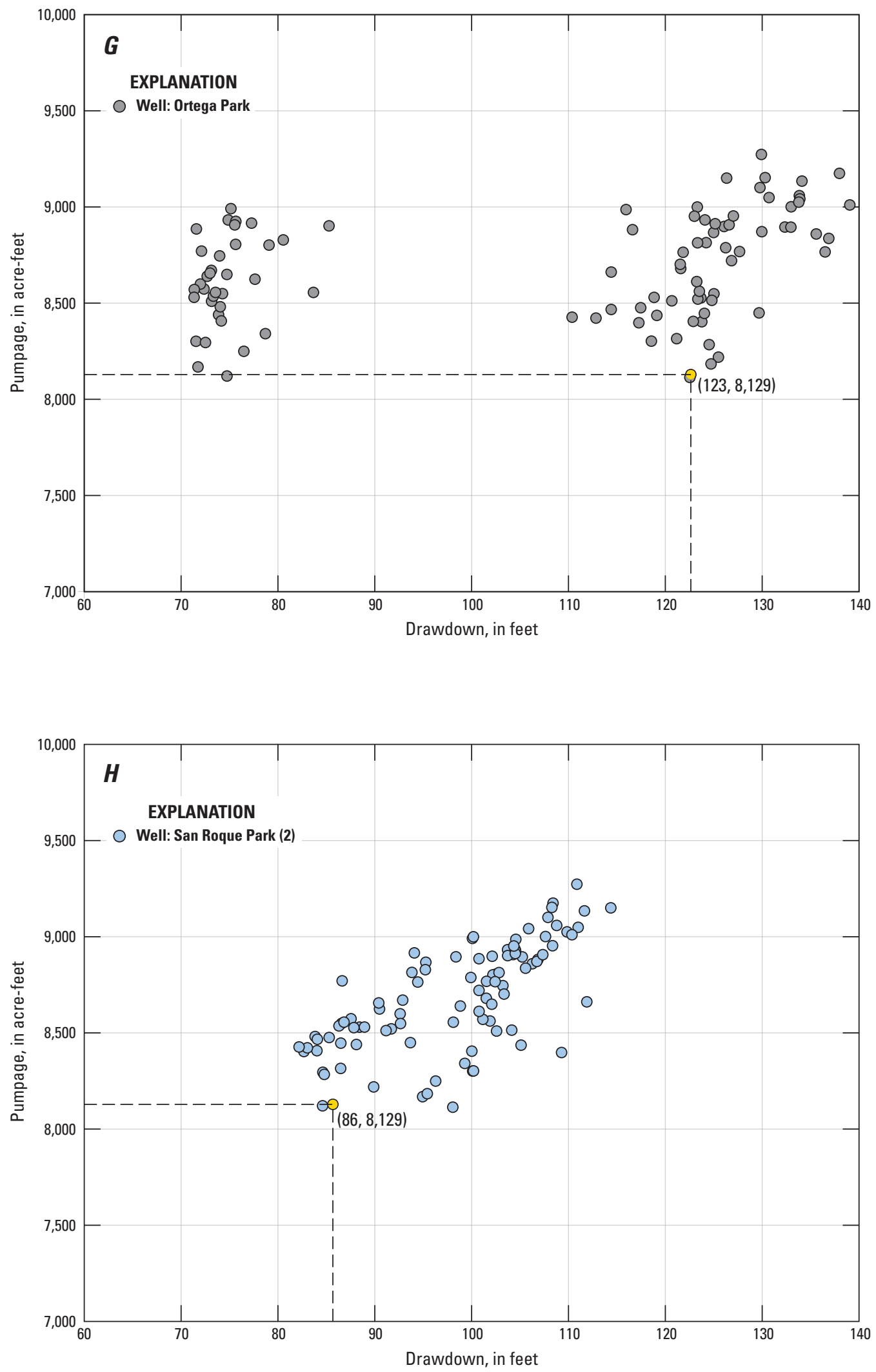

Figure D4-3. - Continued 

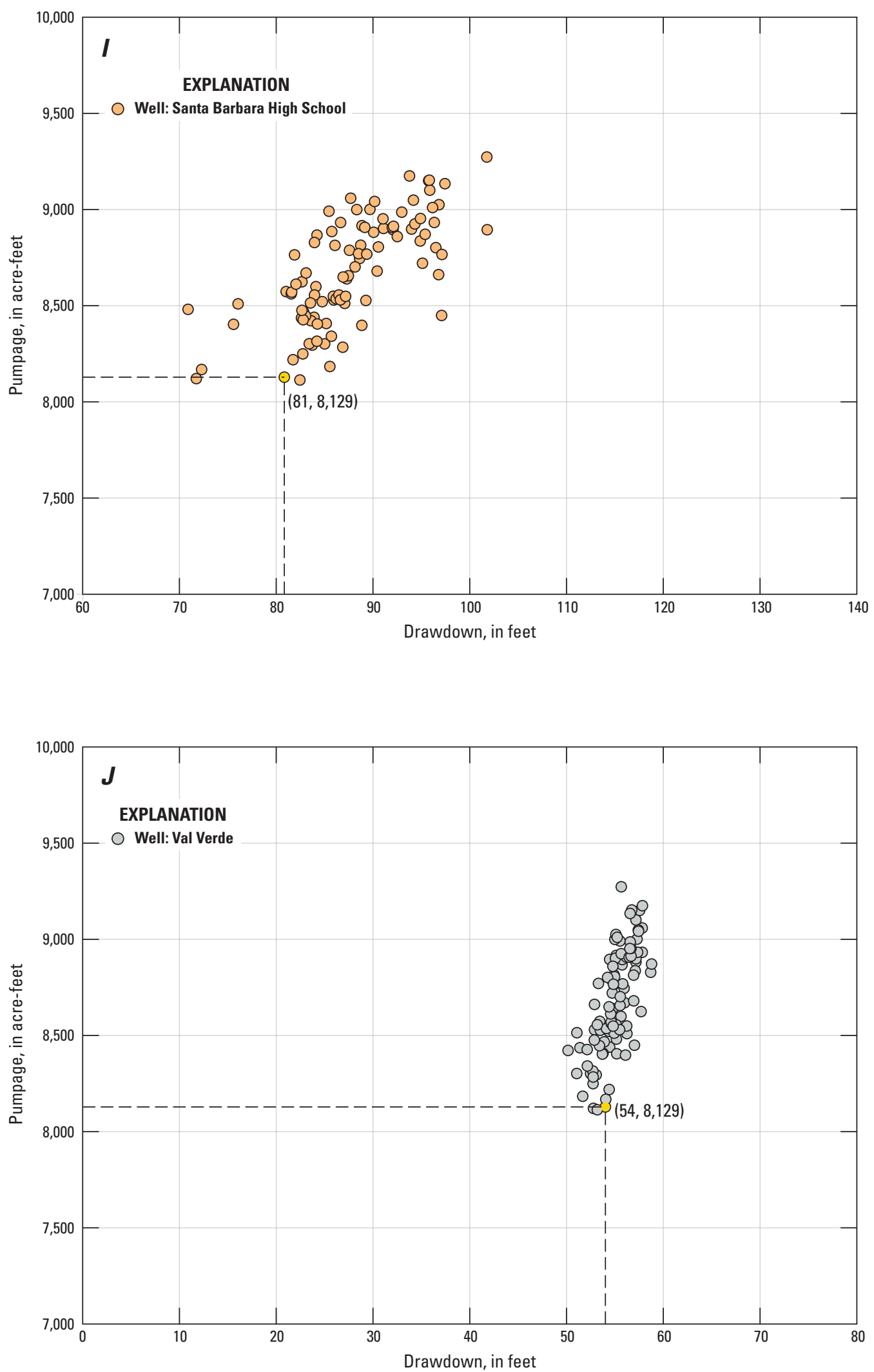

Figure D4-3. - Continued 
378 Santa Barbara and Foothill Groundwater Basins Geohydrology and Optimal Water Resources Management

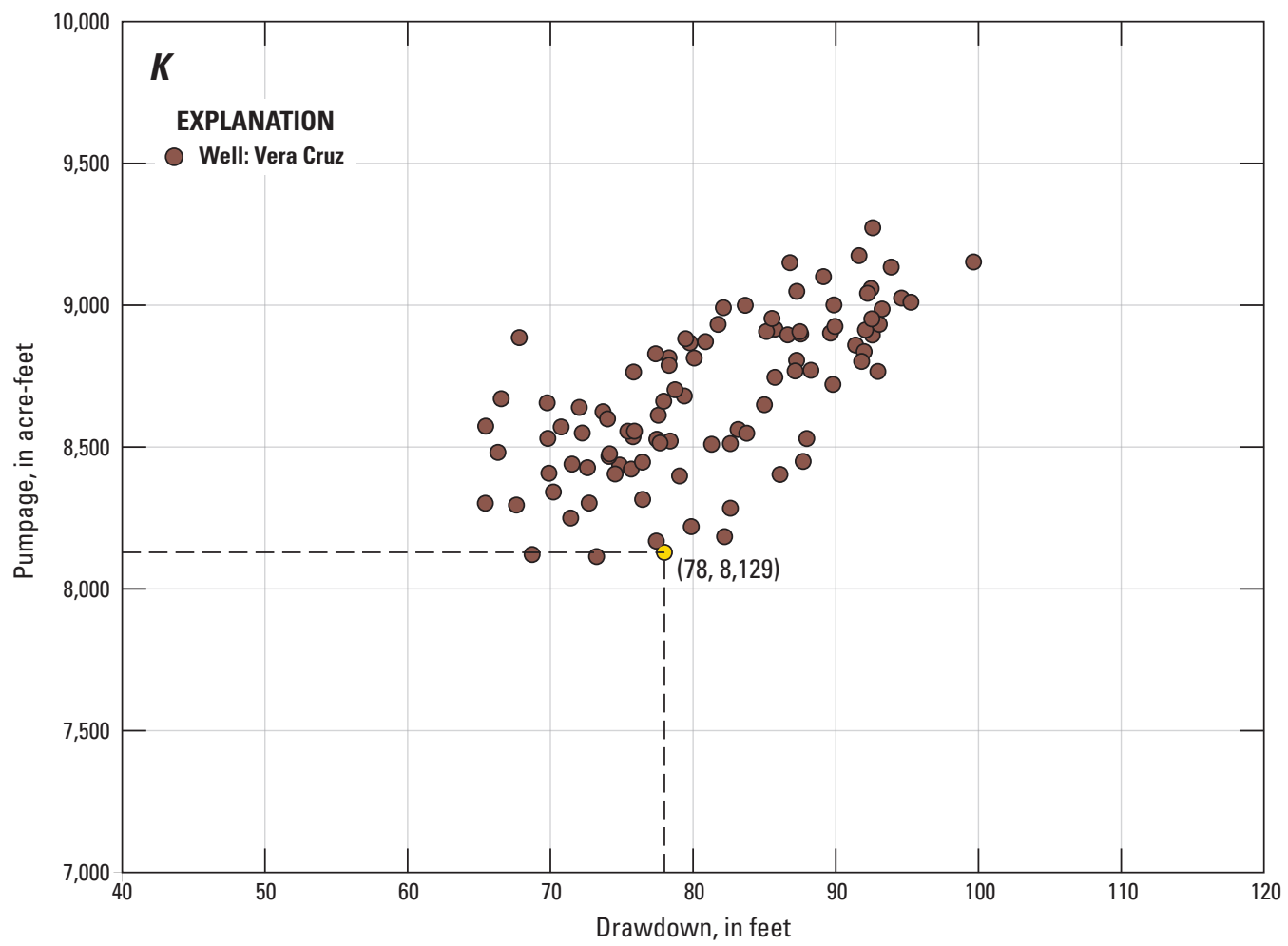

Figure D4-3. - Continued 

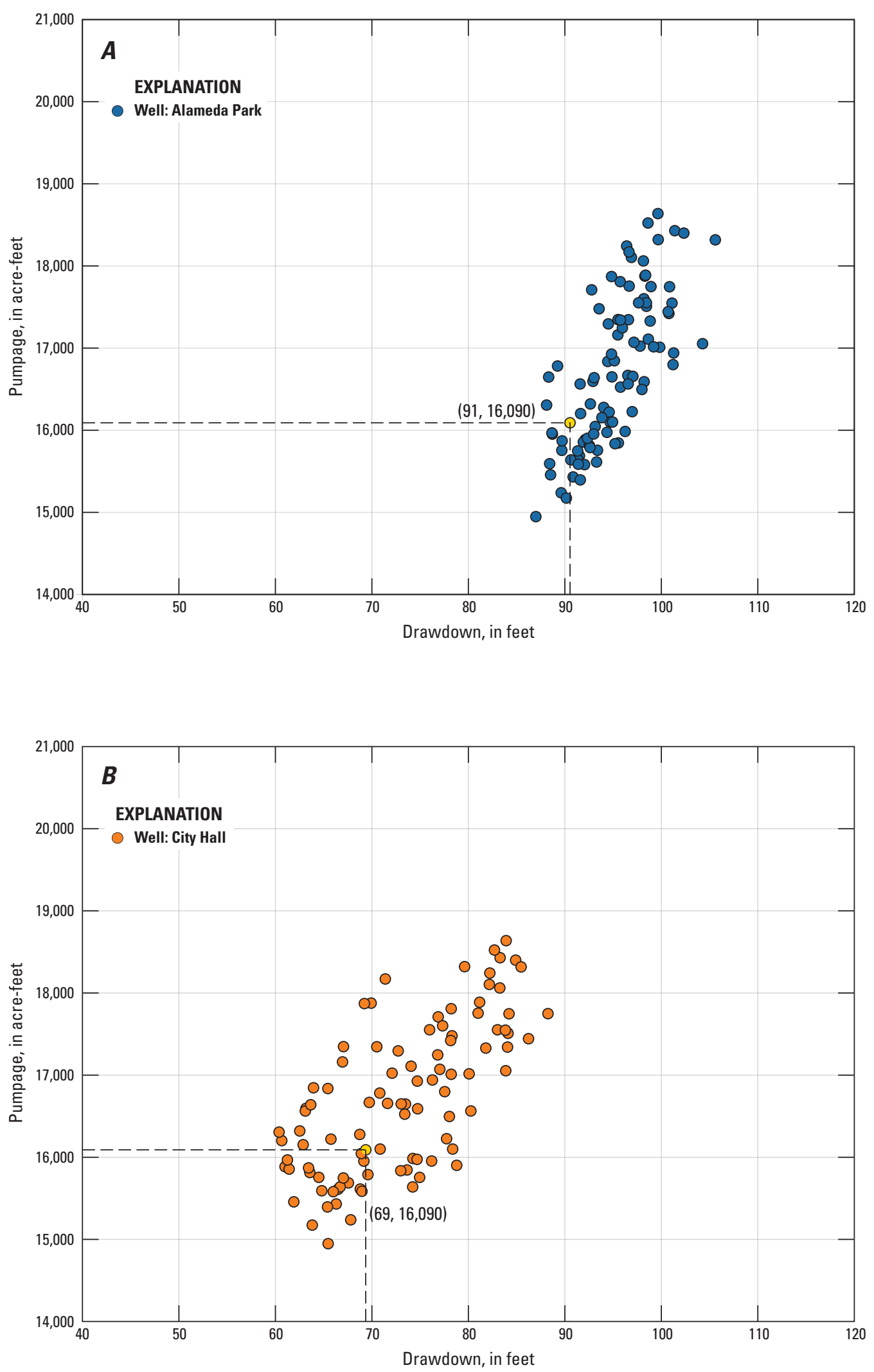

Figure D4-4. Decision rules for total Storage Unit I pumpage as a function of maximum drawdown at the city of Santa Barbara production wells assuming scenario 2 (dry) precipitation, Santa Barbara groundwater basin, Santa Barbara, California (the yellow dot represents the total schedule $2_{D}$ pumpage for Storage Unit I), $A$, Alameda Park; $B$, City Hall; $C$, Corporation Yard; $D$, Hope Avenue; E, Lincolnwood 1; F, Los Robles; G, Ortega Park; H, San Roque Park 2; I, Santa Barbara High School; J, Val Verde; and K, Vera Cruz. 

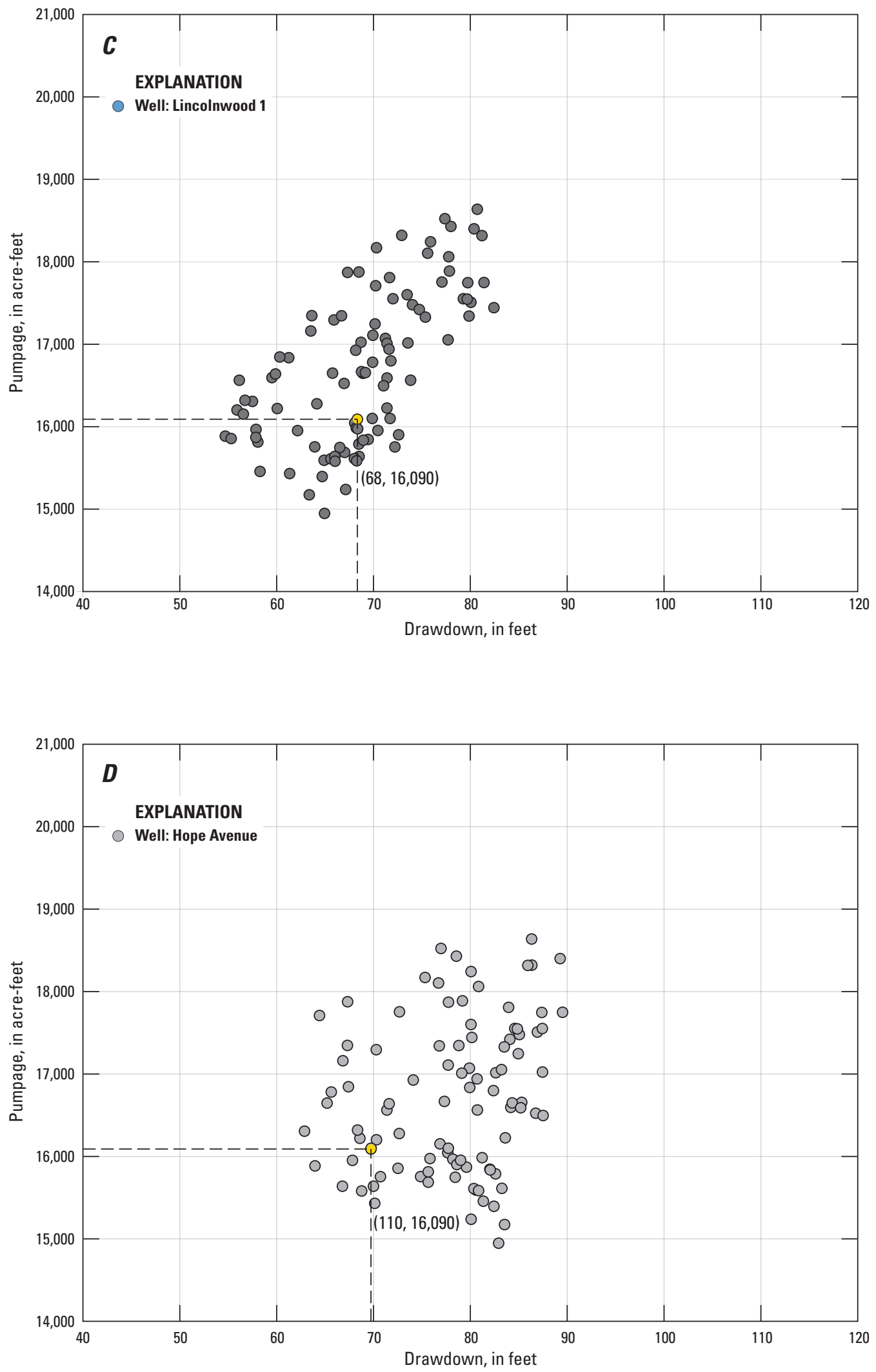

Figure D4-4. - Continued 

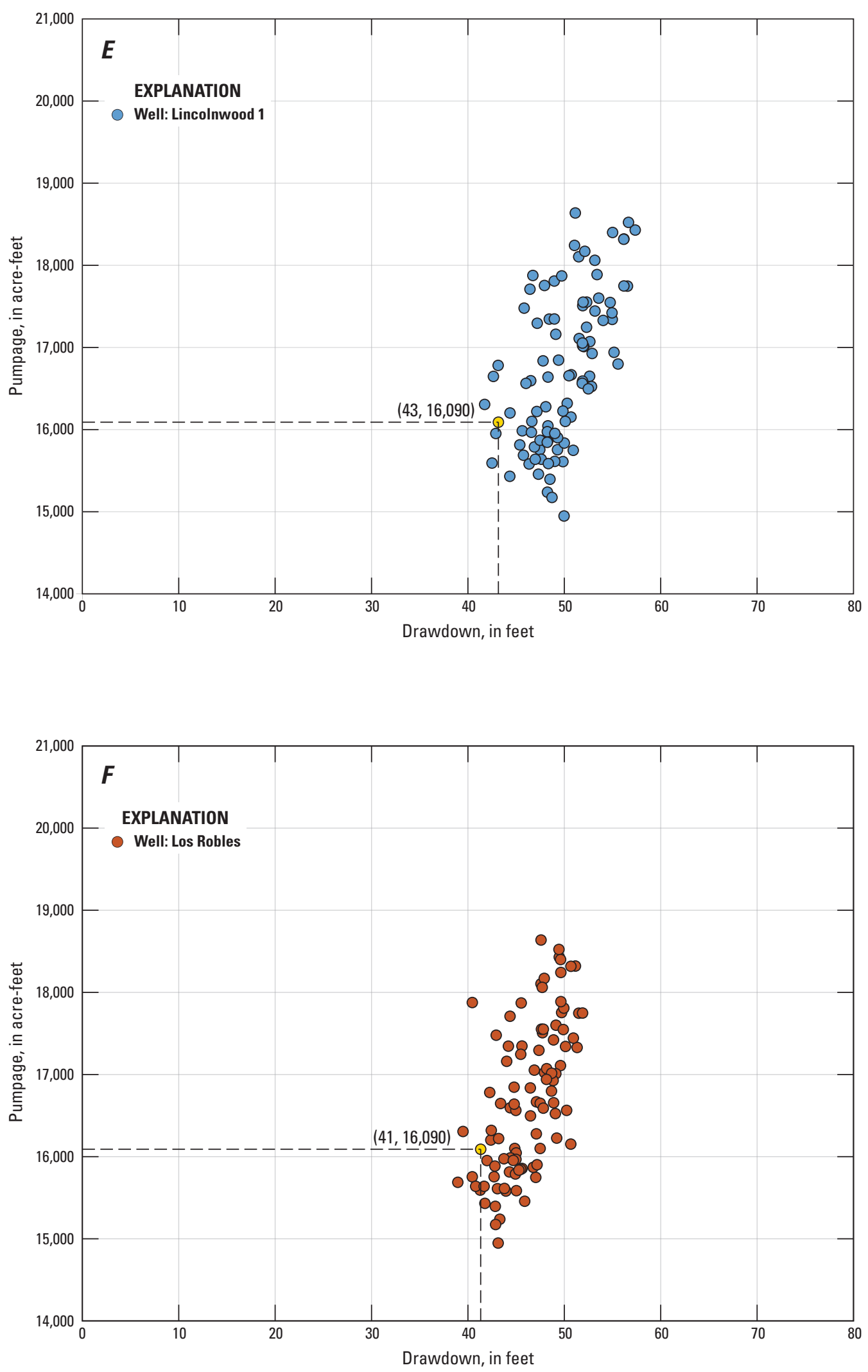

Figure D4-4. - Continued 

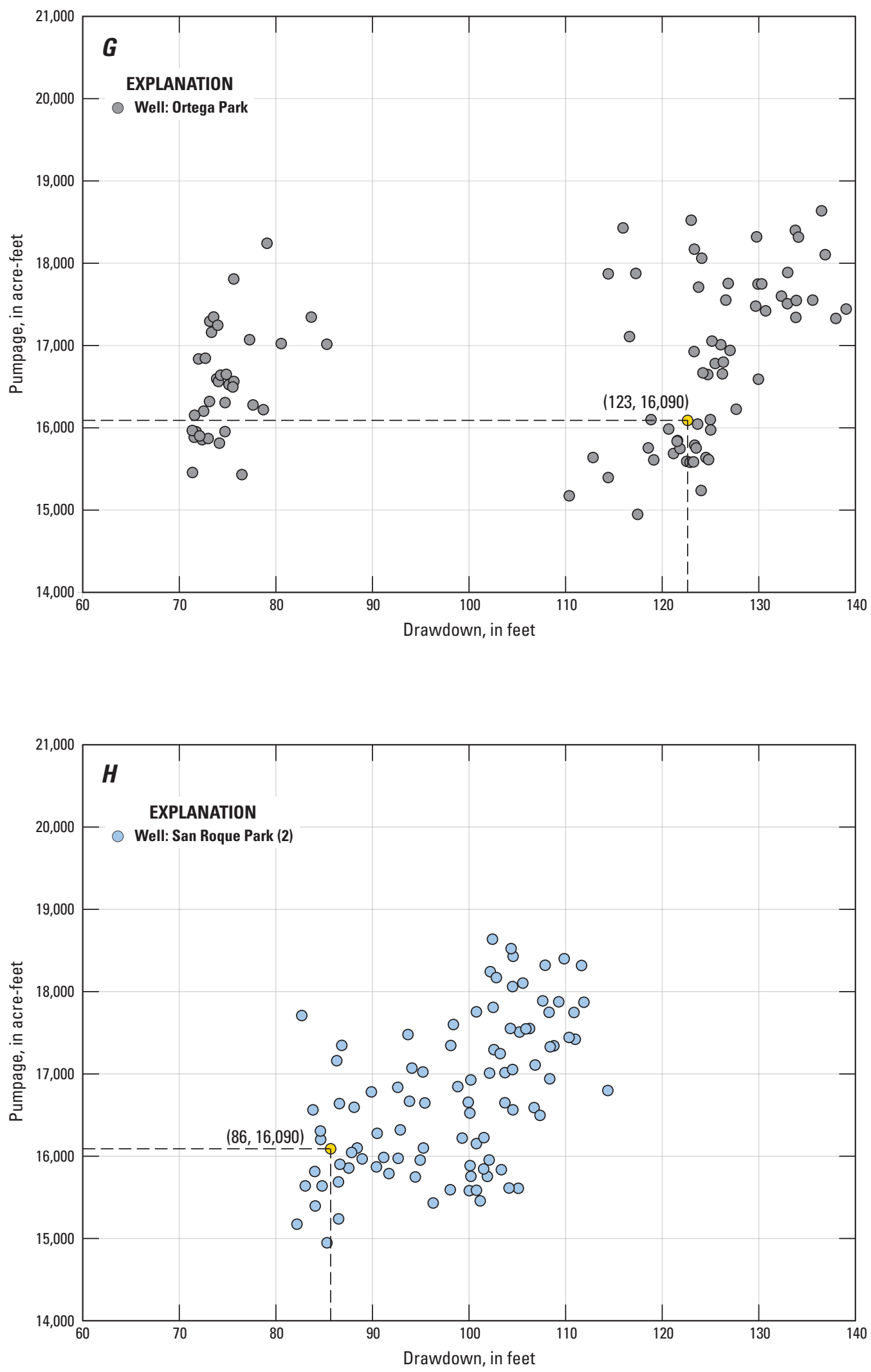

Figure D4-4. - Continued 

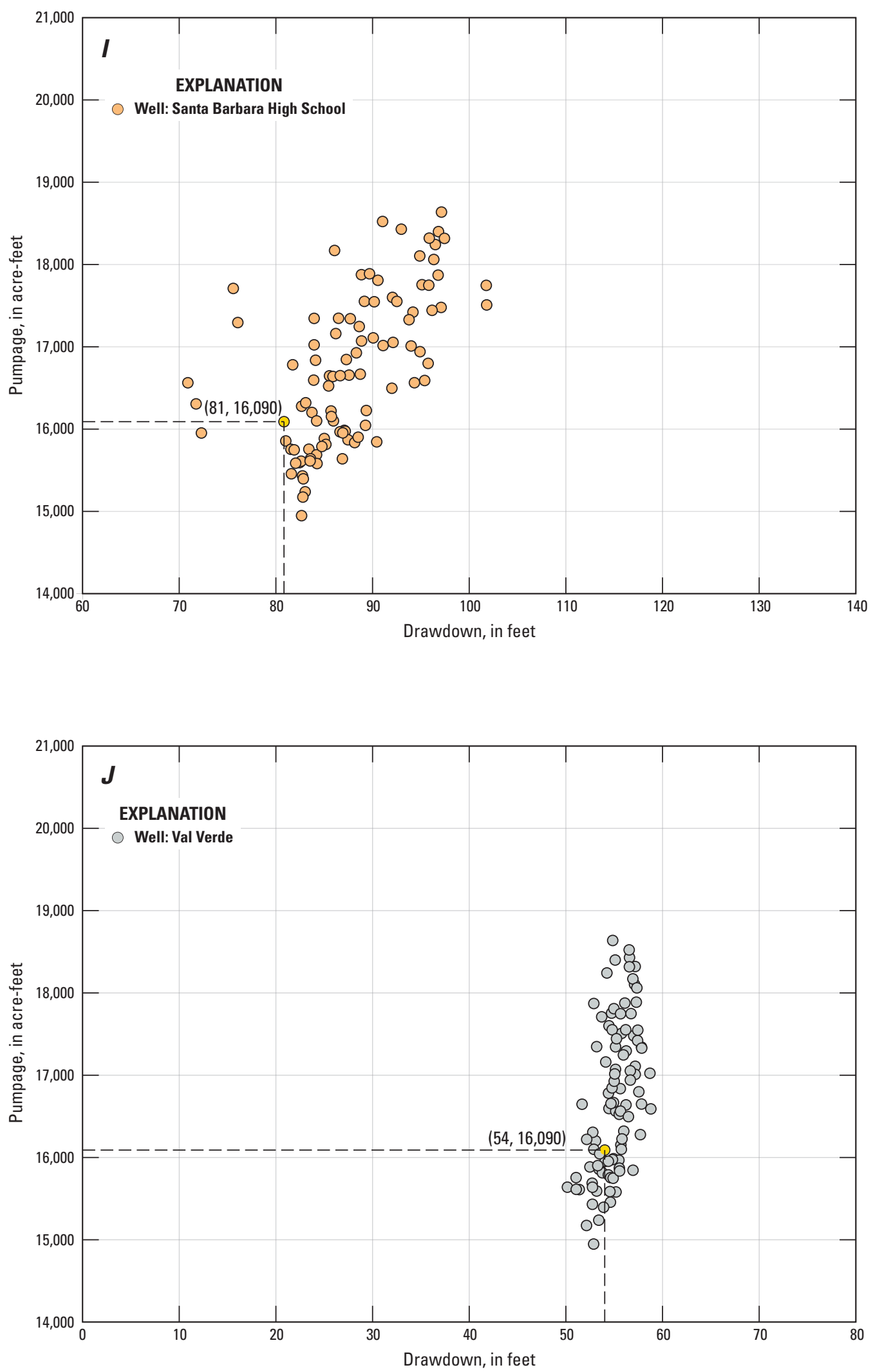

Figure D4-4. - Continued 
384 Santa Barbara and Foothill Groundwater Basins Geohydrology and Optimal Water Resources Management

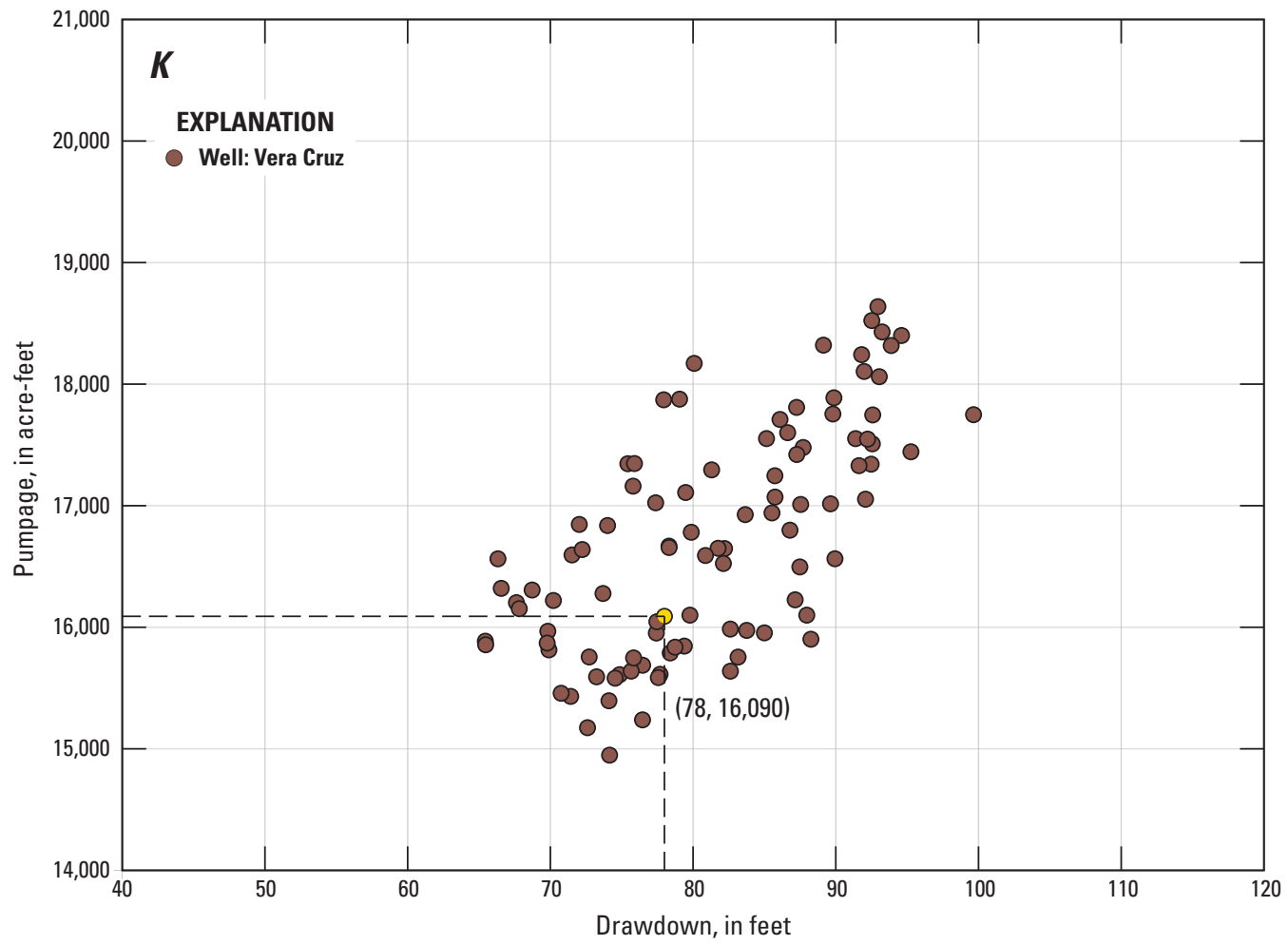

Figure D4-4. - Continued 
Publishing support provided by the U.S. Geological Survey Science Publishing Network, Sacramento Publishing Service Center

For more information concerning the research of this report, contact the Director, California Water Science Center U.S. Geological Survey 6000 J Street, Placer Hall

Sacramento, California 95819

http://ca.water.usgs.gov 


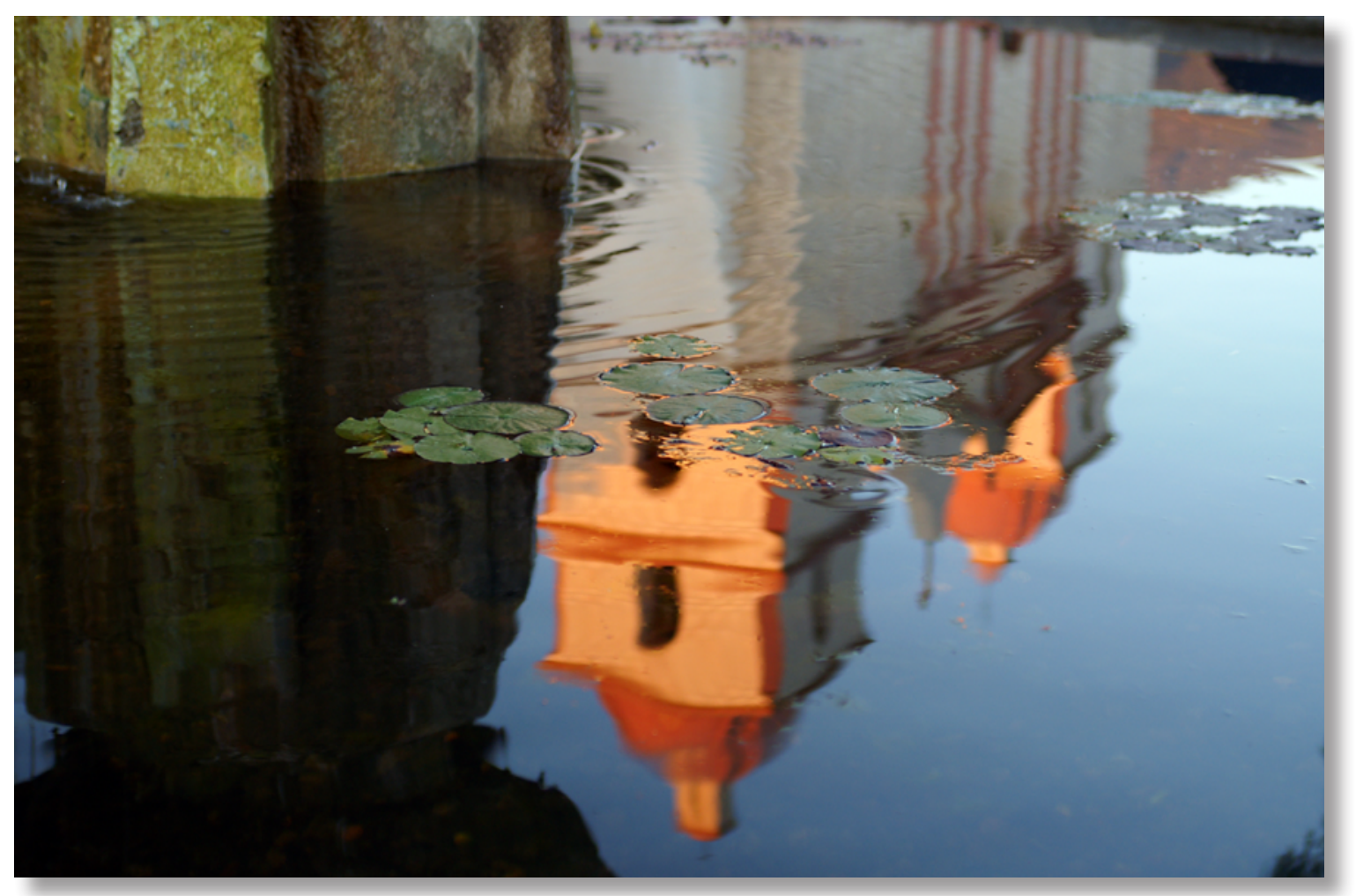

